\title{
Simulation results on the performance of statistical methods in cumulative meta analysis
}

\author{
Elena Kulinskaya and Eung Yaw Mah
}

24 May 2021

\begin{abstract}
Cumulative meta-analysis (CMA) is a process of updating the results of existing meta-analysis to incorporate new study results. This is a popular way to present timevarying evidence. We investigate the properties of CMA, suggest possible improvements and provide the first in-depth simulation study of the use of CMA and CUSUM methods for detection of temporal trends in random-effects meta-analysis. We use the standardized mean difference (SMD) as an effect measure of interest. For CMA, we compare the standard inverse-variance-weighted estimation of the overall effect using REML-estimated of between-study variance $\tau^{2}$ with the sample-size-weighted estimation of the effect combined with Kulinskaya-Dollinger-Bjørkestøl (2011) (KDB) estimation of $\tau^{2}$. For all methods, we consider type 1 error under no shift and power under shift in the mean. To ameliorate the lack of power in CMA, we introduce the two-stage CMA, where the heterogeneity variance $\tau^{2}$ is estimated at stage 1 (first 5-10 studies), and the further CMA monitors a target value of effect, keeping the $\tau^{2}$ value fixed. We recommend the use of this two-stage CMA combined with cumulative testing for positive shift in $\tau^{2}$.
\end{abstract}

\section{Introduction}

Meta-analysis is a statistical methodology that combines estimated effects from multiple studies on the same topic, to arrive at the evidence-based overall effect. It is instrumental in establishing evidence-based practices and in resolving seemingly contradictory research outcomes [Gurevitch et al., 2018]. However, the conduct of 
meta-analysis provides a snapshot of evidence at one time point while the evidence is not static and as it accumulates, new studies often challenge the results of the previous studies. If the evidence changes over time, the conclusions of meta-analysis will strongly depend on when the review was conducted, and any policy-relevant recommendations derived from these reviews will quickly go out of date [Shojania et al., 2007, Gurevitch et al., 2018].

Temporal trends are typically visually explored and often formally detected through cumulative meta-analysis (CMA), originally introduced by Lau et al. [1992]. CMA is a process of updating the results of existing meta-analysis to incorporate new study results. Other available methods for detection of temporal trends are reviewed in Trikalinos and Ioannidis [2005], Kulinskaya and Koricheva [2010] and Koricheva et al. [2013].

It is well understood that repeated testing inherent in CMA results in inflation of type 1 error. This inflation was studied by simulation in Whitehead [1997], Hu et al. [2007], Thorlund et al. [2011] among others, however to our knowledge, it was not systematically quantified. A number of methods addressing this issue are based on the methodology originally developed for sequential clinical trials (Pogue and Yusuf [1997], Brok et al. [2008], Wetterslev et al. [2008], Higgins et al. [2011]). Another approach is to use the quality control methods, in particular CUSUM charts Kulinskaya and Koricheva [2010]. However, the use of random effects model in CMA requires consecutive updating of the between-study variance $\tau^{2}$ as well as the overall effect $\hat{\theta}$, and makes both classes of methods problematic Kulinskaya and Wood [2013], Dogo et al. [2017].

In this paper, we investigate the properties of CMA, suggest possible improvements and provide the in-depth simulation study of the use of CMA and CUSUM methods for detection of temporal trends in random-effects meta-analysis. We use the standardized mean difference (SMD) as an effect measure of interest. For CMA, we consider both the standard inverse-variance-weighted estimation of the overall effect with REML-based estimation of $\tau^{2}$ and a sample-size-weighted (SSW) estimation of $\hat{\theta}$ combined with Kulinskaya-Dollinger-Bjørkestøl (KDB) Kulinskaya et al. [2011] estimation of $\tau^{2}$ recommended by Bakbergenuly et al. [2020]. For all methods, we consider type 1 error under no shift and power under a shift in the mean.

Requisite statistical methods are described in Section 2. Section 3 examines the properties of CMA and identifies the problems due to gross overestimation of 
between-studies variance $\tau^{2}$ resulting from a shift in the mean. Therefore, we suggest cumulative testing of $\tau^{2}$. Also, we propose to modify CMA to two-stage CMA. At stage 1 (first 5 -10 studies), we estimate both effect $\theta$ and $\tau^{2}$, and then we "monitor" either known or this estimated value of effect, keeping the estimated $\tau^{2}$ value fixed. Section 4 includes design and results of our simulations for standard and two-stage CMA methods and for CUSUM charts. Section 5 concludes with discussion.

\section{Preliminaries}

\subsection{Study-level estimation of standardized mean differ- ence}

Consider a meta-analysis of $k$ comparative studies, each consisting of two arms, treatment $(\mathrm{T})$ and control $(\mathrm{C})$, with sample sizes $n_{i T}$ and $n_{i C}$. The total sample size in study $i$ is $n_{i}=n_{i T}+n_{i C}$, and the ratio of the control sample size to the total is denoted by $q_{i}=n_{i C} / n_{i}$. The subject-level data in each arm are assumed to be normally distributed with means $\mu_{i T}$ and $\mu_{i C}$ and equal variances $\sigma_{i}^{2}$. The sample means are $\bar{x}_{i j}$, and the sample variances are $s_{i j}^{2}$, for $i=1, \ldots, k$ and $j=C$ or $T$.

The standardized mean difference (SMD) effect measure is

$$
\delta_{i}=\frac{\mu_{i T}-\mu_{i C}}{\sigma_{i}} .
$$

The unbiased estimator of $\delta_{i}$, sometimes called Hedges' $g$ or Hedges' $d$, is

$$
g_{i}=J\left(m_{i}\right) \frac{\bar{x}_{i T}-\bar{x}_{i C}}{s_{i}},
$$

where $m_{i}=n_{i T}+n_{i C}-2$, and the factor $J(m)=\Gamma\left(\frac{m}{2}\right) / \sqrt{\frac{m}{2}} \Gamma\left(\frac{m-1}{2}\right)$, often approximated by $1-3 /(4 m-1)$, corrects for bias [Hedges, 1983]. The standard deviation, $\sigma_{i}$ is estimated by the square root of the pooled sample variance

$$
s_{i}^{2}=\frac{\left(n_{i T}-1\right) s_{i T}^{2}+\left(n_{i C}-1\right) s_{i C}^{2}}{n_{i T}+n_{i C}-2} .
$$

The variance of $g_{i}$ is $\operatorname{Var}\left(g_{i}\right)=\tilde{n}_{i}^{-1}+\delta^{2} / 2\left(n_{i T}+n_{i C}\right)$, where $\tilde{n}_{i}=n_{i T} n_{i C} /\left(n_{i T}+n_{i C}\right)$ is the effective sample size in study $i$. The unbiased estimator of this variance is Hedges [1983]

$$
v_{i}^{2}=\frac{n_{i T}+n_{i C}}{n_{i T} n_{i C}}+\left(1-\frac{\left(m_{i}-2\right)}{m_{i} J\left(m_{i}\right)^{2}}\right) g_{i}^{2} .
$$


The sample SMD $g_{i}$ has a scaled non-central $t$-distribution with non-centrality parameter $\left[n_{i} q_{i}\left(1-q_{i}\right)\right]^{1 / 2} \delta_{i}$ :

$$
\frac{\sqrt{n_{i} q_{i}\left(1-q_{i}\right)}}{J\left(m_{i}\right)} g_{i} \sim t_{m_{i}}\left(\left[n_{i} q_{i}\left(1-q_{i}\right)\right]^{1 / 2} \delta_{i}\right) .
$$

\subsection{Standard random-effects model}

In meta-analysis of $k$ studies, the standard random-effects model assumes approximately normal distributions of within- and between-study effects. For a generic measure of effect,

$$
\hat{\theta}_{i} \sim N\left(\theta_{i}, \sigma_{i}^{2}\right) \text { and } \theta_{i} \sim N\left(\theta, \tau^{2}\right),
$$

resulting in the marginal distribution $\hat{\theta}_{i} \sim N\left(\theta, \sigma_{i}^{2}+\tau^{2}\right)$. $\hat{\theta}_{i}$ is the estimate of the effect in Study $i$, and its within-study variance is $\sigma_{i}^{2}$, estimated by $\hat{\sigma}_{i}^{2}, i=1, \ldots, k$. $\tau_{k}^{2}$ is the between-study variance, which is estimated (from $k$ studies) by $\hat{\tau}_{k}^{2}$. The overall effect $\theta=\theta_{(k)}$ can be estimated by the weighted mean

$$
\hat{\theta}_{I V,(k)}=\frac{\sum_{i=1}^{k} \hat{w}_{i}\left(\hat{\tau}_{k}^{2}\right) \hat{\theta}_{i}}{\sum_{i=1}^{k} \hat{w}_{i}\left(\hat{\tau}_{k}^{2}\right)},
$$

where the $\hat{w}_{i}\left(\hat{\tau}_{k}^{2}\right)=\left(\hat{\sigma}_{i}^{2}+\hat{\tau}^{2}\right)^{-1}$ are inverse-variance (IV) weights. The fixed effect (FE) model assumes that $\tau^{2} \equiv 0$, and estimate $\hat{\theta}_{k}$ uses IV weights $\hat{w}_{i}=\hat{w}_{i}(0)$.

The variance of $\hat{\theta}_{I V}$ is routinely estimated by

$$
\operatorname{Var}\left(\hat{\theta}_{I V,(k)}\right)=\left[\sum_{i=1}^{k} \hat{w}_{i}\left(\hat{\tau}_{k}^{2}\right)\right]^{-1} .
$$

The standard confidence interval for the overall effect $\hat{\theta}_{I V,(k)}$ uses the IV point estimator as its center, and its half-width equals the estimated standard deviation (square root of the variance (2.6)) times the critical value from the normal distribution.

\subsection{Point and interval estimation of $\tau^{2}$.}

Because the $\hat{w}_{i}\left(\hat{\tau}^{2}\right)$ in Equation (2.5) involve the estimated value of between-study variance $\hat{\tau}^{2}$, we need to choose an estimator of $\tau^{2}$. It is well known, that the maximum likelihood estimator of $\tau^{2}$ is biased, and the restricted maximum likelihood (REML) estimator is a good choice Viechtbauer [2005]. We use $\hat{\tau}_{R E M L}^{2}$ in the IV estimation of $\theta$ and the Q-profile (QP) method Viechtbauer [2007] for accompanying interval 
estimation of $\tau^{2}$. For SMD, this method is one of the best traditional methods available for this purpose Bakbergenuly et al. [2020].

Several alternative estimators of $\tau^{2}$ are based on the moments of Cochran's Q statistic Cochran [1954]. This statistic is often assumed to have a chi-square distribution with $k-1$ degrees of freedom, and its expected value is then approximated by $k-1$. However, this is an adequate approximation for the expected value of Cochran's $Q$ statistic only for very large sample sizes.

For SMD, Kulinskaya et al. [2011] derived $O(1 / n)$ corrections to moments of $Q$. Bakbergenuly et al. [2020] proposed an estimator of $\tau^{2}, \hat{\tau}_{K D B}^{2}$ based on this improved approximation.

An accompanying KDB confidence interval for $\tau^{2}$ combines the Q-profile approach and the improved approximation by Kulinskaya et al. [2011] (i.e., the chi-squared distribution with fractional degrees of freedom based on the corrected first moment of $Q$ ). Bakbergenuly et al. [2020] demonstrated by simulation that $\hat{\tau}_{K D B}^{2}$ is less biased than REML for small sample sizes $(n<100)$, especially for $k \geq 10$, and both estimators become almost unbiased from $n=100$.

There is an evident relationship between a confidence interval for $\tau^{2}$, and a test of the null hypothesis $\tau^{2}=\tau_{0}^{2}$. Values of $\tau_{0}^{2}$ beyond the confidence limits are rejected by a two-sided test, and values above the upper bound of a one-sided interval $(0, u)$ are rejected by a one-sided test.We shall refer to these tests as QP and KDB.

\subsection{Point and interval estimators of $\delta$}

Traditional CMA based on REML uses $\hat{\tau}_{R E M L}^{2}$ in $\hat{\theta}_{I V, k}$ (2.5) and in its variance (2.6), in combination with the critical values from the normal distribution. We refer to this method as IV REML.

For SMD, estimated effects $\hat{\delta}_{i}(2.1)$ and their estimated variances $v_{i}^{2}(2.2)$ are not independent. Because of this, the IV estimates of the overall effects $\hat{\theta}_{I V, k}$ are biased. This bias is eliminated by the use of non-random weights. The use of effective sample size weights (SSW) for estimation of $\theta$ suggested by [Hedges and Olkin, 1985, p.110] was explored and found superior to IV weights in a comprehensive simulation study by Bakbergenuly et al. [2020].

These weights depend only on the studies' sample sizes $w_{i}=\tilde{n}_{i}=n_{i T} n_{i C} /\left(n_{i T}+\right.$ $\left.n_{i C}\right) ; \tilde{n}_{i}$ is the effective sample size in study $i$. They would coincide with the inversevariance weights when $\delta=0$. We refer to the estimator of $\delta$ of the form (2.5) with 
these weights as SSW.

The interval estimator corresponding to SSW (SSW KDB) uses the SSW point estimator as its center, and its half-width equals the estimated standard deviation of SSW under the random-effects model times the critical value from the $t$ distribution on $k-1$ degrees of freedom. The estimator of the variance of SSW is

$$
\widehat{\operatorname{Var}}\left(\hat{\delta}_{S S W}\right)=\frac{\sum \tilde{n}_{i}^{2}\left(v_{i}^{2}+\hat{\tau}^{2}\right)}{\left(\sum \tilde{n}_{i}\right)^{2}}
$$

in which $v_{i}^{2}$ comes from Equation $(2.2)$ and $\hat{\tau}^{2}=\hat{\tau}_{K D B}^{2}$.

Once more, we shall refer to the tests of the hypothesis $\delta=\delta_{0}$ based on the respective confidence intervals as IV REML and SSW KDB.

\section{Estimation and testing in CMA}

Consider a simple shift-in-the-mean $\left(\right.$ at $\left.k_{1}+1\right)$ random effects model for study-level effects:

$$
\hat{\delta}_{i} \sim G\left(\delta_{i}+\Delta I\left(i>k_{1}\right), \sigma_{i}^{2}\right), \text { and } \delta_{i} \sim N\left(\delta, \tau^{2}\right), i=1, \cdots, K,
$$

where $I\left(i>k_{1}\right)$ is the $0 / 1$ indicator for some $k_{1}<K$. In the shift-in-the-mean model (3.1), true SMD shifts from $\delta$ in the first $k_{1}$ observations to $\delta+\Delta$ in observations $k_{1}+1, \cdots, K$. The standard REM (2.4) is a particular case when $k_{1}>K$ (and the distribution $G(\cdot)$ is normal).

In this section, we consider some general CMA patterns under this model and suggest several approaches to testing.

\subsection{Estimation of $\delta$}

In cumulative meta-analysis with IV weights, rolling overall mean effect $\delta_{(k)}$ is reestimated by $\hat{\delta}_{k}(2.5)$ whenever the number of studies $k$ increases.

Assume that the weights $w_{i}$ and estimated effects $\hat{\delta}_{i}$ are independent. If there is no shift in $\delta$, the cumulative mean $\delta_{(k)} \equiv \delta$. However, if there is a shift in $\delta$ at study $k_{1}+1$, the expected value of CMA estimator $\hat{\delta}_{(k)}$ given by equation (2.5) becomes a weighted average of $\delta$ and $\delta+\Delta$ values.

Given a set of weights $w_{i}, i=1, \cdots, K$, and denoting the running sum of weights $W_{k}=\sum_{1}^{k} w_{j}$, the cumulative mean effect for $k \leq k_{1}$ studies is $\delta$, and for $k \geq k_{1}$ studies it is

$$
\delta_{(k)}=\delta+\Delta\left(W_{k}-W_{k_{1}}\right) / W_{k}
$$


Consider, for simplicity, that the sample sizes are equal $n_{i C}+n_{i T}=n / 2$, so that effective sample sizes $\tilde{n}_{i} \equiv n / 4$. Thus, the SSW estimator uses equal weights $w_{i}=$ $n / 4$, and the rolling CMA mean $\delta_{S S W,(k)}=\delta+\Delta\left(1-k_{1} / k\right)$ increases from $\delta$ at $k=k_{1}$ to $\delta_{1}+\Delta$ at $\infty$.

For SMD, the within-study variances are $\operatorname{Var}\left(g_{i}\right)=4 / n+\delta^{2} / 2 n$. Under no shift in $\delta$ or $\tau^{2}$ scenario, the population IV weights should be equal. Given a positive shift in the mean from $\delta$ to $\delta+\Delta$, the variances of $g_{i}, i \geq k_{1}+1$ also increase, and the weights are reduced accordingly. Therefore, the (nominal) cumulative mean effect (3.2) will be reduced for the case of IV weights as compared to SSW. This may reduce the power of the CMA based on the IV weights. Figures in Appendix E1 illustrate changes in $\delta_{S S W,(k)}$ and in $\delta_{I V,(k)}$ under the equal sample sizes scenario. The difference between the two cumulative mean effects is the largest when $\tau^{2}=0$ but it reduces in $\tau^{2}$ and for $\tau^{2}>0$ it also quickly reduces with $n$, so that it is negligible at $n=100$.

However, the estimated variances $v_{i}^{2}$ given by equation (2.2), and therefore, the IV weights are random variables and are not exactly equal. Additionally, these random weights are not independent from the estimated effects $g_{i}$, resulting in the bias of the estimated cumulative mean effect $\hat{\delta}_{I V,(k)}$ even under no shift scenario as we demonstrate in Section 4.4. This order $1 / n$ bias in the nominal rolling cumulative mean is neglected if using (3.2) for SMD. See Appendices C1 and D1 for illustration.

\subsection{Estimation of $\tau^{2}$}

Consider the same simple scenario of equal sample sizes and a shift in the mean from $\delta$ to $\delta+\Delta$ at study $k_{1}+1$ given by (3.1). This shift in the mean effect results in a positive shift in estimated value of $\tau^{2}$ in CMA. It is possible to show that the value of moment estimators of $\tau^{2}$ such as the popular DerSimonian and Laird [1986] (DL) estimator, this shift is proportionate to $\Delta^{2}$ and it does not depend on the sample size.

In our simulation study, we do not use DL, but both the KDB and the popular Paule-Mandel estimator Paule and Mandel [1982] are also based on the first moment of $Q_{(k)}$, and we expect similar behavior from these estimators. REML is not a moment estimator, but our simulation results discussed in Section 4.3 demonstrate this behavior for both KDB and REML. 


\subsection{Testing for shift-in-the-mean in CMA}

Consider testing for a shift at $k$ in the model (3.1) based on the cumulative estimates $\left(\hat{\delta}_{(k)}, \hat{\tau}_{(k)}^{2}\right)$. The standard CMA tests $H_{0}: \delta_{(k)}=\delta_{0}$ against $\delta_{(k)} \neq \delta_{0}$ for a known value of $\delta_{0}$. We consider two of these tests, described at the end of Section 2, IV REML and SSW KDB. As discussed in Section 3.2, the cumulative estimate $\hat{\tau}_{(k)}^{2}$ increases with the shift-in-the-mean. Because of this, we also consider two tests, QP and $\mathrm{KDB}$, of $\left.\tau_{(k)}^{2}\right)=\tau_{0}^{2}$ vs $\tau_{(k)}^{2}>\tau_{0}^{2}$ for a known value of $\tau_{0}^{2}$ as possible tests for shift.

From the findings in Section 3.2, it also follows that for a test of a shift based on $\hat{\delta}_{(k)}$, the accompanying increase in $\hat{\tau}_{(k)}^{2}$ may decrease its power. Because of this, we consider the following modification to traditional CMA which we refer to as the two-stage CMA.

Borrowing the quality control concept of two-stage monitoring, in stage 1 we test for $\delta_{(k)}=\delta_{0}$ for $k=5, \cdots, 10$ using IV REML or SSW KDB. If rejected at $k_{1}+1$, we take $\hat{\tau}_{k_{1}}^{2}$ (estimated by REML or KDB, respectively) as the "true" value $\tau_{0}^{2}$, otherwise we take $\tau_{0}^{2}=\hat{\tau}_{(10)}^{2}$. The rational for this choice is that the estimate of $\tau^{2}$ is reasonably accurate by $k=10$ under no shift, and we do not want to use an inflated value of $\tau^{2}$ in the case of an early shift.

In stage 2 , we monitor the (known) mean effect $\delta_{0}$ using the $\tau_{0}^{2}$ value as known between-study variance $\tau_{(k)}^{2}$ without its further re-estimation. We refer to respective tests and estimators as $\operatorname{IV} \operatorname{REML}\left(\tau_{0}^{2}\right)$ and $\operatorname{SSW} \operatorname{KDB}\left(\tau_{0}^{2}\right)$. We also consider a more realistic scenario of monitoring estimated in stage 1 value of $\delta$ (estimated as described above at $k_{1}+1$ or at $k=10$ ) instead of the "known" $\delta_{0}$. These tests are referred to as IV $\operatorname{REML}(\hat{\delta})$ and $\operatorname{SSW} \operatorname{KDB}(\hat{\delta})$ when further testing proceeds as in the standard $\operatorname{CMA}$, and IV $\operatorname{REML}\left(\hat{\delta}, \tau_{0}^{2}\right) / \operatorname{SSW} \operatorname{KDB}\left(\hat{\delta}, \tau_{0}^{2}\right.$.) when using also estimated $\tau_{0}^{2}$.

For comparison, we also use two-stage CUSUM charts to monitor the known mean $\delta_{0}$ for the effects $\hat{\delta}_{i}$ with variances $v_{i}^{2}+\tau_{0}^{2}$.

\section{Simulation study}

\subsection{Simulation design}

In the majority of our simulations we use $K=50$ as the maximum number of studies, though in some scenarios we also used $K=1000$. For each combination of parameters, we use the equal sample sizes from $n=20$ to 1000 in all $K$ studies. The 
sample sizes in the treatment and control arms are equal.

We use a total of 10,000 repetitions for each combination of parameters. Thus, the simulation standard error for estimated coverage of $\delta$ or $\tau^{2}$ at the $95 \%$ confidence level, or testing at .05 level is roughly $\sqrt{0.95 \times 0.05 / 10,000}=0.00218$. The simulations were programmed in $\mathrm{R}$ version 3.3.2.

For increasing number of studies $k \leq K$, we examine bias and coverage in estimation of $\delta$ and $\tau^{2}$, and the accumulating rejection rates (type 1 error or power) of tests for the shift in the mean effect and in the between-study variance $\tau^{2}$ in CMA. We also consider cumulative signalling rate and the average run length (ARL) in CUSUM analysis. We consider both the constant value of $\delta$ (the null hypothesis of no shift-in-the-mean) and a shift from $\delta=1$ to $\delta=2$ at $0.25,0.5$ and 0.75 of the total number of studies, and also shifts from $\delta=1$ to $\delta=0, \delta=0.5$ and $\delta=1.5$ at study 26 out of 50 studies.

Our summaries of results in Sections 4.3 to 4.7 are based on examination of the figures in the Appendices. We vary five parameters: the overall true $\operatorname{SMD}(\delta)$ and the between-studies variance $\left(\tau^{2}\right)$, in addition to the maximum number of studies $(K)$, the point of shift (if any) $f$ and the studies' total sample size $(n)$. Table 1 lists the values of each parameter.

We generate the true effect sizes $\delta_{i}$ from a normal distribution: $\delta_{i} \sim N\left(\delta, \tau^{2}\right)$. We generate the values of Hedges's estimator $g_{i}$ directly from the appropriately scaled non-central $t$-distribution, given by Equation (2.3), and obtain their estimated withinstudy variances from Equation (2.2).

We study two approaches to point and interval estimation and testing of $\tau^{2}$ (REML/QP and KDB) and two resulting approaches to point and interval estimation and testing of $\delta$ (IV REML and SSW KDB). Each of these two approaches was studied in three settings: traditional CMA setting, the two-stage CMA with known value of $\delta=\delta_{0}$ and the two-stage CMA with the estimated in stage 1 value of $\hat{\delta}$.

\subsection{Outcomes recorded in simulations}

In all simulations, we assumed the shift-in-the-mean model (3.1) and, for the CMA methods of interest, we studied the bias of the point estimators of $\hat{\delta}_{(k)}$ in respect to the cumulative mean $\delta_{(k)}(3.2)$ and (for the standard CMA) the bias of $\hat{\tau}_{k}^{2}$ in respect to $\tau^{2}$ for $5 \leq k \leq K$. We also investigated coverage of the $\delta_{(k)}$ and of $\tau^{2}$ by relevant interval estimators and empirical levels of accompanying two-sided tests for the null 
Table 1: Data patterns in simulations

\begin{tabular}{|l|l|}
\hline Parameters & Input Values \\
\hline$\delta$ (true value of the SMD) & $0,0.5,1$ \\
$\tau^{2}$ (variance of random effect) & $0,0.1,0.25,1$ \\
$K$ (maximum number of studies) & $50,1000(2$-stage CMA) \\
$k_{1}$ (point of shift in $\left.\delta\right)$ & $i\lceil K / 4\rceil, i=1,2,3$ for shift from 1 to $2 ;$ \\
& $K=50$ and $k_{1}=26$ for shifts from 1 to $1.5,1$ to 0.5 \\
& and from 1 to 0. \\
$n$ (sample size - total of the two arms) & $20,50,100,500,1000$ (shift-in-the-mean) \\
& $20,50,100,500$ (no shift-in-the-mean) \\
$M$ (2-sided significance level) & $.05, .01,0.005$ \\
$M$ (repetitions) & 10000 \\
& $1000(2$-stage CMA with $K=1000)$ \\
\hline
\end{tabular}

hypothesis of no shift in $\delta$ and (separately) of one-sided tests of no shift in $\tau^{2}$. We also investigated cumulative type I errors of these tests at $0.05,0.01$ and 0.005 levels for $\delta$ and at twice smaller levels for one-sided tests for $\tau^{2}$.

\subsection{Bias of $\hat{\tau}_{k}^{2}$}

When there is no shift in $\delta$, two estimators of $\tau^{2}$ (REML and KDB) have nonnegligible positive bias for small $k$, especially for small sample sizes $(n \leq 50)$, Appendix A4. KDB retains small positive bias for larger values of $k$, whereas the bias of REML becomes negative when $\tau^{2}>0$. Biases do not depend visibly on the value of $\delta$, but increase in $k$, and considerably increase in $\tau^{2}$. The bias of REML is about -0.04 and bias of $\mathrm{KDB}+0.04$ when $n=20, \tau^{2}=0.25$ and $k=10$ compared to -0.10 and +0.07 when $\tau^{2}=1$. Biases decrease in $n$; when $n=100$, KDB is practically unbiased, and REML has small negative bias of about $2 \%$.

From the moment of shift, both estimators of $\tau^{2}$ rapidly increase, KDB somewhat faster than REML, Appendix C5. However, for larger $n$, behavior of REML converges to that of KDB, and the difference between the estimators is negligible at $n=100$. 


\subsection{Bias and coverage of $\hat{\delta}_{k}$.}

Cumulative effect estimated by SSW is almost unbiased under all simulated conditions, regardless of a value or a shift in $\delta$. In Appendices A1, C1 and C2, SSW coincides with its expected value given by equation (3.2). IV REML is also unbiased when $\delta=0$. However, IV REML has a small negative bias up to about $5 \%$ to $7 \%$ when $n=20$ and $\delta=1$. The bias increases in $\delta$ and in $k$. It also increases, though rather slowly, in $\tau^{2}$. Bias decreases for larger sample sizes, so that when $n=100$ and $\delta=1$, the bias is about $1.5 \%$. After the shift in $\delta$, IV REML is somewhat lower than SSW and it deviates from its nominal mean (3.2), but these differences decrease in sample size, and are practically eliminated by $n=100$.

As illustrated by Appendix A2, coverage of SSW KDB is rather conservative for small number of studies but improves for larger values of $k$ and $\tau^{2}$. When $n=20$, for small number of studies $k \leq 5$, coverage of IV REML is somewhat conservative when $\tau^{2}=0$ and $\delta=0$, but it becomes liberal for larger $k$ when $\delta=1$. For larger sample sizes, IV REML provides stable if somewhat conservative coverage when $\tau^{2}=0$. When $\tau^{2}>0$, IV REML coverage is very liberal for $k \leq 10$, and it does not much improve in $n$. Coverage at nominal .95 level is about $85-90 \%$ when $k=20$ and $\tau^{2} \geq 0.25$, and it still is liberal when $n=100$. Coverage is visibly reduced for $\delta>0$. As we shall see in the next Section, this liberal coverage translates into higher type 1 error in CMA.

\subsection{Level and power of tests for $\delta$ in CMA}

Because of multiple testing over increasing number of studies $k$, empirical levels of SSW KDB and IV REML at the same nominal levels are increasing in $k$, however the empirical levels of SSW KDB are considerably lower. The difference between the two methods is more pronounced for larger values of $\delta$, see Appendix A3. As an example, at the nominal $5 \%$ level, these levels for $\delta=1$ are $4.80 \%$ for SSW KDB vs $11.81 \%$ for IV REML at $k=12$ and $8.89 \%$ vs $18.74 \%$ at $k=25$ for $n=20$ and $\tau^{2}=0$. These levels further increase in $\tau^{2}(7.91 \%$ vs $17.66 \%$ at $k=12$ for $n=20$ and $\tau^{2}=0.1$ ), and somewhat increase in $n$, in this example to $15.03 \%$ vs $25.25 \%$ at $k=12$ for $n=1000$ and $\tau^{2}=0.1$. Testing at the lower nominal levels makes sense for larger values of $k, \tau^{2}$ and/or $n$.

The power of SSW KDB and IV REML is comparatively low, Appendix C3. For both methods, the power is the highest when $\tau^{2}=0$ and considerably deteriorates in $\tau^{2}$. Taking into account the lower level of SSW KDB, it is more powerful than IV 
REML. The power increases in $n$, and for shift at $k=26$, by $n=100$ both methods reach the power of $80 \%$ at 31 studies when $\tau^{2}=0$ and at 36 studies for $\tau^{2}=0.25$. Choosing the nominal level of .01 safeguards the empirical levels about .05 at $k=25$ and reduces the power of CMA accordingly.

\subsection{Level and power of tests for $\tau^{2}$ in CMA}

We studied one-sided tests for $\tau_{(k)}^{2}>\tau_{0}^{2}$ under the null (Appendix A7) and the typical results under shift $\Delta=1$ at $k=13,26$ and 36 are depicted in Appendix C8 for nominal levels .025, .005 and 0.0025. Multiple testing inflates empirical levels, more so for KDB than for QP. The power increases in $n$ and decreases in $\tau^{2}$. When $\tau^{2}=0$, the power is quite high from $n=50$, but when $\tau^{2}=0.25$, and the shift is at $k=26$, the power reaches $80 \%$ for both tests only at $k=41$ for $n=100$. The power is extremely low when $\tau^{2}=1$ even for very large sample sizes; for shift at $k=26$, power barely reaches $30 \%$ at $k=50$ when $n=1000$.

\subsection{Comparing tests for shift in $\delta$ in 1- and 2-stage CMA}

When there is no shift in $\delta$, two-stage CMA performs very similarly to the standard, one-stage CMA, in respect to inflation of the empirical levels (Appendix B). However, it is somewhat more powerful under the shift (Appendix D). This difference in power is clear for $\operatorname{KDB} \operatorname{SSW}\left(\tau_{0}^{2}\right)$ from $n=20$, and for IV REML $\left(\tau_{0}^{2}\right)$ from $n=50$,

Appendix E2. This difference in power is explained by inflation in estimated $\tau_{(k)}^{2}$ in the standard CMA, as discussed in Section 3.3.

For comparison purposes, Figures in Appendix E2 also include CUSUM-based CMA with $h=4,5$ and 6 alongside the CMA tests at levels 0.05, 0.01 and 0.005, respectively (see also Appendices B5 and D7). Under no shift, CUSUM-based analysis results in greater inflation of the empirical levels, but equally, it has more power under shift. Similarly to other tests, its power increases in $n$ and decreases in $\tau^{2}$.

Figures in Appendix E2 also include the 2-stage methods using an estimated in stage 1 mean effect $\hat{\delta}_{0}$. In this scenario, the "standard" CMA methods which re-estimate $\tau_{(k)}^{2}$ have especially inflated levels, and the 2-stage methods which use the estimated $\tau_{0}^{2}$ are clearly the better choice. Unexpectedly, methods using two estimated at stage 1 parameters $\left(\hat{\delta}_{0}, \tau_{0}^{2}\right)$, have somewhat lower type 1 error and somewhat more power than the comparative methods using known $\delta_{0}$, especially so for IV REML. 


\section{Discussion}

Cumulative meta-analysis is a well-established and popular method of evaluating and monitoring accumulating evidence. Multiplicity problems inherent in CMA are well known, and a number of alternative statistical methods aimed at resolving these problems are available to researchers. However, this does not seem to hinder the popularity of CMA in applied research.

Therefore, for a popular effect measure SMD, we investigated, theoretically and by simulations, what are the level and power of CMA and how to improve both. We compared two classes of methods for CMA: IV REML and SSW KDB. Our simulations clearly demonstrate that the SSW KDB analysis is a much better option when $\delta>0$.

We recognised the issues with the variance inflation in CMA when a shift in the mean occurs and suggested therefore a two-stage approach to CMA, as well as testing for shift in $\tau^{2}$. Our simulations show that the two-stage CMA performs better than the standard 1-stage CMA in respect to both the type 1 error and power. Testing for $\tau^{2}$ also works well for small-to moderate values of $\tau^{2} \leq 0.25$, Q-profile method by Viechtbauer [2007] is the preferred option. However this method has very low power for larger $\tau^{2}$.

In our simulations, we also considered CUSUM charts suggested by Kulinskaya and Koricheva [2010], modified for random-effects MA by an addition of estimated at stage one between-study variance $\tau_{0}^{2}$. However, this resulted in a too high type 1 error, and we do not recommend this method.

A practical recommendation is to run simultaneously two analyses: testing for cumulative $\tau^{2}$ at 0.005 level using the Q-profile method, and the two-stage testing for shift in mean effect at 0.01 level using SSW KDB, with either known or estimated in stage one target value of $\delta$, and using the constant value of $\tau_{0}^{2}$ estimated in stage 1 by KDB. However, in the case of high heterogeneity, the power of all CMA methods is very low.

\section{Funding}

Work by E. Kulinskaya was supported by the Economic and Social Research Council [grant number ES/L011859/1]. 


\section{Acknoledgement}

We are grateful to Julia Koricheva and Elizabeth Brisco for interesting discussions of cumulative meta-analyses in ecology.

\section{References}

Ilyas Bakbergenuly, David C. Hoaglin, and Elena Kulinskaya. Estimation in metaanalyses of mean difference and standardized mean difference. Statistics in Medicine, 39(2):171-191, 2020.

J. Brok, K. Thorlund, C. Gluud, and J. Wetterslev. Trial sequential analysis reveals insufficient information size and potentially false positive results in many metaanalyses. Journal of Clinical Epidemiology, 61:763-769, 2008.

William G Cochran. The combination of estimates from different experiments. Biometrics, 10(1):101-129, 1954.

Rebecca DerSimonian and Nan Laird. Meta-analysis in clinical trials. Controlled clinical trials, 7(3):177-188, 1986.

Samson Henry Dogo, Allan Clark, and Elena Kulinskaya. Sequential change detection and monitoring of temporal trends in random-effects meta-analysis. Research Synthesis Methods, 8(2):220-235, 2017. doi: 10.1002/jrsm.1222. URL https://onlinelibrary.wiley.com/doi/abs/10.1002/jrsm. 1222.

J. Gurevitch, J. Koricheva, S. Nakagawa, and G. Stewart. Meta-analysis and the science of research synthesis. Nature, 555:175-182, 2018.

Larry V Hedges. A random effects model for effect sizes. Psychological Bulletin, 93 (2):388-395, 1983.

Larry V Hedges and Ingram Olkin. Statistical Methods for Meta-Analysis. San Diego, California: Academic Press, 1985.

Julian Higgins, Anne Whitehead, and Mark Simmonds. Sequential methods for random-effects meta-analysis. Statistics in medicine, 30(9):903-921, 2011. 
Mingxiu $\mathrm{Hu}$, Joseph C Cappelleri, and KK Gordon Lan. Applying the law of iterated logarithm to control type I error in cumulative meta-analysis of binary outcomes. Clinical Trials, 4(4):329-340, 2007.

J. Koricheva, M.D. Jennions, and J. Lau. Temporal trends in effect sizes: causes, detection, and implications. In J. Koricheva, J. Gurevitch, and K. Mengersen, editors, Handbook of meta-analysis in ecology and evolution, pages 237-254. Princeton University Press, 2013. URL https://doi.org/10.1515/9781400846184.

E. Kulinskaya and J. Koricheva. Use of quality control charts for detection of outliers and temporal trends in cumulative meta-analysis. Research Synthesis Methods, 2010 .

Elena Kulinskaya and John Wood. Trial sequential methods for meta-analysis. Research Synthesis Methods, 2013.

Elena Kulinskaya, Michael B Dollinger, and Kirsten Bjørkestøl. Testing for homogeneity in meta-analysis I. The one-parameter case: standardized mean difference. Biometrics, 67(1):203-212, 2011.

J. Lau, E.M. Antman, J. Jimenez-Silva, B. Kupelnick, F. Mosteller, and T.C. Chalmers. Cumulative meta-analysis of therapeutic trials for myocardial infarction. New England Journal of Medicine, 327(4):248-254, 1992.

Robert C. Paule and John Mandel. Consensus values and weighting factors. Journal of Research of the National Bureau of Standards, 87(5):377-385, 1982.

J.M. Pogue and S. Yusuf. Cumulating evidence from randomized trials: utilizing sequential monitoring boundaries for cumulative meta-analysis. Controlled clinical trials, 18(6):580-593, 1997.

K.G. Shojania, M. Sampson, M.T. Ansari, J. Ji, S. Doucette, and D. Moher. How quickly do systematic reviews go out of date? a survival analysis. Annals of Internal Medicine, 147(4):224-233, 2007.

K. Thorlund, G. Imberger, M. Walsh, R. Chu, C. Gluud, J. Wetterslev, and et al. The number of patients and events required to limit the risk of overestimation of intervention effects in meta-analysis - a simulation study. PLoS ONE, 6(10): e25491, 2011. doi: https://doi.org/10.1371/journal.pone.0025491. 
T.A. Trikalinos and J.P.A. Ioannidis. Assessing the evolution of effect sizes over time. In H.R. Rothstein, A.J. Sutton, and M. Borenstein, editors, Publication bias in meta-analysis - prevention, assessment and adjustments, pages 241-259. Wiley, 2005.

Wolfgang Viechtbauer. Bias and efficiency of meta-analytic variance estimators in the random-effects model. Journal of Educational and Behavioral Statistics, 30(3): 261-293, 2005.

Wolfgang Viechtbauer. Confidence intervals for the amount of heterogeneity in metaanalysis. Statistics in medicine, 26(1):37-52, 2007.

J. Wetterslev, K. Thorlund, J. Brok, and C. Gluud. Trial sequential analysis may establish when firm evidence is reached in cumulative meta-analysis. Journal of Clinical Epidemiology, 61(1):64-75, 2008.

Anne Whitehead. A prospectively planned cumulative meta-analysis applied to a series of concurrent clinical trials. Statistics in medicine, 16(24):2901-2913, 1997. 


\section{APPENDICES}

\section{A. Standard CMA under null hypothesis of no shift-in-the-mean}

- A1. Bias of point estimators of $\delta$

- A2. Coverage of interval estimators of $\delta$

- A3. Coverage of one-sided interval estimators of $\delta$

- A4. Bias of point estimators of $\tau^{2}$

- A5. Coverage of interval estimators of $\tau^{2}$

- A6. Empirical levels of CMA tests for shift-in-the-mean $\delta$

- A7. Empirical levels of CMA tests for positive shift in $\tau^{2}>\tau_{0}^{2}$

B. 2-stage CMA under null hypothesis of no shift-in-the-mean

- B1. Bias of point estimators of $\delta$

- B2. Coverage of interval estimators of $\delta$

- B3. Coverage of one-sided interval estimators of $\delta$

- B4. Empirical levels of 2-Stage CMA tests for shift-in-the-mean $\delta$

- B5. Empirical levels of CUSUM tests for shift-in-the-mean estimated by $\hat{\delta}_{0}$ 


\section{Standard CMA under shift-in-the-mean}

- C1. Comparison of CMA-estimated mean effect $\delta$ to nominal cumulative mean effect

- C2. Bias of point estimators of $\delta$

- C3. Coverage of interval estimators of $\delta$

- C4. Coverage of one-sided interval estimators of $\delta$

- C5. Bias of point estimators of $\tau^{2}$

- C6. Empirical levels and power of CMA tests for shift in $\delta$

- C7. Empirical levels and power of CMA tests for shift in estimated from stage 1 mean effect $\hat{\delta}_{0}$

- C8. Empirical levels of CMA tests for positive shift in $\tau^{2}$

\section{2-Stage CMA under shift-in-the-mean}

- D1. Comparison of 2-Stage CMA-estimated mean effect $\delta$ to nominal cumulative mean effect

- D2. Bias of point estimators of $\delta$

- D3. Coverage of interval estimators of $\delta$

- D4. Coverage of one-sided interval estimators of $\delta$

- D5. Empirical levels and power of 2-Stage CMA tests for shift in $\delta$

- D6. Empirical levels and power of 2-Stage CMA tests for shift in estimated from stage 1 mean effect $\hat{\delta}_{0}$

- D7. Empirical levels and power of CUSUM tests for shift in $\hat{\delta}_{0}$

\section{E. Additional materials}

- E1. Nominal cumulative effects

- E2. Comparison plots - Empirical levels of tests for shift-in-the-mean 


\section{A. Standard CMA under null hypothesis of no shift-in-the-mean}




\section{A1. Bias of point estimators of $\delta$}

For bias of $\delta$ in this section, each figure corresponds to a value of $\delta(=0,0.5,1)$, a set of values of $\tau^{2}(=0,0.1,0.25,1)$, and a set of values of $n(=20,50$ or 100,500$)$ while the study number $k$ increases to maximum number of studies $K=50$.

Each figure contains a panel (with study $k$ on the horizontal axis) for each combination of parameters.

The point estimators of $\delta$ are

- IV REML (Inverse variance weighted mean with the between-study variance $\tau^{2}$ estimated by Restricted maximum likelihood)

- SSW (Effective sample size weighted mean) 

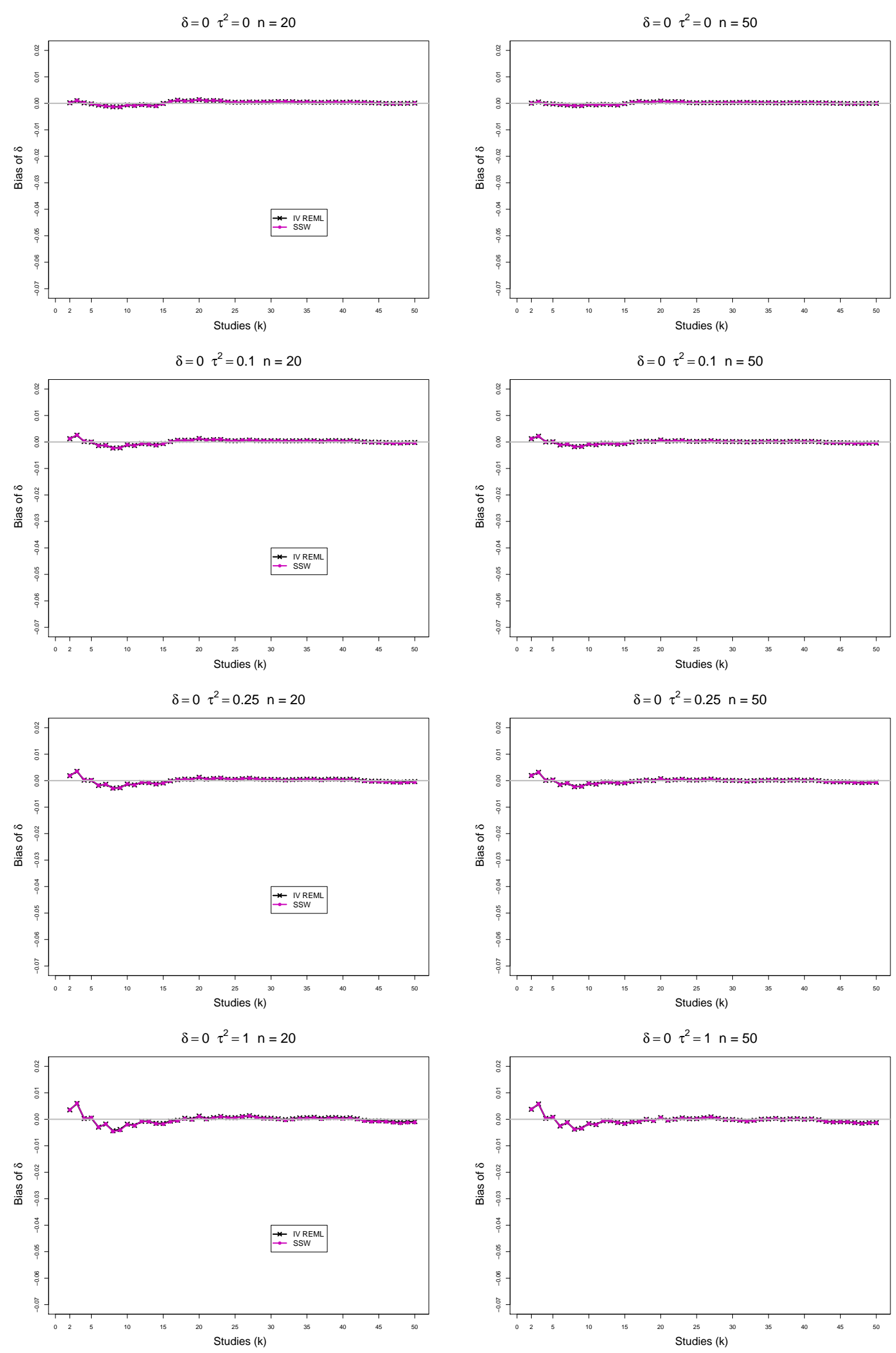

Figure A1. 1: CMA - Bias of IV REML and SSW estimators of $\delta=0$ for $\tau^{2}=0,0.1,0.25,1$, $n=20,50, K=50$. Light grey line at 0 . 

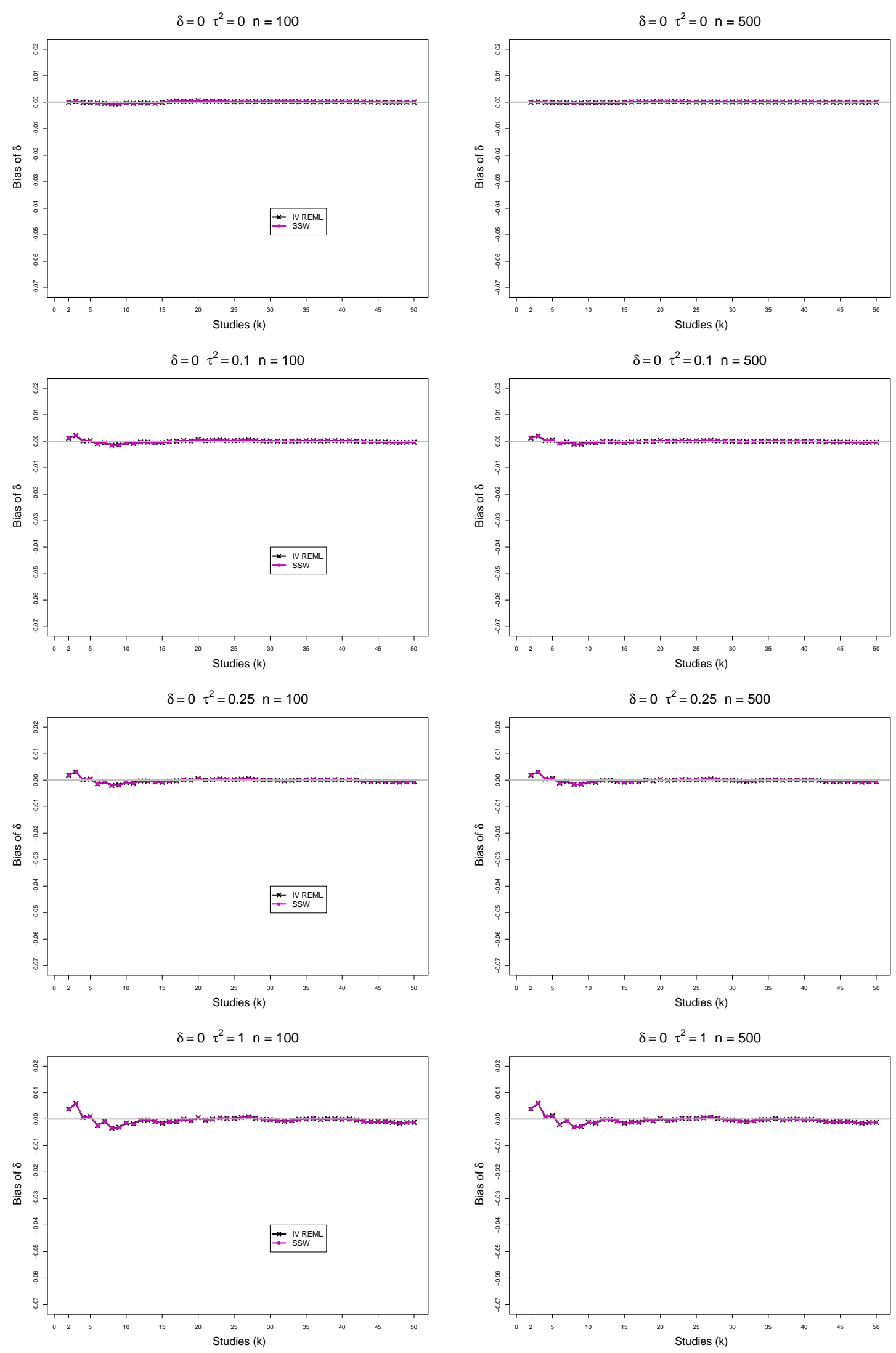

Figure A1. 2: CMA - Bias of IV REML and SSW estimators of $\delta=0$ for $\tau^{2}=0,0.1,0.25,1$, $n=100,500, K=50$. Light grey line at 0 . 

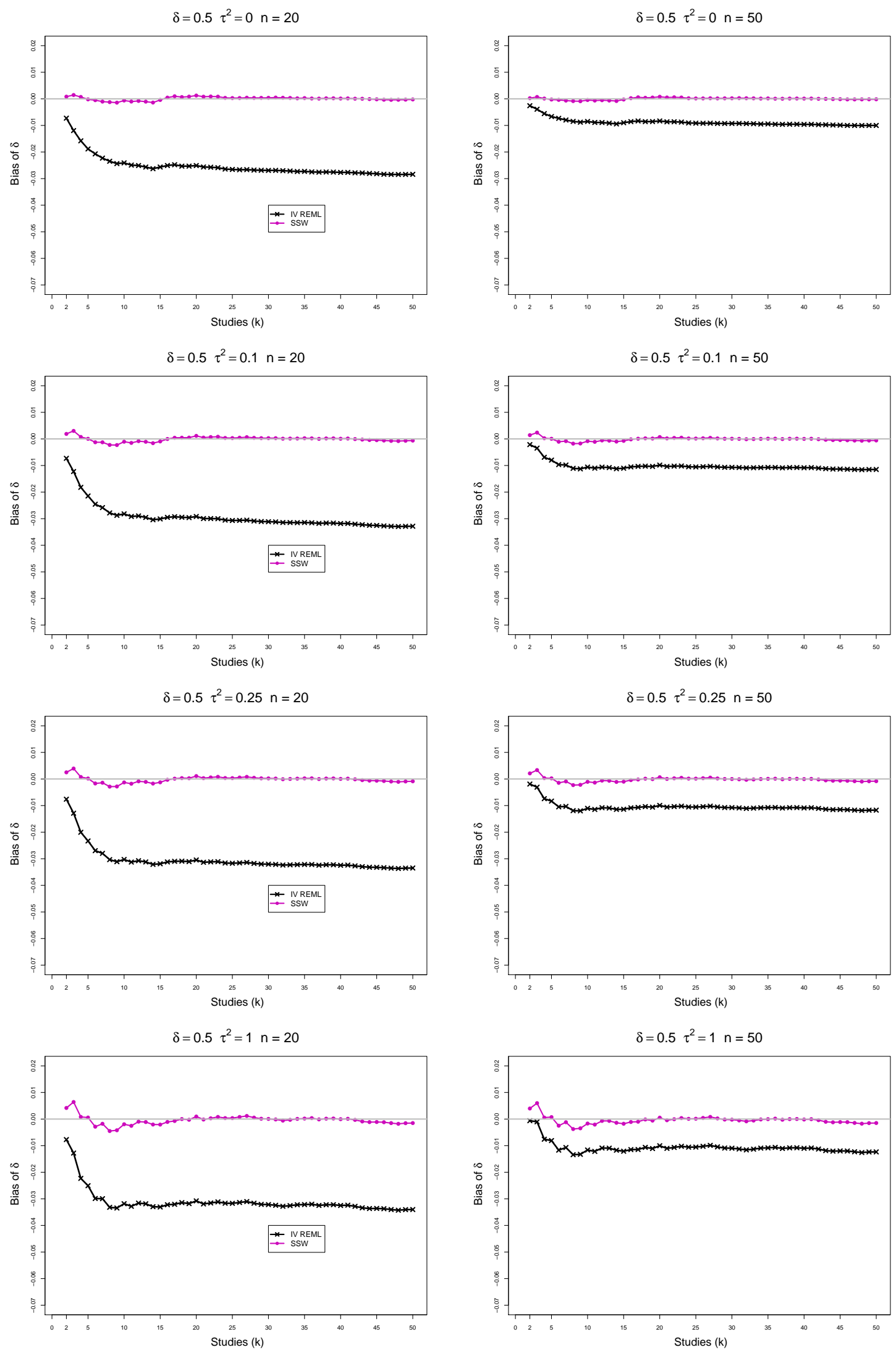

Figure A1. 3: CMA - Bias of IV REML and SSW estimators of $\delta=0.5$ for $\tau^{2}=$ $0,0.1,0.25,1, n=20,50, K=50$. Light grey line at 0 . 

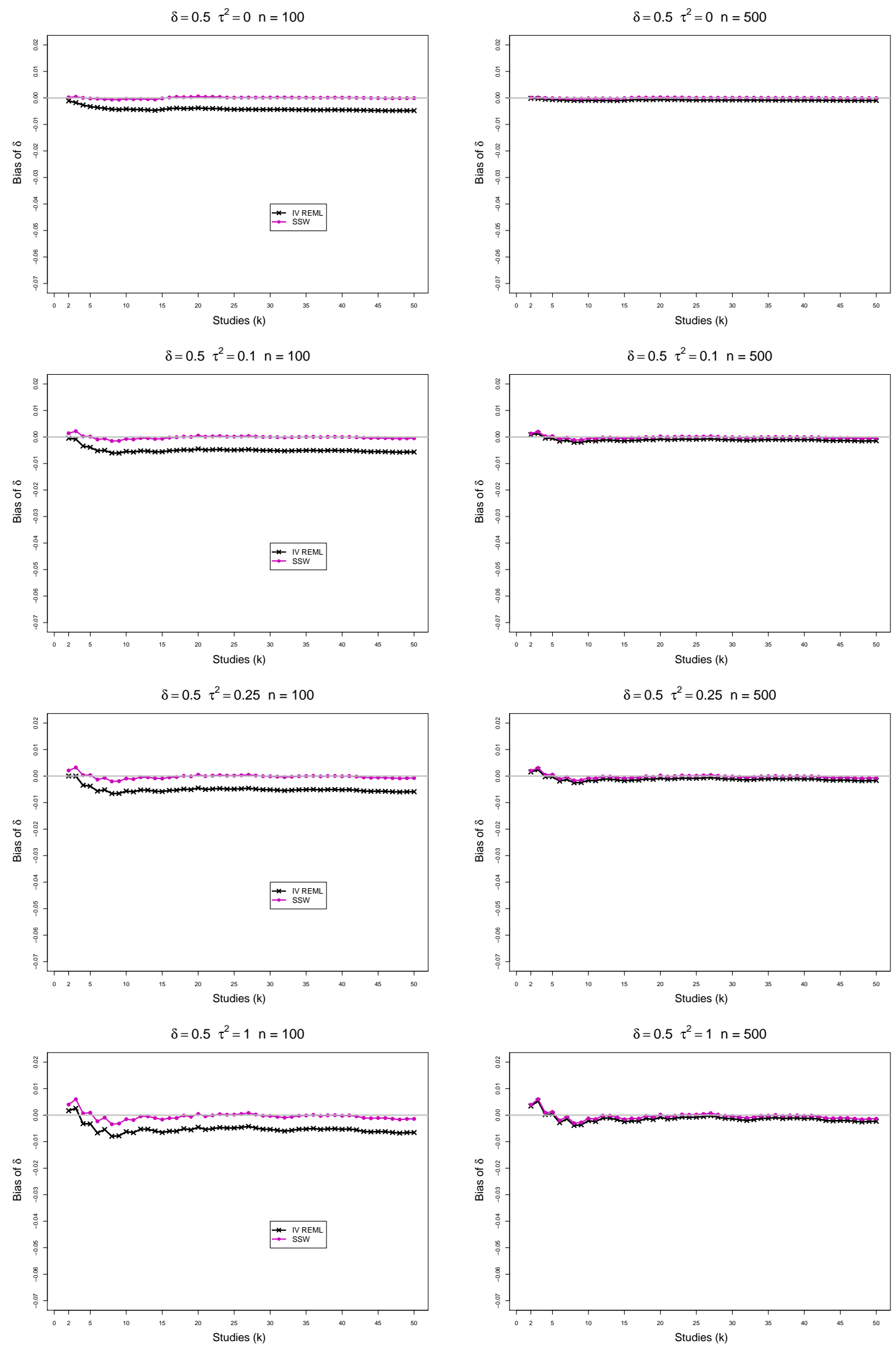

Figure A1. 4: CMA - Bias of IV REML and SSW estimators of $\delta=0.5$ for $\tau^{2}=$ $0,0.1,0.25,1, n=100,500, K=50$. Light grey line at 0 . 

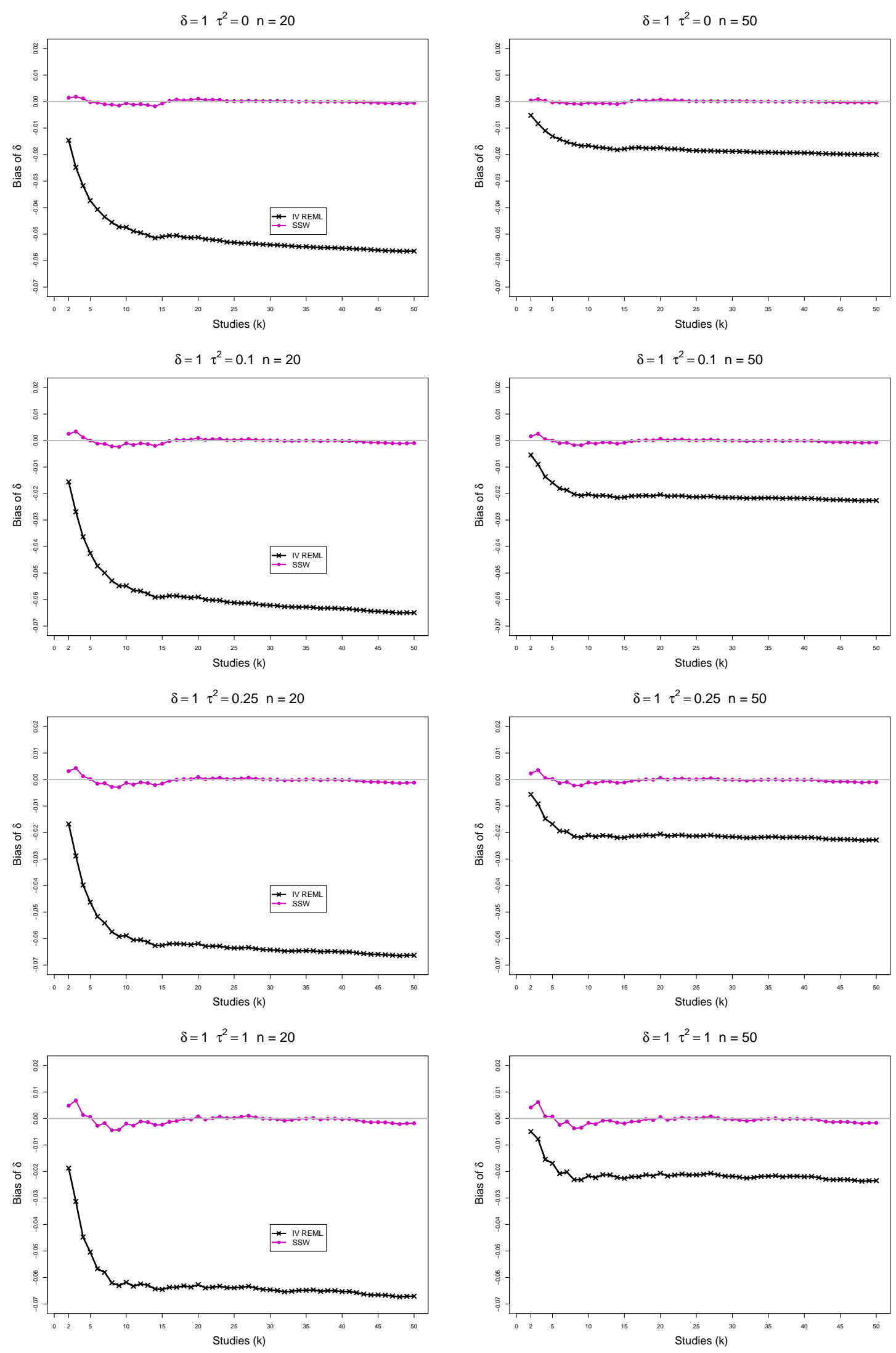

Figure A1. 5: CMA - Bias of IV REML and SSW estimators of $\delta=1$ for $\tau^{2}=0,0.1,0.25,1$, $n=20,50, K=50$. Light grey line at 0 . 

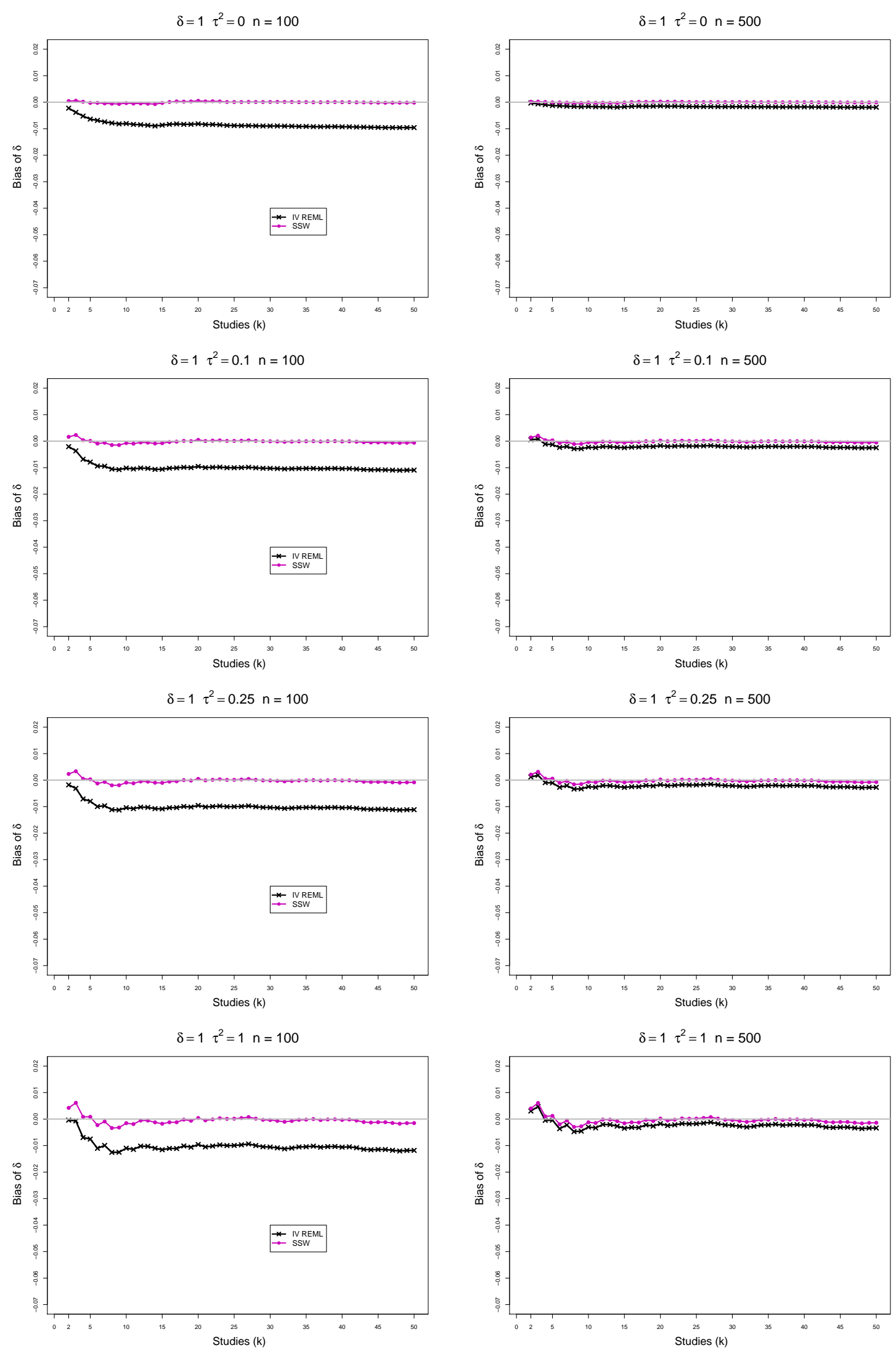

Figure A1. 6: CMA - Bias of IV REML and SSW estimators of $\delta=1$ for $\tau^{2}=0,0.1,0.25,1$, $n=100,500, K=50$. Light grey line at 0 . 


\section{A2. Coverage of interval estimators of $\delta$}

For coverage of $\delta$ in this section, each figure corresponds to a value of $\delta(=0,0.5,1)$, a set of values of $\tau^{2}(=0,0.1,0.25,1)$, a value of $n(=20,50,100,500)$ and a set of values of significance level $(=0.05,0.01,0.005)$ while the study number $k$ increases to maximum number $K=50$.

Each figure contains a panel (with study $k$ on the horizontal axis) for each combination of parameters.

The interval estimators of $\delta$ are

- IV REML (Inverse variance weighted mean with the between-study variance $\tau^{2}$ estimated by Restricted maximum likelihood as center and half-width equal to critical value from Normal distribution times square-root of the inverse sum of weights )

- SSW KDB (Sample size weighted (SSW) as center and half-width equal to critical value from $t_{k-1}$ times estimated standard deviation of SSW with $\hat{\tau}^{2}=$ $\hat{\tau}_{K D B}^{2}$, the Kulinskaya-Dollinger-Bjørkestøl (2011) estimator of $\tau^{2}$ ) 

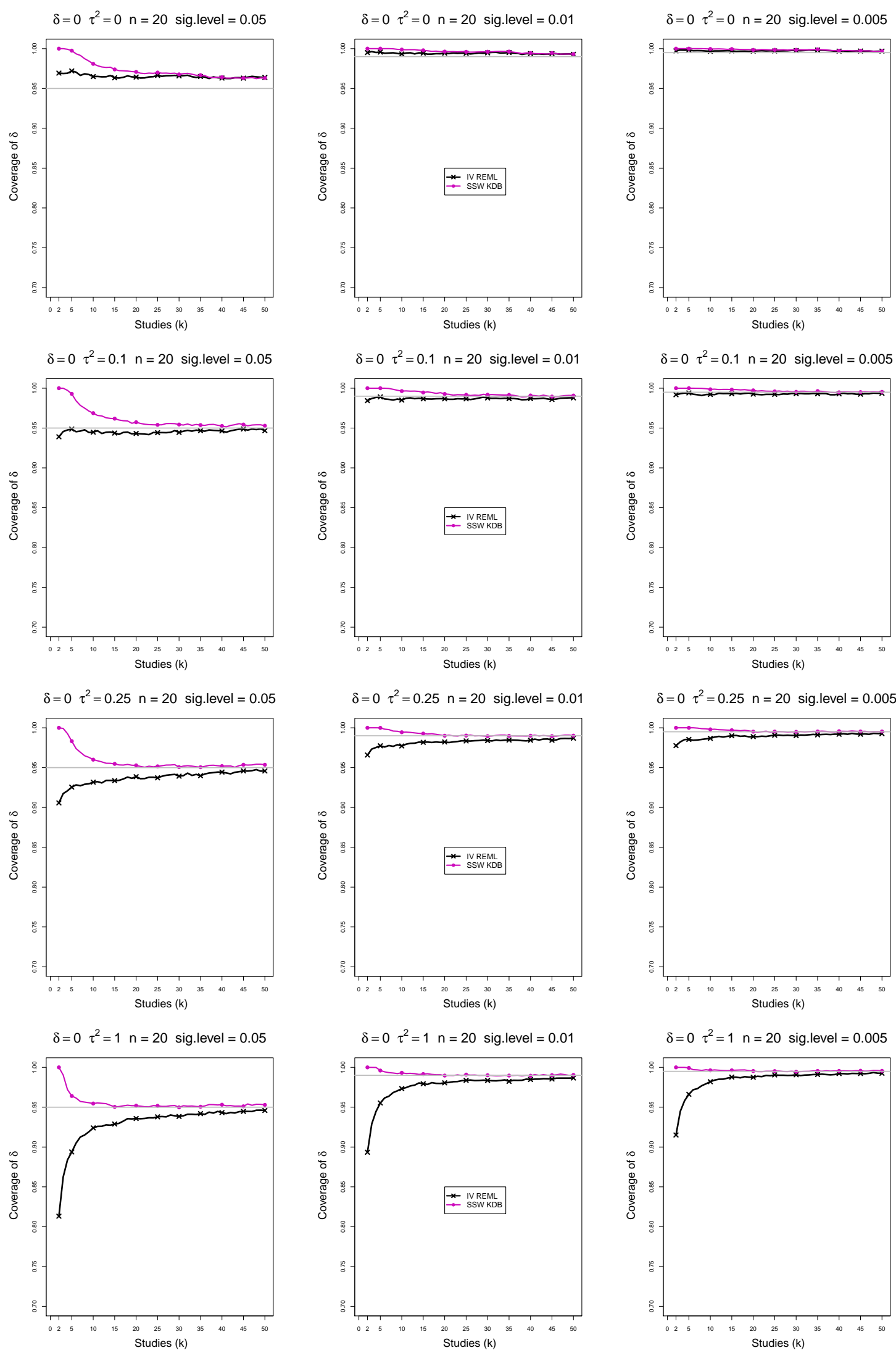

Figure A2. 1: CMA - Coverage of confidence intervals for $\delta=0$ at confidence level $1-\alpha$ for significance levels $\alpha$ of $0.05,0.01,0.005, \tau^{2}=0,0.1,0.25,1, n=20, K=50$. Light grey line at $0.95,0.99,0.995$. 

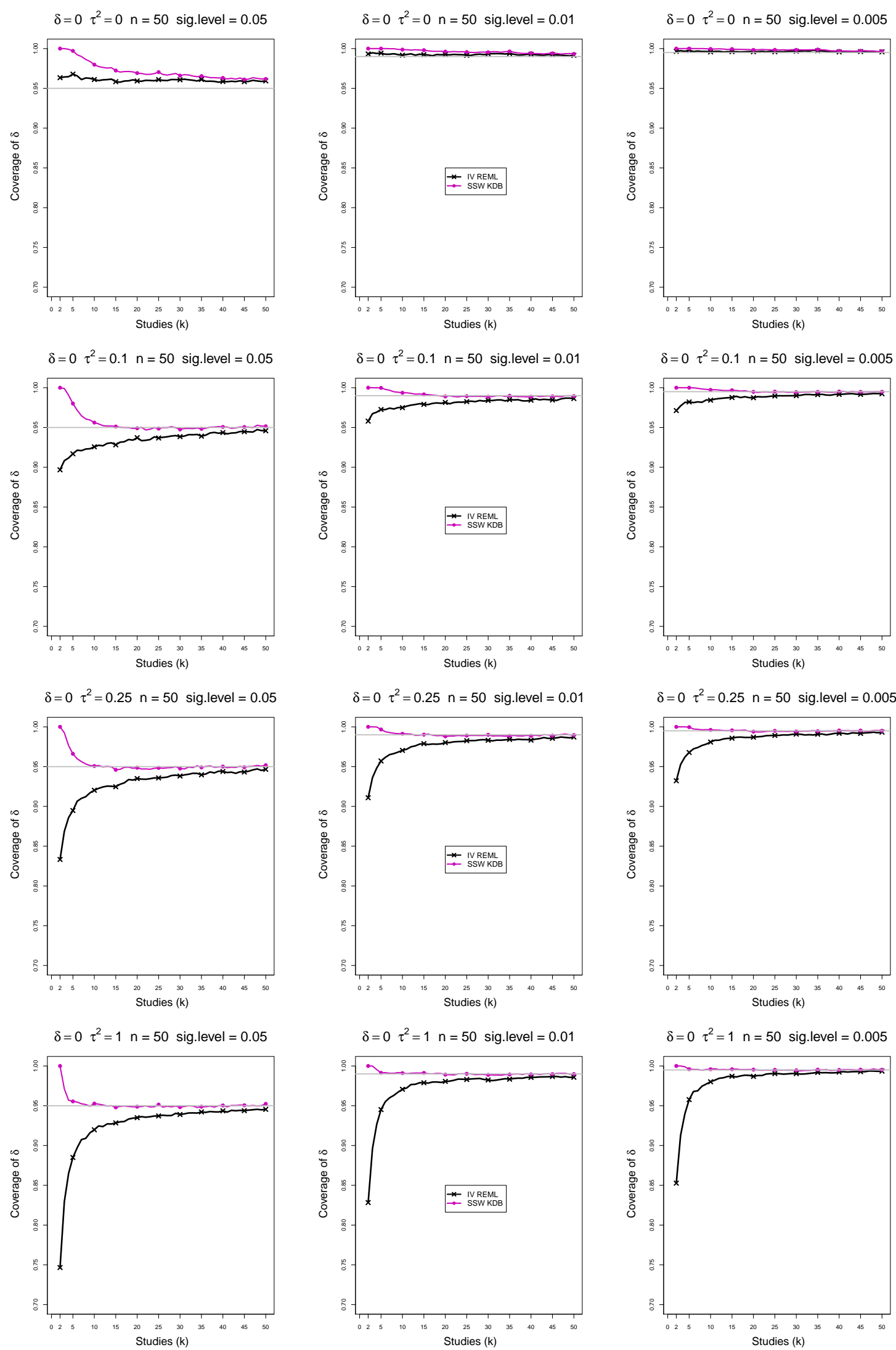

Figure A2. 2: CMA - Coverage of confidence intervals for $\delta=0$ at confidence level $1-\alpha$ for significance levels $\alpha$ of $0.05,0.01,0.005, \tau^{2}=0,0.1,0.25,1, n=50, K=50$. Light grey line at $0.95,0.99,0.995$. 

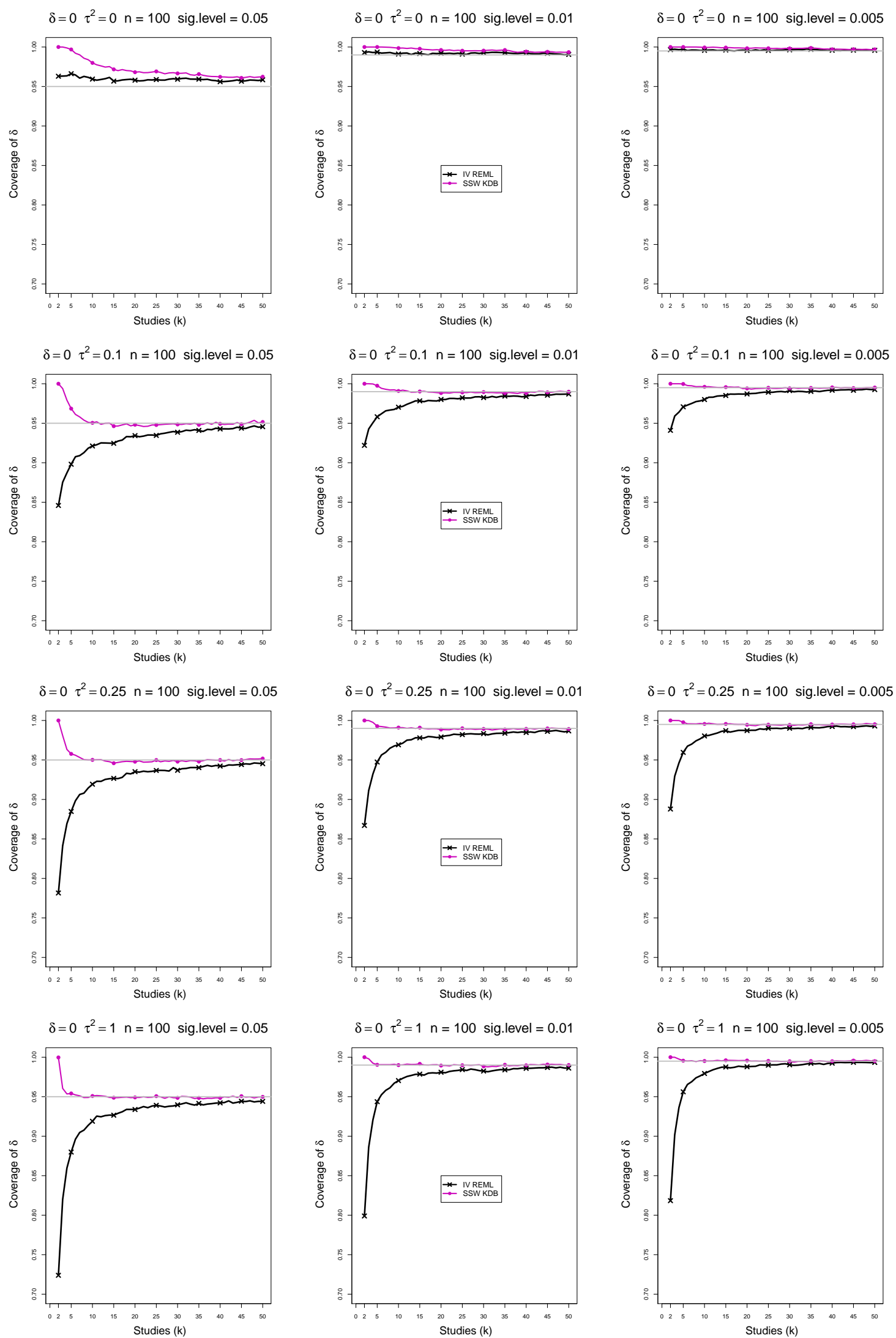

$\delta=0 \quad \tau^{2}=1 \quad n=100$ sig.level $=0.005$

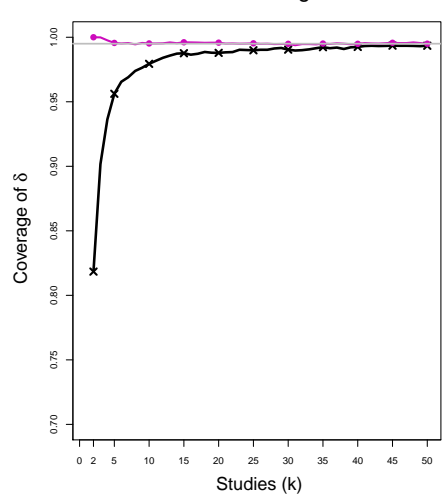

Figure A2. 3: CMA - Coverage of confidence intervals for $\delta=0$ at confidence level $1-\alpha$ for significance levels $\alpha$ of $0.05,0.01,0.005, \tau^{2}=0,0.1,0.25,1, n=100, K=50$. Light grey line at $0.95,0.99,0.995$. 

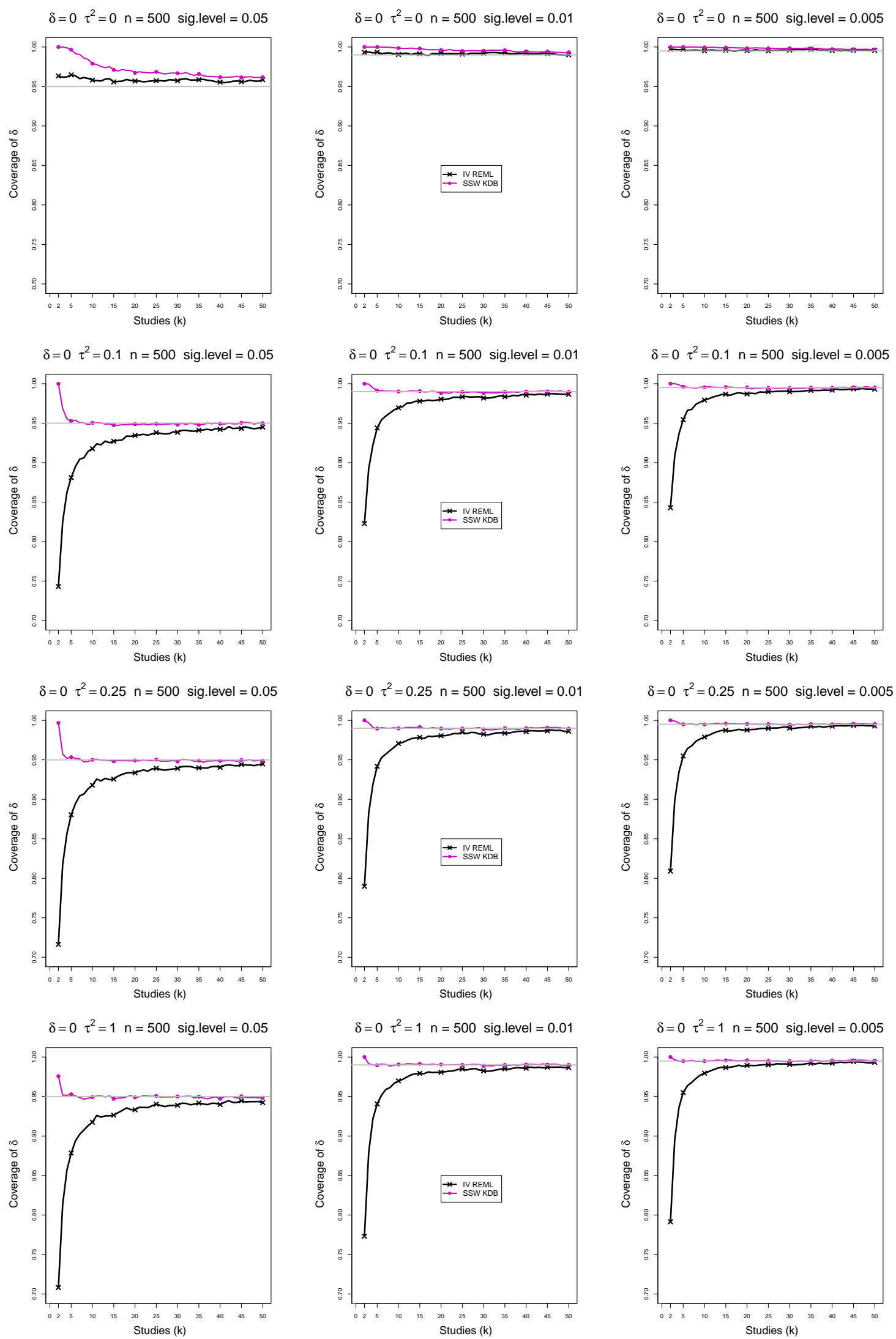

$\delta=0 \quad \tau^{2}=1 \quad n=500$ sig.level $=0.005$

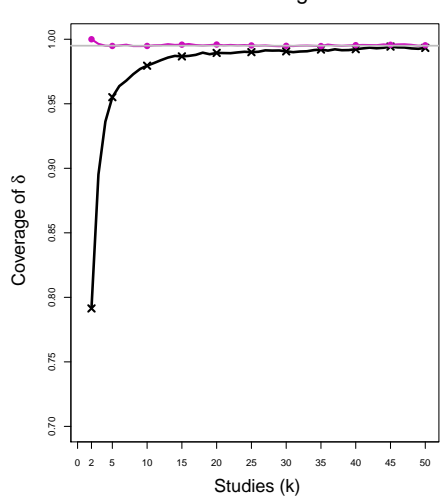

Figure A2. 4: CMA - Coverage of confidence intervals for $\delta=0$ at confidence level $1-\alpha$ for significance levels $\alpha$ of $0.05,0.01,0.005, \tau^{2}=0,0.1,0.25,1, n=500, K=50$. Light grey line at $0.95,0.99,0.995$. 

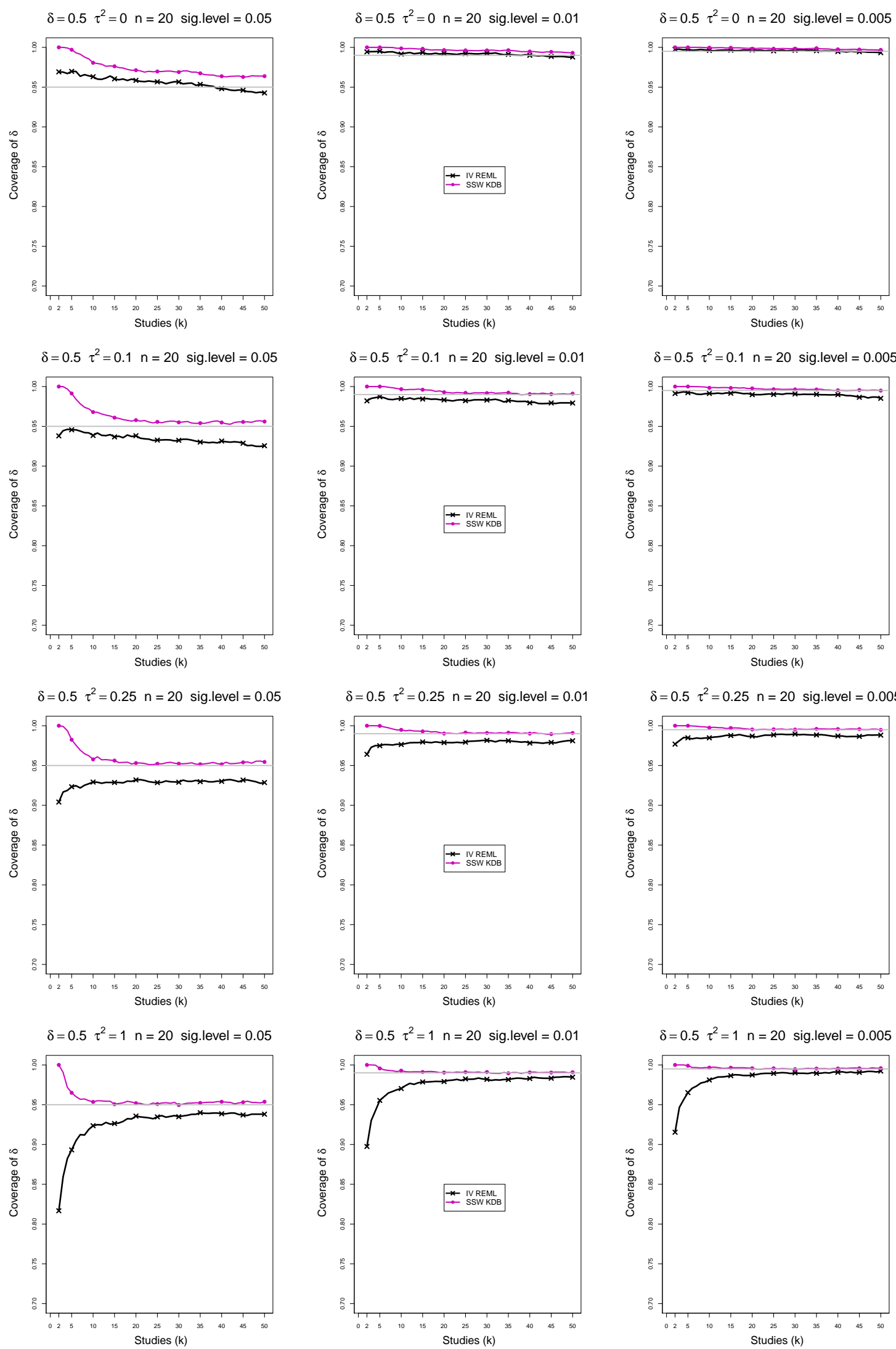

Figure A2. 5: CMA - Coverage of confidence intervals for $\delta=0.5$ at confidence level $1-\alpha$ for significance levels $\alpha$ of $0.05,0.01,0.005, \tau^{2}=0,0.1,0.25,1, n=20, K=50$. Light grey line at $0.95,0.99,0.995$. 

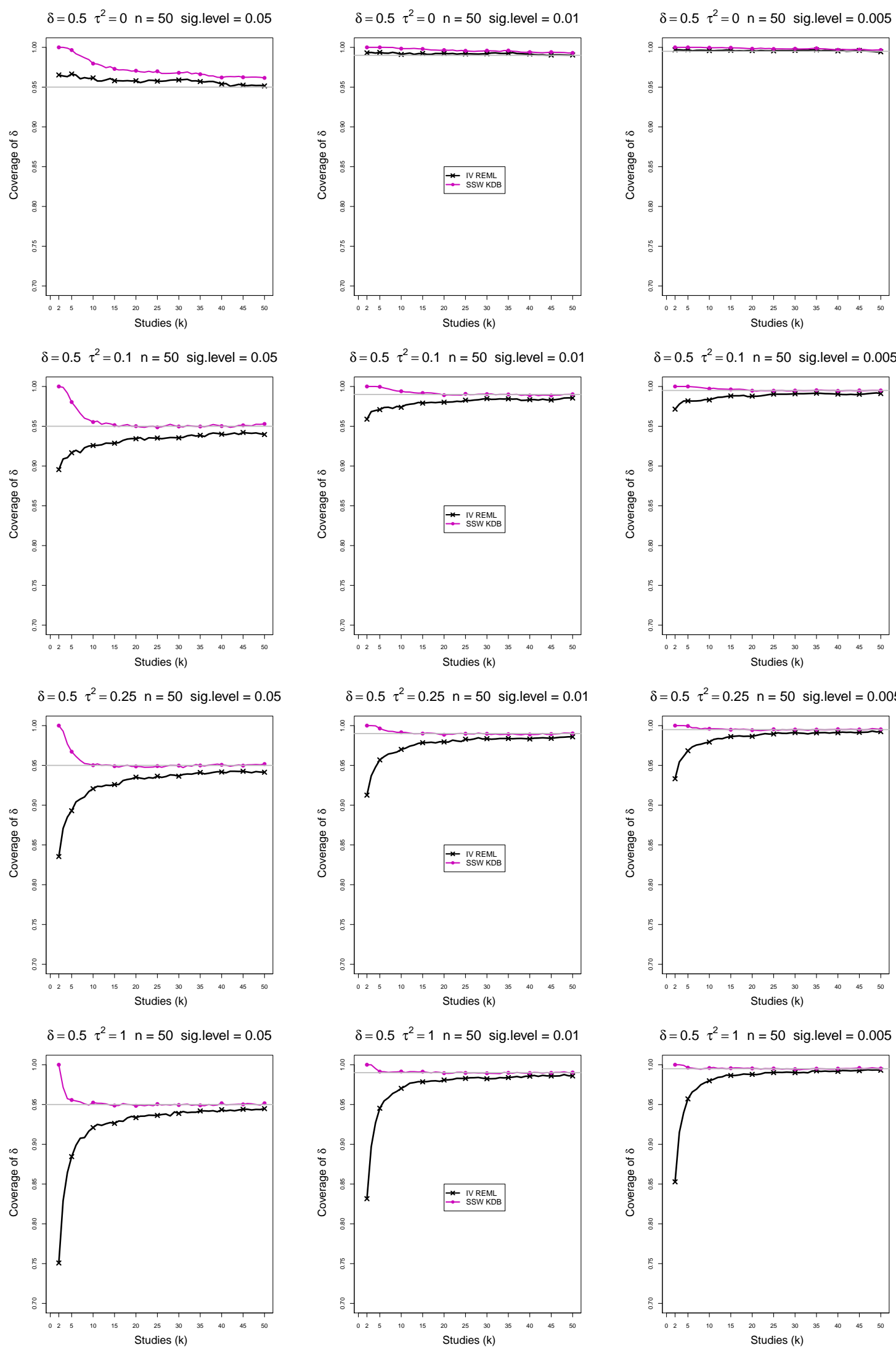

Figure A2. 6: CMA - Coverage of confidence intervals for $\delta=0.5$ at confidence level $1-\alpha$ for significance levels $\alpha$ of $0.05,0.01,0.005, \tau^{2}=0,0.1,0.25,1, n=50, K=50$. Light grey line at $0.95,0.99,0.995$. 

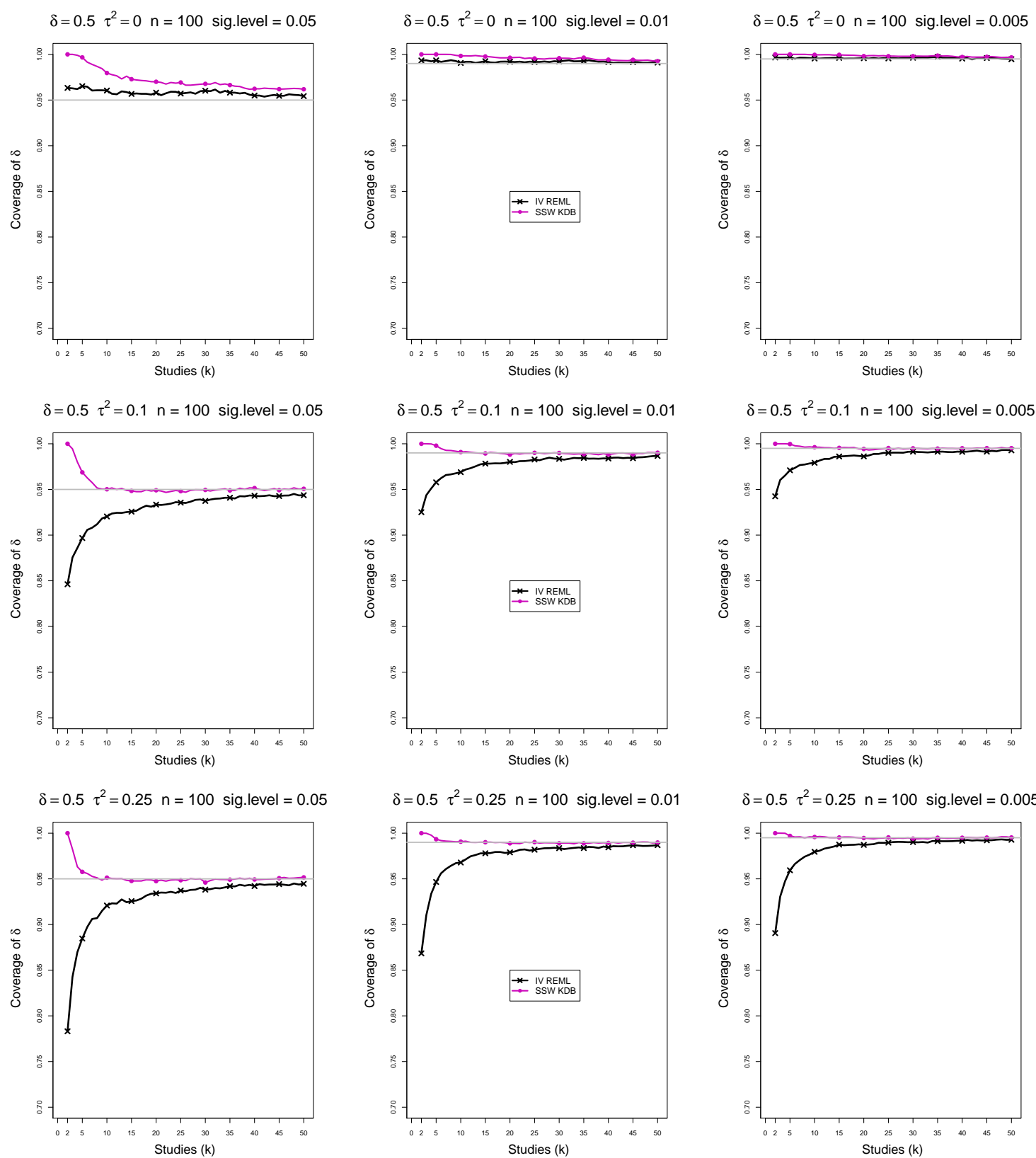

$\delta=0.5 \tau^{2}=0.25 \mathrm{n}=100$ sig.level $=0.005$
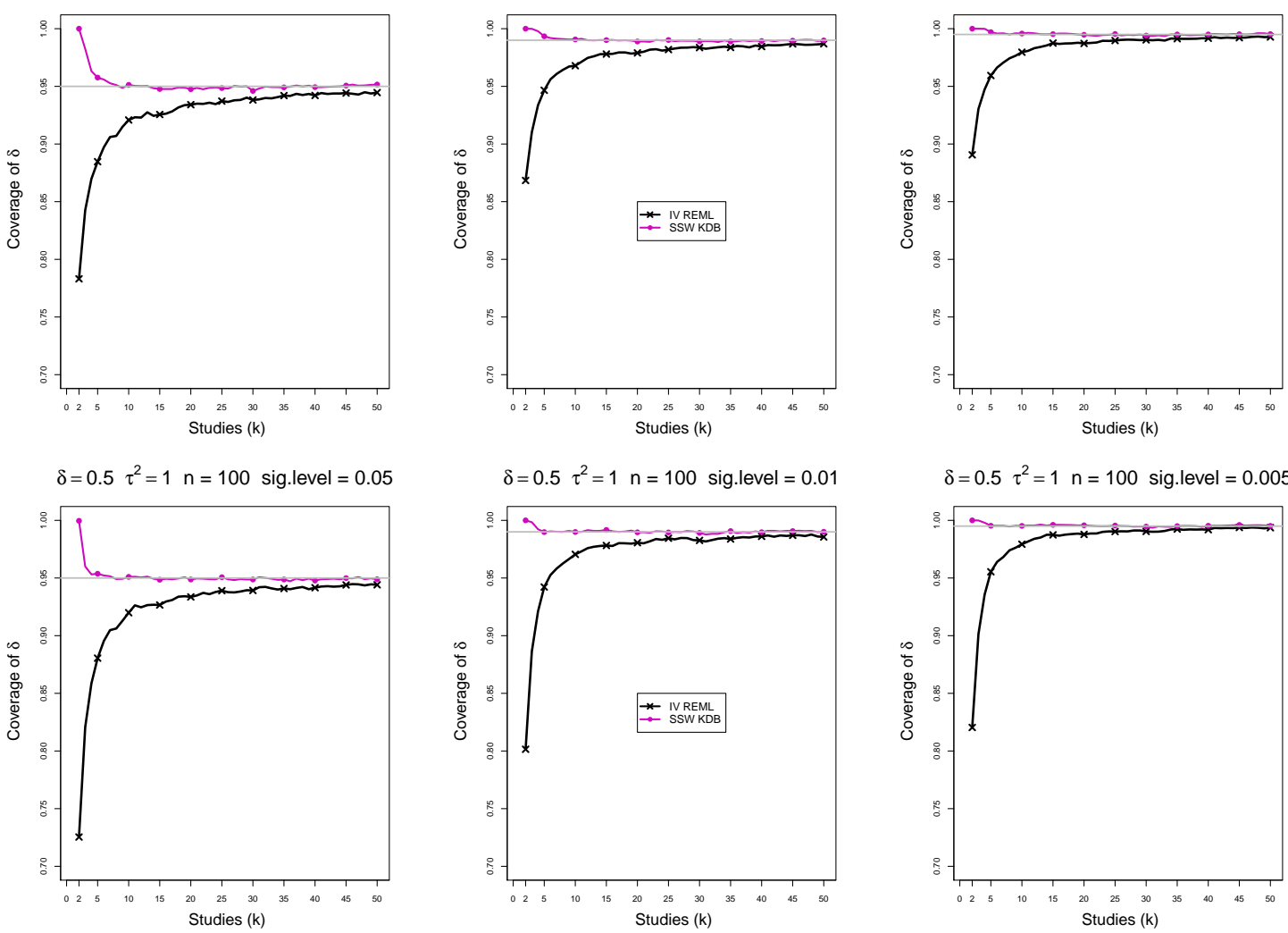

Figure A2. 7: CMA - Coverage of confidence intervals for $\delta=0.5$ at confidence level $1-\alpha$ for significance levels $\alpha$ of $0.05,0.01,0.005, \tau^{2}=0,0.1,0.25,1, n=100, K=50$. Light grey line at $0.95,0.99,0.995$. 

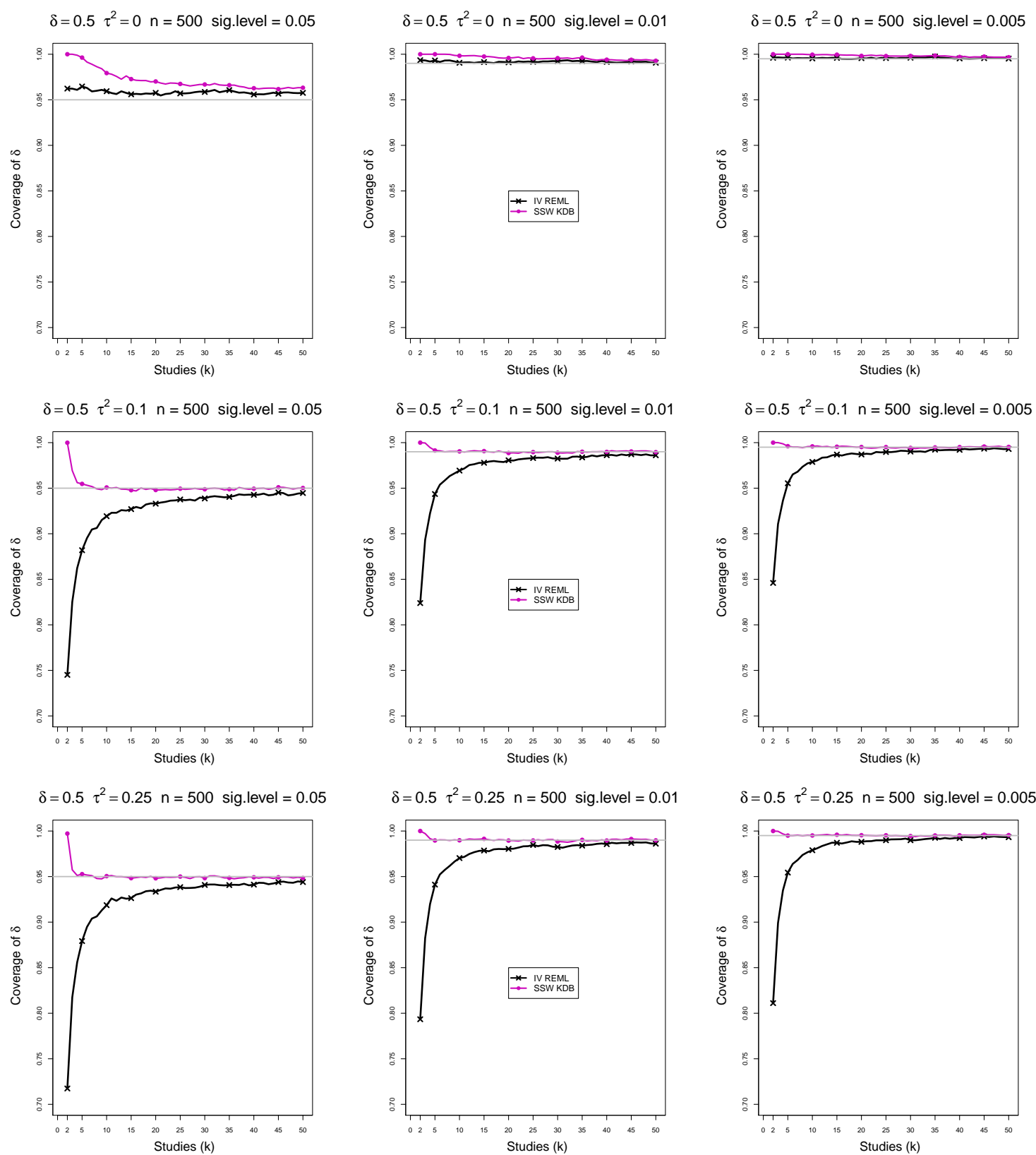

$\delta=0.5 \tau^{2}=0.25 \mathrm{n}=500$ sig.level $=0.005$
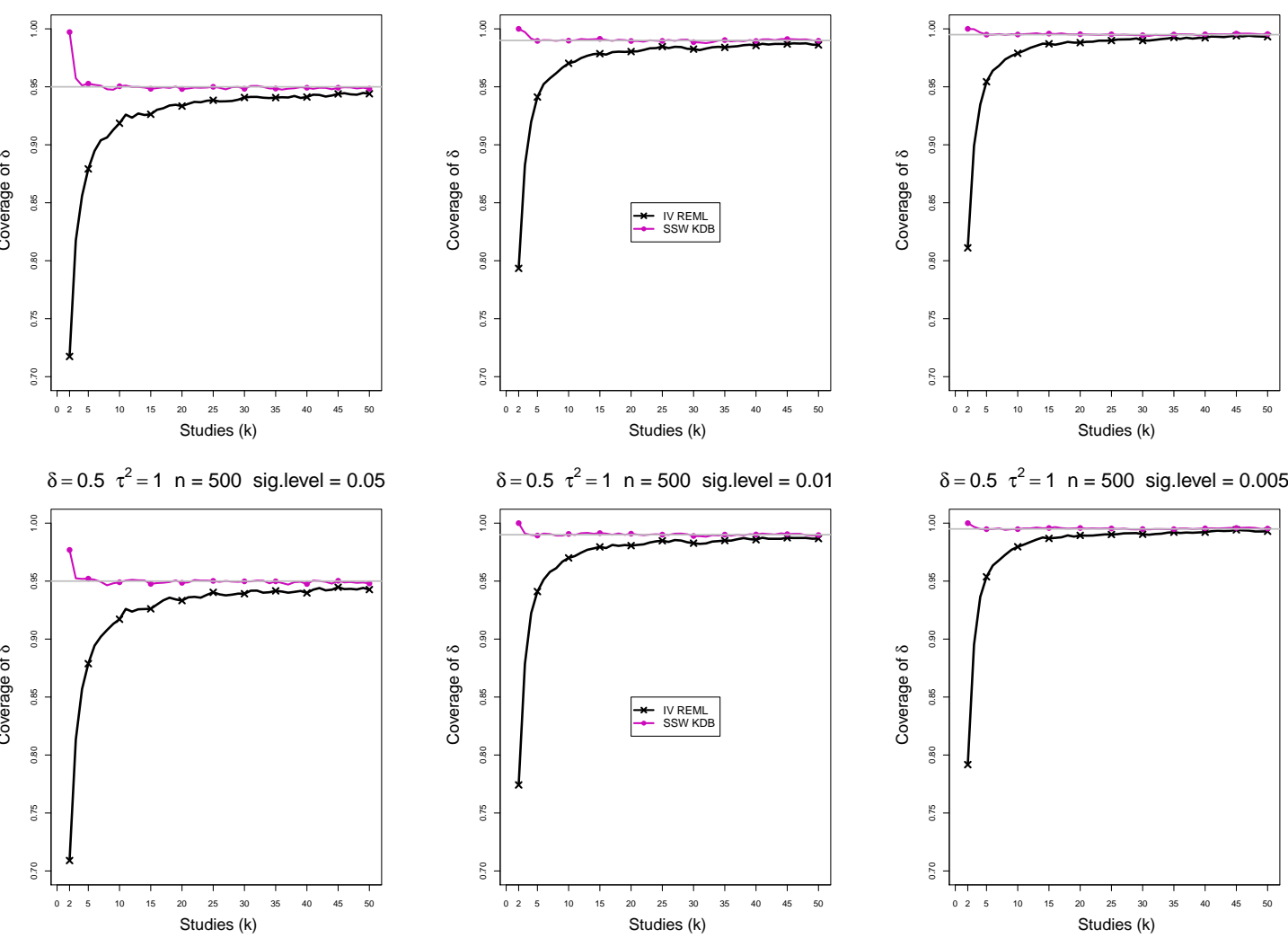

Figure A2. 8: CMA - Coverage of confidence intervals for $\delta=0.5$ at confidence level $1-\alpha$ for significance levels $\alpha$ of $0.05,0.01,0.005, \tau^{2}=0,0.1,0.25,1, n=500, K=50$. Light grey line at $0.95,0.99,0.995$. 

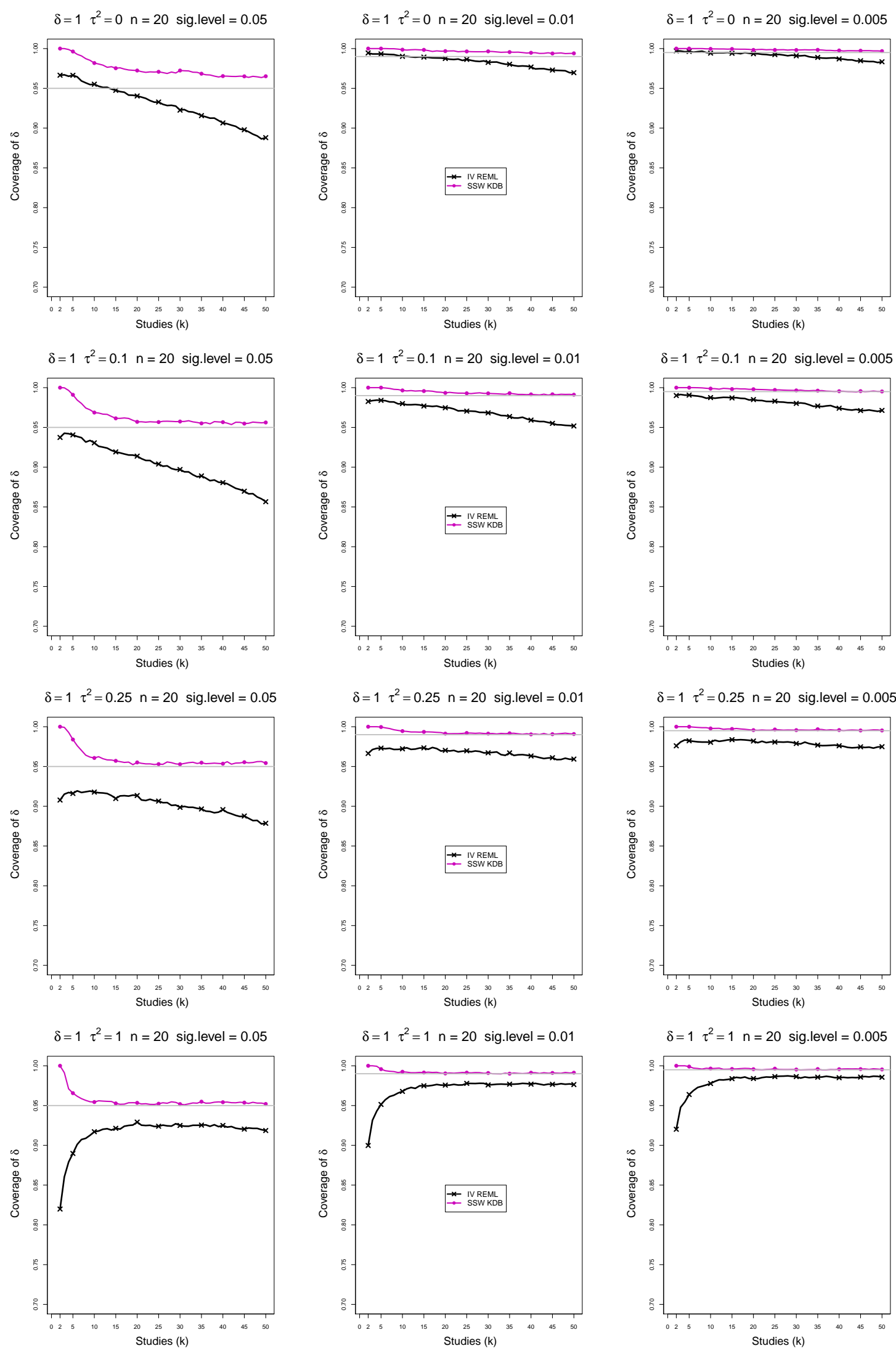

Figure A2. 9: CMA - Coverage of confidence intervals for $\delta=1$ at confidence level $1-\alpha$ for significance levels $\alpha$ of $0.05,0.01,0.005, \tau^{2}=0,0.1,0.25,1, n=20, K=50$. Light grey line at $0.95,0.99,0.995$. 

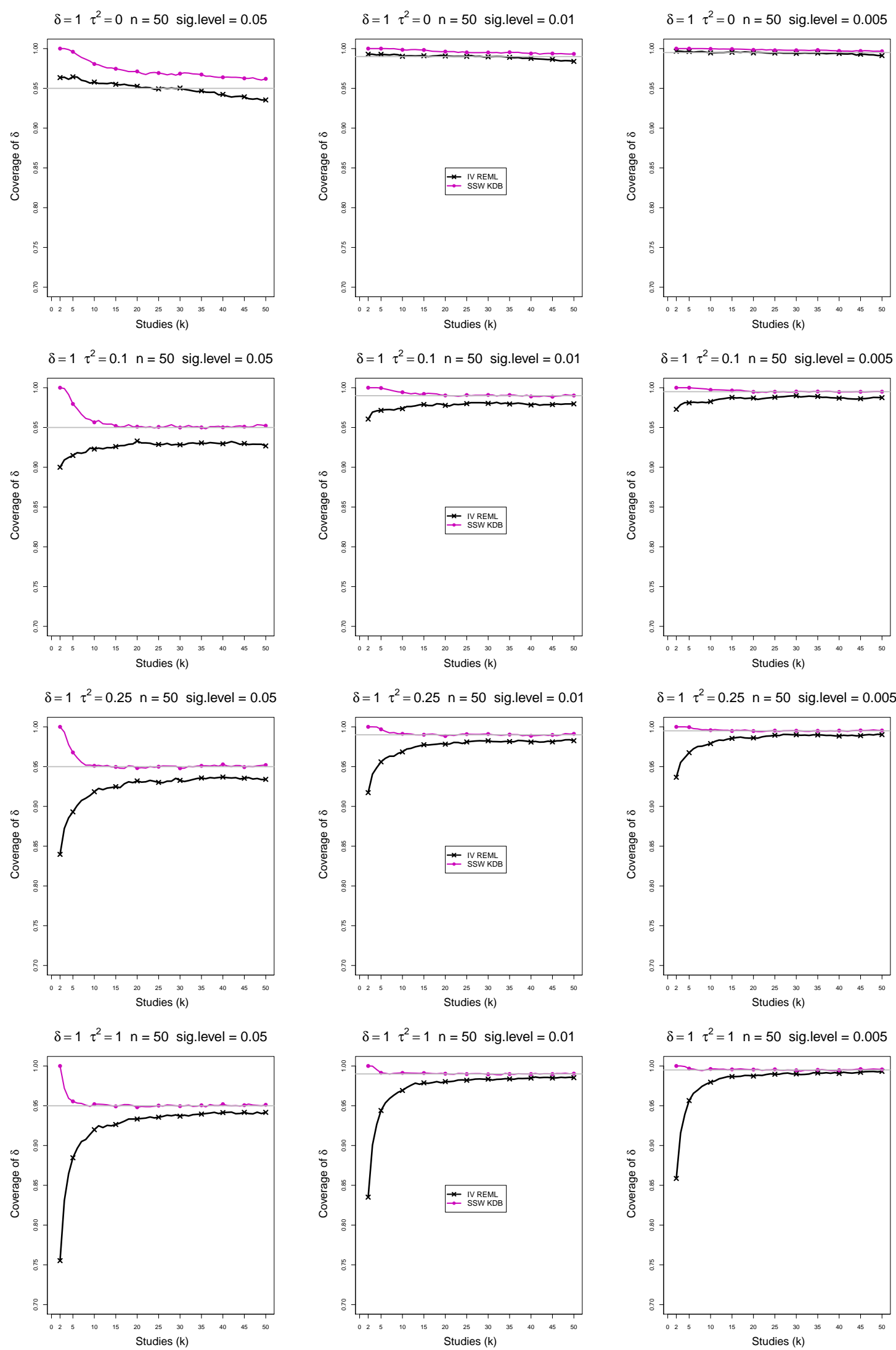

Figure A2. 10: CMA - Coverage of confidence intervals for $\delta=1$ at confidence level $1-\alpha$ for significance levels $\alpha$ of $0.05,0.01,0.005, \tau^{2}=0,0.1,0.25,1, n=50, K=50$. Light grey line at $0.95,0.99,0.995$. 

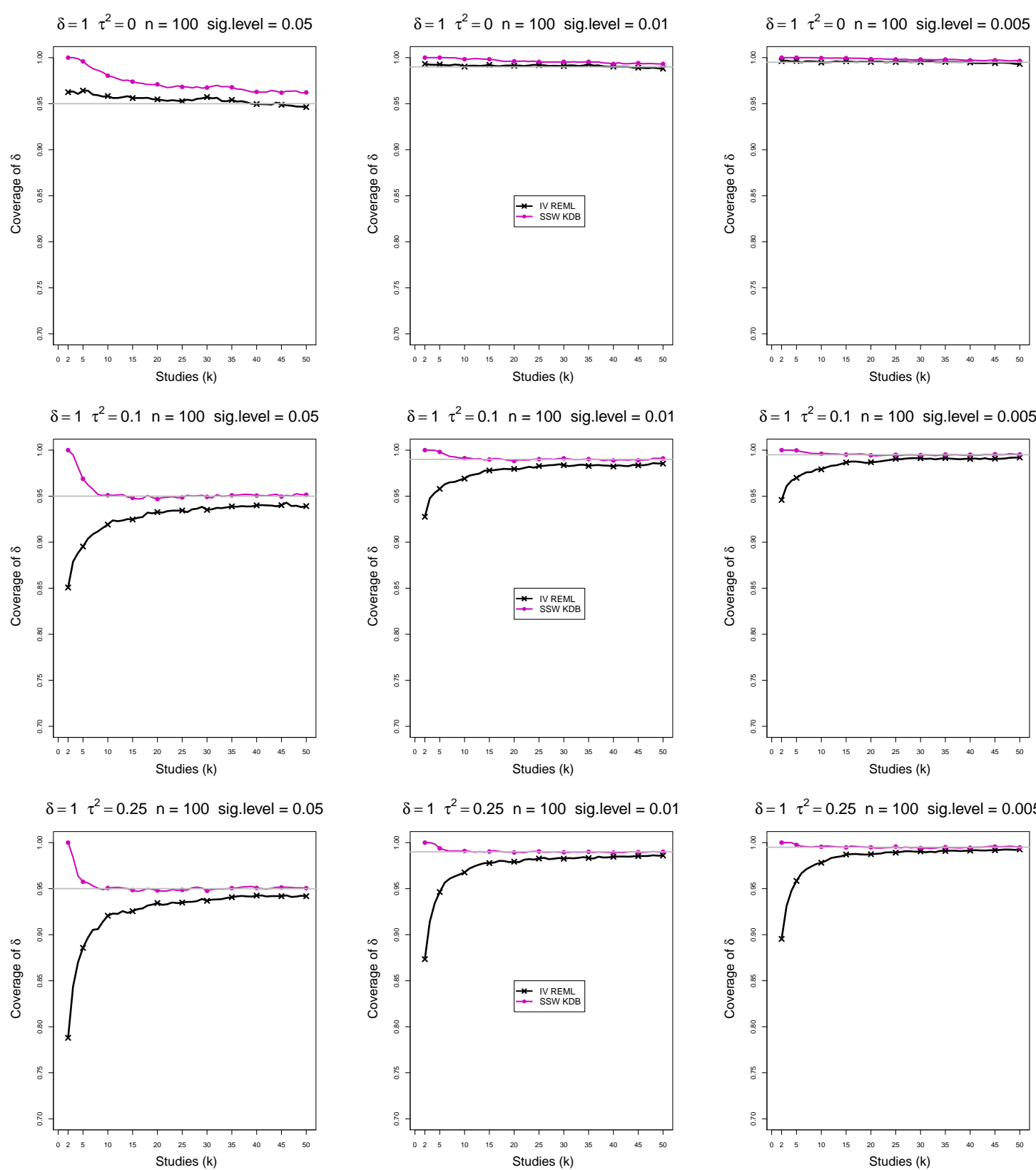

$\delta=1 \tau^{2}=0.25 \mathrm{n}=100$ sig.level $=0.01$

$\delta=1 \tau^{2}=0.25 \mathrm{n}=100$ sig.level $=0.005$
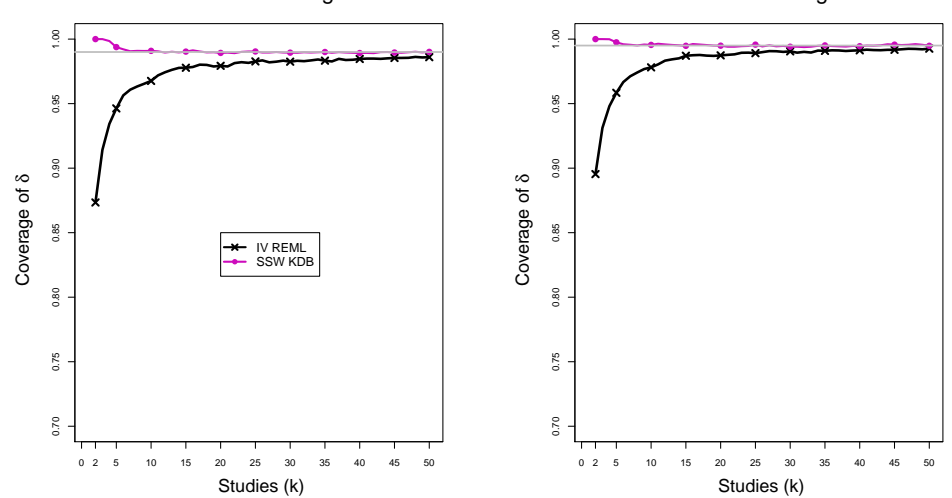

$\delta=1 \quad \tau^{2}=1 \quad \mathrm{n}=100$ sig.level $=0.05$
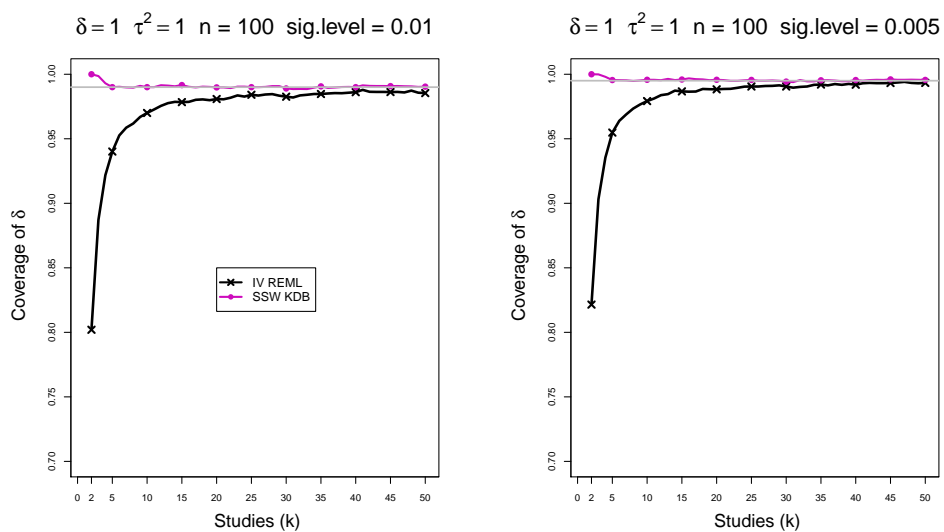

Figure A2. 11: CMA - Coverage of confidence intervals for $\delta=1$ at confidence level $1-\alpha$ for significance levels $\alpha$ of $0.05,0.01,0.005, \tau^{2}=0,0.1,0.25,1, n=100, K=50$. Light grey line at $0.95,0.99,0.995$. 

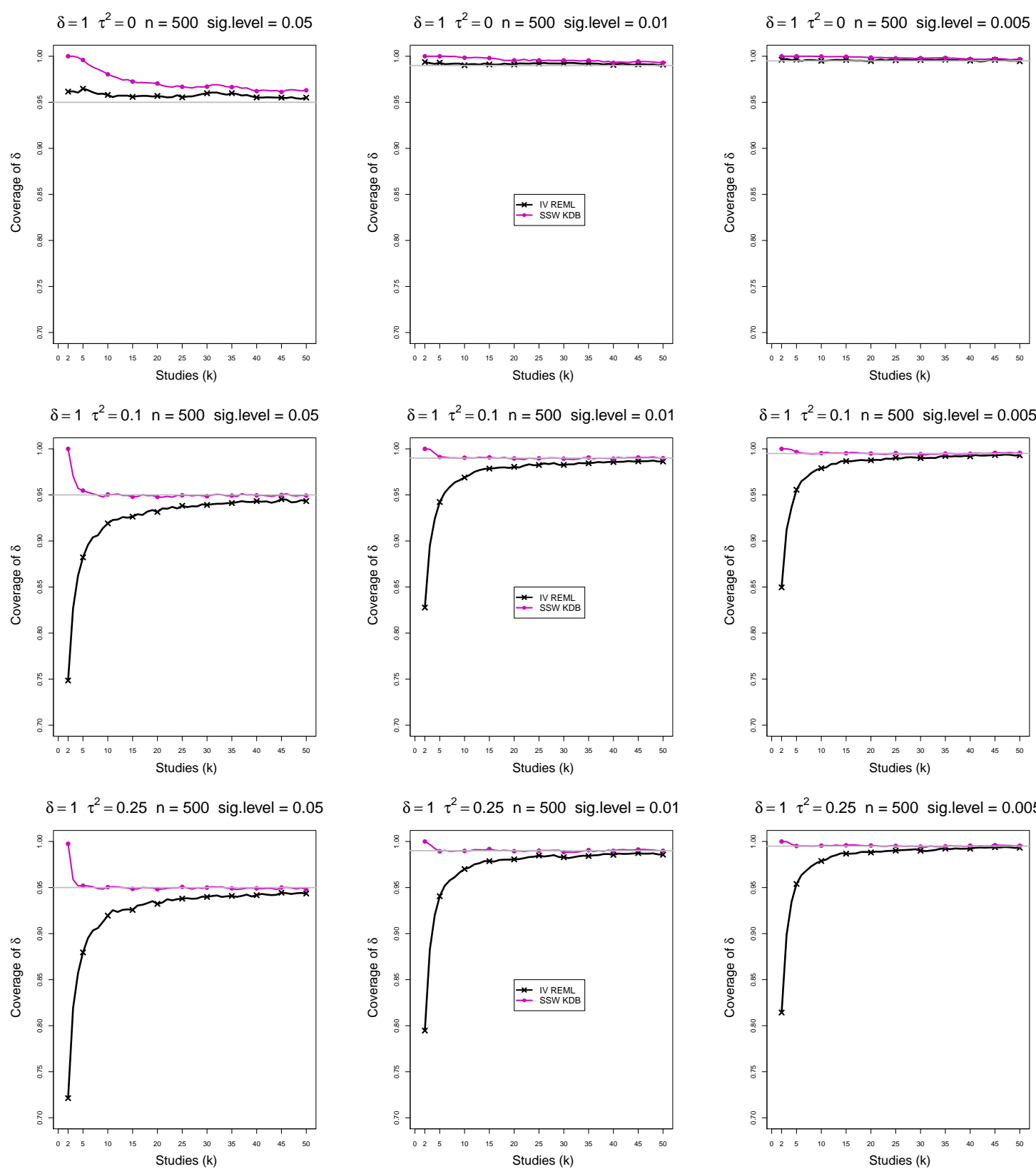

$\delta=1 \quad \tau^{2}=0.25 \quad \mathrm{n}=500$ sig.level $=0.01$

$\delta=1 \tau^{2}=0.25 \mathrm{n}=500$ sig.level $=0.005$
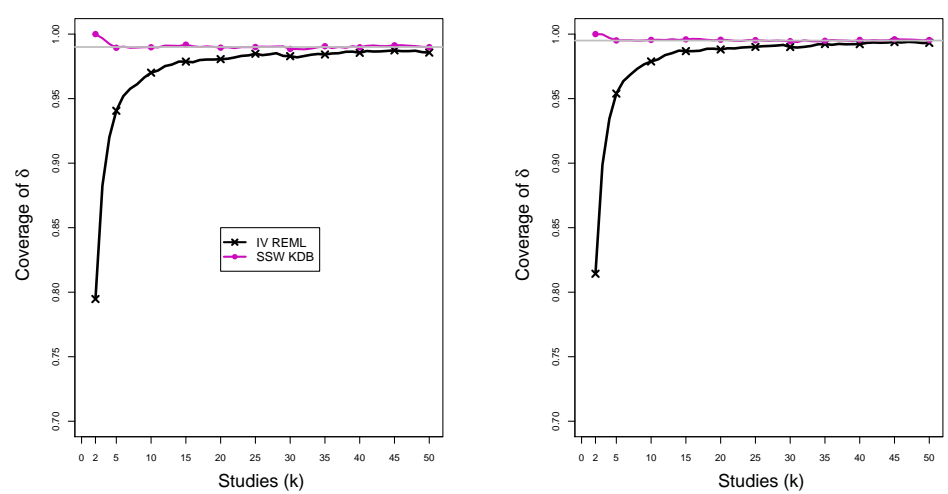

$\delta=1 \quad \tau^{2}=1 \quad \mathrm{n}=500$ sig.level $=0.05$

$\delta=1 \quad \tau^{2}=1 \quad n=500$ sig.level $=0.01$
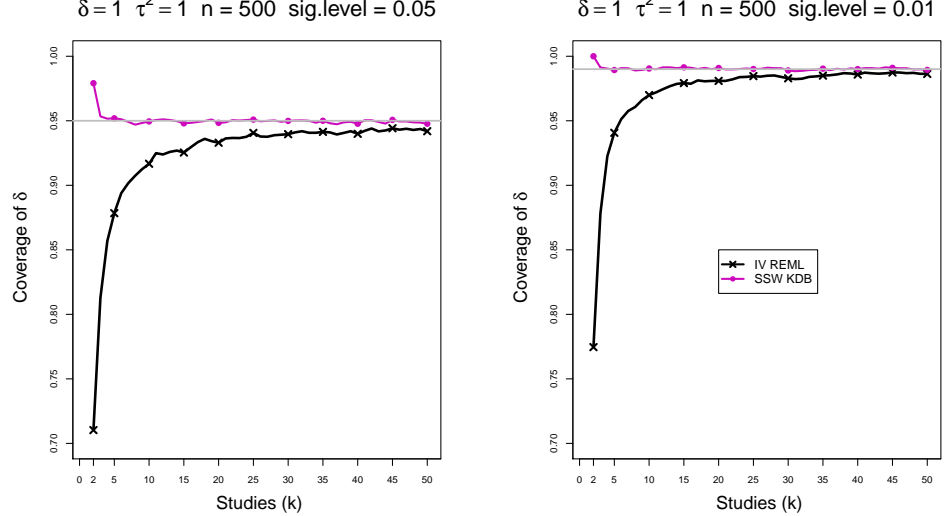

$\delta=1 \quad \tau^{2}=1 \quad \mathrm{n}=500$ sig.level $=0.005$

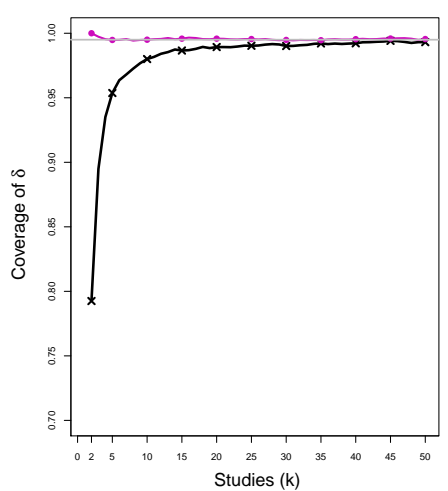

Figure A2. 12: CMA - Coverage of confidence intervals for $\delta=1$ at confidence level $1-\alpha$ for significance levels $\alpha$ of $0.05,0.01,0.005, \tau^{2}=0,0.1,0.25,1, n=500, K=50$. Light grey line at $0.95,0.99,0.995$. 


\section{A3. Coverage of one-sided interval estimators of $\delta$}

For one-sided coverage of $\delta$ in this section, each figure corresponds to a value of $\delta(=0$, $0.5,1)$, a set of values of $\tau^{2}(=0,0.1,0.25,1)$, a value of $n(=20,50,100,500)$ and a set of values of significance level $(=0.05,0.01,0.005)$ while the study number $k$ increases to maximum number $K=50$.

Each figure contains a panel (with study $k$ on the horizontal axis) for each combination of parameters.

The one-sided interval estimators of $\delta$ are

- IV REML (Inverse variance weighted mean with the between-study variance $\tau^{2}$ estimated by Restricted maximum likelihood as center and the lower or the upper confidence limit (as required) defined by taking out or adding critical value from Normal distribution times square-root of the inverse sum of weights )

- SSW KDB (Sample size weighted (SSW) as center and the lower or the upper confidence limit (as required) defined by taking out or adding critical value from $t_{k-1}$ times estimated standard deviation of SSW with $\hat{\tau}^{2}=\hat{\tau}_{K D B}^{2}$, the Kulinskaya-Dollinger-Bjørkestøl (2011) estimator of $\tau^{2}$ ) 

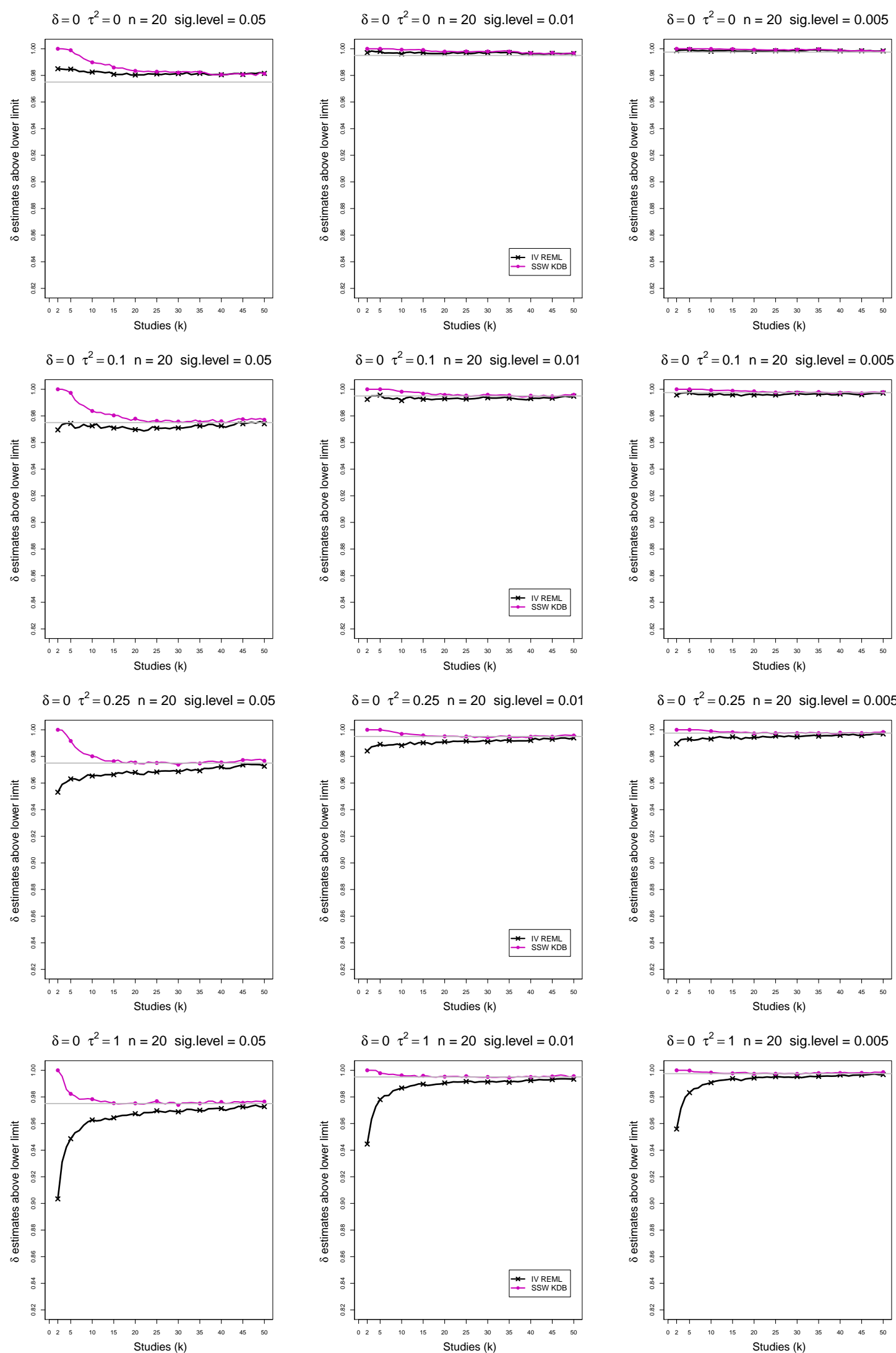

Figure A3. 1: CMA - Coverage of lower confidence intervals of $\delta$ (above lower limit) at confidence limit $1-\alpha / 2$ for 2-sided significance level $\alpha$ of $0.05,0.01,0.005$ for $\delta=0$, $\tau^{2}=0,0.1,0.25,1, n=20, K=50$. Light grey line at $0.975,0.995,0.9975$. 

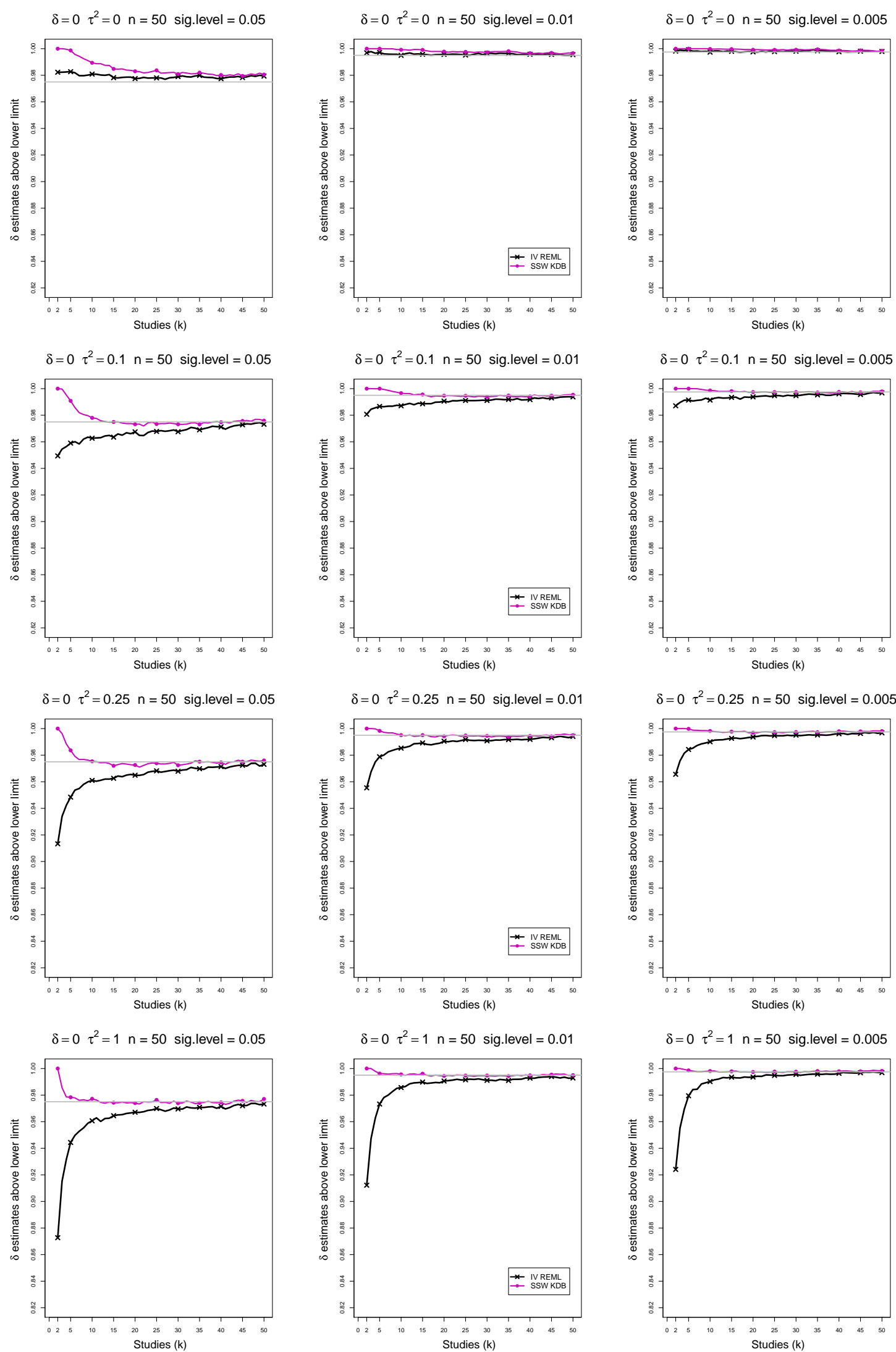

Figure A3. 2: CMA - Coverage of lower confidence intervals of $\delta$ (above lower limit) at confidence limit $1-\alpha / 2$ for 2 -sided significance level $\alpha$ of $0.05,0.01,0.005$ for $\delta=0$, $\tau^{2}=0,0.1,0.25,1, n=50, K=50$. Light grey line at $0.975,0.995,0.9975$. 

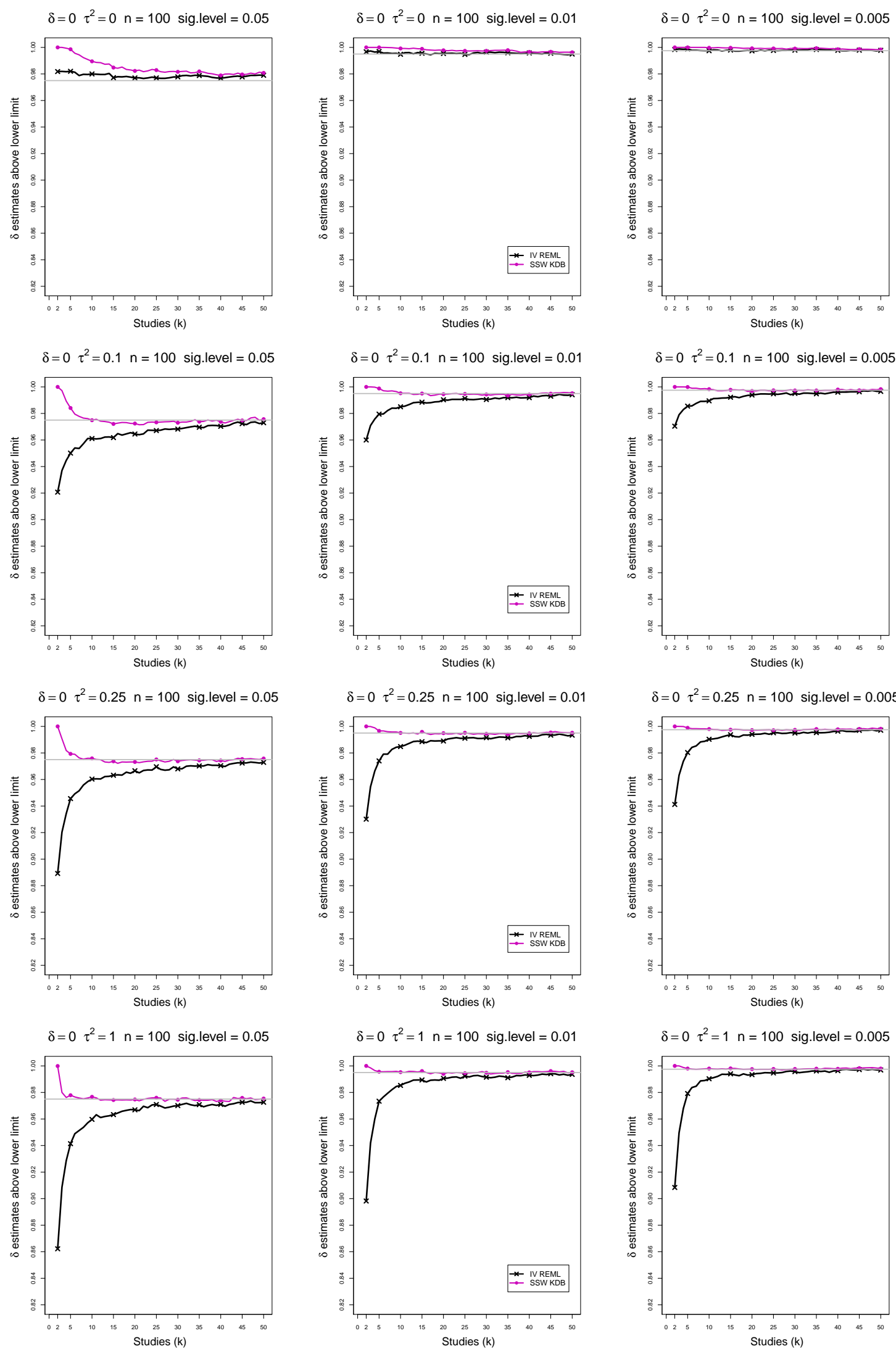

Figure A3. 3: CMA - Coverage of lower confidence intervals of $\delta$ (above lower limit) at confidence limit $1-\alpha / 2$ for 2 -sided significance level $\alpha$ of $0.05,0.01,0.005$ for $\delta=0$, $\tau^{2}=0,0.1,0.25,1, n=100, K=50$. Light grey line at $0.975,0.995,0.9975$. 

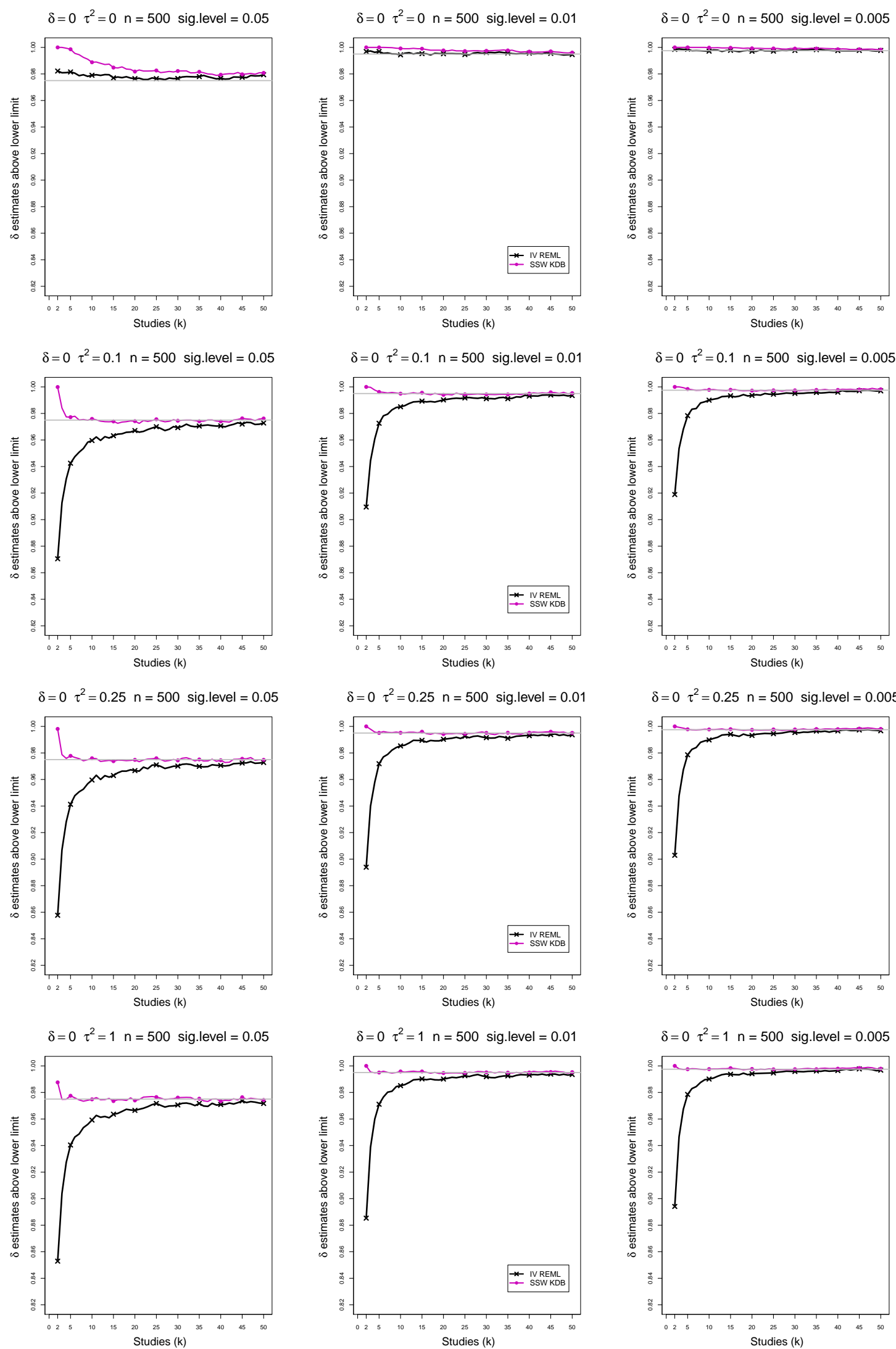

Figure A3. 4: CMA - Coverage of lower confidence intervals of $\delta$ (above lower limit) at confidence limit $1-\alpha / 2$ for 2 -sided significance level $\alpha$ of $0.05,0.01,0.005$ for $\delta=0$, $\tau^{2}=0,0.1,0.25,1, n=500, K=50$. Light grey line at $0.975,0.995,0.9975$. 

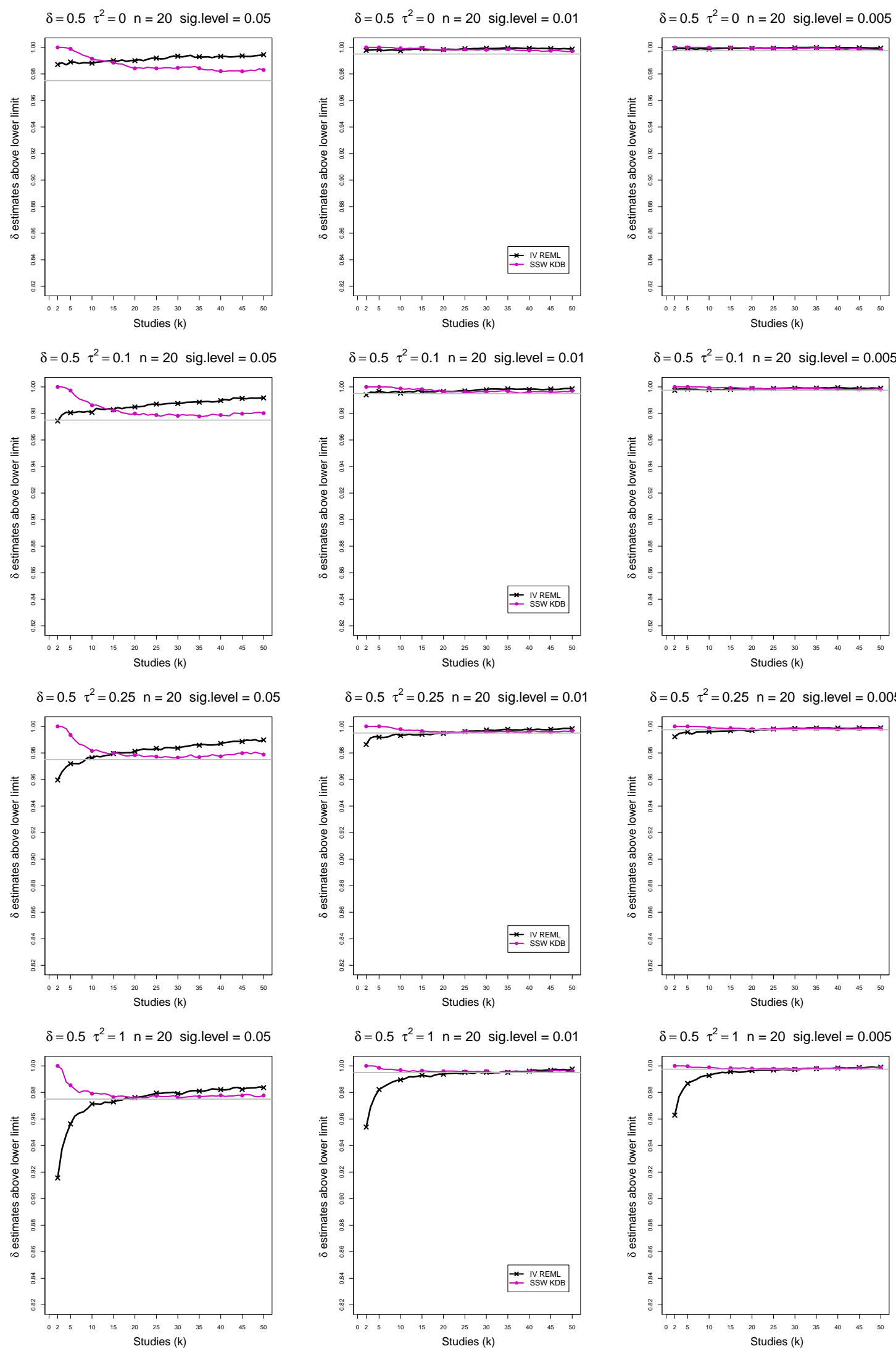

Figure A3. 5: CMA - Coverage of lower confidence intervals of $\delta$ (above lower limit) at confidence limit $1-\alpha / 2$ for 2-sided significance level $\alpha$ of $0.05,0.01,0.005$ for $\delta=0.5$, $\tau^{2}=0,0.1,0.25,1, n=20, K=50$. Light grey line at $0.975,0.995,0.9975$. 

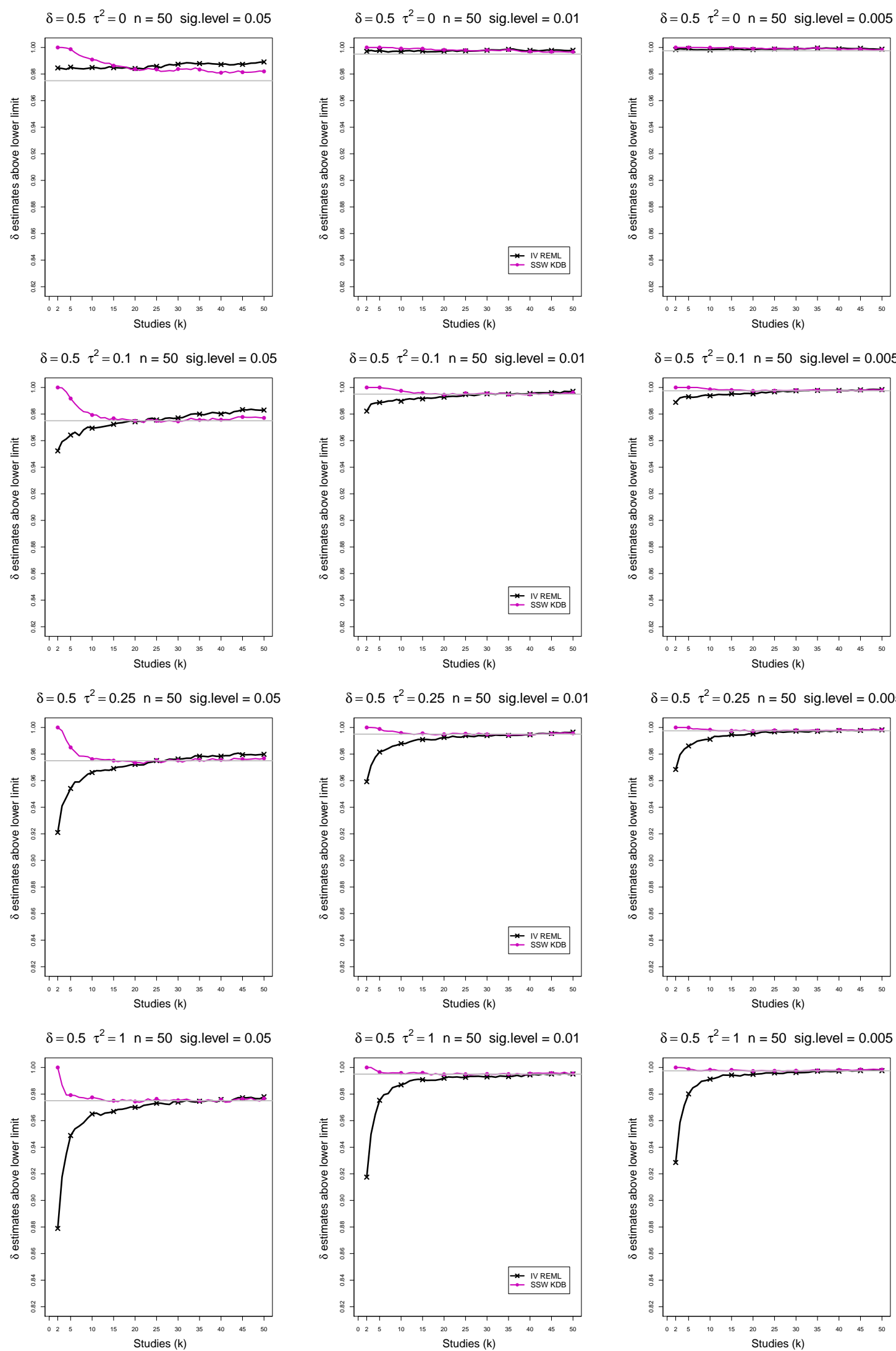

Figure A3. 6: CMA - Coverage of lower confidence intervals of $\delta$ (above lower limit) at confidence limit $1-\alpha / 2$ for 2-sided significance level $\alpha$ of $0.05,0.01,0.005$ for $\delta=0.5$, $\tau^{2}=0,0.1,0.25,1, n=50, K=50$. Light grey line at $0.975,0.995,0.9975$. 

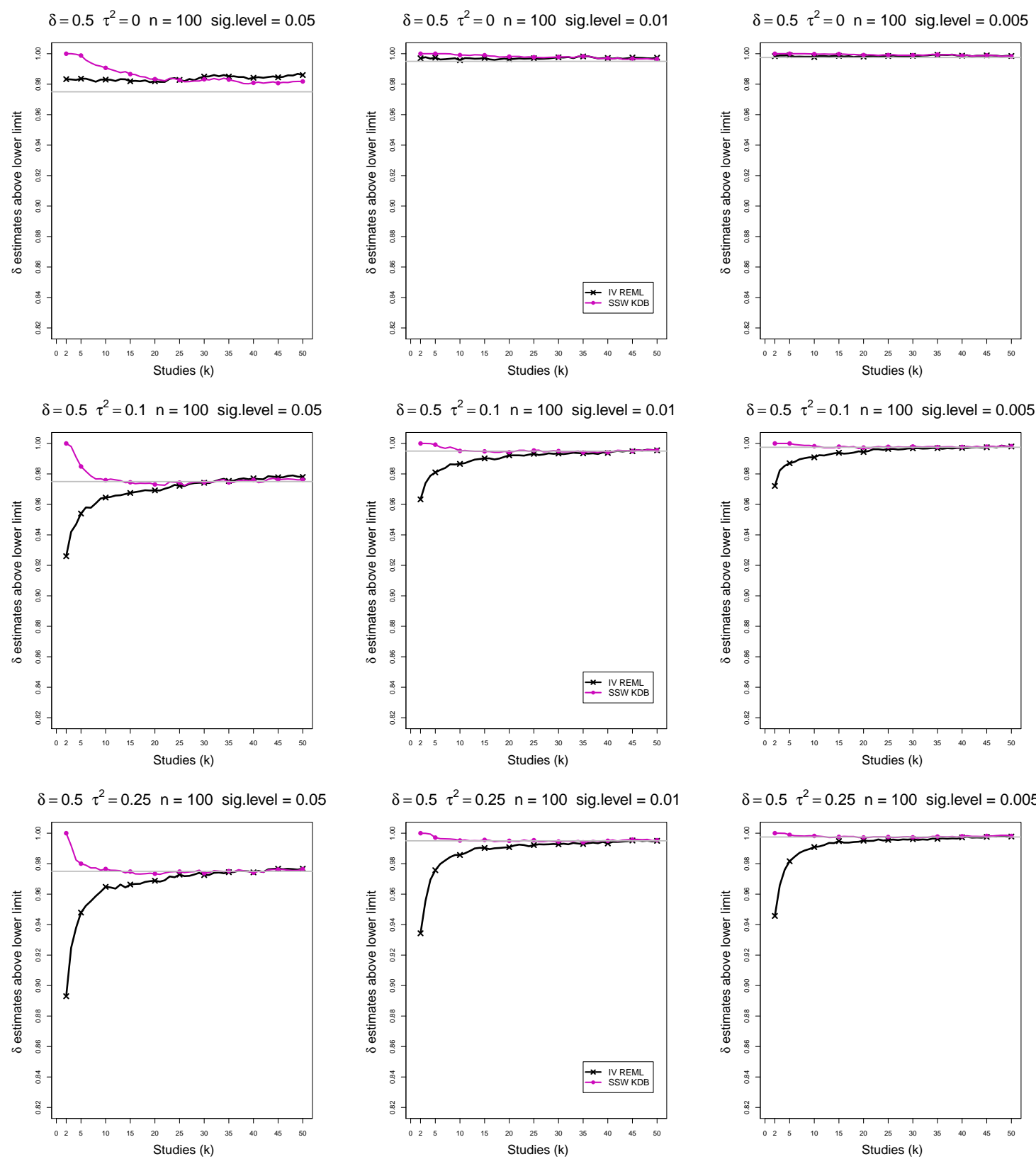

$\delta=0.5 \tau^{2}=0.25 \mathrm{n}=100$ sig.level $=0.005$
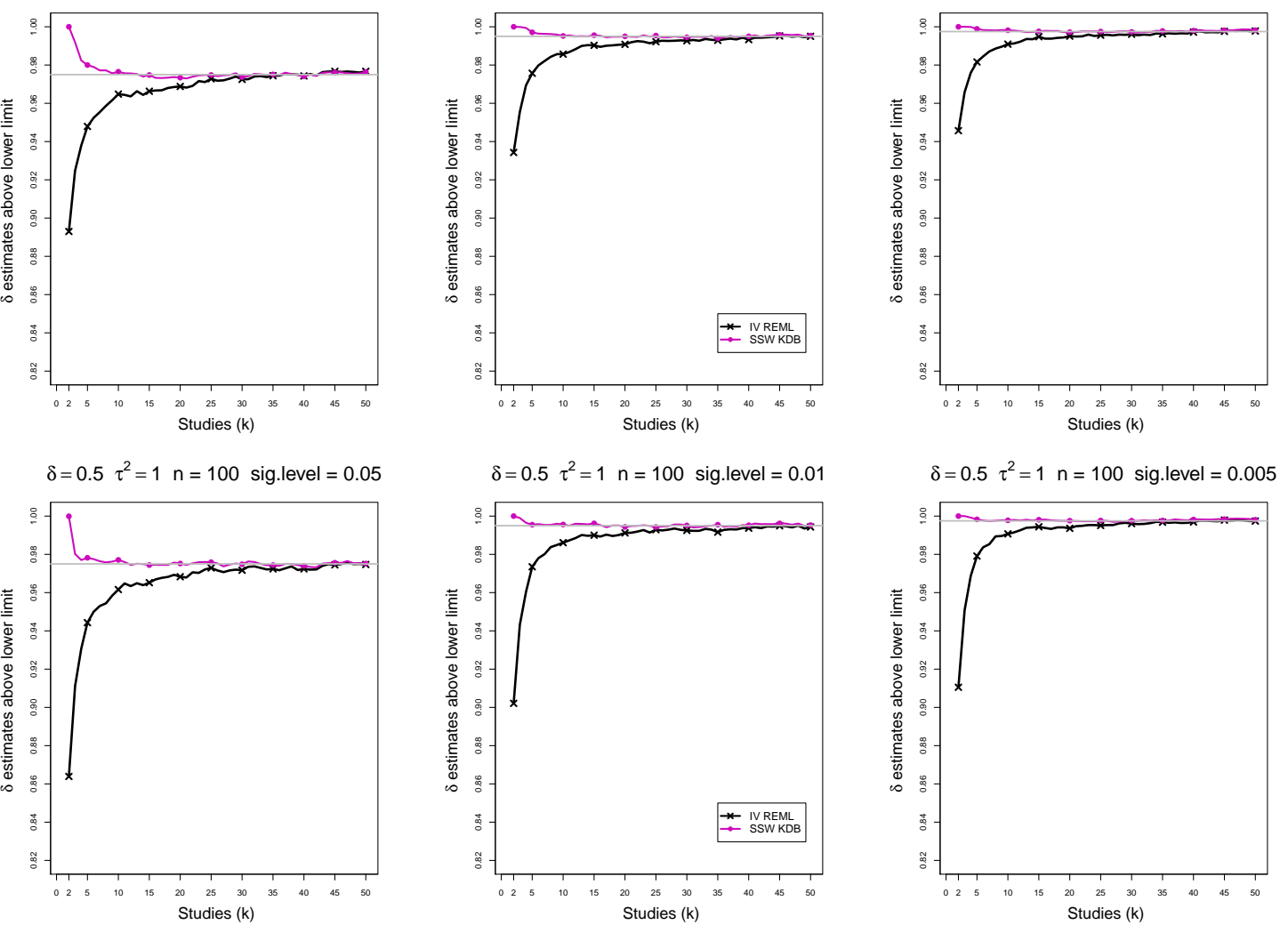

Figure A3. 7: CMA - Coverage of lower confidence intervals of $\delta$ (above lower limit) at confidence limit $1-\alpha / 2$ for 2-sided significance level $\alpha$ of $0.05,0.01,0.005$ for $\delta=0.5$, $\tau^{2}=0,0.1,0.25,1, n=100, K=50$. Light grey line at $0.975,0.995,0.9975$. 

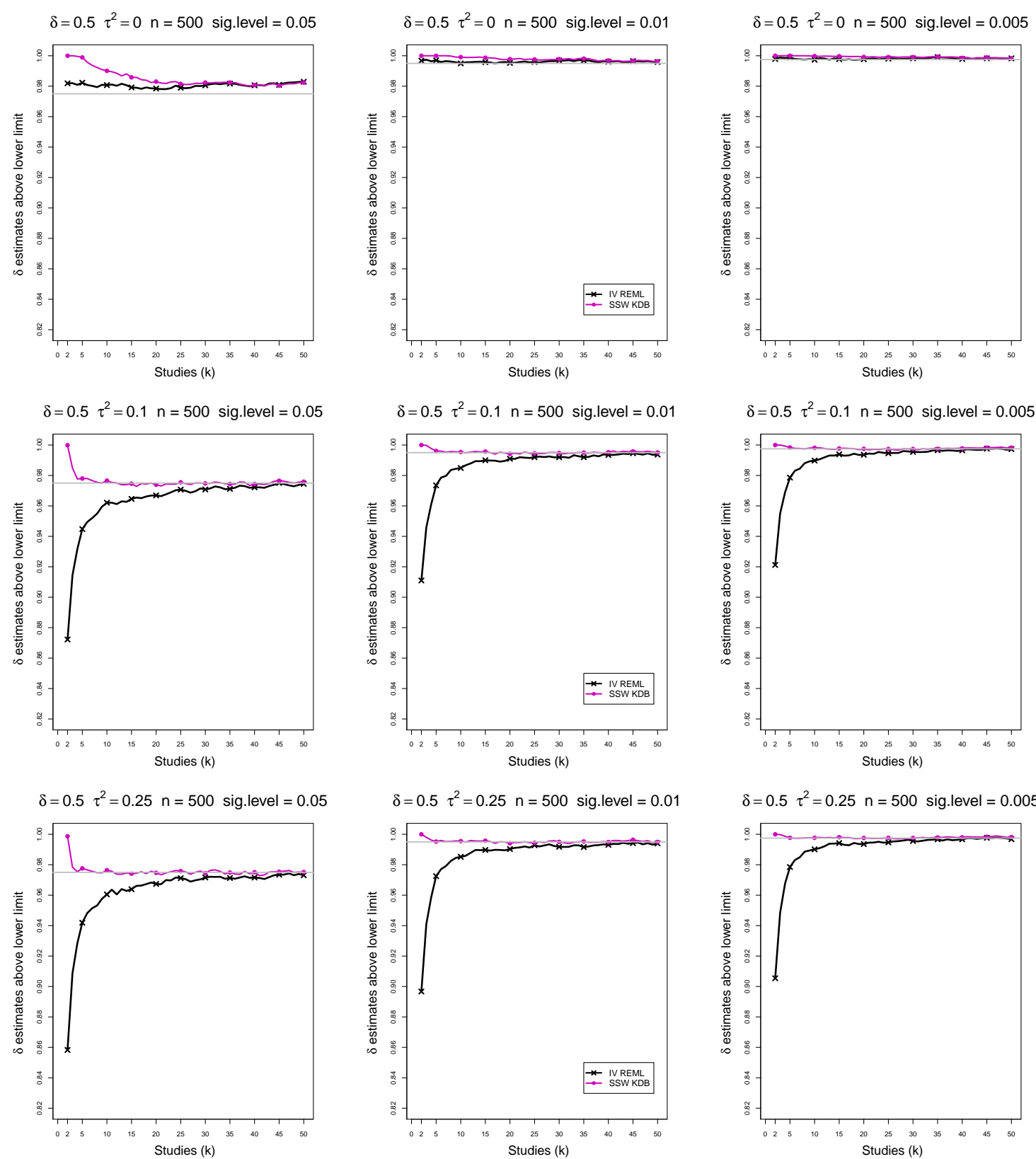

$\delta=0.5 \tau^{2}=0.25 \mathrm{n}=500$ sig.level $=0.005$
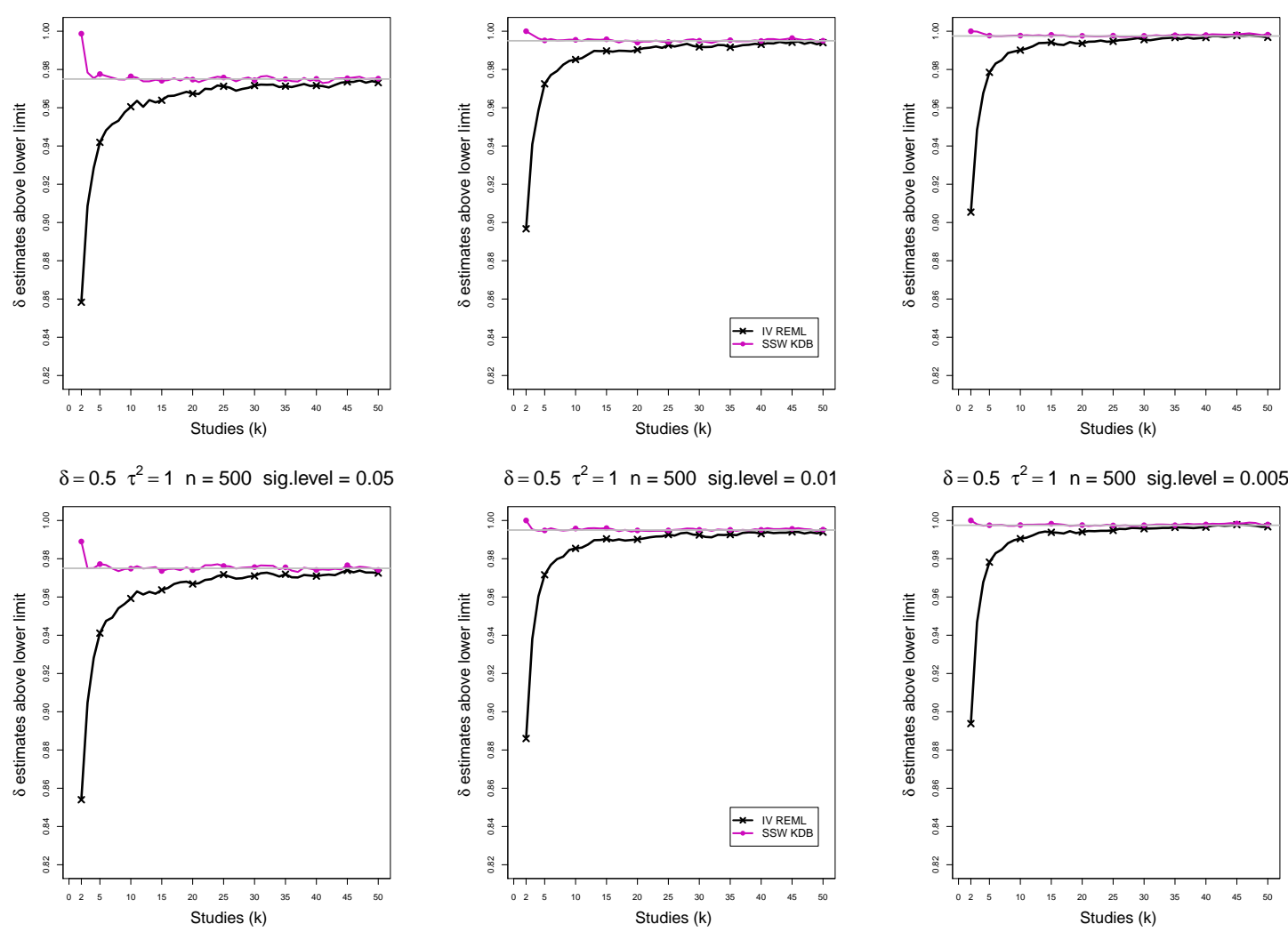

$\delta=0.5 \quad \tau^{2}=1 \quad \mathrm{n}=500$ sig.level $=0.005$

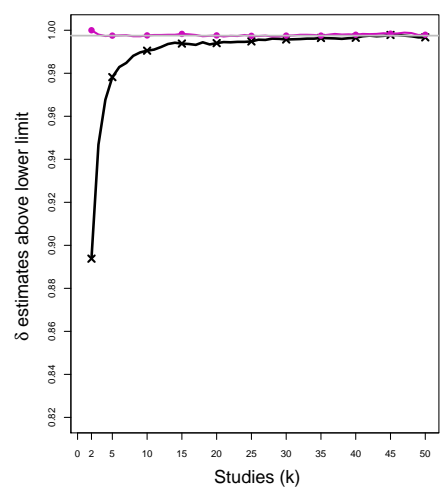

Figure A3. 8: CMA - Coverage of lower confidence intervals of $\delta$ (above lower limit) at confidence limit $1-\alpha / 2$ for 2-sided significance level $\alpha$ of $0.05,0.01,0.005$ for $\delta=0.5$, $\tau^{2}=0,0.1,0.25,1, n=500, K=50$. Light grey line at $0.975,0.995,0.9975$. 

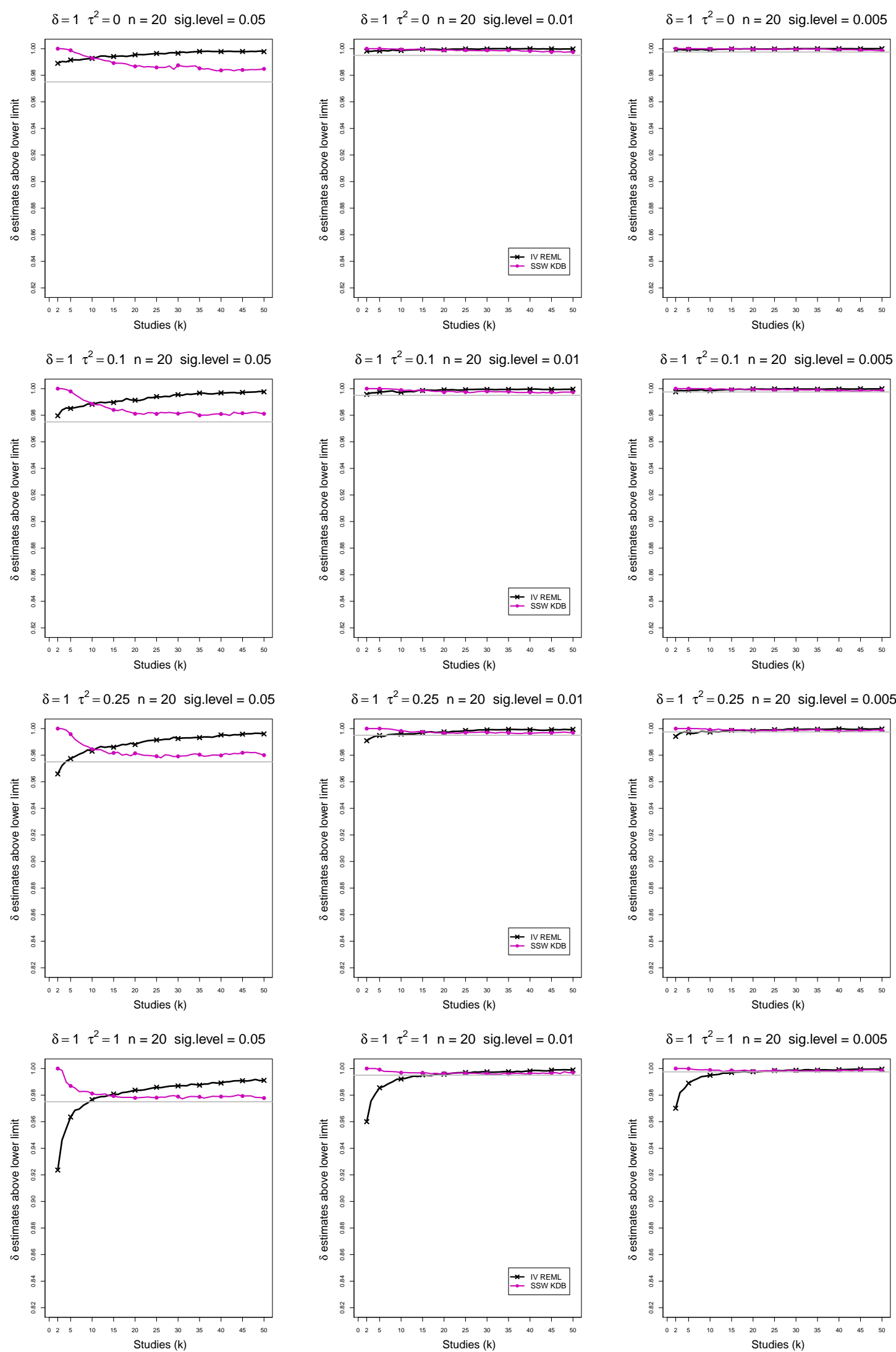

Figure A3. 9: CMA - Coverage of lower confidence intervals of $\delta$ (above lower limit) at confidence limit $1-\alpha / 2$ for 2 -sided significance level $\alpha$ of $0.05,0.01,0.005$ for $\delta=1$, $\tau^{2}=0,0.1,0.25,1, n=20, K=50$. Light grey line at $0.975,0.995,0.9975$. 

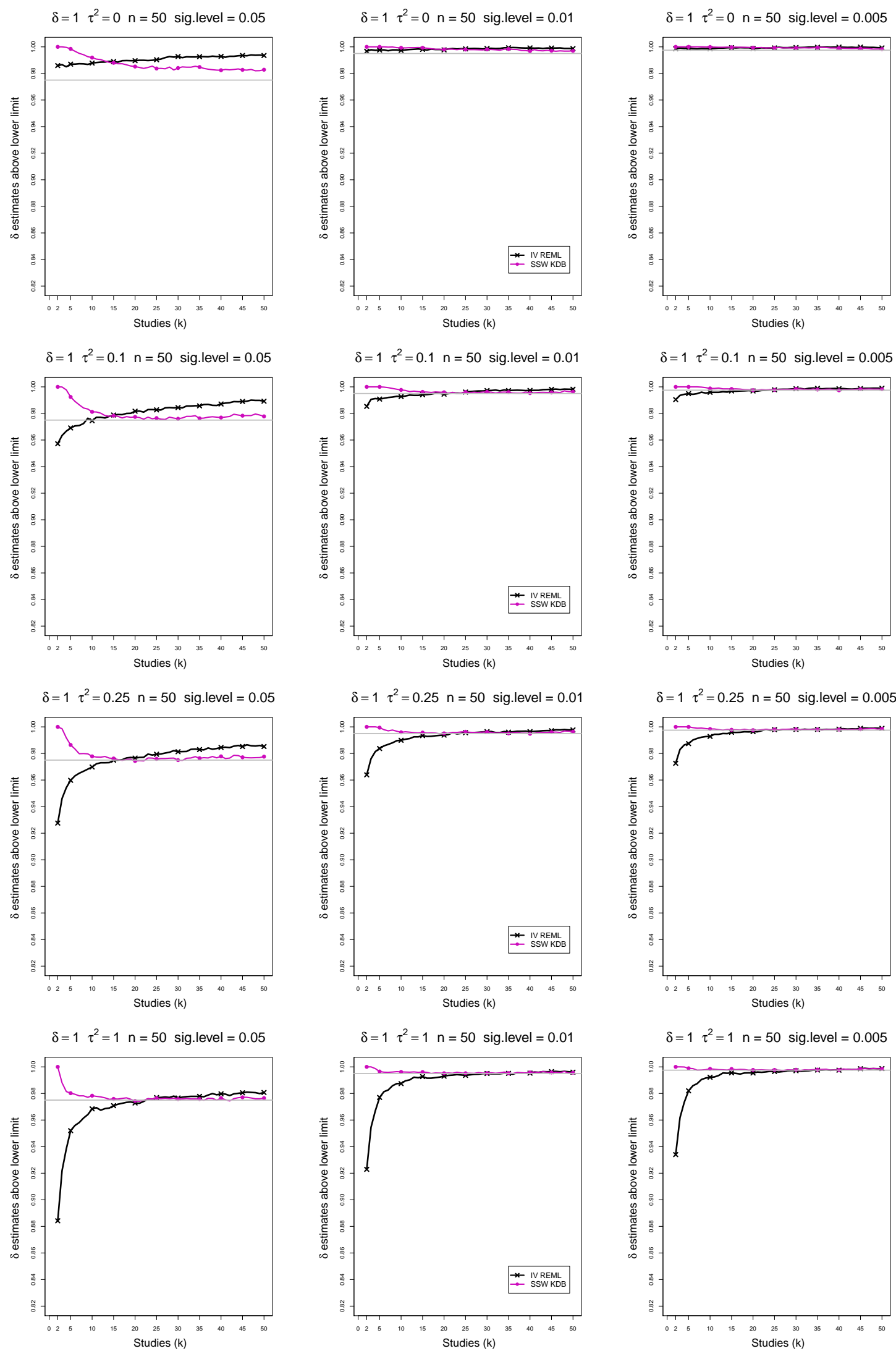

Figure A3. 10: CMA - Coverage of lower confidence intervals of $\delta$ (above lower limit) at confidence limit $1-\alpha / 2$ for 2 -sided significance level $\alpha$ of $0.05,0.01,0.005$ for $\delta=1$, $\tau^{2}=0,0.1,0.25,1, n=50, K=50$. Light grey line at $0.975,0.995,0.9975$. 

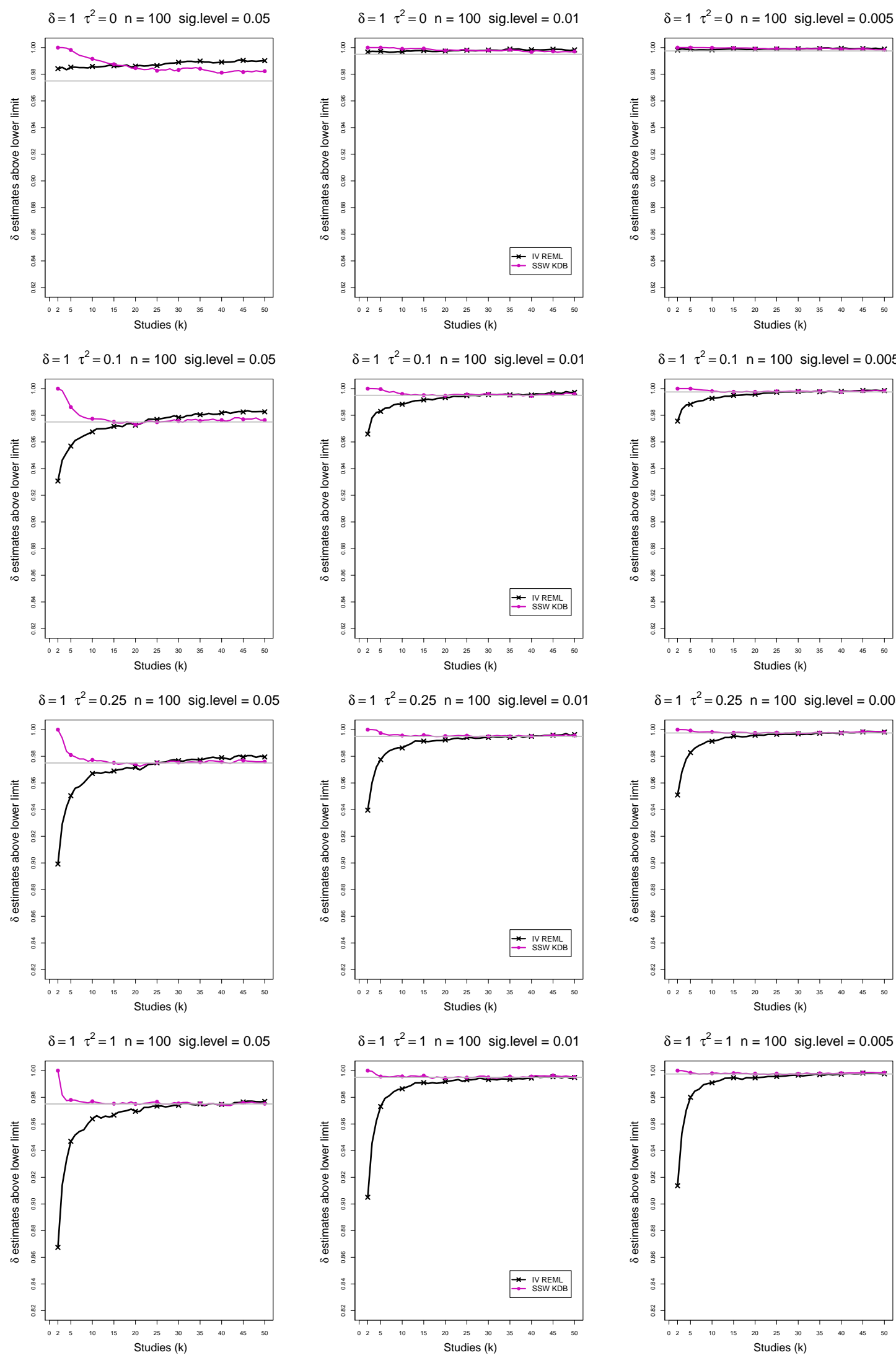

Figure A3. 11: CMA - Coverage of lower confidence intervals of $\delta$ (above lower limit) at confidence limit $1-\alpha / 2$ for 2 -sided significance level $\alpha$ of $0.05,0.01,0.005$ for $\delta=1$, $\tau^{2}=0,0.1,0.25,1, n=100, K=50$. Light grey line at $0.975,0.995,0.9975$. 

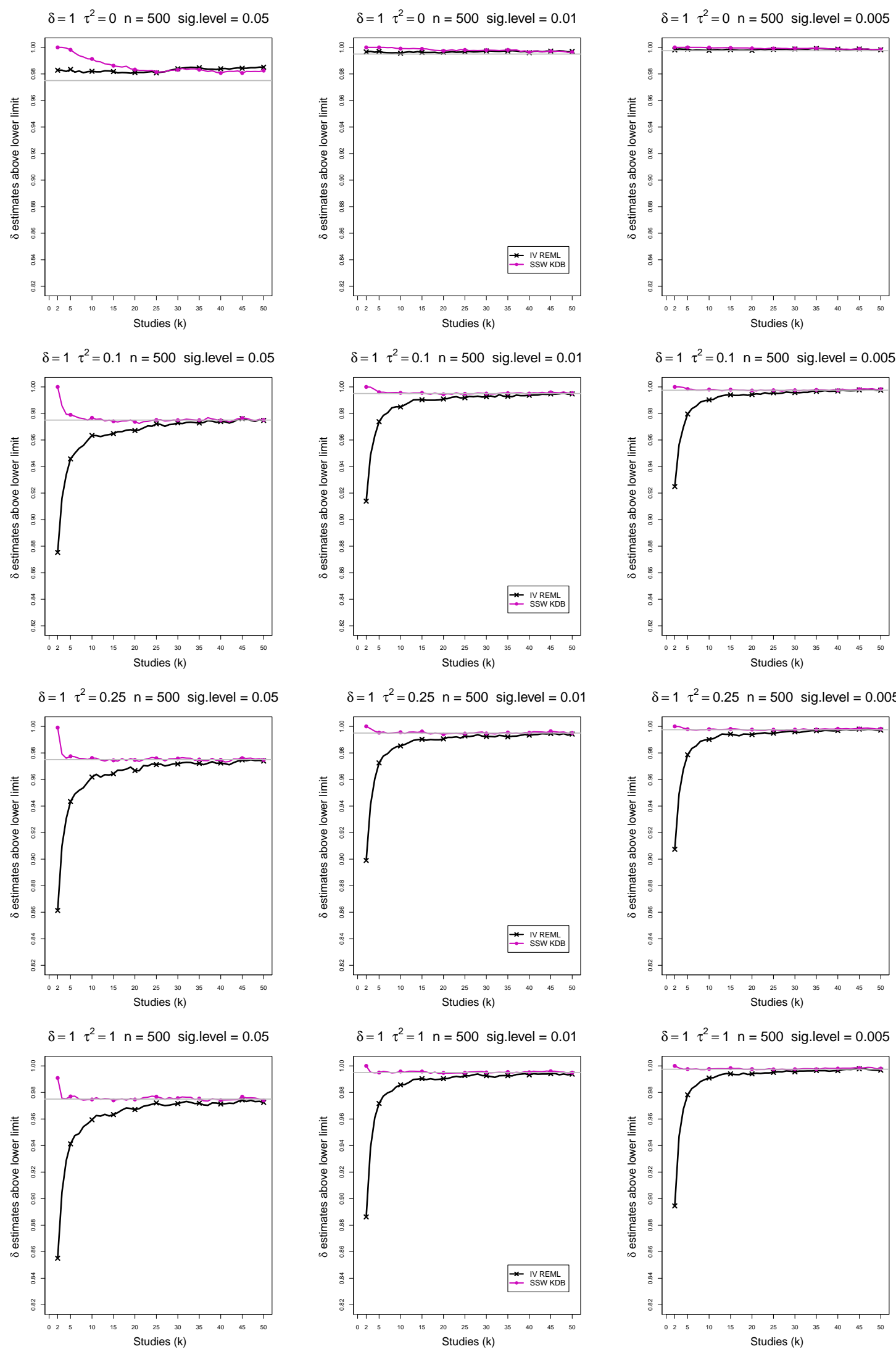

Figure A3. 12: CMA - Coverage of lower confidence intervals of $\delta$ (above lower limit) at confidence limit $1-\alpha / 2$ for 2 -sided significance level $\alpha$ of $0.05,0.01,0.005$ for $\delta=1$, $\tau^{2}=0,0.1,0.25,1, n=500, K=50$. Light grey line at $0.975,0.995,0.9975$. 

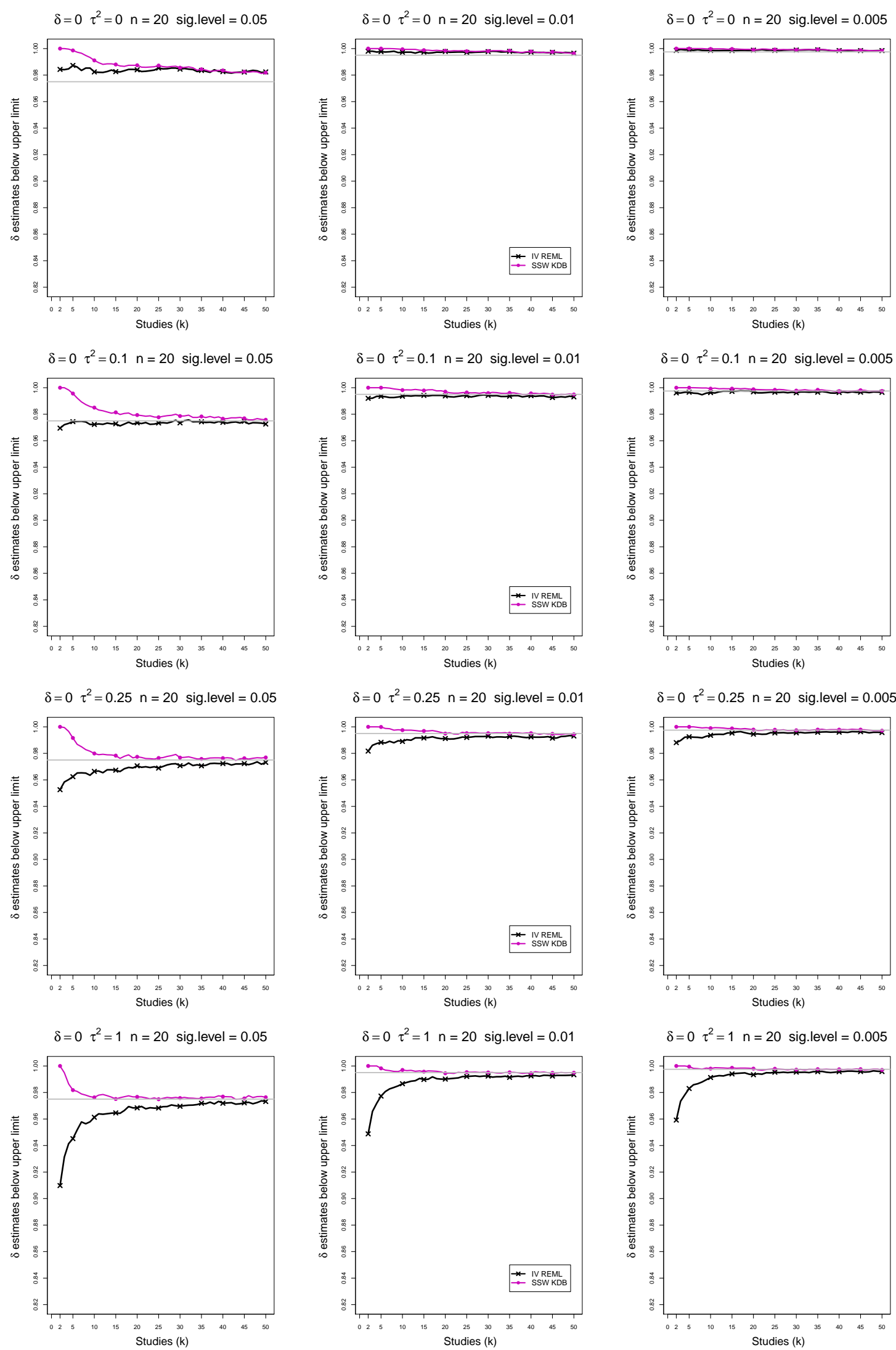

Figure A3. 13: CMA - Coverage of upper confidence intervals of $\delta$ (below upper limit) at confidence limit $1-\alpha / 2$ for 2 -sided significance level $\alpha$ of $0.05,0.01,0.005$ for $\delta=0$, $\tau^{2}=0,0.1,0.25,1, n=20, K=50$. Light grey line at $0.975,0.995,0.9975$. 

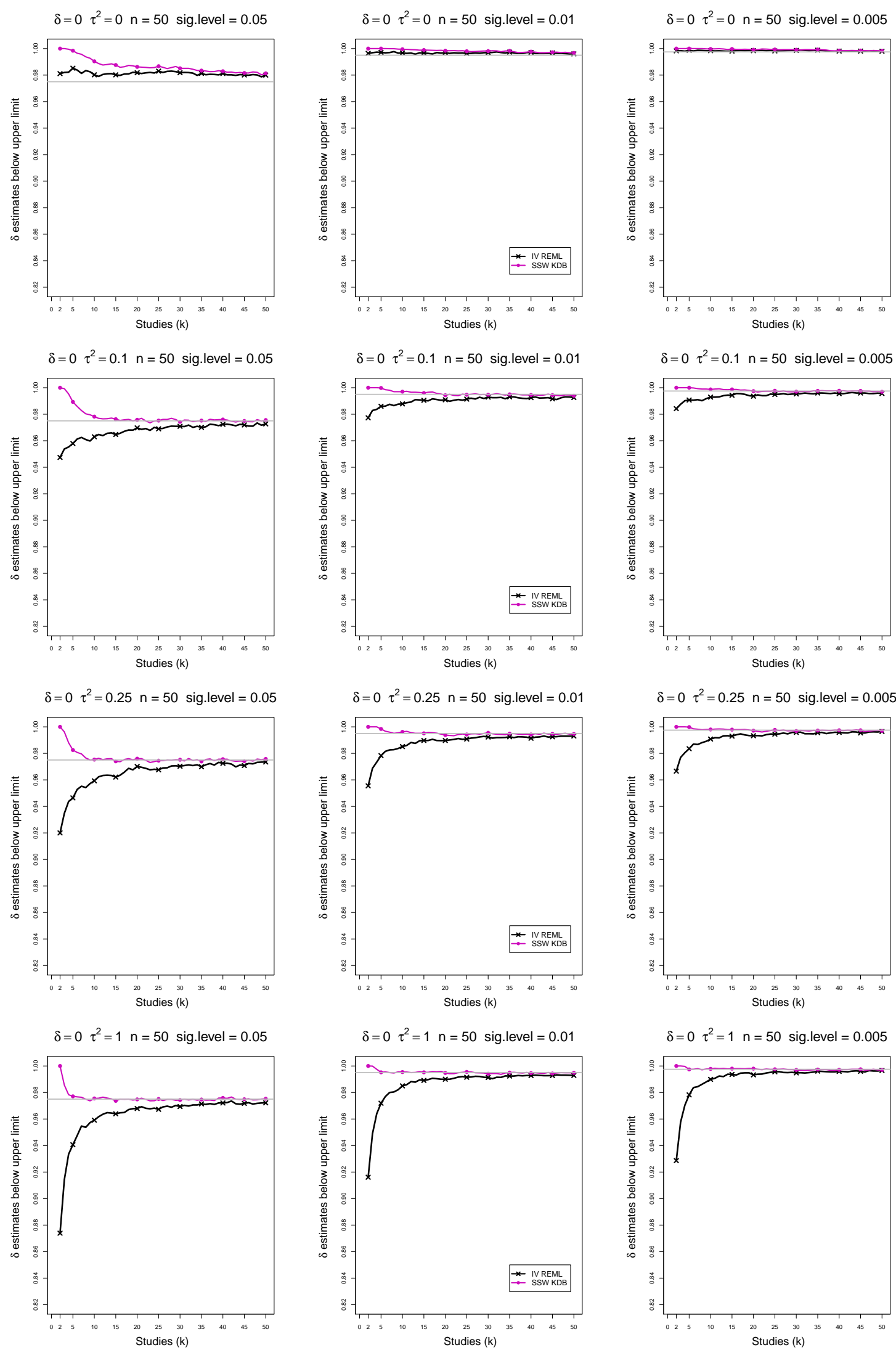

Figure A3. 14: CMA - Coverage of upper confidence intervals of $\delta$ (below upper limit) at confidence limit $1-\alpha / 2$ for 2-sided significance level $\alpha$ of $0.05,0.01,0.005$ for $\delta=0$, $\tau^{2}=0,0.1,0.25,1, n=50, K=50$. Light grey line at $0.975,0.995,0.9975$. 

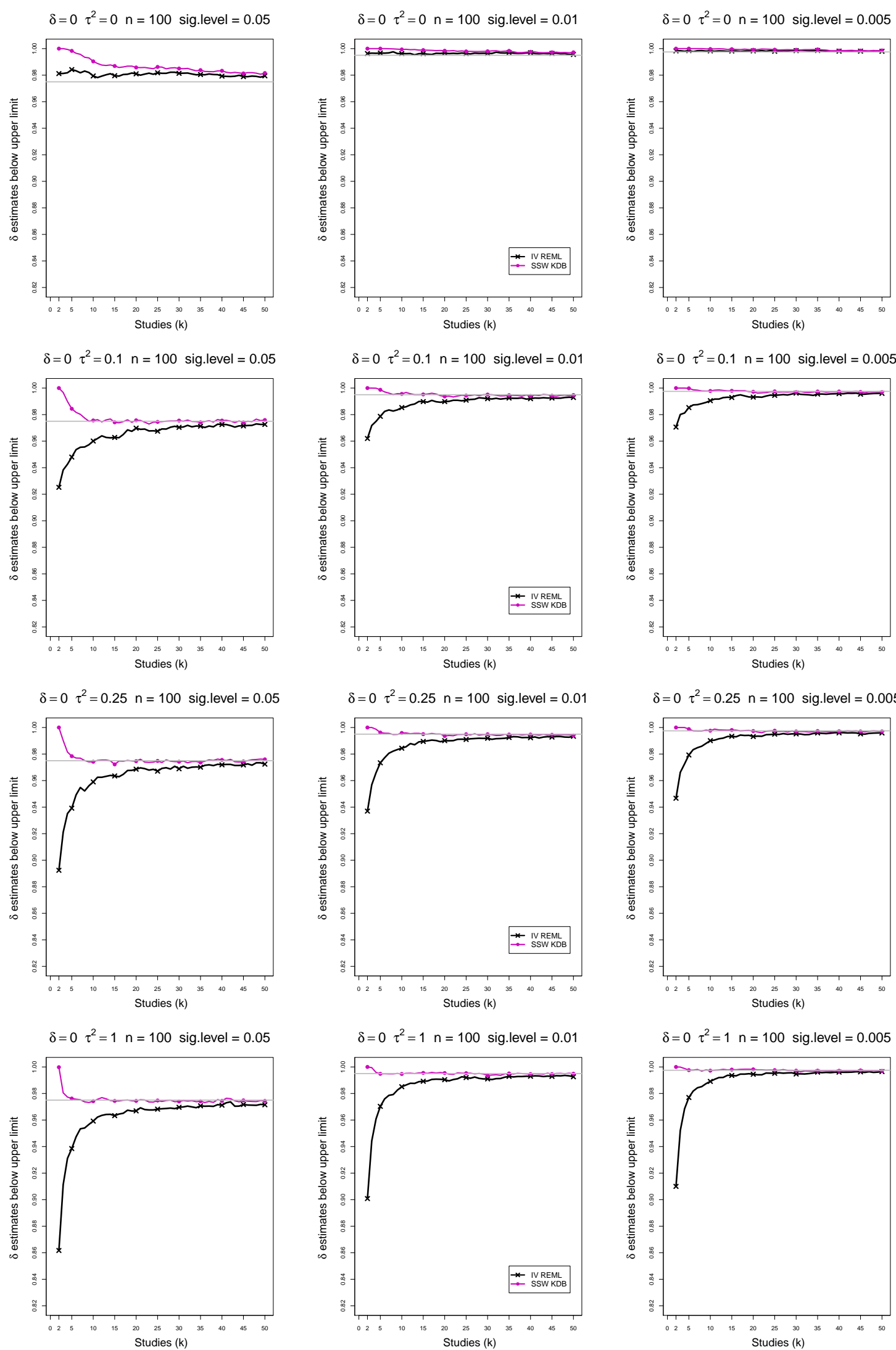

Figure A3. 15: CMA - Coverage of upper confidence intervals of $\delta$ (below upper limit) at confidence limit $1-\alpha / 2$ for 2 -sided significance level $\alpha$ of $0.05,0.01,0.005$ for $\delta=0$, $\tau^{2}=0,0.1,0.25,1, n=100, K=50$. Light grey line at $0.975,0.995,0.9975$. 

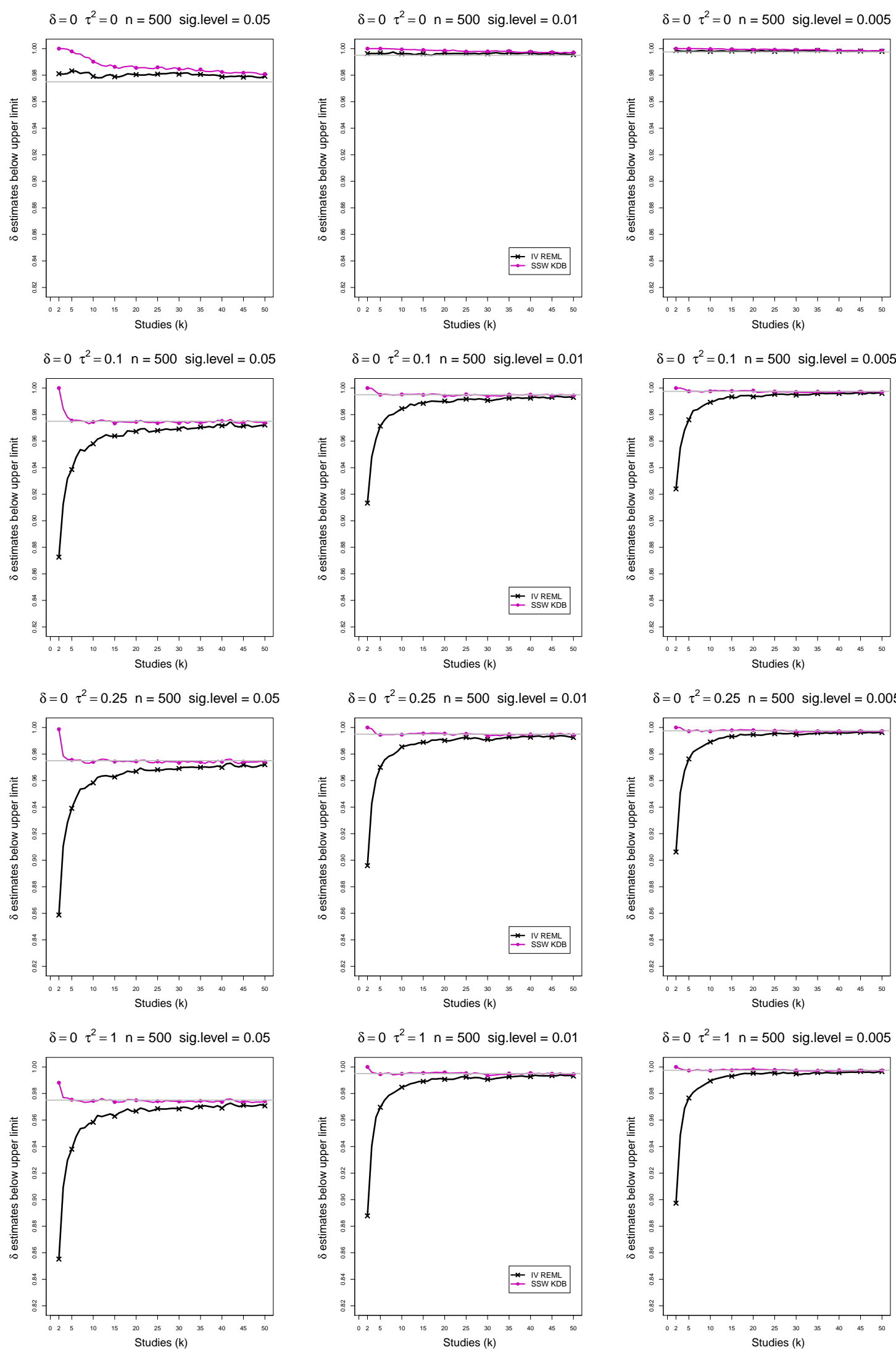

Figure A3. 16: CMA - Coverage of upper confidence intervals of $\delta$ (below upper limit) at confidence limit $1-\alpha / 2$ for 2 -sided significance level $\alpha$ of $0.05,0.01,0.005$ for $\delta=0$, $\tau^{2}=0,0.1,0.25,1, n=500, K=50$. Light grey line at $0.975,0.995,0.9975$. 

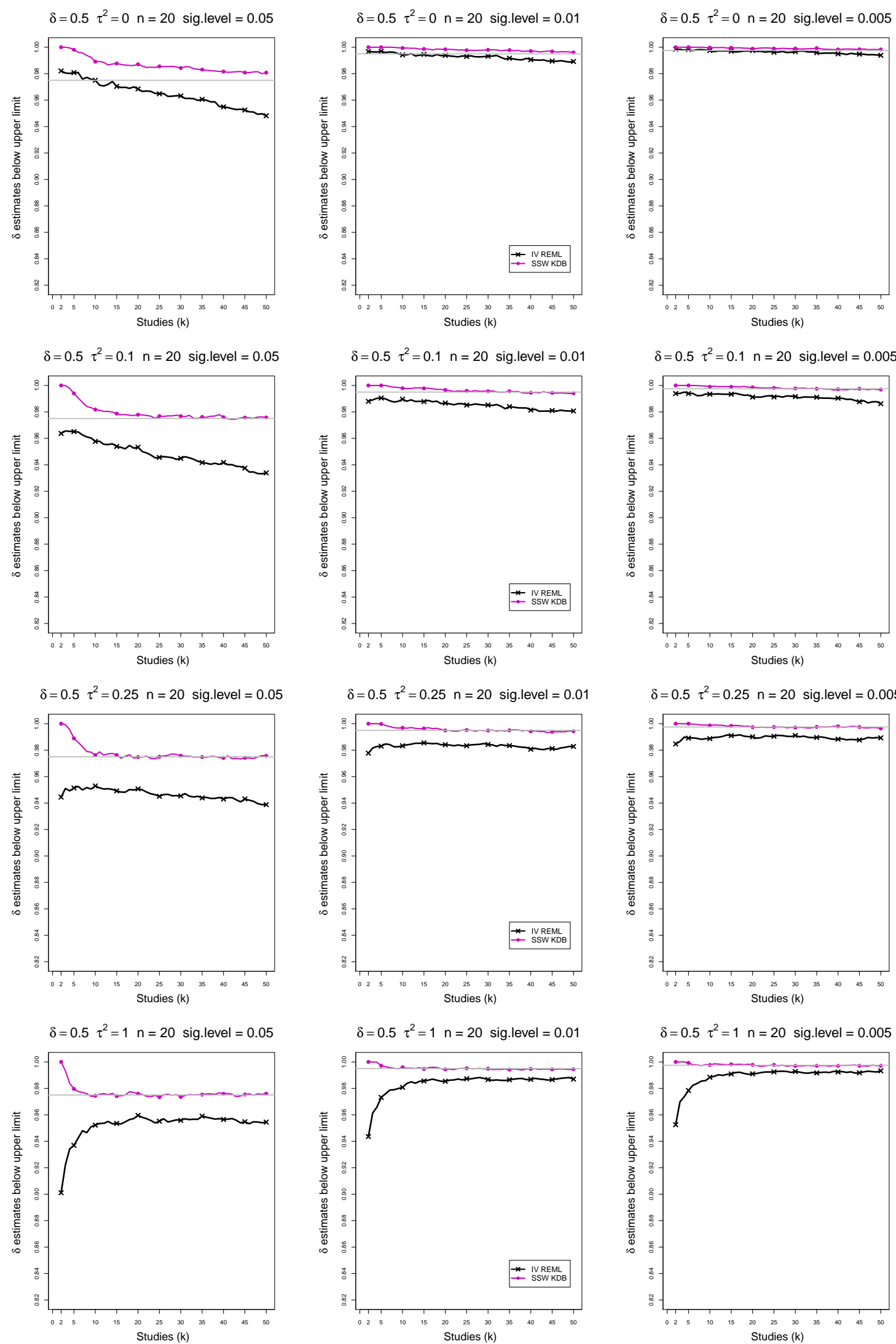

Figure A3. 17: CMA - Coverage of upper confidence intervals of $\delta$ (below upper limit) at confidence limit $1-\alpha / 2$ for 2-sided significance level $\alpha$ of 0.05, 0.01,0.005 for $\delta=0.5$, $\tau^{2}=0,0.1,0.25,1, n=20, K=50$. Light grey line at $0.975,0.995,0.9975$. 

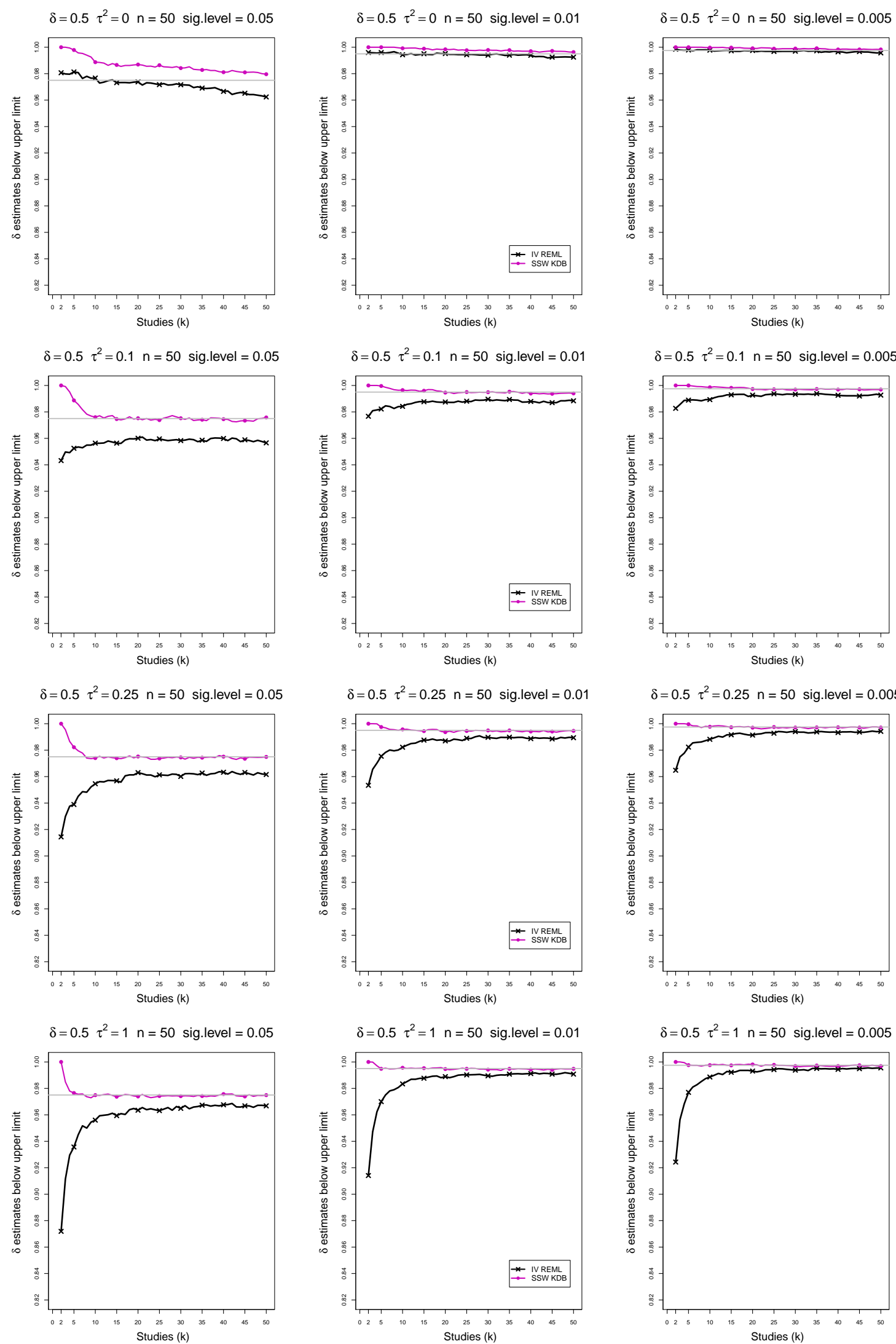

Figure A3. 18: CMA - Coverage of upper confidence intervals of $\delta$ (below upper limit) at confidence limit $1-\alpha / 2$ for 2-sided significance level $\alpha$ of $0.05,0.01,0.005$ for $\delta=0.5$, $\tau^{2}=0,0.1,0.25,1, n=50, K=50$. Light grey line at $0.975,0.995,0.9975$. 

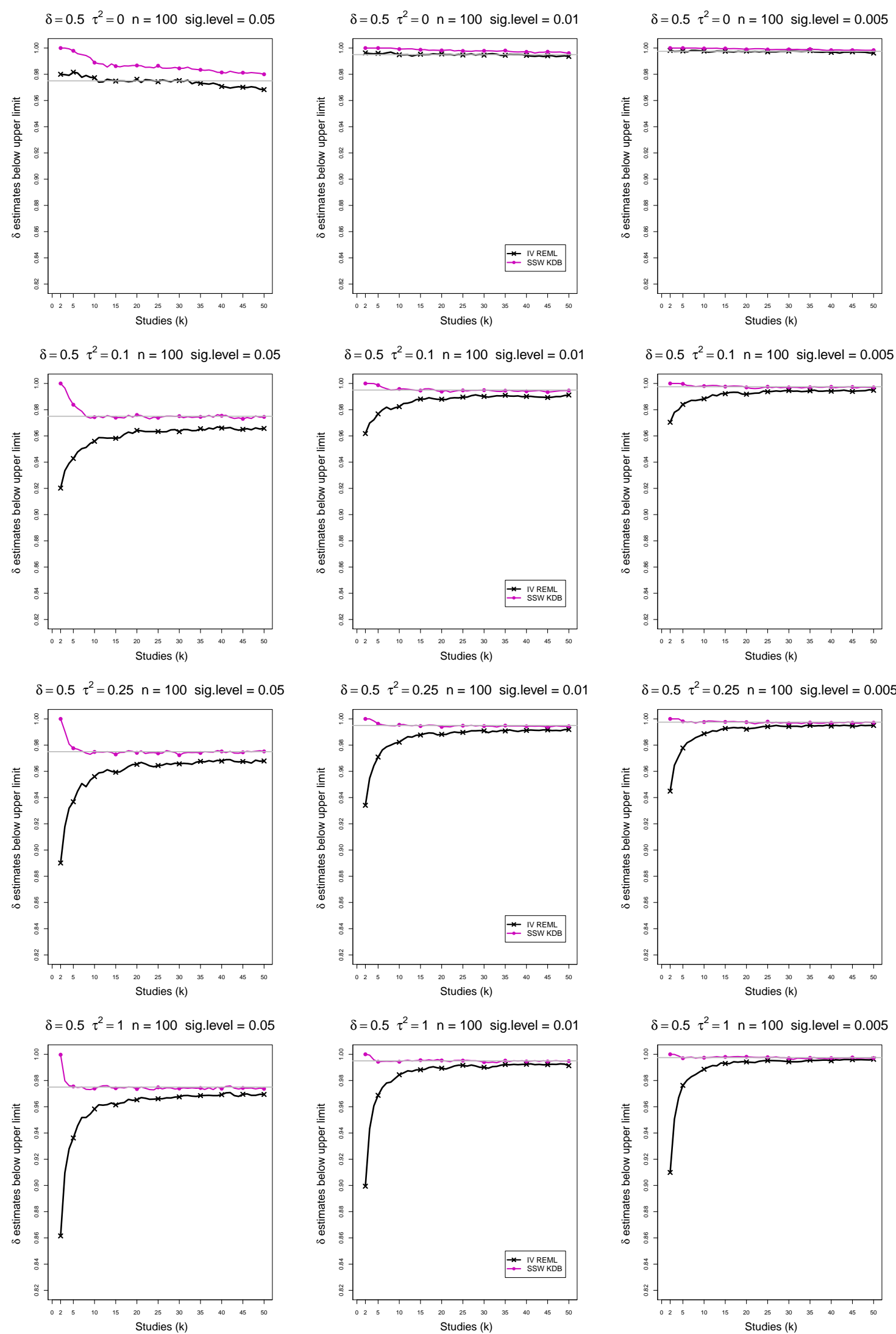

Figure A3. 19: CMA - Coverage of upper confidence intervals of $\delta$ (below upper limit) at confidence limit $1-\alpha / 2$ for 2-sided significance level $\alpha$ of $0.05,0.01,0.005$ for $\delta=0.5$, $\tau^{2}=0,0.1,0.25,1, n=100, K=50$. Light grey line at $0.975,0.995,0.9975$. 

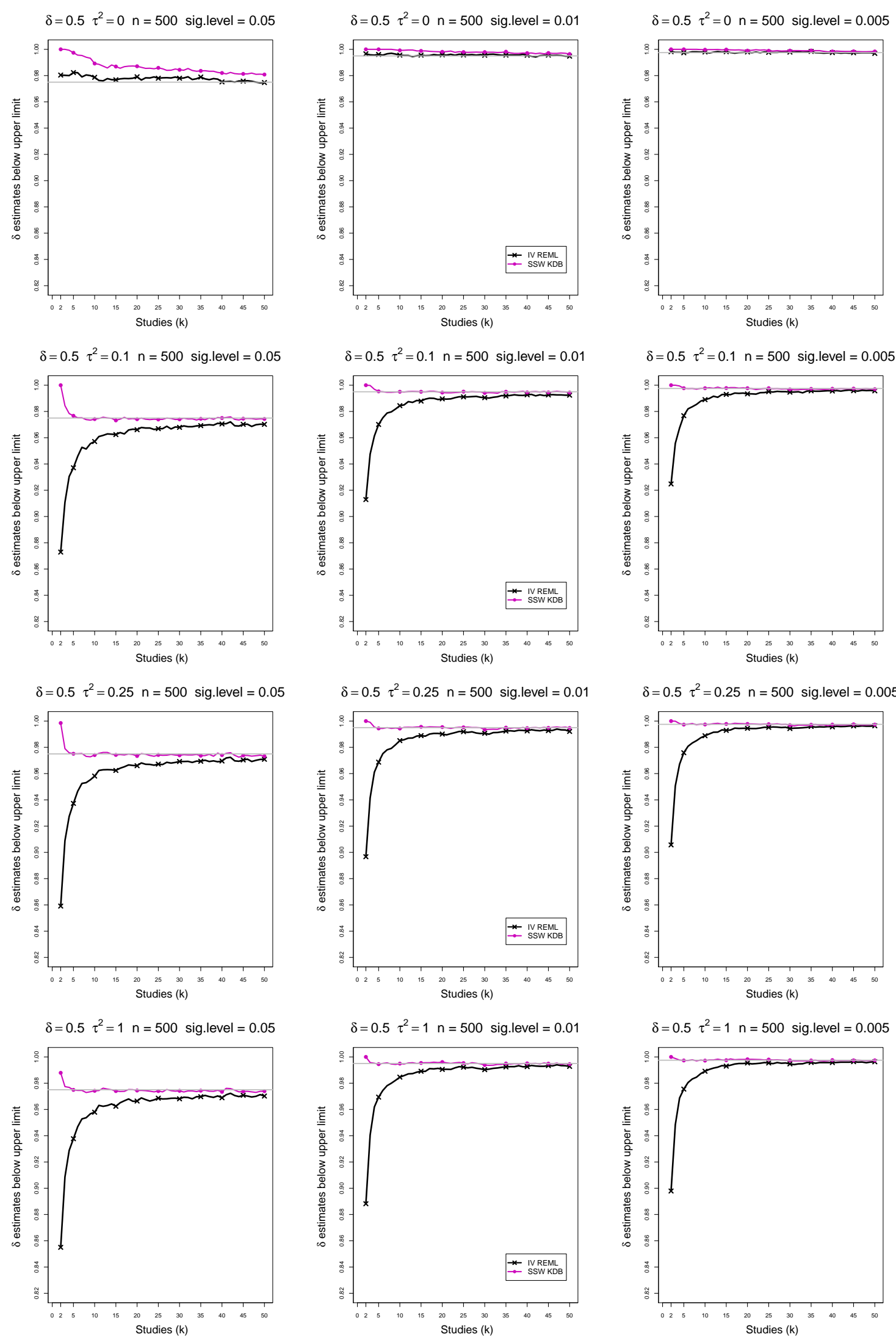

Figure A3. 20: CMA - Coverage of upper confidence intervals of $\delta$ (below upper limit) at confidence limit $1-\alpha / 2$ for 2-sided significance level $\alpha$ of $0.05,0.01,0.005$ for $\delta=0.5$, $\tau^{2}=0,0.1,0.25,1, n=500, K=50$. Light grey line at $0.975,0.995,0.9975$. 

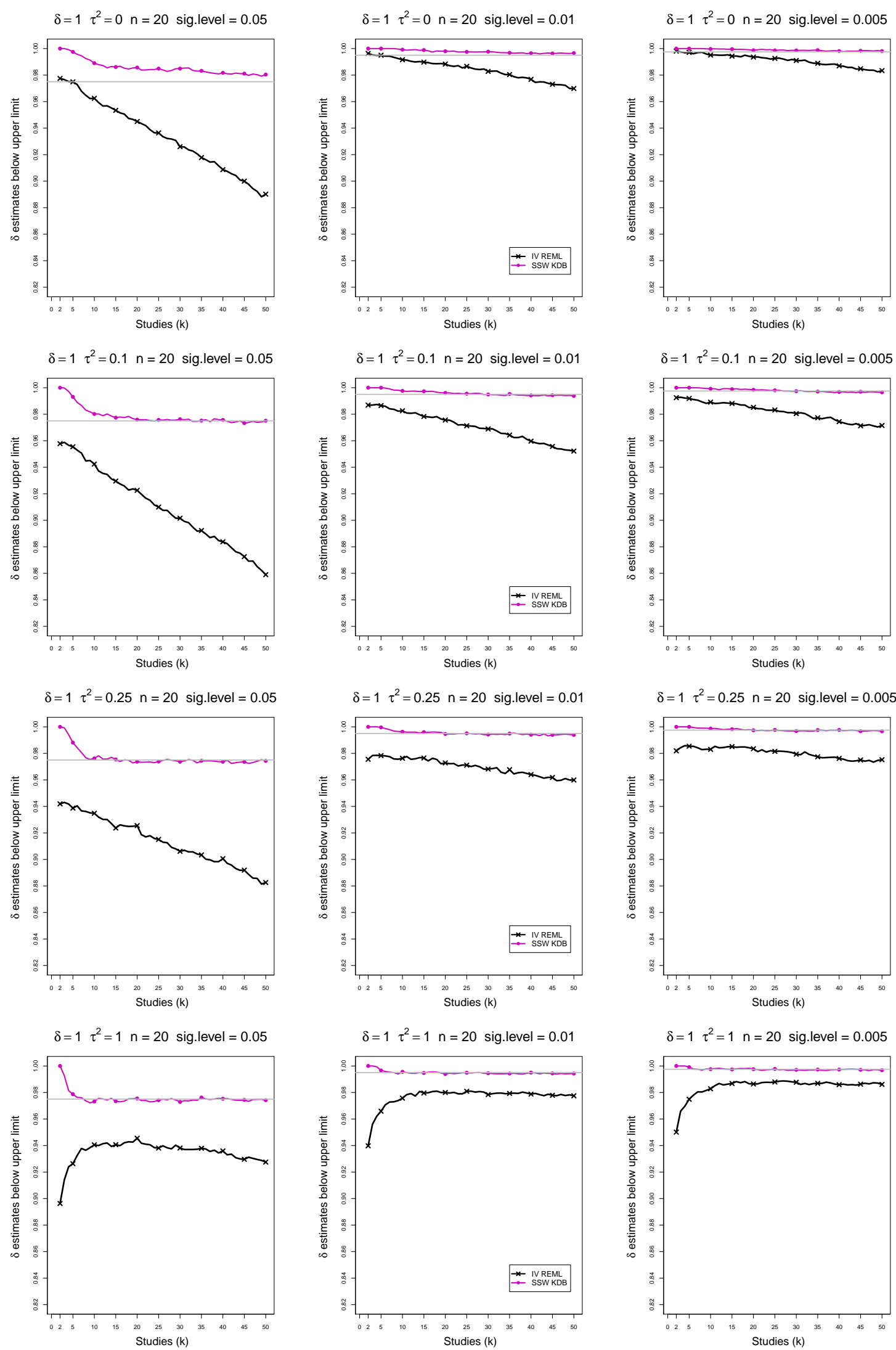

Figure A3. 21: CMA - Coverage of upper confidence intervals of $\delta$ (below upper limit) at confidence limit $1-\alpha / 2$ for 2 -sided significance level $\alpha$ of $0.05,0.01,0.005$ for $\delta=1$, $\tau^{2}=0,0.1,0.25,1, n=20, K=50$. Light grey line at $0.975,0.995,0.9975$. 

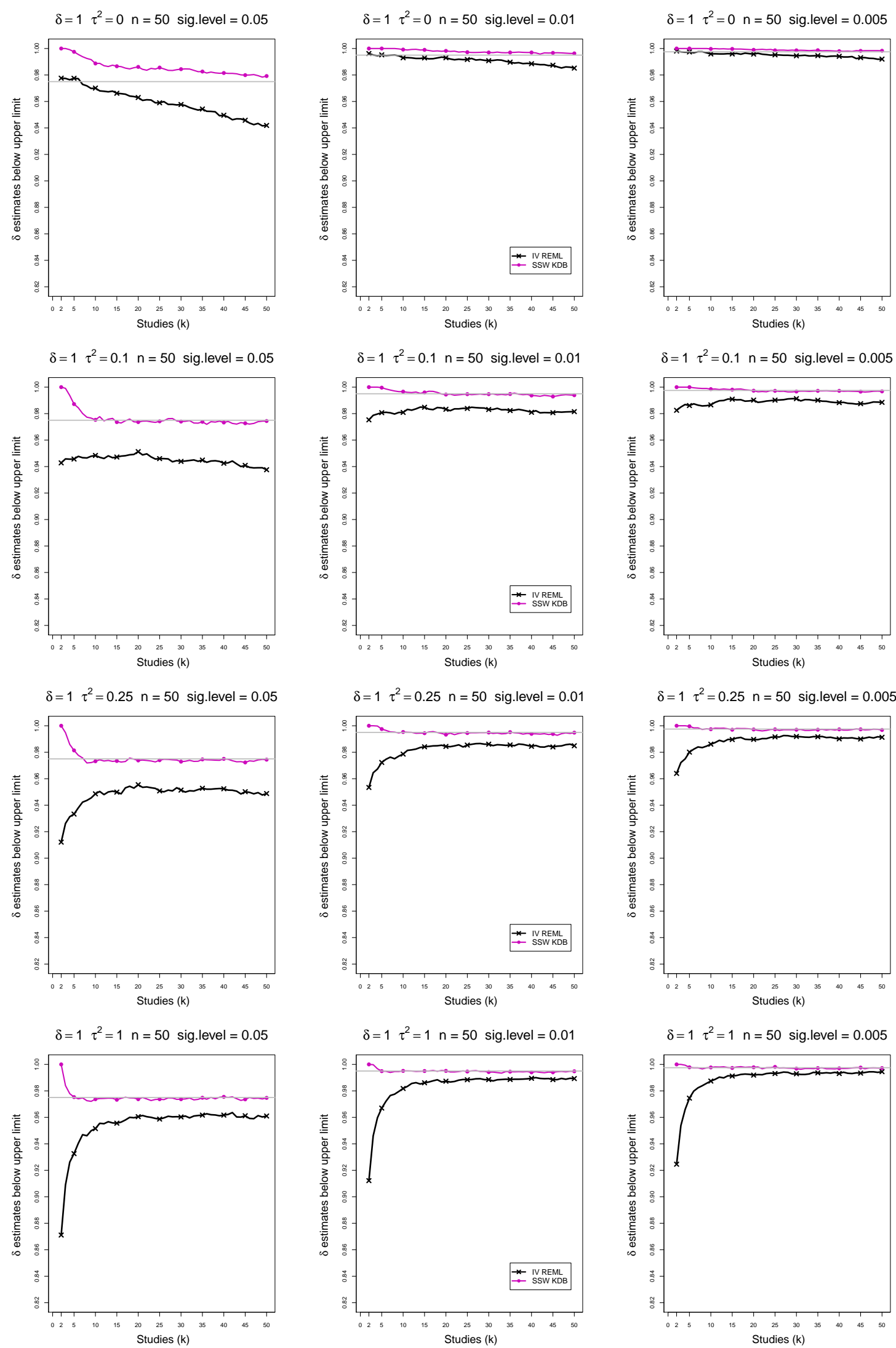

Figure A3. 22: CMA - Coverage of upper confidence intervals of $\delta$ (below upper limit) at confidence limit $1-\alpha / 2$ for 2 -sided significance level $\alpha$ of $0.05,0.01,0.005$ for $\delta=1$, $\tau^{2}=0,0.1,0.25,1, n=50, K=50$. Light grey line at $0.975,0.995,0.9975$. 

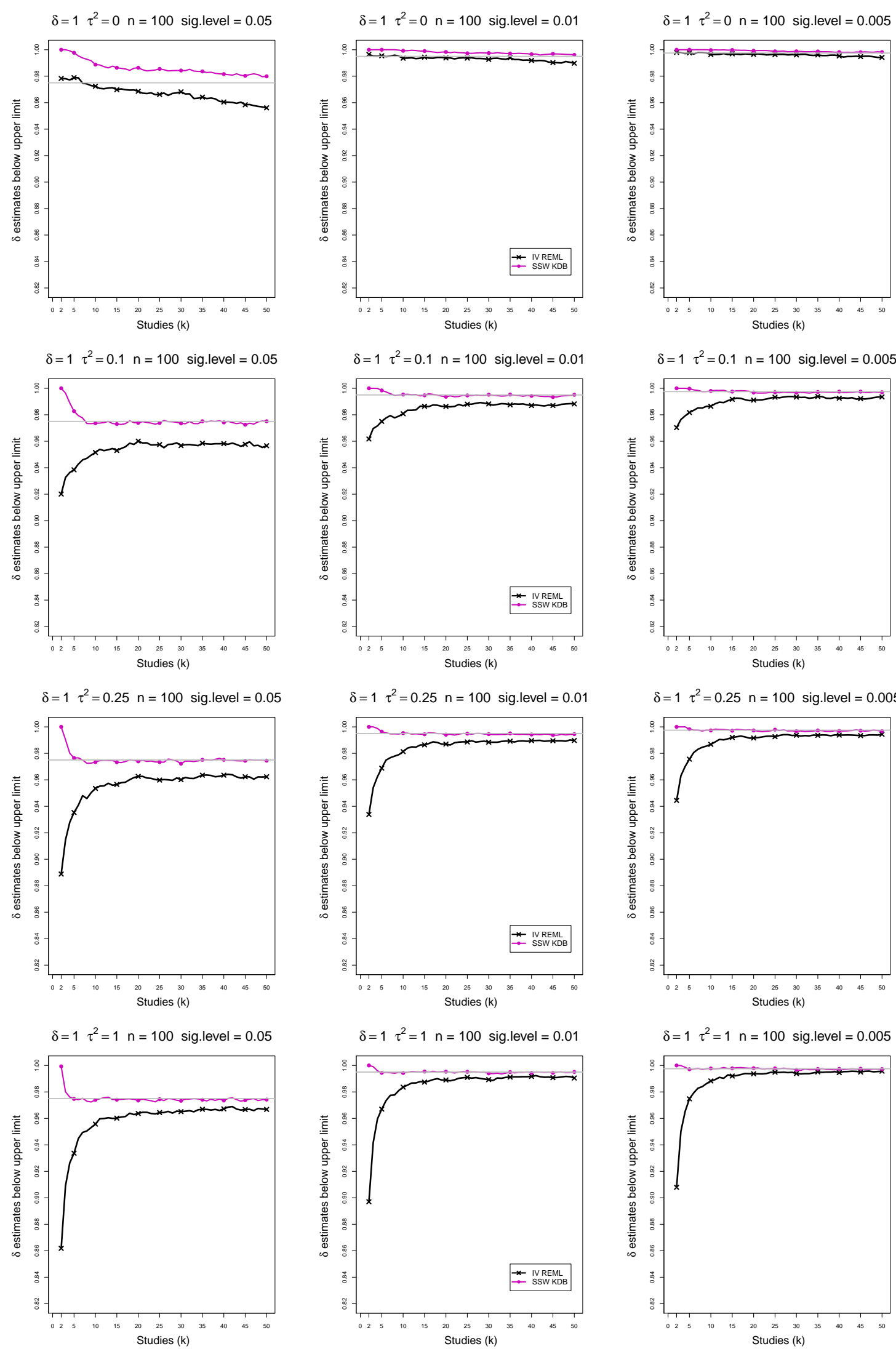

Figure A3. 23: CMA - Coverage of upper confidence intervals of $\delta$ (below upper limit) at confidence limit $1-\alpha / 2$ for 2-sided significance level $\alpha$ of $0.05,0.01,0.005$ for $\delta=1$, $\tau^{2}=0,0.1,0.25,1, n=100, K=50$. Light grey line at $0.975,0.995,0.9975$. 

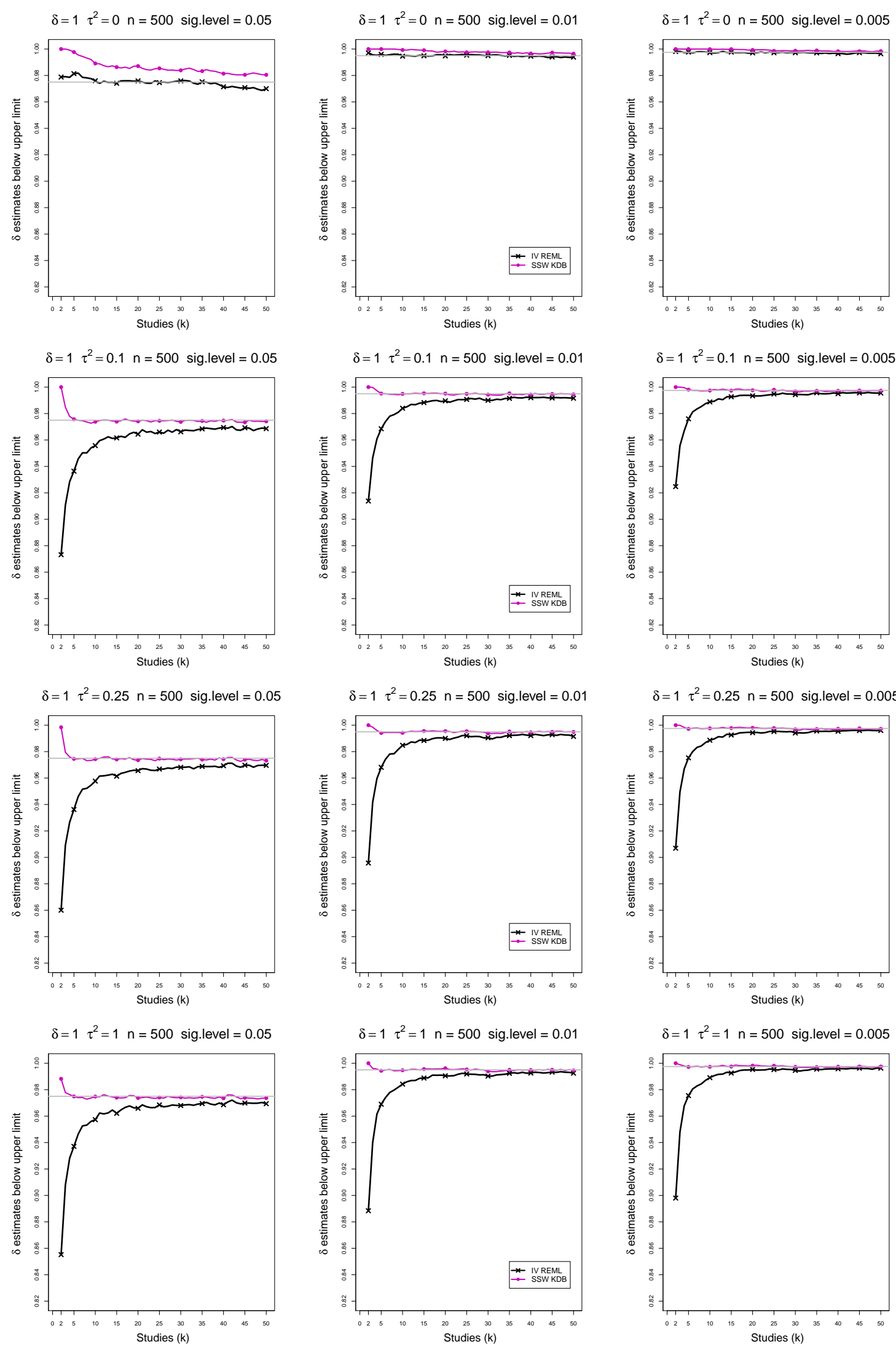

Figure A3. 24: CMA - Coverage of upper confidence intervals of $\delta$ (below upper limit) at confidence limit $1-\alpha / 2$ for 2 -sided significance level $\alpha$ of $0.05,0.01,0.005$ for $\delta=1$, $\tau^{2}=0,0.1,0.25,1, n=500, K=50$. Light grey line at $0.975,0.995,0.9975$. 


\section{A4. Bias of point estimators of $\tau^{2}$}

For bias of $\tau^{2}$ in this section, each figure corresponds to a value of $\delta(=0,0.5,1)$, a set of values of $\tau^{2}(=0,0.1,0.25,1)$, and a set of values of $n(=20,50$ or 100,500$)$ while the study number $k$ increases to maximum number $K=50$.

Each figure contains a panel (with study $k$ on the horizontal axis) for each combination of parameters.

The point estimators of $\tau^{2}$ are

- REML (Restricted maximum likelihood)

- KDB (Kulinskaya-Dollinger-Bjørkestøl) (2011) 

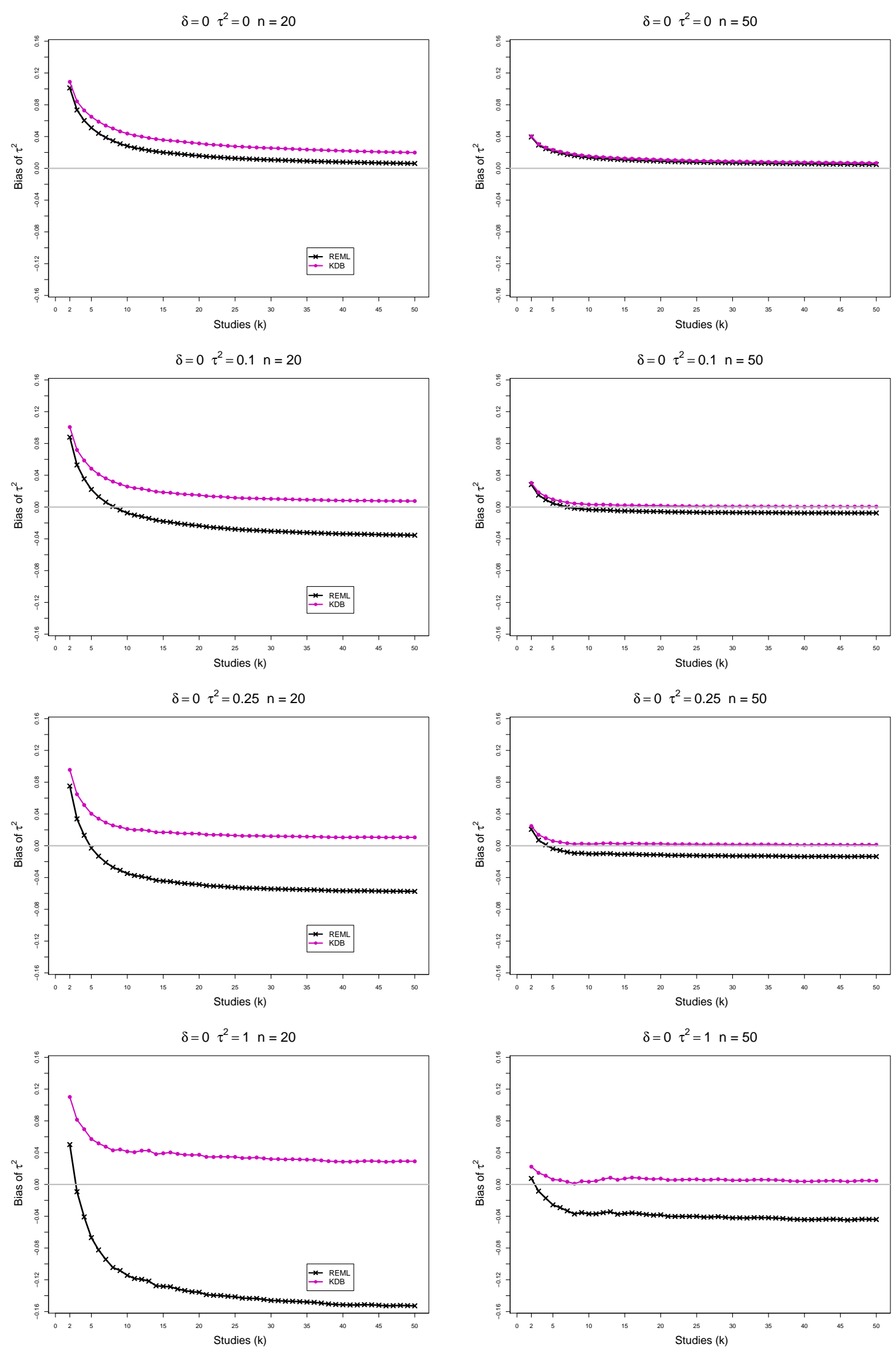

Figure A4. 1: CMA - Bias of the estimation of between-studies variance $\tau^{2}$ with REML and KDB for $\delta=0, \tau^{2}=0,0.1,0.25,1, n=20,50, K=50$. Light grey line at 0 . 

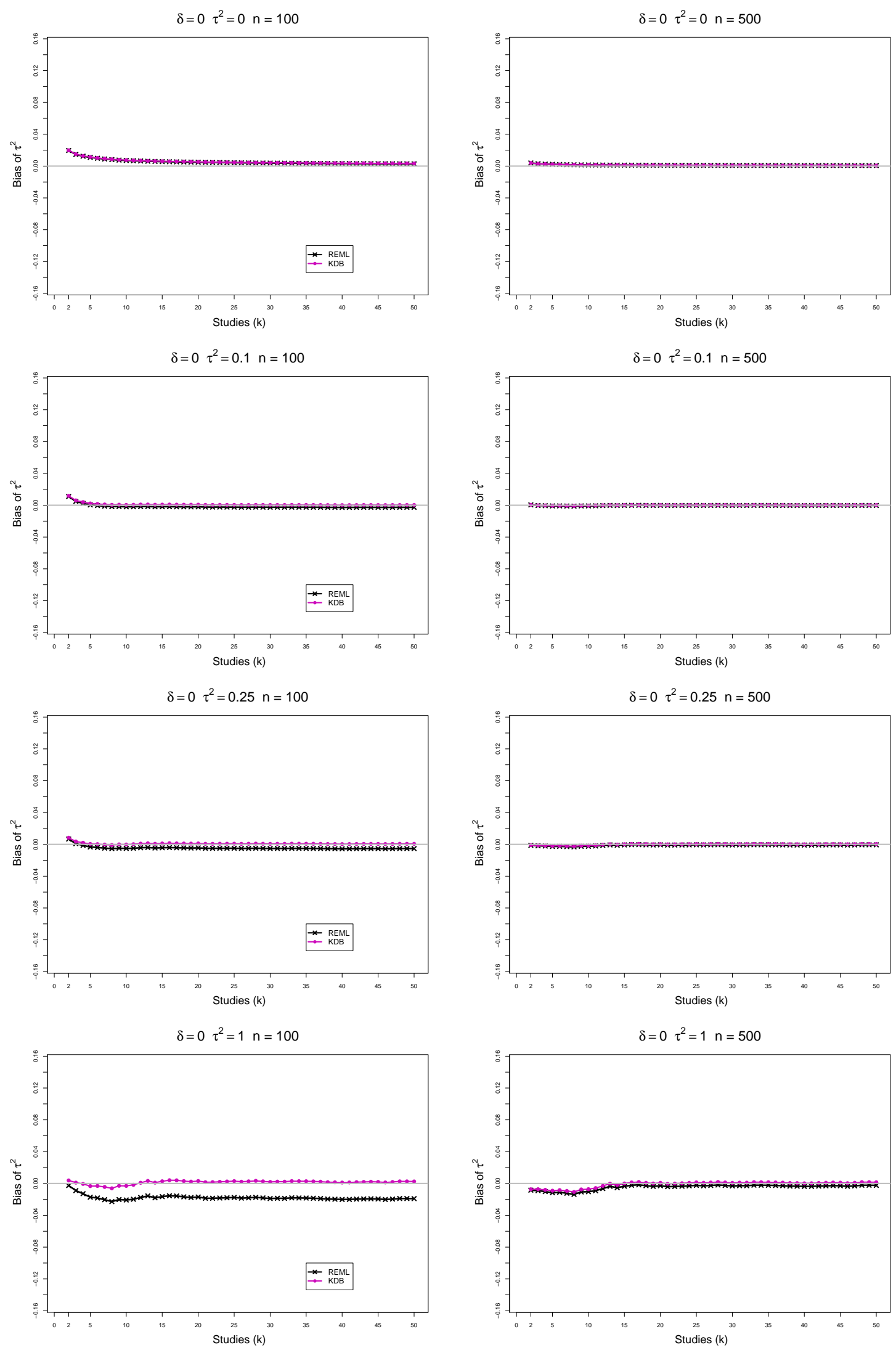

Figure A4. 2: CMA - Bias of the estimation of between-studies variance $\tau^{2}$ with REML and $\mathrm{KDB}$ for $\delta=0, \tau^{2}=0,0.1,0.25,1, n=100,500, K=50$. Light grey line at 0 . 

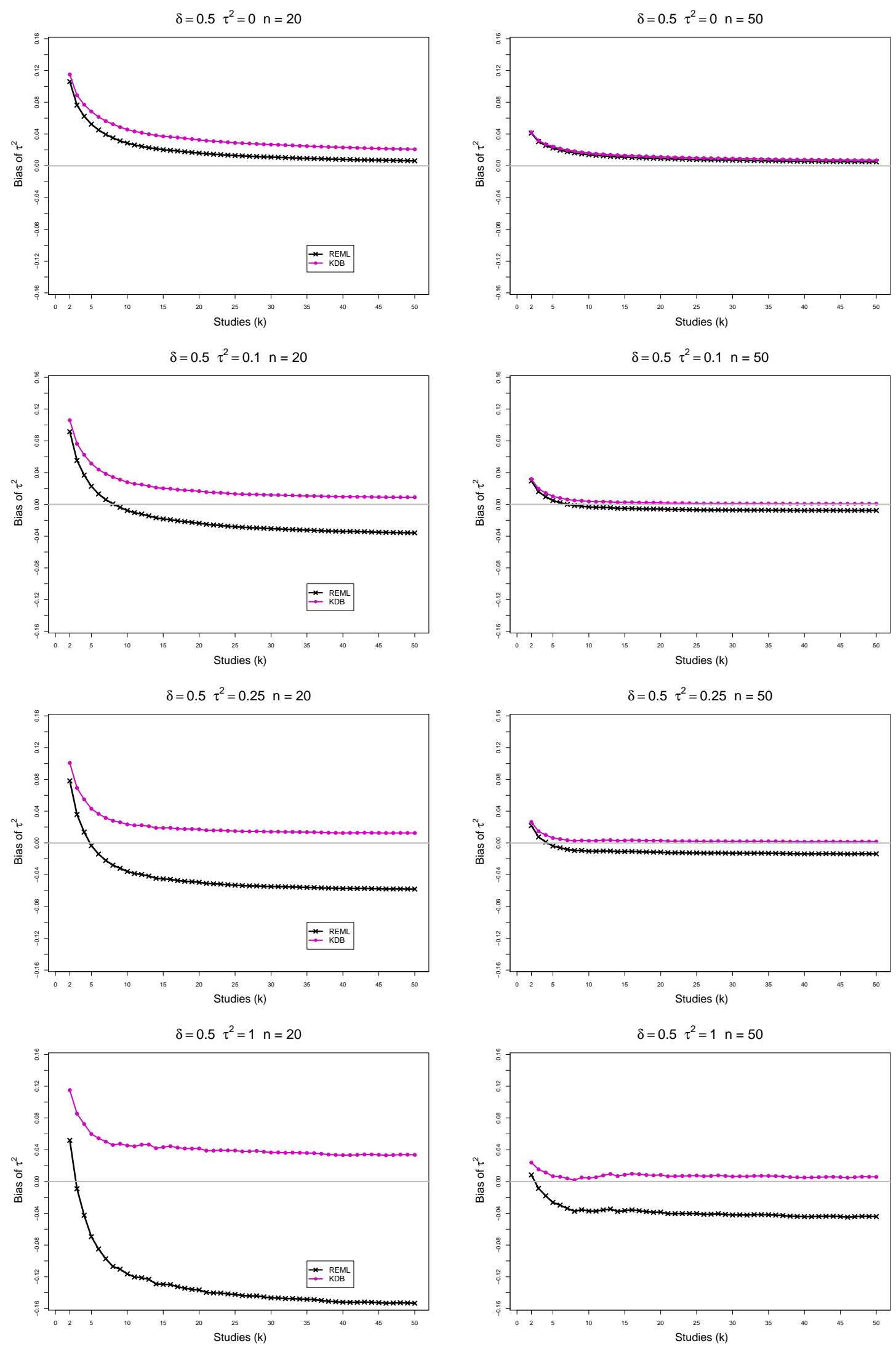

Figure A4. 3: CMA - Bias of the estimation of between-studies variance $\tau^{2}$ with REML and $\mathrm{KDB}$ for $\delta=0.5, \tau^{2}=0,0.1,0.25,1, n=20,50, K=50$. Light grey line at 0 . 

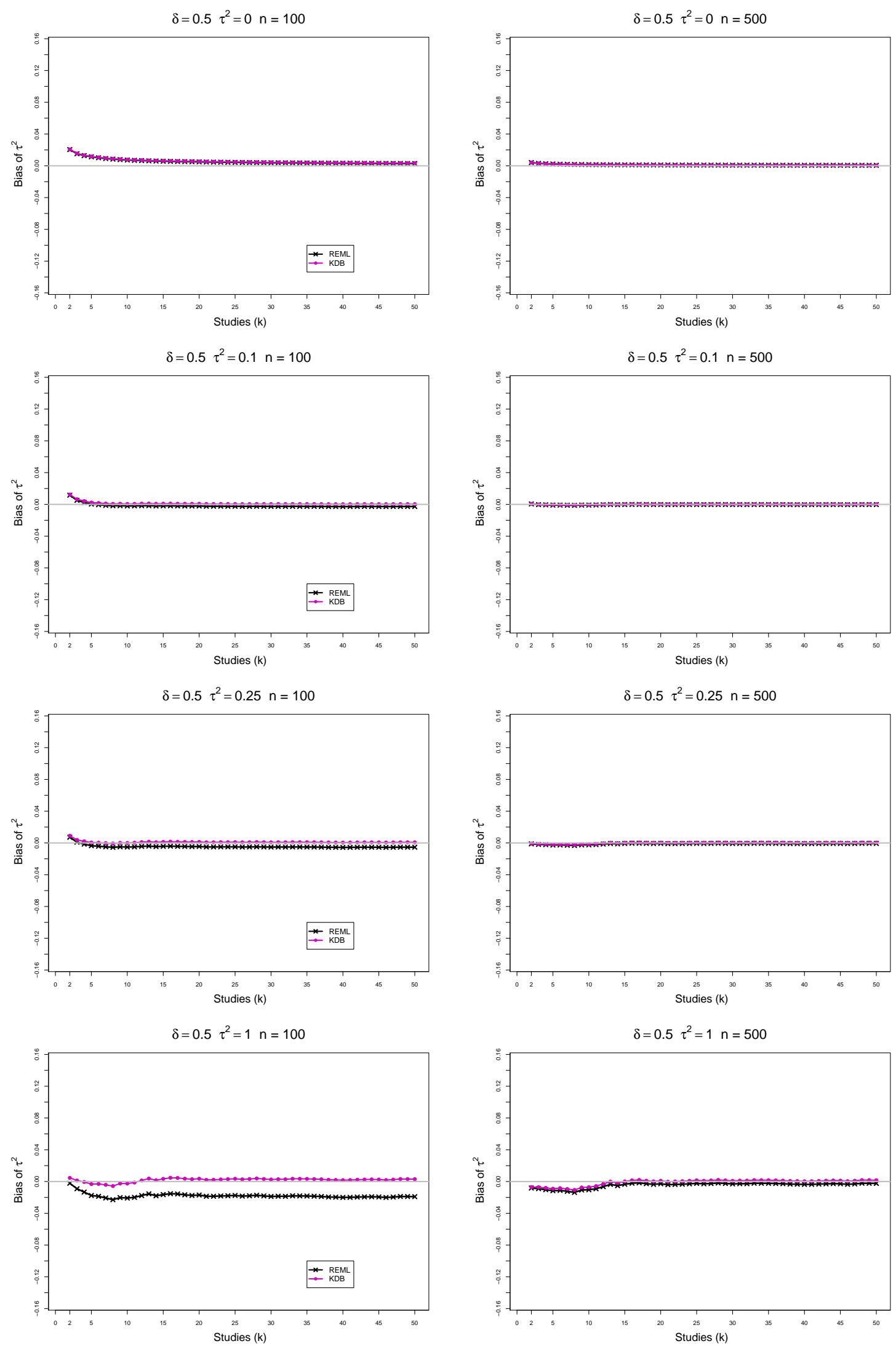

Figure A4. 4: CMA - Bias of the estimation of between-studies variance $\tau^{2}$ with REML and KDB for $\delta=0.5, \tau^{2}=0,0.1,0.25,1, n=100,500, K=50$. Light grey line at 0 . 

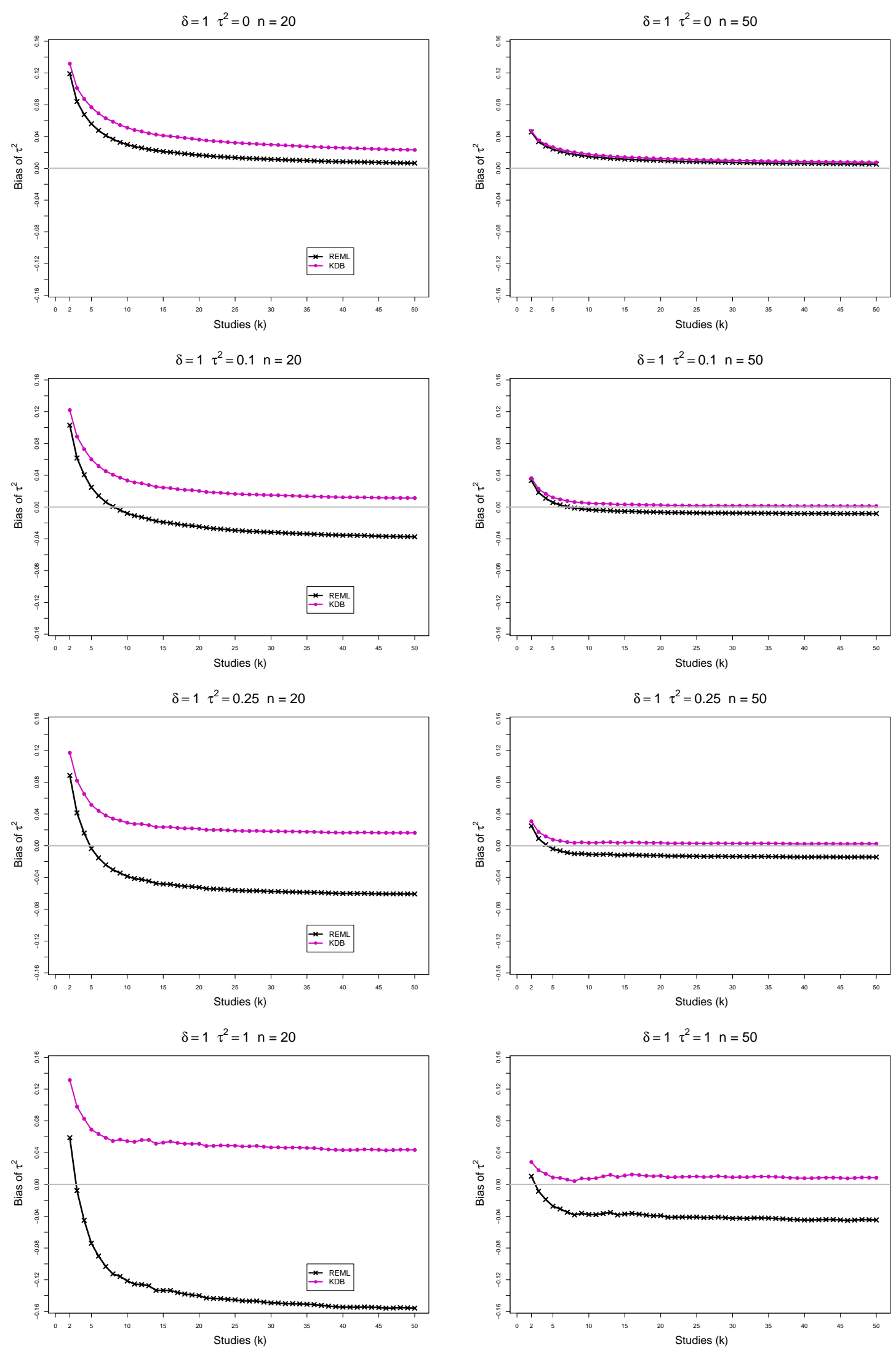

Figure A4. 5: CMA - Bias of the estimation of between-studies variance $\tau^{2}$ with REML and KDB for $\delta=1, \tau^{2}=0,0.1,0.25,1, n=20,50, K=50$. Light grey line at 0 . 

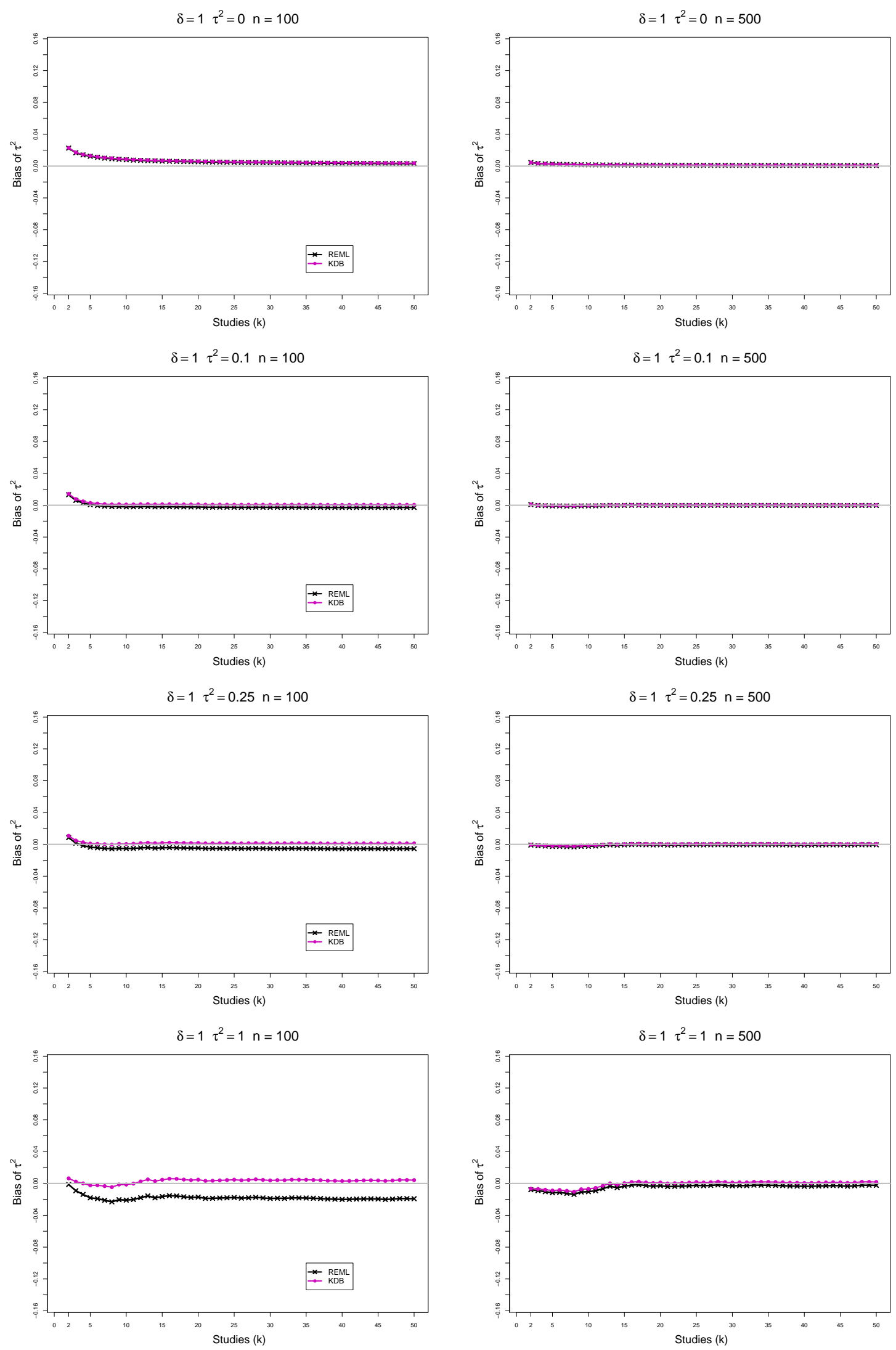

Figure A4. 6: CMA - Bias of the estimation of between-studies variance $\tau^{2}$ with REML and $\mathrm{KDB}$ for $\delta=1, \tau^{2}=0,0.1,0.25,1, n=100,500, K=50$. Light grey line at 0 . 


\section{A5. Coverage of interval estimators of $\tau^{2}$}

For coverage of $\tau^{2}$ in this section, each figure corresponds to a value of $\delta(=0,0.5,1)$, a set of values of $\tau^{2}(=0,0.1,0.25,1)$, a value of $n(=20,50,100,500)$ and a set of values of significance levels $\alpha(=0.05,0.01,0.005)$ while the study number $k$ increases to maximum number $K=50$.

Each figure contains a panel (with study $k$ on the horizontal axis) for each combination of parameters.

The confidence estimators of $\delta$ are

- QP (Q-profile confidence interval) by Viechtbauer (2007)

- KDB (improved Q-profile method based on Kulinskaya, Dollinger, Bjørkestøl (2011)) 

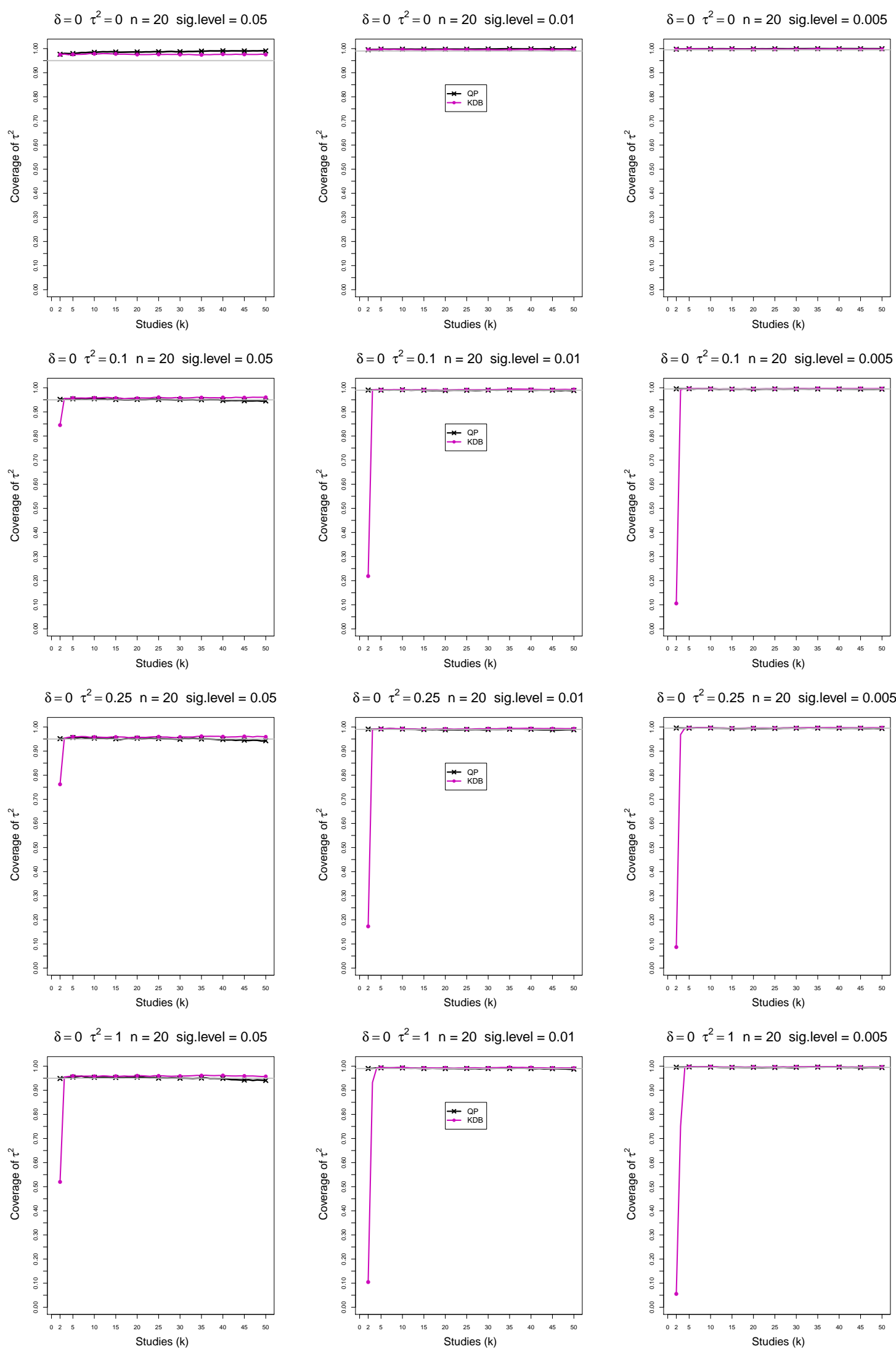

Figure A5. 1: CMA - Coverage of interval estimators of between-study variance $\tau^{2}$ at confidence level $1-\alpha$ for $\alpha$ of $0.05,0.01,0.005$ for $\delta=0, \tau^{2}=0,0.1,0.25,1, n=20$, $K=50$. Light grey line at 0.95,0.99,0.995. 

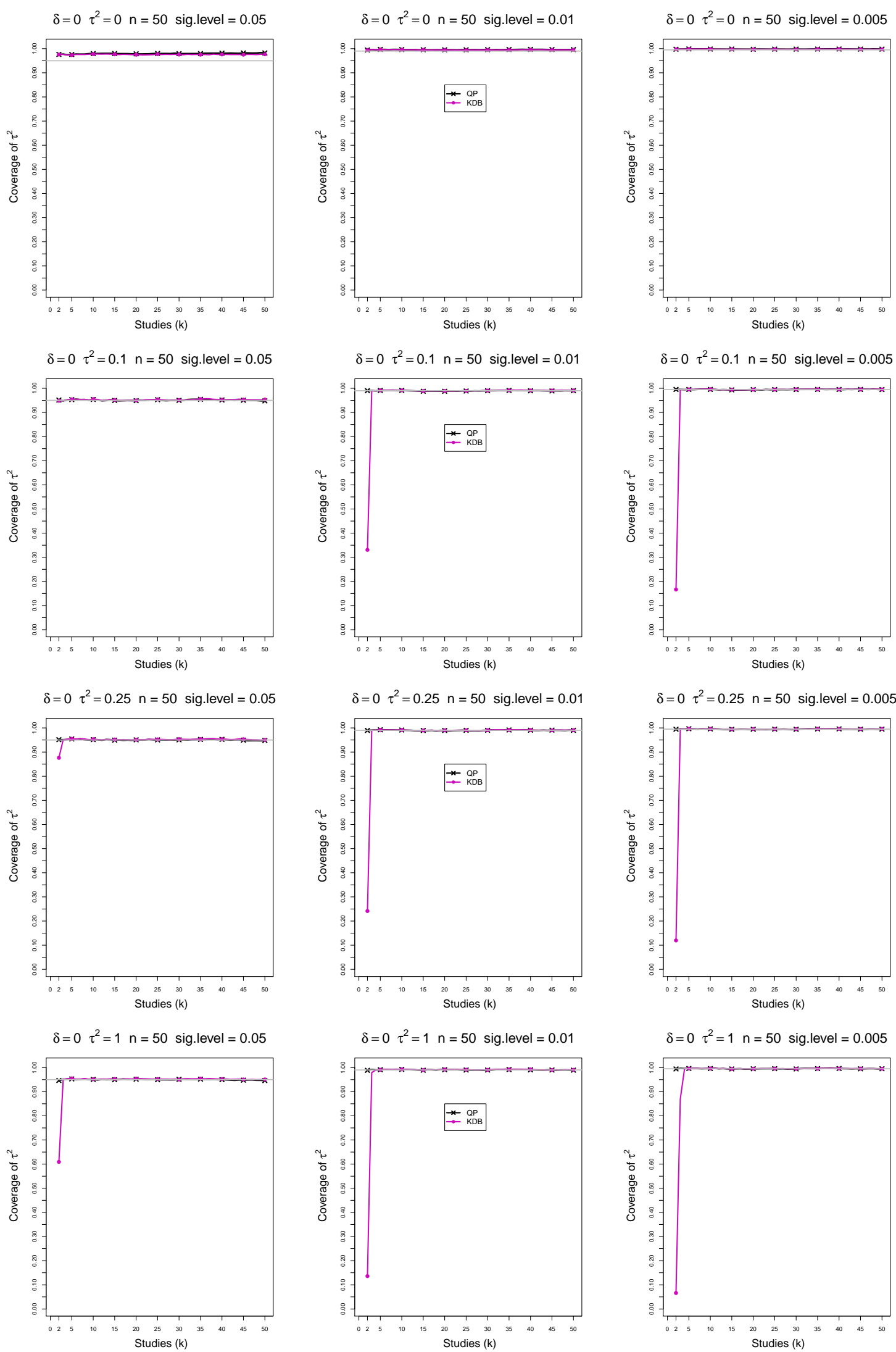

Figure A5. 2: CMA - Coverage of interval estimators of between-study variance $\tau^{2}$ at confidence level $1-\alpha$ for alpha of 0.05, 0.01,0.005 for $\delta=0, \tau^{2}=0,0.1,0.25,1, n=50$, $K=50$. Light grey line at 0.95,0.99,0.995. 

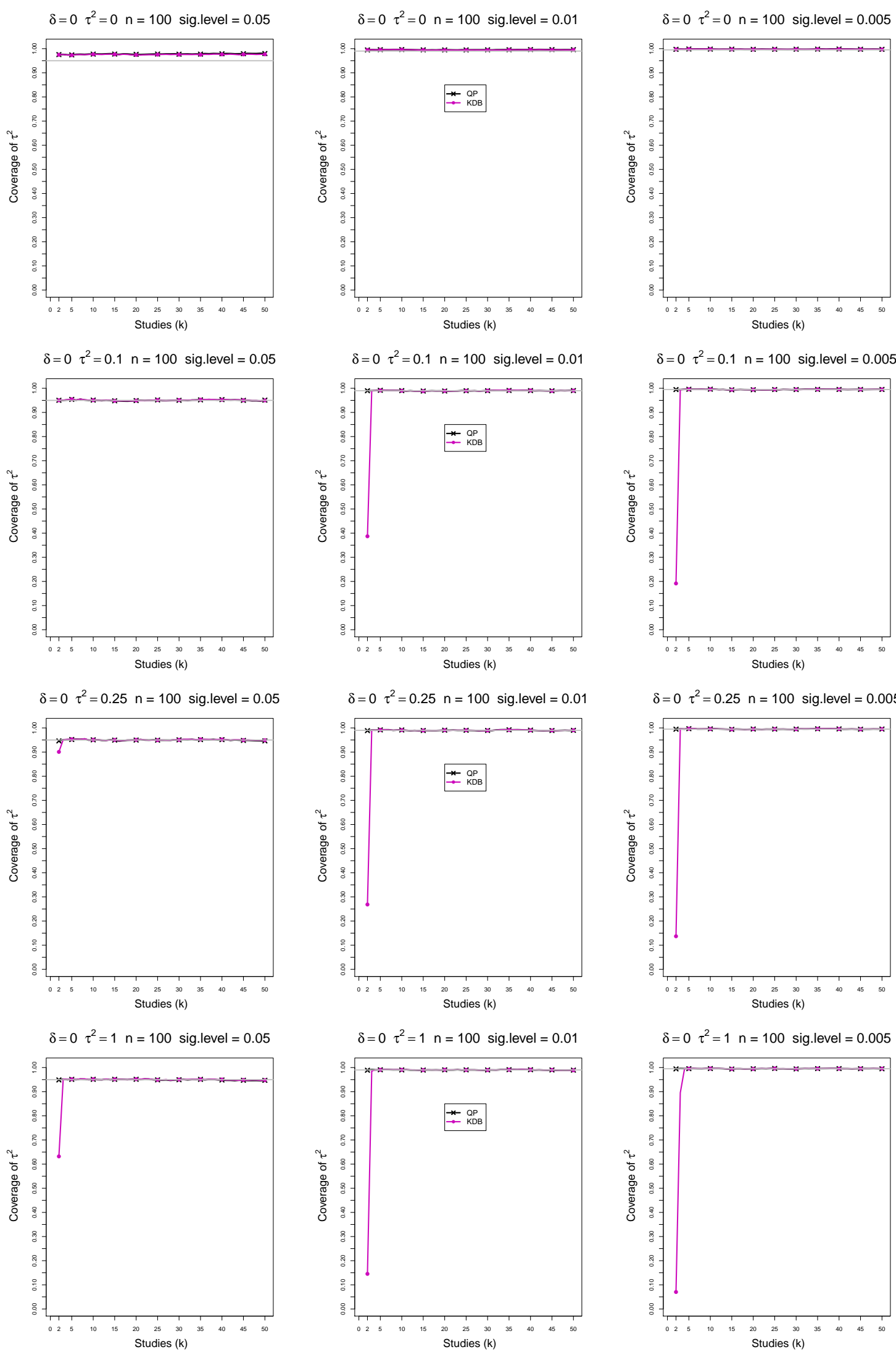

Figure A5. 3: CMA - Coverage of interval estimators of between-study variance $\tau^{2}$ at confidence level $1-\alpha$ for alpha of $0.05,0.01,0.005$ for $\delta=0, \tau^{2}=0,0.1,0.25,1, n=100$, $K=50$. Light grey line at $0.95,0.99,0.995$. 

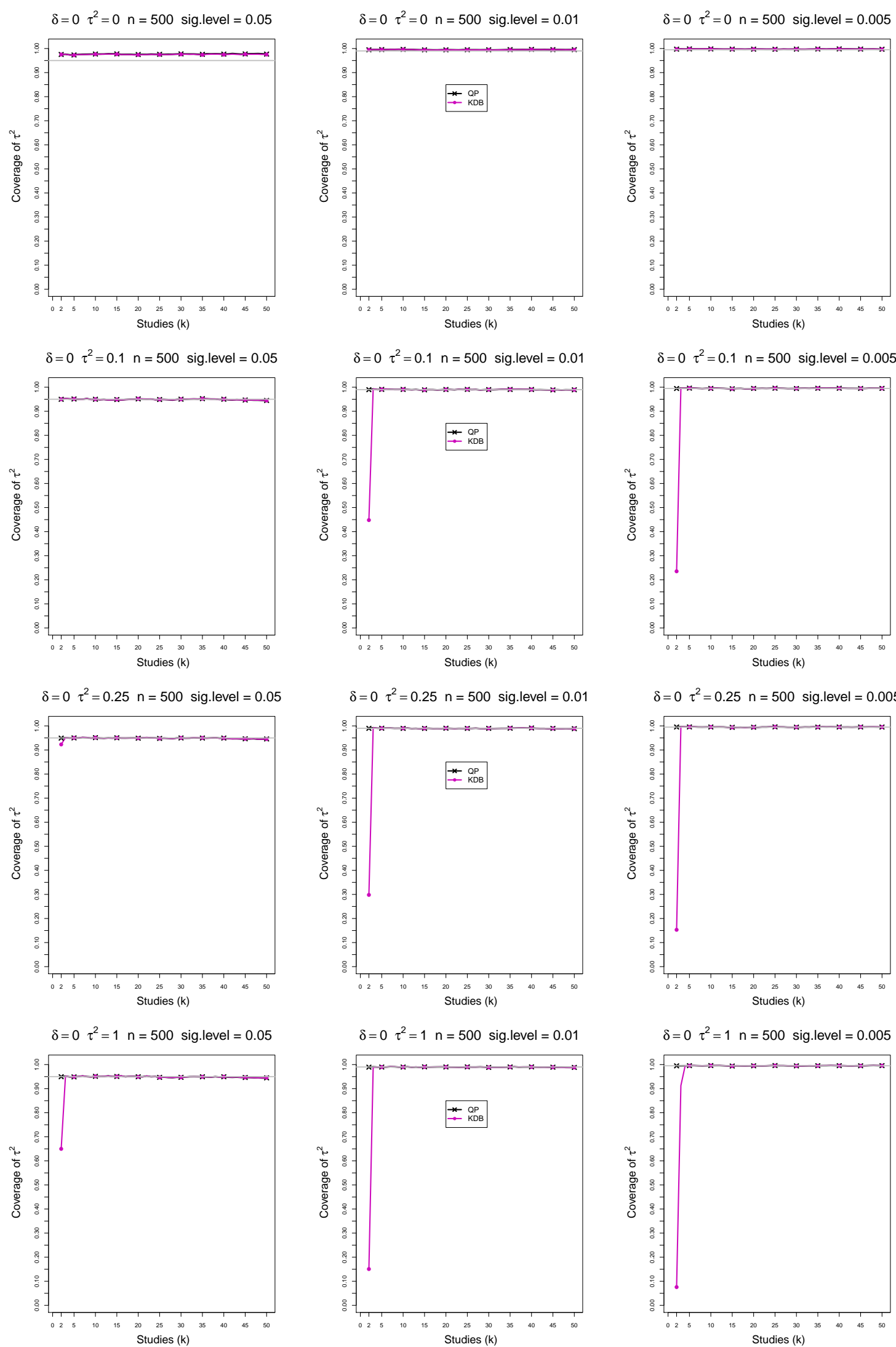

Figure A5. 4: CMA - Coverage of interval estimators of between-study variance $\tau^{2}$ at confidence level $1-\alpha$ for alpha of $0.05,0.01,0.005$ for $\delta=0, \tau^{2}=0,0.1,0.25,1, n=500$, $K=50$. Light grey line at $0.95,0.99,0.995$. 

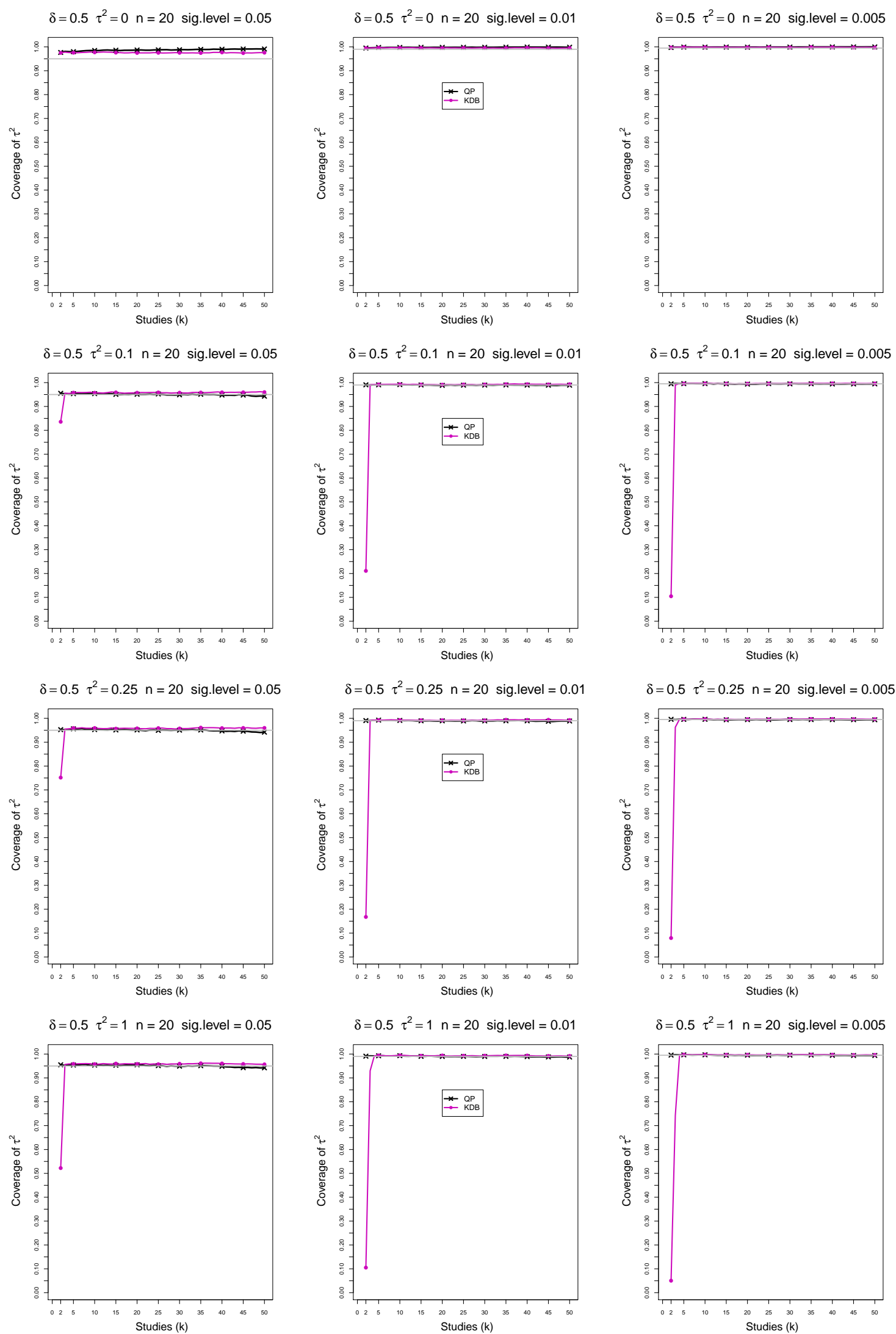

Figure A5. 5: CMA - Coverage of interval estimators of between-study variance $\tau^{2}$ at confidence level $1-\alpha$ for alpha of $0.05,0.01,0.005$ for $\delta=0.5, \tau^{2}=0,0.1,0.25,1, n=20$, $K=50$. Light grey line at $0.95,0.99,0.995$. 

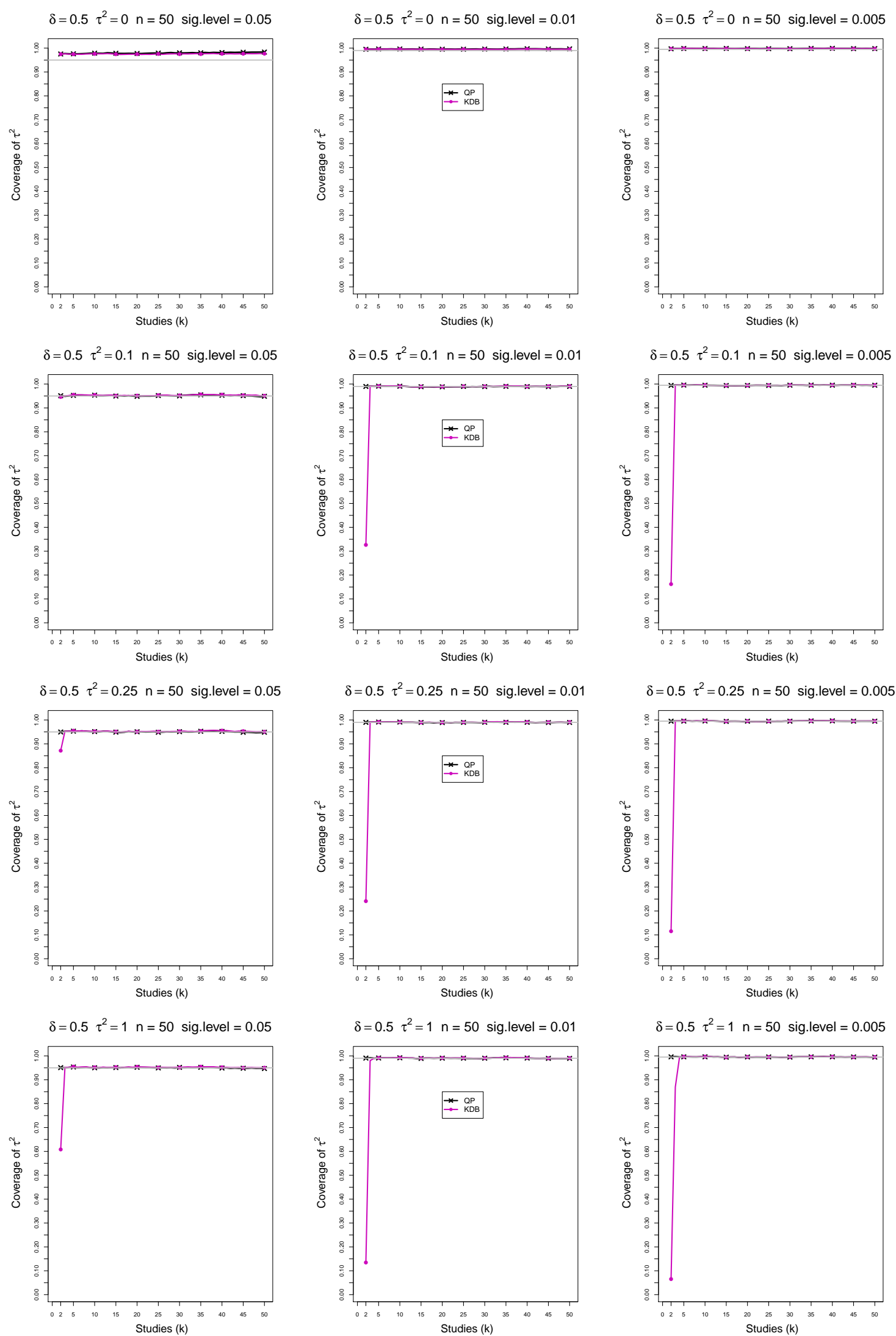

Figure A5. 6: CCMA - Coverage of interval estimators of between-study variance $\tau^{2}$ at confidence level $1-\alpha$ for alpha of 0.05, 0.01,0.005 for $\delta=0.5, \tau^{2}=0,0.1,0.25,1, n=50$, $K=50$. Light grey line at $0.95,0.99,0.995$. 

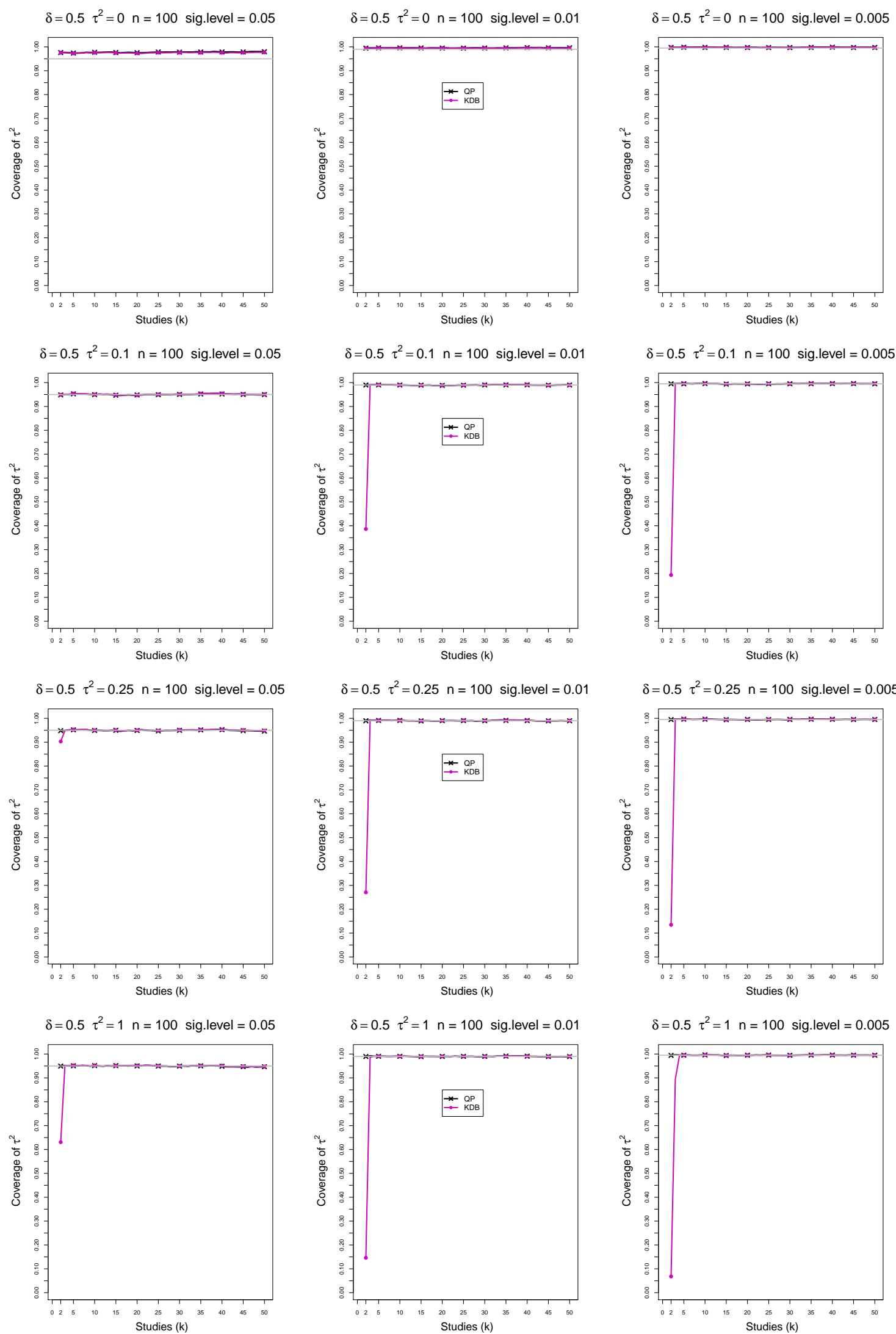

Figure A5. 7: CMA - Coverage of interval estimators of between-study variance $\tau^{2}$ at confidence level $1-\alpha$ for alpha of $0.05,0.01,0.005$ for $\delta=0.5, \tau^{2}=0,0.1,0.25,1, n=100$, $K=50$. Light grey line at $0.95,0.99,0.995$. 

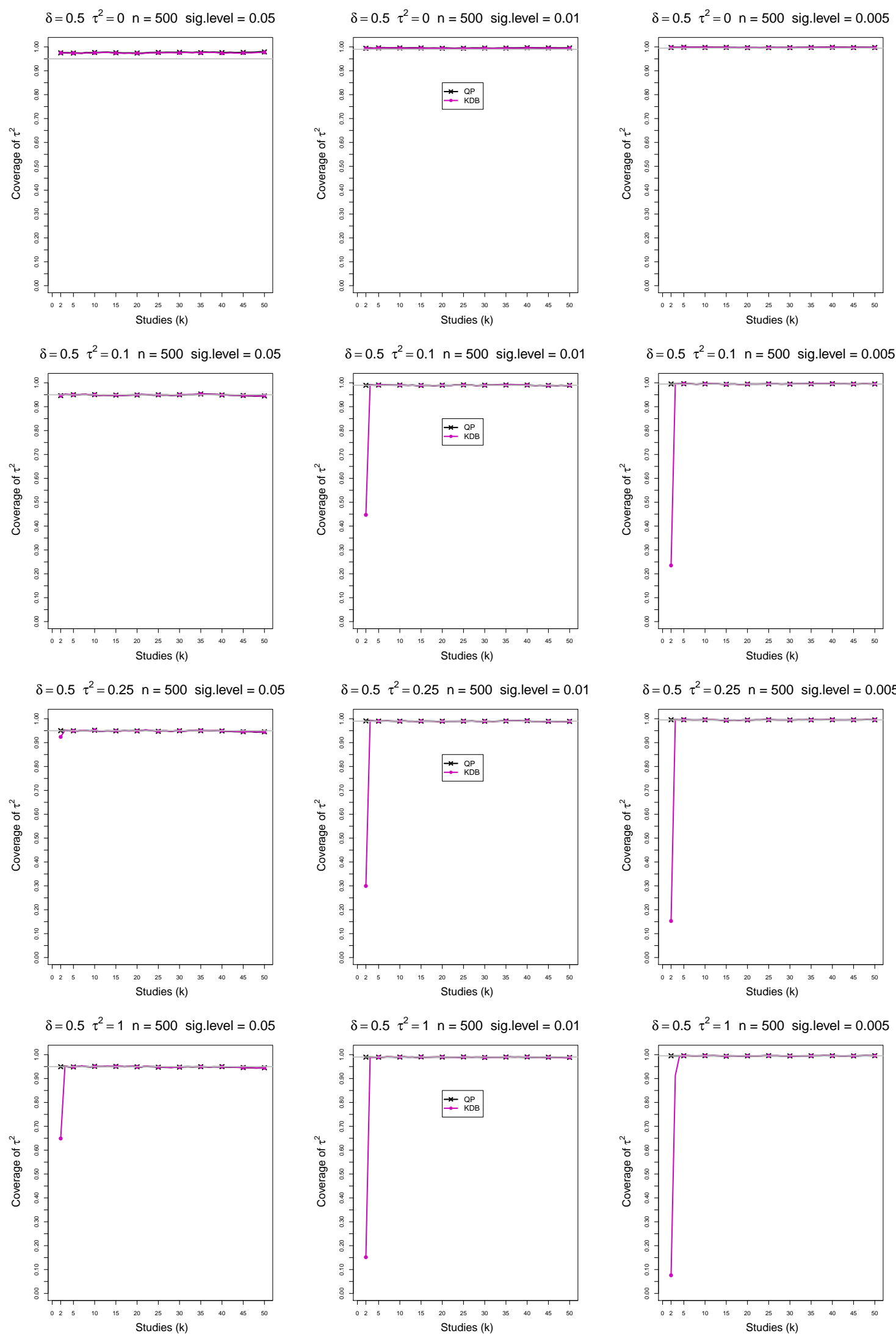

Figure A5. 8: CMA - Coverage of interval estimators of between-study variance $\tau^{2}$ at confidence level $1-\alpha$ for alpha of $0.05,0.01,0.005$ for $\delta=0.5, \tau^{2}=0,0.1,0.25,1, n=500$, $K=50$. Light grey line at $0.95,0.99,0.995$. 

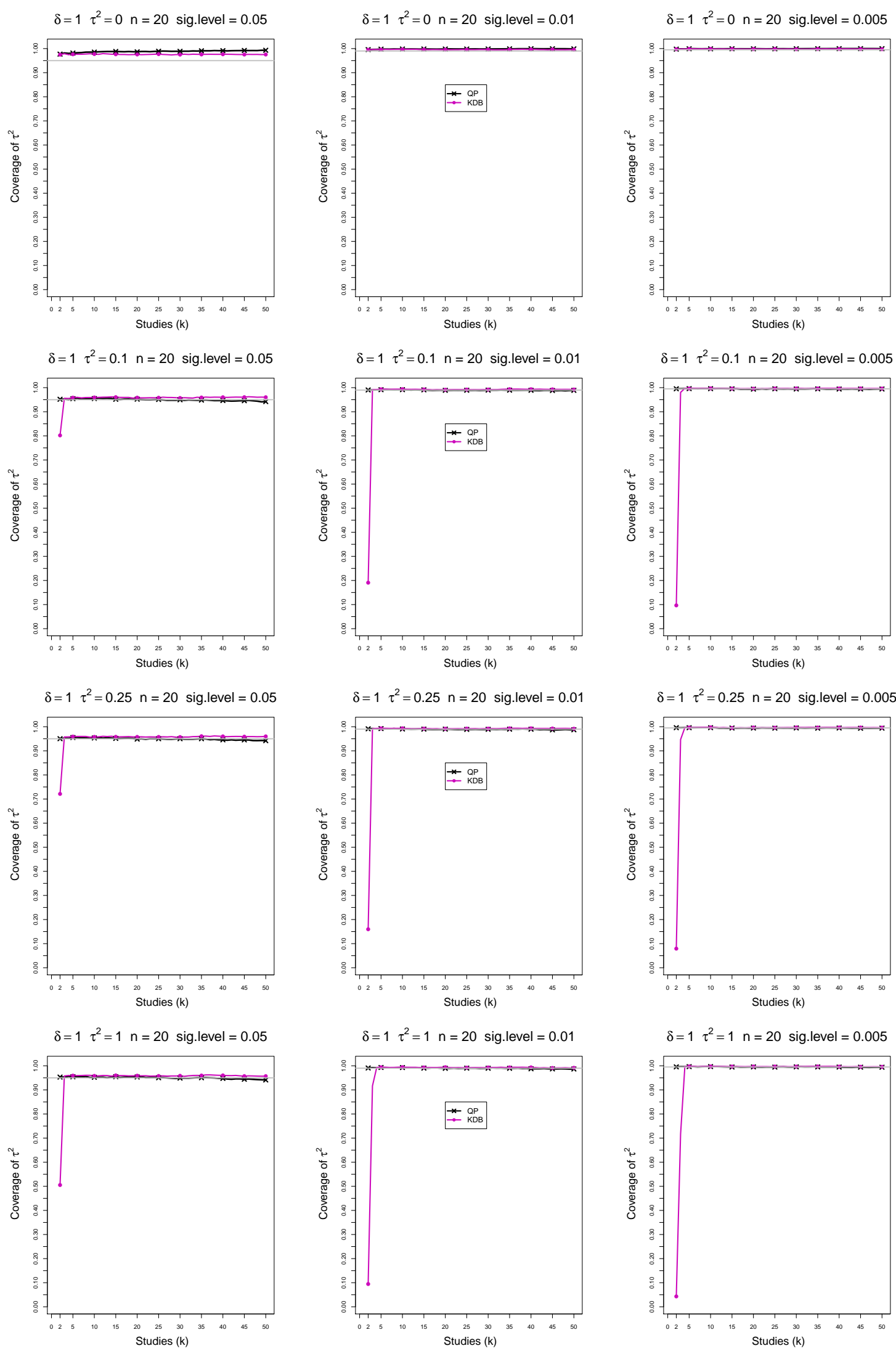

Figure A5. 9: CMA - Coverage of interval estimators of between-study variance $\tau^{2}$ at confidence level $1-\alpha$ for alpha of 0.05, 0.01, 0.005 for $\delta=1, \tau^{2}=0,0.1,0.25,1, n=20$, $K=50$. Light grey line at 0.95,0.99,0.995. 

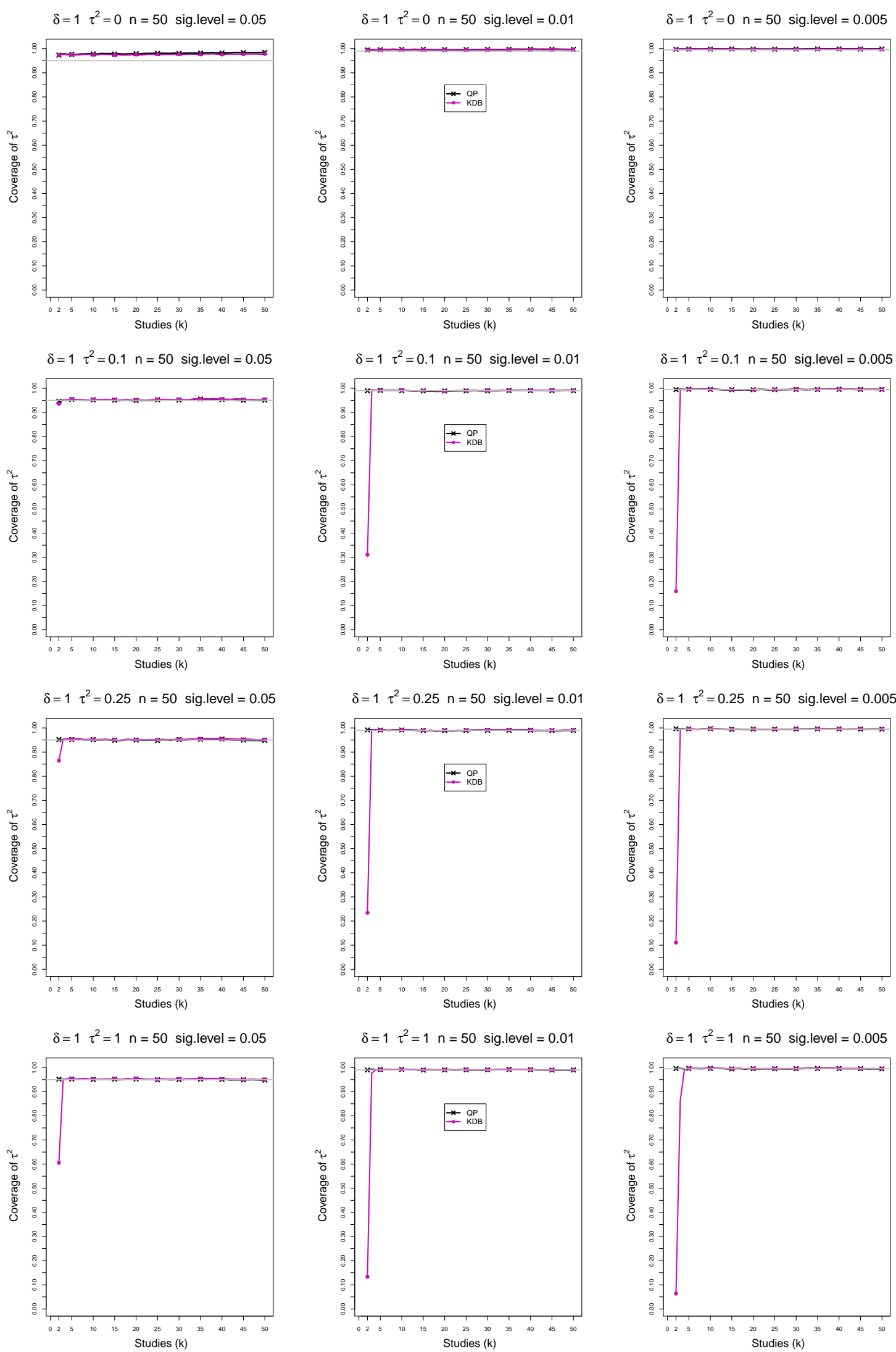

Figure A5. 10: CMA - Coverage of interval estimators of between-study variance $\tau^{2}$ at confidence level $1-\alpha$ for alpha of 0.05, 0.01, 0.005 for $\delta=1, \tau^{2}=0,0.1,0.25,1, n=50$, $K=50$. Light grey line at 0.95,0.99,0.995. 

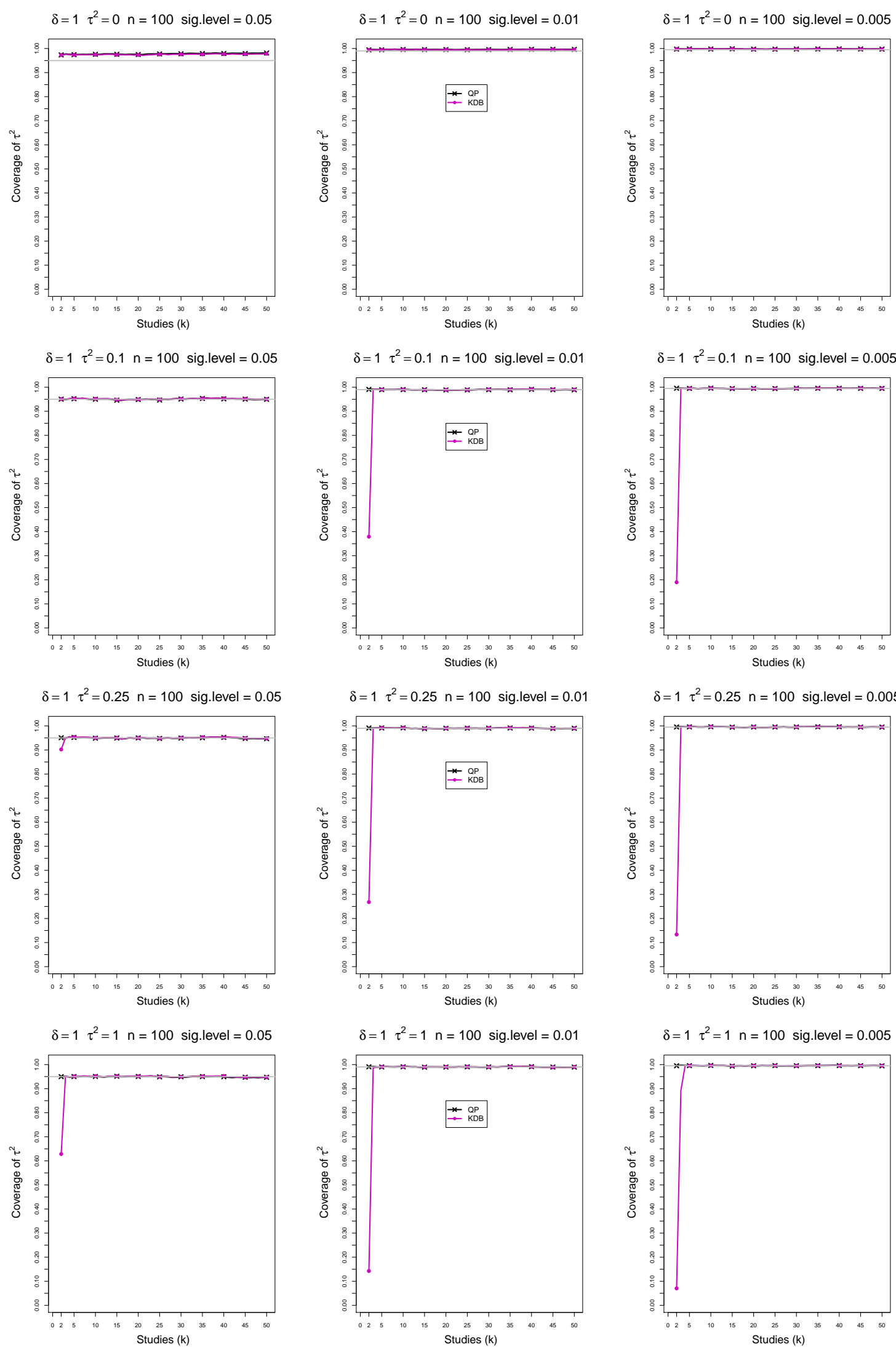

Figure A5. 11: CMA - Coverage of interval estimators of between-study variance $\tau^{2}$ at confidence level $1-\alpha$ for alpha of $0.05,0.01,0.005$ for $\delta=1, \tau^{2}=0,0.1,0.25,1, n=100$, $K=50$. Light grey line at $0.95,0.99,0.995$. 

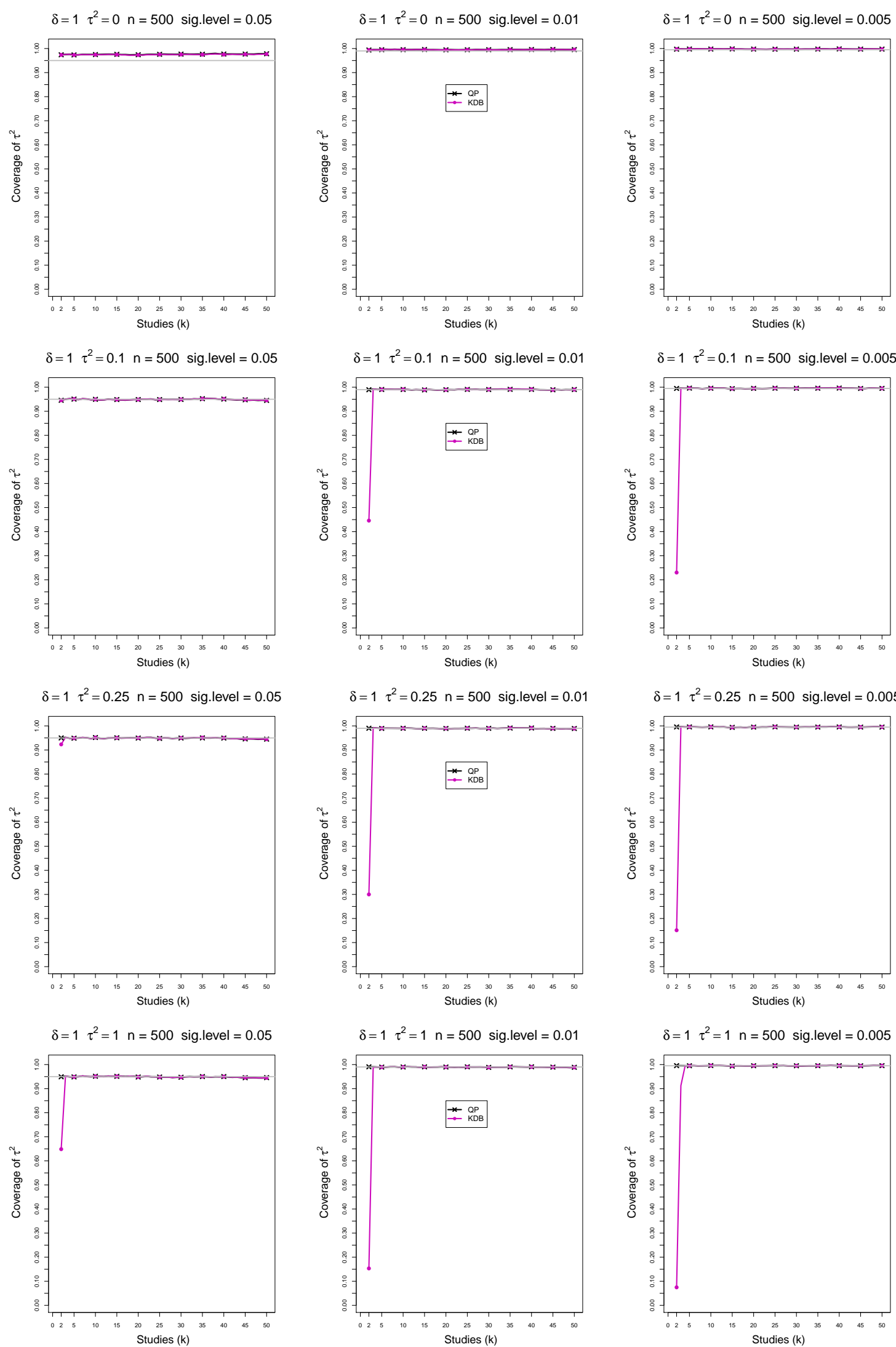

Figure A5. 12: CMA - Coverage of interval estimators of between-study variance $\tau^{2}$ at confidence level $1-\alpha$ for alpha of $0.05,0.01,0.005$ for $\delta=1, \tau^{2}=0,0.1,0.25,1, n=500$, $K=50$. Light grey line at $0.95,0.99,0.995$. 


\section{A6. Empirical levels of CMA tests for shift-in- the-mean $\delta$}

For empirical levels of test for shift-in-the-mean $\delta$ in this section, each figure corresponds to one of the two tested meta-analysis methods, a value of $\delta(=0,0.5,1)$, a set of values of $\tau^{2}(=0,0.1,0.25,1)$, a value of $n(=20,50,100,500)$ and a set of values of nominal significance levels $(=0.05,0.01,0.005)$ while the study number $k$ increases to maximum number $K=50$. Each figure contains a panel (with study $k$ on the horizontal axis) for each combination of parameters.

The tested methods are

- IV REML (Wald test based on the inverse variance weighted mean with the between-study variance $\tau^{2}$ estimated by Restricted maximum likelihood)

- SSW (Test based on effective sample size weighted mean with $t_{K-1}$ critical values) 

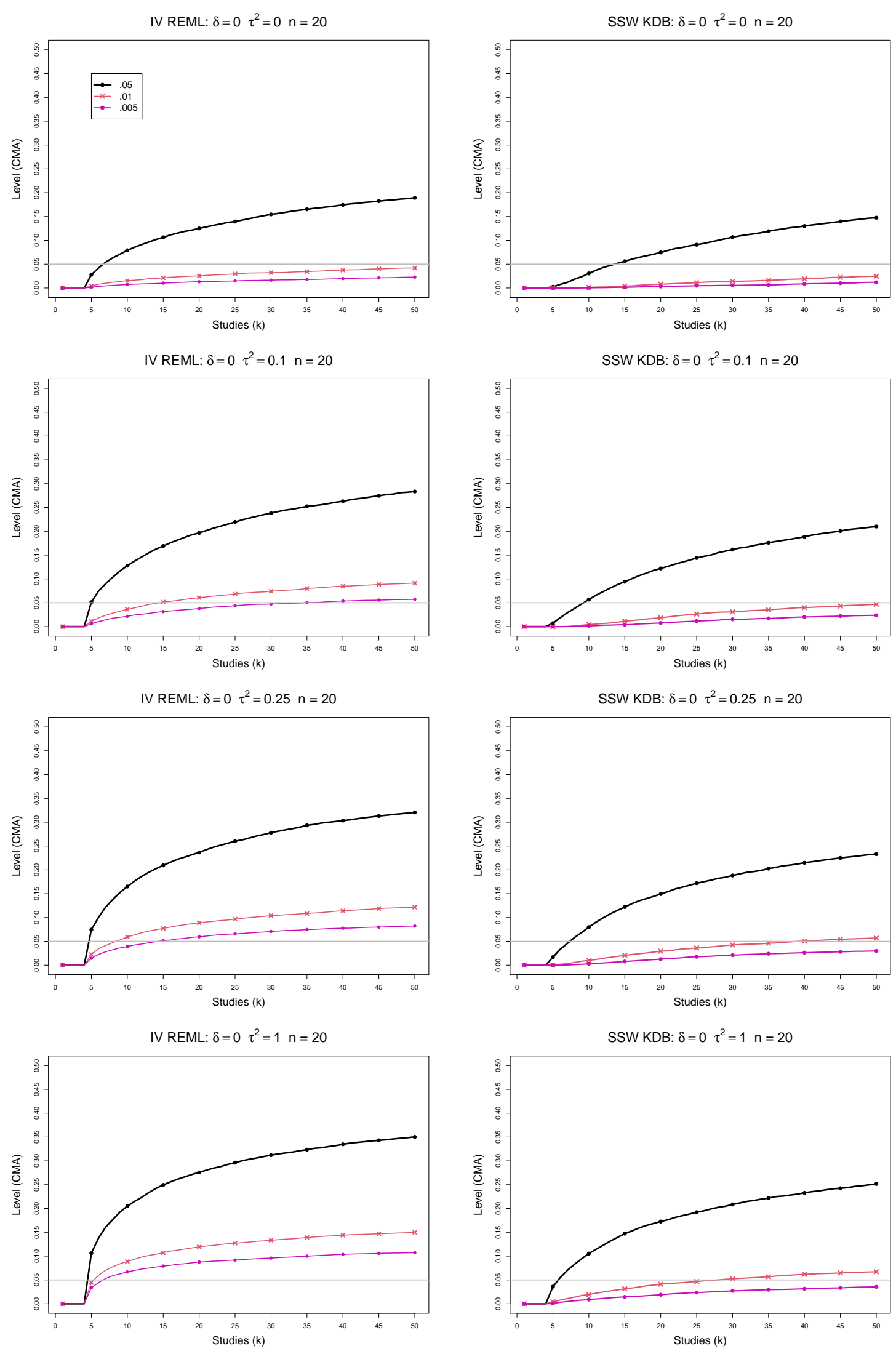

Figure A6. 1: Empirical levels of CMA tests for shift in $\delta=0$ based on SSW KDB and IV REML at .05, .01 and .005 nominal levels for $k$ up to $K=50$ for equal sample sizes $n_{i C}+n_{i T}=n=20, \tau^{2}=0,0.1,0.25,1$. Light grey line at .05. 

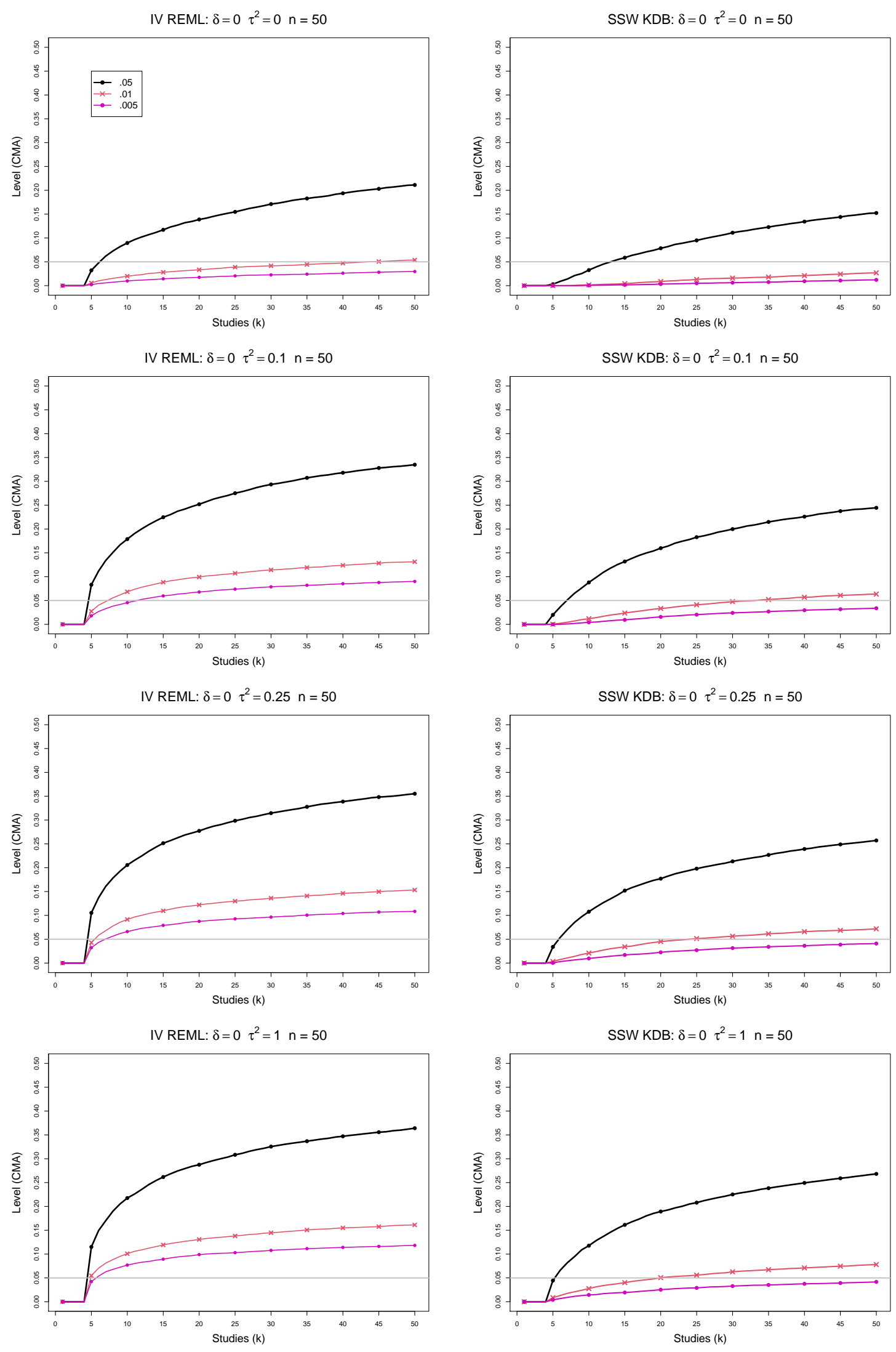

Figure A6. 2: Empirical levels of CMA tests for shift in $\delta=0$ based on SSW KDB and IV REML at .05, .01 and .005 nominal levels for $k$ up to $K=50$ for equal sample sizes $n_{i C}+n_{i T}=n=50, \tau^{2}=0,0.1,0.25,1$. Light grey line at .05. 

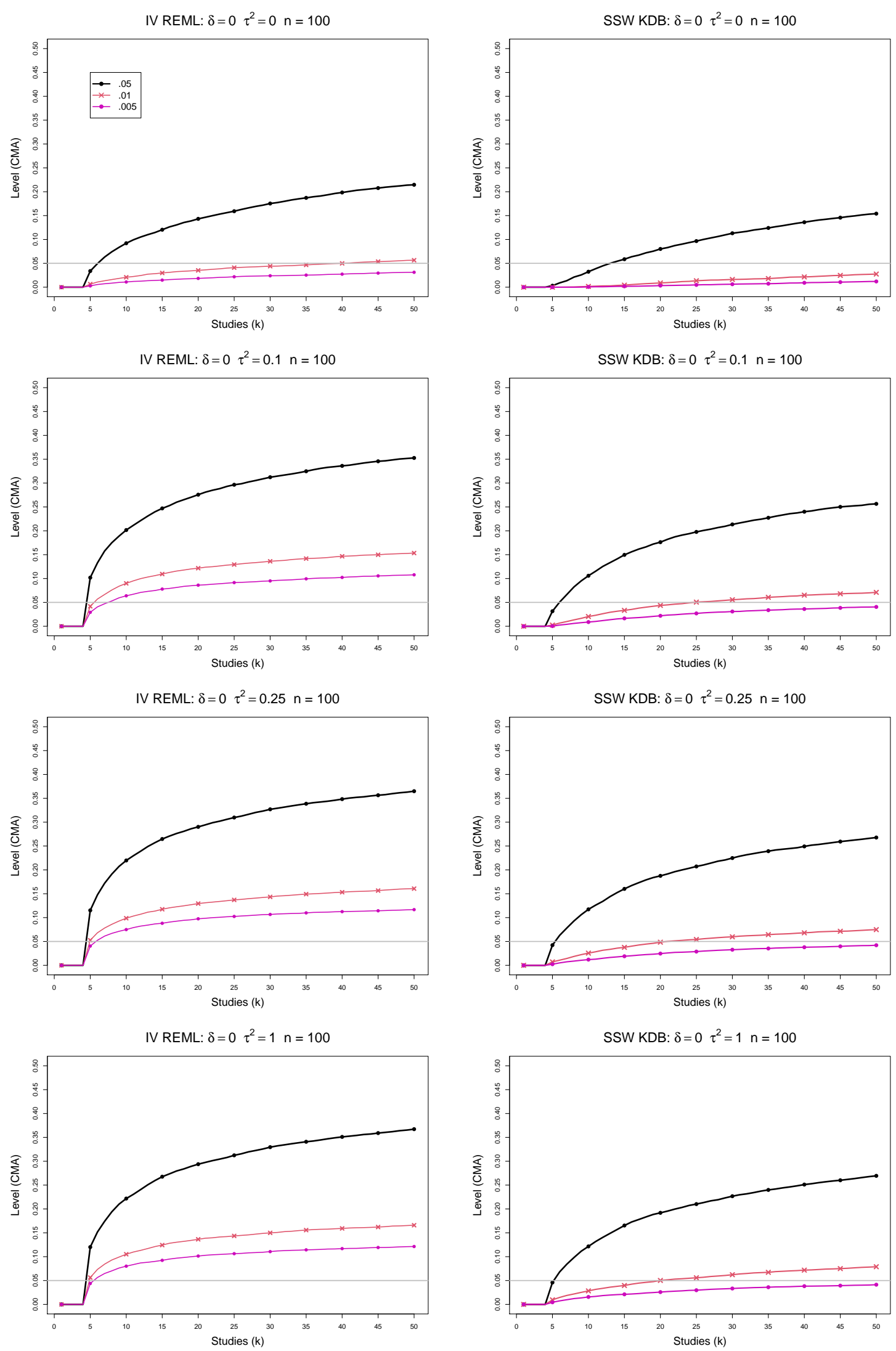

Figure A6. 3: Empirical levels of CMA tests for shift in $\delta=0$ based on SSW KDB and IV REML at .05, .01 and .005 nominal levels for $k$ up to $K=50$ for equal sample sizes $n_{i C}+n_{i T}=n=100, \tau^{2}=0,0.1,0.25,1$. Light grey line at .05. 

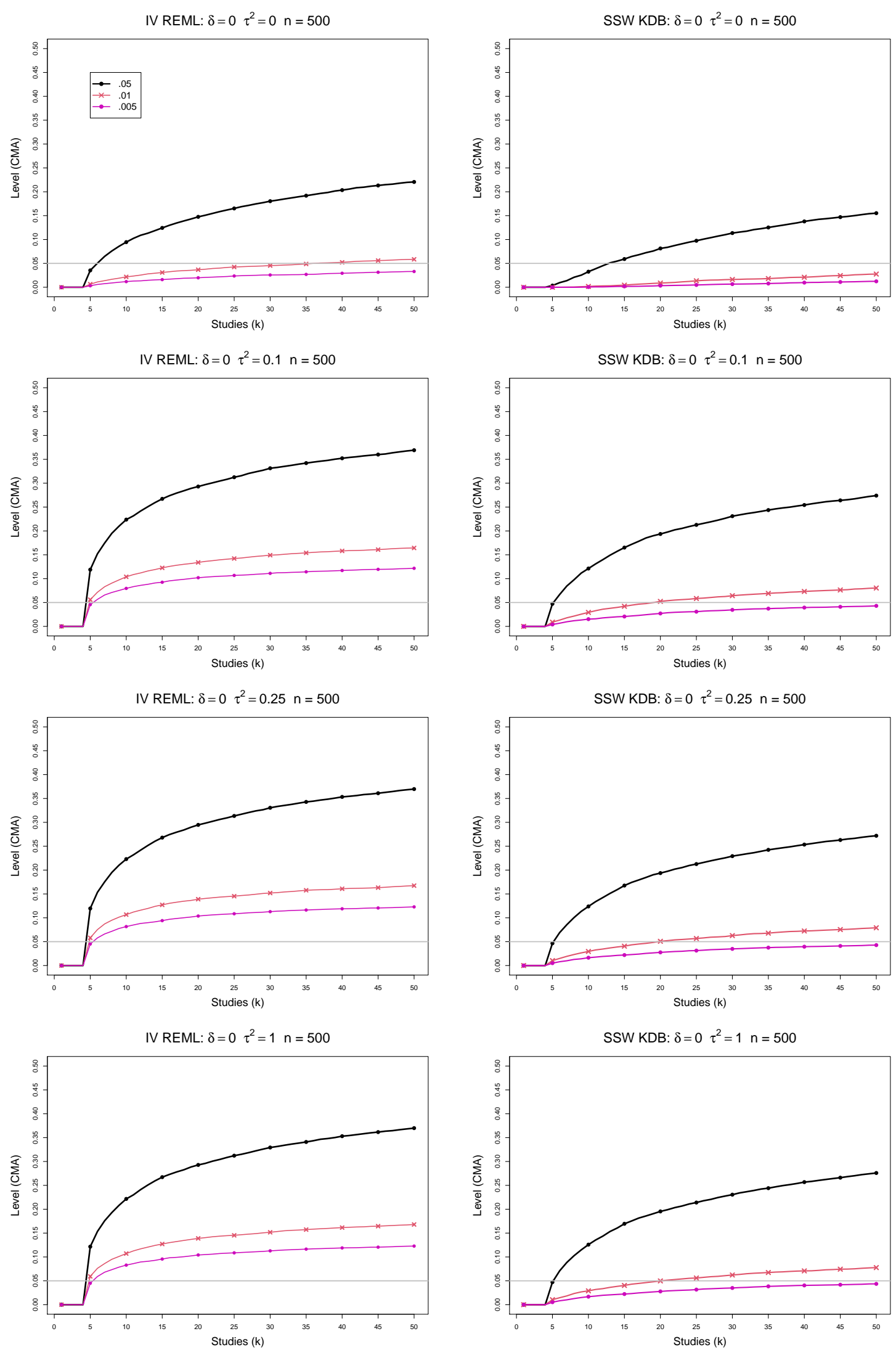

Figure A6. 4: Empirical levels of CMA tests for shift in $\delta=0$ based on SSW KDB and IV REML at .05, .01 and .005 nominal levels for $k$ up to $K=50$ for equal sample sizes $n_{i C}+n_{i T}=n=500, \tau^{2}=0,0.1,0.25,1$. Light grey line at .05. 

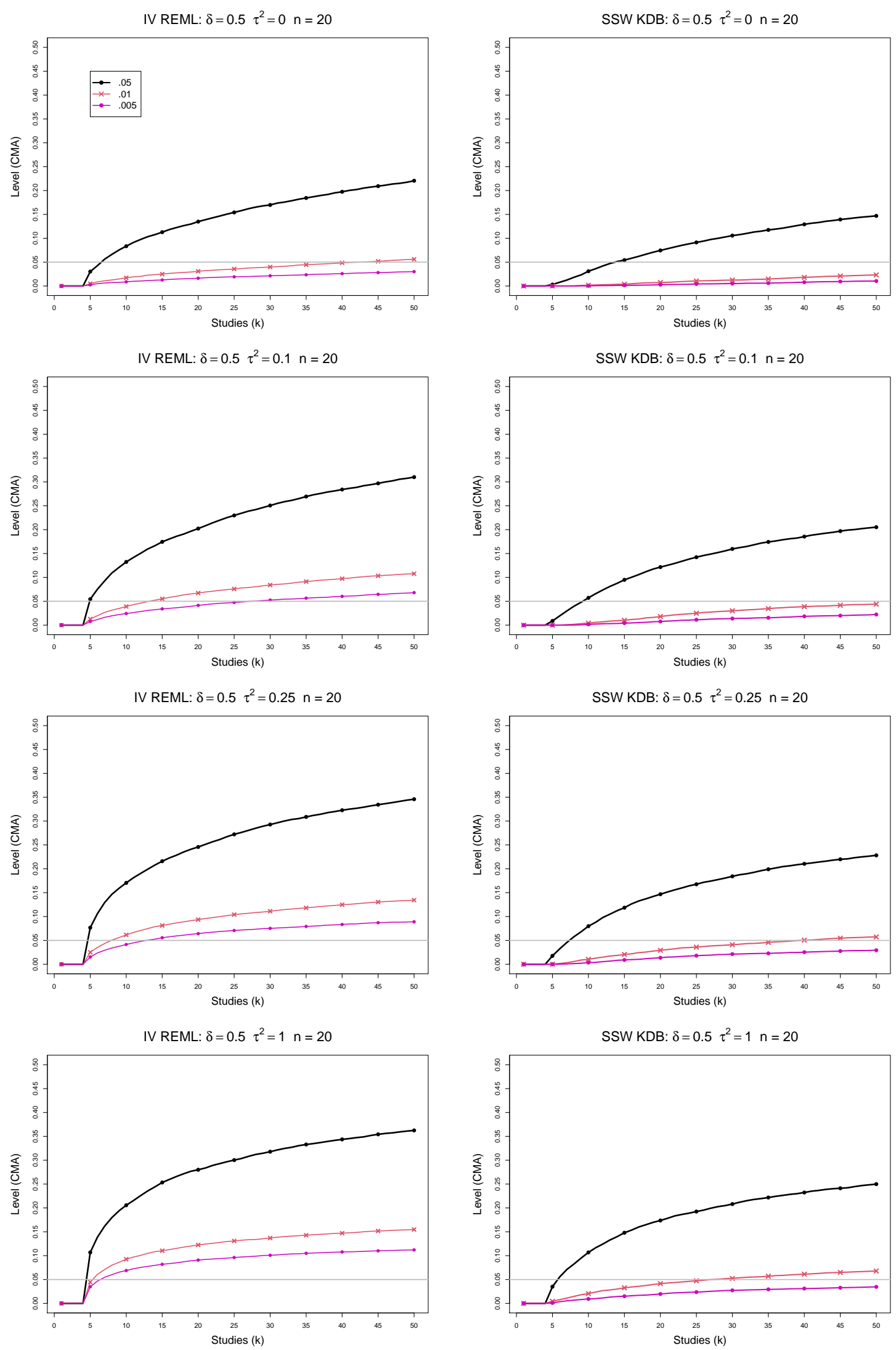

Figure A6. 5: Empirical levels of CMA tests for shift in $\delta=0.5$ based on SSW KDB and IV REML at .05, .01 and .005 nominal levels for $k$ up to $K=50$ for equal sample sizes $n_{i C}+n_{i T}=n=20, \tau^{2}=0,0.1,0.25,1$. Light grey line at .05. 

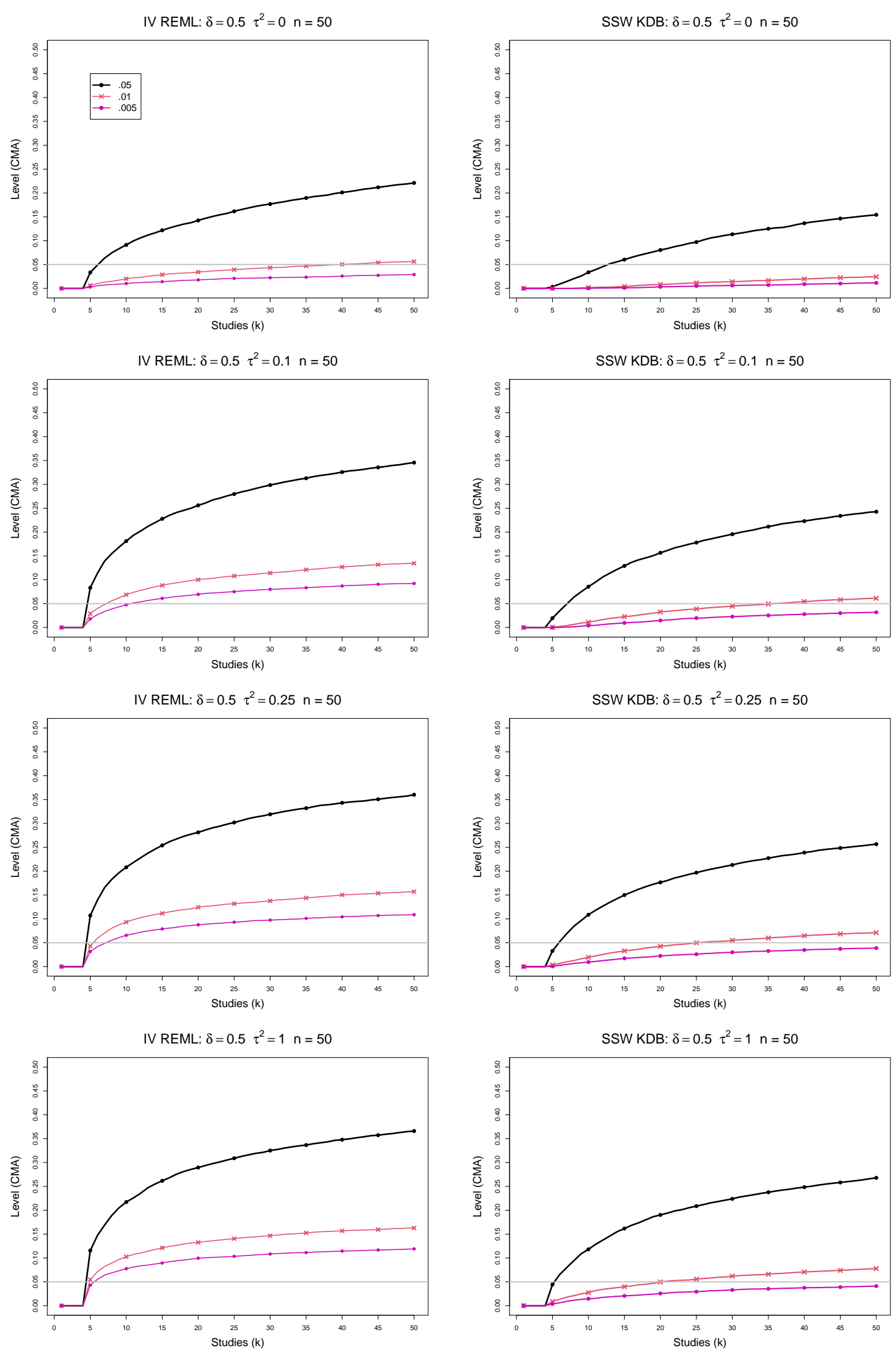

Figure A6. 6: Empirical levels of CMA tests for shift in $\delta=0.5$ based on SSW KDB and IV REML at .05, .01 and .005 nominal levels for $k$ up to $K=50$ for equal sample sizes $n_{i C}+n_{i T}=n=50, \tau^{2}=0,0.1,0.25,1$. Light grey line at .05. 

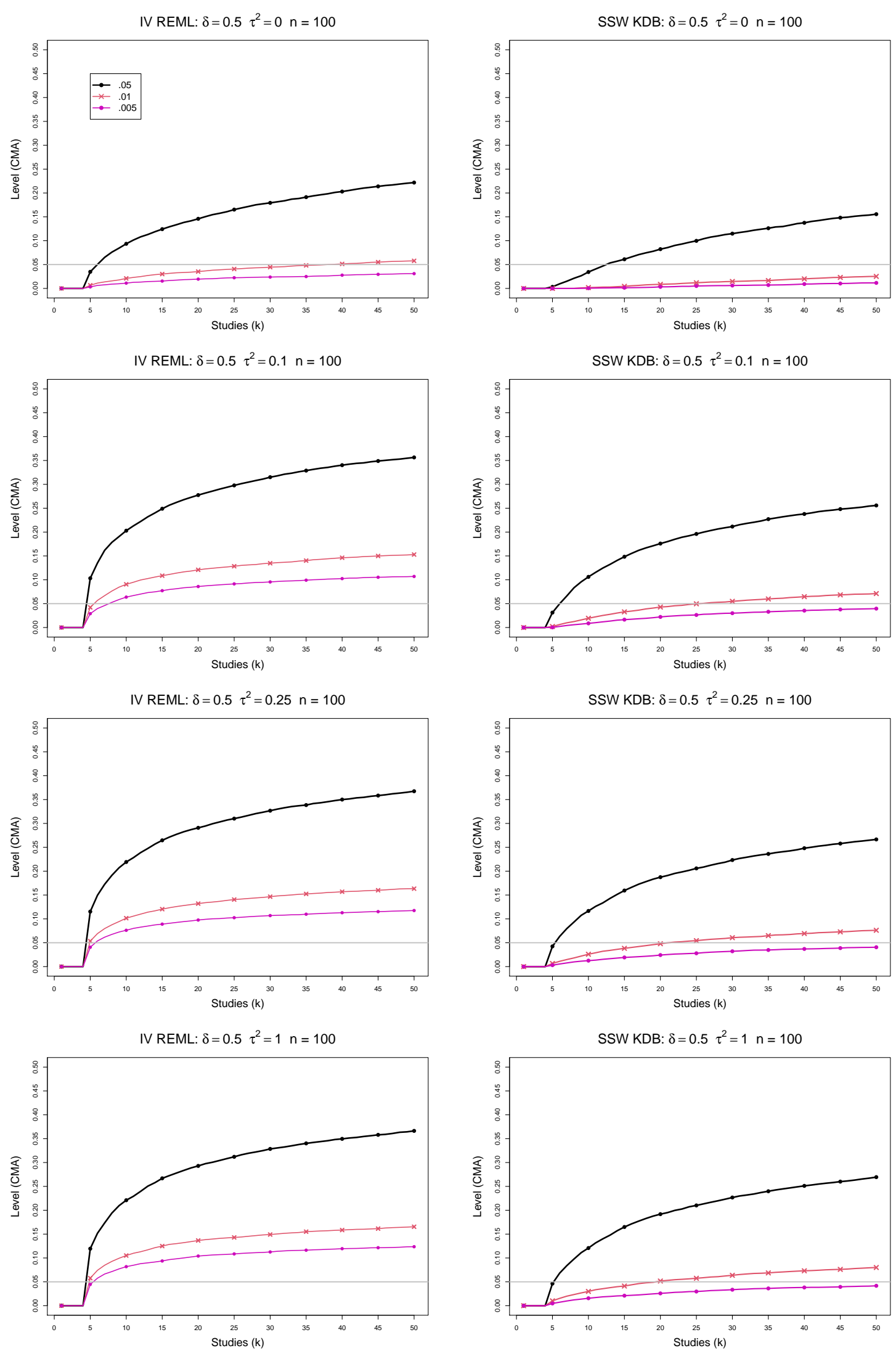

Figure A6. 7: Empirical levels of CMA tests for shift in $\delta=0.5$ based on SSW KDB and IV REML at .05, .01 and .005 nominal levels for $k$ up to $K=50$ for equal sample sizes $n_{i C}+n_{i T}=n=100, \tau^{2}=0,0.1,0.25,1$. Light grey line at .05. 

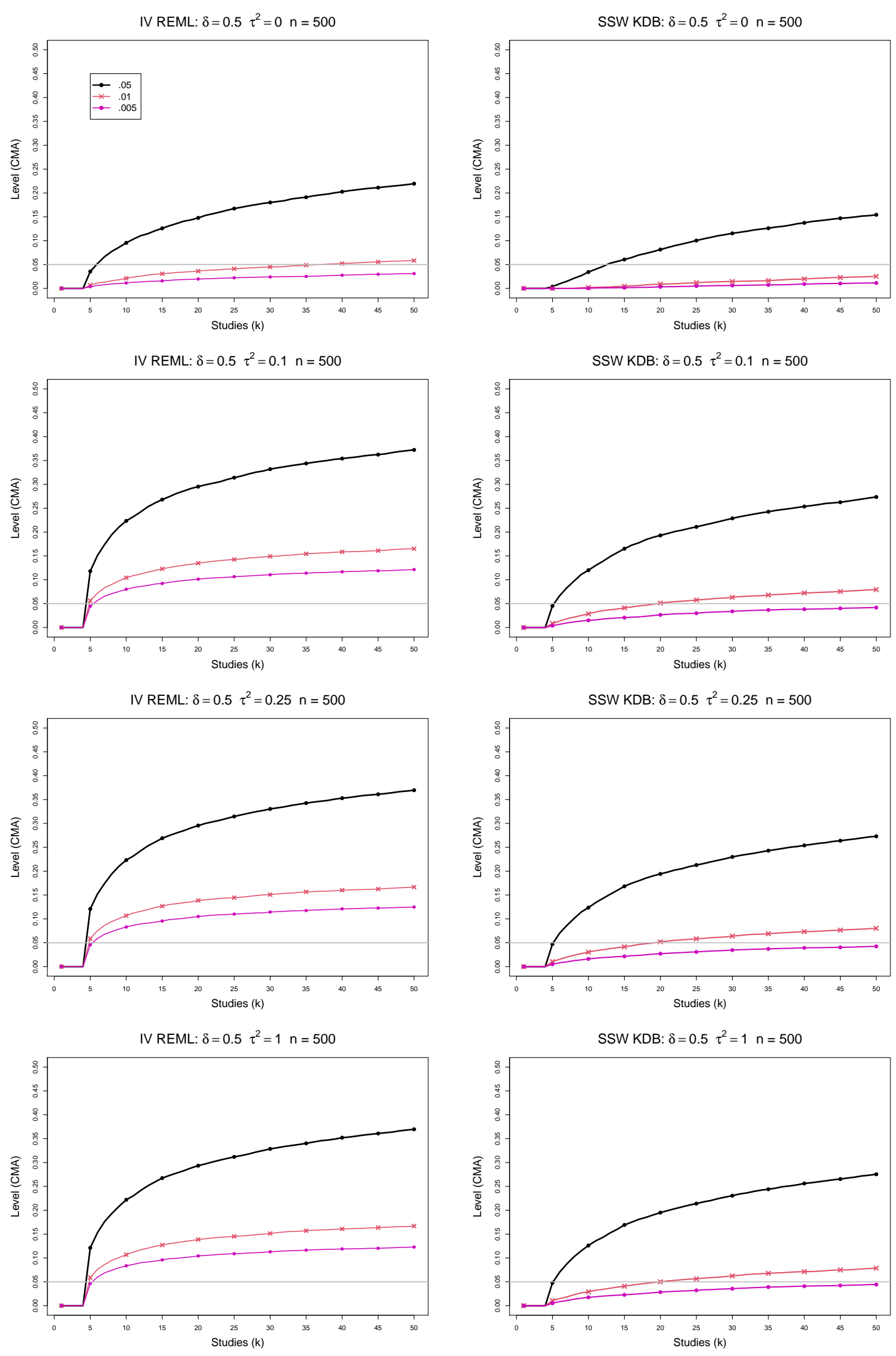

Figure A6. 8: Empirical levels of CMA tests for shift in $\delta=0.5$ based on SSW KDB and IV REML at .05, .01 and .005 nominal levels for $k$ up to $K=50$ for equal sample sizes $n_{i C}+n_{i T}=n=500, \tau^{2}=0,0.1,0.25,1$. Light grey line at .05. 

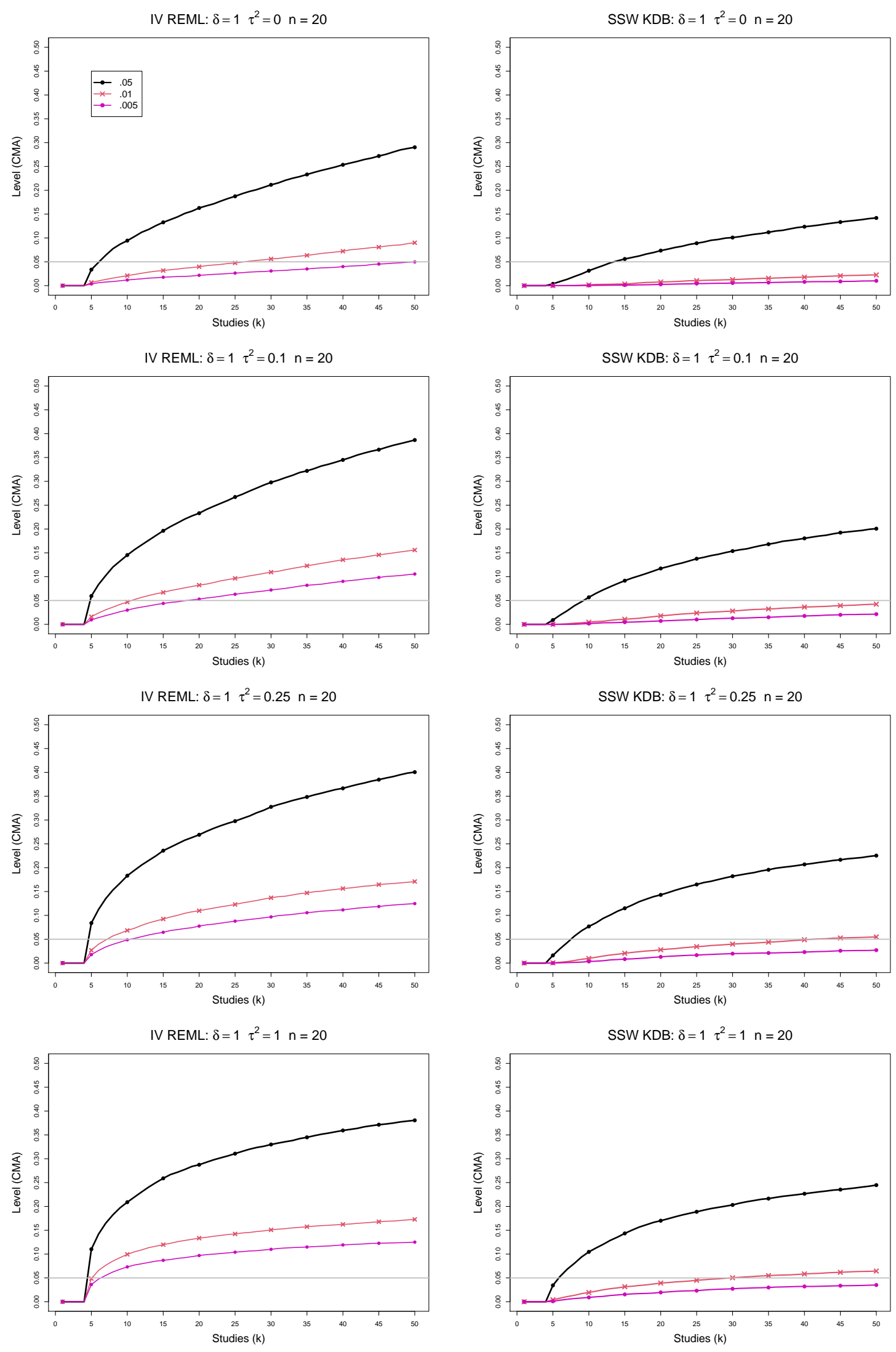

Figure A6. 9: Empirical levels of CMA tests for shift in $\delta=1$ based on SSW KDB and IV REML at .05, .01 and .005 nominal levels for $k$ up to $K=50$ for equal sample sizes $n_{i C}+n_{i T}=n=20, \tau^{2}=0,0.1,0.25,1$. Light grey line at .05. 

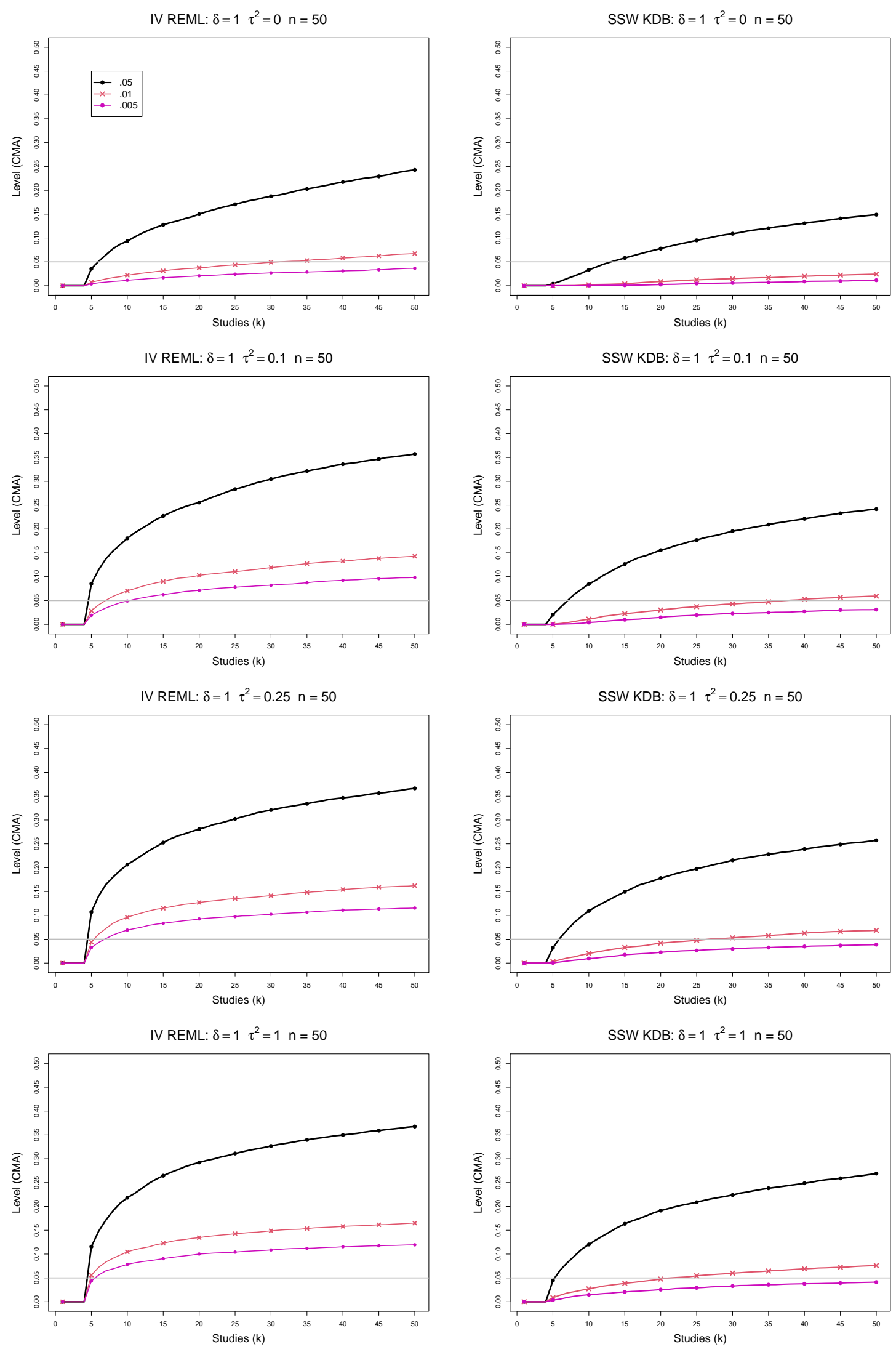

Figure A6. 10: Empirical levels of CMA tests for shift in $\delta=1$ based on SSW KDB and IV REML at .05, .01 and .005 nominal levels for $k$ up to $K=50$ for equal sample sizes $n_{i C}+n_{i T}=n=50, \tau^{2}=0,0.1,0.25,1$. Light grey line at .05. 

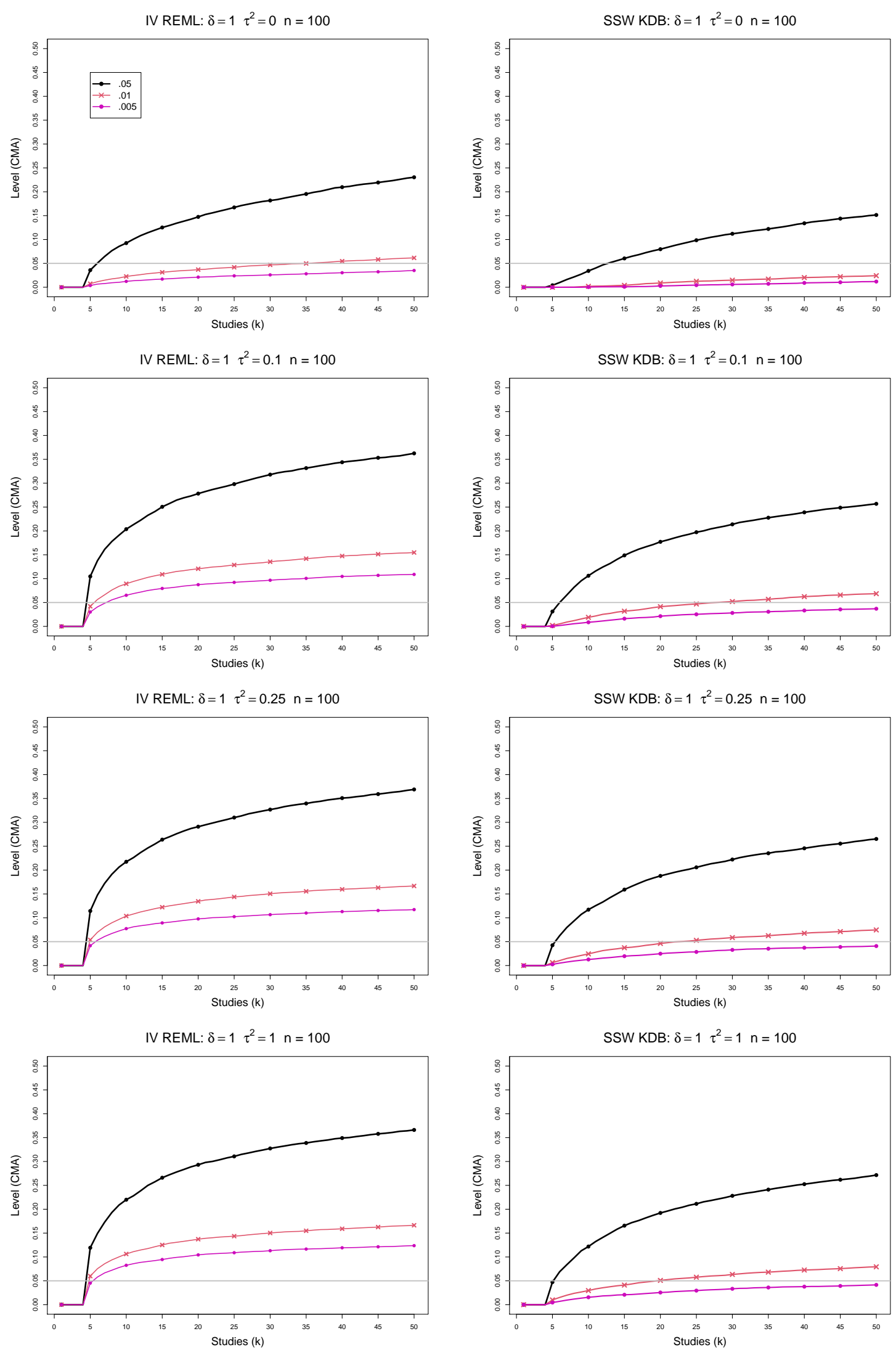

Figure A6. 11: Empirical levels of CMA tests for shift in $\delta=1$ based on SSW KDB and IV REML at .05, .01 and .005 nominal levels for $k$ up to $K=50$ for equal sample sizes $n_{i C}+n_{i T}=n=100, \tau^{2}=0,0.1,0.25,1$. Light grey line at .05. 

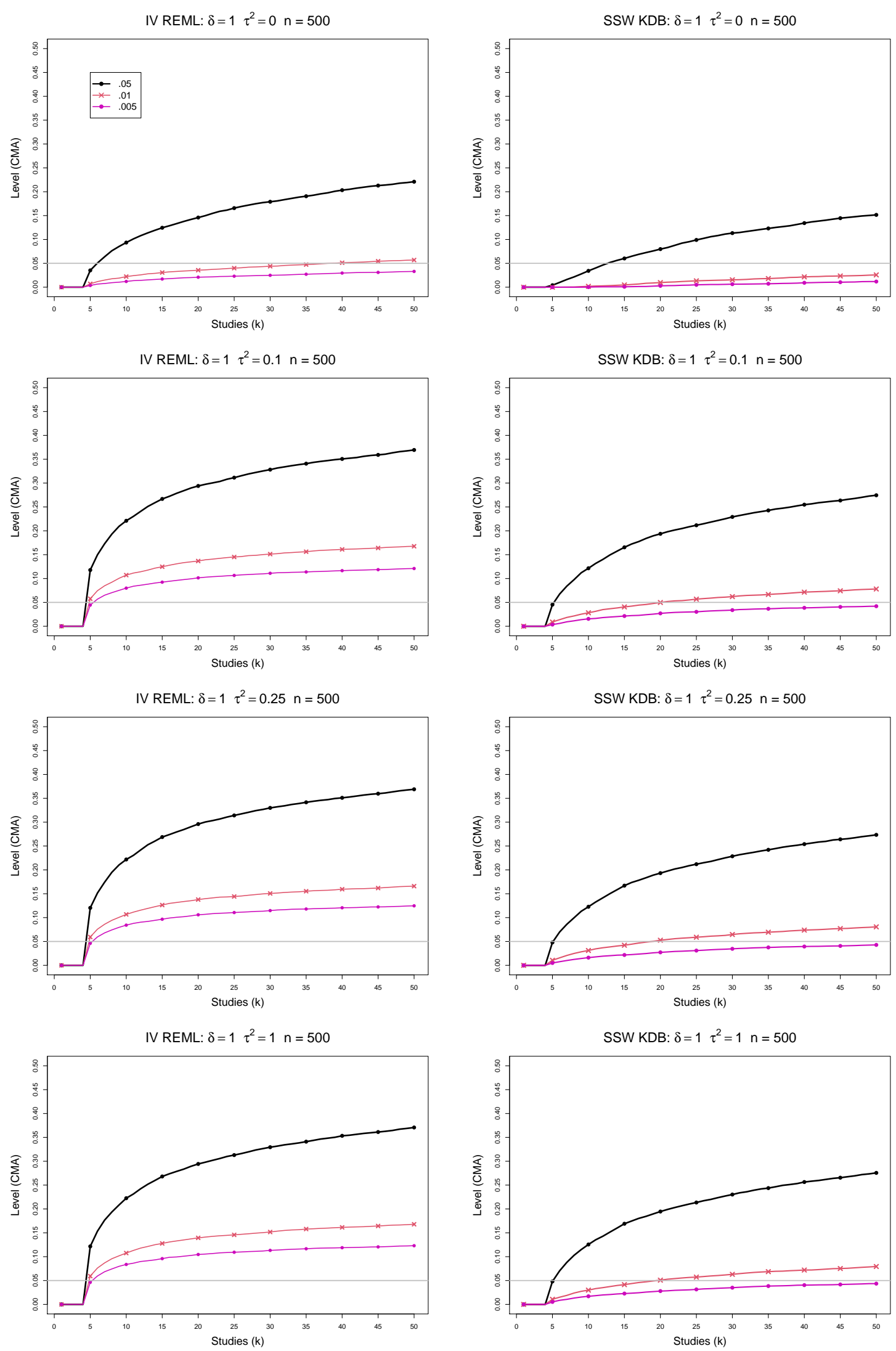

Figure A6. 12: Empirical levels of CMA tests for shift in $\delta=1$ based on SSW KDB and IV REML at .05, .01 and .005 nominal levels for $k$ up to $K=50$ for equal sample sizes $n_{i C}+n_{i T}=n=500, \tau^{2}=0,0.1,0.25,1$. Light grey line at .05. 


\section{A7. Empirical levels of CMA tests for positive shift in $\tau^{2}$}

For empirical levels of one-sided tests for positive shift in known value of $\tau^{2}$ in this section, each figure corresponds to one of the two tested methods, a value of $\delta(=0$, $0.5,1)$, a set of values of $\tau^{2}(=0,0.1,0.25,1)$, a value of $n(=20,50,100,500)$ and a set of values of significance level $(=0.025,0.005,0.0025)$ while the study number $k$ increases to maximum number $K=50$.

Each figure contains a panel (with study $k$ on the horizontal axis) for each combination of parameters.

The tested methods are

- QP (one-sided test based on Q-profile confidence interval by Viechtbauer (2007))

- KDB (one-sided test based on improved Q-profile method by Kulinskaya, Dollinger, Bjørkestøl (2011)) 

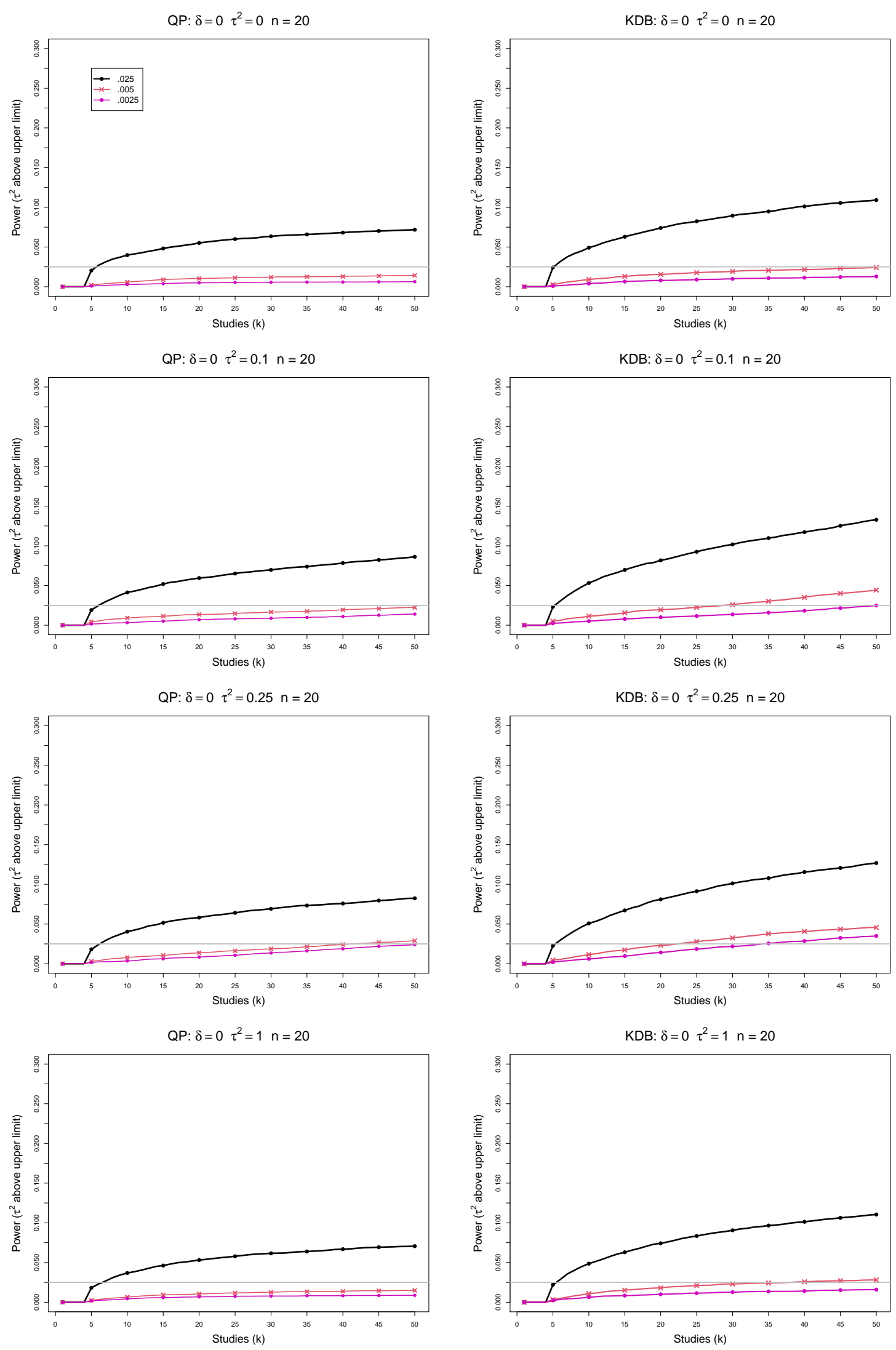

Figure A7. 1: Empirical levels of CMA tests for positive shift in $\tau^{2}$ based on KDB and QP at $.025, .005$ and .0025 nominal levels for $k$ up to $K=50$ for equal sample sizes $n_{i C}+n_{i T}=n=20, \delta=0, \tau^{2}=0,0.1,0.25,1$. Light grey line at .025. 

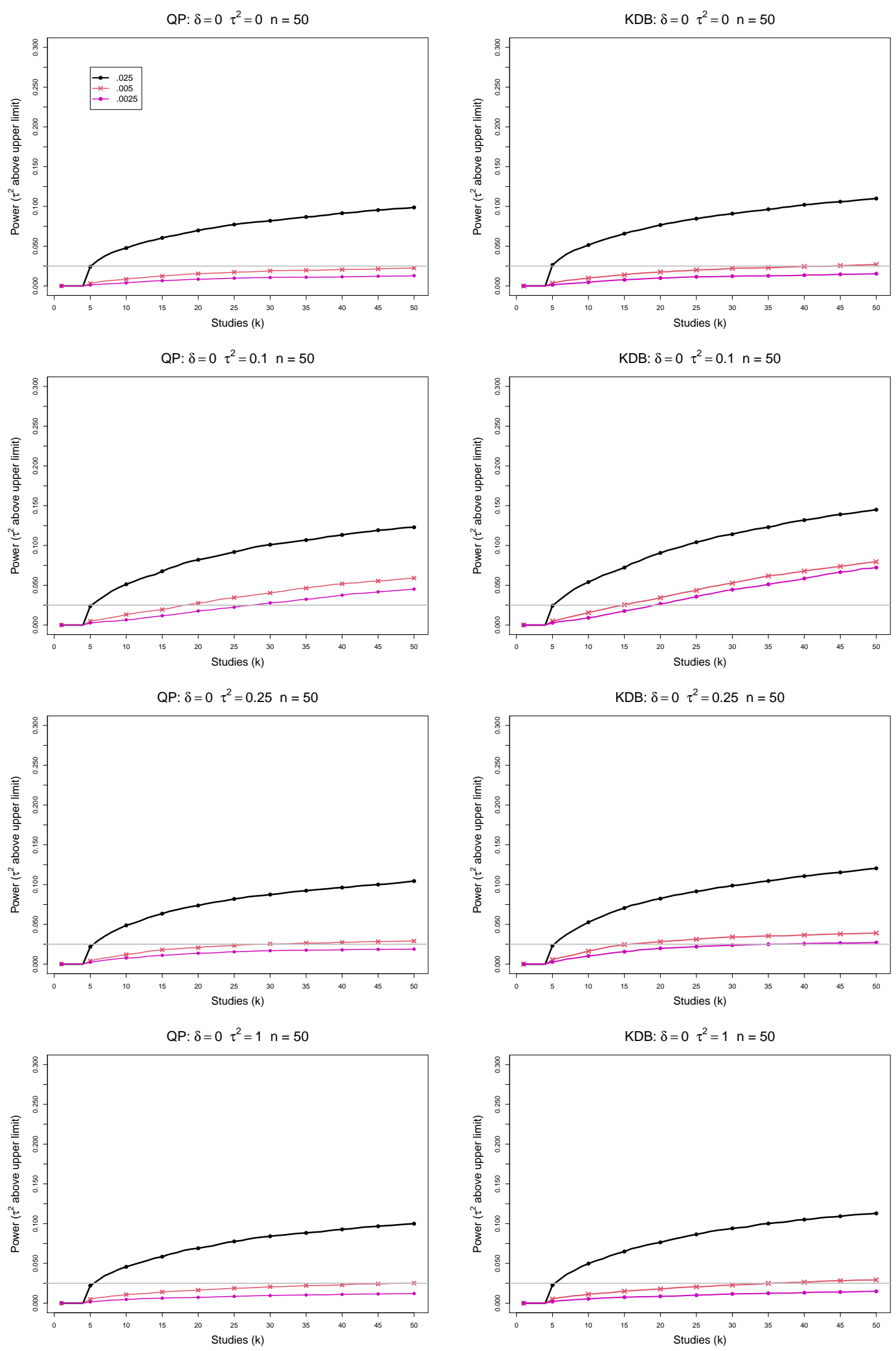

Figure A7. 2: Empirical levels of CMA tests for positive shift in $\tau^{2}$ based on KDB and QP at $.025, .005$ and .0025 nominal levels for $k$ up to $K=50$ for equal sample sizes $n_{i C}+n_{i T}=n=50, \delta=0, \tau^{2}=0,0.1,0.25,1$. Light grey line at .025. 
QP: $\delta=0 \quad \tau^{2}=0 \quad n=100$

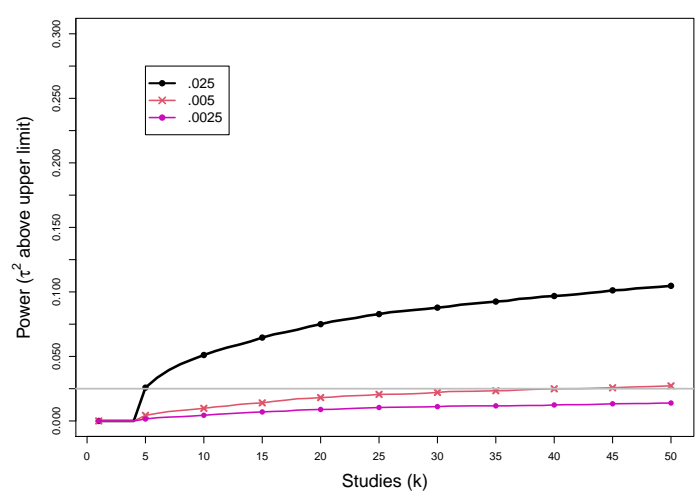

QP: $\delta=0 \quad \tau^{2}=0.1 \quad n=100$

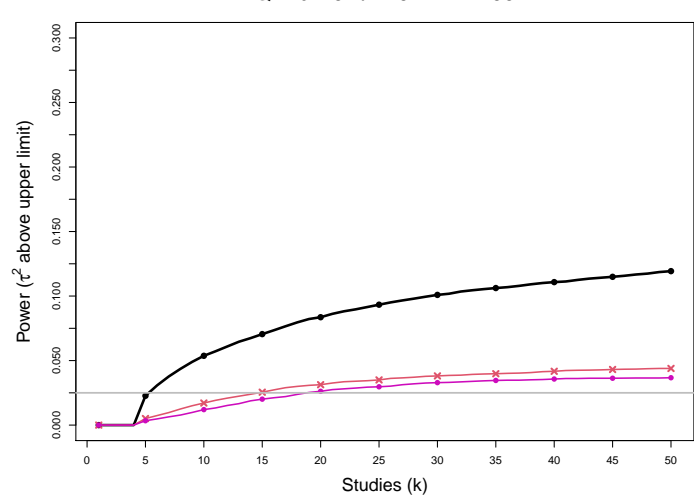

QP: $\delta=0 \quad \tau^{2}=0.25 \quad n=100$

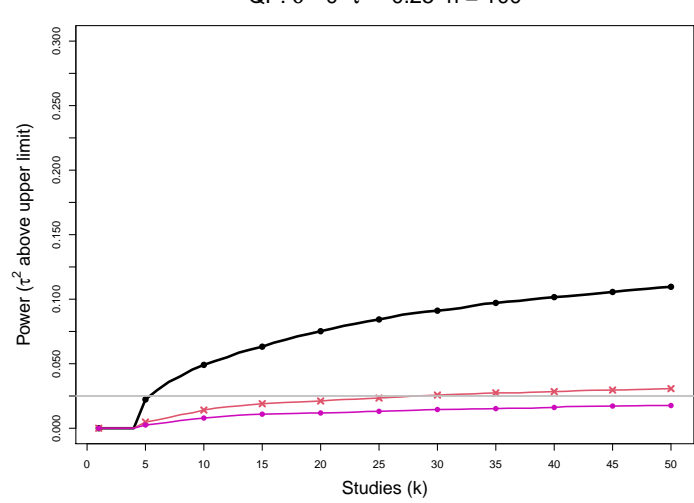

QP: $\delta=0 \quad \tau^{2}=1 \quad n=100$

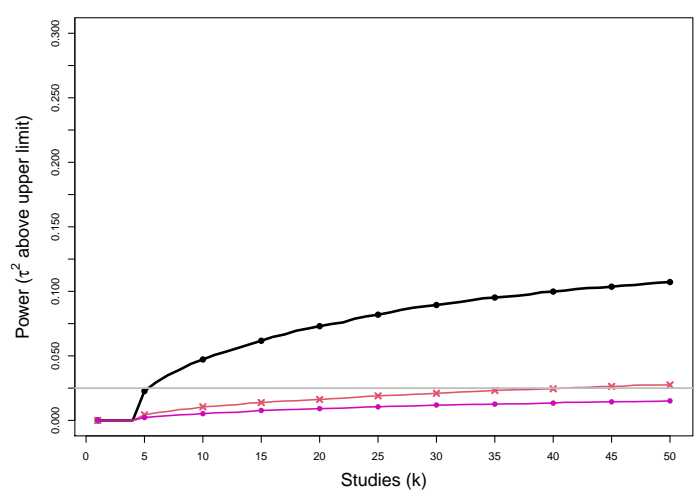

KDB: $\delta=0 \quad \tau^{2}=0 \quad n=100$

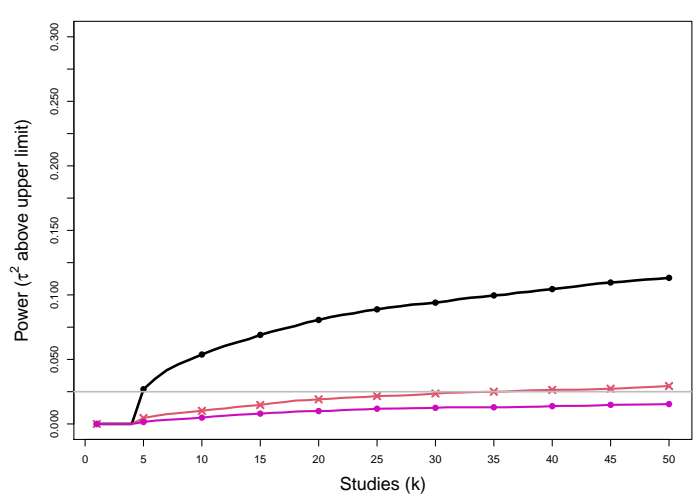

KDB: $\delta=0 \quad \tau^{2}=0.1 \quad \mathrm{n}=100$

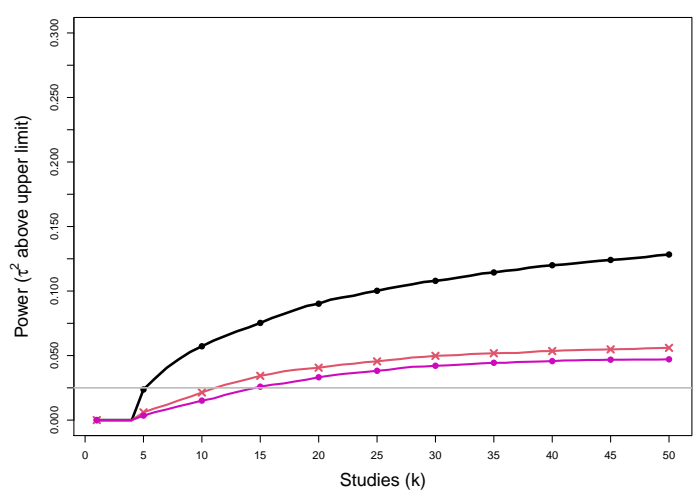

KDB: $\delta=0 \tau^{2}=0.25 n=100$

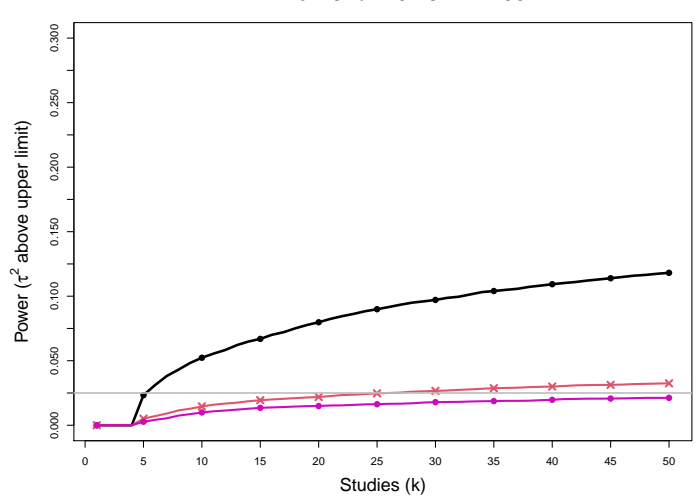

KDB: $\delta=0 \quad \tau^{2}=1 \quad n=100$

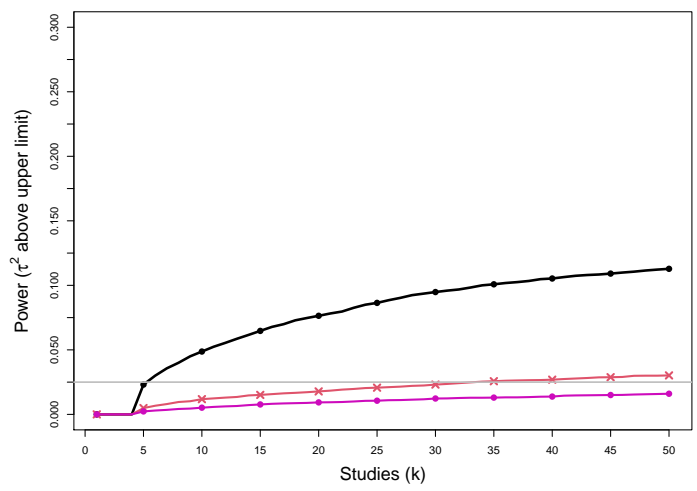

Figure A7. 3: Empirical levels of CMA tests for positive shift in $\tau^{2}$ based on KDB and QP at .025, .005 and .0025 nominal levels for $k$ up to $K=50$ for equal sample sizes $n_{i C}+n_{i T}=n=100, \delta=0, \tau^{2}=0,0.1,0.25,1$. Light grey line at .025. 

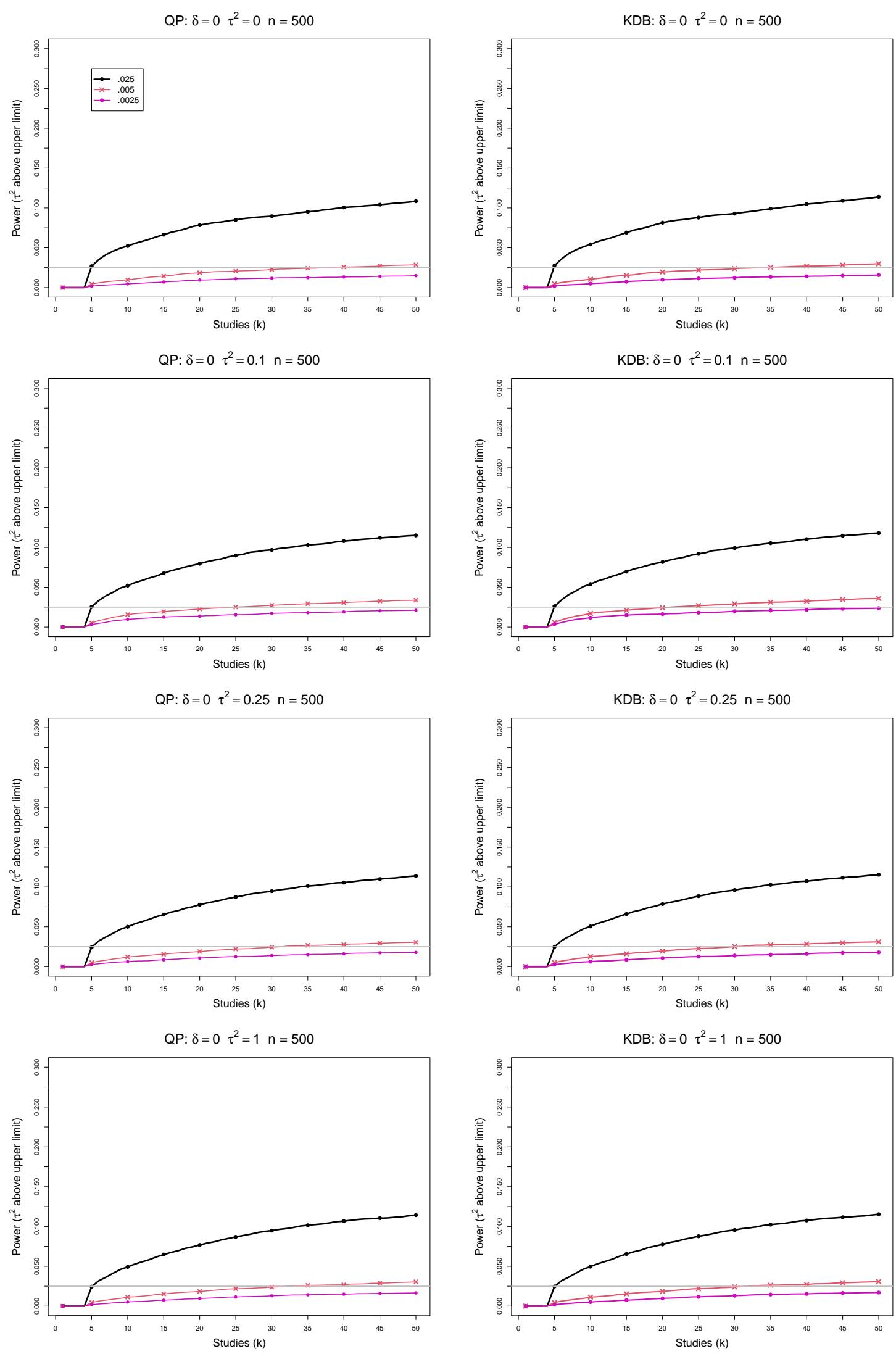

Figure A7. 4: Empirical levels of CMA tests for positive shift in $\tau^{2}$ based on KDB and QP at $.025, .005$ and .0025 nominal levels for $k$ up to $K=50$ for equal sample sizes $n_{i C}+n_{i T}=n=500, \delta=0, \tau^{2}=0,0.1,0.25,1$. Light grey line at .025. 
QP: $\delta=0.5 \tau^{2}=0 \quad n=20$

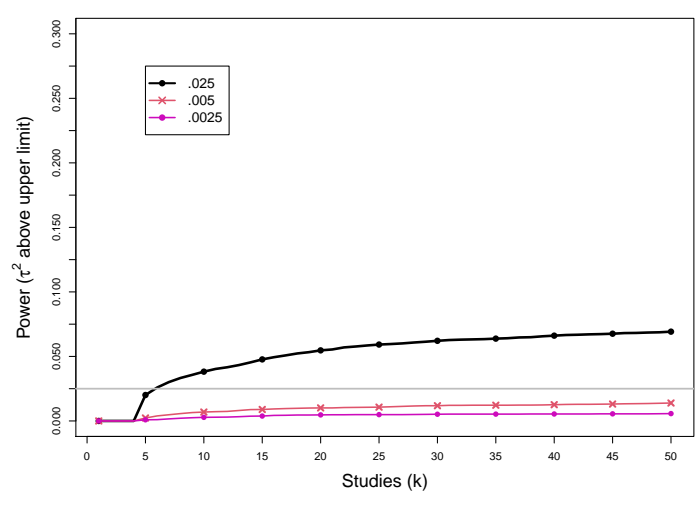

QP: $\delta=0.5 \tau^{2}=0.1 \mathrm{n}=20$

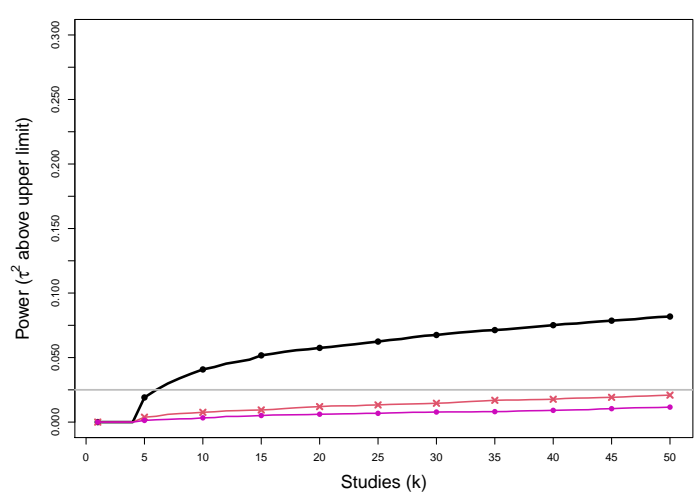

QP: $\delta=0.5 \tau^{2}=0.25 n=20$

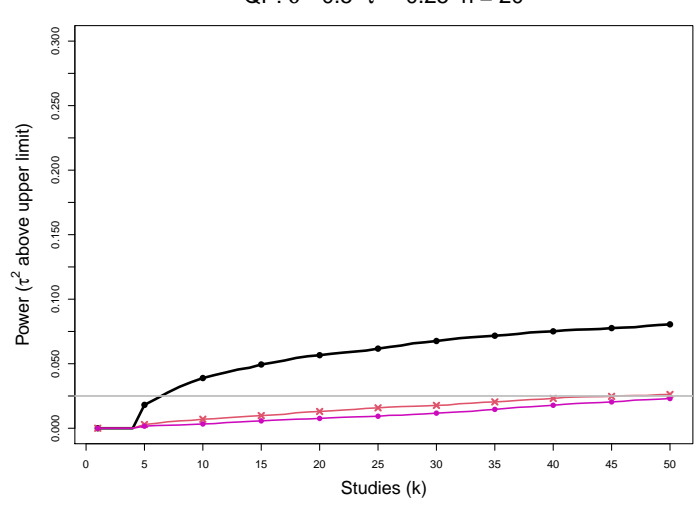

QP: $\delta=0.5 \tau^{2}=1 \mathrm{n}=20$

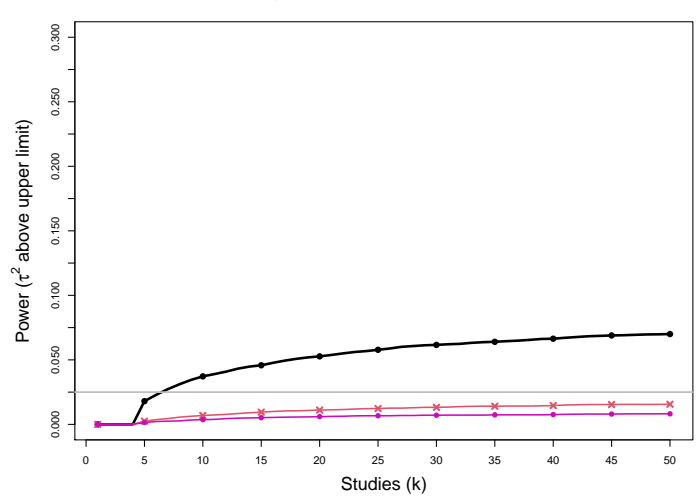

KDB: $\delta=0.5 \tau^{2}=0 \quad n=20$

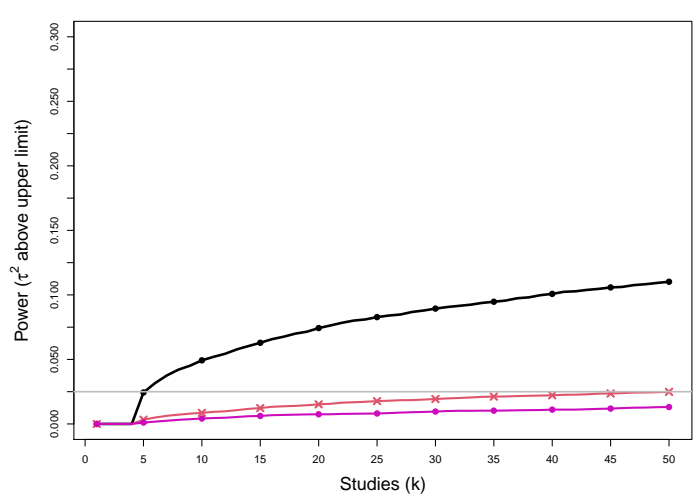

KDB: $\delta=0.5 \tau^{2}=0.1 \quad n=20$

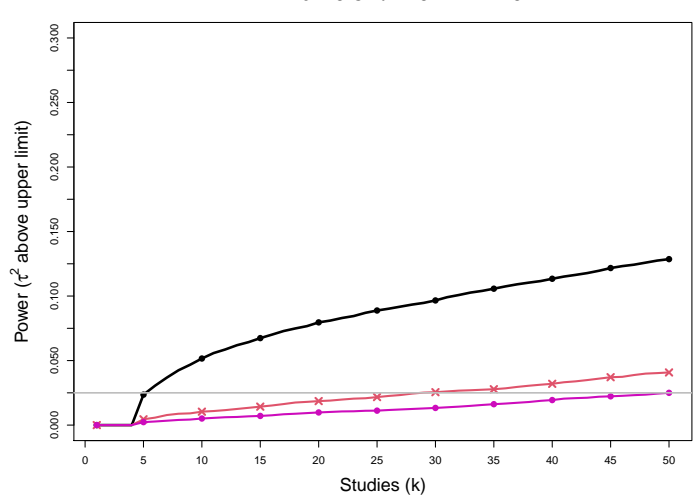

KDB: $\delta=0.5 \tau^{2}=0.25 \mathrm{n}=20$

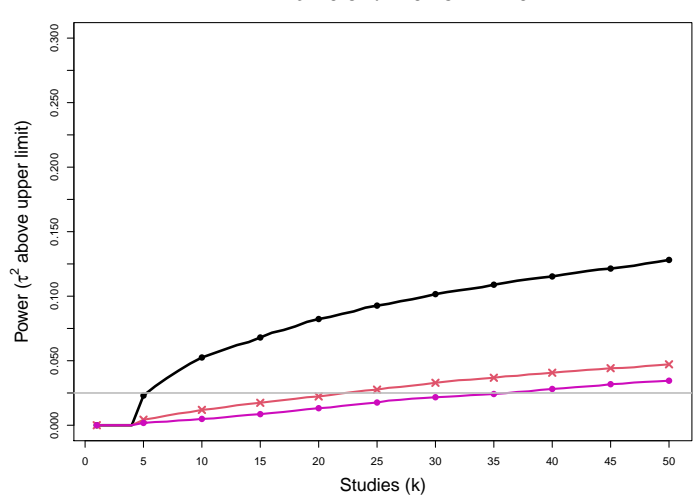

KDB: $\delta=0.5 \tau^{2}=1 \quad n=20$

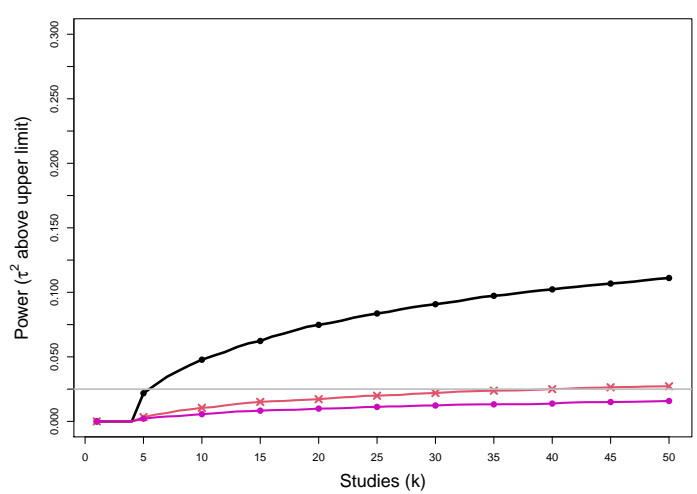

Figure A7. 5: Empirical levels of CMA tests for positive shift in $\tau^{2}$ based on KDB and QP at .025, .005 and .0025 nominal levels for $k$ up to $K=50$ for equal sample sizes $n_{i C}+n_{i T}=n=20, \delta=0.5, \tau^{2}=0,0.1,0.25,1$. Light grey line at .025 . 
QP: $\delta=0.5 \tau^{2}=0 \quad n=50$

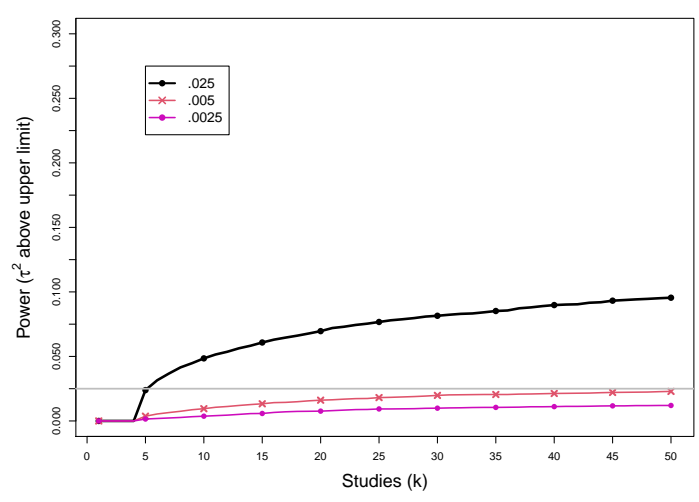

QP: $\delta=0.5 \tau^{2}=0.1 \mathrm{n}=50$

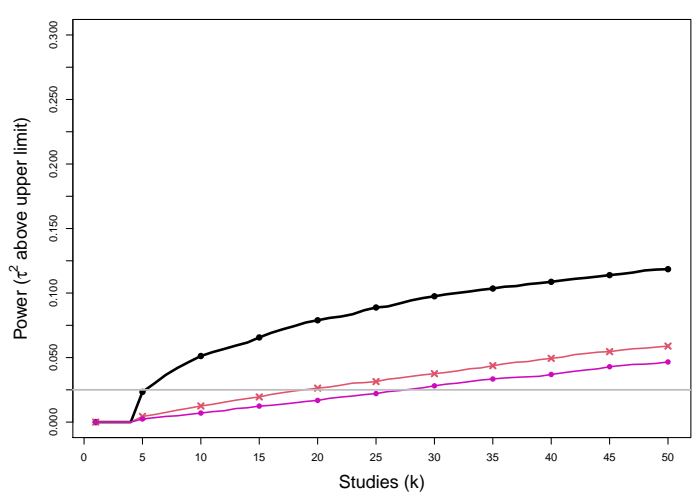

QP: $\delta=0.5 \tau^{2}=0.25 n=50$

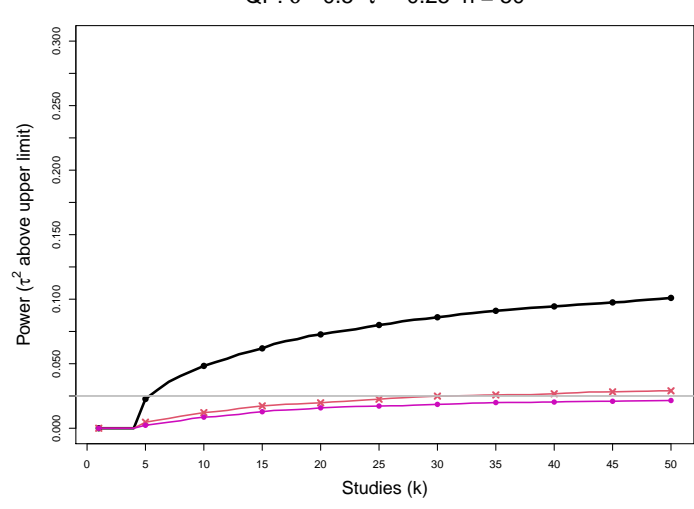

QP: $\delta=0.5 \tau^{2}=1 \quad n=50$

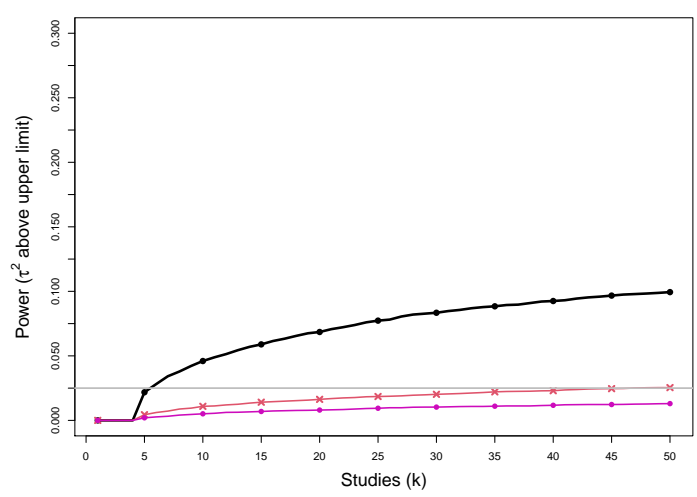

KDB: $\delta=0.5 \tau^{2}=0 \quad n=50$

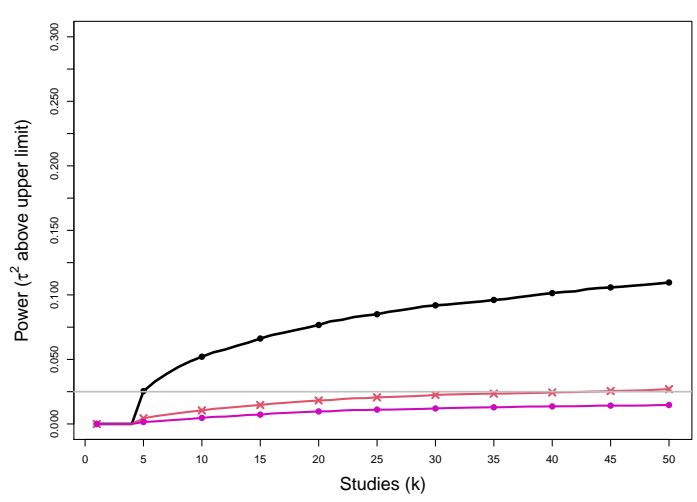

KDB: $\delta=0.5 \tau^{2}=0.1 \quad n=50$

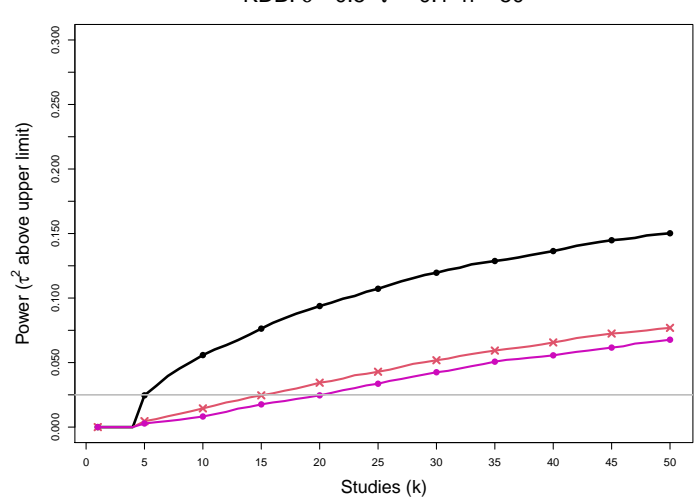

KDB: $\delta=0.5 \tau^{2}=0.25 \mathrm{n}=50$

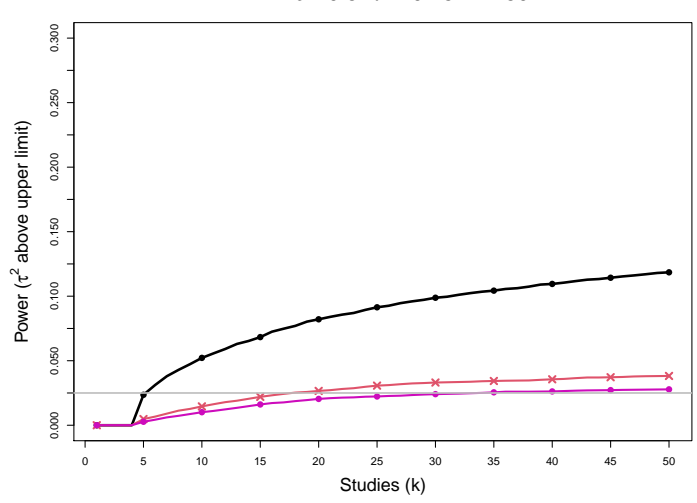

KDB: $\delta=0.5 \tau^{2}=1 \quad n=50$

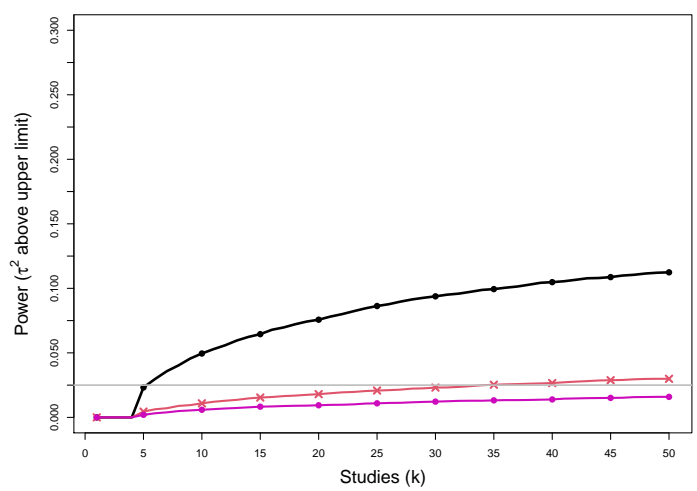

Figure A7. 6: Empirical levels of CMA tests for positive shift in $\tau^{2}$ based on KDB and QP at .025, .005 and .0025 nominal levels for $k$ up to $K=50$ for equal sample sizes $n_{i C}+n_{i T}=n=50, \delta=0.5, \tau^{2}=0,0.1,0.25,1$. Light grey line at .025 . 

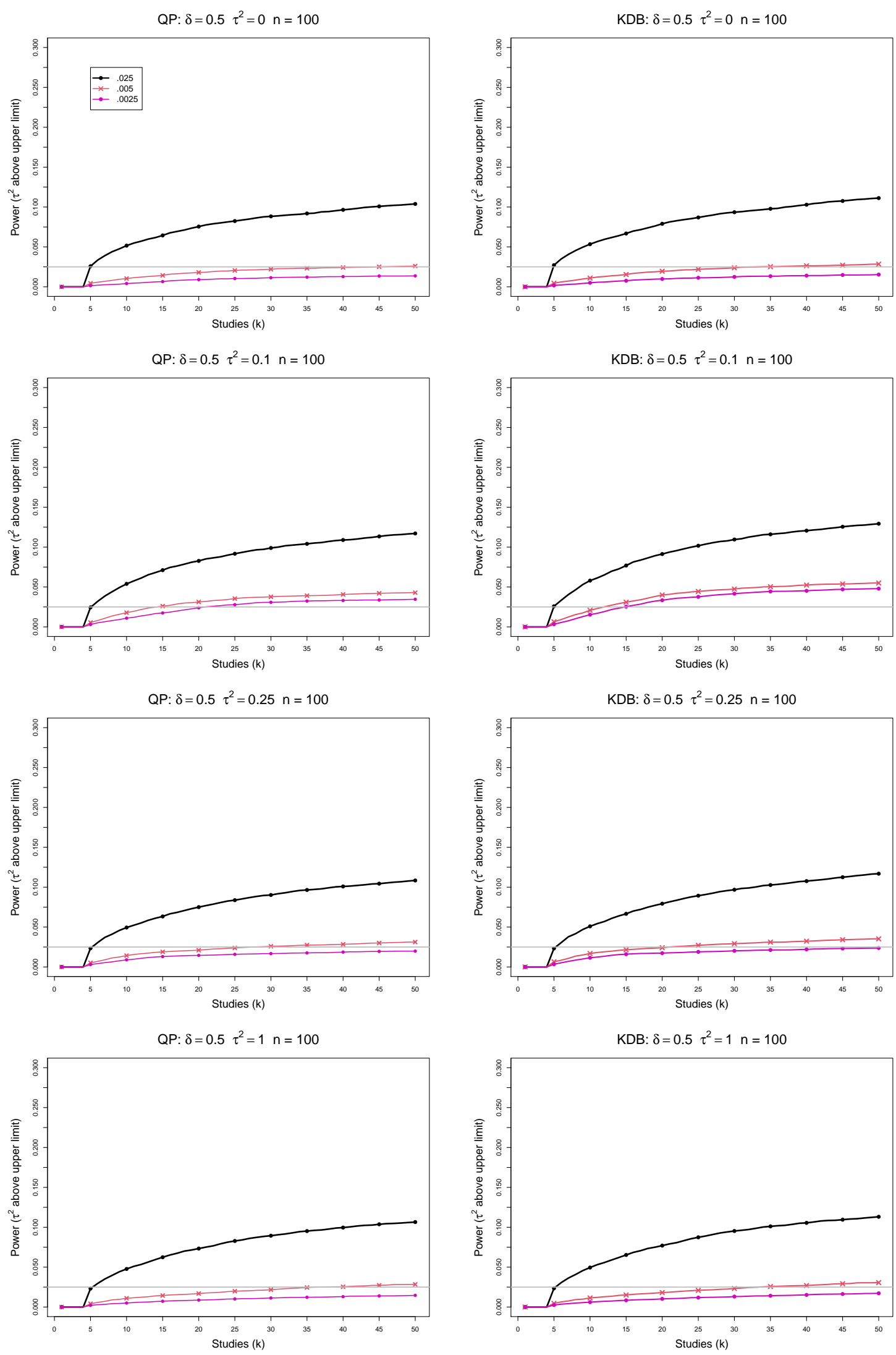

Figure A7. 7: Empirical levels of CMA tests for positive shift in $\tau^{2}$ based on KDB and QP at $.025, .005$ and .0025 nominal levels for $k$ up to $K=50$ for equal sample sizes $n_{i C}+n_{i T}=n=100, \delta=0.5, \tau^{2}=0,0.1,0.25,1$. Light grey line at .025. 

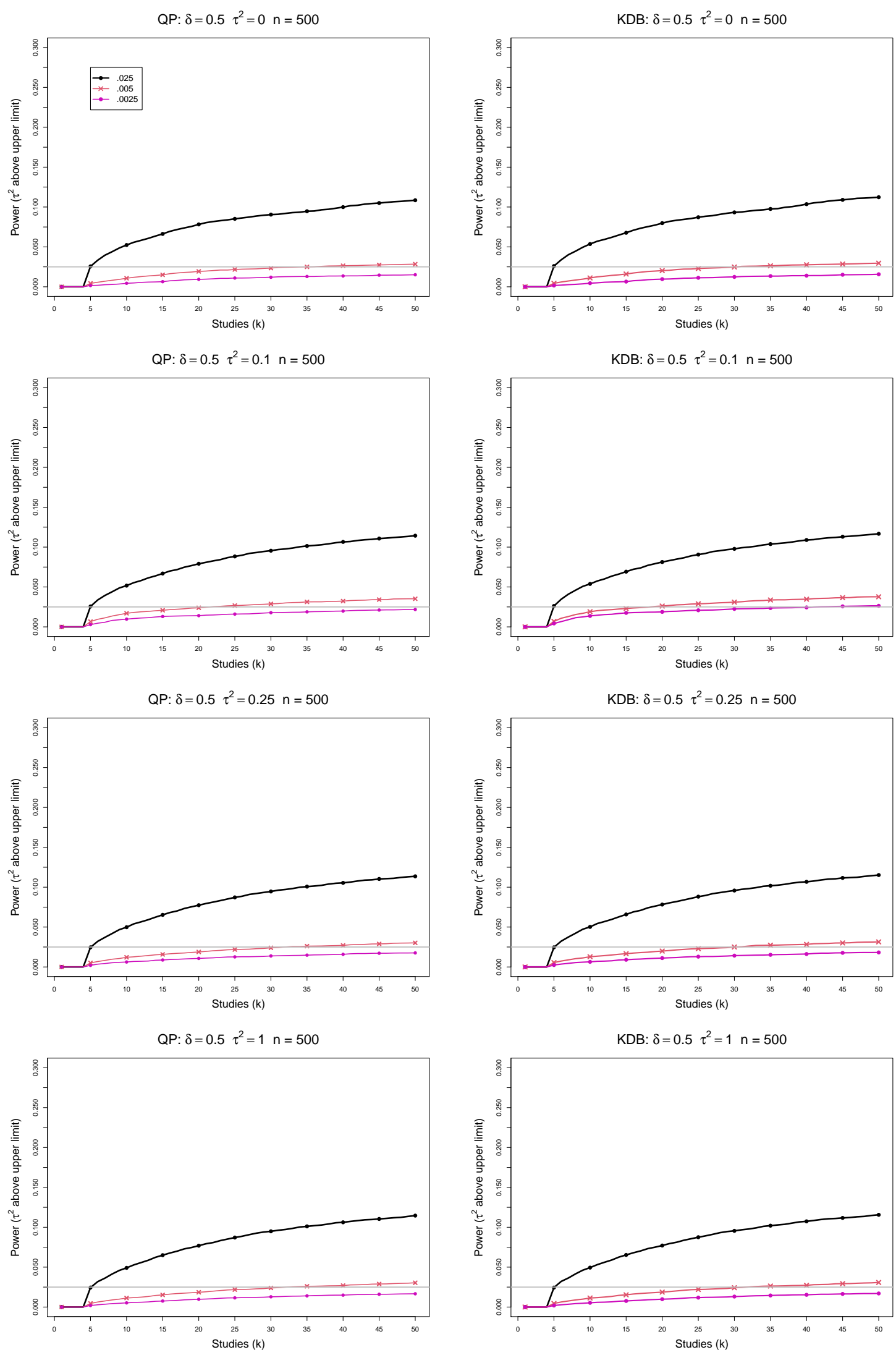

Figure A7. 8: Empirical levels of CMA tests for positive shift in $\tau^{2}$ based on KDB and QP at $.025, .005$ and .0025 nominal levels for $k$ up to $K=50$ for equal sample sizes $n_{i C}+n_{i T}=n=500, \delta=0.5, \tau^{2}=0,0.1,0.25,1$. Light grey line at .025 . 

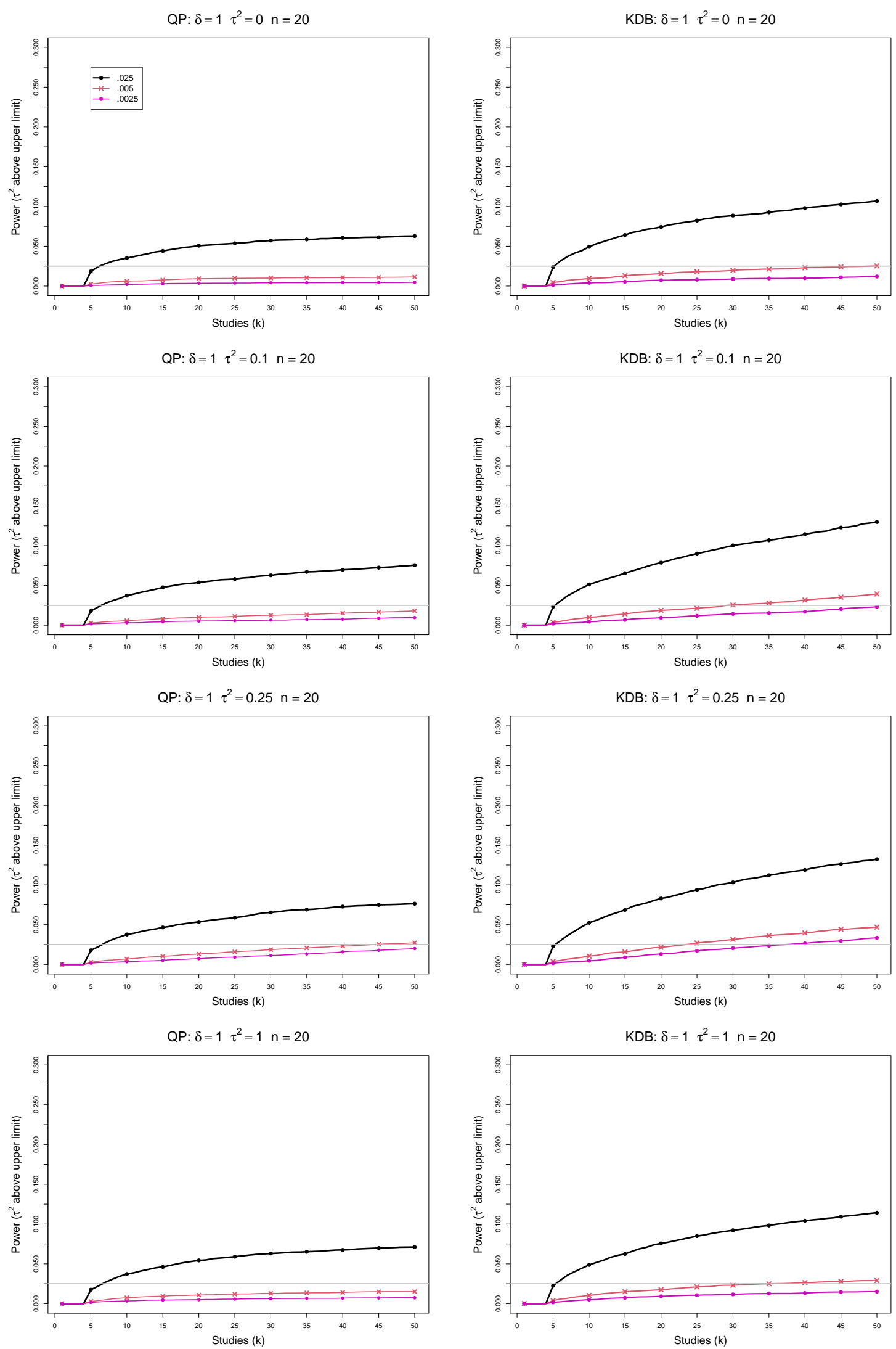

Figure A7. 9: Empirical levels of CMA tests for positive shift in $\tau^{2}$ based on KDB and QP at $.025, .005$ and .0025 nominal levels for $k$ up to $K=50$ for equal sample sizes $n_{i C}+n_{i T}=n=20, \delta=1, \tau^{2}=0,0.1,0.25,1$. Light grey line at .025. 

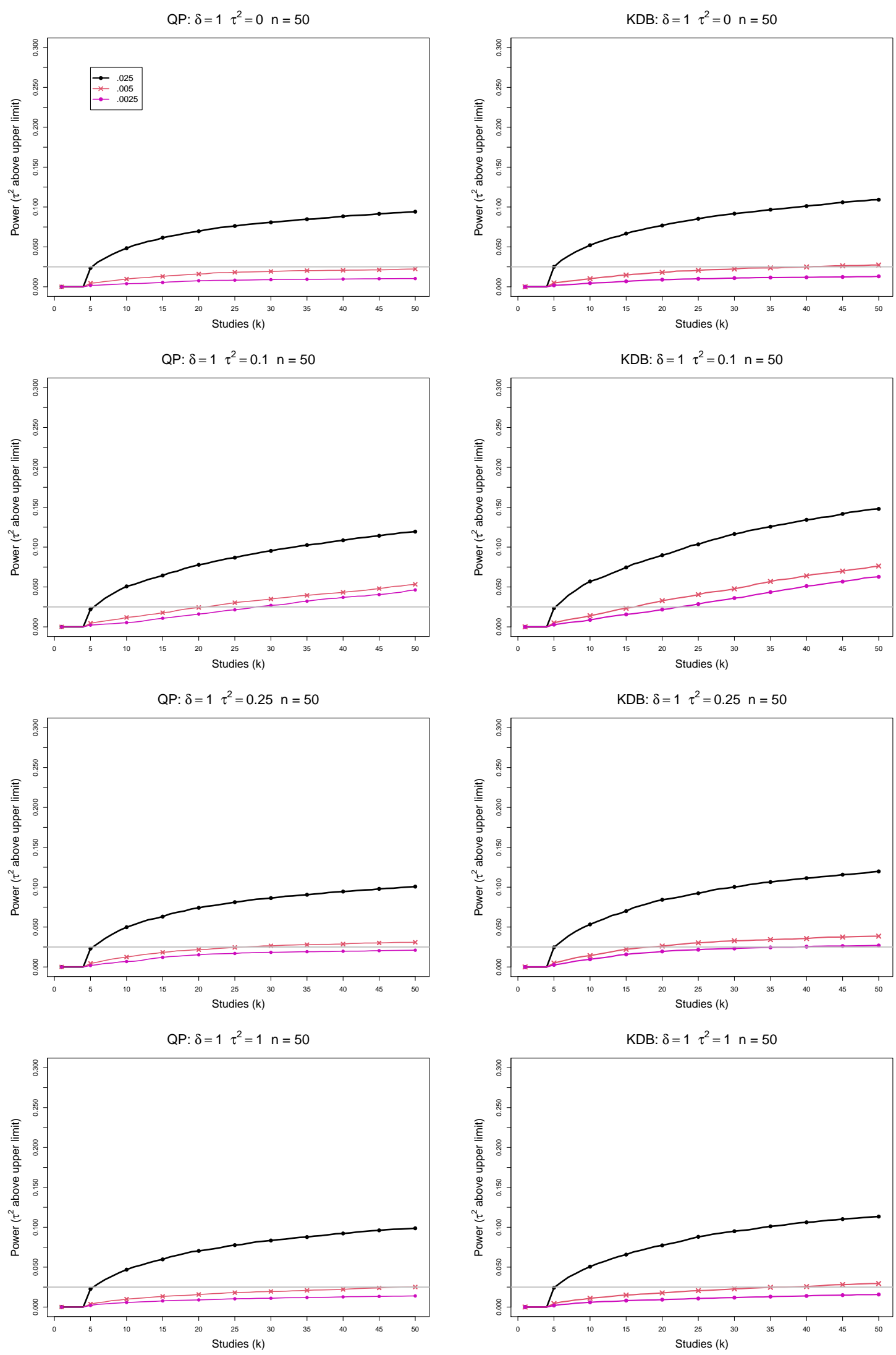

Figure A7. 10: Empirical levels of CMA tests for positive shift in $\tau^{2}$ based on KDB and QP at $.025, .005$ and .0025 nominal levels for $k$ up to $K=50$ for equal sample sizes $n_{i C}+n_{i T}=n=50, \delta=1, \tau^{2}=0,0.1,0.25,1$. Light grey line at .025. 

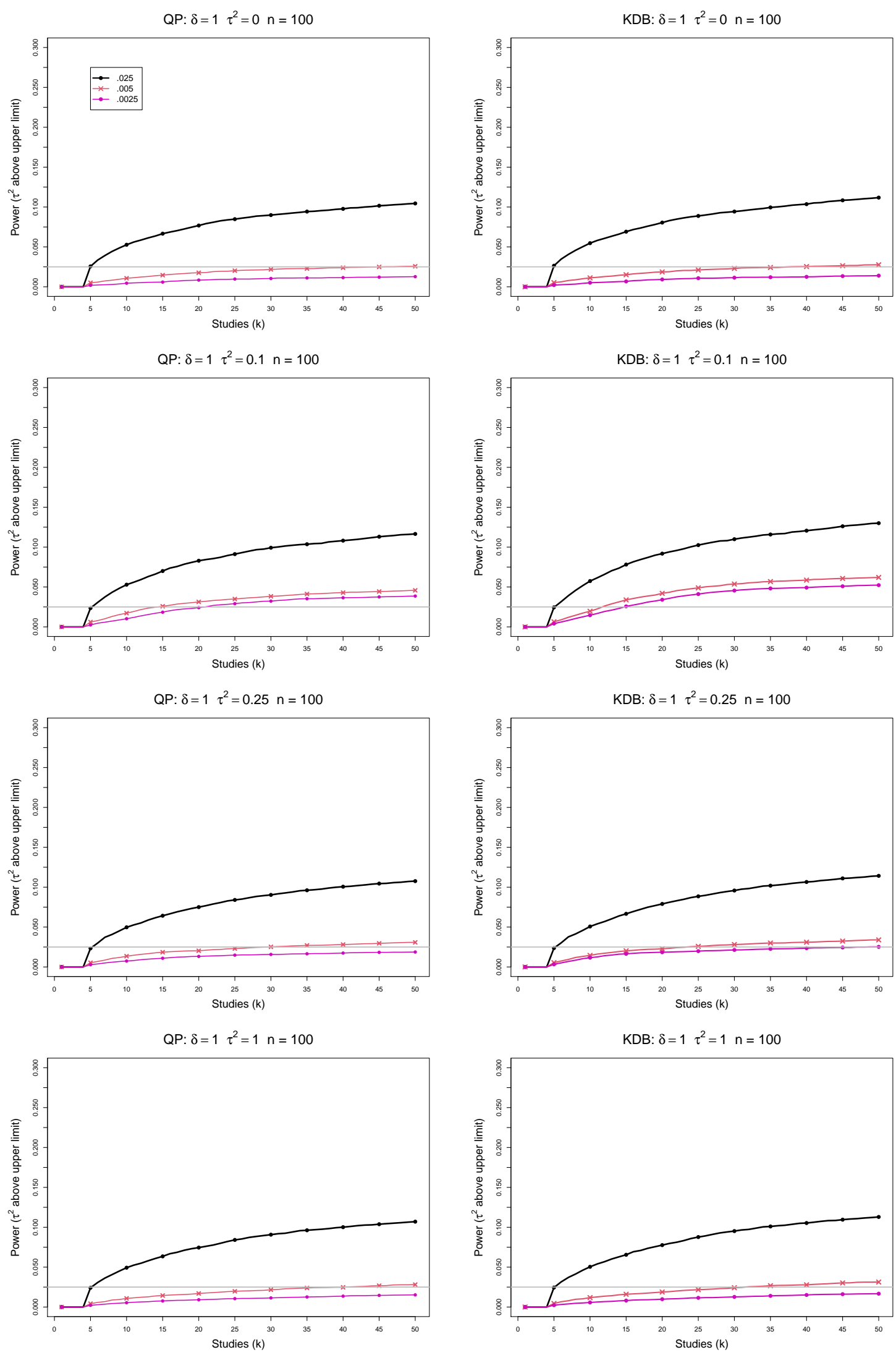

Figure A7. 11: Empirical levels of CMA tests for positive shift in $\tau^{2}$ based on KDB and QP at $.025, .005$ and .0025 nominal levels for $k$ up to $K=50$ for equal sample sizes $n_{i C}+n_{i T}=n=100, \delta=1, \tau^{2}=0,0.1,0.25,1$. Light grey line at .025. 

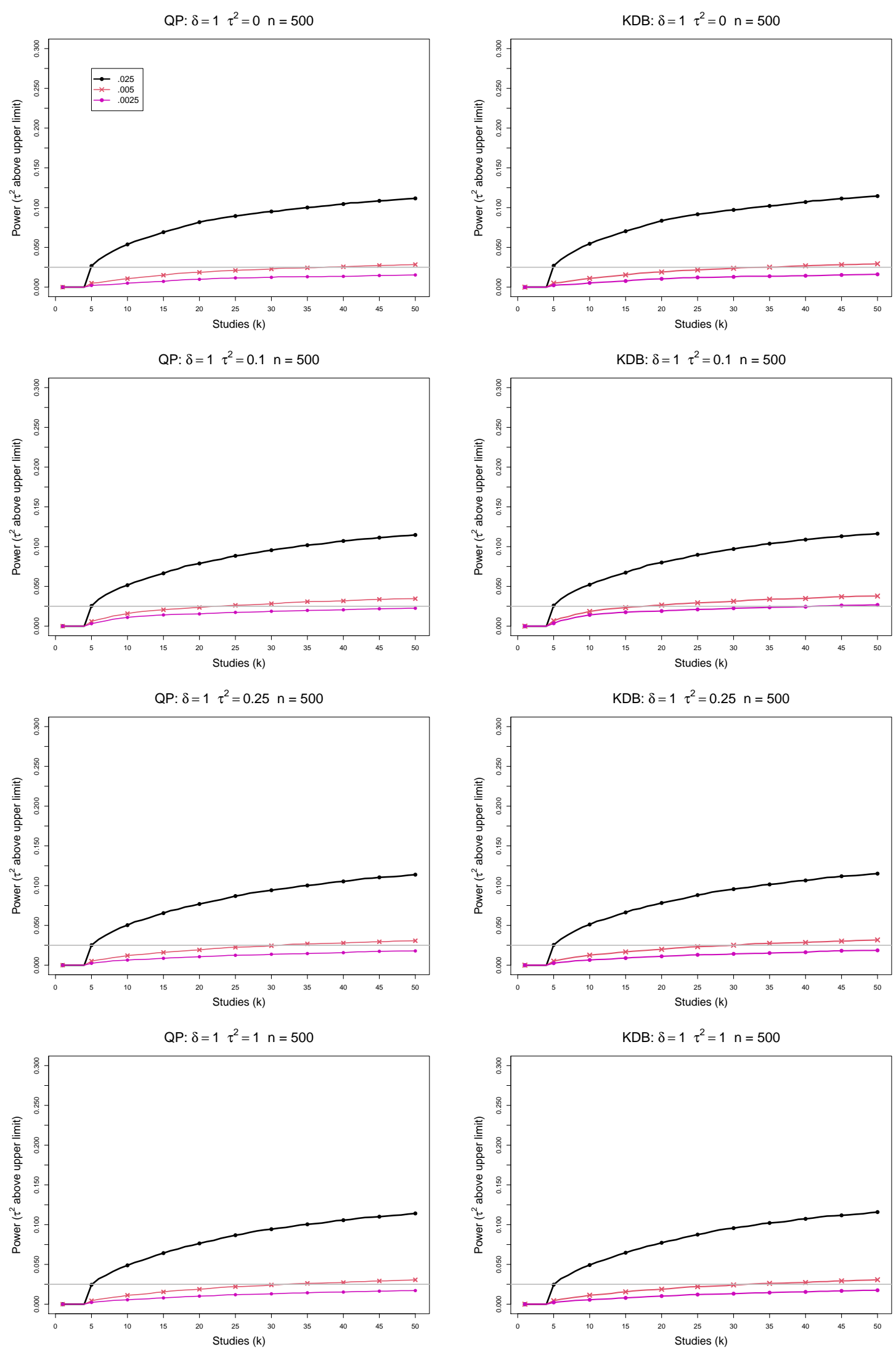

Figure A7. 12: Empirical levels of CMA tests for positive shift in $\tau^{2}$ based on KDB and QP at $.025, .005$ and .0025 nominal levels for $k$ up to $K=50$ for equal sample sizes $n_{i C}+n_{i T}=n=500, \delta=1, \tau^{2}=0,0.1,0.25,1$. Light grey line at .025. 
B. 2-stage CMA under null hypothesis of no shift-in-the-mean 


\section{B1. Bias of point estimators of $\delta$}

For bias of $\delta$ in this section, each figure corresponds to a set of values of $\tau^{2}(=0,0.1$, $0.25,1)$, and a set of values of $n(=20,50$ or 100,500$)$ while the study number $k$ increases to maximum number $K=1000$ and $\delta=1$.

Each figure contains a panel (with study $k$ on the horizontal axis) for each combination of parameters.

The point estimators of $\delta$ are

- IV REML (Inverse variance weighted mean with the between-study variance $\tau^{2}$ estimated by Restricted maximum likelihood)

- SSW (Effective sample size weighted mean) 

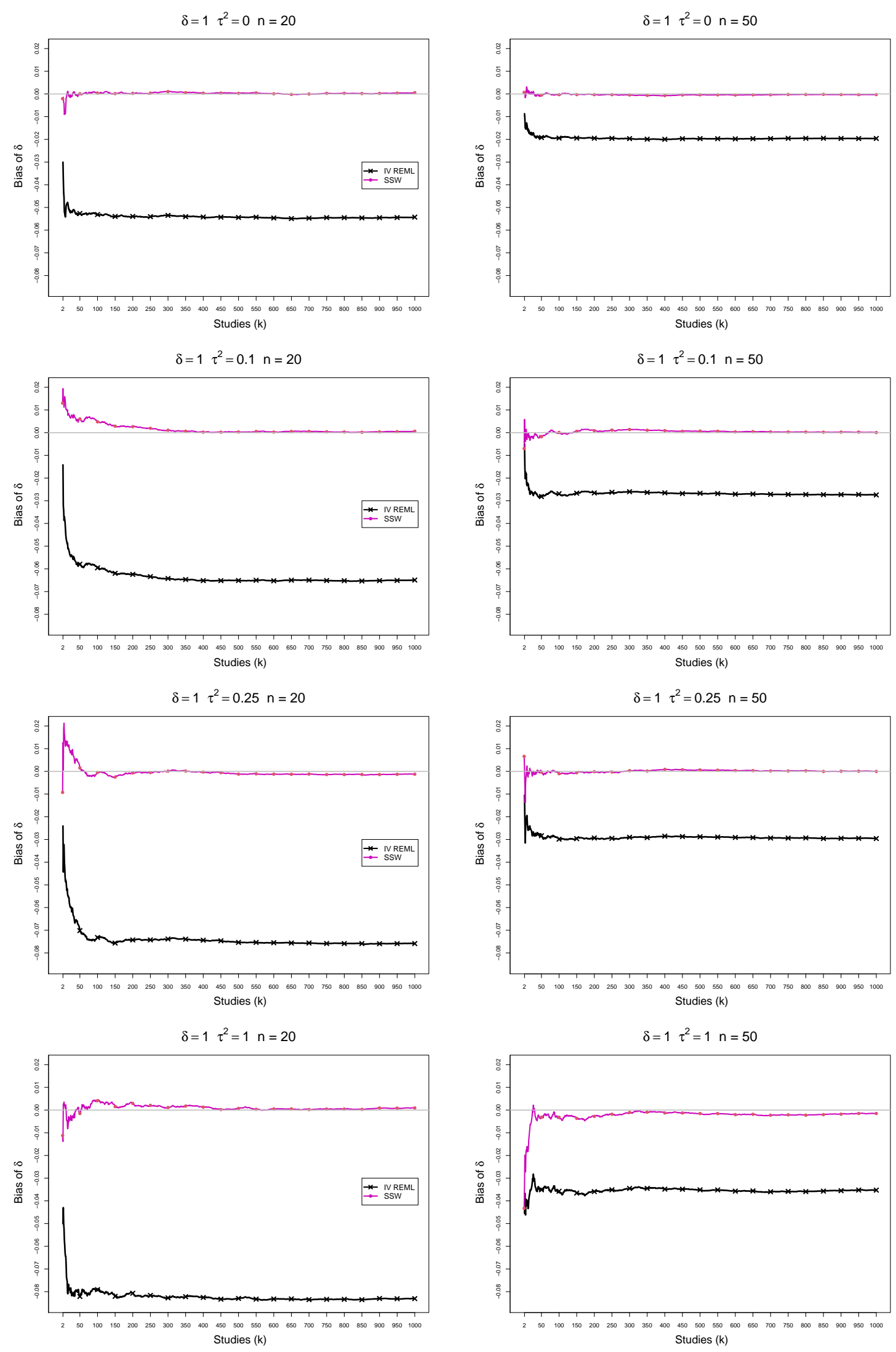

Figure B1. 1: 2-Stage CMA - Bias of the REML and SSW estimators of $\delta=1$ for $\tau^{2}=$ $0,0.1,0.25,1, n=20,50, K=1000$. Light grey line at 0 . 

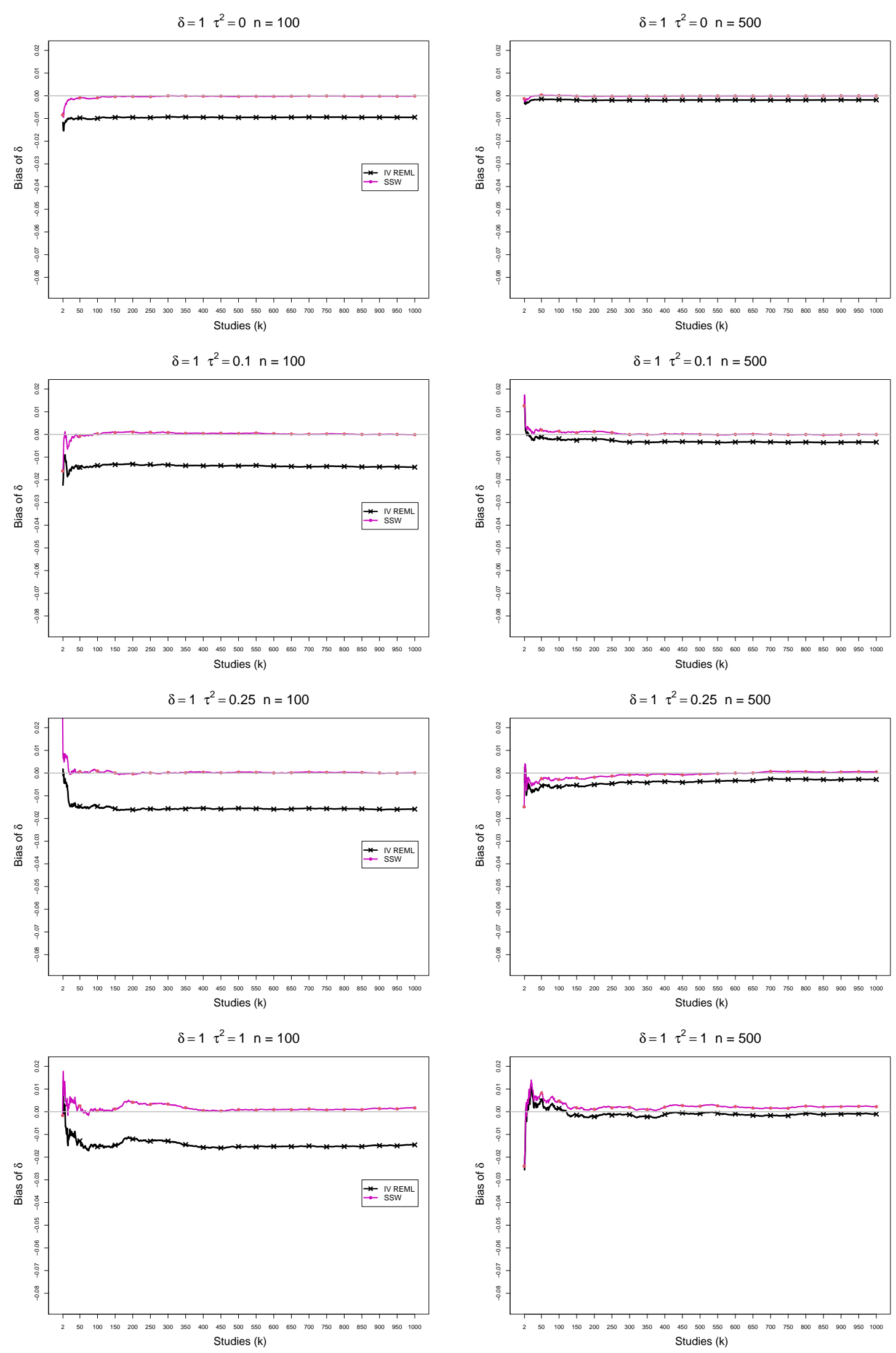

Figure B1. 2: 2-Stage CMA - Bias of the REML and SSW estimators of $\delta=1$ for $\tau^{2}=$ $0,0.1,0.25,1, n=100,500, K=1000$. Light grey line at 0 . 


\section{B2. Coverage of interval estimators of $\delta$}

For coverage of $\delta$ in this section, each figure corresponds to a set of values of $\tau^{2}(=0$, $0.1,0.25,1)$, a value of $n(=20,50,100,500)$ and a set of values of significance levels $(=0.05,0.01,0.005)$ while the study number $k$ increases to maximum number $K=1000$ and $\delta=1$.

Each figure contains a panel (with study $k$ on the horizontal axis) for each combinations.

The interval estimators of $\delta$ are

- IV REML (Inverse variance weighted mean with the between-study variance $\tau^{2}$ estimated by REML as center and normal critical values)

- SSW KDB (Sample size weighted (SSW) as center and half-width equal to critical value from $t_{k-1}$ times estimated standard deviation of SSW with $\hat{\tau}^{2}=$ $\hat{\tau}_{K D B}^{2}$, the Kulinskaya-Dollinger-Bjørkestøl estimator of $\tau^{2}$ ) 

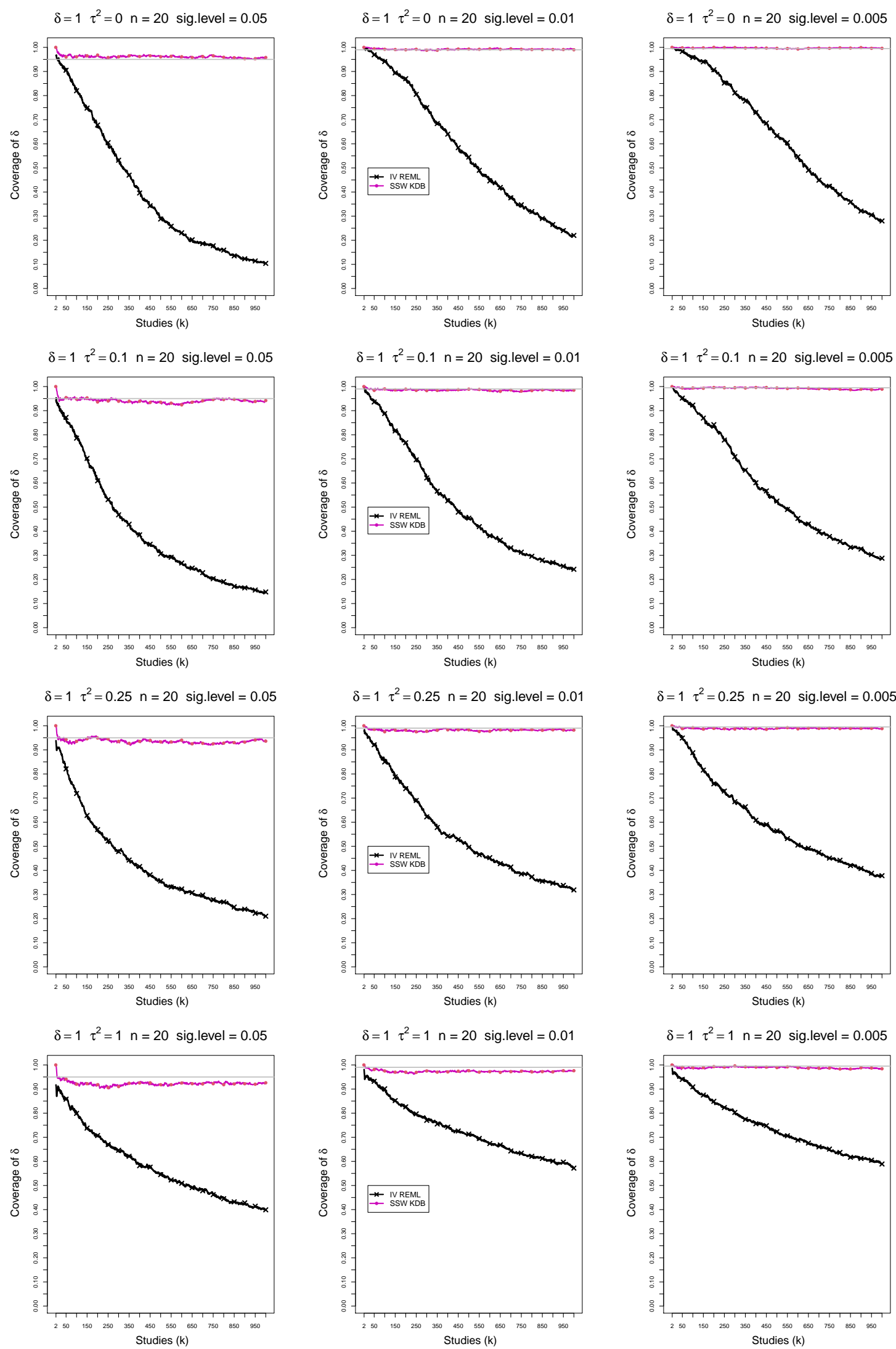

Figure B2. 1: 2-Stage CMA - Coverage of interval estimators of $\delta$ at confidence level of $1-\alpha$ for $\alpha=0.05,0.01,0.005$ for $\delta=1, \tau^{2}=0,0.1,0.25,1, n=20, K=1000$. Light grey line at $0.95,0.99,0.995$. 

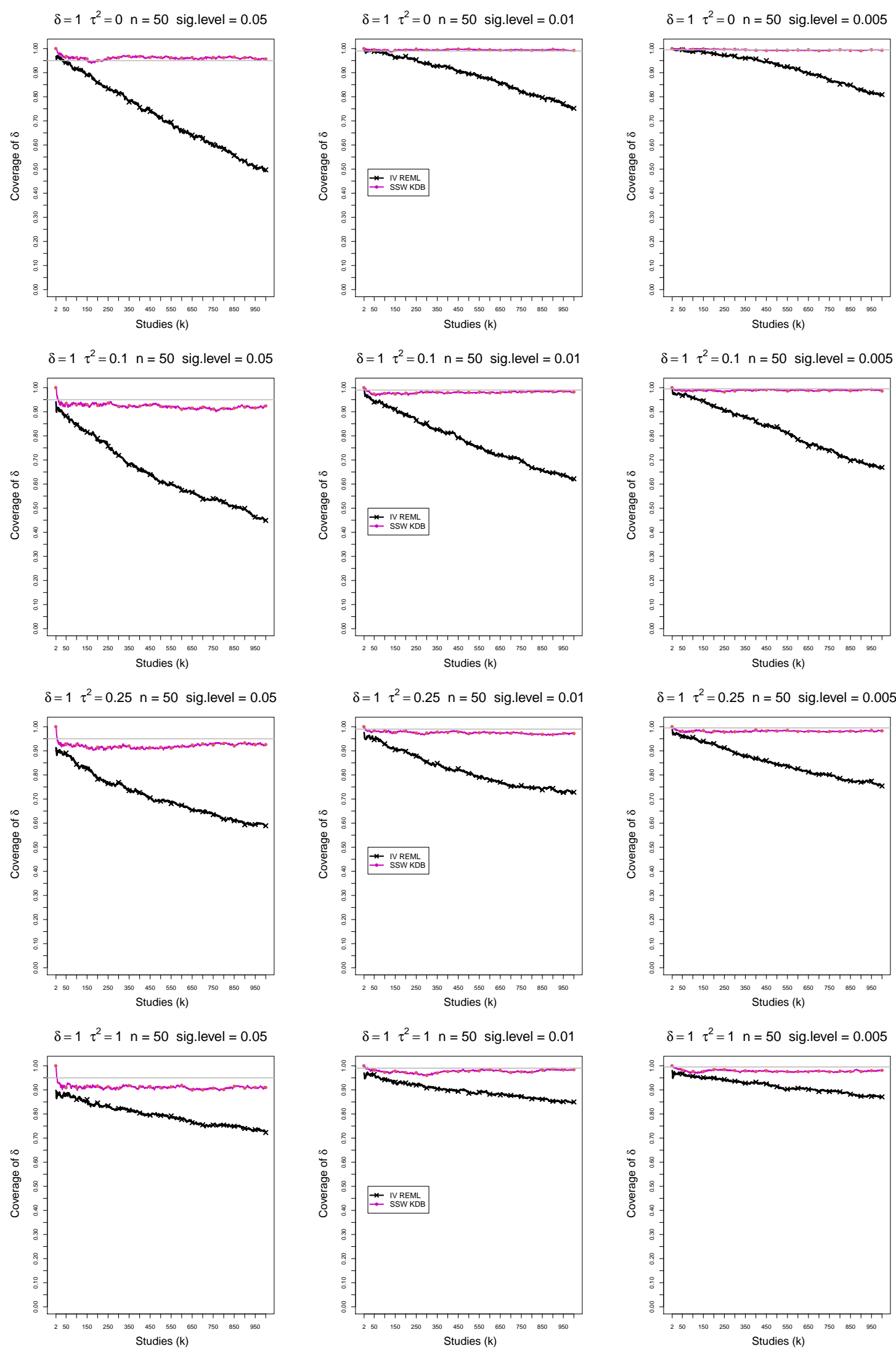

Figure B2. 2: 2-Stage CMA - Coverage of interval estimators of $\delta$ at confidence level of $1-\alpha$ for $\alpha=0.05,0.01,0.005$ for $\delta=1, \tau^{2}=0,0.1,0.25,1, n=50, K=1000$. Light grey line at $0.95,0.99,0.995$. 

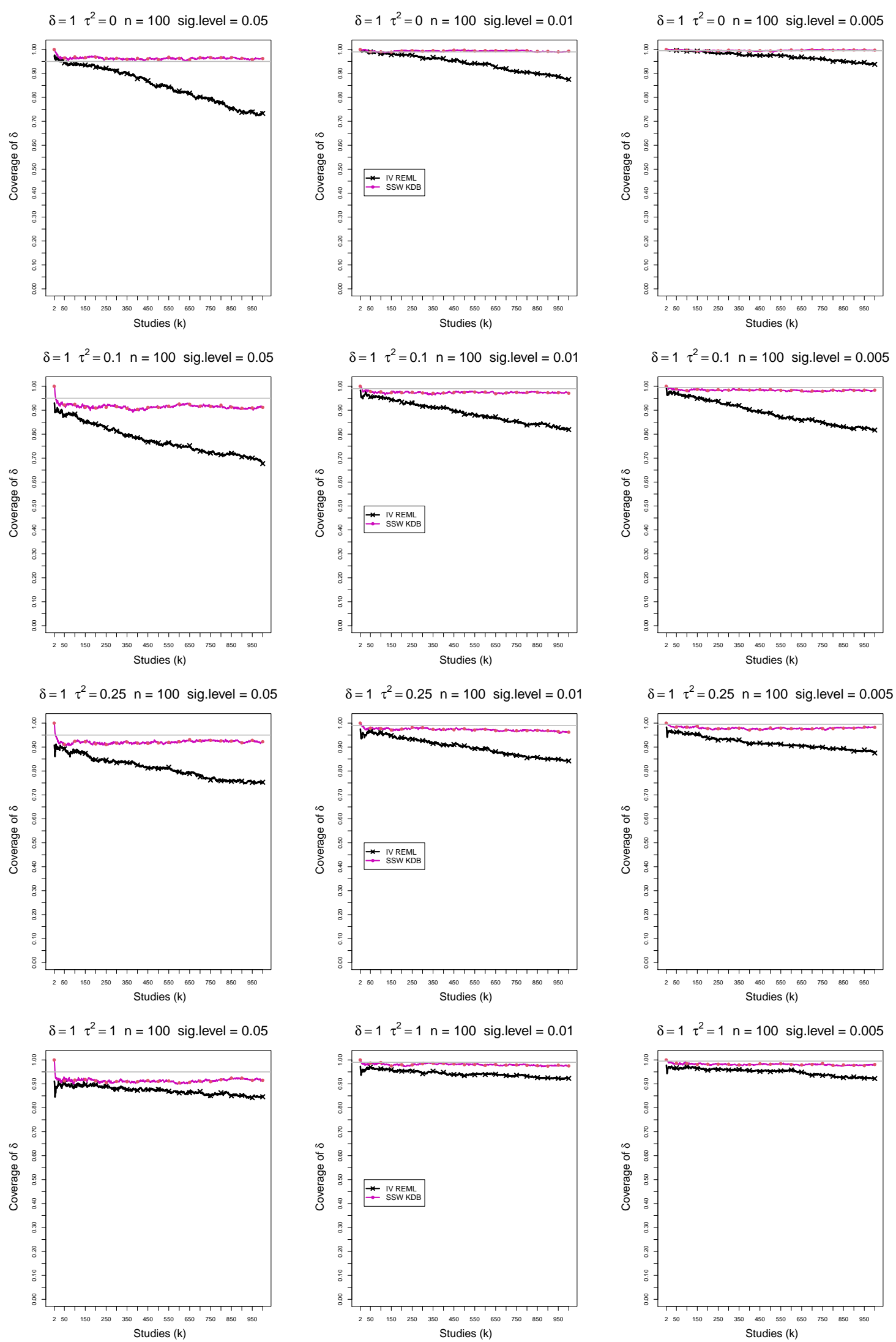

Figure B2. 3: 2-Stage CMA - Coverage of interval estimators of $\delta$ at confidence level of $1-\alpha$ for $\alpha=0.05,0.01,0.005$ for $\delta=1, \tau^{2}=0,0.1,0.25,1, n=100, K=1000$. Light grey line at $0.95,0.99,0.995$. 

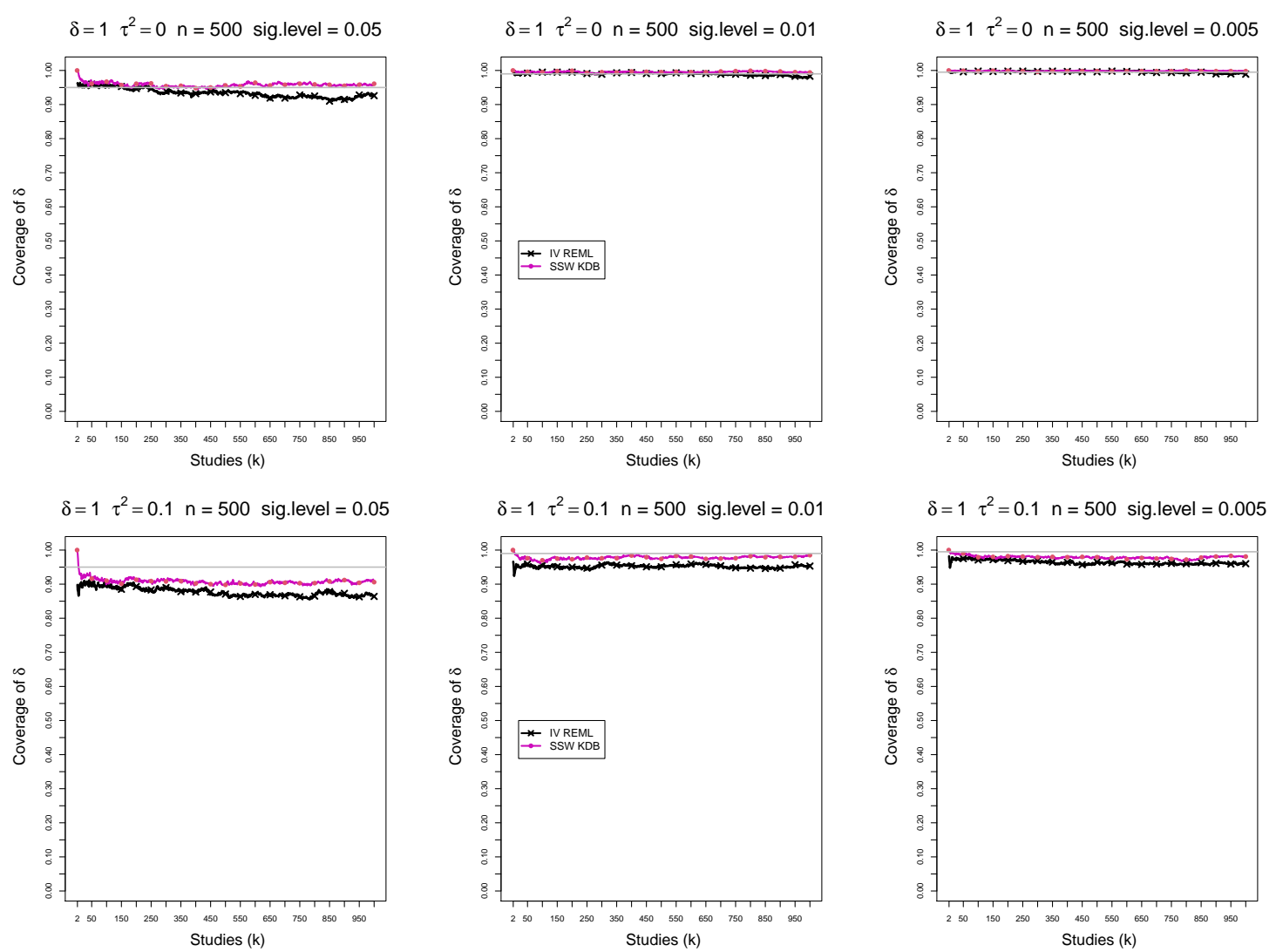

$\delta=1 \quad \tau^{2}=0.25 \mathrm{n}=500$ sig.level $=0.05$

$\delta=1 \quad \tau^{2}=0.25 \quad \mathrm{n}=500$ sig.level $=0.01$

$\delta=1 \tau^{2}=0.25 \quad \mathrm{n}=500$ sig.level $=0.005$
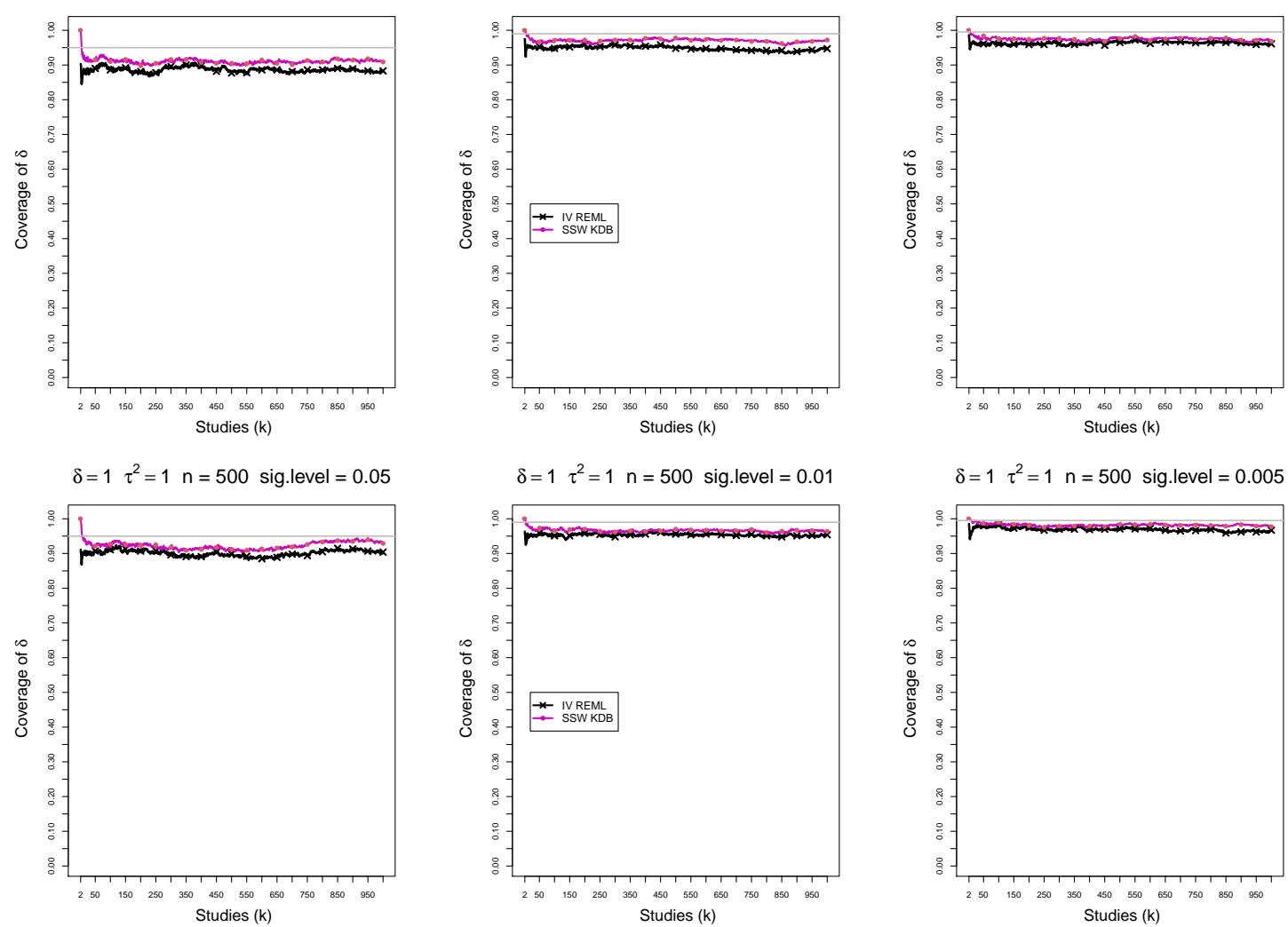

$\delta=1 \quad \tau^{2}=1 \quad \mathrm{n}=500$ sig.level $=0.005$

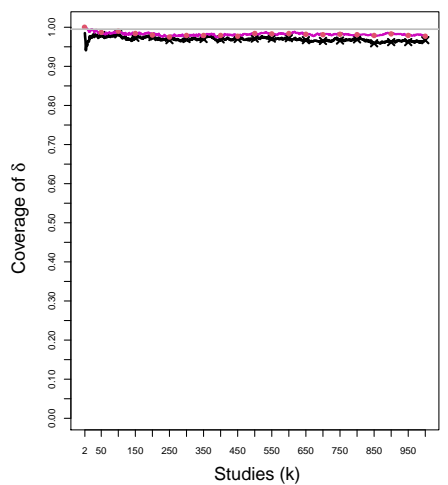

Figure B2. 4: 2-Stage CMA - Coverage of interval estimators of $\delta$ at confidence level of $1-\alpha$ for $\alpha=0.05,0.01,0.005$ for $\delta=1, \tau^{2}=0,0.1,0.25,1, n=500, K=1000$. Light grey line at $0.95,0.99,0.995$. 


\section{B3. Coverage of one-sided interval estimators of $\delta$}

For coverage of one-sided (lower or upper) confidence intervals of $\delta$ in this section, each figure corresponds to a set of values of $\tau^{2}(=0,0.1,0.25,1)$, a value of $n(=20$, $50,100,500)$ and a set of values of 2 -sided significance level $(=0.05,0.01,0.005)$ while maximum number of studies $K=1000$ and $\delta=1$,

Each figure contains a panel (with study $k$ on the horizontal axis) for each combination of parameters.

The interval estimators of $\delta$ are

- IV REML (Inverse variance weighted mean with the between-study variance $\tau^{2}$ estimated by Restricted maximum likelihood as center and the lower or the upper confidence limit (as required) defined by taking out or adding critical value from Normal distribution times square-root of the inverse sum of weights )

- SSW KDB (Sample size weighted (SSW) as center and the lower or the upper confidence limit (as required) defined by taking out or adding critical value from $t_{k-1}$ times estimated standard deviation of SSW with $\hat{\tau}^{2}=\hat{\tau}_{K D B}^{2}$, the Kulinskaya-Dollinger-Bjørkestøl (2011) estimator of $\tau^{2}$ ) 

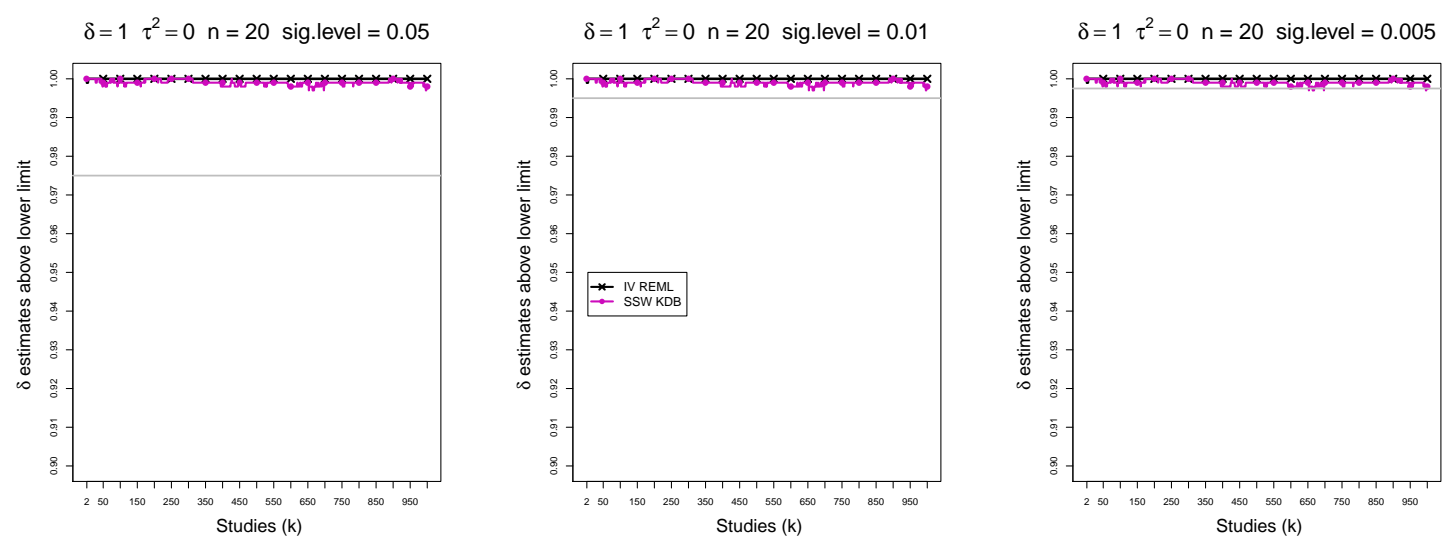

$\delta=1 \tau^{2}=0.1 \quad \mathrm{n}=20$ sig.level $=0.05$

$\delta=1 \tau^{2}=0.1 \mathrm{n}=20$ sig.level $=0.01$
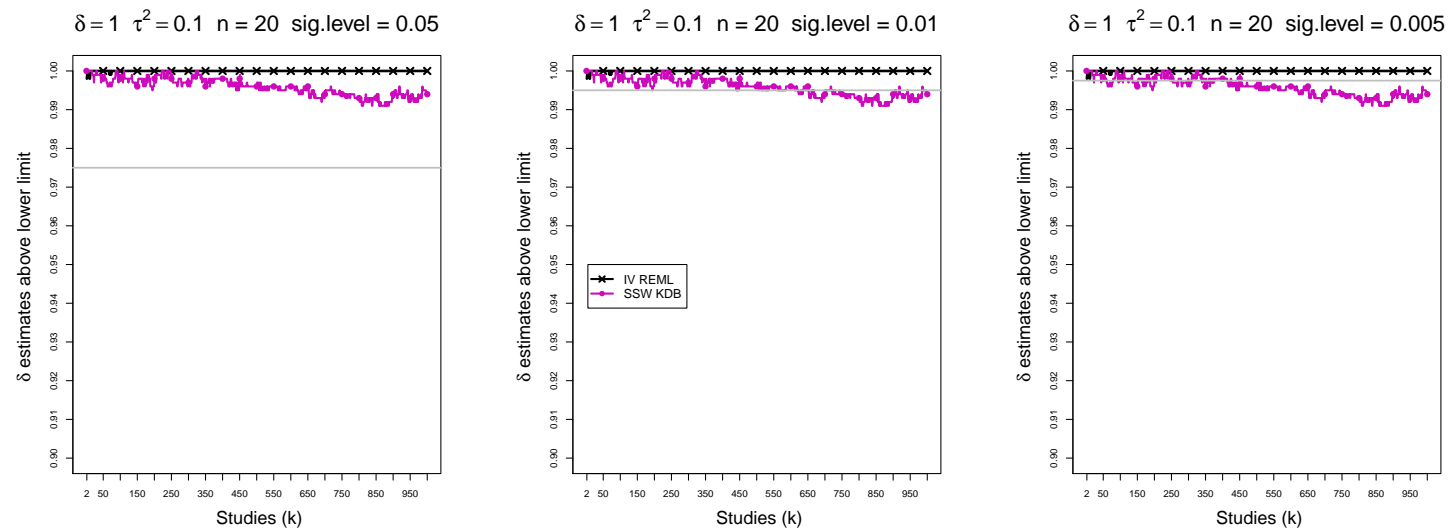

$\delta=1 \tau^{2}=0.25 \quad n=20$ sig.level $=0.05$
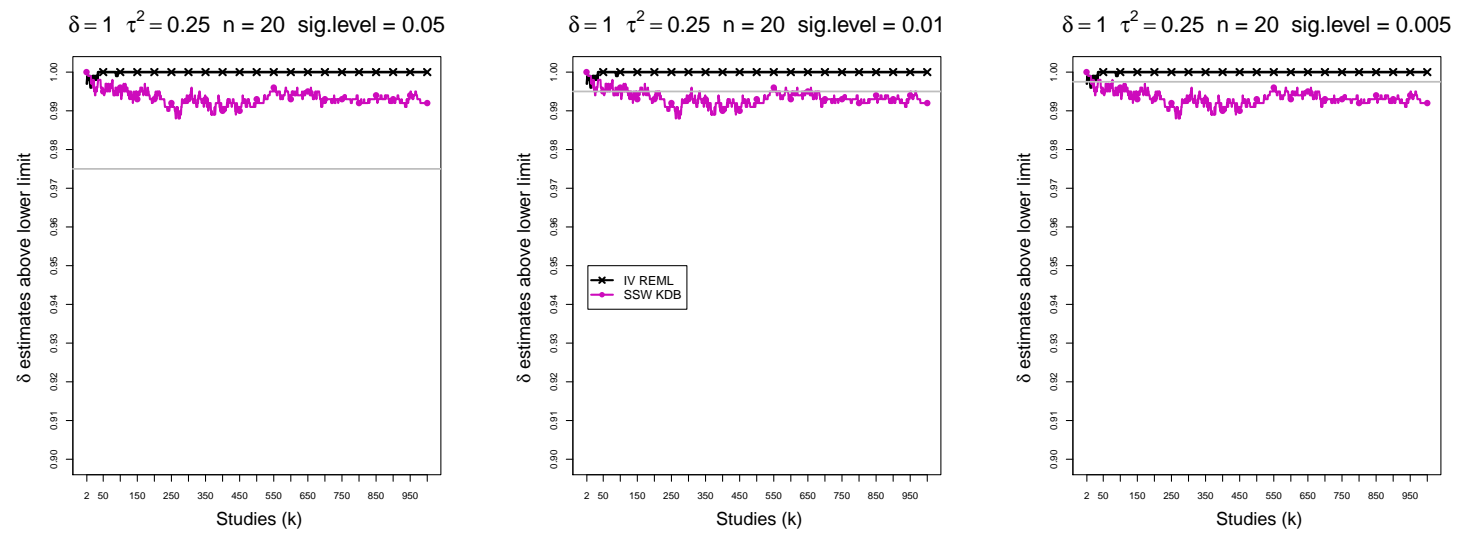

$\delta=1 \tau^{2}=1 \quad \mathrm{n}=20$ sig.level $=0.05$

$\delta=1 \tau^{2}=1 \quad \mathrm{n}=20$ sig.level $=0.01$
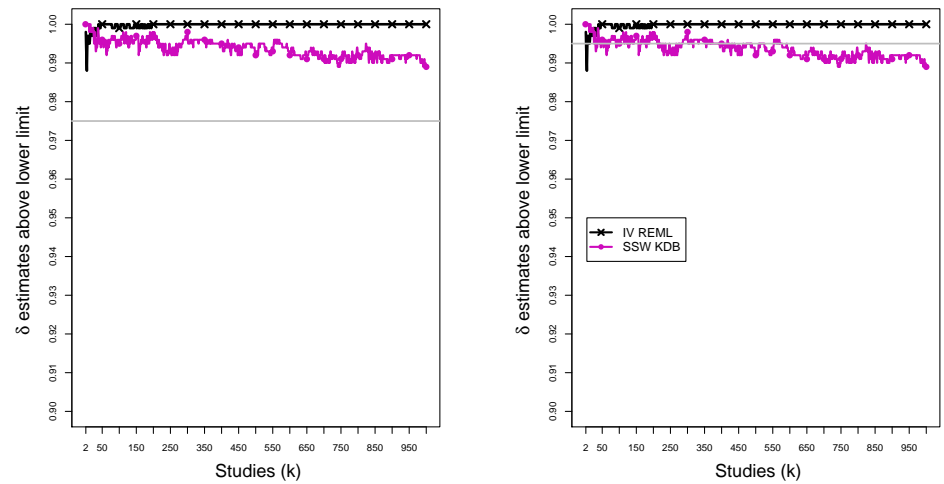

$\delta=1 \tau^{2}=1 \quad \mathrm{n}=20$ sig.level $=0.005$

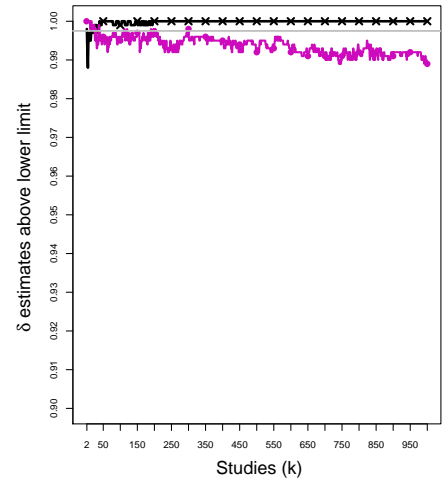

Figure B3. 1: 2-Stage CMA - Coverage of the lower confidence interval of $\delta$ (above lower limit) at the confidence level $1-\alpha$ for $\alpha=0.05,0.01,0.005$ for $\delta=1, \tau^{2}=0,0.1,0.25,1$, $n=20, K=1000$. Light grey line at $0.975,0.995,0.9975$. 

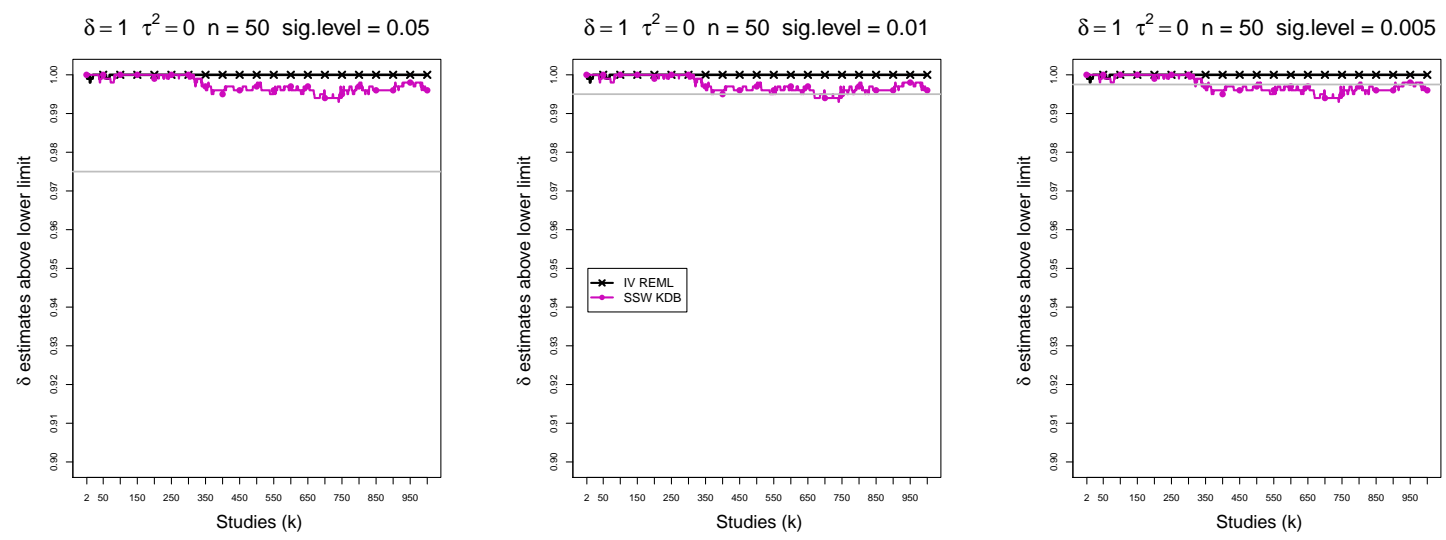

$\delta=1 \quad \tau^{2}=0.1 \quad \mathrm{n}=50$ sig.level $=0.05$
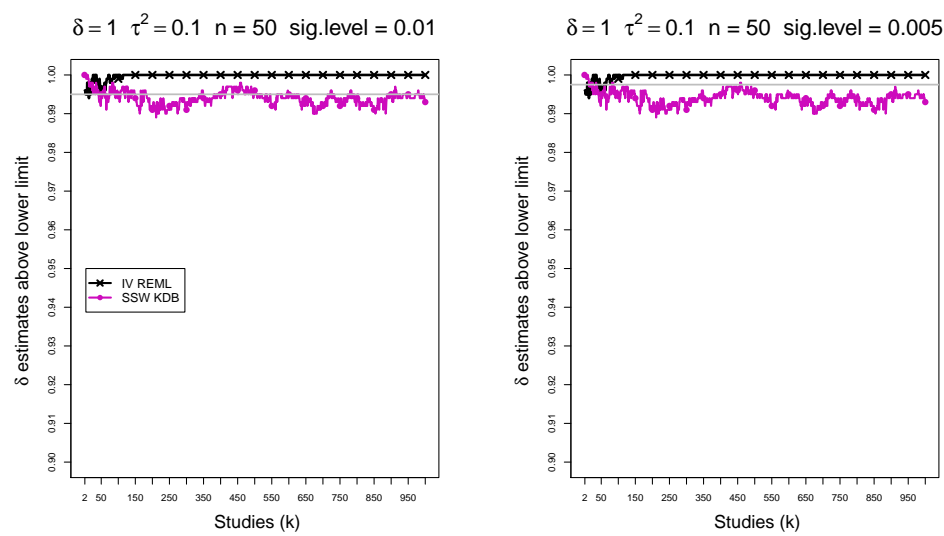

$\delta=1 \quad \tau^{2}=0.25 \quad \mathrm{n}=50$ sig.level $=0.05$

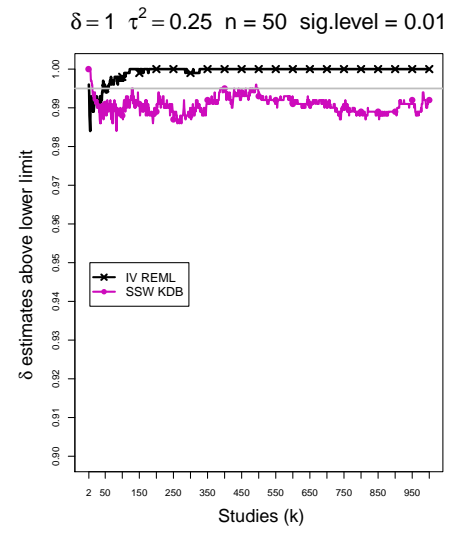

$\delta=1 \quad \tau^{2}=0.25 \quad \mathrm{n}=50$ sig.level $=0.005$
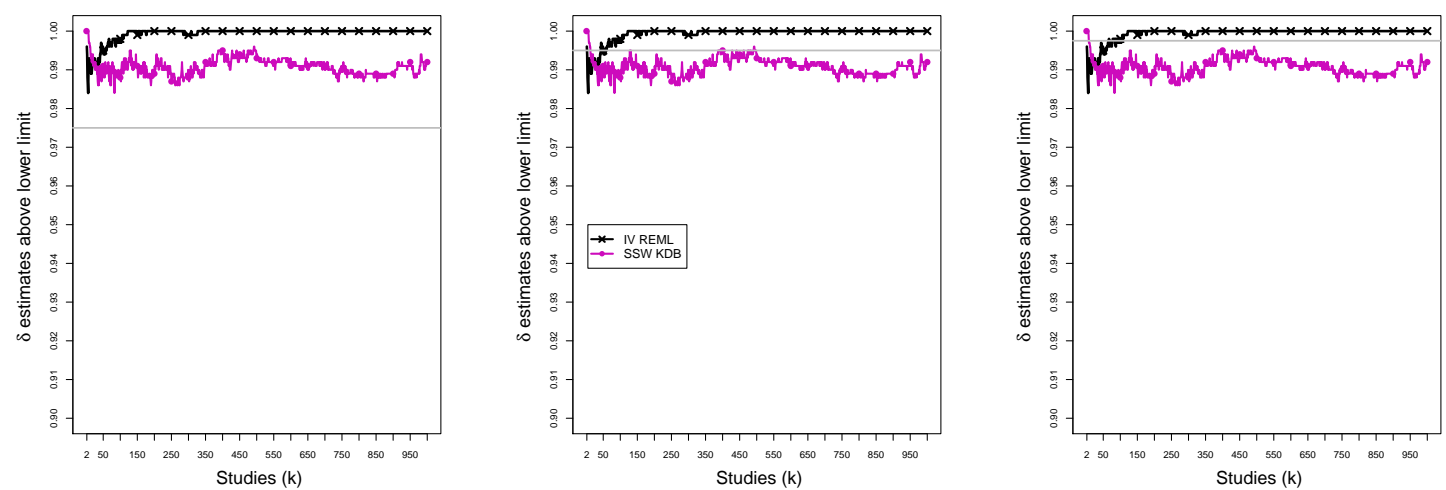

$\delta=1 \quad \tau^{2}=1 \mathrm{n}=50$ sig.level $=0.05$
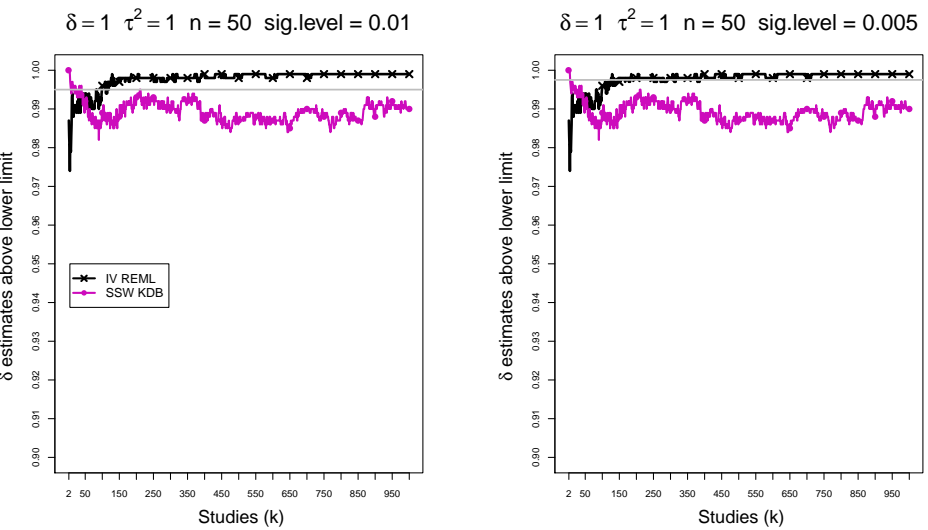

Figure B3. 2: 2-Stage CMA - Coverage of the lower confidence interval of $\delta$ (above lower limit) at the confidence level $1-\alpha$ for $\alpha=0.05,0.01,0.005$ for $\delta=1, \tau^{2}=0,0.1,0.25,1$, $n=50, K=1000$. Light grey line at $0.975,0.995,0.9975$. 

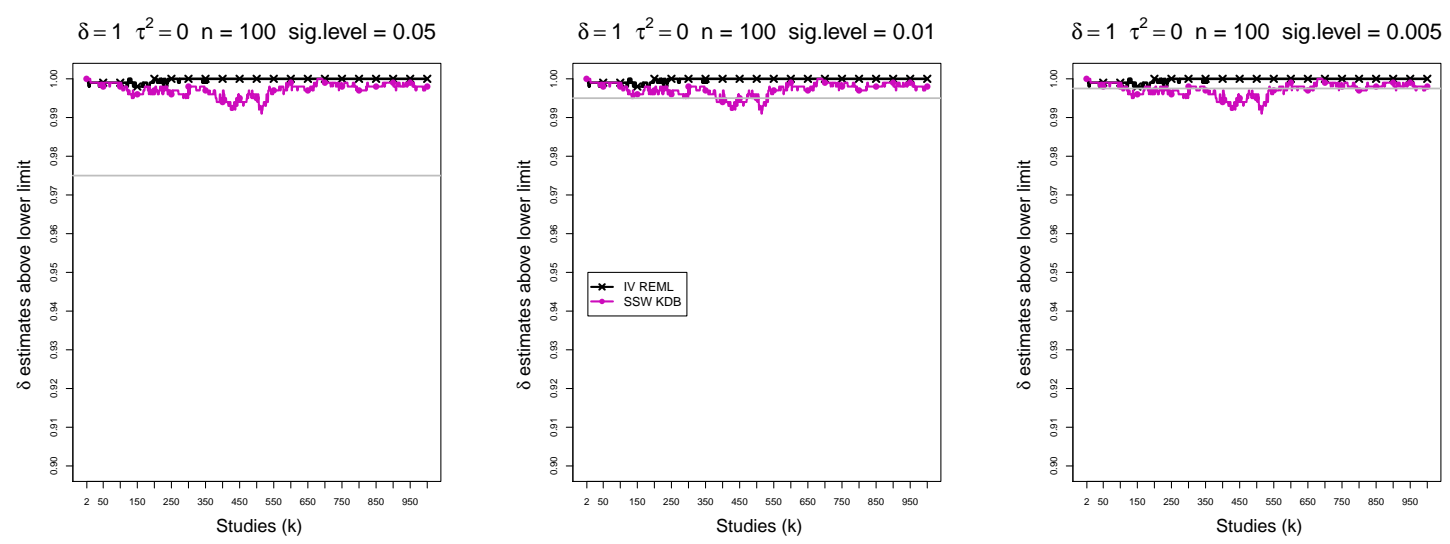

$\delta=1 \quad \tau^{2}=0.1 \quad \mathrm{n}=100$ sig.level $=0.05$
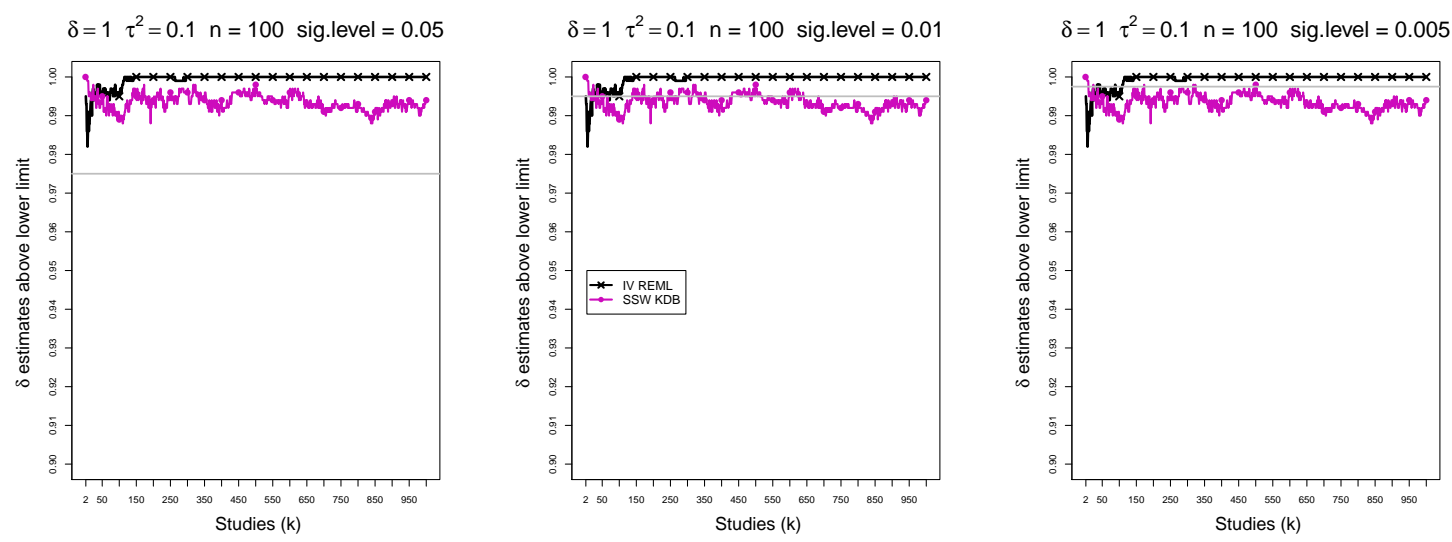

$\delta=1 \tau^{2}=0.25 \mathrm{n}=100$ sig.level $=0.05$

$\delta=1 \tau^{2}=0.25 \mathrm{n}=100$ sig.level $=0.01$

$\delta=1 \tau^{2}=0.25 \mathrm{n}=100$ sig.level $=0.005$
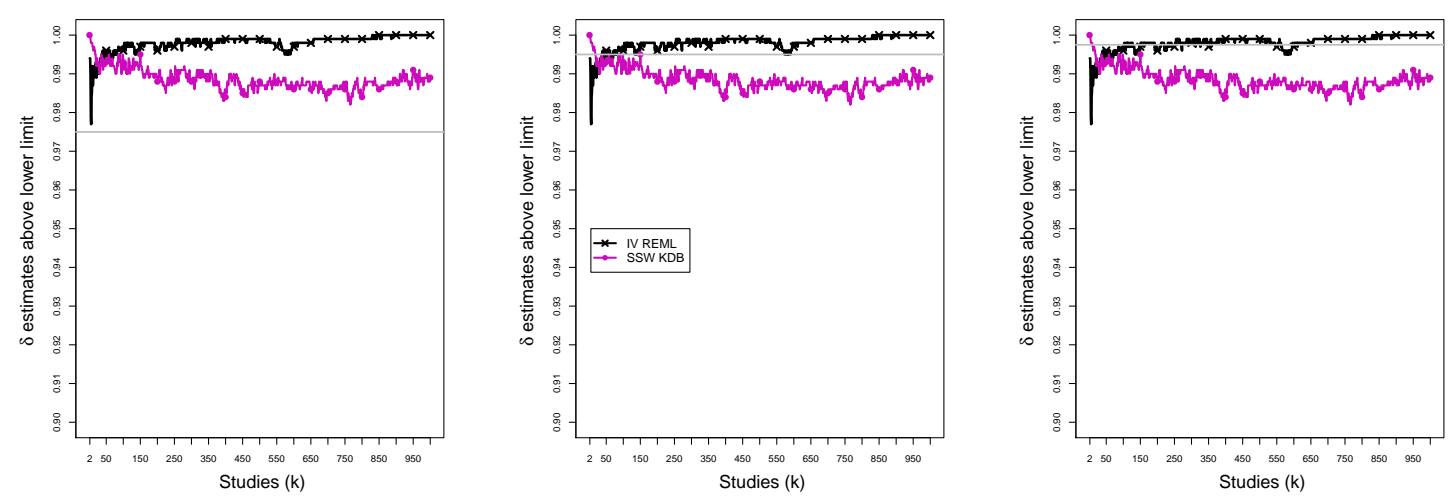

$\delta=1 \quad \tau^{2}=1 \quad \mathrm{n}=100$ sig.level $=0.05$
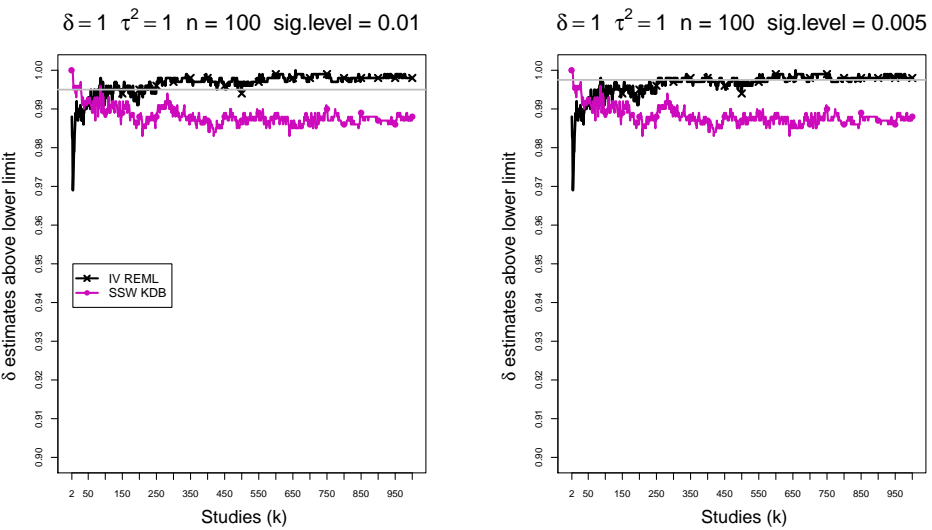

Figure B3. 3: 2-Stage CMA - Coverage of the lower confidence interval of $\delta$ (above lower limit) at the confidence level $1-\alpha$ for $\alpha=0.05,0.01,0.005$ for $\delta=1, \tau^{2}=0,0.1,0.25,1$, $n=100, K=1000$. Light grey line at $0.975,0.995,0.9975$. 

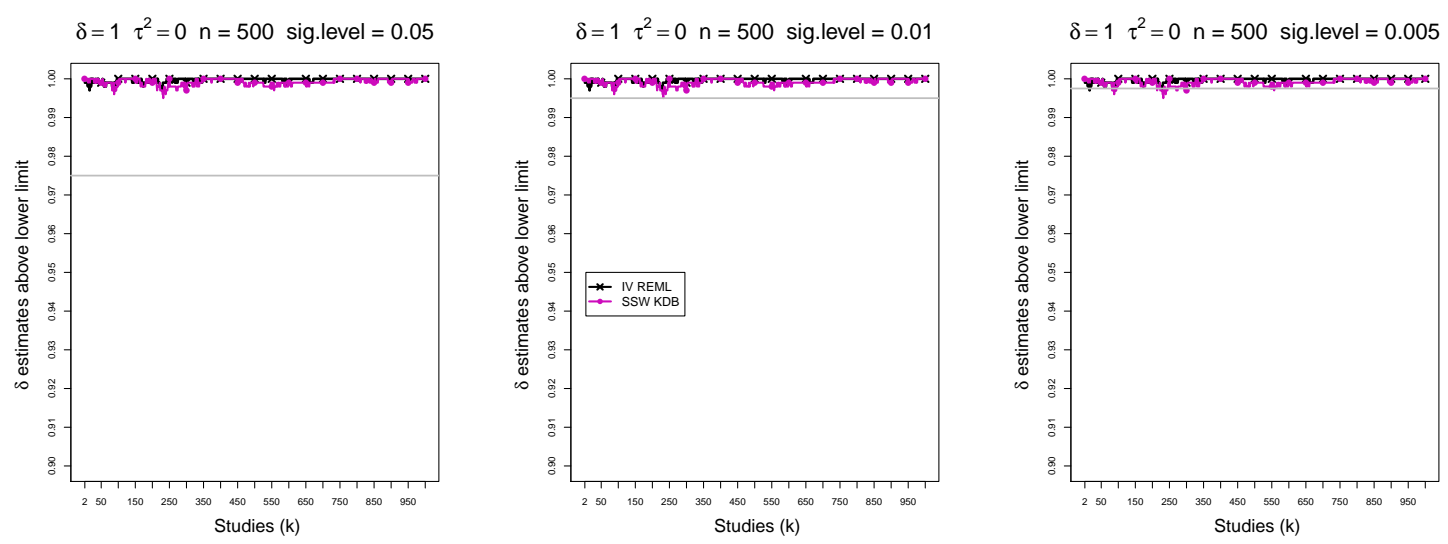

$\delta=1 \quad \tau^{2}=0.1 \quad \mathrm{n}=500$ sig.level $=0.05$
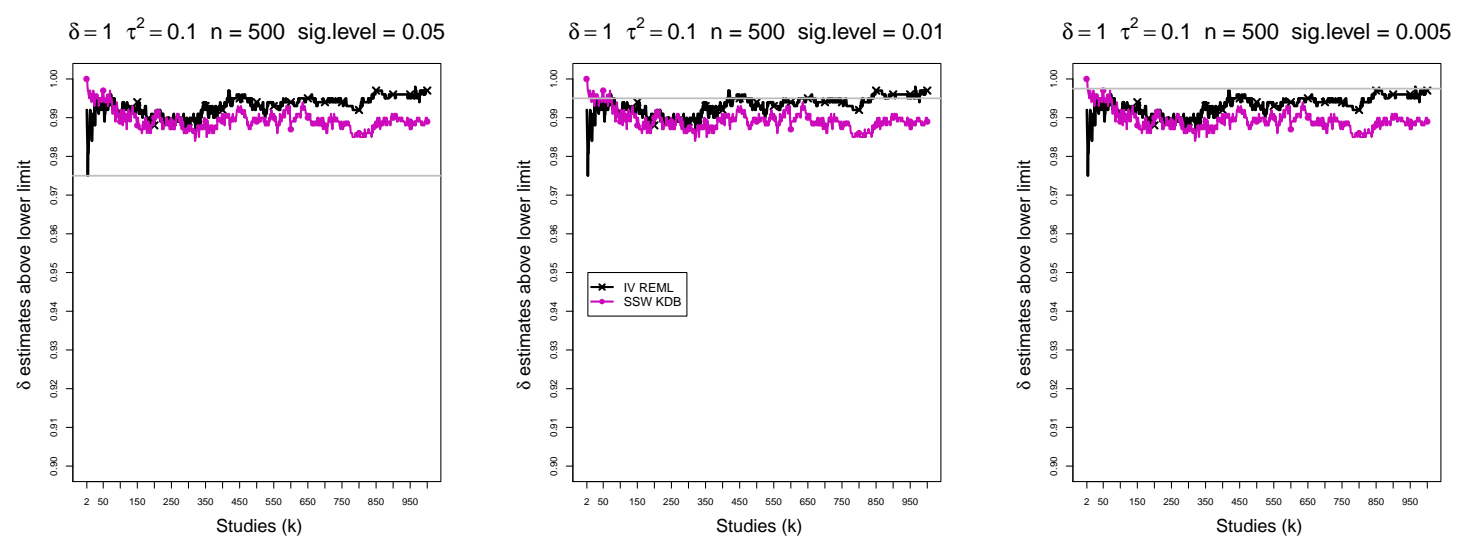

$\delta=1 \tau^{2}=0.25 \mathrm{n}=500$ sig.level $=0.05$

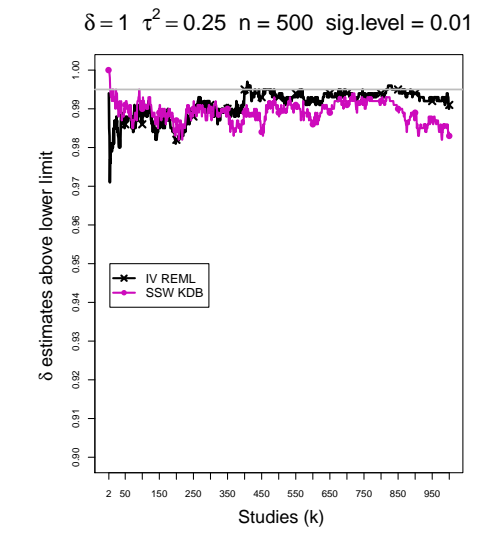

$\delta=1 \quad \tau^{2}=0.25 \mathrm{n}=500$ sig.level $=0.005$
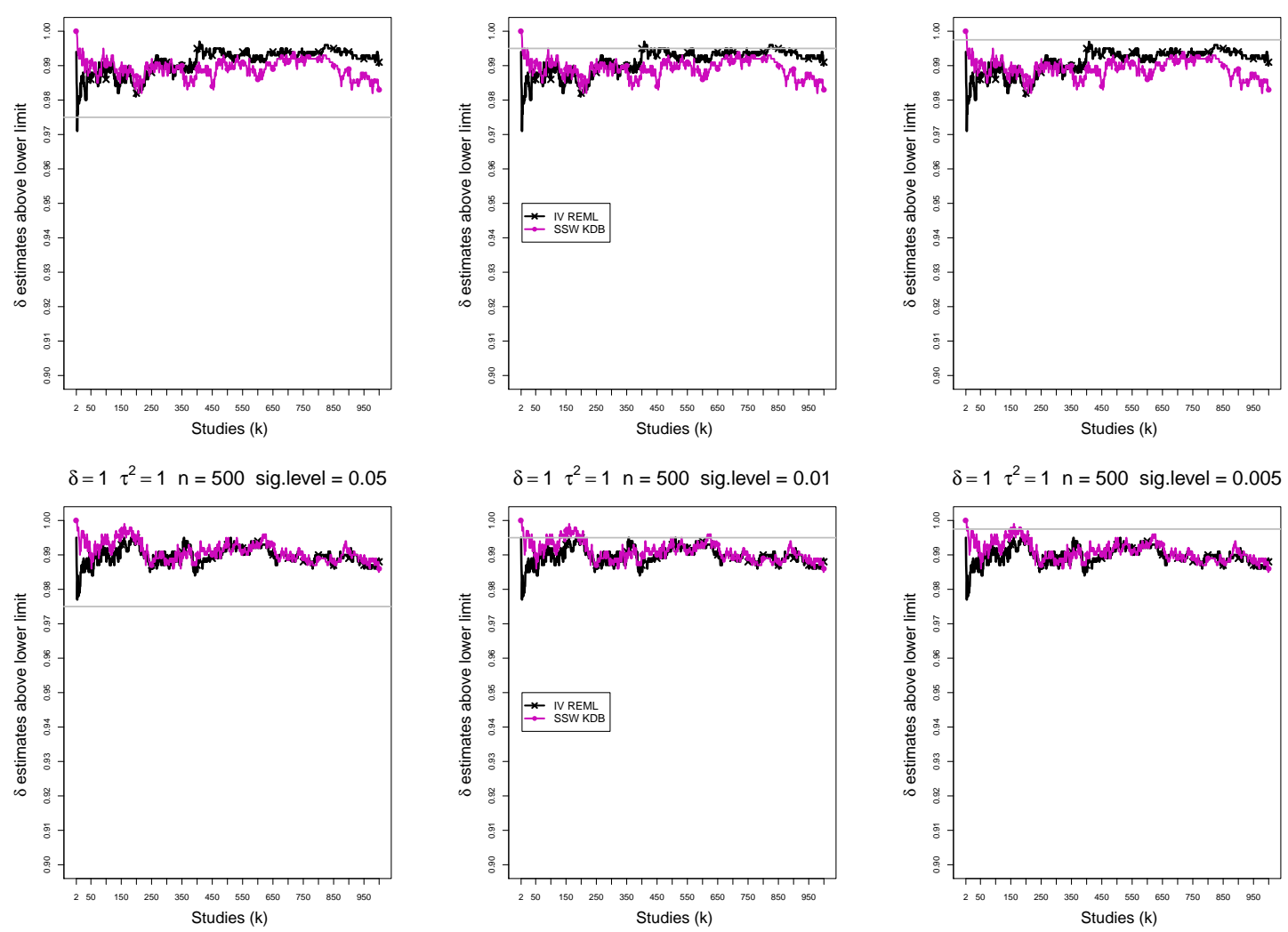

Figure B3. 4: 2-Stage CMA - Coverage of the lower confidence interval of $\delta$ (above lower limit) at the confidence level $1-\alpha$ for $\alpha=0.05,0.01,0.005$ for $\delta=1, \tau^{2}=0,0.1,0.25,1$, $n=500, K=1000$. Light grey line at $0.975,0.995,0.9975$. 

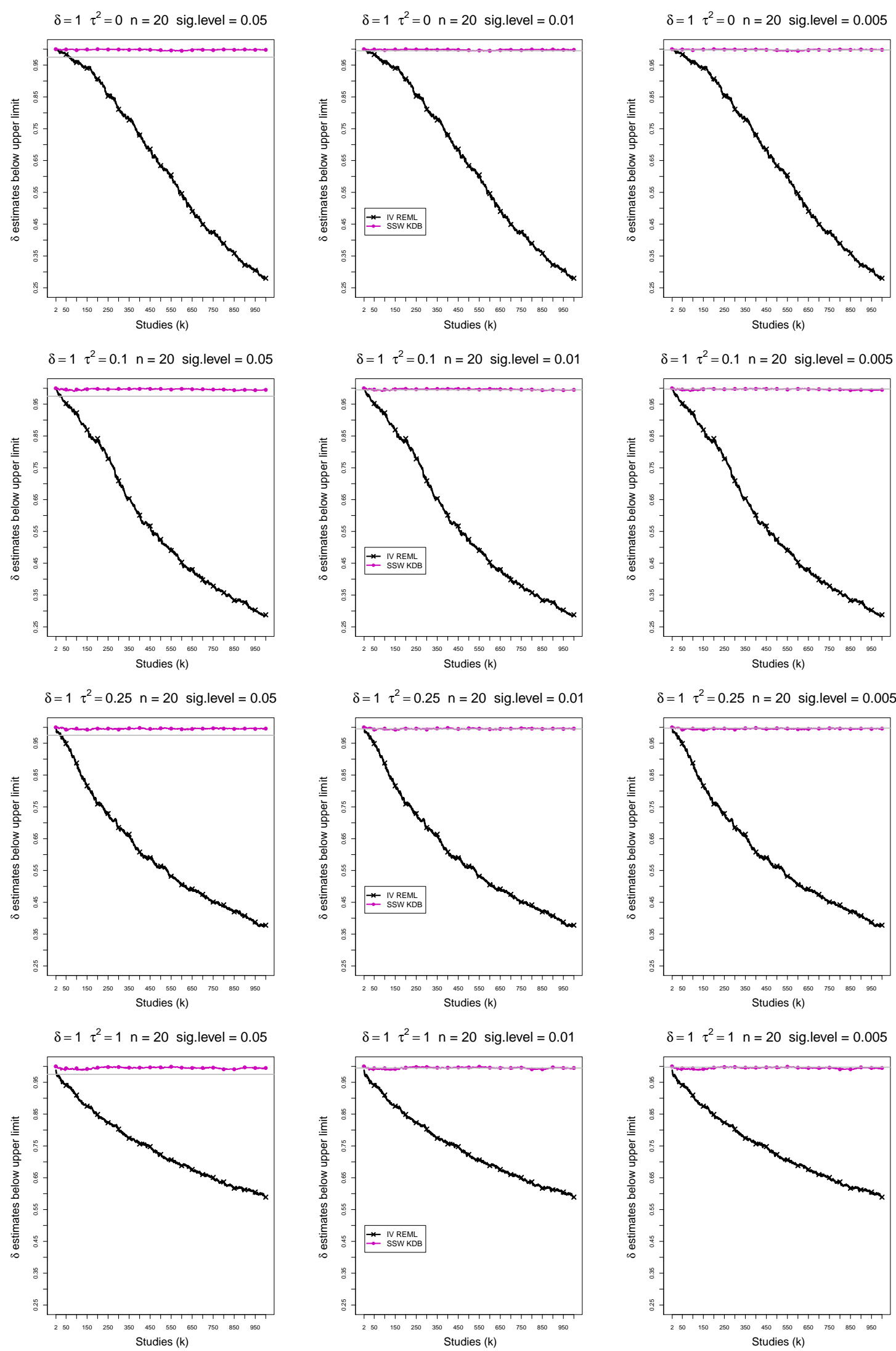

Figure B3. 5: 2-Stage CMA - Coverage of the upper confidence interval of $\delta$ (below upper limit) at the confidence level $1-\alpha$ for $\alpha=0.05,0.01,0.005$ for $\delta=1, \tau^{2}=0,0.1,0.25,1$, $n=20, K=1000$. Light grey line at $0.975,0.995,0.9975$. 

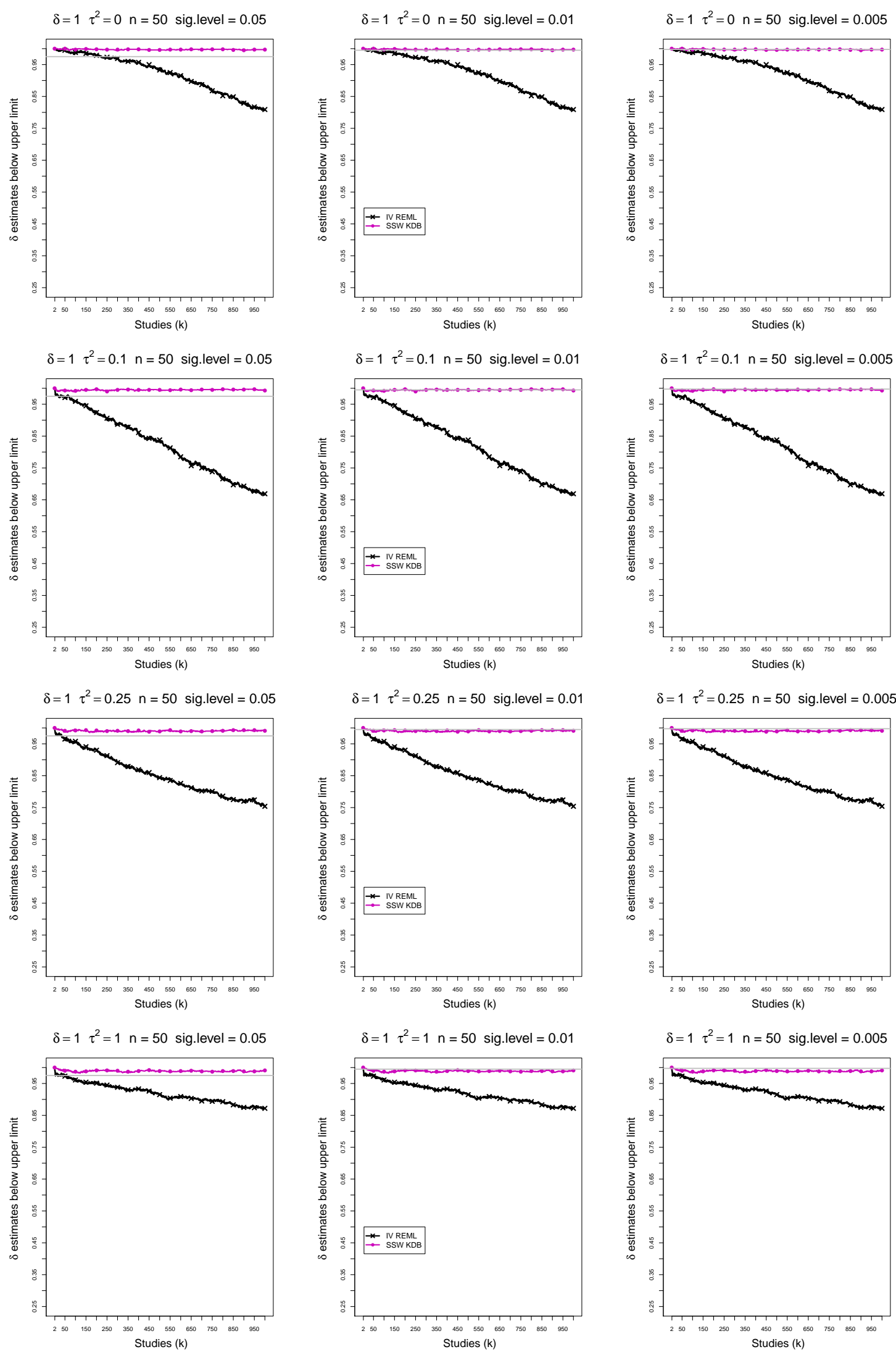

Figure B3. 6: 2-Stage CMA - Coverage of the upper confidence interval of $\delta$ (below upper limit) at the confidence level $1-\alpha$ for $\alpha=0.05,0.01,0.005$ for $\delta=1, \tau^{2}=0,0.1,0.25,1$, $n=50, K=1000$. Light grey line at $0.975,0.995,0.9975$. 

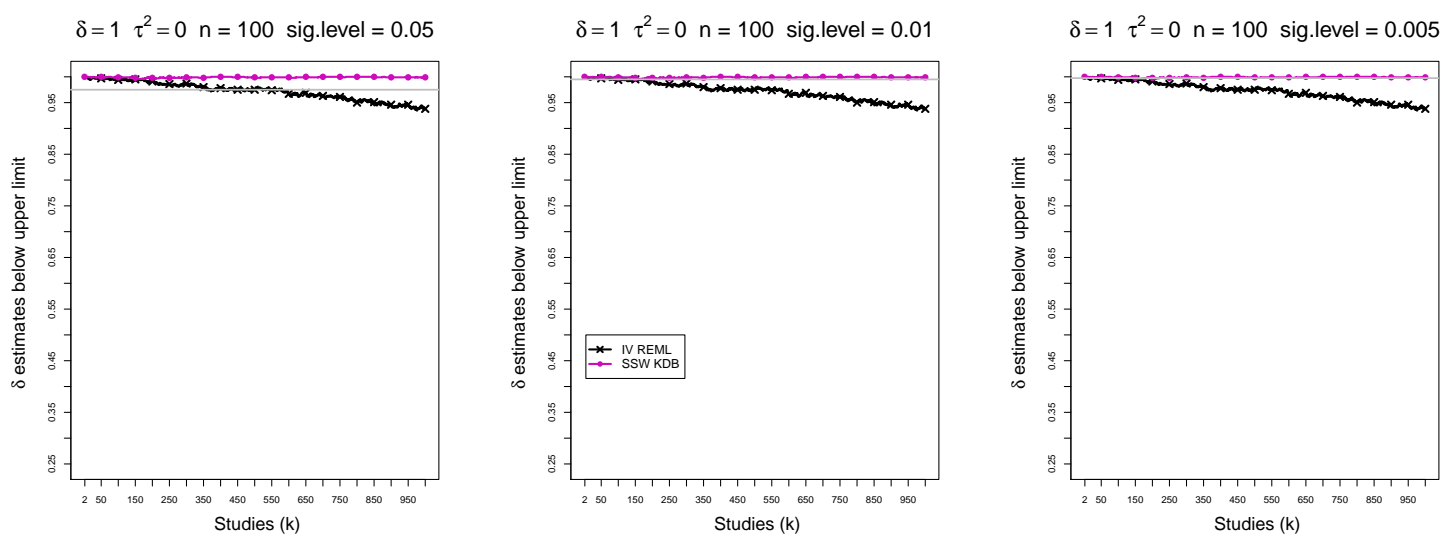

$\delta=1 \quad \tau^{2}=0.1 \quad \mathrm{n}=100$ sig.level $=0.05$

$\delta=1 \quad \tau^{2}=0.1 \quad \mathrm{n}=100$ sig.level $=0.01$
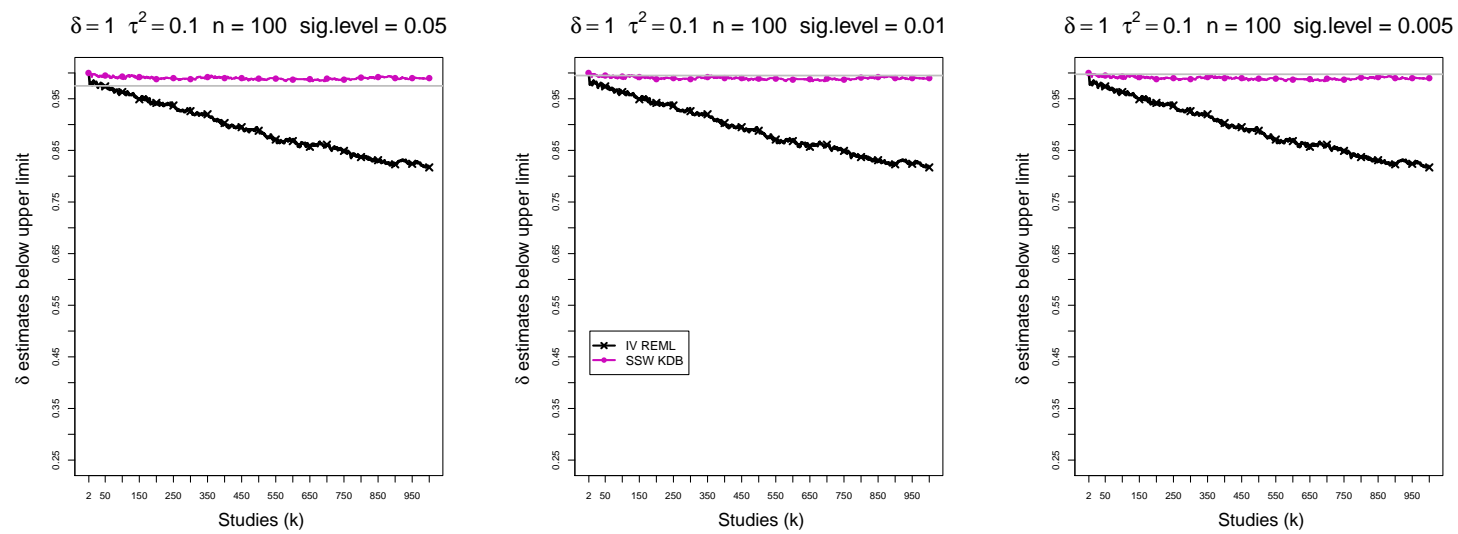

$\delta=1 \tau^{2}=0.25 \mathrm{n}=100$ sig.level $=0.05$

$\delta=1 \tau^{2}=0.25 \mathrm{n}=100$ sig.level $=0.01$

$\delta=1 \tau^{2}=0.25 \quad n=100$ sig.level $=0.005$
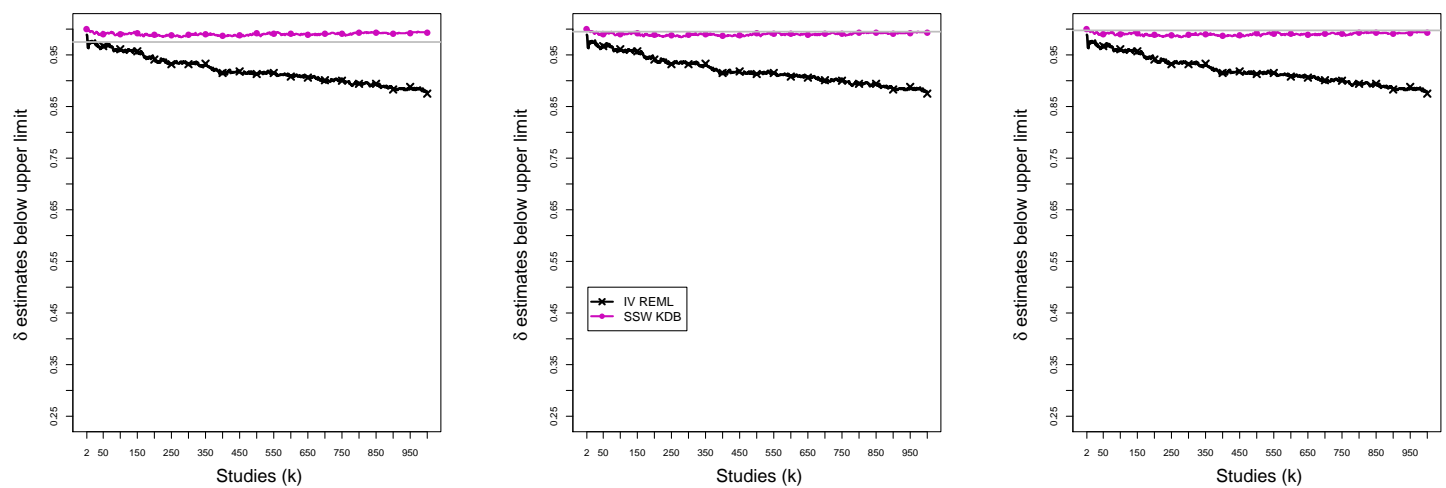

$\delta=1 \tau^{2}=1 \quad \mathrm{n}=100$ sig.level $=0.05$
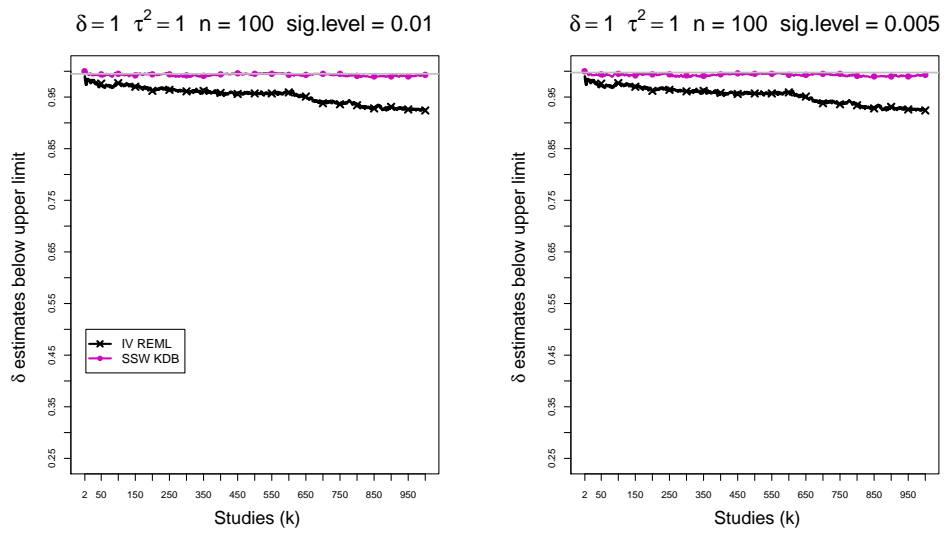

Figure B3. 7: 2-Stage CMA - Coverage of the upper confidence interval of $\delta$ (below upper limit) at the confidence level $1-\alpha$ for $\alpha=0.05,0.01,0.005$ for $\delta=1, \tau^{2}=0,0.1,0.25,1$, $n=100, K=1000$. Light grey line at $0.975,0.995,0.9975$. 

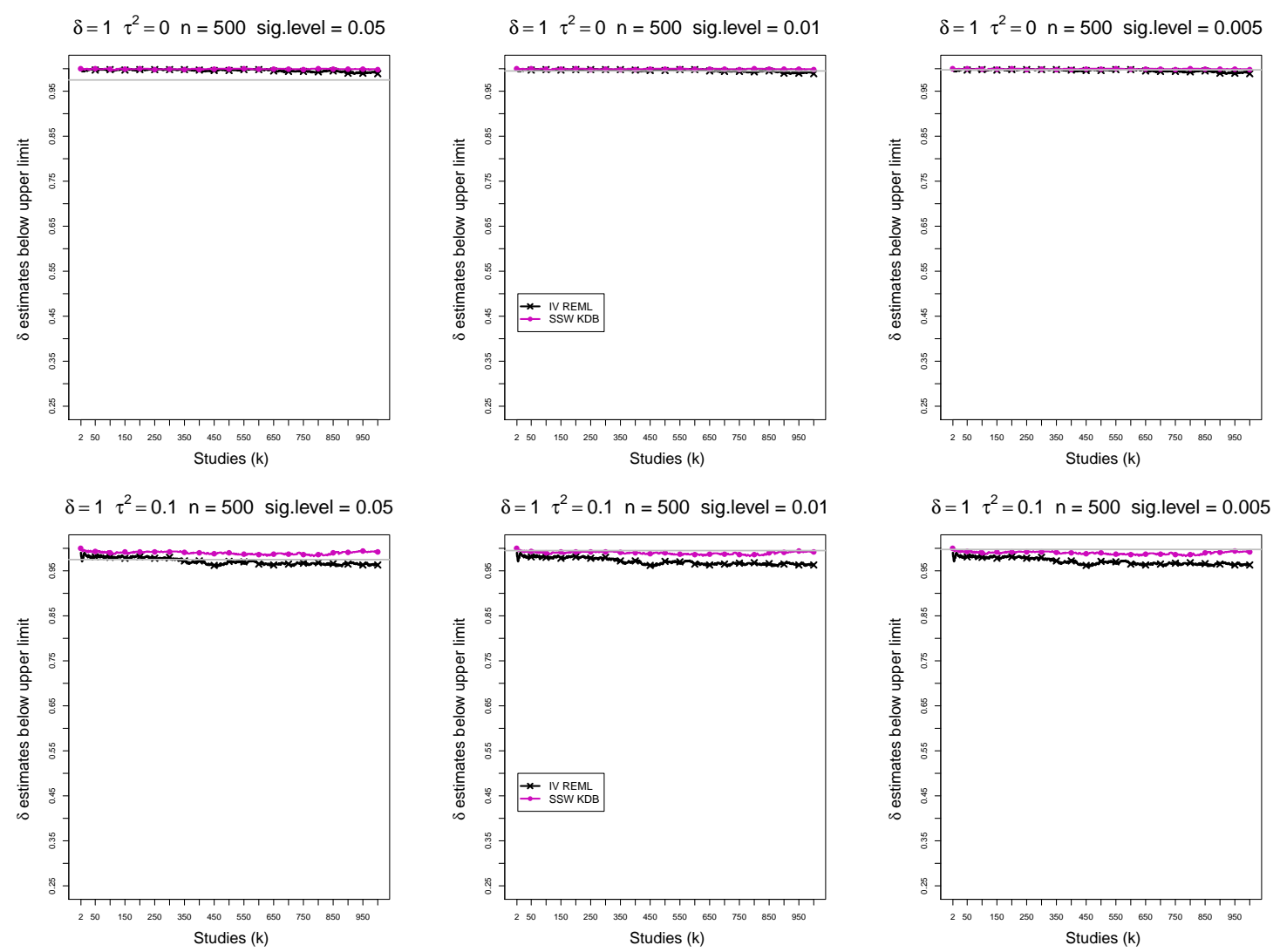

$\delta=1 \quad \tau^{2}=0.25 \quad n=500$ sig.level $=0.05$

$\delta=1 \quad \tau^{2}=0.25 \mathrm{n}=500$ sig.level $=0.01$

$\delta=1 \tau^{2}=0.25 \mathrm{n}=500$ sig.level $=0.005$
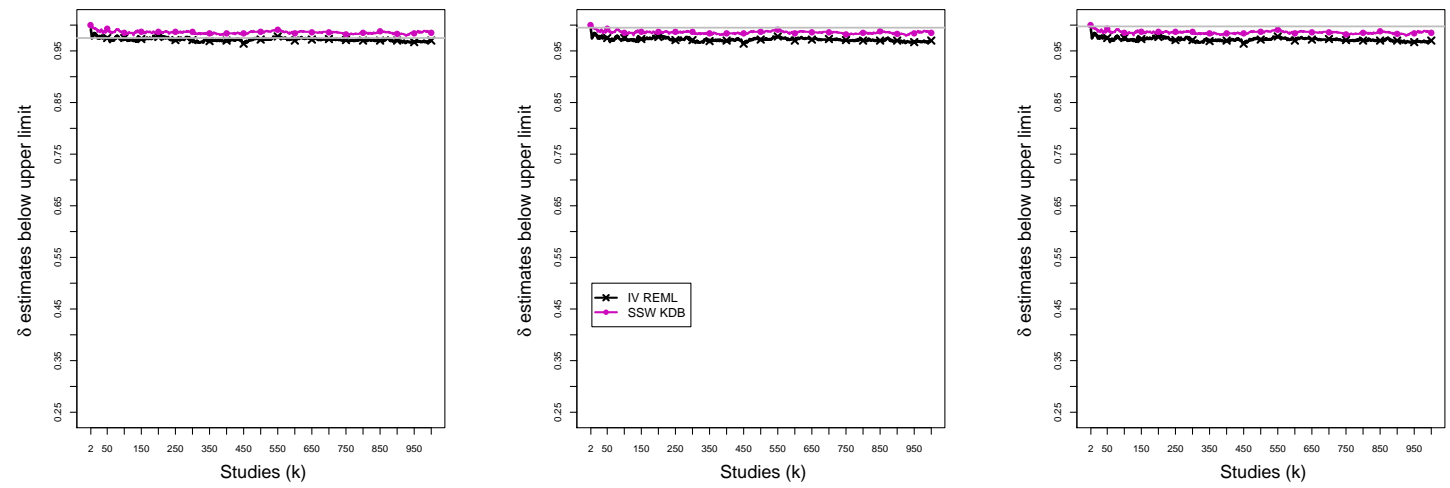

$\delta=1 \quad \tau^{2}=1 \quad n=500$ sig.level $=0.05$
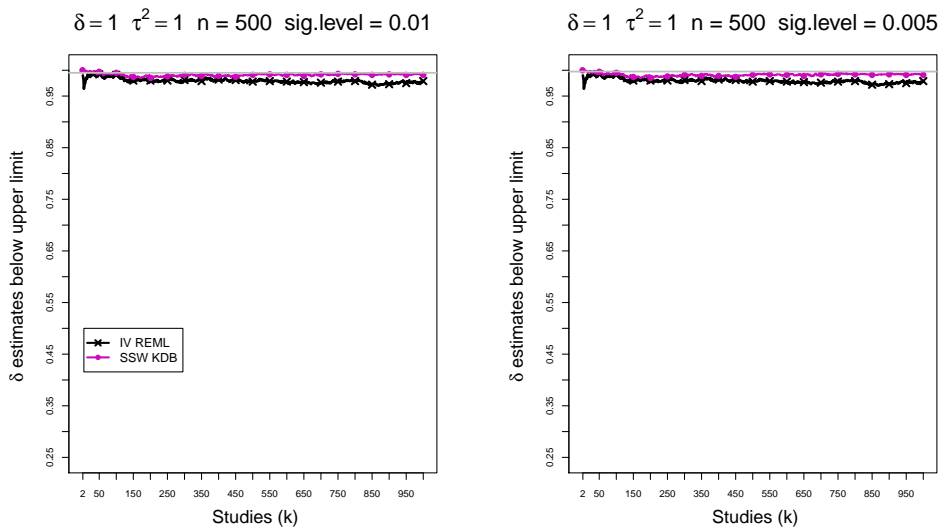

Figure B3. 8: 2-Stage CMA - Coverage of the upper confidence interval of $\delta$ (below upper limit) at the confidence level $1-\alpha$ for $\alpha=0.05,0.01,0.005$ for $\delta=1, \tau^{2}=0,0.1,0.25,1$, $n=500, K=1000$. Light grey line at 0.975,0.995,0.9975. 


\section{B4. Empirical levels of 2-Stage CMA tests for shift-in-the-mean $\delta$}

For empirical levels of tests for shift in known mean $\delta$ in this section, each figure corresponds to one of the two tested meta-analysis methods, a set of values of $\tau^{2}(=0$, $0.1,0.25,1)$, a value of $n(=20,50,100,500)$ and a set of values of significance level $(=0.05,0.01,0.005)$ while maximum number of studies $K=1000$ and $\delta=1$.

Each figure contains a panel (with study $k$ on the horizontal axis) for each combination of parameters.

The tested methods are

- IV REML (Wald test based on the inverse variance weighted mean with the between-study variance $\tau^{2}$ estimated by Restricted maximum likelihood)

- SSW (Test based on effective sample size weighted mean with $t_{K-1}$ critical values) 
IV REML: $\delta=1 \quad \tau^{2}=0 \quad \mathrm{n}=20$

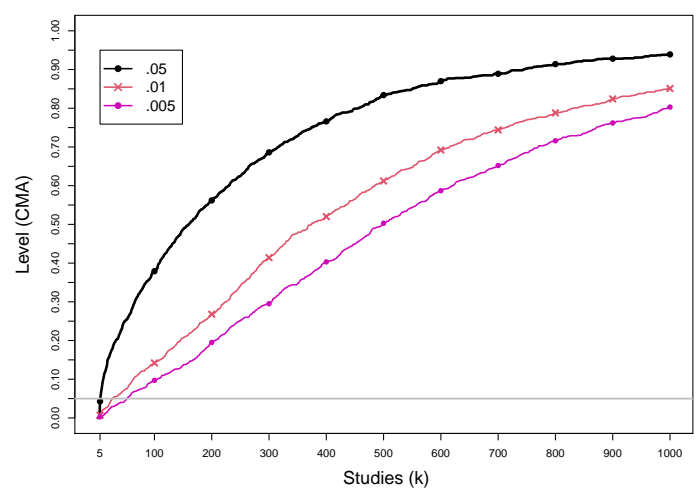

IV REML: $\delta=1 \tau^{2}=0.1 \quad \mathrm{n}=20$

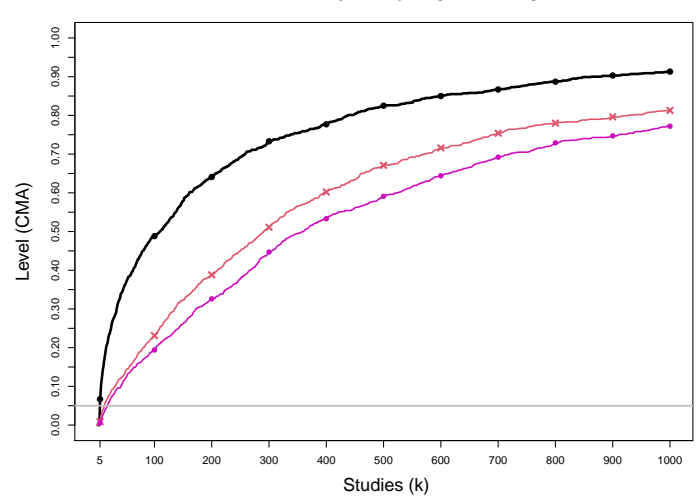

IV REML: $\delta=1 \tau^{2}=0.25 \mathrm{n}=20$

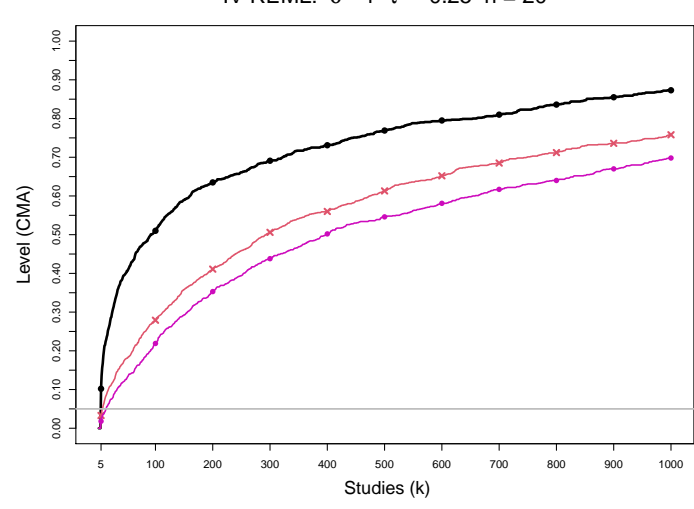

IV REML: $\delta=1 \quad \tau^{2}=1 \quad \mathrm{n}=20$

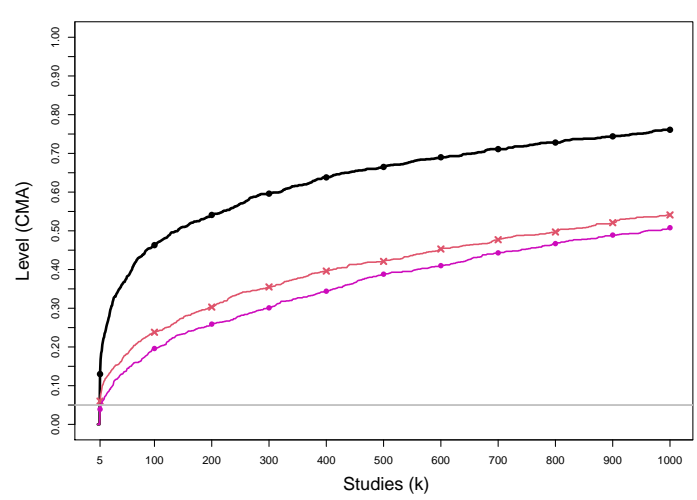

SSW KDB: $\delta=1 \quad \tau^{2}=0 \quad n=20$

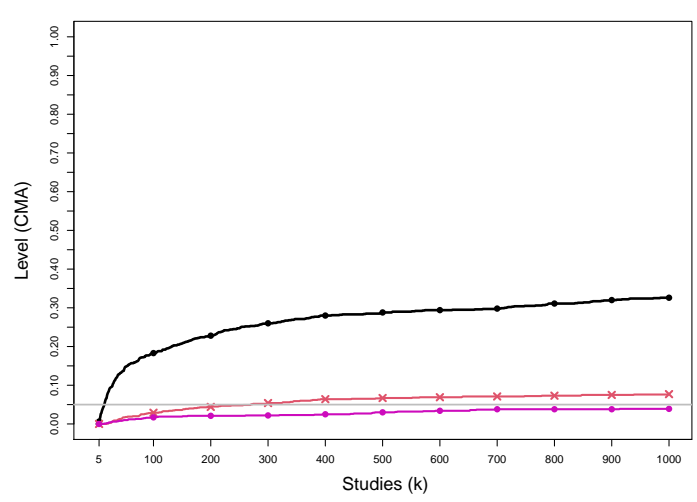

SSW KDB: $\delta=1 \quad \tau^{2}=0.1 \quad \mathrm{n}=20$

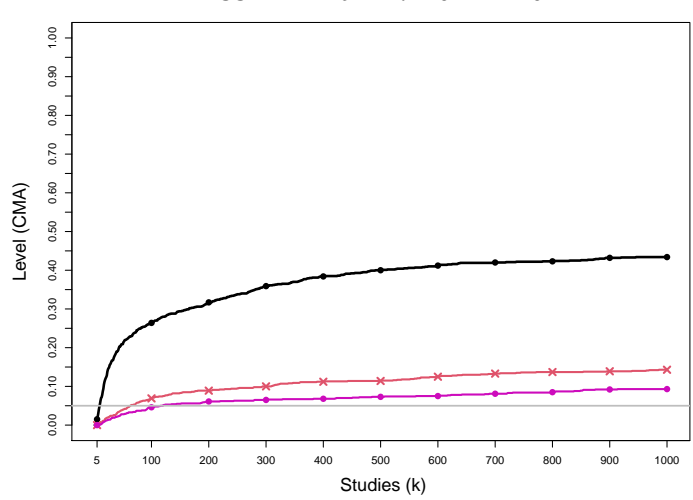

SSW KDB: $\delta=1 \quad \tau^{2}=0.25 \mathrm{n}=20$

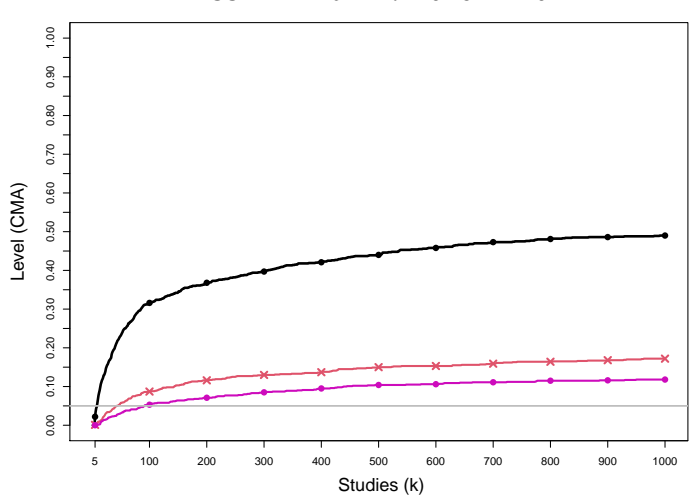

SSW KDB: $\delta=1 \quad \tau^{2}=1 \quad \mathrm{n}=20$

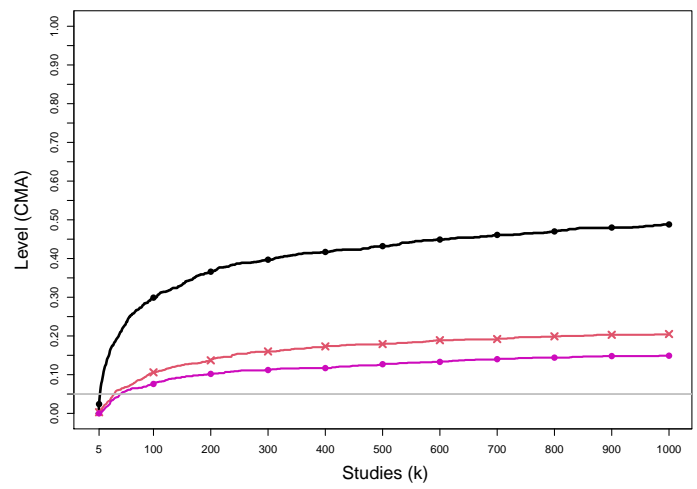

Figure B4. 1: Empirical levels of 2-Stage CMA tests for shift in $\delta=1$ based on SSW KDB and IV REML at .05, .01 and .005 nominal levels for number of studies $k$ up to $K=1000$ for equal sample sizes $n_{i C}+n_{i T}=n=20, \tau^{2}=0,0.1,0.25,1$. Light grey line at .05 . 

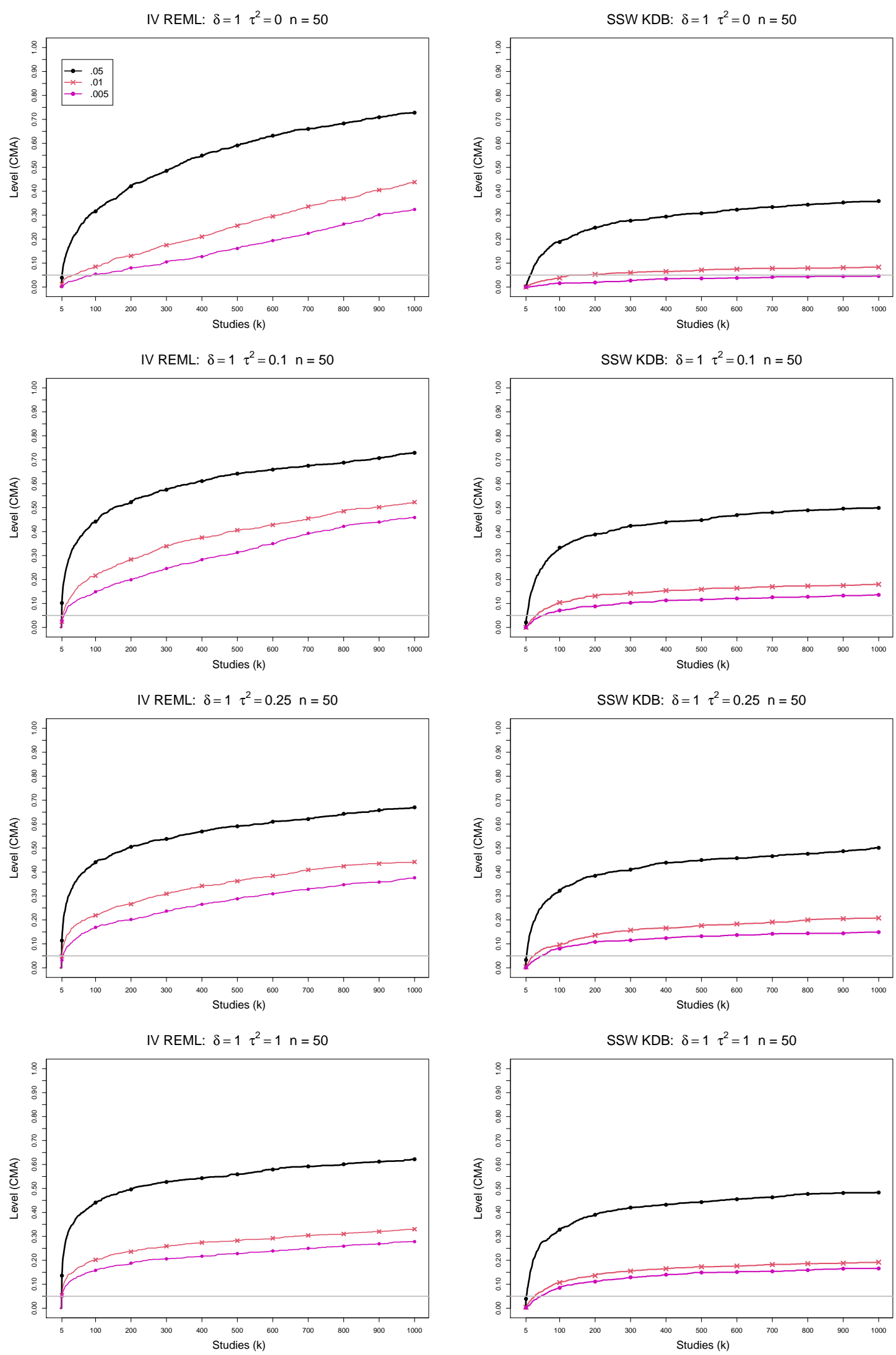

Figure B4. 2: Empirical levels of 2-Stage CMA tests for shift in $\delta=1$ based on SSW KDB and IV REML at .05, .01 and .005 nominal levels for number of studies $k$ up to $K=1000$ for equal sample sizes $n_{i C}+n_{i T}=n=50, \tau^{2}=0,0.1,0.25,1$. Light grey line at .05 . 

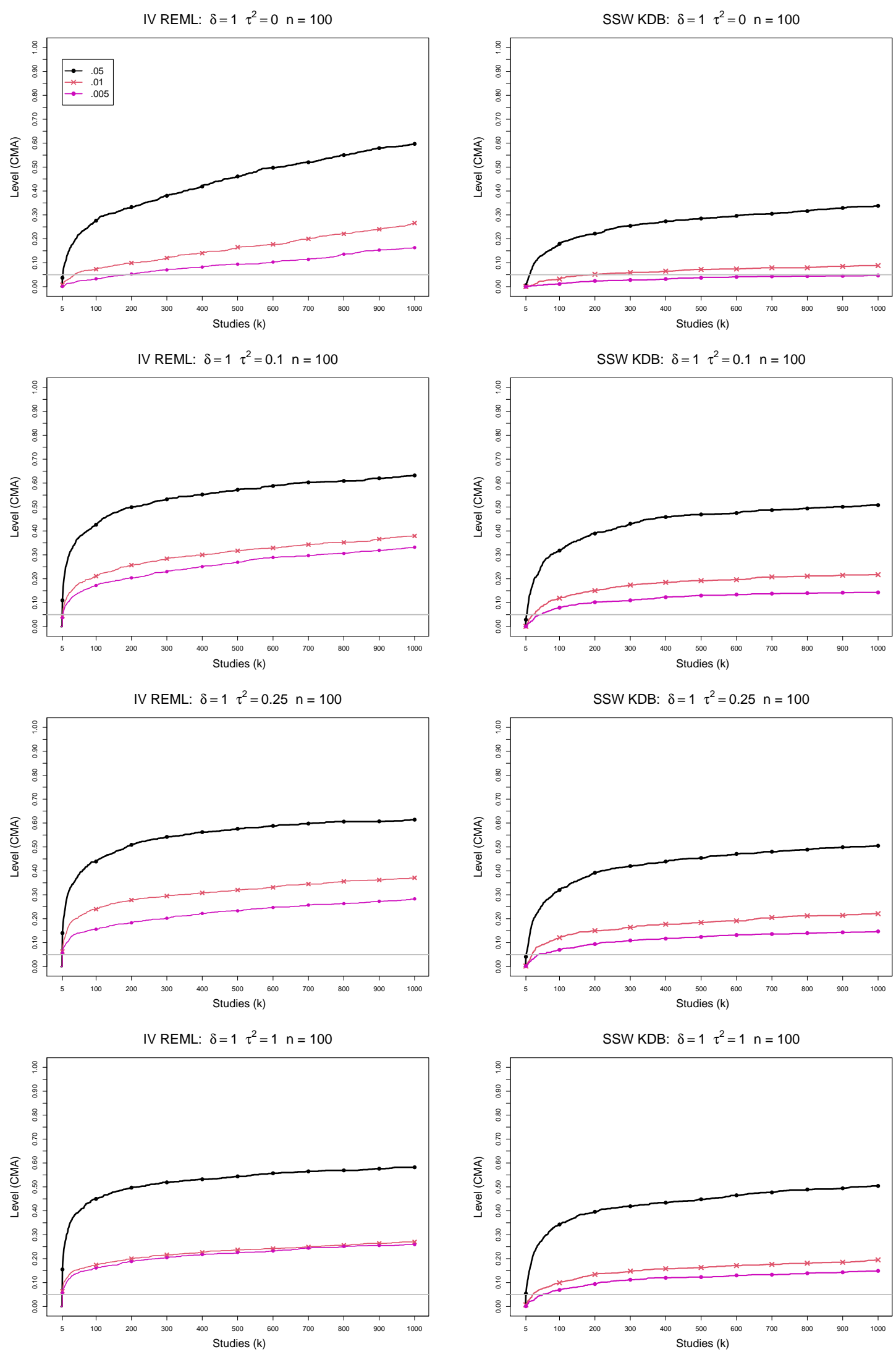

Figure B4. 3: Empirical levels of 2-Stage CMA tests for shift in $\delta=1$ based on SSW KDB and IV REML at .05, .01 and .005 nominal levels for number of studies $k$ up to $K=1000$ for equal sample sizes $n_{i C}+n_{i T}=n=100, \tau^{2}=0,0.1,0.25,1$. Light grey line at .05. 

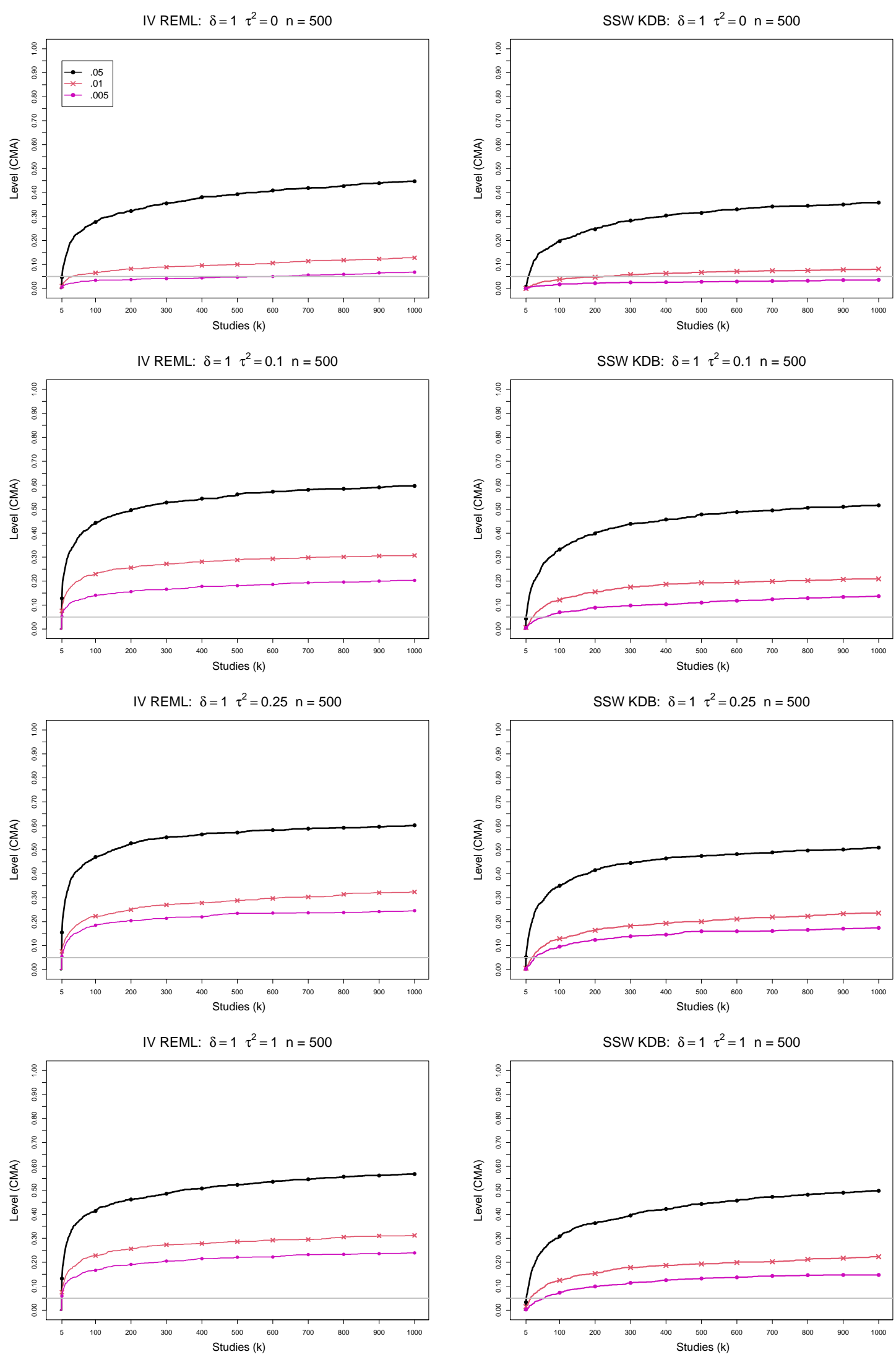

Figure B4. 4: Empirical levels of 2-Stage CMA tests for shift in $\delta=1$ based on SSW KDB and IV REML at .05, .01 and .005 nominal levels for number of studies $k$ up to $K=1000$ for equal sample sizes $n_{i C}+n_{i T}=n=500, \tau^{2}=0,0.1,0.25,1$. Light grey line at .05. 


\section{B5. Empirical levels of CUSUM tests for shift in $\hat{\delta}_{0}$}

In this section, for empirical levels of tests for shift in estimated in stage 1 value of $\delta$ using also estimated in stage 1 value of $\tau^{2}$, each figure corresponds to one of the two tested meta-analysis methods, a set of values of $\tau^{2}(=0,0.1,0.25,1)$, a value of $n(=20,50,100,500)$ and a set of values of decision interval $h(=4,5,6)$ while maximum number of studies $K=1000$ and $\delta=1$.

Each figure contains a panel (with study $k$ on the horizontal axis) for each combination of parameters.

The tested methods are

- IV REML (Inverse variance weighted mean with the between-study variance $\tau^{2}$ estimated by Restricted maximum likelihood)

- SSW KDB(Effective sample size weighted mean and KDB-estimated $\tau^{2}$ ) 
IV REML: $\delta=1 \quad \tau^{2}=0 \quad \mathrm{n}=20$

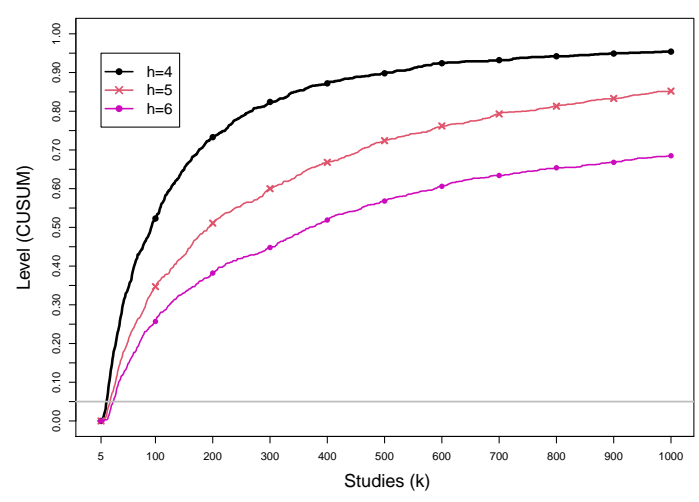

IV REML: $\delta=1 \quad \tau^{2}=0.1 \quad \mathrm{n}=20$

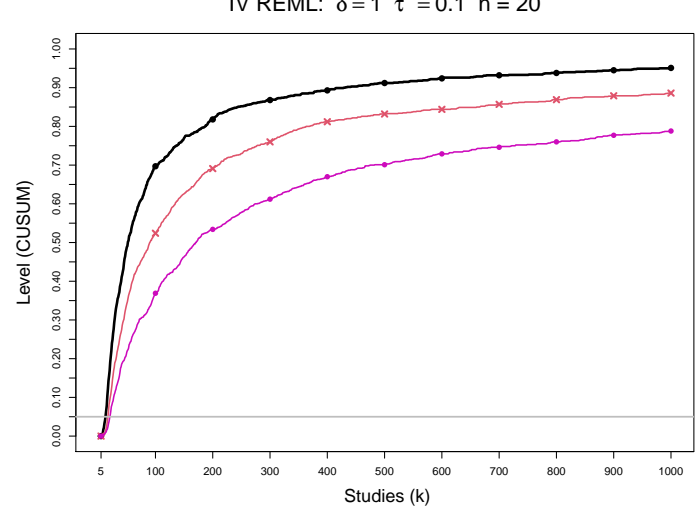

IV REML: $\delta=1 \quad \tau^{2}=0.25 \quad \mathrm{n}=20$

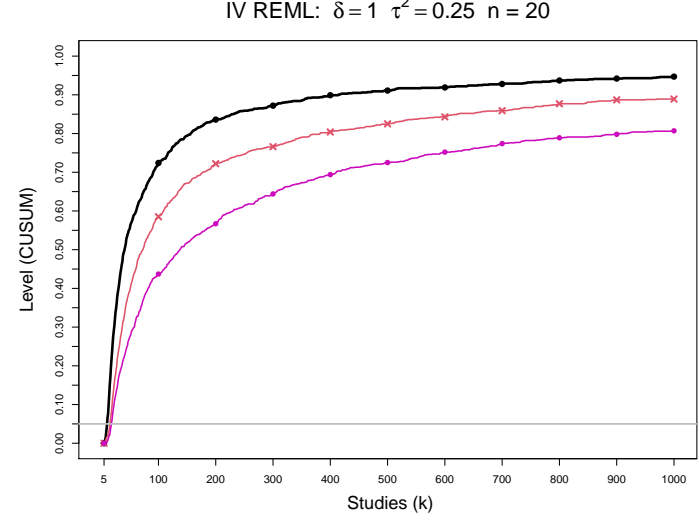

IV REML: $\delta=1 \quad \tau^{2}=1 \quad \mathrm{n}=20$

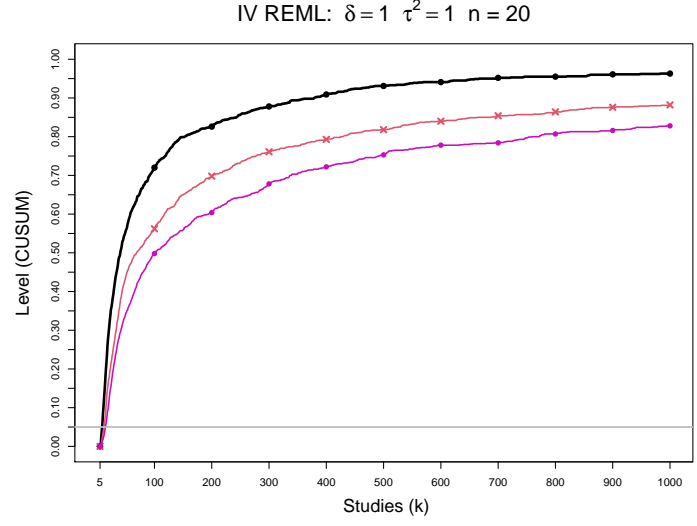

SSW KDB: $\delta=1 \quad \tau^{2}=0 \quad n=20$

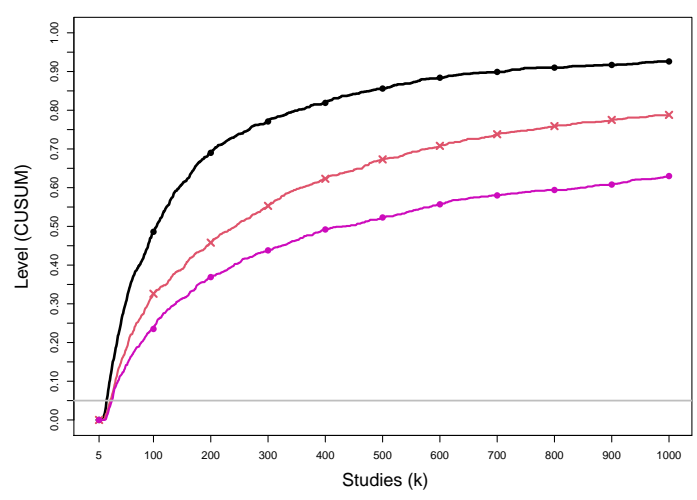

SSW KDB: $\delta=1 \quad \tau^{2}=0.1 \quad \mathrm{n}=20$

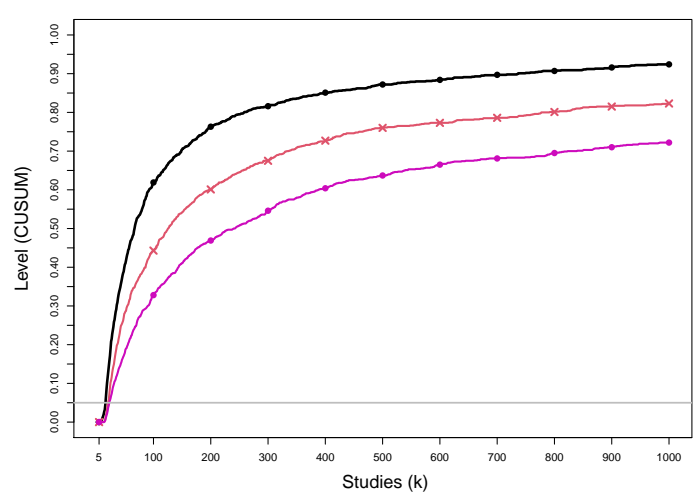

SSW KDB: $\delta=1 \quad \tau^{2}=0.25 \mathrm{n}=20$

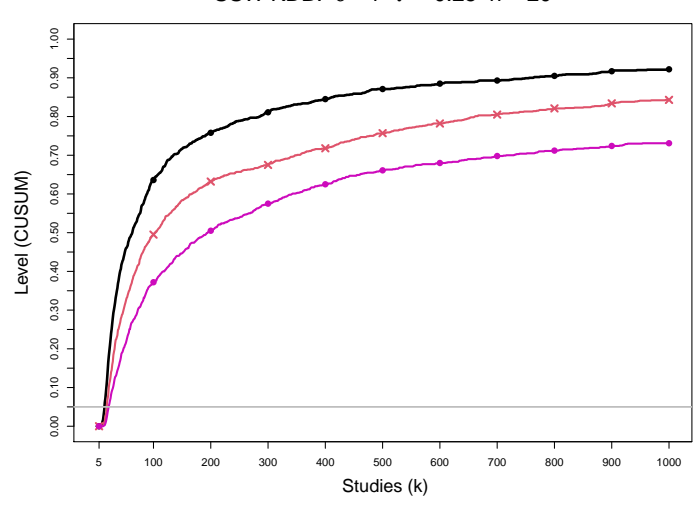

SSW KDB: $\delta=1 \quad \tau^{2}=1 \quad \mathrm{n}=20$

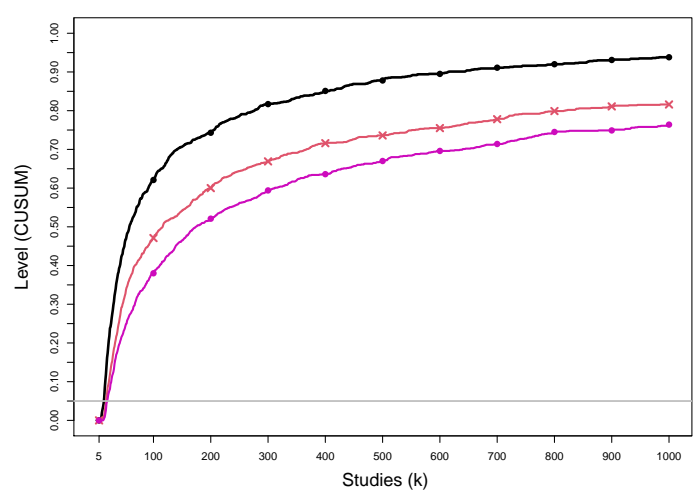

Figure B5. 1: Empirical levels of CUSUM tests for shift in $\delta=1$ based on SSW KDB and IV REML at $h=4,5,6$ for $k$ up to $K=1000$ for equal sample sizes $n_{i C}+n_{i T}=n=20$, $\tau^{2}=0,0.1,0.25,1$. Light grey line at .05 . 
IV REML: $\delta=1 \quad \tau^{2}=0 \quad \mathrm{n}=50$

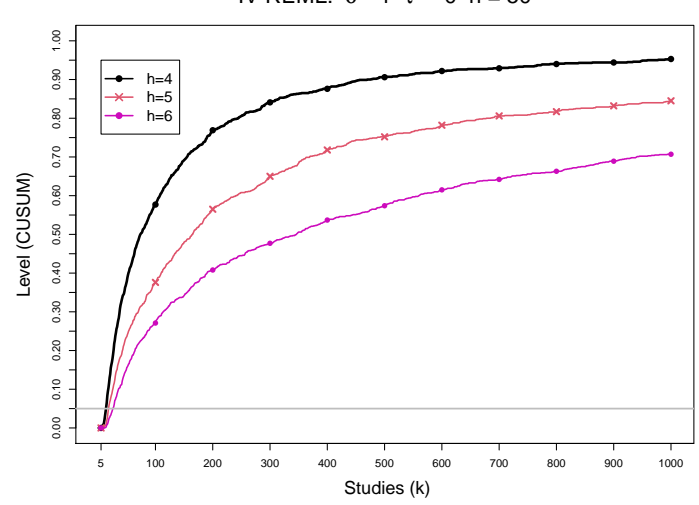

IV REML: $\delta=1 \quad \tau^{2}=0.1 \quad \mathrm{n}=50$

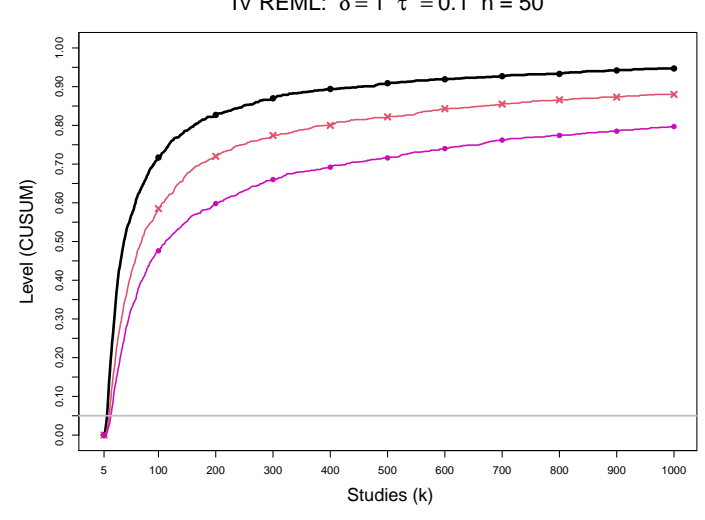

IV REML: $\delta=1 \quad \tau^{2}=0.25 \quad \mathrm{n}=50$

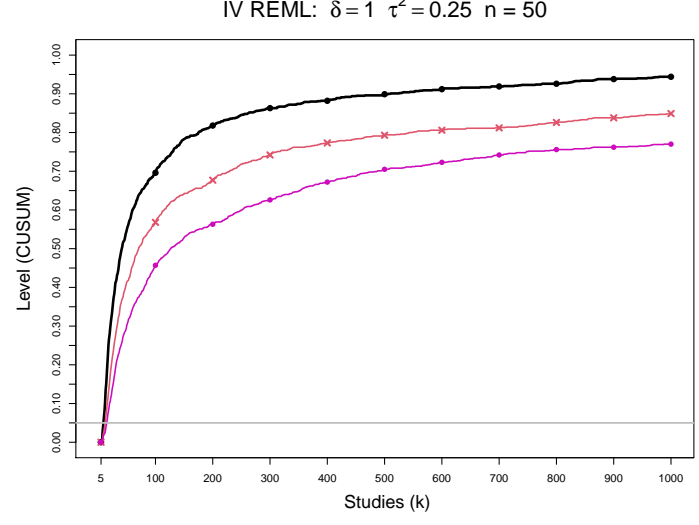

IV REML: $\delta=1 \quad \tau^{2}=1 \quad \mathrm{n}=50$

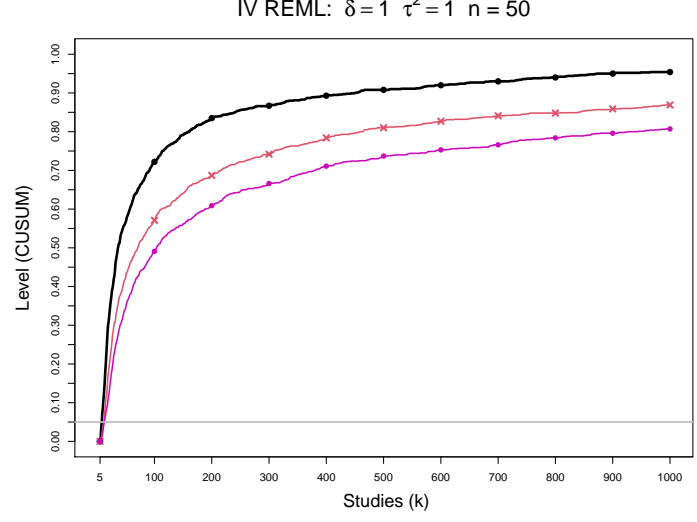

SSW KDB: $\delta=1 \quad \tau^{2}=0 \quad n=50$

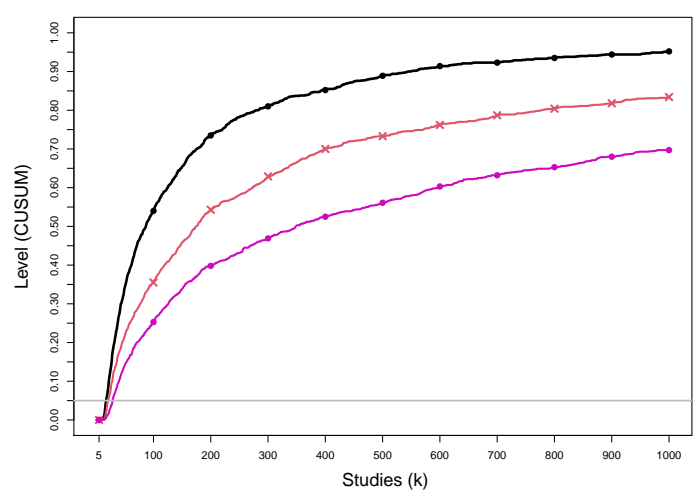

SSW KDB: $\delta=1 \quad \tau^{2}=0.1 \quad \mathrm{n}=50$

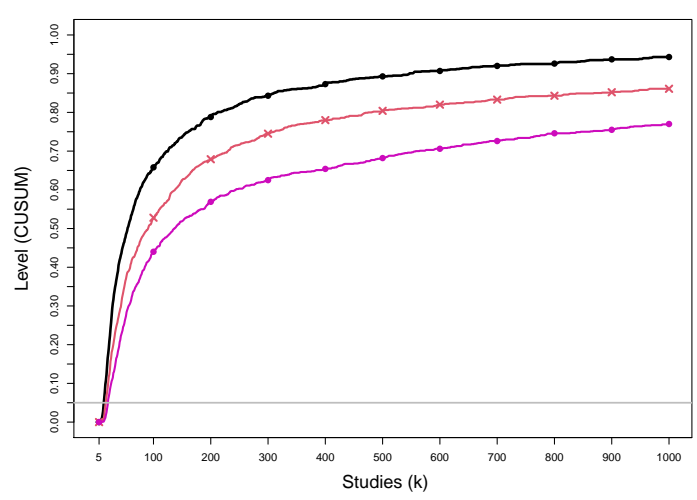

SSW KDB: $\delta=1 \quad \tau^{2}=0.25 \mathrm{n}=50$

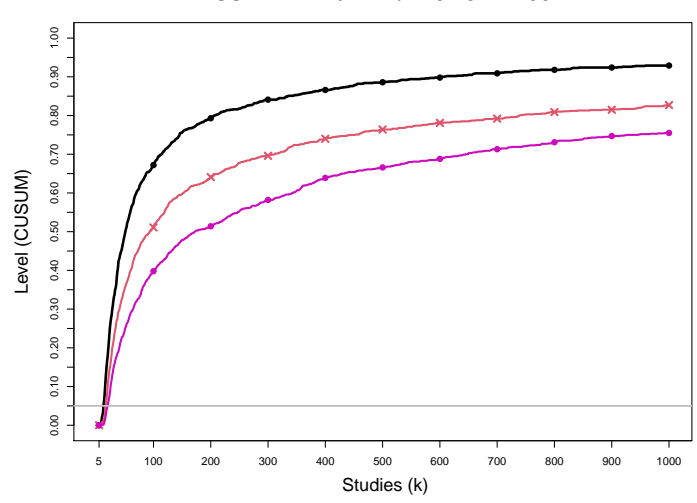

SSW KDB: $\delta=1 \quad \tau^{2}=1 \quad \mathrm{n}=50$

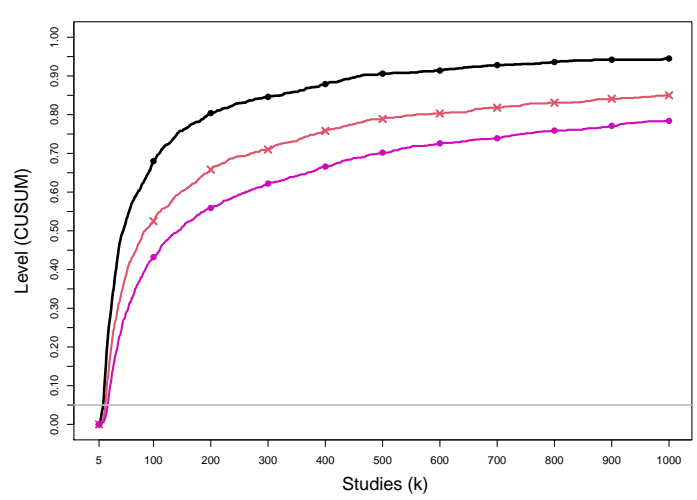

Figure B5. 2: Empirical levels of CUSUM tests for shift in $\delta=1$ based on SSW KDB and IV REML at $h=4,5,6$ for $k$ up to $K=1000$ for equal sample sizes $n_{i C}+n_{i T}=n=50$, $\tau^{2}=0,0.1,0.25,1$. Light grey line at .05 . 

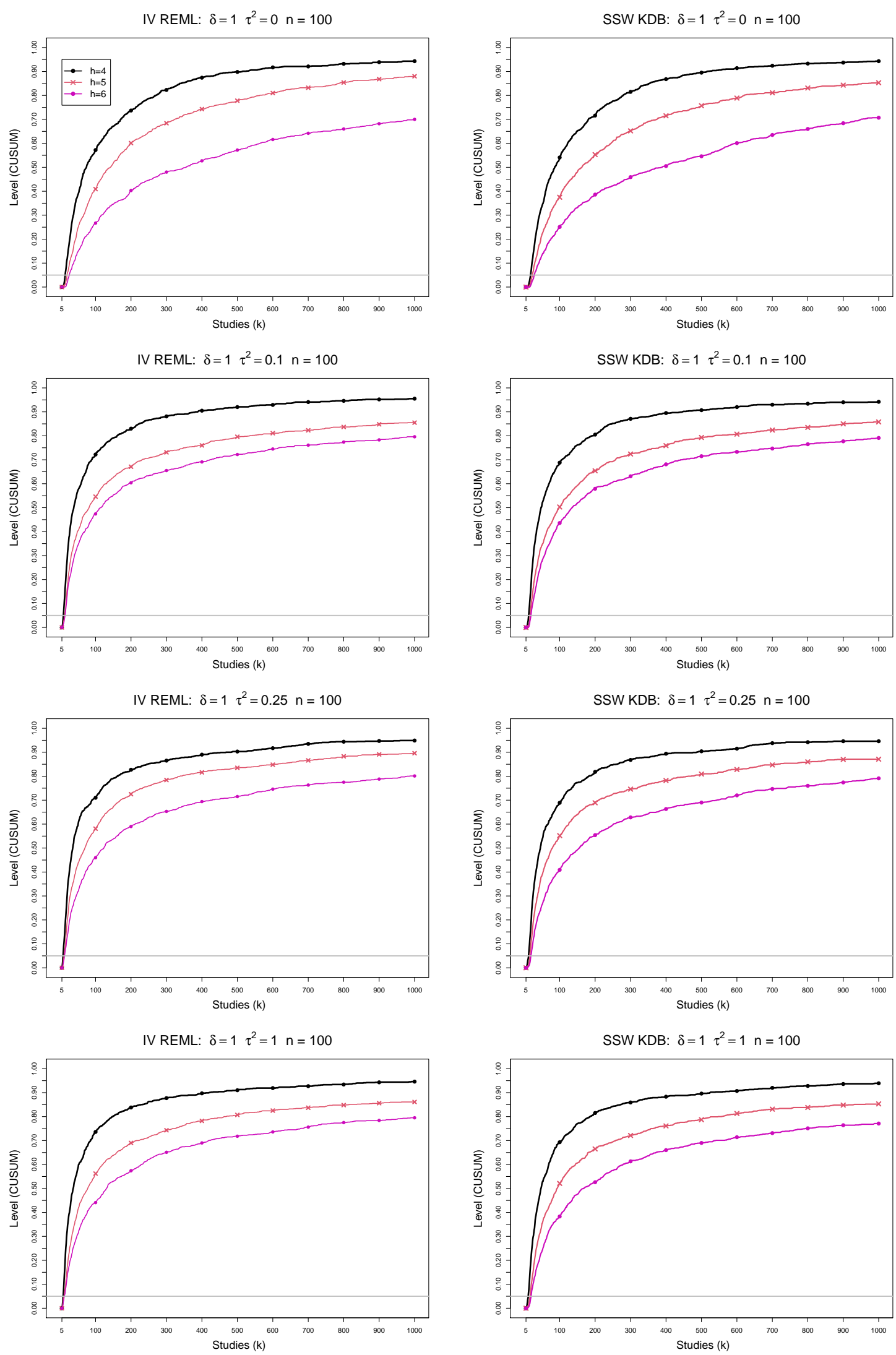

Figure B5. 3: Empirical levels of CUSUM tests for shift in $\delta=1$ based on SSW KDB and IV REML at $h=4,5,6$ for $k$ up to $K=1000$ for equal sample sizes $n_{i C}+n_{i T}=n=100$, $\tau^{2}=0,0.1,0.25,1$. Light grey line at .05. 

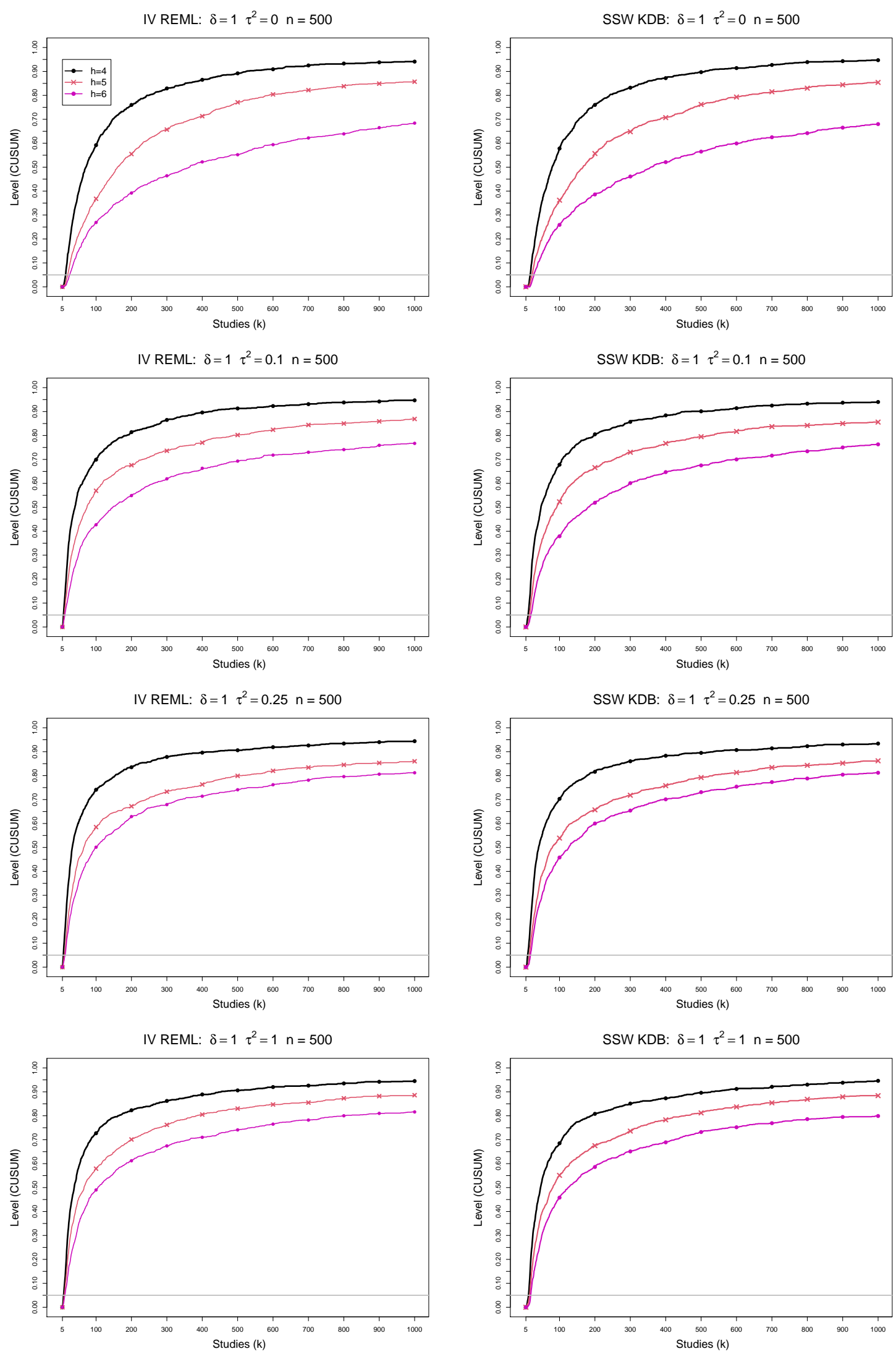

Figure B5. 4: Empirical levels of CUSUM tests for shift in $\delta=1$ based on SSW KDB and IV REML at $h=4,5,6$ for $k$ up to $K=1000$ for equal sample sizes $n_{i C}+n_{i T}=n=500$, $\tau^{2}=0,0.1,0.25,1$. Light grey line at .05. 


\section{Standard CMA under shift-in-the-mean}




\section{C1. Comparison of CMA-estimated mean ef- fects $\delta$ to nominal cumulative mean effects}

In this section, we compare CMA-estimated mean effects $\delta$ to nominal cumulative effects. Each figure corresponds to a set of values of $\tau^{2}(=0,0.1,0.25,1)$, a set of values of $n(=20,50,100$ or 500,1000$)$ and a value of point of shift $f(=13,26,39)$ while $\delta$ shifts from 1 to 2 and number of studies $K=50$.

Each figure contains a panel (with study $k$ on the horizontal axis) for each combination of parameters.

The point estimators of $\delta$ are

- IV REML (Inverse variance weighted mean with the between-study variance $\tau^{2}$ estimated by Restricted maximum likelihood)

- SSW (Effective sample size weighted mean)

The nominal values of $\delta$ are calculated with

- $\delta$ IV (Inverse variance weights)

- $\delta \mathrm{SSW}$ (Effective sample size weights) 

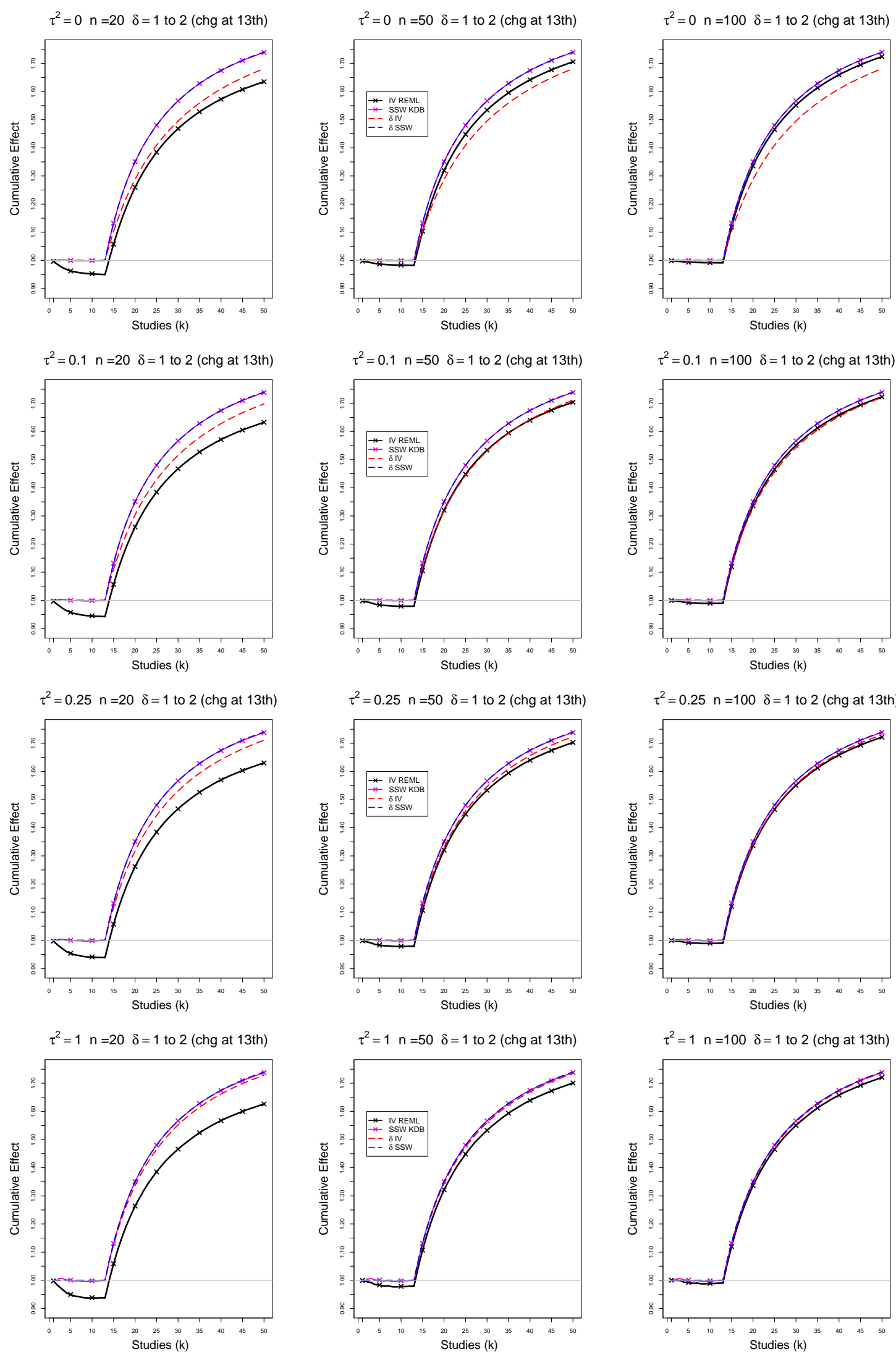

Figure C1. 1: CMA: Nominal cumulative effects $\delta_{S S W,(k)}$ and $\delta_{I V,(k)}$ (dashed lines) and estimated by SSW and IV REML cumulative effects $\hat{\delta}_{(k)}$ (solid lines) when $\delta=1$ for $k \leq 12$ followed by a shift to $\delta=2$ for $k \geq 13, n=20,50,100, K=50$ and $\tau^{2}=0,0.1,0.25,1$. Light grey line at 1. 

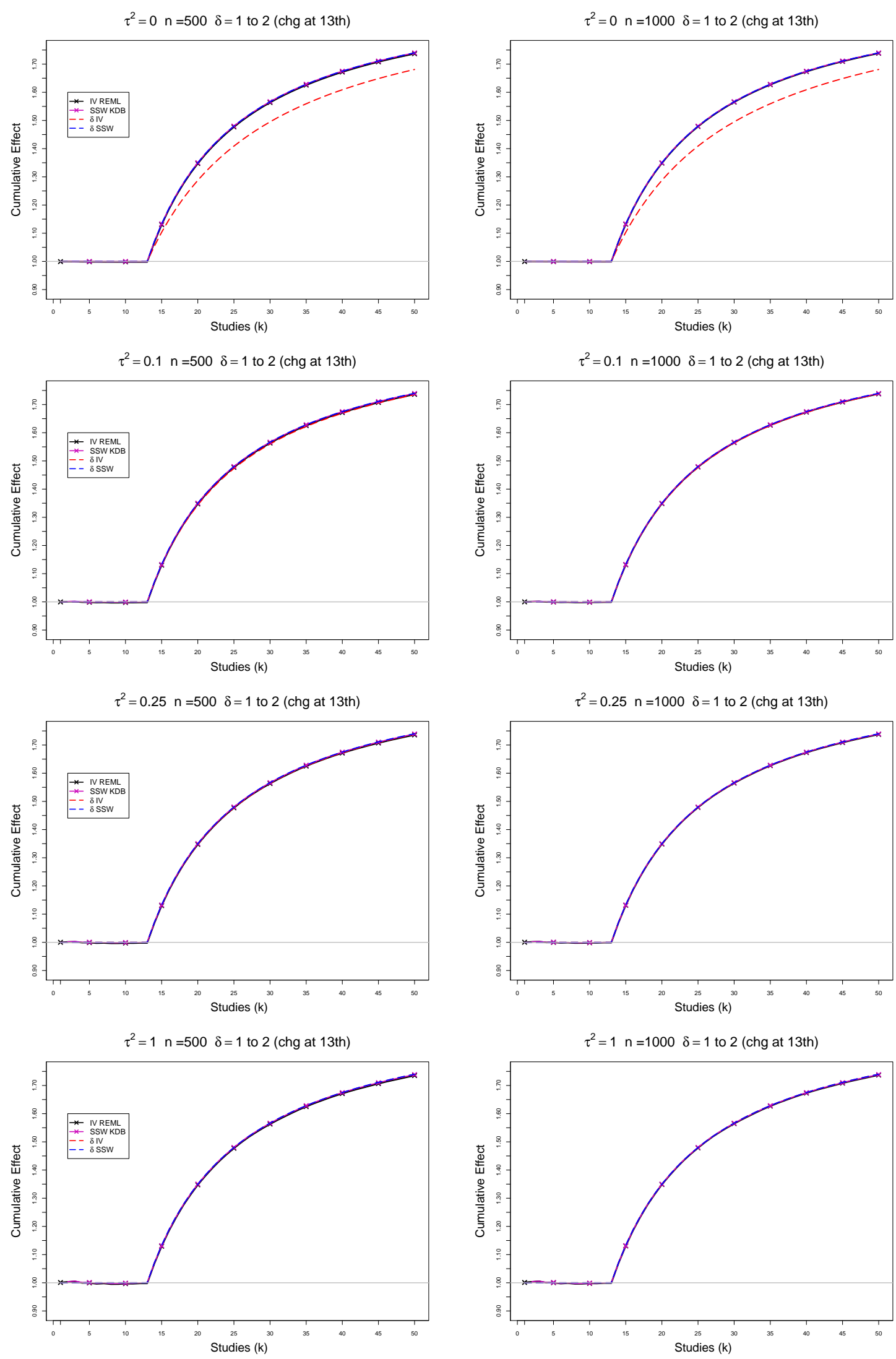

Figure C1. 2: CMA: Nominal cumulative effects $\delta_{S S W,(k)}$ and $\delta_{I V,(k)}$ (dashed lines) and estimated by SSW and IV REML cumulative effects $\hat{\delta}_{(k)}$ (solid lines) when $\delta=1$ for $k \leq 12$ followed by a shift to $\delta=2$ for $k \geq 13, n=500,1000, K=50$ and $\tau^{2}=0,0.1,0.25,1$. Light grey line at 1. 

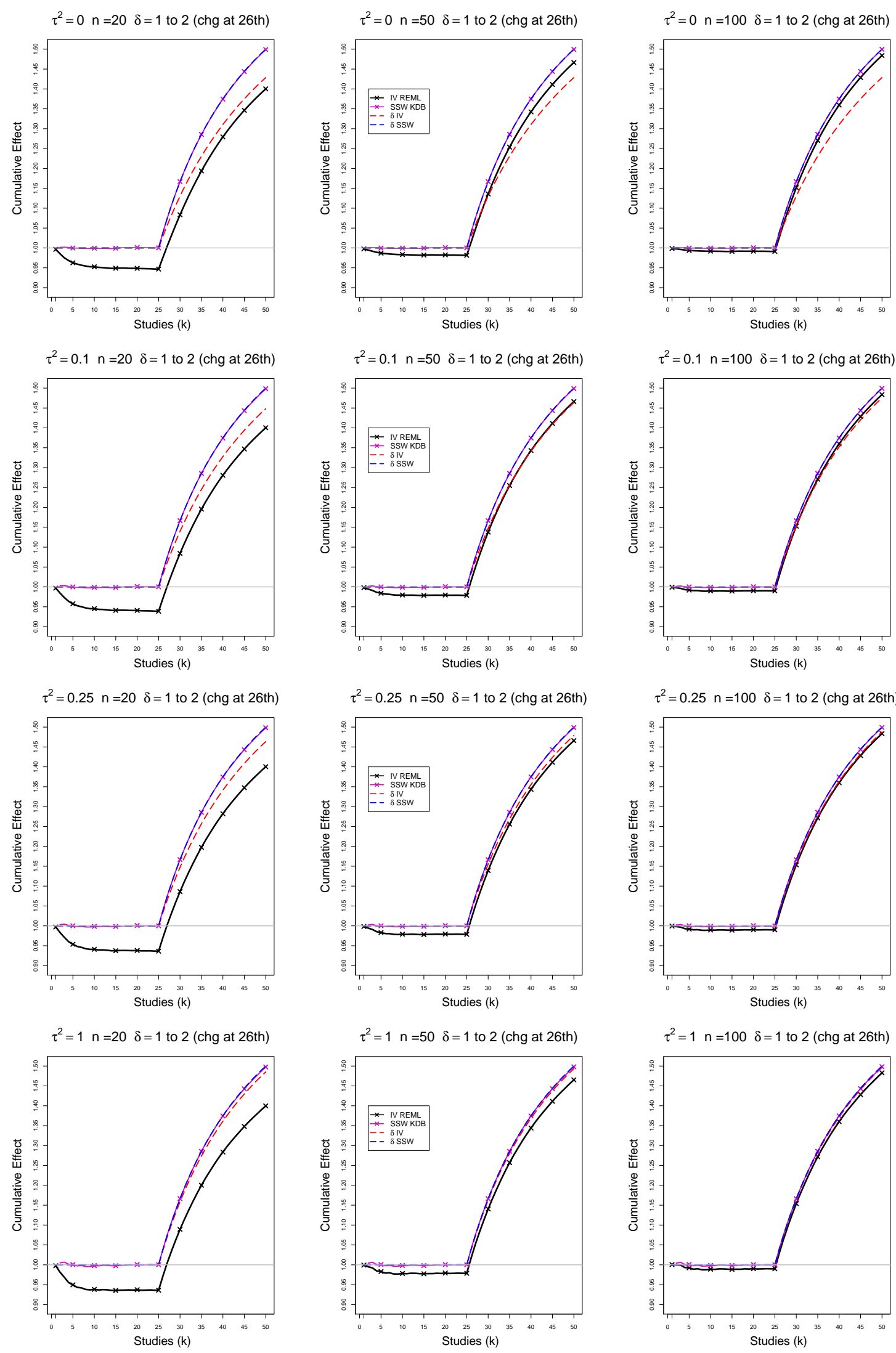

Figure C1. 3: CMA: Nominal cumulative effects $\delta_{S S W,(k)}$ and $\delta_{I V,(k)}$ (dashed lines) and estimated by SSW and IV REML cumulative effects $\hat{\delta}_{(k)}$ (solid lines) when $\delta=1$ for $k \leq 25$ followed by a shift to $\delta=2$ for $k \geq 26, n=20,50,100, K=50$ and $\tau^{2}=0,0.1,0.25,1$. Light grey line at 1. 

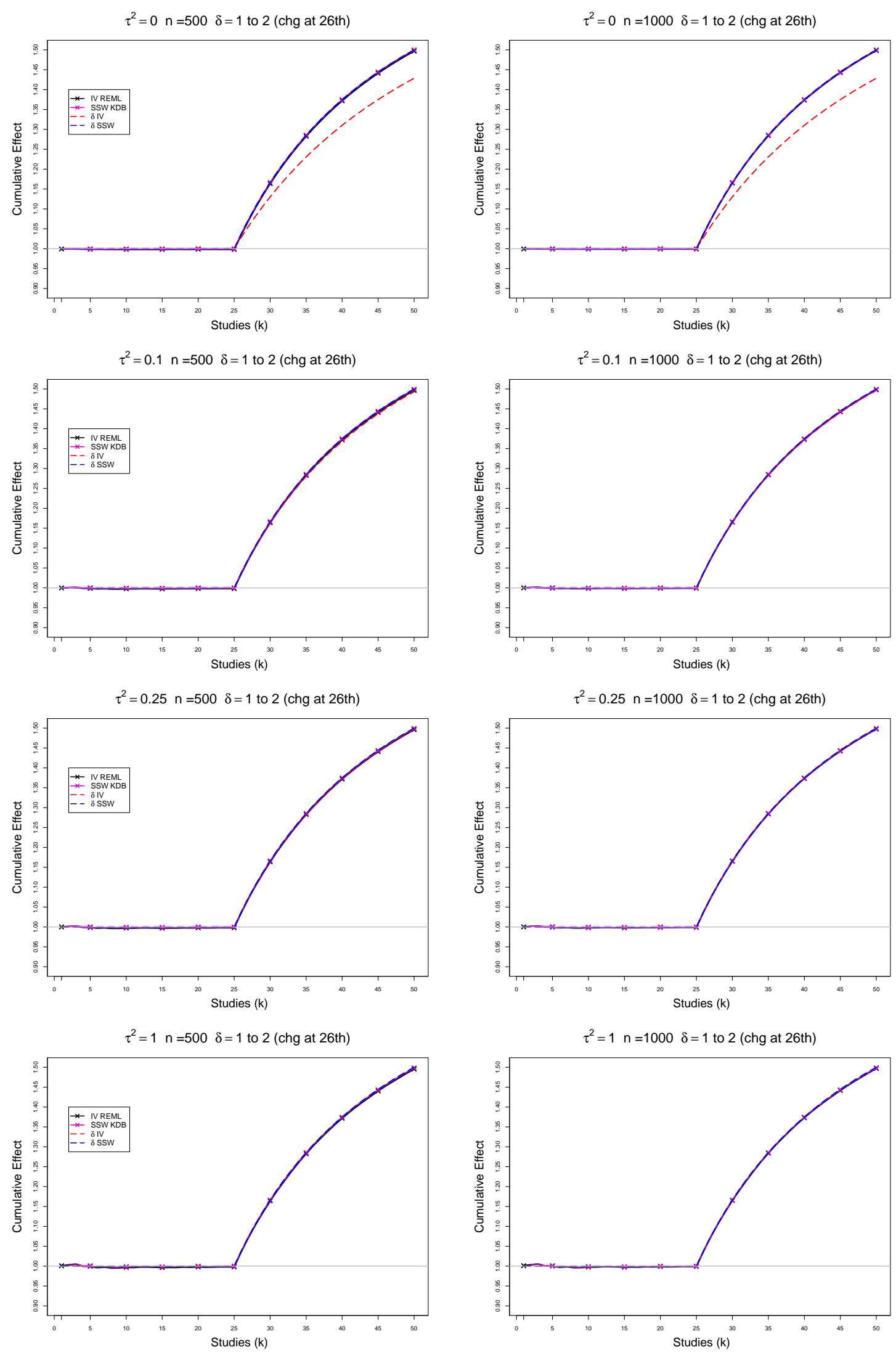

Figure C1. 4: CMA: Nominal cumulative effects $\delta_{S S W,(k)}$ and $\delta_{I V,(k)}$ (dashed lines) and estimated by SSW and IV REML cumulative effects $\hat{\delta}_{(k)}$ (solid lines) when $\delta=1$ for $k \leq 25$ followed by a shift to $\delta=2$ for $k \geq 26, n=500,1000, K=50$ and $\tau^{2}=0,0.1,0.25,1$. Light grey line at 1. 

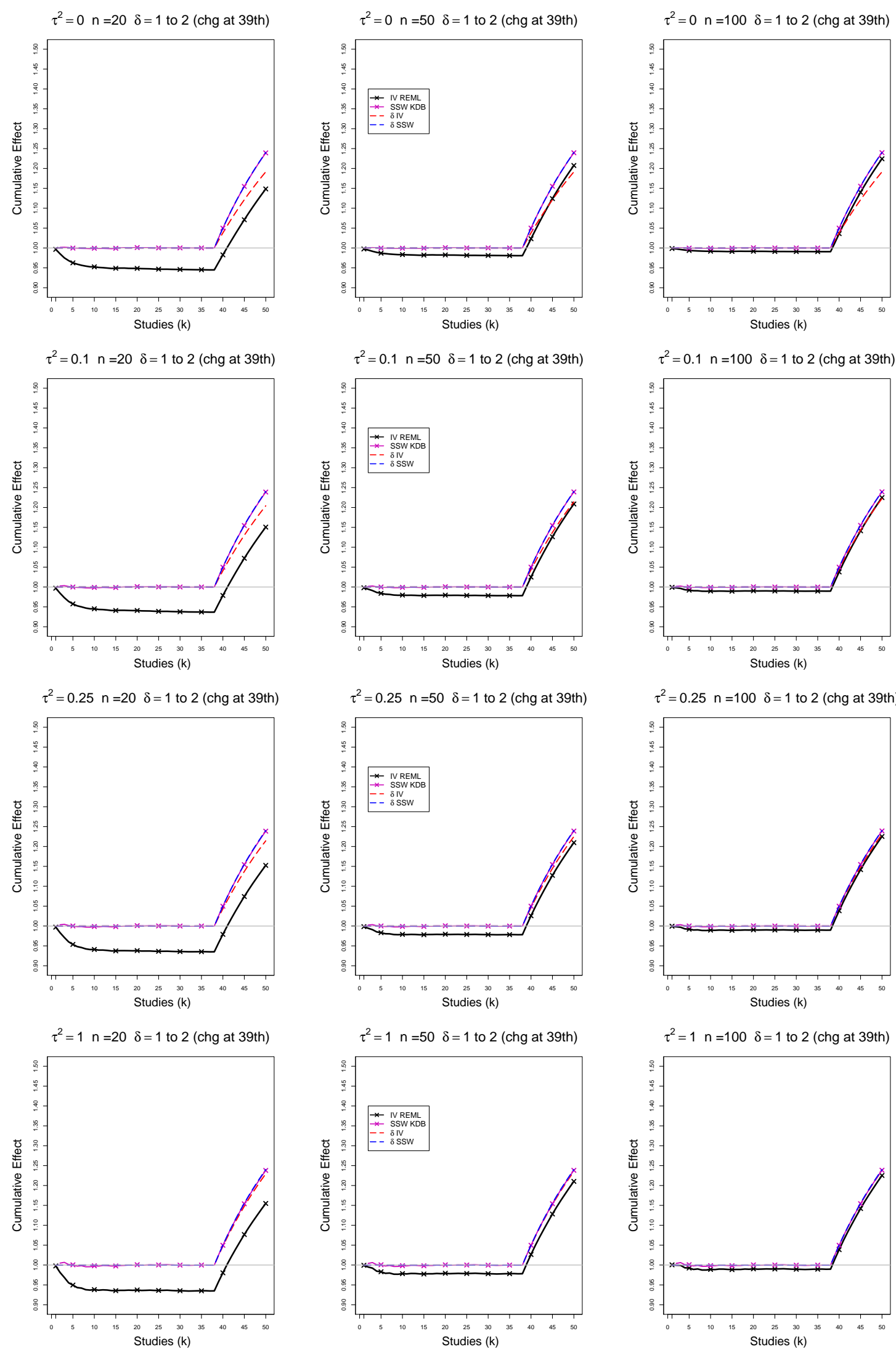

Figure C1. 5: CMA: Nominal cumulative effects $\delta_{S S W,(k)}$ and $\delta_{I V,(k)}$ (dashed lines) and estimated by SSW and IV REML cumulative effects $\hat{\delta}_{(k)}$ (solid lines) when $\delta=1$ for $k \leq 38$ followed by a shift to $\delta=2$ for $k \geq 39, n=20,50,100, K=50$ and $\tau^{2}=0,0.1,0.25,1$. Light grey line at 1. 

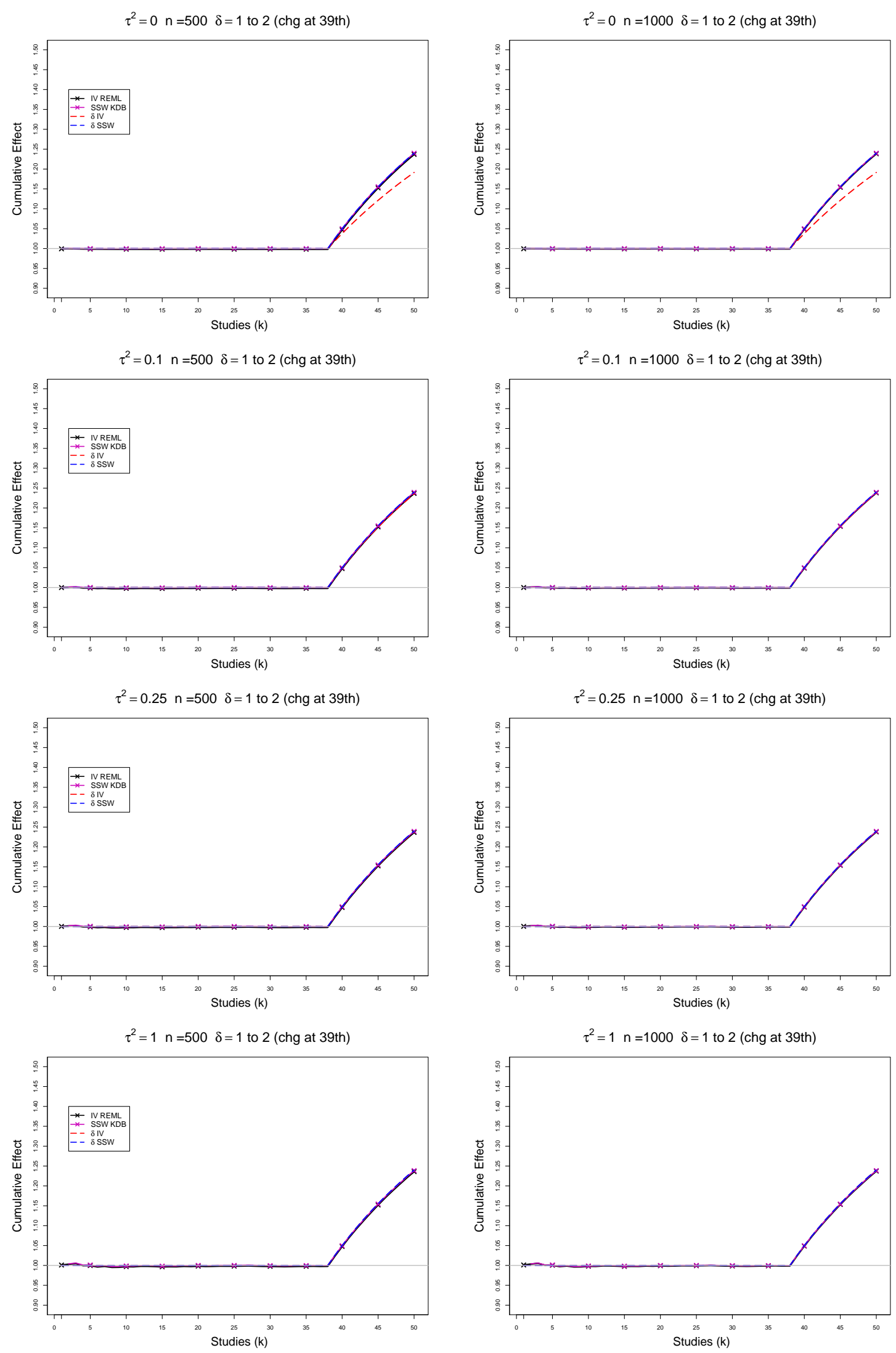

Figure C1. 6: CMA: Nominal cumulative effects $\delta_{S S W,(k)}$ and $\delta_{I V,(k)}$ (dashed lines) and estimated by SSW and IV REML cumulative effects $\hat{\delta}_{(k)}$ (solid lines) when $\delta=1$ for $k \leq 38$ followed by a shift to $\delta=2$ for $k \geq 39, n=500,1000, K=50$ and $\tau^{2}=0,0.1,0.25,1$. Light grey line at 1. 


\section{C2. Bias of point estimators of $\delta$}

For bias of $\delta$ in this section, each figure corresponds to a set of values of $\tau^{2}(=0,0.1$, $0.25,1)$, a set of values of $n(=20,50,100$ or 500,1000$)$ and a value of point of shift $f(=13,26,39)$ while $\delta$ shifts from 1 to 2 and maximum number of studies $K=50$.

Each figure contains a panel (with study $k$ on the horizontal axis) for each combination of parameters.

The point estimators of $\delta$ are

- IV REML (Inverse variance weighted mean with the between-study variance $\tau^{2}$ estimated by Restricted maximum likelihood)

- SSW (Effective sample size weighted) 

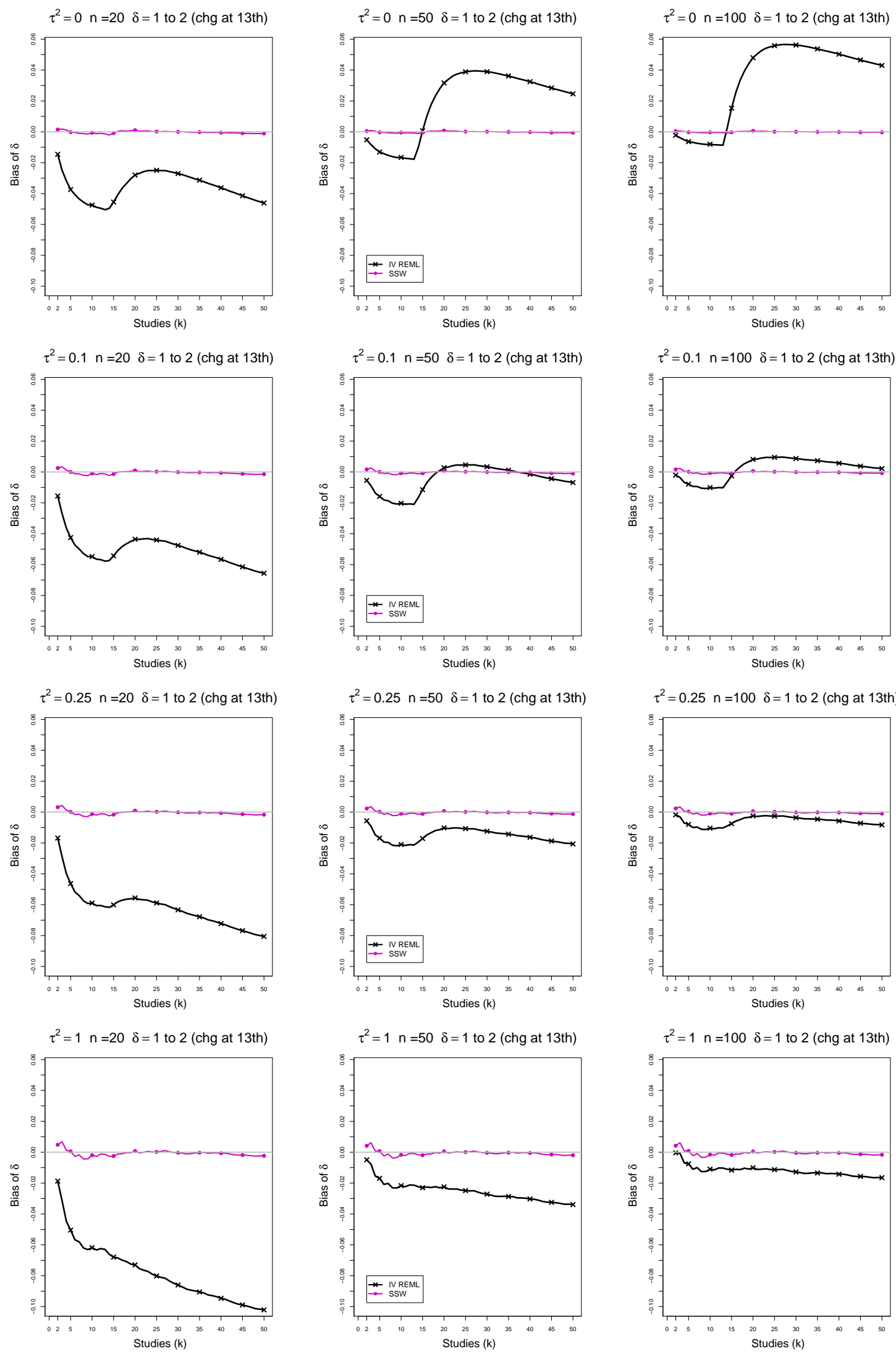

Figure C2. 1: CMA - Bias of inverse-variance (REML) and sample-size-weighted estimator of $\delta$ when $\delta=1$ for $k \leq 12$ followed by a shift to $\delta=2$ for $k \geq 13, \tau^{2}=0,0.1,0.25,1$, $n=20,50,100, K=50$. Light grey line at 0 . 

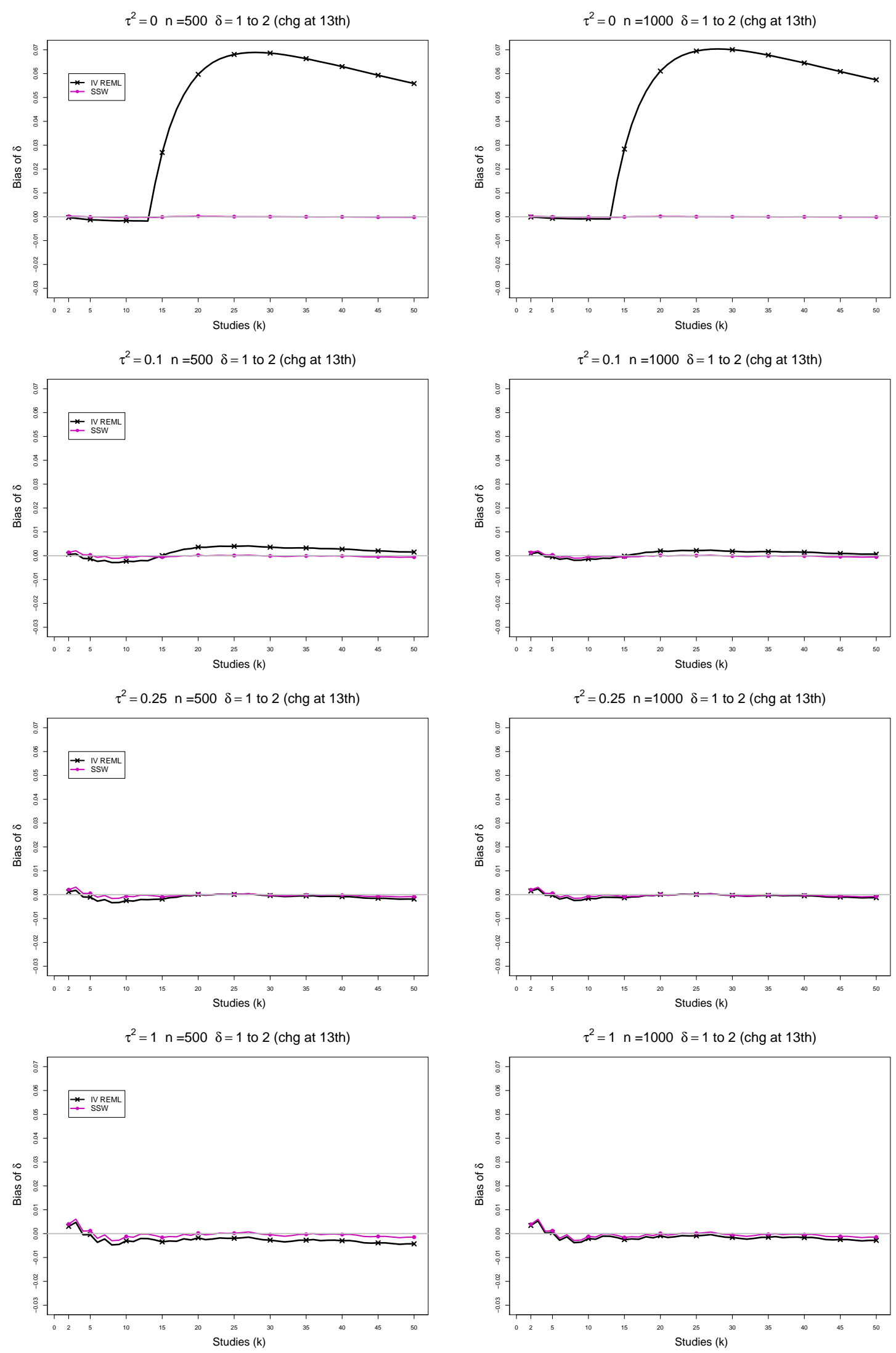

Figure C2. 2: CMA - Bias of inverse-variance (REML) and sample-size-weighted estimator of $\delta$ when $\delta=1$ for $k \leq 12$ followed by a shift to $\delta=2$ for $k \geq 13, \tau^{2}=0,0.1,0.25,1$, $n=500,1000, K=50$. Light grey line at 0 . 

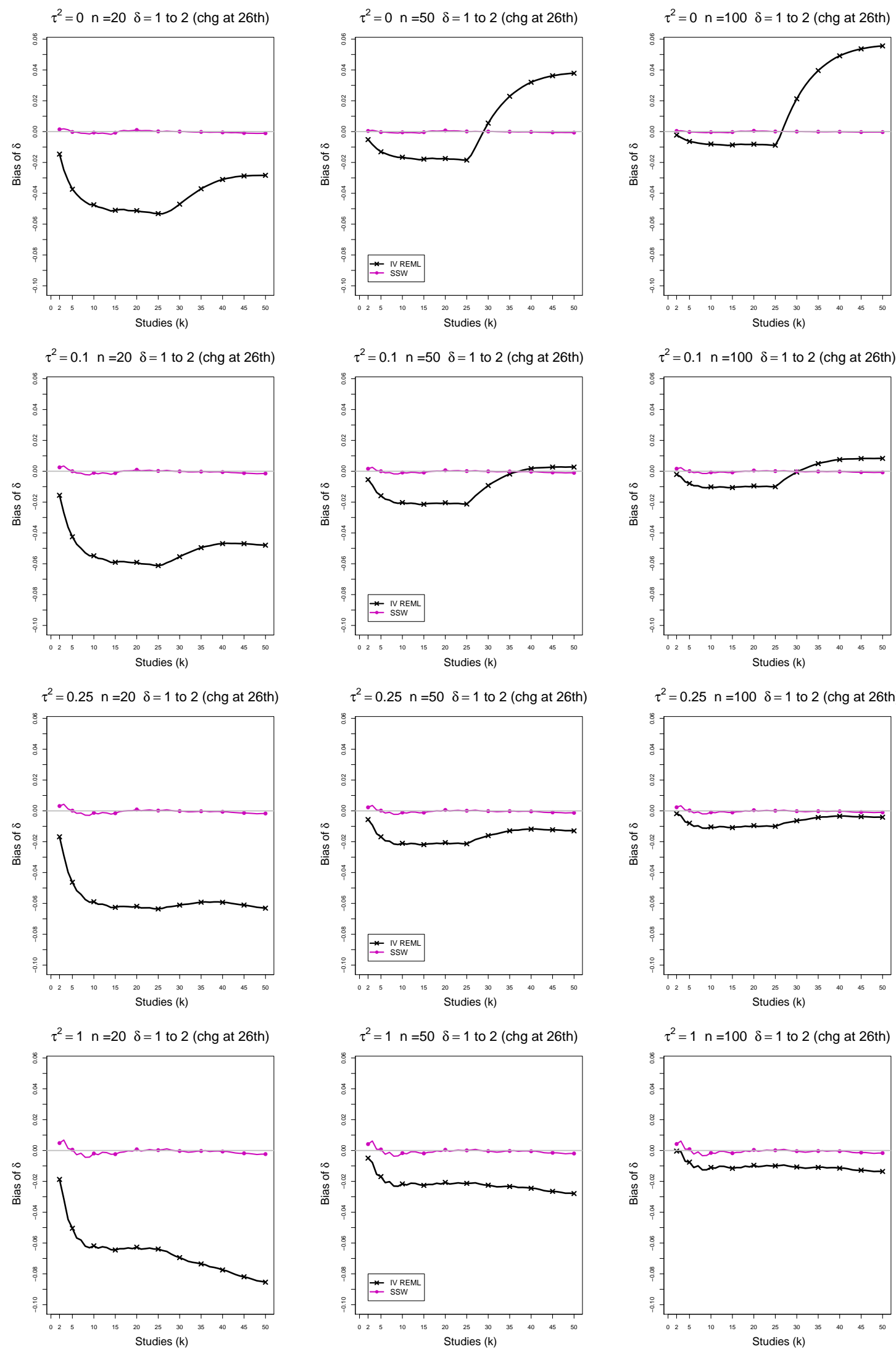

Figure C2. 3: CMA - Bias of inverse-variance (REML) and sample-size-weighted estimator of $\delta$ when $\delta=1$ for $k \leq 25$ followed by a shift to $\delta=2$ for $k \geq 26, \tau^{2}=0,0.1,0.25,1$, $n=20,50,100, K=50$. Light grey line at 0 . 

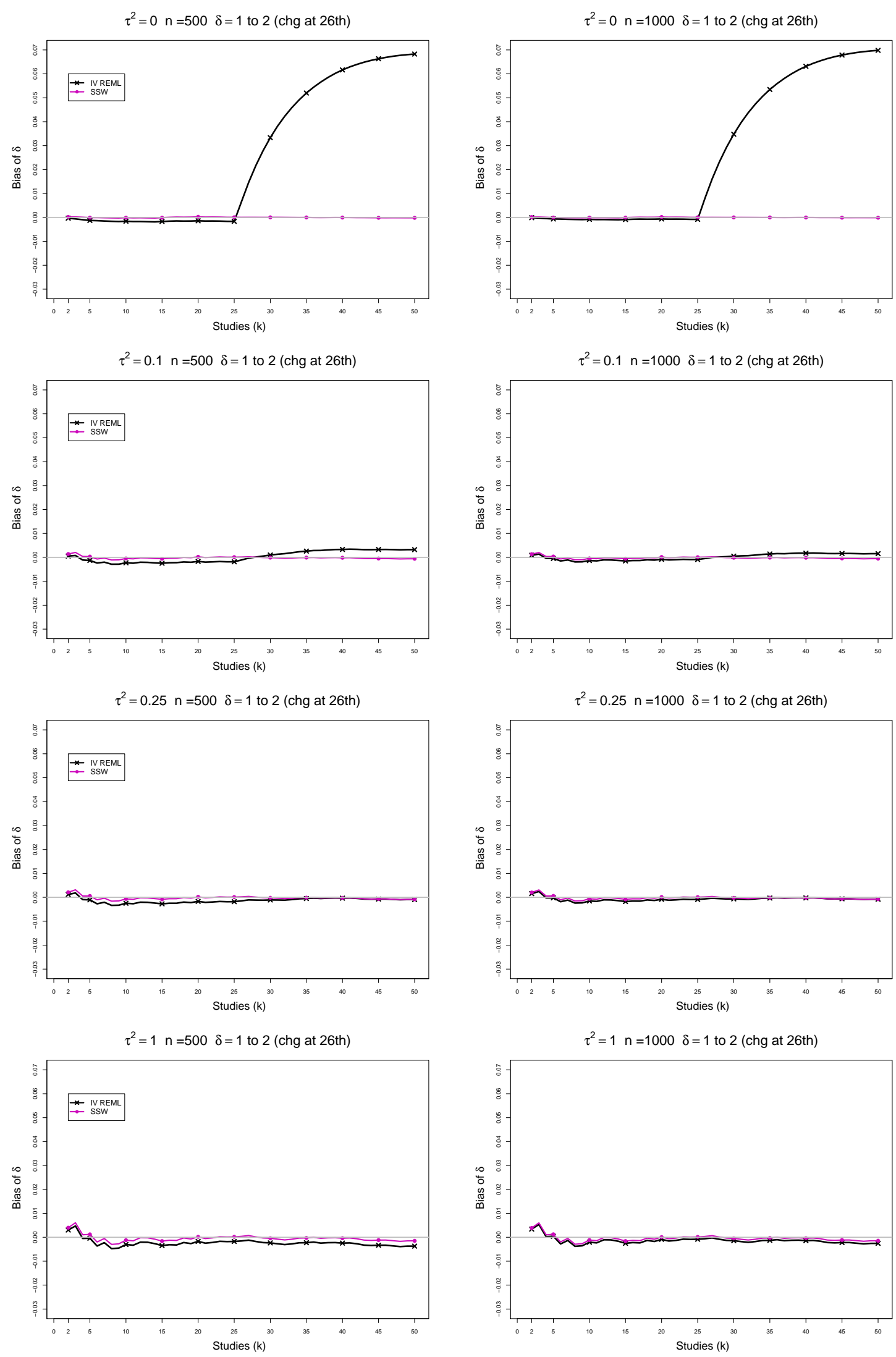

Figure C2. 4: CMA - Bias of inverse-variance (REML) and sample-size-weighted estimator of $\delta$ when $\delta=1$ for $k \leq 25$ followed by a shift to $\delta=2$ for $k \geq 26, \tau^{2}=0,0.1,0.25,1$, $n=500,1000, K=50$. Light grey line at 0 . 

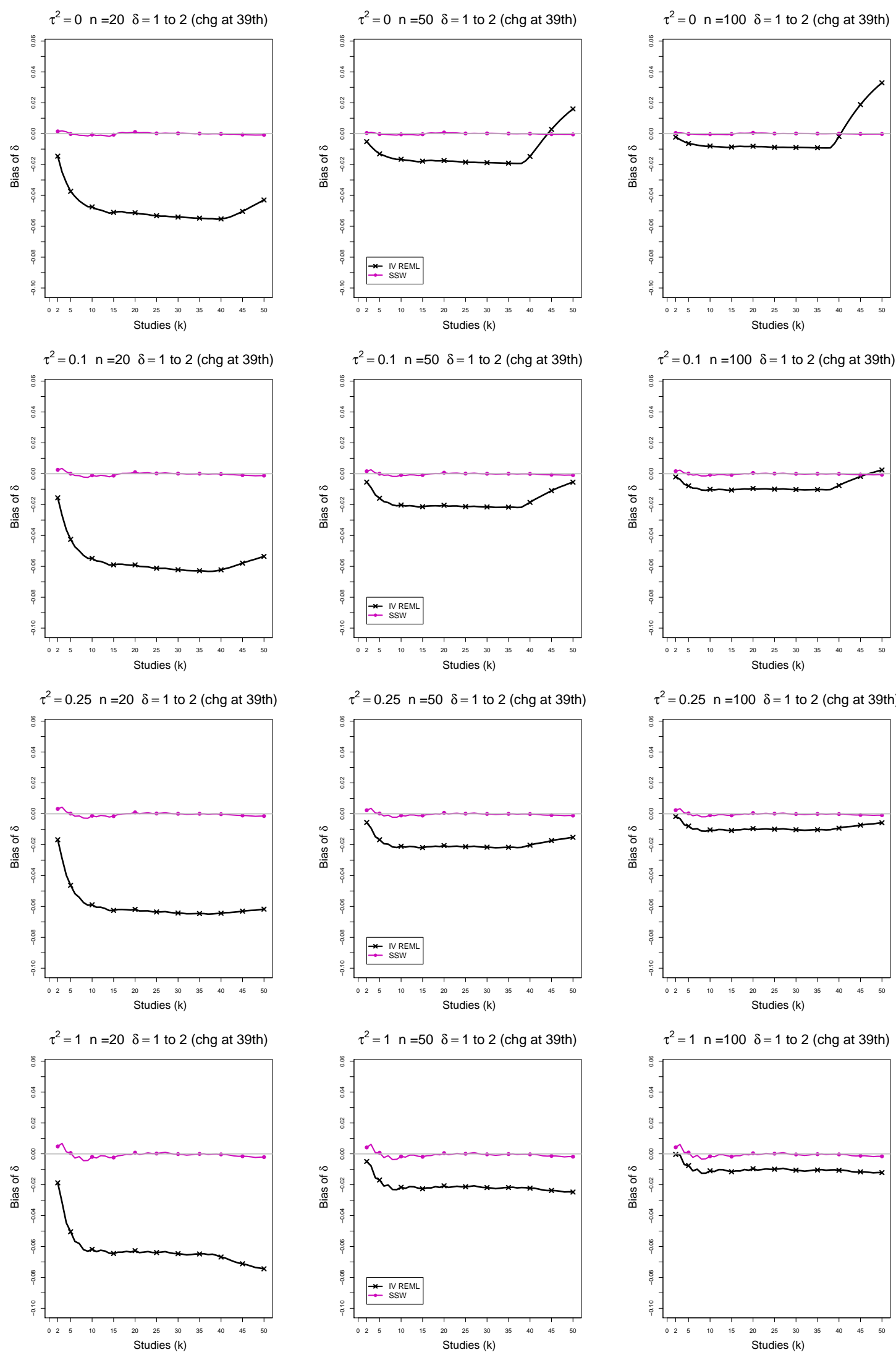

Figure C2. 5: CMA - Bias of inverse-variance (REML) and sample-size-weighted estimator of $\delta$ when $\delta=1$ for $k \leq 38$ followed by a shift to $\delta=2$ for $k \geq 39, \tau^{2}=0,0.1,0.25,1$, $n=20,50,100, K=50$. Light grey line at 0 . 

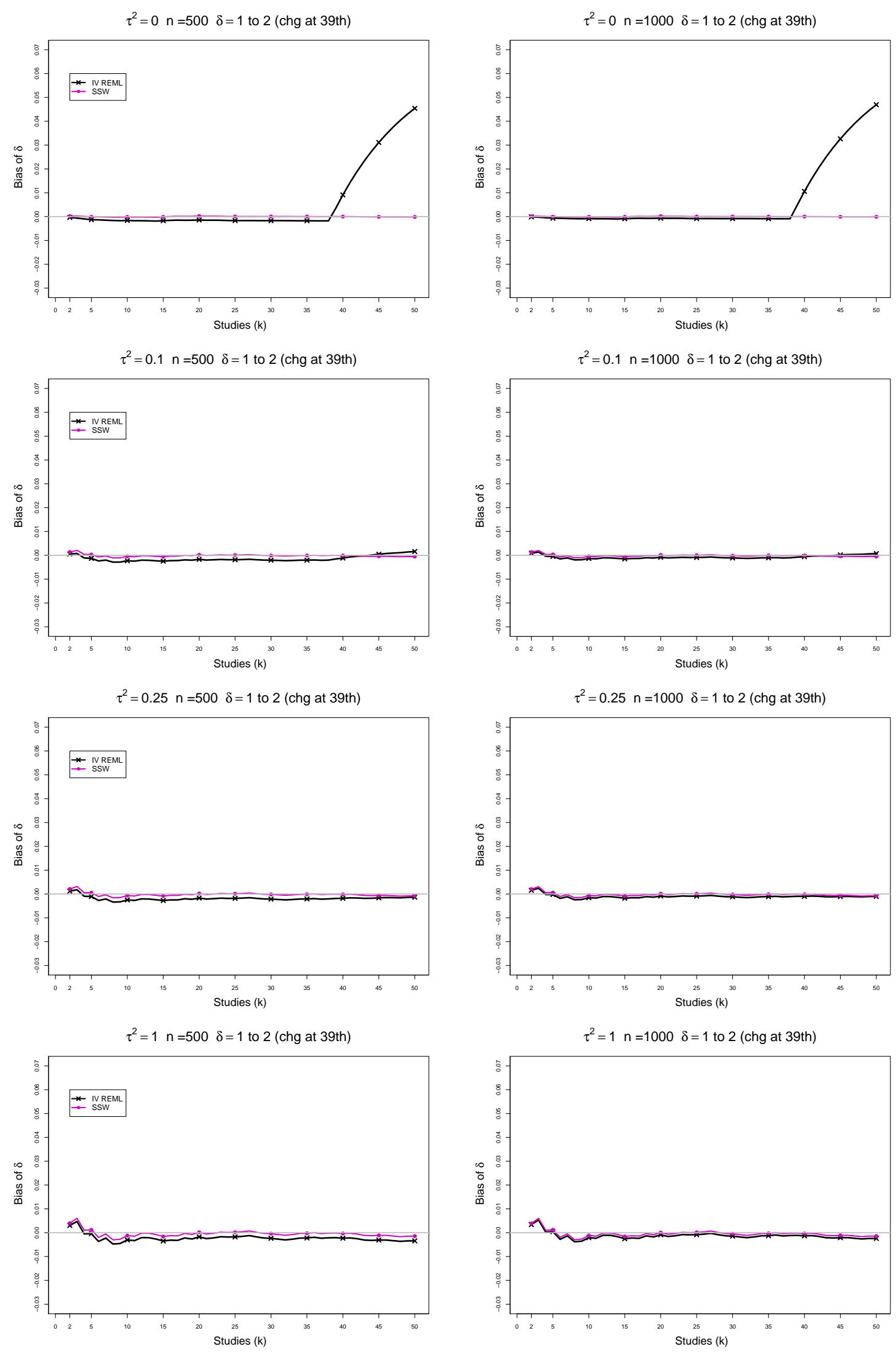

Figure C2. 6: CMA - Bias of inverse-variance (REML) and sample-size-weighted estimator of $\delta$ when $\delta=1$ for $k \leq 38$ followed by a shift to $\delta=2$ for $k \geq 39, \tau^{2}=0,0.1,0.25,1$, $n=500,1000, K=50$. Light grey line at 0 . 


\section{C3. Coverage of interval estimators of $\delta$}

For coverage of $\delta$ in this section, each figure corresponds to a value of a set of values of $\tau^{2}(=0,0.1,0.25,1)$, a value of $n(=20,50,100,500,1000)$, a set of values of significance level $(=0.05,0.01,0.005)$ and a value of point of shift $f(=13,26,39)$ while $\delta$ shifts from 1 to 2 and maximum number of studies $K=50$.

Each figure contains a panel (with study $k$ on the horizontal axis) for each combination of parameters.

The interval estimators of $\delta$ are

- IV REML (Inverse variance weighted mean with the between-study variance $\tau^{2}$ estimated by REML as center and normal critical values)

- SSW KDB (Sample size weighted (SSW) as center and half-width equal to critical value from $t_{k-1}$ times estimated standard deviation of SSW with $\hat{\tau}^{2}=$ $\hat{\tau}_{K D B}^{2}$, the Kulinskaya-Dollinger-Bjørkestøl (2011) estimator of $\left.\tau^{2}\right)$ 

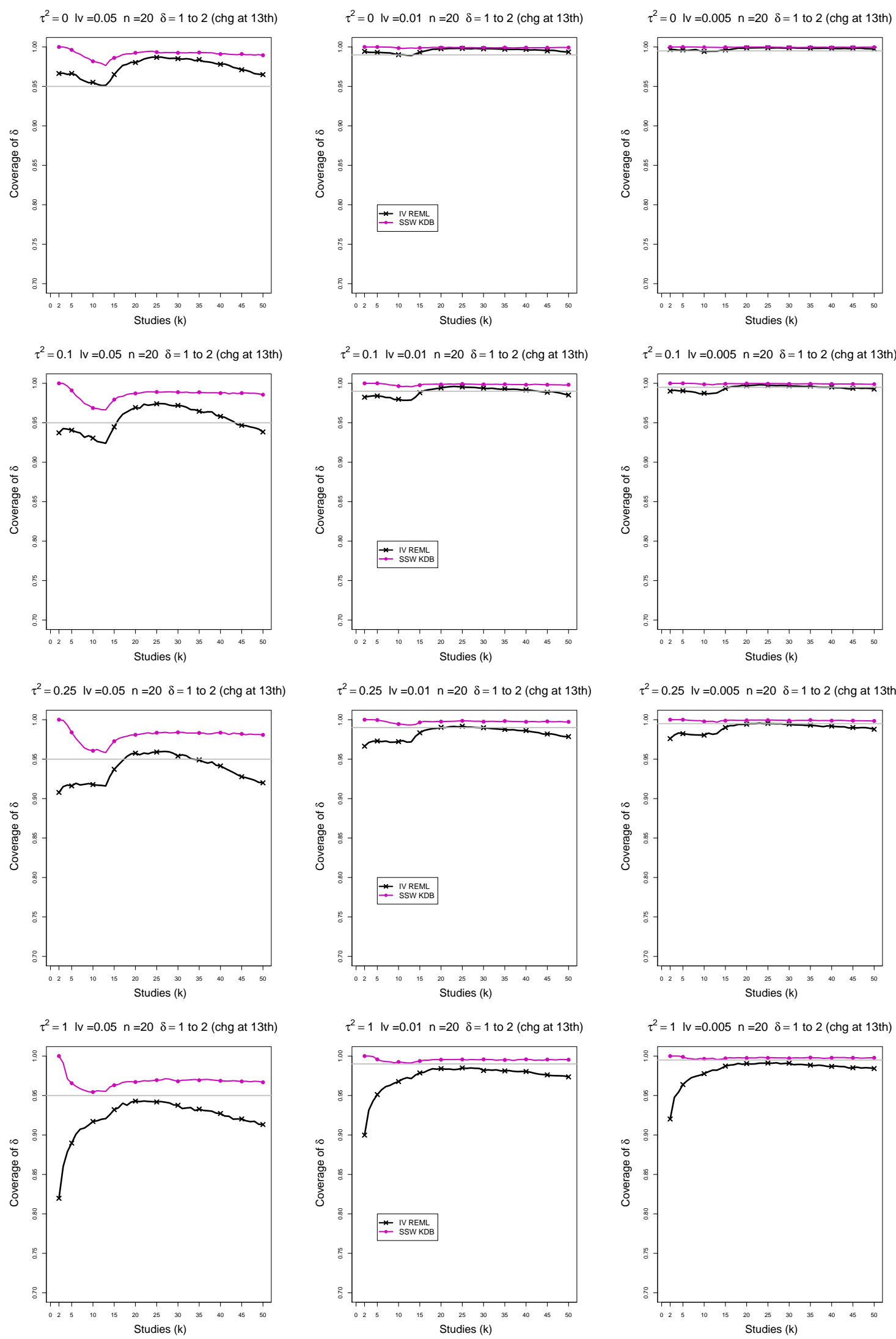

Figure C3. 1: CMA - Coverage of interval estimators of $\delta$ at confidence level of $1-\alpha$ for $\alpha=0.05,0.01,0.005$ when $\delta=1$ for $k \leq 12$ followed by a shift to $\delta=2$ for $k \geq 13$, $\tau^{2}=0,0.1,0.25,1, n=20, K=50$. Light grey line at $0.95,0.99,0.995$. 

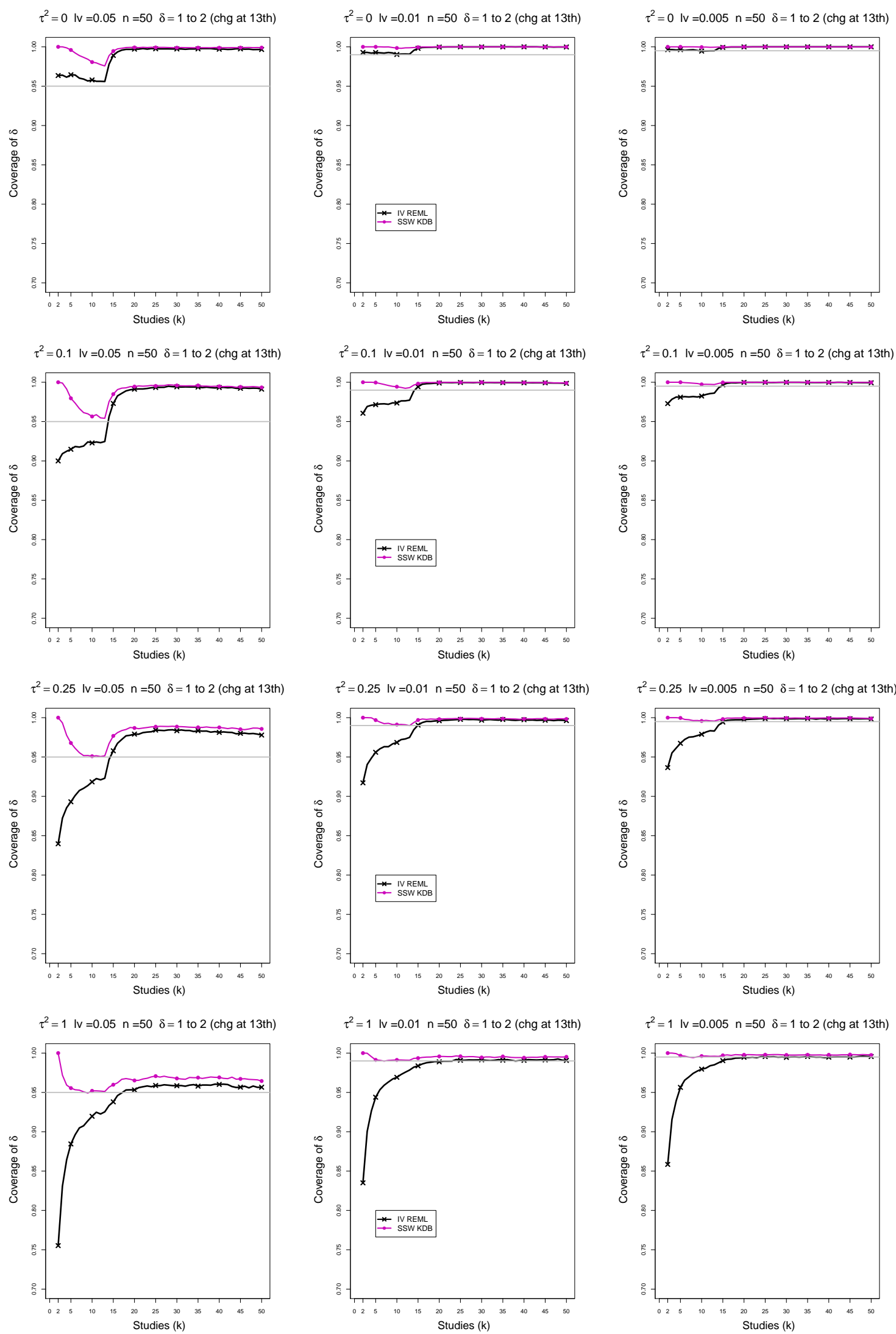

Figure C3. 2: CMA - Coverage of interval estimators of $\delta$ at confidence level of $1-\alpha$ for $\alpha=0.05,0.01,0.005$ when $\delta=1$ for $k \leq 12$ followed by a shift to $\delta=2$ for $k \geq 13$, $\tau^{2}=0,0.1,0.25,1, n=50, K=50$. Light grey line at $0.95,0.99,0.995$. 

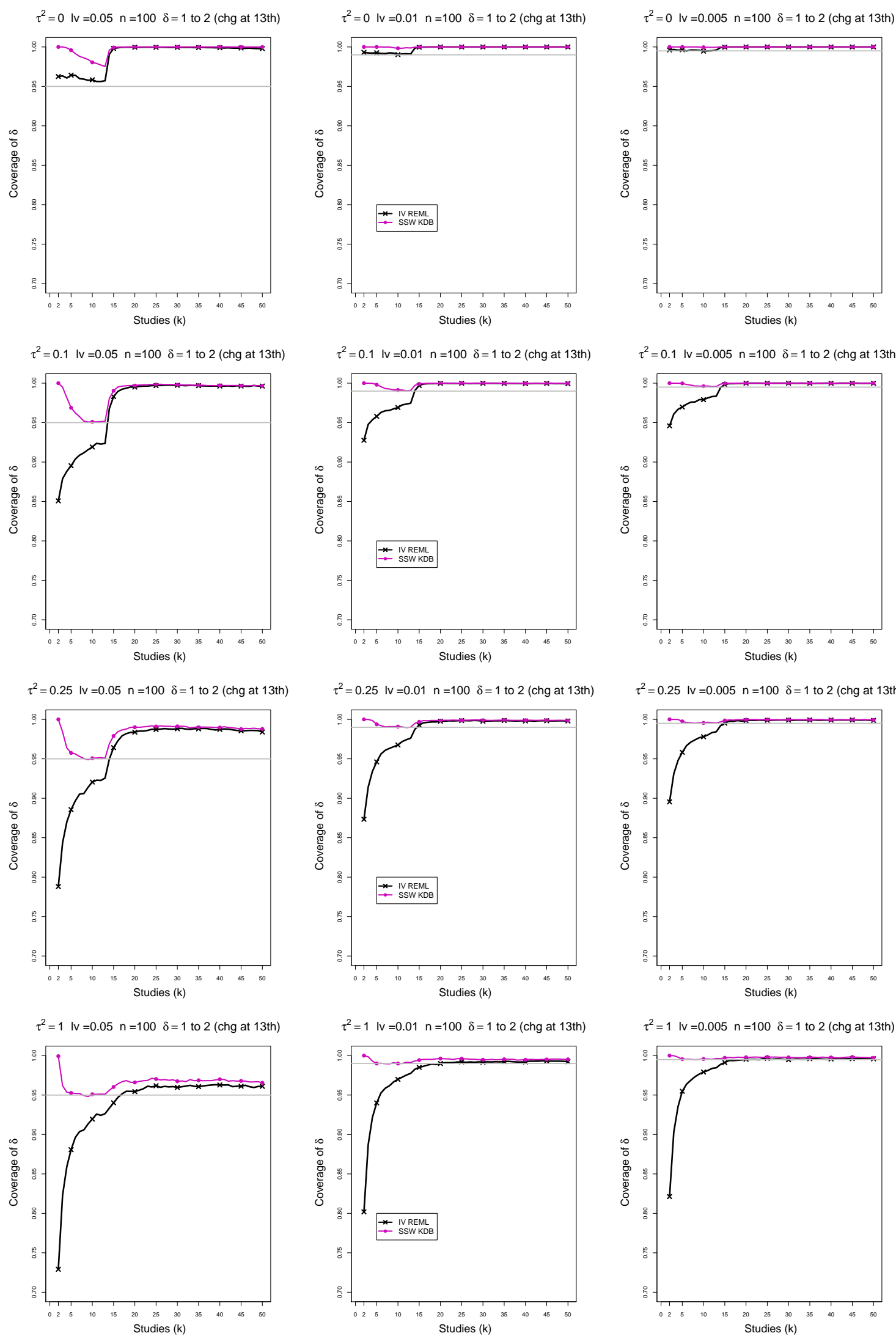

Figure C3. 3: CMA - Coverage of interval estimators of $\delta$ at confidence level of $1-\alpha$ for $\alpha=0.05,0.01,0.005$ when $\delta=1$ for $k \leq 12$ followed by a shift to $\delta=2$ for $k \geq 13$, $\tau^{2}=0,0.1,0.25,1, n=100, K=50$. Light grey line at $0.95,0.99,0.995$. 

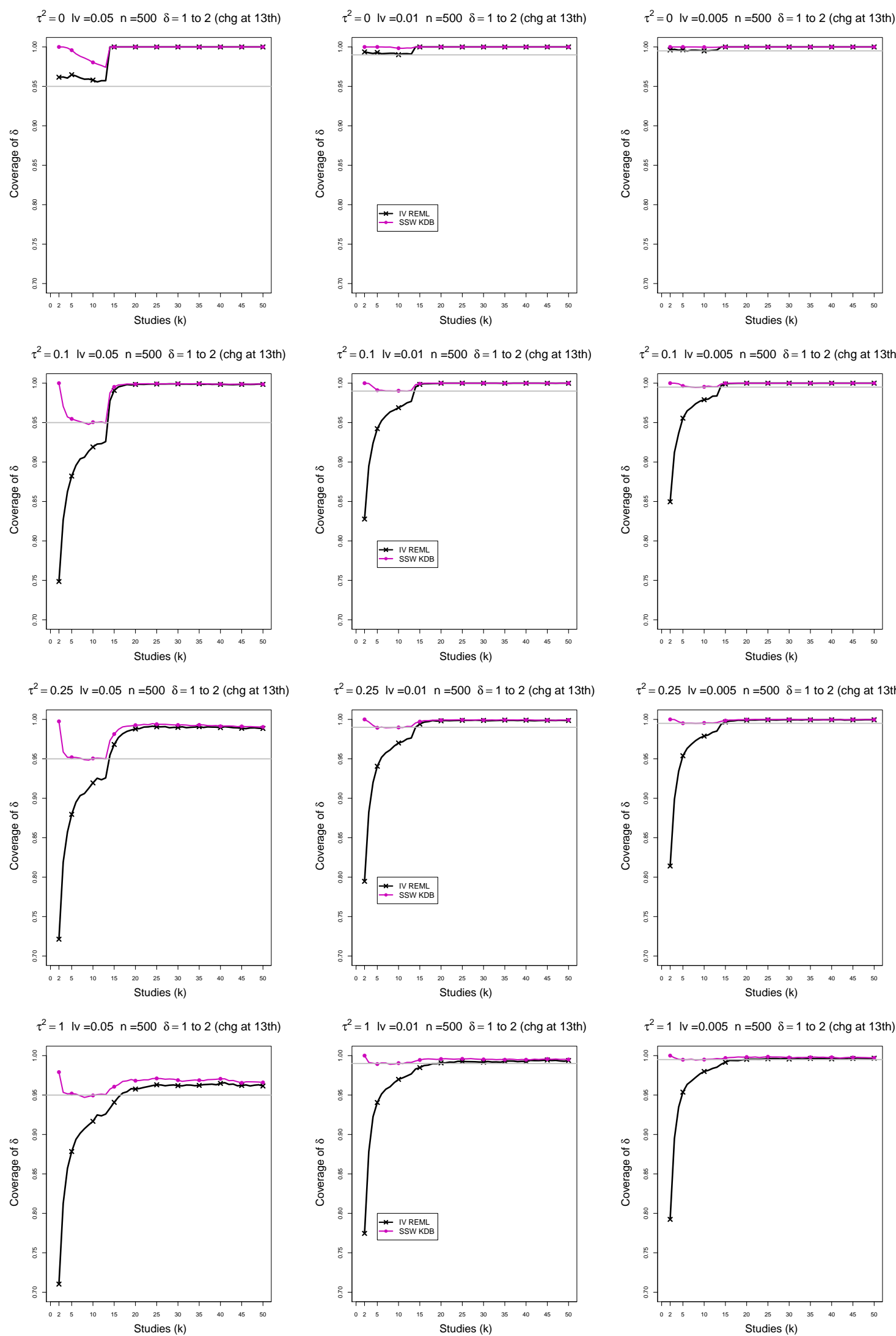

Figure C3. 4: CMA - Coverage of interval estimators of $\delta$ at confidence level of $1-\alpha$ for $\alpha=0.05,0.01,0.005$ when $\delta=1$ for $k \leq 12$ followed by a shift to $\delta=2$ for $k \geq 13$, $\tau^{2}=0,0.1,0.25,1, n=500, K=50$. Light grey line at $0.95,0.99,0.995$. 

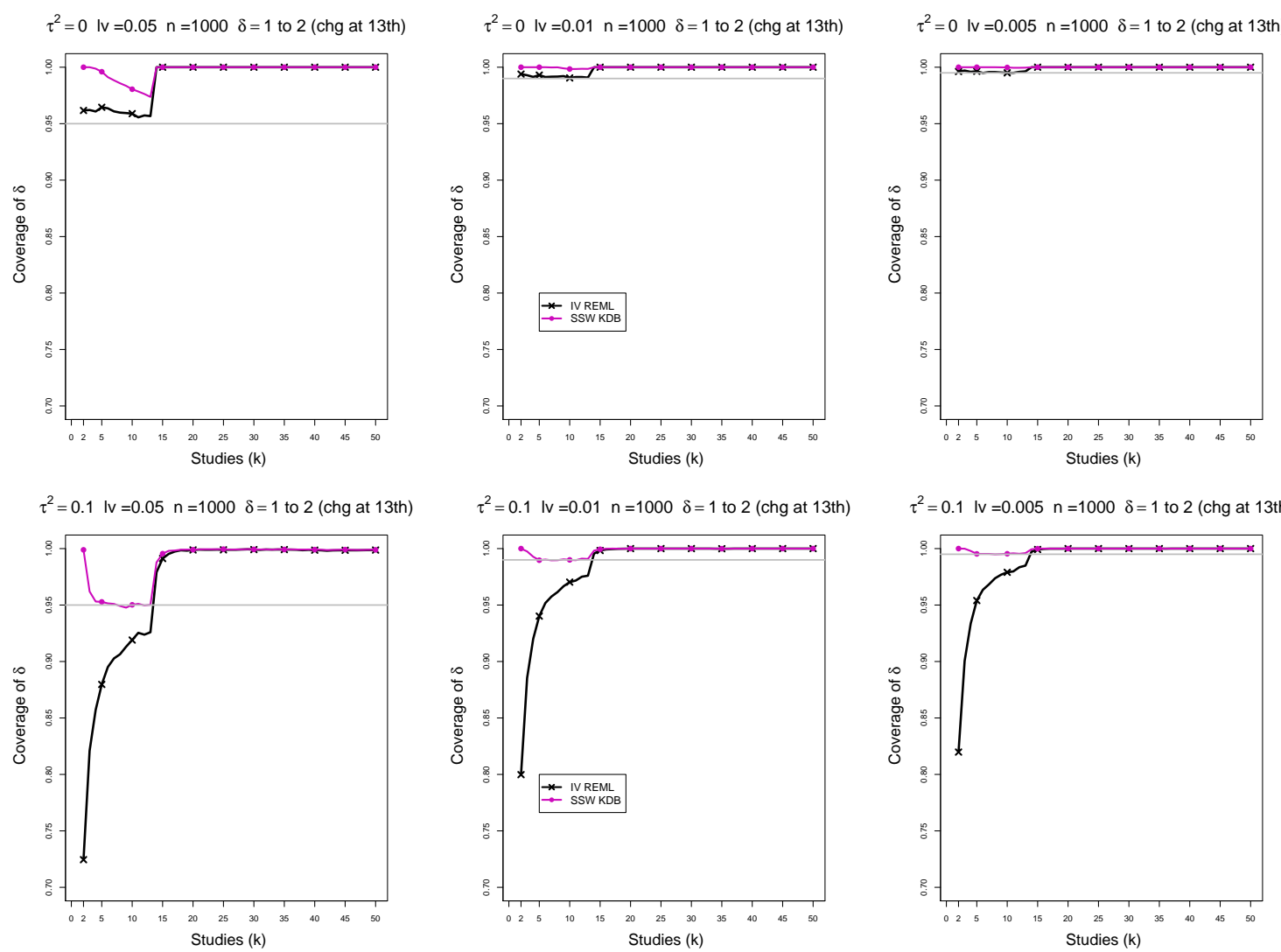

$\tau^{2}=0.1 \mathrm{lv}=0.005 \mathrm{n}=1000 \delta=1$ to 2 (chg at 13 th)

$\tau^{2}=0.25 \mathrm{lv}=0.05 \mathrm{n}=1000 \delta=1$ to 2 (chg at 13 th)

$\tau^{2}=0.25 \mathrm{IV}=0.01 \mathrm{n}=1000 \delta=1$ to 2 (chg at 13 th)
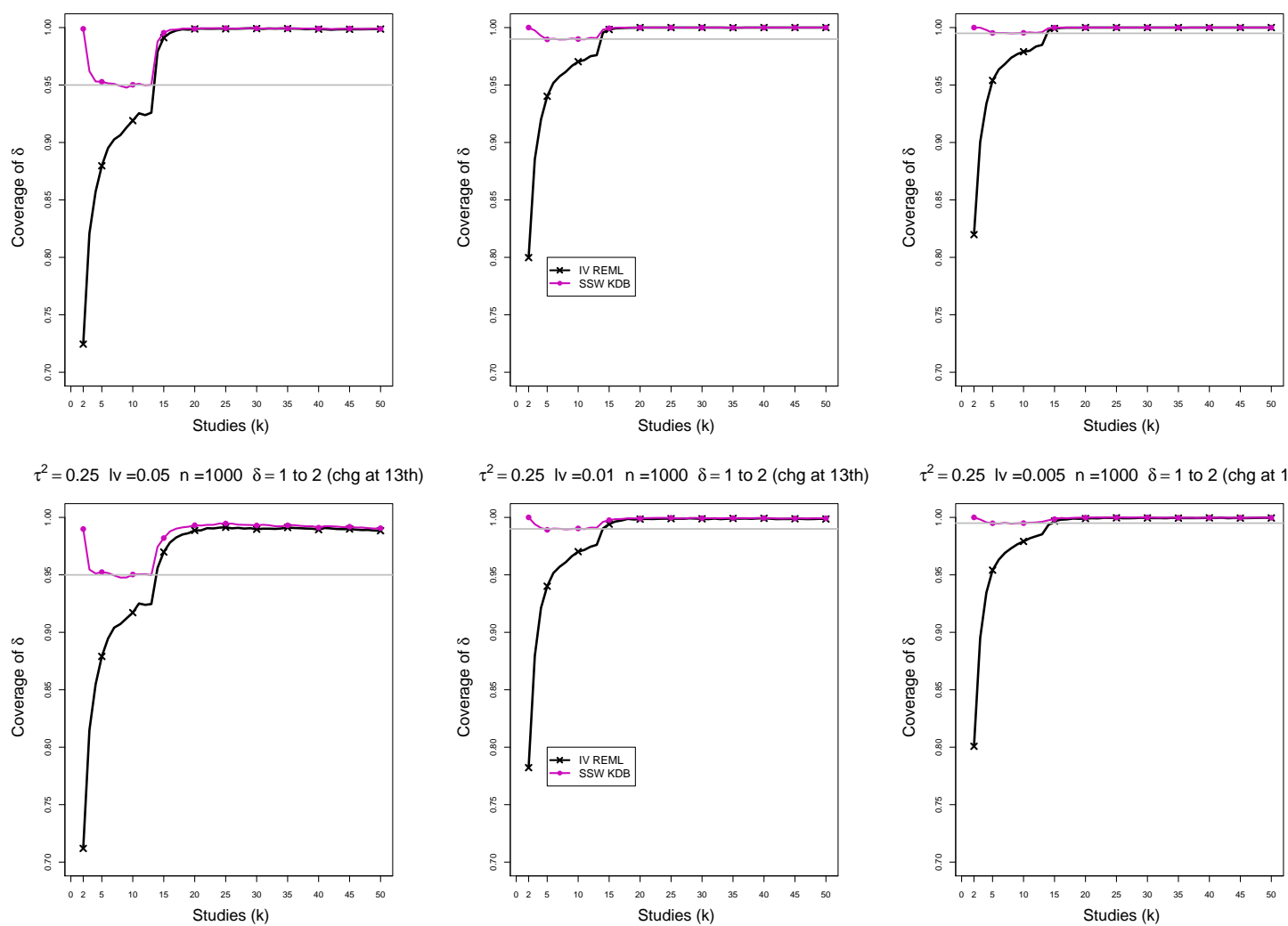

$\tau^{2}=0.25 \mathrm{lv}=0.005 \mathrm{n}=1000 \delta=1$ to 2 (chg at 13 th)

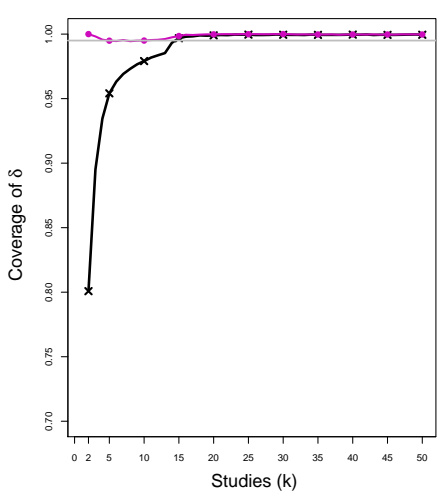

$\tau^{2}=1 \mathrm{IV}=0.05 \mathrm{n}=1000 \delta=1$ to 2 (chg at 13 th)

$\tau^{2}=1 \mathrm{IV}=0.01 \mathrm{n}=1000 \quad \delta=1$ to 2 (chg at 13 th)

$\tau^{2}=1 \mathrm{IV}=0.005 \mathrm{n}=1000 \delta=1$ to 2 (chg at 13 th)
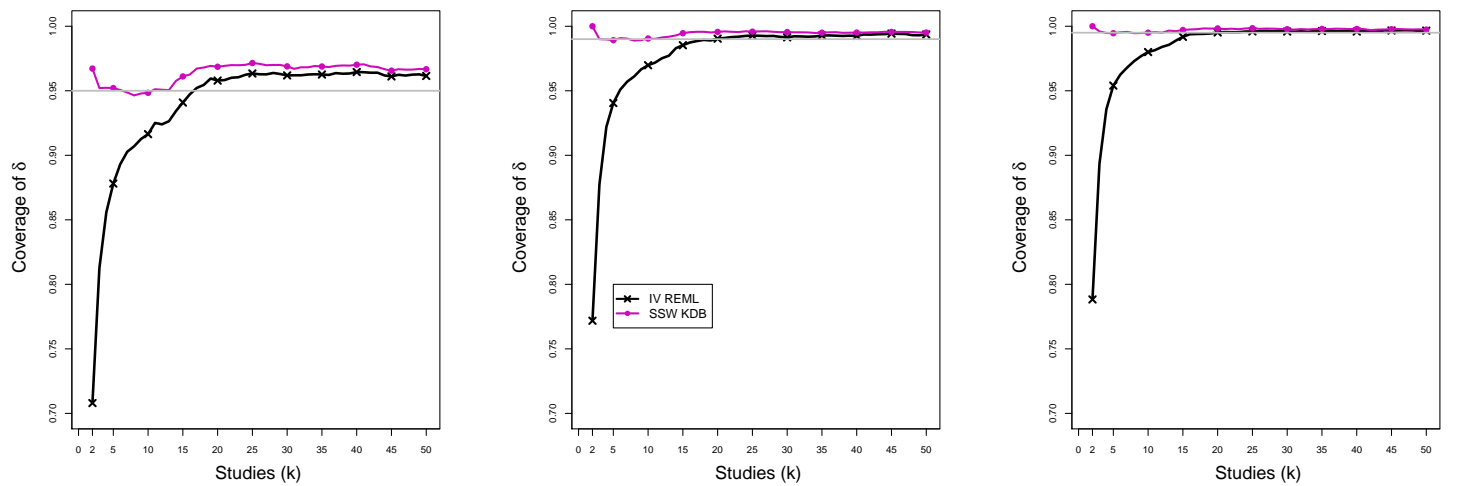

Figure C3. 5: CMA - Coverage of interval estimators of $\delta$ at confidence level of $1-\alpha$ for $\alpha=0.05,0.01,0.005$ when $\delta=1$ for $k \leq 12$ followed by a shift to $\delta=2$ for $k \geq 13$, $\tau^{2}=0,0.1,0.25,1, n=1000, K=50$. Light grey line at $0.95,0.99,0.995$. 

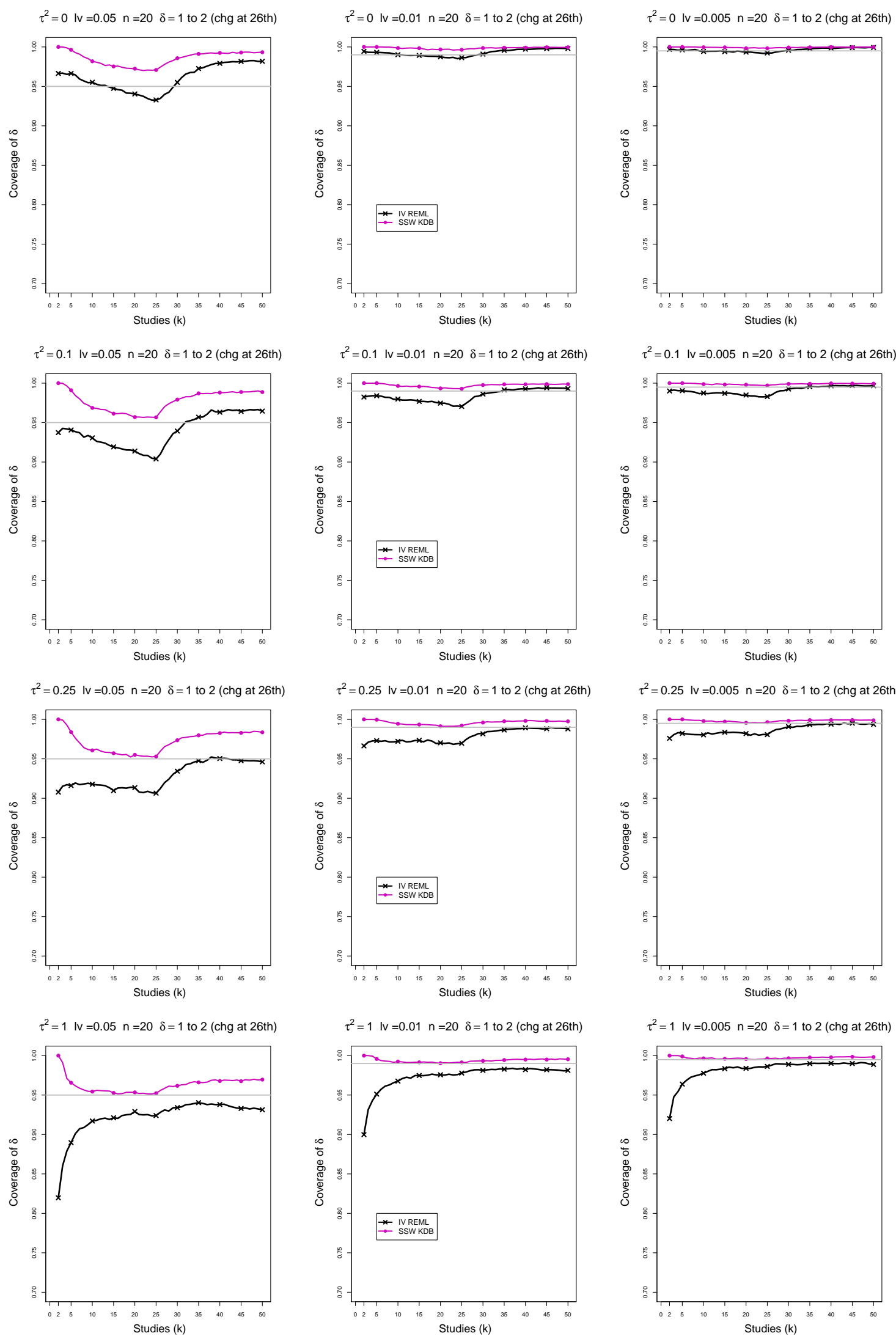

Figure C3. 6: CMA - Coverage of interval estimators of $\delta$ at confidence level of $1-\alpha$ for $\alpha=0.05,0.01,0.005$ when $\delta=1$ for $k \leq 25$ followed by a shift to $\delta=2$ for $k \geq 26$, $\tau^{2}=0,0.1,0.25,1, n=20, K=50$. Light grey line at $0.95,0.99,0.995$. 

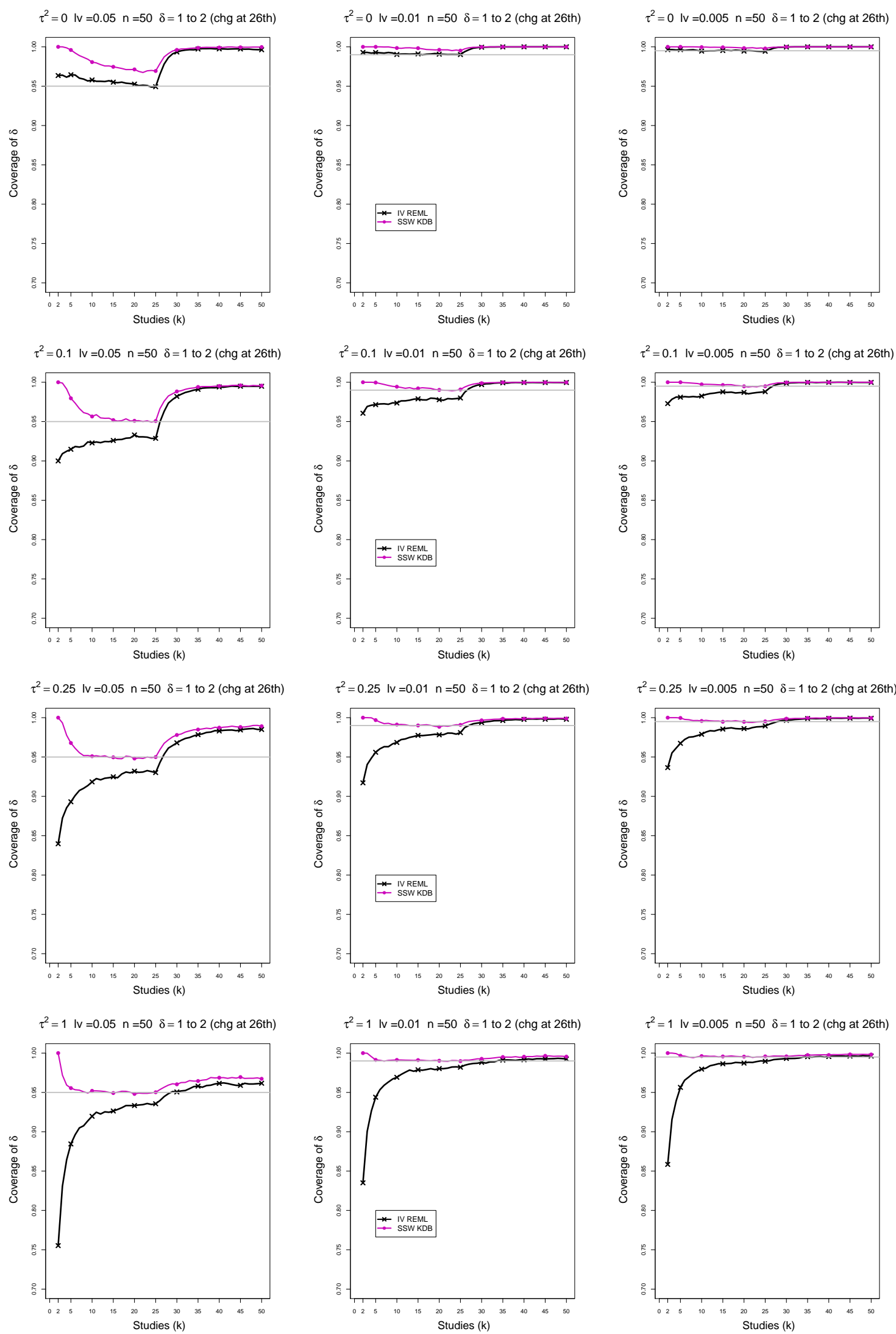

Figure C3. 7: CMA - Coverage of interval estimators of $\delta$ at confidence level of $1-\alpha$ for $\alpha=0.05,0.01,0.005$ when $\delta=1$ for $k \leq 25$ followed by a shift to $\delta=2$ for $k \geq 26$, $\tau^{2}=0,0.1,0.25,1, n=50, K=50$. Light grey line at $0.95,0.99,0.995$. 

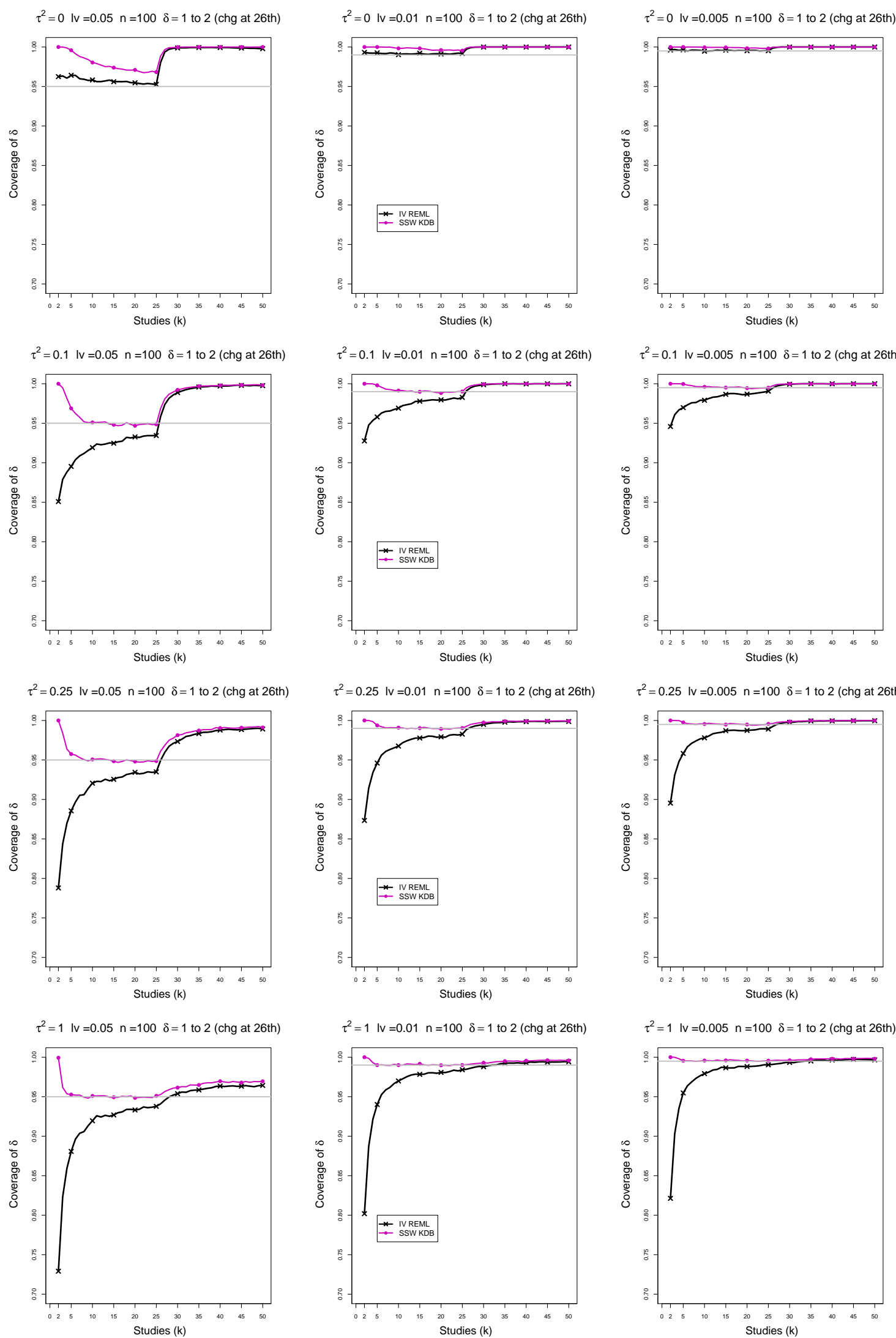

Figure C3. 8: CMA - Coverage of interval estimators of $\delta$ at confidence level of $1-\alpha$ for $\alpha=0.05,0.01,0.005$ when $\delta=1$ for $k \leq 25$ followed by a shift to $\delta=2$ for $k \geq 26$, $\tau^{2}=0,0.1,0.25,1, n=100, K=50$. Light grey line at $0.95,0.99,0.995$. 

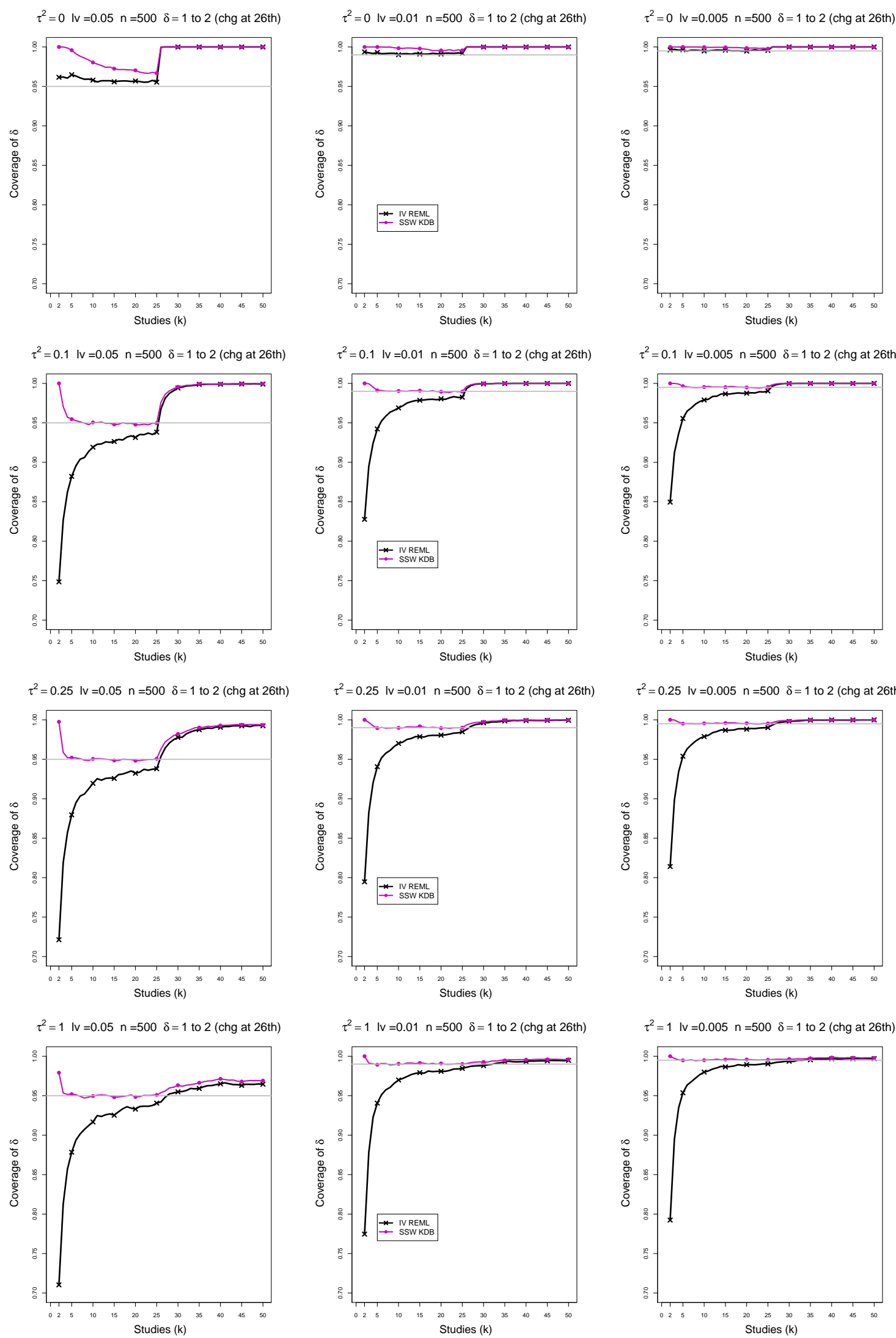

Figure C3. 9: CMA - Coverage of interval estimators of $\delta$ at confidence level of $1-\alpha$ for $\alpha=0.05,0.01,0.005$ when $\delta=1$ for $k \leq 25$ followed by a shift to $\delta=2$ for $k \geq 26$, $\tau^{2}=0,0.1,0.25,1, n=500, K=50$. Light grey line at $0.95,0.99,0.995$. 

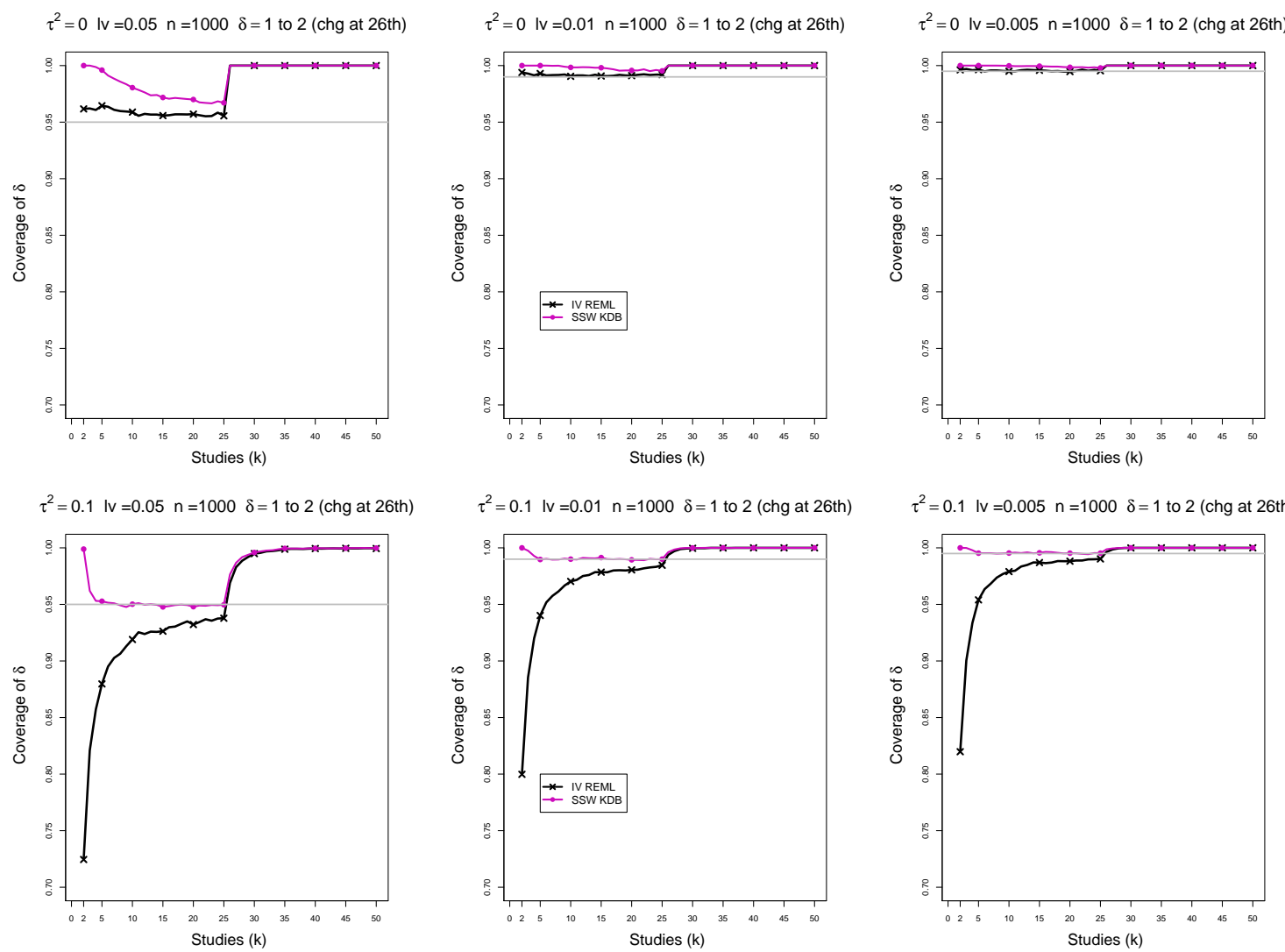

$\tau^{2}=0.1 \mathrm{lv}=0.005 \mathrm{n}=1000 \quad \delta=1$ to 2 (chg at 26th)

$\tau^{2}=0.25 \mathrm{lv}=0.05 \mathrm{n}=1000 \delta=1$ to 2 (chg at 26 th)

$\tau^{2}=0.25 \mathrm{IV}=0.01 \mathrm{n}=1000 \delta=1$ to 2 (chg at 26th)
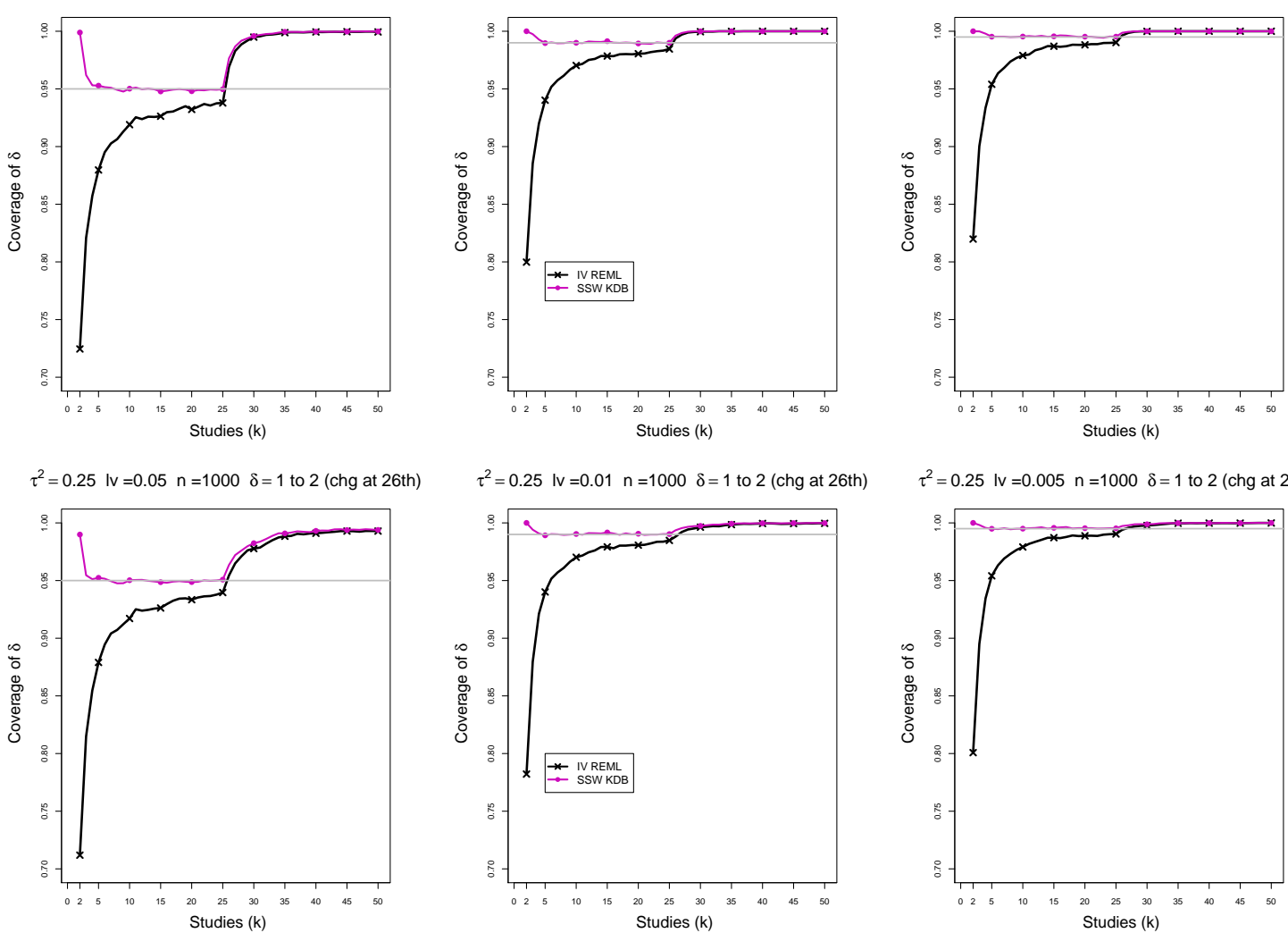

$\tau^{2}=0.25 \mathrm{lv}=0.005 \mathrm{n}=1000 \delta=1$ to 2 (chg at 26 th)
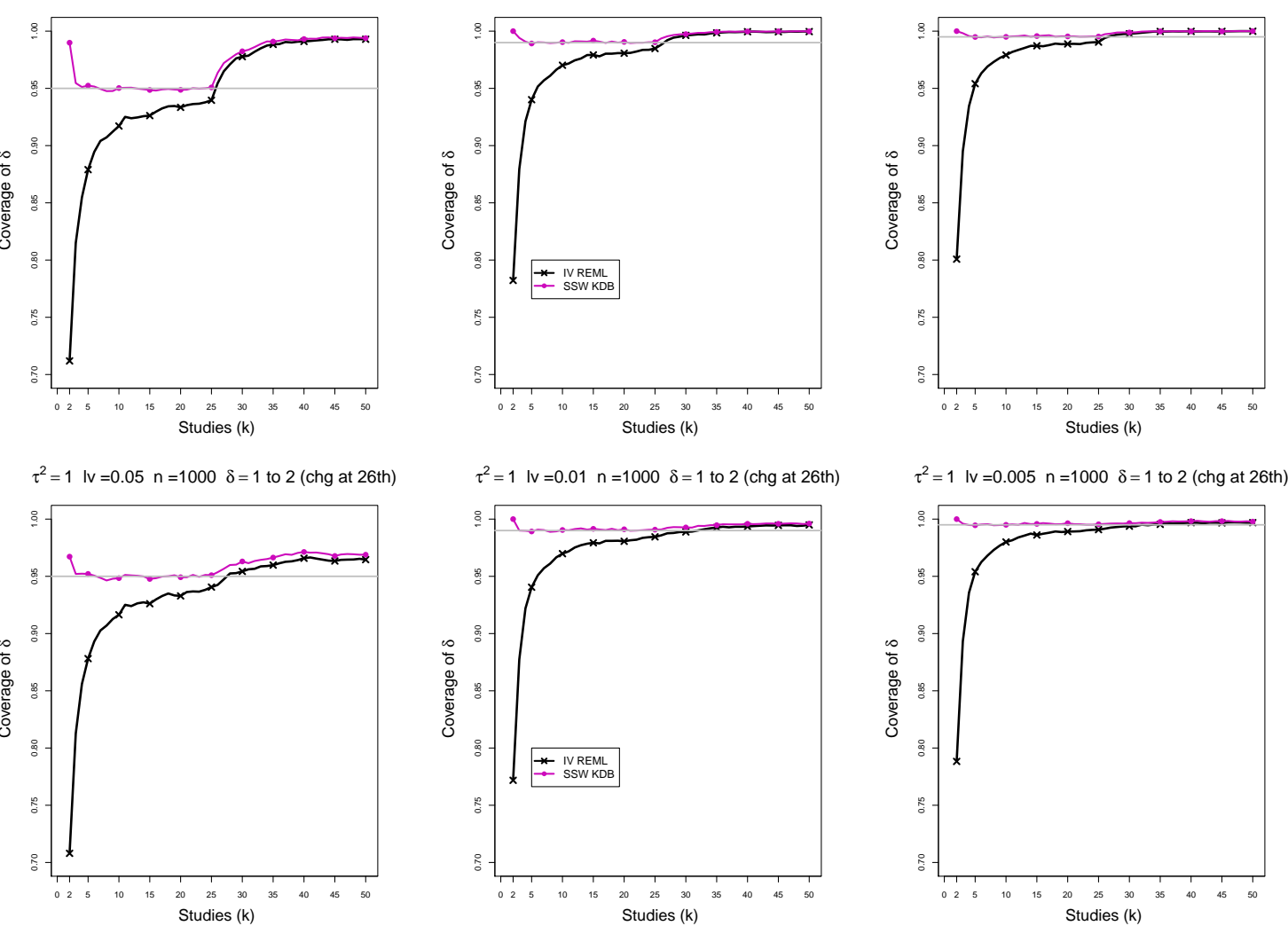

Figure C3. 10: CMA - Coverage of interval estimators of $\delta$ at confidence level of $1-\alpha$ for $\alpha=0.05,0.01,0.005$ when $\delta=1$ for $k \leq 25$ followed by a shift to $\delta=2$ for $k \geq 26$, $\tau^{2}=0,0.1,0.25,1, n=1000, K=50$. Light grey line at $0.95,0.99,0.995$. 

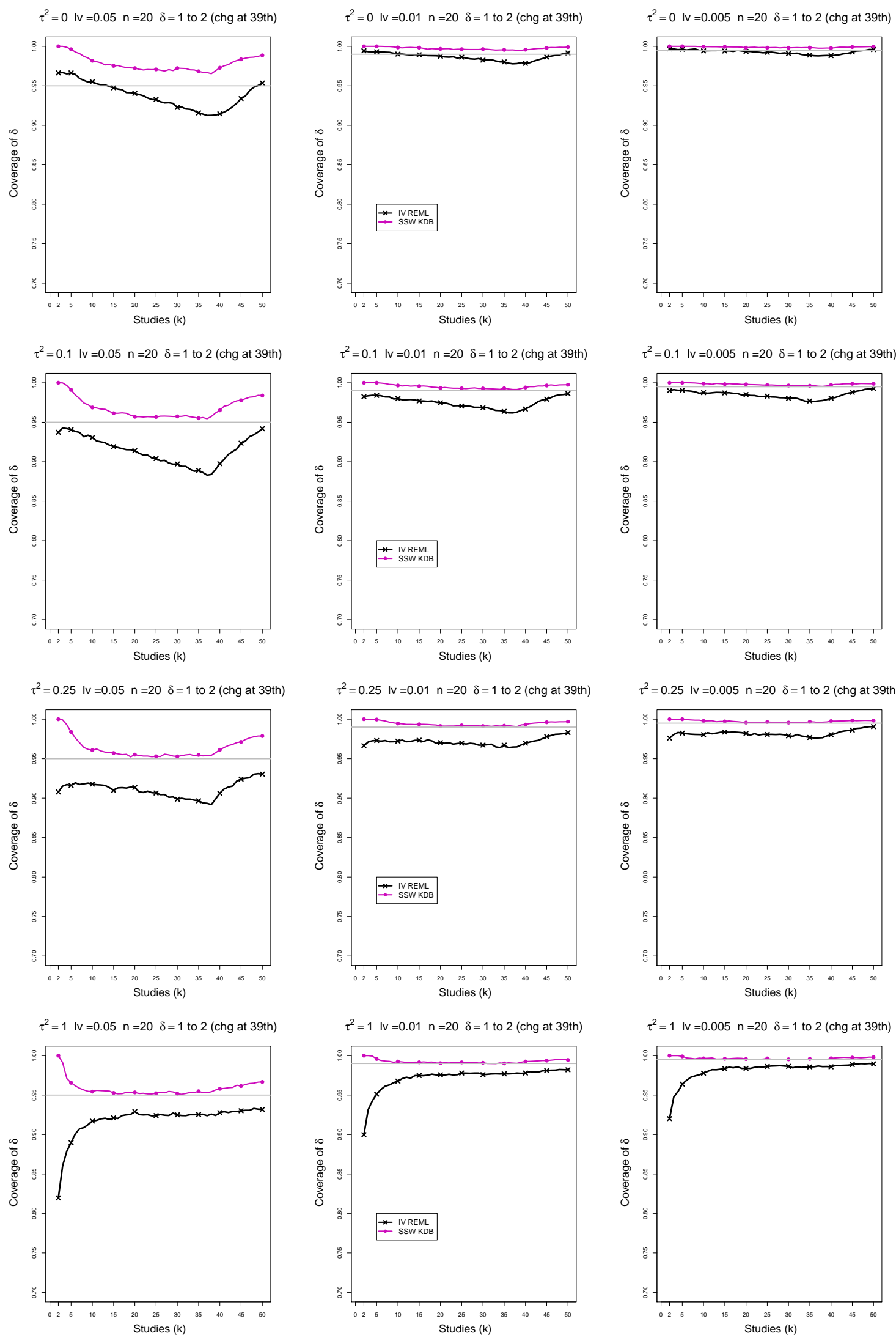

Figure C3. 11: CMA - Coverage of interval estimators of $\delta$ at confidence level of $1-\alpha$ for $\alpha=0.05,0.01,0.005$ when $\delta=1$ for $k \leq 38$ followed by a shift to $\delta=2$ for $k \geq 39$, $\tau^{2}=0,0.1,0.25,1, n=20, K=50$. Light grey line at $0.95,0.99,0.995$. 

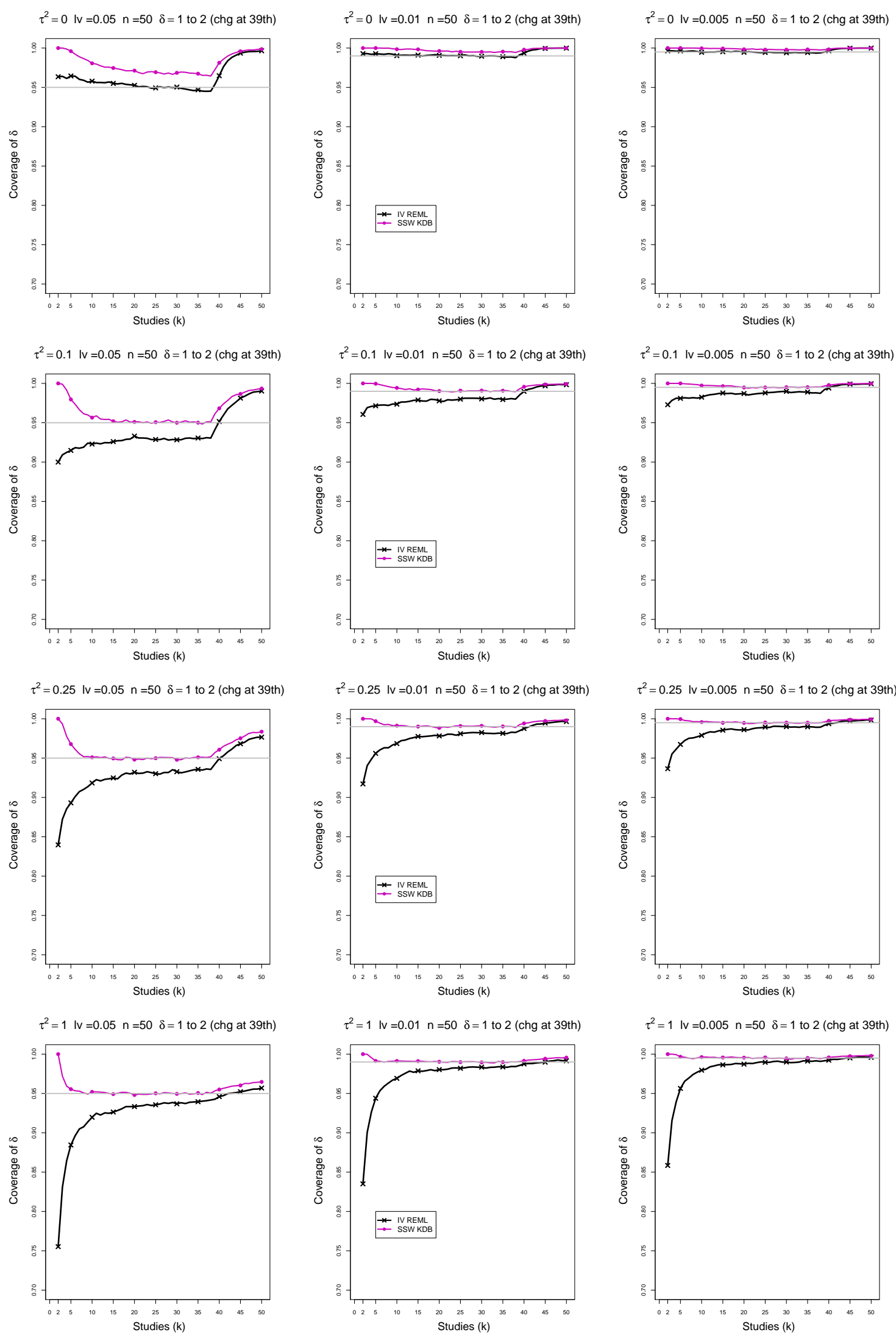

Figure C3. 12: CMA - Coverage of interval estimators of $\delta$ at confidence level of $1-\alpha$ for $\alpha=0.05,0.01,0.005$ when $\delta=1$ for $k \leq 38$ followed by a shift to $\delta=2$ for $k \geq 39$, $\tau^{2}=0,0.1,0.25,1, n=50, K=50$. Light grey line at $0.95,0.99,0.995$. 

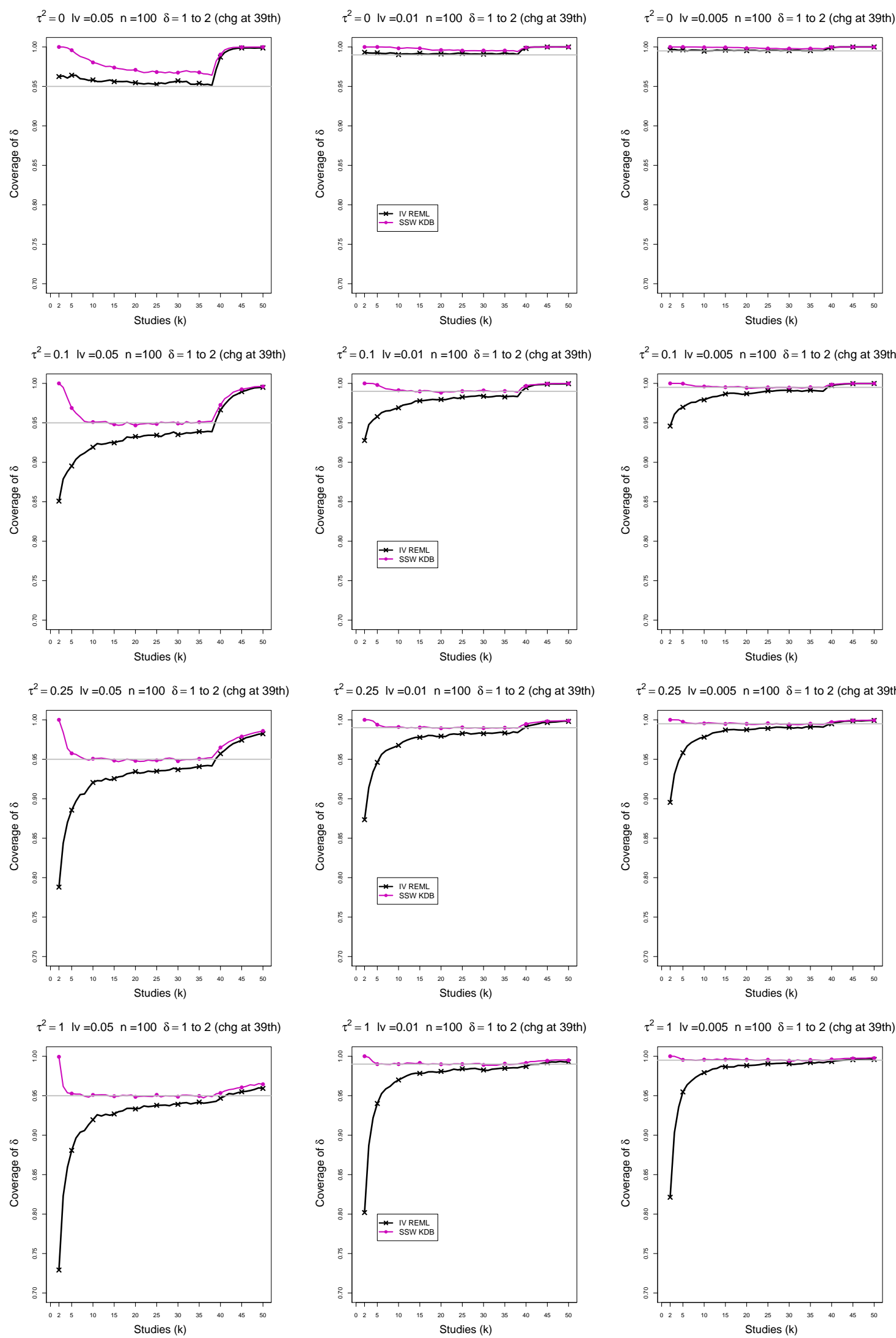

Figure C3. 13: CMA - Coverage of interval estimators of $\delta$ at confidence level of $1-\alpha$ for $\alpha=0.05,0.01,0.005$ when $\delta=1$ for $k \leq 38$ followed by a shift to $\delta=2$ for $k \geq 39$, $\tau^{2}=0,0.1,0.25,1, n=100, K=50$. Light grey line at $0.95,0.99,0.995$. 

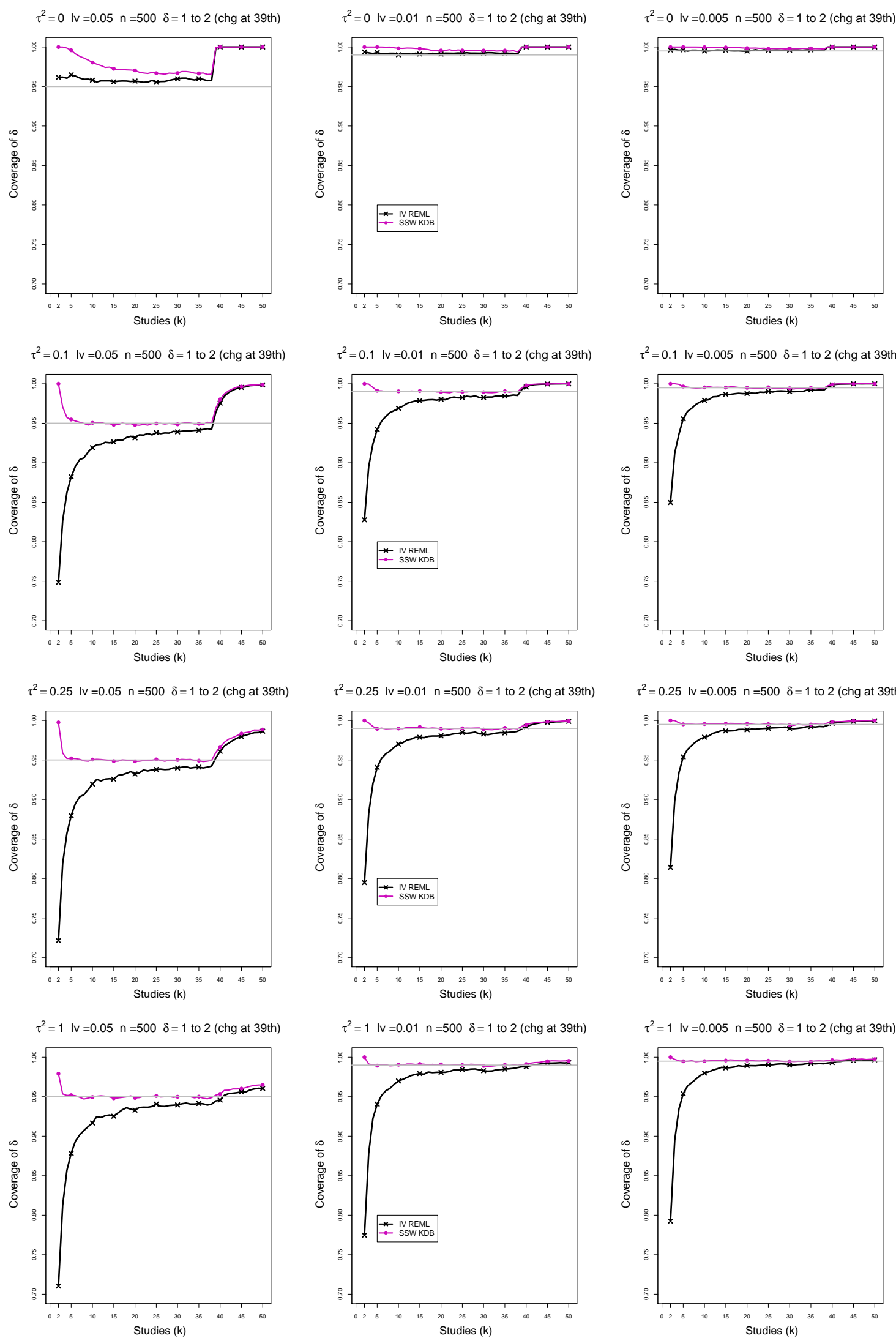

Figure C3. 14: CMA - Coverage of interval estimators of $\delta$ at confidence level of $1-\alpha$ for $\alpha=0.05,0.01,0.005$ when $\delta=1$ for $k \leq 38$ followed by a shift to $\delta=2$ for $k \geq 39$, $\tau^{2}=0,0.1,0.25,1, n=500, K=50$. Light grey line at $0.95,0.99,0.995$. 

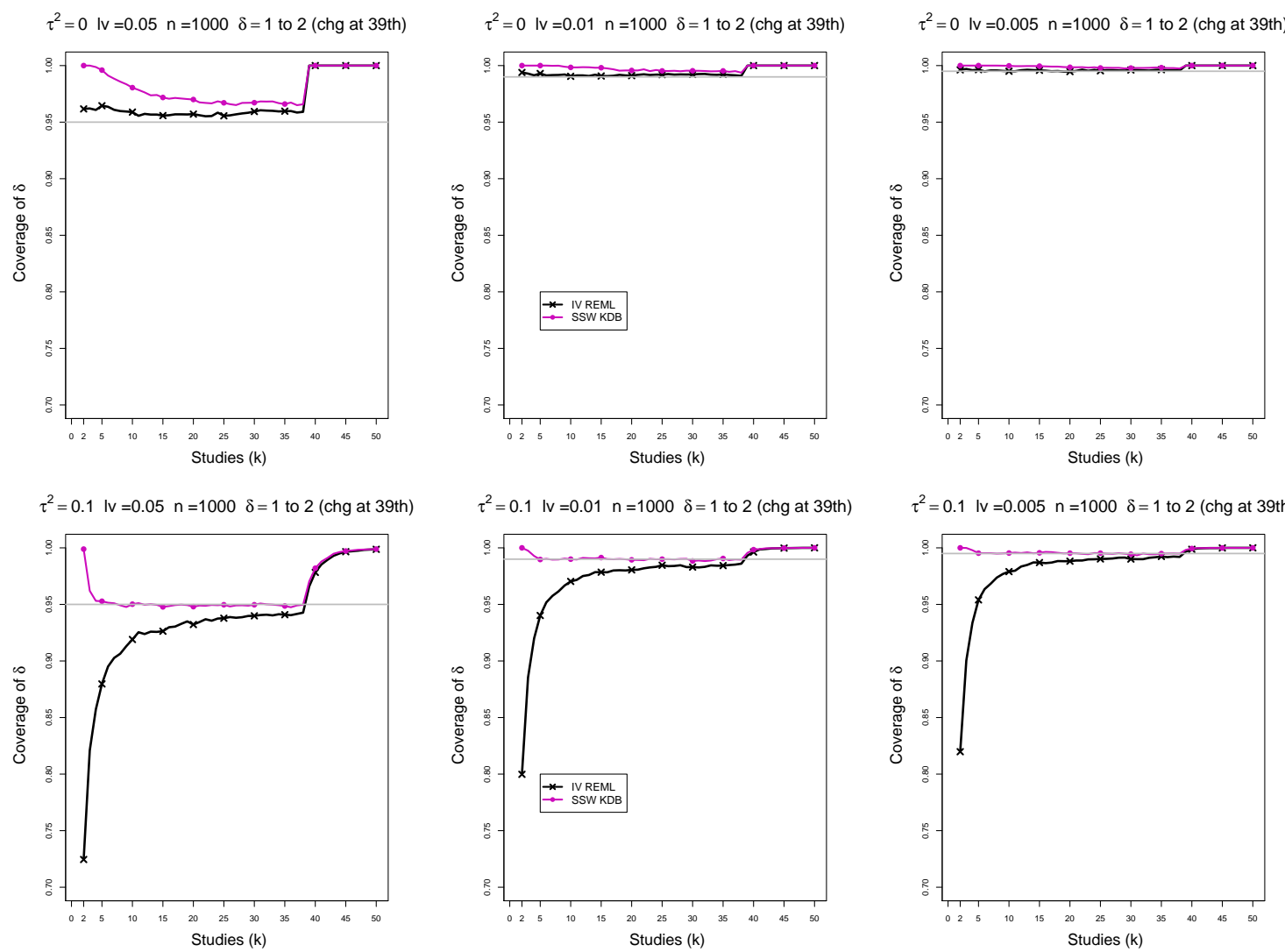

$\tau^{2}=0.1 \mathrm{lv}=0.005 \mathrm{n}=1000 \delta=1$ to 2 (chg at 39th)

$\tau^{2}=0.25 \mathrm{IV}=0.05 \mathrm{n}=1000 \delta=1$ to 2 (chg at 39 th)

$\tau^{2}=0.25 \mathrm{IV}=0.01 \mathrm{n}=1000 \delta=1$ to 2 (chg at 39th)
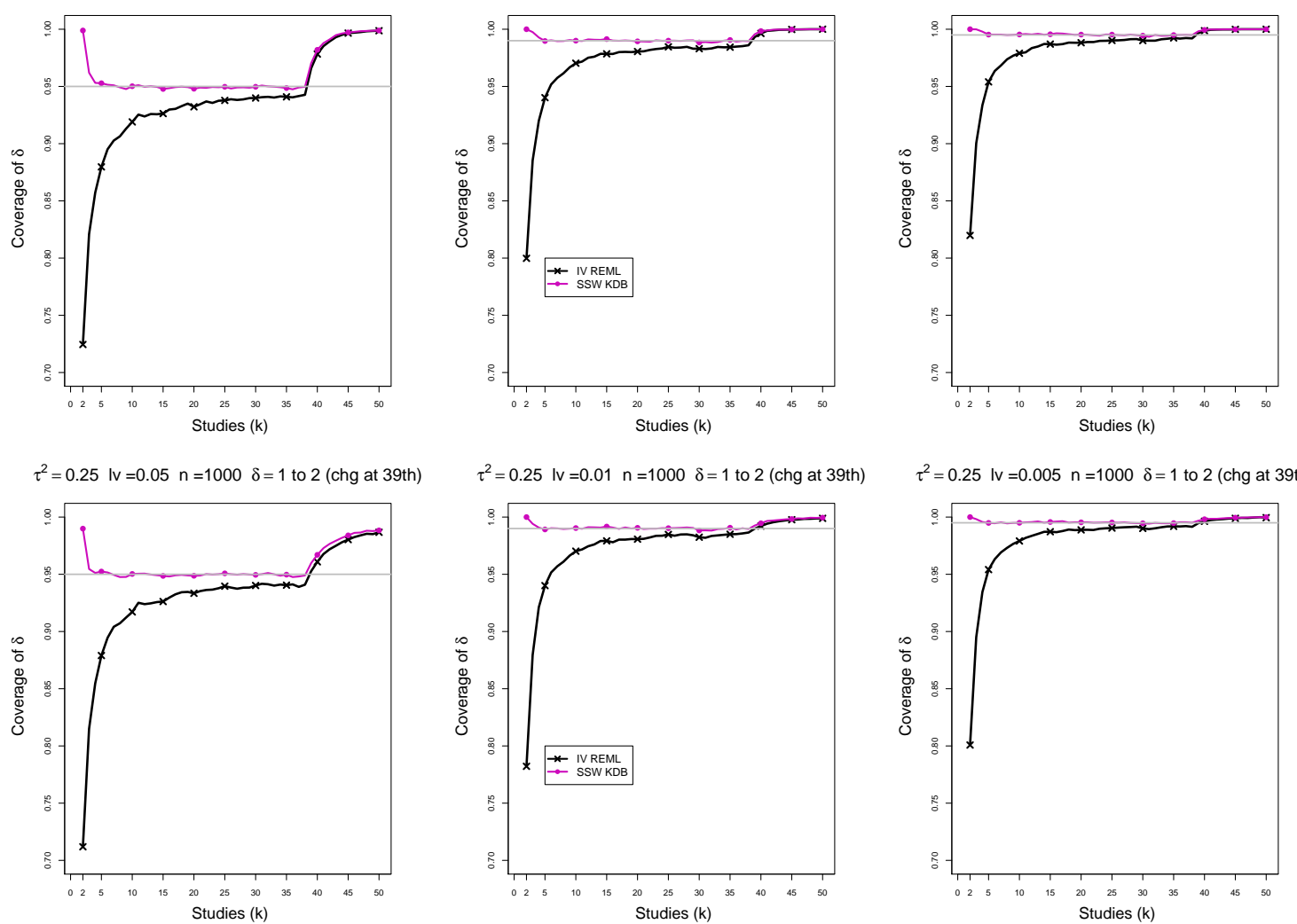

$\tau^{2}=0.25 \mathrm{lv}=0.005 \mathrm{n}=1000 \delta=1$ to 2 (chg at 39th)

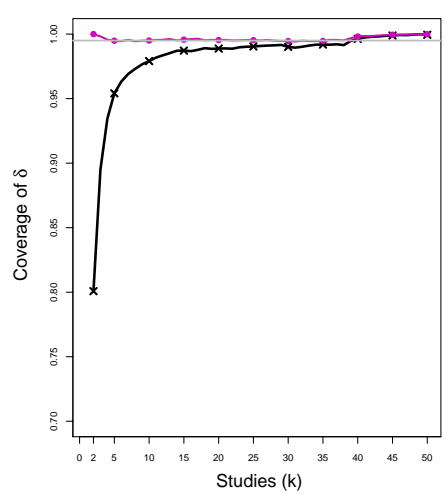

$\tau^{2}=1 \mathrm{lv}=0.05 \mathrm{n}=1000 \delta=1$ to 2 (chg at 39 th)

$\tau^{2}=1 \mathrm{IV}=0.01 \mathrm{n}=1000 \delta=1$ to 2 (chg at 39th)

$\tau^{2}=1 \mathrm{IV}=0.005 \mathrm{n}=1000 \delta=1$ to 2 (chg at 39 th)
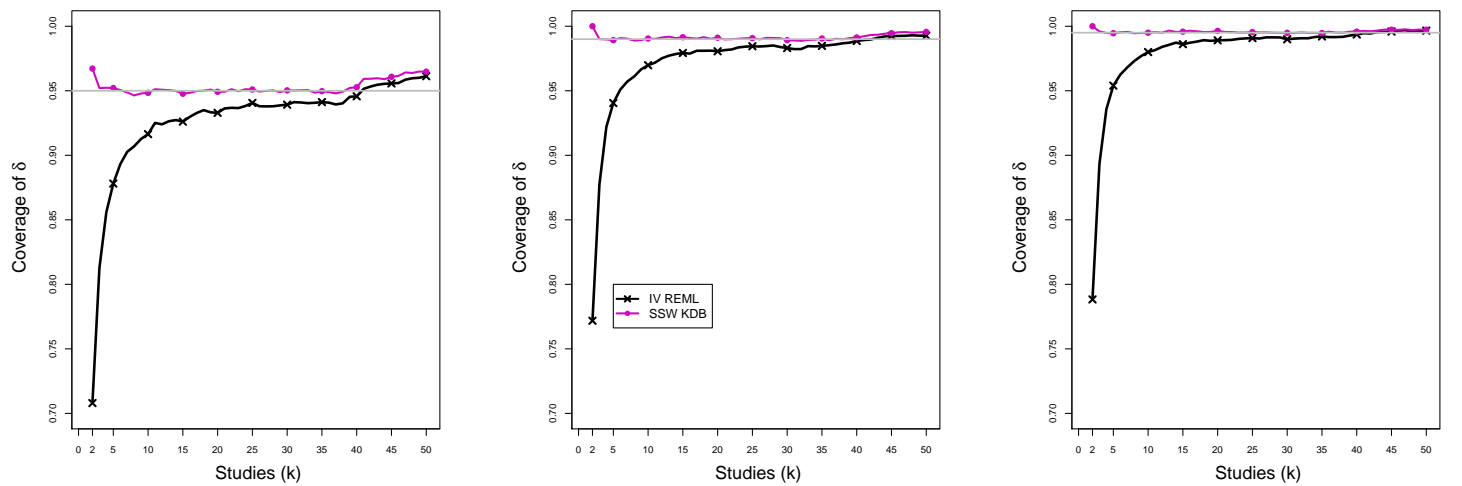

Figure C3. 15: CMA - Coverage of interval estimators of $\delta$ at confidence level of $1-\alpha$ for $\alpha=0.05,0.01,0.005$ when $\delta=1$ for $k \leq 38$ followed by a shift to $\delta=2$ for $k \geq 39$, $\tau^{2}=0,0.1,0.25,1, n=1000, K=50$. Light grey line at $0.95,0.99,0.995$. 


\section{C4. Coverage of one-sided interval estimators of $\delta$}

For one-sided coverage of $\delta$ in this section, each figure corresponds to a value of a set of values of $\tau^{2}(=0,0.1,0.25,1)$, a value of $n(=20,50,100,500,1000)$, a set of values of 2 -sided significance level $(=0.05,0.01,0.005)$ and a value of point of shift $f(=13$, $26,39)$ while $\delta$ shift from 1 to 2 and number of studies $K=50$.

Each figure contains a panel (with study $k$ on the horizontal axis) for each combination of parameters.

The interval estimators of $\delta$ are

- IV REML (Inverse variance weighted with REML-estimated $\tau^{2}$ as center and normal critical values, )

- $\mathrm{SSW}$ KDB (Sample size weighted (SSW) as center with $\hat{\tau}^{2}=\hat{\tau}_{K D B}^{2}$, the Kulinskaya-Dollinger-Bjørkest $\varnothing$ l estimator of $\tau^{2}$ and critical value from $t_{k-1}$.) 

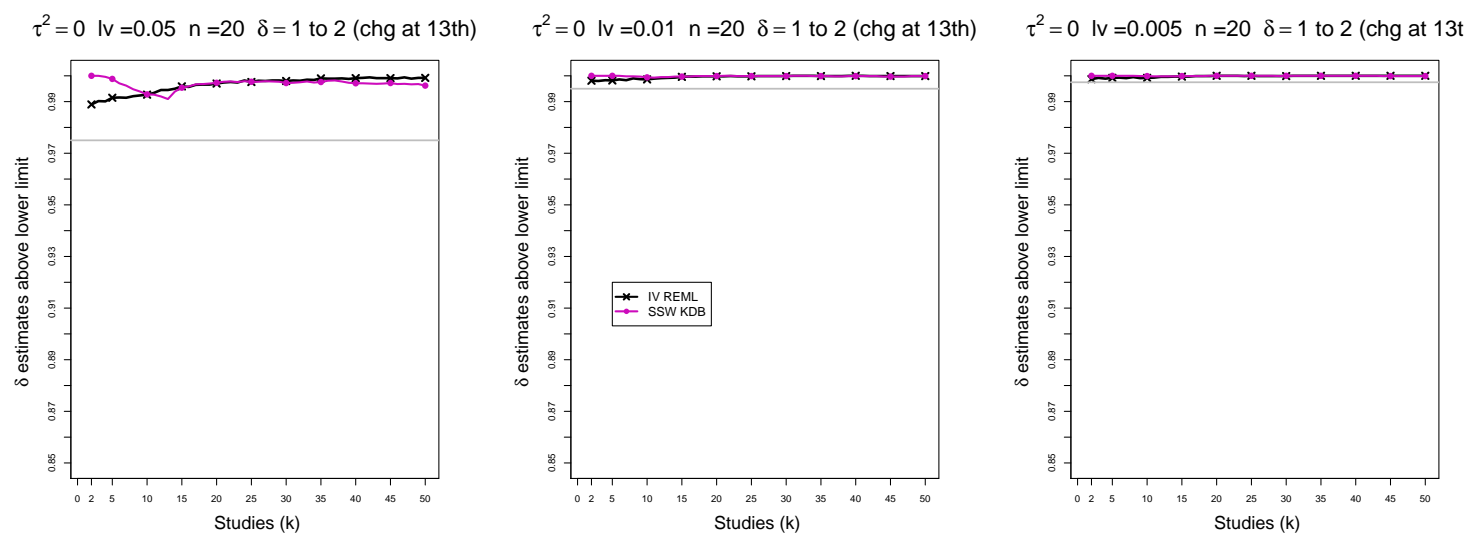

$\tau^{2}=0.1 \mathrm{IV}=0.05 \mathrm{n}=20 \quad \delta=1$ to 2 (chg at 13 th)
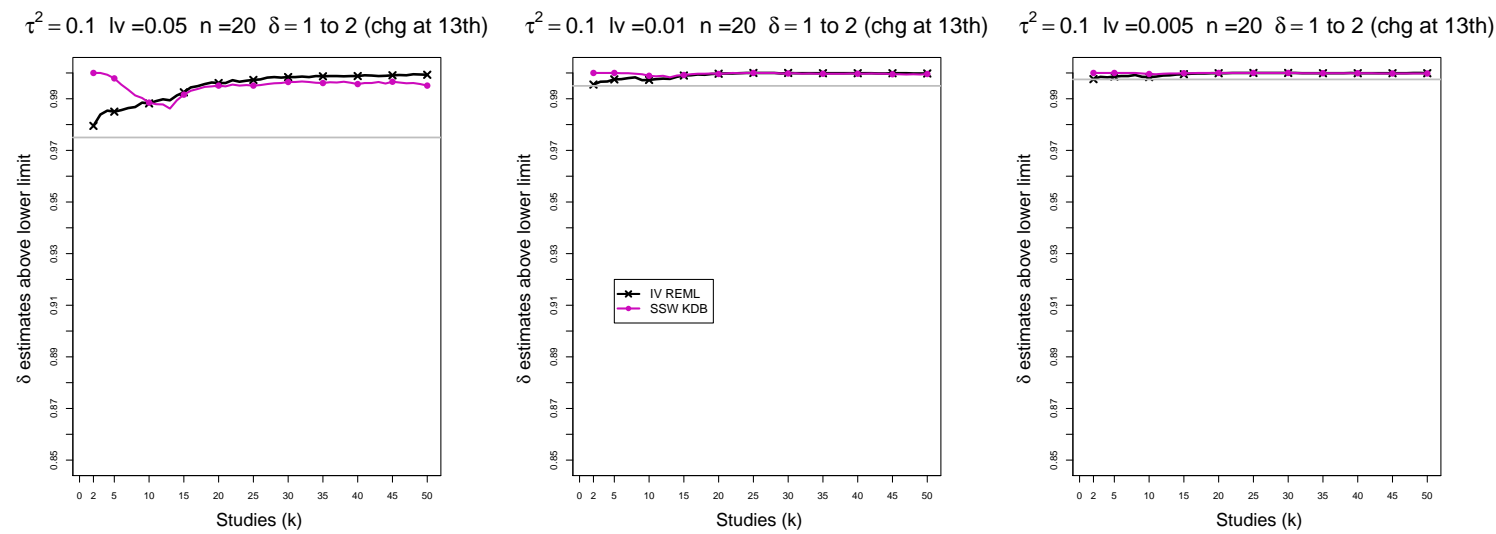

$\tau^{2}=0.25 \mathrm{lv}=0.05 \mathrm{n}=20 \quad \delta=1$ to 2 (chg at 13 th)
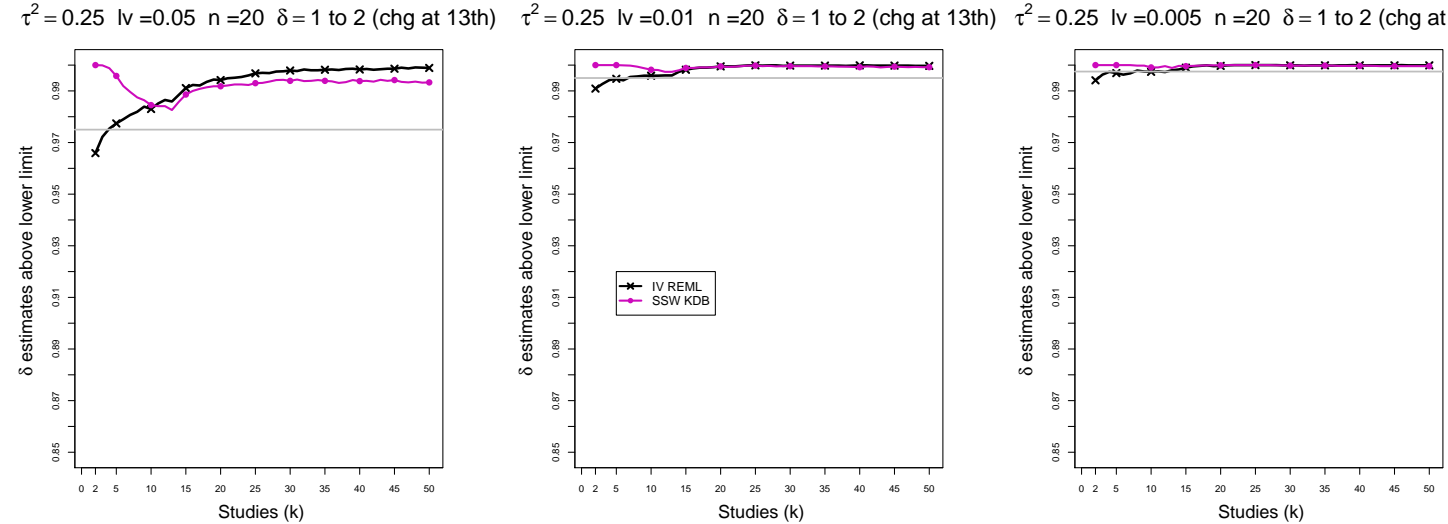

$\tau^{2}=1 \mathrm{IV}=0.01 \mathrm{n}=20 \quad \delta=1$ to 2 (chg at 13 th)

$\tau^{2}=1 \mathrm{lv}=0.005 \mathrm{n}=20 \delta=1$ to 2 (chg at 13 th)
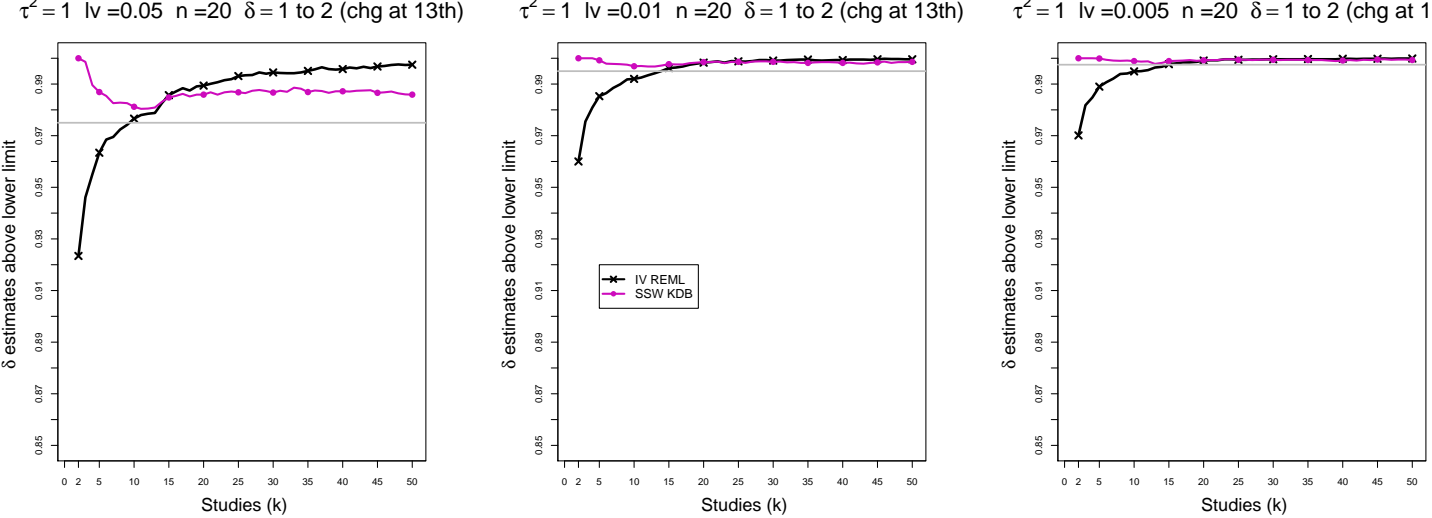

Figure C4. 1: CMA - Coverage of the lower confidence interval of $\delta$ (above lower limit) at the confidence level $1-\alpha / 2$ for $\alpha=0.05,0.01,0.005$ when $\delta=1$ for $k \leq 12$ followed by a shift to $\delta=2$ for $k \geq 13, \tau^{2}=0,0.1,0.25,1, n=20, K=50$. Light grey line at $0.975,0.995,0.9975$. 

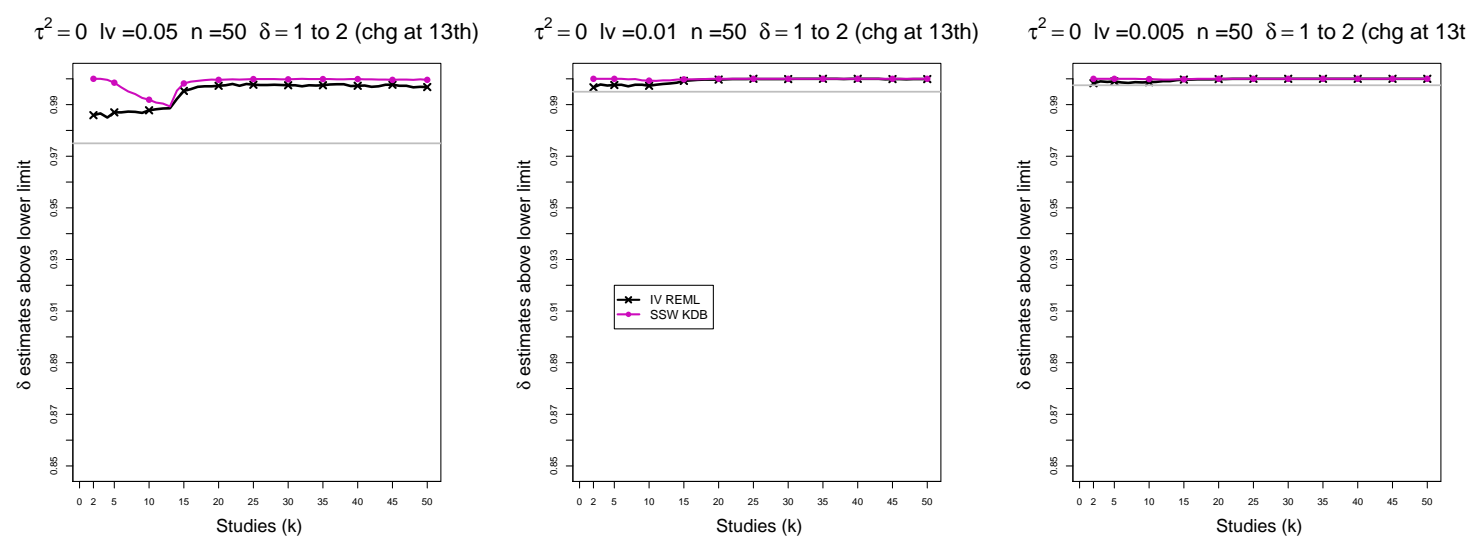

$\tau^{2}=0.1 \mathrm{IV}=0.05 \mathrm{n}=50 \quad \delta=1$ to 2 (chg at 13 th)
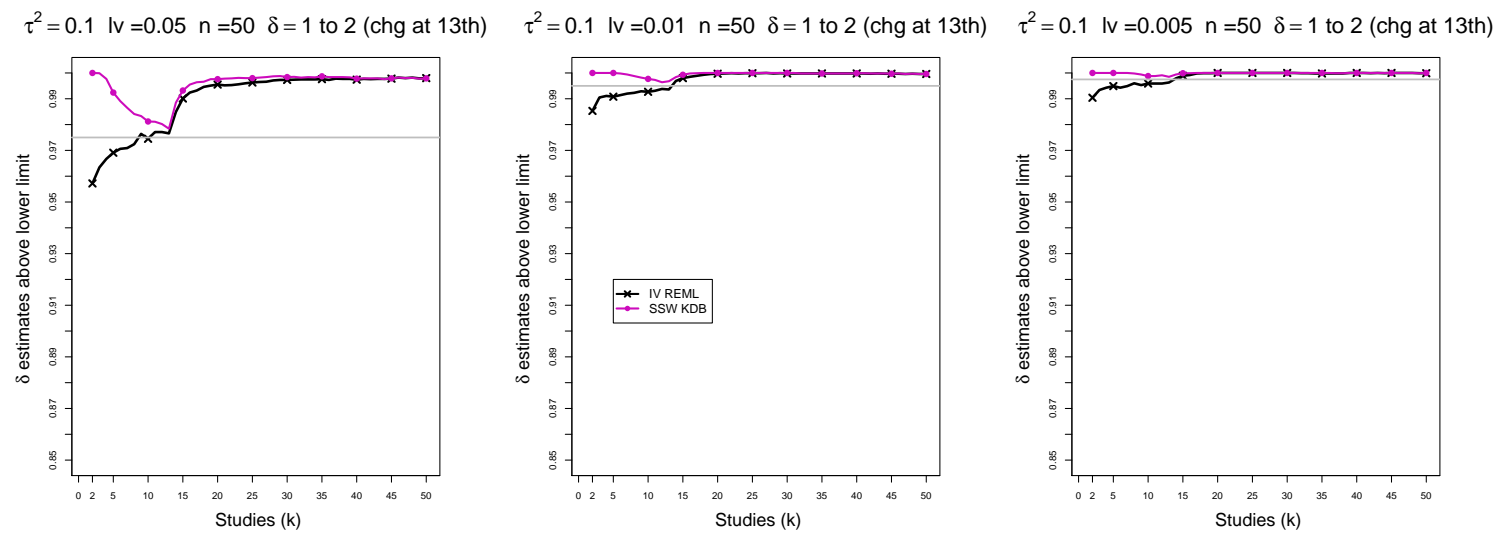

$\tau^{2}=0.25 \mathrm{IV}=0.05 \mathrm{n}=50 \quad \delta=1$ to 2 (chg at 13 th)
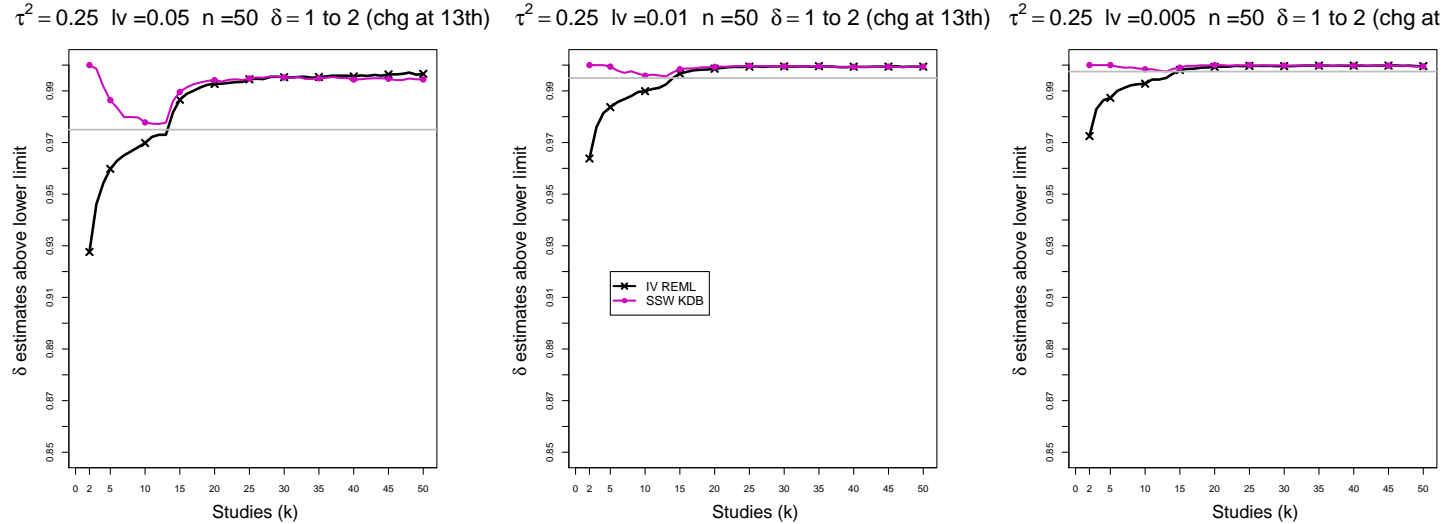

$\tau^{2}=1 \mathrm{lv}=0.01 \mathrm{n}=50 \quad \delta=1$ to 2 (chg at 13 th)

$\tau^{2}=1 \mathrm{lv}=0.005 \mathrm{n}=50 \delta=1$ to 2 (chg at 13 th)
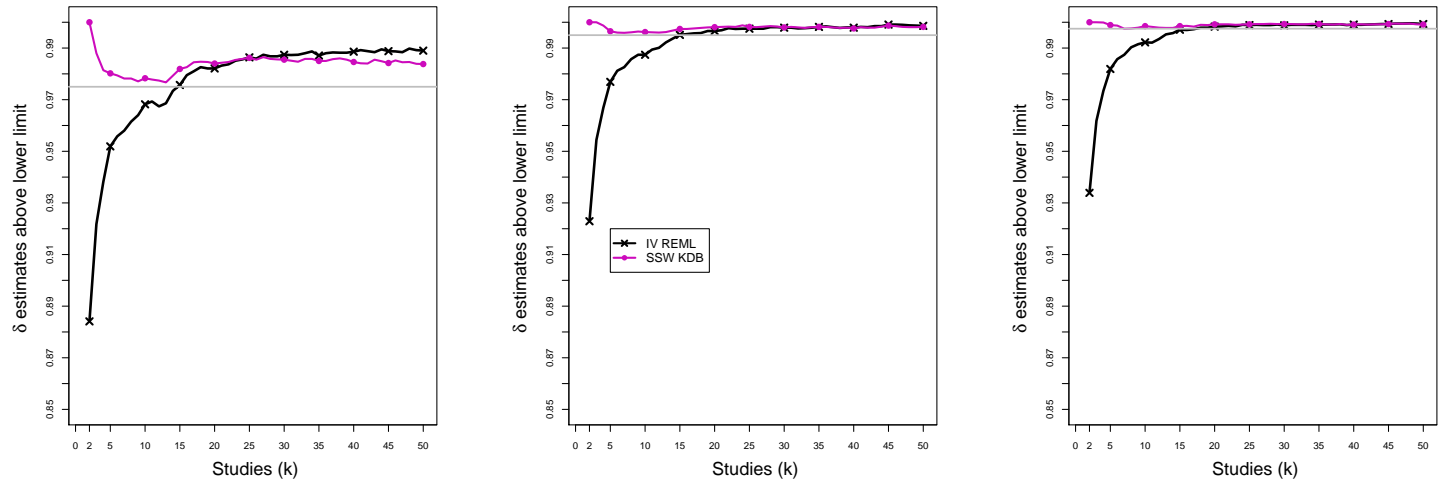

Figure C4. 2: CMA - Coverage of the lower confidence interval of $\delta$ (above lower limit) at the confidence level $1-\alpha / 2$ for $\alpha=0.05,0.01,0.005$ when $\delta=1$ for $k \leq 12$ followed by a shift to $\delta=2$ for $k \geq 13, \tau^{2}=0,0.1,0.25,1, n=50, K=50$. Light grey line at $0.975,0.995,0.9975$. 

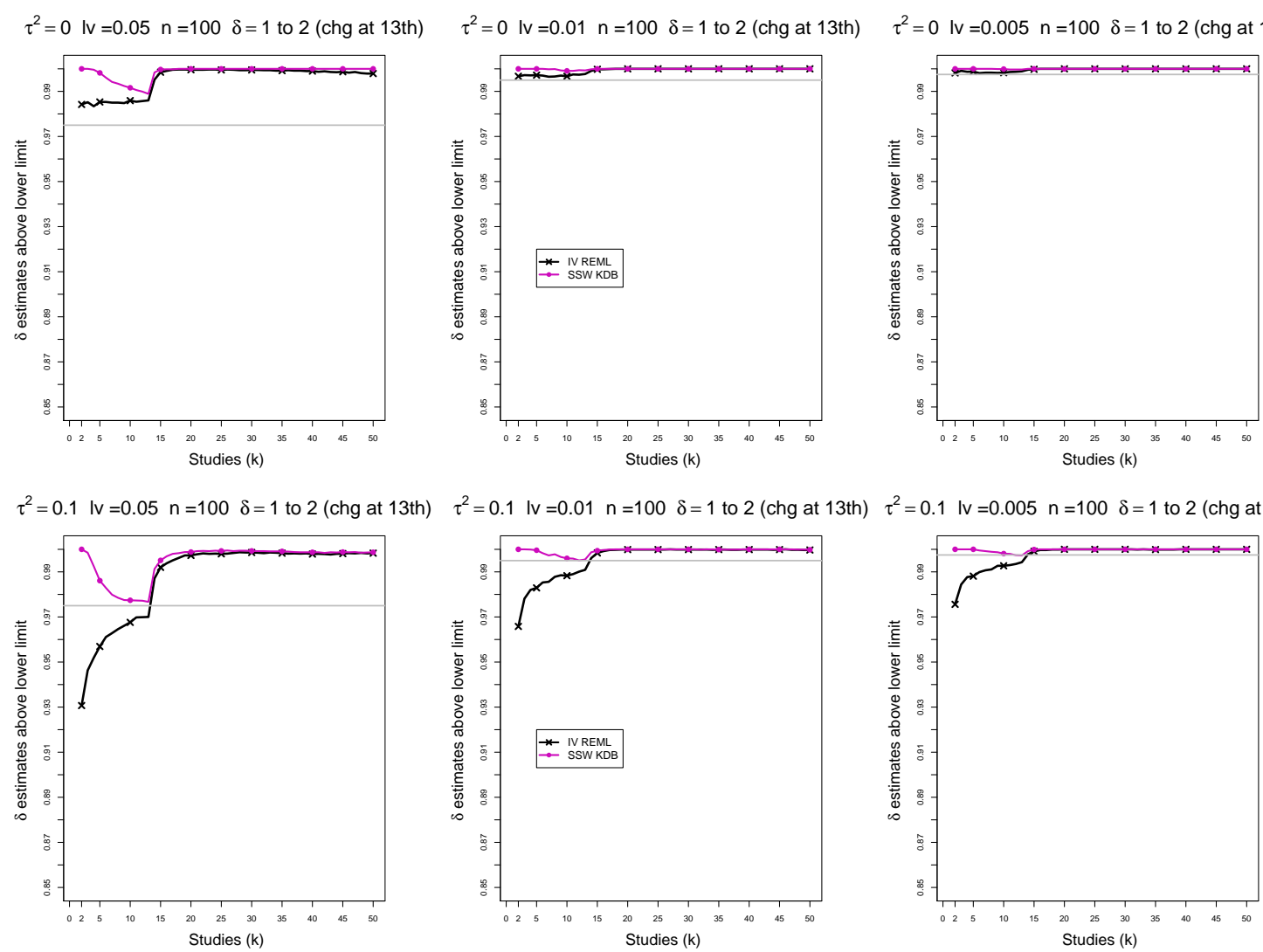

$\tau^{2}=0.25 \mathrm{IV}=0.05 \mathrm{n}=100 \delta=1$ to 2 (chg at 13 th) $\tau^{2}=0.25 \mathrm{IV}=0.01 \mathrm{n}=100 \delta=1$ to 2 (chg at 13 th) $\tau^{2}=0.25 \mathrm{IV}=0.005 \mathrm{n}=100 \quad \delta=1$ to 2 (chg at 13 th)
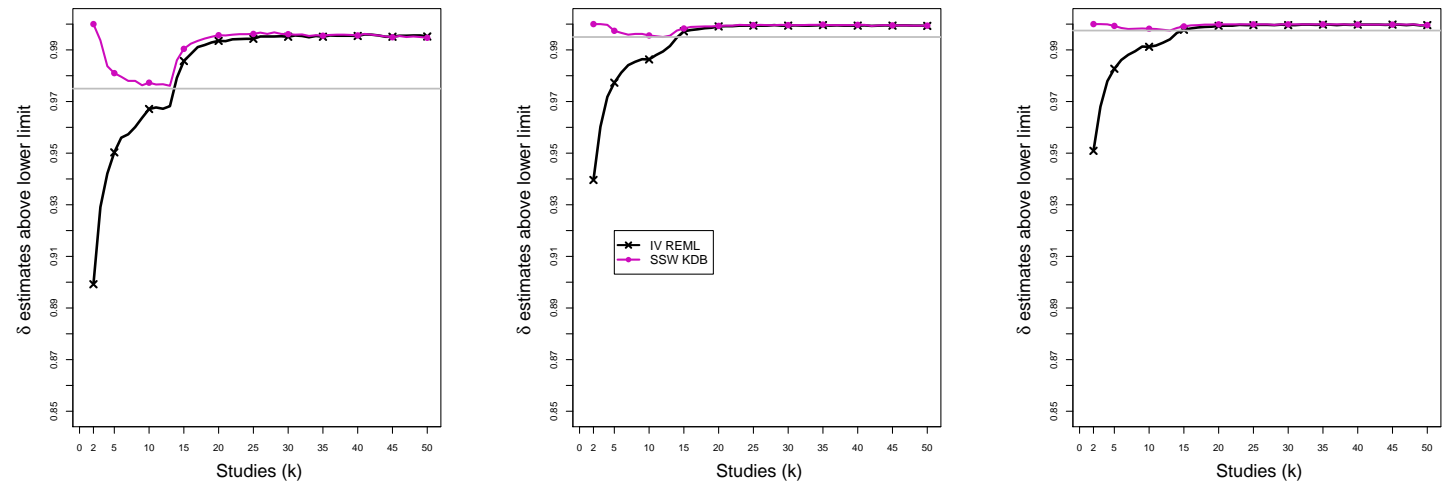

$\tau^{2}=1 \mathrm{IV}=0.05 \mathrm{n}=100 \delta=1$ to 2 (chg at 13 th)

$\tau^{2}=1 \mathrm{lv}=0.01 \mathrm{n}=100 \delta=1$ to 2 (chg at 13 th)

$\tau^{2}=1 \mathrm{lv}=0.005 \mathrm{n}=100 \delta=1$ to 2 (chg at 13th)
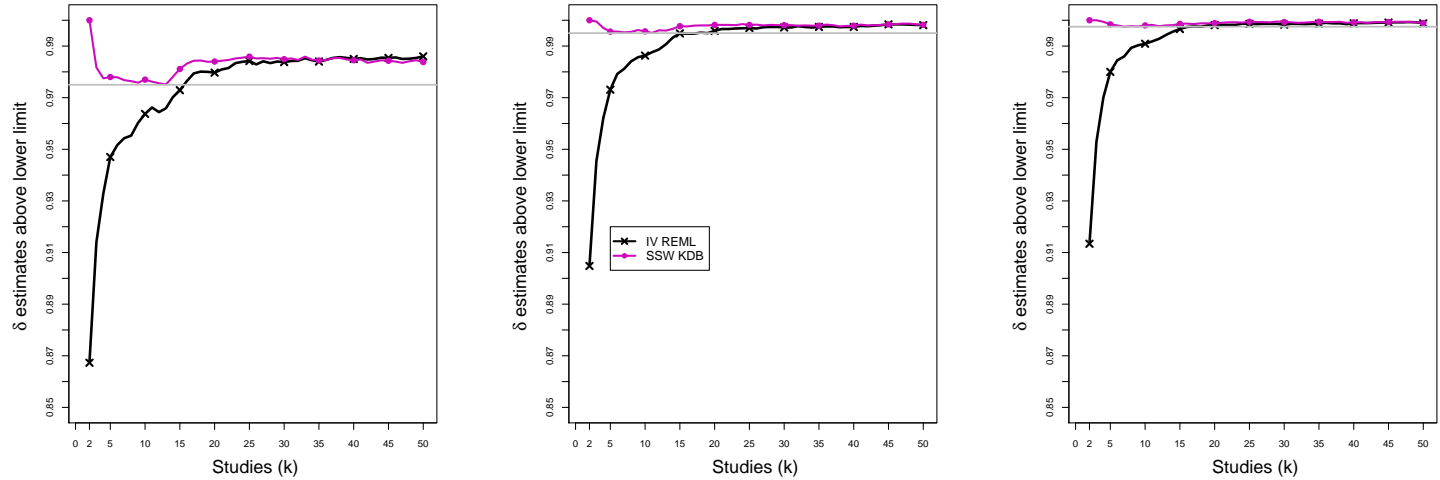

Figure C4. 3: CMA - Coverage of the lower confidence interval of $\delta$ (above lower limit) at the confidence level $1-\alpha / 2$ for $\alpha=0.05,0.01,0.005$ when $\delta=1$ for $k \leq 12$ followed by a shift to $\delta=2$ for $k \geq 13, \tau^{2}=0,0.1,0.25,1, n=100, K=50$. Light grey line at 

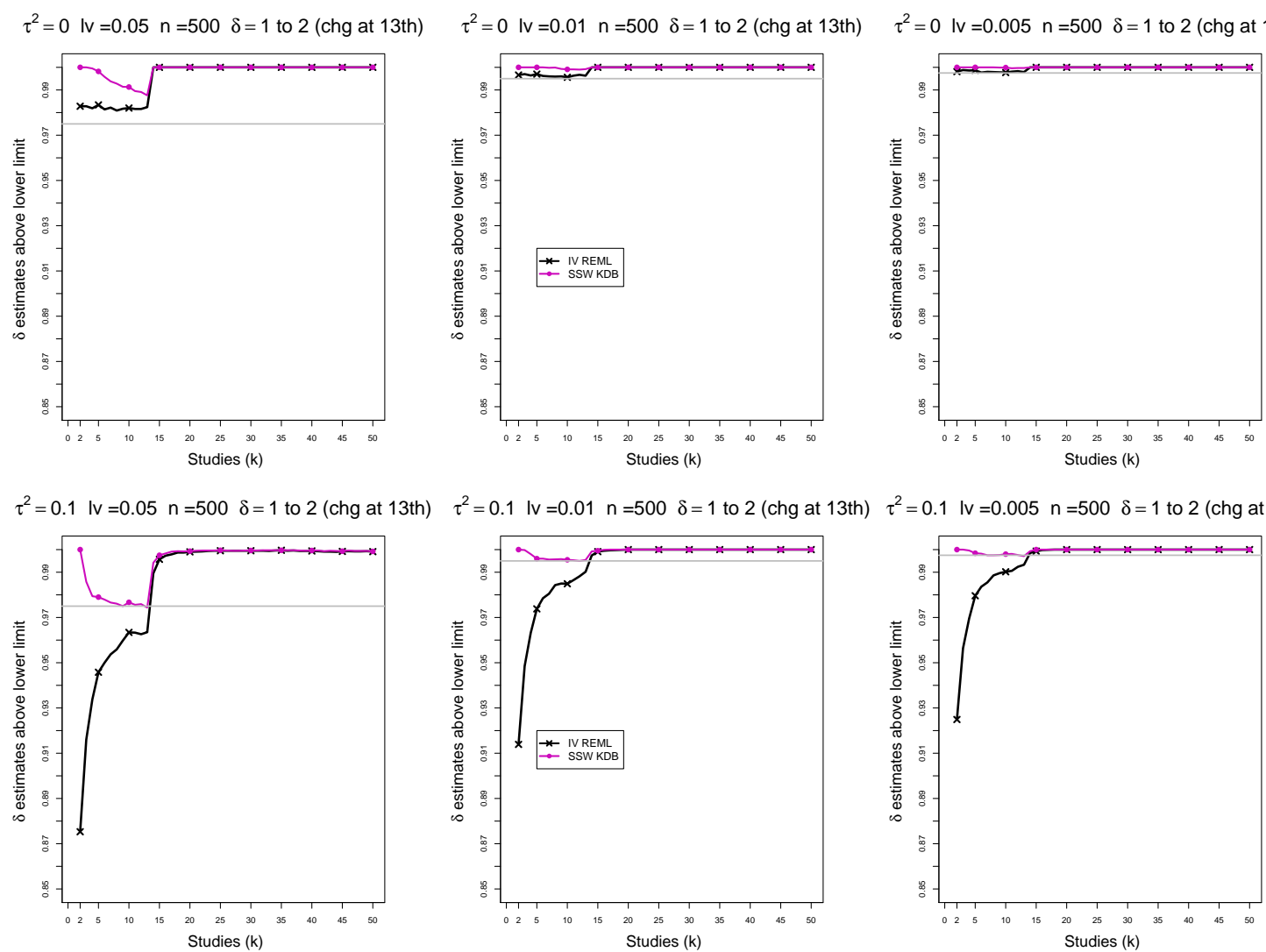

$\tau^{2}=0.25 \mathrm{IV}=0.05 \mathrm{n}=500 \delta=1$ to 2 (chg at 13 th) $\tau^{2}=0.25 \mathrm{IV}=0.01 \mathrm{n}=500 \delta=1$ to 2 (chg at 13 th) $\tau^{2}=0.25 \mathrm{IV}=0.005 \mathrm{n}=500 \quad \delta=1$ to 2 (chg at 13 th)
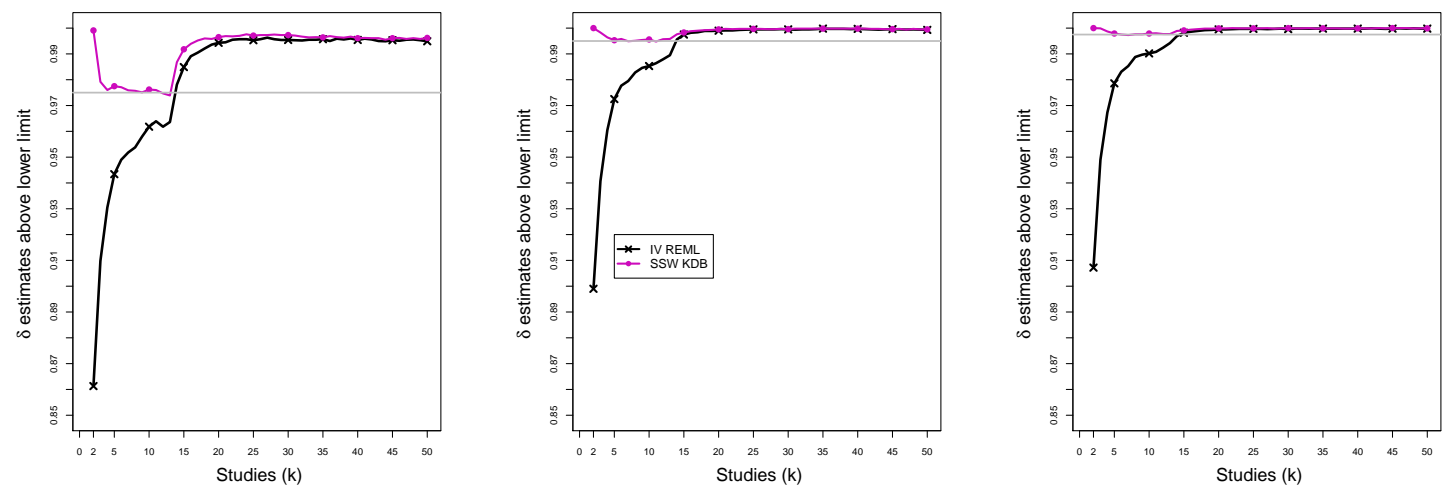

$\tau^{2}=1 \mathrm{IV}=0.05 \mathrm{n}=500 \delta=1$ to 2 (chg at 13 th)

$\tau^{2}=1 \mathrm{lv}=0.01 \mathrm{n}=500 \delta=1$ to 2 (chg at 13 th)

$\tau^{2}=1 \mathrm{lv}=0.005 \mathrm{n}=500 \delta=1$ to 2 (chg at 13 th)
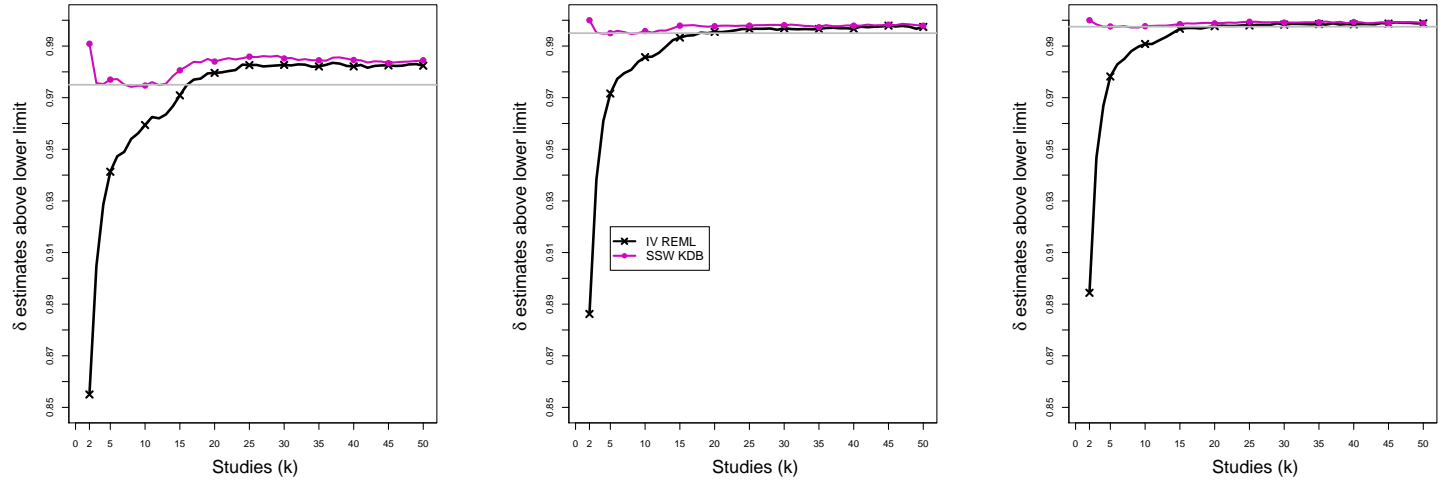

Figure C4. 4: CMA - Coverage of the lower confidence interval of $\delta$ (above lower limit) at the confidence level $1-\alpha / 2 / 2$ for $\alpha=0.05,0.01,0.005$ when $\delta=1$ for $k \leq 12$ followed by a shift to $\delta=2$ for $k \geq 13, \tau^{2}=0,0.1,0.25,1, n=500, K=50$. Light grey line at 

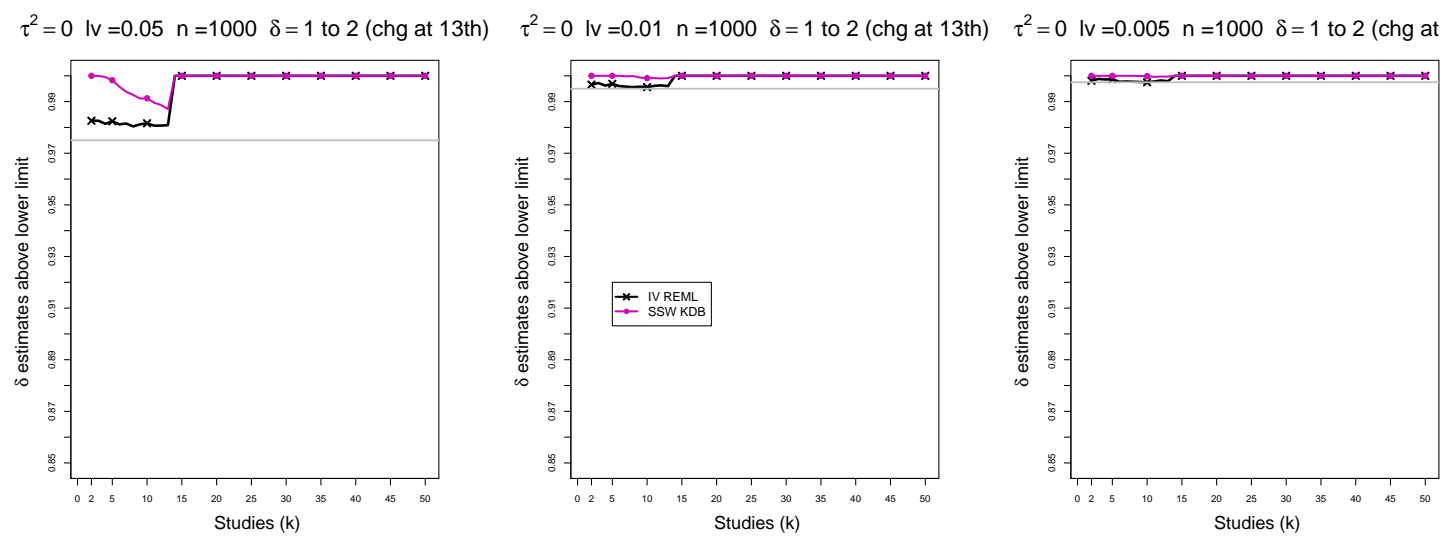

$\tau^{2}=0.1 \mathrm{lv}=0.05 \mathrm{n}=1000 \delta=1$ to 2 (chg at 13 th) $\tau^{2}=0.1 \mathrm{lv}=0.01 \mathrm{n}=1000 \delta=1$ to 2 (chg at 13 th) $\tau^{2}=0.1 \mathrm{lv}=0.005 \mathrm{n}=1000 \quad \delta=1$ to 2 (chg at 13 th)
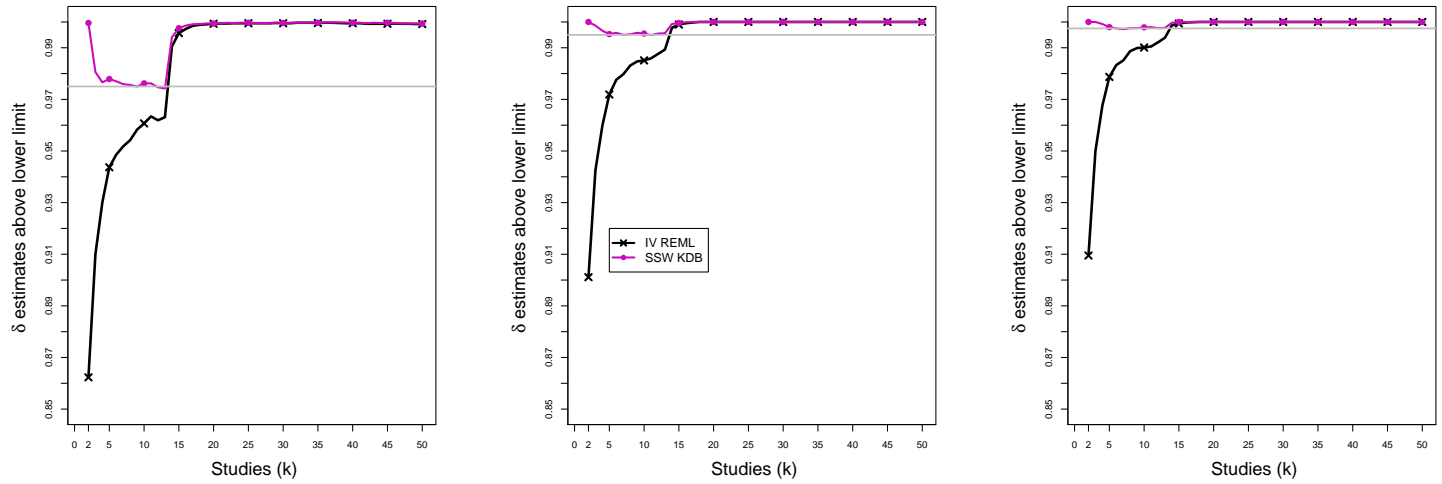

$\tau^{2}=0.25 \mathrm{lv}=0.05 \mathrm{n}=1000 \delta=1$ to $2(\mathrm{chg}$ at 13 th $) \tau^{2}=0.25 \mathrm{lv}=0.01 \mathrm{n}=1000 \quad \delta=1$ to $2(\text { chg at } 13 \text { th) })^{2}=0.25 \mathrm{lv}=0.005 \mathrm{n}=1000 \quad \delta=1$ to 2 (chg at 13 th
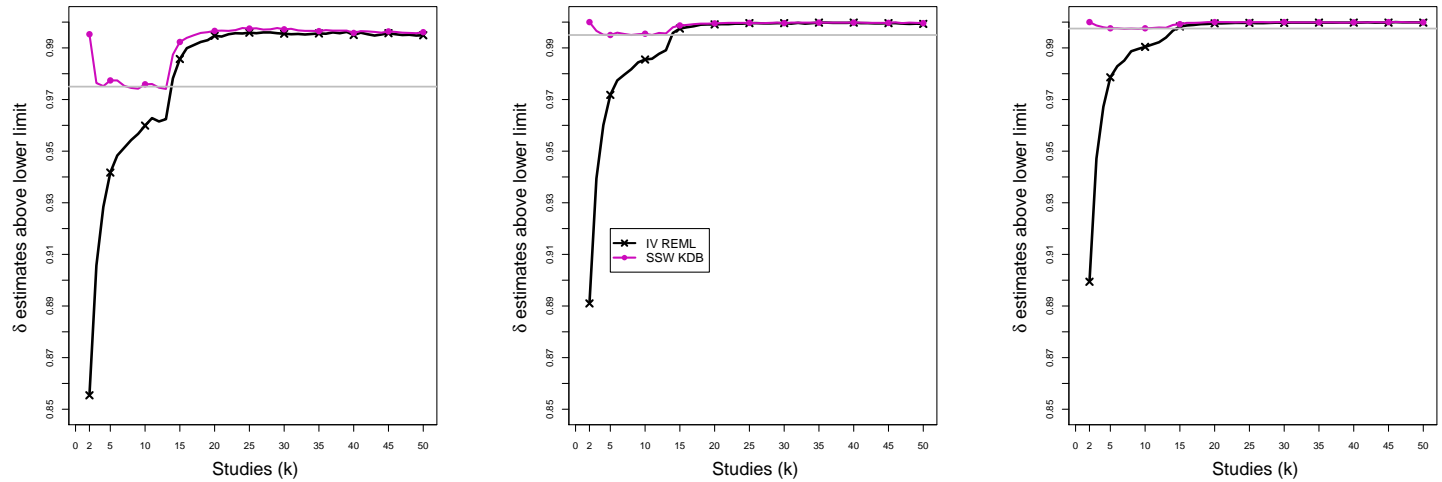

$\tau^{2}=1 \mathrm{lv}=0.05 \mathrm{n}=1000 \delta=1$ to 2 (chg at 13 th)
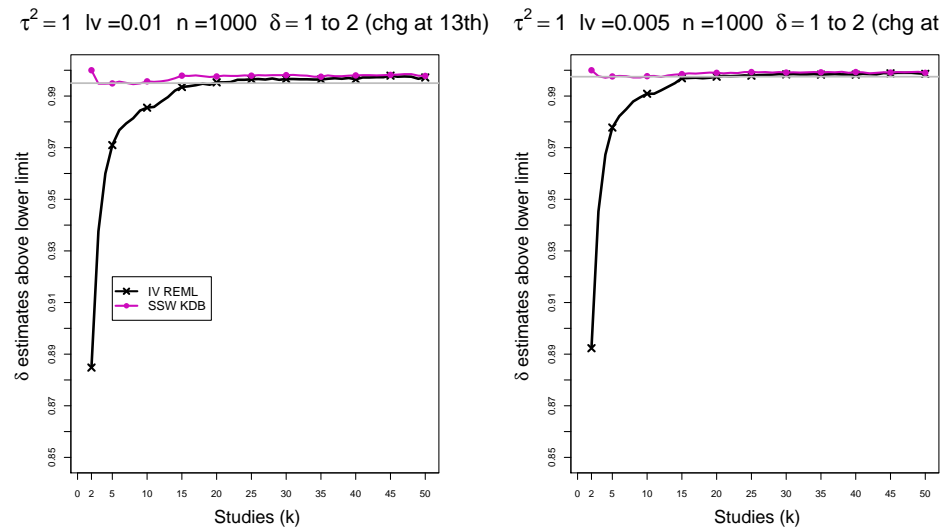

Figure C4. 5: CMA - Coverage of the lower confidence interval of $\delta$ (above lower limit) at the confidence level $1-\alpha / 2$ for $\alpha=0.05,0.01,0.005$ when $\delta=1$ for $k \leq 12$ followed by a shift to $\delta=2$ for $k \geq 13, \tau^{2}=0,0.1,0.25,1, n=1000, K=50$. Light grey line at 

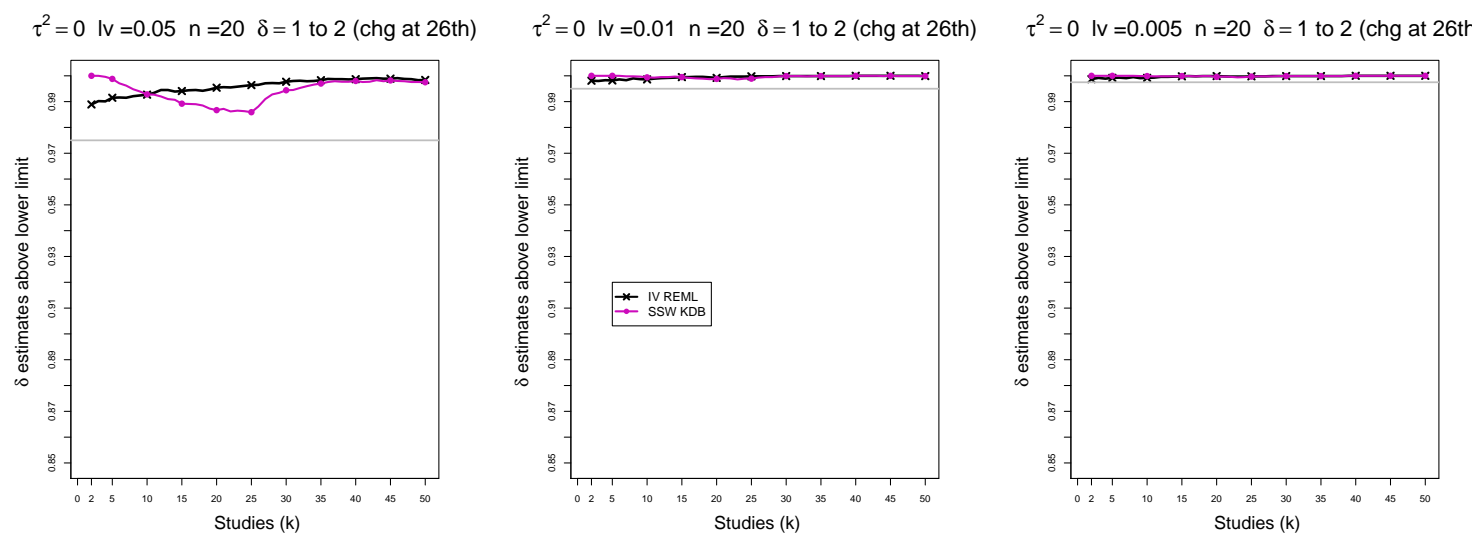

$\tau^{2}=0.1 \mathrm{lv}=0.05 \mathrm{n}=20 \quad \delta=1$ to 2 (chg at 26th)
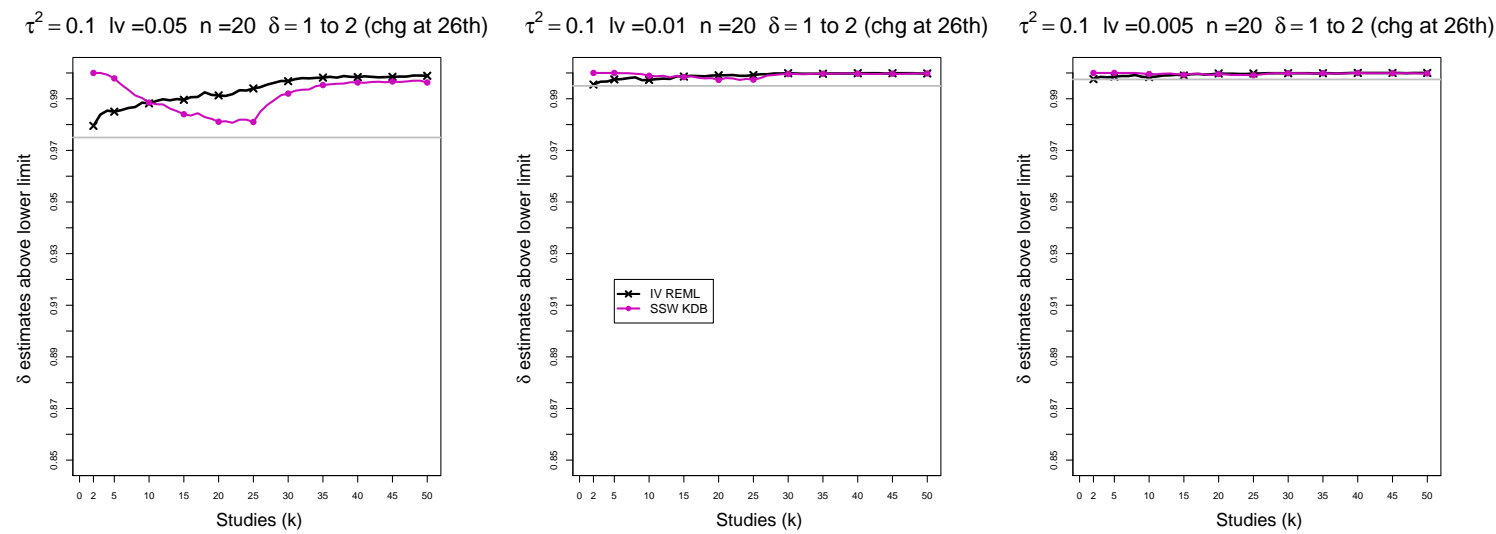

$\tau^{2}=0.25 \mathrm{lv}=0.05 \mathrm{n}=20 \quad \delta=1$ to 2 (chg at 26th)
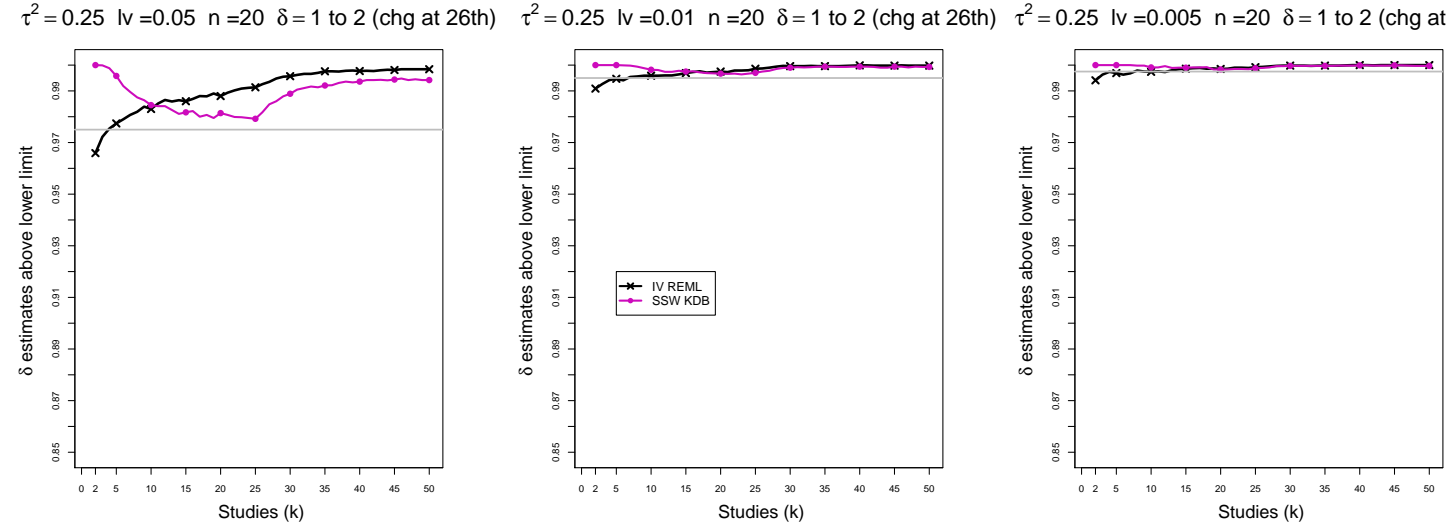

$\tau^{2}=1 \mathrm{IV}=0.01 \mathrm{n}=20 \quad \delta=1$ to 2 (chg at 26th)

$\tau^{2}=1 \mathrm{lv}=0.005 \mathrm{n}=20 \delta=1$ to 2 (chg at 26th)
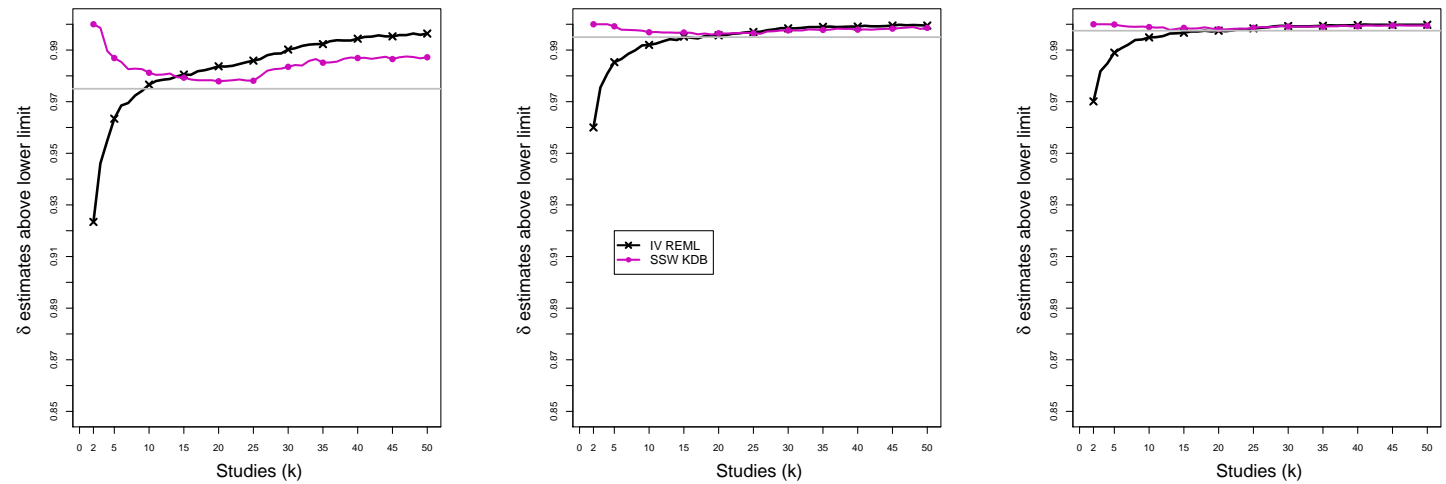

Figure C4. 6: CMA - Coverage of the lower confidence interval of $\delta$ (above lower limit) at the confidence level $1-\alpha / 2$ for $\alpha=0.05,0.01,0.005$ when $\delta=1$ for $k \leq 25$ followed by a shift to $\delta=2$ for $k \geq 26, \tau^{2}=0,0.1,0.25,1, n=20, K=50$. Light grey line at $0.975,0.995,0.9975$. 

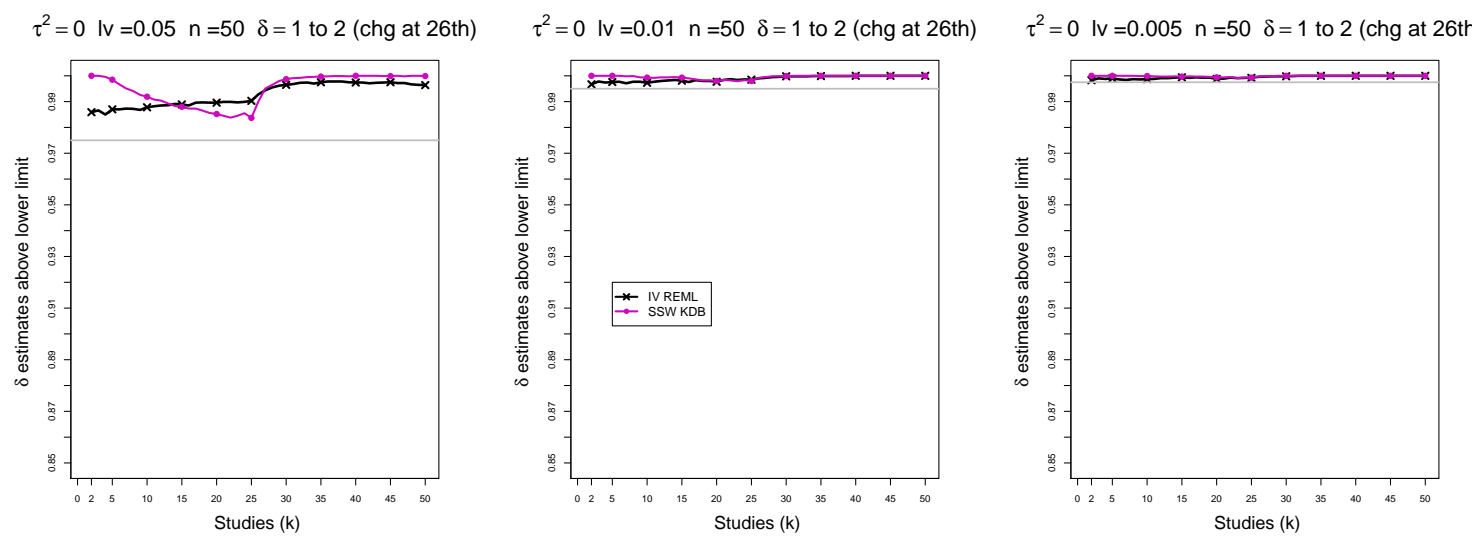

$\tau^{2}=0.1 \mathrm{lv}=0.05 \mathrm{n}=50 \quad \delta=1$ to 2 (chg at 26th)
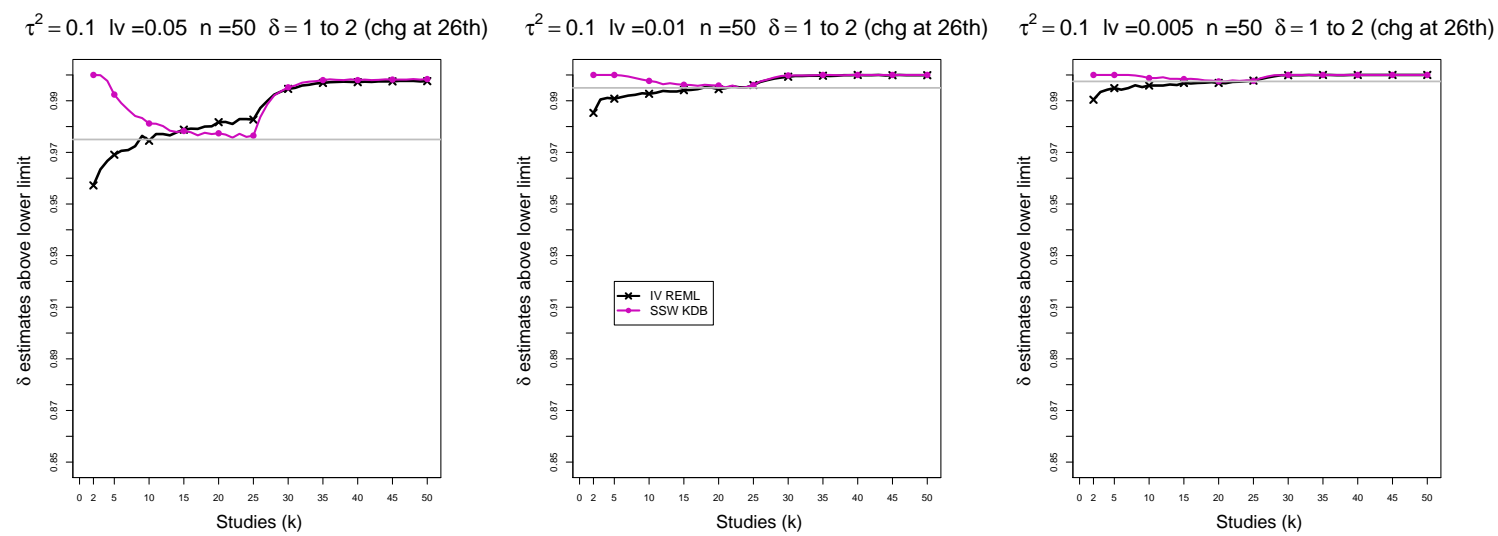

$\tau^{2}=0.25 \mathrm{lv}=0.05 \mathrm{n}=50 \quad \delta=1$ to 2 (chg at 26th)
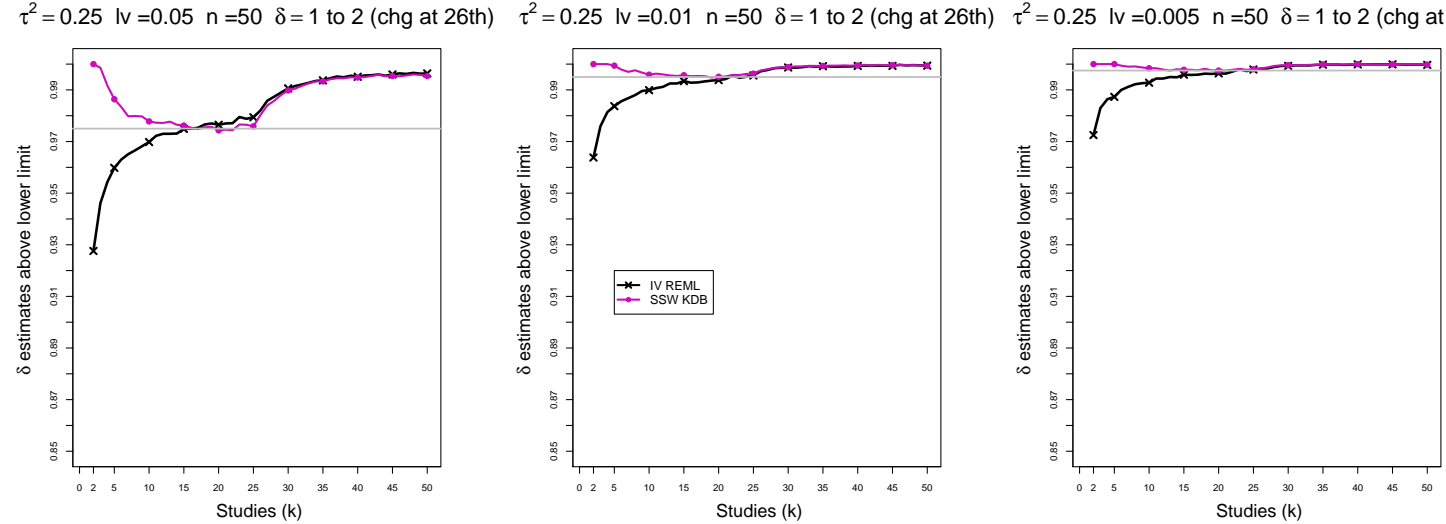

$\tau^{2}=1 \mathrm{lv}=0.01 \mathrm{n}=50 \delta=1$ to 2 (chg at 26 th)

$\tau^{2}=1 \mathrm{lv}=0.005 \mathrm{n}=50 \delta=1$ to 2 (chg at 26th)
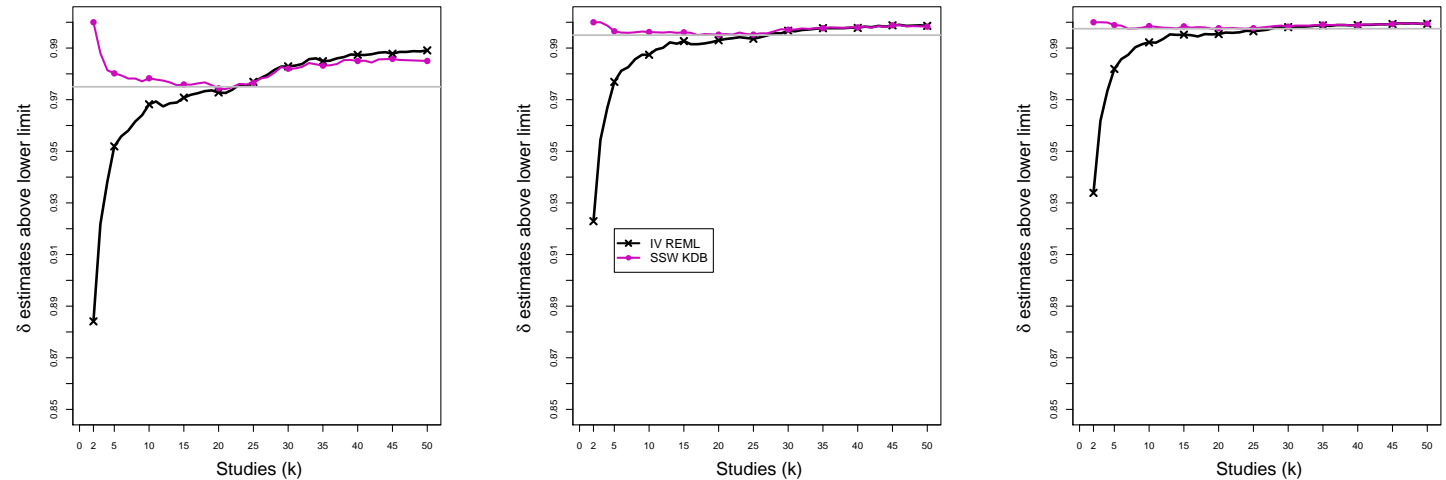

Figure C4. 7: CMA - CCoverage of the lower confidence interval of $\delta$ (above lower limit) at the confidence level $1-\alpha / 2$ for $\alpha=0.05,0.01,0.005$ when $\delta=1$ for $k \leq 25$ followed by a shift to $\delta=2$ for $k \geq 26, \tau^{2}=0,0.1,0.25,1, n=50, K=50$. Light grey line at $0.975,0.995,0.9975$. 

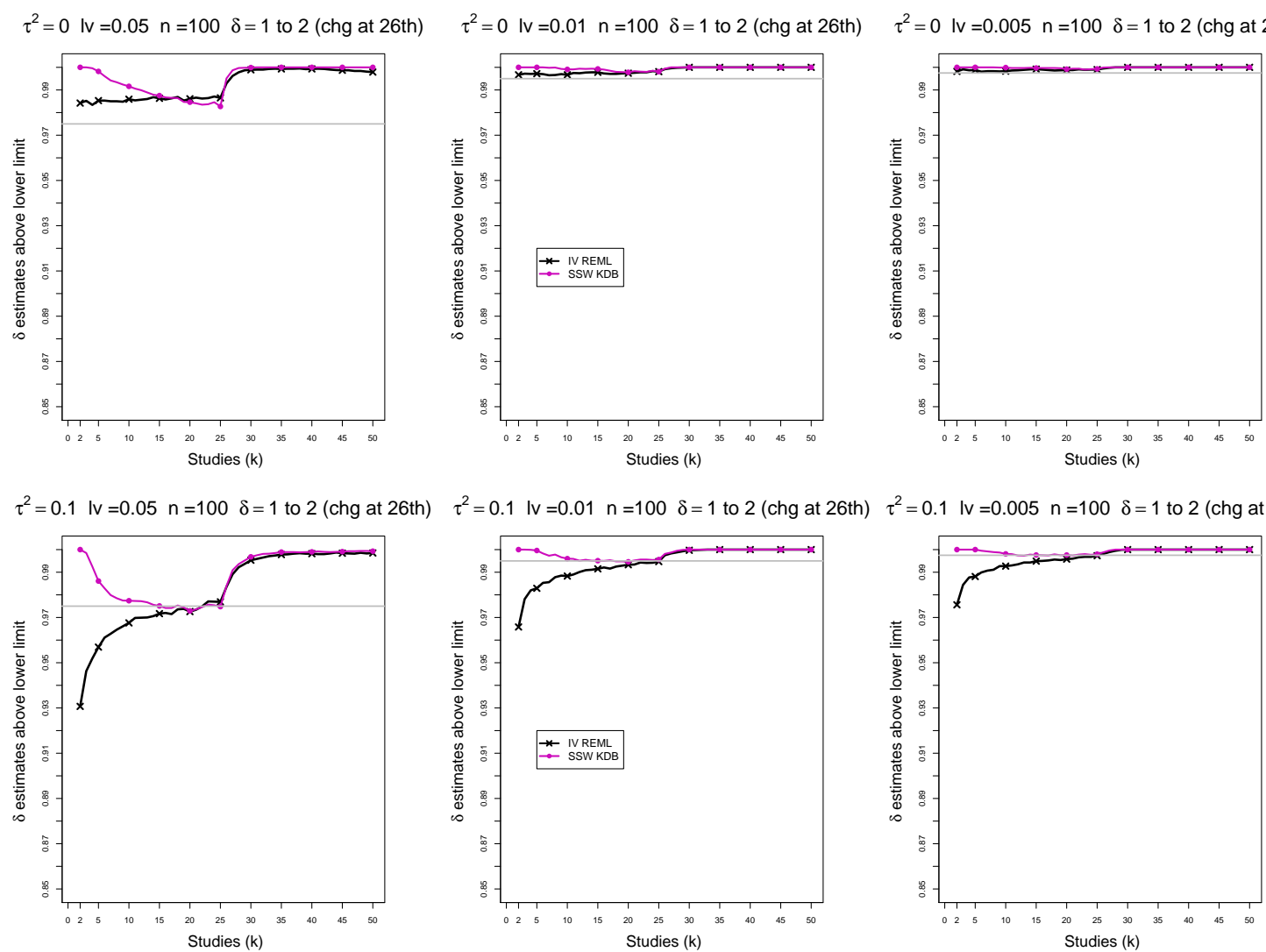

$\tau^{2}=0.25 \mathrm{IV}=0.05 \mathrm{n}=100 \delta=1$ to 2 (chg at 26 th) $\tau^{2}=0.25 \mathrm{IV}=0.01 \mathrm{n}=100 \delta=1$ to 2 (chg at 26th) $\tau^{2}=0.25 \mathrm{IV}=0.005 \mathrm{n}=100 \quad \delta=1$ to 2 (chg at 26 th)
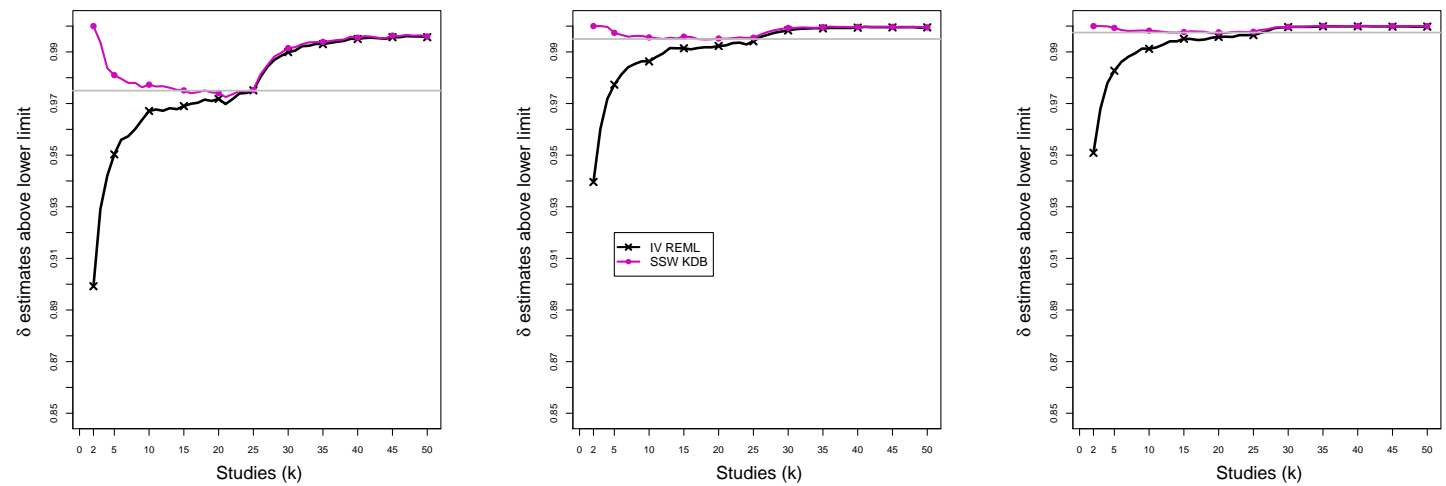

$\tau^{2}=1 \mathrm{IV}=0.05 \mathrm{n}=100 \delta=1$ to 2 (chg at 26th)

$\tau^{2}=1 \mathrm{lv}=0.01 \mathrm{n}=100 \delta=1$ to 2 (chg at 26 th)

$\tau^{2}=1 \mathrm{lv}=0.005 \mathrm{n}=100 \delta=1$ to 2 (chg at 26th)
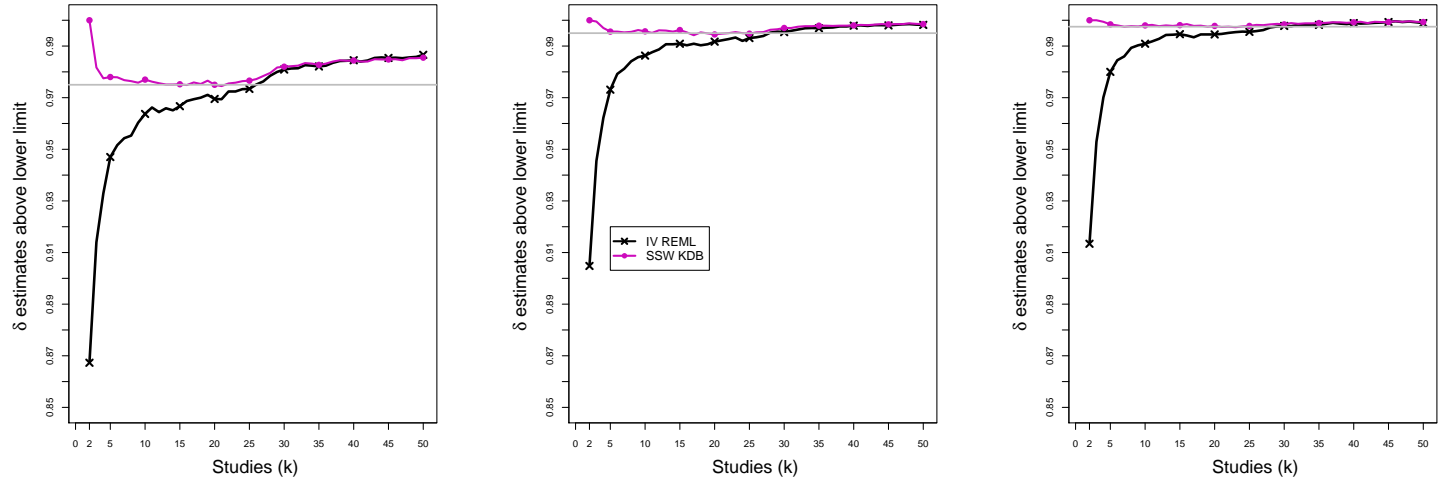

Figure C4. 8: CMA - Coverage of the lower confidence interval of $\delta$ (above lower limit) at the confidence level $1-\alpha / 2$ for $\alpha=0.05,0.01,0.005$ when $\delta=1$ for $k \leq 25$ followed by a shift to $\delta=2$ for $k \geq 26, \tau^{2}=0,0.1,0.25,1, n=100, K=50$. Light grey line at 

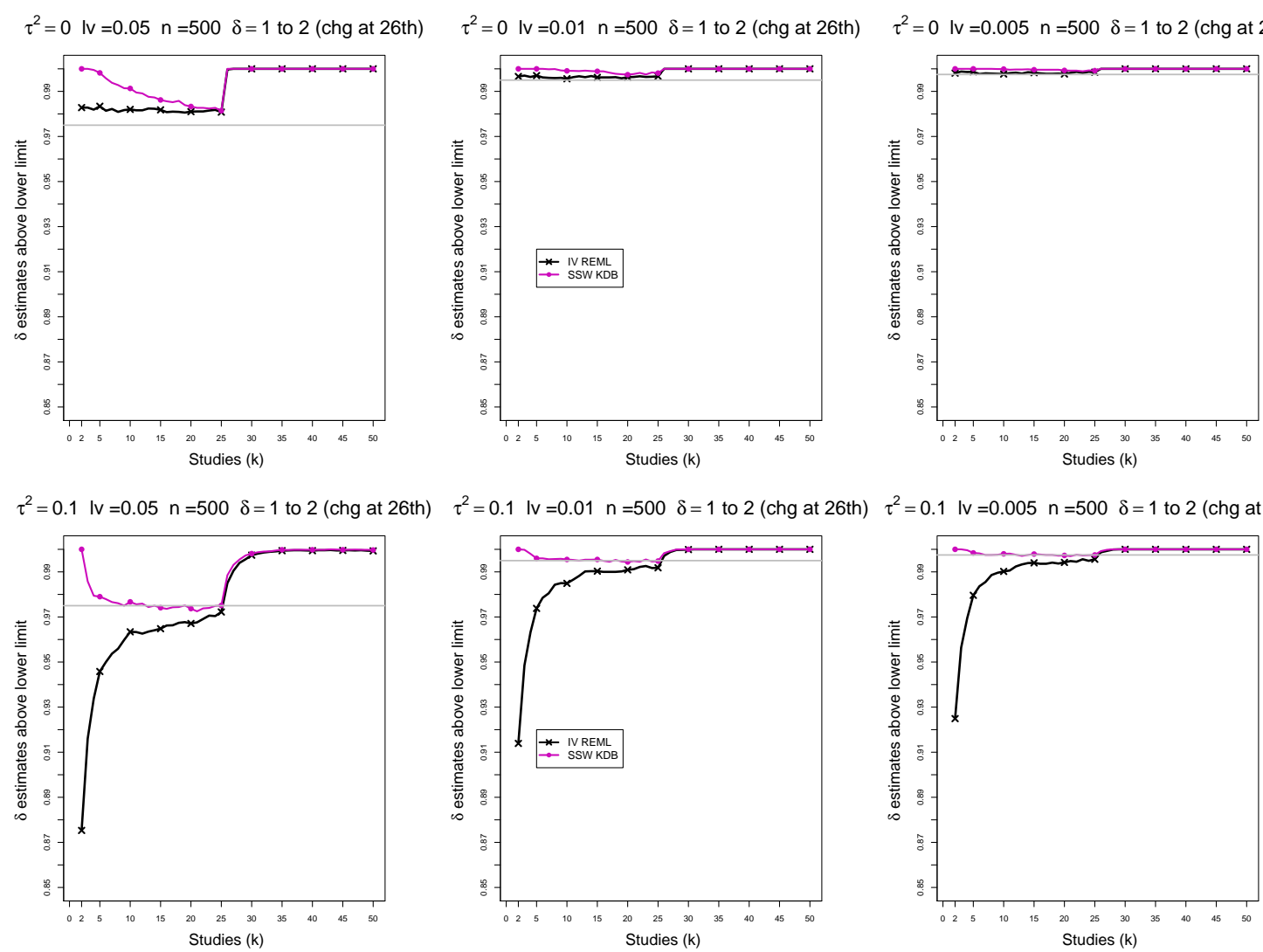

$\tau^{2}=0.25 \mathrm{IV}=0.05 \mathrm{n}=500 \delta=1$ to 2 (chg at 26 th) $\tau^{2}=0.25 \mathrm{IV}=0.01 \mathrm{n}=500 \delta=1$ to 2 (chg at 26th) $\tau^{2}=0.25 \mathrm{IV}=0.005 \mathrm{n}=500 \quad \delta=1$ to 2 (chg at 26 th)
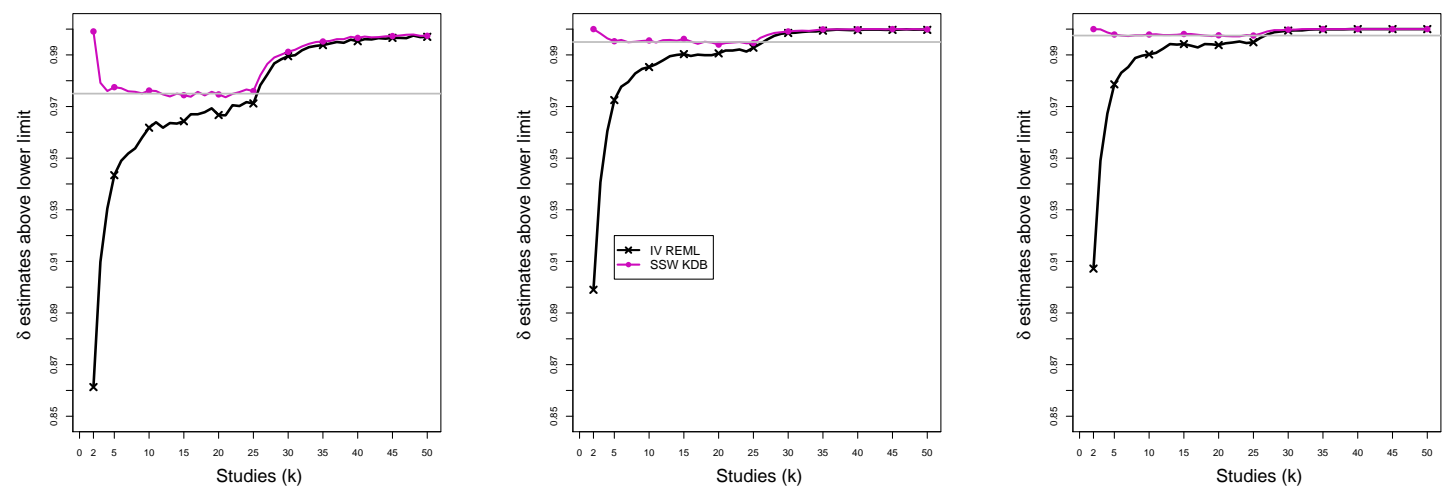

$\tau^{2}=1 \mathrm{IV}=0.05 \mathrm{n}=500 \delta=1$ to 2 (chg at $26 \mathrm{th}$ )

$\tau^{2}=1 \mathrm{lv}=0.01 \mathrm{n}=500 \delta=1$ to 2 (chg at 26 th)

$\tau^{2}=1 \mathrm{lv}=0.005 \mathrm{n}=500 \delta=1$ to 2 (chg at 26th)
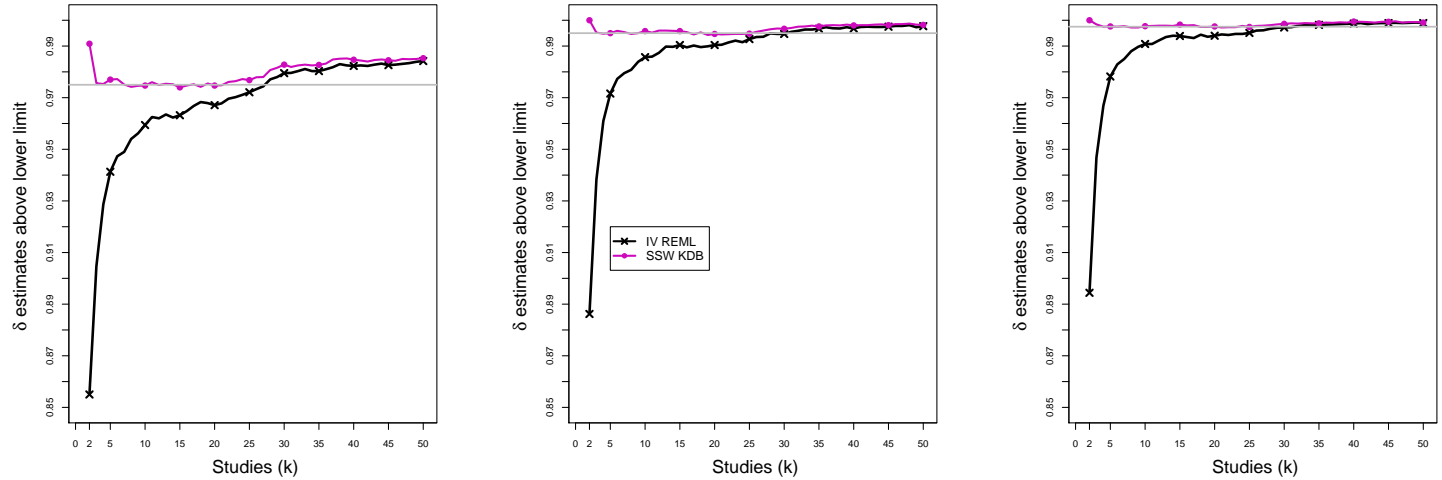

Figure C4. 9: CMA - Coverage of the lower confidence interval of $\delta$ (above lower limit) at the confidence level $1-\alpha / 2$ for $\alpha=0.05,0.01,0.005$ when $\delta=1$ for $k \leq 25$ followed by a shift to $\delta=2$ for $k \geq 26, \tau^{2}=0,0.1,0.25,1, n=500, K=50$. Light grey line at $0.975,0.995,0.9975$. 

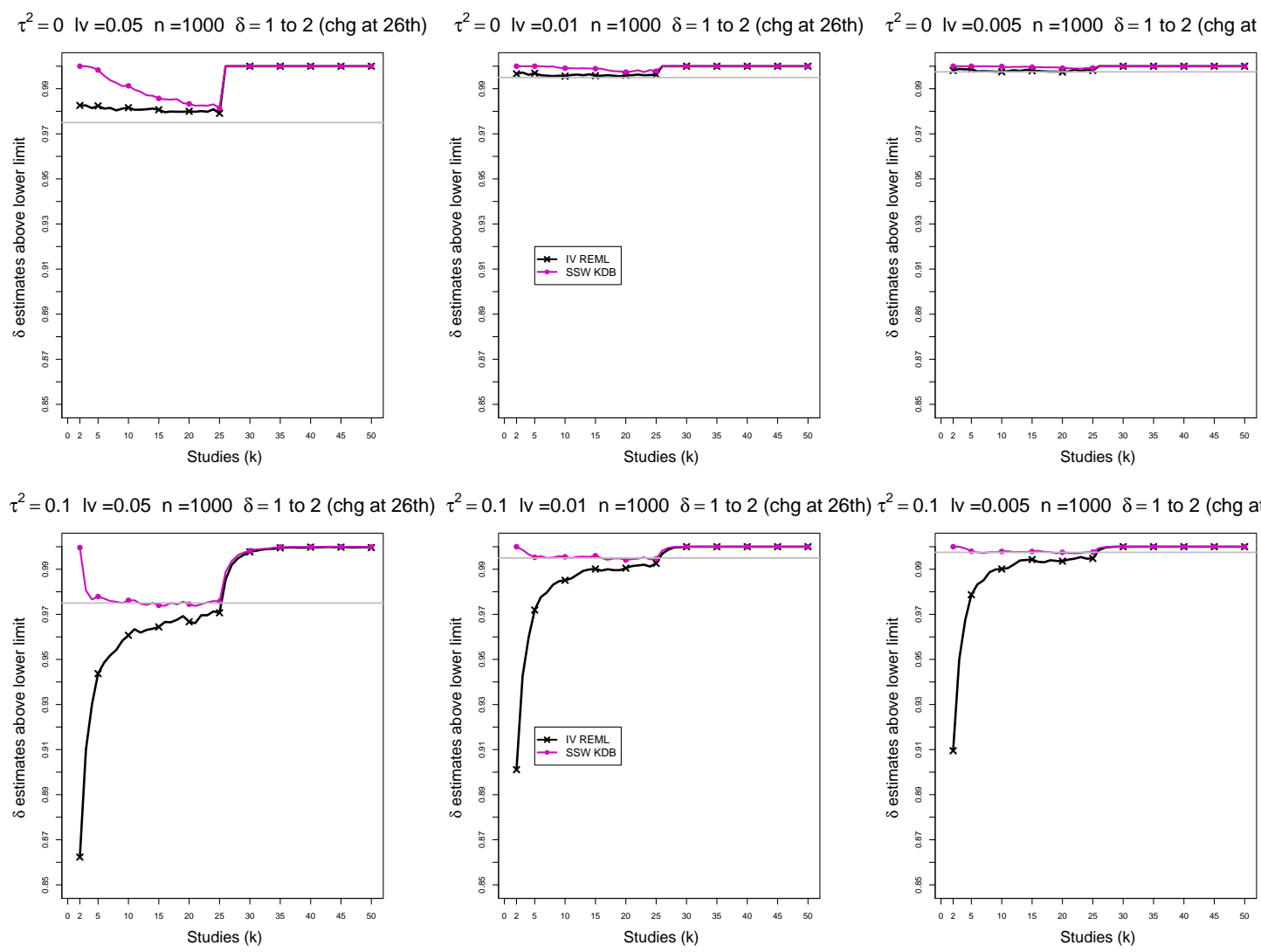

$\tau^{2}=0.25 \mathrm{lv}=0.05 \mathrm{n}=1000 \delta=1$ to 2 (chg at 26 th) $\tau^{2}=0.25 \mathrm{lv}=0.01 \mathrm{n}=1000 \quad \delta=1$ to 2 (chg at 26 th) $:^{2}=0.25 \mathrm{lv}=0.005 \mathrm{n}=1000 \delta=1$ to 2 (chg at 26 th
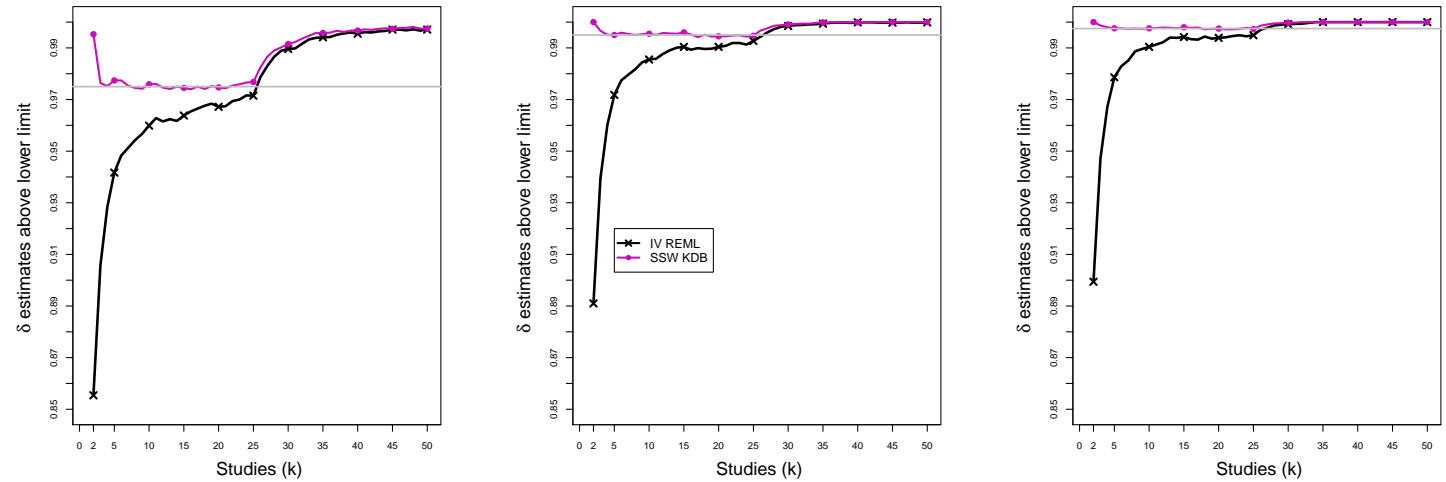

$\tau^{2}=1 \mathrm{lv}=0.05 \mathrm{n}=1000 \delta=1$ to 2 (chg at 26th)
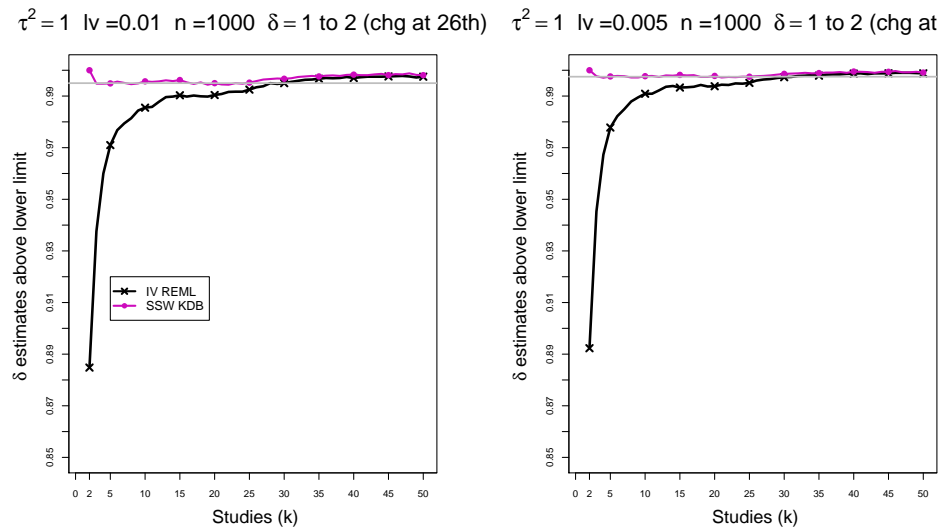

Figure C4. 10: CMA - Coverage of the lower confidence interval of $\delta$ (above lower limit) at the confidence level $1-\alpha / 2$ for $\alpha=0.05,0.01,0.005$ when $\delta=1$ for $k \leq 25$ followed by a shift to $\delta=2$ for $k \geq 26, \tau^{2}=0,0.1,0.25,1, n=1000, K=50$. Light grey line at 

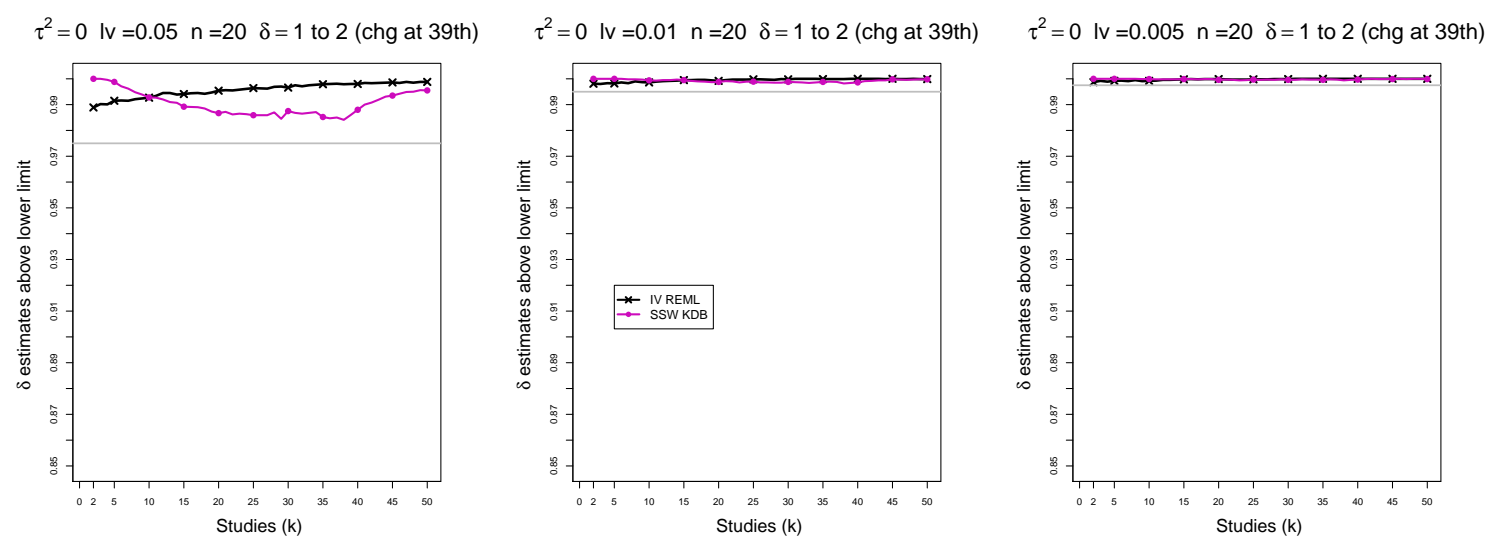

$\tau^{2}=0.1 \mathrm{lv}=0.05 \mathrm{n}=20 \quad \delta=1$ to 2 (chg at 39 th)
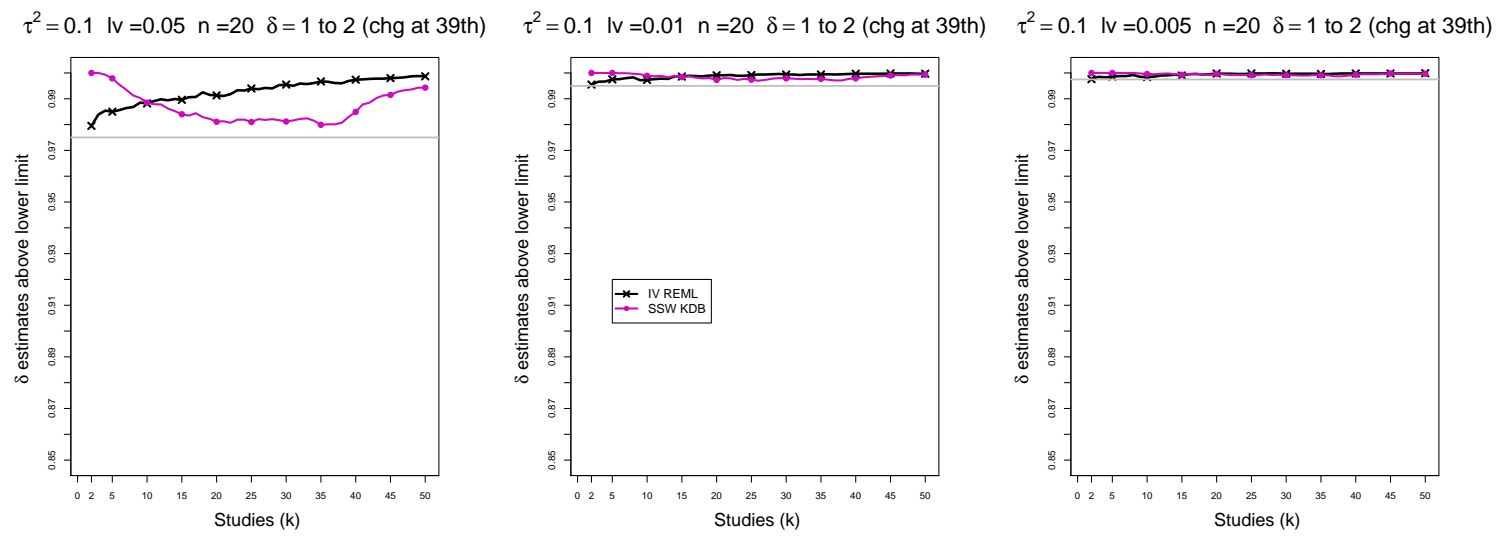

$\tau^{2}=0.25 \mathrm{lv}=0.05 \mathrm{n}=20 \quad \delta=1$ to 2 (chg at 39 th)

$\tau^{2}=0.25 \mathrm{IV}=0.01 \mathrm{n}=20 \delta=1$ to 2 (chg at 39th) $\tau^{2}=0.25 \mathrm{IV}=0.005 \mathrm{n}=20 \delta=1$ to 2 (chg at 39th)
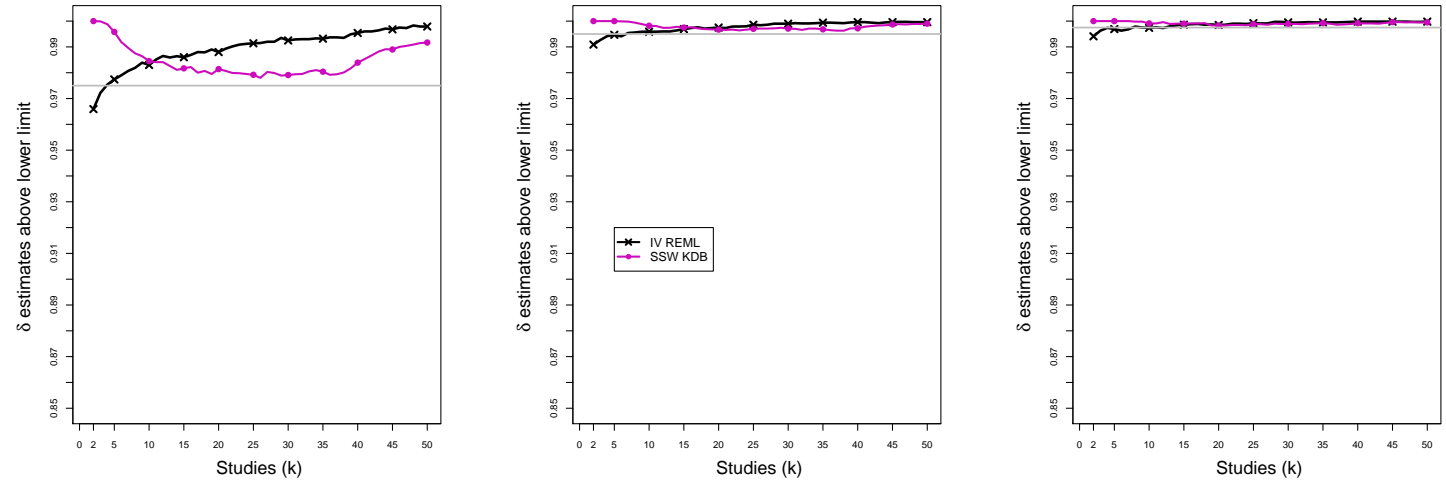

$\tau^{2}=1 \mathrm{lv}=0.05 \mathrm{n}=20 \quad \delta=1$ to 2 (chg at 39 th)

$\tau^{2}=1 \mathrm{lv}=0.01 \mathrm{n}=20 \quad \delta=1$ to 2 (chg at 39 th)

$\tau^{2}=1 \mathrm{lv}=0.005 \mathrm{n}=20 \delta=1$ to 2 (chg at 39th)
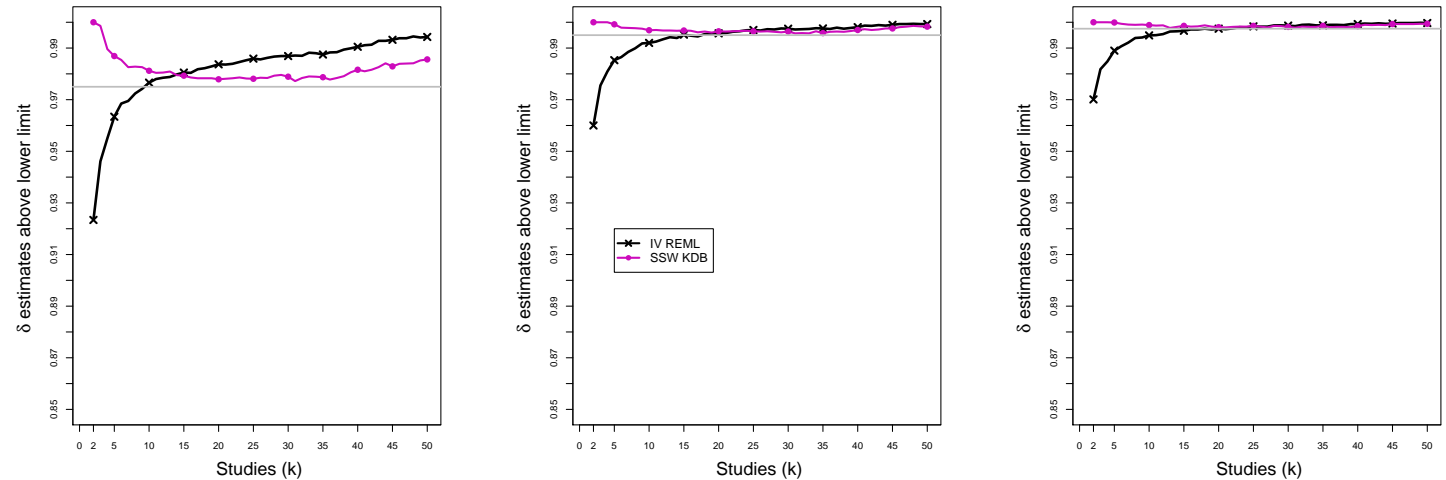

Figure C4. 11: CMA - Coverage of the lower confidence interval of $\delta$ (above lower limit) at the confidence level $1-\alpha / 2$ for $\alpha=0.05,0.01,0.005$ when $\delta=1$ for $k \leq 38$ followed by a shift to $\delta=2$ for $k \geq 39, \tau^{2}=0,0.1,0.25,1, n=20, K=50$. Light grey line at $0.975,0.995,0.9975$. 

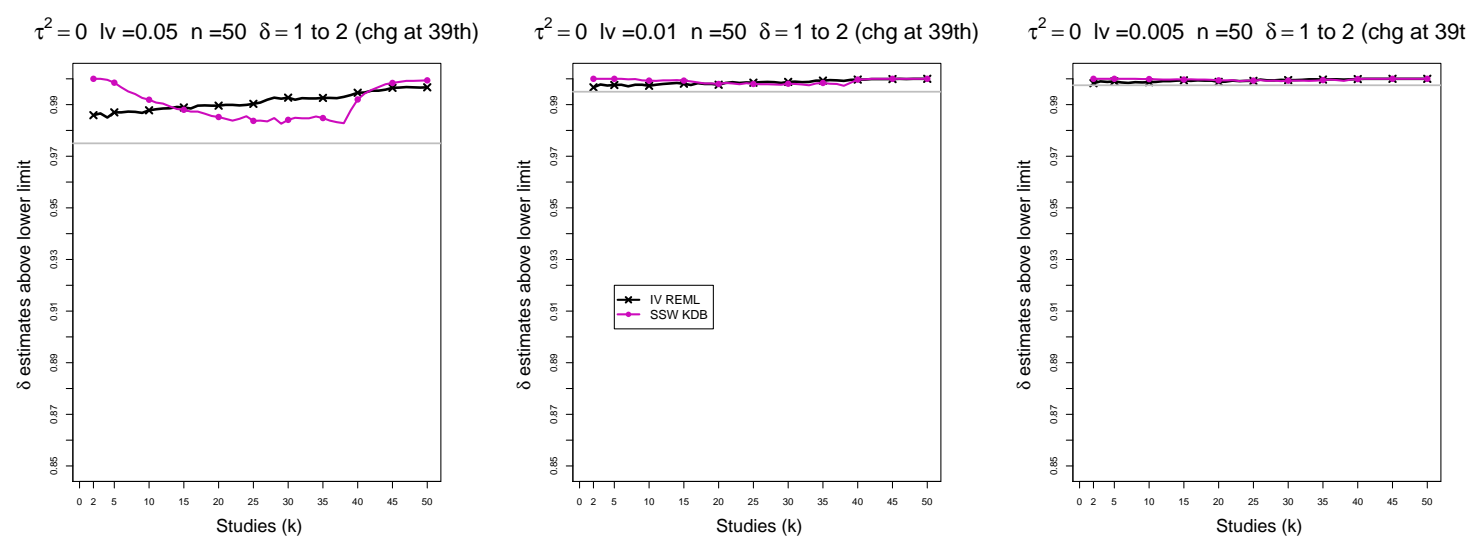

$\tau^{2}=0.1 \mathrm{IV}=0.05 \mathrm{n}=50 \quad \delta=1$ to 2 (chg at 39 th)
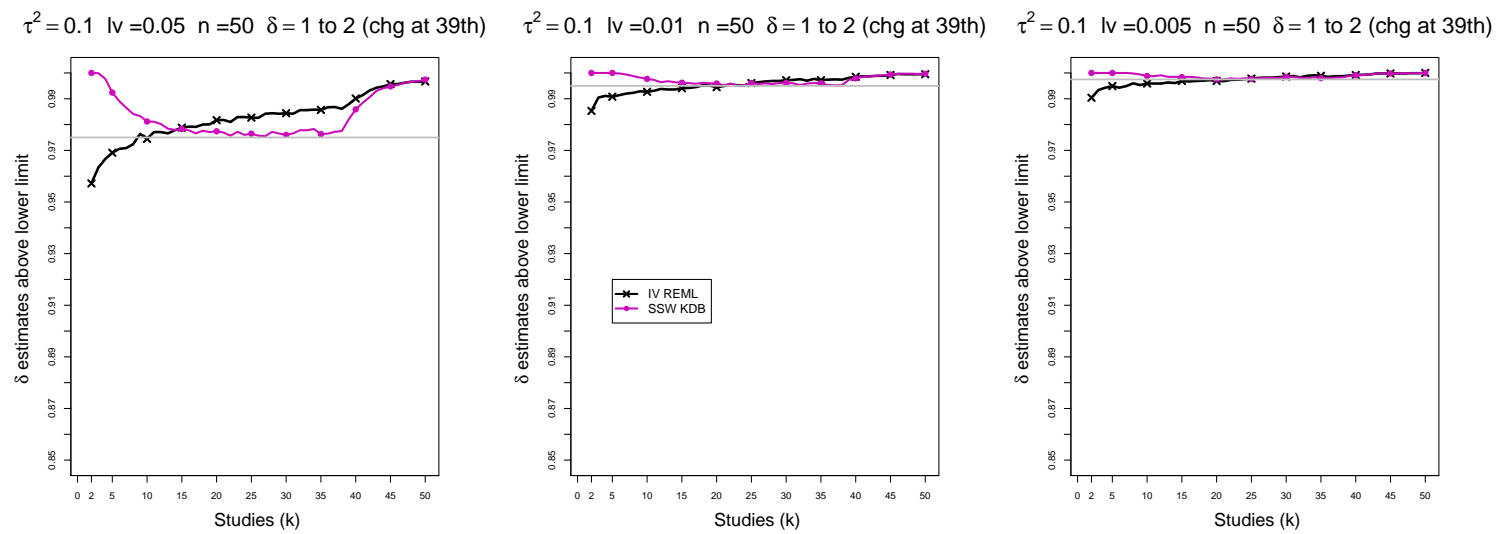

$\tau^{2}=0.25 \mathrm{lv}=0.05 \mathrm{n}=50 \quad \delta=1$ to 2 (chg at 39 th)
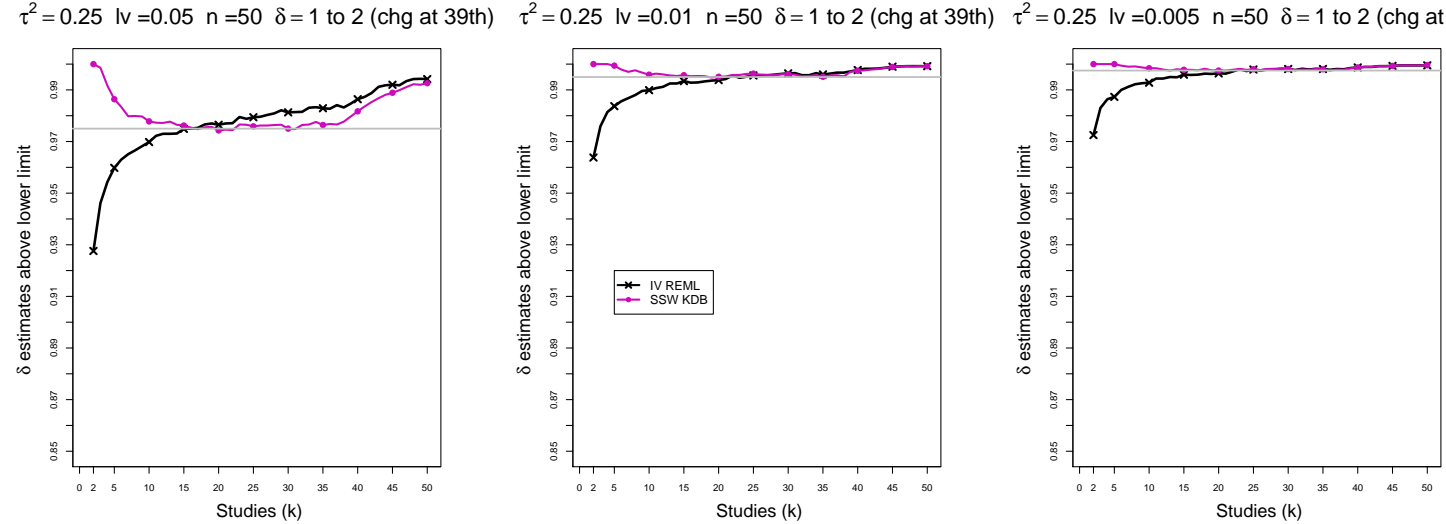

$\tau^{2}=1 \mathrm{lv}=0.01 \mathrm{n}=50 \delta=1$ to 2 (chg at 39 th)

$\tau^{2}=1 \mathrm{lv}=0.005 \mathrm{n}=50 \delta=1$ to 2 (chg at 39th)
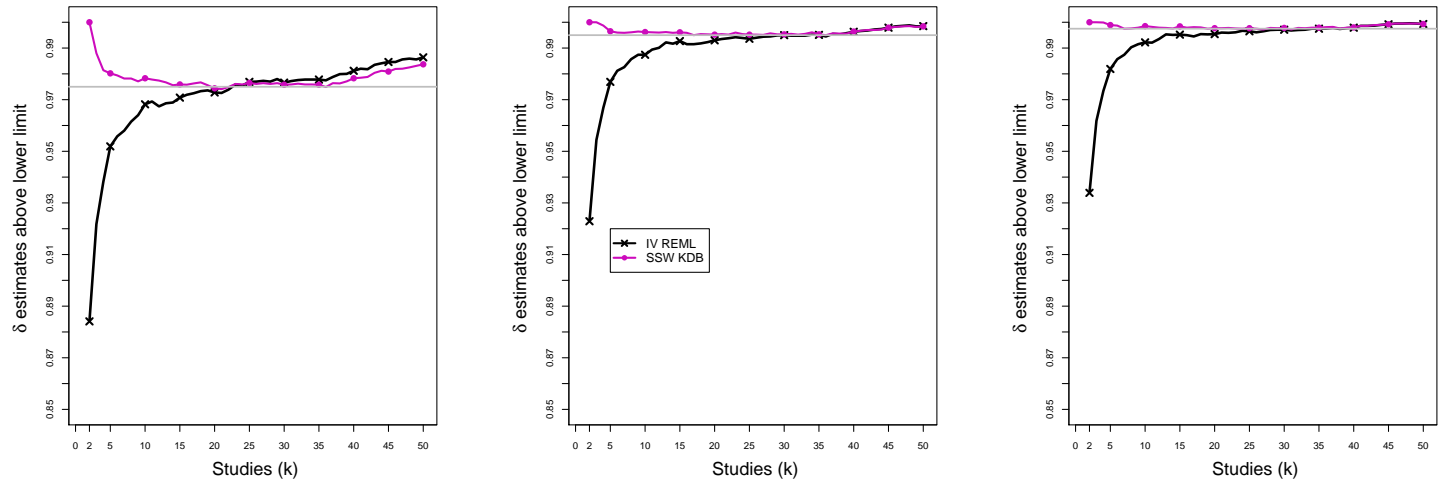

Figure C4. 12: CMA - Coverage of the lower confidence interval of $\delta$ (above lower limit) at the confidence level $1-\alpha / 2$ for $\alpha=0.05,0.01,0.005$ when $\delta=1$ for $k \leq 38$ followed by a shift to $\delta=2$ for $k \geq 39, \tau^{2}=0,0.1,0.25,1, n=50, K=50$. Light grey line at $0.975,0.995,0.9975$. 

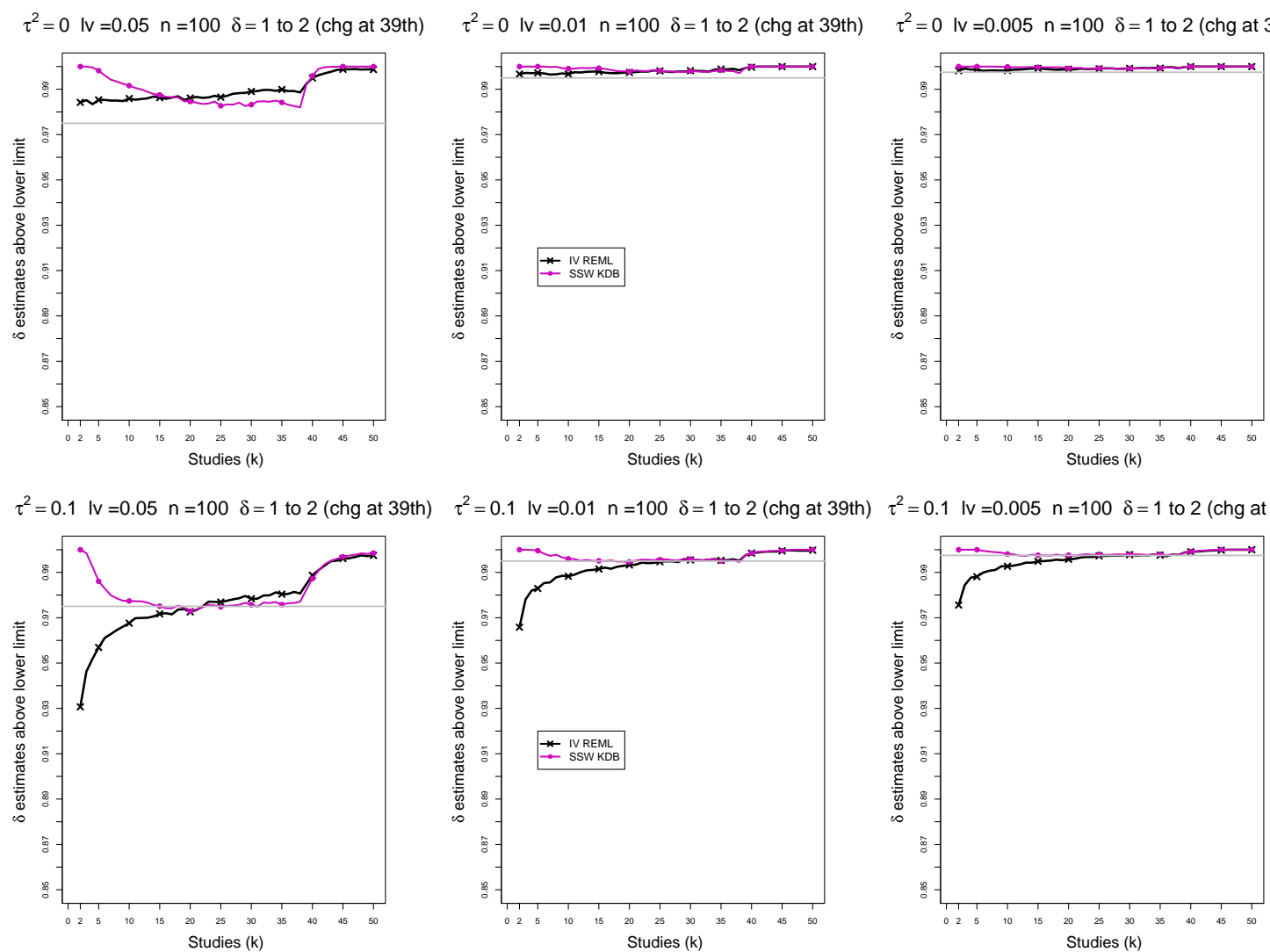

$\tau^{2}=0.25 \mathrm{IV}=0.05 \mathrm{n}=100 \delta=1$ to 2 (chg at 39 th) $\tau^{2}=0.25 \mathrm{IV}=0.01 \mathrm{n}=100 \delta=1$ to 2 (chg at 39 th) $\tau^{2}=0.25 \mathrm{IV}=0.005 \mathrm{n}=100 \quad \delta=1$ to 2 (chg at 39 th)
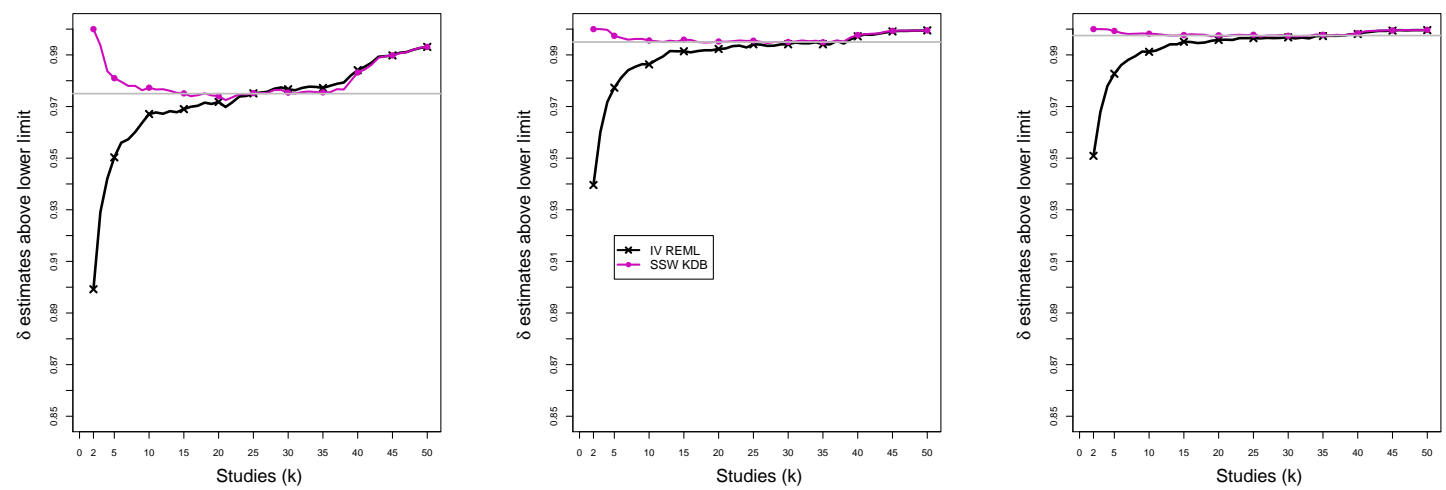

$\tau^{2}=1 \mathrm{IV}=0.05 \mathrm{n}=100 \delta=1$ to 2 (chg at 39 th)

$\tau^{2}=1 \mathrm{lv}=0.01 \mathrm{n}=100 \delta=1$ to 2 (chg at 39 th)

$\tau^{2}=1 \mathrm{lv}=0.005 \mathrm{n}=100 \delta=1$ to 2 (chg at 39th)
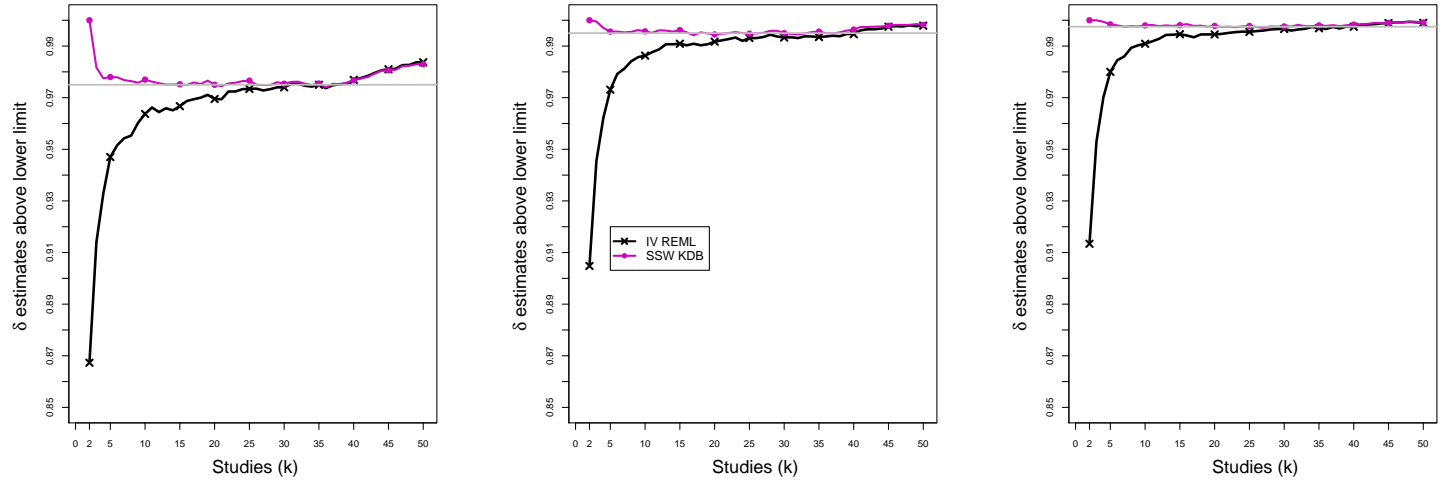

Figure C4. 13: CMA - Coverage of the lower confidence interval of $\delta$ (above lower limit) at the confidence level $1-\alpha / 2$ for $\alpha=0.05,0.01,0.005$ when $\delta=1$ for $k \leq 38$ followed by a shift to $\delta=2$ for $k \geq 39, \tau^{2}=0,0.1,0.25,1, n=100, K=50$. Light grey line at $0.975,0.995,0.9975$. 

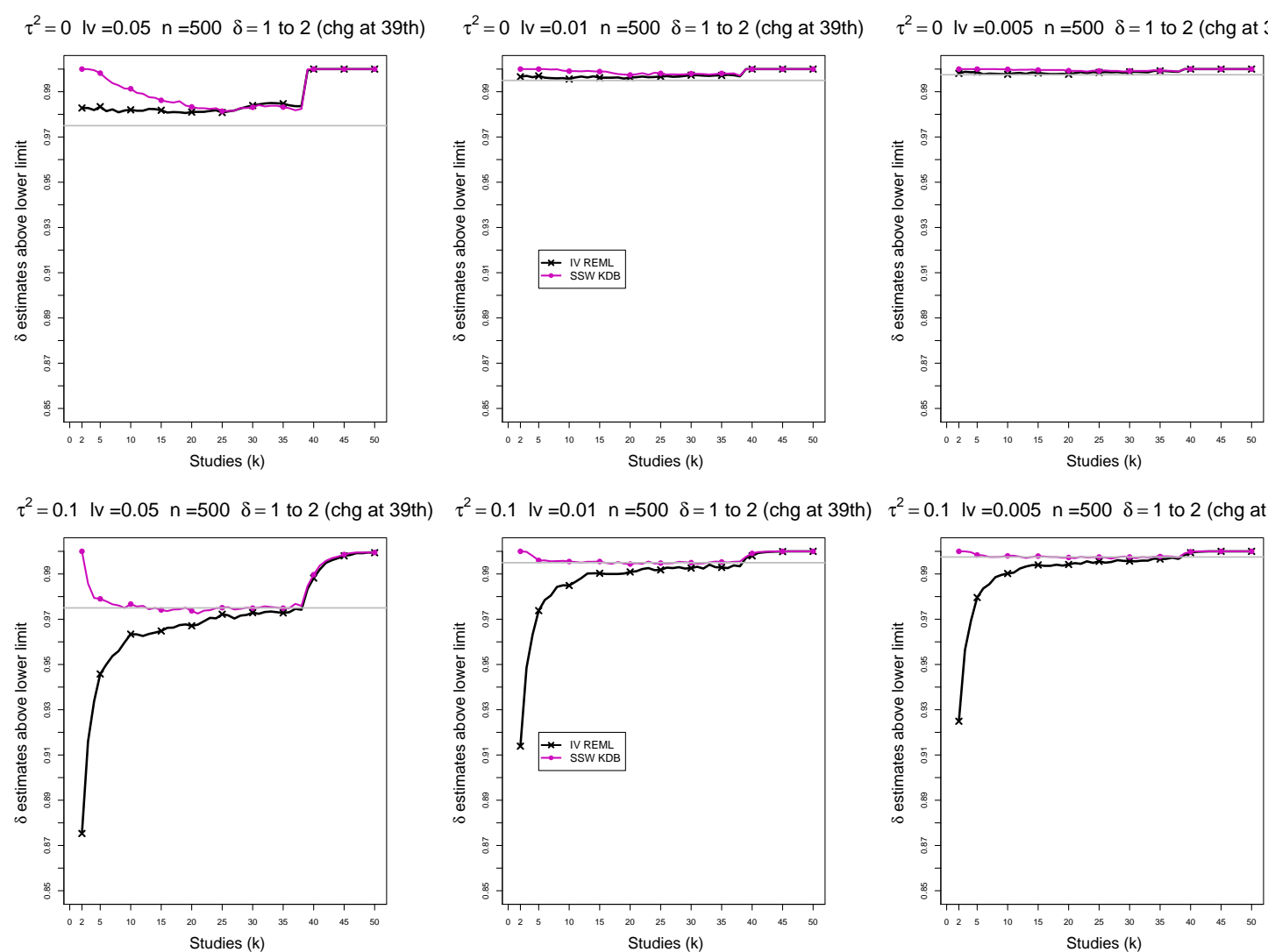

$\tau^{2}=0.25 \mathrm{IV}=0.05 \mathrm{n}=500 \delta=1$ to 2 (chg at 39 th) $\tau^{2}=0.25 \mathrm{IV}=0.01 \mathrm{n}=500 \delta=1$ to 2 (chg at 39 th) $\tau^{2}=0.25 \mathrm{lv}=0.005 \mathrm{n}=500 \quad \delta=1$ to 2 (chg at 39 th)
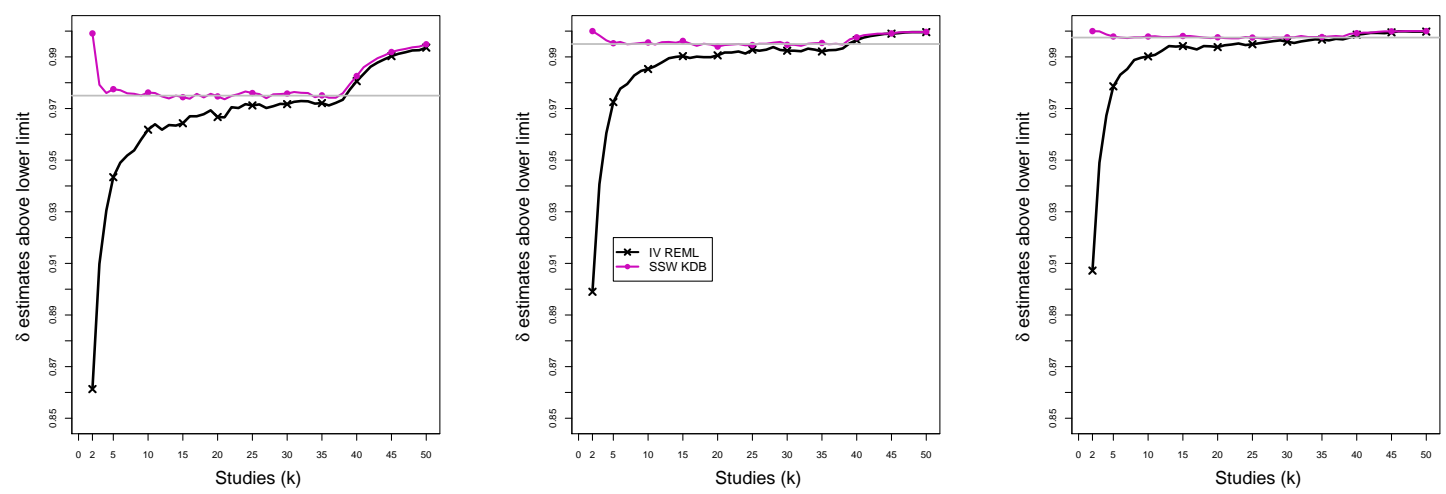

$\tau^{2}=1 \mathrm{IV}=0.05 \mathrm{n}=500 \delta=1$ to 2 (chg at 39 th)

$\tau^{2}=1 \mathrm{IV}=0.01 \mathrm{n}=500 \quad \delta=1$ to 2 (chg at 39th)

$\tau^{2}=1 \mathrm{lv}=0.005 \mathrm{n}=500 \delta=1$ to 2 (chg at 39th)
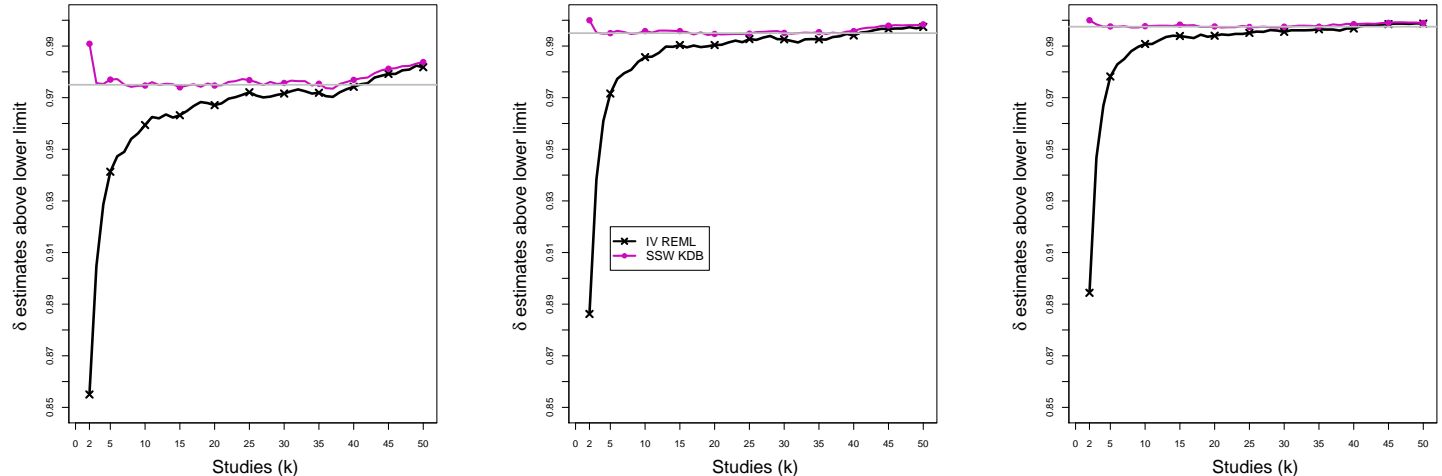

Figure C4. 14: CMA - Coverage of the lower confidence interval of $\delta$ (above lower limit) at the confidence level $1-\alpha / 2$ for $\alpha=0.05,0.01,0.005$ when $\delta=1$ for $k \leq 38$ followed by a shift to $\delta=2$ for $k \geq 39, \tau^{2}=0,0.1,0.25,1, n=500, K=50$. Light grey line at $0.975,0.995,0.9975$. 

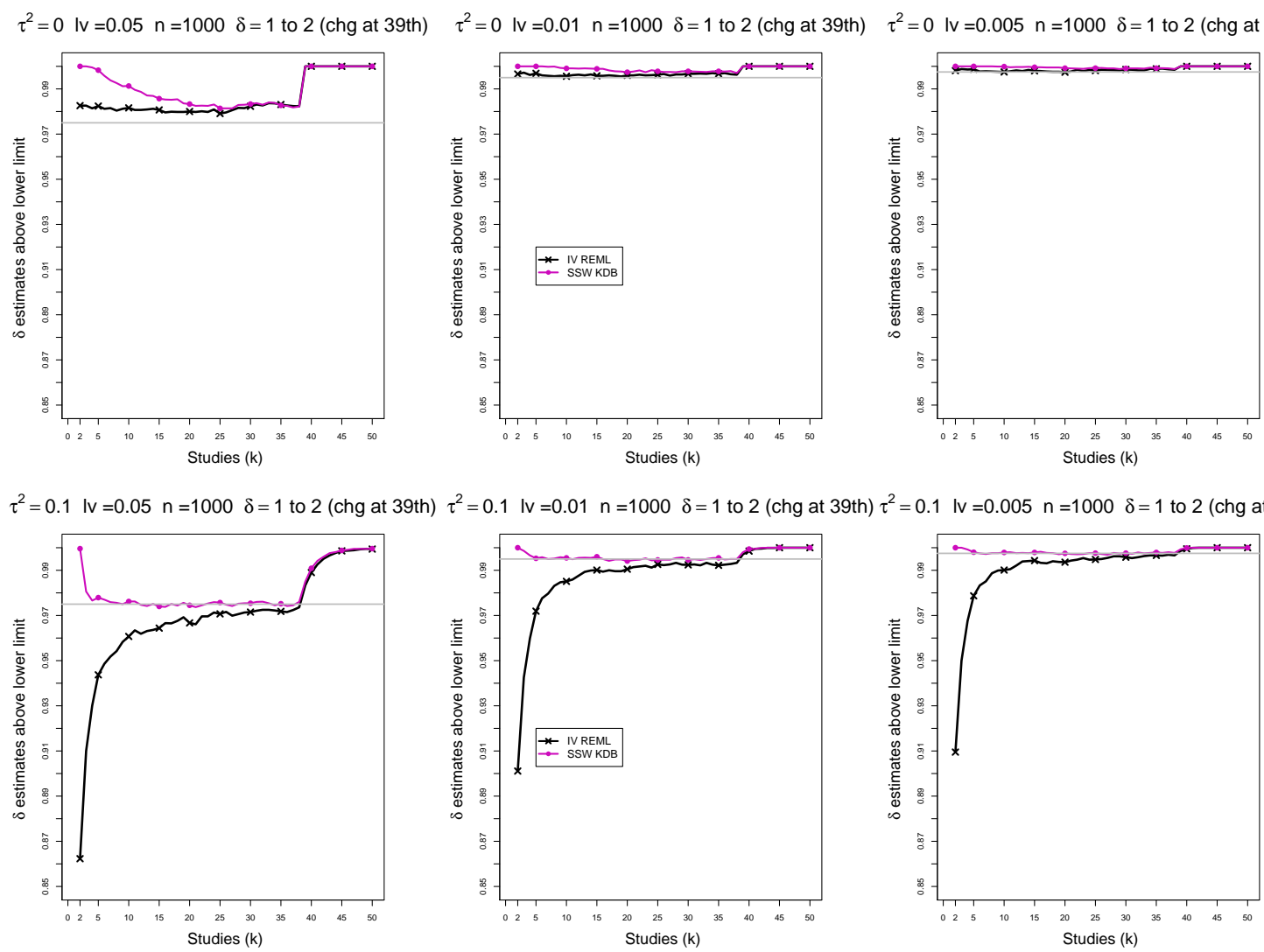

$\tau^{2}=0.25 \mathrm{lv}=0.05 \mathrm{n}=1000 \delta=1$ to 2 (chg at 39 th) $\tau^{2}=0.25 \mathrm{lv}=0.01 \mathrm{n}=1000 \quad \delta=1$ to 2 (chg at 39 th) $:^{2}=0.25 \mathrm{lv}=0.005 \mathrm{n}=1000 \delta=1$ to 2 (chg at 39 th
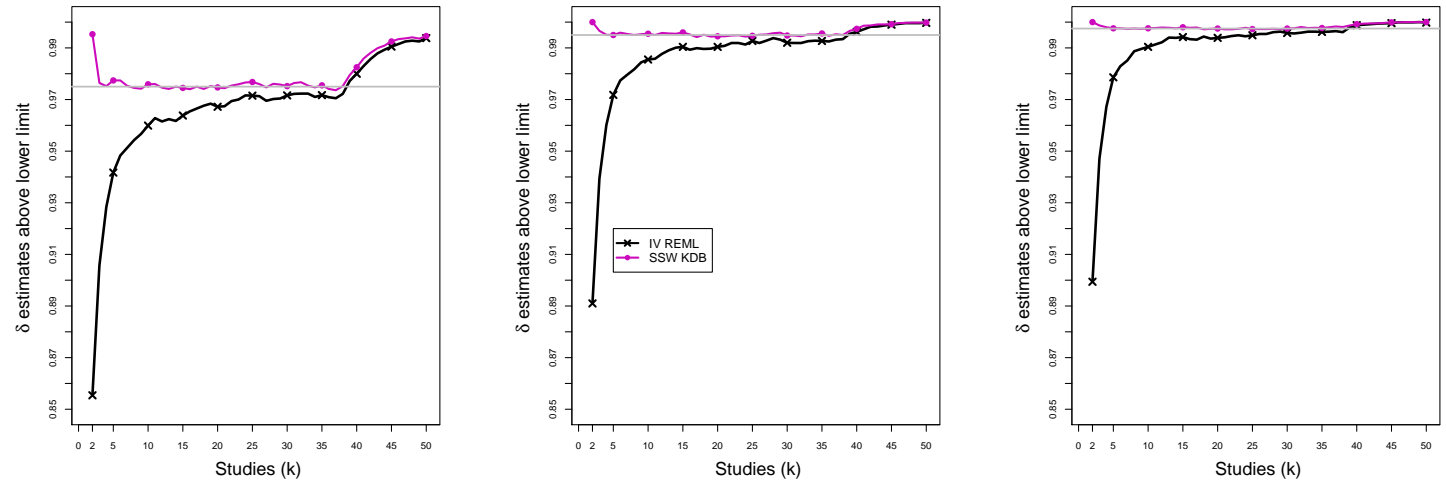

$\tau^{2}=1 \mathrm{IV}=0.05 \mathrm{n}=1000 \delta=1$ to 2 (chg at 39 th)

$\tau^{2}=1 \mathrm{lv}=0.01 \mathrm{n}=1000 \delta=1$ to 2 (chg at 39 th) $\tau^{2}=1 \mathrm{lv}=0.005 \mathrm{n}=1000 \delta=1$ to 2 (chg at 39th)
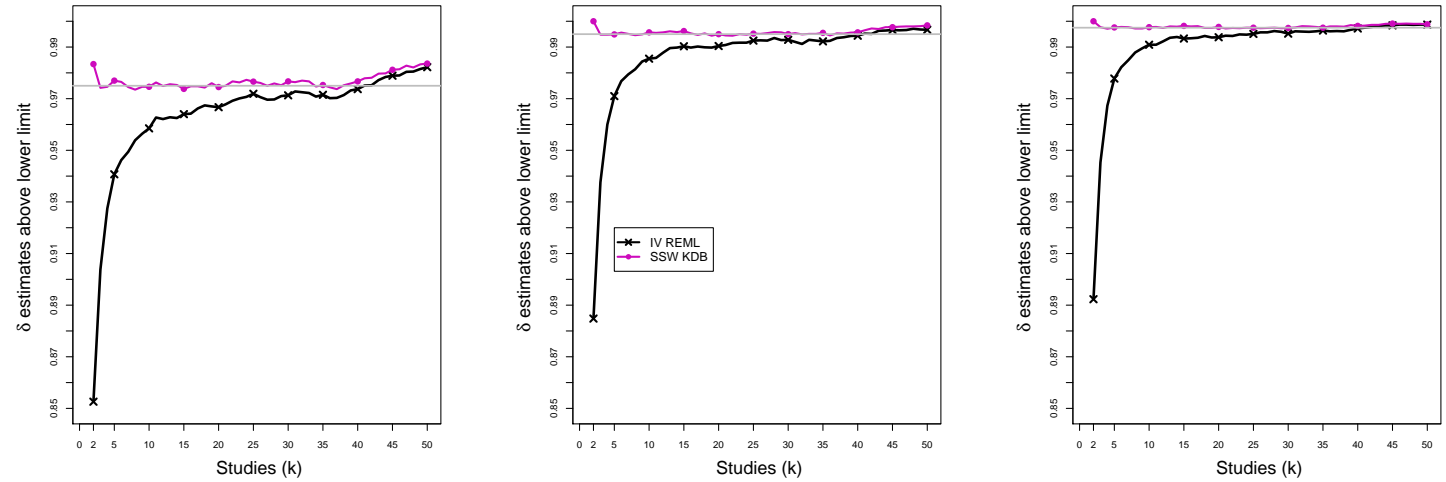

Figure C4. 15: CMA - Coverage of the lower confidence interval of $\delta$ (above lower limit) at the confidence level $1-\alpha / 2$ for $\alpha=0.05,0.01,0.005$ when $\delta=1$ for $k \leq 38$ followed by a shift to $\delta=2$ for $k \geq 39, \tau^{2}=0,0.1,0.25,1, n=1000, K=50$. Light grey line at 

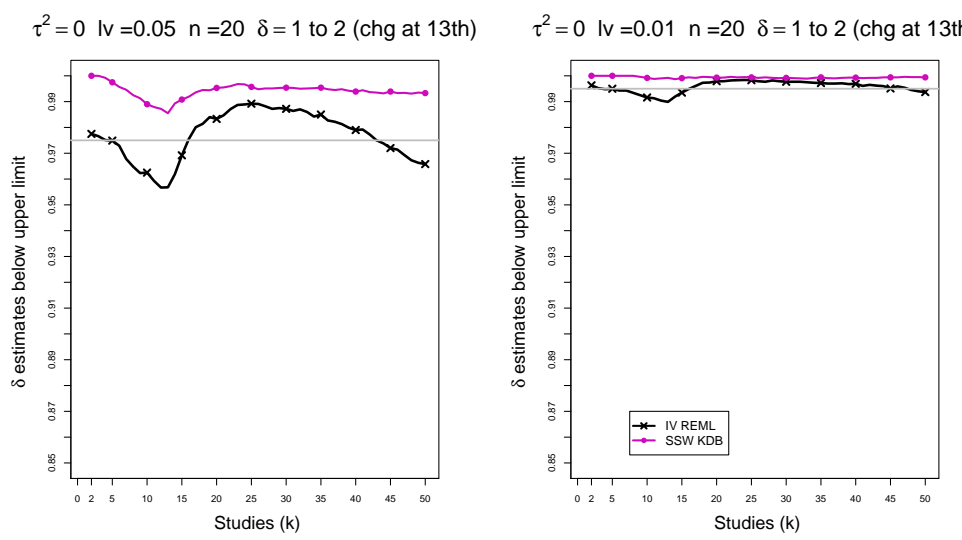

$\tau^{2}=0 \mathrm{lv}=0.005 \mathrm{n}=20 \delta=1$ to 2 (chg at 13 th)

$\tau^{2}=0.1 \mathrm{lv}=0.05 \mathrm{n}=20 \delta=1$ to 2 (chg at 13 th)

$\tau^{2}=0.1 \mathrm{lv}=0.01 \mathrm{n}=20 \quad \delta=1$ to 2 (chg at 13 th)
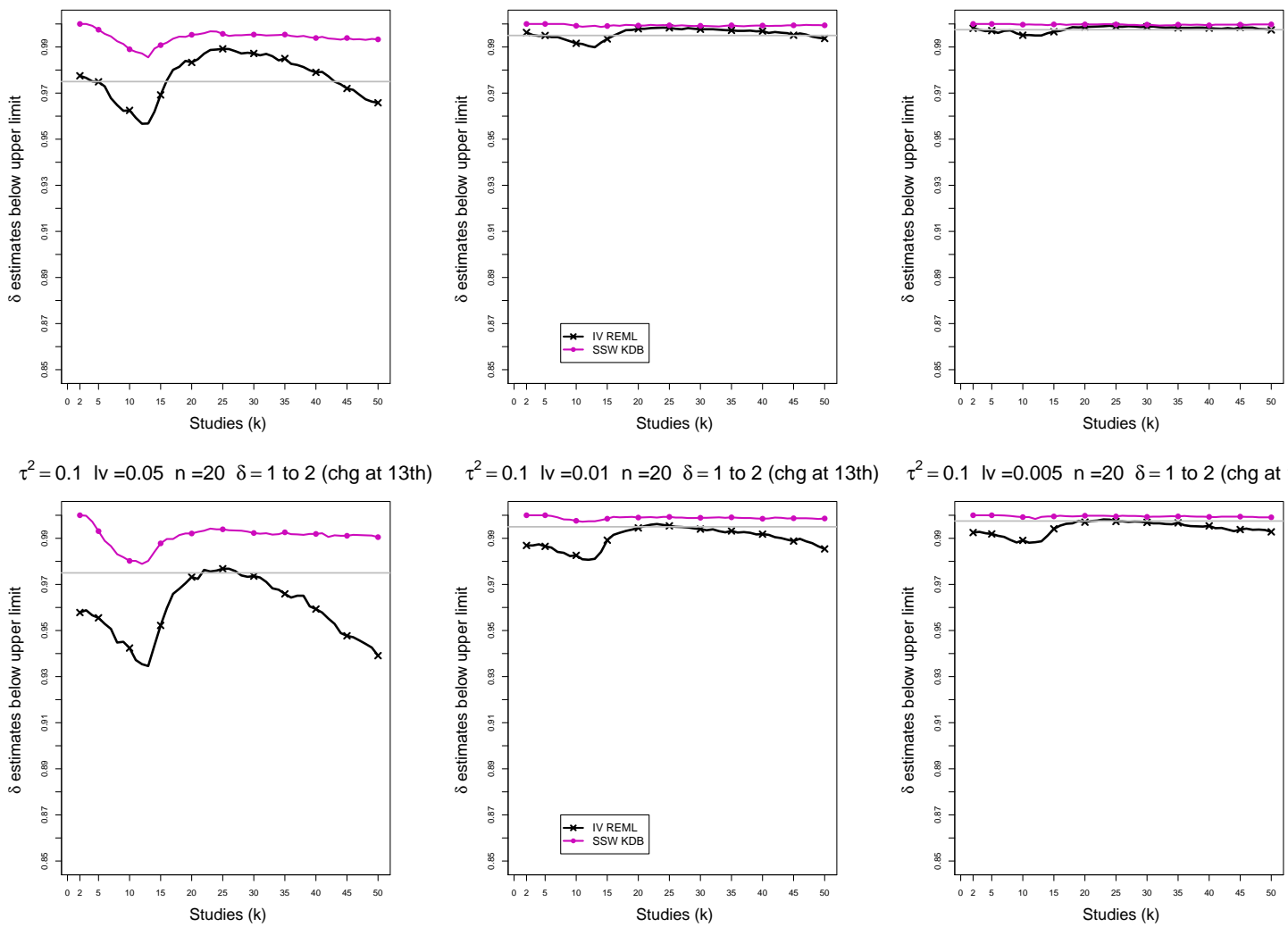

$\tau^{2}=0.1 \mathrm{lv}=0.005 \mathrm{n}=20 \delta=1$ to 2 (chg at 13 th $)$

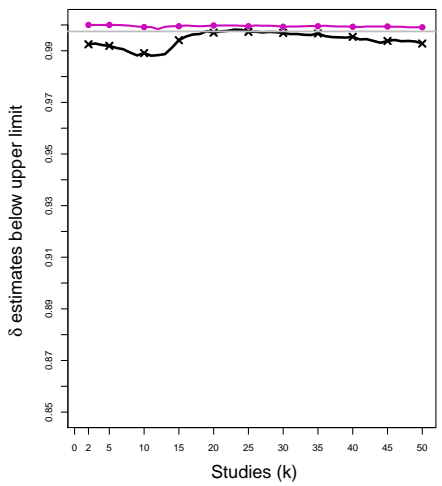

$\tau^{2}=0.25 \mathrm{IV}=0.05 \mathrm{n}=20 \quad \delta=1$ to 2 (chg at 13 th)
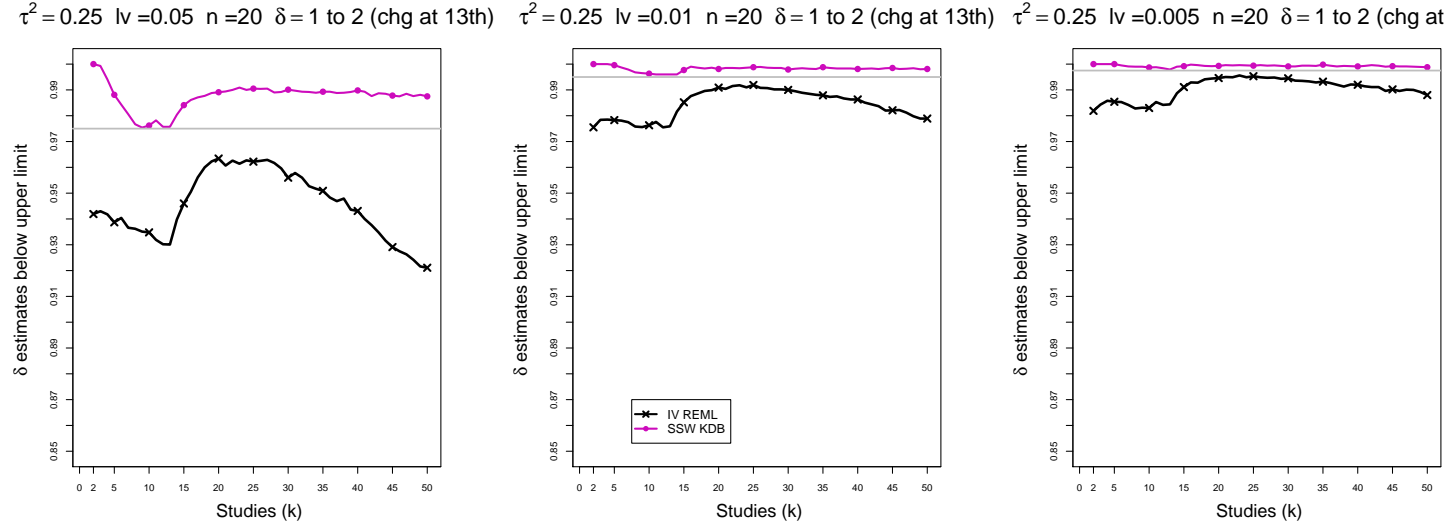

$\tau^{2}=1 \mathrm{lv}=0.01 \mathrm{n}=20 \quad \delta=1$ to 2 (chg at 13 th)

$\tau^{2}=1 \mathrm{lv}=0.005 \mathrm{n}=20 \delta=1$ to 2 (chg at 13 th)
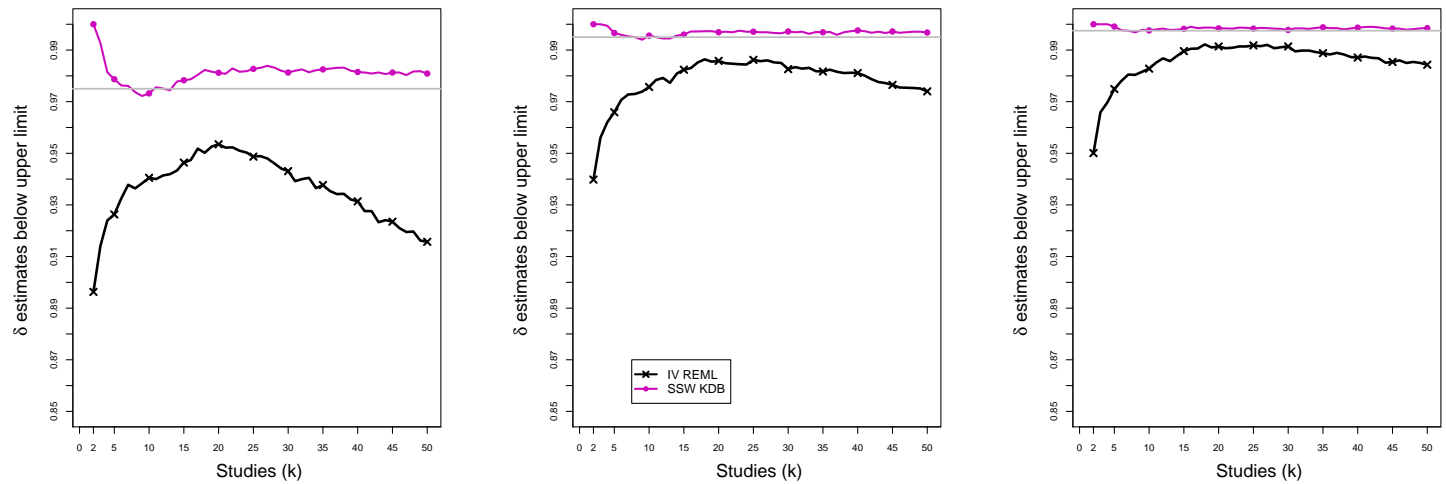

Figure C4. 16: CMA - Coverage of the upper confidence interval of $\delta$ (below upper limit) at the confidence level $1-\alpha / 2$ for $\alpha=0.05,0.01,0.005$ when $\delta=1$ for $k \leq 12$ followed by a shift to $\delta=2$ for $k \geq 13, \tau^{2}=0,0.1,0.25,1, n=20, K=50$. Light grey line at $0.975,0.995,0.9975$. 

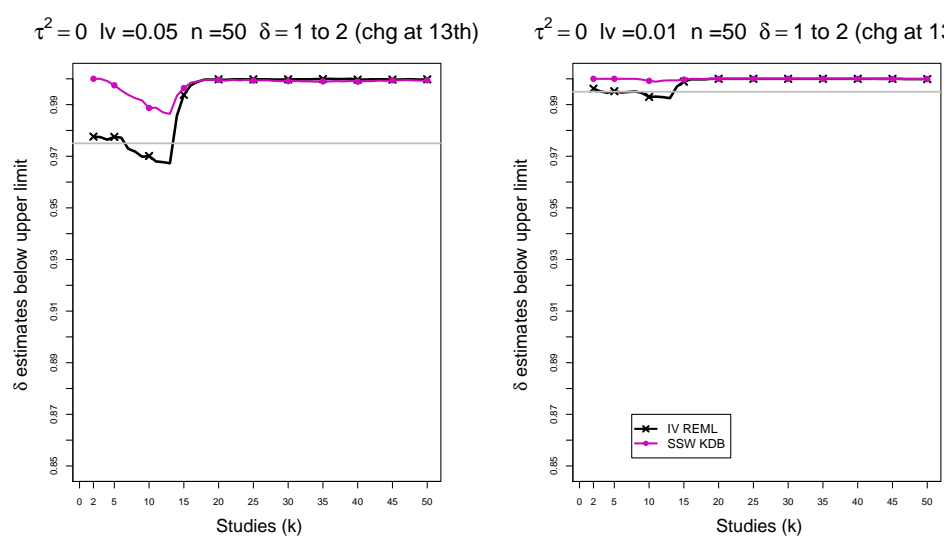

$\tau^{2}=0 \mathrm{IV}=0.005 \mathrm{n}=50 \quad \delta=1$ to 2 (chg at 13 th)

$\tau^{2}=0.1 \mathrm{lv}=0.05 \mathrm{n}=50 \delta=1$ to 2 (chg at 13 th)
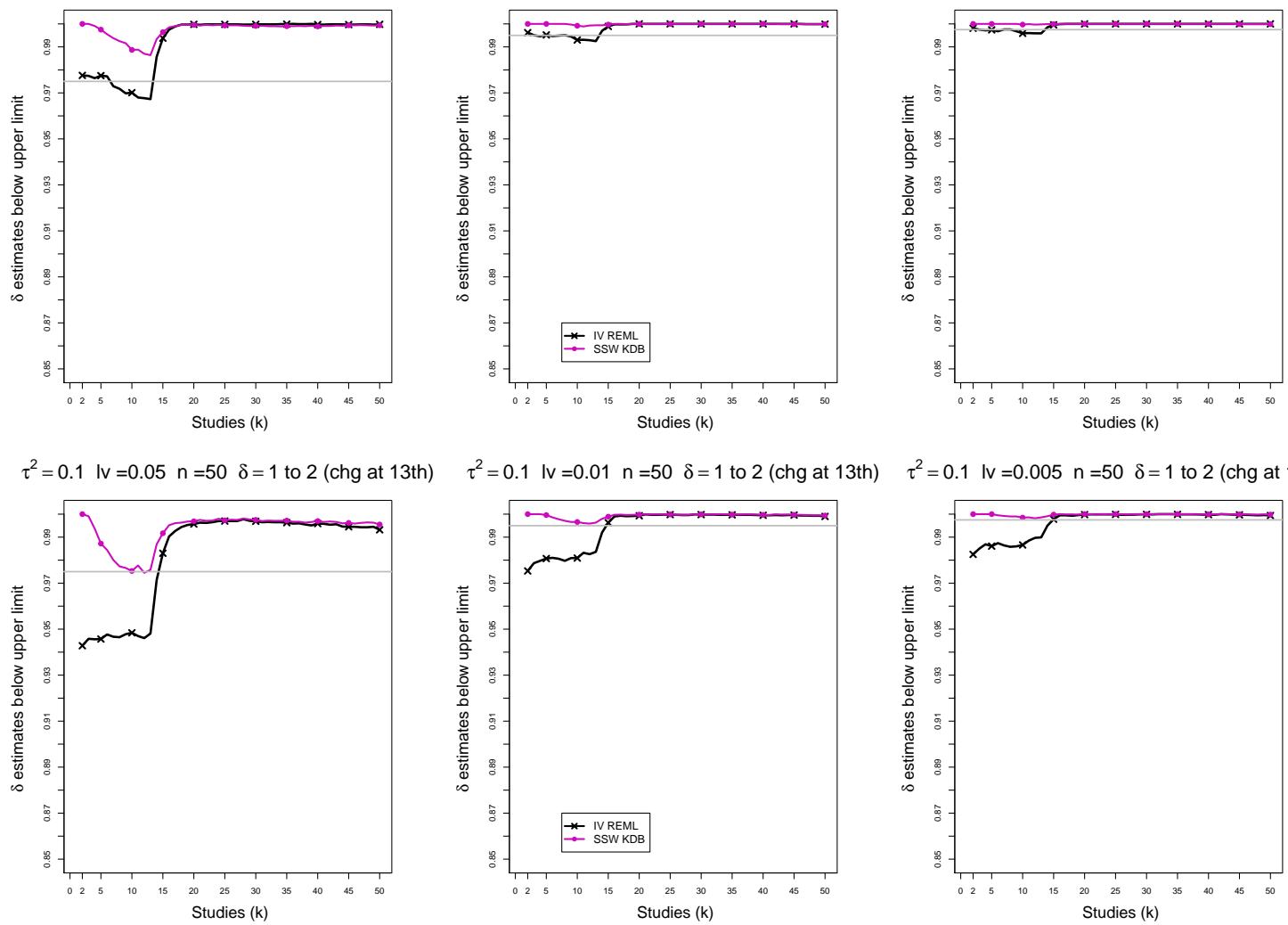

$\tau^{2}=0.1 \mathrm{lv}=0.005 \mathrm{n}=50 \delta=1$ to 2 (chg at 13 th)

$\tau^{2}=0.25 \mathrm{IV}=0.05 \mathrm{n}=50 \delta=1$ to 2 (chg at 13 th)
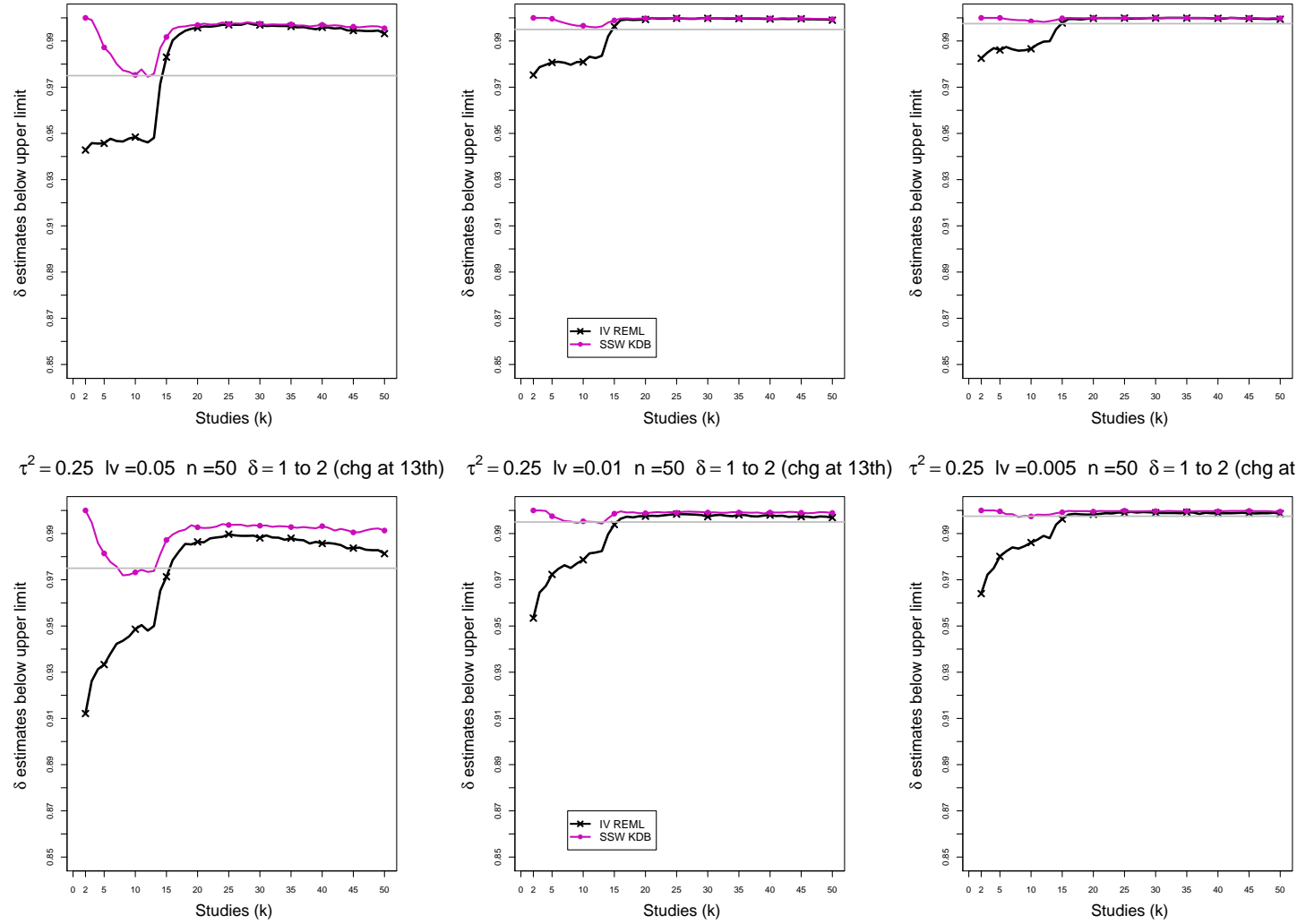

$\tau^{2}=1 \mathrm{IV}=0.05 \mathrm{n}=50 \delta=1$ to 2 (chg at 13 th)

$\tau^{2}=1 \mathrm{IV}=0.01 \mathrm{n}=50 \delta=1$ to 2 (chg at 13 th)

$\tau^{2}=1 \mathrm{lv}=0.005 \mathrm{n}=50 \delta=1$ to 2 (chg at 13th)
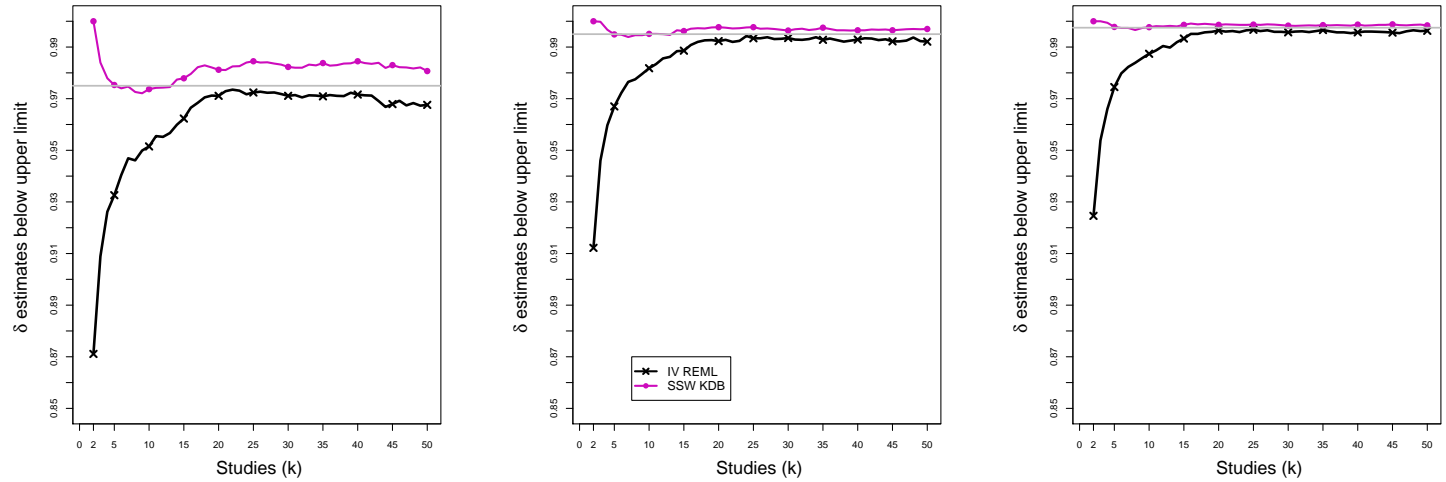

Figure C4. 17: CMA - Coverage of the upper confidence interval of $\delta$ (below upper limit) at the confidence level $1-\alpha / 2$ for $\alpha=0.05,0.01,0.005$ when $\delta=1$ for $k \leq 12$ followed by a shift to $\delta=2$ for $k \geq 13, \tau^{2}=0,0.1,0.25,1, n=50, K=50$. Light grey line at $0.975,0.995,0.9975$. 

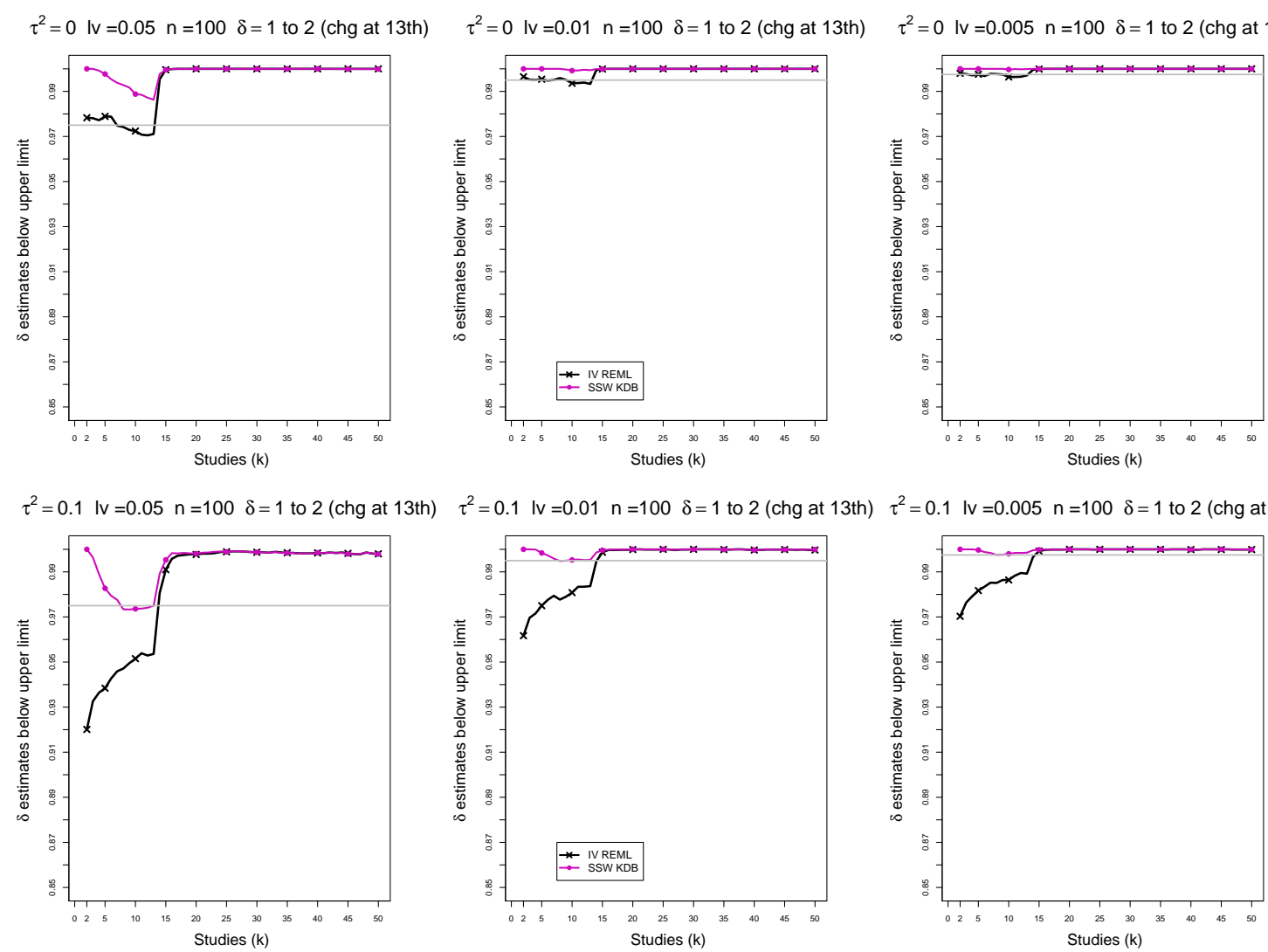

$\tau^{2}=0.25 \mathrm{IV}=0.05 \mathrm{n}=100 \delta=1$ to 2 (chg at 13 th) $\tau^{2}=0.25 \mathrm{IV}=0.01 \mathrm{n}=100 \delta=1$ to 2 (chg at 13 th) $\tau^{2}=0.25 \mathrm{IV}=0.005 \mathrm{n}=100 \quad \delta=1$ to 2 (chg at 13 th)
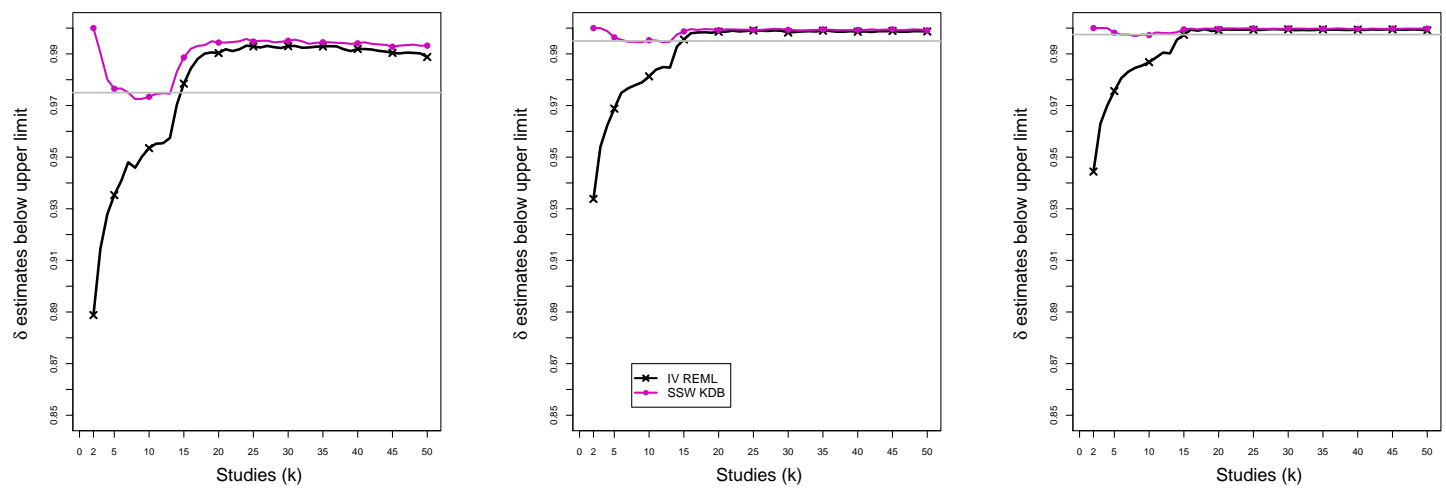

$\tau^{2}=1 \mathrm{IV}=0.05 \mathrm{n}=100 \delta=1$ to 2 (chg at 13 th)

$\tau^{2}=1 \mathrm{lv}=0.01 \mathrm{n}=100 \delta=1$ to 2 (chg at 13 th)

$\tau^{2}=1 \mathrm{lv}=0.005 \mathrm{n}=100 \delta=1$ to 2 (chg at 13th)
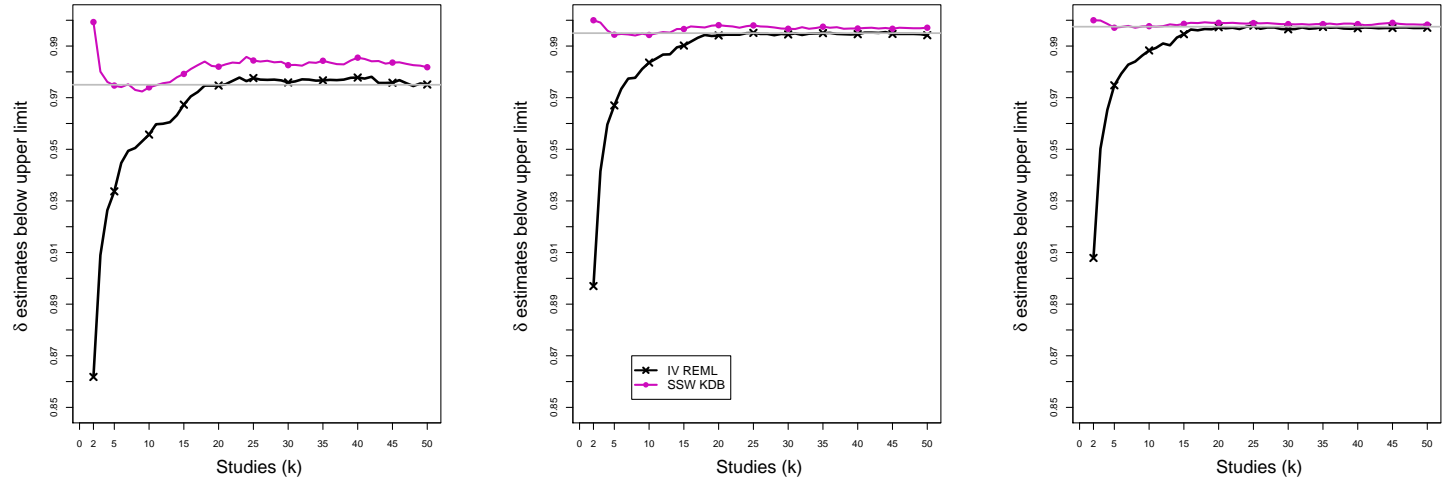

Figure C4. 18: CMA - Coverage of the upper confidence interval of $\delta$ (below upper limit) at the confidence level $1-\alpha / 2$ for $\alpha=0.05,0.01,0.005$ when $\delta=1$ for $k \leq 12$ followed by a shift to $\delta=2$ for $k \geq 13, \tau^{2}=0,0.1,0.25,1, n=100, K=50$. Light grey line at $0.975,0.995,0.9975$. 

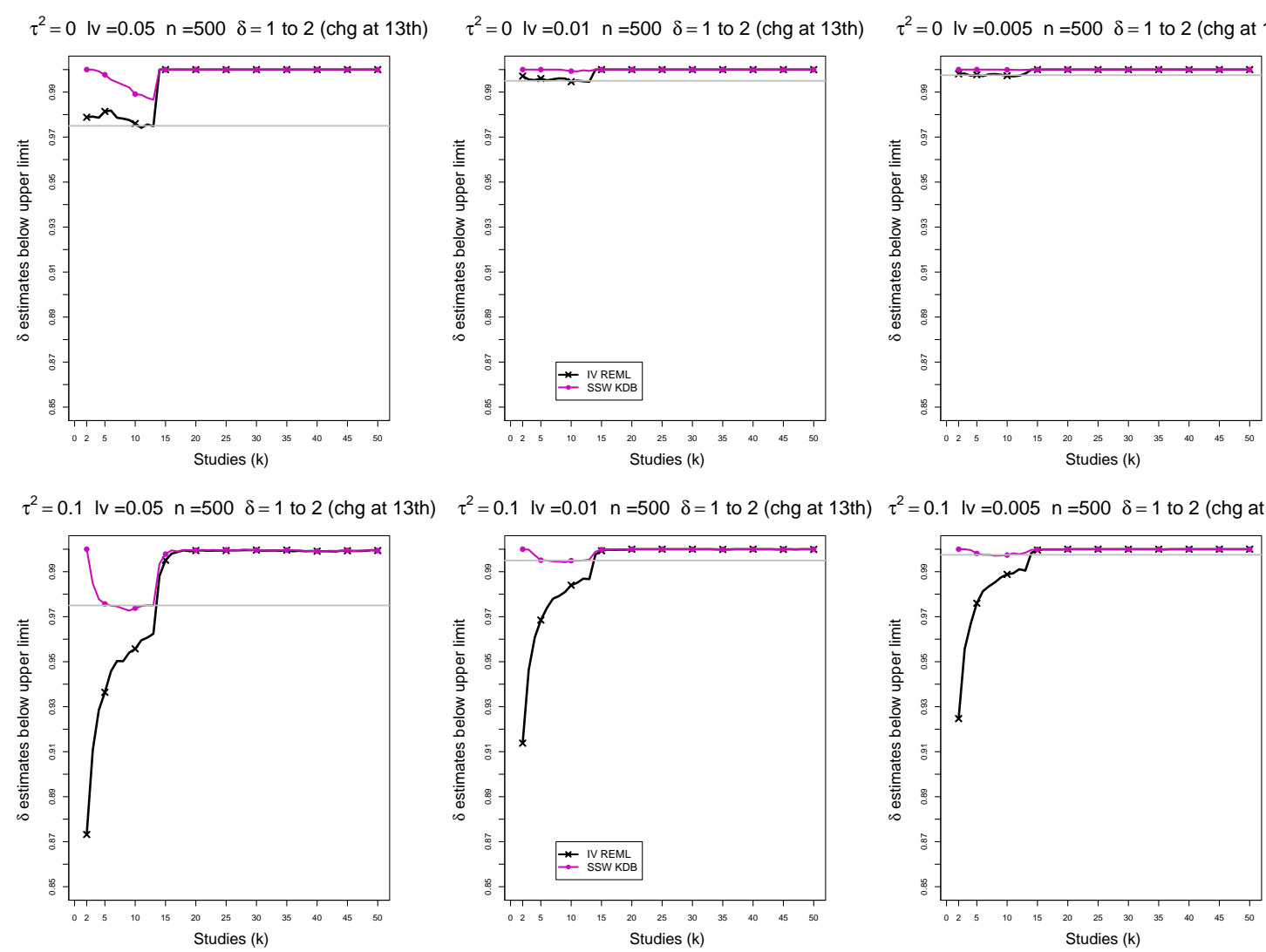

$\tau^{2}=0.25 \mathrm{lv}=0.05 \mathrm{n}=500 \delta=1$ to 2 (chg at 13 th) $\tau^{2}=0.25 \mathrm{lv}=0.01 \mathrm{n}=500 \delta=1$ to 2 (chg at 13 th) $\tau^{2}=0.25 \mathrm{lv}=0.005 \mathrm{n}=500 \quad \delta=1$ to 2 (chg at 13 th)
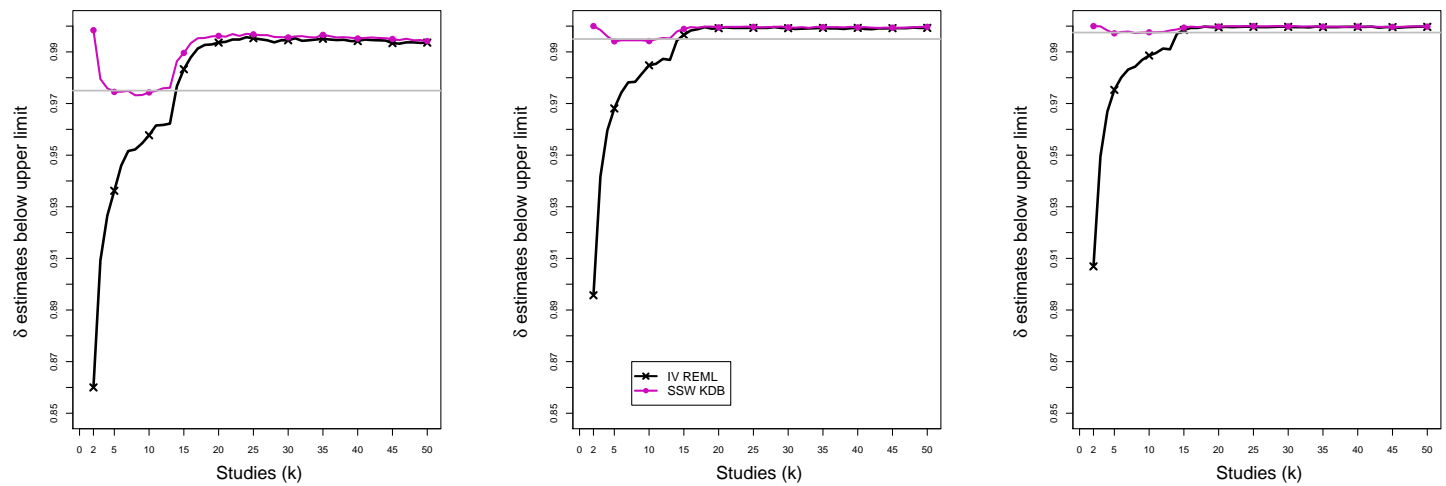

$\tau^{2}=1 \mathrm{IV}=0.05 \mathrm{n}=500 \delta=1$ to 2 (chg at 13 th)

$\tau^{2}=1 \mathrm{lv}=0.01 \mathrm{n}=500 \delta=1$ to 2 (chg at 13 th)

$\tau^{2}=1 \mathrm{lv}=0.005 \mathrm{n}=500 \delta=1$ to 2 (chg at 13th)
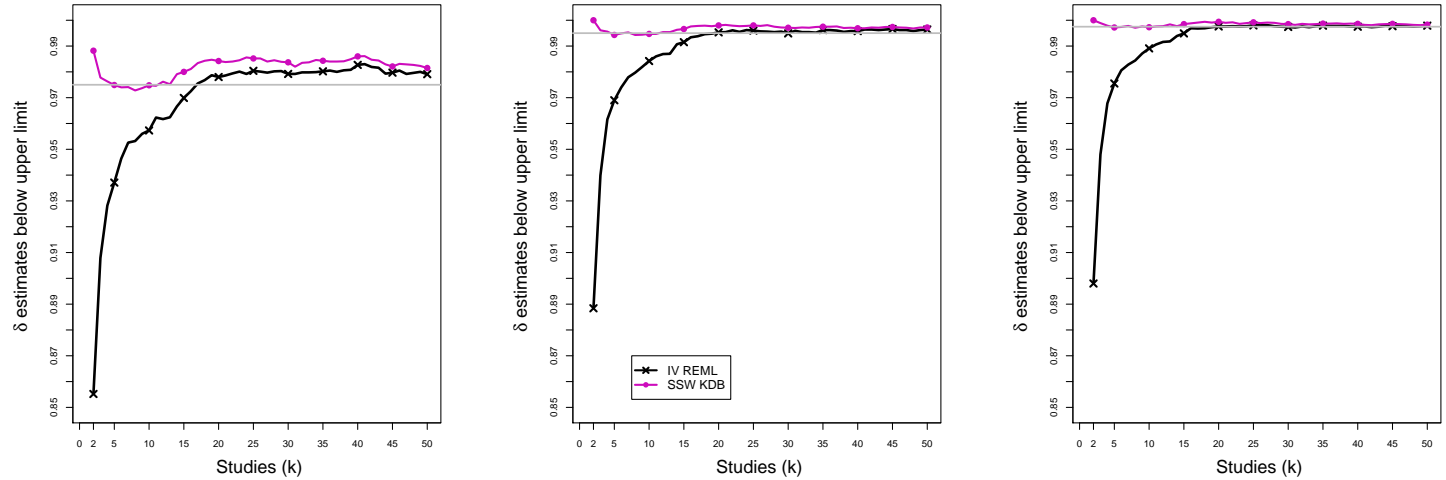

Figure C4. 19: CMA - Coverage of the upper confidence interval of $\delta$ (below upper limit) at the confidence level $1-\alpha / 2$ for $\alpha=0.05,0.01,0.005$ when $\delta=1$ for $k \leq 12$ followed by a shift to $\delta=2$ for $k \geq 13, \tau^{2}=0,0.1,0.25,1, n=500, K=50$. Light grey line at $0.975,0.995,0.9975$. 

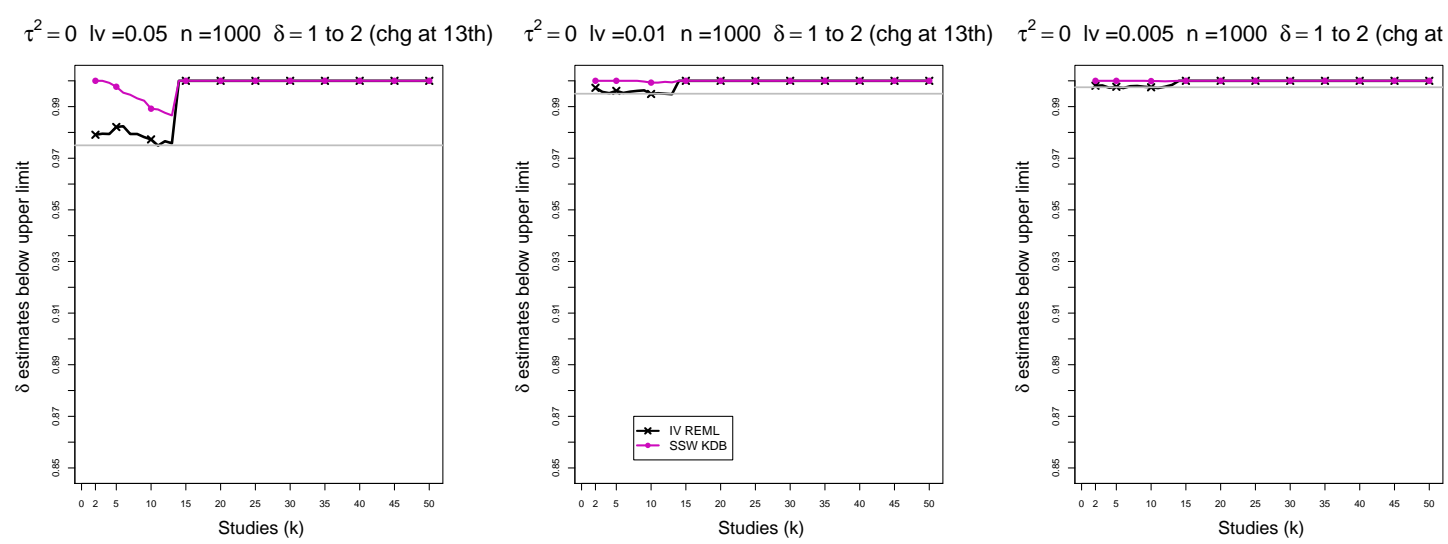

$\tau^{2}=0.1 \mathrm{lv}=0.05 \mathrm{n}=1000 \delta=1$ to 2 (chg at 13th) $\tau^{2}=0.1 \mathrm{lv}=0.01 \mathrm{n}=1000 \delta=1$ to 2 (chg at 13 th) $\tau^{2}=0.1 \mathrm{lv}=0.005 \mathrm{n}=1000 \delta=1$ to 2 (chg at 13 th)
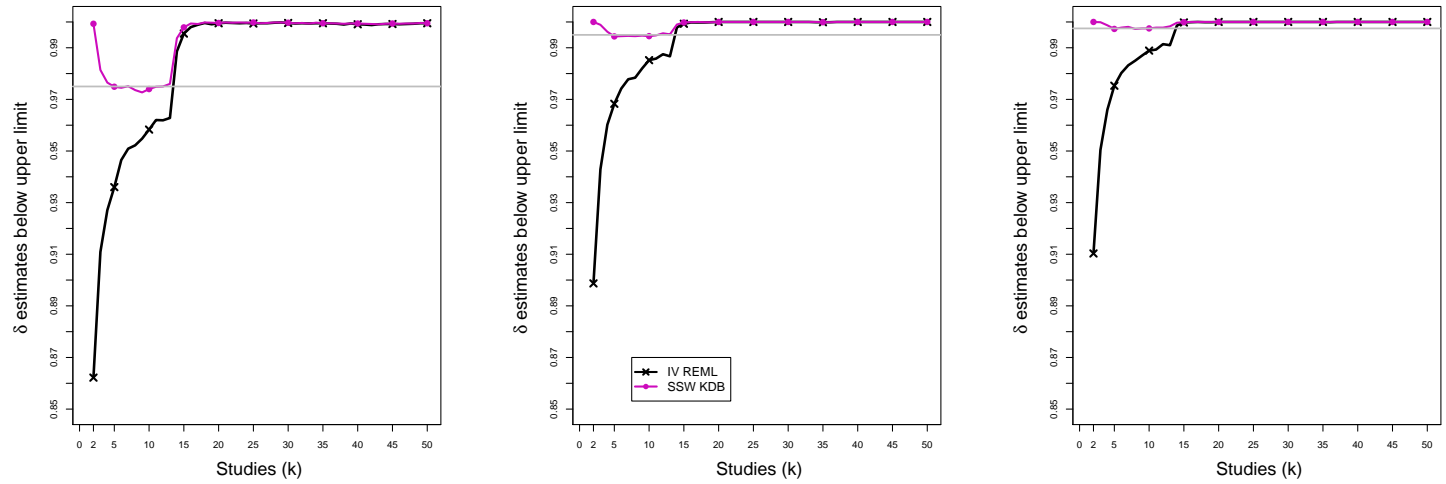

$\tau^{2}=0.25 \mathrm{lv}=0.05 \mathrm{n}=1000 \delta=1$ to $2(\mathrm{chg}$ at 13 th $) \tau^{2}=0.25 \mathrm{lv}=0.01 \mathrm{n}=1000 \quad \delta=1$ to $2(\text { chg at } 13 \text { th })^{2}=0.25 \mathrm{lv}=0.005 \mathrm{n}=1000 \quad \delta=1$ to 2 (chg at 13 th
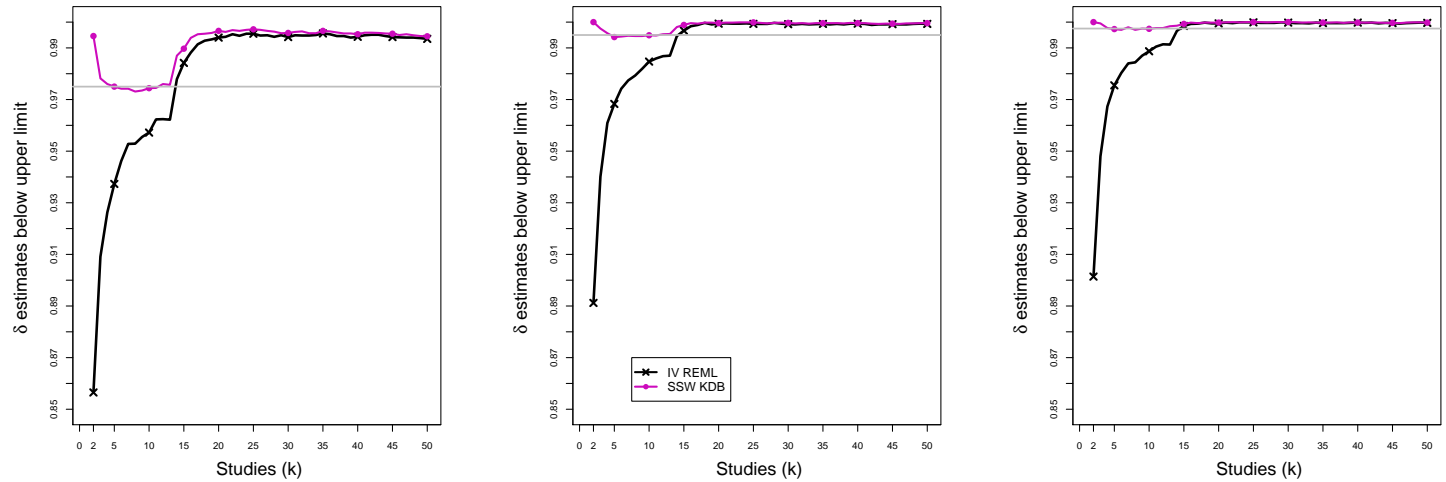

$\tau^{2}=1 \mathrm{IV}=0.05 \mathrm{n}=1000 \delta=1$ to 2 (chg at 13 th)
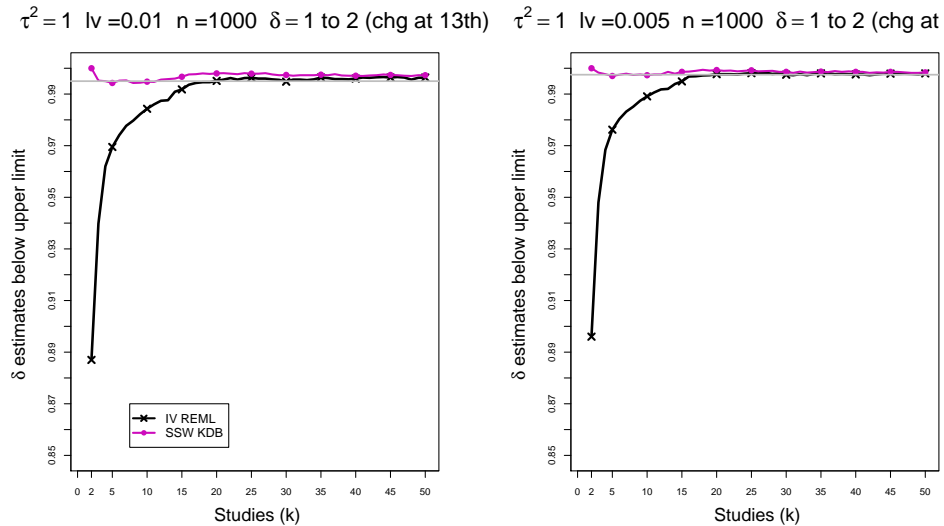

Figure C4. 20: CMA - Coverage of the upper confidence interval of $\delta$ (below upper limit) at the confidence level $1-\alpha / 2$ for $\alpha=0.05,0.01,0.005$ when $\delta=1$ for $k \leq 12$ followed by a shift to $\delta=2$ for $k \geq 13, \tau^{2}=0,0.1,0.25,1, n=1000, K=50$. Light grey line at $0.975,0.995,0.9975$. 

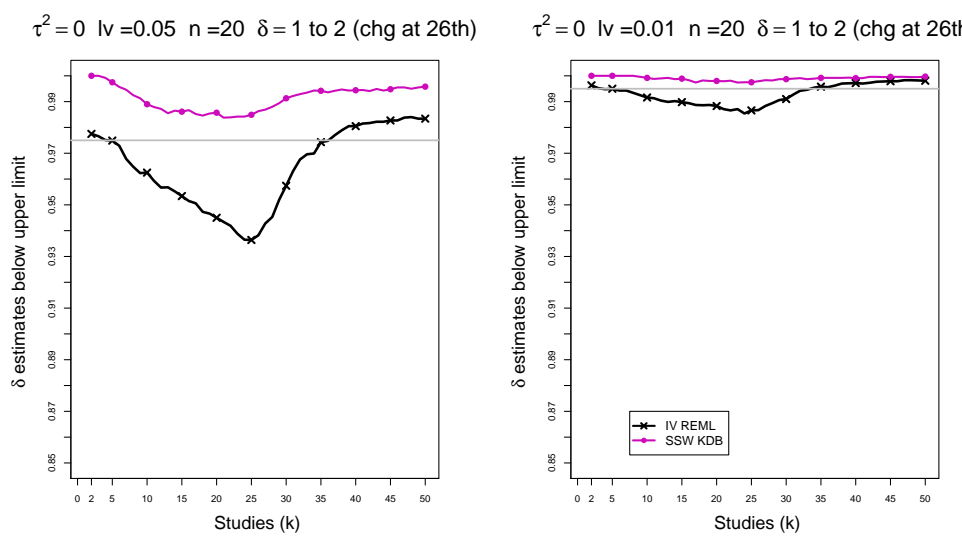

$\tau^{2}=0 \mathrm{IV}=0.005 \mathrm{n}=20 \quad \delta=1$ to 2 (chg at 26th)

$\tau^{2}=0.1 \mathrm{lv}=0.05 \mathrm{n}=20 \quad \delta=1$ to 2 (chg at 26th)

$\tau^{2}=0.1 \mathrm{IV}=0.01 \mathrm{n}=20 \quad \delta=1$ to 2 (chg at 26th)
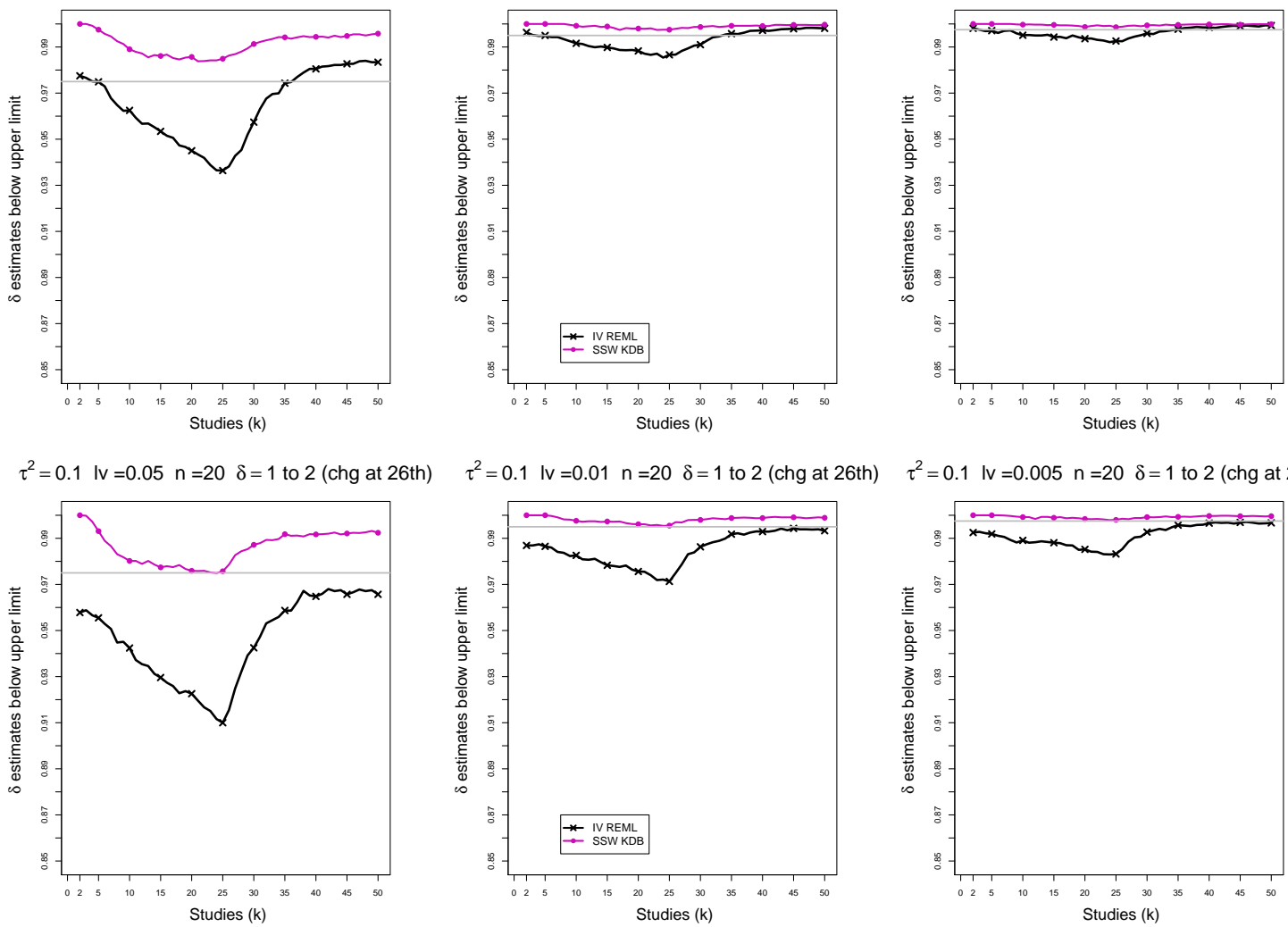

$\tau^{2}=0.1 \mathrm{IV}=0.005 \mathrm{n}=20 \delta=1$ to 2 (chg at 26 th)

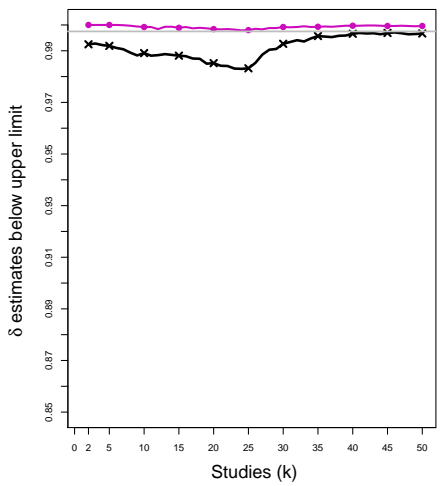

$\tau^{2}=0.25 \mathrm{lv}=0.05 \mathrm{n}=20 \quad \delta=1$ to 2 (chg at 26th)
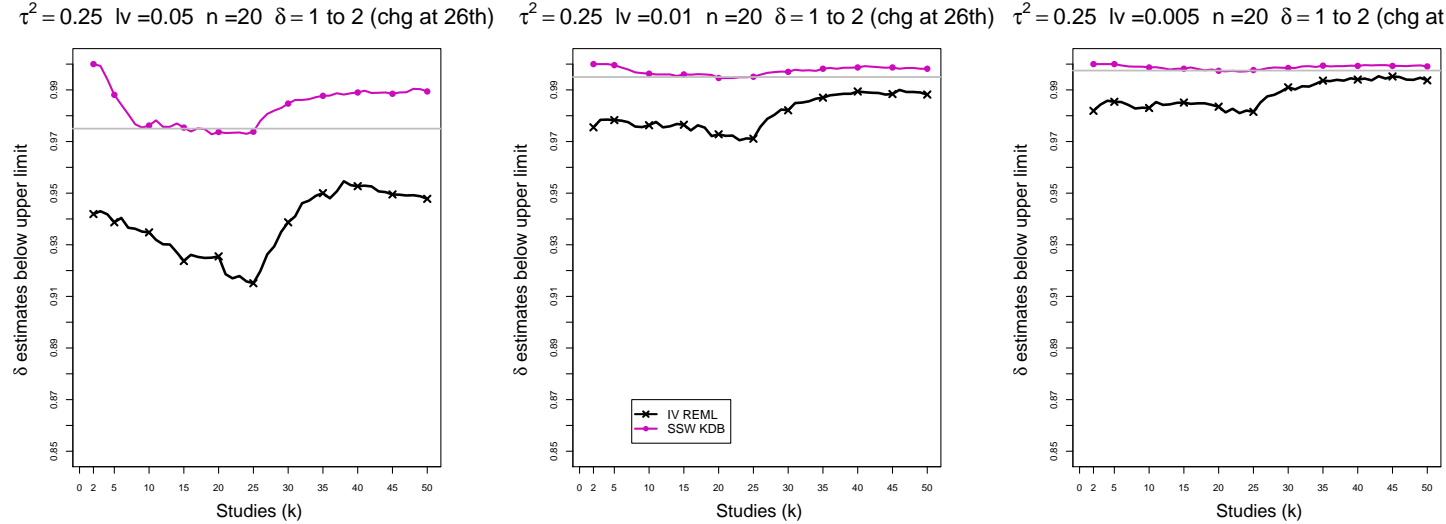

$\tau^{2}=1 \mathrm{lv}=0.01 \mathrm{n}=20 \quad \delta=1$ to 2 (chg at 26 th)

$\tau^{2}=1 \mathrm{lv}=0.005 \mathrm{n}=20 \delta=1$ to 2 (chg at 26th)
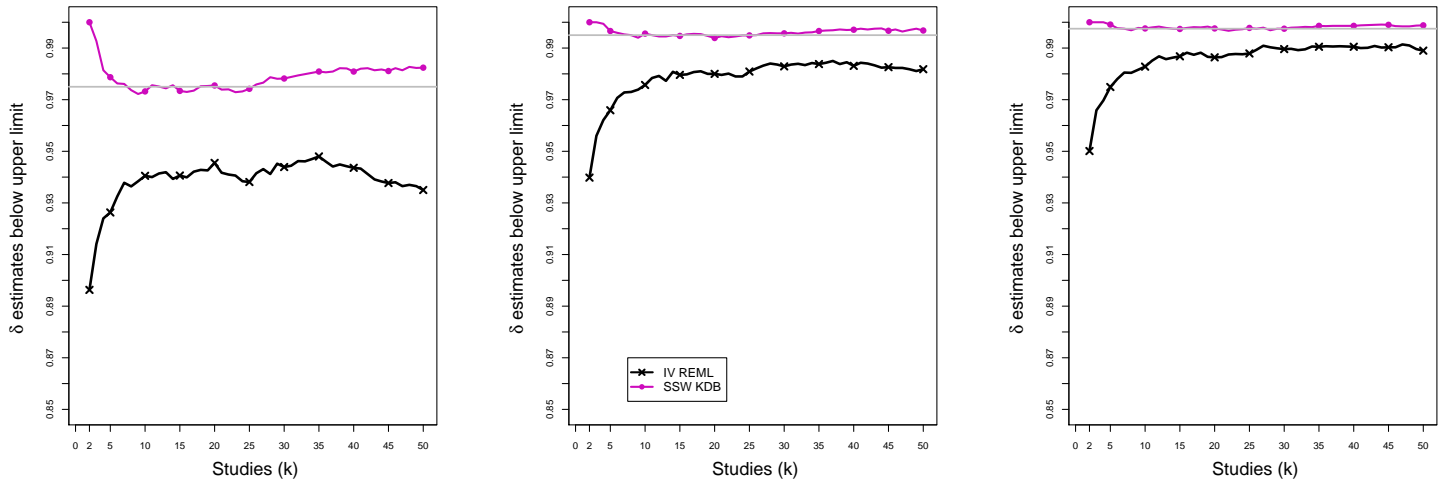

Figure C4. 21: CMA - Coverage of the upper confidence interval of $\delta$ (below upper limit) at the confidence level $1-\alpha / 2$ for $\alpha=0.05,0.01,0.005$ when $\delta=1$ for $k \leq 25$ followed by a shift to $\delta=2$ for $k \geq 26, \tau^{2}=0,0.1,0.25,1, n=20, K=50$. Light grey line at $0.975,0.995,0.9975$. 

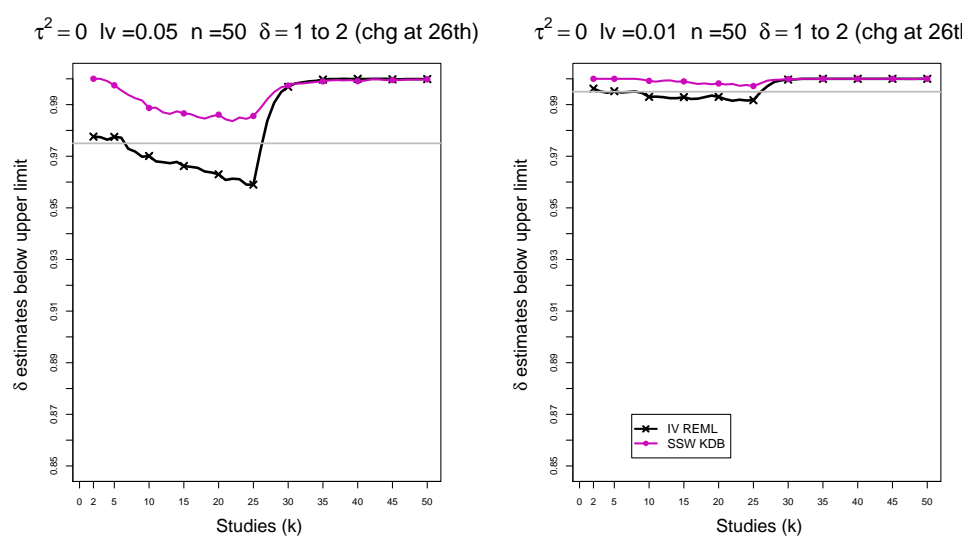

$\tau^{2}=0 \mathrm{lv}=0.005 \mathrm{n}=50 \delta=1$ to 2 (chg at 26th)

$\tau^{2}=0.1 \mathrm{lv}=0.05 \mathrm{n}=50 \delta=1$ to 2 (chg at 26th)

$\tau^{2}=0.1 \mathrm{lv}=0.01 \mathrm{n}=50 \quad \delta=1$ to 2 (chg at 26th)
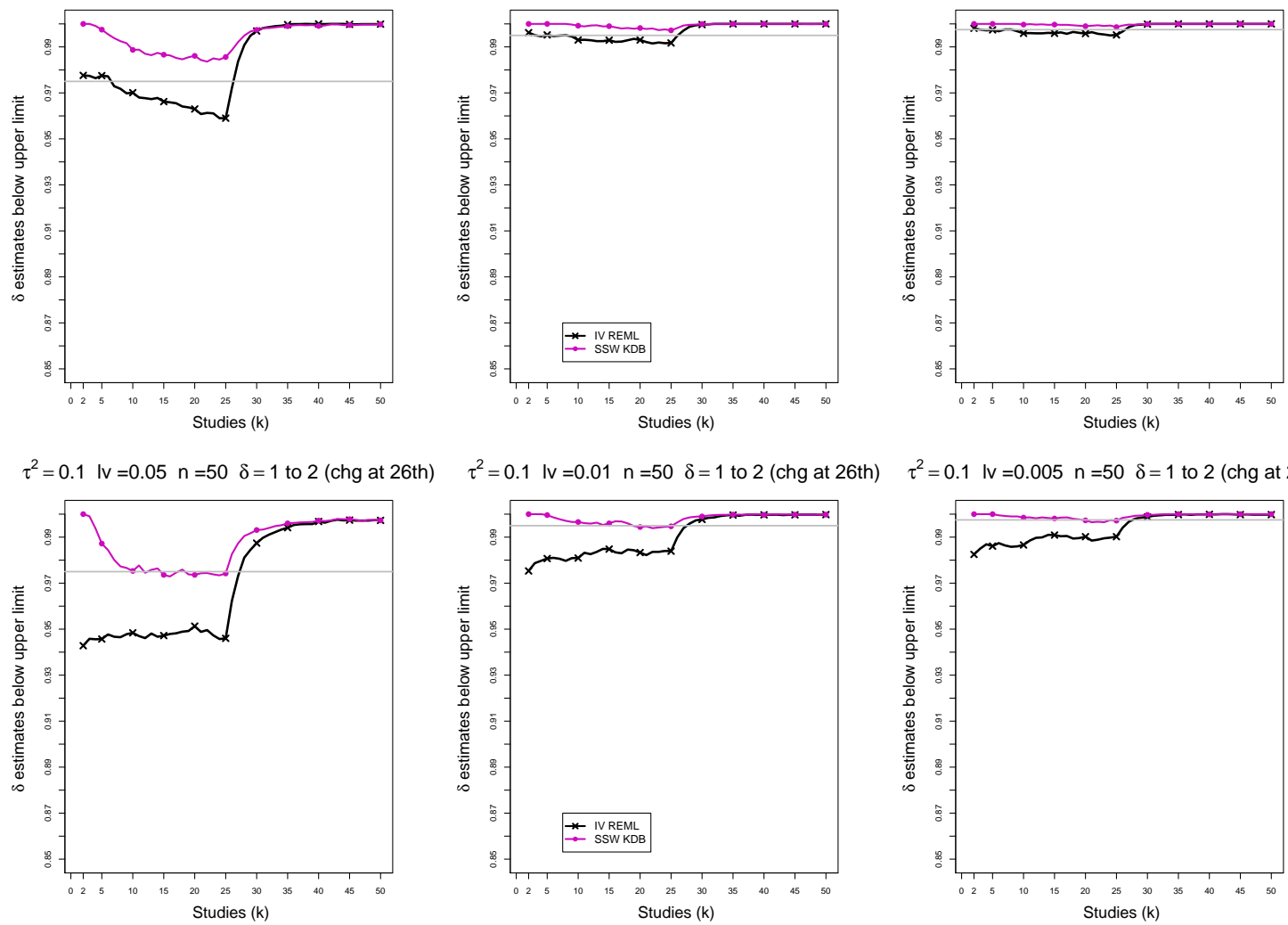

$\tau^{2}=0.1 \mathrm{IV}=0.005 \mathrm{n}=50 \delta=1$ to 2 (chg at 26 th)

$\tau^{2}=0.25 \mathrm{lv}=0.05 \mathrm{n}=50 \quad \delta=1$ to 2 (chg at 26th)
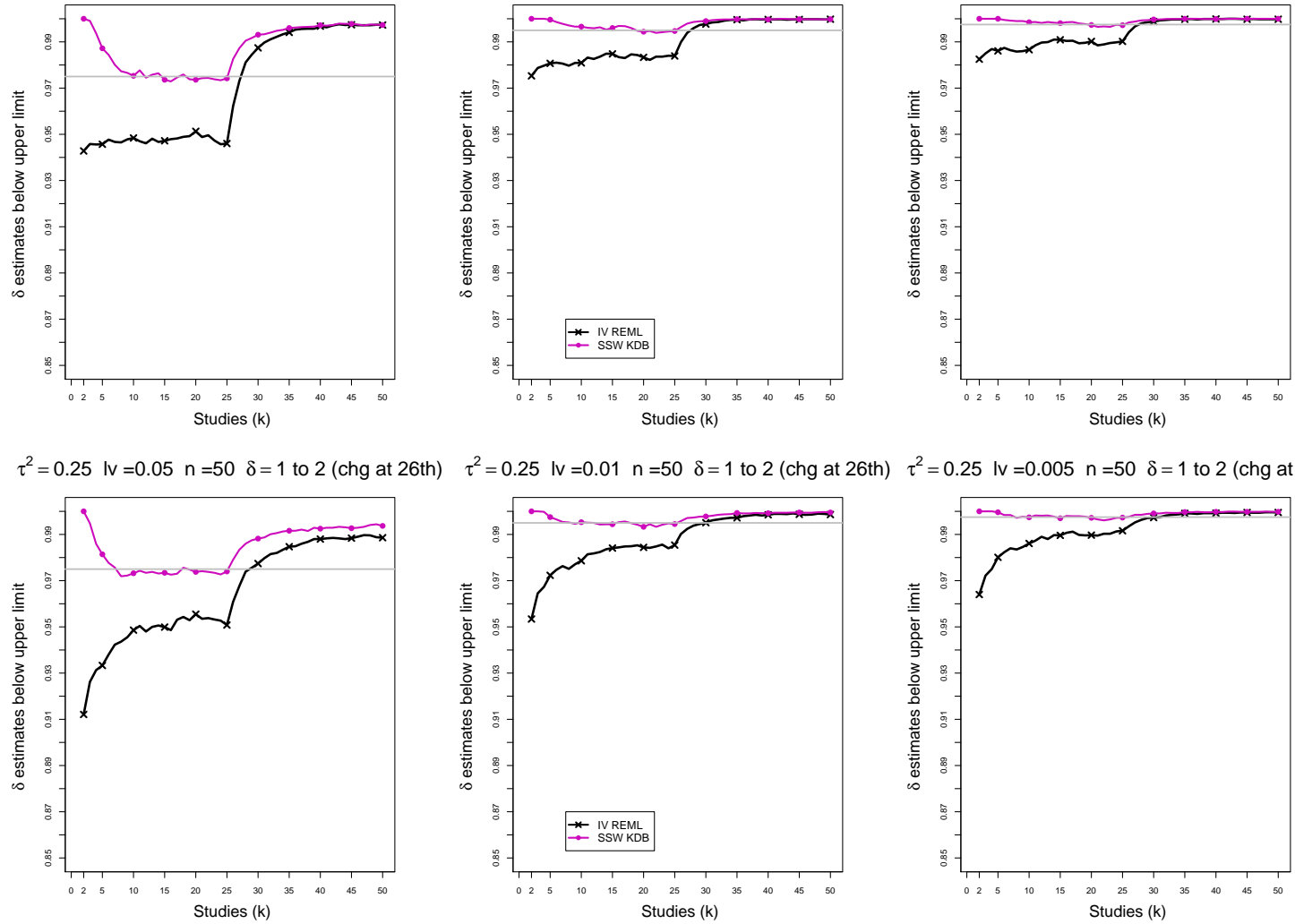

$\tau^{2}=1 \mathrm{lv}=0.05 \mathrm{n}=50 \delta=1$ to 2 (chg at 26th)

$\tau^{2}=1 \mathrm{lv}=0.01 \mathrm{n}=50 \quad \delta=1$ to 2 (chg at 26 th)

$\tau^{2}=1 \mathrm{lv}=0.005 \mathrm{n}=50 \delta=1$ to 2 (chg at 26th)
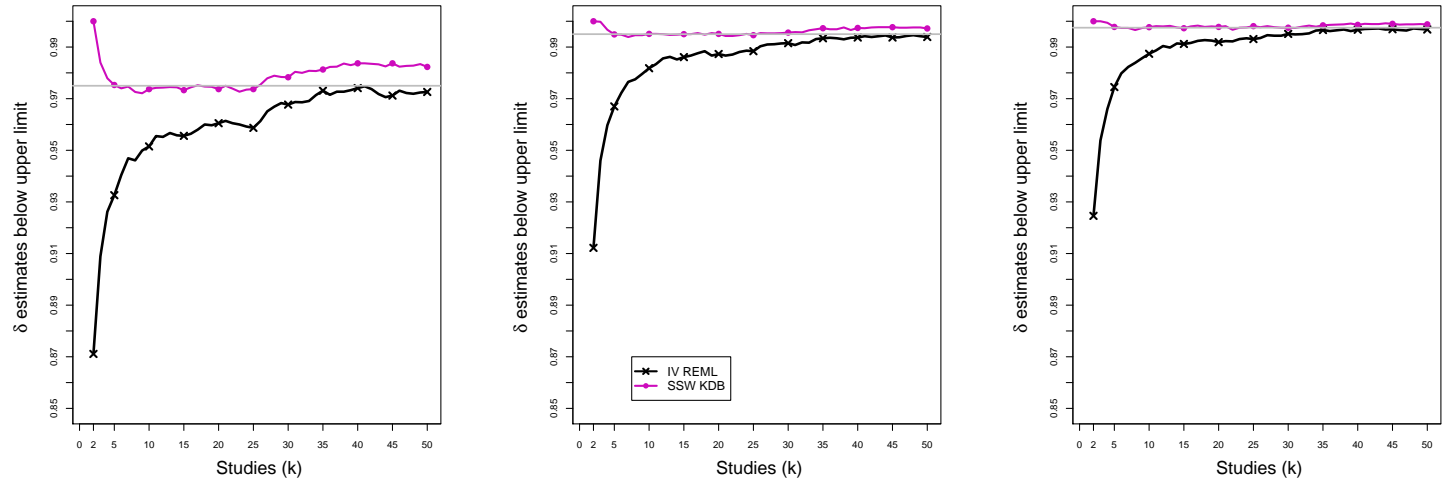

Figure C4. 22: CMA - Coverage of the upper confidence interval of $\delta$ (below upper limit) at the confidence level $1-\alpha / 2$ for $\alpha=0.05,0.01,0.005$ when $\delta=1$ for $k \leq 25$ followed by a shift to $\delta=2$ for $k \geq 26, \tau^{2}=0,0.1,0.25,1, n=50, K=50$. Light grey line at $0.975,0.995,0.9975$. 

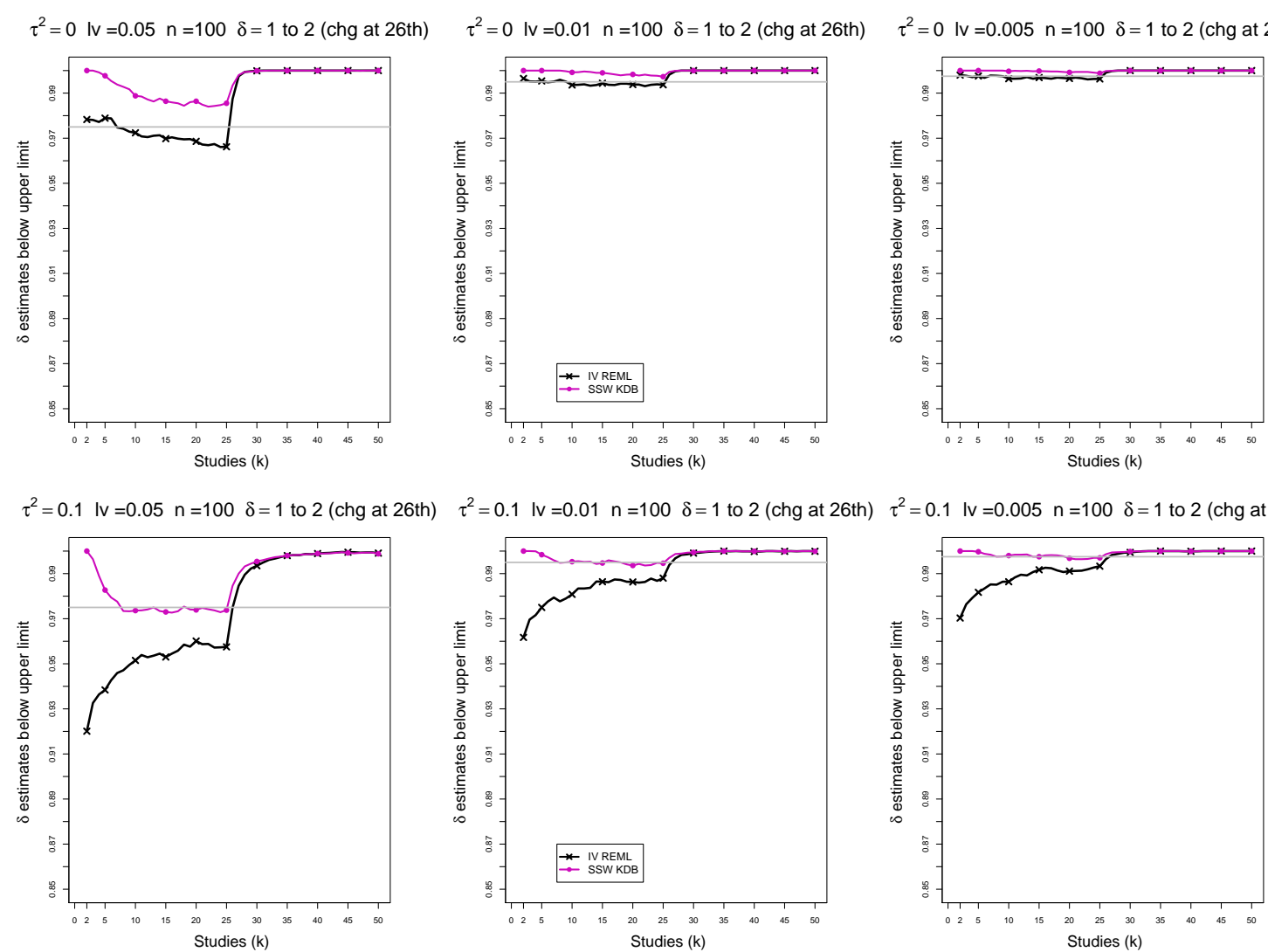

$\tau^{2}=0.25 \mathrm{lv}=0.05 \mathrm{n}=100 \delta=1$ to 2 (chg at 26 th) $\tau^{2}=0.25 \mathrm{lv}=0.01 \mathrm{n}=100 \quad \delta=1$ to 2 (chg at 26th) $\tau^{2}=0.25 \mathrm{lv}=0.005 \mathrm{n}=100 \quad \delta=1$ to 2 (chg at 26 th)
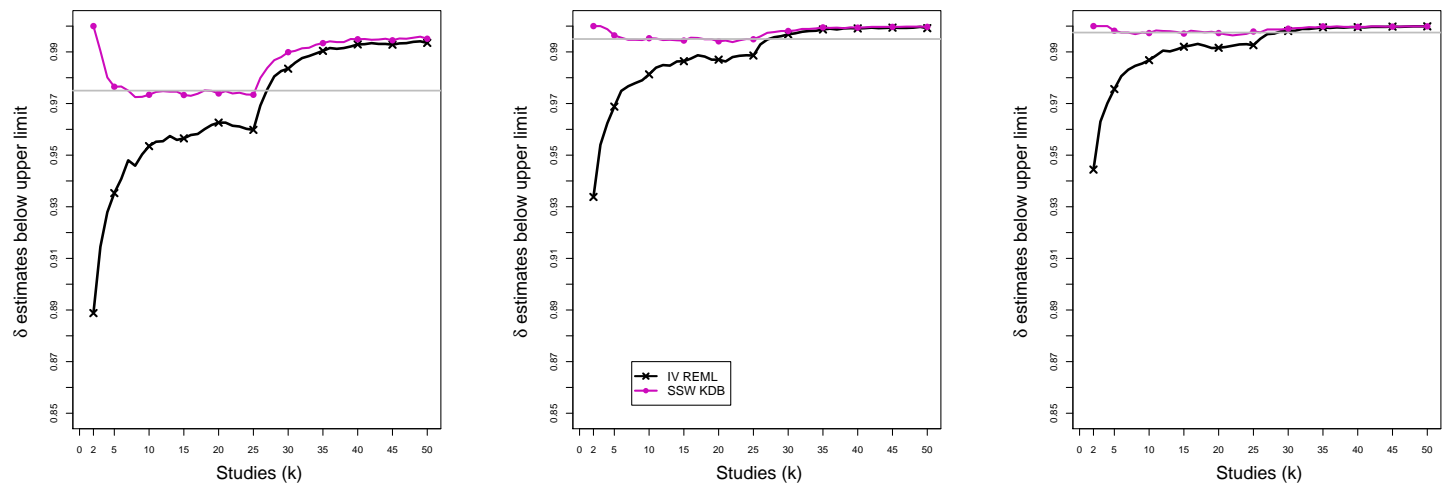

$\tau^{2}=1 \mathrm{IV}=0.05 \mathrm{n}=100 \delta=1$ to 2 (chg at $26 \mathrm{th}$ )

$\tau^{2}=1 \mathrm{lv}=0.01 \mathrm{n}=100 \delta=1$ to 2 (chg at 26 th)

$\tau^{2}=1 \mathrm{lv}=0.005 \mathrm{n}=100 \delta=1$ to 2 (chg at 26th)
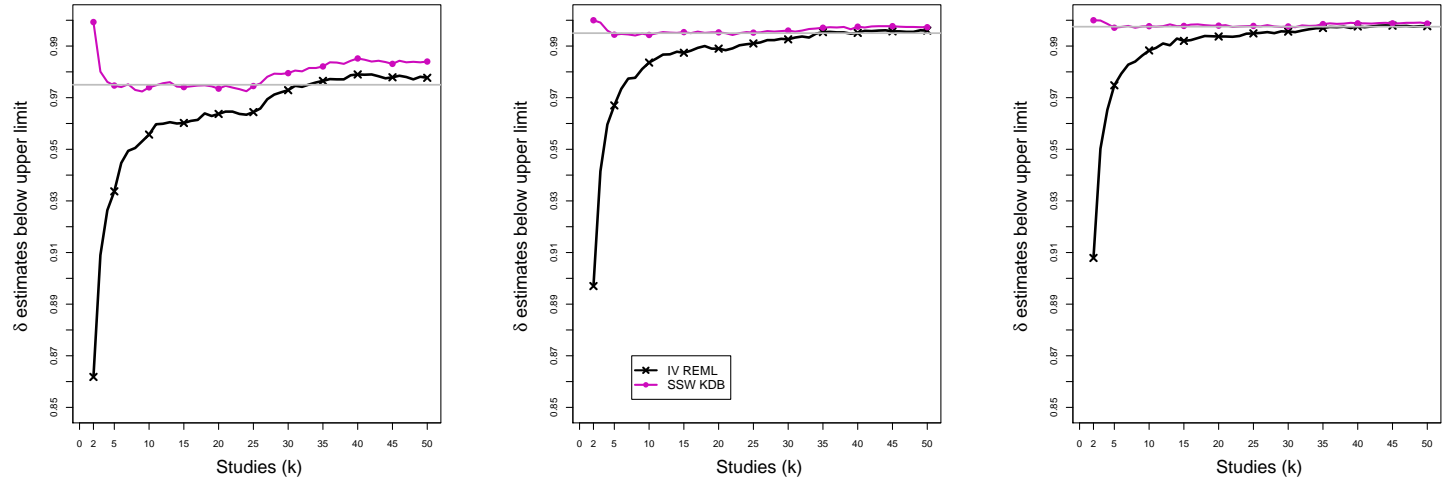

Figure C4. 23: CMA - Coverage of the upper confidence interval of $\delta$ (below upper limit) at the confidence level $1-\alpha / 2$ for $\alpha=0.05,0.01,0.005$ when $\delta=1$ for $k \leq 25$ followed by a shift to $\delta=2$ for $k \geq 26, \tau^{2}=0,0.1,0.25,1, n=100, K=50$. Light grey line at $0.975,0.995,0.9975$. 

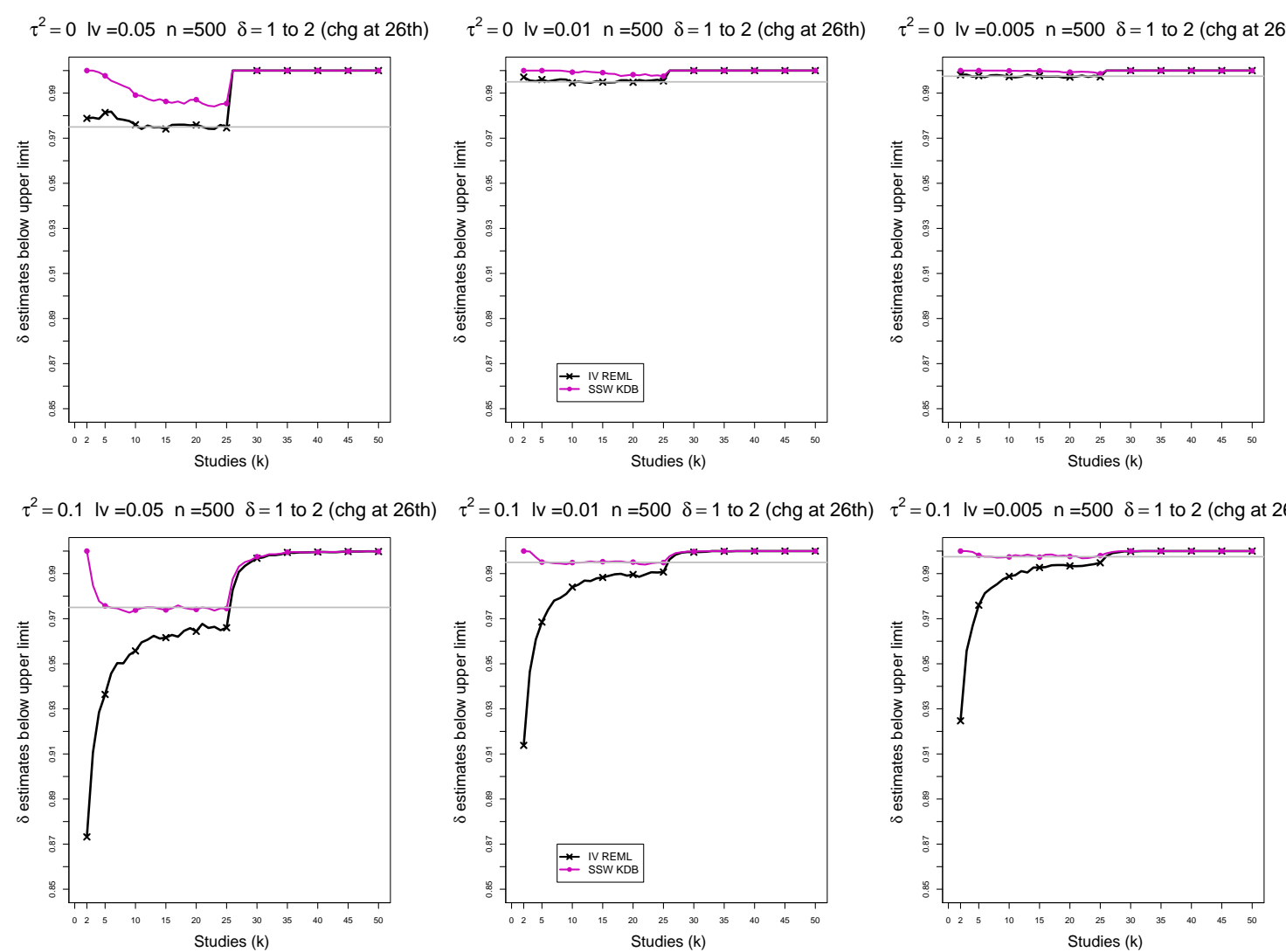

$\tau^{2}=0.25 \mathrm{lv}=0.05 \mathrm{n}=500 \delta=1$ to 2 (chg at 26 th) $\tau^{2}=0.25 \mathrm{lv}=0.01 \mathrm{n}=500 \delta=1$ to 2 (chg at 26th) $\tau^{2}=0.25 \mathrm{lv}=0.005 \mathrm{n}=500 \quad \delta=1$ to 2 (chg at 26 th)
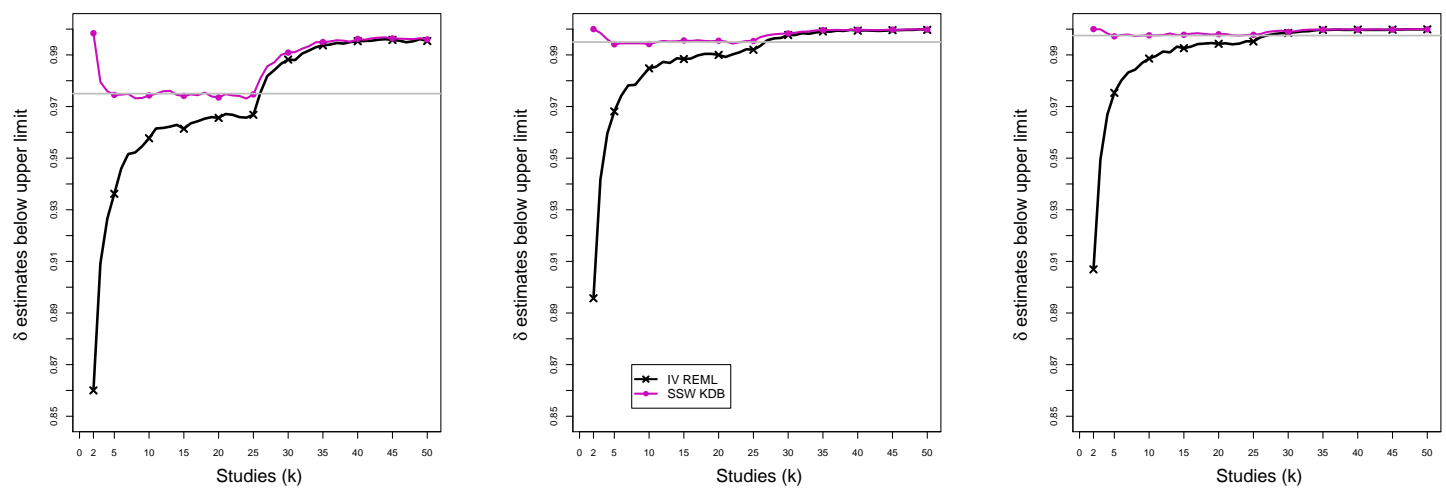

$\tau^{2}=1 \mathrm{IV}=0.05 \mathrm{n}=500 \delta=1$ to 2 (chg at $26 \mathrm{th}$ )

$\tau^{2}=1 \mathrm{IV}=0.01 \mathrm{n}=500 \delta=1$ to 2 (chg at $26 \mathrm{th}$ )

$\tau^{2}=1 \mathrm{lv}=0.005 \mathrm{n}=500 \delta=1$ to 2 (chg at 26th)
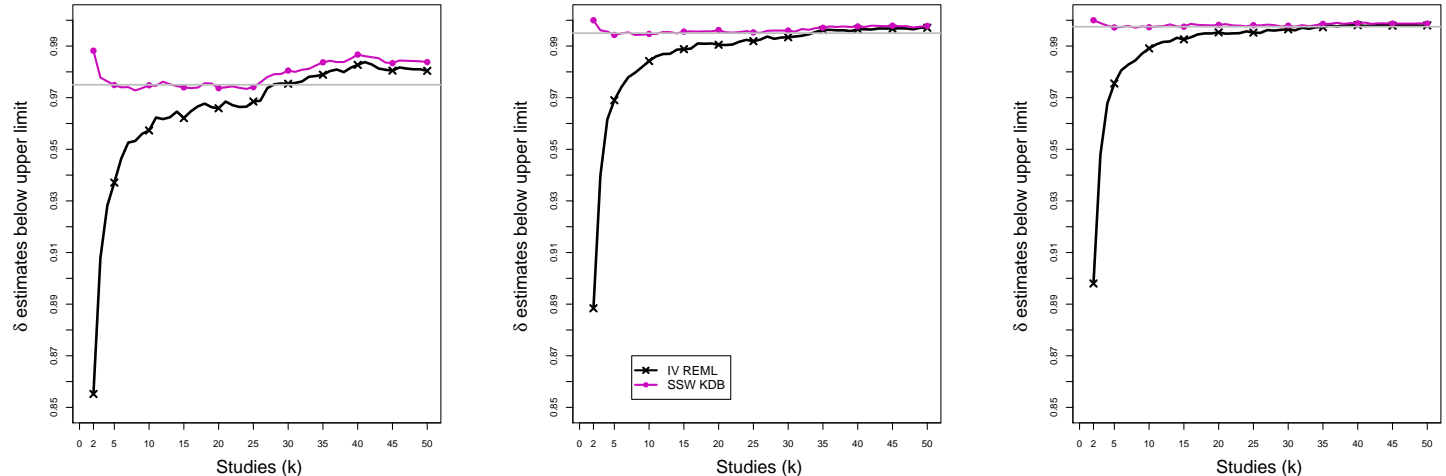

Figure C4. 24: CMA - Coverage of the upper confidence interval of $\delta$ (below upper limit) at the confidence level $1-\alpha / 2$ for $\alpha=0.05,0.01,0.005$ when $\delta=1$ for $k \leq 25$ followed by a shift to $\delta=2$ for $k \geq 26, \tau^{2}=0,0.1,0.25,1, n=500, K=50$. Light grey line at 

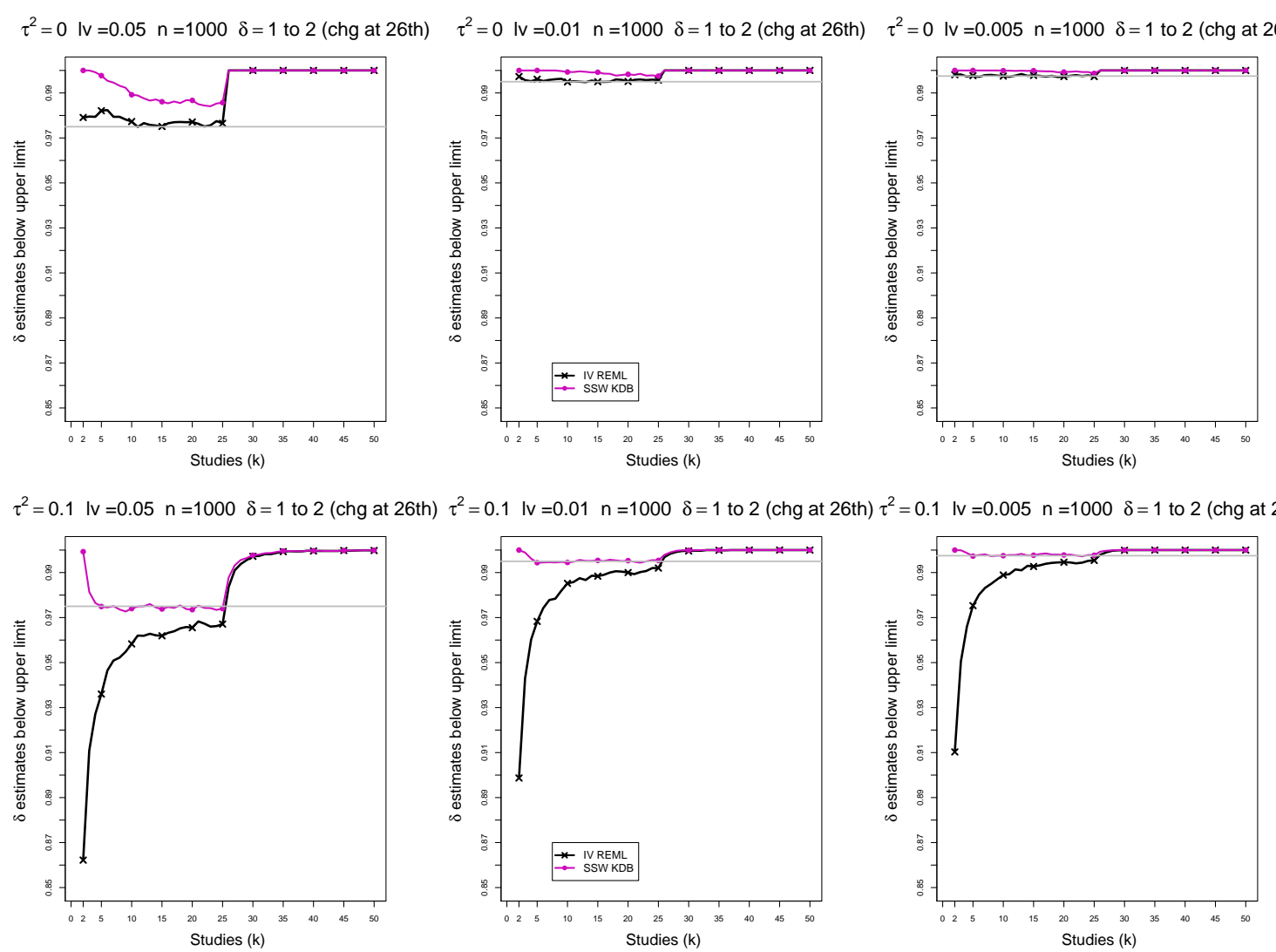

$\tau^{2}=0.25 \mathrm{lv}=0.05 \mathrm{n}=1000 \delta=1$ to 2 (chg at 26 th) $\tau^{2}=0.25 \mathrm{lv}=0.01 \mathrm{n}=1000 \quad \delta=1$ to 2 (chg at 26 th) $:^{2}=0.25 \mathrm{lv}=0.005 \mathrm{n}=1000 \quad \delta=1$ to 2 (chg at 26 th
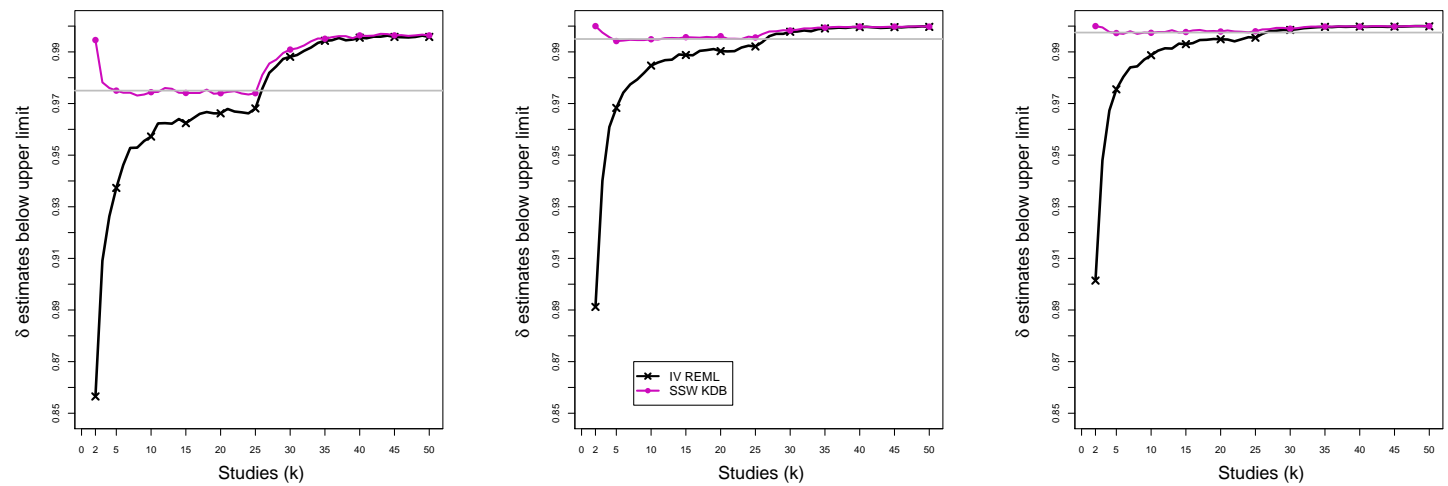

$\tau^{2}=1 \mathrm{lv}=0.05 \mathrm{n}=1000 \delta=1$ to 2 (chg at $\left.26 \mathrm{th}\right)$

$\tau^{2}=1 \mathrm{IV}=0.01 \mathrm{n}=1000 \delta=1$ to 2 (chg at 26th) $\tau^{2}=1 \mathrm{IV}=0.005 \mathrm{n}=1000 \delta=1$ to 2 (chg at 26th)
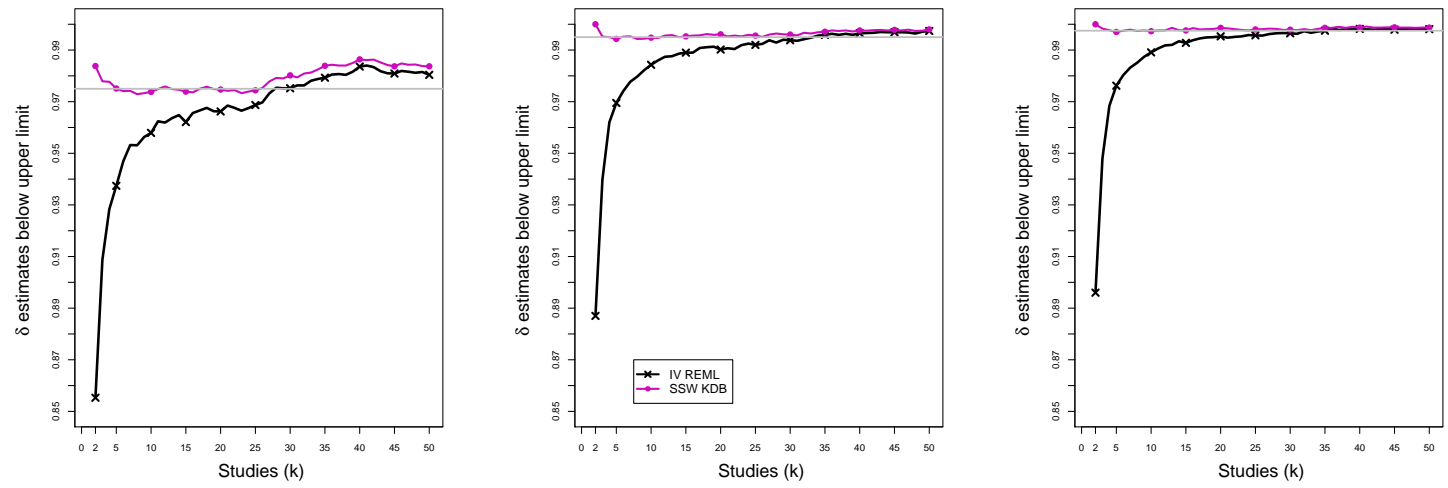

Figure C4. 25: CMA - Coverage of the upper confidence interval of $\delta$ (below upper limit) at the confidence level $1-\alpha / 2$ for $\alpha=0.05,0.01,0.005$ when $\delta=1$ for $k \leq 25$ followed by a shift to $\delta=2$ for $k \geq 26, \tau^{2}=0,0.1,0.25,1, n=1000, K=50$. Light grey line at 

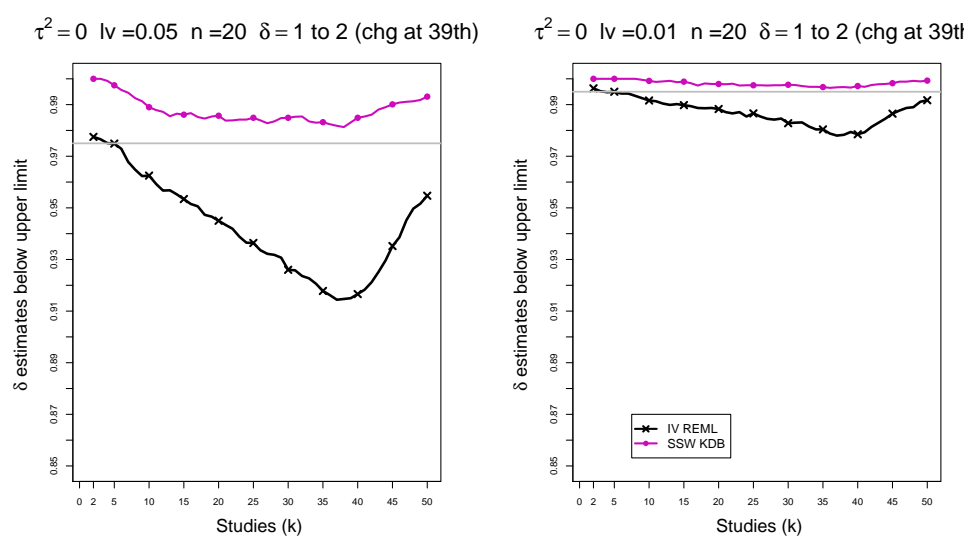

$\tau^{2}=0 \mathrm{IV}=0.005 \mathrm{n}=20 \quad \delta=1$ to 2 (chg at 39 th)

$\tau^{2}=0.1 \mathrm{lv}=0.05 \mathrm{n}=20 \quad \delta=1$ to 2 (chg at 39 th)

$\tau^{2}=0.1 \mathrm{lv}=0.01 \mathrm{n}=20 \quad \delta=1$ to 2 (chg at 39 th)
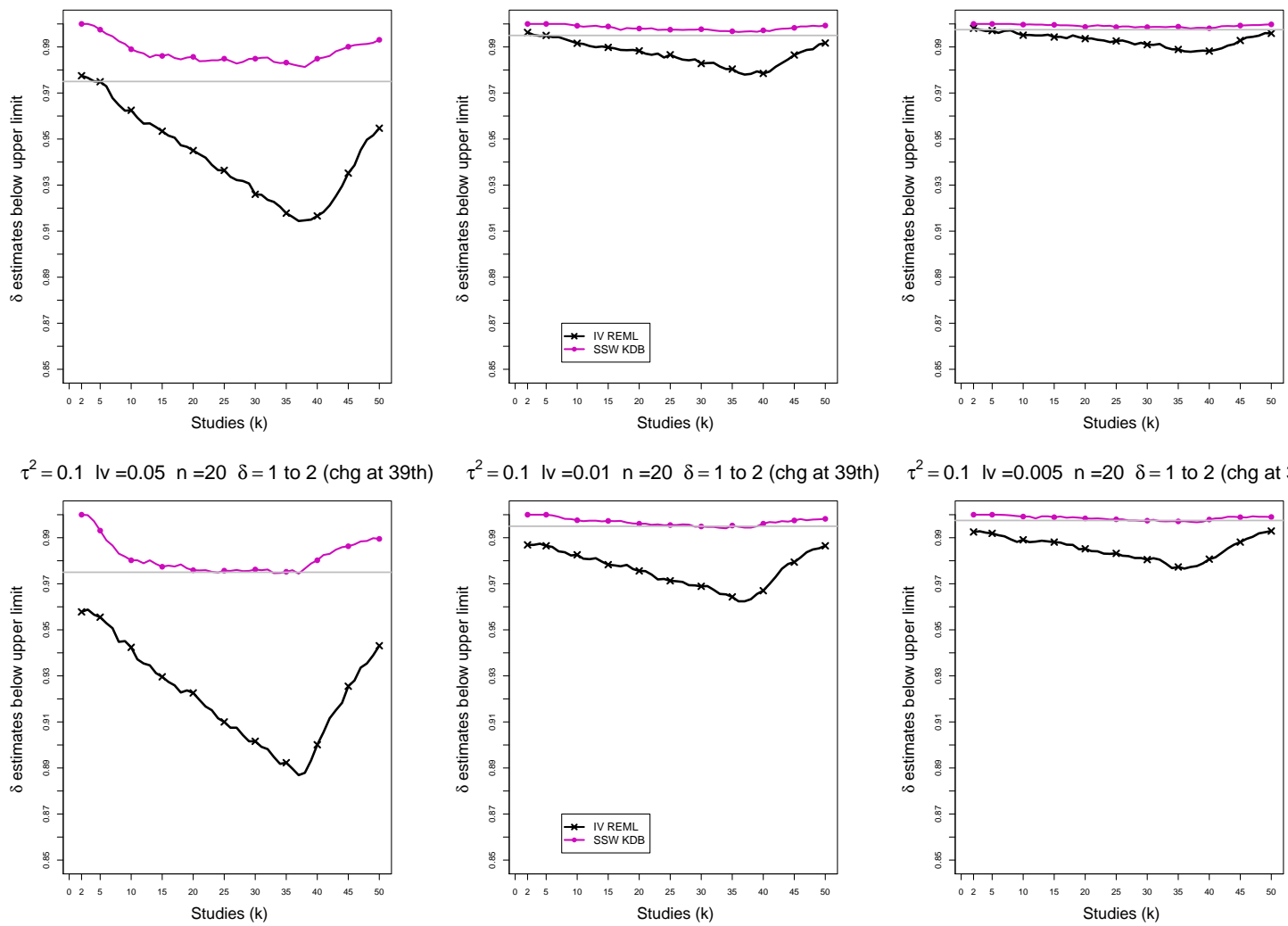

$\tau^{2}=0.1 \mathrm{IV}=0.005 \mathrm{n}=20 \delta=1$ to 2 (chg at 39 th)

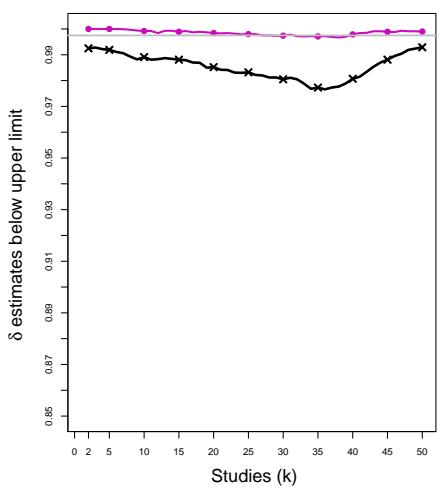

$\tau^{2}=0.25 \mathrm{IV}=0.05 \mathrm{n}=20 \delta=1$ to 2 (chg at 39th)

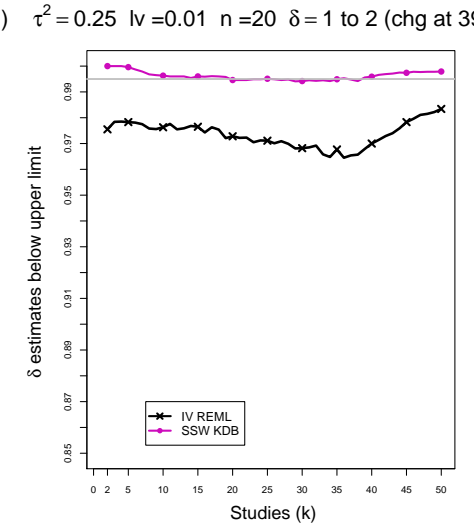

$\tau^{2}=0.25 \mathrm{IV}=0.005 \mathrm{n}=20 \quad \delta=1$ to 2 (chg at 39 th)
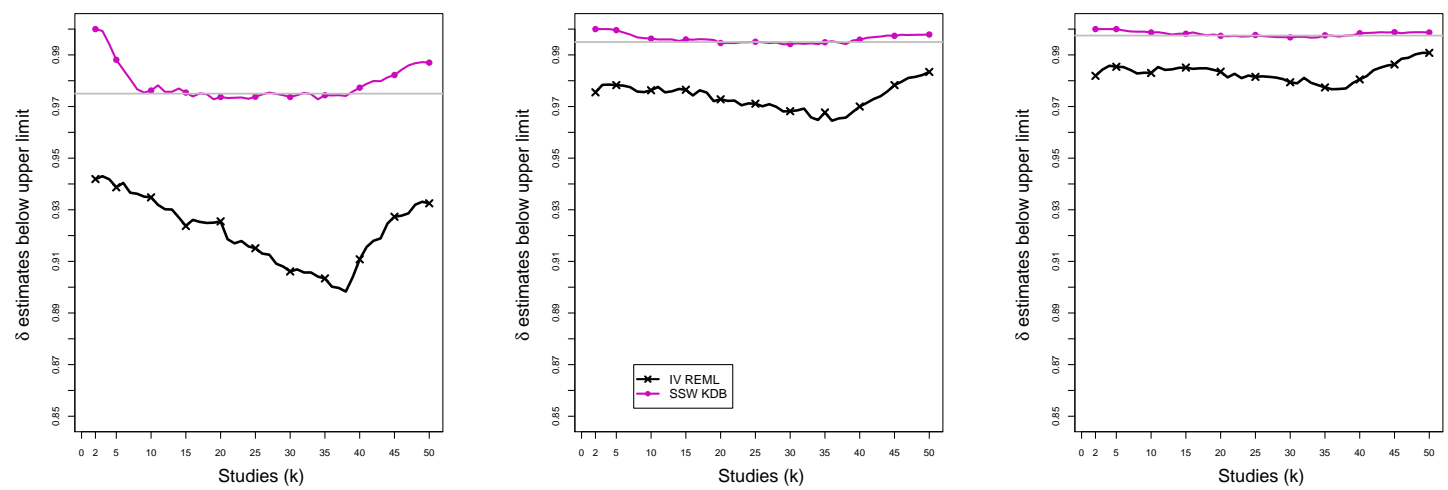

$\tau^{2}=1 \mathrm{IV}=0.05 \mathrm{n}=20 \quad \delta=1$ to 2 (chg at 39 th)

$\tau^{2}=1 \mathrm{lv}=0.01 \mathrm{n}=20 \delta=1$ to 2 (chg at 39 th)
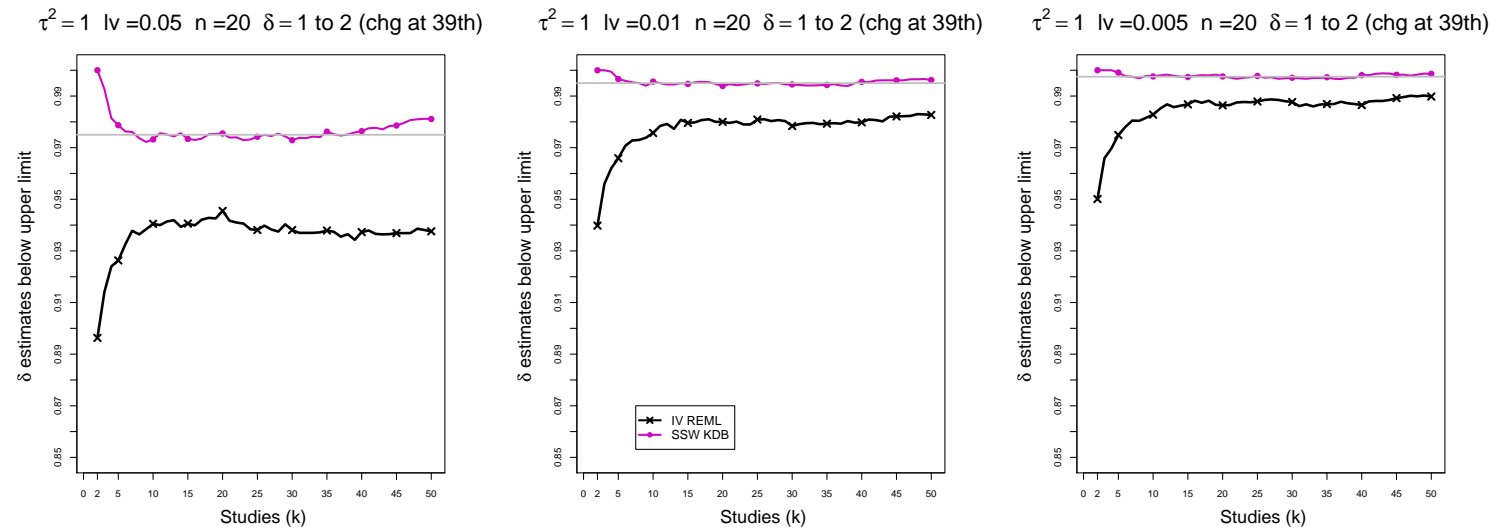

Figure C4. 26: CMA - Coverage of the upper confidence interval of $\delta$ (below upper limit) at the confidence level $1-\alpha / 2$ for $\alpha=0.05,0.01,0.005$ when $\delta=1$ for $k \leq 38$ followed by a shift to $\delta=2$ for $k \geq 39, \tau^{2}=0,0.1,0.25,1, n=20, K=50$. Light grey line at $0.975,0.995,0.9975$. 

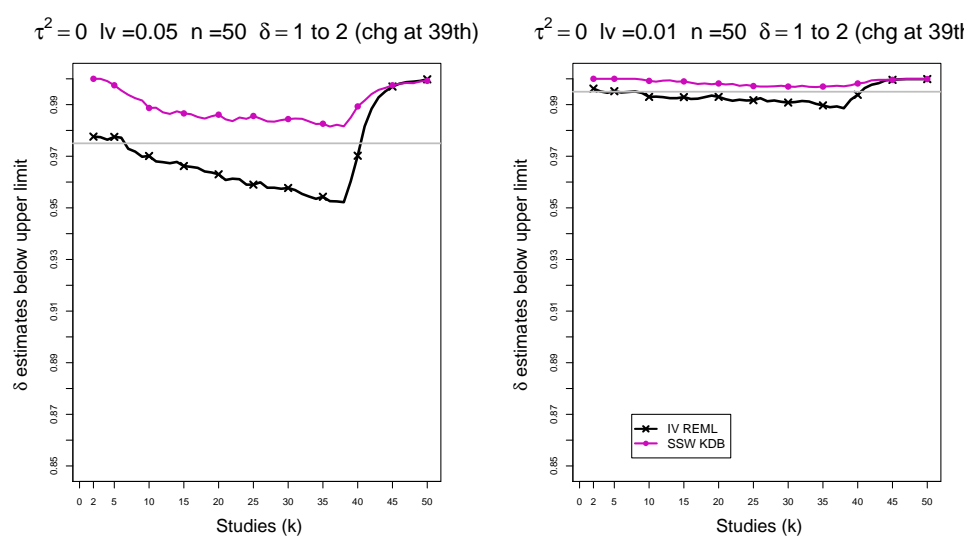

$\tau^{2}=0 \mathrm{IV}=0.005 \mathrm{n}=50 \quad \delta=1$ to 2 (chg at 39 th)

$\tau^{2}=0.1 \mathrm{lv}=0.05 \mathrm{n}=50 \delta=1$ to 2 (chg at 39 th)

$\tau^{2}=0.1 \mathrm{lv}=0.01 \quad \mathrm{n}=50 \quad \delta=1$ to 2 (chg at 39 th)
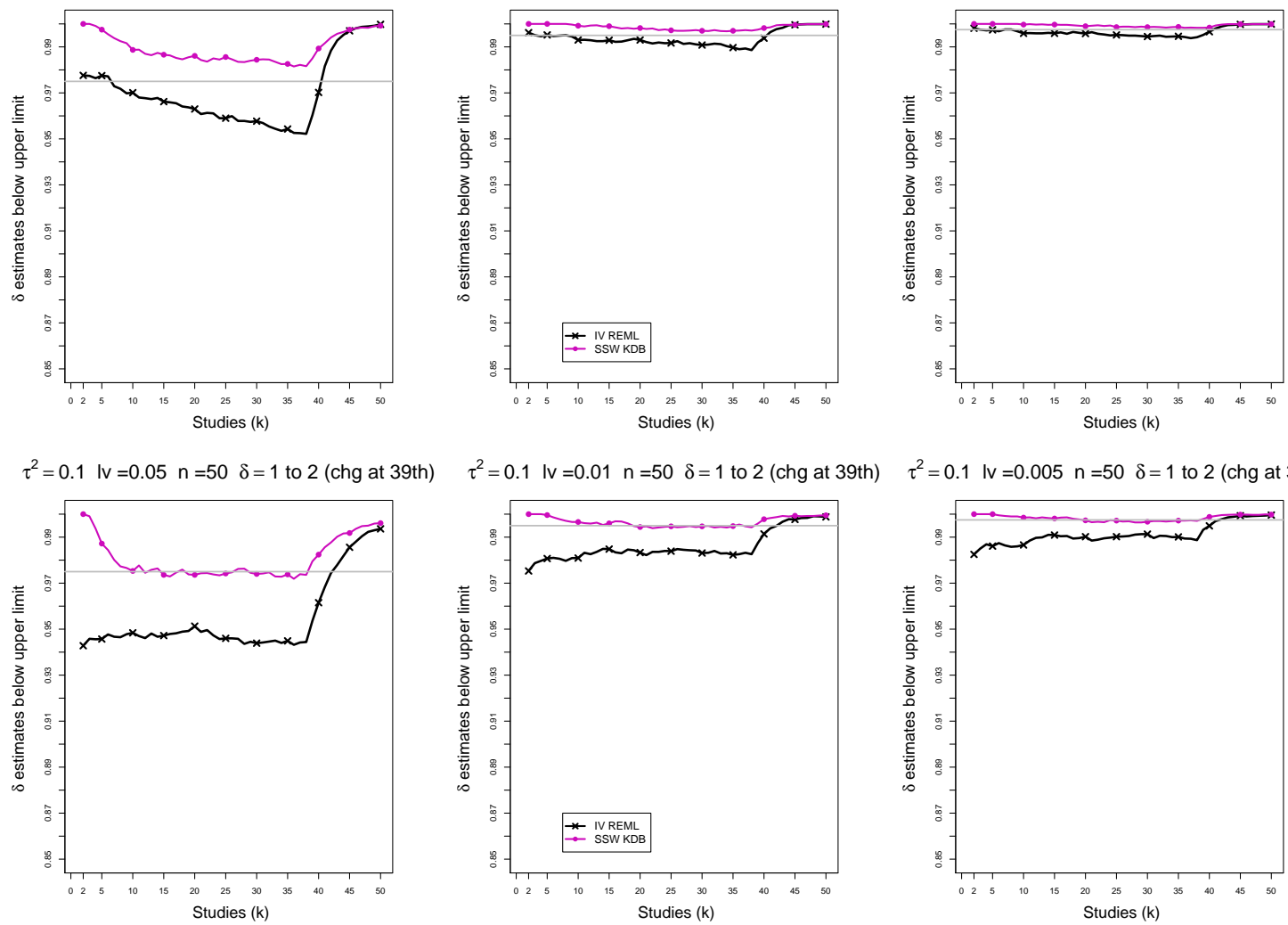

$\tau^{2}=0.1 \mathrm{IV}=0.005 \mathrm{n}=50 \delta=1$ to 2 (chg at 39 th)

$\tau^{2}=0.25 \mathrm{lv}=0.05 \mathrm{n}=50 \quad \delta=1$ to 2 (chg at 39 th)
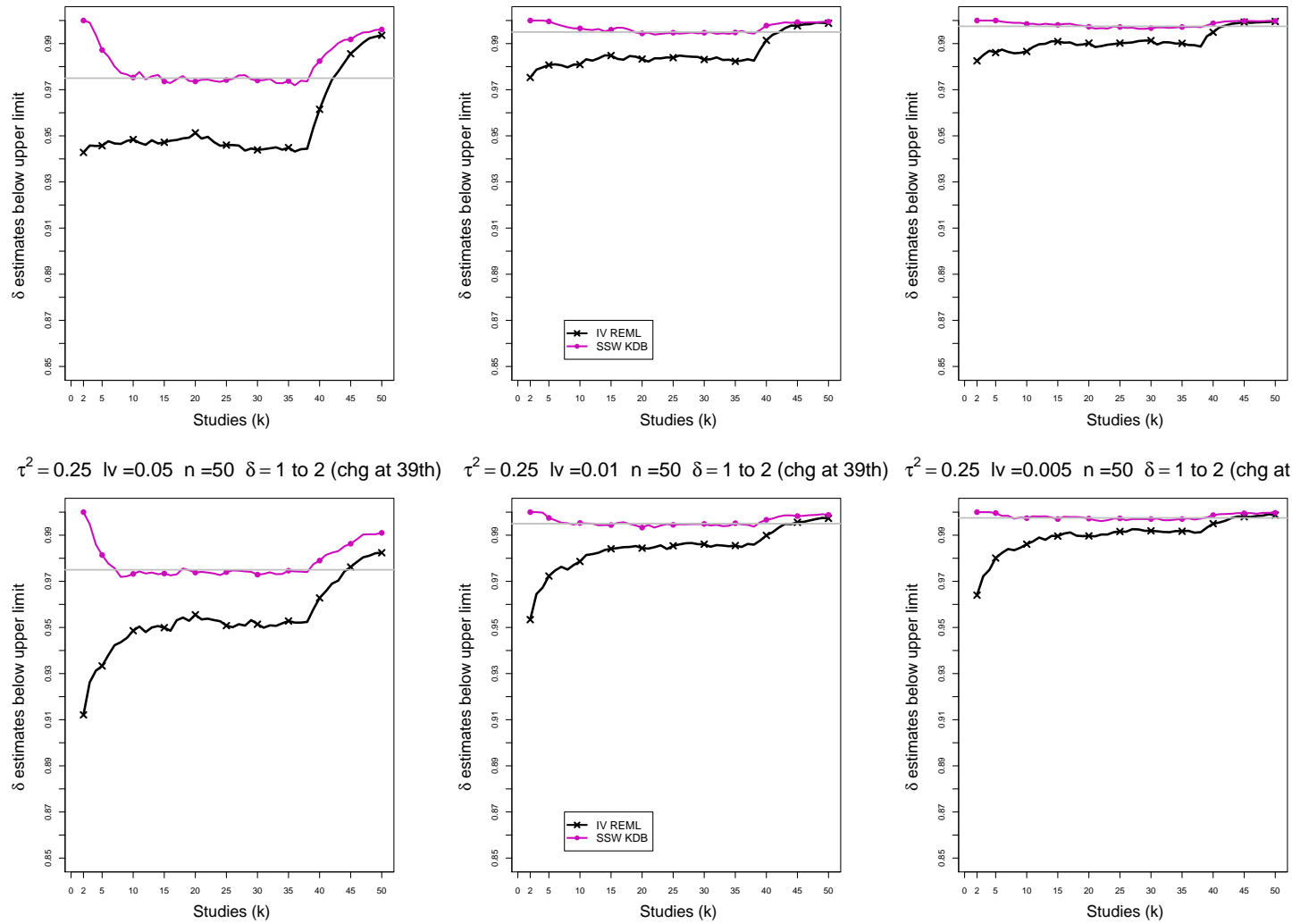

$\tau^{2}=1 \mathrm{lv}=0.05 \mathrm{n}=50 \delta=1$ to 2 (chg at 39 th)

$\tau^{2}=1 \mathrm{IV}=0.01 \mathrm{n}=50 \quad \delta=1$ to 2 (chg at 39 th)

$\tau^{2}=1 \mathrm{lv}=0.005 \mathrm{n}=50 \delta=1$ to 2 (chg at 39th)
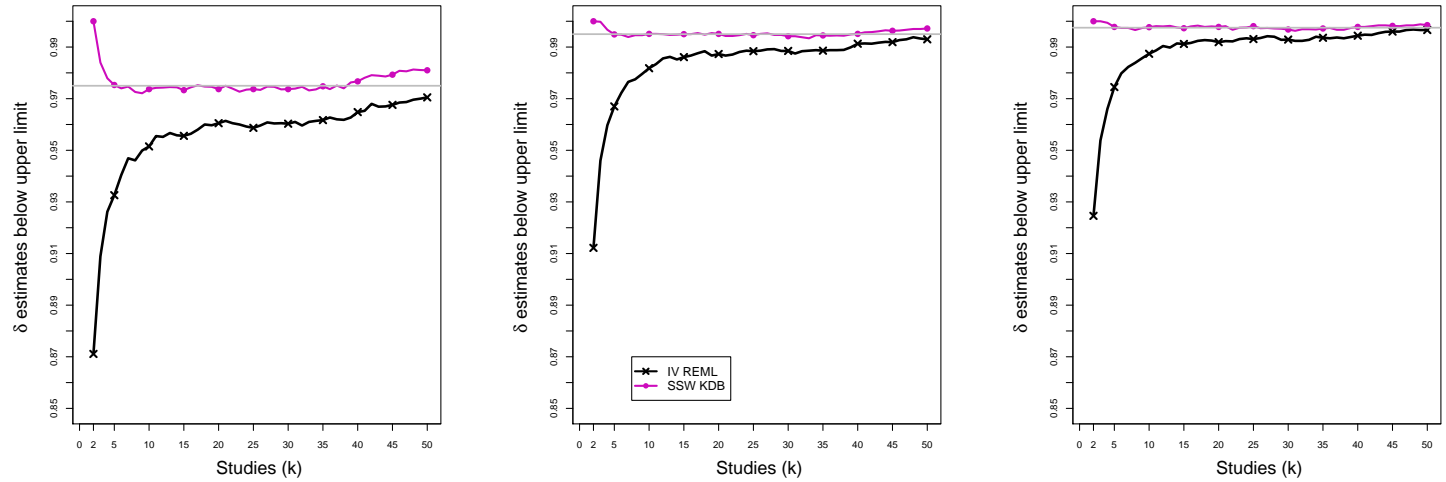

Figure C4. 27: CMA - Coverage of the upper confidence interval of $\delta$ (below upper limit) at the confidence level $1-\alpha / 2$ for $\alpha=0.05,0.01,0.005$ when $\delta=1$ for $k \leq 38$ followed by a shift to $\delta=2$ for $k \geq 39, \tau^{2}=0,0.1,0.25,1, n=50, K=50$. Light grey line at $0.975,0.995,0.9975$. 

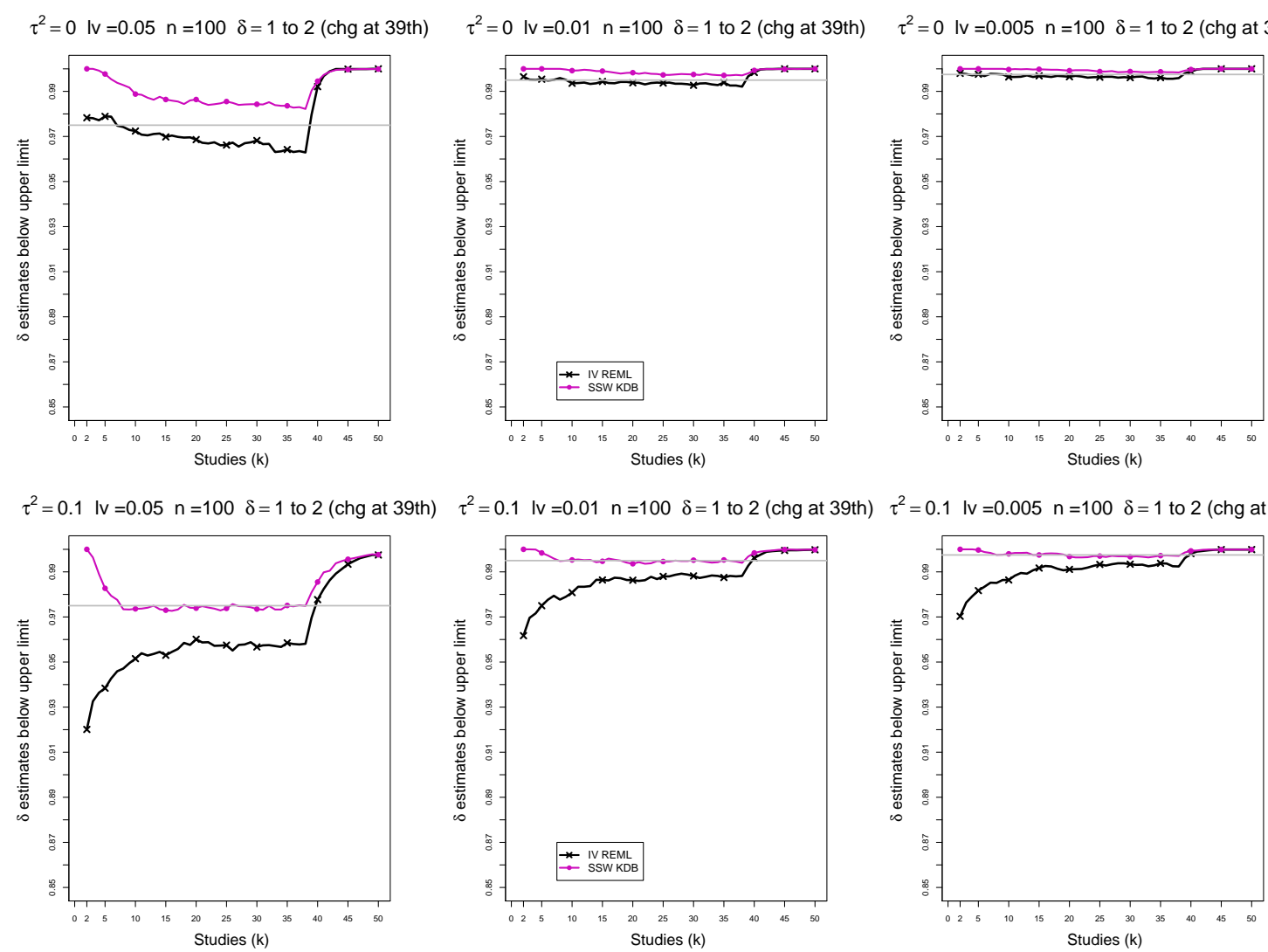

$\tau^{2}=0.25 \mathrm{lv}=0.05 \mathrm{n}=100 \delta=1$ to 2 (chg at 39 th) $\tau^{2}=0.25 \mathrm{lv}=0.01 \mathrm{n}=100 \quad \delta=1$ to 2 (chg at 39th) $\tau^{2}=0.25 \mathrm{lv}=0.005 \mathrm{n}=100 \quad \delta=1$ to 2 (chg at 39 th)
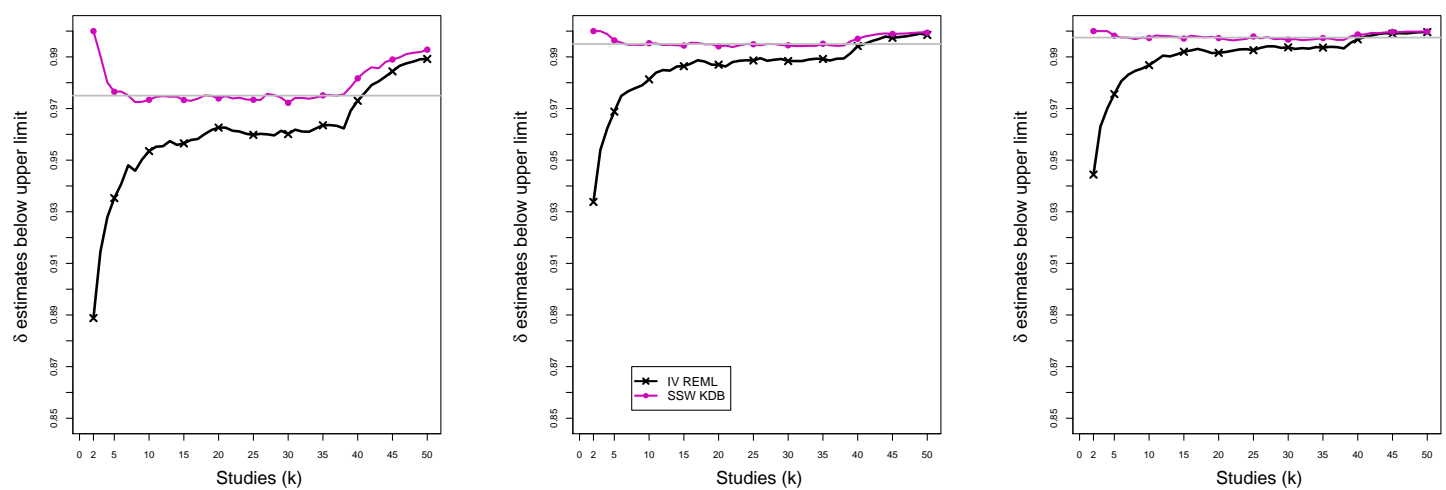

$\tau^{2}=1 \mathrm{IV}=0.05 \mathrm{n}=100 \delta=1$ to 2 (chg at 39 th)

$\tau^{2}=1 \mathrm{lv}=0.01 \mathrm{n}=100 \delta=1$ to 2 (chg at 39 th)

$\tau^{2}=1 \mathrm{lv}=0.005 \mathrm{n}=100 \delta=1$ to 2 (chg at 39th)
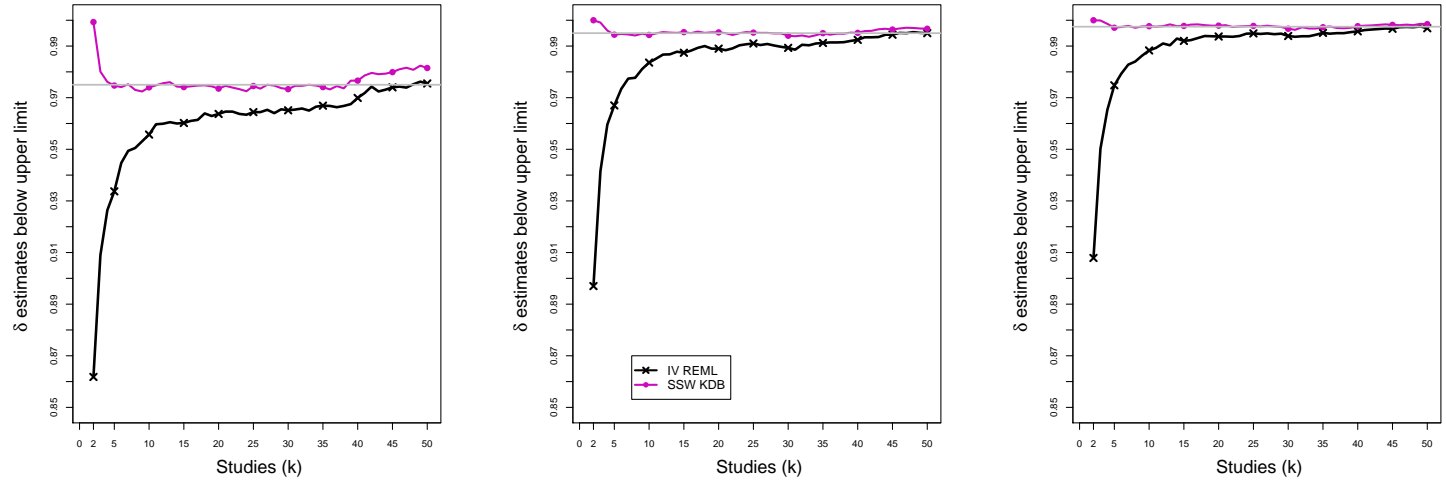

Figure C4. 28: CMA - Coverage of the upper confidence interval of $\delta$ (below upper limit) at the confidence level $1-\alpha / 2$ for $\alpha=0.05,0.01,0.005$ when $\delta=1$ for $k \leq 38$ followed by a shift to $\delta=2$ for $k \geq 39, \tau^{2}=0,0.1,0.25,1, n=100, K=50$. Light grey line at $0.975,0.995,0.9975$. 

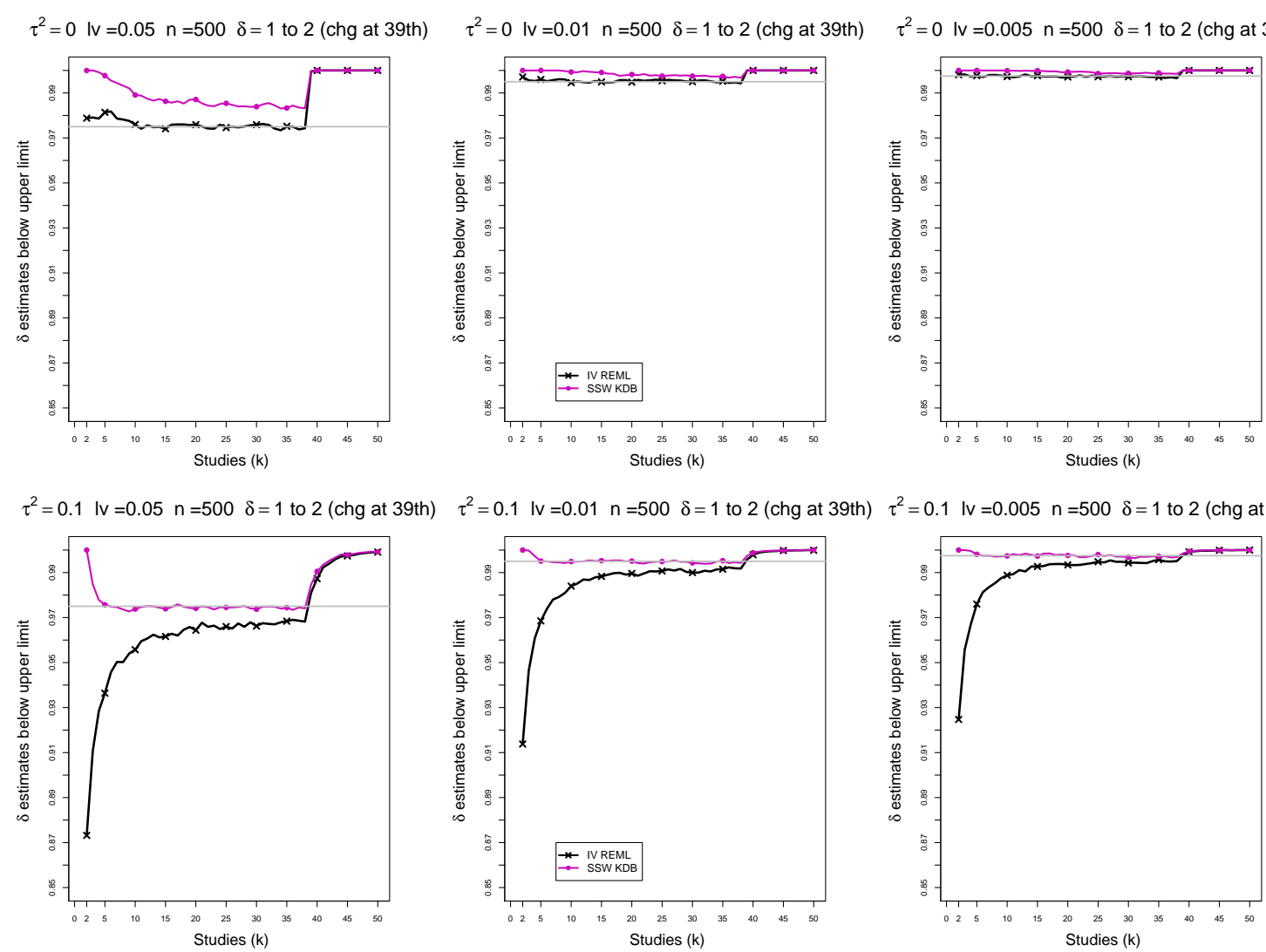

$\tau^{2}=0.25 \mathrm{lv}=0.05 \mathrm{n}=500 \delta=1$ to 2 (chg at 39 th) $\tau^{2}=0.25 \mathrm{lv}=0.01 \mathrm{n}=500 \quad \delta=1$ to 2 (chg at 39th) $\tau^{2}=0.25 \mathrm{lv}=0.005 \mathrm{n}=500 \quad \delta=1$ to 2 (chg at 39 th)
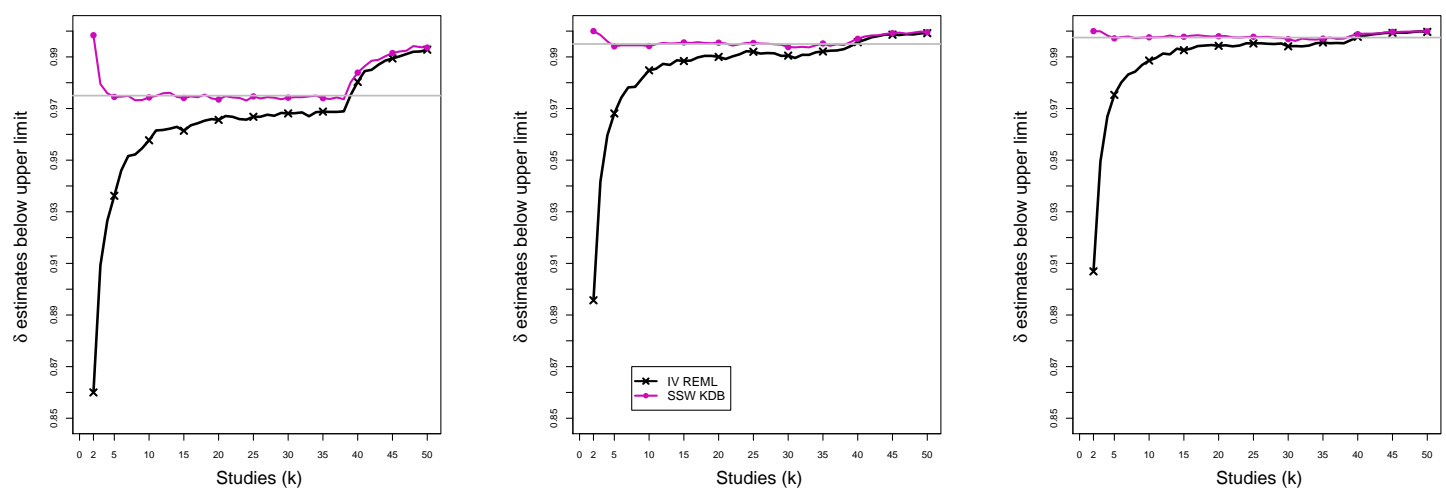

$\tau^{2}=1 \mathrm{IV}=0.05 \mathrm{n}=500 \delta=1$ to 2 (chg at 39 th)

$\tau^{2}=1 \mathrm{lv}=0.01 \mathrm{n}=500 \delta=1$ to 2 (chg at 39 th)

$\tau^{2}=1 \mathrm{lv}=0.005 \mathrm{n}=500 \delta=1$ to 2 (chg at 39 th)
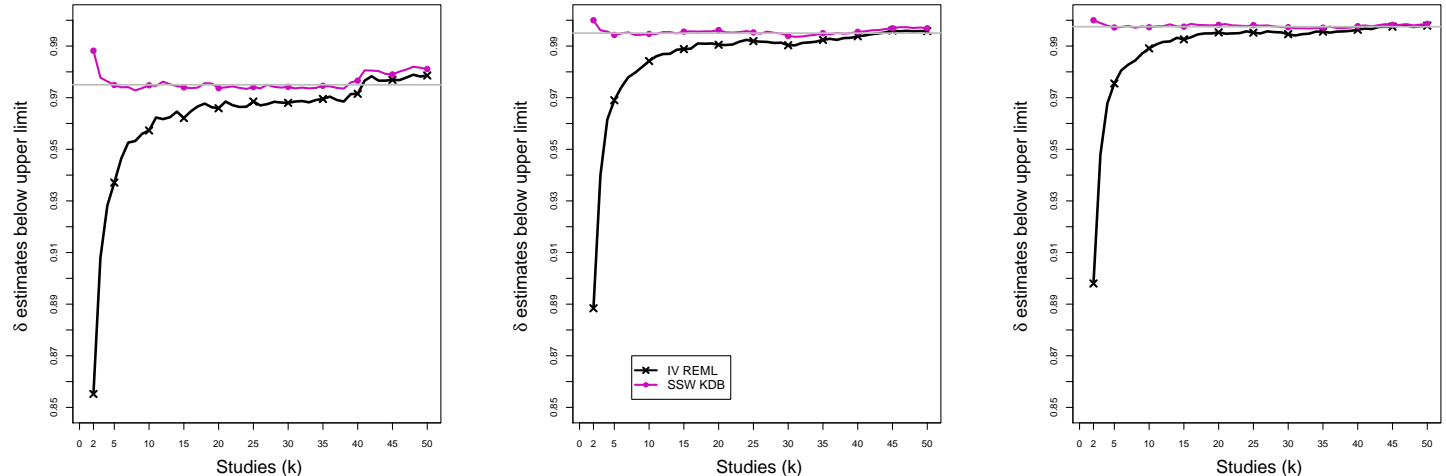

Figure C4. 29: CMA - Coverage of the upper confidence interval of $\delta$ (below upper limit) at the confidence level $1-\alpha / 2$ for $\alpha=0.05,0.01,0.005$ when $\delta=1$ for $k \leq 38$ followed by a shift to $\delta=2$ for $k \geq 39, \tau^{2}=0,0.1,0.25,1, n=500, K=50$. Light grey line at $0.975,0.995,0.9975$. 

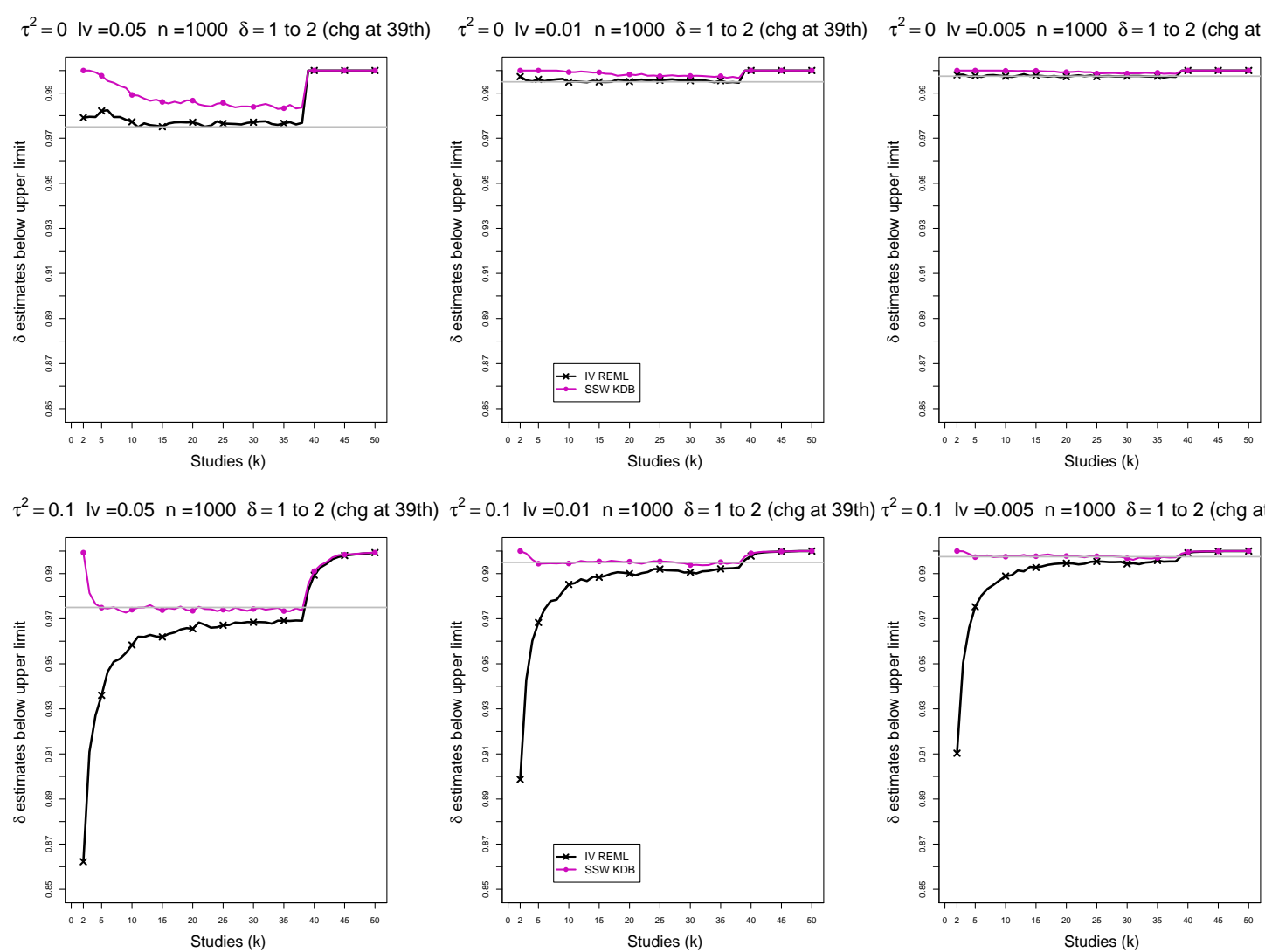

$\tau^{2}=0.25 \mathrm{lv}=0.05 \mathrm{n}=1000 \quad \delta=1$ to 2 (chg at 39 th) $\tau^{2}=0.25 \mathrm{lv}=0.01 \mathrm{n}=1000 \quad \delta=1$ to 2 (chg at 39 th) $:^{2}=0.25 \mathrm{lv}=0.005 \mathrm{n}=1000 \quad \delta=1$ to 2 (chg at 39 th
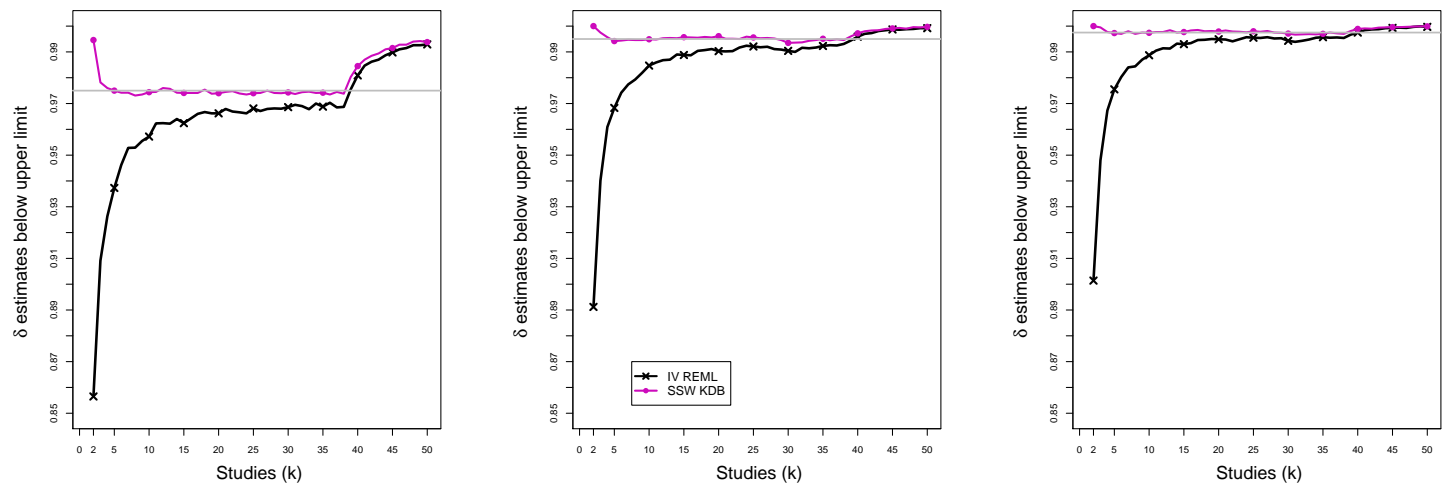

$\tau^{2}=1 \mathrm{lv}=0.05 \mathrm{n}=1000 \delta=1$ to 2 (chg at 39th)

$\tau^{2}=1 \mathrm{lv}=0.01 \mathrm{n}=1000 \delta=1$ to 2 (chg at 39 th) $\tau^{2}=1 \mathrm{lv}=0.005 \mathrm{n}=1000 \delta=1$ to 2 (chg at 39th)
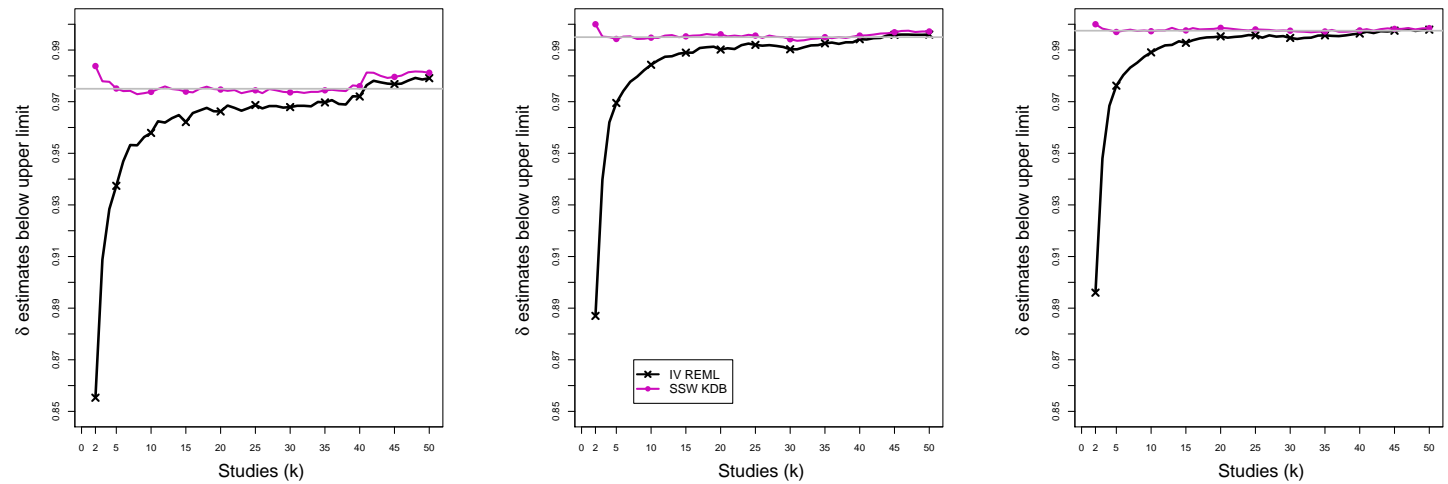

Figure C4. 30: CMA - Coverage of the upper confidence interval of $\delta$ (below upper limit) at the confidence level $1-\alpha / 2$ for $\alpha=0.05,0.01,0.005$ when $\delta=1$ for $k \leq 38$ followed by a shift to $\delta=2$ for $k \geq 39, \tau^{2}=0,0.1,0.25,1, n=1000, K=50$. Light grey line at $0.975,0.995,0.9975$. 


\section{C5. Bias of point estimators of $\tau^{2}$}

For bias of $\tau^{2}$ in this section, each figure corresponds to a set of values of $\tau^{2}(=0,0.1$, $0.25,1)$, a set of values of $n(=20,50,100$ or 500,1000$)$ and a value of point of shift $f(=13,26,39)$ while $\delta$ shifts from 1 to 2 and number of studies $K=50$.

Each figure contains a panel (with study $k$ on the horizontal axis) for each combination of parameters.

The point estimators of $\tau^{2}$ are

- REML (Restricted maximum likelihood)

- KDB (Kulinskaya-Dollinger-Bjørkestøl (2011)) 

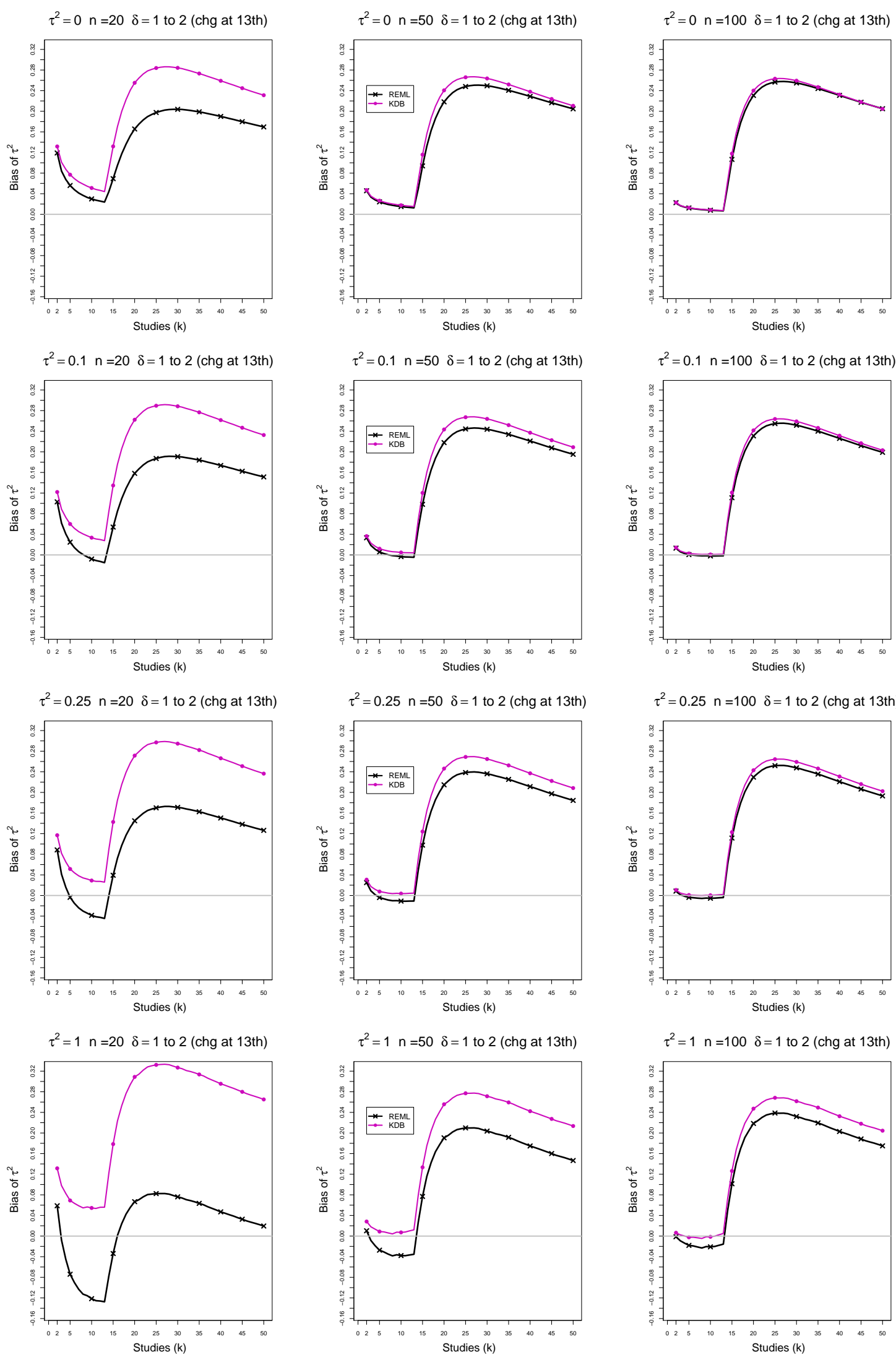

Figure C5. 1: CMA - Bias of the estimation of between-studies variance $\tau^{2}$ with REML and $\mathrm{KDB}$ when $\delta=1$ for $k \leq 12$ followed by a shift to $\delta=2$ for $k \geq 13, \tau^{2}=0,0.1,0.25,1$, $n=20,50,100, K=50$. Light grey line at 0 . 

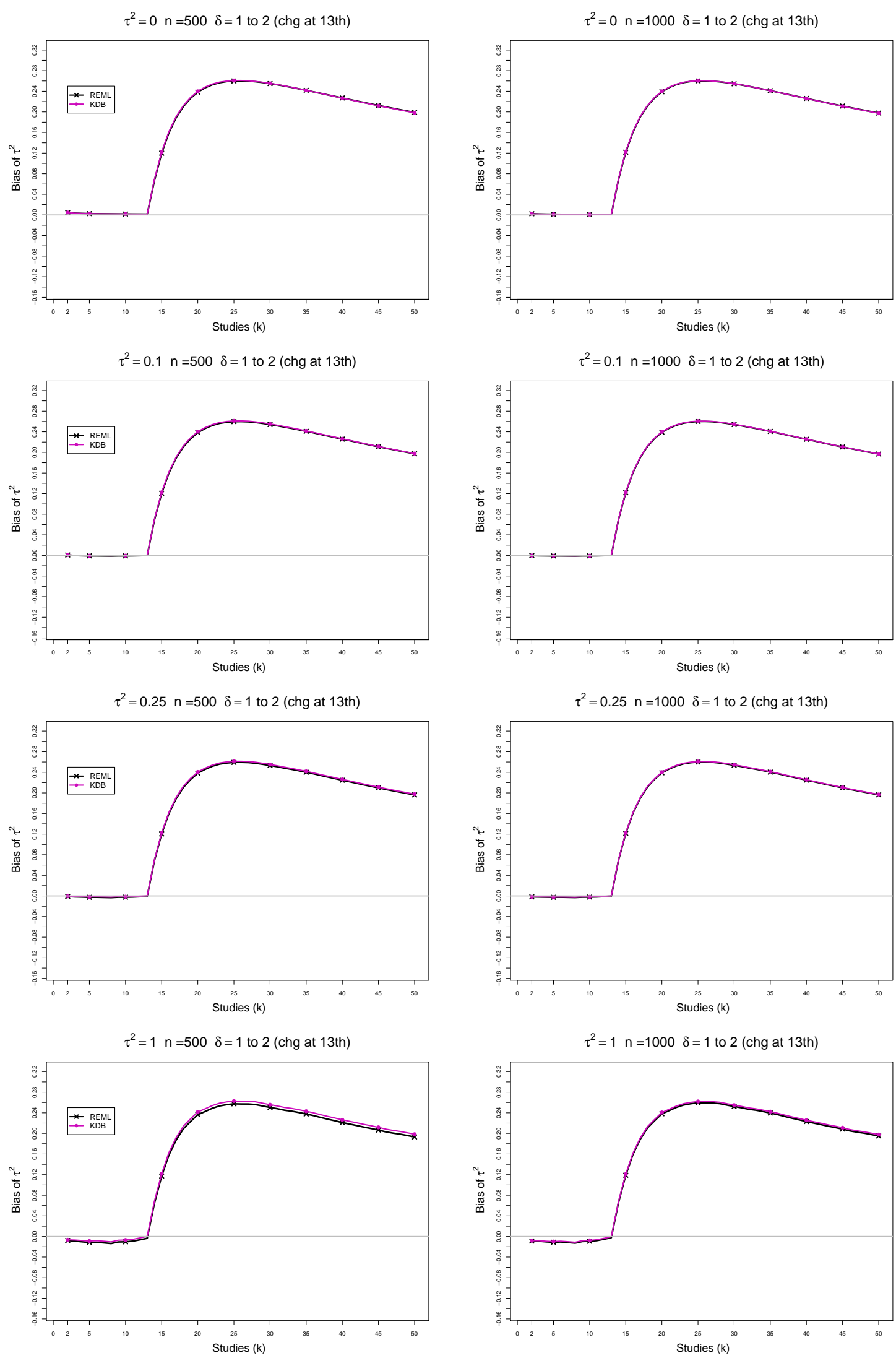

Figure C5. 2: CMA - Bias of the estimation of between-studies variance $\tau^{2}$ with REML and $\mathrm{KDB}$ when $\delta=1$ for $k \leq 12$ followed by a shift to $\delta=2$ for $k \geq 13, \tau^{2}=0,0.1,0.25,1$, $n=500,1000, K=50$. Light grey line at 0 . 

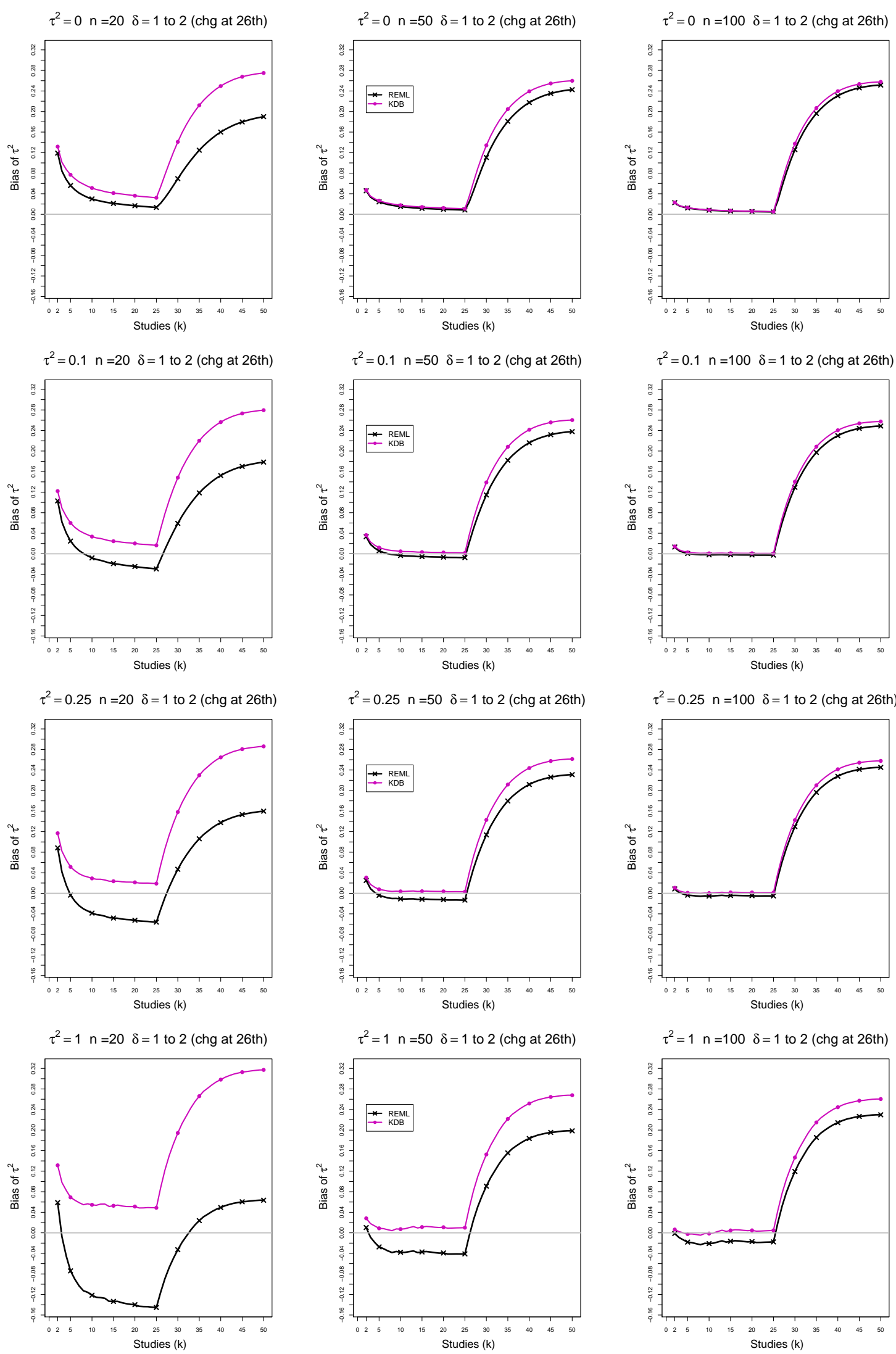

Figure C5. 3: CMA - Bias of the estimation of between-studies variance $\tau^{2}$ with REML and $\mathrm{KDB}$ when $\delta=1$ for $k \leq 25$ followed by a shift to $\delta=2$ for $k \geq 26, \tau^{2}=0,0.1,0.25,1$, $n=20,50,100, K=50$. Light grey line at 0 . 

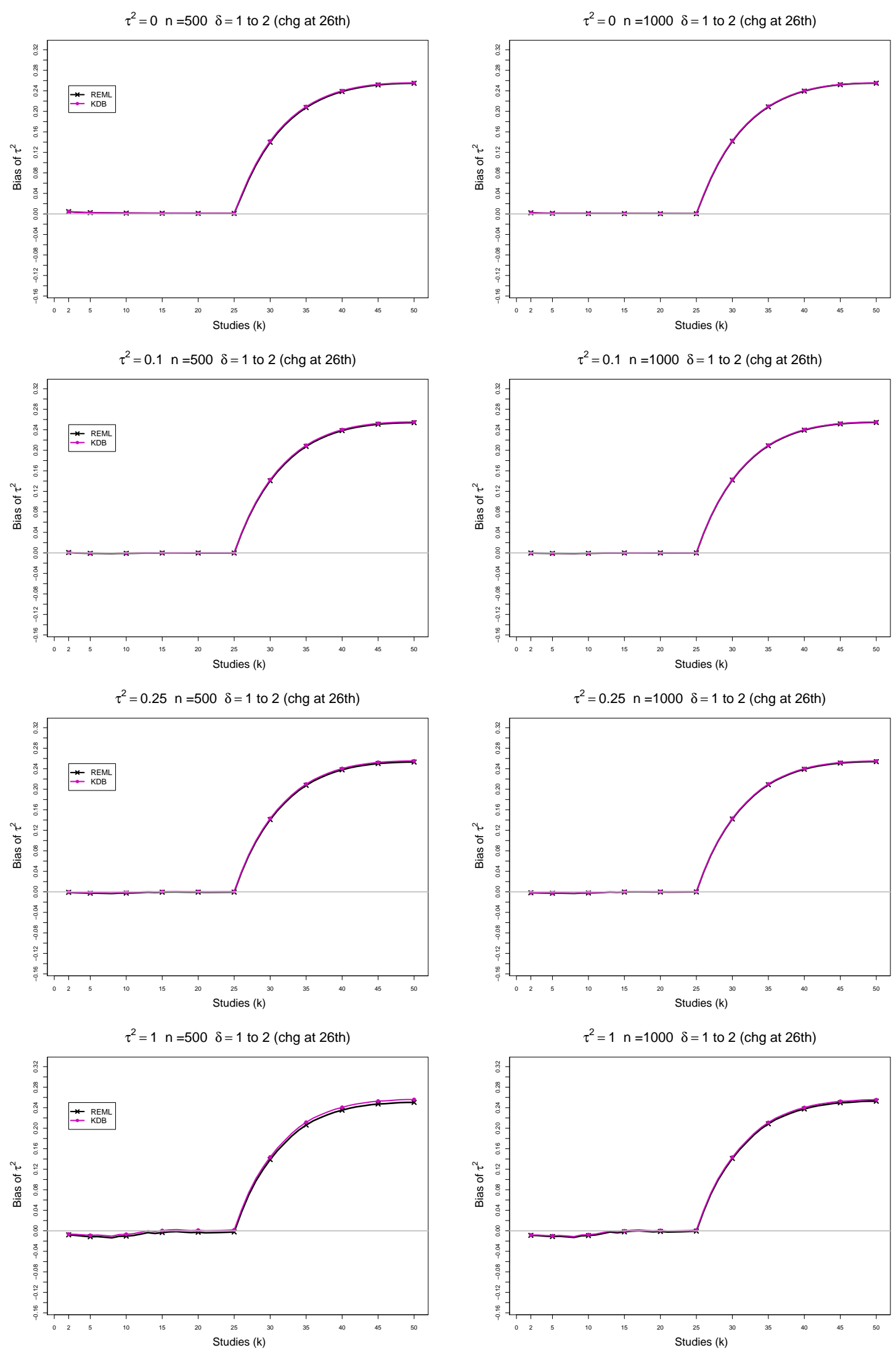

Figure C5. 4: CMA - Bias of the estimation of between-studies variance $\tau^{2}$ with REML and $\mathrm{KDB}$ when $\delta=1$ for $k \leq 25$ followed by a shift to $\delta=2$ for $k \geq 26, \tau^{2}=0,0.1,0.25,1$, $n=500,1000, K=50$. Light grey line at 0 . 

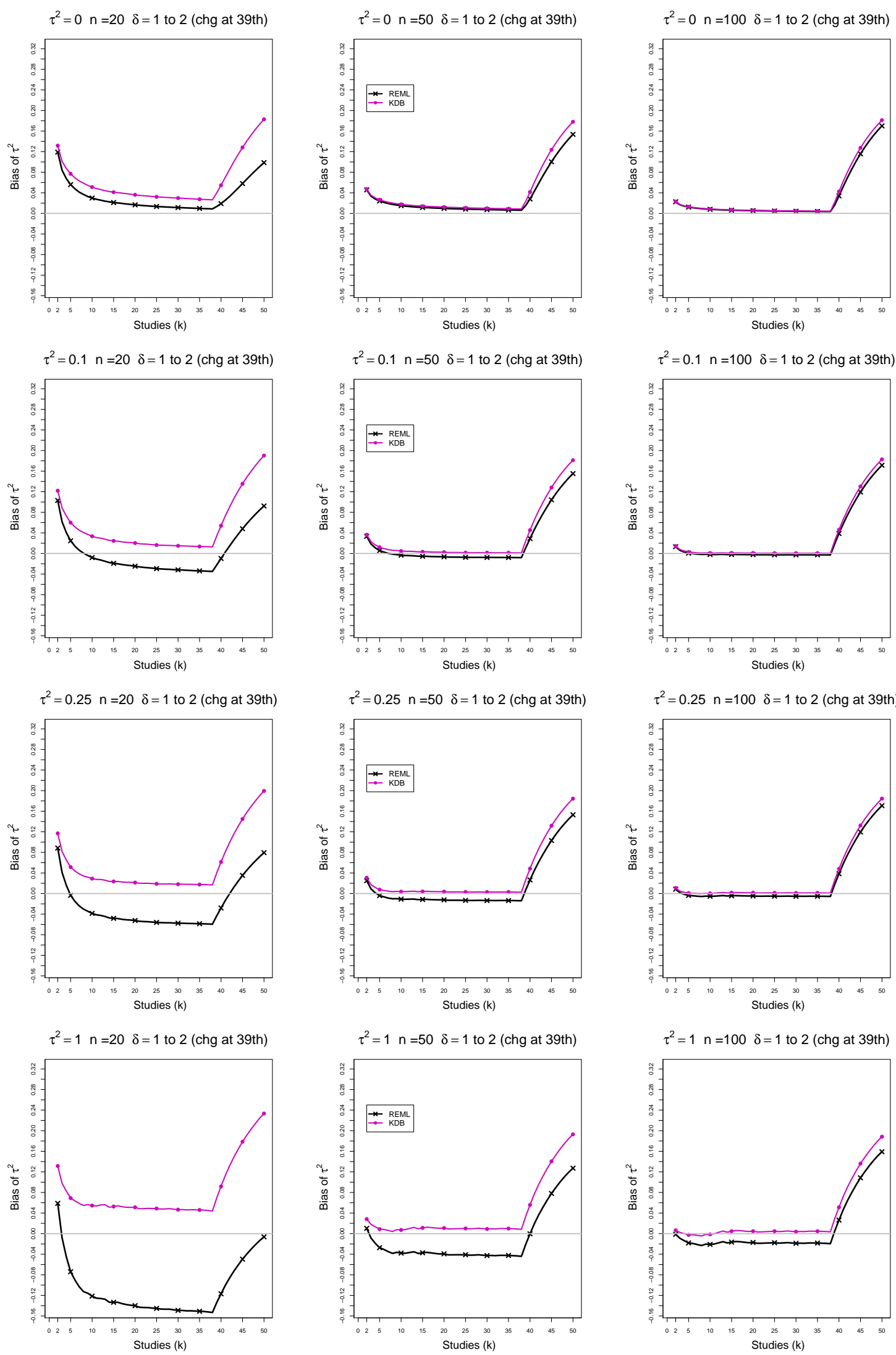

Figure C5. 5: CMA - Bias of the estimation of between-studies variance $\tau^{2}$ with REML and $\mathrm{KDB}$ when $\delta=1$ for $k \leq 38$ followed by a shift to $\delta=2$ for $k \geq 39, \tau^{2}=0,0.1,0.25,1$, $n=20,50,100, K=50$. Light grey line at 0 . 

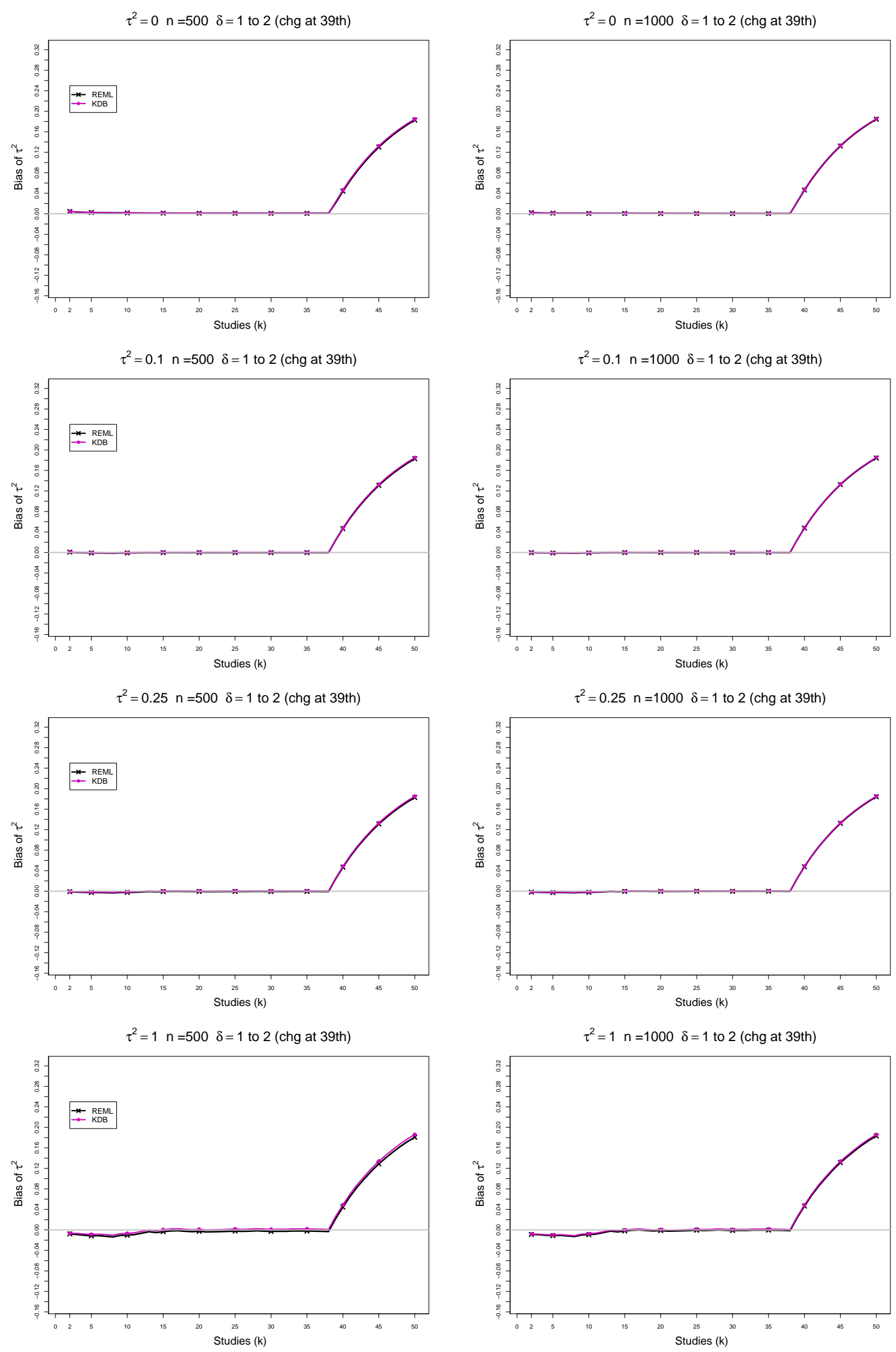

Figure C5. 6: CMA - Bias of the estimation of between-studies variance $\tau^{2}$ with REML and $\mathrm{KDB}$ when $\delta=1$ for $k \leq 38$ followed by a shift to $\delta=2$ for $k \geq 39, \tau^{2}=0,0.1,0.25,1$, $n=500,1000, K=50$. Light grey line at 0 . 


\section{C6. Empirical levels and power of CMA tests for shift in $\delta$}

For empirical levels (power) of tests for shift in $\delta$ in this section, each figure corresponds to one of the two tested meta-analysis methods, a set of values of $\tau^{2}(=0$, $0.1,0.25,1)$, a value of $n(=20,50,100,500,1000)$, a set of values of significance level $(=0.05,0.01,0.005)$ and a value of point of shift $f(=13,26,39)$ while $\delta$ shifts from 1 to 2 and number of studies $K=50$.

Each figure contains a panel (with study $k$ on the horizontal axis) for each combination of parameters.

The tested methods are

- IV REML (Restricted maximum likelihood, inverse variance weighted)

- SSW KDB (Kulinskaya-Dollinger-Bjørkestøl estimator of $\tau^{2}$, sample size weighted) 

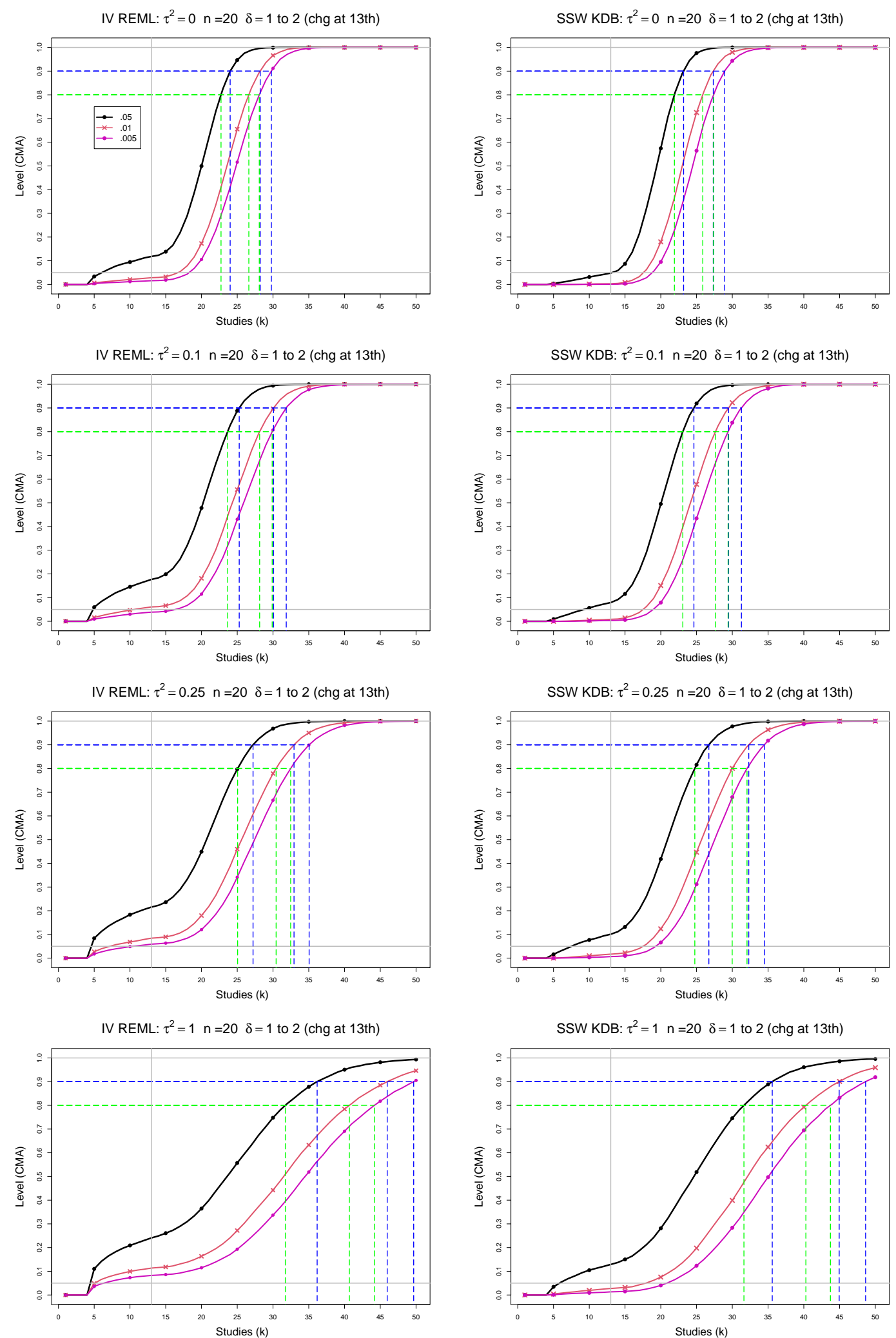

Figure C6. 1: Empirical levels of CMA tests for shift in $\delta$ based on SSW KDB and IV REML at .05, .01 and .005 nominal levels for equal sample sizes $n_{i C}+n_{i T}=n=20$, $\tau^{2}=0,0.1,0.25,1$ and a shift from $\delta=1$ to $\delta=2$ at study number 13. Light grey line at .05. Green and blue dashed lines correspond to power $80 \%$ and $90 \%$, respectively. 

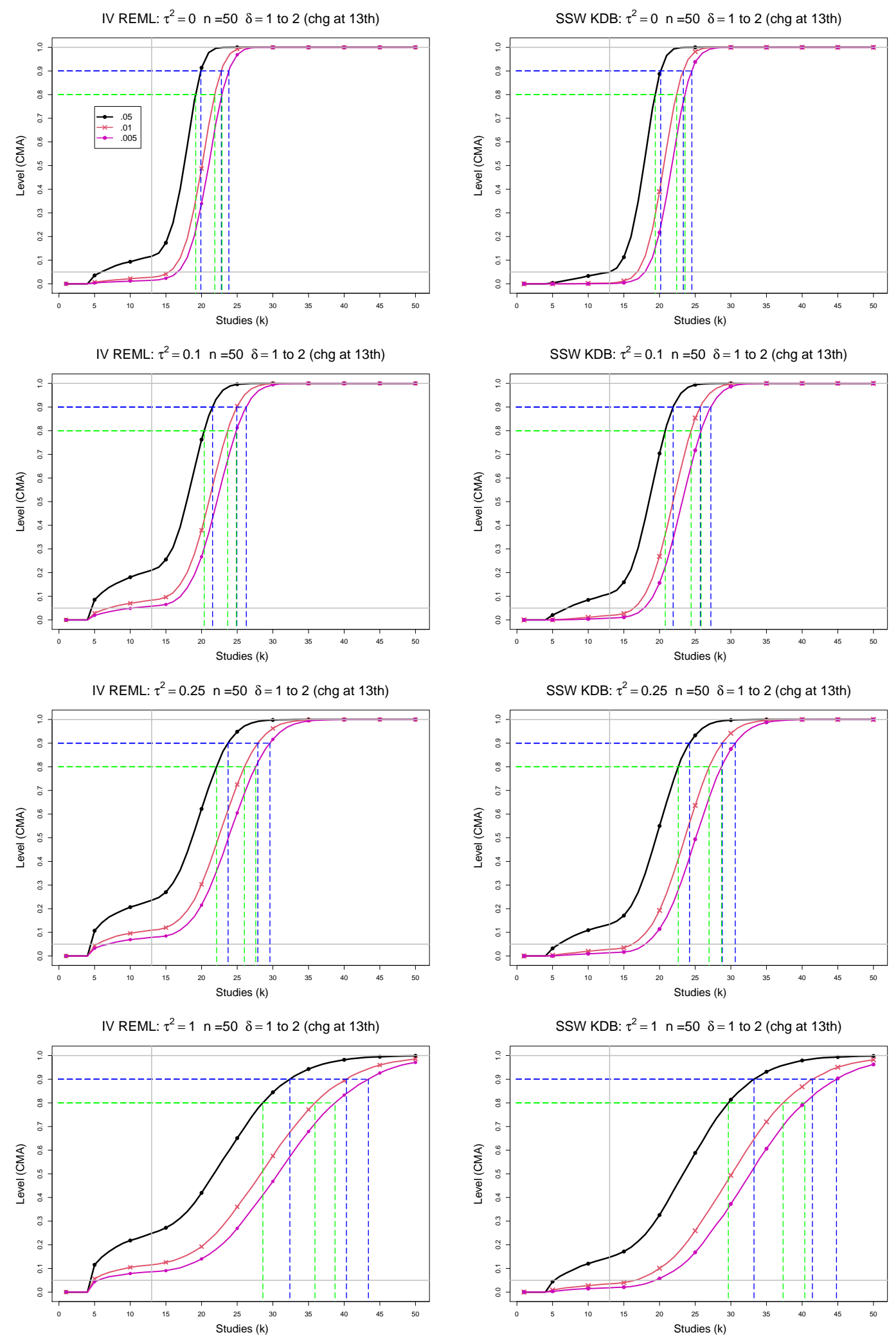

Figure C6. 2: Empirical levels of CMA tests for shift in $\delta$ based on SSW KDB and IV REML at .05, .01 and .005 nominal levels for equal sample sizes $n_{i C}+n_{i T}=n=50$, $\tau^{2}=0,0.1,0.25,1$ and a shift from $\delta=1$ to $\delta=2$ at study number 13. Light grey line at .05. Green and blue dashed lines correspond to power $80 \%$ and $90 \%$, respectively. 

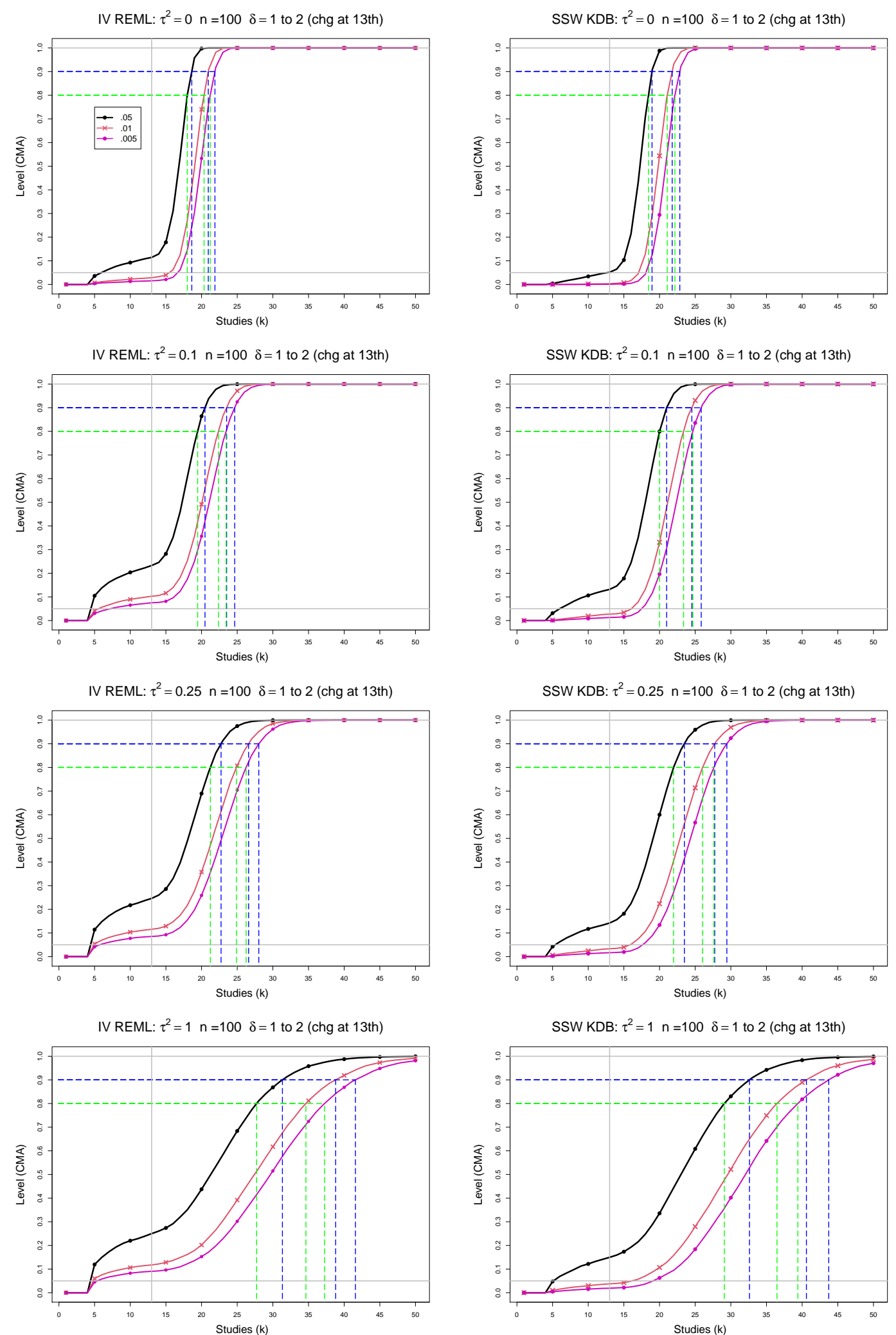

Figure C6. 3: Empirical levels of CMA tests for shift in $\delta$ based on SSW KDB and IV REML at .05, .01 and .005 nominal levels for equal sample sizes $n_{i C}+n_{i T}=n=100$, $\tau^{2}=0,0.1,0.25,1$ and a shift from $\delta=1$ to $\delta=2$ at study number 13. Light grey line at .05. Green and blue dashed lines correspond to power $80 \%$ and $90 \%$, respectively. 

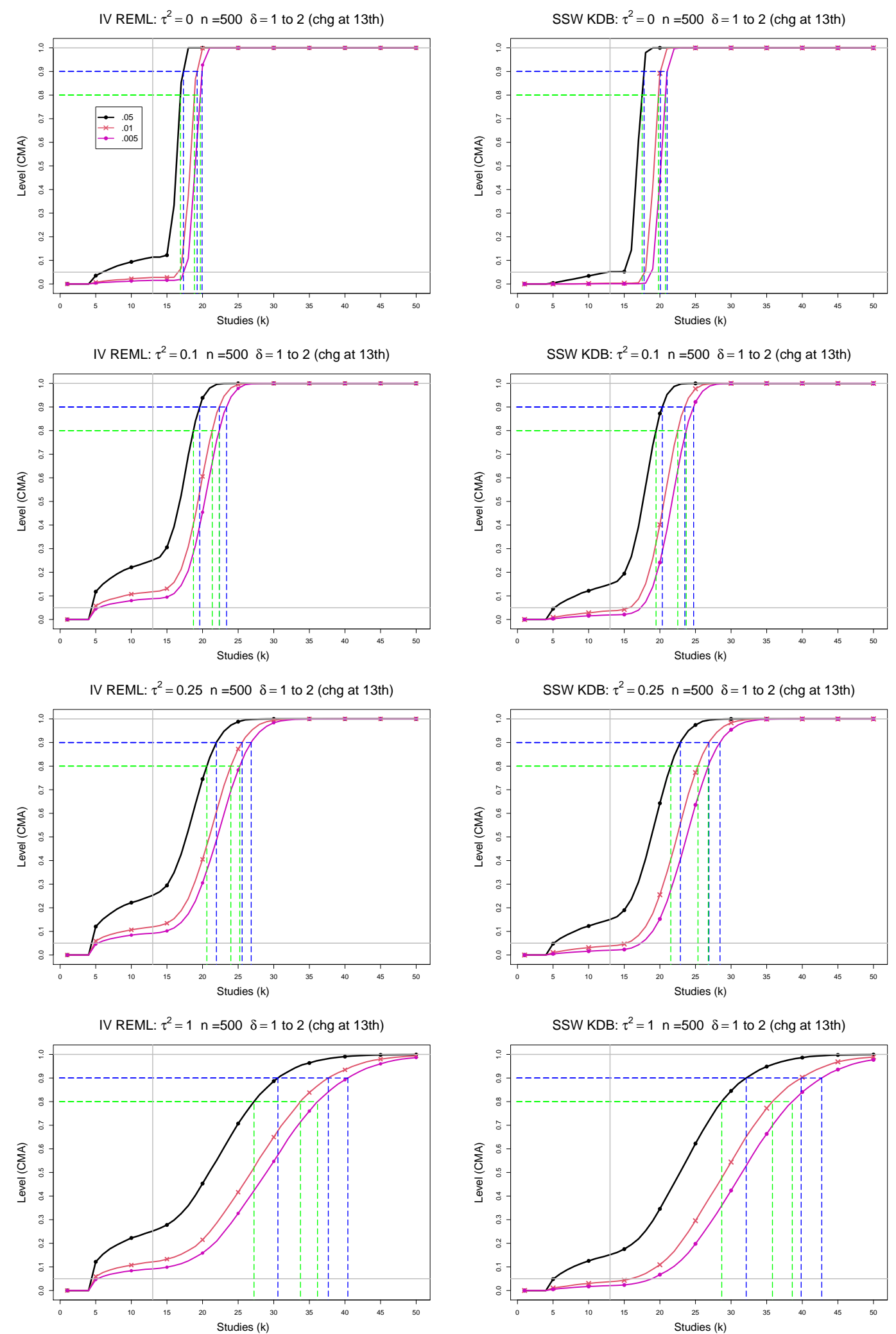

Figure C6. 4: Empirical levels of CMA tests for shift in $\delta$ based on SSW KDB and IV REML at .05, .01 and .005 nominal levels for equal sample sizes $n_{i C}+n_{i T}=n=500$, $\tau^{2}=0,0.1,0.25,1$ and a shift from $\delta=1$ to $\delta=2$ at study number 13. Light grey line at .05. Green and blue dashed lines correspond to power $80 \%$ and $90 \%$, respectively. 

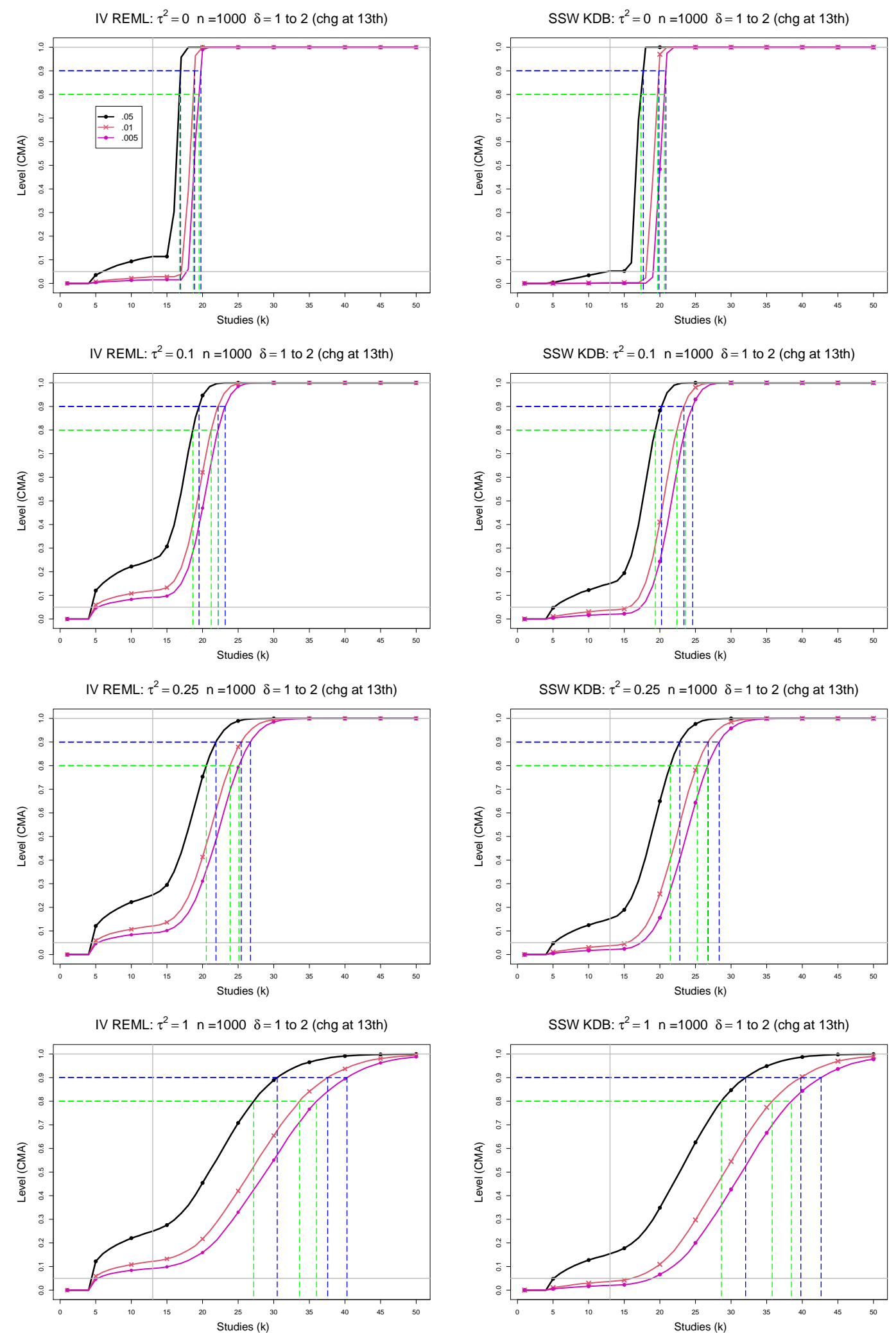

Figure C6. 5: Empirical levels of CMA tests for shift in $\delta$ based on SSW KDB and IV REML at .05, .01 and .005 nominal levels for equal sample sizes $n_{i C}+n_{i T}=n=1000$, $\tau^{2}=0,0.1,0.25,1$ and a shift from $\delta=1$ to $\delta=2$ at study number 13 . Light grey line at .05. Green and blue dashed lines correspond to power $80 \%$ and $90 \%$, respectively. 

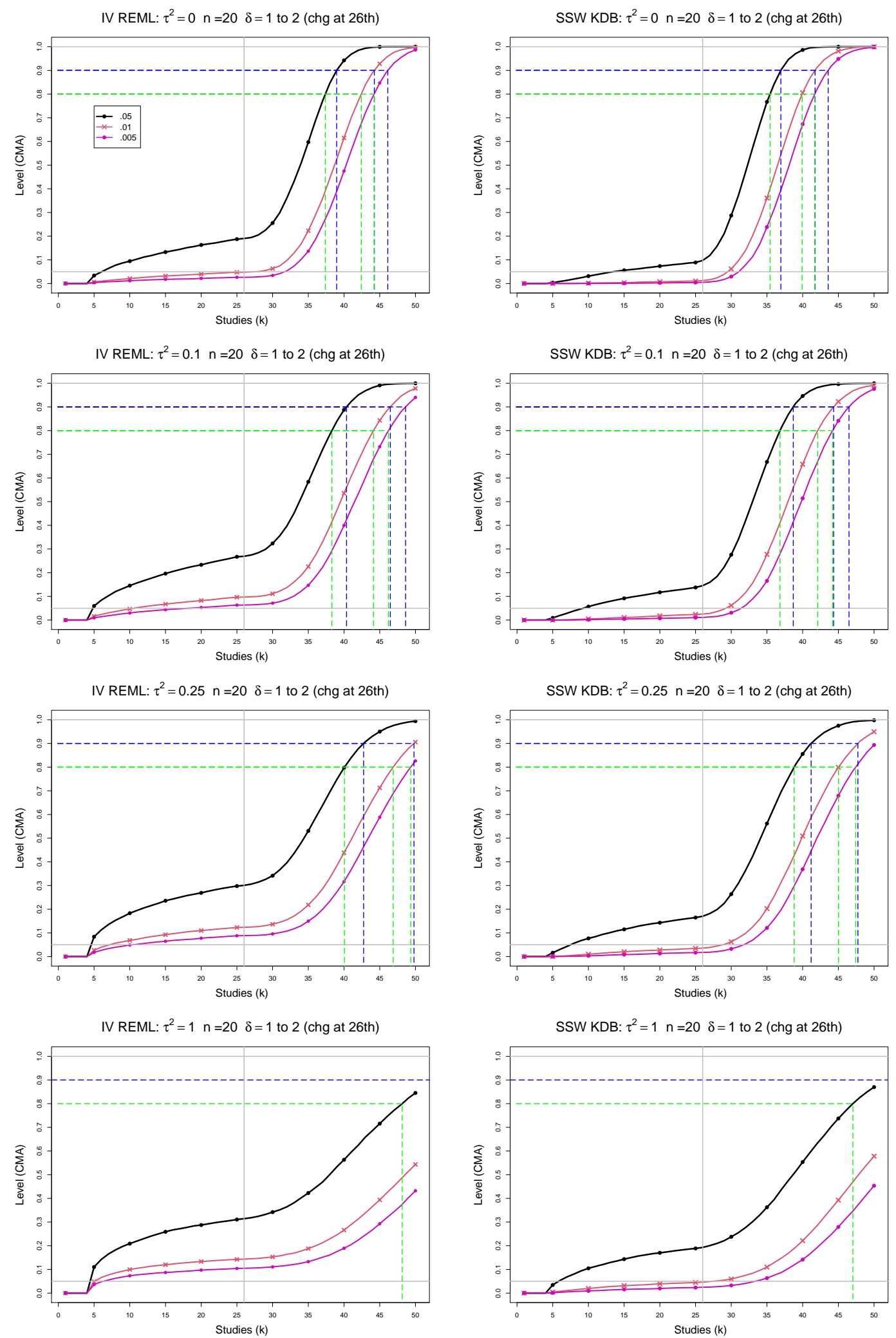

Figure C6. 6: Empirical levels of CMA tests for shift in $\delta$ based on SSW KDB and IV REML at .05, .01 and .005 nominal levels for equal sample sizes $n_{i C}+n_{i T}=n=20$, $\tau^{2}=0,0.1,0.25,1$ and a shift from $\delta=1$ to $\delta=2$ at study number 26. Light grey line at .05. Green and blue dashed lines correspond to power $80 \%$ and $90 \%$, respectively. 

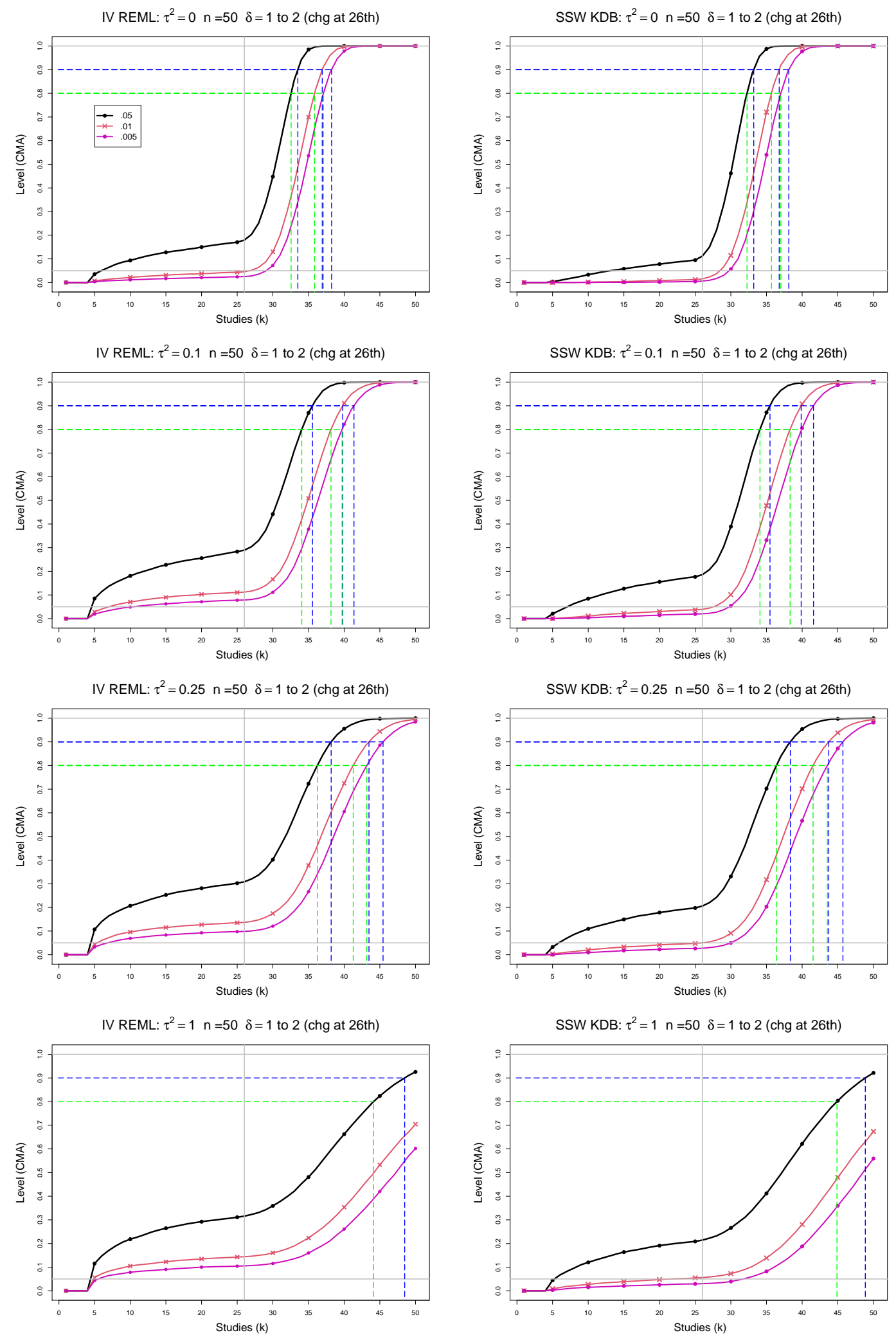

Figure C6. 7: Empirical levels of CMA tests for shift in $\delta$ based on SSW KDB and IV REML at .05, .01 and .005 nominal levels for equal sample sizes $n_{i C}+n_{i T}=n=50$, $\tau^{2}=0,0.1,0.25,1$ and a shift from $\delta=1$ to $\delta=2$ at study number 26. Light grey line at .05. Green and blue dashed lines correspond to power $80 \%$ and $90 \%$, respectively. 

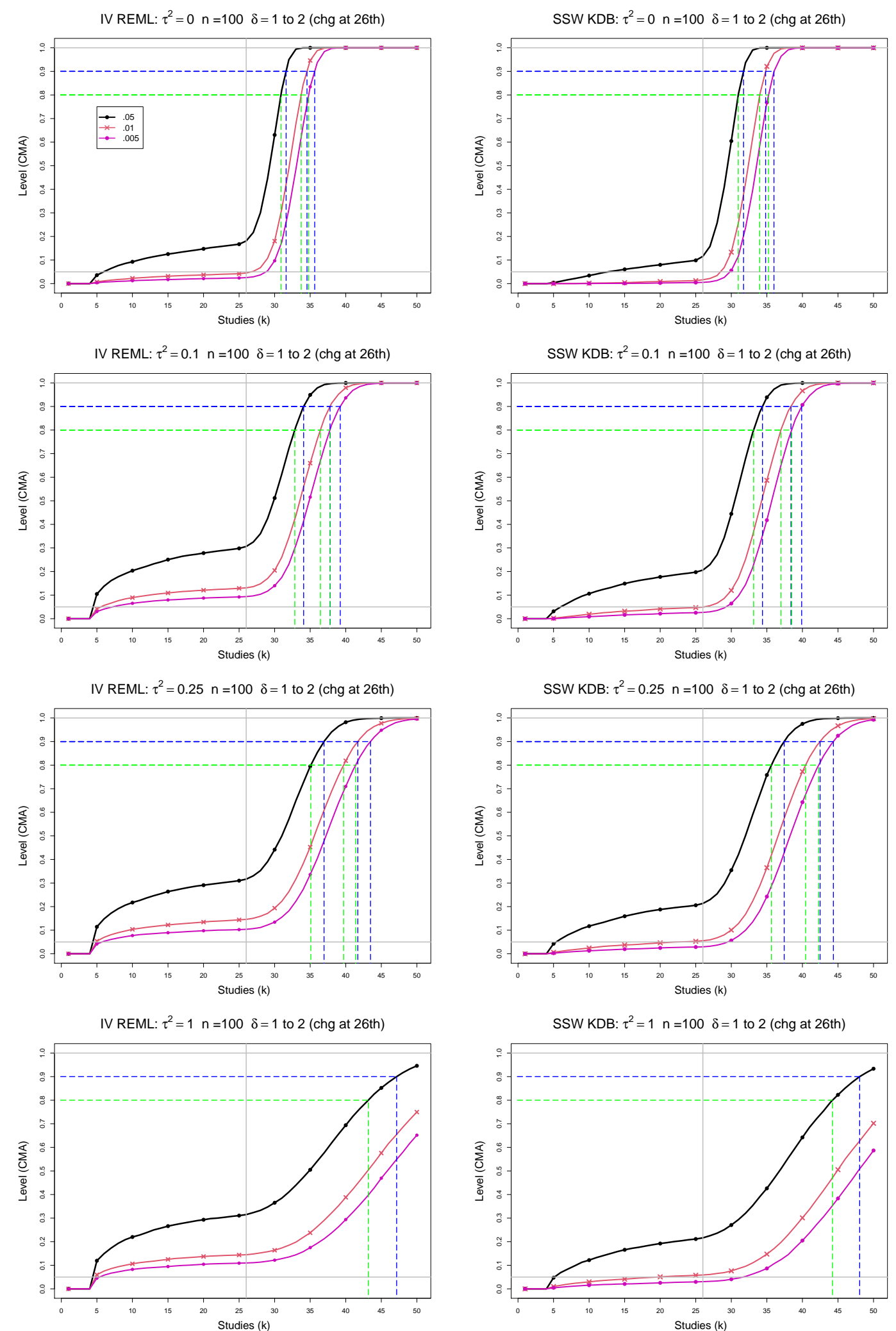

Figure C6. 8: Empirical levels of CMA tests for shift in $\delta$ based on SSW KDB and IV REML at .05, .01 and .005 nominal levels for equal sample sizes $n_{i C}+n_{i T}=n=100$, $\tau^{2}=0,0.1,0.25,1$ and a shift from $\delta=1$ to $\delta=2$ at study number 26. Light grey line at .05. Green and blue dashed lines correspond to power $80 \%$ and $90 \%$, respectively. 

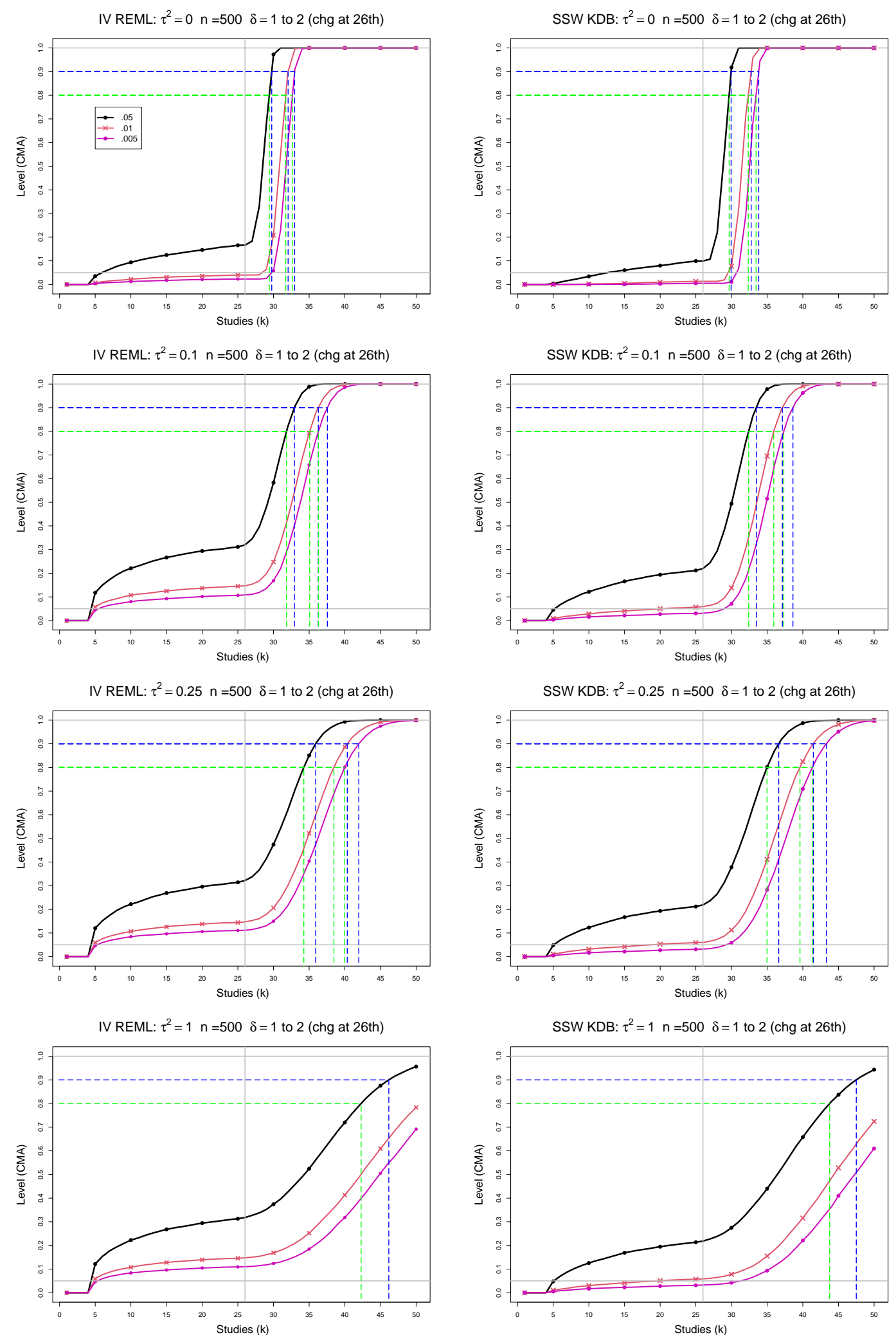

Figure C6. 9: Empirical levels of CMA tests for shift in $\delta$ based on SSW KDB and IV REML at .05, .01 and .005 nominal levels for equal sample sizes $n_{i C}+n_{i T}=n=500$, $\tau^{2}=0,0.1,0.25,1$ and a shift from $\delta=1$ to $\delta=2$ at study number 26. Light grey line at .05. Green and blue dashed lines correspond to power $80 \%$ and $90 \%$, respectively. 

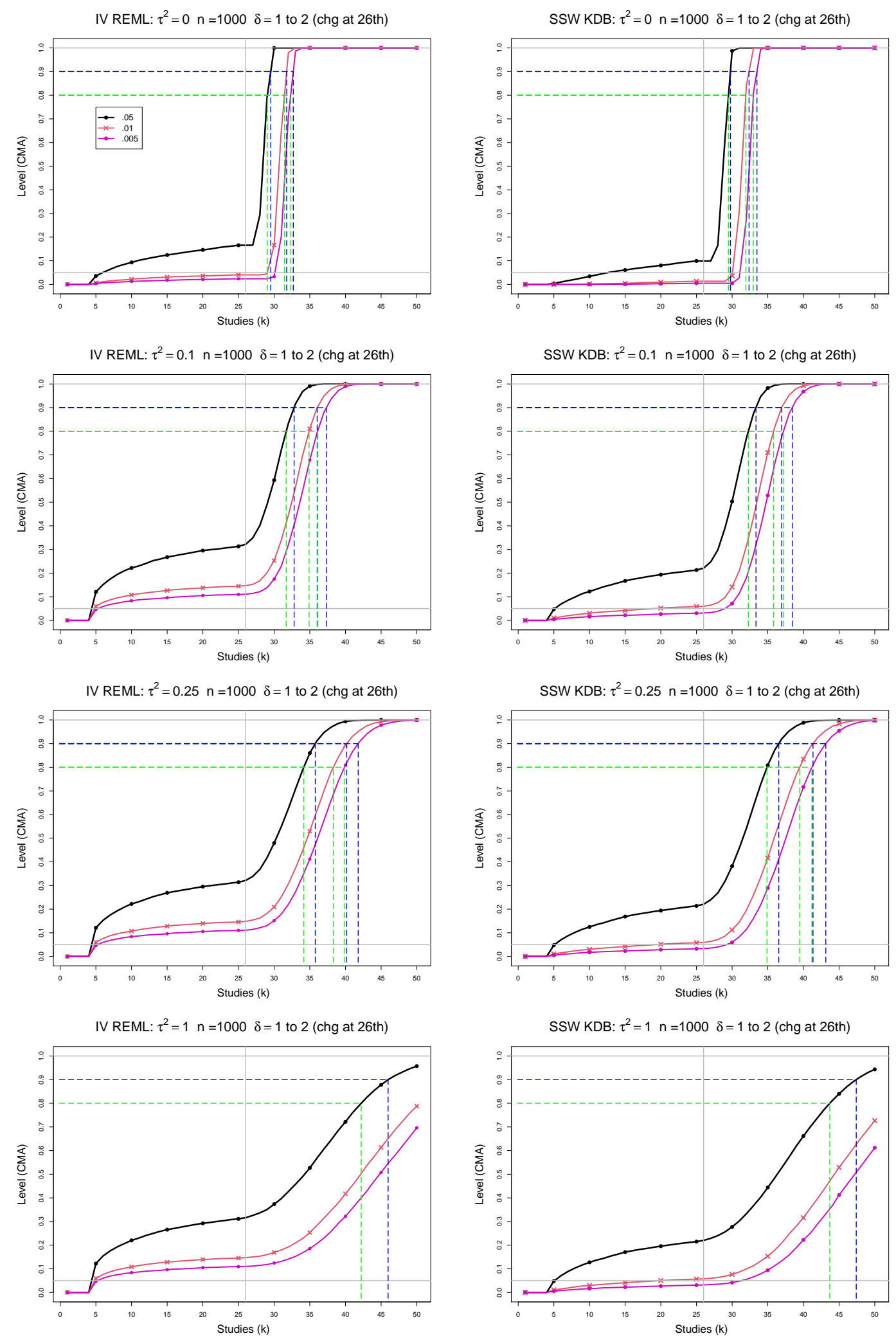

Figure C6. 10: Empirical levels of CMA tests for shift in $\delta$ based on SSW KDB and IV REML at .05, .01 and .005 nominal levels for equal sample sizes $n_{i C}+n_{i T}=n=1000$, $\tau^{2}=0,0.1,0.25,1$ and a shift from $\delta=1$ to $\delta=2$ at study number 26. Light grey line at .05. Green and blue dashed lines correspond to power $80 \%$ and $90 \%$, respectively. 

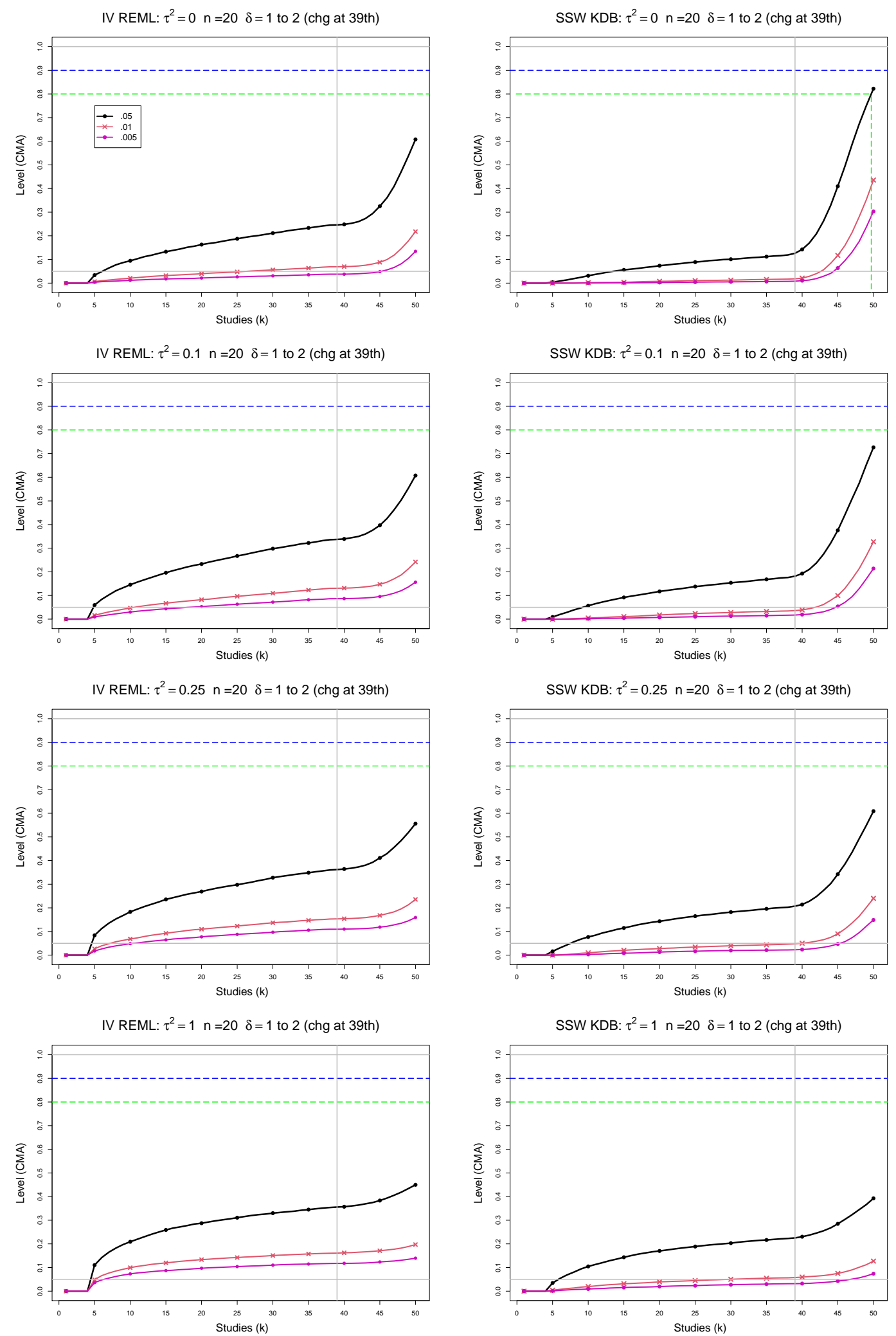

Figure C6. 11: Empirical levels of CMA tests for shift in $\delta$ based on SSW KDB and IV REML at .05, .01 and .005 nominal levels for equal sample sizes $n_{i C}+n_{i T}=n=20$, $\tau^{2}=0,0.1,0.25,1$ and a shift from $\delta=1$ to $\delta=2$ at study number 39. Light grey line at .05. Green and blue dashed lines correspond to power $80 \%$ and $90 \%$, respectively. 

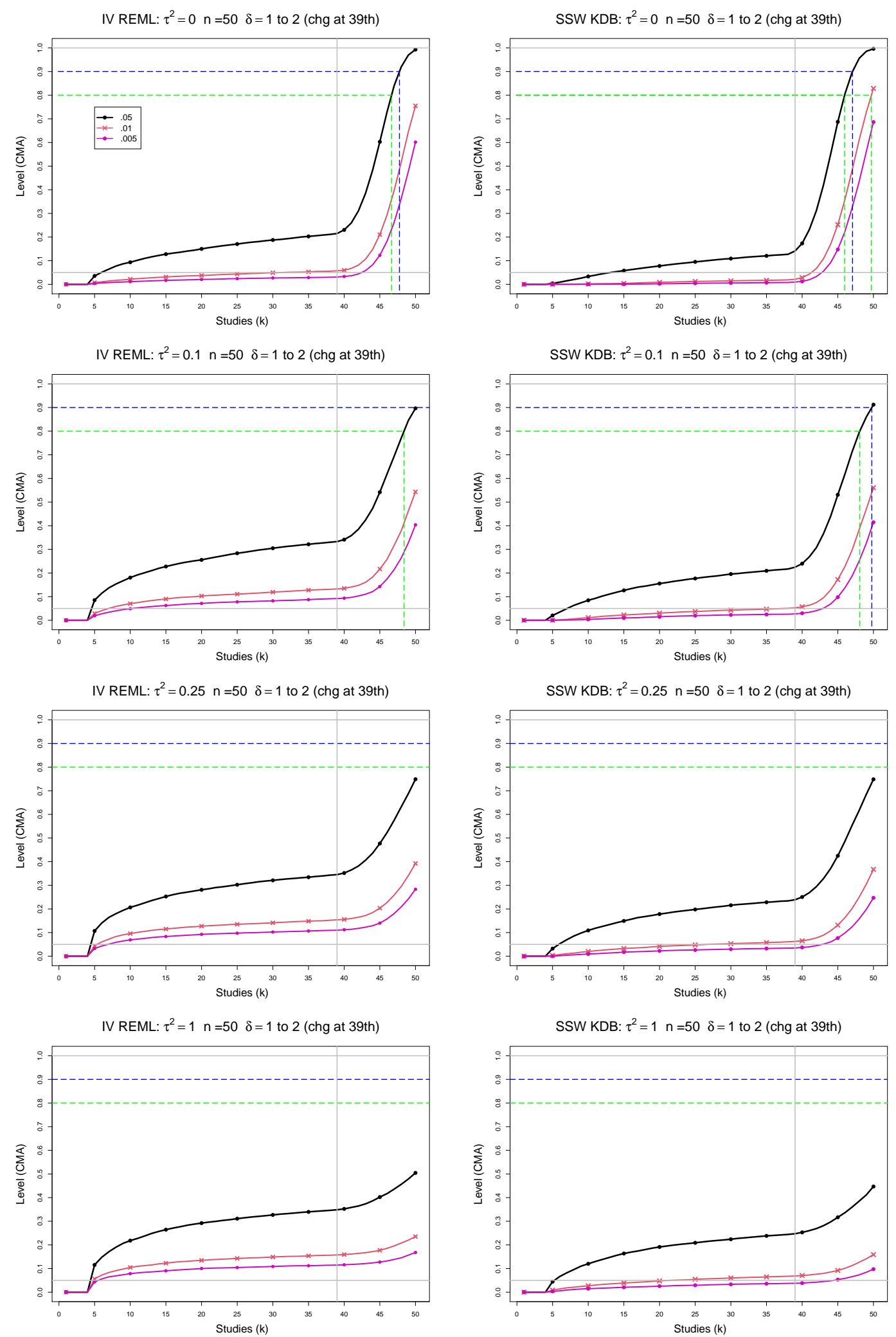

Figure C6. 12: Empirical levels of CMA tests for shift in $\delta$ based on SSW KDB and IV REML at .05, .01 and .005 nominal levels for equal sample sizes $n_{i C}+n_{i T}=n=50$, $\tau^{2}=0,0.1,0.25,1$ and a shift from $\delta=1$ to $\delta=2$ at study number 39. Light grey line at .05. Green and blue dashed lines correspond to power $80 \%$ and $90 \%$, respectively. 

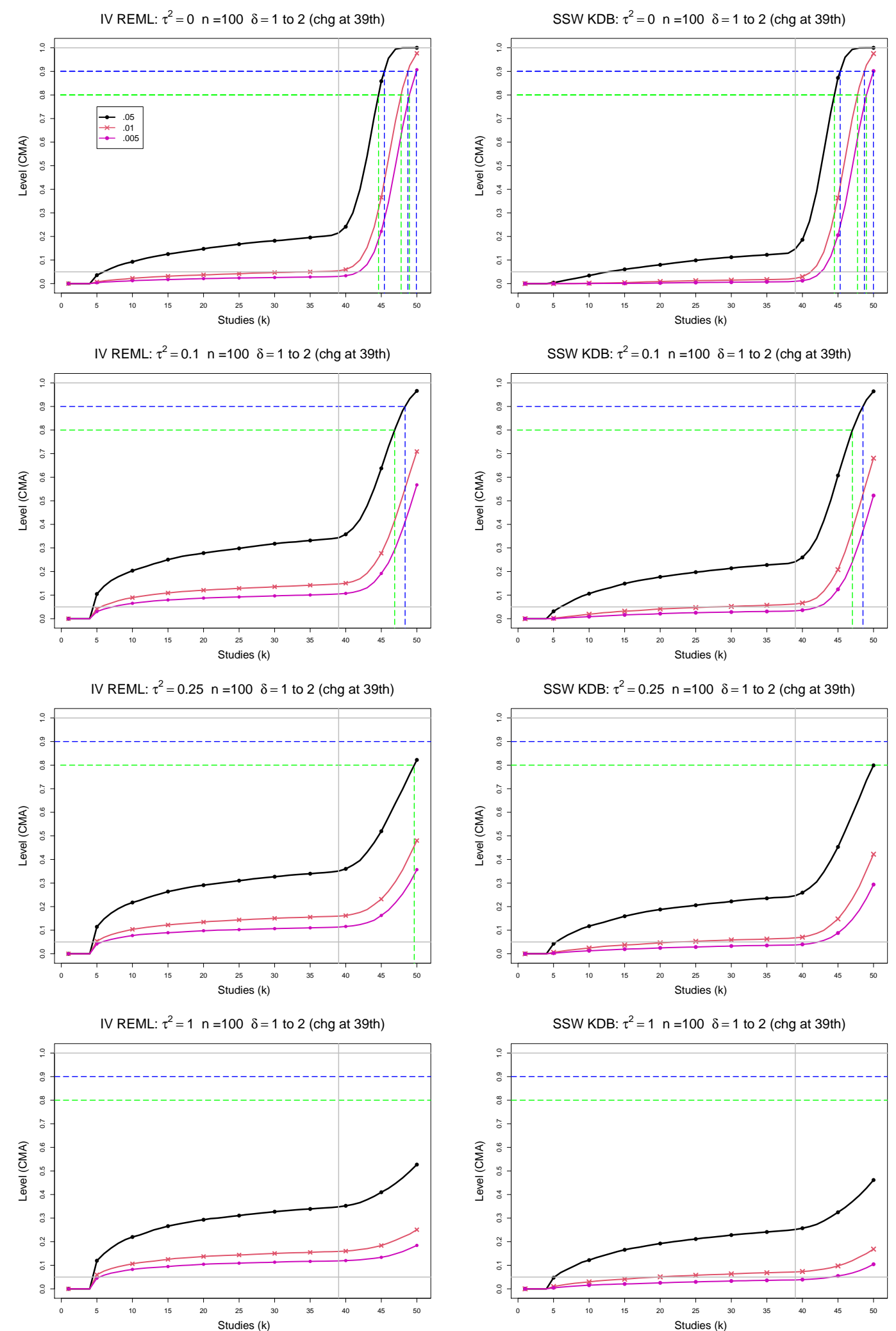

Figure C6. 13: Empirical levels of CMA tests for shift in $\delta$ based on SSW KDB and IV REML at .05, .01 and .005 nominal levels for equal sample sizes $n_{i C}+n_{i T}=n=100$, $\tau^{2}=0,0.1,0.25,1$ and a shift from $\delta=1$ to $\delta=2$ at study number 39. Light grey line at .05. Green and blue dashed lines correspond to power $80 \%$ and $90 \%$, respectively. 

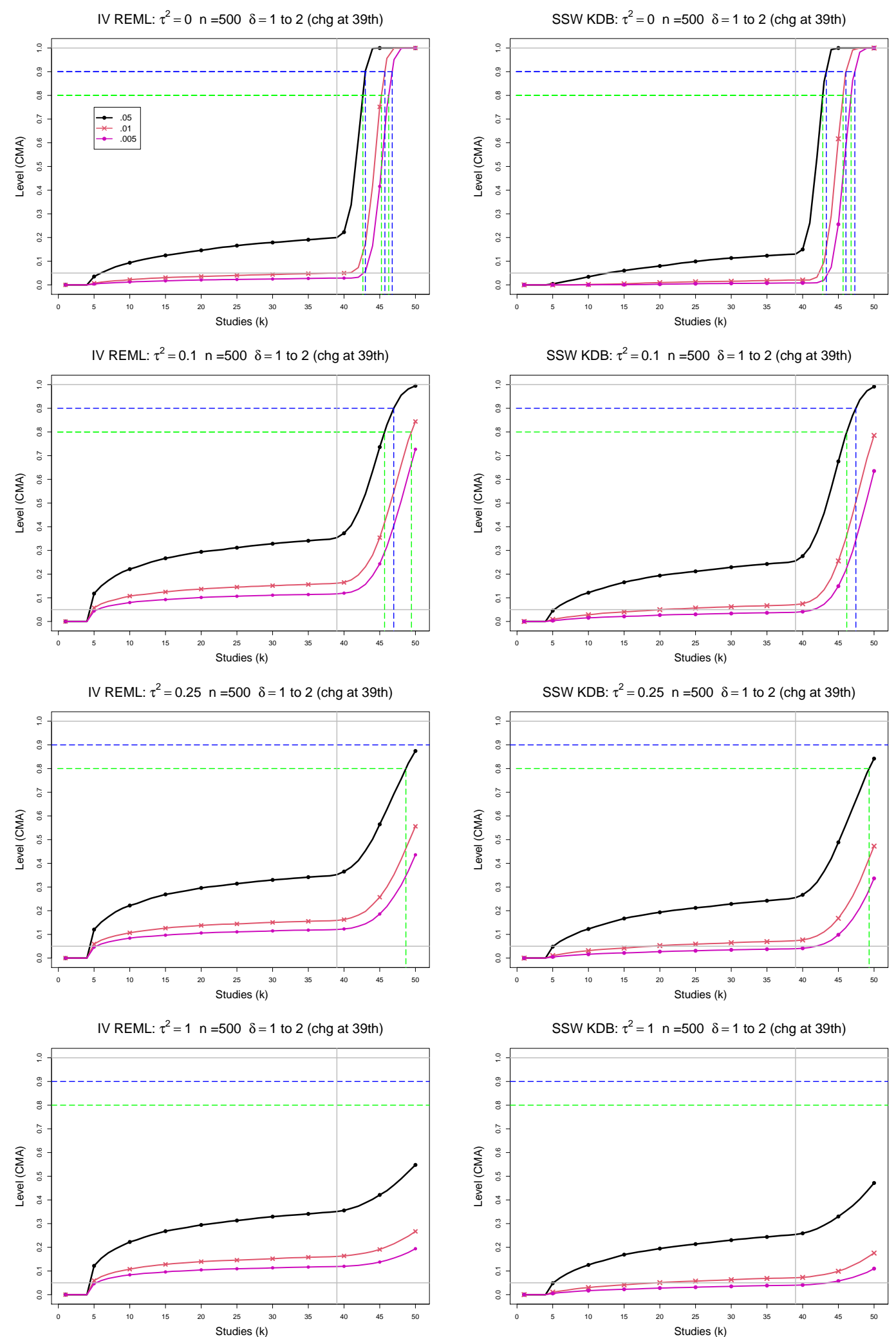

Figure C6. 14: Empirical levels of CMA tests for shift in $\delta$ based on SSW KDB and IV REML at .05, .01 and .005 nominal levels for equal sample sizes $n_{i C}+n_{i T}=n=500$, $\tau^{2}=0,0.1,0.25,1$ and a shift from $\delta=1$ to $\delta=2$ at study number 39. Light grey line at .05. Green and blue dashed lines correspond to power $80 \%$ and $90 \%$, respectively. 

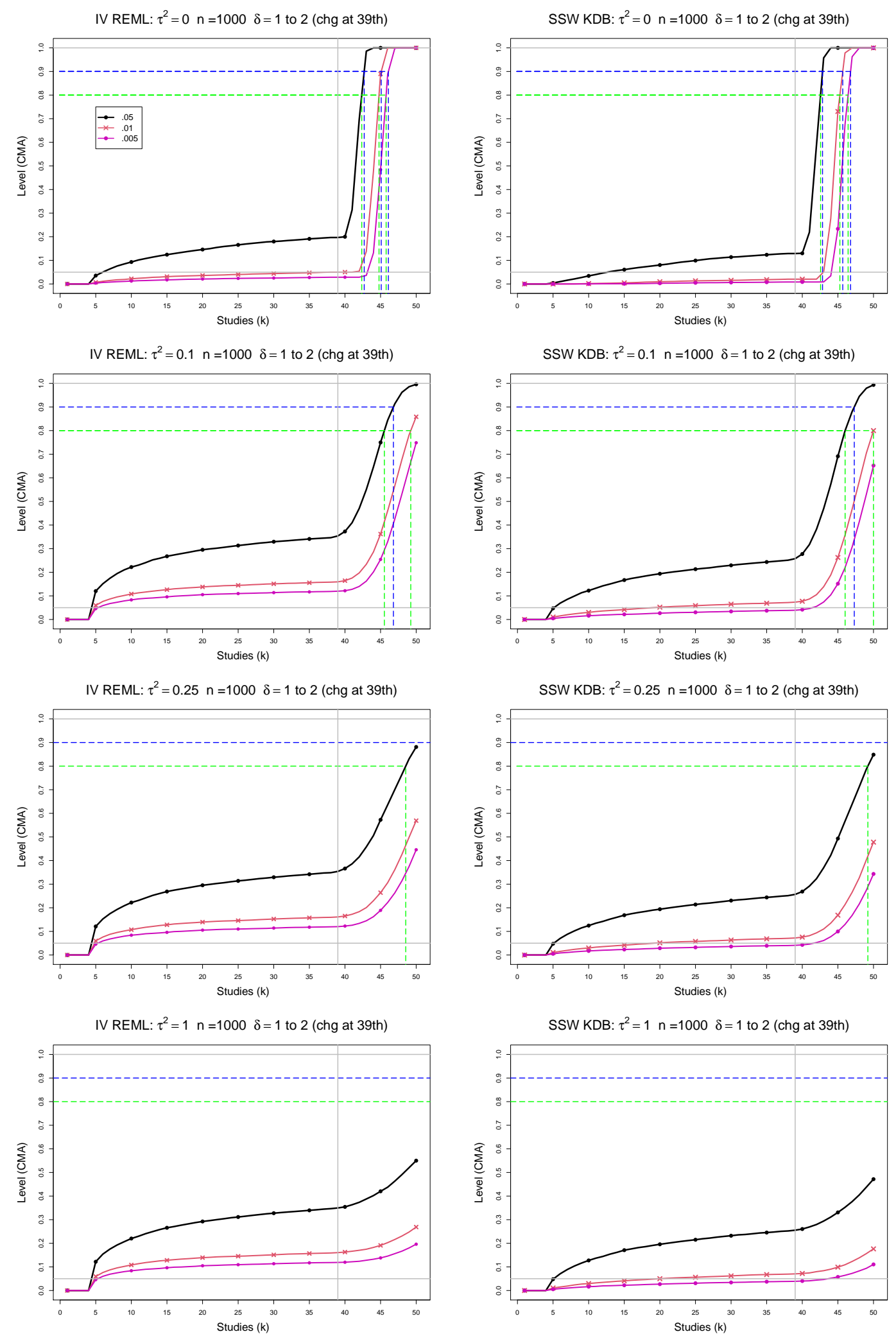

Figure C6. 15: Empirical levels of CMA tests for shift in $\delta$ based on SSW KDB and IV REML at .05, .01 and .005 nominal levels for equal sample sizes $n_{i C}+n_{i T}=n=1000$, $\tau^{2}=0,0.1,0.25,1$ and a shift from $\delta=1$ to $\delta=2$ at study number 39. Light grey line at .05. Green and blue dashed lines correspond to power $80 \%$ and $90 \%$, respectively. 


\section{C7. Empirical levels and power of CMA tests for shift in estimated from stage 1 mean effect $\hat{\delta}_{0}$}

For empirical levels of test for shift in $\hat{\delta}_{0}$ in this section, each figure corresponds to one of the two tested meta-analysis methods, a set of values of $\tau^{2}(=0,0.1,0.25,1)$, a value of $n(=20,50,100,500,1000)$, a set of values of significance level $(=0.05,0.01$, $0.005)$ and a value of point of shift $f(=13,26,39)$ while $\delta$ shifts from 1 to 2 and maximum number of studies $K=50$.

Each figure contains a panel (with study $k$ on the horizontal axis) for each combination of parameters.

The tested methods are

- IV REML (Restricted maximum likelihood, inverse variance weighted)

- SSW KDB (Kulinskaya-Dollinger-Bjørkest øl estimator of $\tau^{2}$, sample size weighted) 

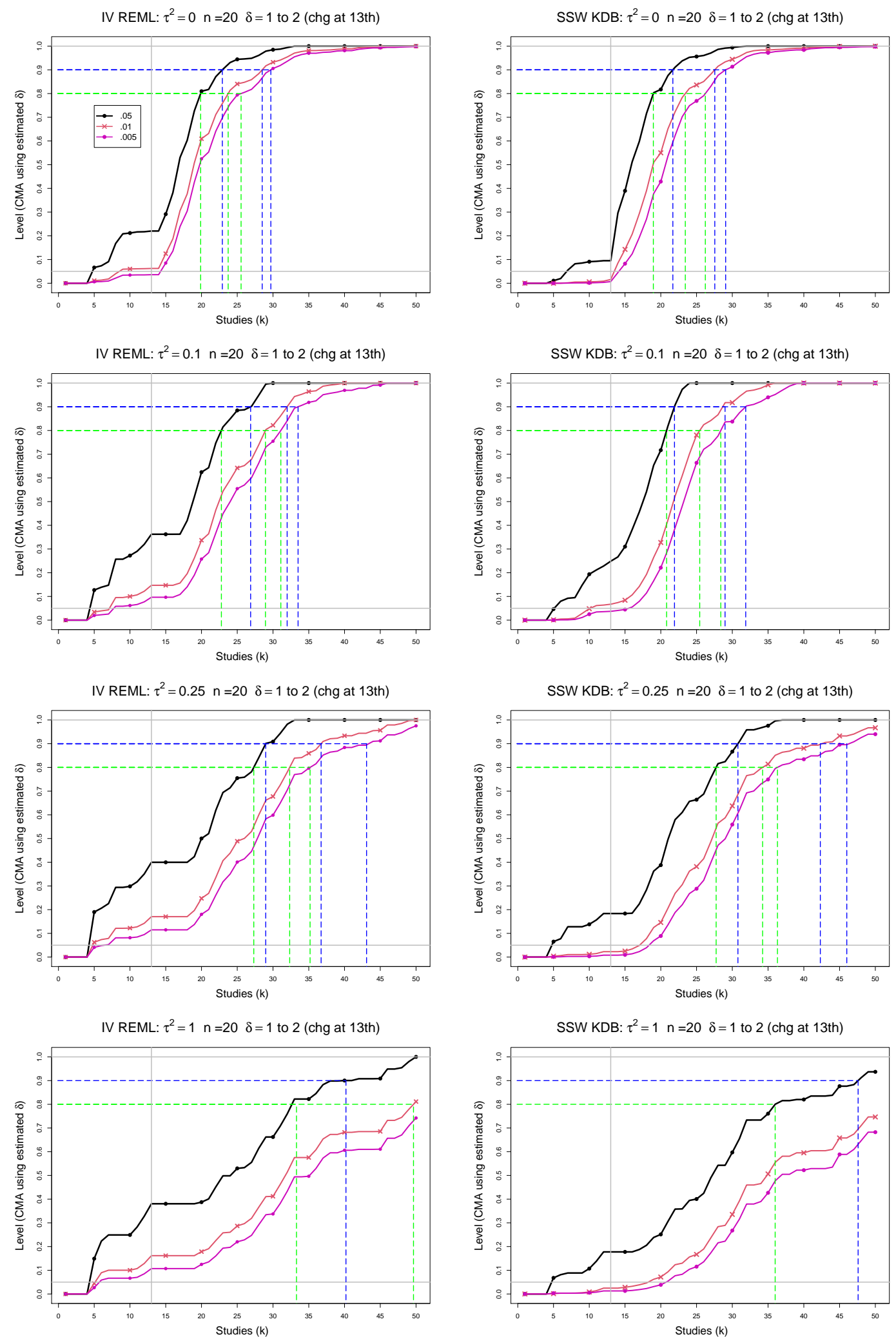

Figure C7. 1: Empirical levels of CMA tests for shift in $\hat{\delta}_{0}$ based on SSW KDB and IV REML at .05, .01 and .005 nominal levels for equal sample sizes $n_{i C}+n_{i T}=n=20$, $\tau^{2}=0,0.1,0.25,1$ and a shift from $\delta=1$ to $\delta=2$ at study number 13. Light grey line at .05. Green and blue dashed lines correspond to power $80 \%$ and $90 \%$, respectively. 

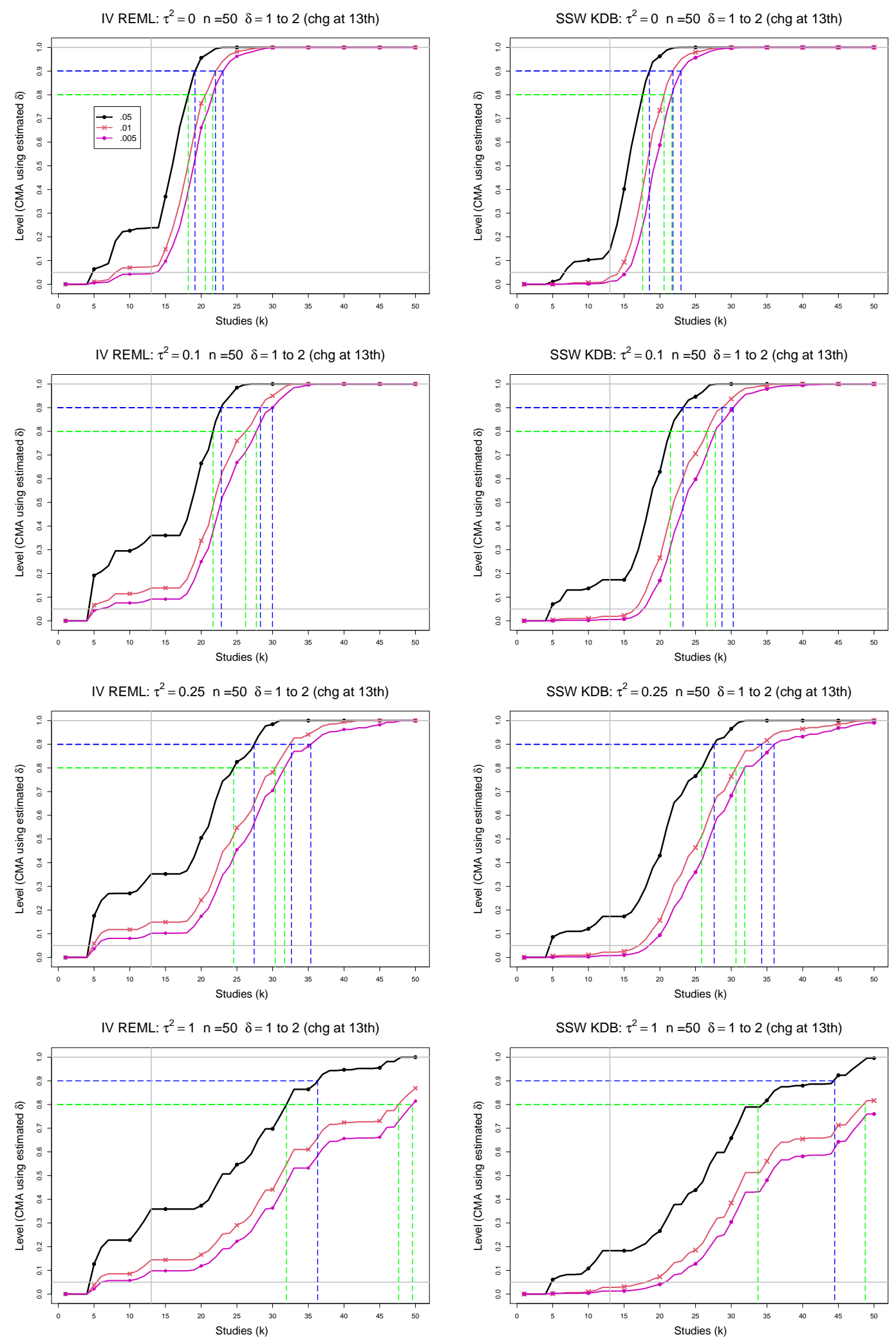

Figure C7. 2: Empirical levels of CMA tests for shift in $\hat{\delta}_{0}$ based on SSW KDB and IV REML at .05, .01 and .005 nominal levels for equal sample sizes $n_{i C}+n_{i T}=n=50$, $\tau^{2}=0,0.1,0.25,1$ and a shift from $\delta=1$ to $\delta=2$ at study number 13. Light grey line at .05. Green and blue dashed lines correspond to power $80 \%$ and $90 \%$, respectively. 

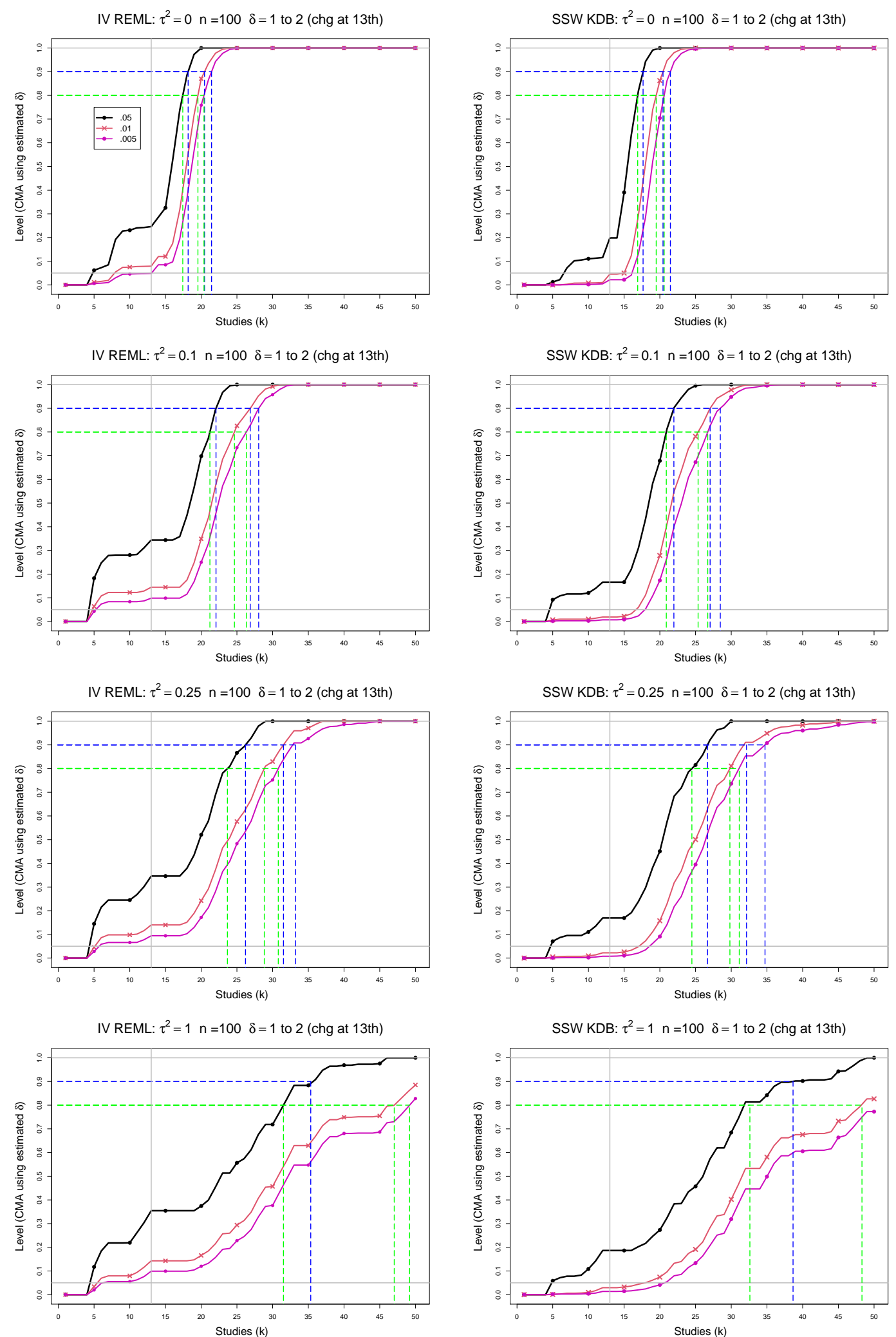

Figure C7. 3: Empirical levels of CMA tests for shift in $\hat{\delta}_{0}$ based on SSW KDB and IV REML at .05, .01 and .005 nominal levels for equal sample sizes $n_{i C}+n_{i T}=n=100$, $\tau^{2}=0,0.1,0.25,1$ and a shift from $\delta=1$ to $\delta=2$ at study number 13 . Light grey line at .05. Green and blue dashed lines correspond to power $80 \%$ and $90 \%$, respectively. 

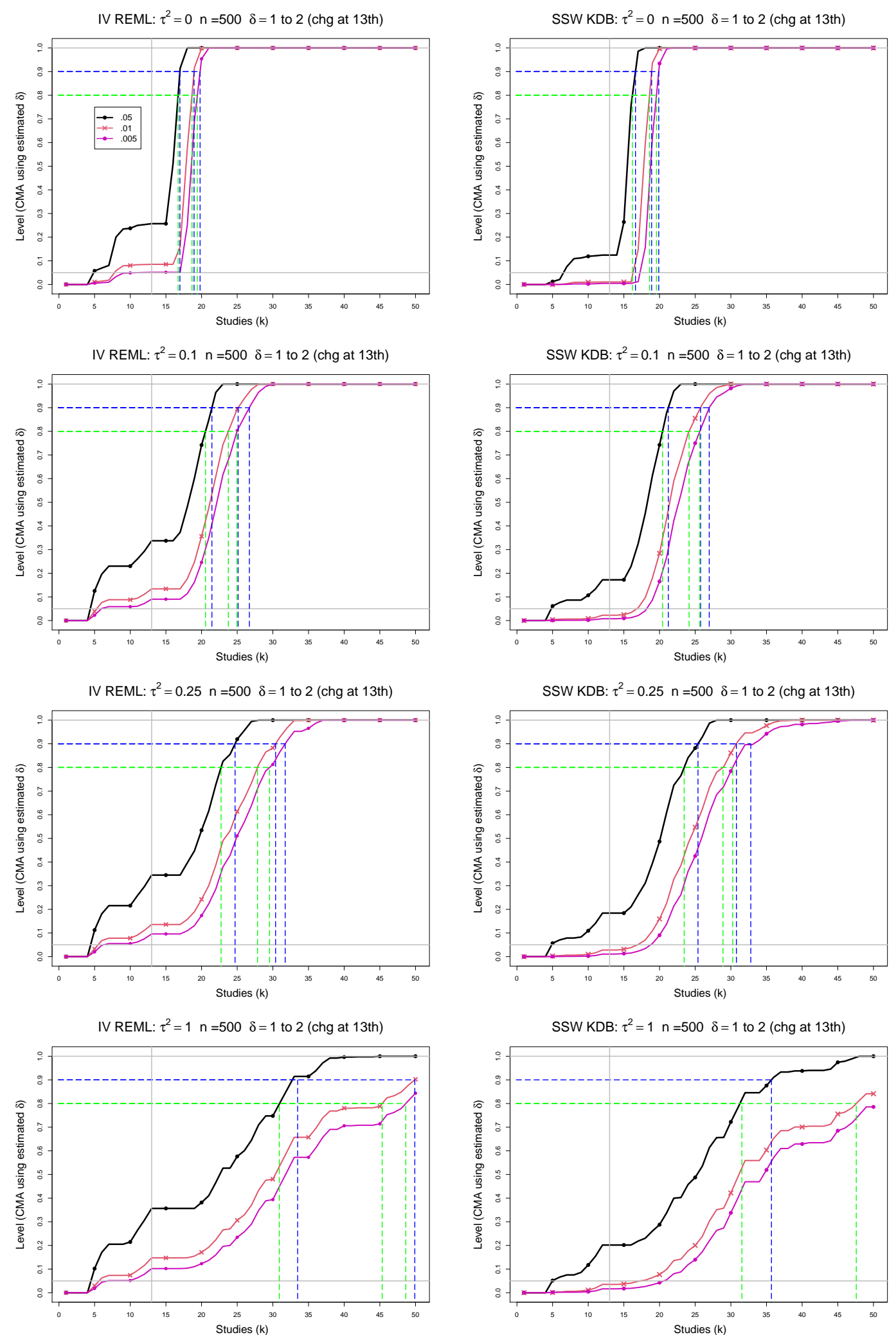

Figure C7. 4: Empirical levels of CMA tests for shift in $\hat{\delta}_{0}$ based on SSW KDB and IV REML at .05, .01 and .005 nominal levels for equal sample sizes $n_{i C}+n_{i T}=n=500$, $\tau^{2}=0,0.1,0.25,1$ and a shift from $\delta=1$ to $\delta=2$ at study number 13. Light grey line at .05. Green and blue dashed lines correspond to power $80 \%$ and $90 \%$, respectively. 

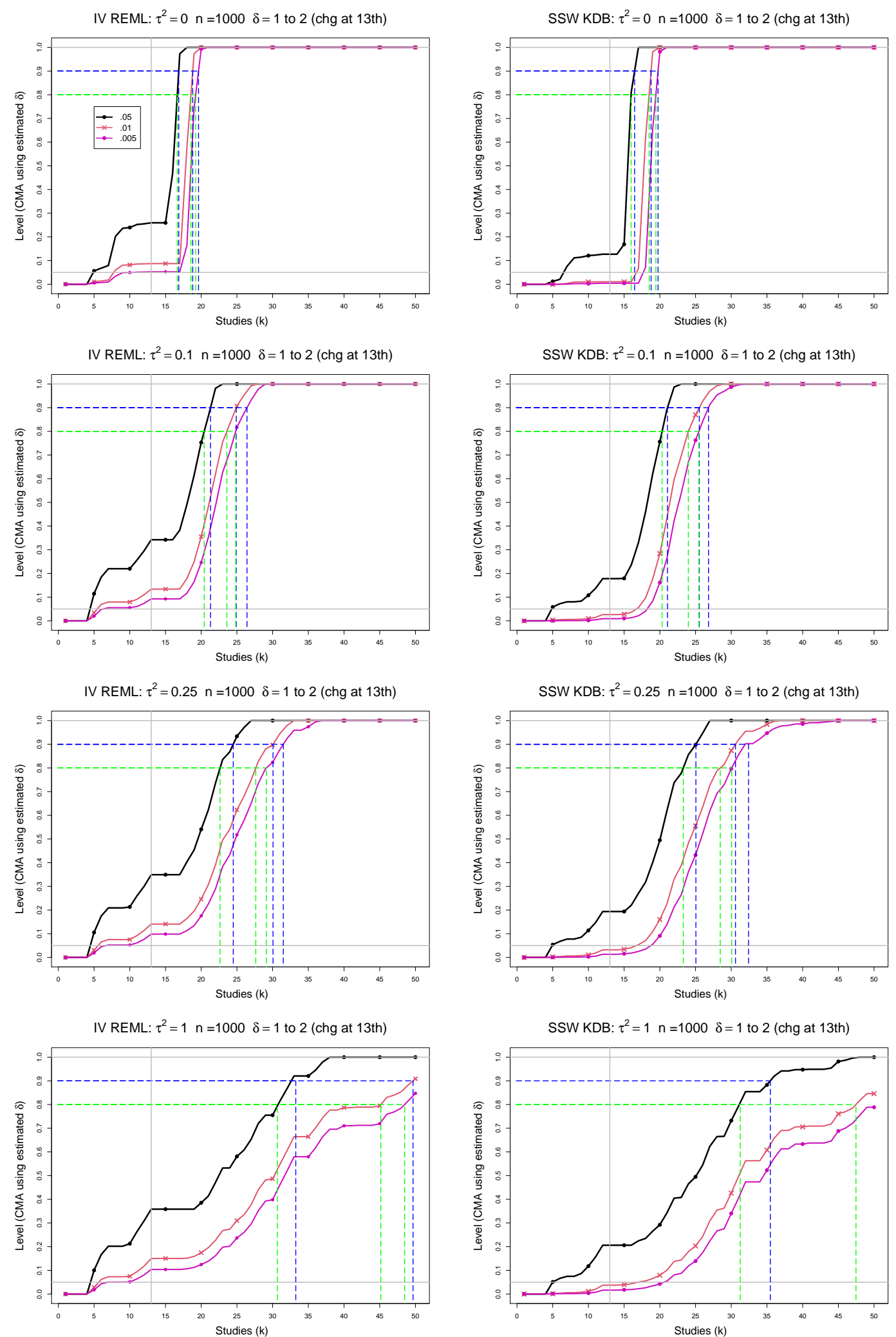

Figure C7. 5: Empirical levels of CMA tests for shift in $\hat{\delta}_{0}$ based on SSW KDB and IV REML at .05, .01 and .005 nominal levels for equal sample sizes $n_{i C}+n_{i T}=n=1000$, $\tau^{2}=0,0.1,0.25,1$ and a shift from $\delta=1$ to $\delta=2$ at study number 13 . Light grey line at .05. Green and blue dashed lines correspond to power $80 \%$ and $90 \%$, respectively. 

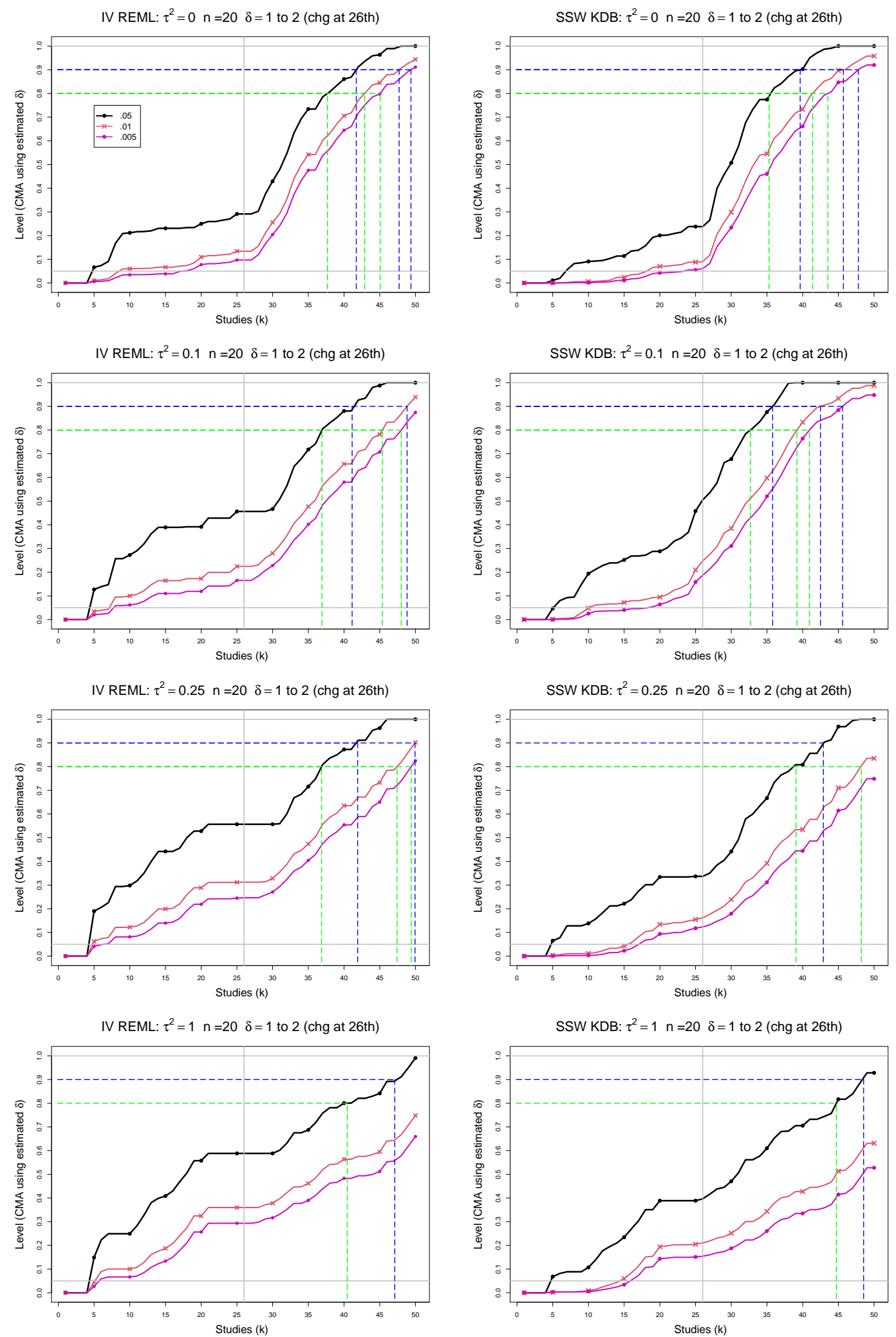

Figure C7. 6: Empirical levels of CMA tests for shift in $\hat{\delta}_{0}$ based on SSW KDB and IV REML at .05, .01 and .005 nominal levels for equal sample sizes $n_{i C}+n_{i T}=n=20$, $\tau^{2}=0,0.1,0.25,1$ and a shift from $\delta=1$ to $\delta=2$ at study number 26. Light grey line at .05. Green and blue dashed lines correspond to power $80 \%$ and $90 \%$, respectively. 

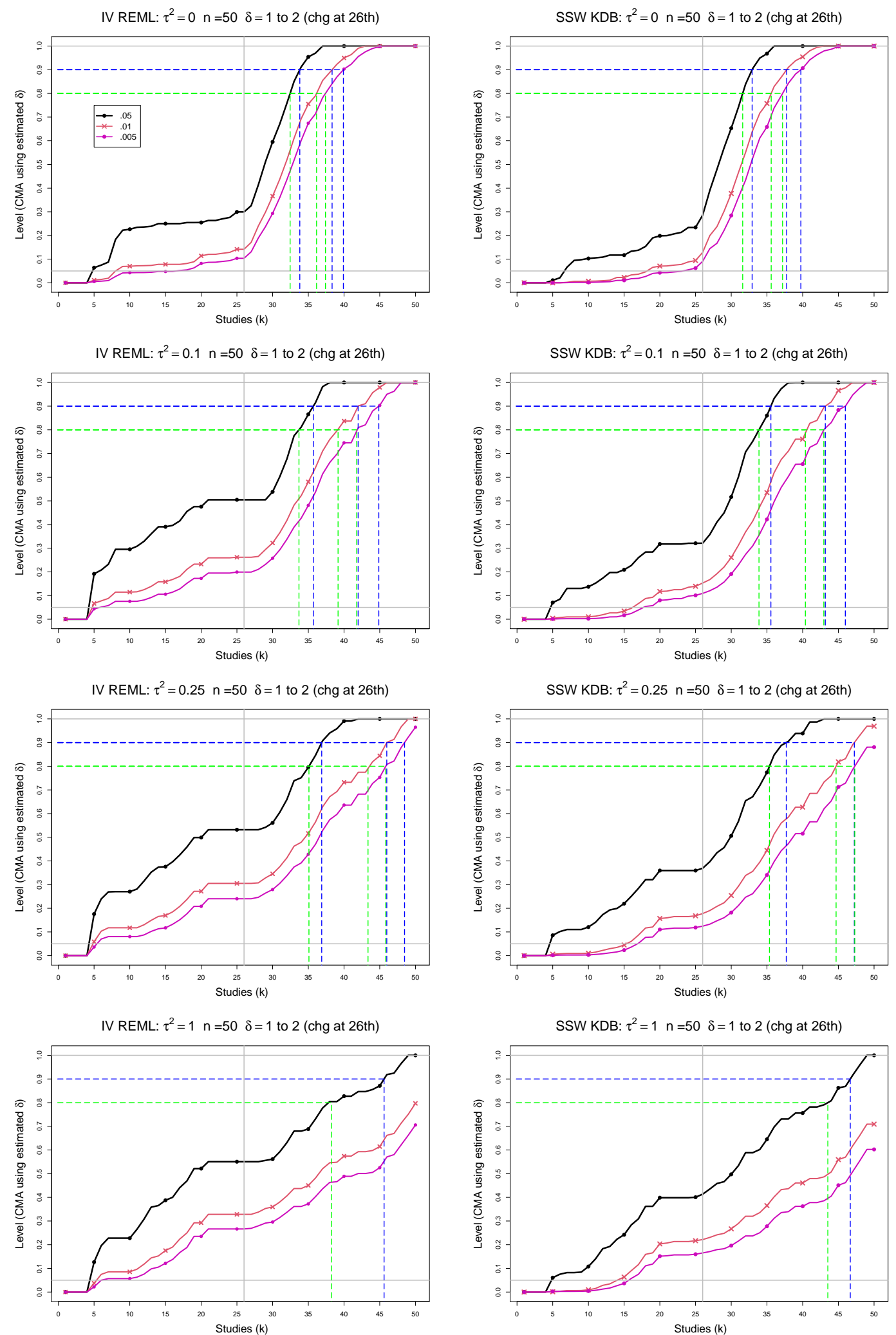

Figure C7. 7: Empirical levels of CMA tests for shift in $\hat{\delta}_{0}$ based on SSW KDB and IV REML at .05, .01 and .005 nominal levels for equal sample sizes $n_{i C}+n_{i T}=n=50$, $\tau^{2}=0,0.1,0.25,1$ and a shift from $\delta=1$ to $\delta=2$ at study number 26. Light grey line at .05. Green and blue dashed lines correspond to power $80 \%$ and $90 \%$, respectively. 

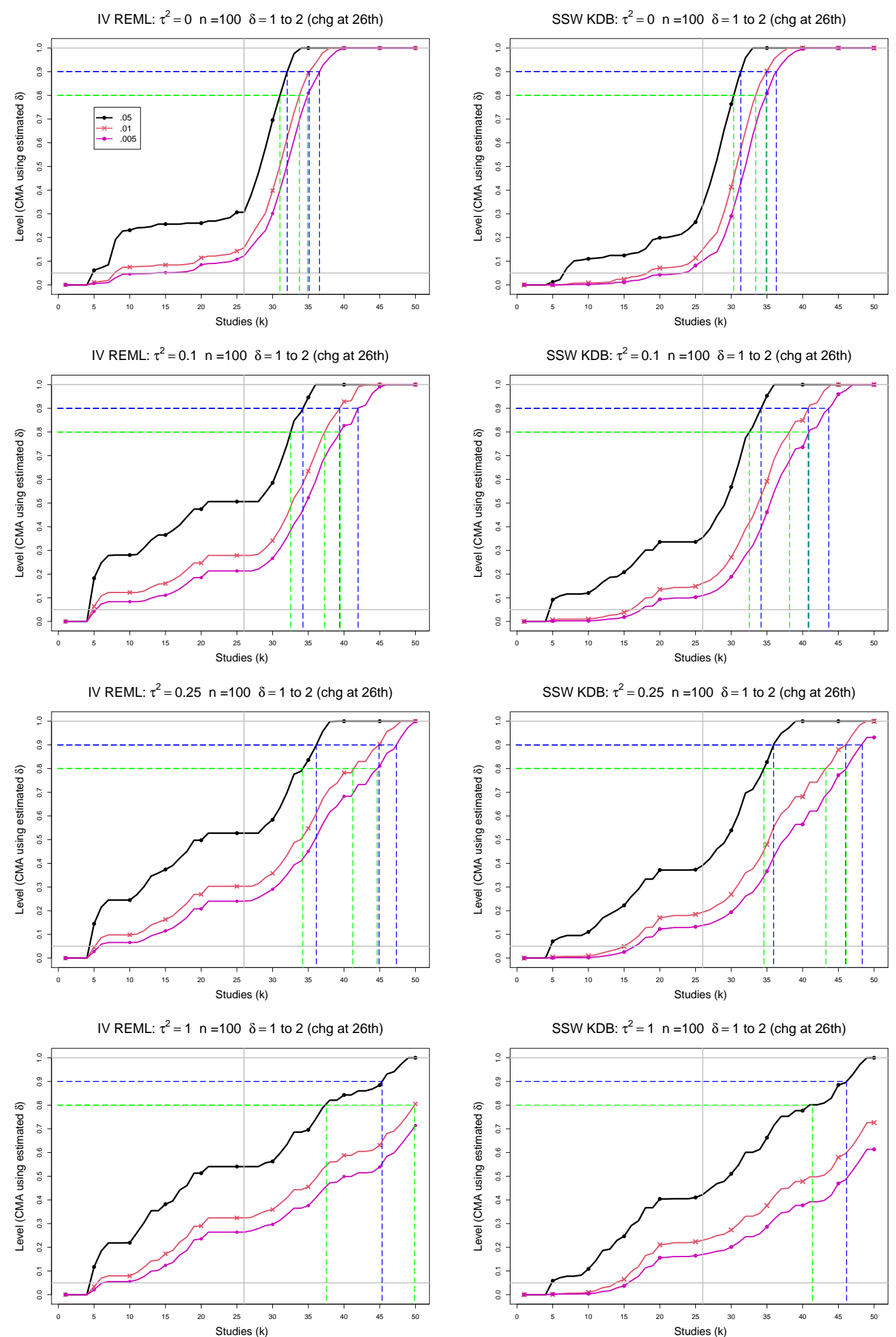

Figure C7. 8: Empirical levels of CMA tests for shift in $\hat{\delta}_{0}$ based on SSW KDB and IV REML at .05, .01 and .005 nominal levels for equal sample sizes $n_{i C}+n_{i T}=n=100$, $\tau^{2}=0,0.1,0.25,1$ and a shift from $\delta=1$ to $\delta=2$ at study number 26. Light grey line at .05. Green and blue dashed lines correspond to power $80 \%$ and $90 \%$, respectively. 

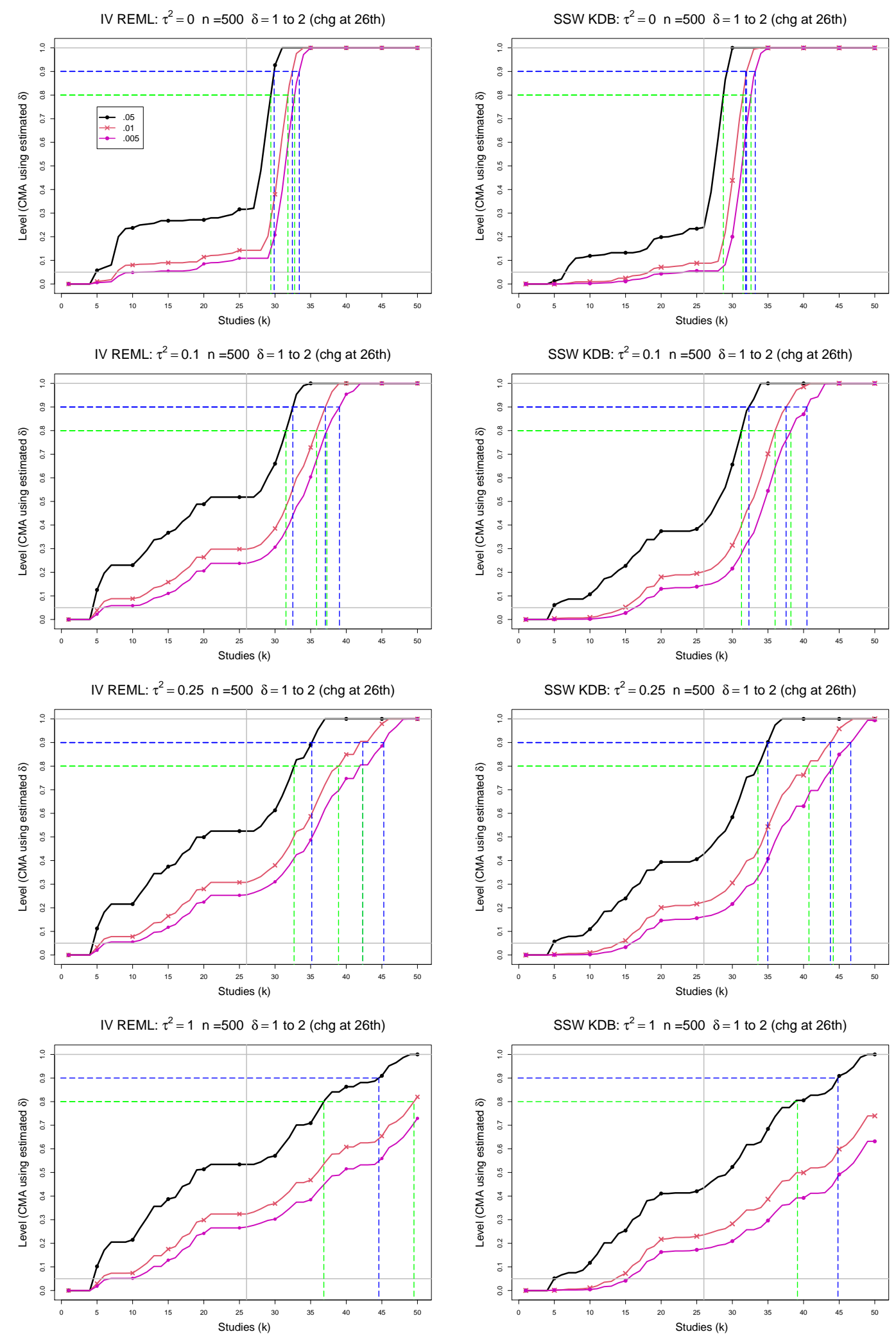

Figure C7. 9: Empirical levels of CMA tests for shift in $\hat{\delta}_{0}$ based on SSW KDB and IV REML at .05, .01 and .005 nominal levels for equal sample sizes $n_{i C}+n_{i T}=n=500$, $\tau^{2}=0,0.1,0.25,1$ and a shift from $\delta=1$ to $\delta=2$ at study number 26. Light grey line at .05. Green and blue dashed lines correspond to power $80 \%$ and $90 \%$, respectively. 

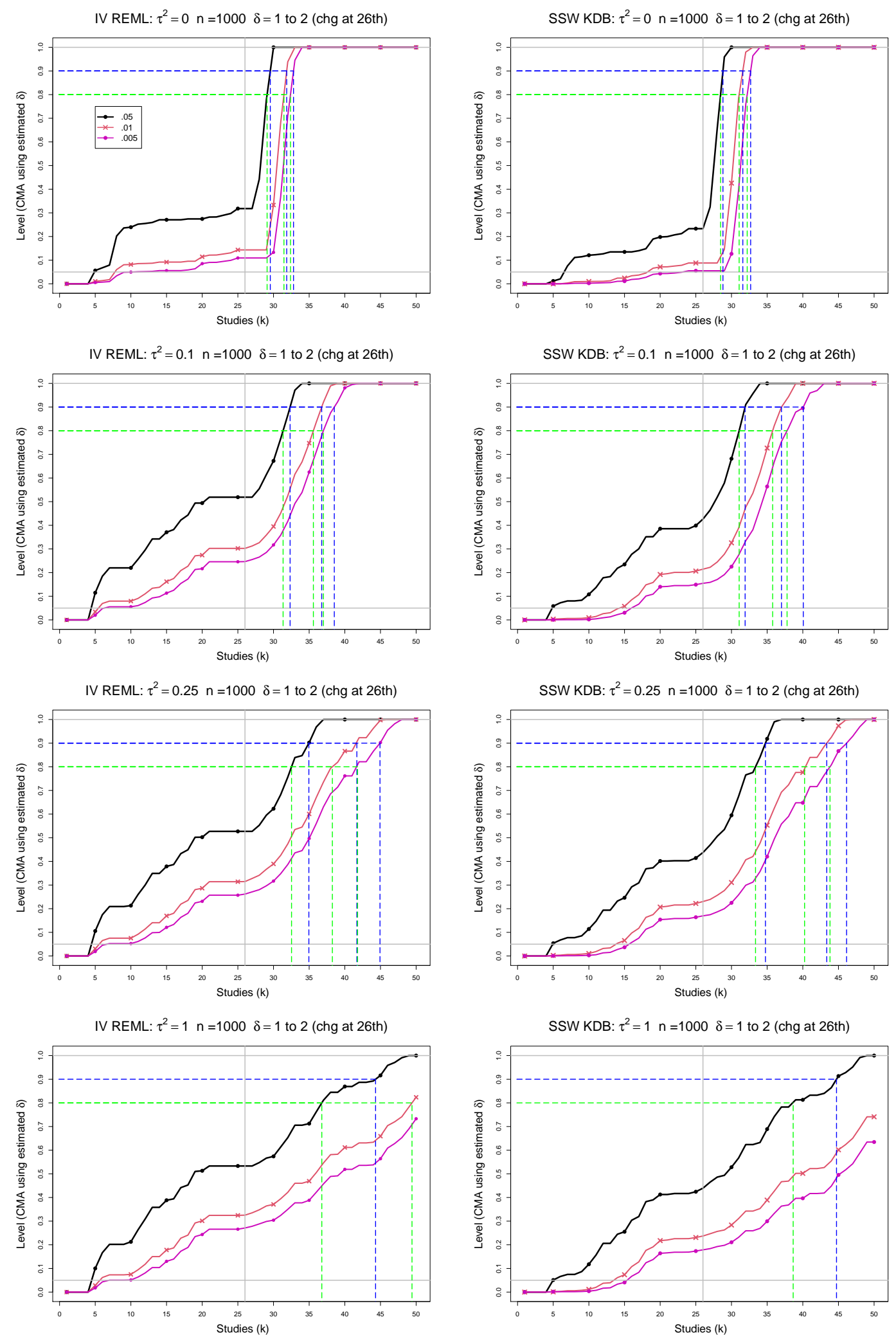

Figure C7. 10: Empirical levels of CMA tests for shift in $\hat{\delta}_{0}$ based on SSW KDB and IV REML at .05, .01 and .005 nominal levels for equal sample sizes $n_{i C}+n_{i T}=n=1000$, $\tau^{2}=0,0.1,0.25,1$ and a shift from $\delta=1$ to $\delta=2$ at study number 26. Light grey line at .05. Green and blue dashed lines correspond to power $80 \%$ and $90 \%$, respectively. 

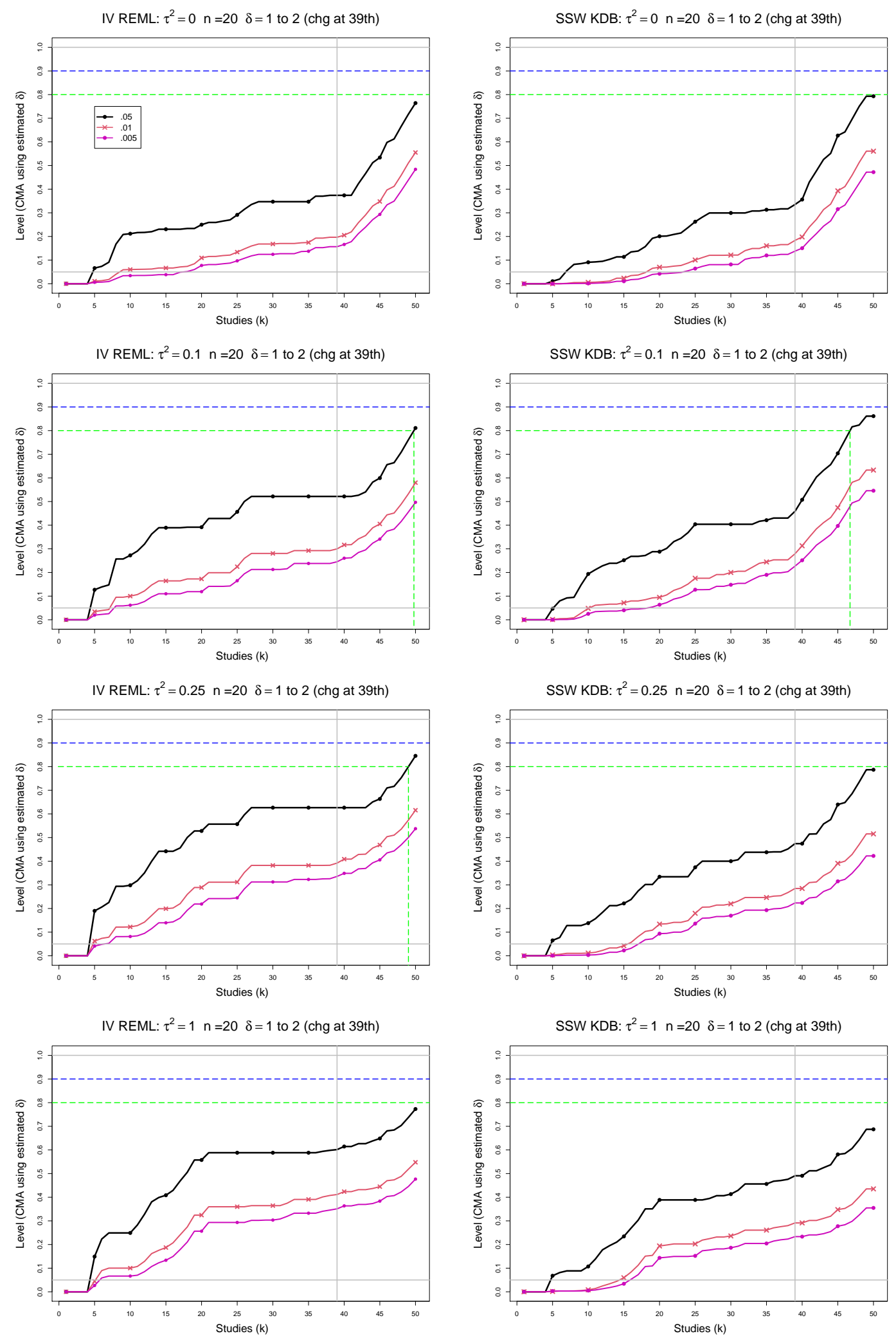

Figure C7. 11: Empirical levels of CMA tests for shift in $\hat{\delta}_{0}$ based on SSW KDB and IV REML at .05, .01 and .005 nominal levels for equal sample sizes $n_{i C}+n_{i T}=n=20$, $\tau^{2}=0,0.1,0.25,1$ and a shift from $\delta=1$ to $\delta=2$ at study number 39. Light grey line at .05. Green and blue dashed lines correspond to power $80 \%$ and $90 \%$, respectively. 

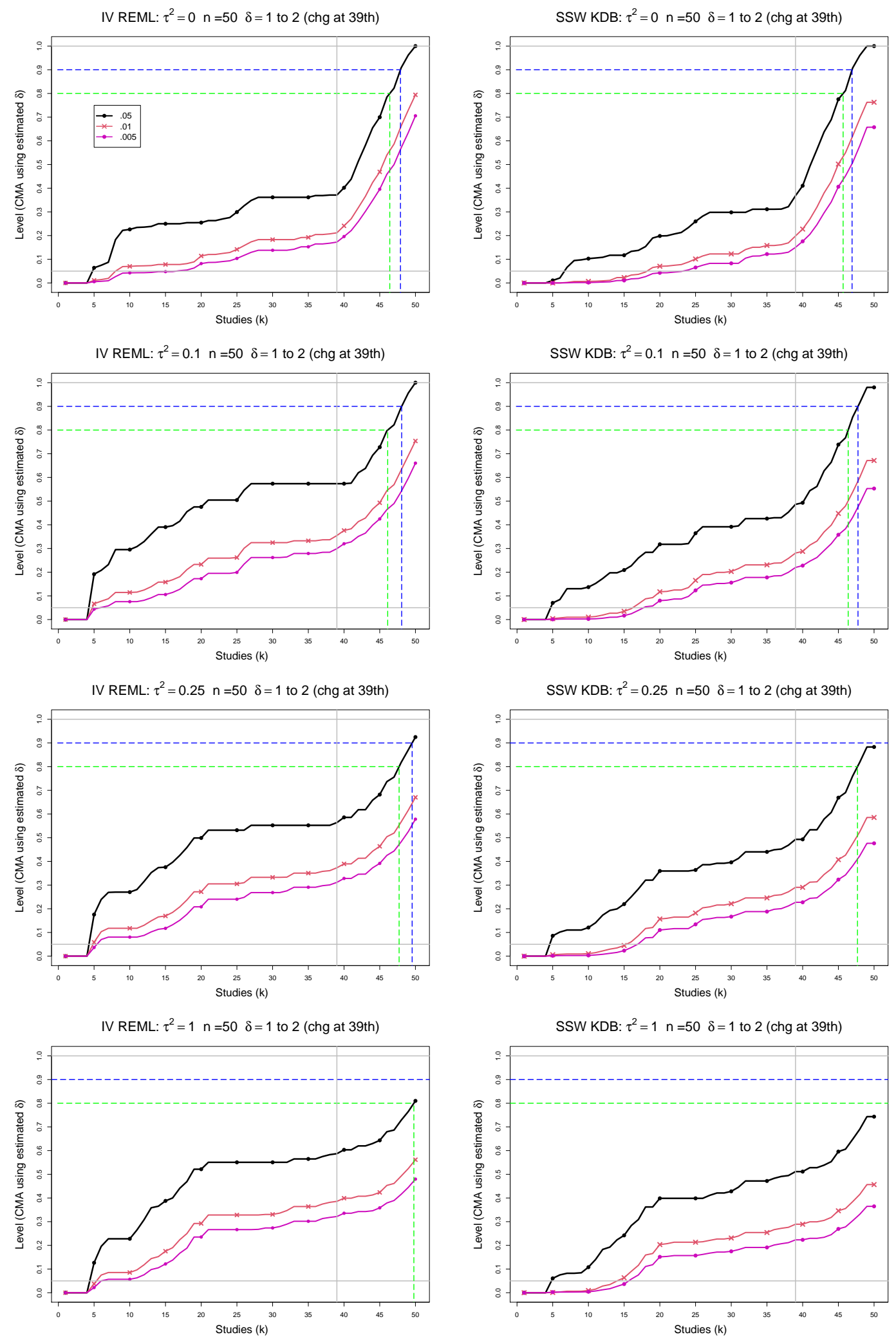

Figure C7. 12: Empirical levels of CMA tests for shift in $\hat{\delta}_{0}$ based on SSW KDB and IV REML at .05, .01 and .005 nominal levels for equal sample sizes $n_{i C}+n_{i T}=n=50$, $\tau^{2}=0,0.1,0.25,1$ and a shift from $\delta=1$ to $\delta=2$ at study number 39. Light grey line at .05. Green and blue dashed lines correspond to power $80 \%$ and $90 \%$, respectively. 

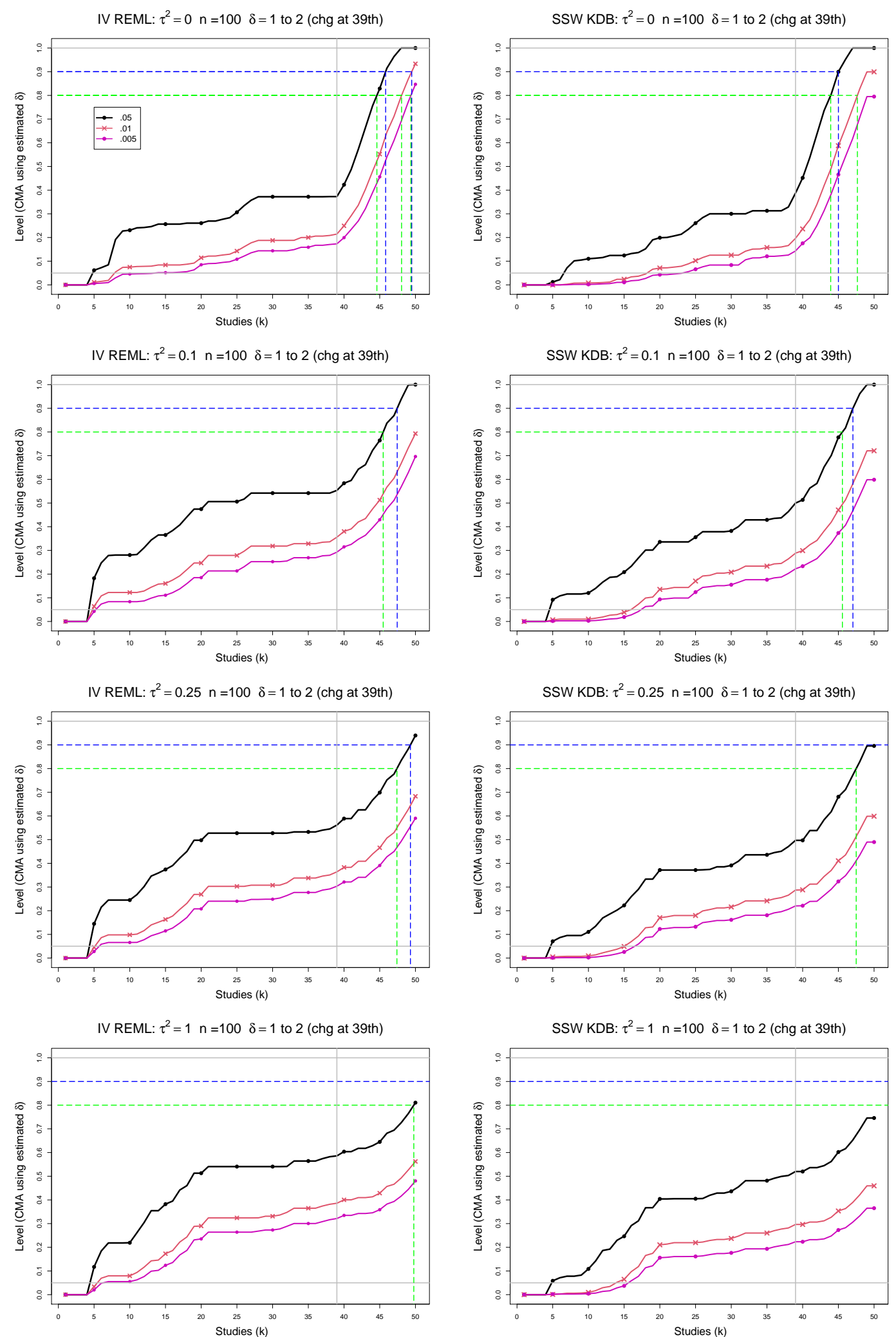

Figure C7. 13: Empirical levels of CMA tests for shift in $\hat{\delta}_{0}$ based on SSW KDB and IV REML at .05, .01 and .005 nominal levels for equal sample sizes $n_{i C}+n_{i T}=n=100$, $\tau^{2}=0,0.1,0.25,1$ and a shift from $\delta=1$ to $\delta=2$ at study number 39. Light grey line at .05. Green and blue dashed lines correspond to power $80 \%$ and $90 \%$, respectively. 

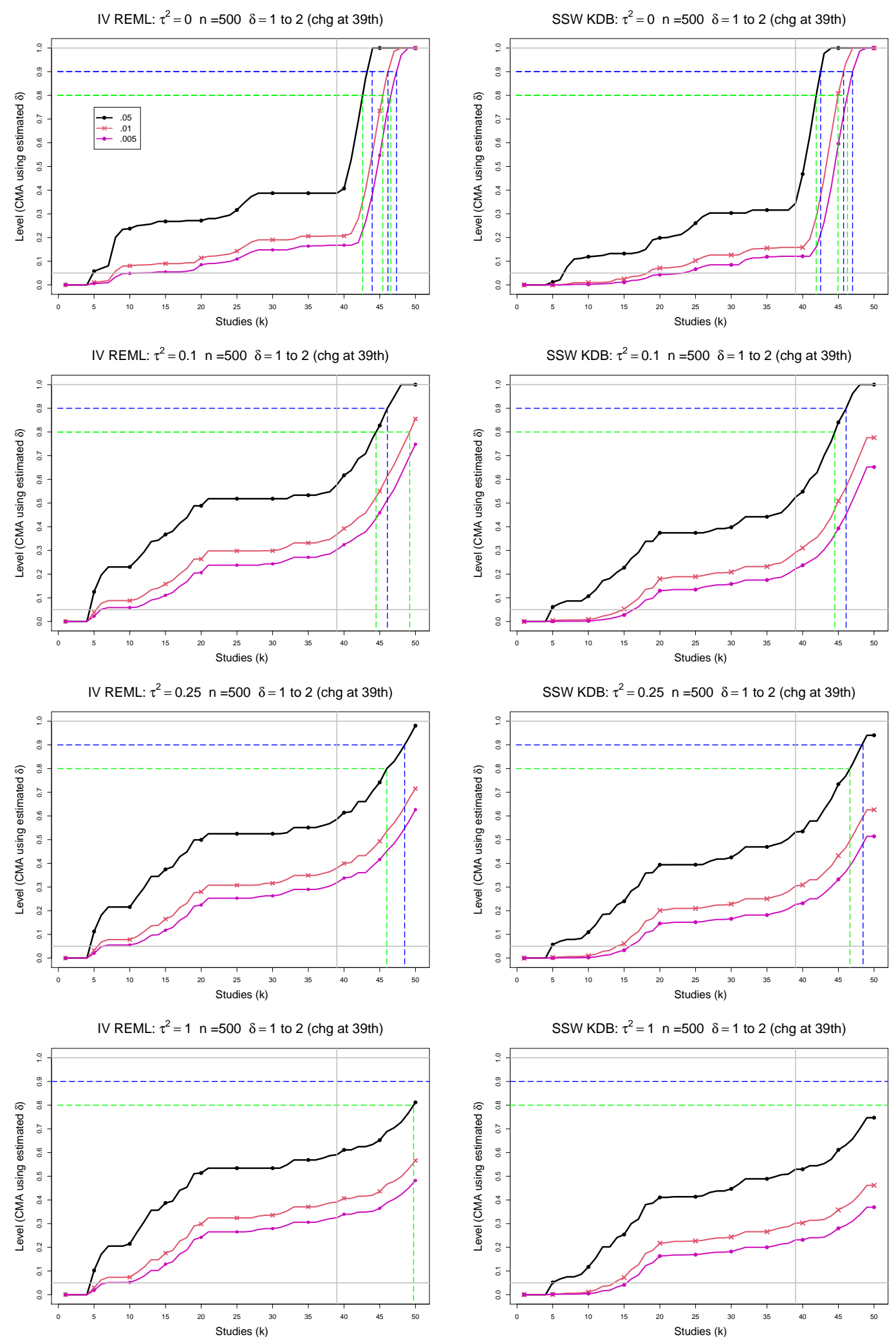

Figure C7. 14: Empirical levels of CMA tests for shift in $\hat{\delta}_{0}$ based on SSW KDB and IV REML at .05, .01 and .005 nominal levels for equal sample sizes $n_{i C}+n_{i T}=n=500$, $\tau^{2}=0,0.1,0.25,1$ and a shift from $\delta=1$ to $\delta=2$ at study number 39. Light grey line at .05. Green and blue dashed lines correspond to power $80 \%$ and $90 \%$, respectively. 

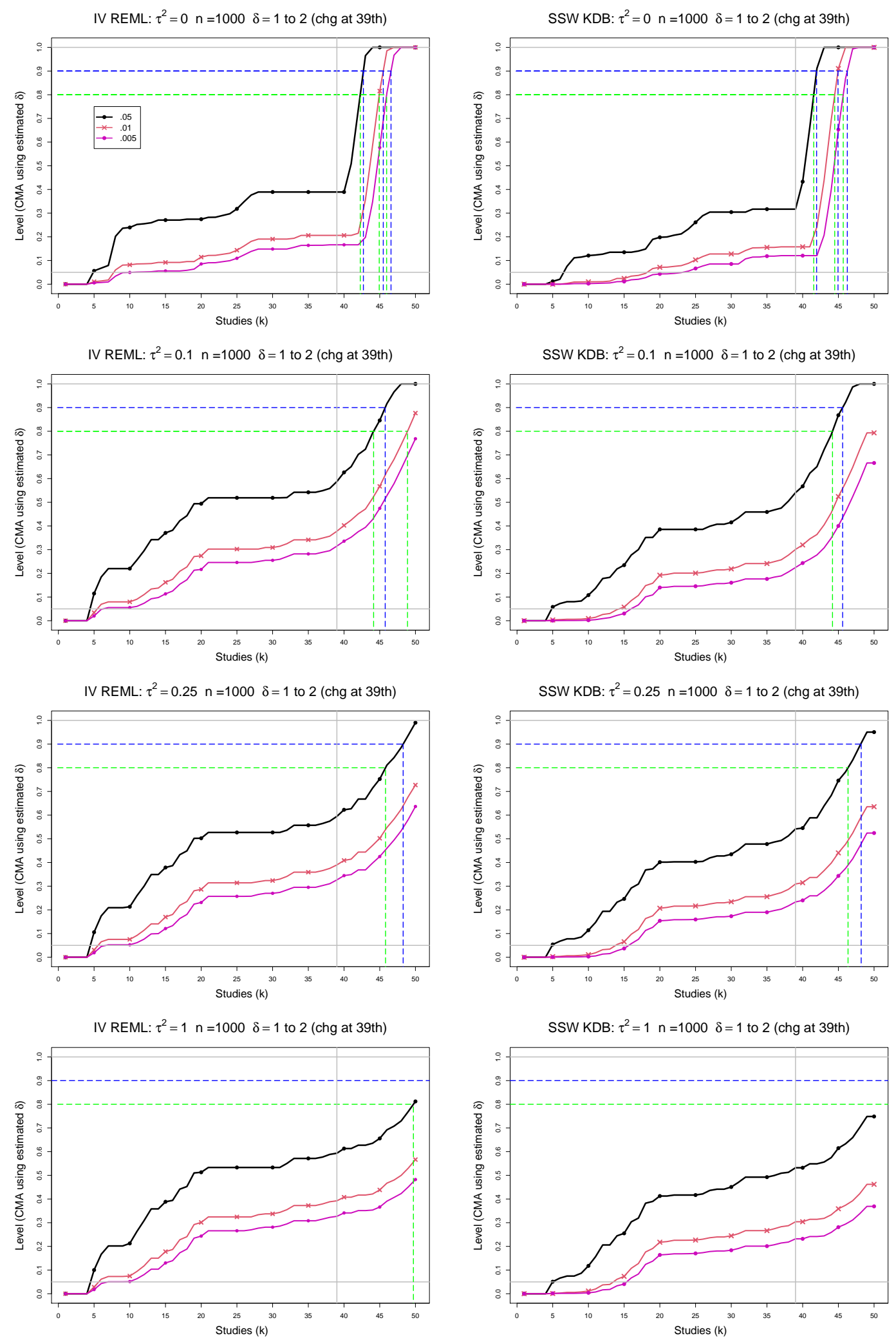

Figure C7. 15: Empirical levels of CMA tests for shift in $\hat{\delta}_{0}$ based on SSW KDB and IV REML at .05, .01 and .005 nominal levels for equal sample sizes $n_{i C}+n_{i T}=n=1000$, $\tau^{2}=0,0.1,0.25,1$ and a shift from $\delta=1$ to $\delta=2$ at study number 39. Light grey line at .05. Green and blue dashed lines correspond to power $80 \%$ and $90 \%$, respectively. 


\section{C8. Empirical levels of CMA tests for positive}

\section{shift in $\tau^{2}$}

For empirical levels of tests for positive shift in $\tau^{2}$ in this section, each figure corresponds to one of the two tested methods, a set of values of $\tau^{2}(=0,0.1,0.25,1)$, a value of $n(=20,50,100,500,1000)$, a set of values of significance level $(=0.025,0.005$, $0.0025)$ and a value of point of shift $f(=13,26,39)$ while $\delta$ shifts from 1 to 2 and maximum number of studies $K=50$.

Each figure contains a panel (with study $k$ on the horizontal axis) for each combination of parameters.

The tested methods are

- QP (A test based on Q-profile confidence interval by Viechtbauer (2007))

- KDB (A test based on improved Q-profile method by Kulinskaya, Dollinger, Bjørkestøl (2011)) 

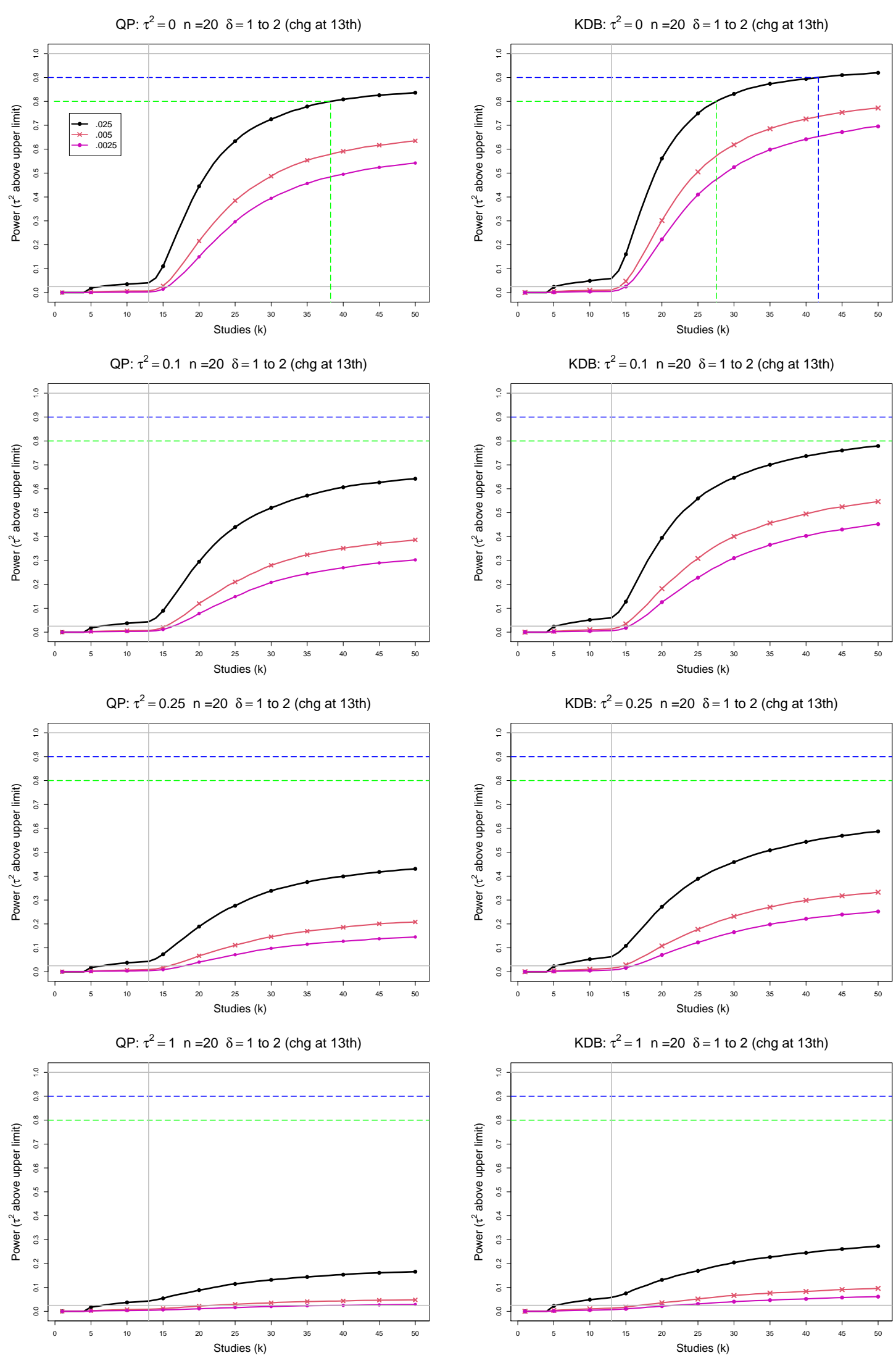

Figure C8. 1: Empirical levels of CMA tests for positive shift in $\tau^{2}$ based on KDB and QP at $.025, .005$ and .0025 nominal levels for $k$ up to $K=50$ for equal sample sizes $n_{i C}+n_{i T}=n=20, \tau^{2}=0,0.1,0.25,1$ and a shift from $\delta=1$ to $\delta=2$ at study number 13. Light grey line at .025. 

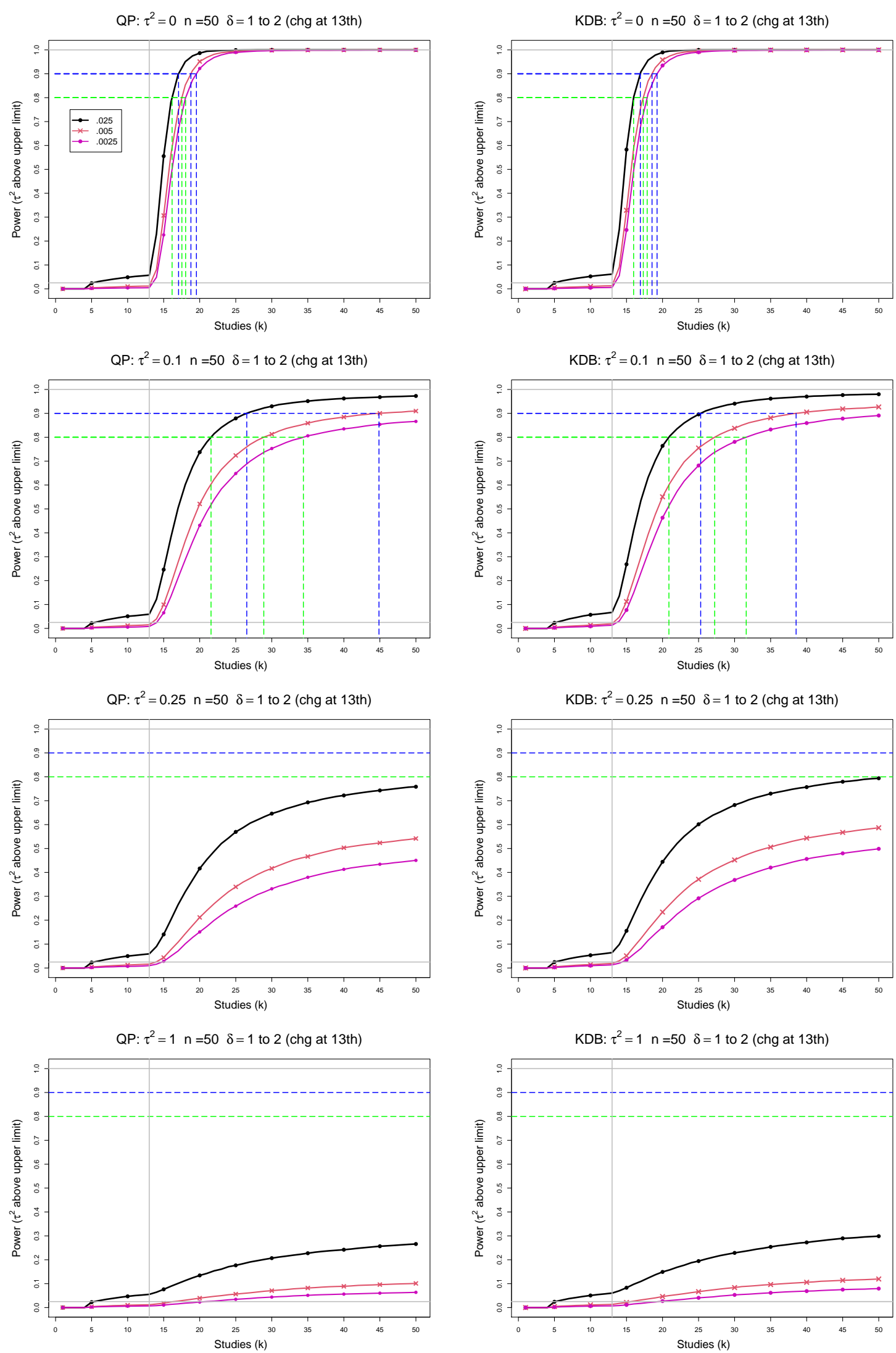

Figure C8. 2: Empirical levels of CMA tests for positive shift in $\tau^{2}$ based on KDB and QP at .025, .005 and .0025 nominal levels for $k$ up to $K=50$ for equal sample sizes $n_{i C}+n_{i T}=n=50, \tau^{2}=0,0.1,0.25,1$ and a shift from $\delta=1$ to $\delta=2$ at study number 13. Light grey line at .025. 

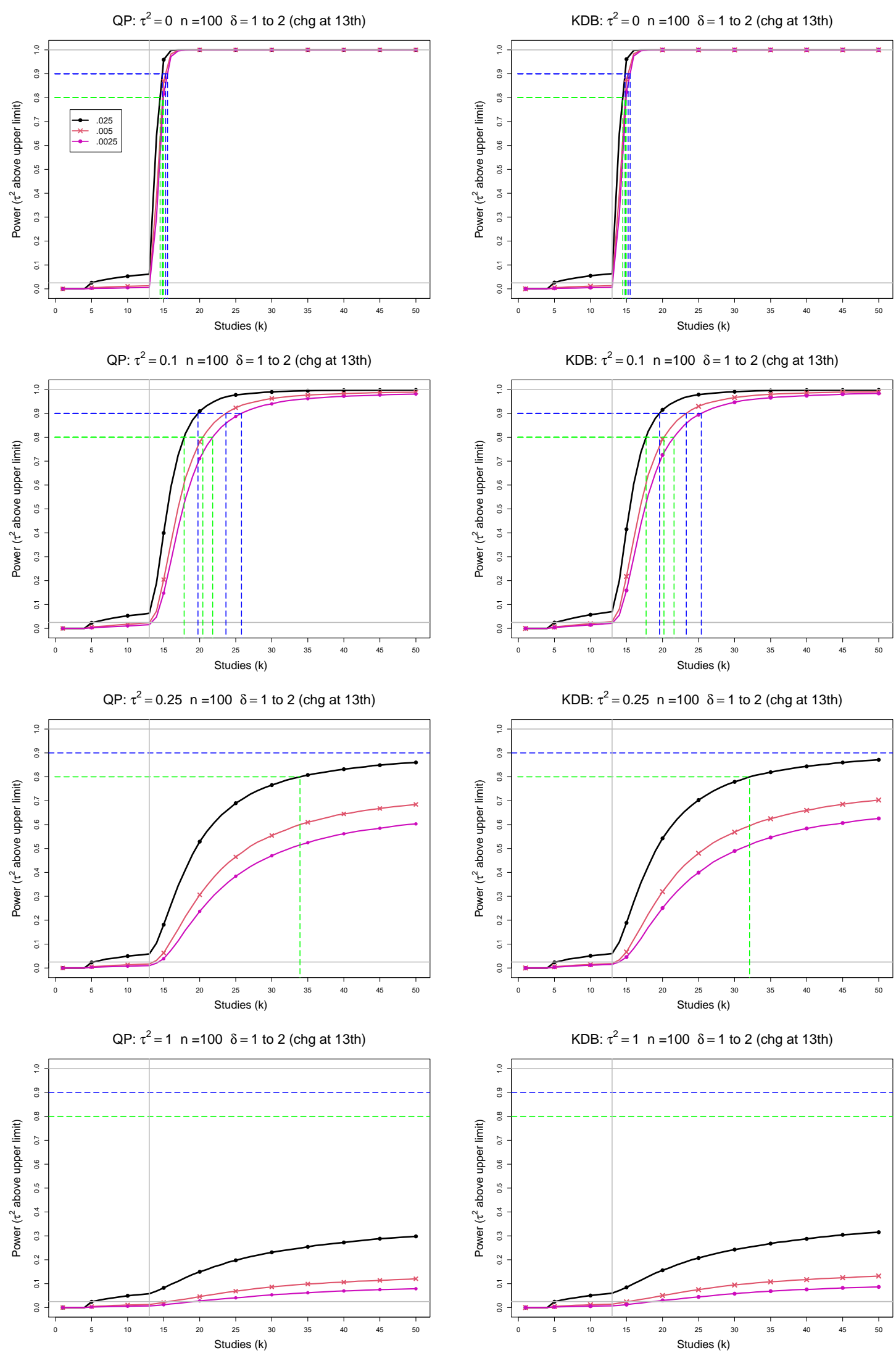

Figure C8. 3: Empirical levels of CMA tests for positive shift in $\tau^{2}$ based on KDB and QP at .025, .005 and .0025 nominal levels for $k$ up to $K=50$ for equal sample sizes $n_{i C}+n_{i T}=n=100, \tau^{2}=0,0.1,0.25,1$ and a shift from $\delta=1$ to $\delta=2$ at study number 13. Light grey line at .025. 

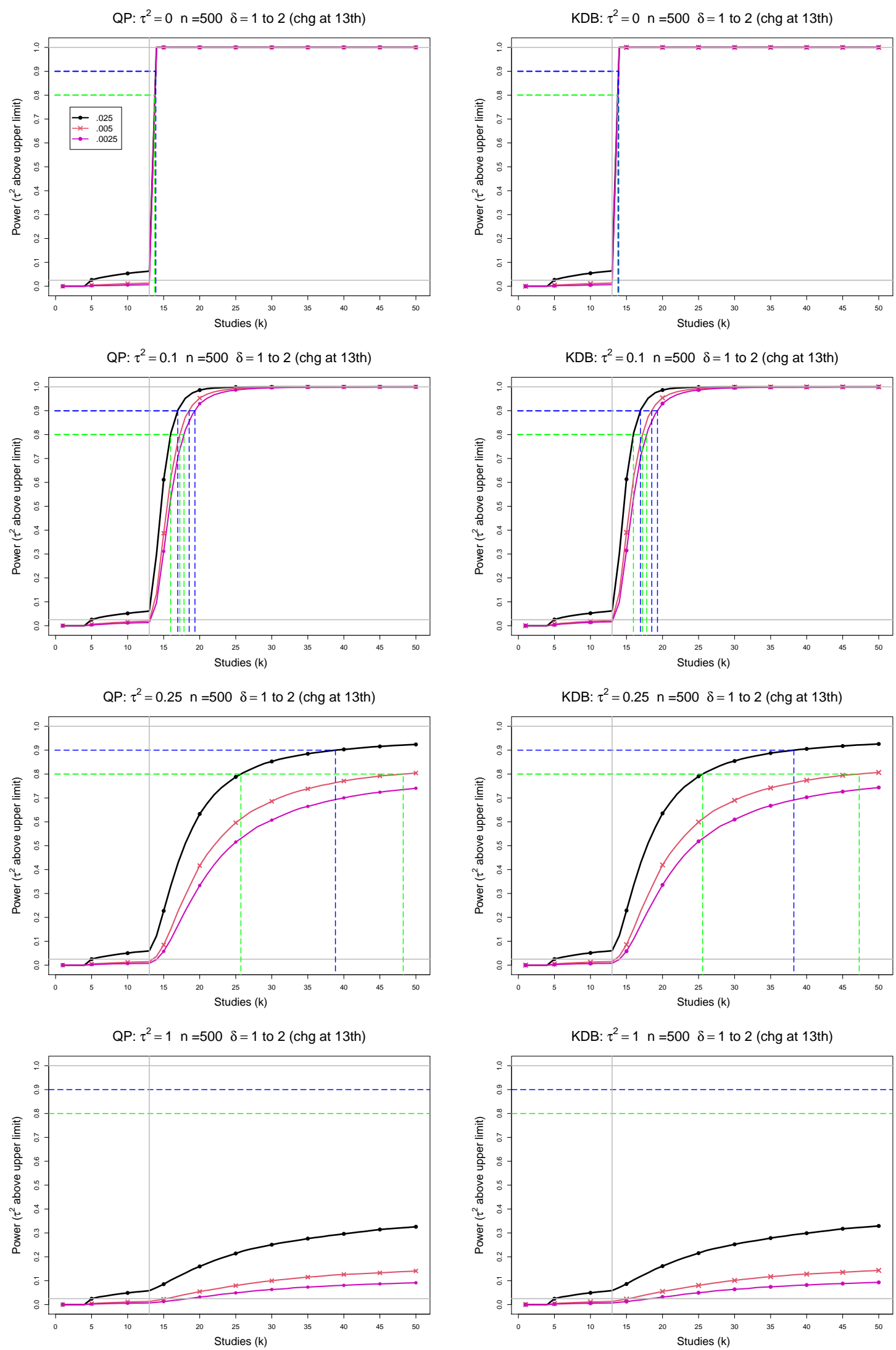

Figure C8. 4: Empirical levels of CMA tests for positive shift in $\tau^{2}$ based on KDB and QP at .025, .005 and .0025 nominal levels for $k$ up to $K=50$ for equal sample sizes $n_{i C}+n_{i T}=n=500, \tau^{2}=0,0.1,0.25,1$ and a shift from $\delta=1$ to $\delta=2$ at study number 13. Light grey line at .025. 

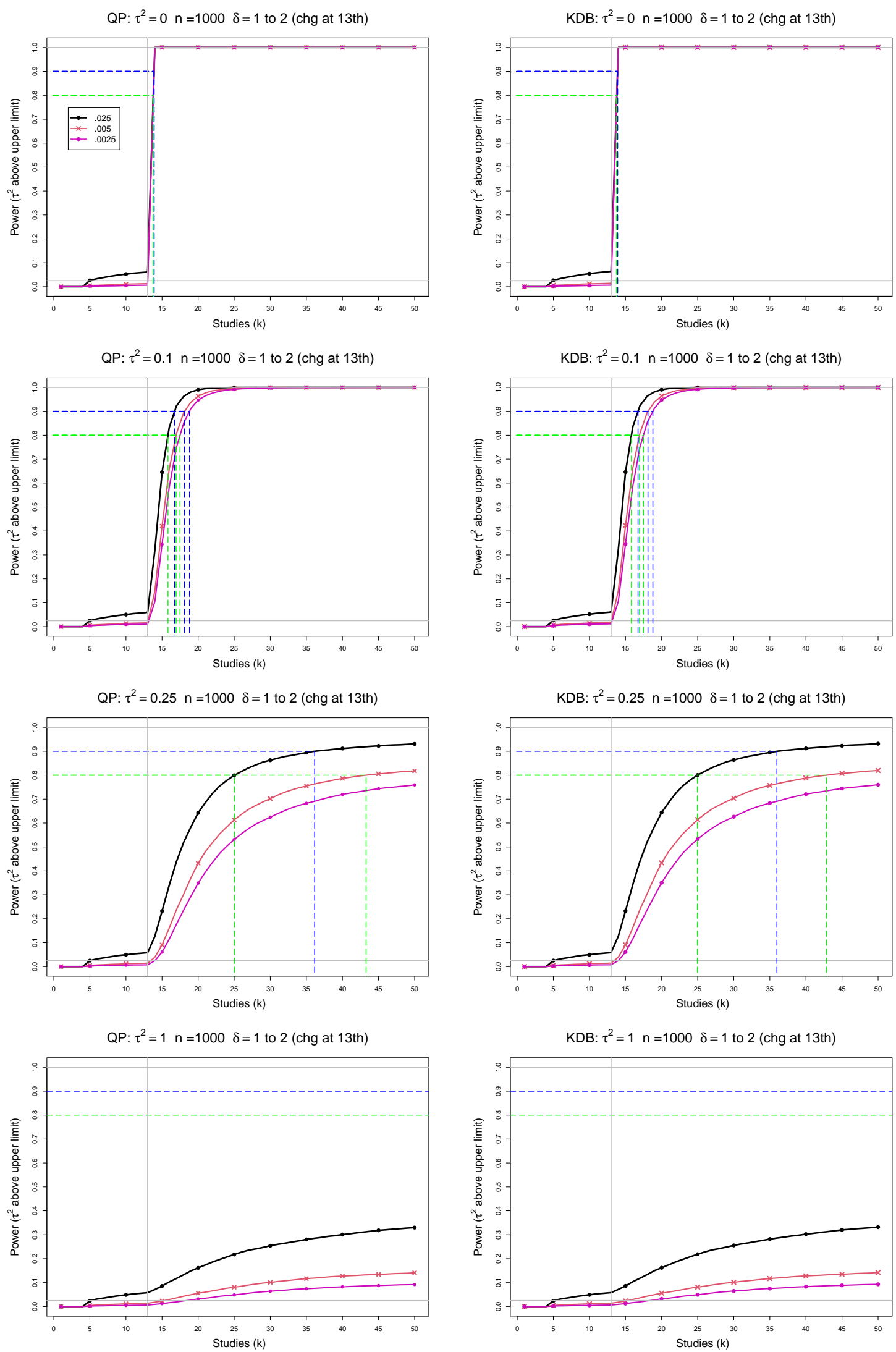

Figure C8. 5: Empirical levels of CMA tests for positive shift in $\tau^{2}$ based on KDB and QP at .025, .005 and .0025 nominal levels for $k$ up to $K=50$ for equal sample sizes $n_{i C}+n_{i T}=n=1000, \tau^{2}=0,0.1,0.25,1$ and a shift from $\delta=1$ to $\delta=2$ at study number 13. Light grey line at .025. 

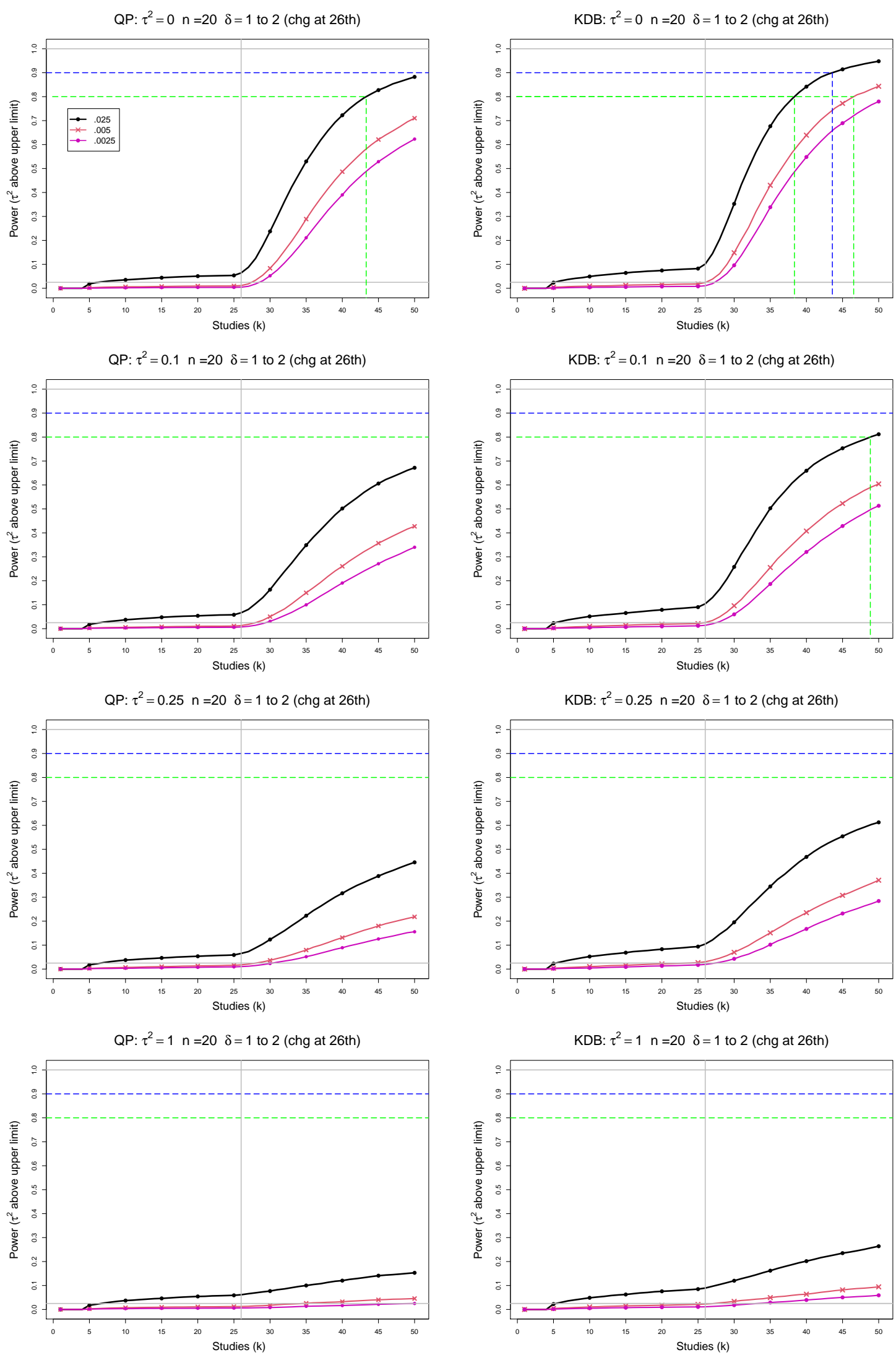

Figure C8. 6: Empirical levels of CMA tests for positive shift in $\tau^{2}$ based on KDB and QP at .025, .005 and .0025 nominal levels for $k$ up to $K=50$ for equal sample sizes $n_{i C}+n_{i T}=n=20, \tau^{2}=0,0.1,0.25,1$ and a shift from $\delta=1$ to $\delta=2$ at study number 26. Light grey line at .025. 

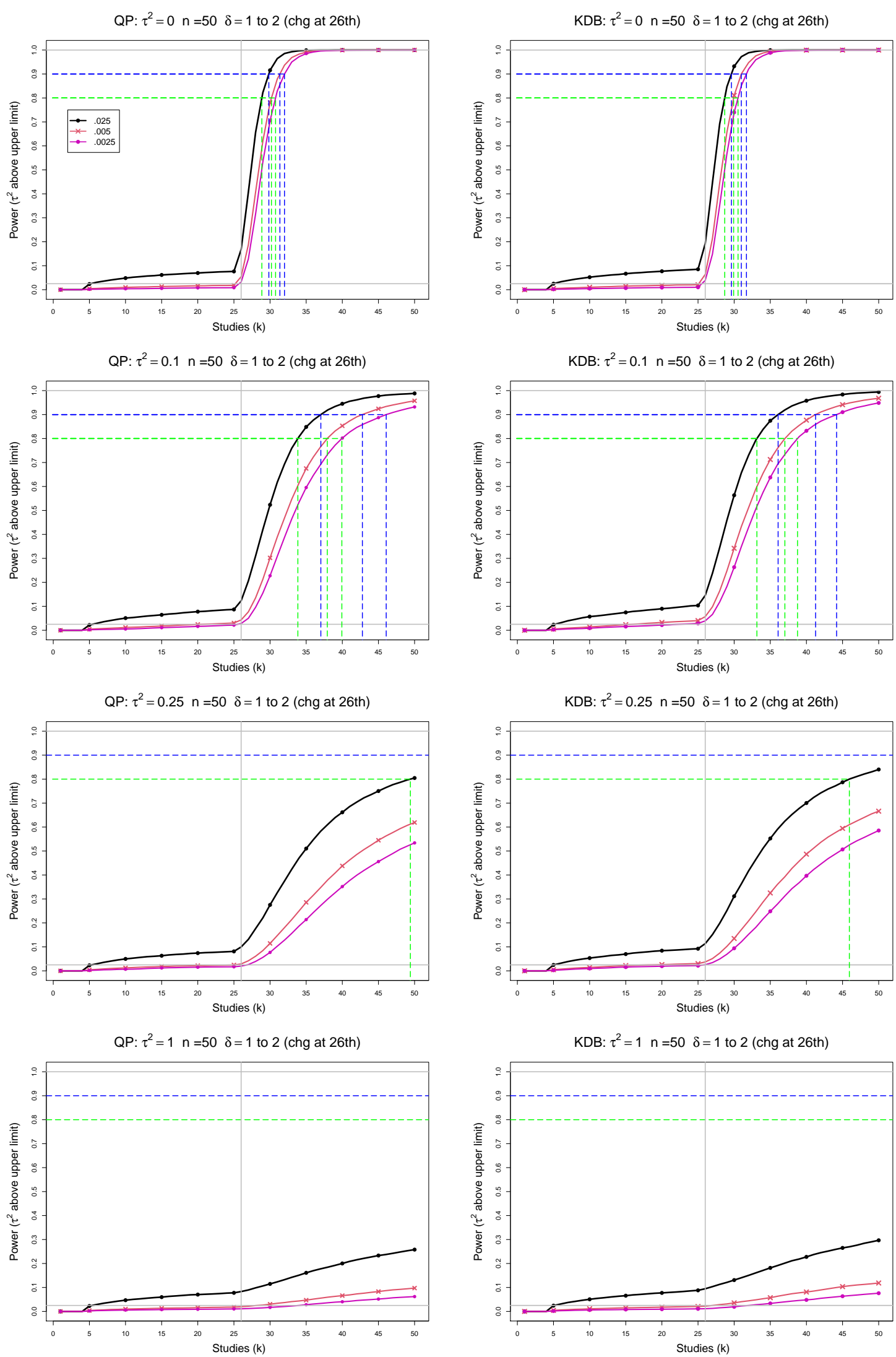

Figure C8. 7: Empirical levels of CMA tests for positive shift in $\tau^{2}$ based on KDB and QP at .025, .005 and .0025 nominal levels for $k$ up to $K=50$ for equal sample sizes $n_{i C}+n_{i T}=n=50, \tau^{2}=0,0.1,0.25,1$ and a shift from $\delta=1$ to $\delta=2$ at study number 26. Light grey line at .025. 

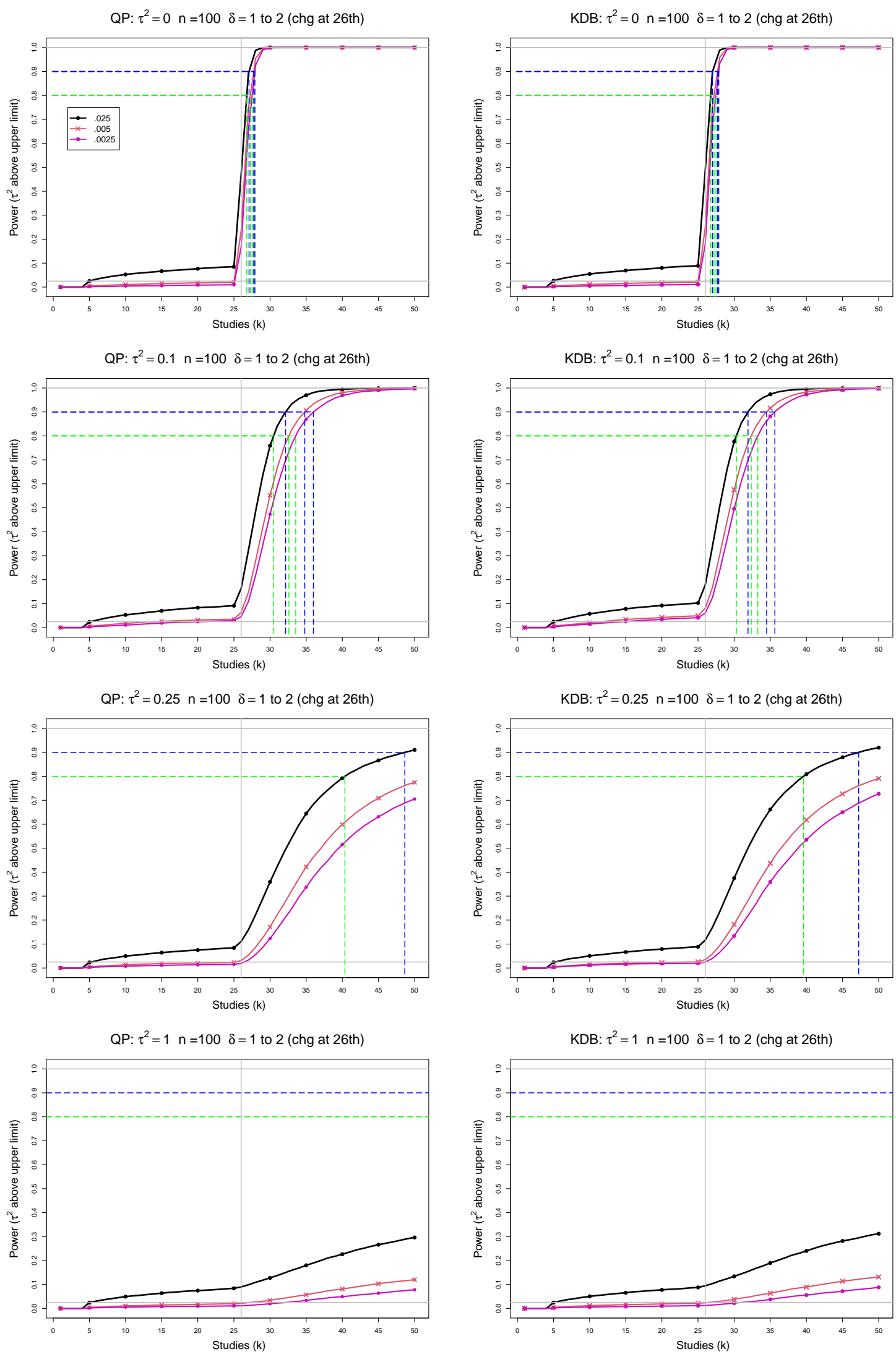

Figure C8. 8: Empirical levels of CMA tests for positive shift in $\tau^{2}$ based on KDB and QP at .025, .005 and .0025 nominal levels for $k$ up to $K=50$ for equal sample sizes $n_{i C}+n_{i T}=n=100, \tau^{2}=0,0.1,0.25,1$ and a shift from $\delta=1$ to $\delta=2$ at study number 26. Light grey line at .025. 

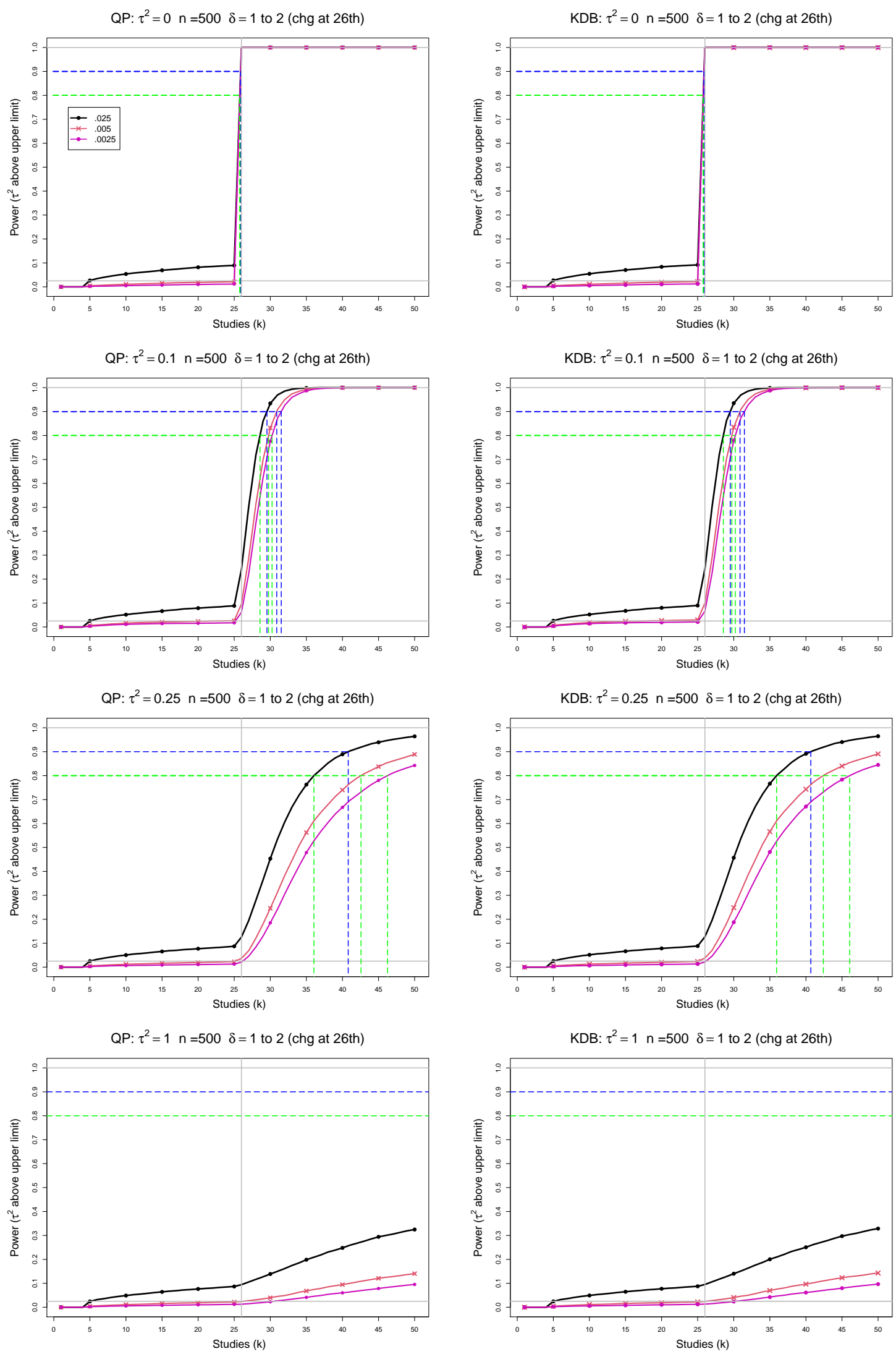

Figure C8. 9: Empirical levels of CMA tests for positive shift in $\tau^{2}$ based on KDB and QP at .025, .005 and .0025 nominal levels for $k$ up to $K=50$ for equal sample sizes $n_{i C}+n_{i T}=n=500, \tau^{2}=0,0.1,0.25,1$ and a shift from $\delta=1$ to $\delta=2$ at study number 26. Light grey line at .025. 

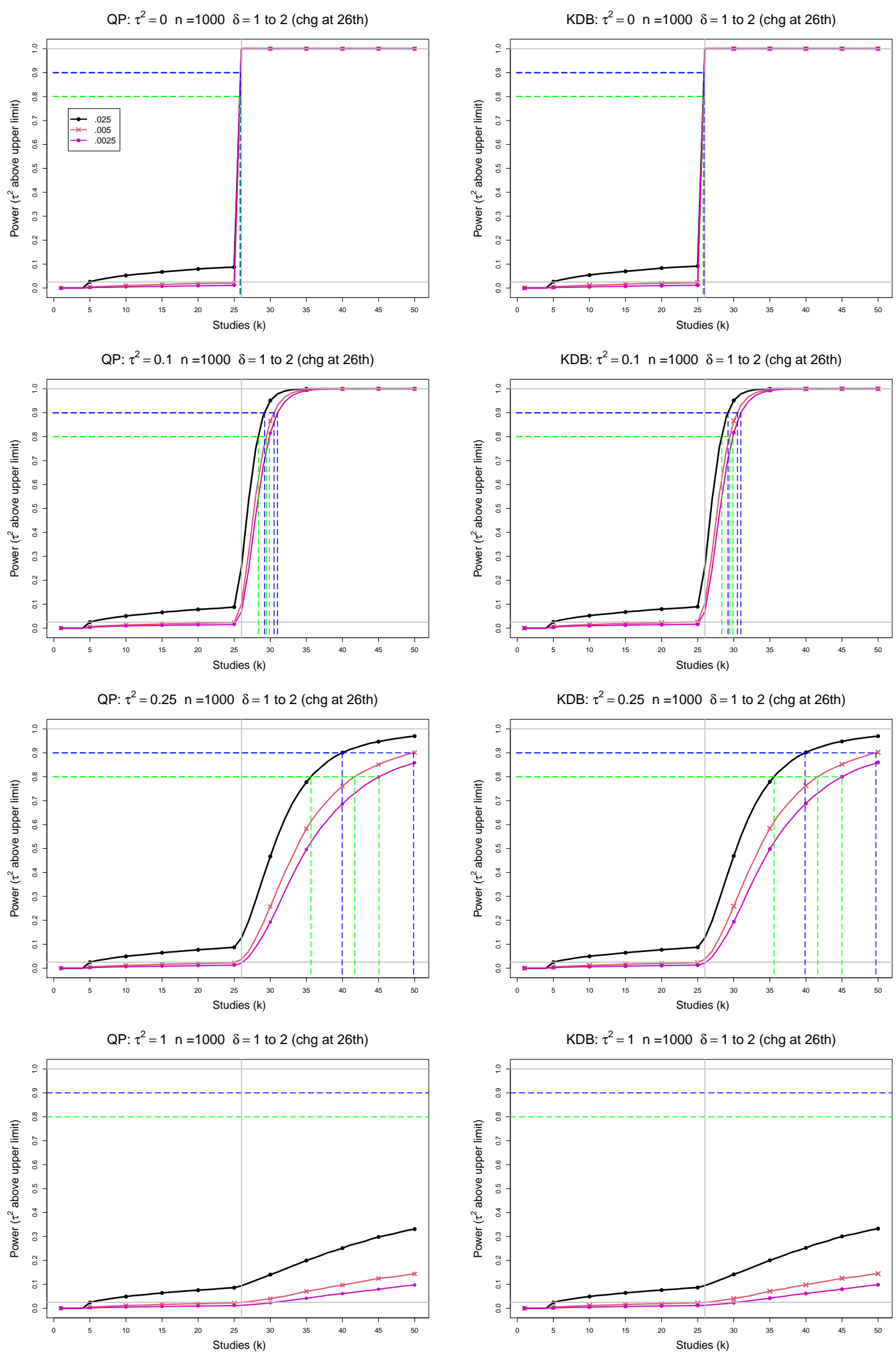

Figure C8. 10: Empirical levels of CMA tests for positive shift in $\tau^{2}$ based on KDB and QP at .025, .005 and .0025 nominal levels for $k$ up to $K=50$ for equal sample sizes $n_{i C}+n_{i T}=n=1000, \tau^{2}=0,0.1,0.25,1$ and a shift from $\delta=1$ to $\delta=2$ at study number 26. Light grey line at .025. 

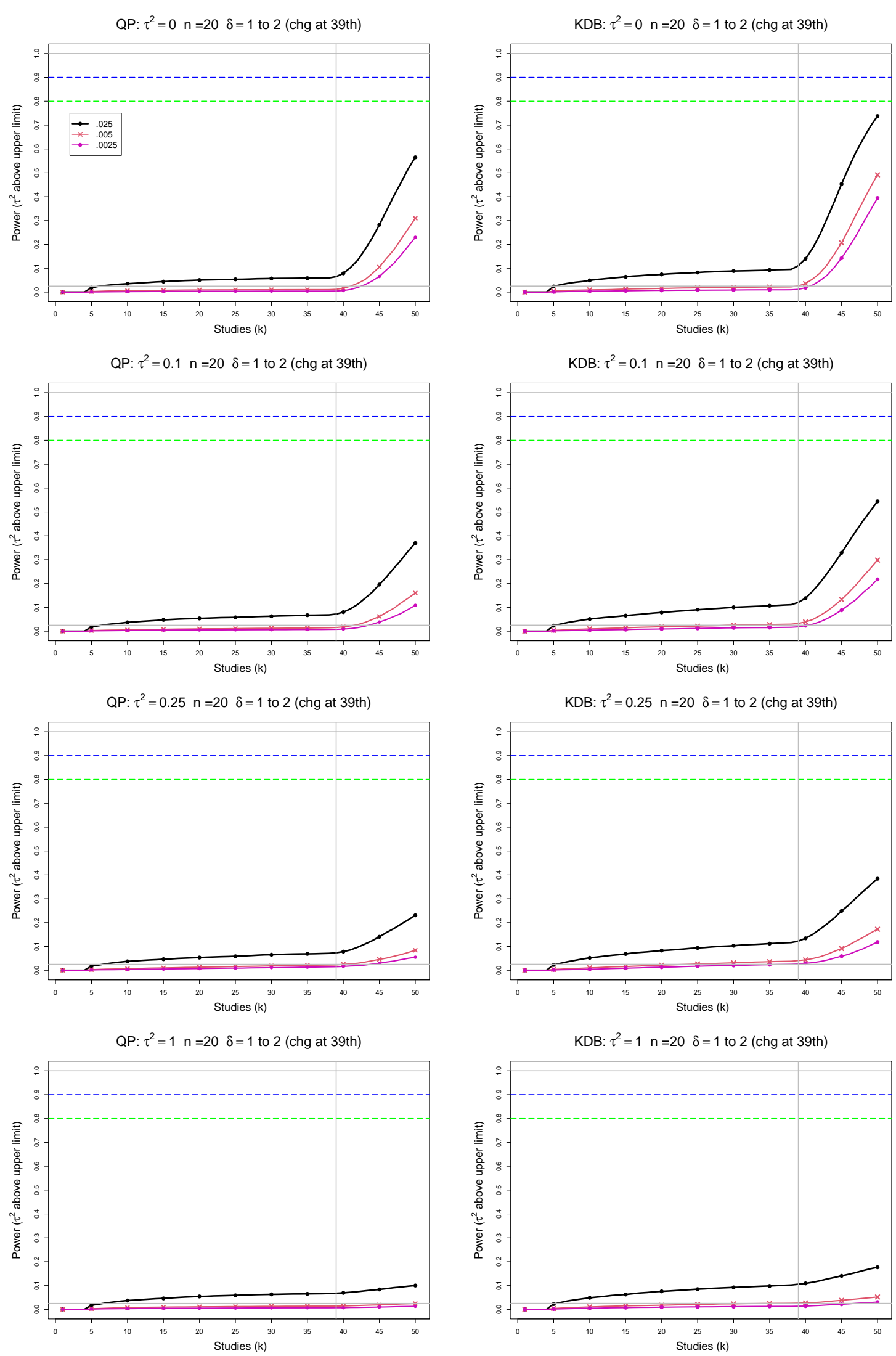

Figure C8. 11: Empirical levels of CMA tests for positive shift in $\tau^{2}$ based on KDB and QP at .025, .005 and .0025 nominal levels for $k$ up to $K=50$ for equal sample sizes $n_{i C}+n_{i T}=n=20 \tau^{2}=0,0.1,0.25,1$ and a shift from $\delta=1$ to $\delta=2$ at study number 39. 

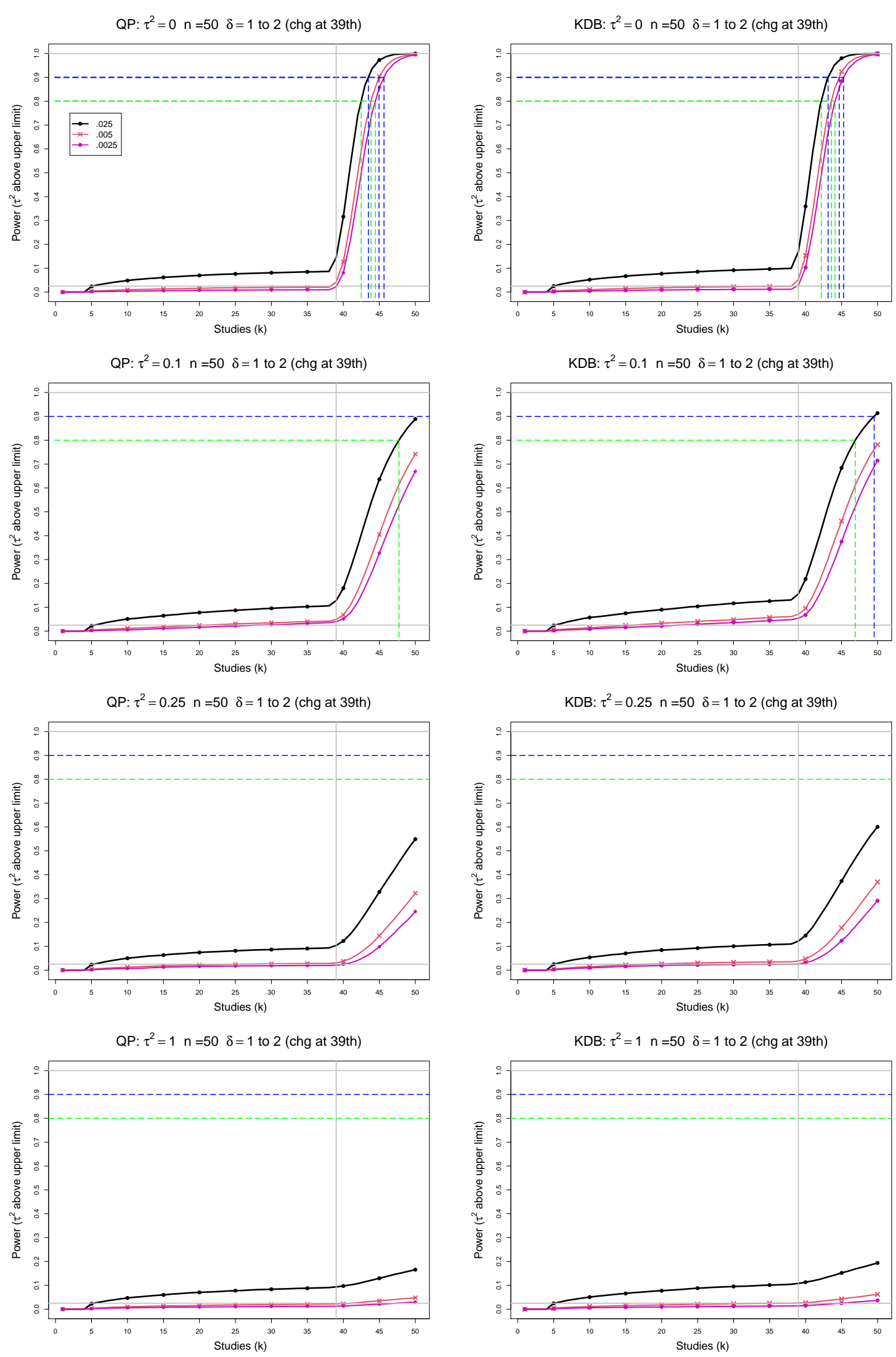

Figure C8. 12: Empirical levels of CMA tests for positive shift in $\tau^{2}$ based on KDB and QP at $.025, .005$ and .0025 nominal levels for $k$ up to $K=50$ for equal sample sizes $n_{i C}+n_{i T}=n=50, \tau^{2}=0,0.1,0.25,1$ and a shift from $\delta=1$ to $\delta=2$ at study number 39. Light grey line at .025. 

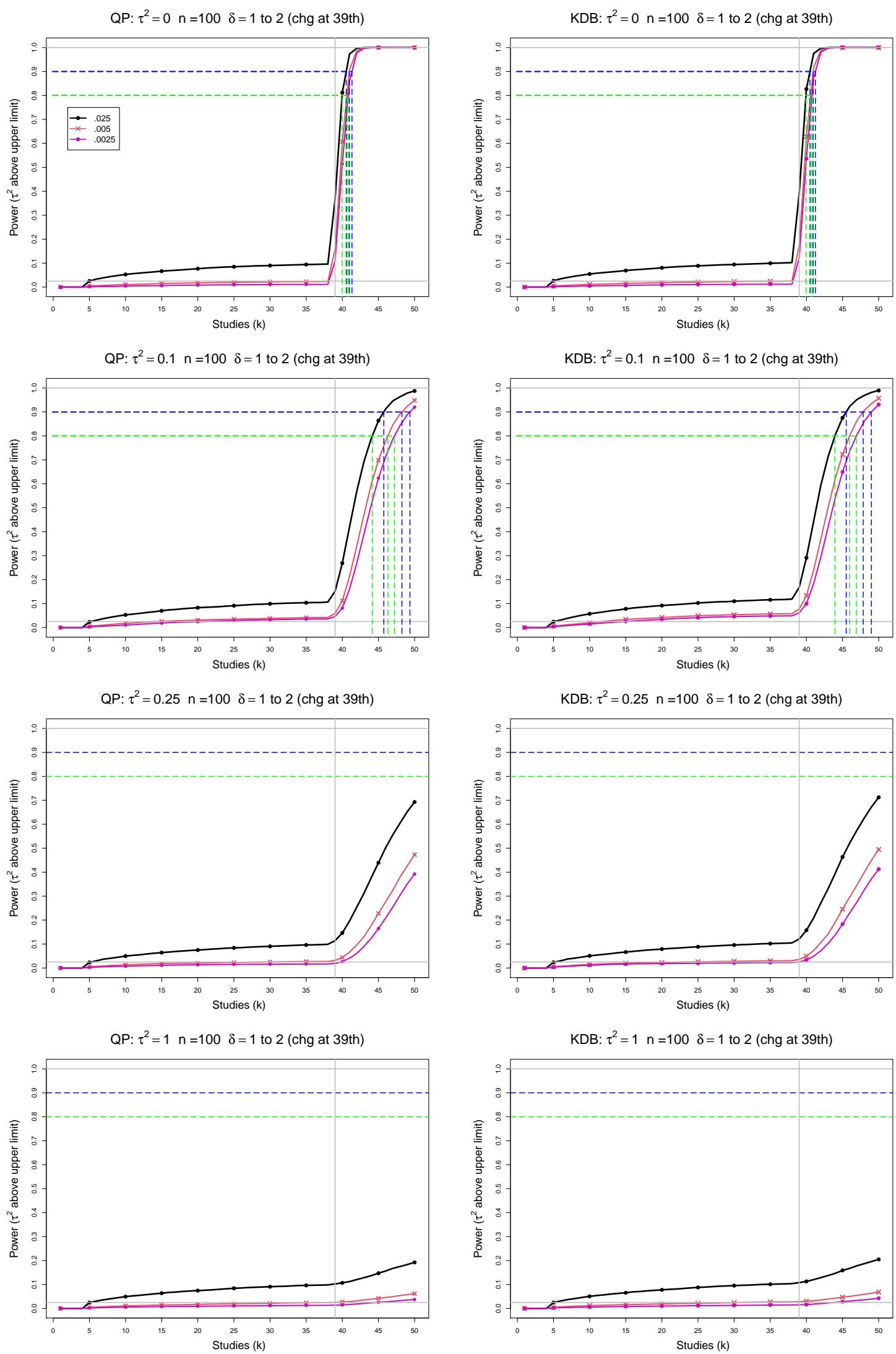

Figure C8. 13: Empirical levels of CMA tests for positive shift in $\tau^{2}$ based on KDB and QP at .025, .005 and .0025 nominal levels for $k$ up to $K=50$ for equal sample sizes $n_{i C}+n_{i T}=n=100, \tau^{2}=0,0.1,0.25,1$ and a shift from $\delta=1$ to $\delta=2$ at study number 39. Light grey line at .025. 

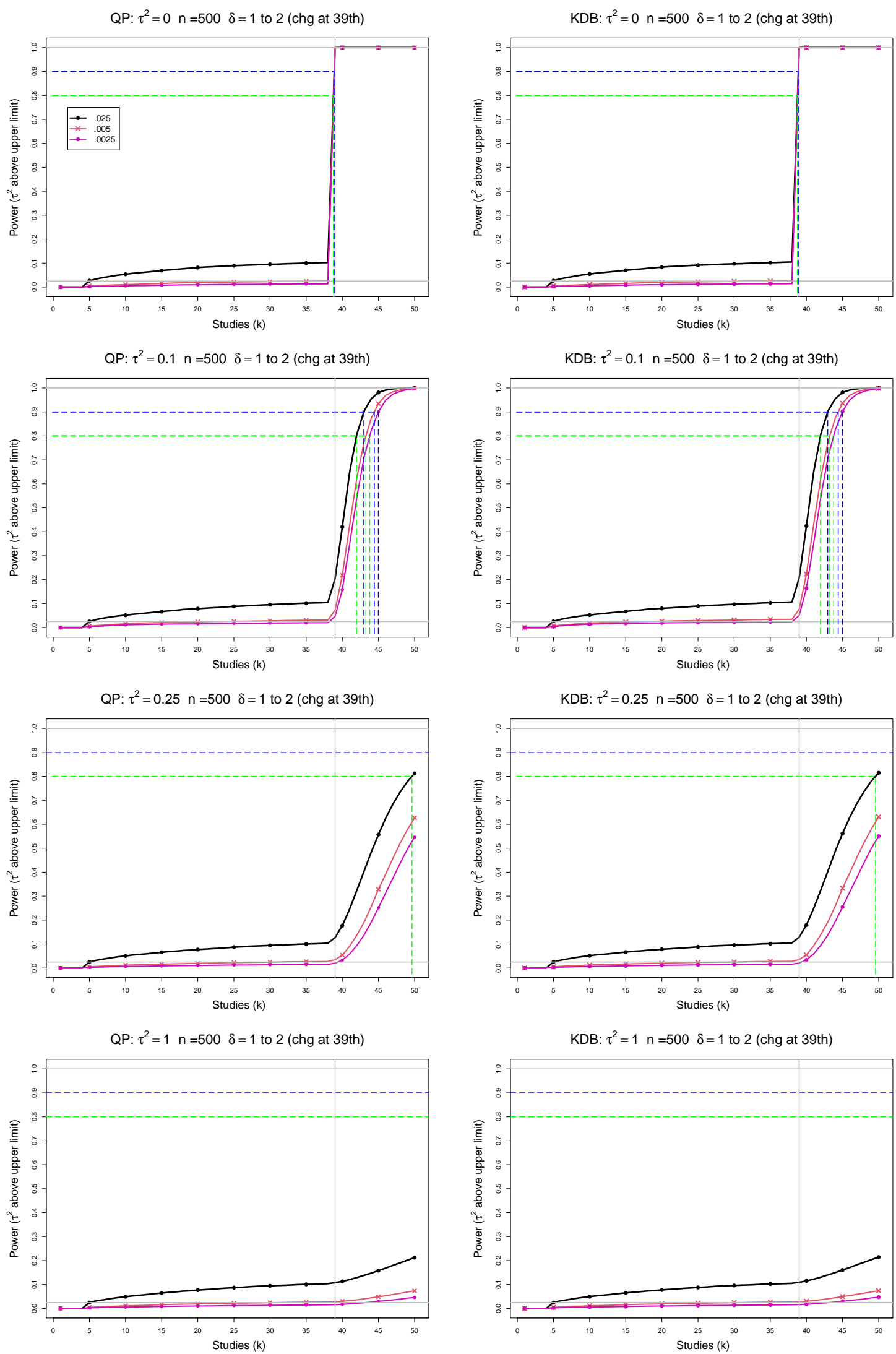

Figure C8. 14: Empirical levels of CMA tests for positive shift in $\tau^{2}$ based on KDB and QP at .025, .005 and .0025 nominal levels for $k$ up to $K=50$ for equal sample sizes $n_{i C}+n_{i T}=n=500, \tau^{2}=0,0.1,0.25,1$ and a shift from $\delta=1$ to $\delta=2$ at study number 36. Light grey line at .025. 

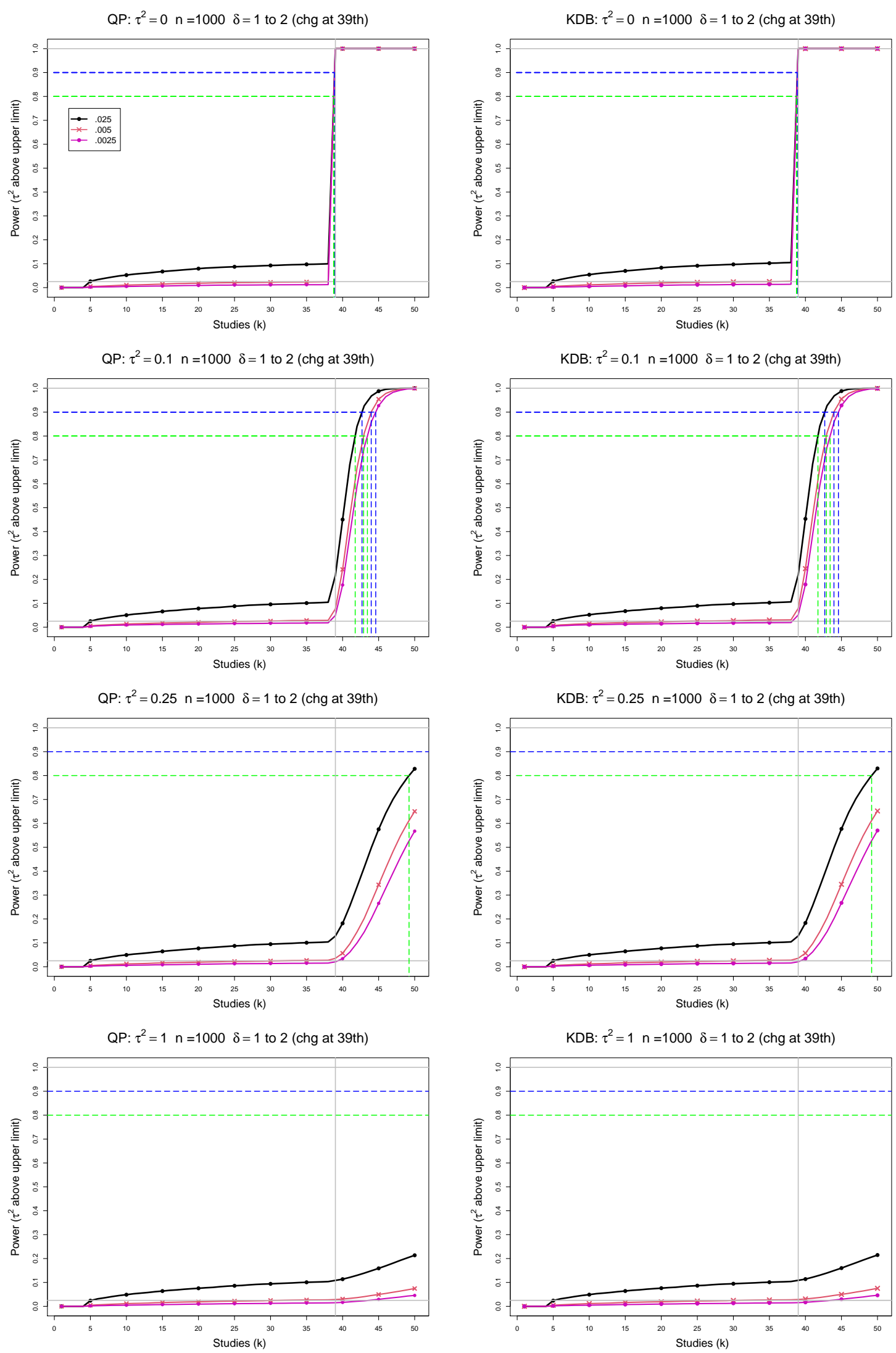

Figure C8. 15: Empirical levels of CMA tests for positive shift in $\tau^{2}$ based on KDB and QP at .025, .005 and .0025 nominal levels for $k$ up to $K=50$ for equal sample sizes $n_{i C}+n_{i T}=n=1000, \tau^{2}=0,0.1,0.25,1$ and a shift from $\delta=1$ to $\delta=2$ at study number 36. Light grey line at .025. 


\section{2-Stage CMA under shift-in-the-mean $\delta$}




\section{D1. Comparison of 2-Stage CMA-estimated mean effect $\delta$ to nominal cumulative mean effect}

In this section, we compare 2-stage CMA-estimated value of $\delta$ to nominal cumulative effects. Each figure corresponds to a set of values of $\tau^{2}(=0,0.1,0.25,1)$, a set of values of $n(=20,50,100$ or 500,1000$)$ and a value of point of shift $f(=13,26,39)$ while $\delta$ shifts from 1 to 2 and number of studies $K=50$.

Each figure contains a panel (with study $k$ on the horizontal axis) for each combination of parameters.

The point estimators of $\delta$ are

- IV REML (Restricted maximum likelihood, inverse variance weighted)

- SSW (Kulinskaya-Dollinger-Bjørkestøl estimator of $\tau^{2}$, sample size weighted)

The nominal $\delta$ values are calculated with

- $\delta$ IV (Inverse variance weights)

- $\delta \mathrm{SSW}$ (effective sample size weights) 

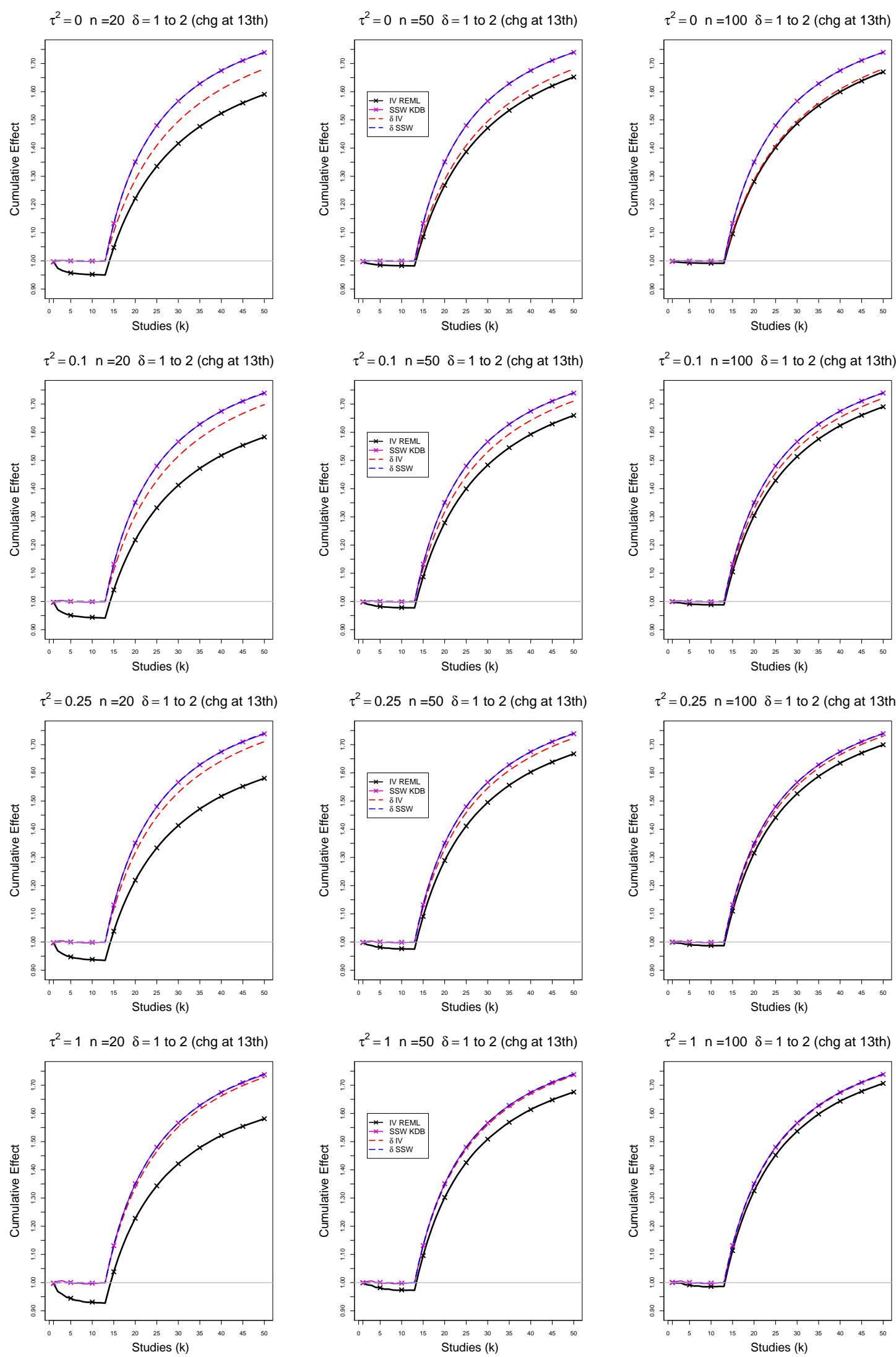

Figure D1. 1: 2-Stage CMA: Nominal cumulative effects $\delta_{S S W,(k)}$ and $\delta_{I V,(k)}$ (dashed lines) and estimated by SSW and IV REML cumulative effects $\hat{\delta}_{(k)}$ (solid lines) when $\delta=1$ for $k \leq 12$ followed by a shift to $\delta=2$ for $k \geq 13, n=20,50,100, K=50$ and $\tau^{2}=$ $0,0.1,0.25,1$. Light grey line at 1. 

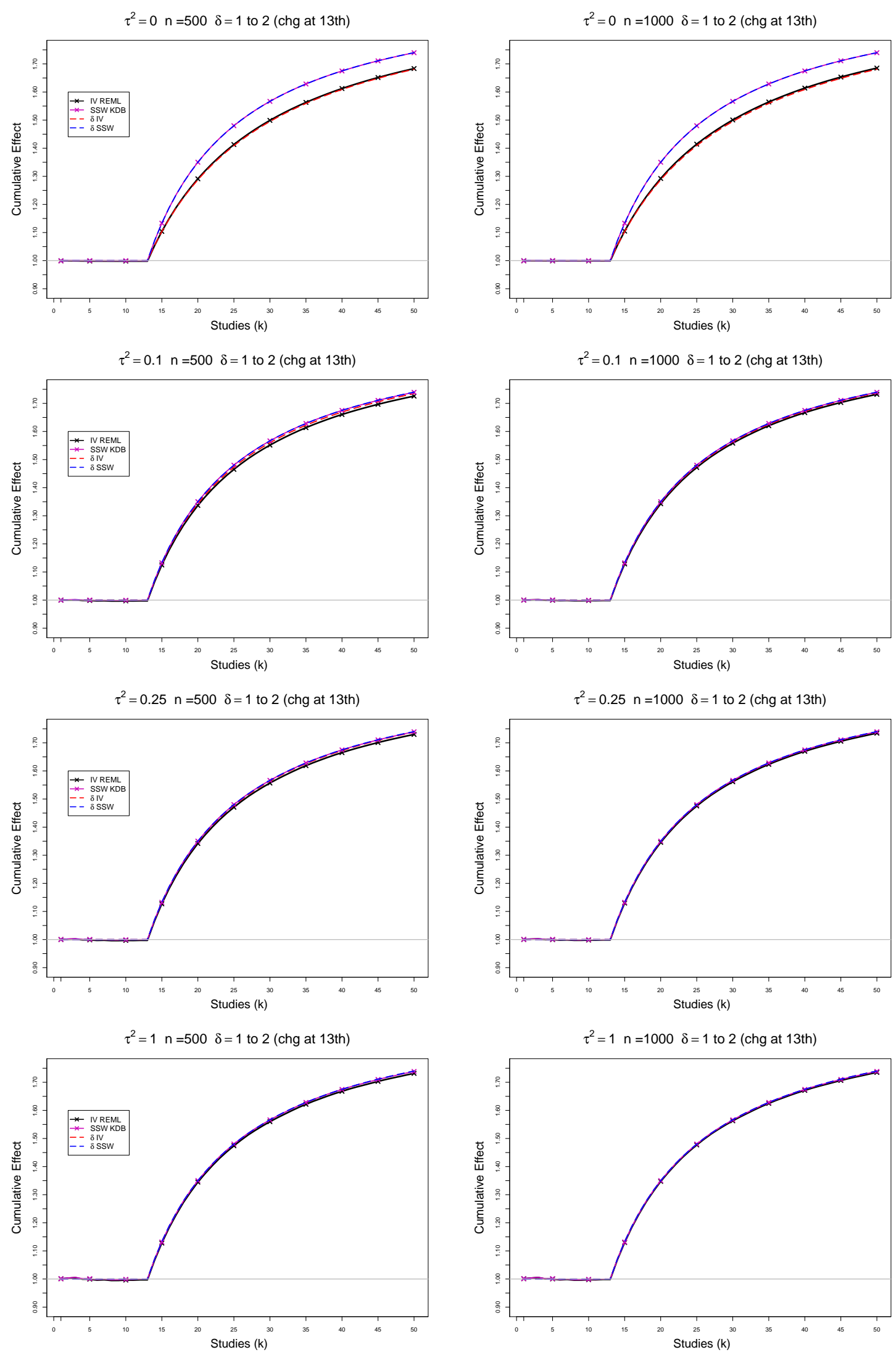

Figure D1. 2: 2-Stage CMA: Nominal cumulative effects $\delta_{S S W,(k)}$ and $\delta_{I V,(k)}$ (dashed lines) and estimated by SSW and IV REML cumulative effects $\hat{\delta}_{(k)}$ (solid lines) when $\delta=1$ for $k \leq 12$ followed by a shift to $\delta=2$ for $k \geq 13, n=500,1000, K=50$ and $\tau^{2}=$ $0,0.1,0.25,1$. Light grey line at 1 . 

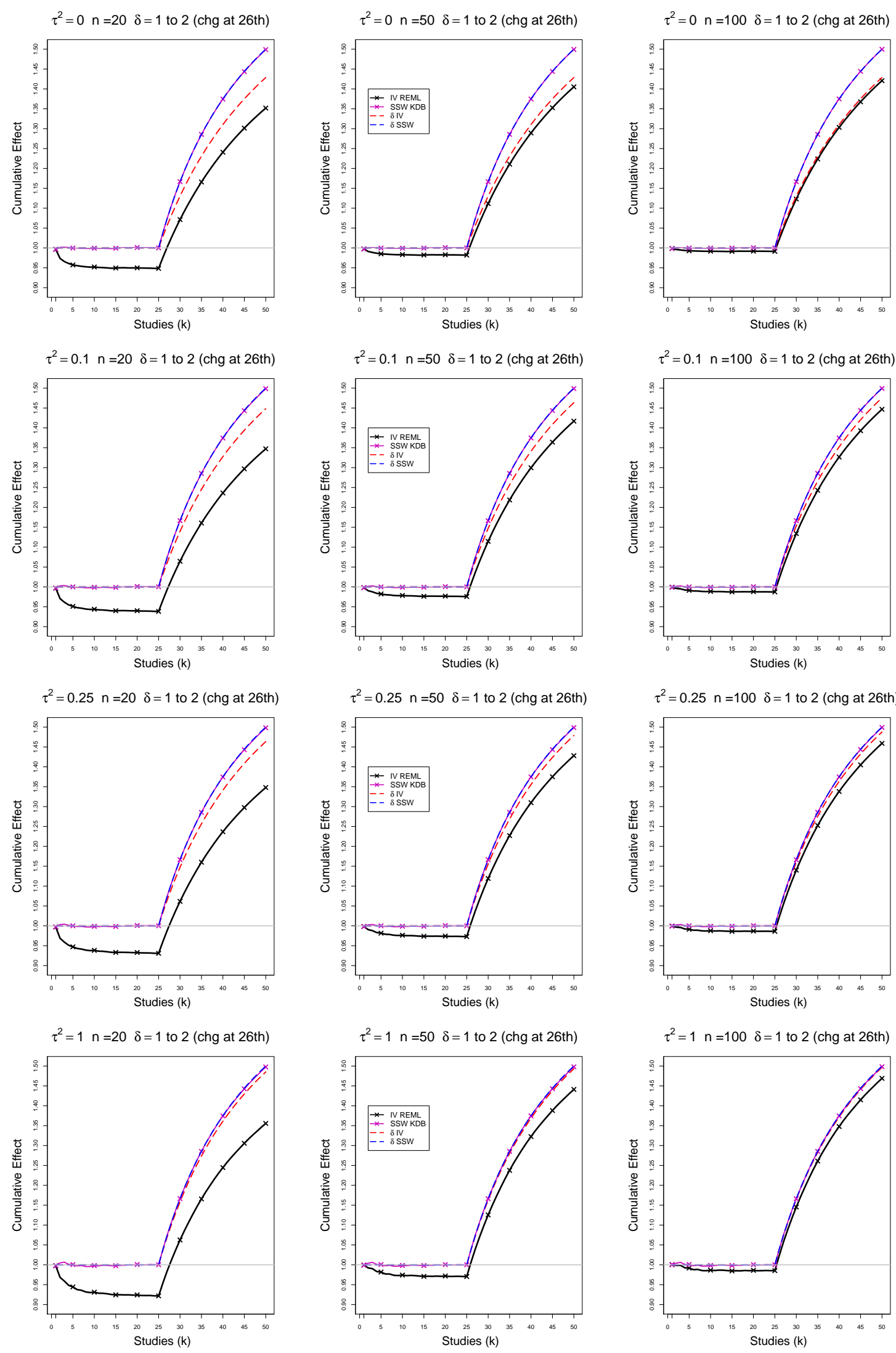

Figure D1. 3: 2-Stage CMA: Nominal cumulative effects $\delta_{S S W,(k)}$ and $\delta_{I V,(k)}$ (dashed lines) and estimated by SSW and IV REML cumulative effects $\hat{\delta}_{(k)}$ (solid lines) when $\delta=1$ for $k \leq 25$ followed by a shift to $\delta=2$ for $k \geq 26, n=20,50,100, K=50$ and $\tau^{2}=$ $0,0.1,0.25,1$. Light grey line at 1. 

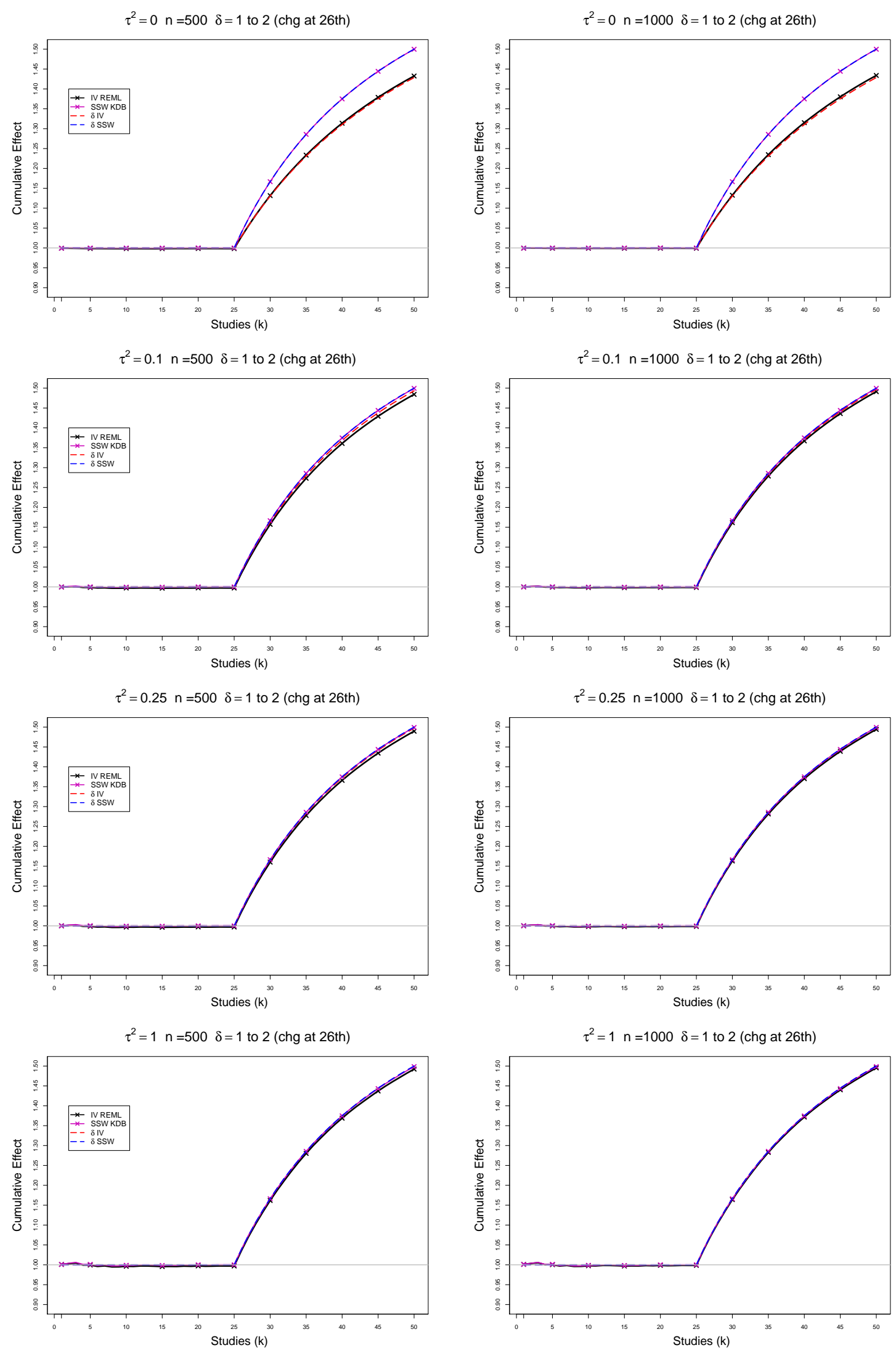

Figure D1. 4: 2-Stage CMA: Nominal cumulative effects $\delta_{S S W,(k)}$ and $\delta_{I V,(k)}$ (dashed lines) and estimated by SSW and IV REML cumulative effects $\hat{\delta}_{(k)}$ (solid lines) when $\delta=1$ for $k \leq 25$ followed by a shift to $\delta=2$ for $k \geq 26, n=500,1000, K=50$ and $\tau^{2}=$ $0,0.1,0.25,1$. Light grey line at 1 . 

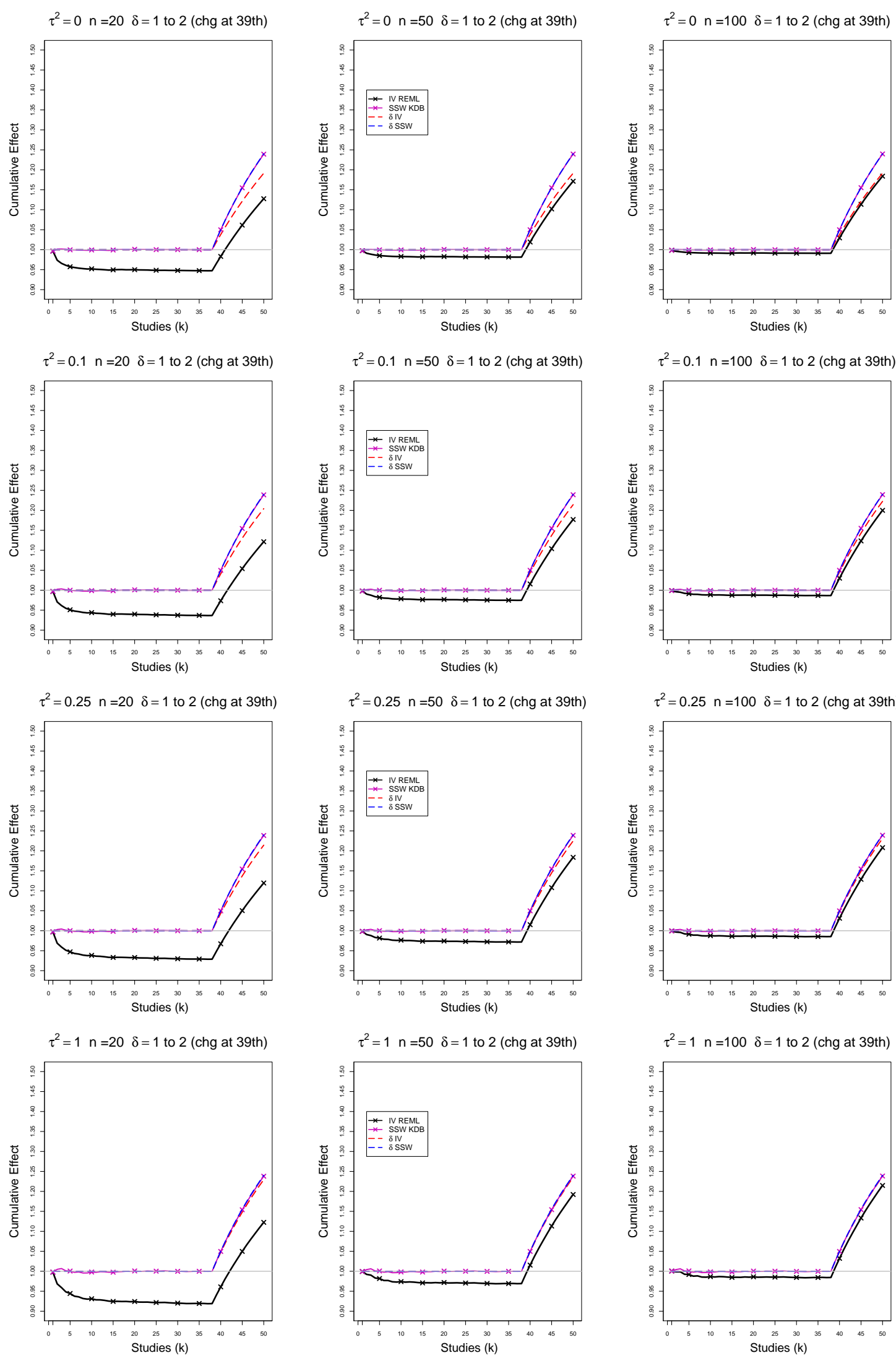

Figure D1. 5: 2-Stage CMA: Nominal cumulative effects $\delta_{S S W,(k)}$ and $\delta_{I V,(k)}$ (dashed lines) and estimated by SSW and IV REML cumulative effects $\hat{\delta}_{(k)}$ (solid lines) when $\delta=1$ for $k \leq 38$ followed by a shift to $\delta=2$ for $k \geq 39, n=20,50,100, K=50$ and $\tau^{2}=$ $0,0.1,0.25,1$. Light grey line at 1 . 

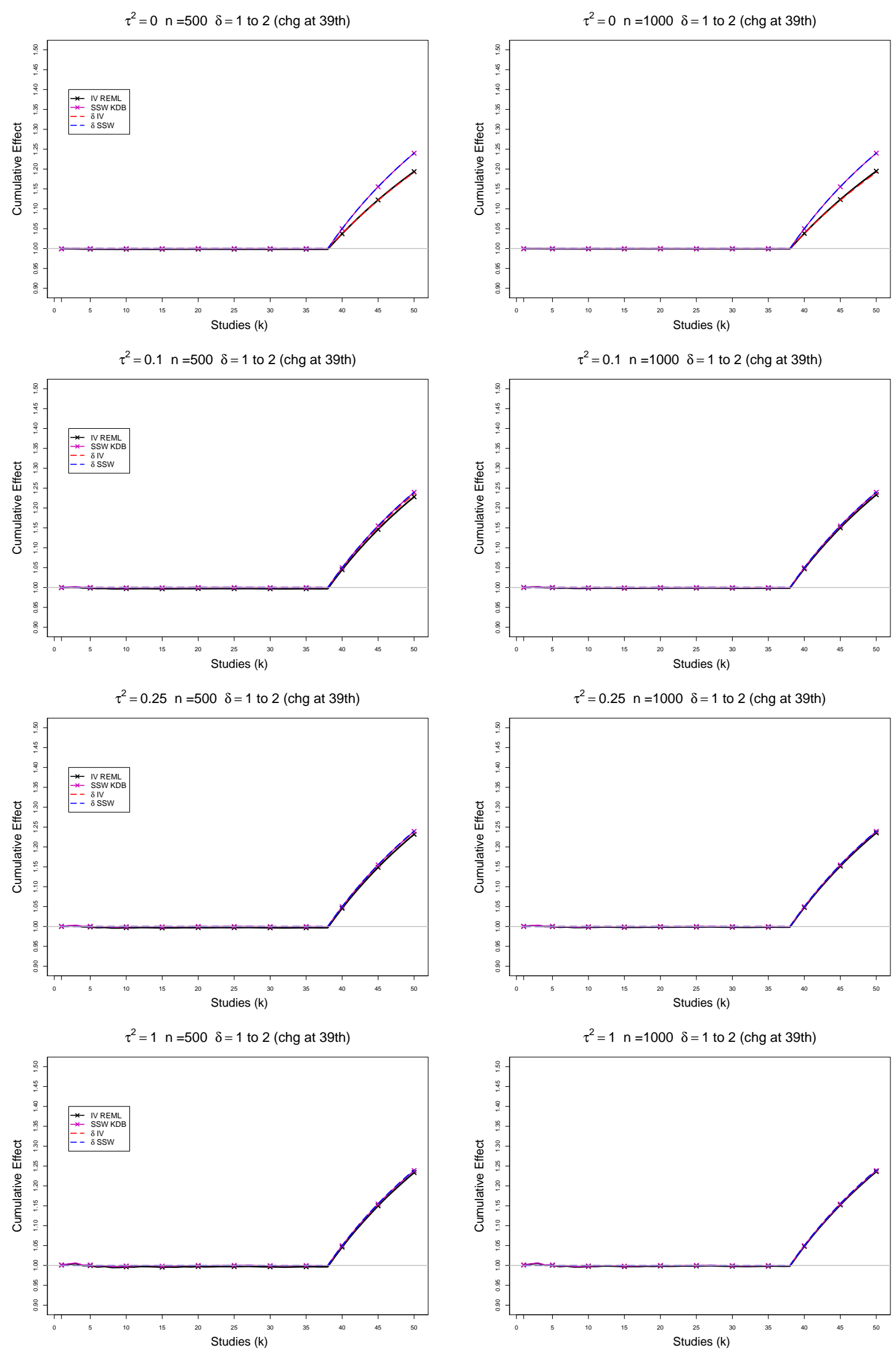

Figure D1. 6: 2-Stage CMA: Nominal cumulative effects $\delta_{S S W,(k)}$ and $\delta_{I V,(k)}$ (dashed lines) and estimated by SSW and IV REML cumulative effects $\hat{\delta}_{(k)}$ (solid lines) when $\delta=1$ for $k \leq 38$ followed by a shift to $\delta=2$ for $k \geq 39, n=500,1000, K=50$ and $\tau^{2}=$ $0,0.1,0.25,1$. Light grey line at 1 . 


\section{D2. Bias of point estimators of $\delta$}

For bias of $\delta$ in this section, each figure corresponds to a set of values of $\tau^{2}(=0,0.1$, $0.25,1)$, a set of values of $n(=20,50,100$ or 500,1000$)$ and a value of point of shift $f(=13,26,39)$ while $\delta$ shifts from 1 to 2 and maximum number of studies $K=50$.

Each figure contains a panel (with study $k$ on the horizontal axis) for each combination of parameters.

The point estimators of $\delta$ are

- IV REML (Restricted maximum likelihood, inverse variance weighted)

- SSW (Sample size weighted) 

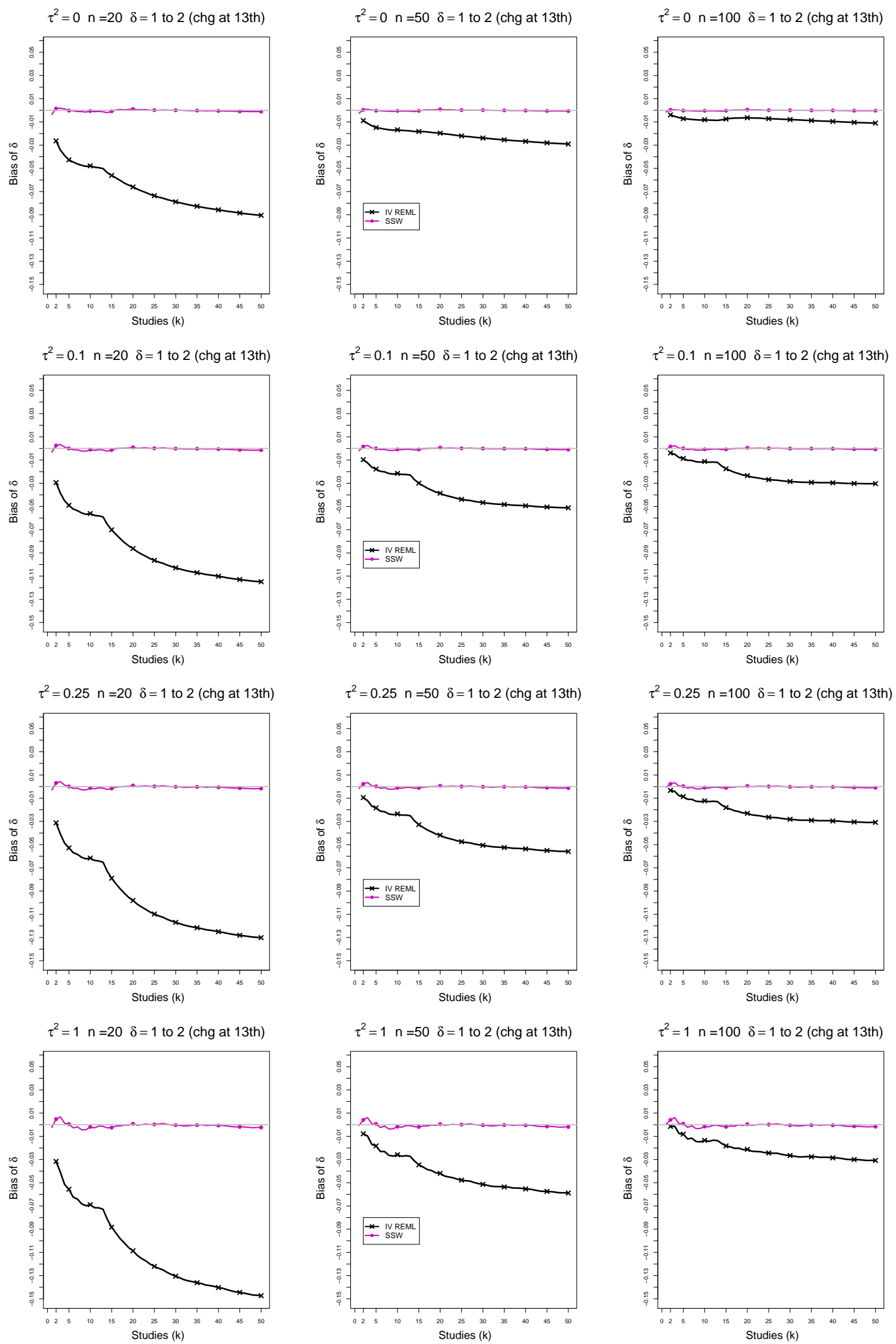

Figure D2. 1: 2-Stage CMA - Bias of IV REML and SSW estimators of $\delta$ when $\delta=1$ for $k \leq 12$ followed by a shift to $\delta=2$ for $k \geq 13, \tau^{2}=0,0.1,0.25,1, n=20,50,100, K=50$. Light grey line at 0 . 

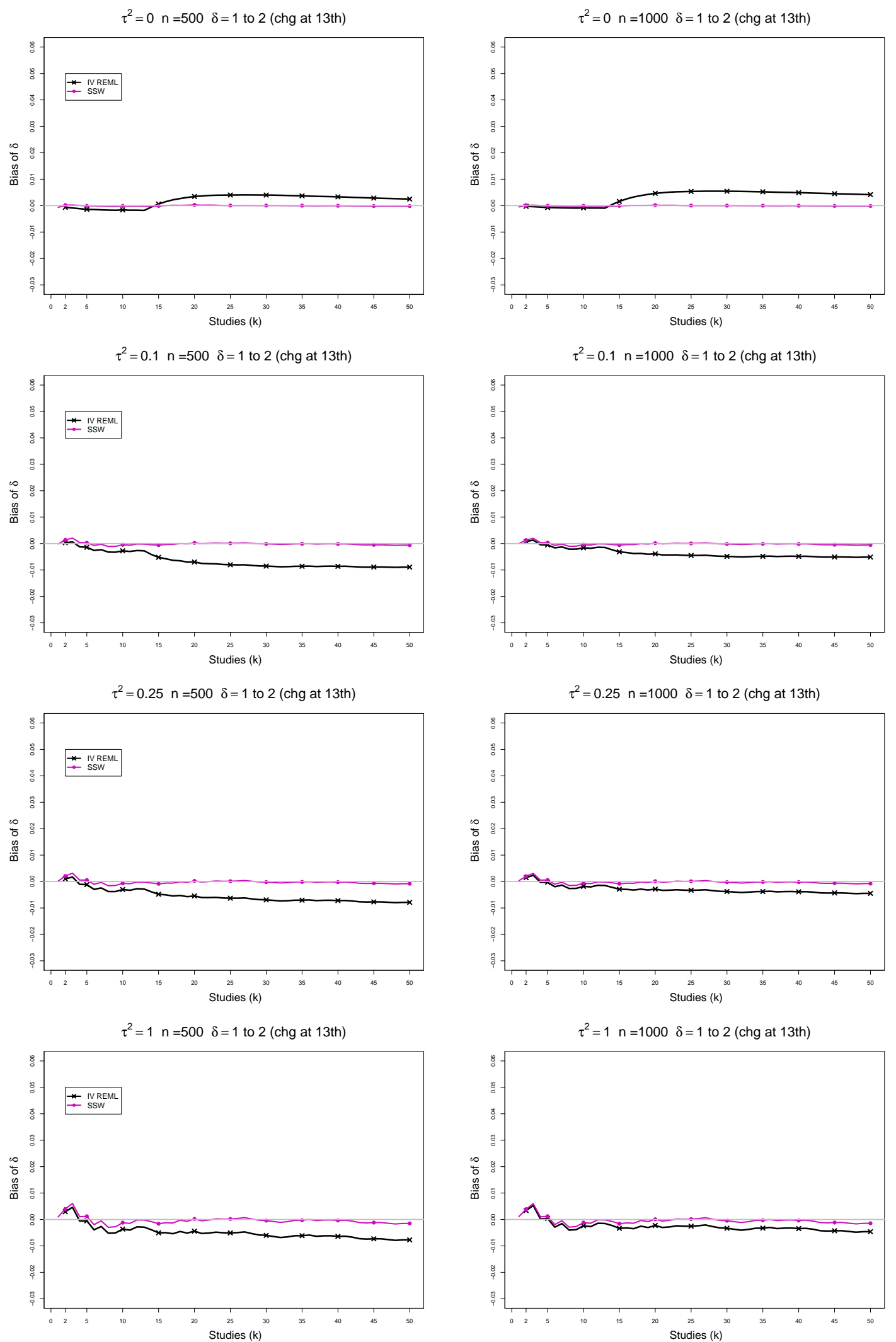

Figure D2. 2: 2-Stage CMA - Bias of IV REML and SSW estimators of $\delta$ when $\delta=1$ for $k \leq 12$ followed by a shift to $\delta=2$ for $k \geq 13, \tau^{2}=0,0.1,0.25,1, n=500,1000, K=50$. Light grey line at 0 . 

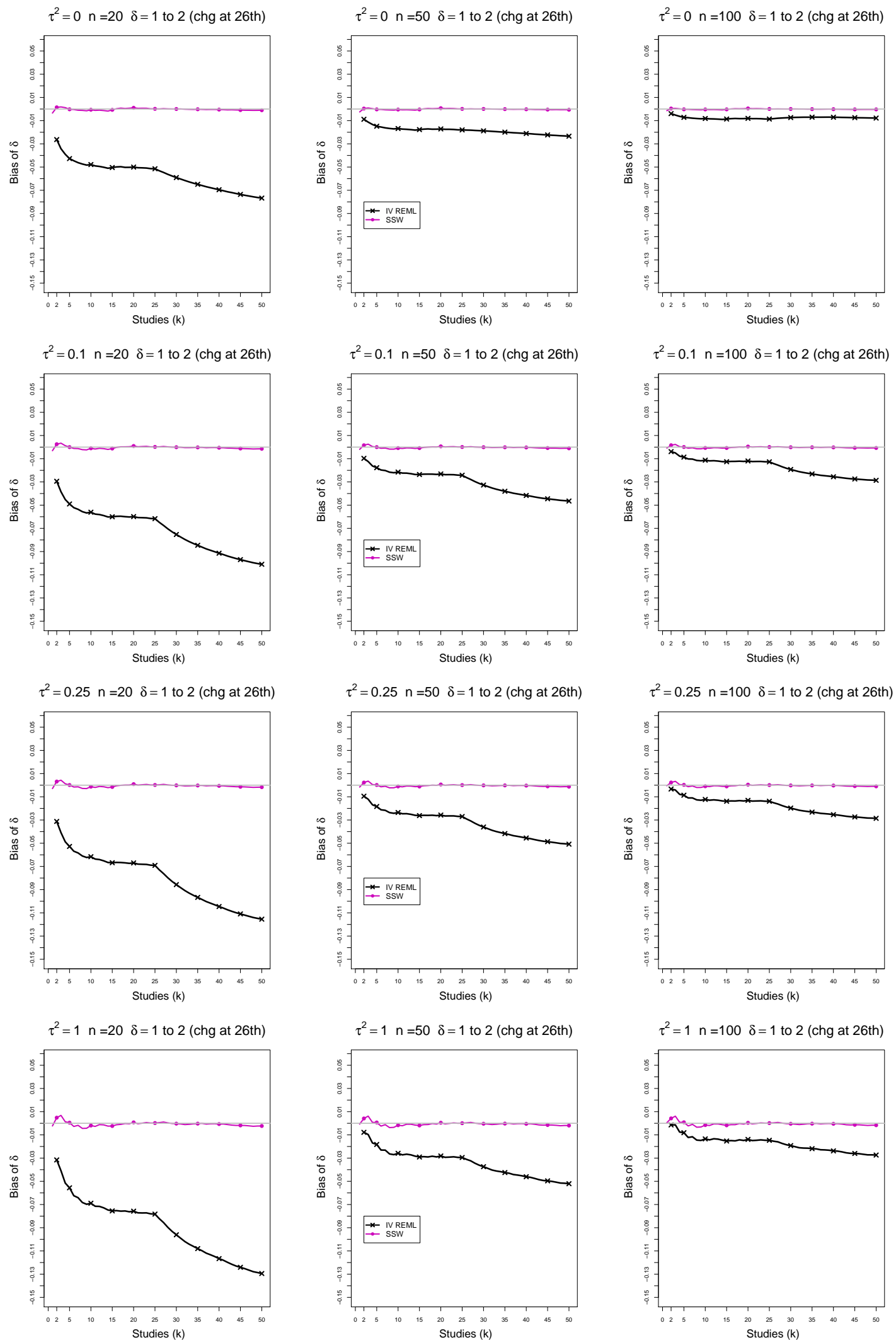

Figure D2. 3: 2-Stage CMA - Bias of IV REML and SSW estimators of $\delta$ when $\delta=1$ for $k \leq 25$ followed by a shift to $\delta=2$ for $k \geq 26, \tau^{2}=0,0.1,0.25,1, n=20,50,100, K=50$. Light grey line at 0 . 

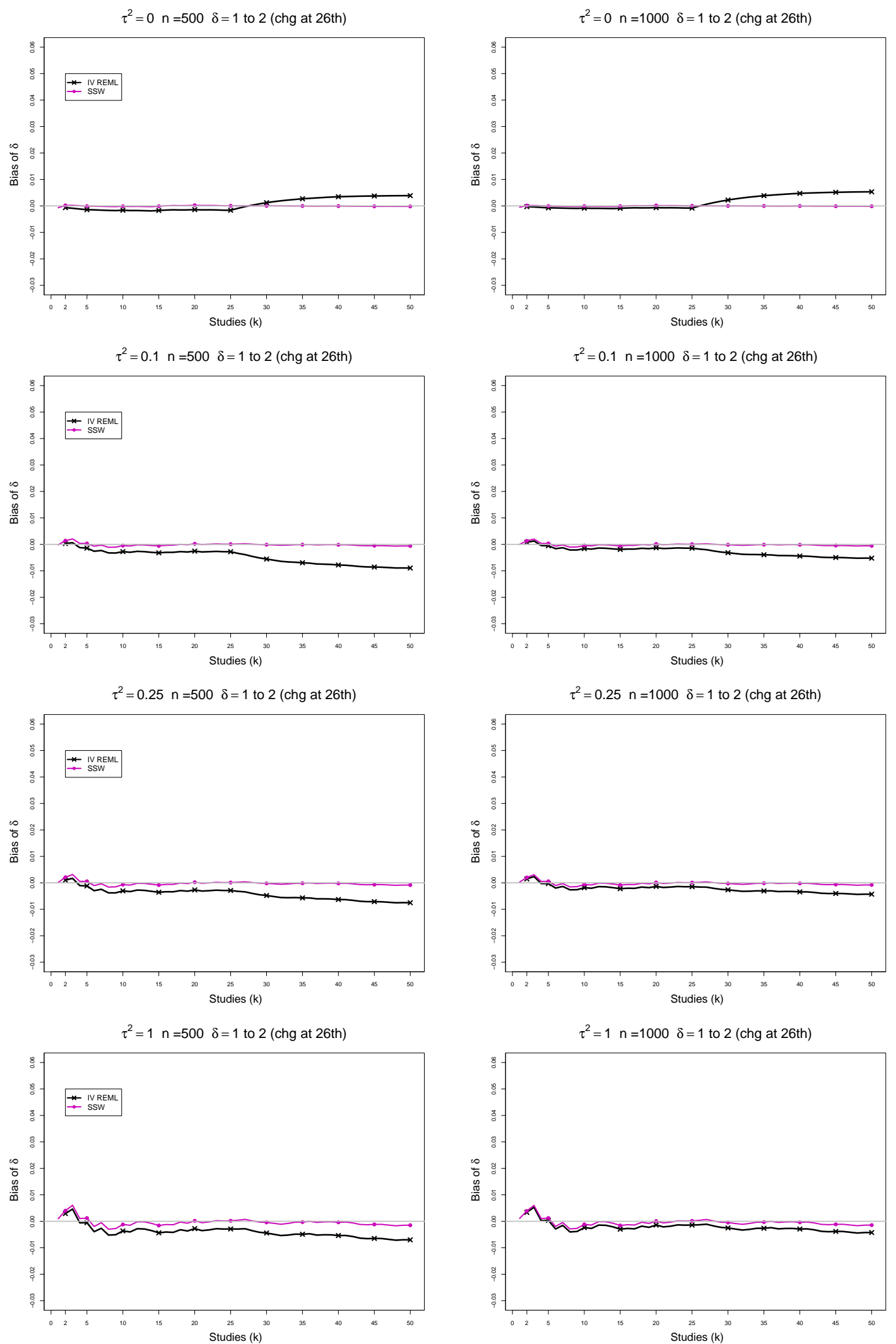

Figure D2. 4: 2-Stage CMA - Bias of IV REML and SSW estimators of $\delta$ when $\delta=1$ for $k \leq 25$ followed by a shift to $\delta=2$ for $k \geq 26, \tau^{2}=0,0.1,0.25,1, n=500,1000, K=50$. Light grey line at 0 . 

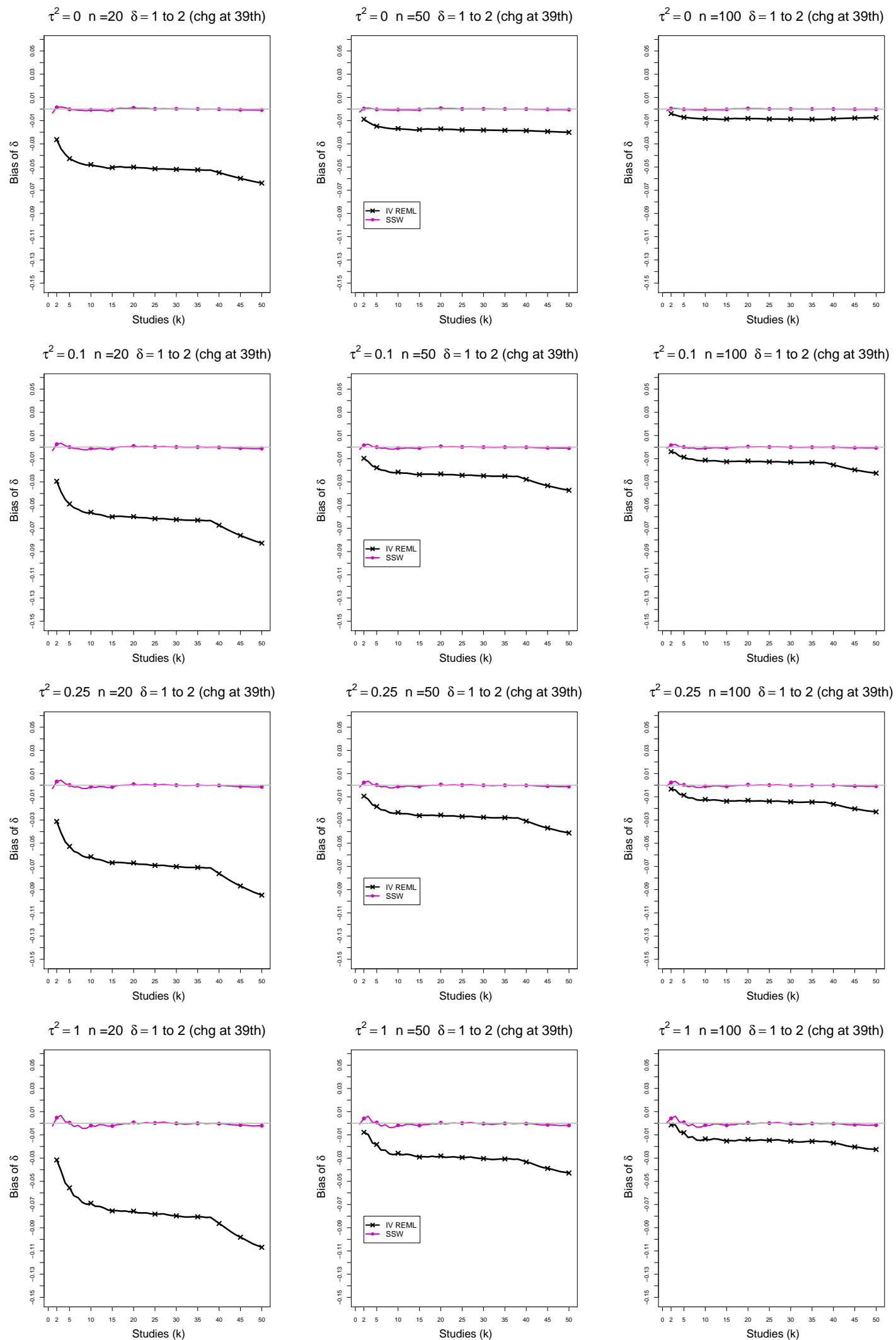

Figure D2. 5: 2-Stage CMA - Bias of IV REML and SSW estimators of $\delta$ when $\delta=1$ for $k \leq 38$ followed by a shift to $\delta=2$ for $k \geq 39, \tau^{2}=0,0.1,0.25,1, n=20,50,100, K=50$. Light grey line at 0 . 

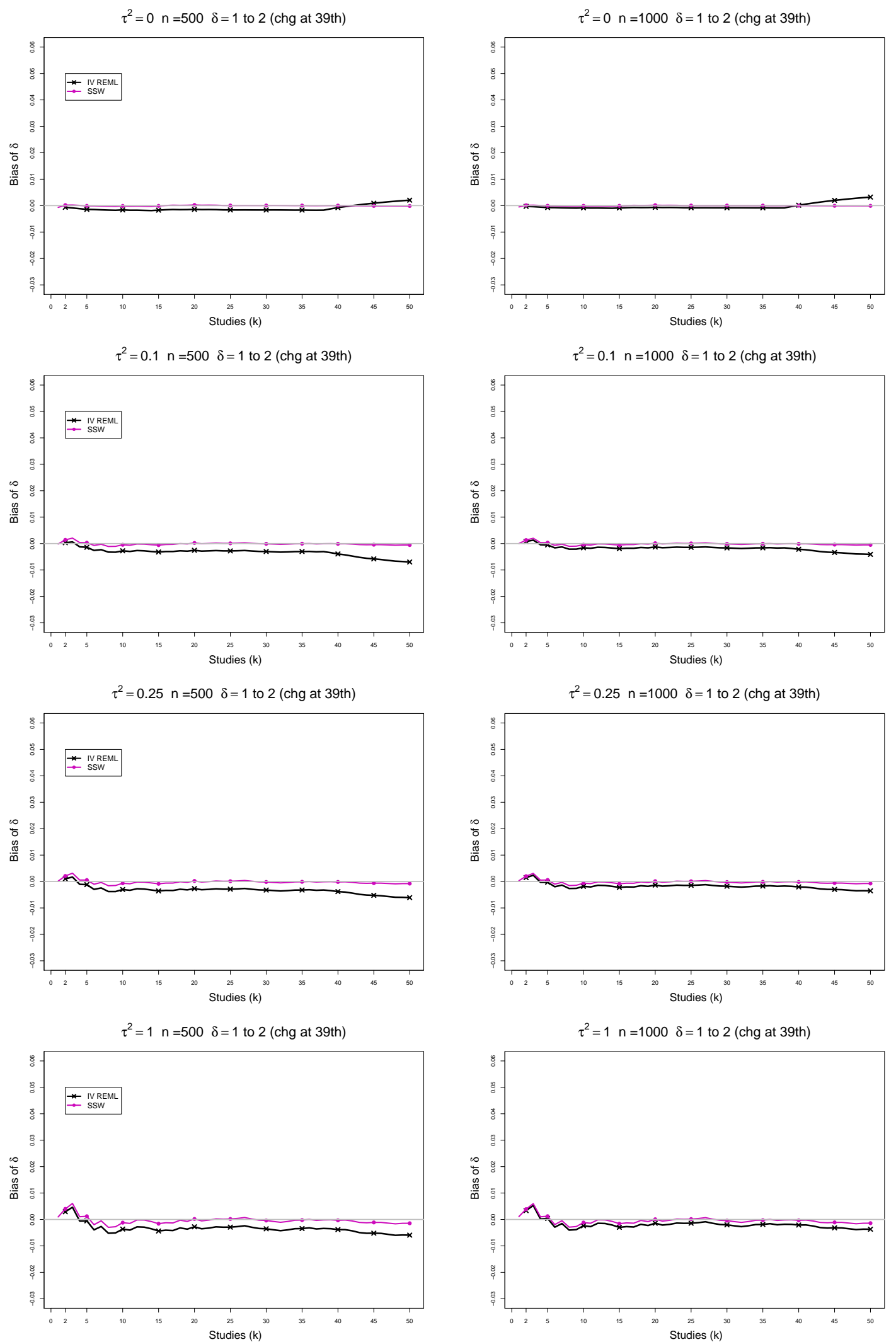

Figure D2. 6: 2-Stage CMA - Bias of IV REML and SSW estimators of $\delta$ when $\delta=1$ for $k \leq 38$ followed by a shift to $\delta=2$ for $k \geq 39, \tau^{2}=0,0.1,0.25,1, n=500,1000, K=50$. Light grey line at 0 . 


\section{D3. Coverage of interval estimators of $\delta$}

For coverage of $\delta$ in this section, each figure corresponds to a value of a set of values of $\tau^{2}(=0,0.1,0.25,1)$, a value of $n(=20,50,100,500,1000)$, a set of values of significance level $(=0.05,0.01,0.005)$ and a value of point of shift $f(=13,26,39)$ while $\delta$ shifts from 1 to 2 and maximum number of studies $K=50$.

Each figure contains a panel (with study $k$ on the horizontal axis) for each combination of parameters.

The interval estimators of $\delta$ are

- IV REML (Restricted maximum likelihood estimation of $\tau^{2}$, inverse variance weighted mean, normal critical values)

- SSW KDB (Effective sample size weighted (SSW) as center and half-width equal to critical value from $t_{k-1}$ times estimated standard deviation of SSW with $\hat{\tau}^{2}=\hat{\tau}_{K D B}^{2}$, the Kulinskaya-Dollinger-Bjørkest $\varnothing$ l estimator of $\tau^{2}$ ) 

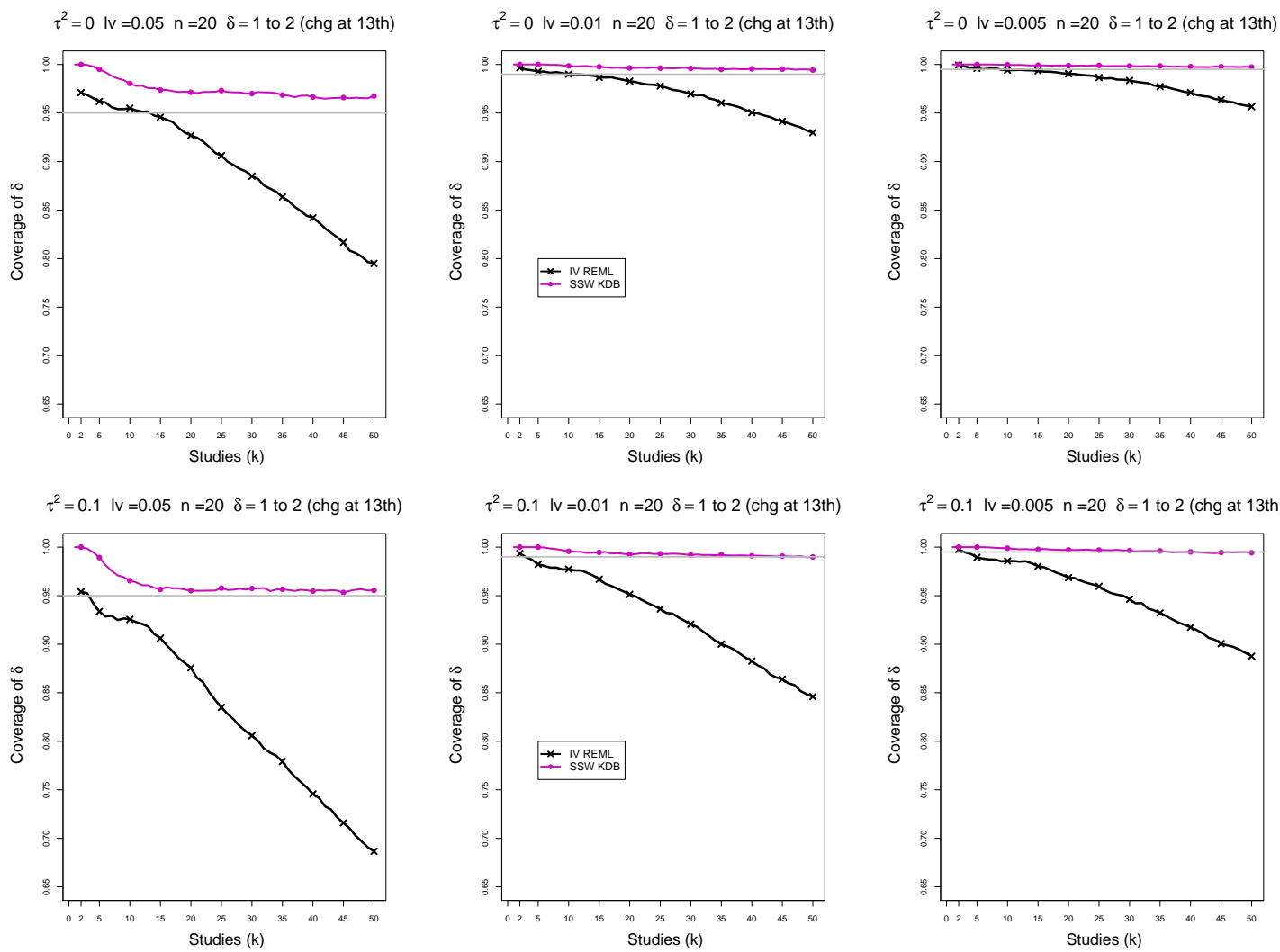

$\tau^{2}=0.1 \mathrm{lv}=0.005 \mathrm{n}=20 \delta=1$ to 2 (chg at 13 th)
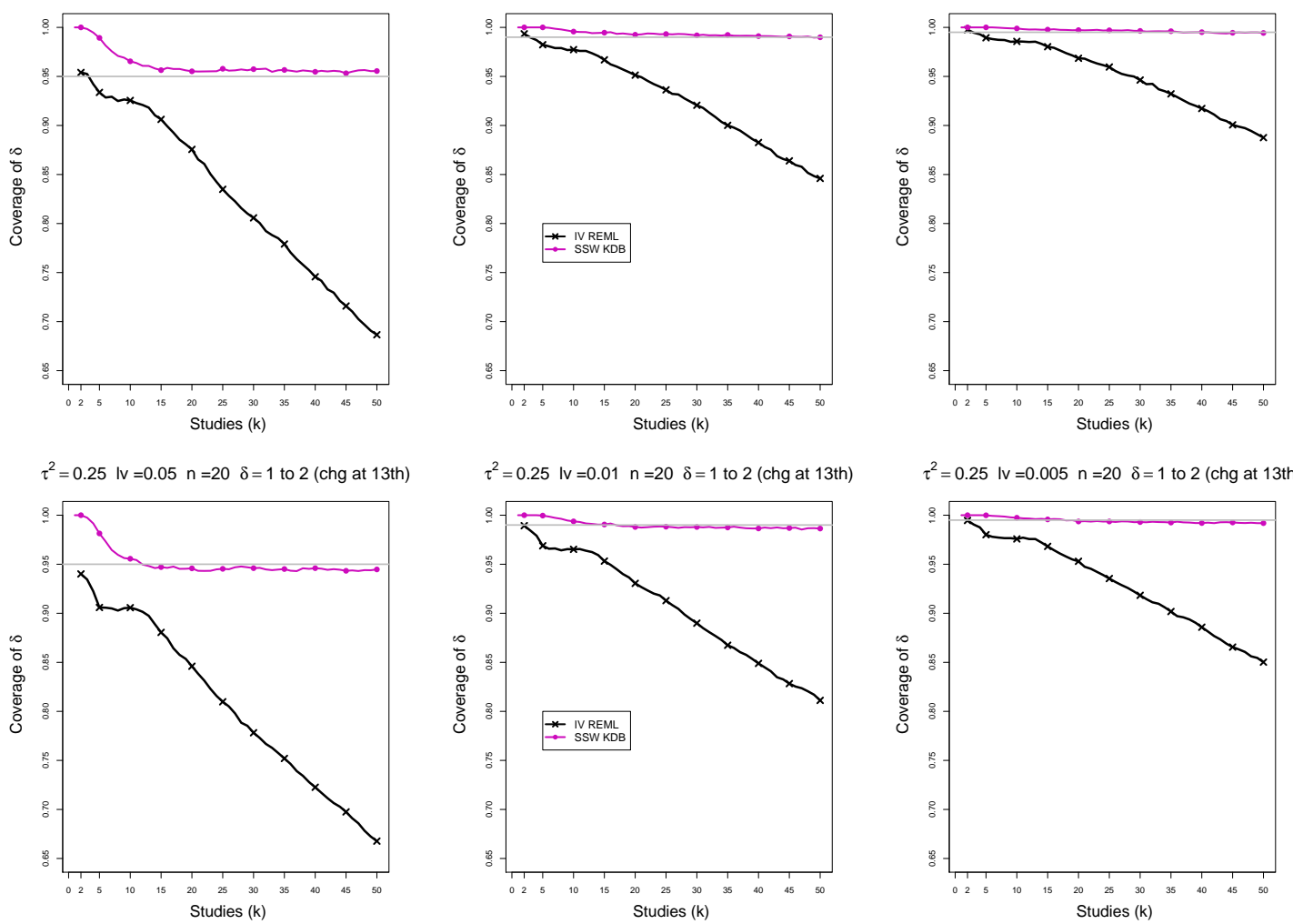

$\tau^{2}=0.25 \mathrm{lv}=0.005 \mathrm{n}=20 \quad \delta=1$ to 2 (chg at 13 th)

$\tau^{2}=1 \mathrm{lv}=0.05 \mathrm{n}=20 \quad \delta=1$ to 2 (chg at 13 th)
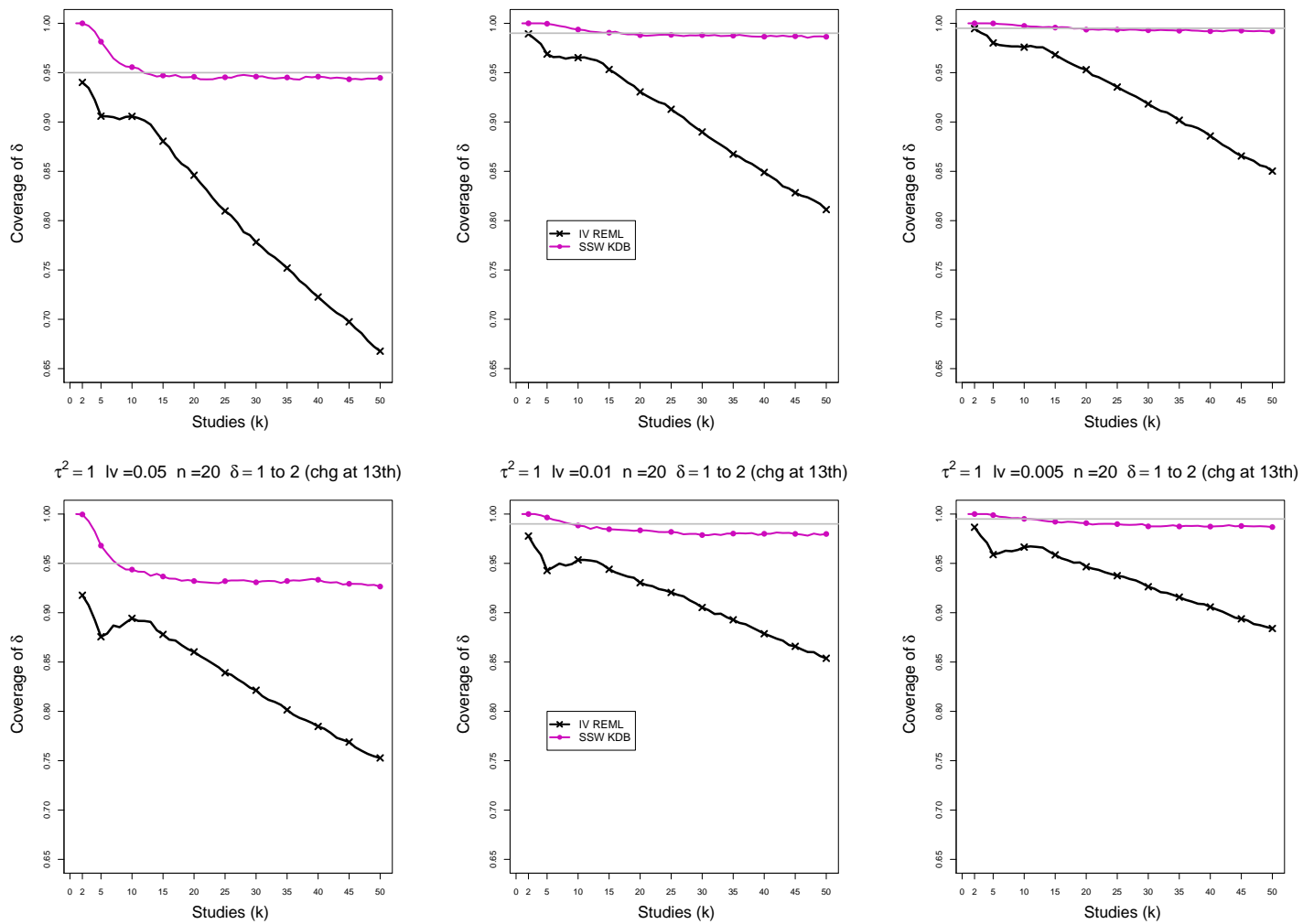

Figure D3. 1: 2-Stage CMA - Coverage of interval estimators of $\delta$ at confidence level of $1-\alpha$ for $\alpha=0.05,0.01,0.005$ when $\delta=1$ for $k \leq 12$ followed by a shift to $\delta=2$ for $k \geq 13, \tau^{2}=0,0.1,0.25,1, n=20, K=50$. Light grey line at $0.95,0.99,0.995$. 

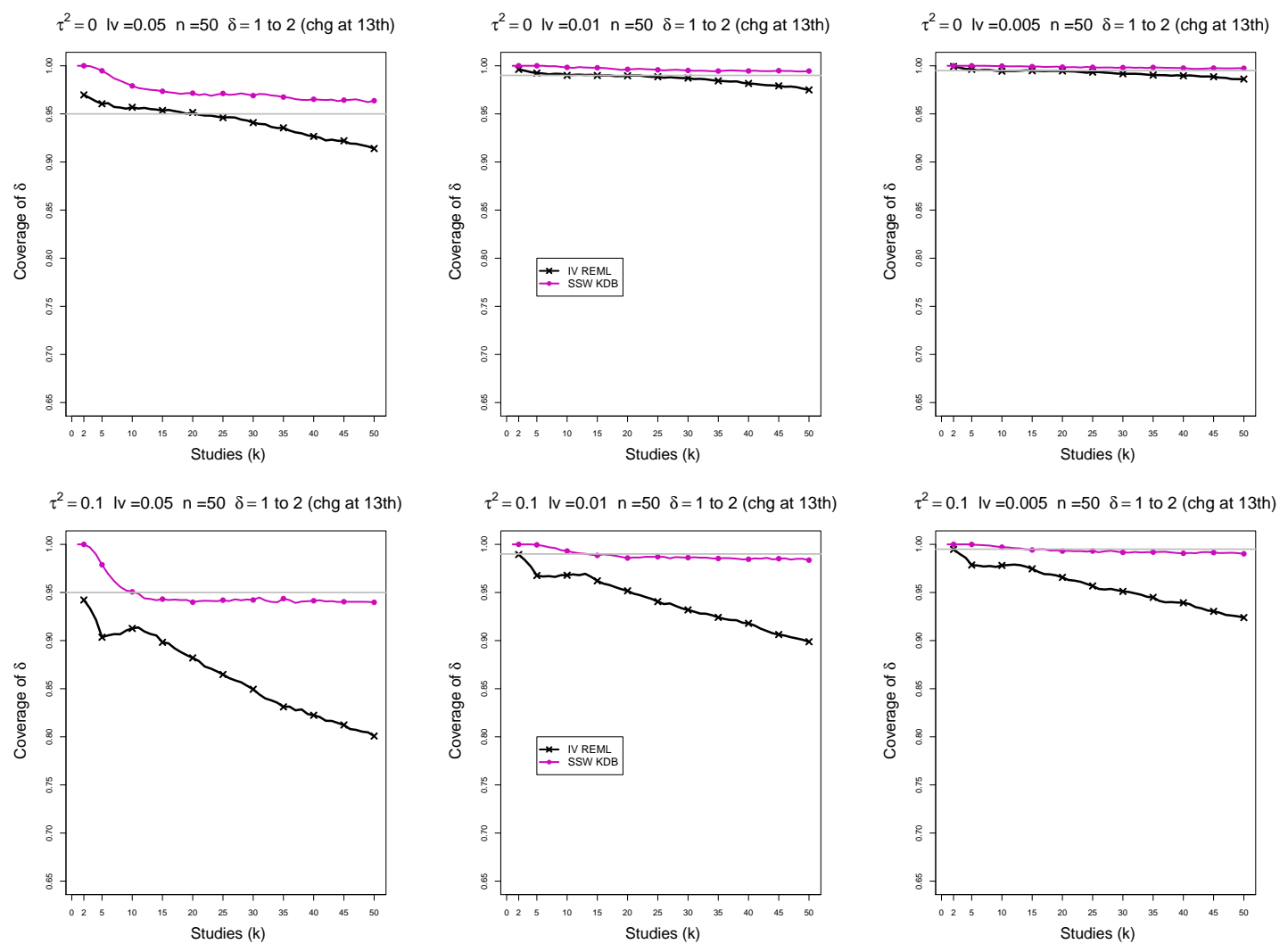

$\tau^{2}=0.25 \mathrm{lv}=0.05 \mathrm{n}=50 \quad \delta=1$ to 2 (chg at 13 th)
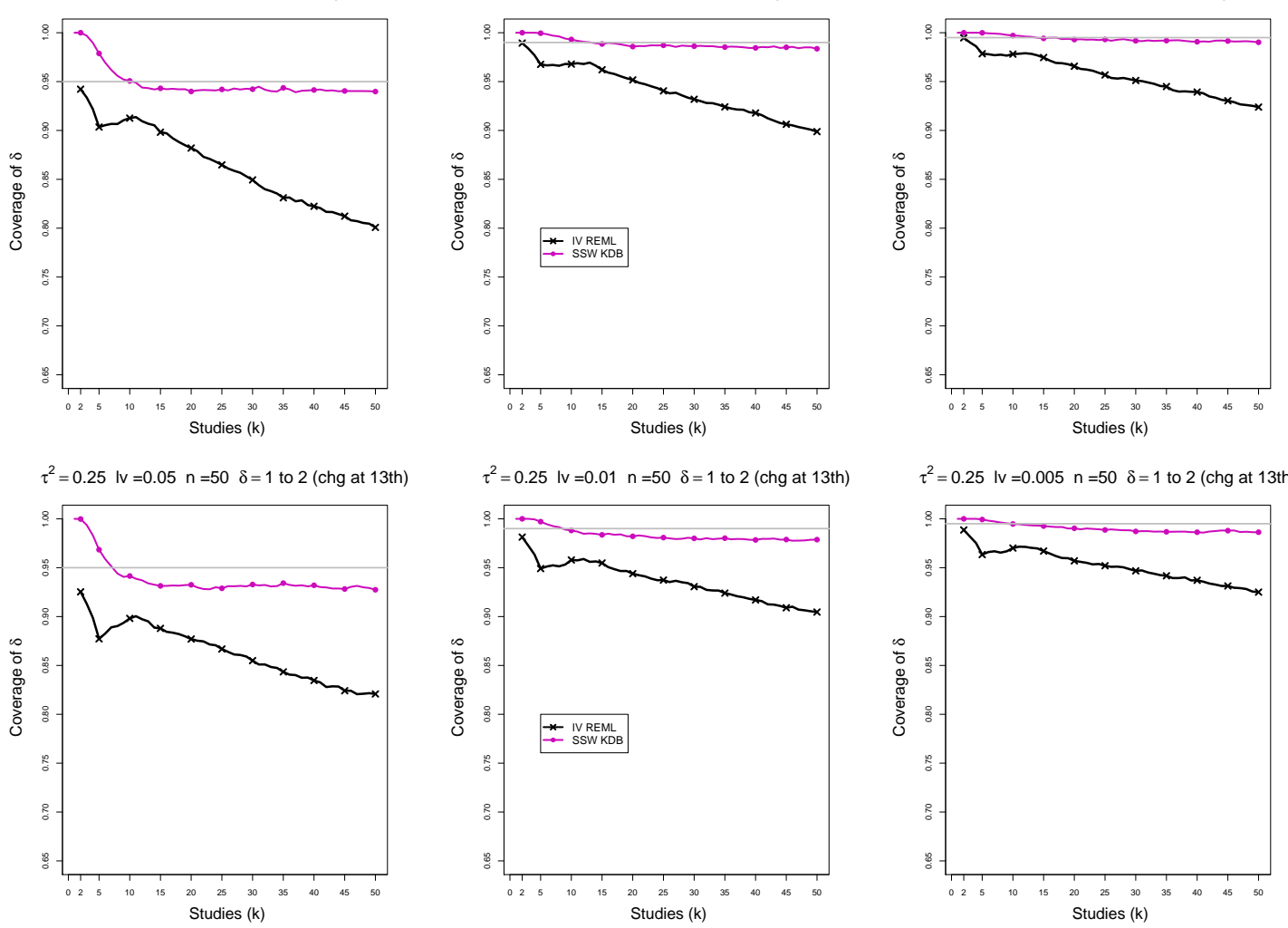

$\tau^{2}=0.25 \mathrm{lv}=0.005 \mathrm{n}=50 \quad \delta=1$ to 2 (chg at 13 th)

$\tau^{2}=1 \mathrm{lv}=0.05 \mathrm{n}=50 \quad \delta=1$ to 2 (chg at 13 th)
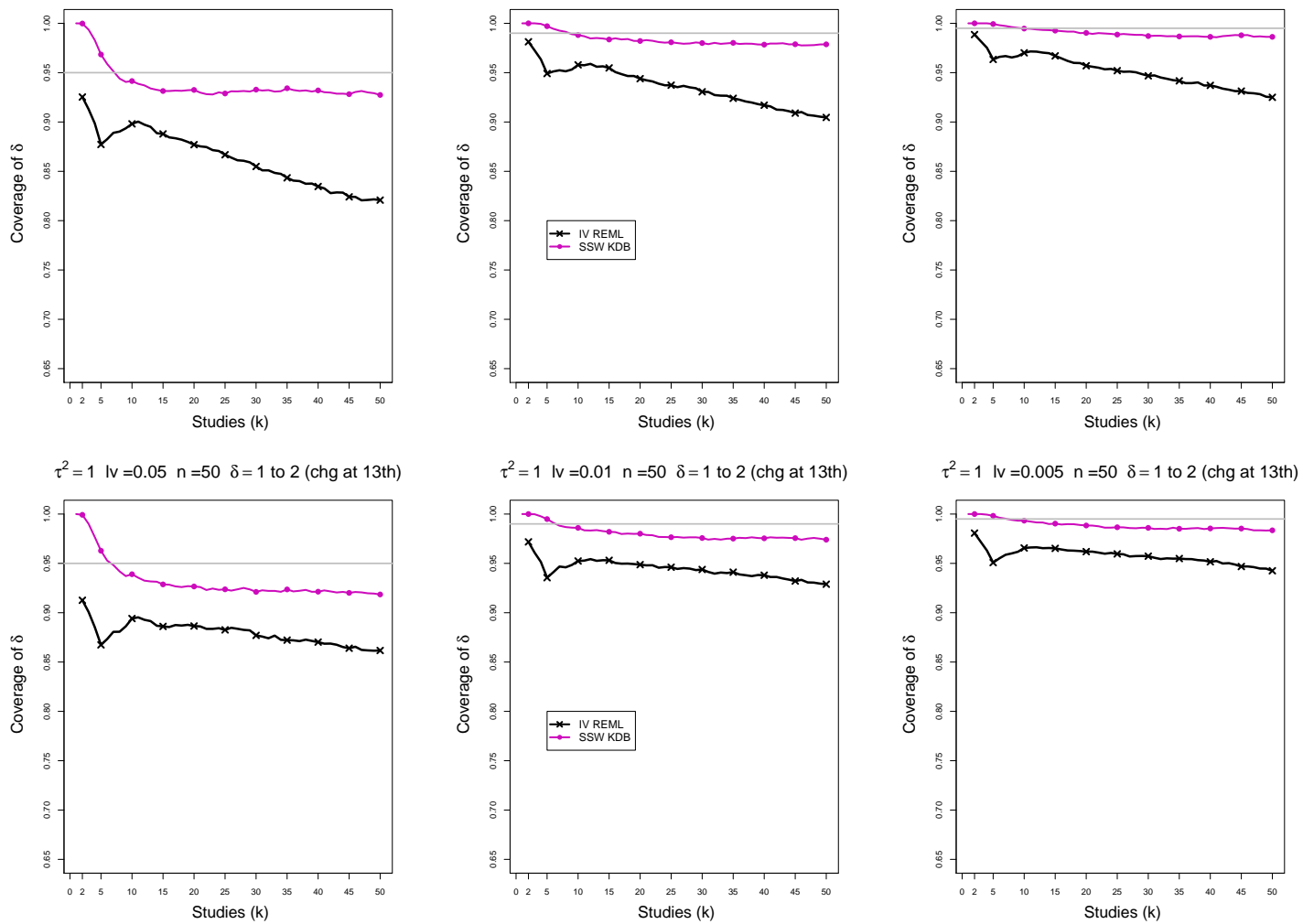

Figure D3. 2: 2-Stage CMA - Coverage of interval estimators of $\delta$ at confidence level of $1-\alpha$ for $\alpha=0.05,0.01,0.005$ when $\delta=1$ for $k \leq 12$ followed by a shift to $\delta=2$ for $k \geq 13, \tau^{2}=0,0.1,0.25,1, n=50, K=50$. Light grey line at $0.95,0.99,0.995$. 

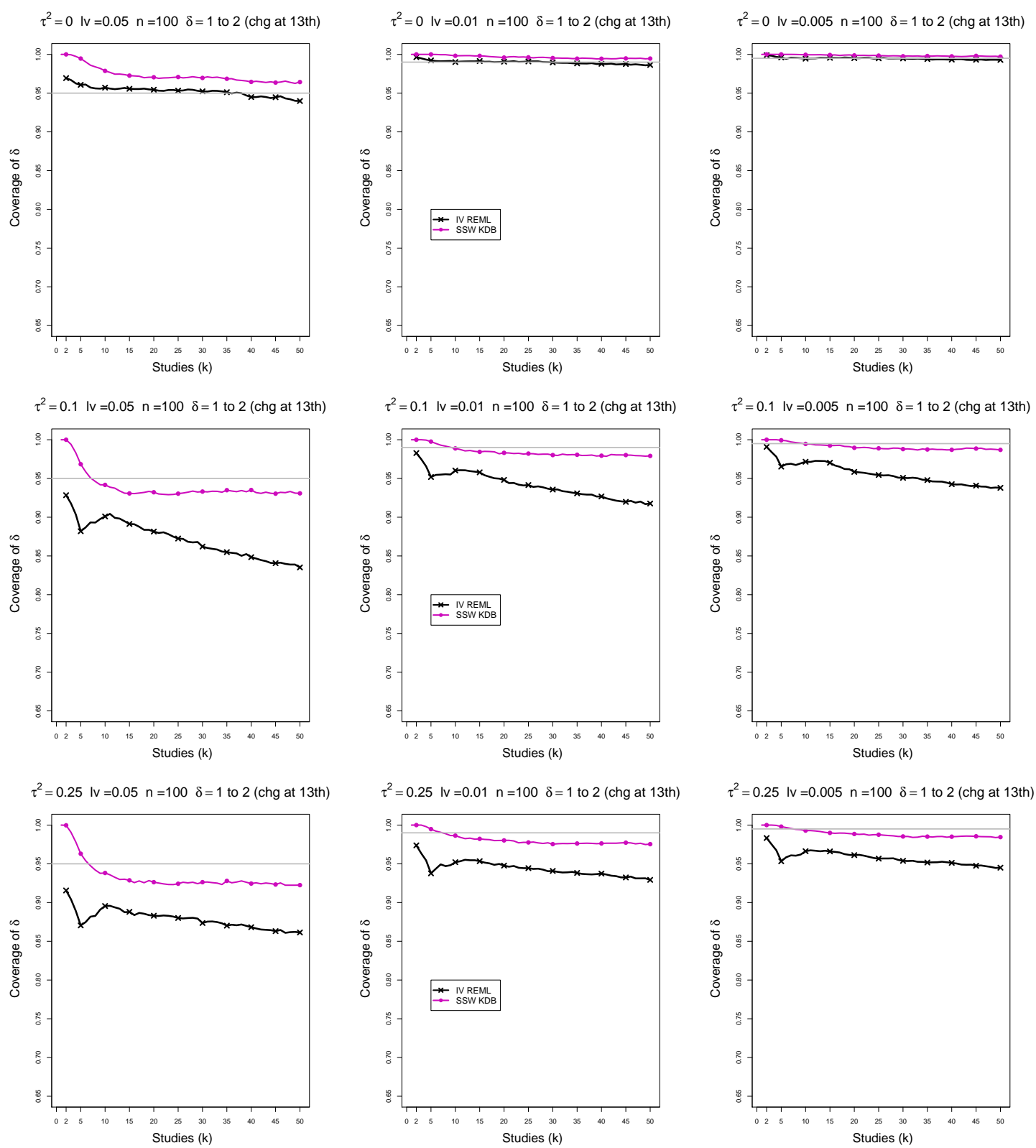

$\tau^{2}=1 \mathrm{lv}=0.05 \mathrm{n}=100 \quad \delta=1$ to 2 (chg at 13 th)

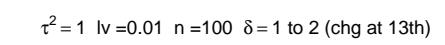

$\tau^{2}=0.25 \mathrm{lv}=0.005 \mathrm{n}=100 \quad \delta=1$ to 2 (chg at 13 th)
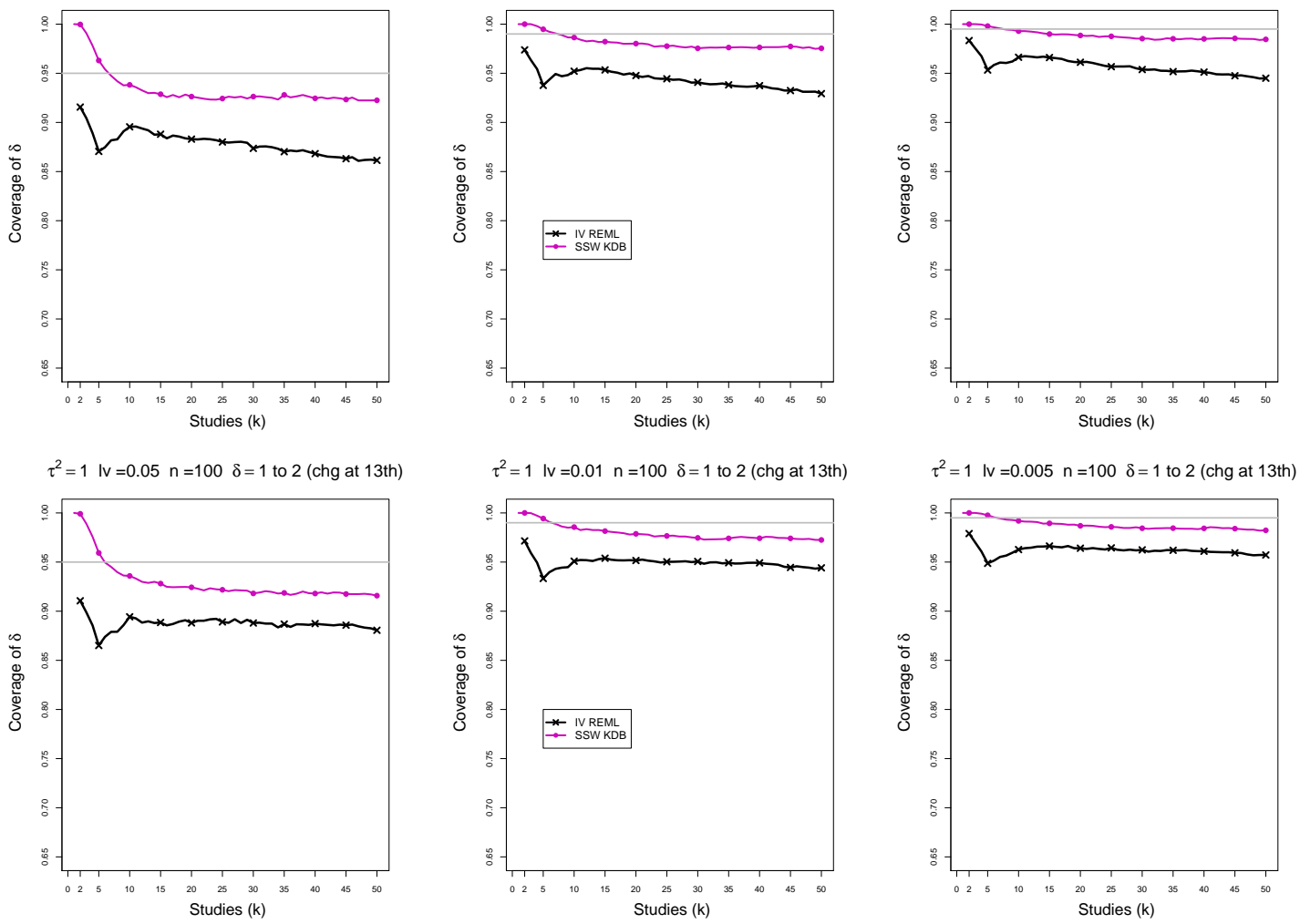

Figure D3. 3: 2-Stage CMA - Coverage of interval estimators of $\delta$ at confidence level of $1-\alpha$ for $\alpha=0.05,0.01,0.005$ when $\delta=1$ for $k \leq 12$ followed by a shift to $\delta=2$ for $k \geq 13, \tau^{2}=0,0.1,0.25,1, n=100, K=50$. Light grey line at $0.95,0.99,0.995$. 

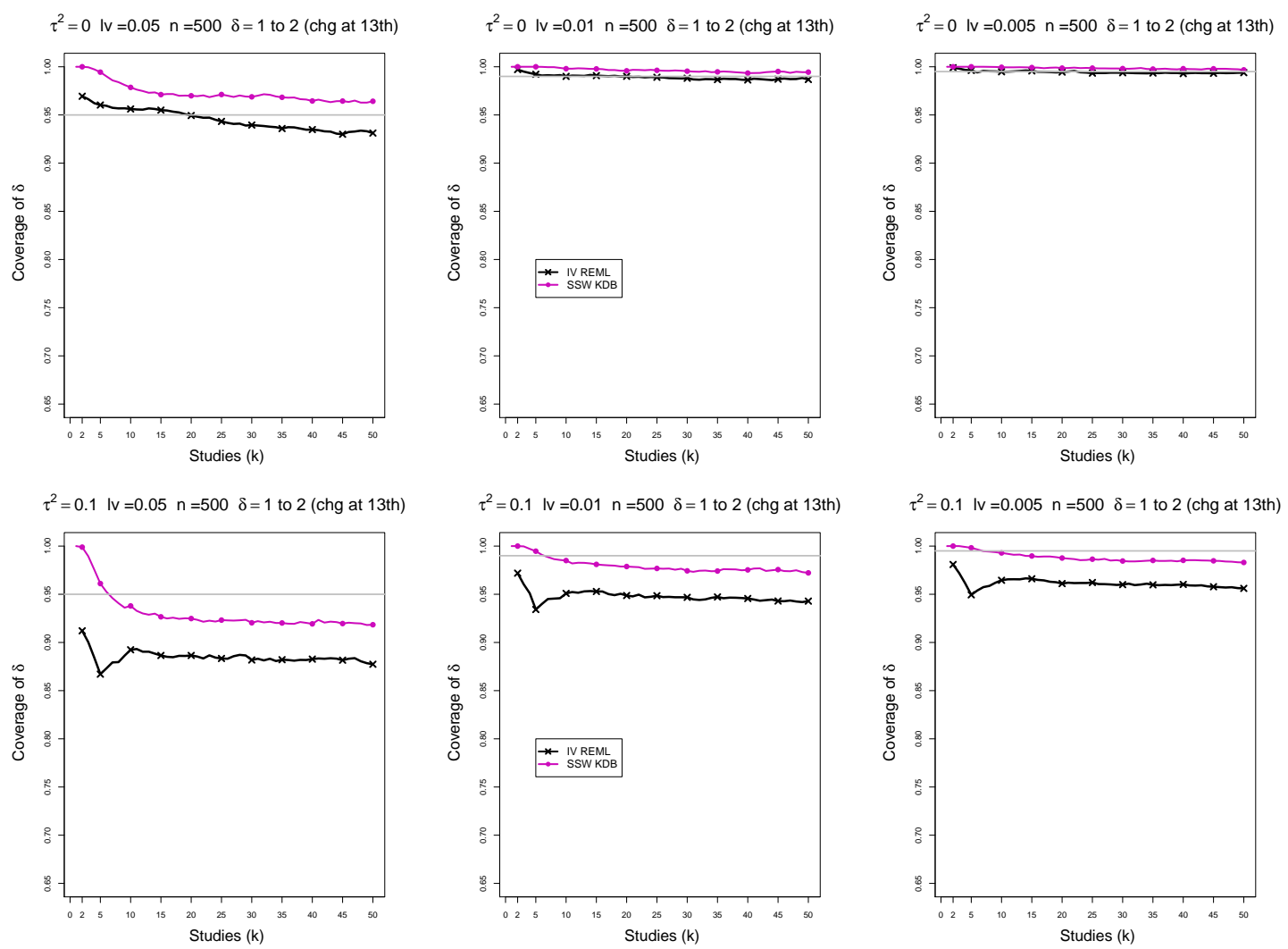

$\tau^{2}=0.25 \mathrm{lv}=0.05 \mathrm{n}=500 \quad \delta=1$ to 2 (chg at 13 th)
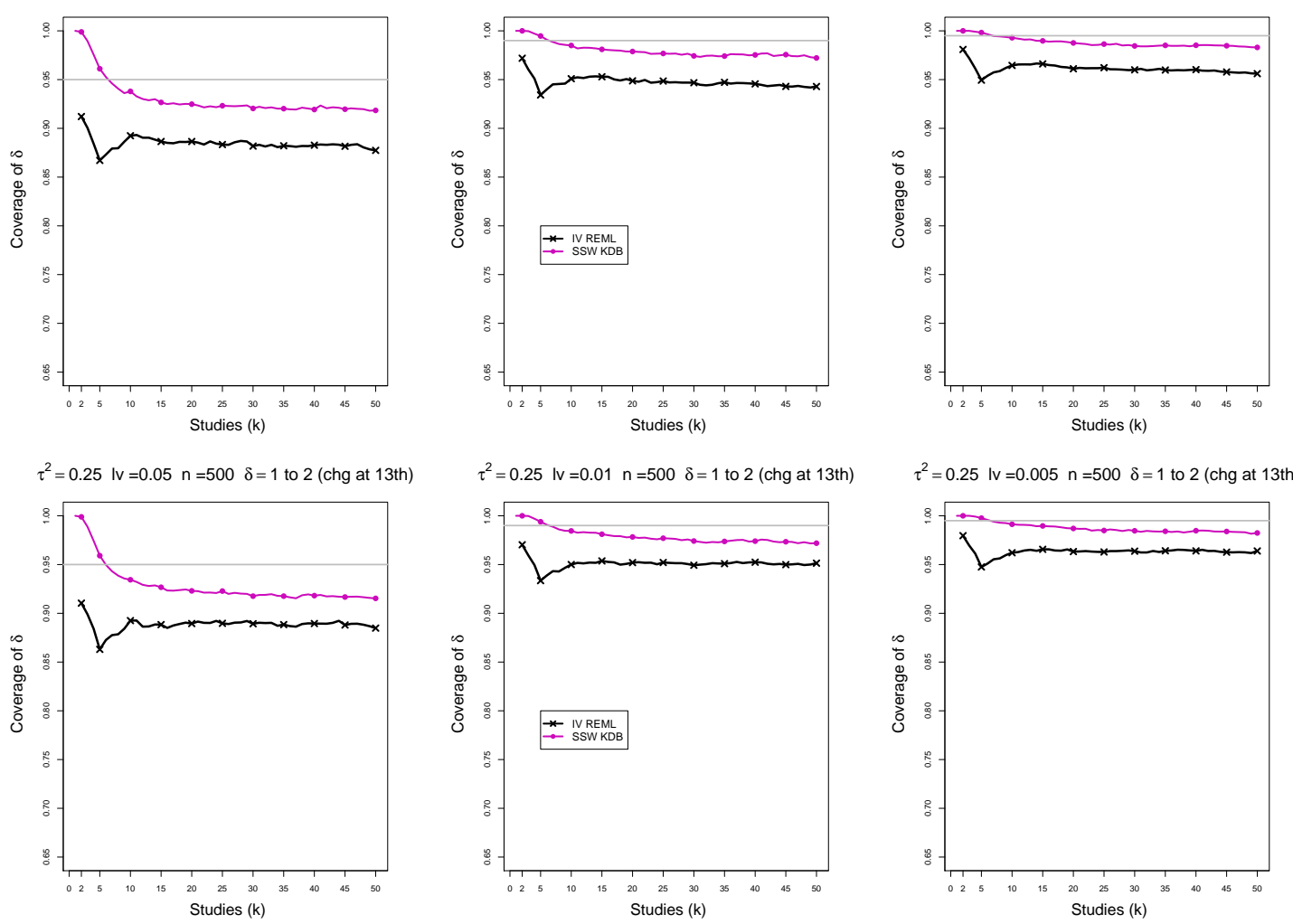

$\tau^{2}=0.25 \mathrm{lv}=0.005 \mathrm{n}=500 \delta=1$ to 2 (chg at 13 th)

$\tau^{2}=1 \mathrm{lv}=0.05 \mathrm{n}=500 \quad \delta=1$ to 2 (chg at 13 th)
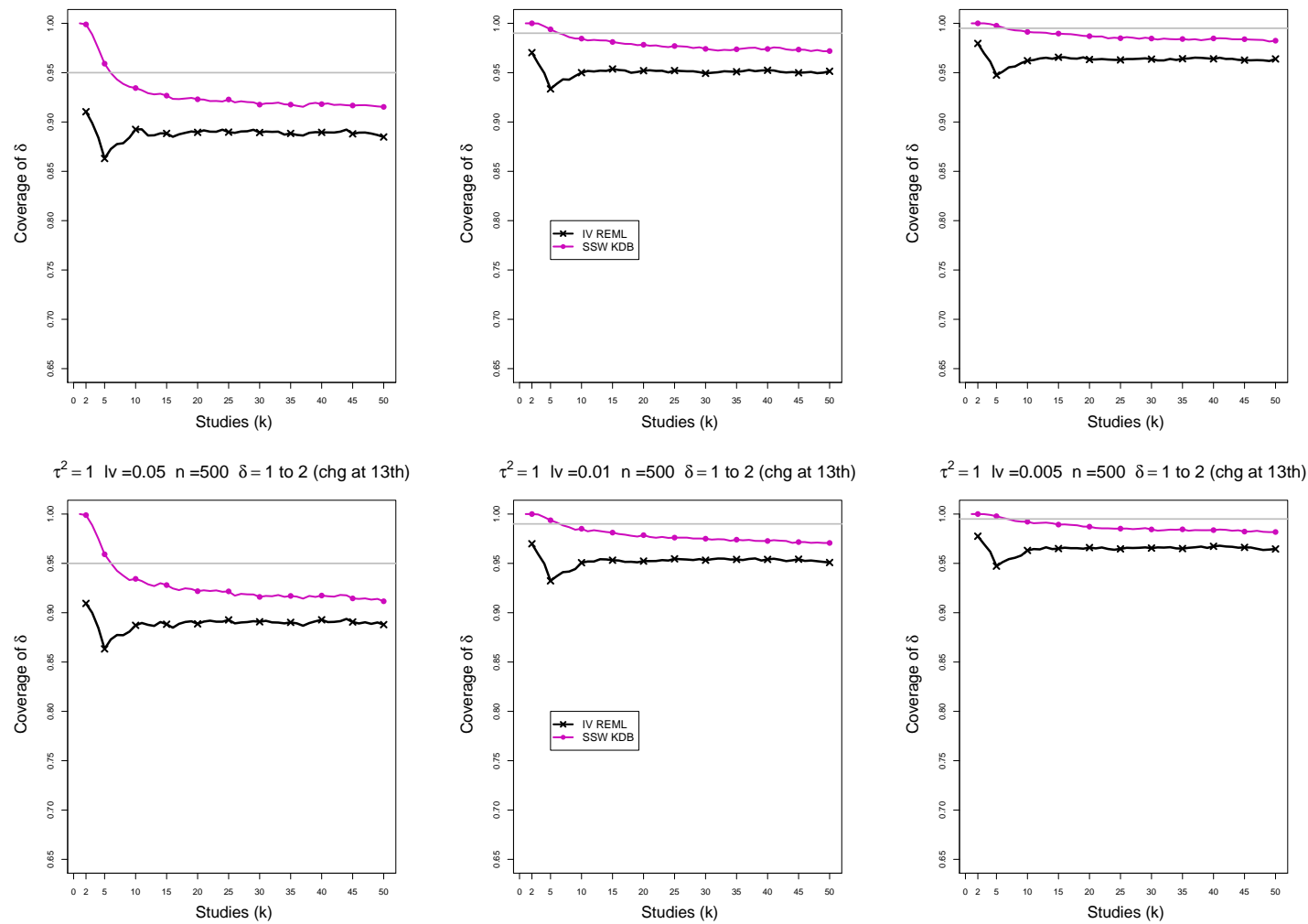

Figure D3. 4: 2-Stage CMA - Coverage of interval estimators of $\delta$ at confidence level of $1-\alpha$ for $\alpha=0.05,0.01,0.005$ when $\delta=1$ for $k \leq 12$ followed by a shift to $\delta=2$ for $k \geq 13, \tau^{2}=0,0.1,0.25,1, n=500, K=50$. Light grey line at 0.95,0.99,0.995 

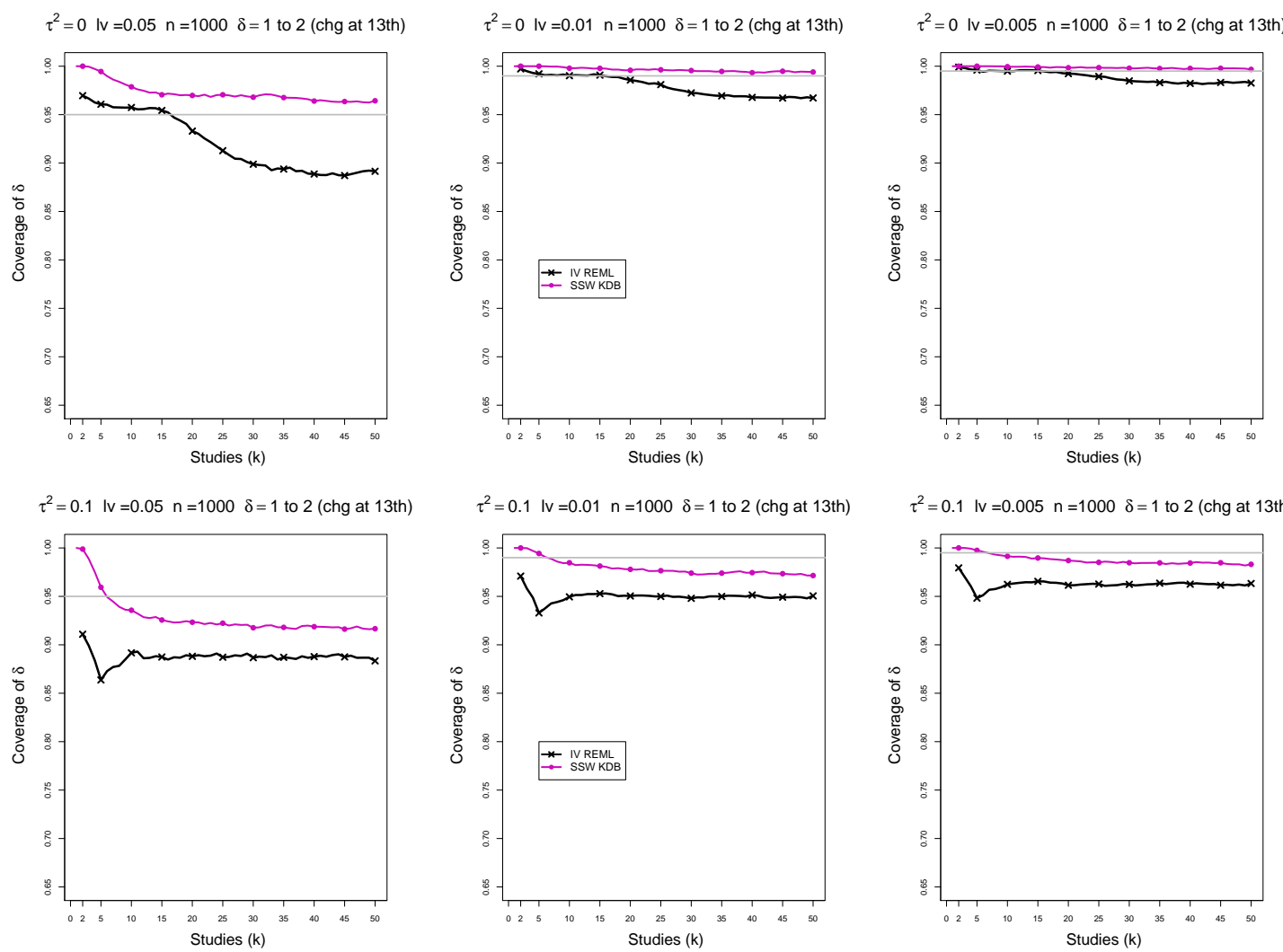

$\tau^{2}=0.1 \mathrm{lv}=0.005 \mathrm{n}=1000 \delta=1$ to 2 (chg at 13 th)

$\tau^{2}=0.25 \mathrm{lv}=0.05 \mathrm{n}=1000 \delta=1$ to 2 (chg at 13 th)
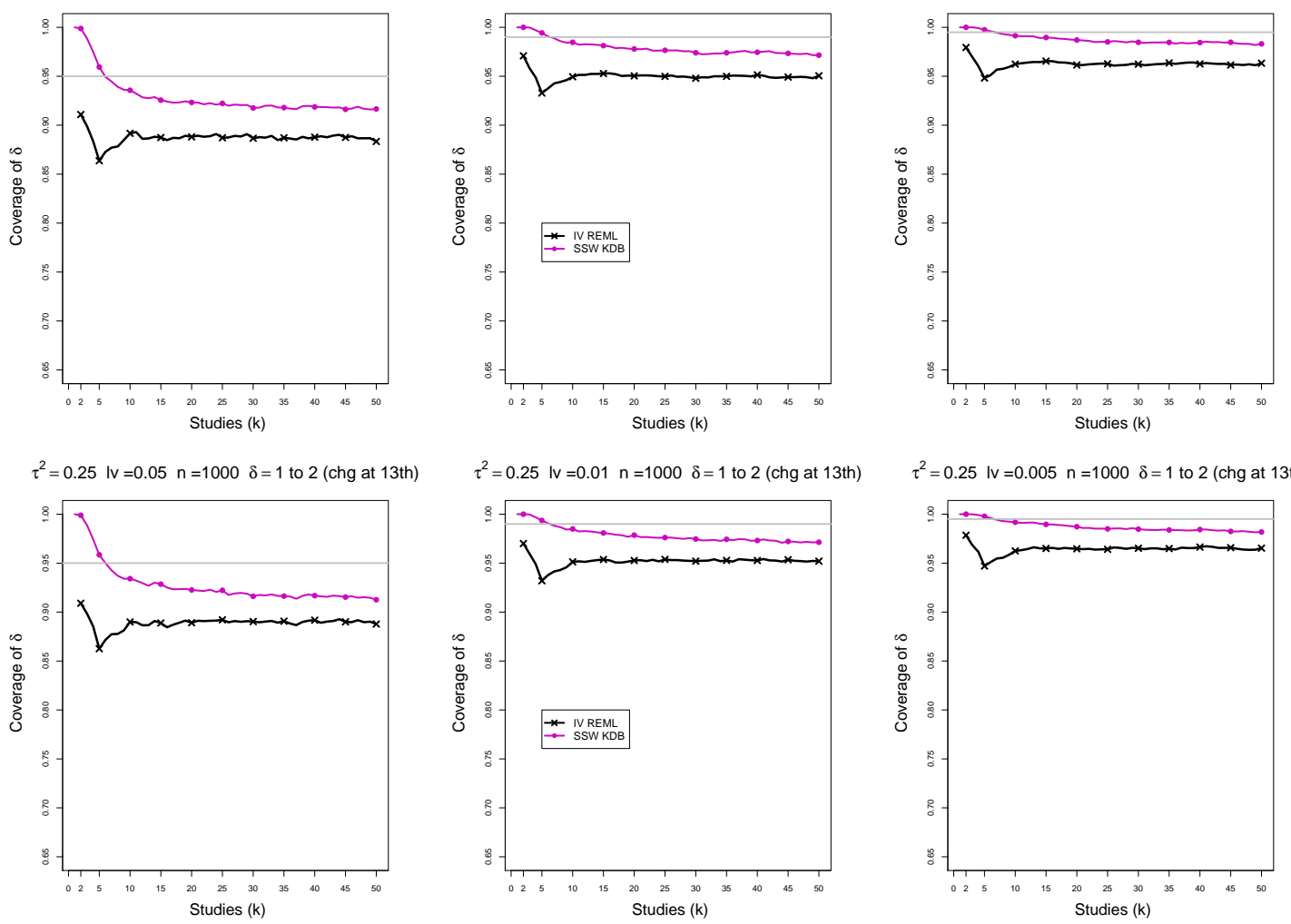

$\tau^{2}=0.25 \mathrm{lv}=0.005 \mathrm{n}=1000 \delta=1$ to 2 (chg at 13 th)

$\tau^{2}=1 \mathrm{lv}=0.05 \mathrm{n}=1000 \delta=1$ to 2 (chg at 13 th)
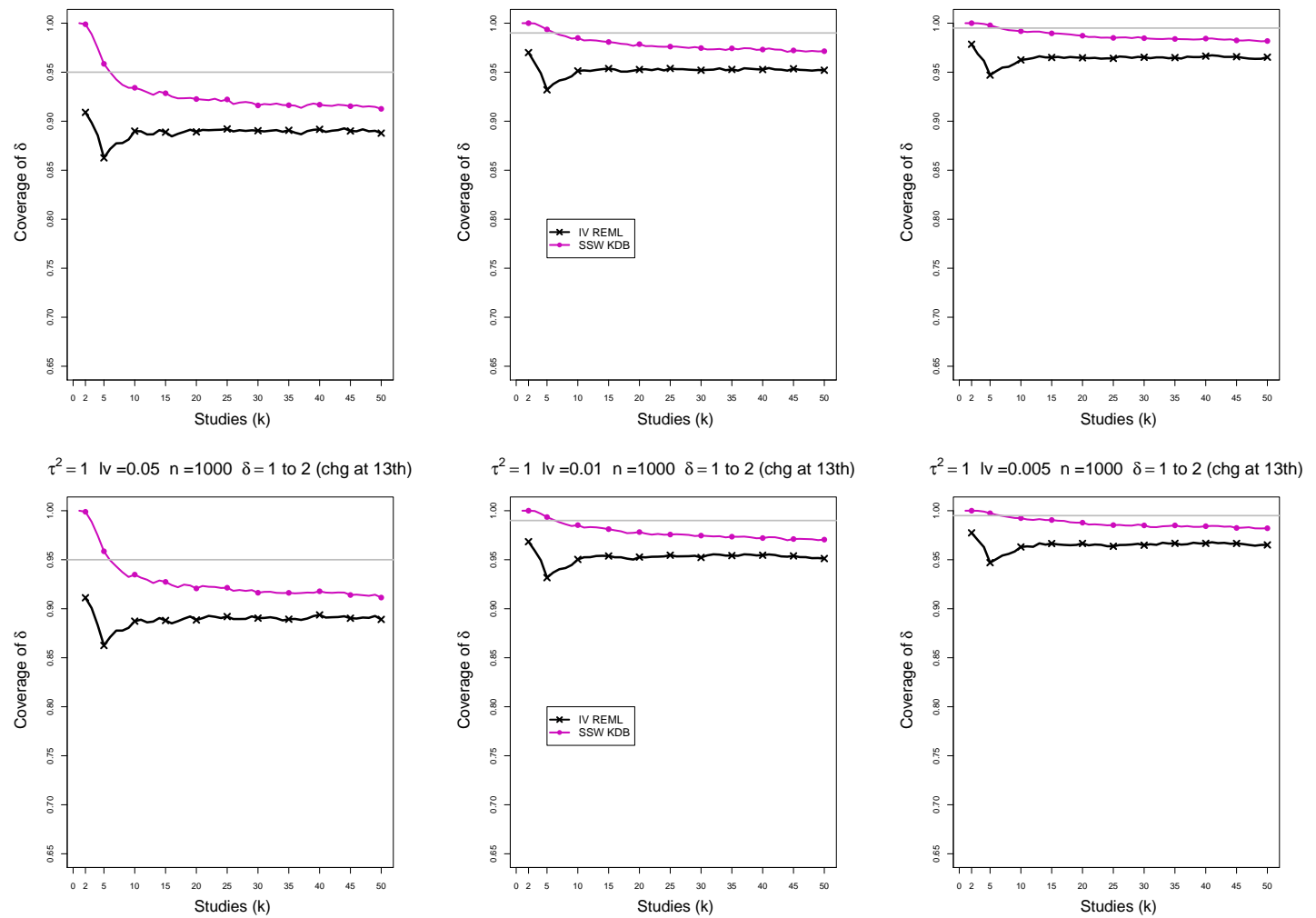

Figure D3. 5: 2-Stage CMA - Coverage of interval estimators of $\delta$ at confidence level of $1-\alpha$ for $\alpha=0.05,0.01,0.005$ when $\delta=1$ for $k \leq 12$ followed by a shift to $\delta=2$ for $k \geq 13, \tau^{2}=0,0.1,0.25,1, n=1000, K=50$. Light grey line at 0.95,0.99,0.995. 

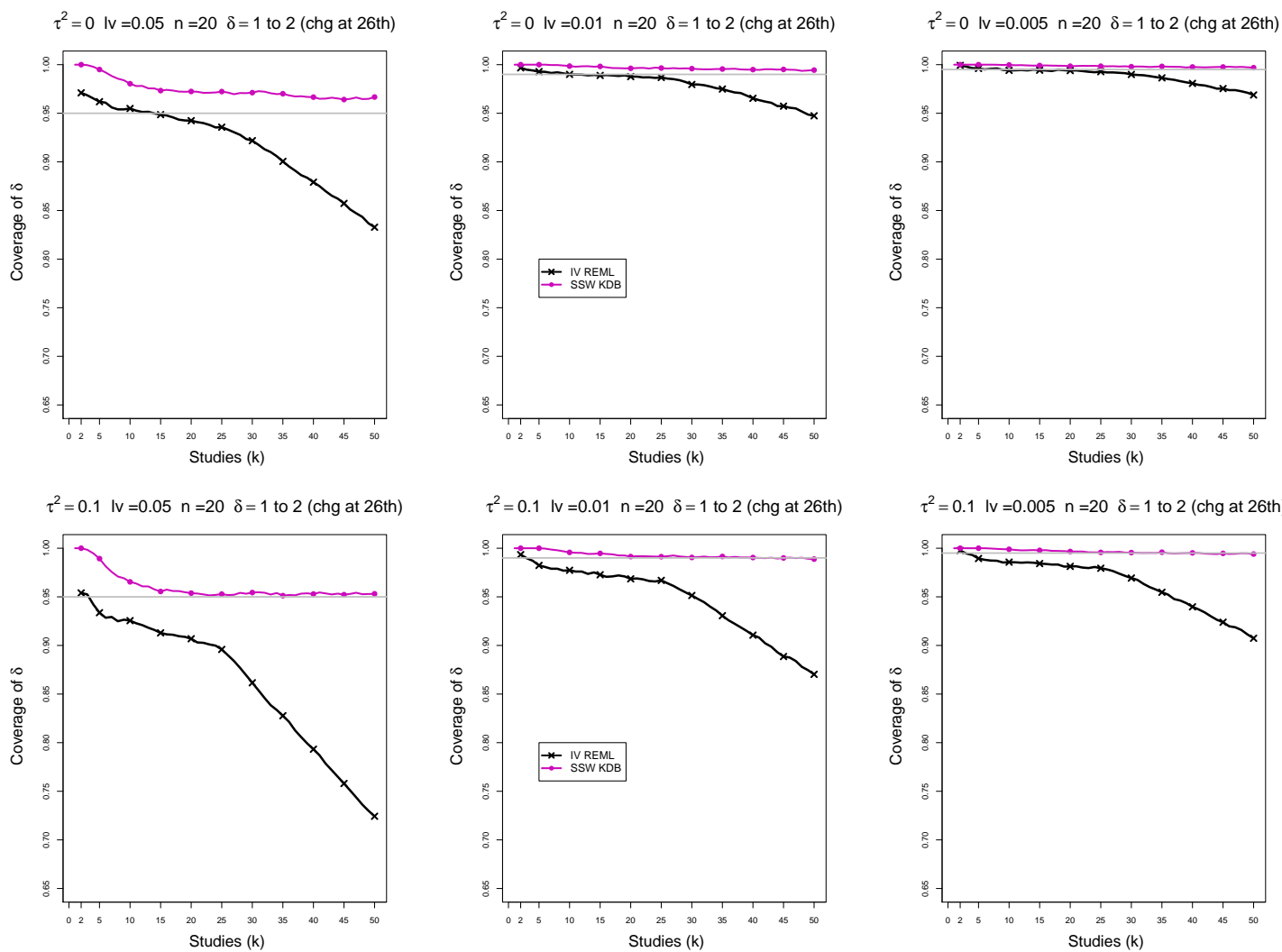

$\tau^{2}=0.1 \mathrm{lv}=0.005 \mathrm{n}=20 \delta=1$ to 2 (chg at 26 th)

$\tau^{2}=0.25 \mathrm{lv}=0.05 \mathrm{n}=20 \quad \delta=1$ to 2 (chg at $26 \mathrm{th}$ )
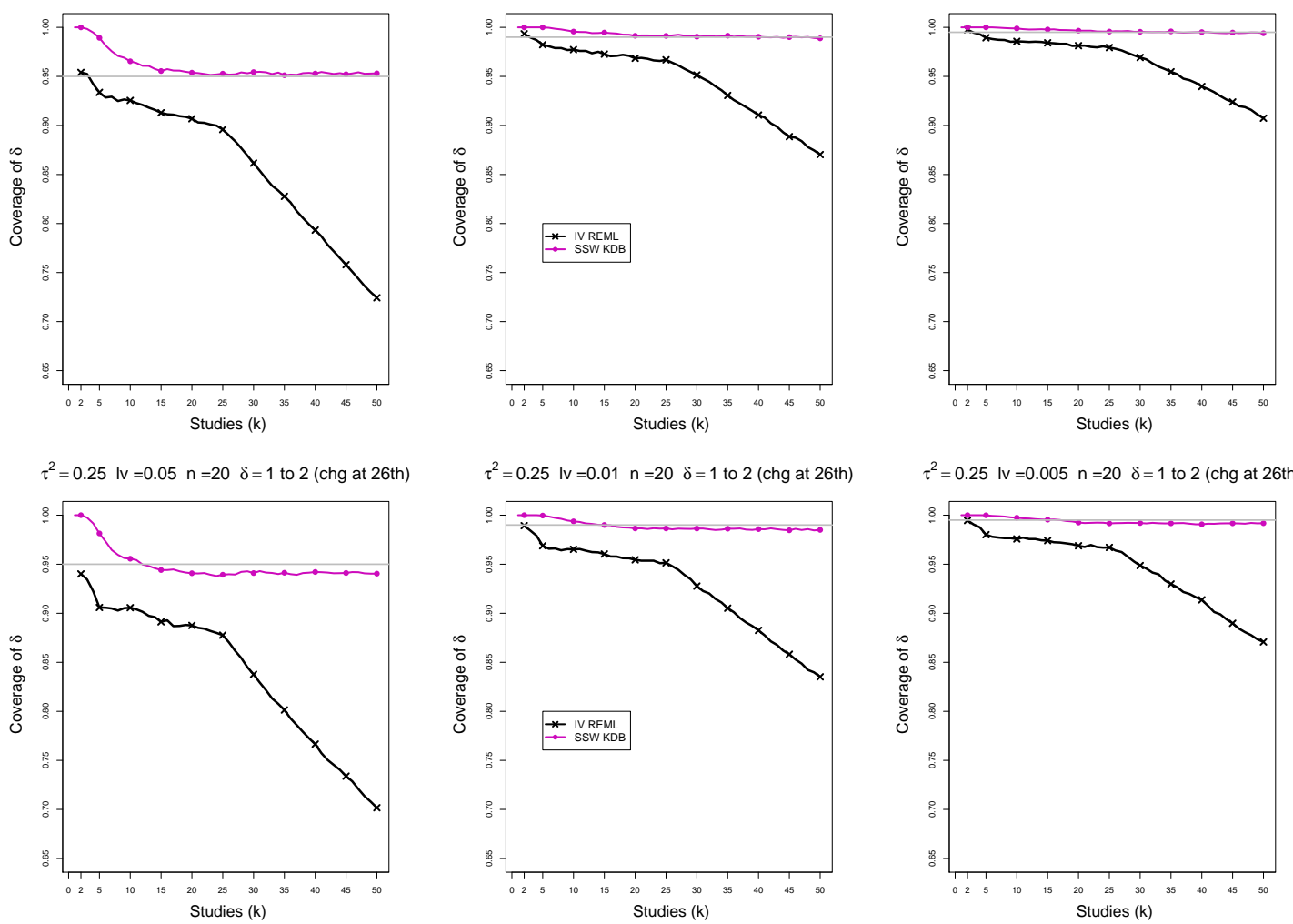

$\tau^{2}=0.25 \mathrm{lv}=0.005 \mathrm{n}=20 \quad \delta=1$ to 2 (chg at 26 th)

$\tau^{2}=1 \mathrm{lv}=0.05 \mathrm{n}=20 \quad \delta=1$ to 2 (chg at 26th)
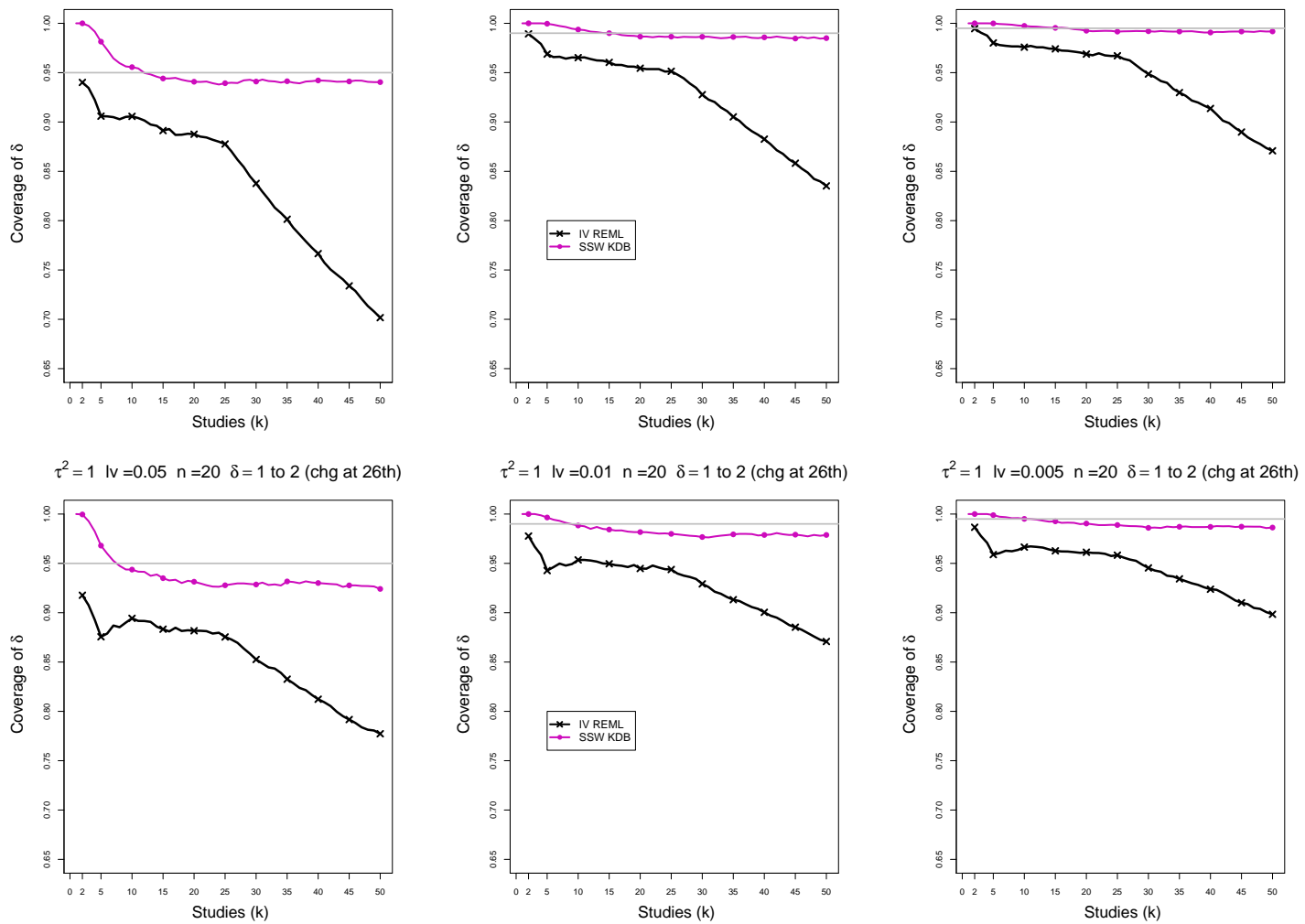

Figure D3. 6: 2-Stage CMA - Coverage of interval estimators of $\delta$ at confidence level of $1-\alpha$ for $\alpha=0.05,0.01,0.005$ when $\delta=1$ for $k \leq 25$ followed by a shift to $\delta=2$ for $k \geq 26, \tau^{2}=0,0.1,0.25,1, n=20, K=50$. Light grey line at $0.95,0.99,0.995$. 

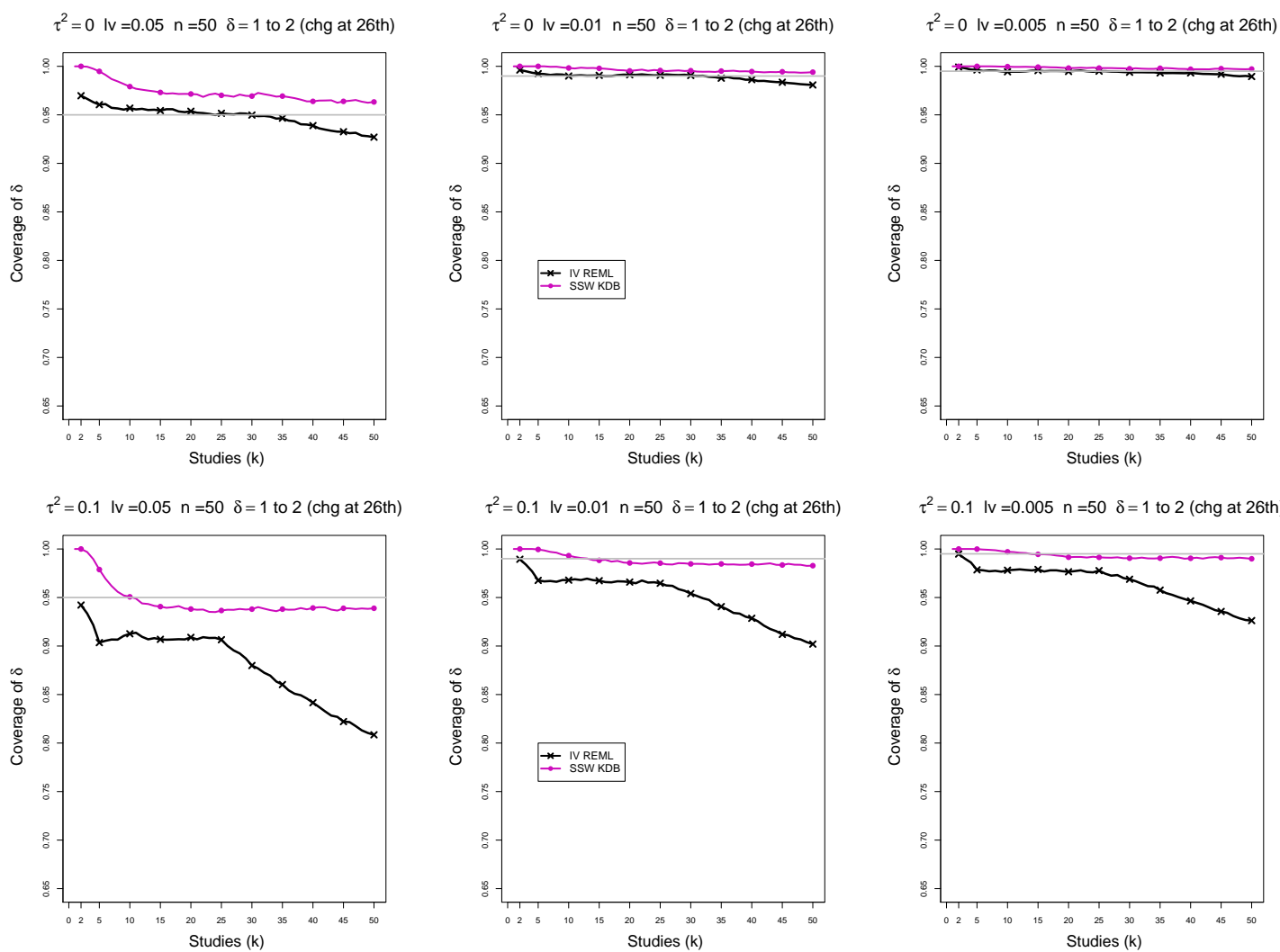

$\tau^{2}=0.1 \mathrm{lv}=0.005 \mathrm{n}=50 \quad \delta=1$ to 2 (chg at $26 \mathrm{th}$ )
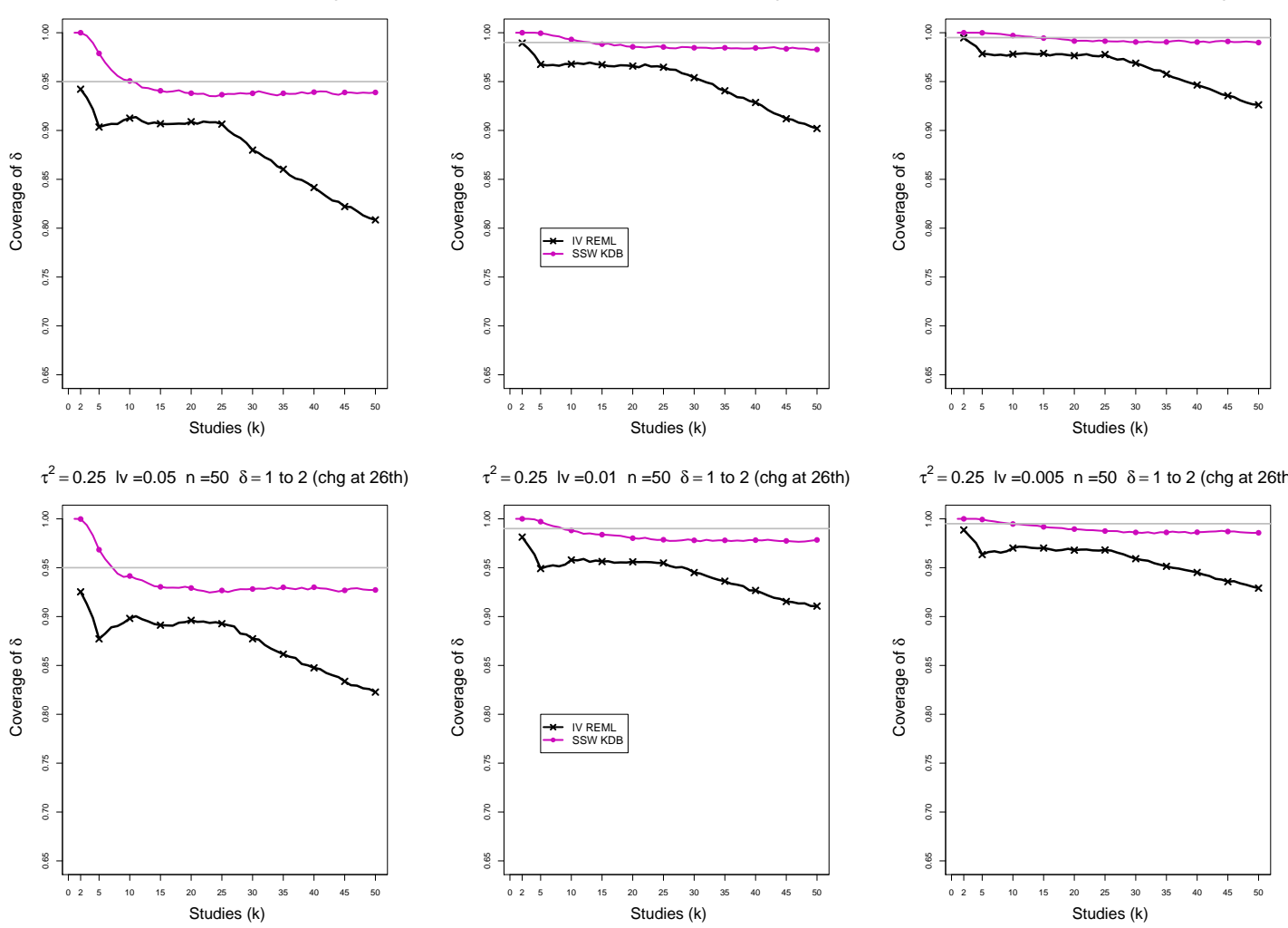

$\tau^{2}=0.25 \mathrm{lv}=0.005 \mathrm{n}=50 \quad \delta=1$ to 2 (chg at 26 th)

$\tau^{2}=1 \mathrm{lv}=0.05 \mathrm{n}=50 \quad \delta=1$ to 2 (chg at 26th)
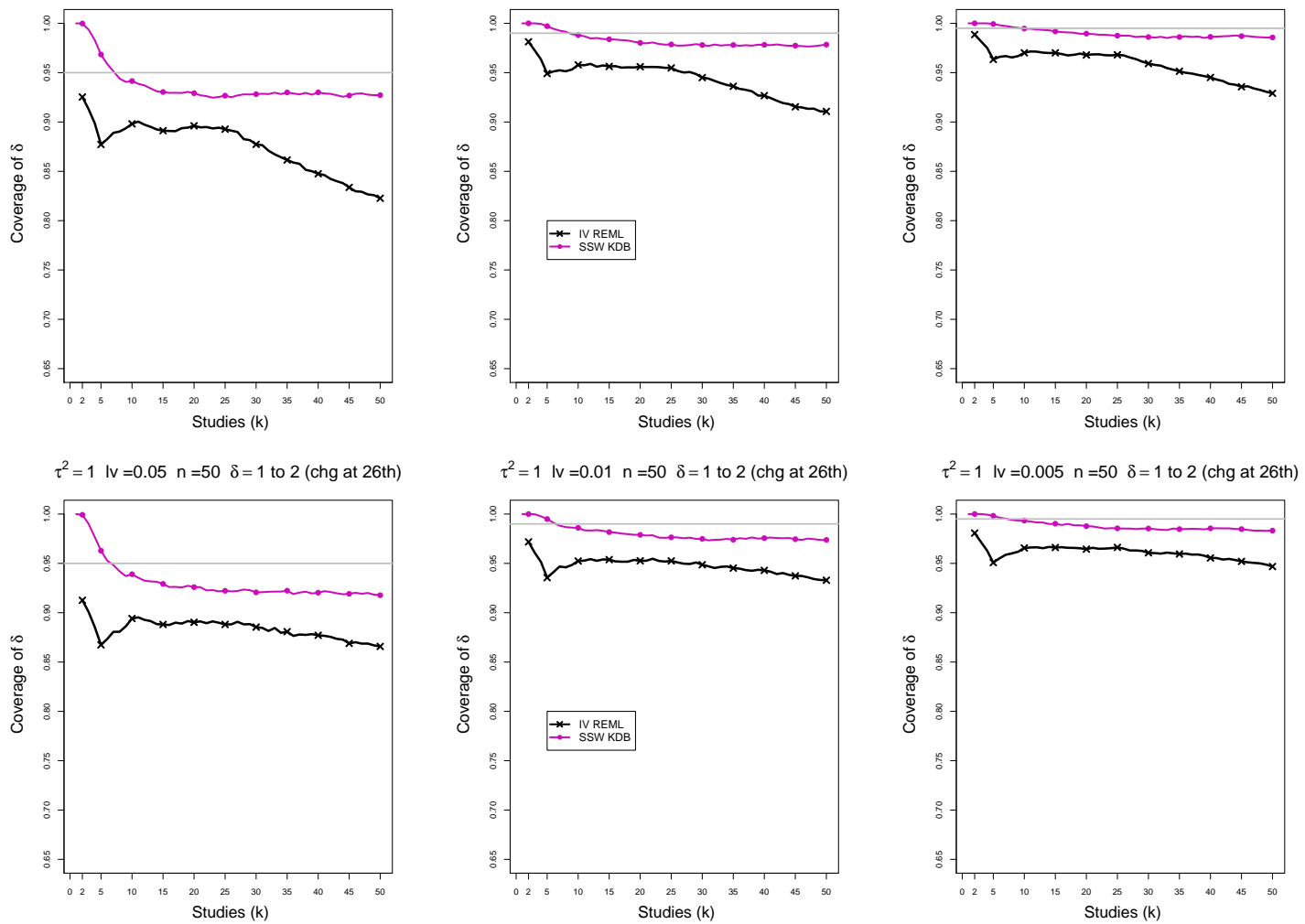

Figure D3. 7: 2-Stage CMA - Coverage of interval estimators of $\delta$ at confidence level of $1-\alpha$ for $\alpha=0.05,0.01,0.005$ when $\delta=1$ for $k \leq 25$ followed by a shift to $\delta=2$ for $k \geq 26, \tau^{2}=0,0.1,0.25,1, n=50, K=50$. Light grey line at $0.95,0.99,0.995$. 

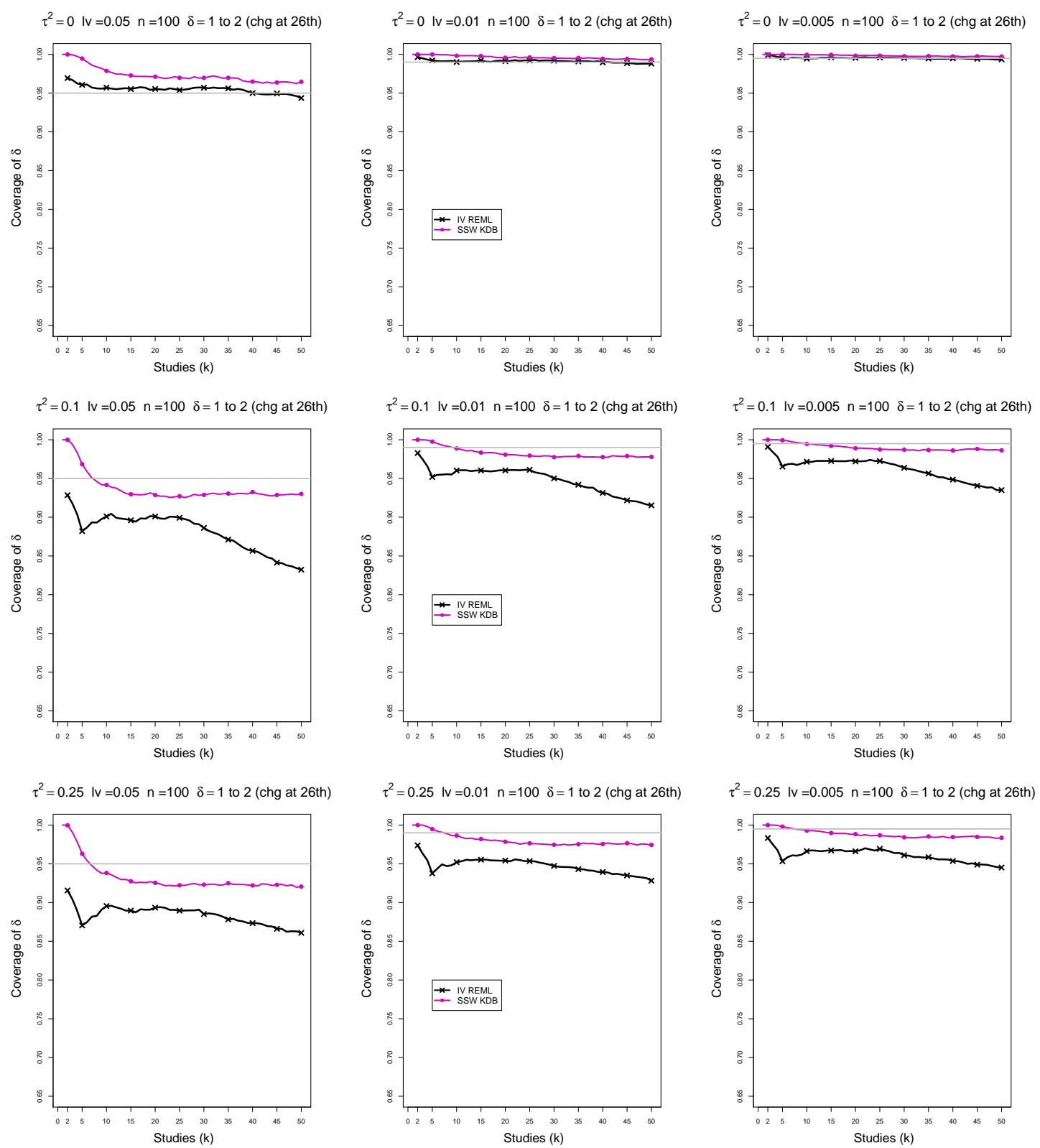

$\tau^{2}=1 \mathrm{lv}=0.05 \mathrm{n}=100 \quad \delta=1$ to 2 (chg at 26th)

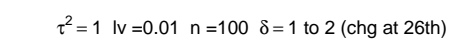

$\tau^{2}=0.25 \mathrm{lv}=0.005 \mathrm{n}=100 \delta=1$ to 2 (chg at 26 th)
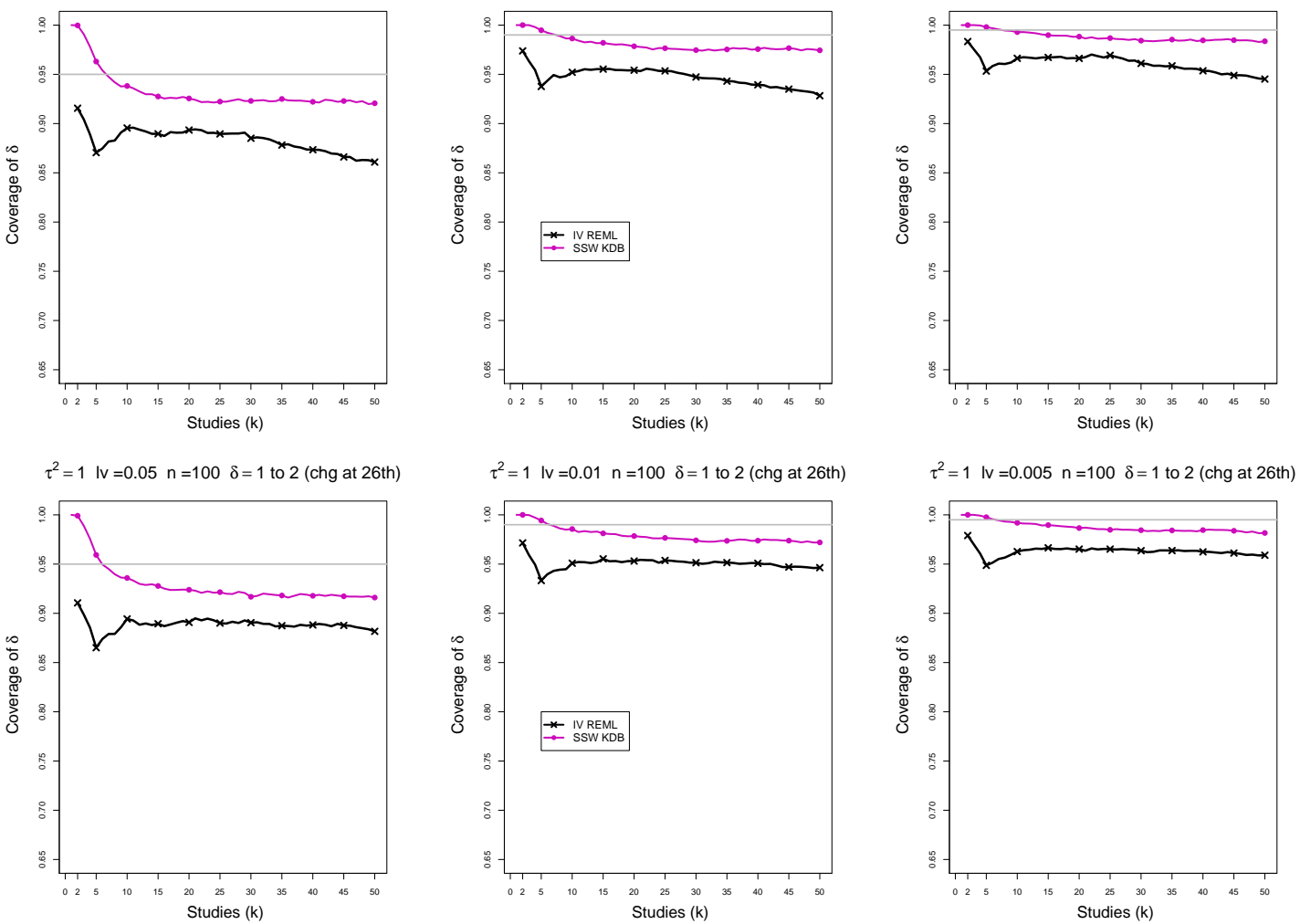

Figure D3. 8: 2-Stage CMA - Coverage of interval estimators of $\delta$ at confidence level of $1-\alpha$ for $\alpha=0.05,0.01,0.005$ when $\delta=1$ for $k \leq 25$ followed by a shift to $\delta=2$ for $k \geq 26, \tau^{2}=0,0.1,0.25,1, n=100, K=50$. Light grey line at 0.95,0.99,0.995. 

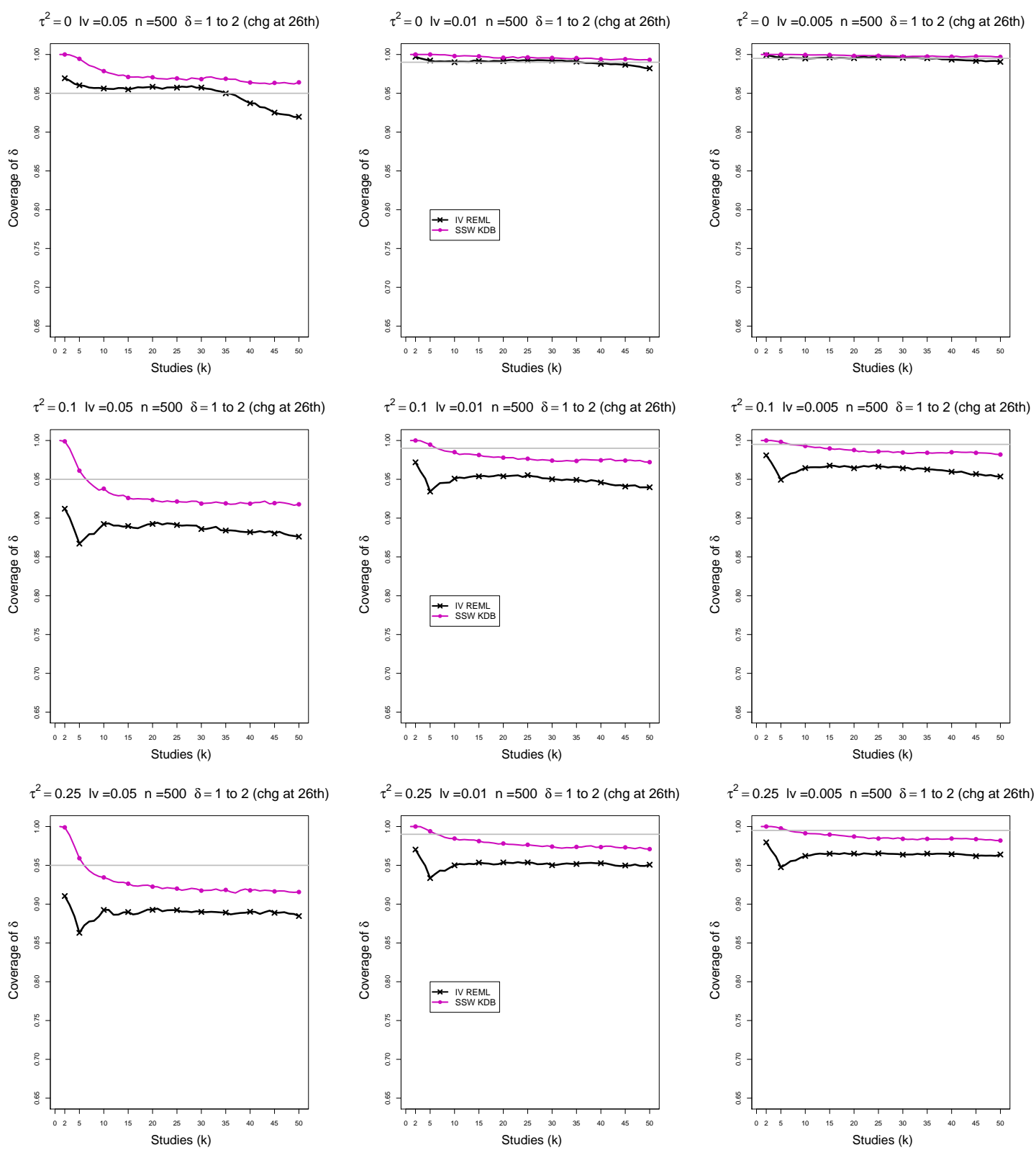

$\tau^{2}=1 \mathrm{lv}=0.05 \mathrm{n}=500 \quad \delta=1$ to 2 (chg at 26 th)

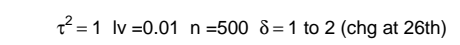

$\tau^{2}=0.25 \mathrm{lv}=0.005 \mathrm{n}=500 \delta=1$ to 2 (chg at 26th)
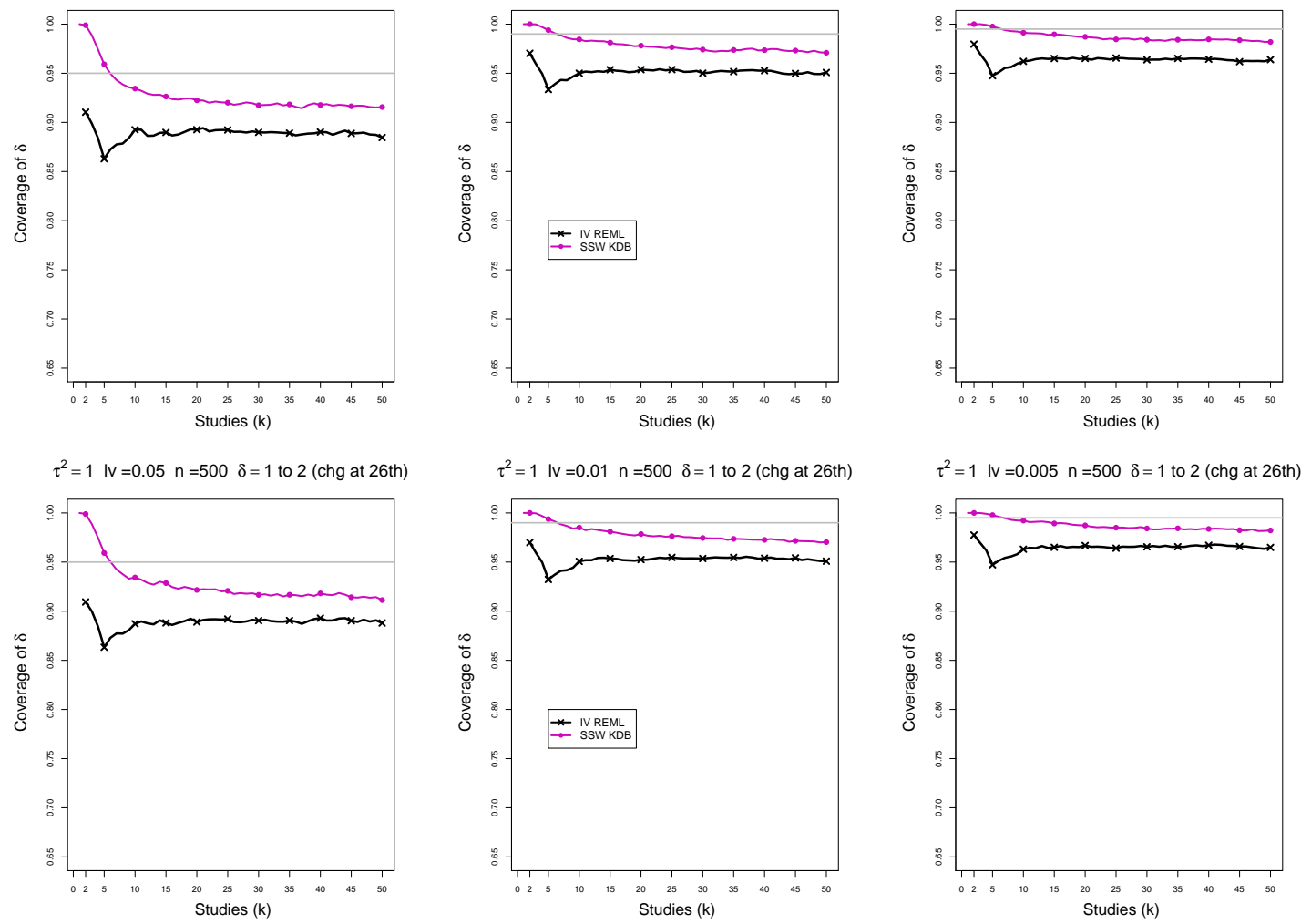

Figure D3. 9: 2-Stage CMA - Coverage of interval estimators of $\delta$ at confidence level of $1-\alpha$ for $\alpha=0.05,0.01,0.005$ when $\delta=1$ for $k \leq 25$ followed by a shift to $\delta=2$ for $k \geq 26, \tau^{2}=0,0.1,0.25,1, n=500, K=50$. Light grey line at 0.95,0.99,0.995. 

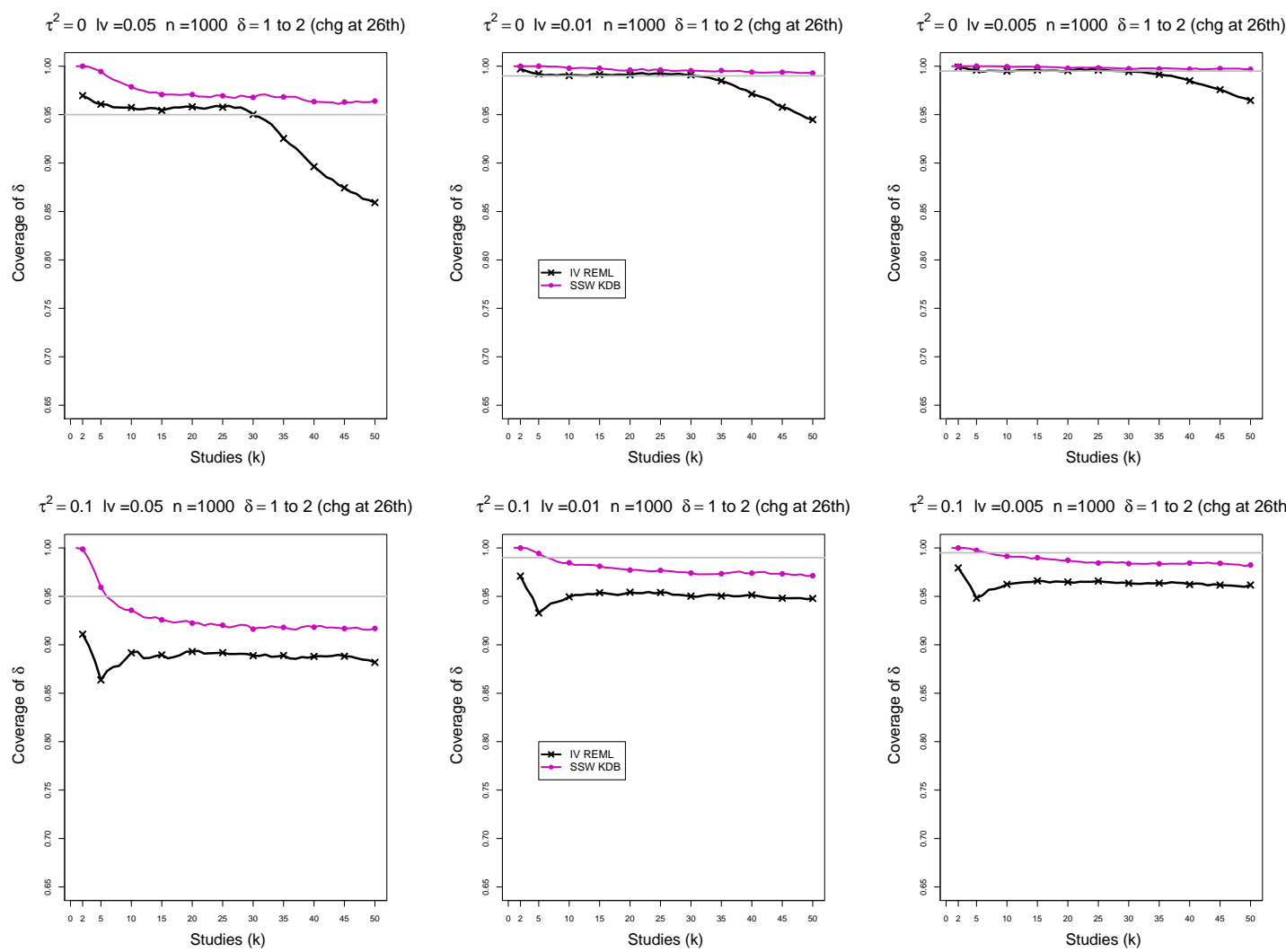

$\tau^{2}=0.1 \mathrm{lv}=0.005 \mathrm{n}=1000 \delta=1$ to 2 (chg at 26th)

$\tau^{2}=0.25 \mathrm{lv}=0.05 \mathrm{n}=1000 \quad \delta=1$ to 2 (chg at 26 th)
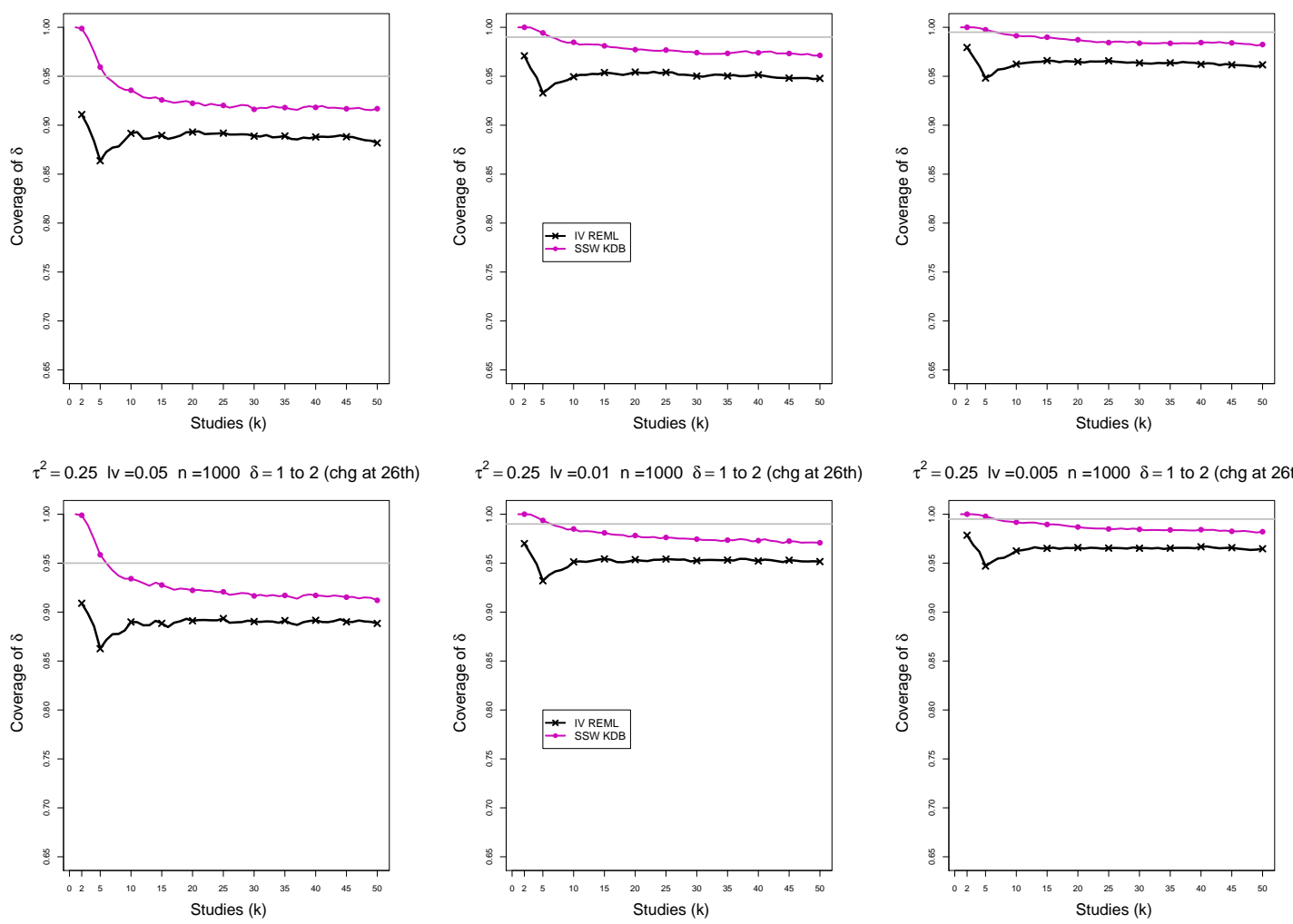

$\tau^{2}=0.25 \mathrm{lv}=0.005 \mathrm{n}=1000 \delta=1$ to 2 (chg at 26th)
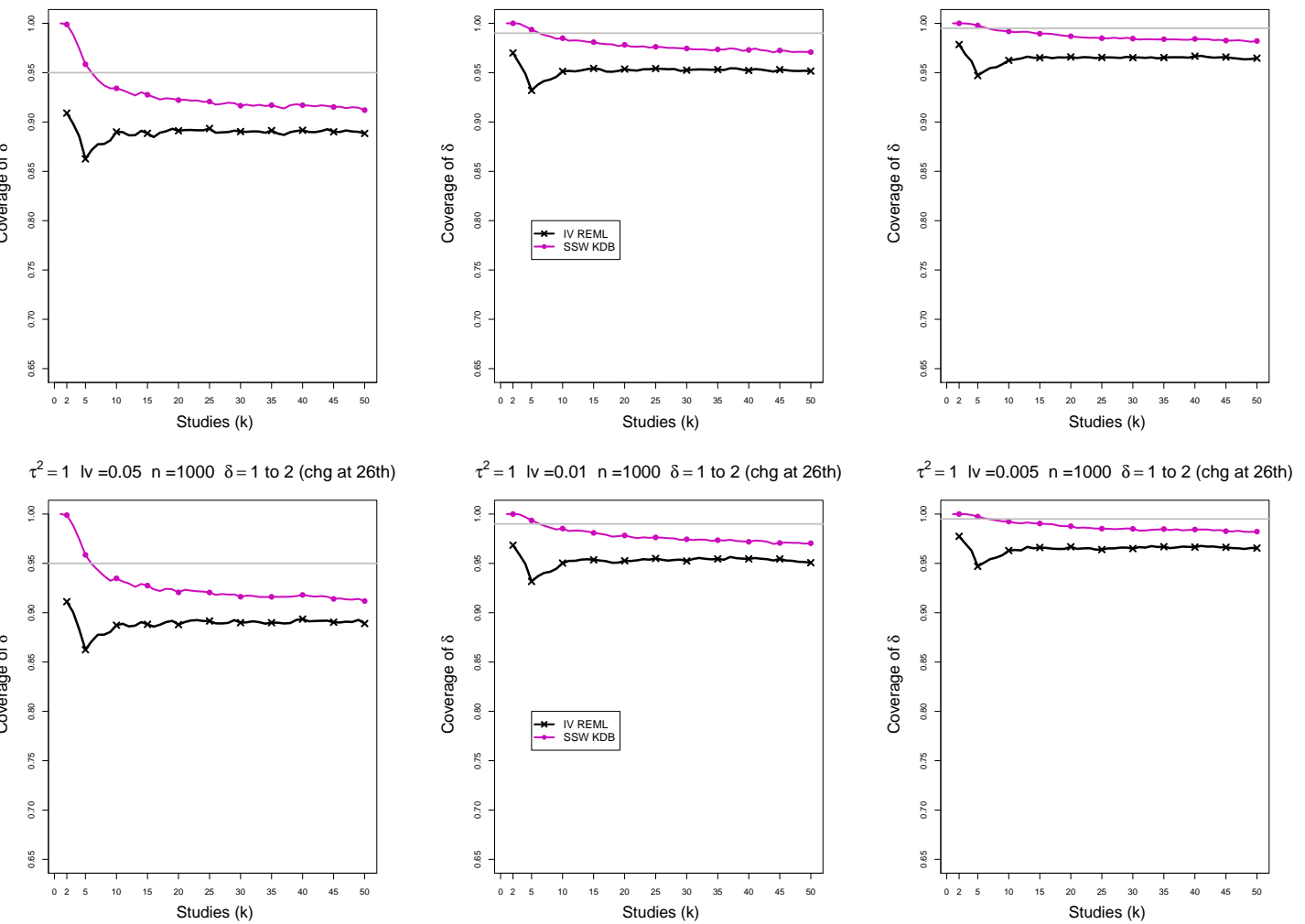

Figure D3. 10: 2-Stage CMA - Coverage of interval estimators of $\delta$ at confidence level of $1-\alpha$ for $\alpha=0.05,0.01,0.005$ when $\delta=1$ for $k \leq 25$ followed by a shift to $\delta=2$ for $k \geq 26, \tau^{2}=0,0.1,0.25,1, n=1000, K=50$. Light grey line at $0.95,0.99,0.995$. 

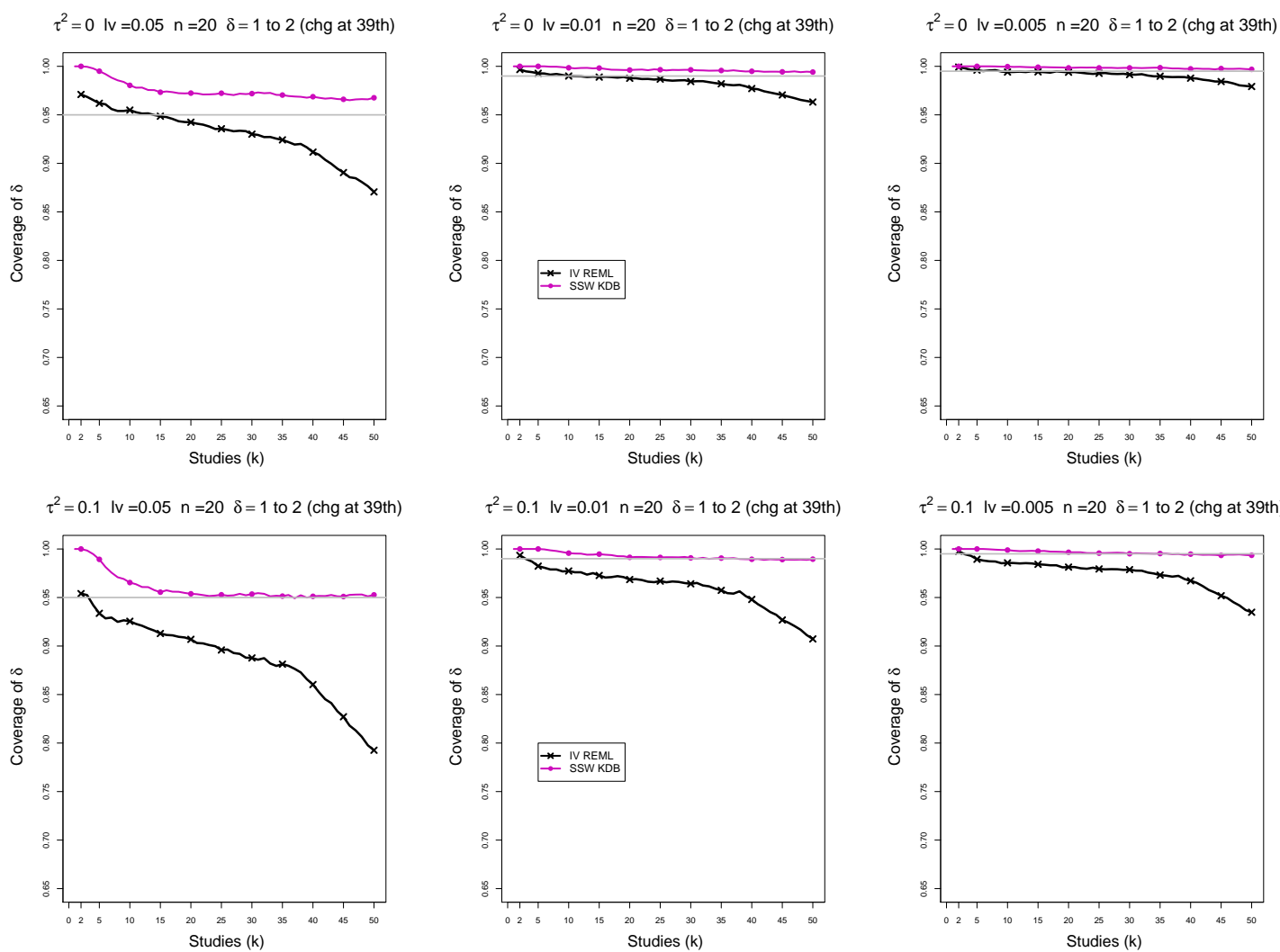

$\tau^{2}=0.1 \mathrm{lv}=0.005 \mathrm{n}=20 \quad \delta=1$ to 2 (chg at 39 th)
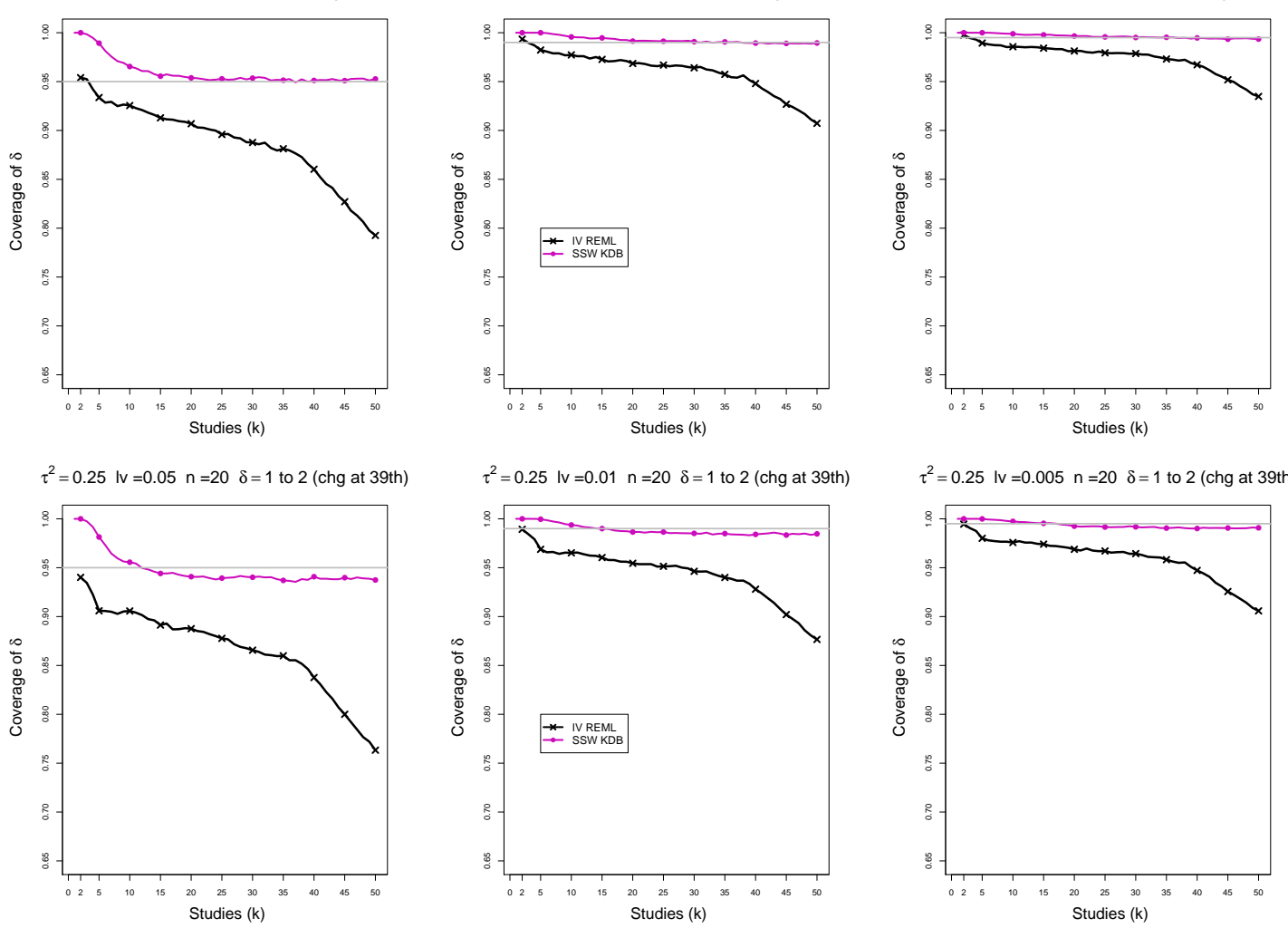

$\tau^{2}=0.25 \mathrm{lv}=0.005 \mathrm{n}=20 \quad \delta=1$ to 2 (chg at 39 th)

$\tau^{2}=1 \mathrm{lv}=0.05 \mathrm{n}=20 \quad \delta=1$ to 2 (chg at 39 th)
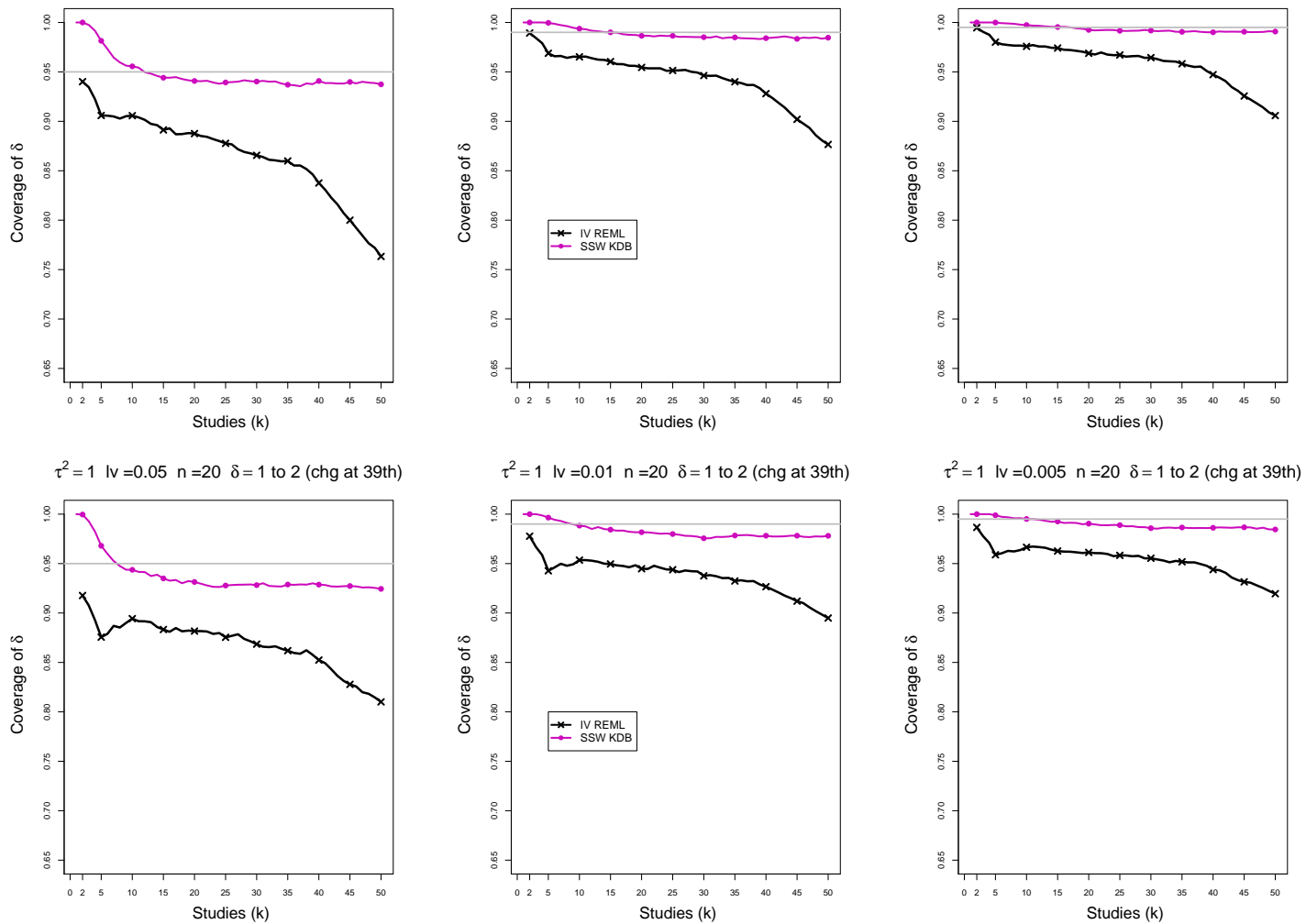

Figure D3. 11: 2-Stage CMA - Coverage of interval estimators of $\delta$ at confidence level of $1-\alpha$ for $\alpha=0.05,0.01,0.005$ when $\delta=1$ for $k \leq 38$ followed by a shift to $\delta=2$ for $k \geq 39, \tau^{2}=0,0.1,0.25,1, n=20, K=50$. Light grey line at $0.95,0.99,0.995$. 

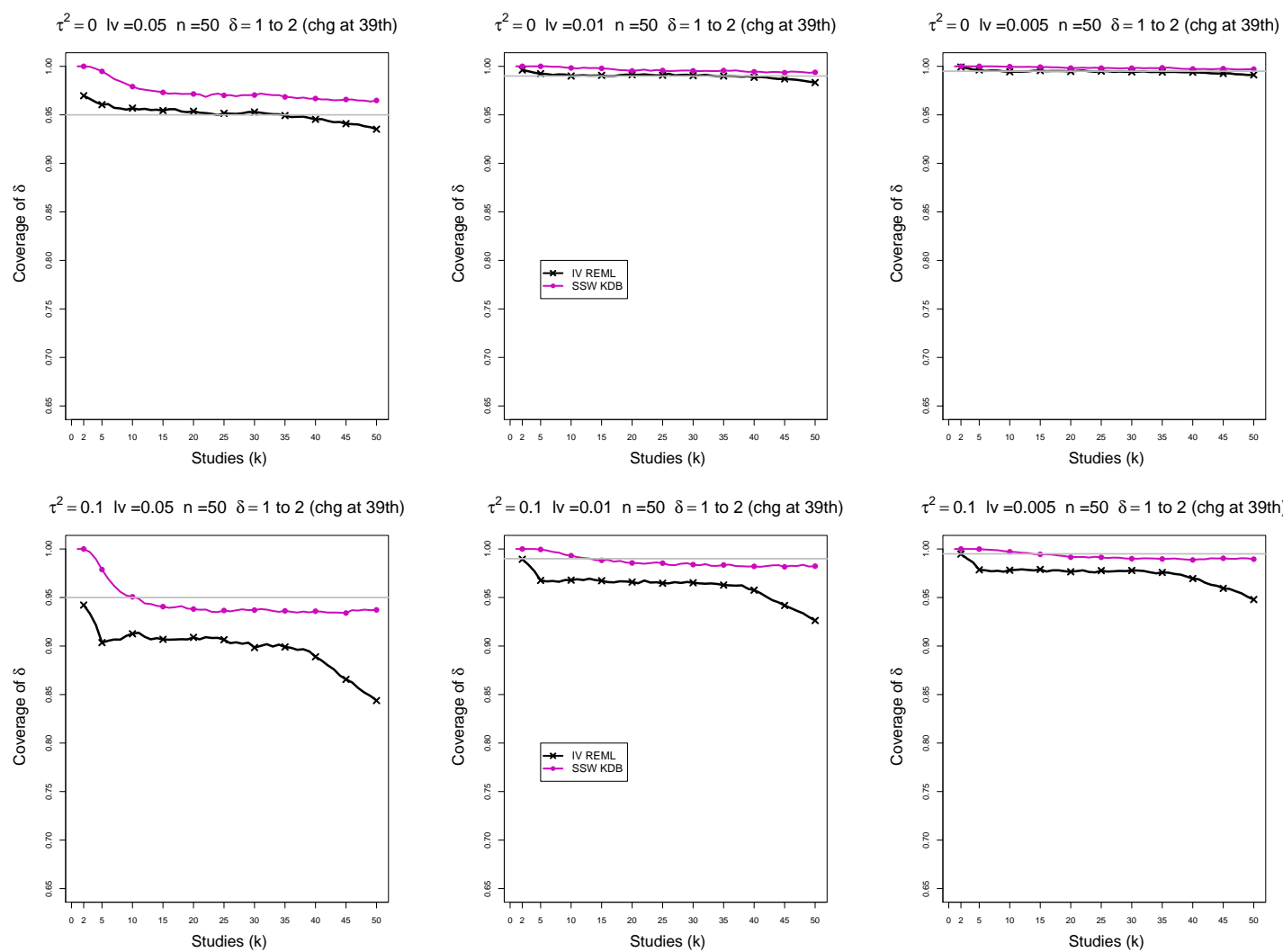

$\tau^{2}=0.1 \mathrm{lv}=0.005 \mathrm{n}=50 \quad \delta=1$ to 2 (chg at 39 th)

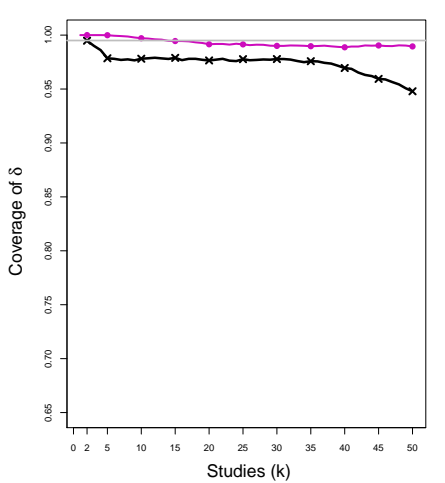

$\tau^{2}=0.25 \mathrm{lv}=0.05 \mathrm{n}=50 \quad \delta=1$ to 2 (chg at 39 th)

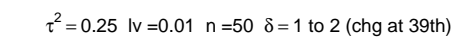

$\tau^{2}=0.25 \mathrm{lv}=0.005 \mathrm{n}=50 \delta=1$ to 2 (chg at 39 th)
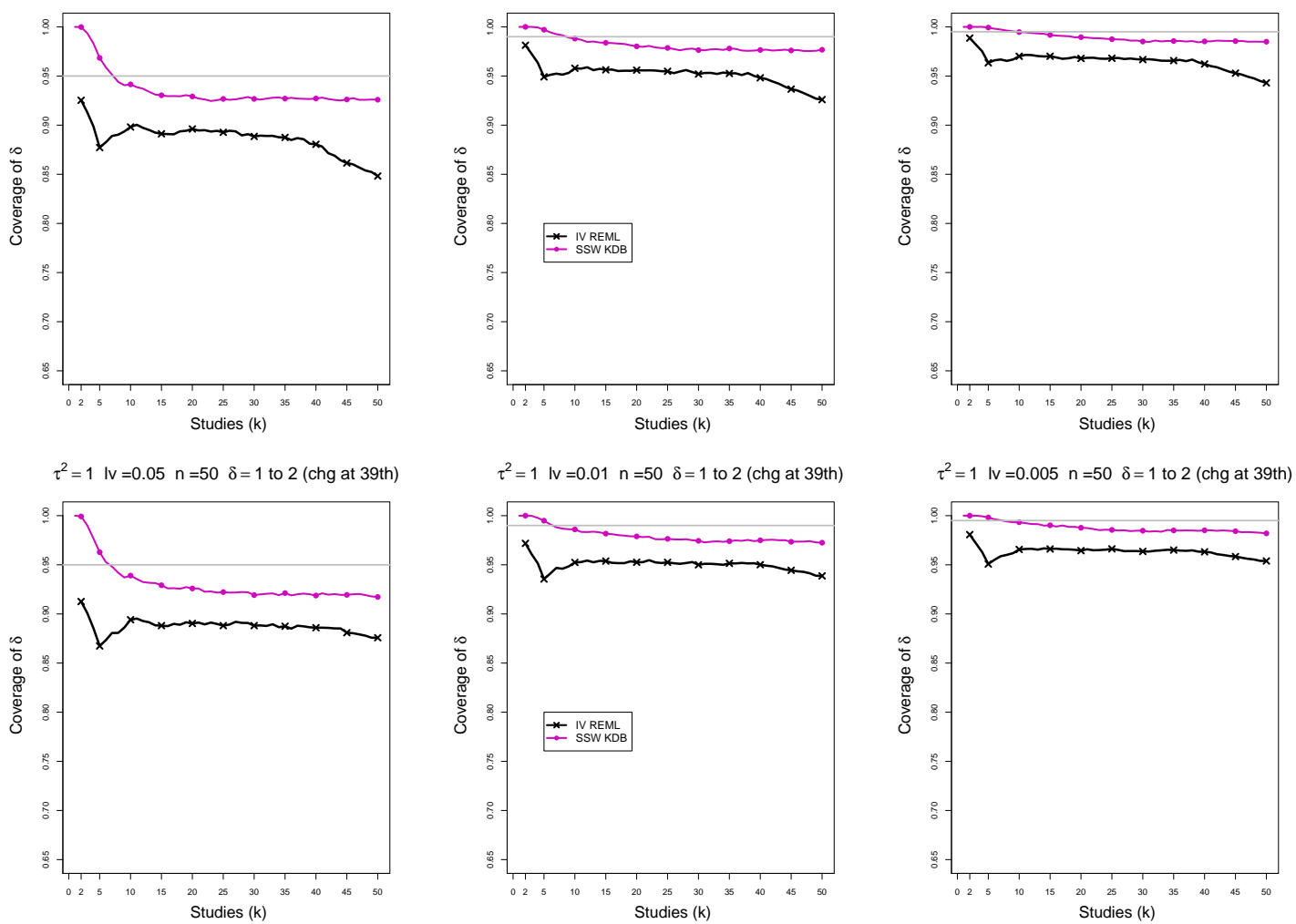

Figure D3. 12: 2-Stage CMA - Coverage of interval estimators of $\delta$ at confidence level of $1-\alpha$ for $\alpha=0.05,0.01,0.005$ when $\delta=1$ for $k \leq 38$ followed by a shift to $\delta=2$ for $k \geq 39, \tau^{2}=0,0.1,0.25,1, n=50, K=50$. Light grey line at $0.95,0.99,0.995$. 

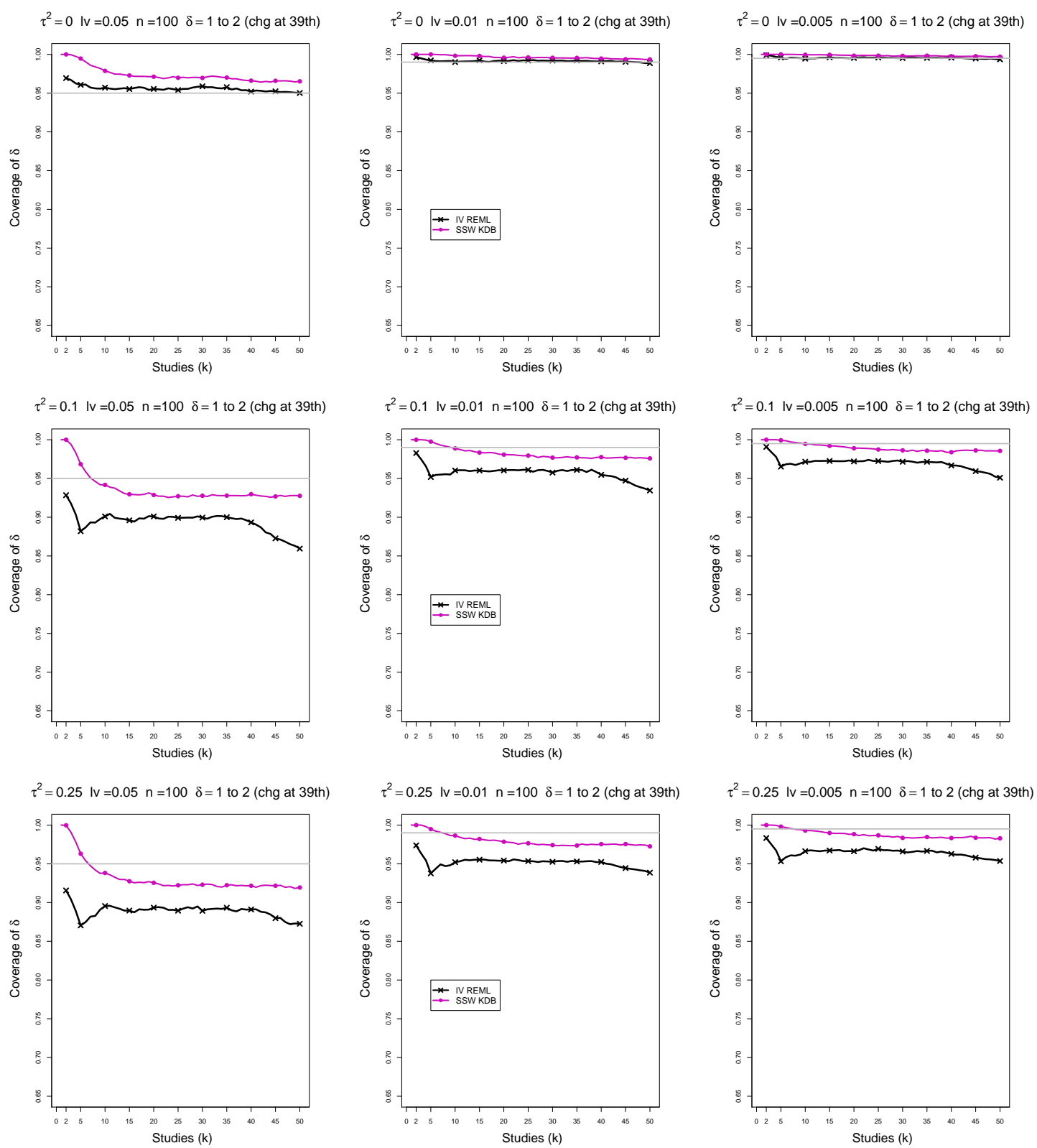

$\tau^{2}=1 \mathrm{lv}=0.05 \mathrm{n}=100 \quad \delta=1$ to 2 (chg at 39 th)

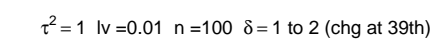

$\tau^{2}=0.25 \mathrm{lv}=0.005 \mathrm{n}=100 \delta=1$ to 2 (chg at 39 th)
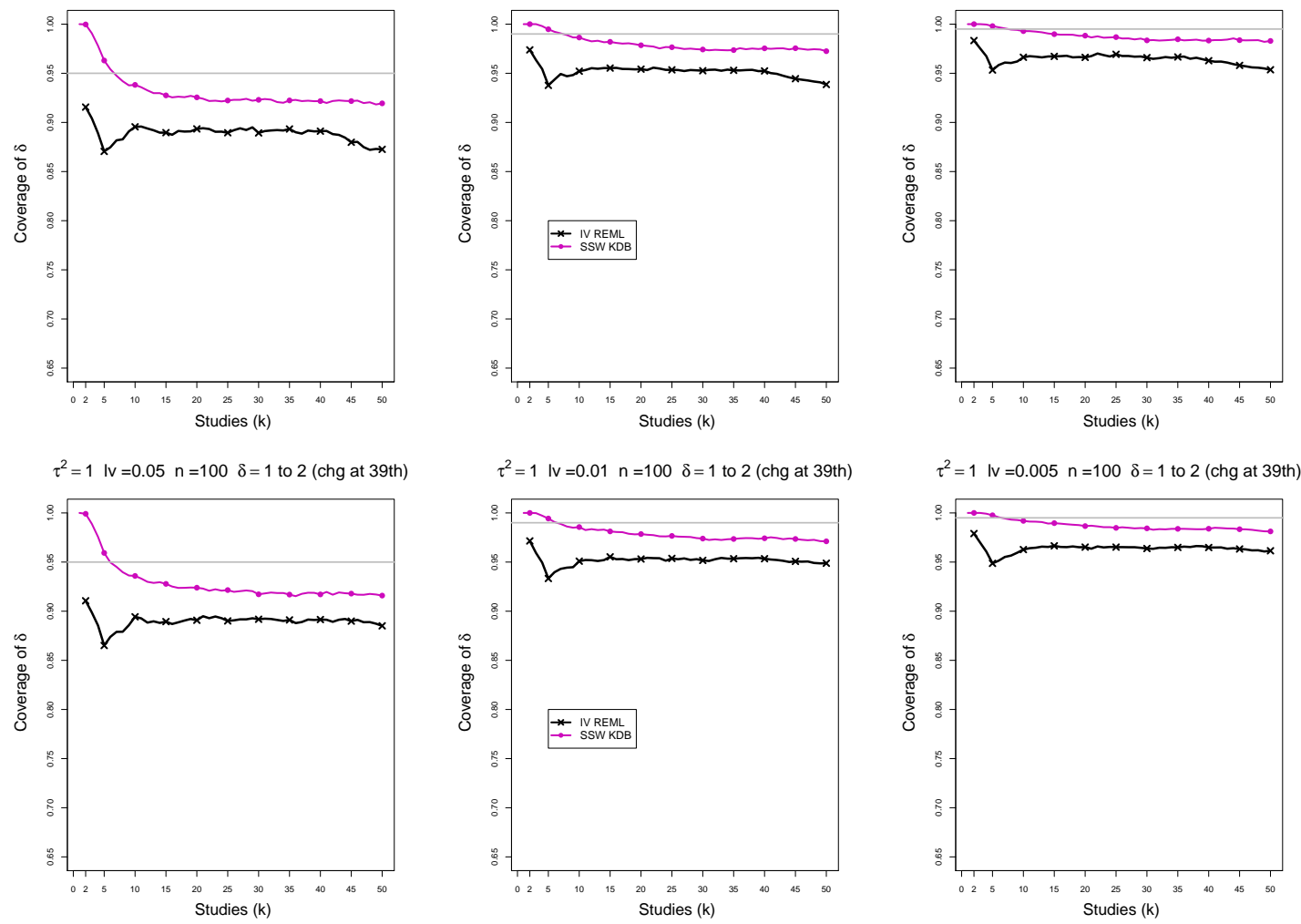

Figure D3. 13: 2-Stage CMA - Coverage of interval estimators of $\delta$ at confidence level of $1-\alpha$ for $\alpha=0.05,0.01,0.005$ when $\delta=1$ for $k \leq 38$ followed by a shift to $\delta=2$ for $k \geq 39, \tau^{2}=0,0.1,0.25,1, n=100, K=50$. Light grey line at 0.95,0.99,0.995. 

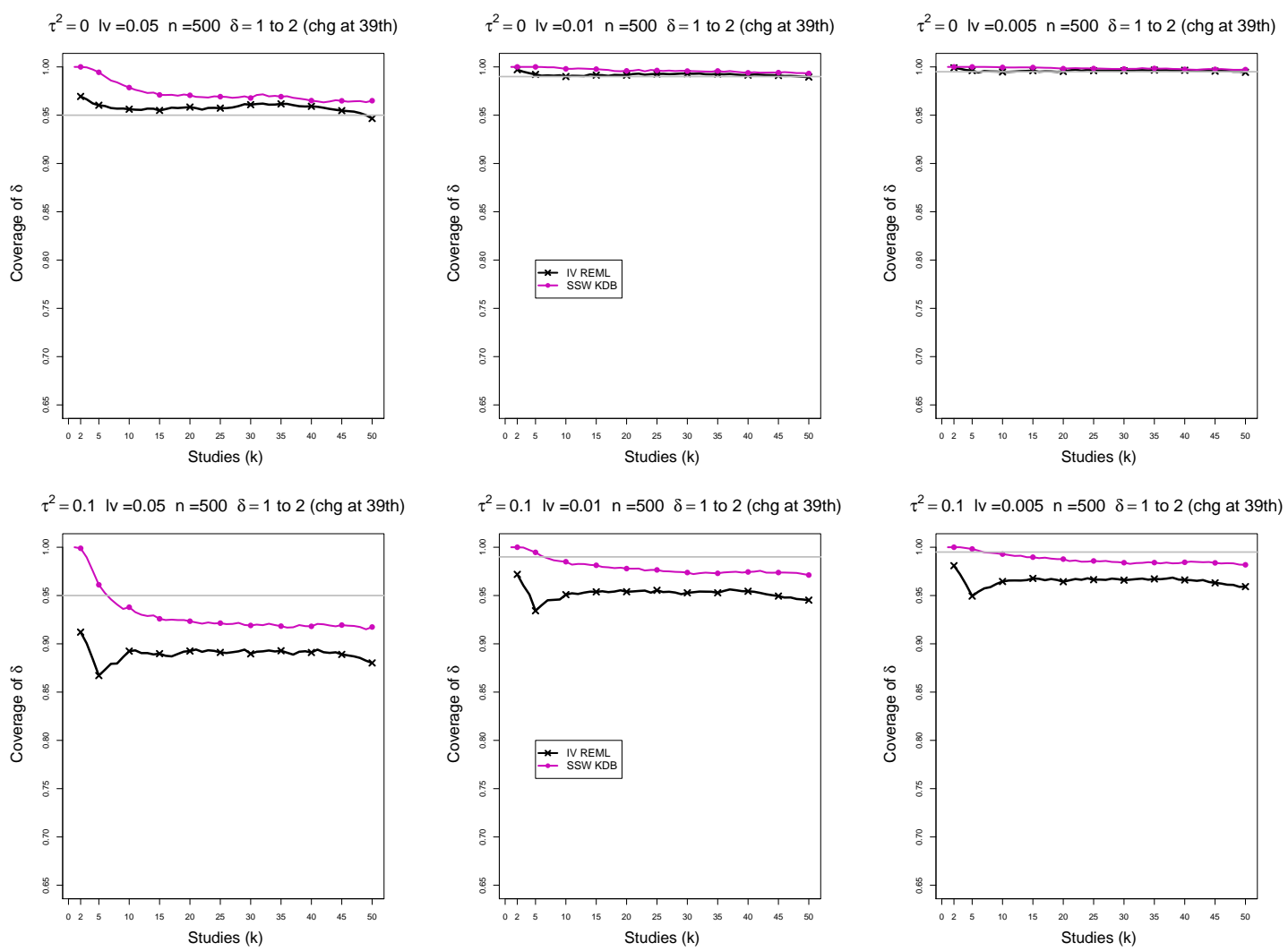

$\tau^{2}=0.25 \mathrm{lv}=0.05 \mathrm{n}=500 \quad \delta=1$ to 2 (chg at 39 th)
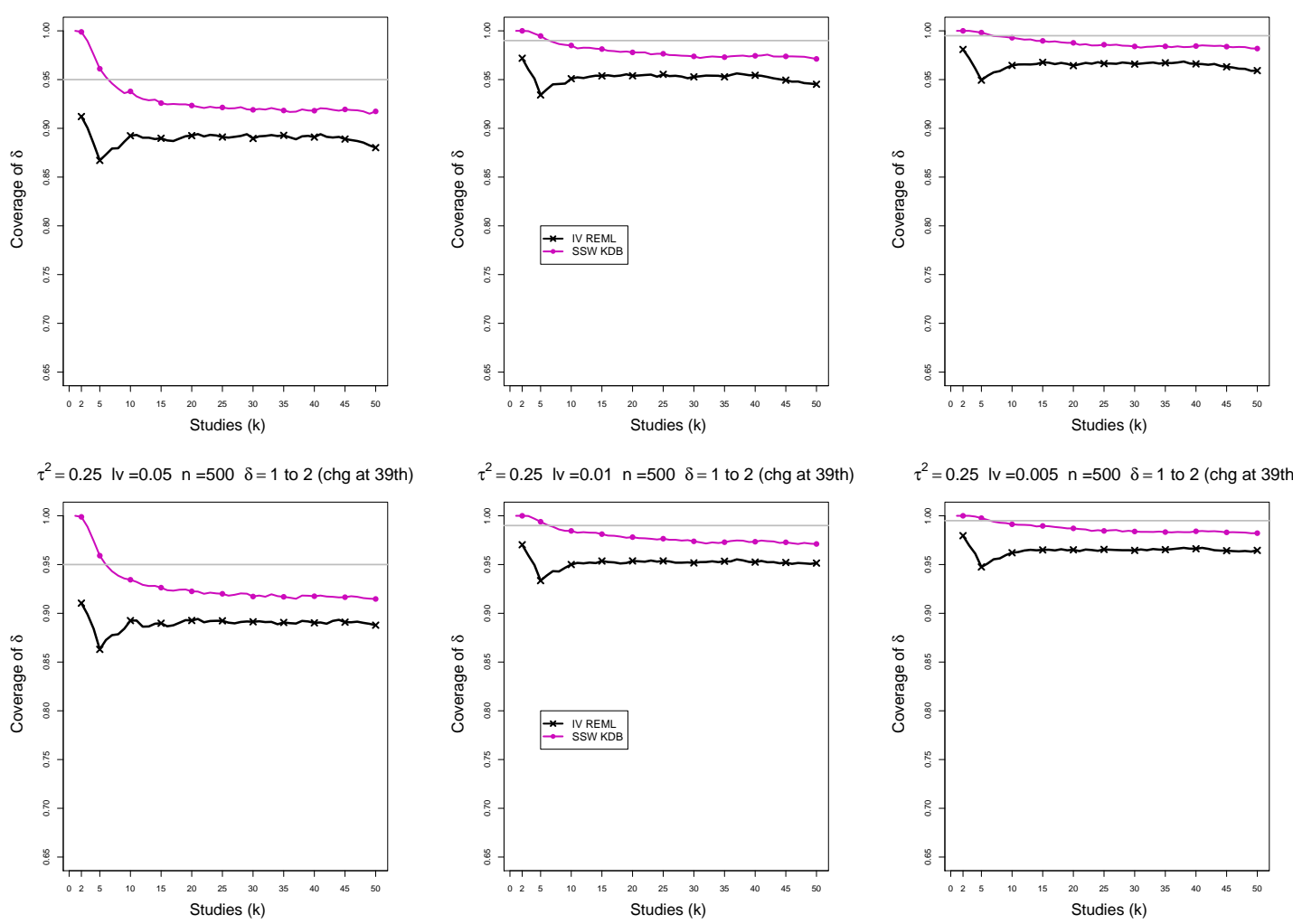

$\tau^{2}=0.25 \mathrm{lv}=0.005 \mathrm{n}=500 \delta=1$ to 2 (chg at 39 th)

$\tau^{2}=1 \mathrm{lv}=0.05 \mathrm{n}=500 \quad \delta=1$ to 2 (chg at 39 th)
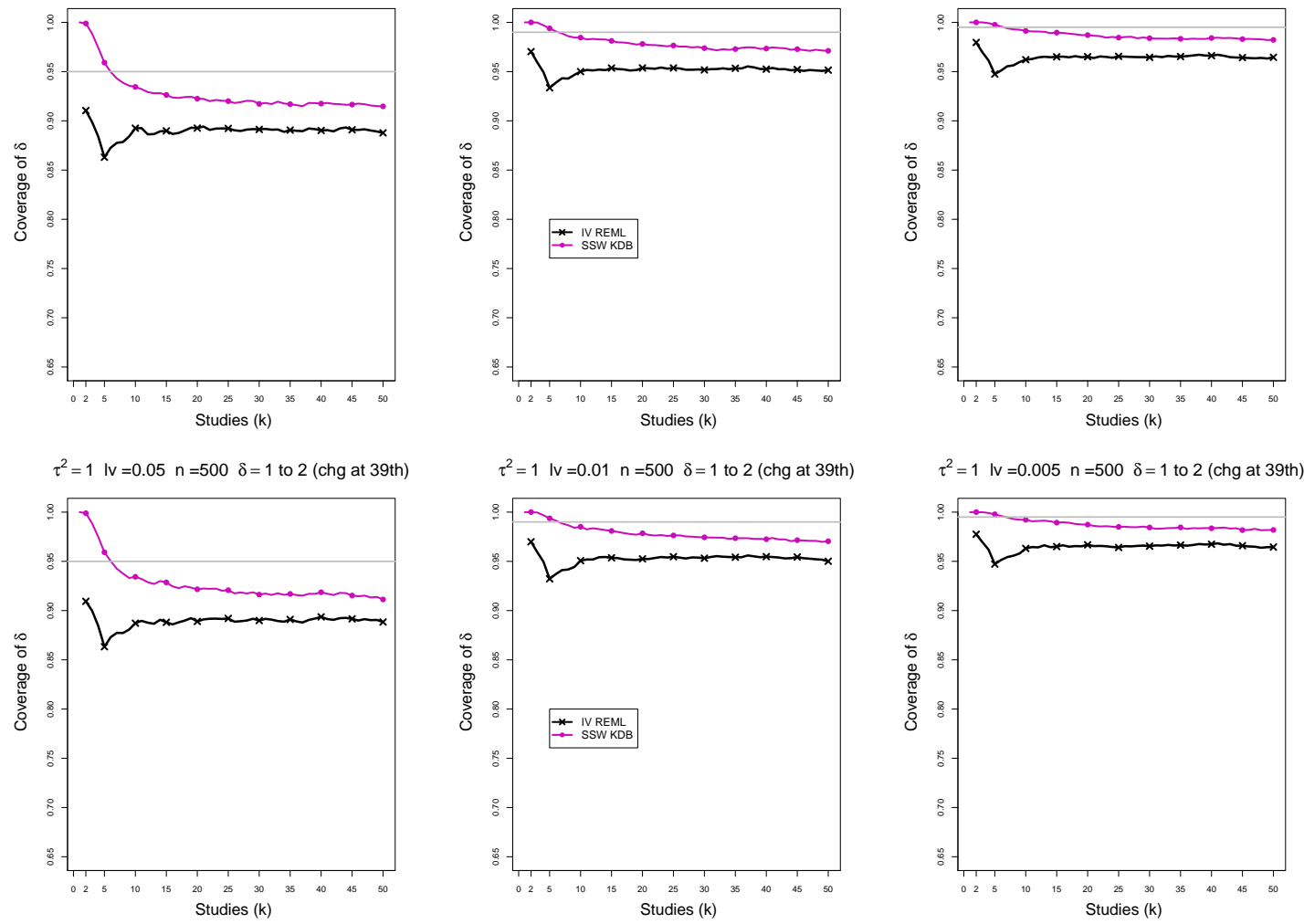

Figure D3. 14: 2-Stage CMA - Coverage of interval estimators of $\delta$ at confidence level of $1-\alpha$ for $\alpha=0.05,0.01,0.005$ when $\delta=1$ for $k \leq 38$ followed by a shift to $\delta=2$ for $k \geq 39, \tau^{2}=0,0.1,0.25,1, n=500, K=50$. Light grey line at 0.95,0.99,0.995. 

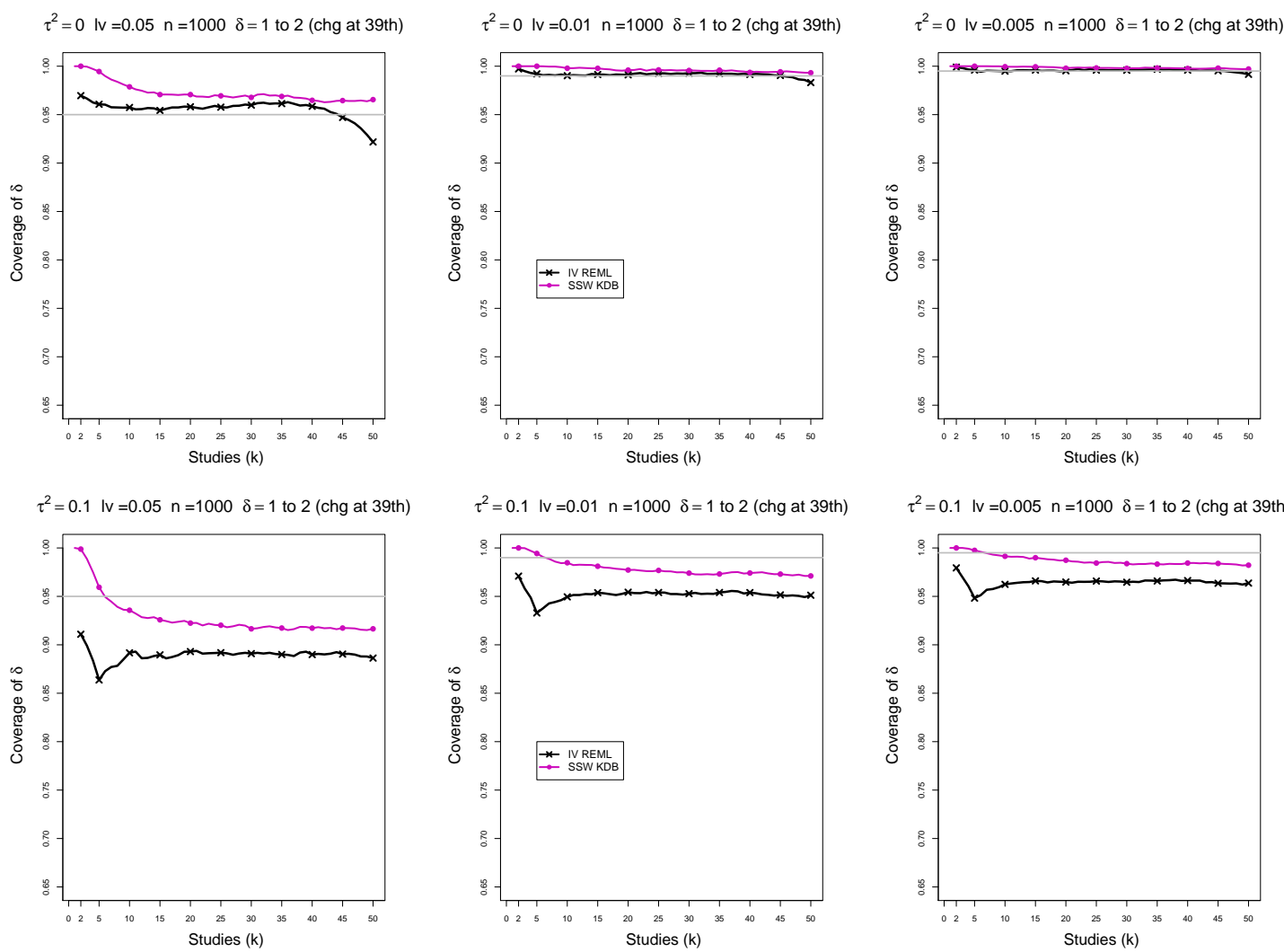

$\tau^{2}=0.1 \mathrm{lv}=0.005 \mathrm{n}=1000 \delta=1$ to 2 (chg at 39 th)

$\tau^{2}=0.25 \mathrm{lv}=0.05 \mathrm{n}=1000 \quad \delta=1$ to 2 (chg at 39 th)
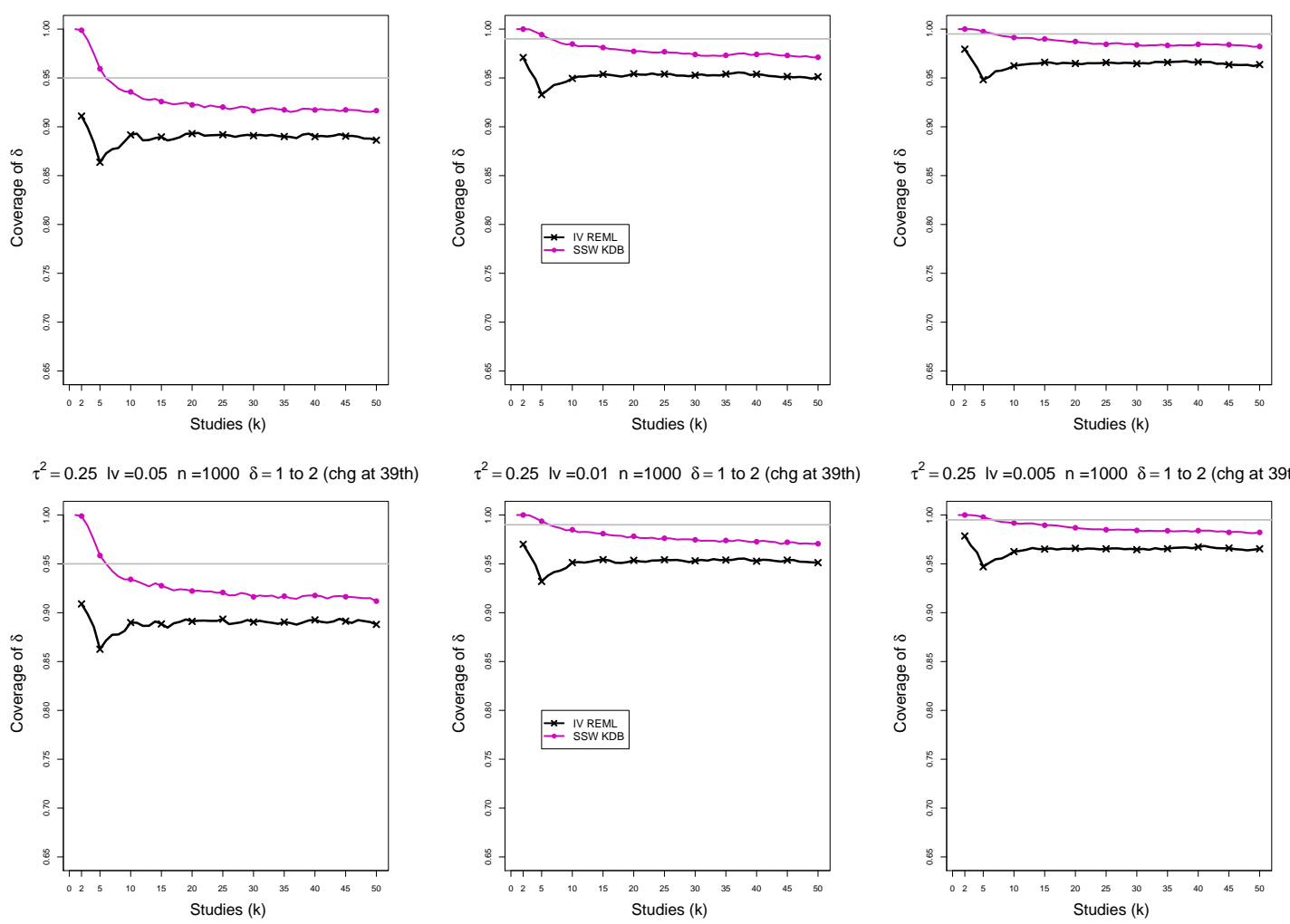

$\tau^{2}=0.25 \mathrm{lv}=0.005 \mathrm{n}=1000 \delta=1$ to 2 (chg at 39 th)
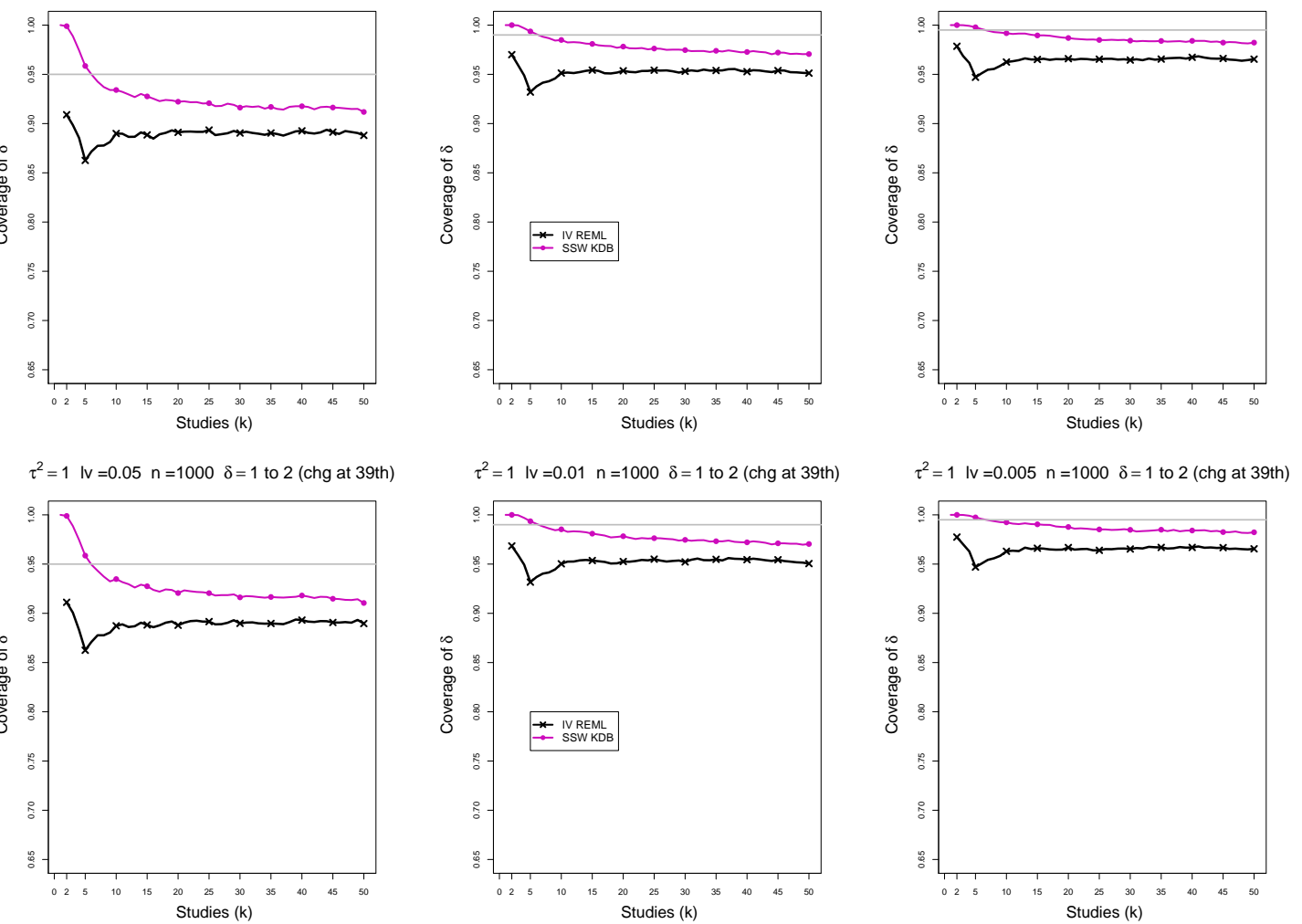

Figure D3. 15: 2-Stage CMA - Coverage of interval estimators of $\delta$ at confidence level of $1-\alpha$ for $\alpha=0.05,0.01,0.005$ when $\delta=1$ for $k \leq 38$ followed by a shift to $\delta=2$ for $k \geq 39, \tau^{2}=0,0.1,0.25,1, n=1000, K=50$. Light grey line at $0.95,0.99,0.995$. 


\section{D4. Coverage of one-sided interval estimators of $\delta$}

For coverage of one-sided interval estimators of $\delta$ in this section, each figure corresponds to a value of a set of values of $\tau^{2}(=0,0.1,0.25,1)$, a value of $n(=20,50,100$, $500,1000)$, a set of values of 2 -sided significance level $(=0.05,0.01,0.005)$ and a value of point of shift $f(=13,26,39)$ while $\delta$ shifts from 1 to 2 and maximum number of studies $K=50$.

Each figure contains a panel (with study $k$ on the horizontal axis) for each combination of parameters.

The interval estimators of $\delta$ are

- IV REML (Restricted maximum likelihood, inverse variance weighted, normal critical values)

- SSW KDB (Effective sample size weighted (SSW), critical value from $t_{k-1}$ with $\hat{\tau}^{2}=\hat{\tau}_{K D B}^{2}$, the Kulinskaya-Dollinger-Bjørkestøl estimator of $\tau^{2}$ ) 

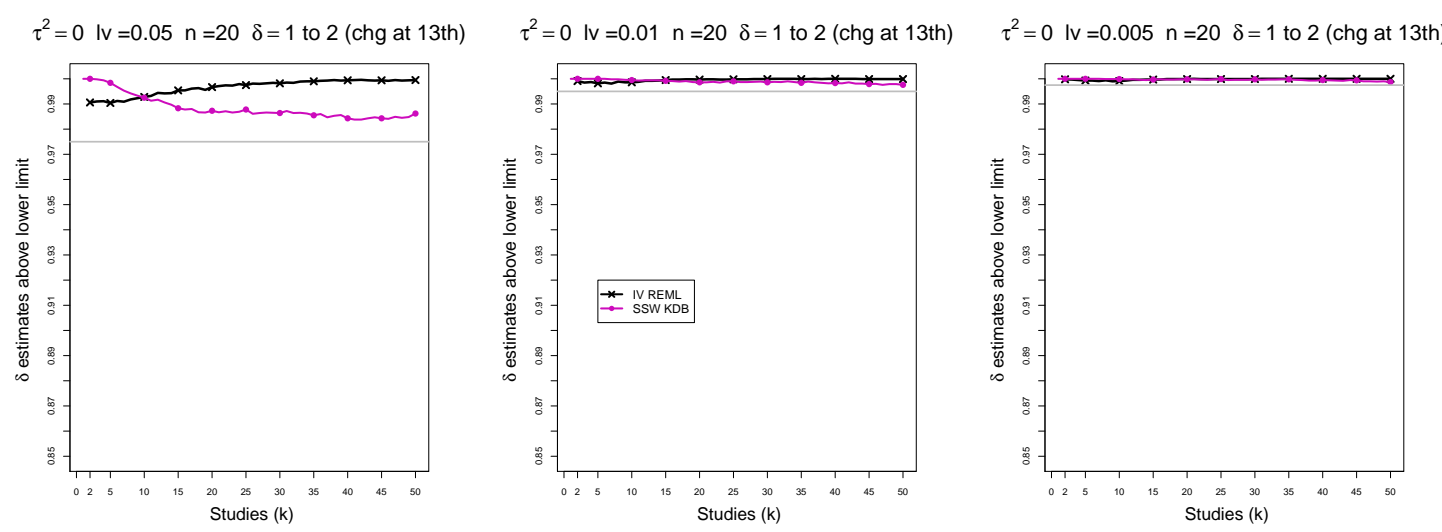

$\tau^{2}=0.1 \mathrm{IV}=0.05 \mathrm{n}=20 \delta=1$ to 2 (chg at 13 th)

$\tau^{2}=0.1 \mathrm{lV}=0.01 \mathrm{n}=20 \quad \delta=1$ to 2 (chg at 13 th)

$\tau^{2}=0.1 \mathrm{lv}=0.005 \mathrm{n}=20 \quad \delta=1$ to 2 (chg at 13 th)
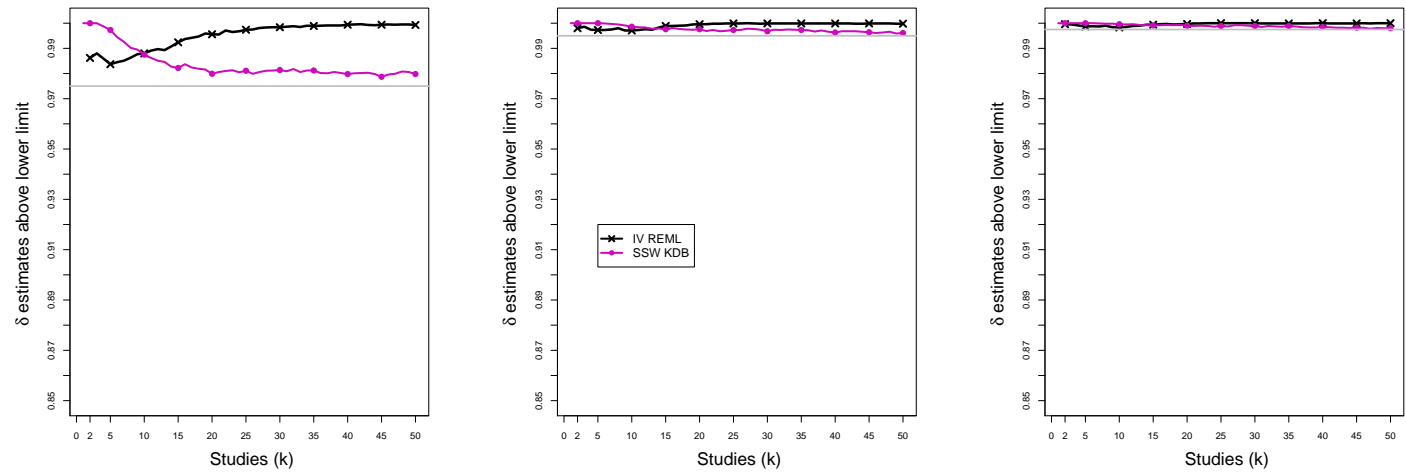

$\tau^{2}=0.25$ Iv $=0.05 \mathrm{n}=20 \quad \delta=1$ to 2 (chg at 13 th)
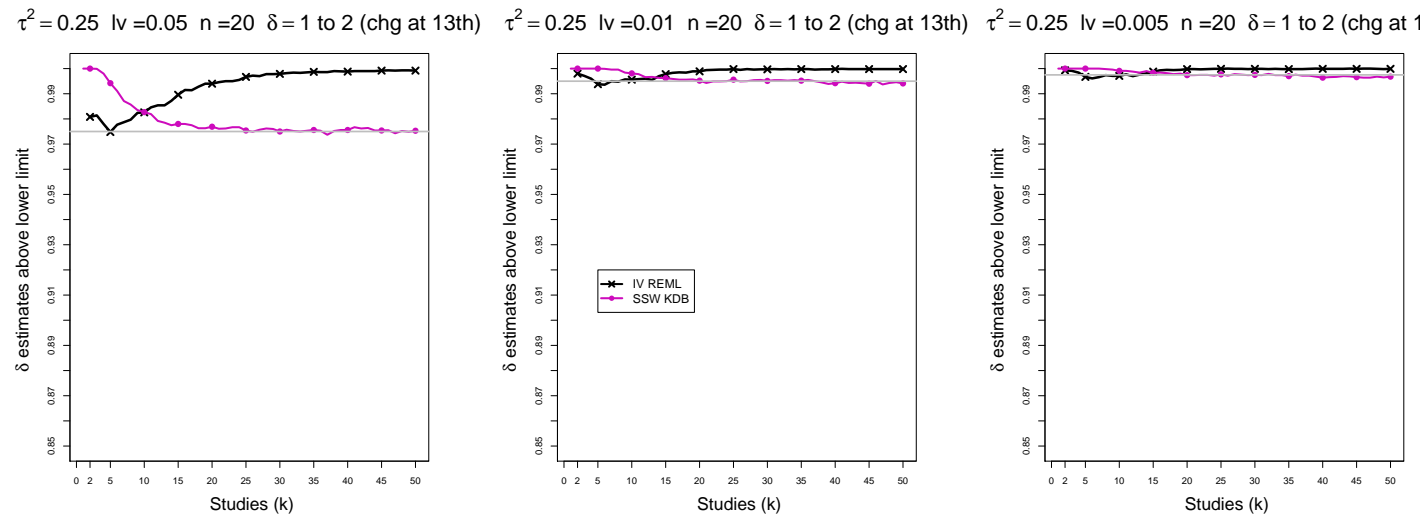

$\tau^{2}=1 \mathrm{lv}=0.05 \mathrm{n}=20 \quad \delta=1$ to 2 (chg at 13 th)
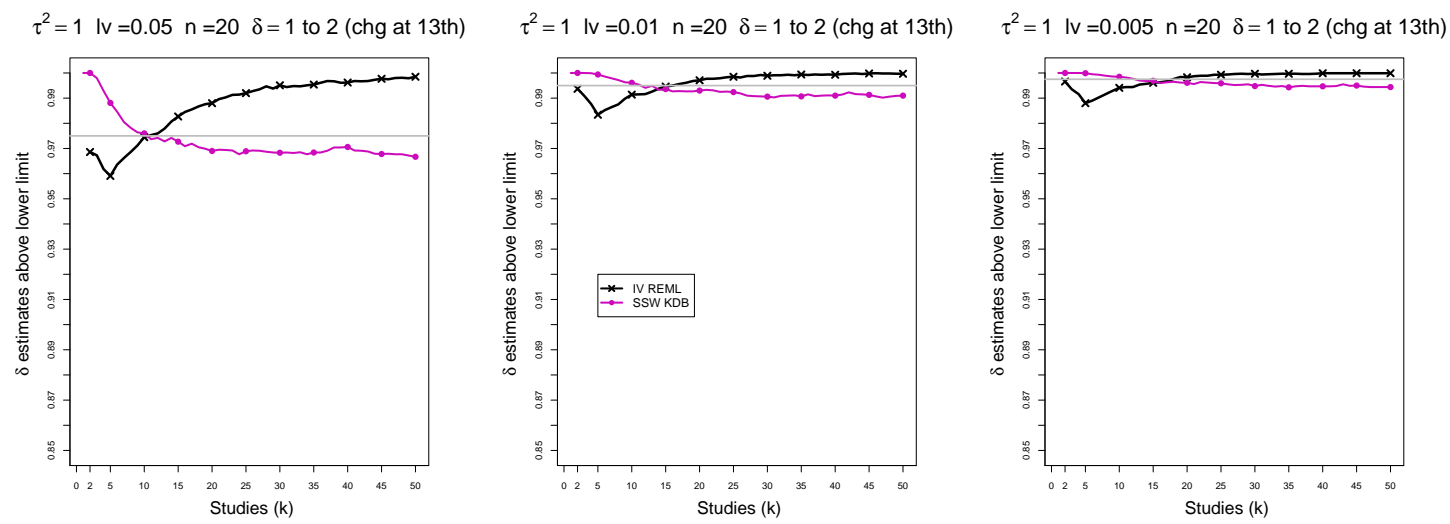

Figure D4. 1: 2-Stage CMA - Coverage of the lower confidence interval of $\delta$ (above lower limit) at the confidence level $1-\alpha / 2$ for $\alpha=0.05,0.01,0.005$ when $\delta=1$ for $k \leq 12$ followed by a shift to $\delta=2$ for $k \geq 13, \tau^{2}=0,0.1,0.25,1, n=20, K=50$. Light grey line at $0.975,0.995,0.9975$. 

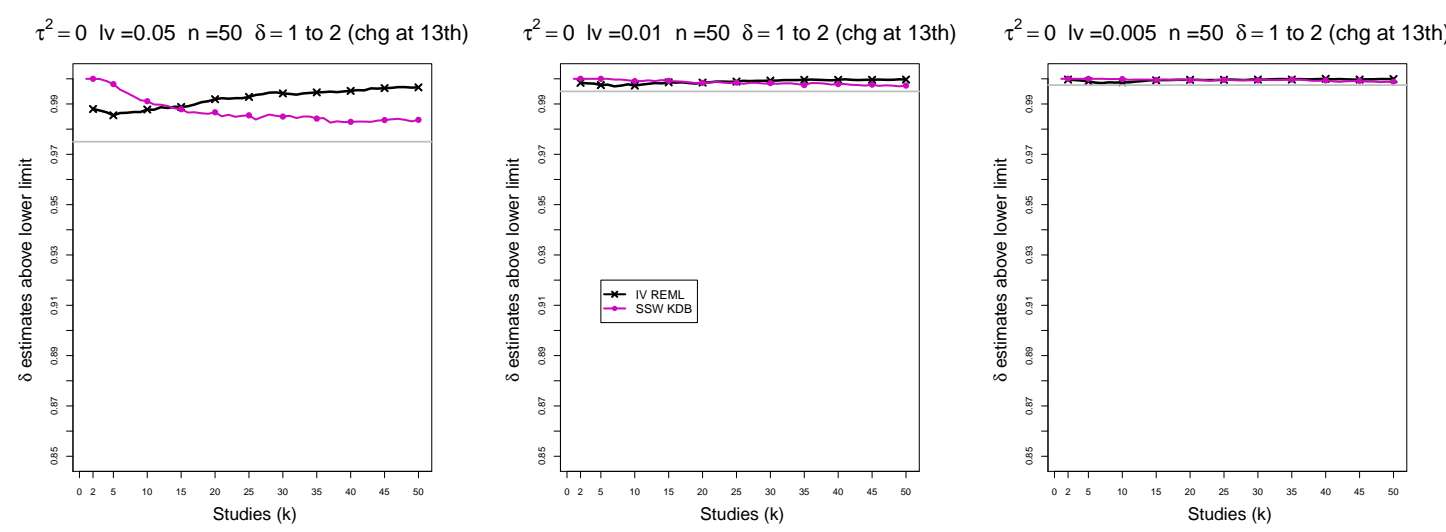

$\tau^{2}=0.1 \mathrm{IV}=0.05 \mathrm{n}=50 \delta=1$ to 2 (chg at 13 th)

$\tau^{2}=0.1 \mathrm{lV}=0.01 \mathrm{n}=50 \delta=1$ to 2 (chg at 13 th)

$\tau^{2}=0.1 \mathrm{lv}=0.005 \mathrm{n}=50 \delta=1$ to 2 (chg at 13 th)
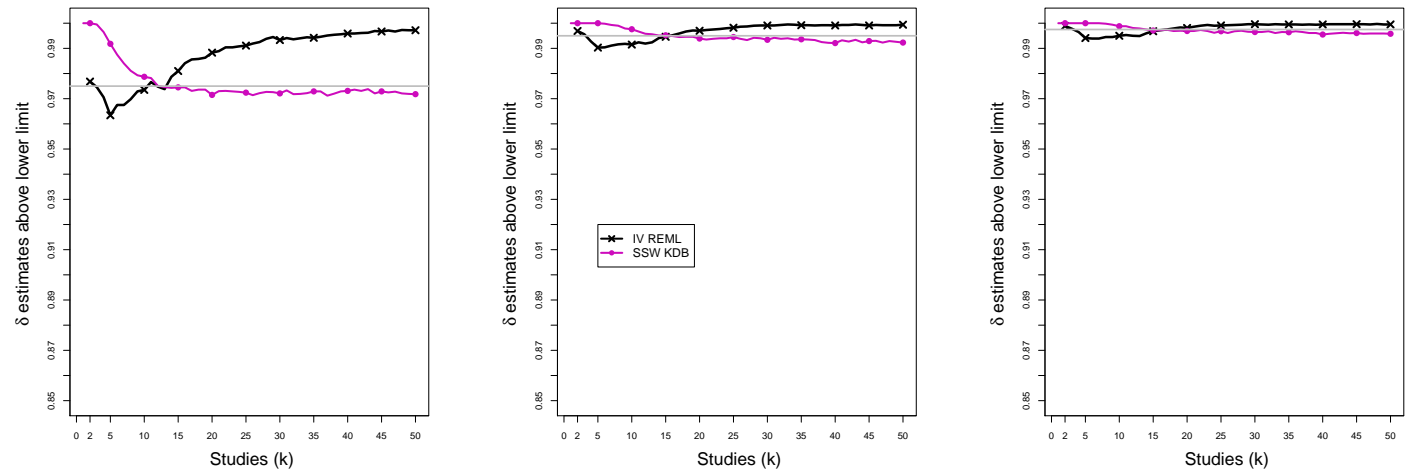

$\tau^{2}=0.25$ IV $=0.05 \mathrm{n}=50 \quad \delta=1$ to 2 (chg at 13 th)
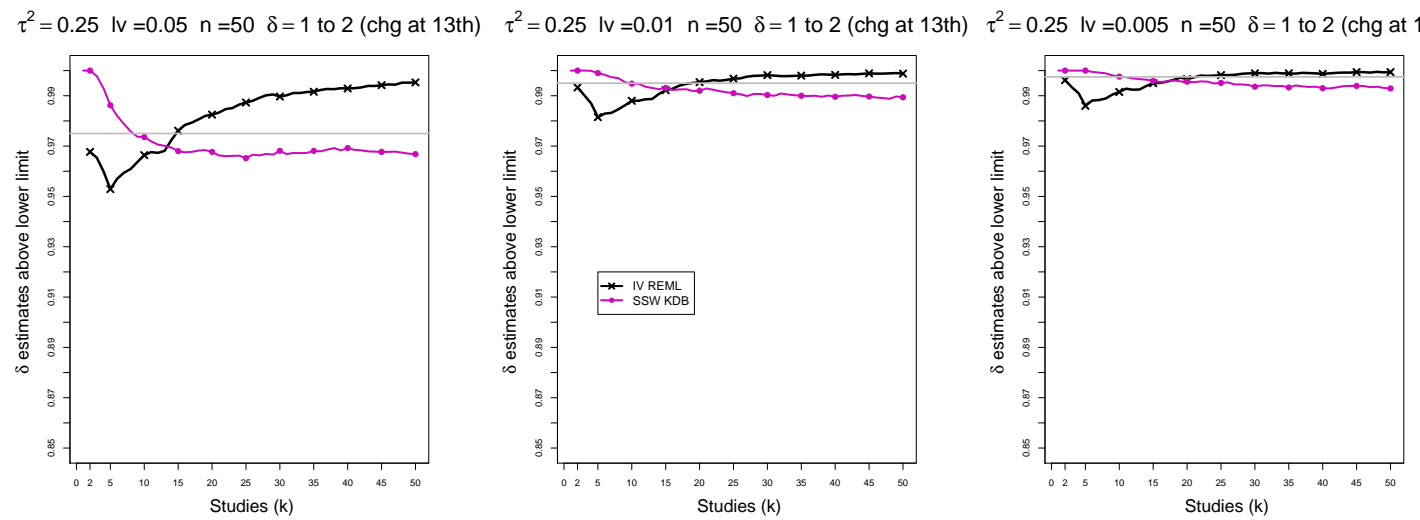

$\tau^{2}=1 \mathrm{lv}=0.05 \mathrm{n}=50 \quad \delta=1$ to 2 (chg at 13 th)
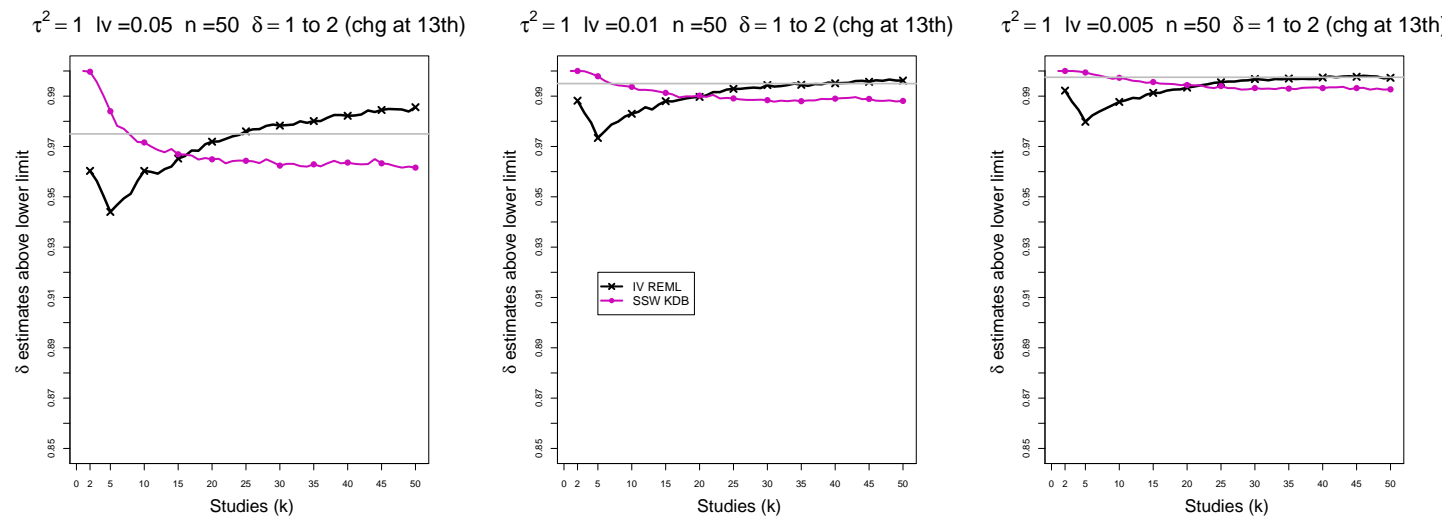

Figure D4. 2: 2-Stage CMA - Coverage of the lower confidence interval of $\delta$ (above lower limit) at the confidence level $1-\alpha / 2$ for $\alpha=0.05,0.01,0.005$ when $\delta=1$ for $k \leq 12$ followed by a shift to $\delta=2$ for $k \geq 13, \tau^{2}=0,0.1,0.25,1, n=50, K=50$. Light grey line at $0.975,0.995,0.9975$. 

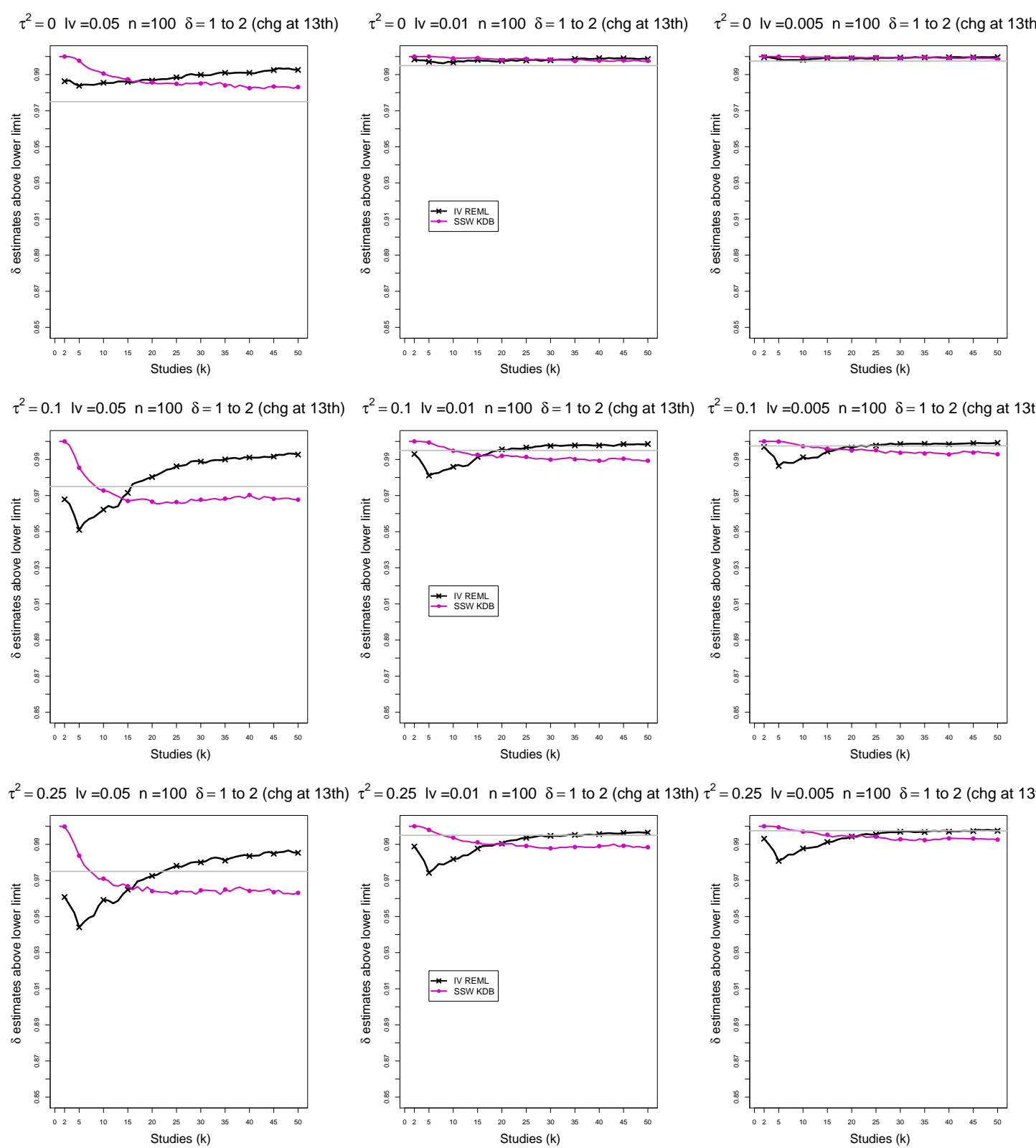

$\tau^{2}=1 \mathrm{lv}=0.05 \mathrm{n}=100 \delta=1$ to 2 (chg at 13 th)
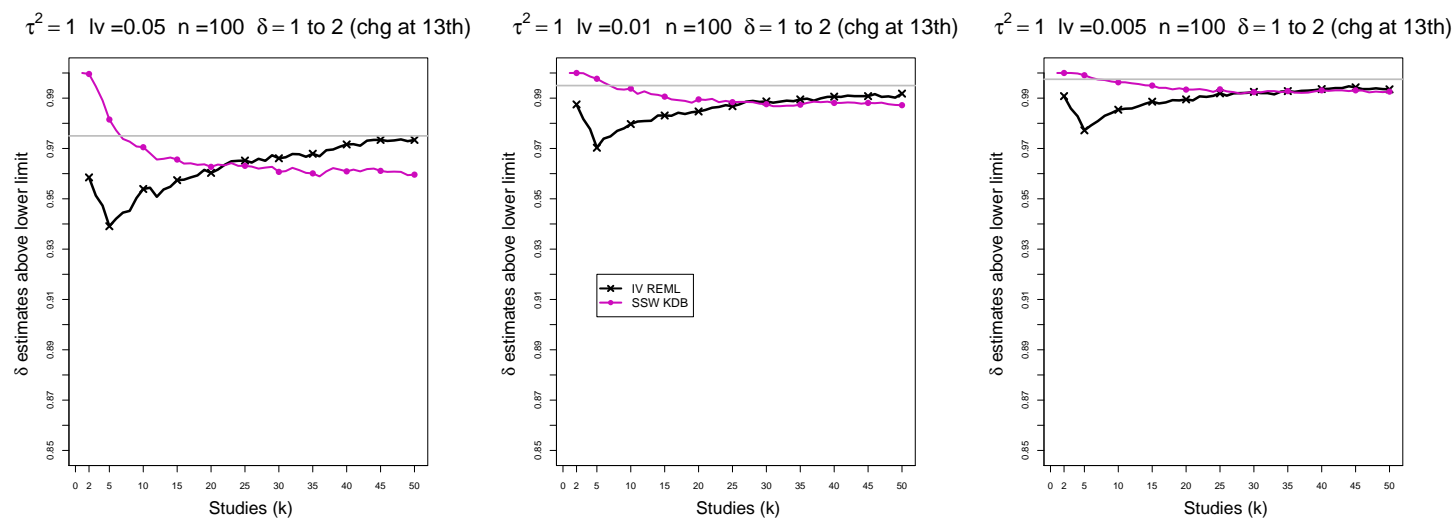

Figure D4. 3: 2-Stage CMA - Coverage of the lower confidence interval of $\delta$ (above lower limit) at the confidence level $1-\alpha / 2$ for $\alpha=0.05,0.01,0.005$ when $\delta=1$ for $k \leq 12$ followed by a shift to $\delta=2$ for $k \geq 13, \tau^{2}=0,0.1,0.25,1, n=100, K=50$. Light grey line at $0.975,0.995,0.9975$. 

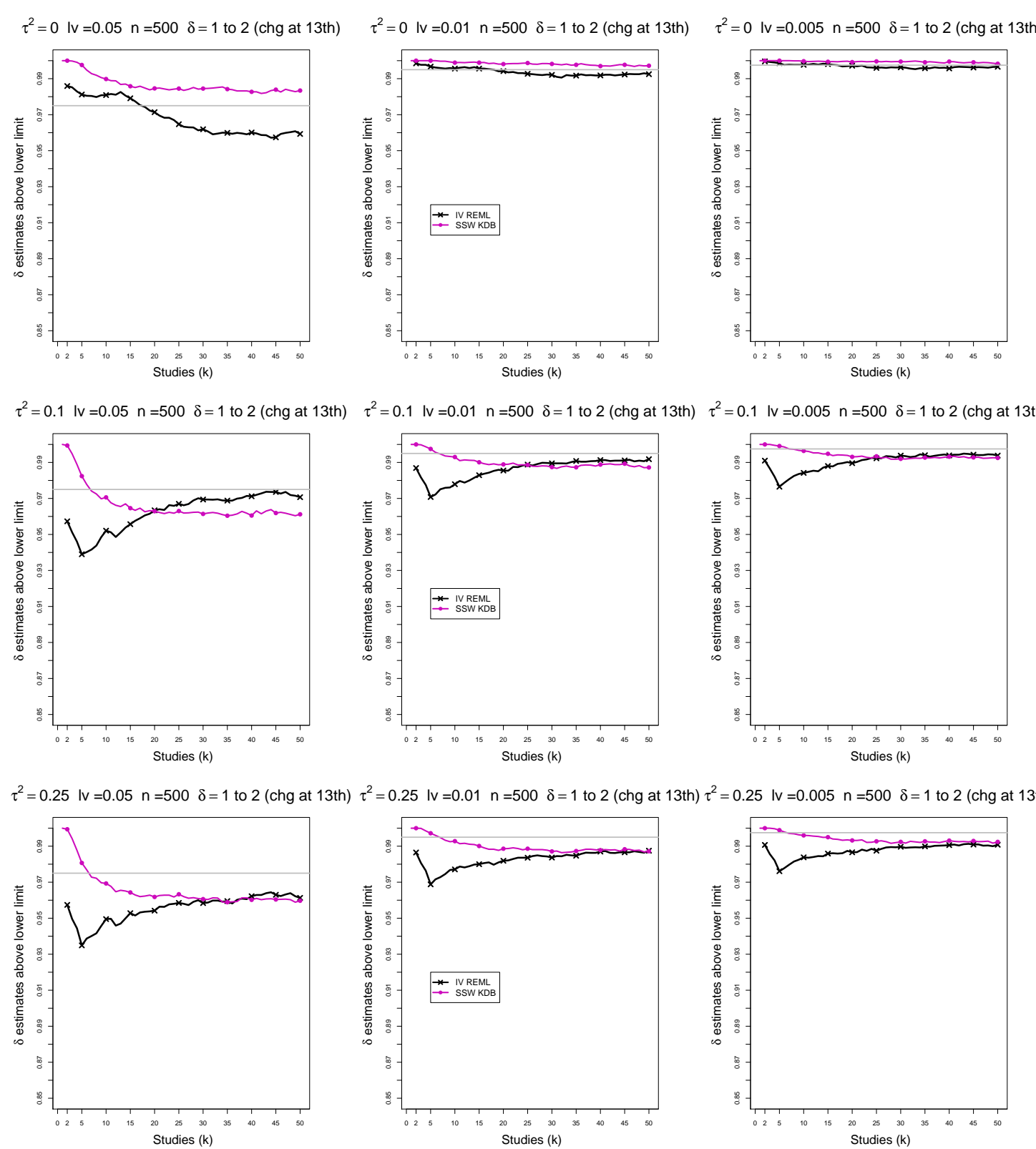

$\tau^{2}=1 \mathrm{lv}=0.05 \mathrm{n}=500 \delta=1$ to 2 (chg at 13 th)
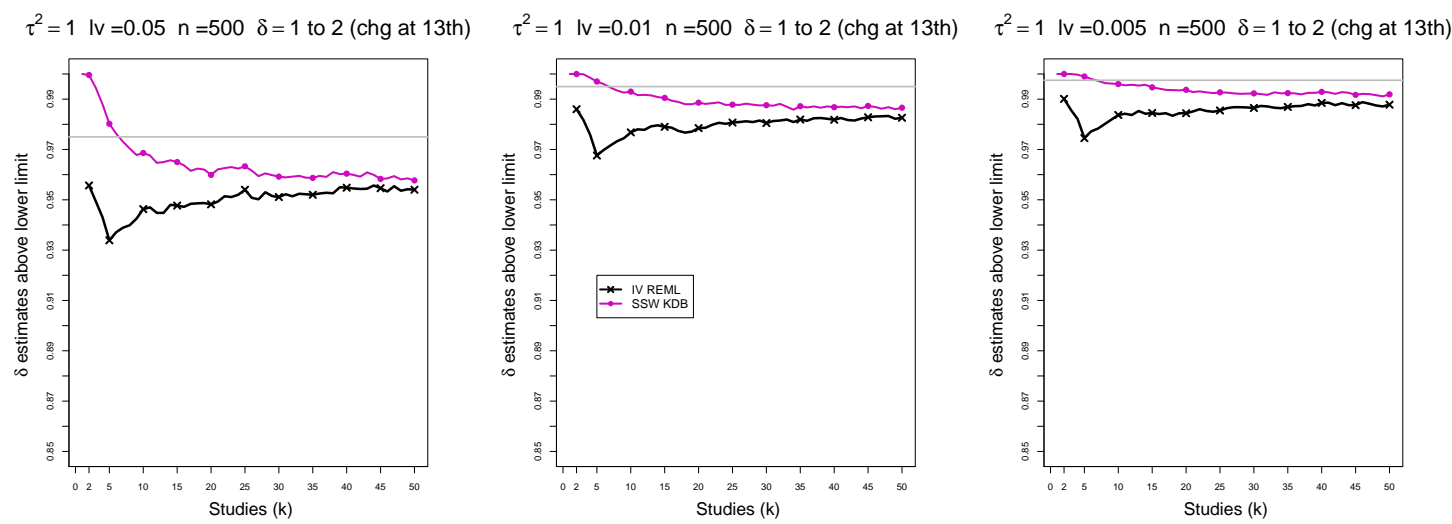

Figure D4. 4: 2-Stage CMA - Coverage of the lower confidence interval of $\delta$ (above lower limit) at the confidence level $1-\alpha / 2$ for $\alpha=0.05,0.01,0.005$ when $\delta=1$ for $k \leq 12$ followed by a shift to $\delta=2$ for $k \geq 13, \tau^{2}=0,0.1,0.25,1, n=500, K=50$. Light grey line at $0.975,0.995,0.9975$. 

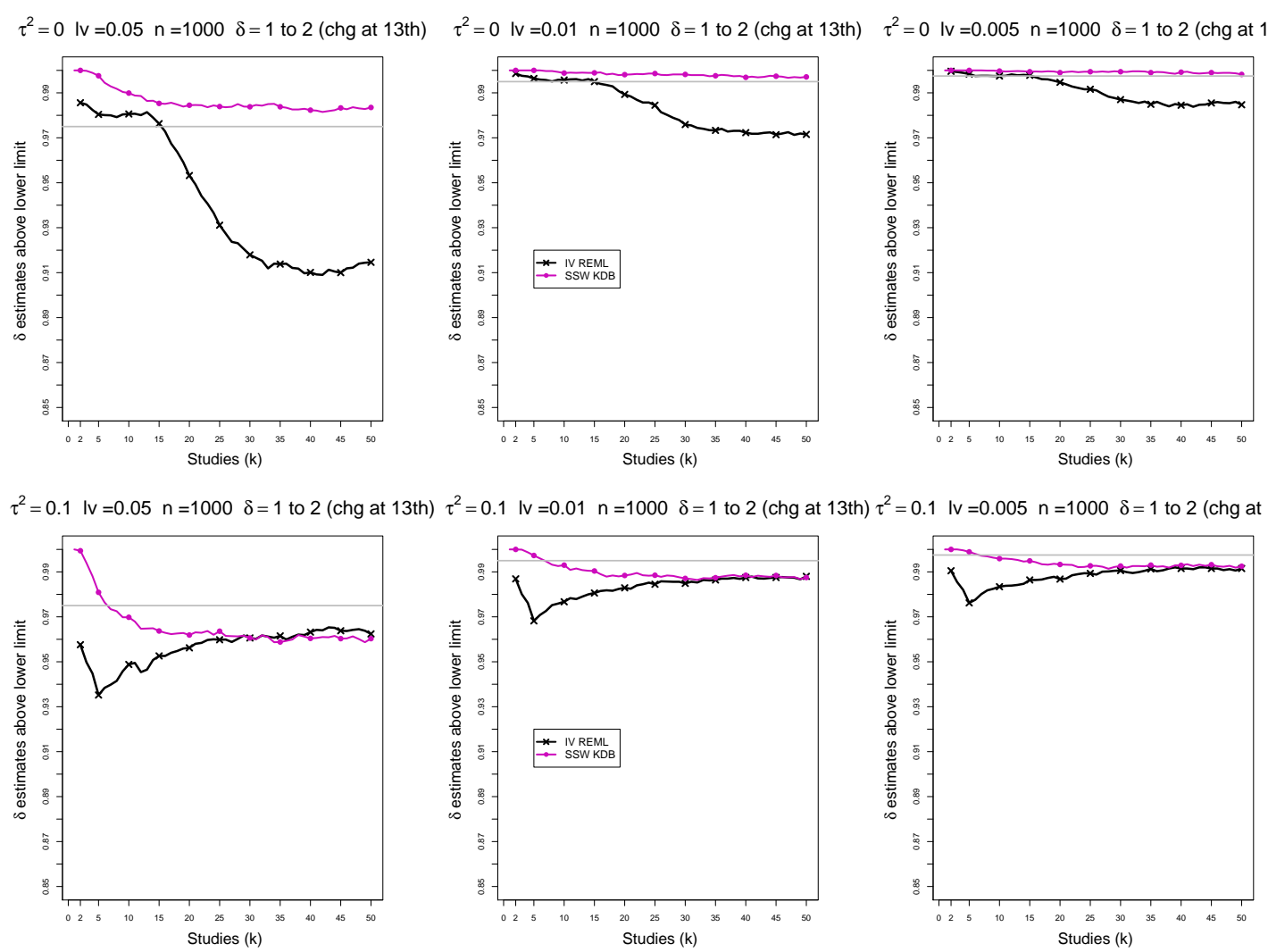

$\tau^{2}=0.25 \mathrm{lv}=0.05 \mathrm{n}=1000 \quad \delta=1$ to 2 (chg at 13 th $) \tau^{2}=0.25 \mathrm{lv}=0.01 \mathrm{n}=1000 \quad \delta=1$ to 2 (chg at 13 th) $)^{2}=0.25 \mathrm{lv}=0.005 \mathrm{n}=1000 \quad \delta=1$ to 2 (chg at 13 th
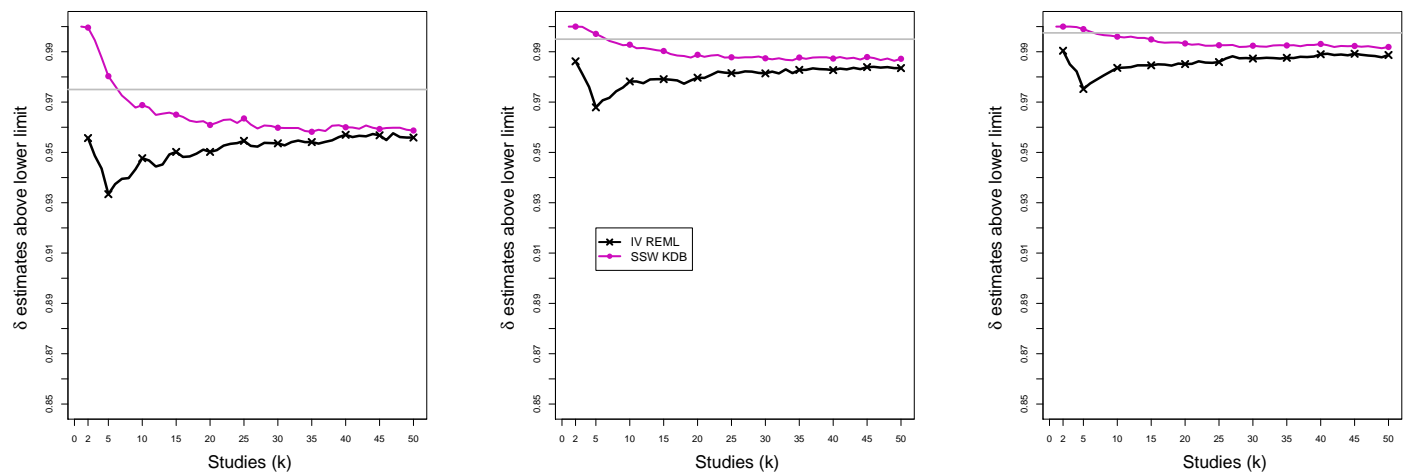

$\tau^{2}=1 \mathrm{lv}=0.05 \mathrm{n}=1000 \delta=1$ to 2 (chg at 13 th)
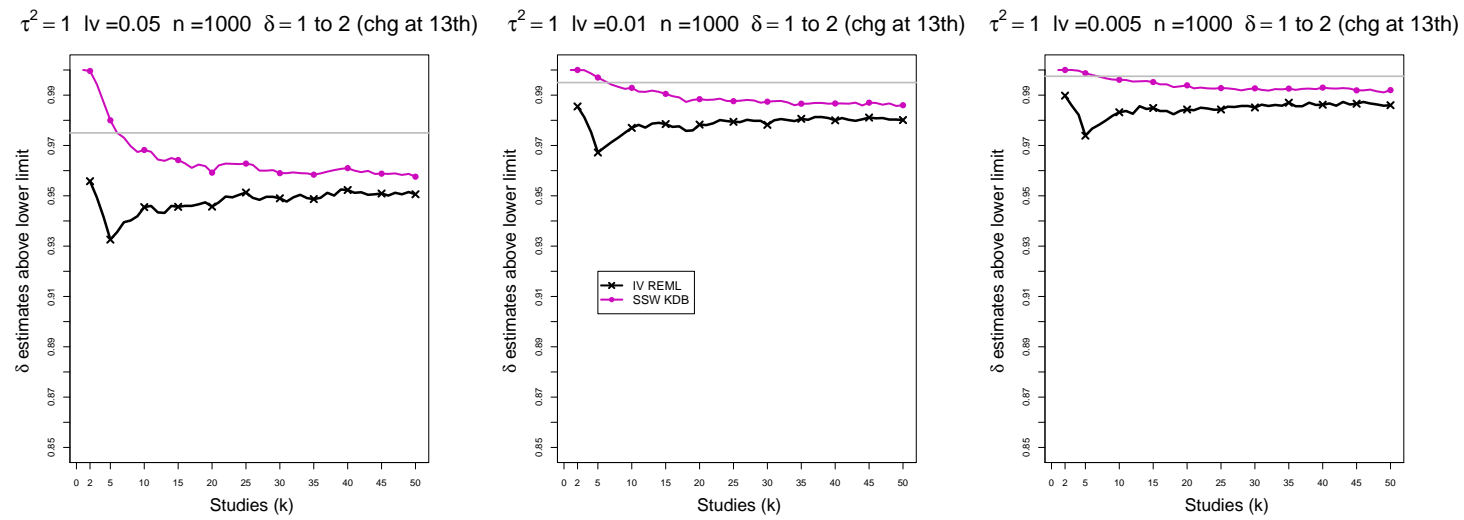

Figure D4. 5: 2-Stage CMA - Coverage of the lower confidence interval of $\delta$ (above lower limit) at the confidence level $1-\alpha / 2$ for $\alpha=0.05,0.01,0.005$ when $\delta=1$ for $k \leq 12$ followed by a shift to $\delta=2$ for $k \geq 13, \tau^{2}=0,0.1,0.25,1, n=1000, K=50$. Light grey line at $0.975,0.995,0.9975$. 

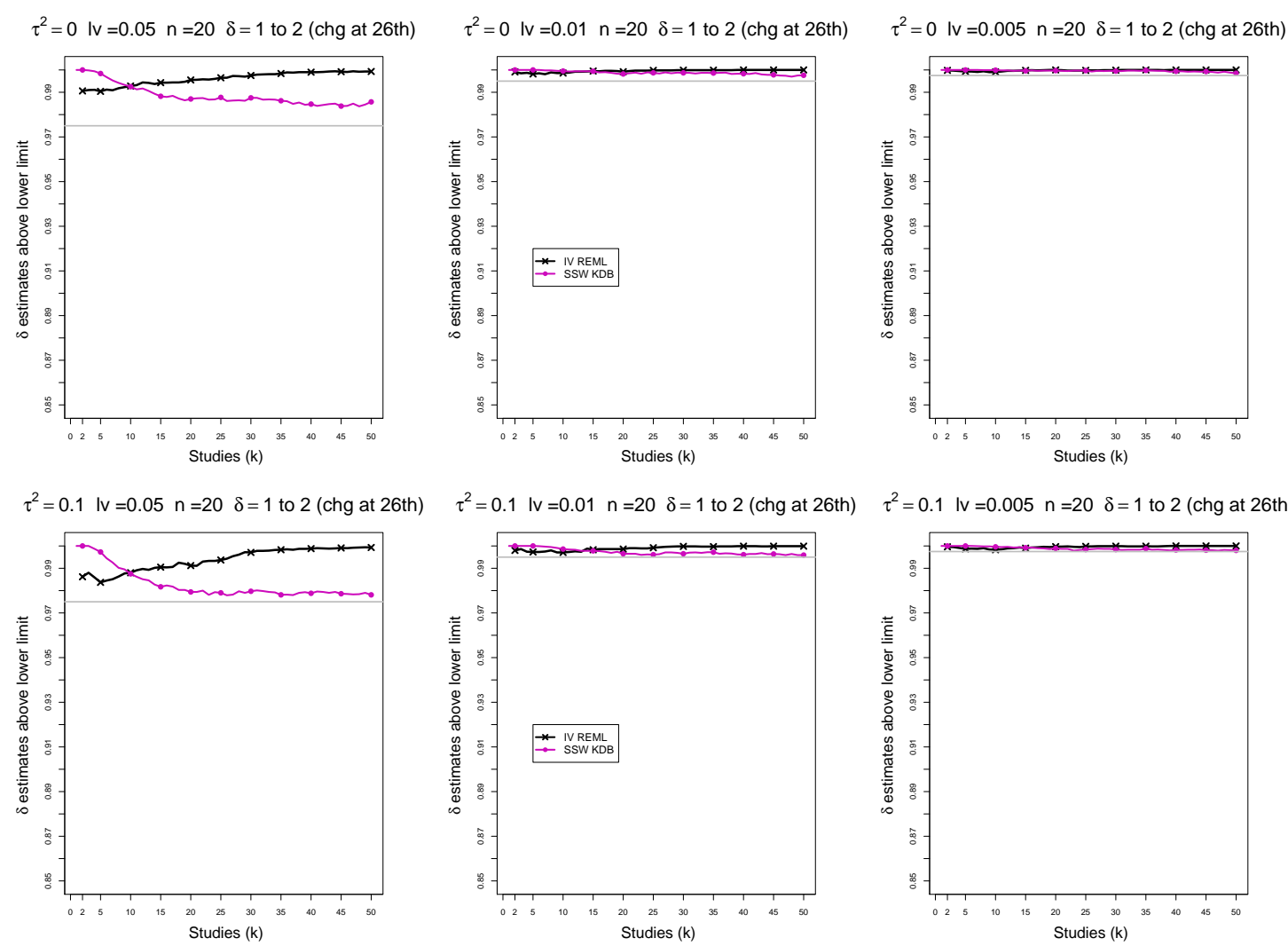

$\tau^{2}=0.1 \mathrm{lv}=0.005 \mathrm{n}=20 \delta=1$ to 2 (chg at 26th)
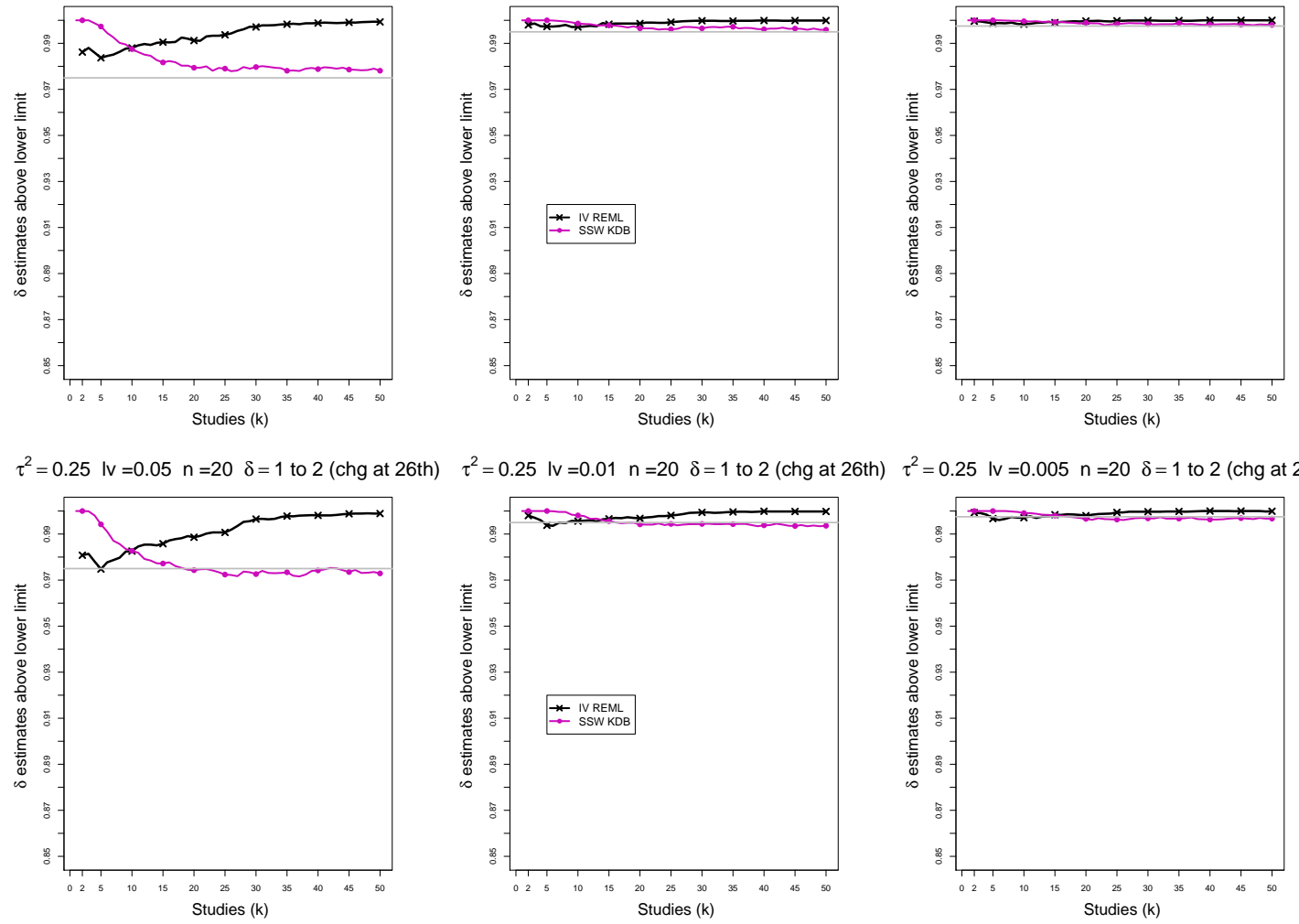

$\tau^{2}=1 \mathrm{lv}=0.05 \mathrm{n}=20 \quad \delta=1$ to 2 (chg at 26th)
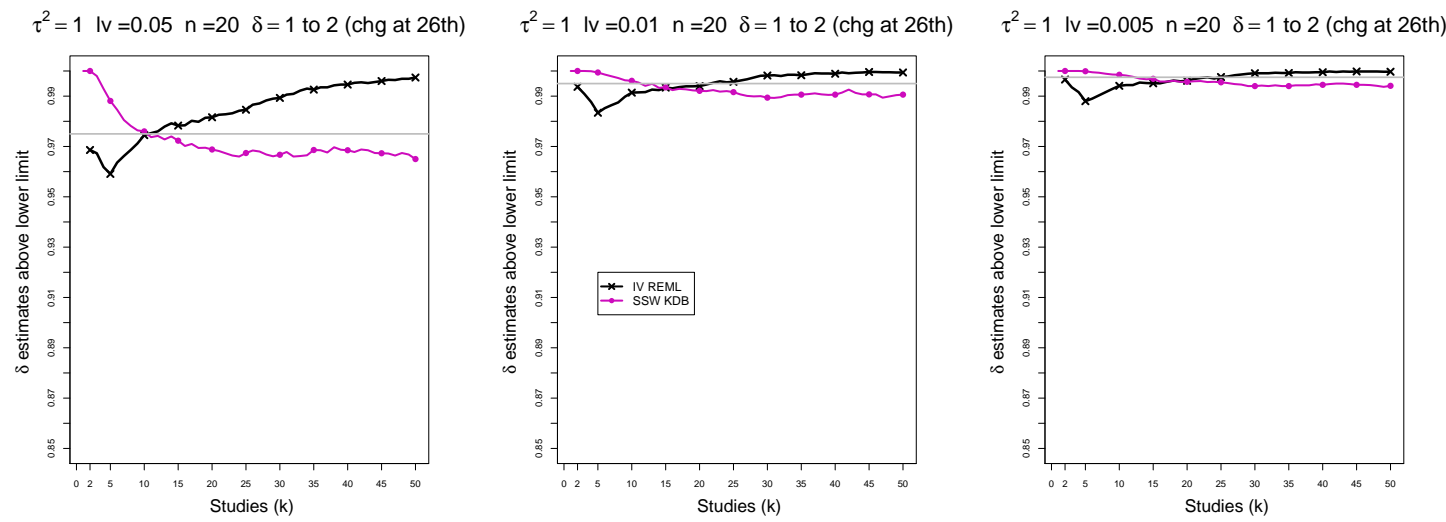

Figure D4. 6: 2-Stage CMA - Coverage of the lower confidence interval of $\delta$ (above lower limit) at the confidence level $1-\alpha / 2$ for $\alpha=0.05,0.01,0.005$ when $\delta=1$ for $k \leq 25$ followed by a shift to $\delta=2$ for $k \geq 26, \tau^{2}=0,0.1,0.25,1, n=20, K=50$. Light grey line at $0.975,0.995,0.9975$. 

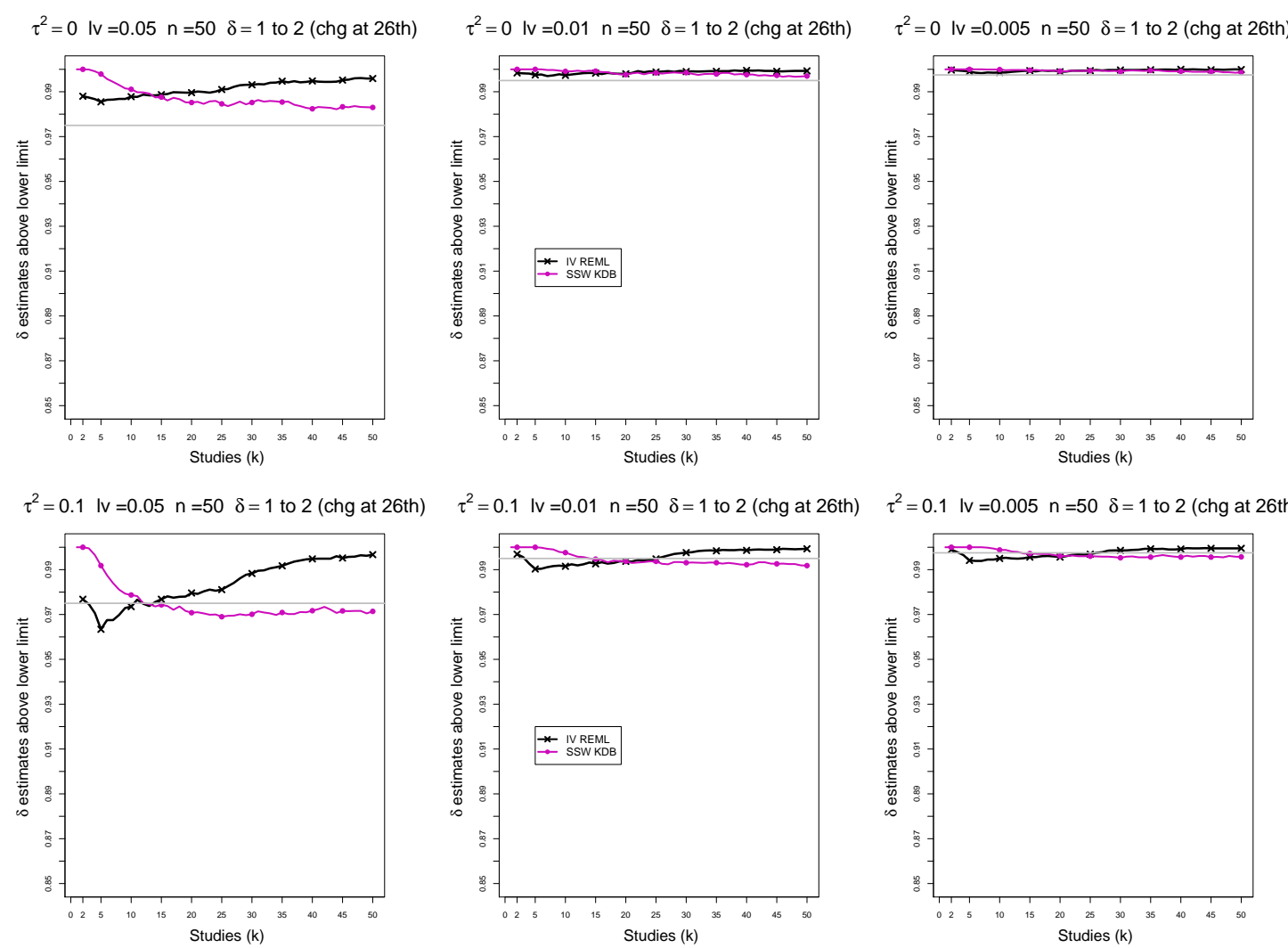

$\tau^{2}=0.1 \mathrm{lv}=0.005 \mathrm{n}=50 \delta=1$ to 2 (chg at 26th)

$\tau^{2}=0.25 \mathrm{lv}=0.05 \mathrm{n}=50 \quad \delta=1$ to 2 (chg at 26 th)
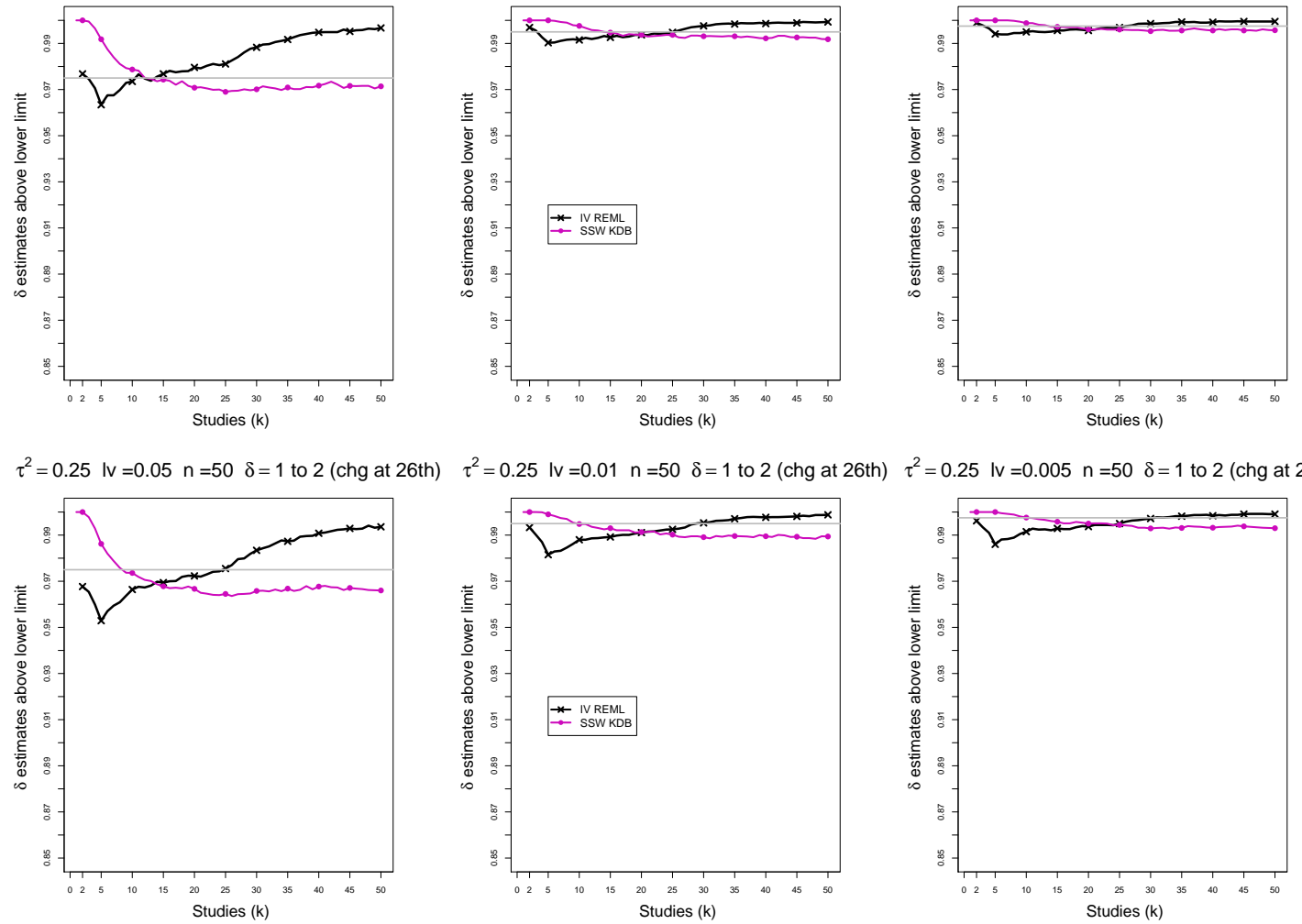

$\tau^{2}=1 \mathrm{lv}=0.05 \mathrm{n}=50 \quad \delta=1$ to 2 (chg at 26 th)
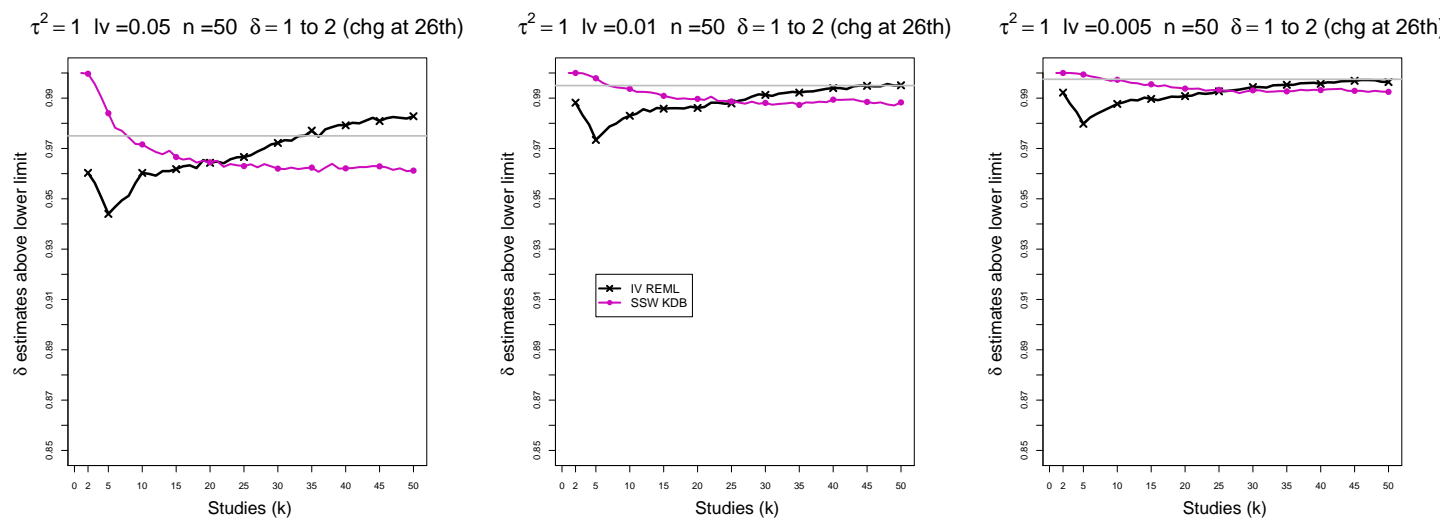

Figure D4. 7: 2-Stage CMA - Coverage of the lower confidence interval of $\delta$ (above lower limit) at the confidence level $1-\alpha / 2$ for $\alpha=0.05,0.01,0.005$ when $\delta=1$ for $k \leq 25$ followed by a shift to $\delta=2$ for $k \geq 26, \tau^{2}=0,0.1,0.25,1, n=50, K=50$. Light grey line at $0.975,0.995,0.9975$. 

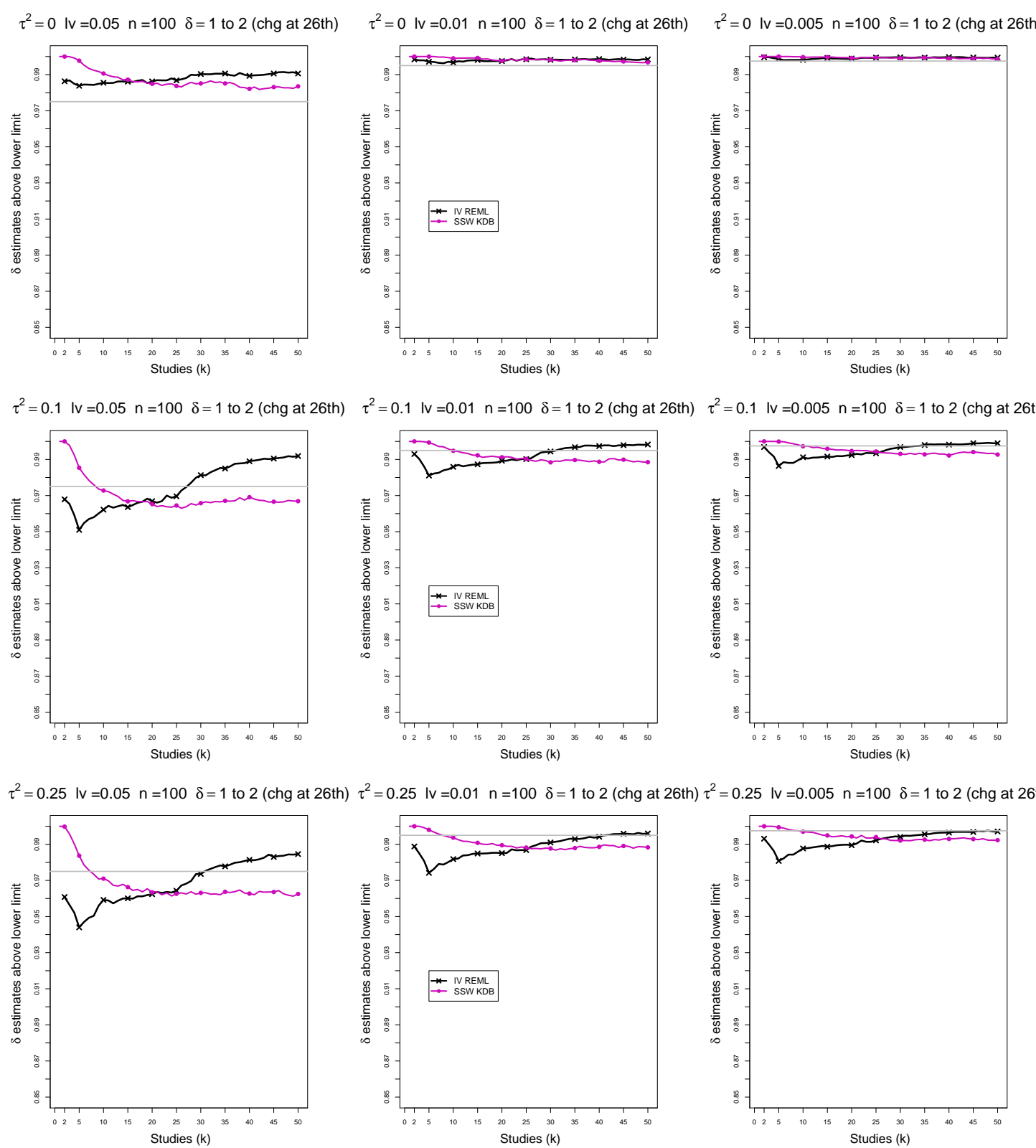

$\tau^{2}=1 \mathrm{lv}=0.05 \mathrm{n}=100 \delta=1$ to 2 (chg at 26 th)
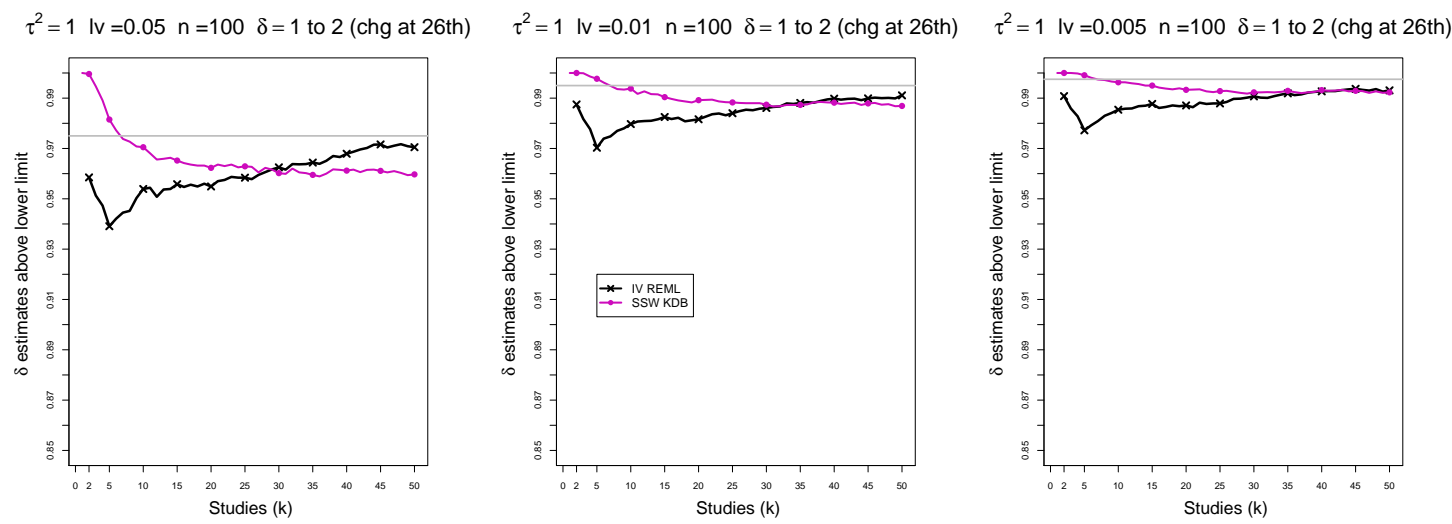

Figure D4. 8: 2-Stage CMA - Coverage of the lower confidence interval of $\delta$ (above lower limit) at the confidence level $1-\alpha / 2$ for $\alpha=0.05,0.01,0.005$ when $\delta=1$ for $k \leq 25$ followed by a shift to $\delta=2$ for $k \geq 26, \tau^{2}=0,0.1,0.25,1, n=100, K=50$. Light grey line at $0.975,0.995,0.9975$. 

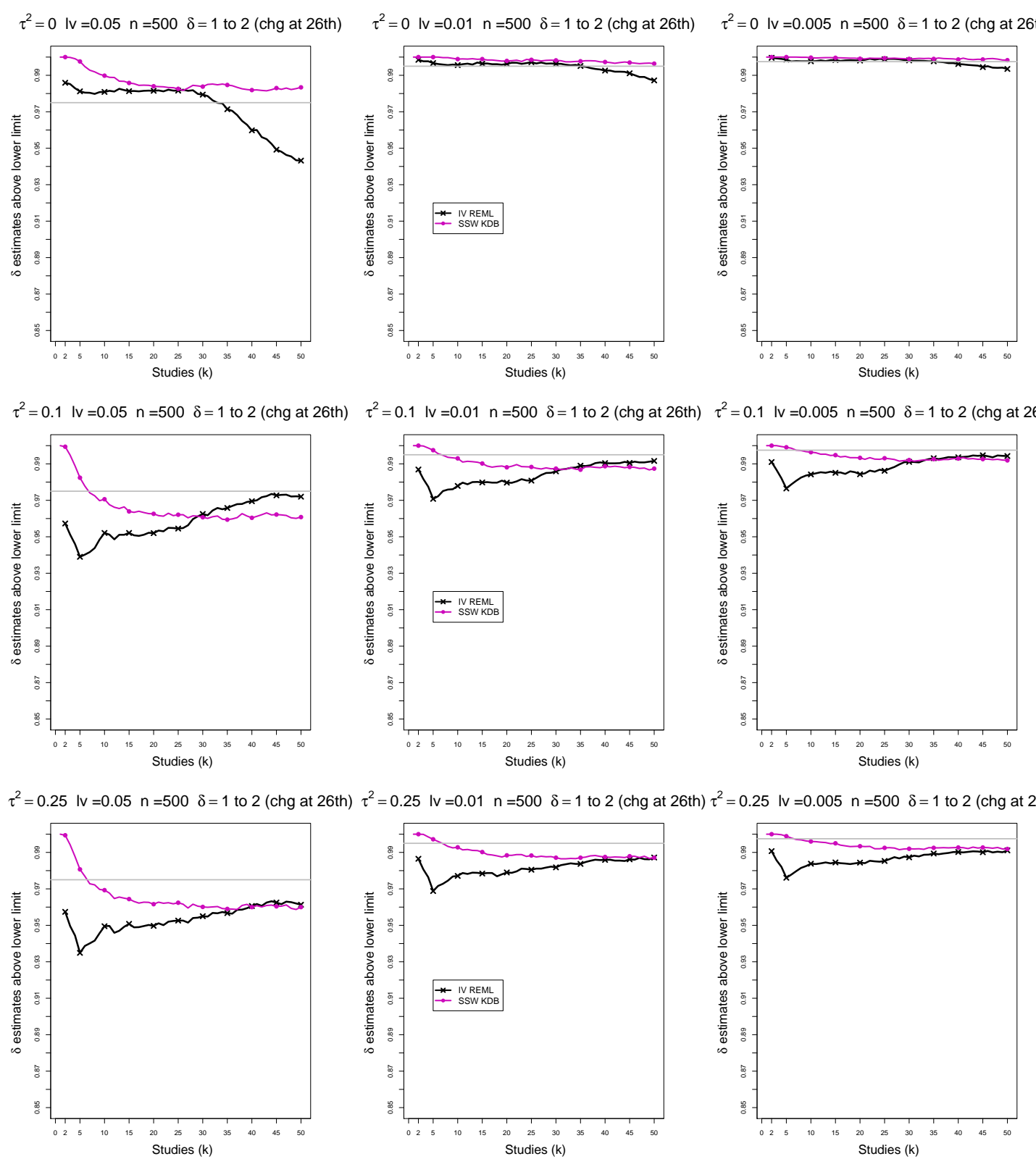

$\tau^{2}=1 \mathrm{lv}=0.05 \mathrm{n}=500 \delta=1$ to 2 (chg at 26 th)
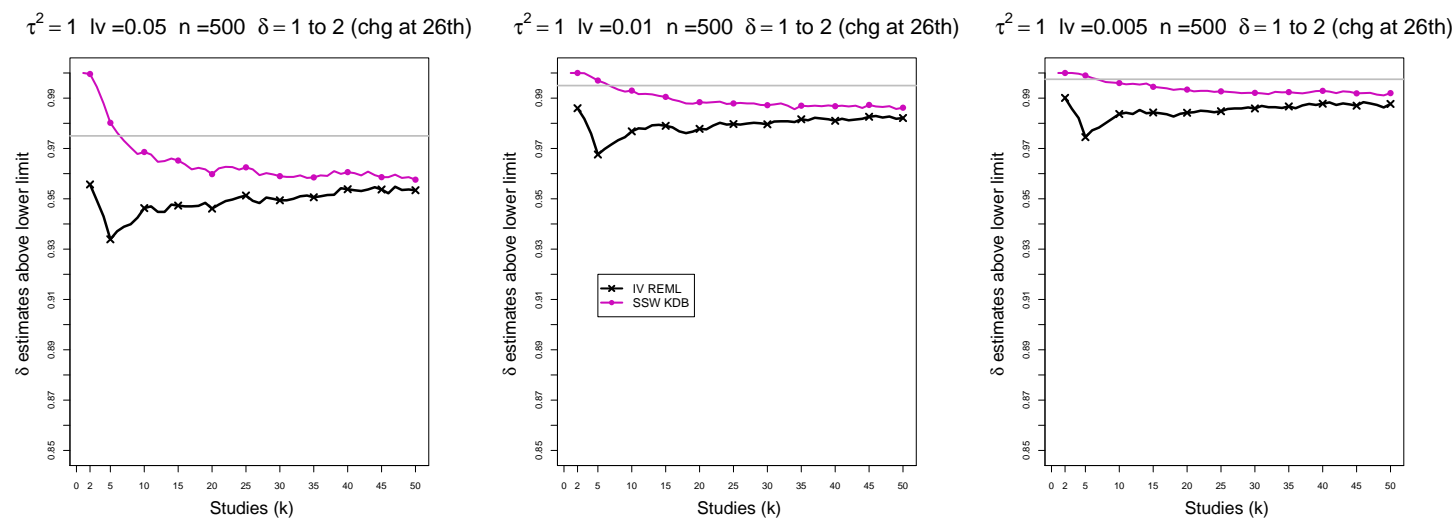

Figure D4. 9: 2-Stage CMA - Coverage of the lower confidence interval of $\delta$ (above lower limit) at the confidence level $1-\alpha / 2$ for $\alpha=0.05,0.01,0.005$ when $\delta=1$ for $k \leq 25$ followed by a shift to $\delta=2$ for $k \geq 26, \tau^{2}=0,0.1,0.25,1, n=500, K=50$. Light grey line at $0.975,0.995,0.9975$. 

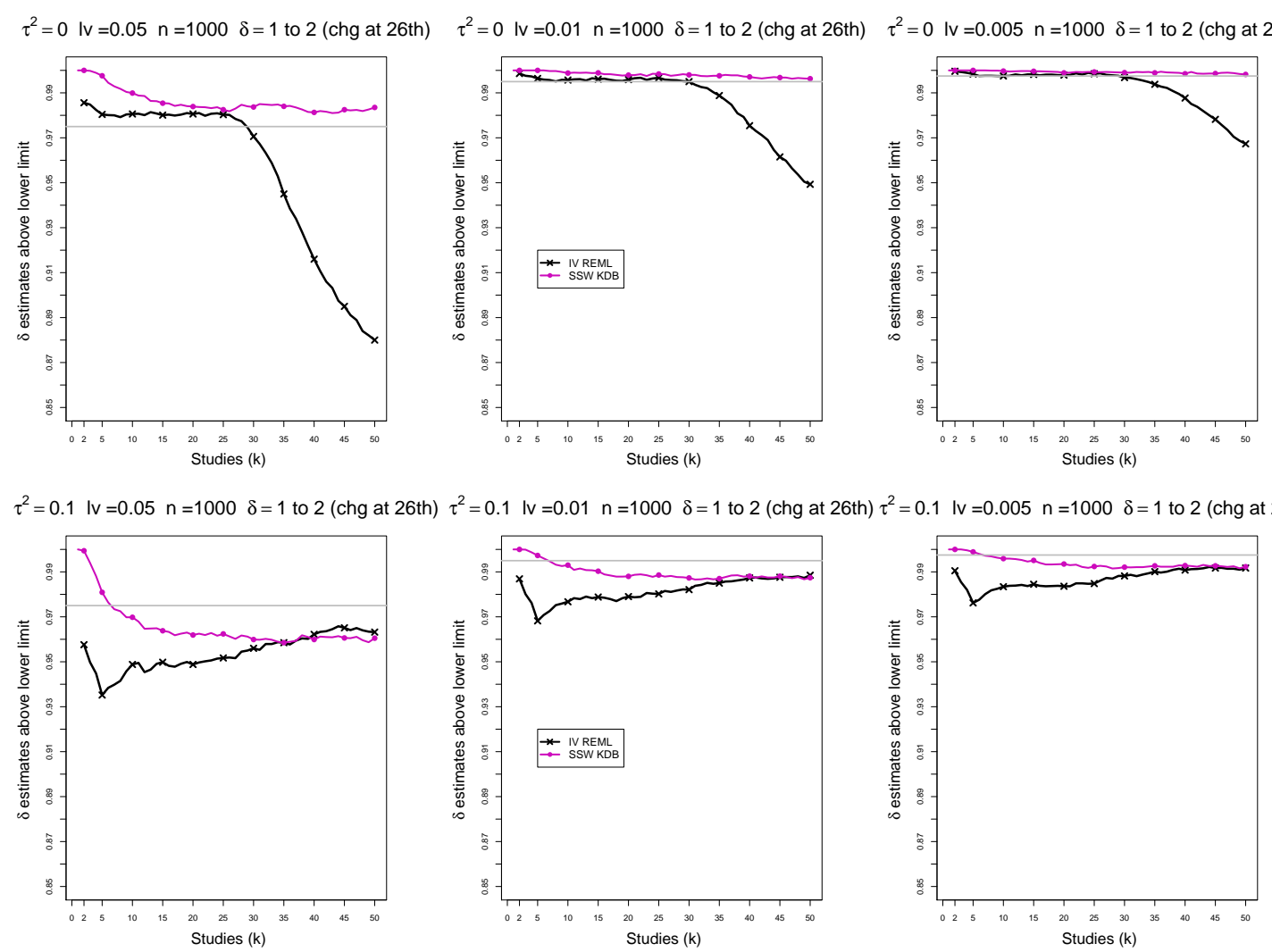

$\tau^{2}=0.25 \mathrm{IV}=0.05 \mathrm{n}=1000 \delta=1$ to 2 (chg at 26 th) $\tau^{2}=0.25 \mathrm{IV}=0.01 \mathrm{n}=1000 \quad \delta=1$ to 2 (chg at 26 th) $:^{2}=0.25 \mathrm{IV}=0.005 \mathrm{n}=1000 \quad \delta=1$ to 2 (chg at 26 th
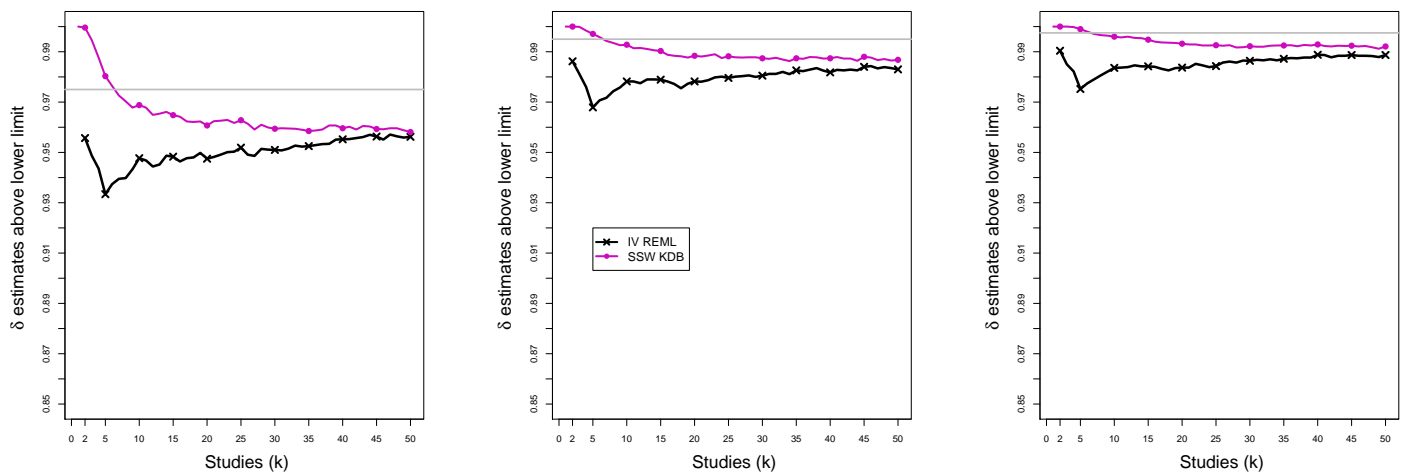

$\tau^{2}=1 \mathrm{IV}=0.05 \mathrm{n}=1000 \delta=1$ to 2 (chg at 26th)

$\tau^{2}=1$ IV $=0.01 \mathrm{n}=1000 \delta=1$ to 2 (chg at 26th)
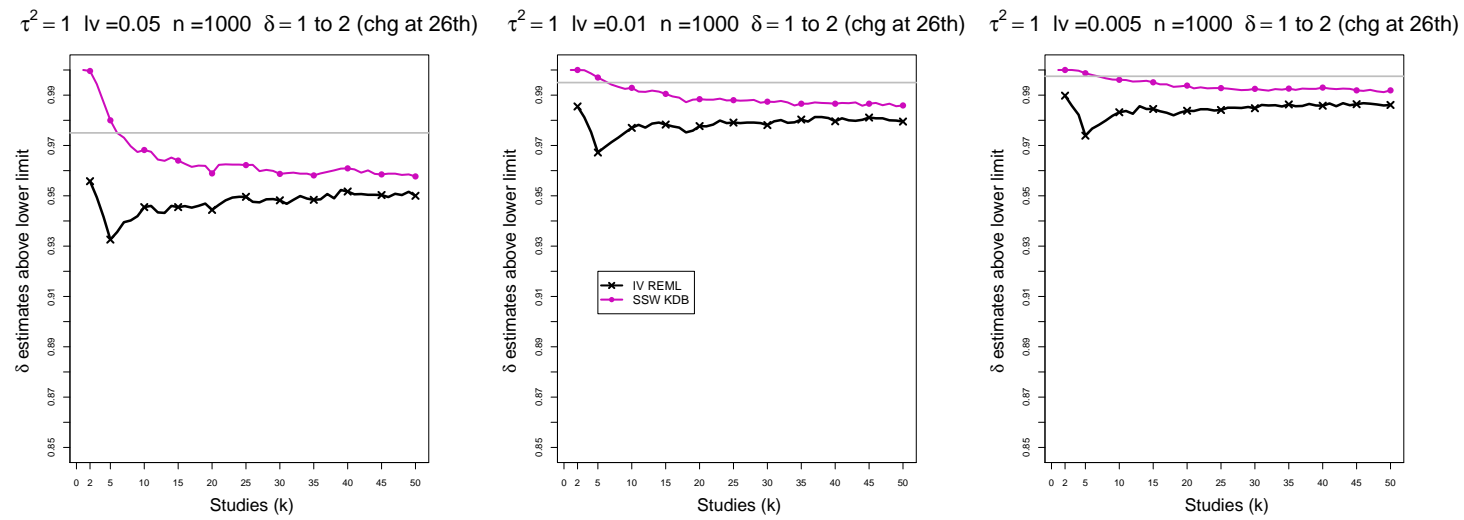

Figure D4. 10: 2-Stage CMA - Coverage of the lower confidence interval of $\delta$ (above lower limit) at the confidence level $1-\alpha / 2$ for $\alpha=0.05,0.01,0.005$ when $\delta=1$ for $k \leq 25$ followed by a shift to $\delta=2$ for $k \geq 26, \tau^{2}=0,0.1,0.25,1, n=1000, K=50$. Light grey line at $0.975,0.995,0.9975$. 

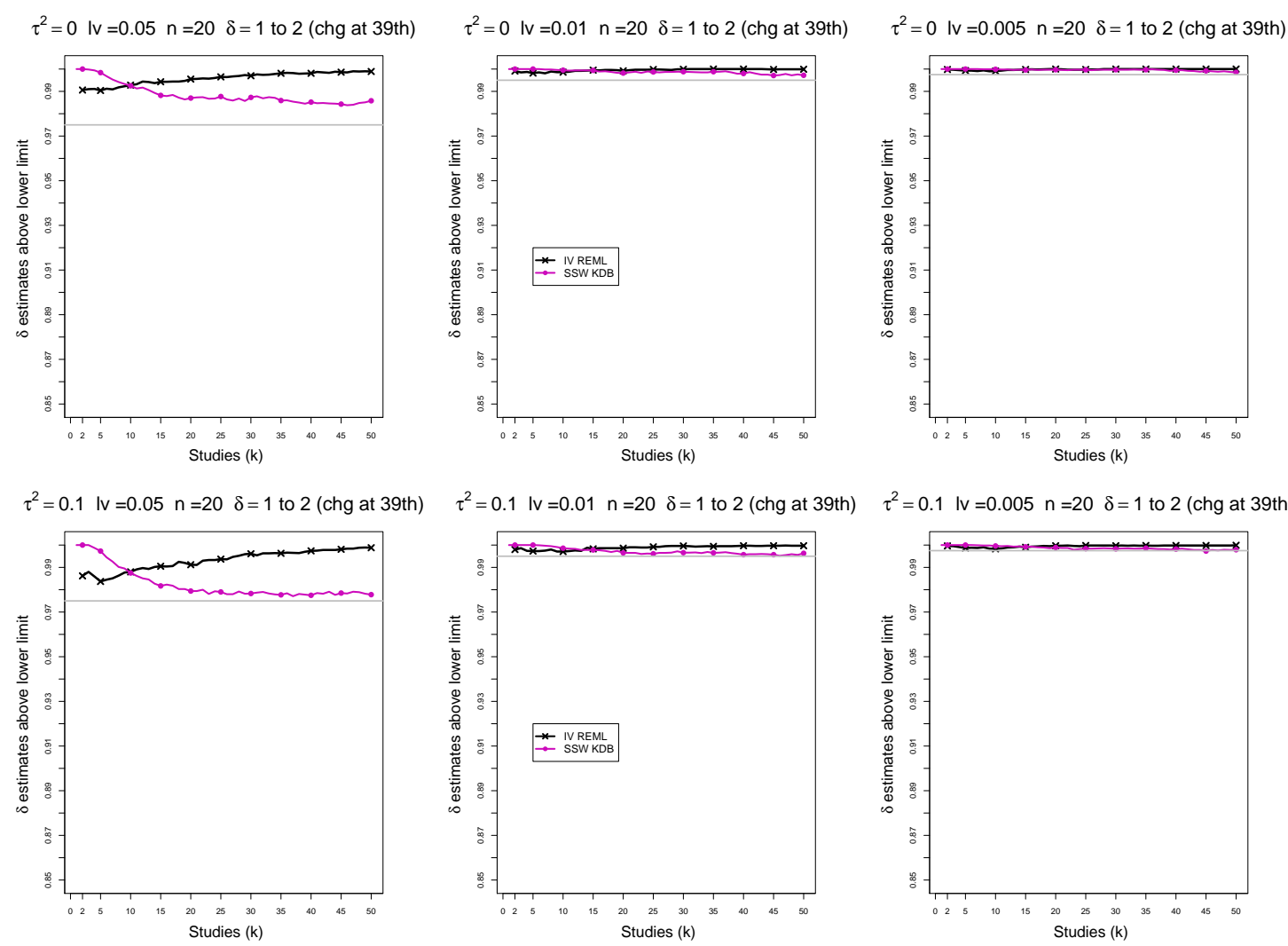

$\tau^{2}=0.1 \mathrm{lv}=0.005 \mathrm{n}=20 \quad \delta=1$ to 2 (chg at 39 th)

$\tau^{2}=0.25 \mathrm{lv}=0.05 \mathrm{n}=20 \quad \delta=1$ to 2 (chg at 39 th)
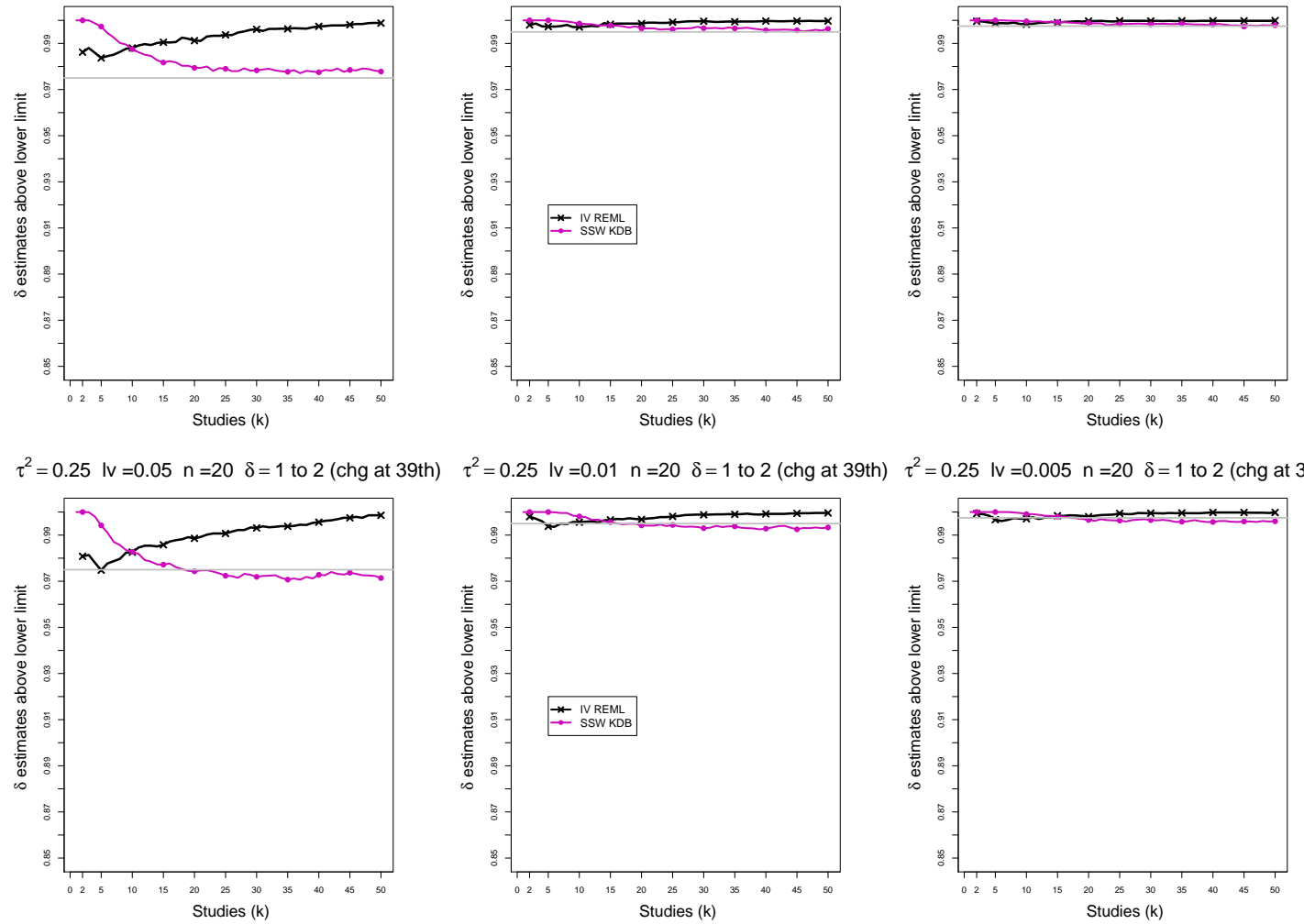

$\tau^{2}=1 \mathrm{lv}=0.05 \mathrm{n}=20 \quad \delta=1$ to 2 (chg at 39 th)
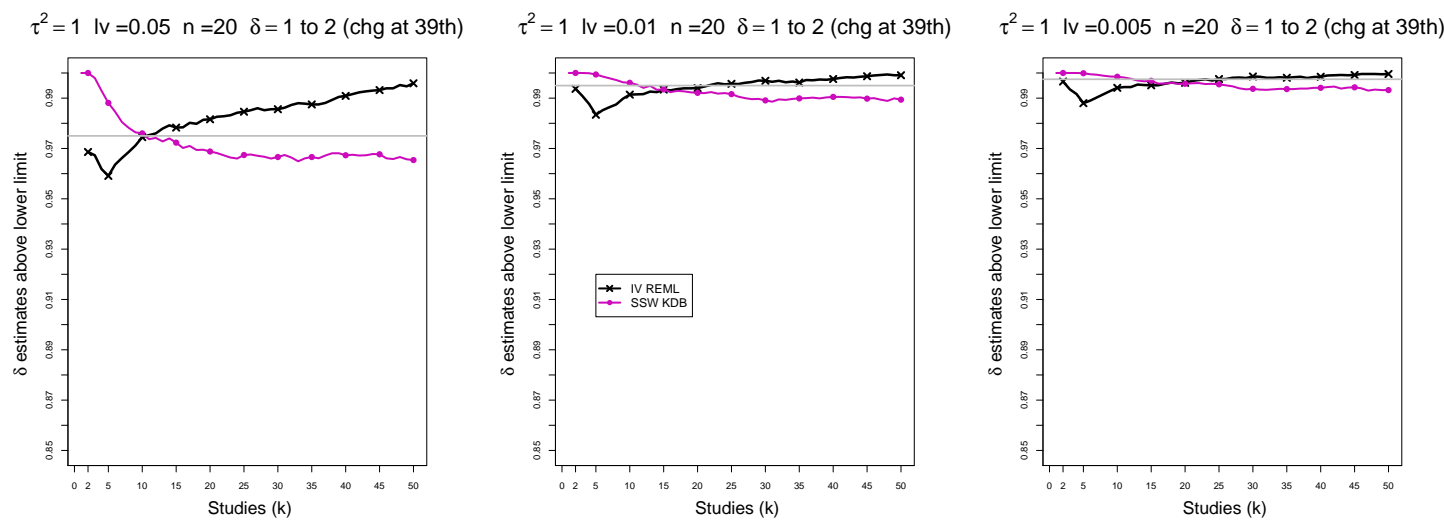

Figure D4. 11: 2-Stage CMA - Coverage of the lower confidence interval of $\delta$ (above lower limit) at the confidence level $1-\alpha / 2$ for $\alpha=0.05,0.01,0.005$ when $\delta=1$ for $k \leq 38$ followed by a shift to $\delta=2$ for $k \geq 39, \tau^{2}=0,0.1,0.25,1, n=20, K=50$. Light grey line at $0.975,0.995,0.9975$. 

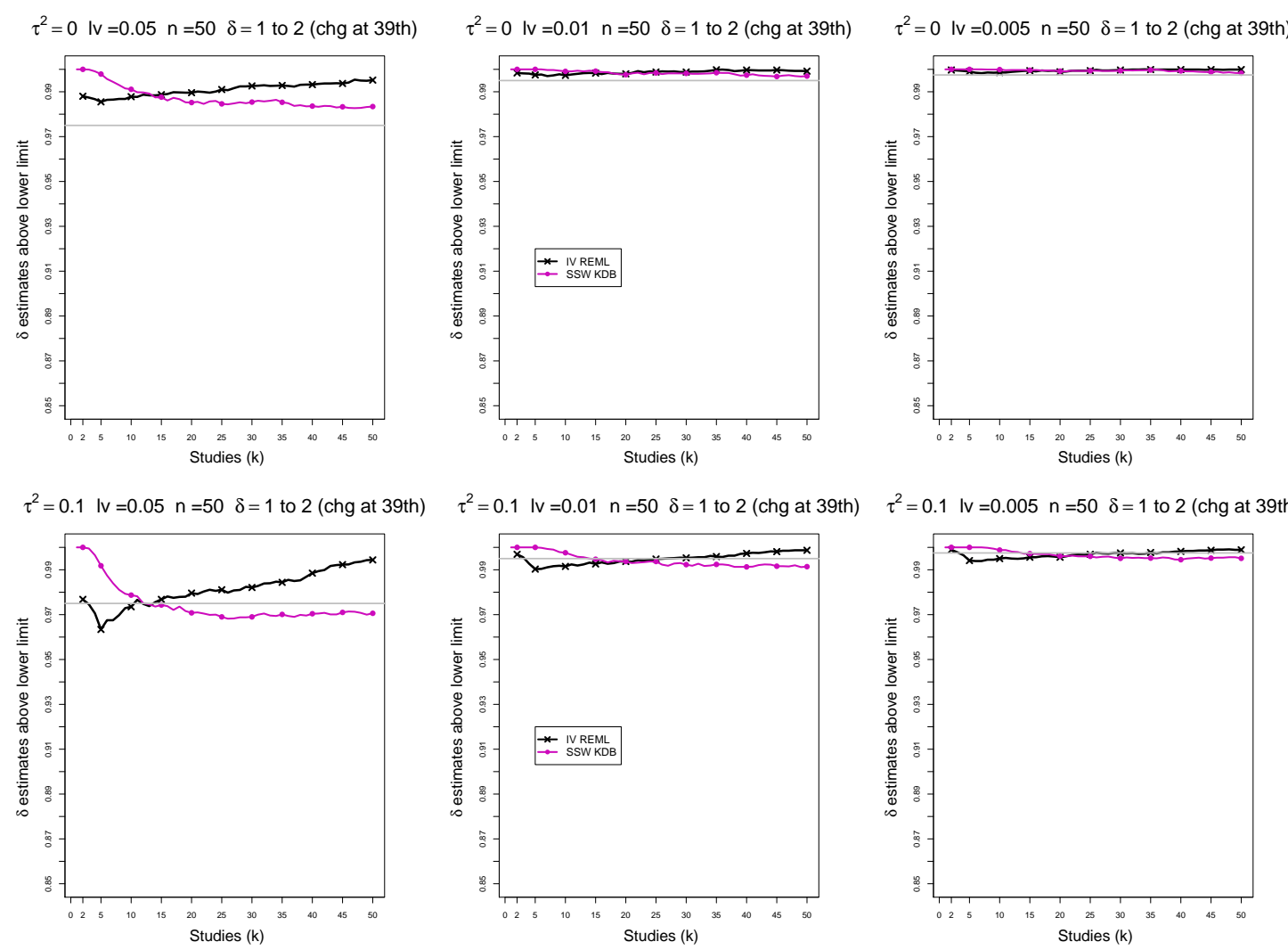

$\tau^{2}=0.1 \mathrm{lv}=0.005 \mathrm{n}=50 \delta=1$ to 2 (chg at 39 th)

$\tau^{2}=0.25 \mathrm{lv}=0.05 \mathrm{n}=50 \quad \delta=1$ to 2 (chg at 39 th)
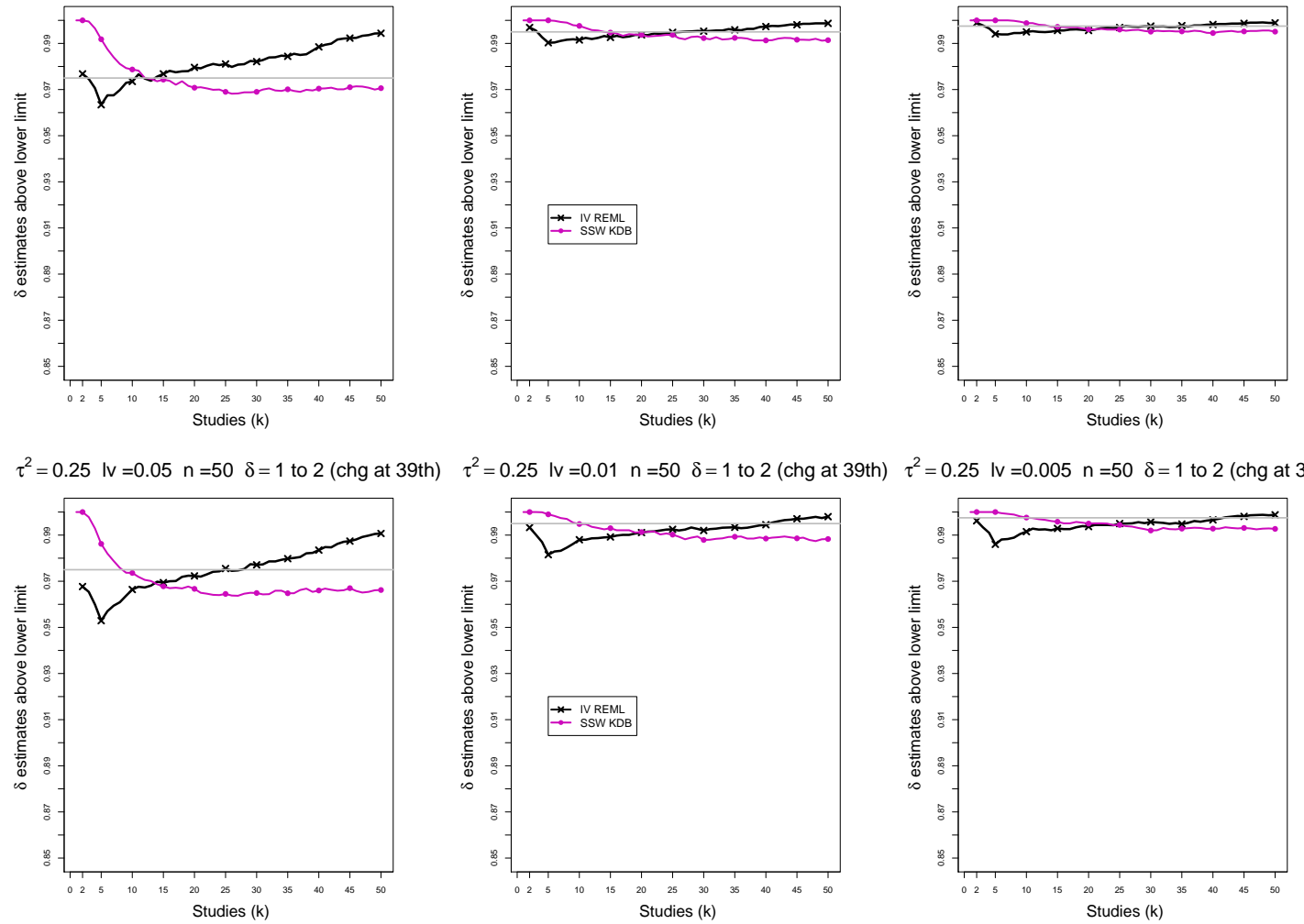

$\tau^{2}=1 \mathrm{lv}=0.05 \mathrm{n}=50 \quad \delta=1$ to 2 (chg at 39 th)
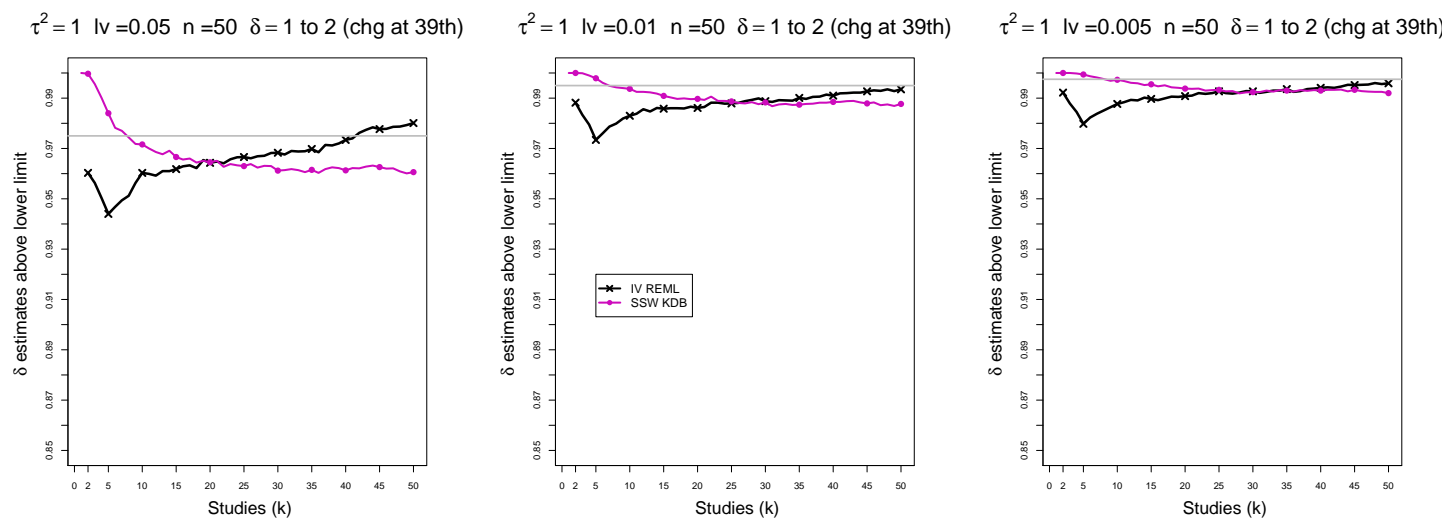

Figure D4. 12: 2-Stage CMA - Coverage of the lower confidence interval of $\delta$ (above lower limit) at the confidence level $1-\alpha / 2$ for $\alpha=0.05,0.01,0.005$ when $\delta=1$ for $k \leq 38$ followed by a shift to $\delta=2$ for $k \geq 39, \tau^{2}=0,0.1,0.25,1, n=50, K=50$. Light grey line at $0.975,0.995,0.9975$. 

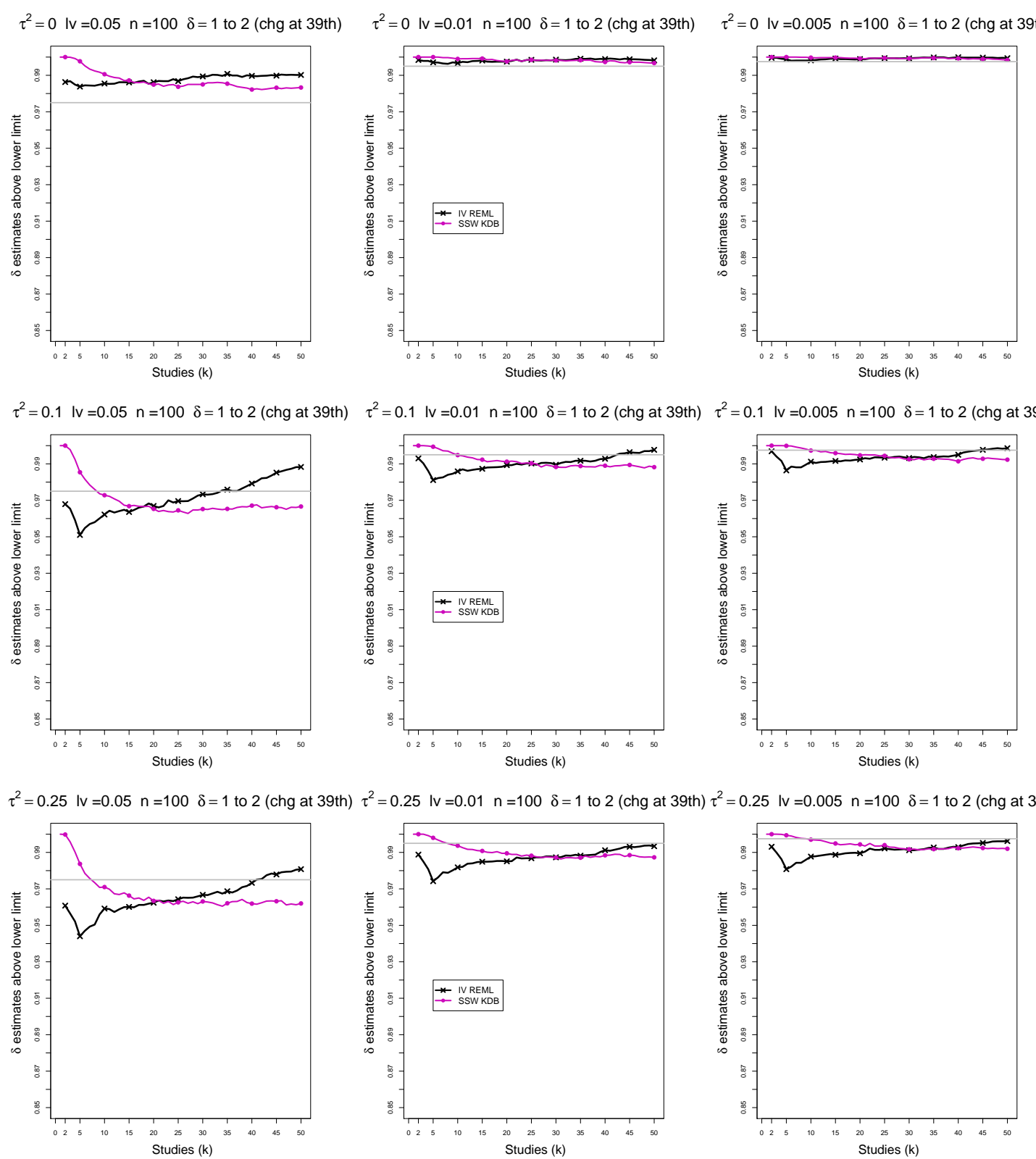

$\tau^{2}=1 \mathrm{lv}=0.05 \mathrm{n}=100 \delta=1$ to 2 (chg at 39 th)
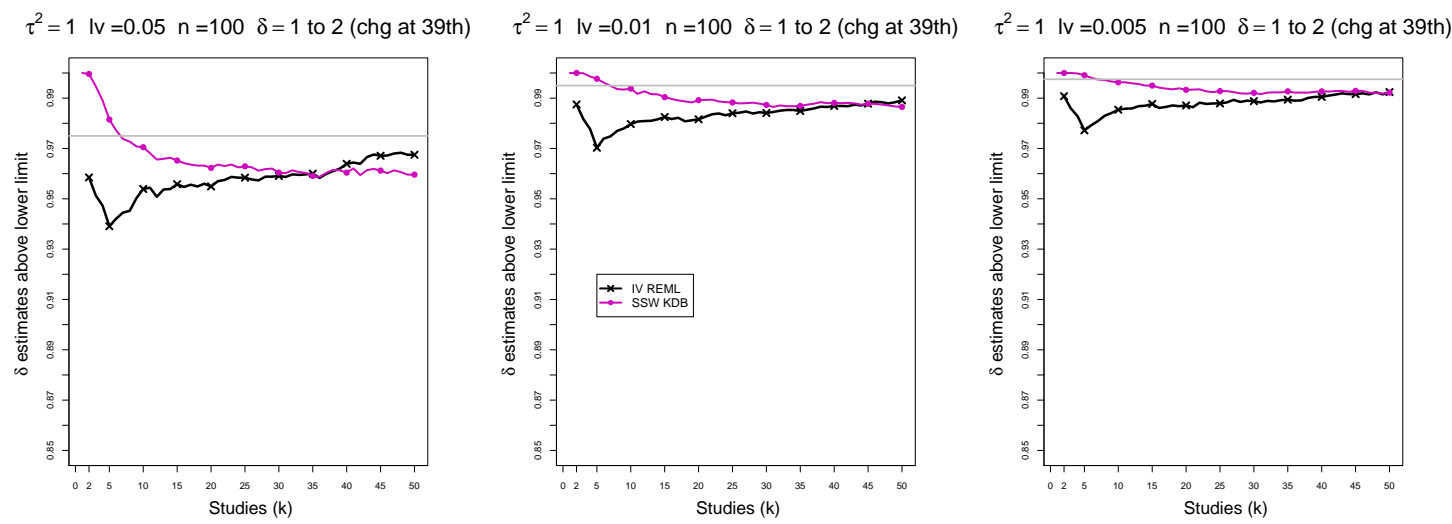

Figure D4. 13: 2-Stage CMA - Coverage of the lower confidence interval of $\delta$ (above lower limit) at the confidence level $1-\alpha / 2$ for $\alpha=0.05,0.01,0.005$ when $\delta=1$ for $k \leq 38$ followed by a shift to $\delta=2$ for $k \geq 39, \tau^{2}=0,0.1,0.25,1, n=100, K=50$. Light grey line at $0.975,0.995,0.9975$. 

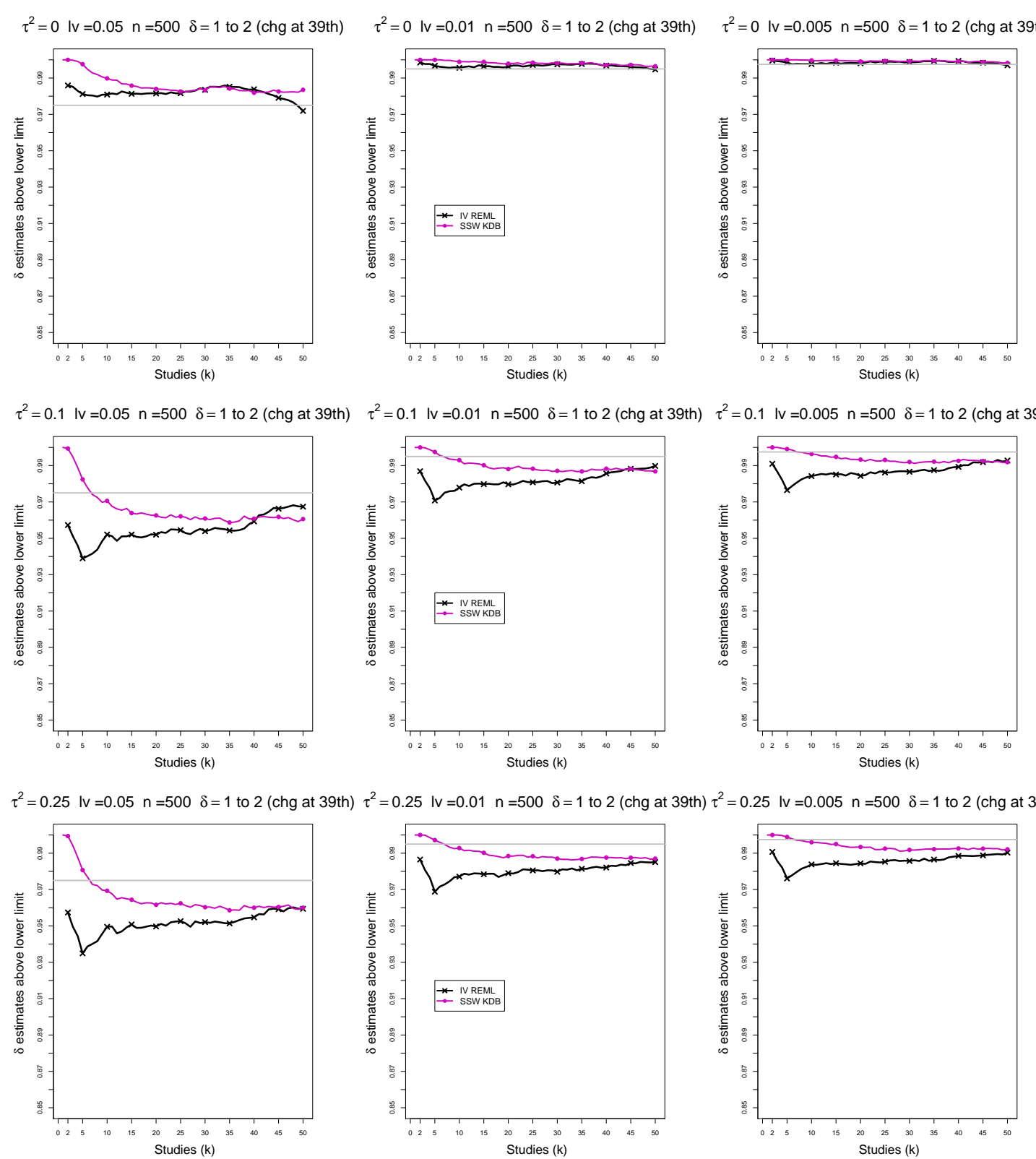

$\tau^{2}=1 \mathrm{lv}=0.05 \mathrm{n}=500 \delta=1$ to 2 (chg at 39 th)
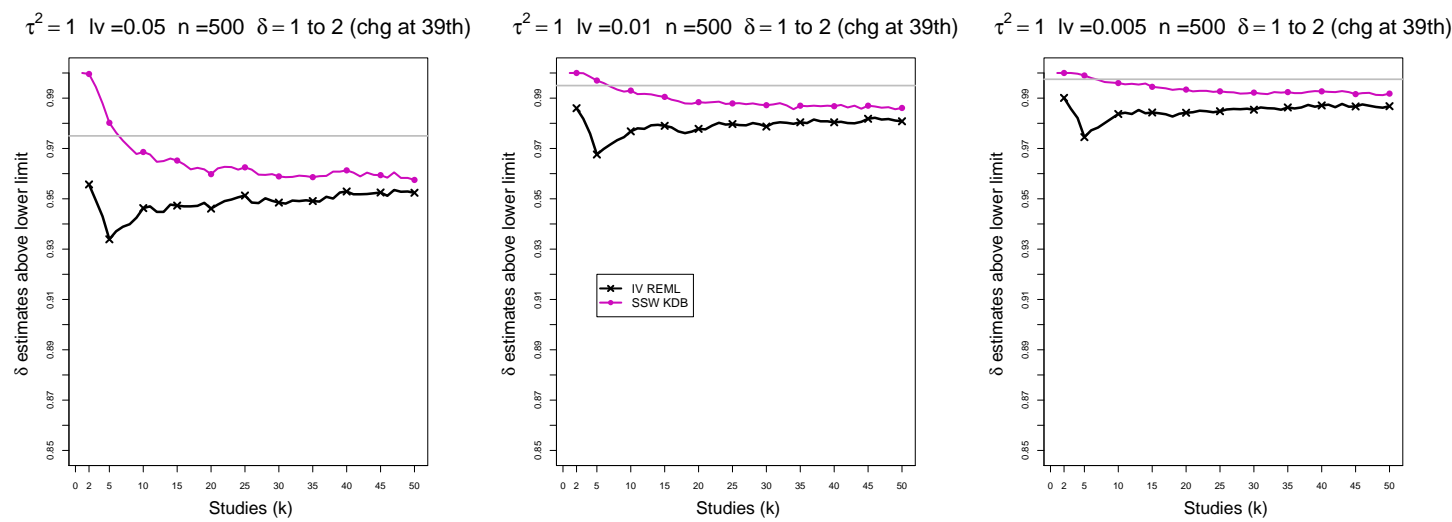

Figure D4. 14: 2-Stage CMA - Coverage of the lower confidence interval of $\delta$ (above lower limit) at the confidence level $1-\alpha / 2$ for $\alpha=0.05,0.01,0.005$ when $\delta=1$ for $k \leq 38$ followed by a shift to $\delta=2$ for $k \geq 39, \tau^{2}=0,0.1,0.25,1, n=500, K=50$. Light grey line at $0.975,0.995,0.9975$. 

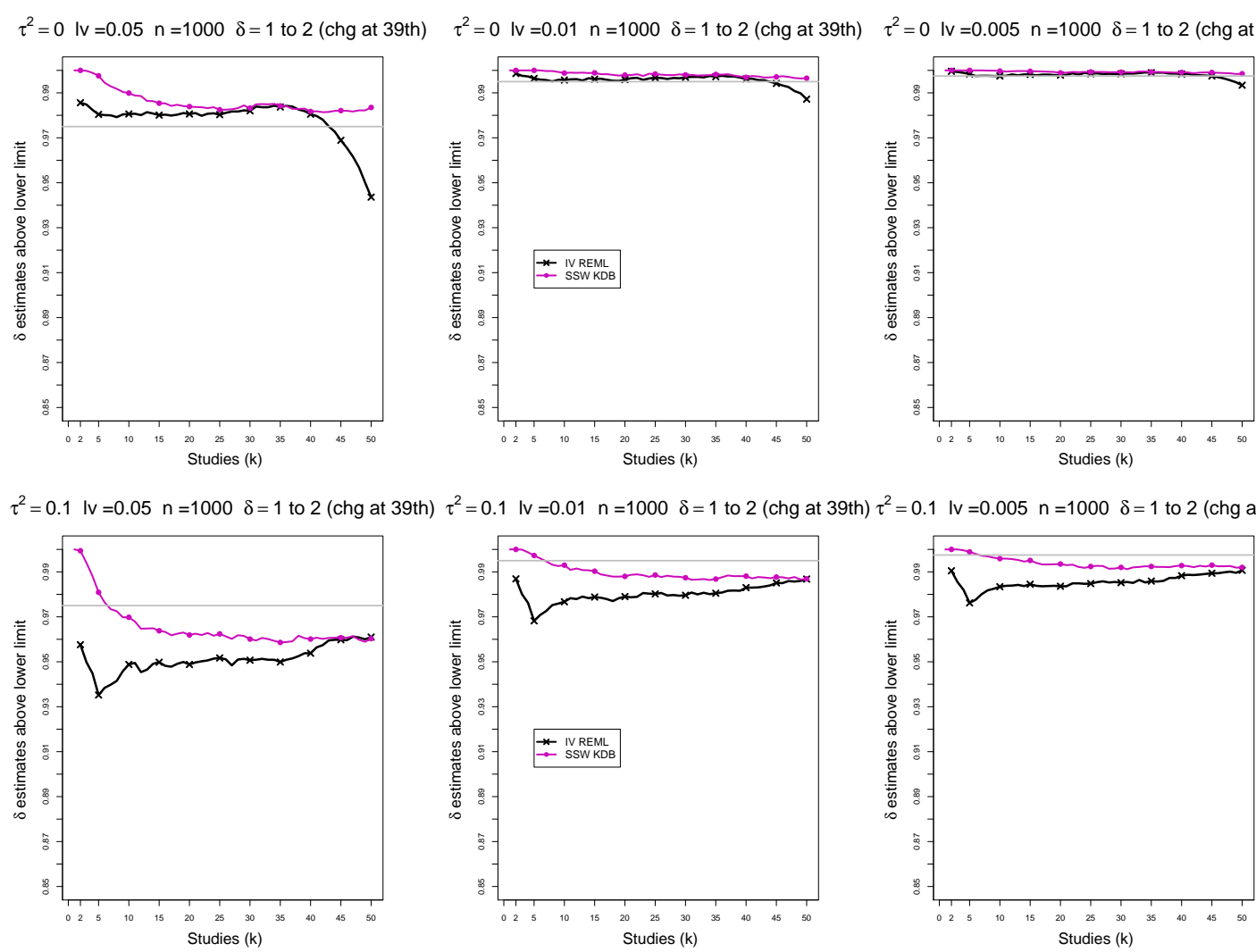

$\tau^{2}=0.25 \mathrm{IV}=0.05 \mathrm{n}=1000 \delta=1$ to 2 (chg at 39 th) $\tau^{2}=0.25 \mathrm{IV}=0.01 \mathrm{n}=1000 \quad \delta=1$ to 2 (chg at 39 th) $:^{2}=0.25 \mathrm{IV}=0.005 \mathrm{n}=1000 \quad \delta=1$ to 2 (chg at 39 th
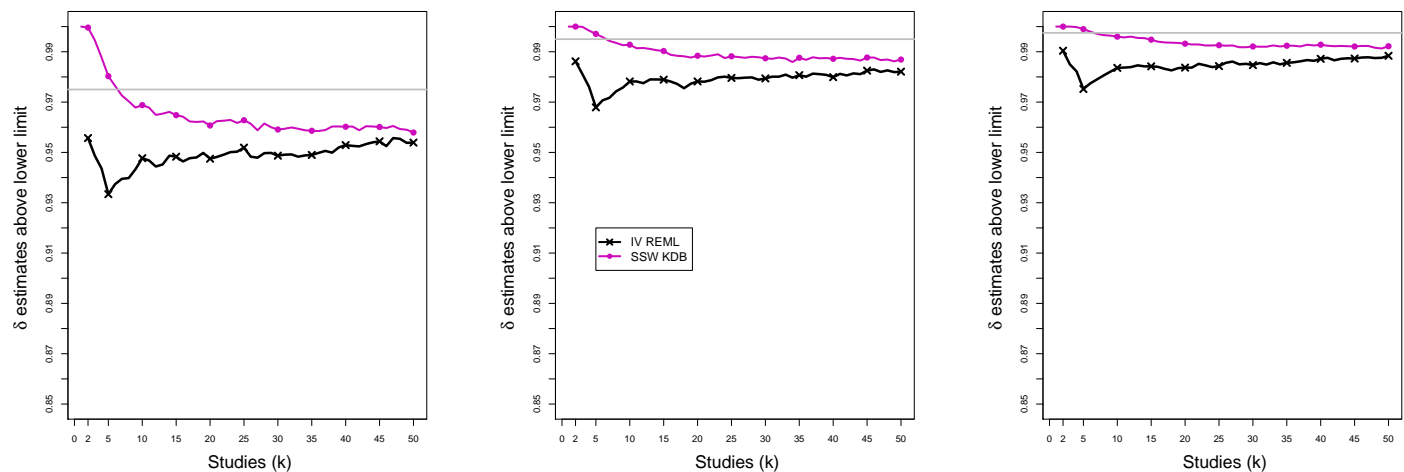

$\tau^{2}=1 \mathrm{IV}=0.05 \mathrm{n}=1000 \delta=1$ to 2 (chg at 39 th)

$\tau^{2}=1$ IV $=0.01 \mathrm{n}=1000 \delta=1$ to 2 (chg at 39 th)
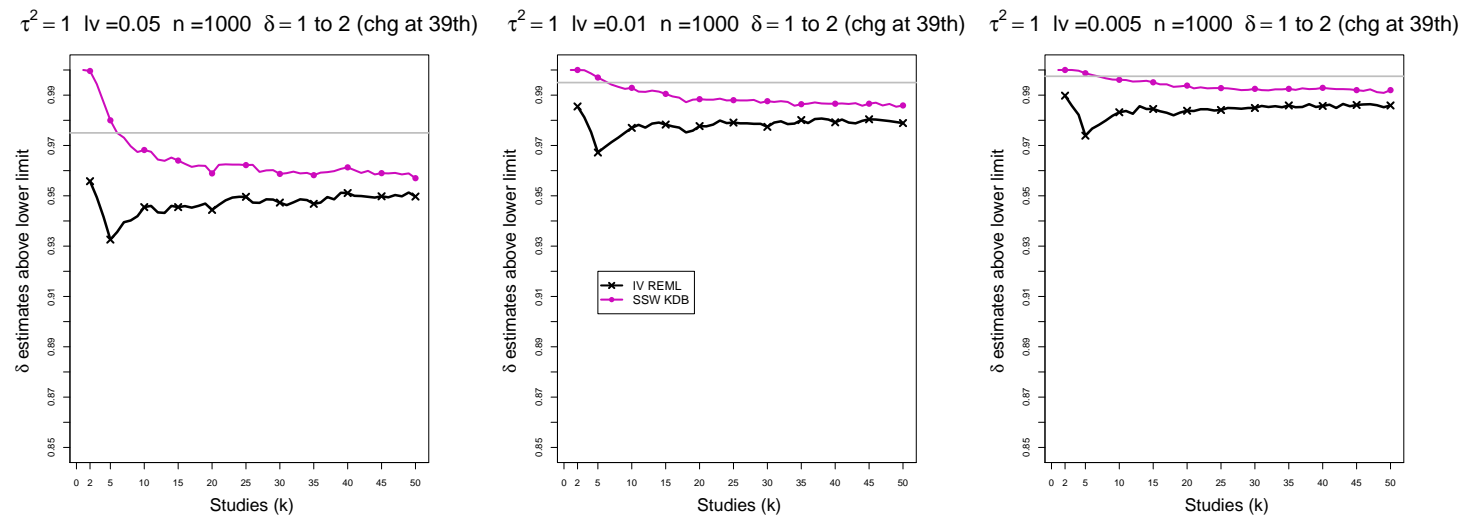

Figure D4. 15: 2-Stage CMA - Coverage of the lower confidence interval of $\delta$ (above lower limit) at the confidence level $1-\alpha / 2$ for $\alpha=0.05,0.01,0.005$ when $\delta=1$ for $k \leq 38$ followed by a shift to $\delta=2$ for $k \geq 39, \tau^{2}=0,0.1,0.25,1, n=1000, K=50$. Light grey line at $0.975,0.995,0.9975$. 

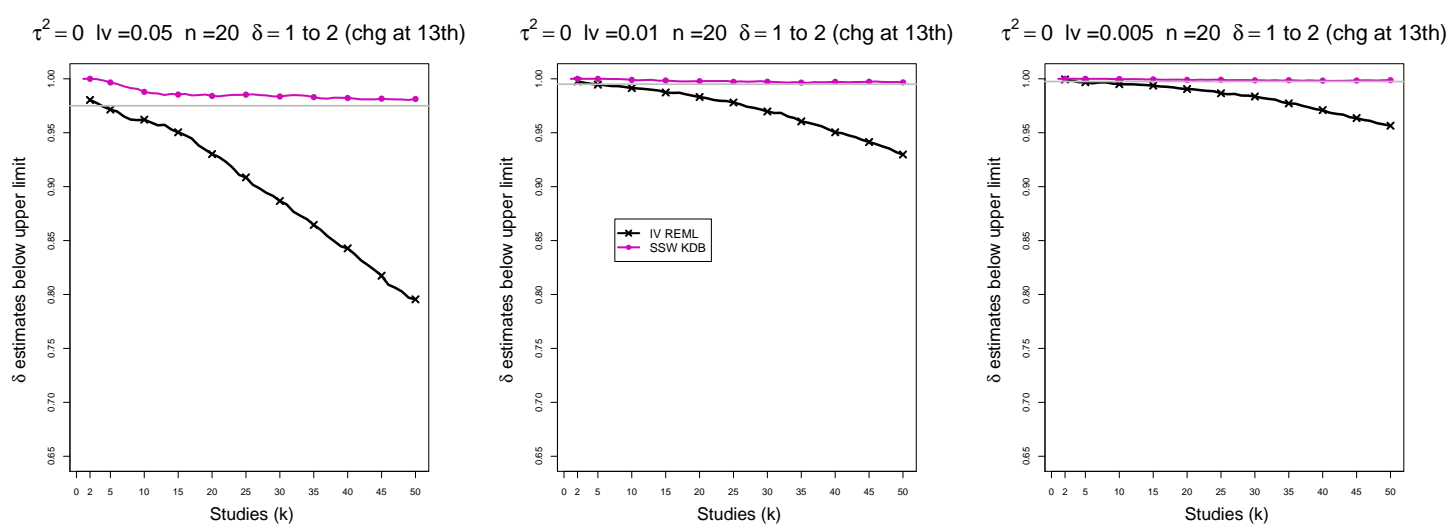

$\tau^{2}=0.1 \mathrm{lv}=0.05 \mathrm{n}=20 \delta=1$ to 2 (chg at 13 th)

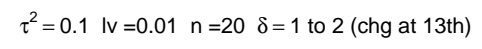

$\tau^{2}=0.1 \mathrm{lv}=0.005 \mathrm{n}=20 \quad \delta=1$ to 2 (chg at 13 th)
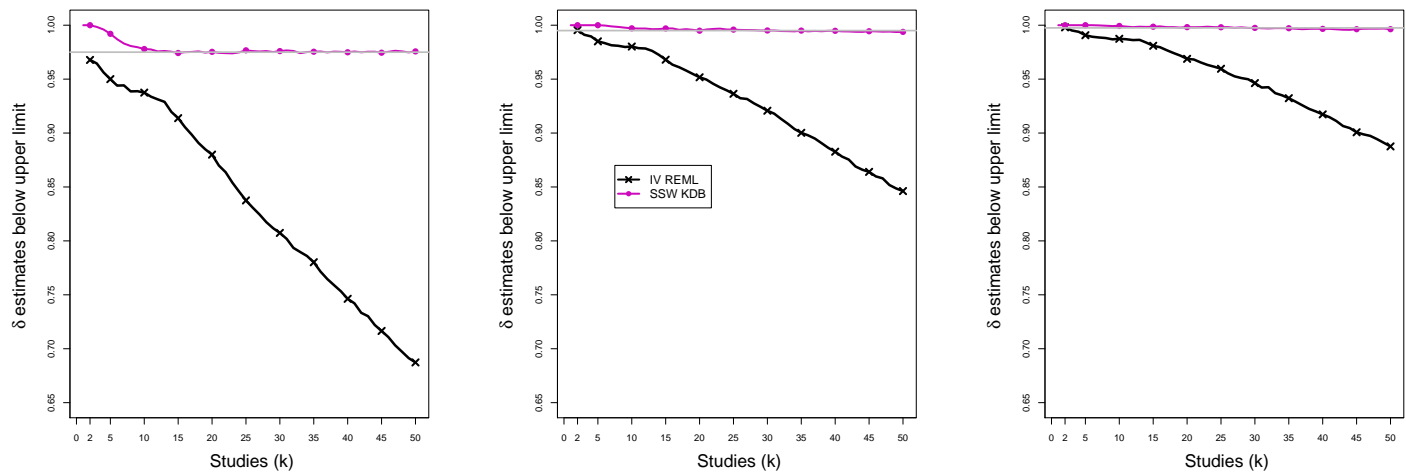

$\tau^{2}=0.25 \mathrm{lv}=0.05 \mathrm{n}=20 \quad \delta=1$ to 2 (chg at 13 th)
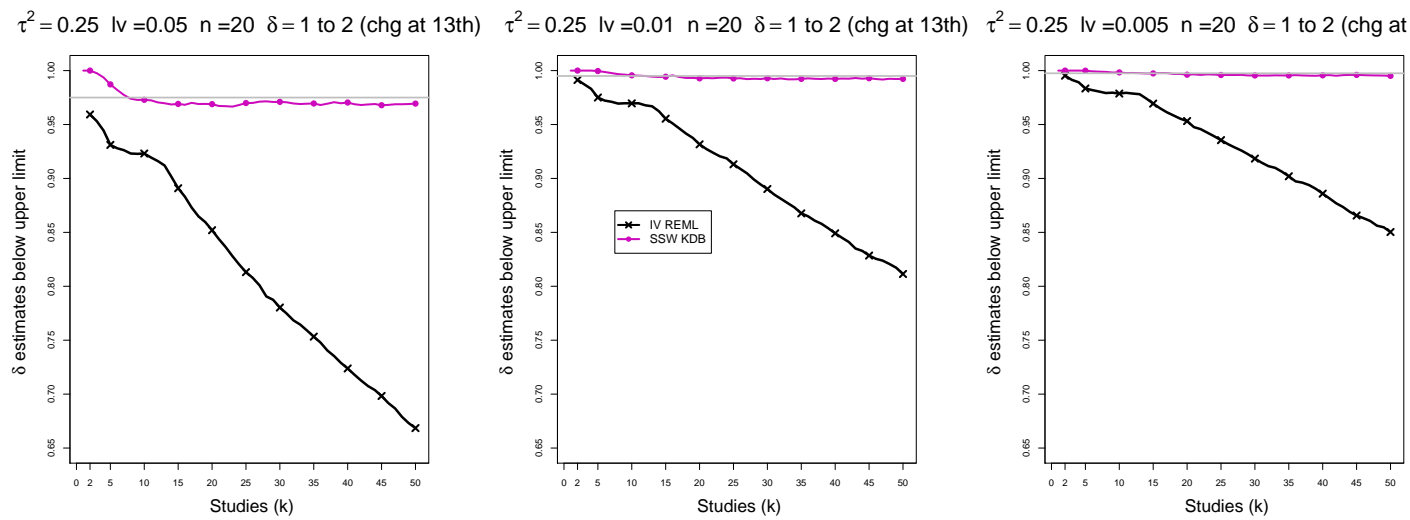

$\tau^{2}=1 \mathrm{IV}=0.01 \mathrm{n}=20 \delta=1$ to 2 (chg at 13 th)

$\tau^{2}=1 \mathrm{IV}=0.005 \mathrm{n}=20 \delta=1$ to 2 (chg at 13 th)
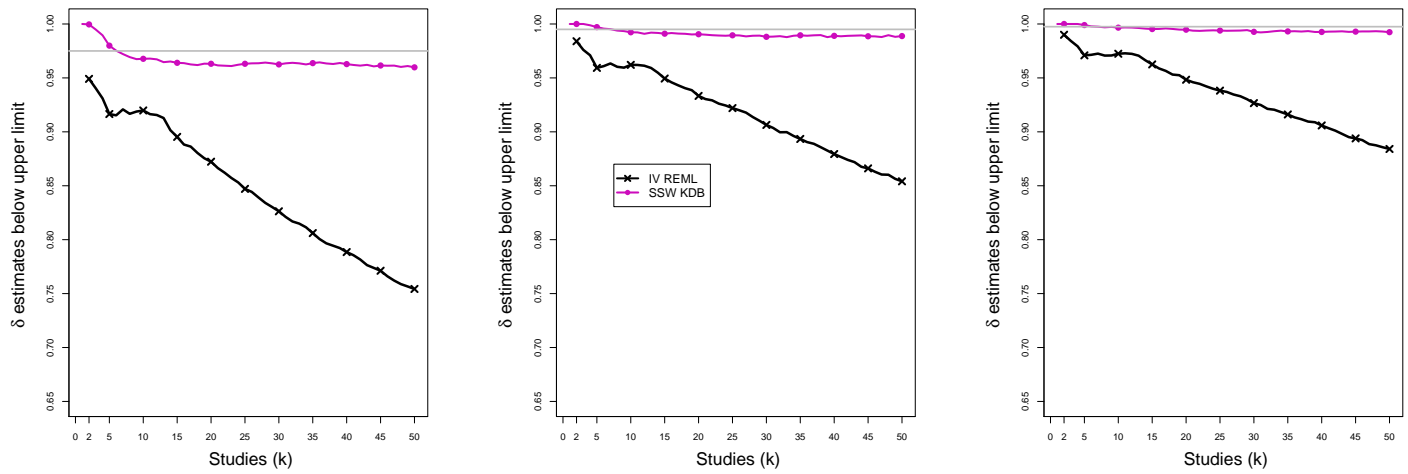

Figure D4. 16: 2-Stage CMA - Coverage of the upper confidence interval of $\delta$ (below upper limit) at the confidence level $1-\alpha / 2$ for $\alpha=0.05,0.01,0.005$ when $\delta=1$ for $k \leq 12$ followed by a shift to $\delta=2$ for $k \geq 13, \tau^{2}=0,0.1,0.25,1, n=20, K=50$. Light grey line at $0.975,0.995,0.9975$. 

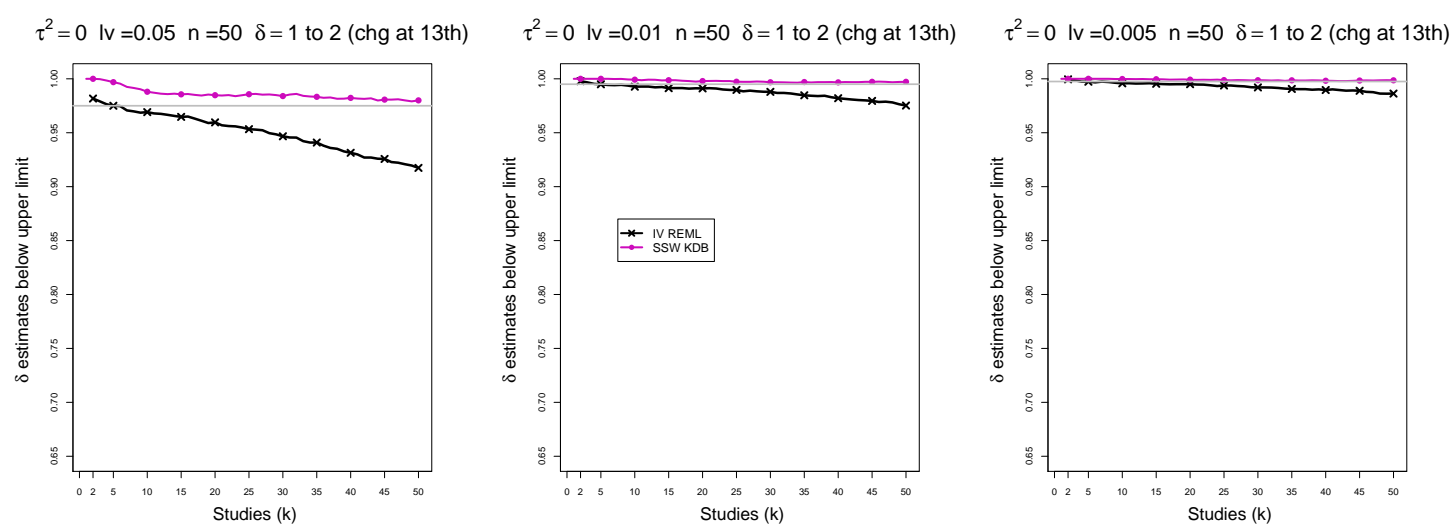

$\tau^{2}=0.1 \mathrm{lv}=0.05 \mathrm{n}=50 \delta=1$ to 2 (chg at 13 th)

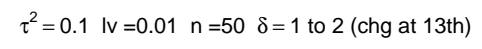

$\tau^{2}=0.1 \mathrm{lv}=0.005 \mathrm{n}=50 \quad \delta=1$ to 2 (chg at 13 th)
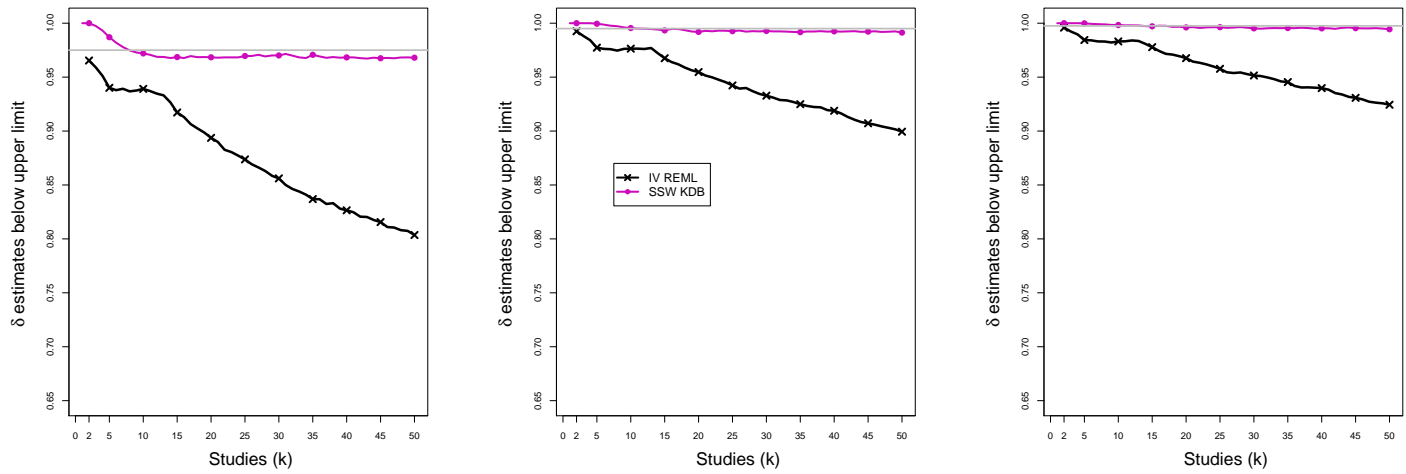

$\tau^{2}=0.25 \mathrm{lv}=0.05 \mathrm{n}=50 \quad \delta=1$ to 2 (chg at 13 th)
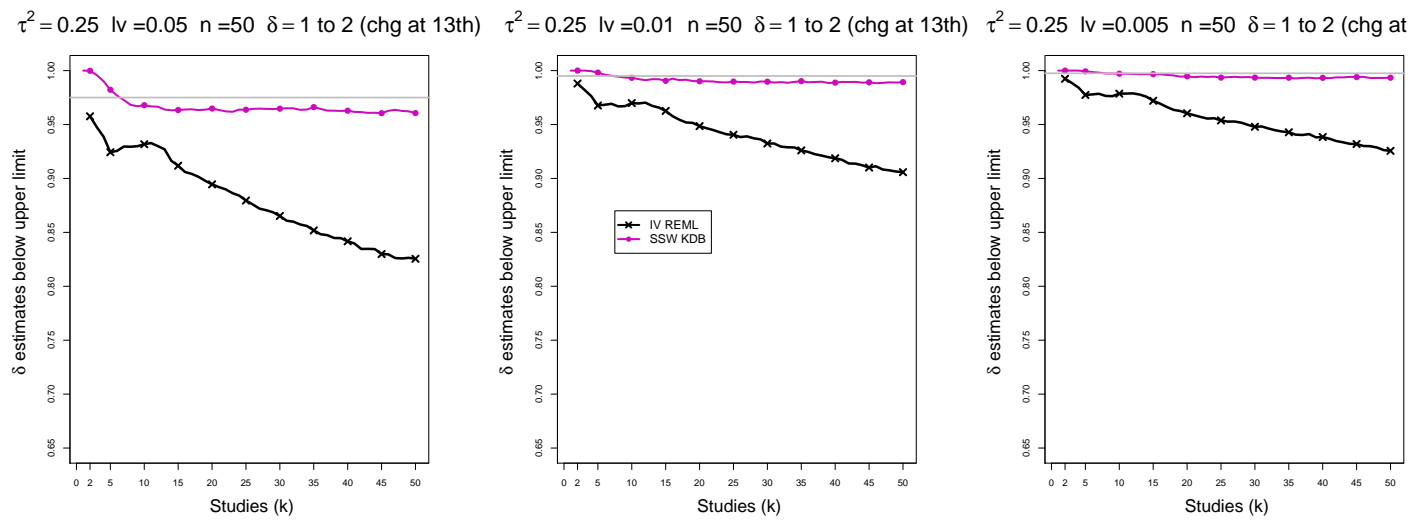

$\tau^{2}=1 \mathrm{IV}=0.01 \mathrm{n}=50 \quad \delta=1$ to 2 (chg at 13 th)
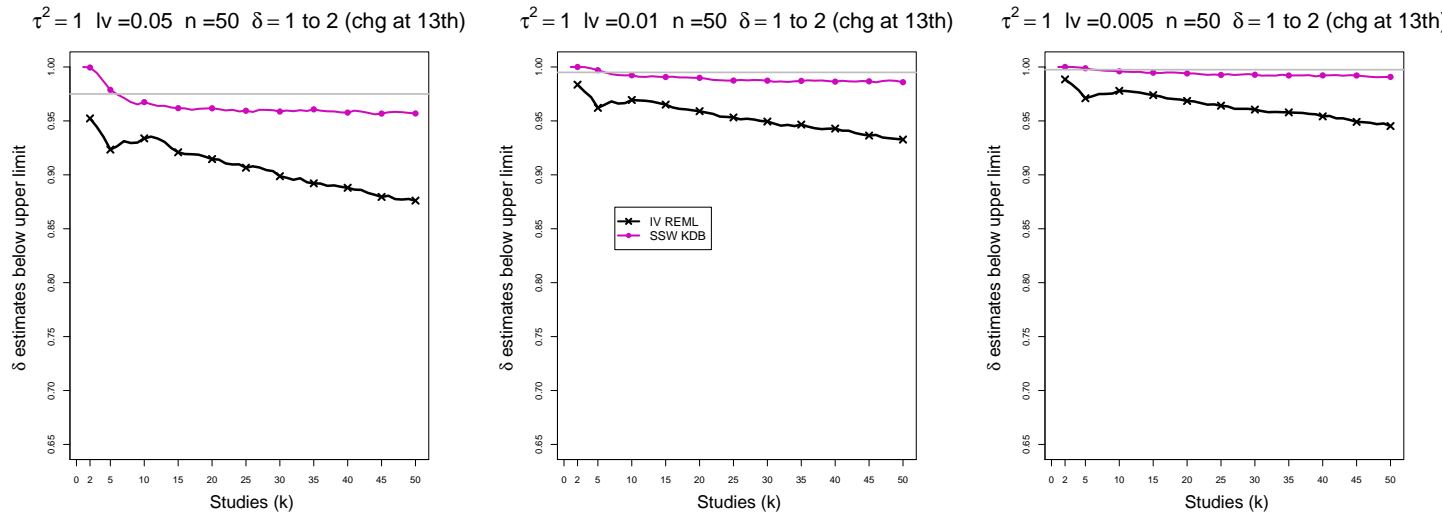

Figure D4. 17: 2-Stage CMA - Coverage of the upper confidence interval of $\delta$ (below upper limit) at the confidence level $1-\alpha / 2$ for $\alpha=0.05,0.01,0.005$ when $\delta=1$ for $k \leq 12$ followed by a shift to $\delta=2$ for $k \geq 13, \tau^{2}=0,0.1,0.25,1, n=50, K=50$. Light grey line at $0.975,0.995,0.9975$. 

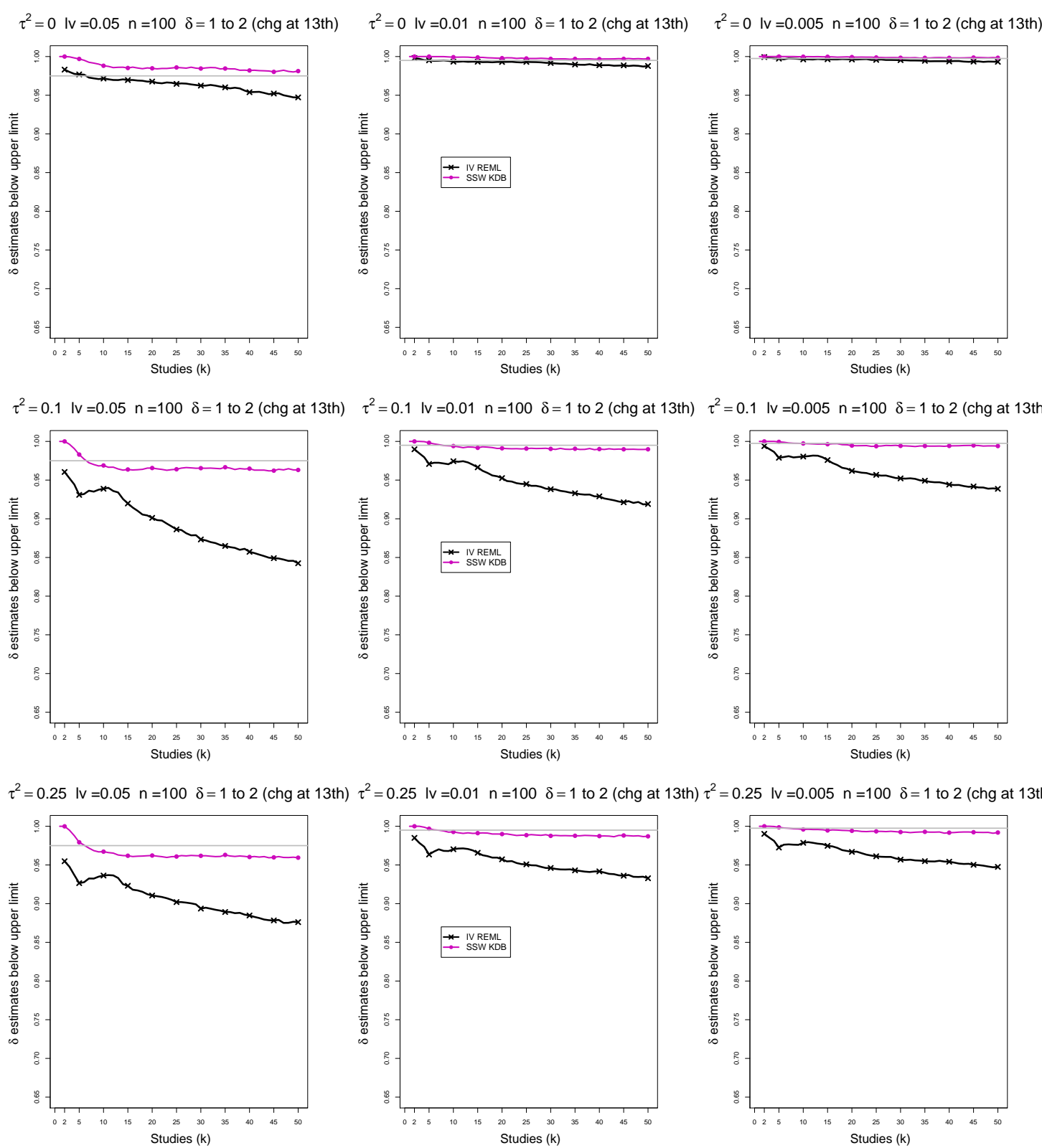

$\tau^{2}=1 \mathrm{lv}=0.05 \mathrm{n}=100 \delta=1$ to 2 (chg at 13 th)

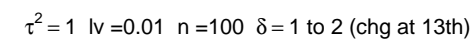

$\tau^{2}=1 \mathrm{lv}=0.005 \mathrm{n}=100 \delta=1$ to 2 (chg at 13th)
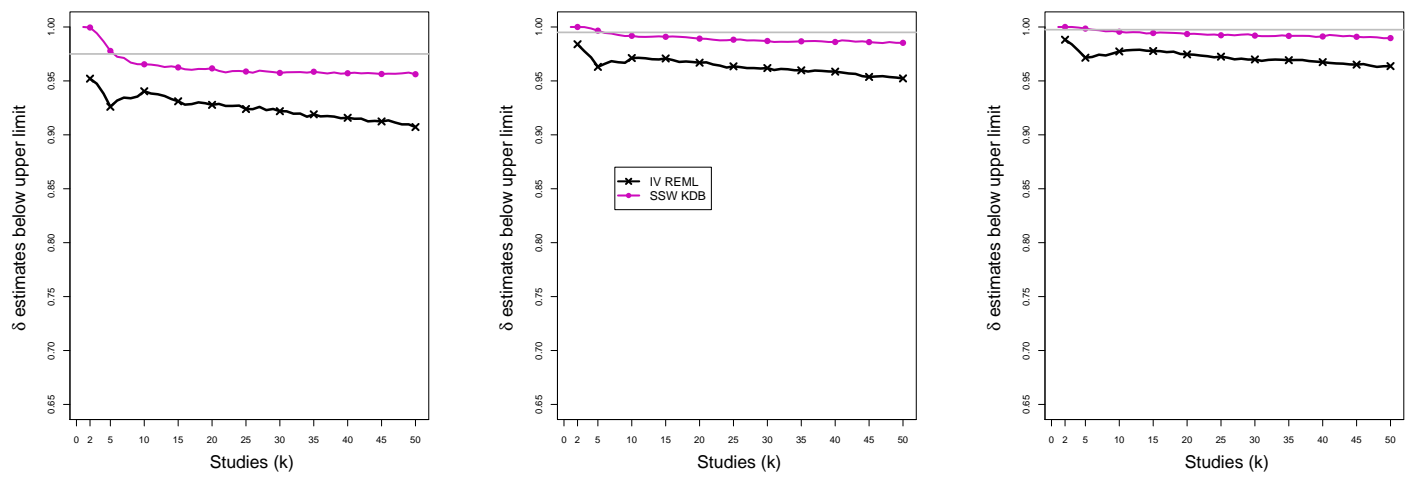

Figure D4. 18: 2-Stage CMA - Coverage of the upper confidence interval of $\delta$ (below upper limit) at the confidence level $1-\alpha / 2$ for $\alpha=0.05,0.01,0.005$ when $\delta=1$ for $k \leq 12$ followed by a shift to $\delta=2$ for $k \geq 13, \tau^{2}=0,0.1,0.25,1, n=100, K=50$. Light grey line at $0.975,0.995,0.9975$. 

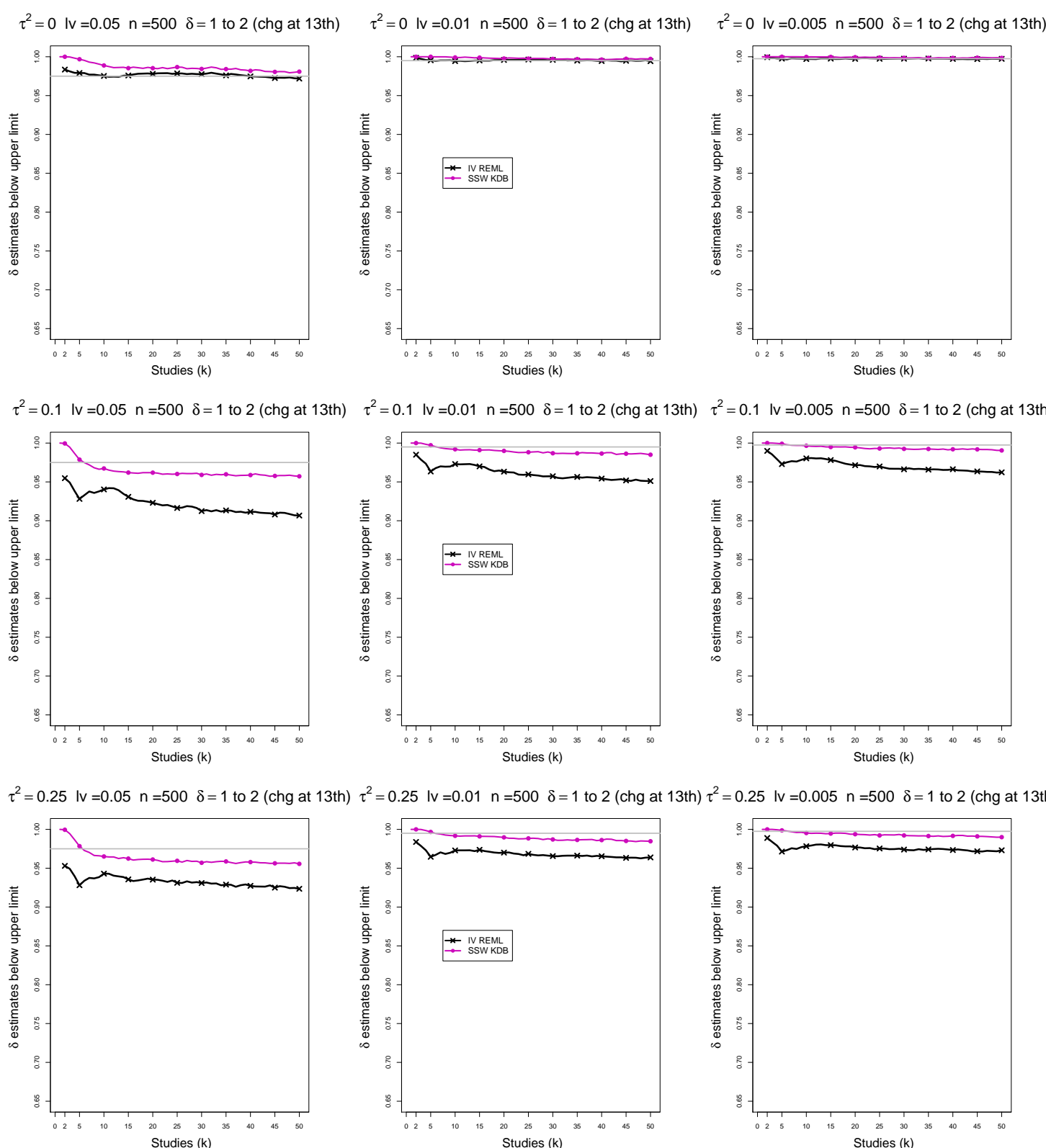

$\tau^{2}=1 \mathrm{lv}=0.05 \mathrm{n}=500 \delta=1$ to 2 (chg at 13 th)

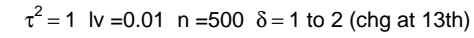

$\tau^{2}=1 \mathrm{lv}=0.005 \mathrm{n}=500 \delta=1$ to 2 (chg at 13th)
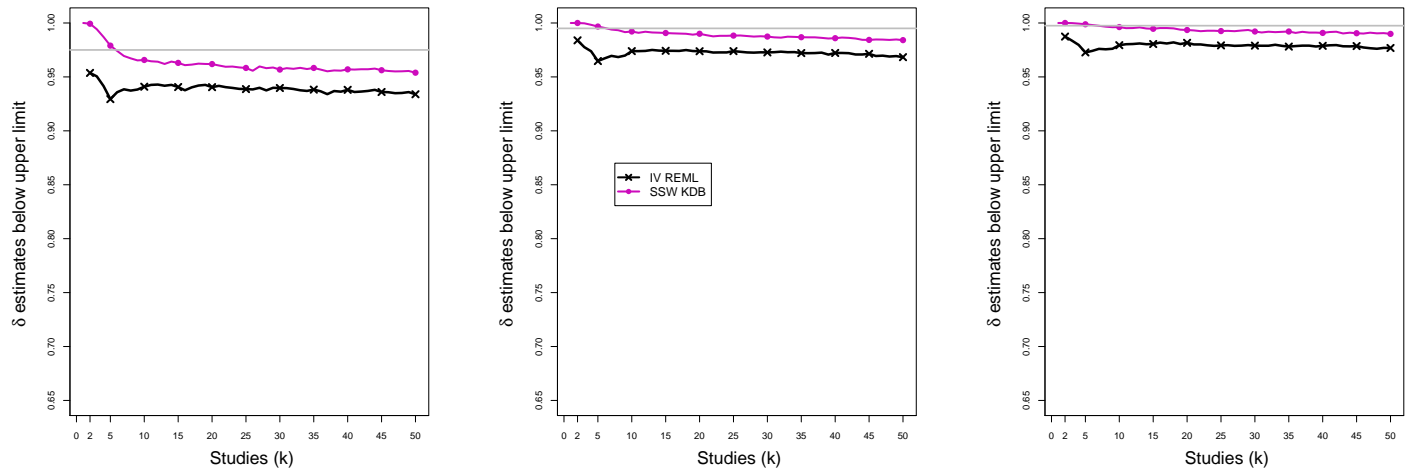

Figure D4. 19: 2-Stage CMA - Coverage of the upper confidence interval of $\delta$ (below upper limit) at the confidence level $1-\alpha / 2$ for $\alpha=0.05,0.01,0.005$ when $\delta=1$ for $k \leq 12$ followed by a shift to $\delta=2$ for $k \geq 13, \tau^{2}=0,0.1,0.25,1, n=500, K=50$. Light grey line at $0.975,0.995,0.9975$. 

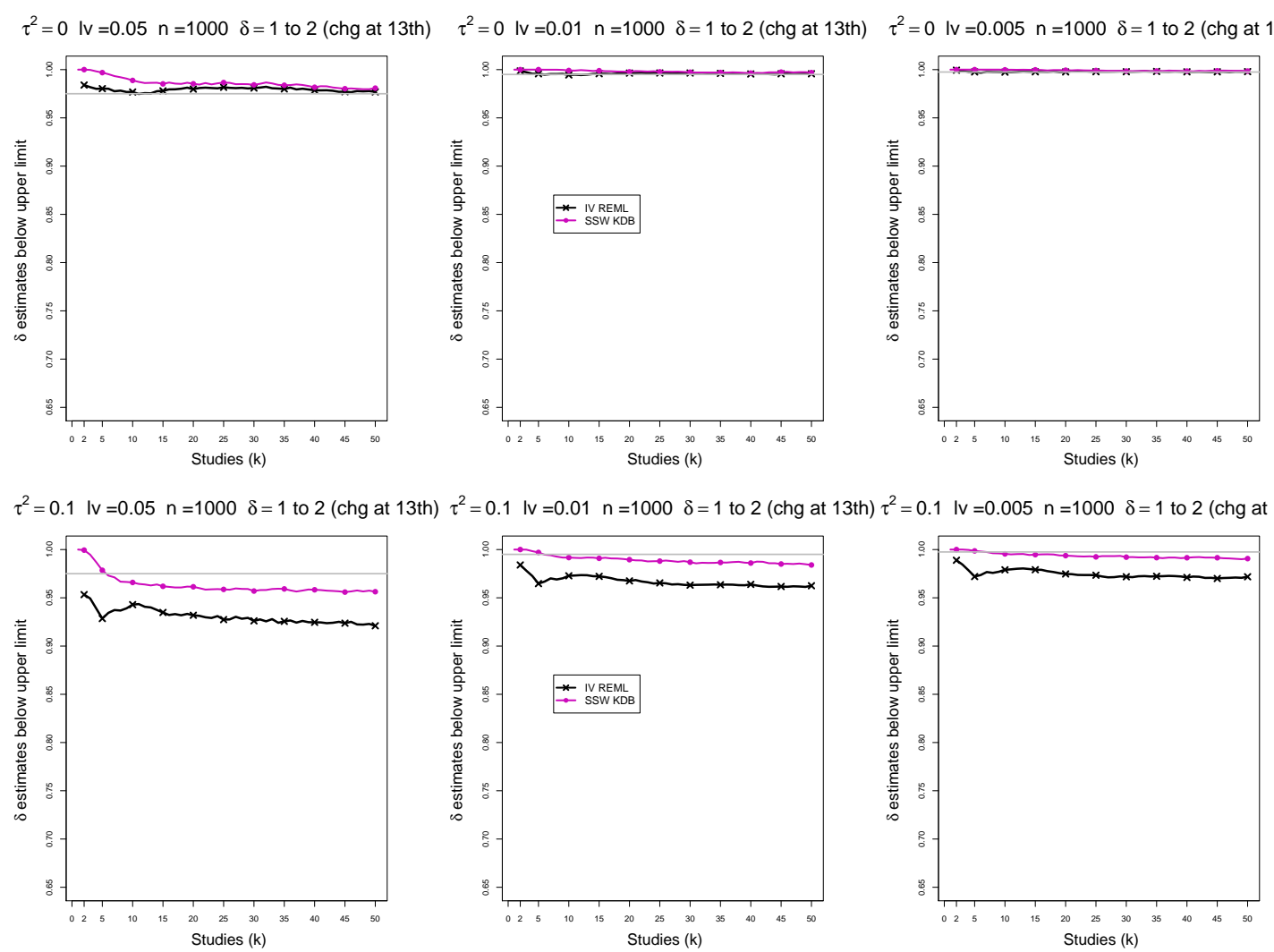

$\tau^{2}=0.25 \mathrm{lv}=0.05 \mathrm{n}=1000 \delta=1$ to 2 (chg at 13 th) $\tau^{2}=0.25 \mathrm{IV}=0.01 \mathrm{n}=1000 \quad \delta=1$ to 2 (chg at 13 th) $:^{2}=0.25 \mathrm{IV}=0.005 \mathrm{n}=1000 \quad \delta=1$ to 2 (chg at 13 th
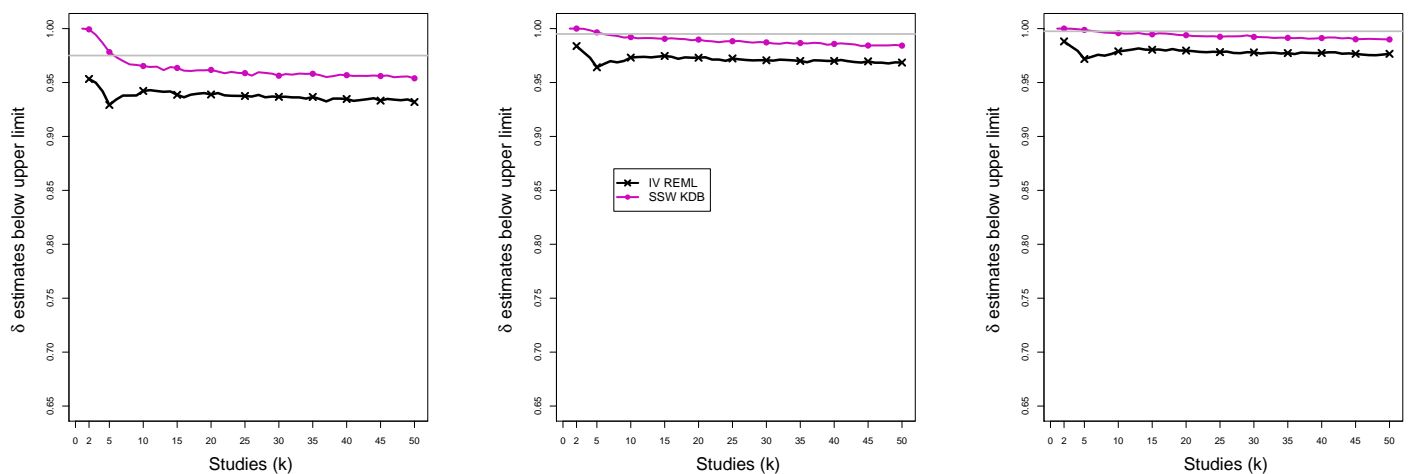

$\tau^{2}=1 \mathrm{IV}=0.05 \mathrm{n}=1000 \delta=1$ to 2 (chg at 13 th)

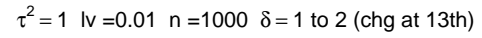

$\tau^{2}=1$ Iv $=0.005 \mathrm{n}=1000 \delta=1$ to 2 (chg at 13 th)
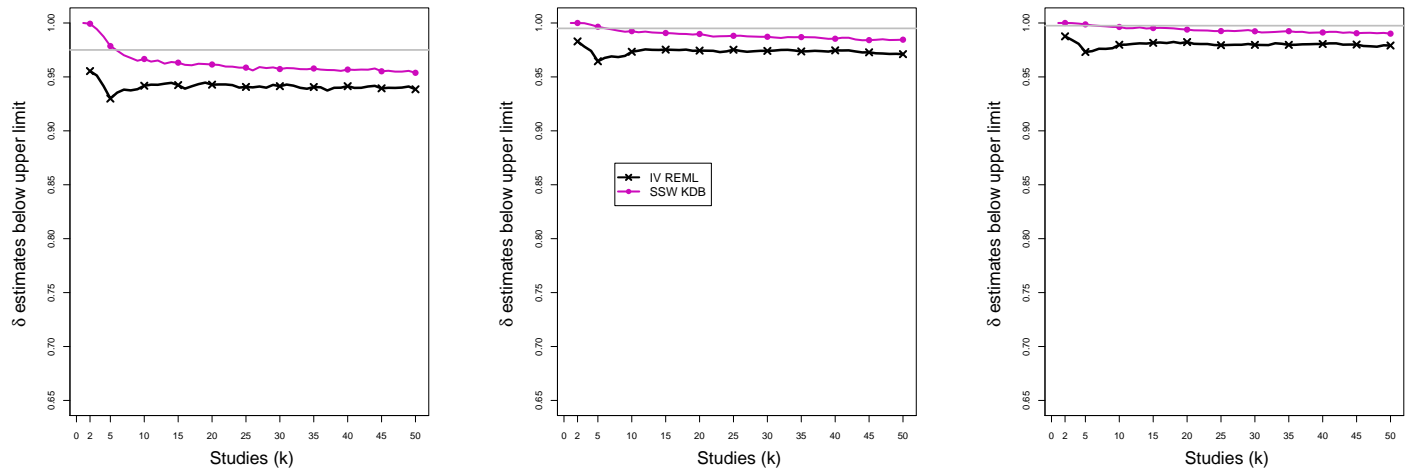

Figure D4. 20: 2-Stage CMA - Coverage of the upper confidence interval of $\delta$ (below upper limit) at the confidence level $1-\alpha / 2$ for $\alpha=0.05,0.01,0.005$ when $\delta=1$ for $k \leq 12$ followed by a shift to $\delta=2$ for $k \geq 13, \tau^{2}=0,0.1,0.25,1, n=1000, K=50$. Light grey line at $0.975,0.995,0.9975$. 

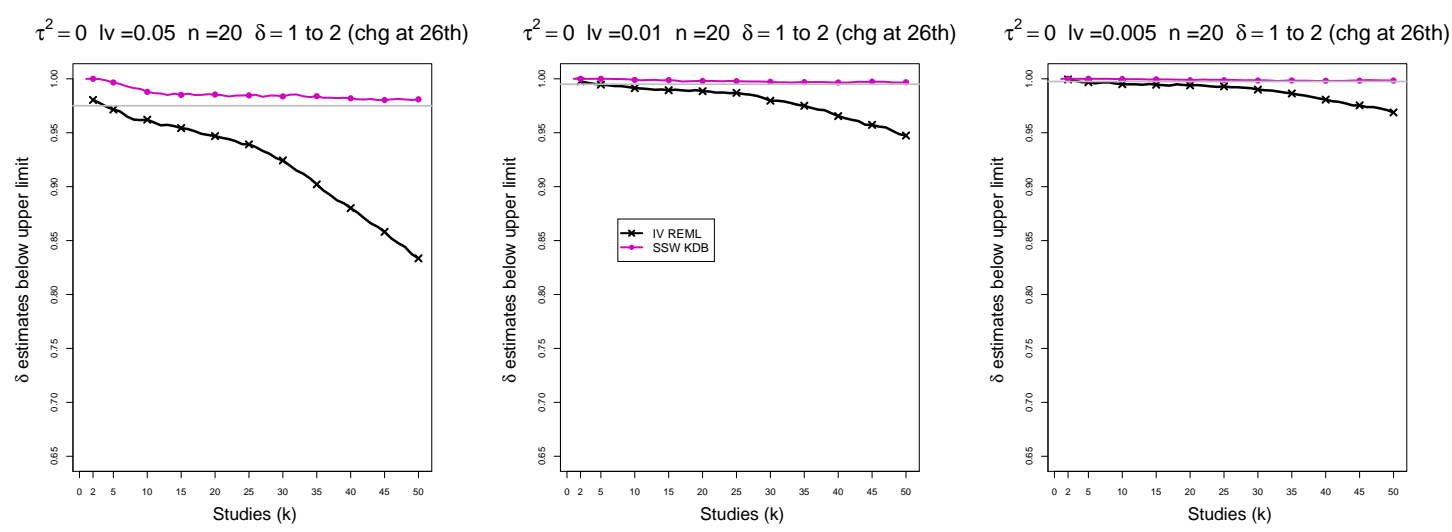

$\tau^{2}=0.1 \mathrm{lv}=0.05 \mathrm{n}=20 \delta=1$ to 2 (chg at $26 \mathrm{th}$ )

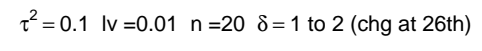

$\tau^{2}=0.1 \mathrm{IV}=0.005 \mathrm{n}=20 \quad \delta=1$ to 2 (chg at 26 th)
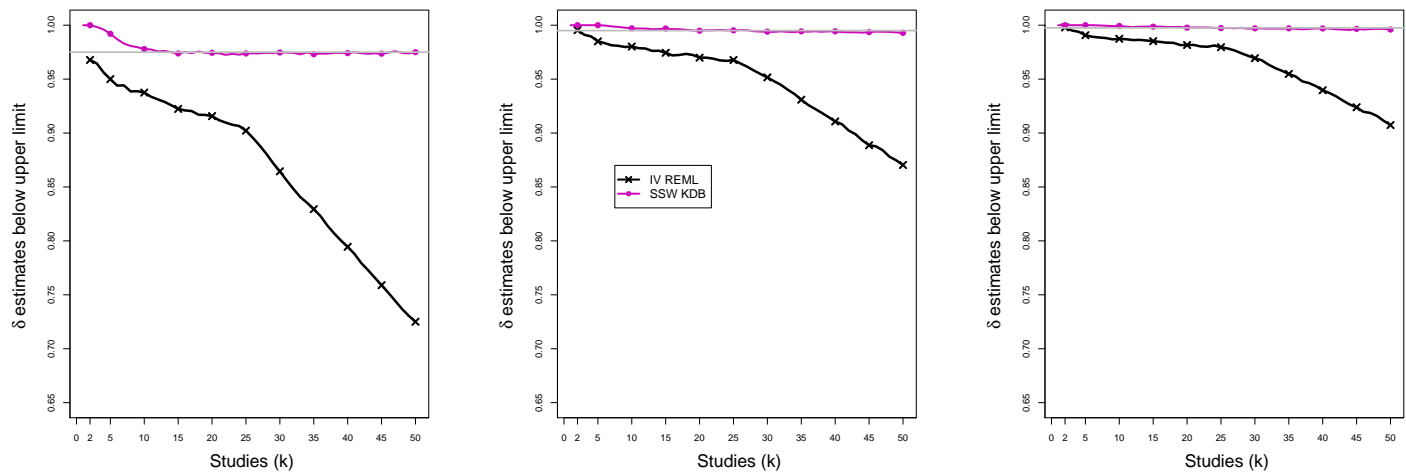

$\tau^{2}=0.25 \mathrm{lv}=0.05 \mathrm{n}=20 \quad \delta=1$ to 2 (chg at 26th)
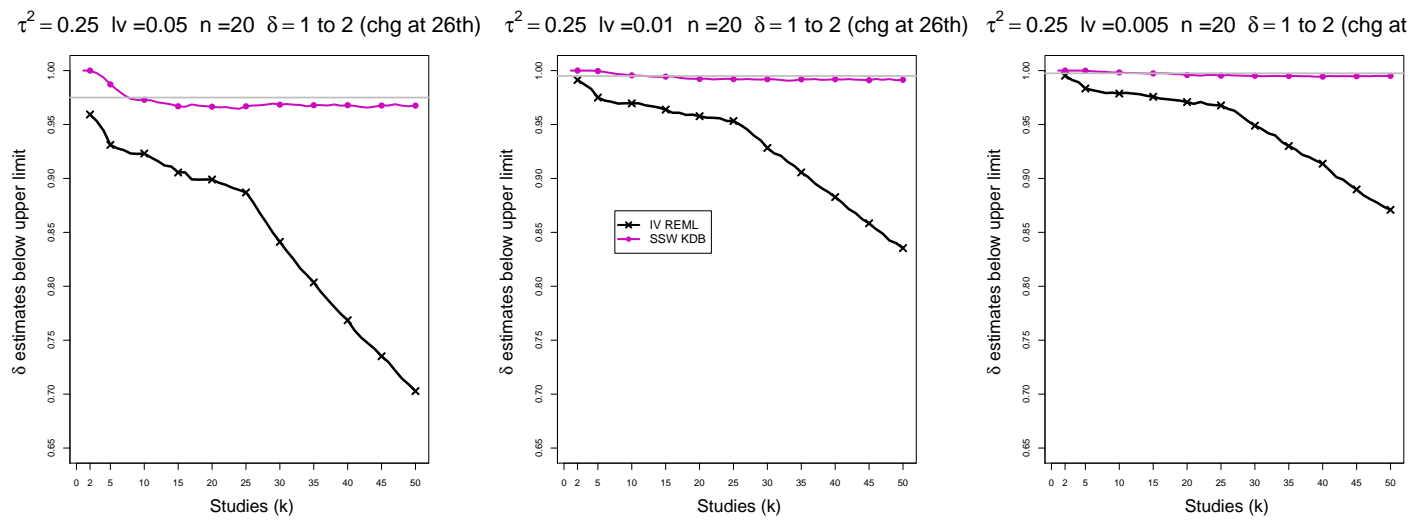

$\tau^{2}=1 \mathrm{IV}=0.01 \mathrm{n}=20 \delta=1$ to 2 (chg at 26 th)

$\tau^{2}=1 \mathrm{lv}=0.005 \mathrm{n}=20 \delta=1$ to 2 (chg at 26 th)
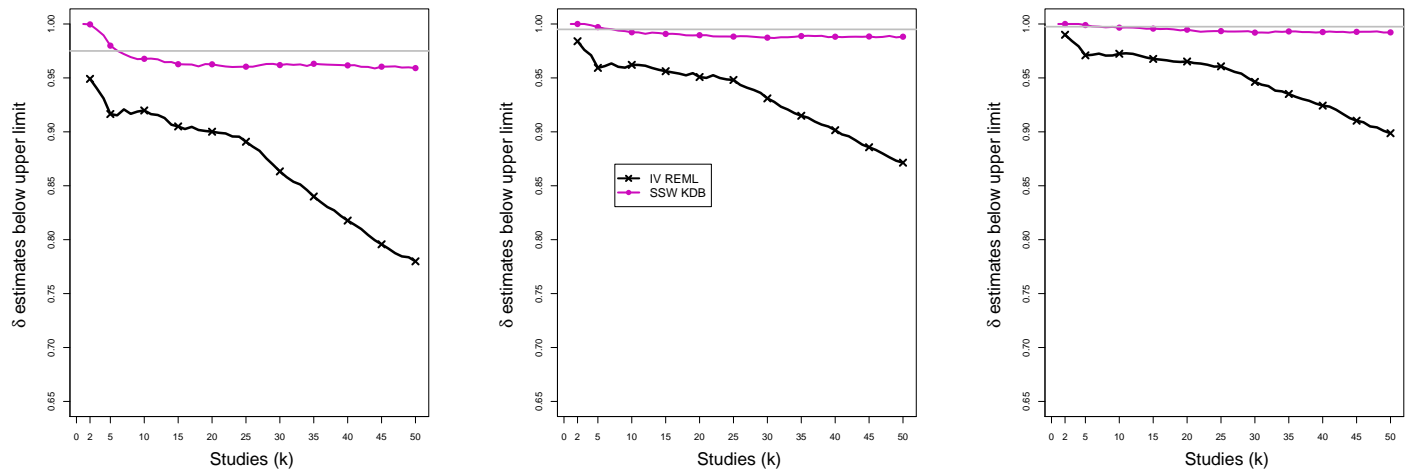

Figure D4. 21: 2-Stage CMA - Coverage of the upper confidence interval of $\delta$ (below upper limit) at the confidence level $1-\alpha / 2$ for $\alpha=0.05,0.01,0.005$ when $\delta=1$ for $k \leq 25$ followed by a shift to $\delta=2$ for $k \geq 26, \tau^{2}=0,0.1,0.25,1, n=20, K=50$. Light grey line at $0.975,0.995,0.9975$. 

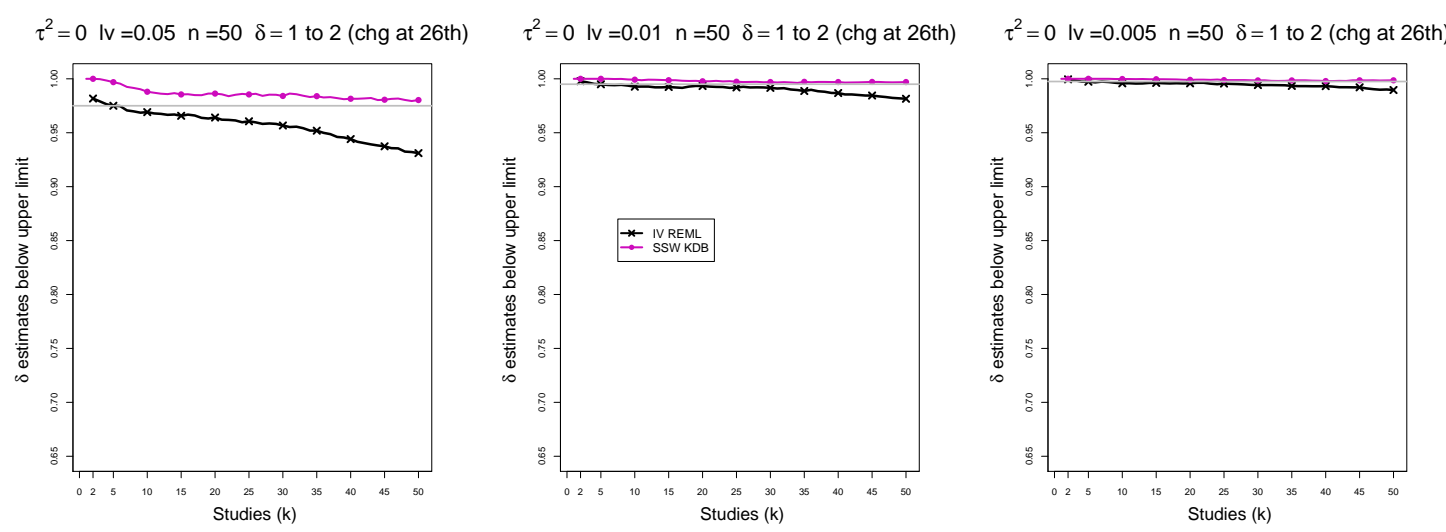

$\tau^{2}=0.1 \mathrm{lv}=0.05 \mathrm{n}=50 \delta=1$ to 2 (chg at $\left.26 \mathrm{th}\right)$

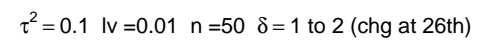

$\tau^{2}=0.1 \mathrm{lv}=0.005 \mathrm{n}=50 \delta=1$ to 2 (chg at 26th)
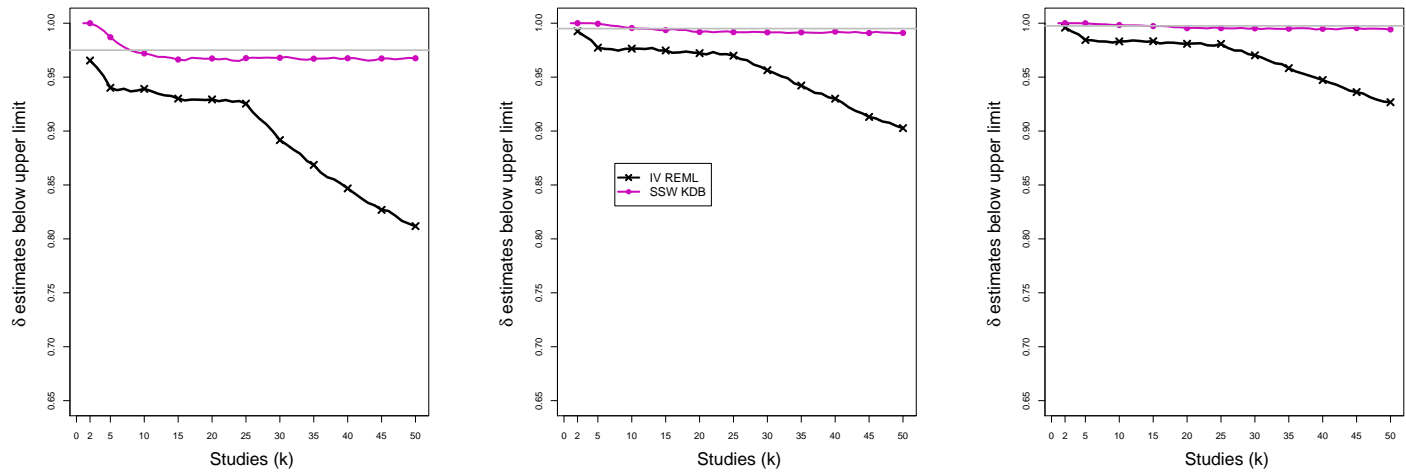

$\tau^{2}=0.25 \mathrm{lv}=0.05 \mathrm{n}=50 \quad \delta=1$ to 2 (chg at 26th)
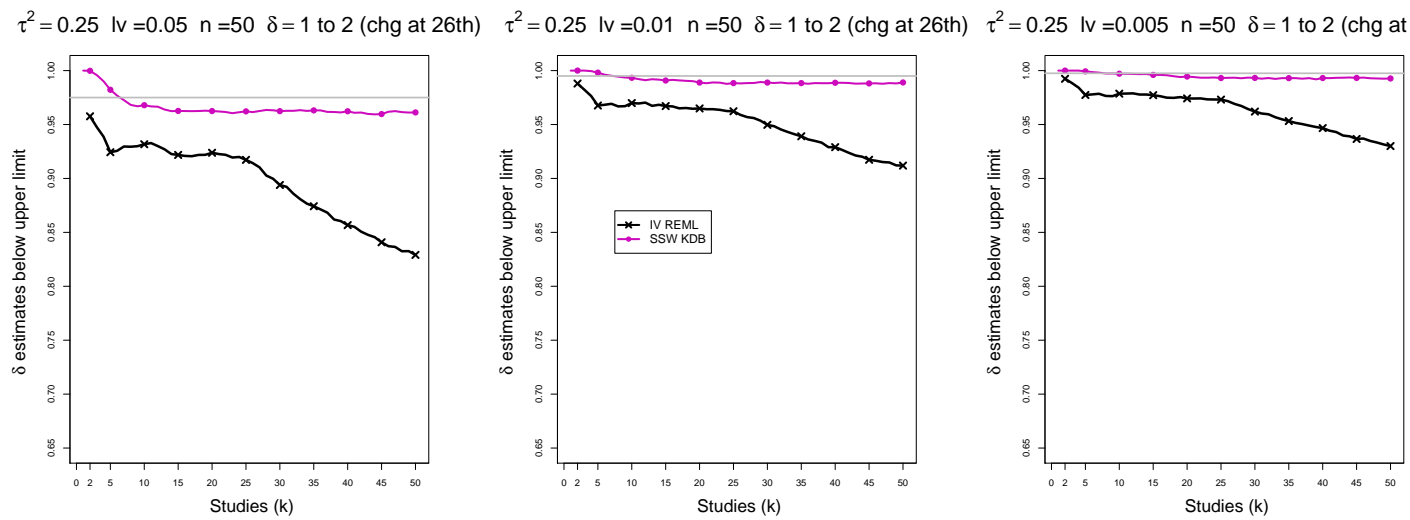

$\tau^{2}=1 \mathrm{IV}=0.01 \mathrm{n}=50 \quad \delta=1$ to 2 (chg at $\left.26 \mathrm{th}\right)$

$\tau^{2}=1 \mathrm{lv}=0.005 \mathrm{n}=50 \delta=1$ to 2 (chg at 26 th)
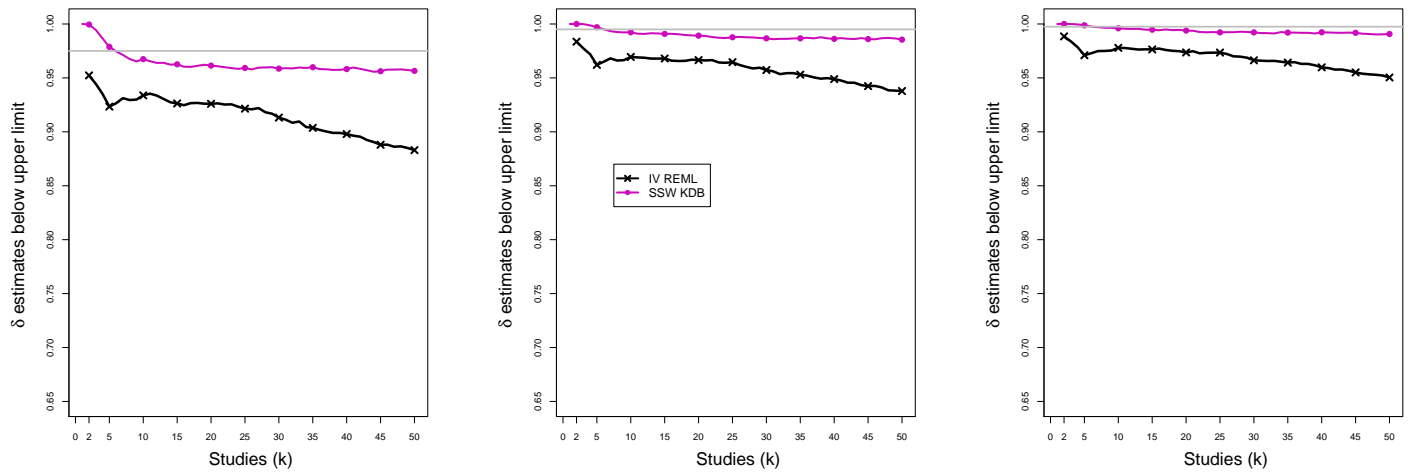

Figure D4. 22: 2-Stage CMA - Coverage of the upper confidence interval of $\delta$ (below upper limit) at the confidence level $1-\alpha / 2$ for $\alpha=0.05,0.01,0.005$ when $\delta=1$ for $k \leq 25$ followed by a shift to $\delta=2$ for $k \geq 26, \tau^{2}=0,0.1,0.25,1, n=50, K=50$. Light grey line at $0.975,0.995,0.9975$. 

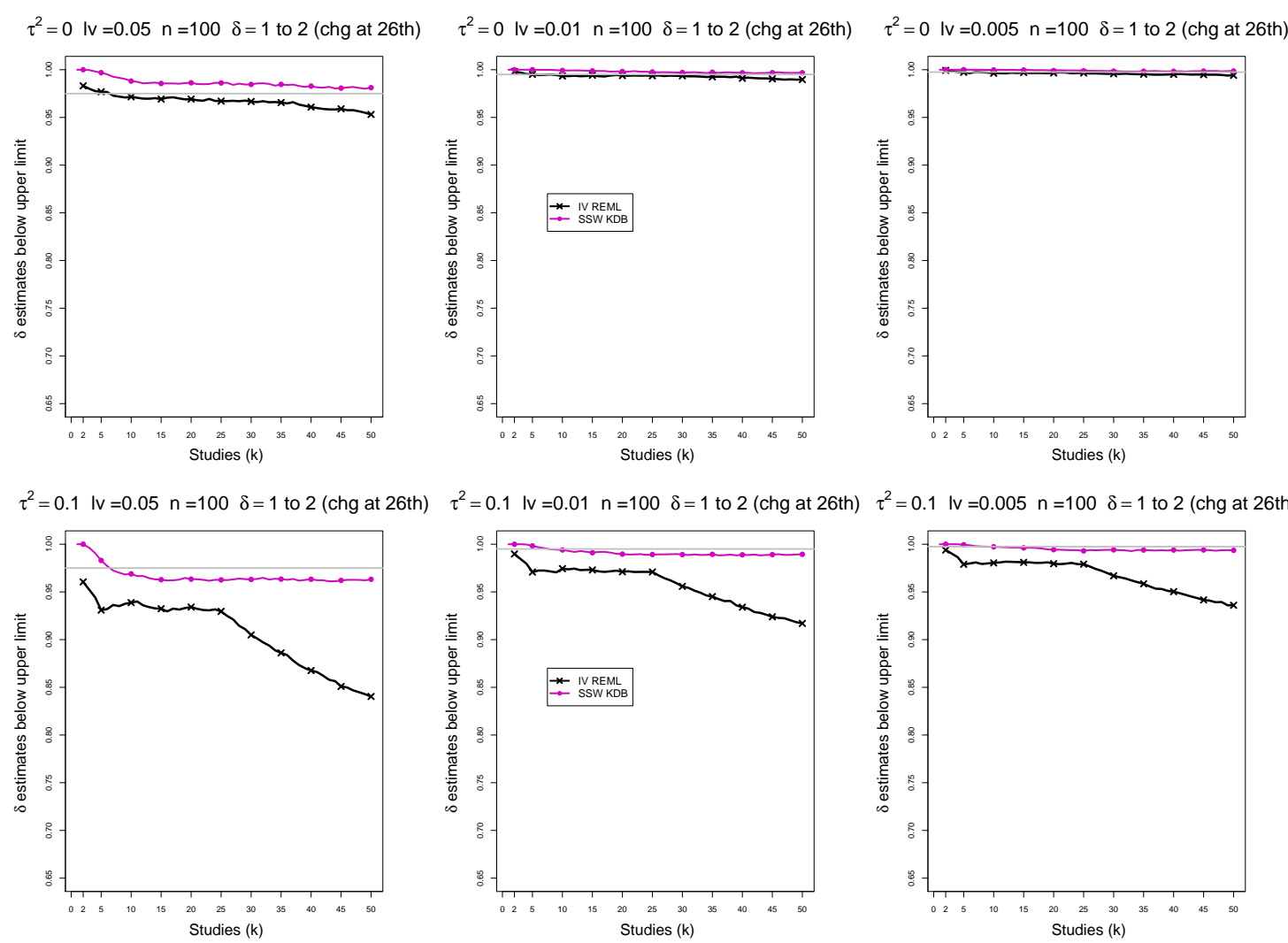

$\tau^{2}=0.25 \mathrm{lv}=0.05 \mathrm{n}=100 \quad \delta=1$ to 2 (chg at 26 th) $\tau^{2}=0.25 \mathrm{lv}=0.01 \mathrm{n}=100 \quad \delta=1$ to 2 (chg at 26 th) $\tau^{2}=0.25 \mathrm{lv}=0.005 \mathrm{n}=100 \quad \delta=1$ to 2 (chg at 26th)
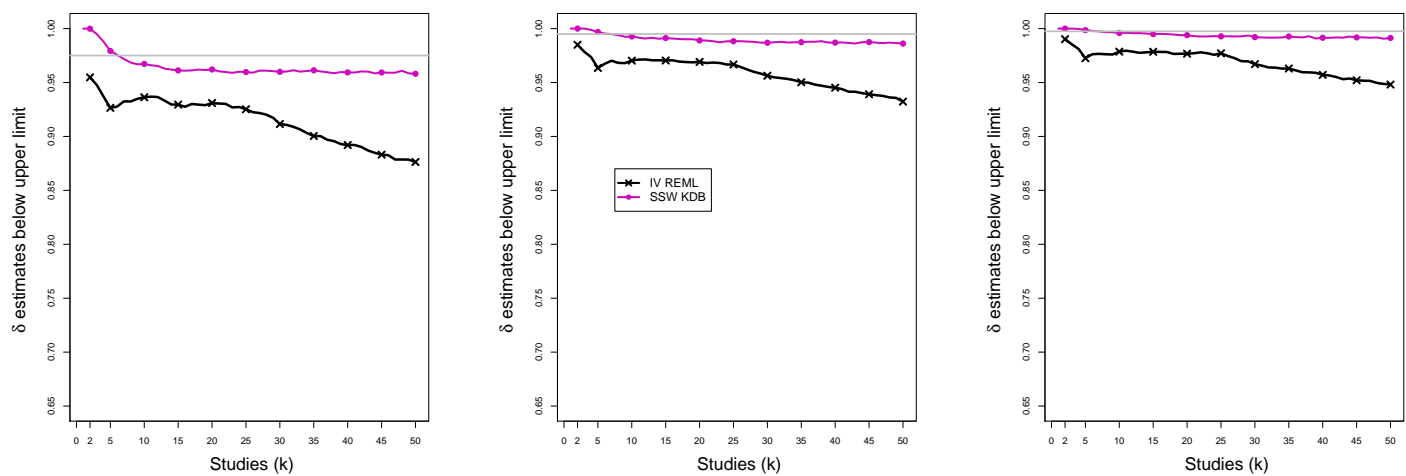

$\tau^{2}=1 \mathrm{lv}=0.05 \mathrm{n}=100 \delta=1$ to 2 (chg at 26 th)

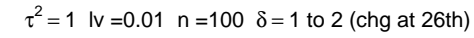

$\tau^{2}=1$ IV $=0.005 \quad n=100 \quad \delta=1$ to 2 (chg at 26th)
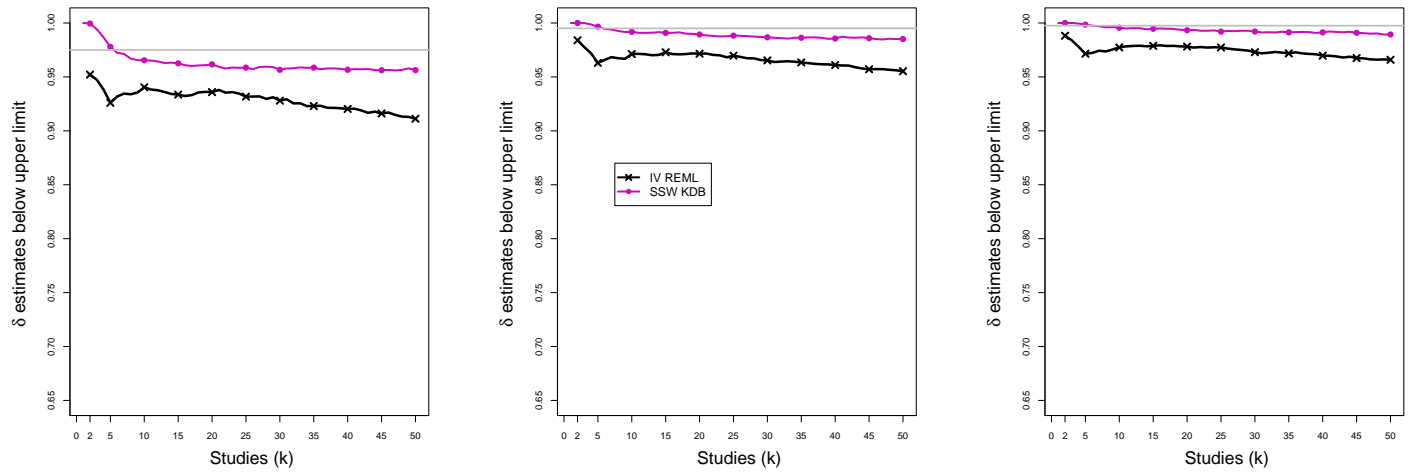

Figure D4. 23: 2-Stage CMA - Coverage of the upper confidence interval of $\delta$ (below upper limit) at the confidence level $1-\alpha / 2$ for $\alpha=0.05,0.01,0.005$ when $\delta=1$ for $k \leq 25$ followed by a shift to $\delta=2$ for $k \geq 26, \tau^{2}=0,0.1,0.25,1, n=100, K=50$. Light grey line at $0.975,0.995,0.9975$. 

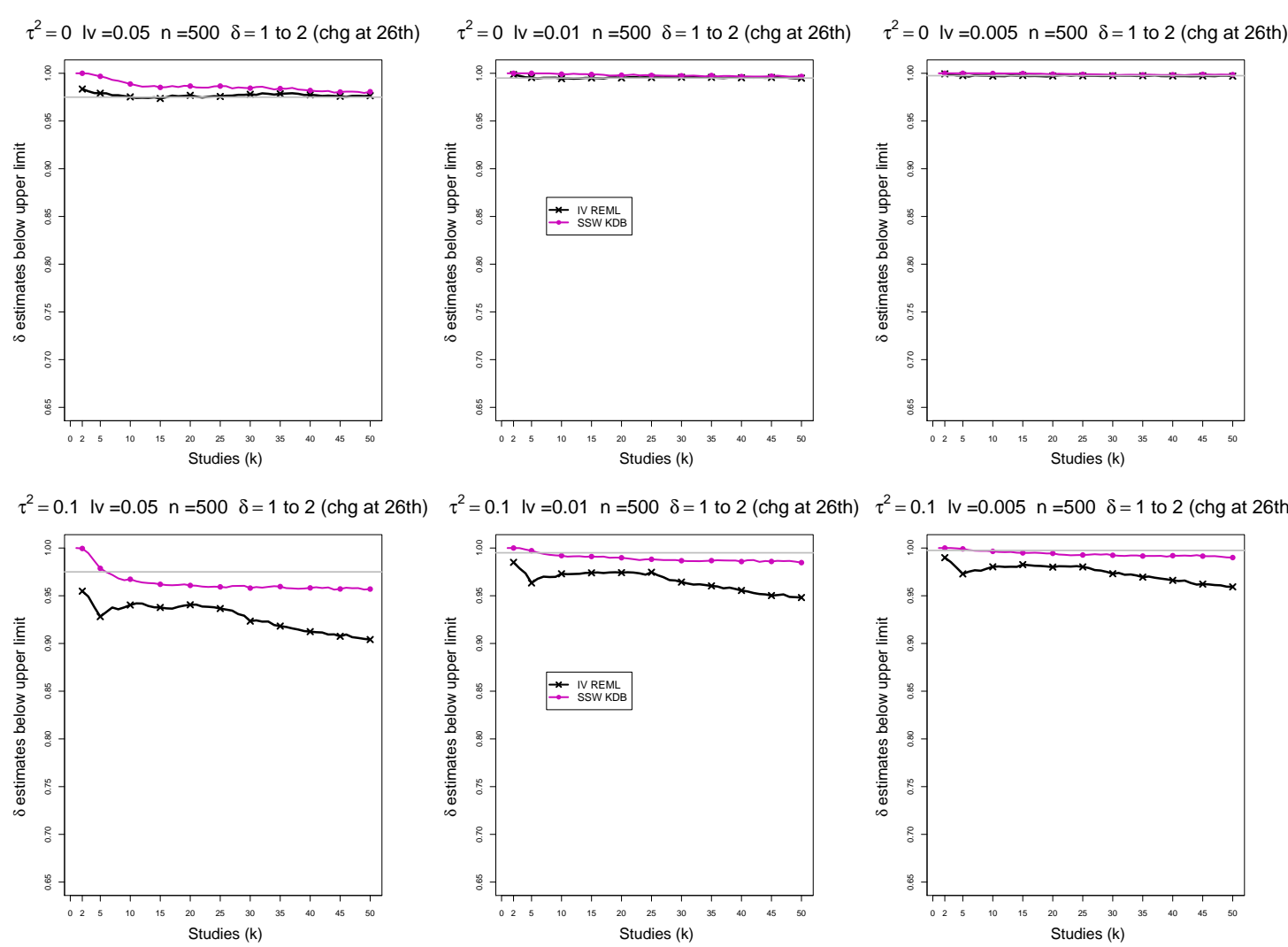

$\tau^{2}=0.25 \mathrm{lv}=0.05 \mathrm{n}=500 \quad \delta=1$ to 2 (chg at 26 th) $\tau^{2}=0.25 \mathrm{lv}=0.01 \mathrm{n}=500 \quad \delta=1$ to 2 (chg at 26 th) $\tau^{2}=0.25 \mathrm{lv}=0.005 \mathrm{n}=500 \quad \delta=1$ to 2 (chg at 26th)
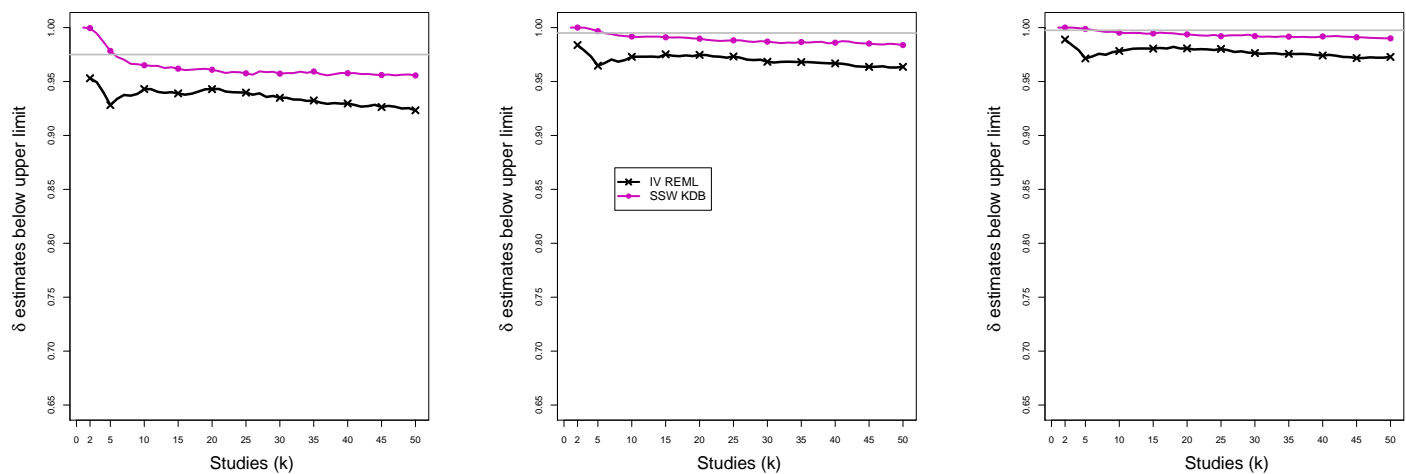

$\tau^{2}=1 \mathrm{lv}=0.05 \mathrm{n}=500 \delta=1$ to 2 (chg at 26 th)

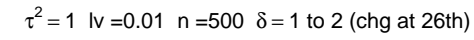

$\tau^{2}=1$ IV $=0.005 \quad n=500 \delta=1$ to 2 (chg at 26th)
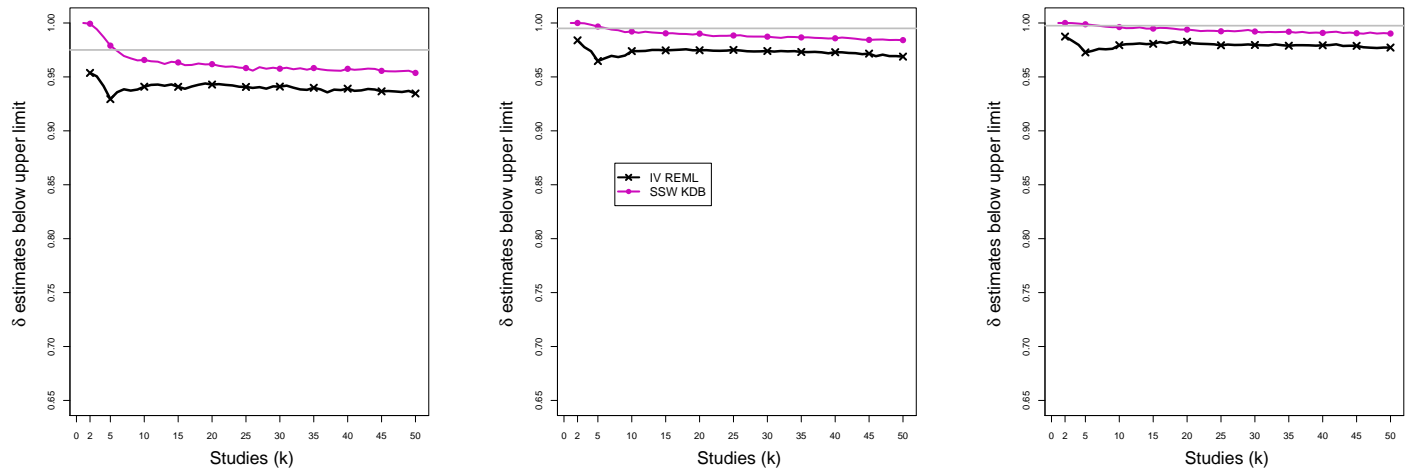

Figure D4. 24: 2-Stage CMA - Coverage of the upper confidence interval of $\delta$ (below upper limit) at the confidence level $1-\alpha / 2$ for $\alpha=0.05,0.01,0.005$ when $\delta=1$ for $k \leq 25$ followed by a shift to $\delta=2$ for $k \geq 26, \tau^{2}=0,0.1,0.25,1, n=500, K=50$. Light grey line at $0.975,0.995,0.9975$. 

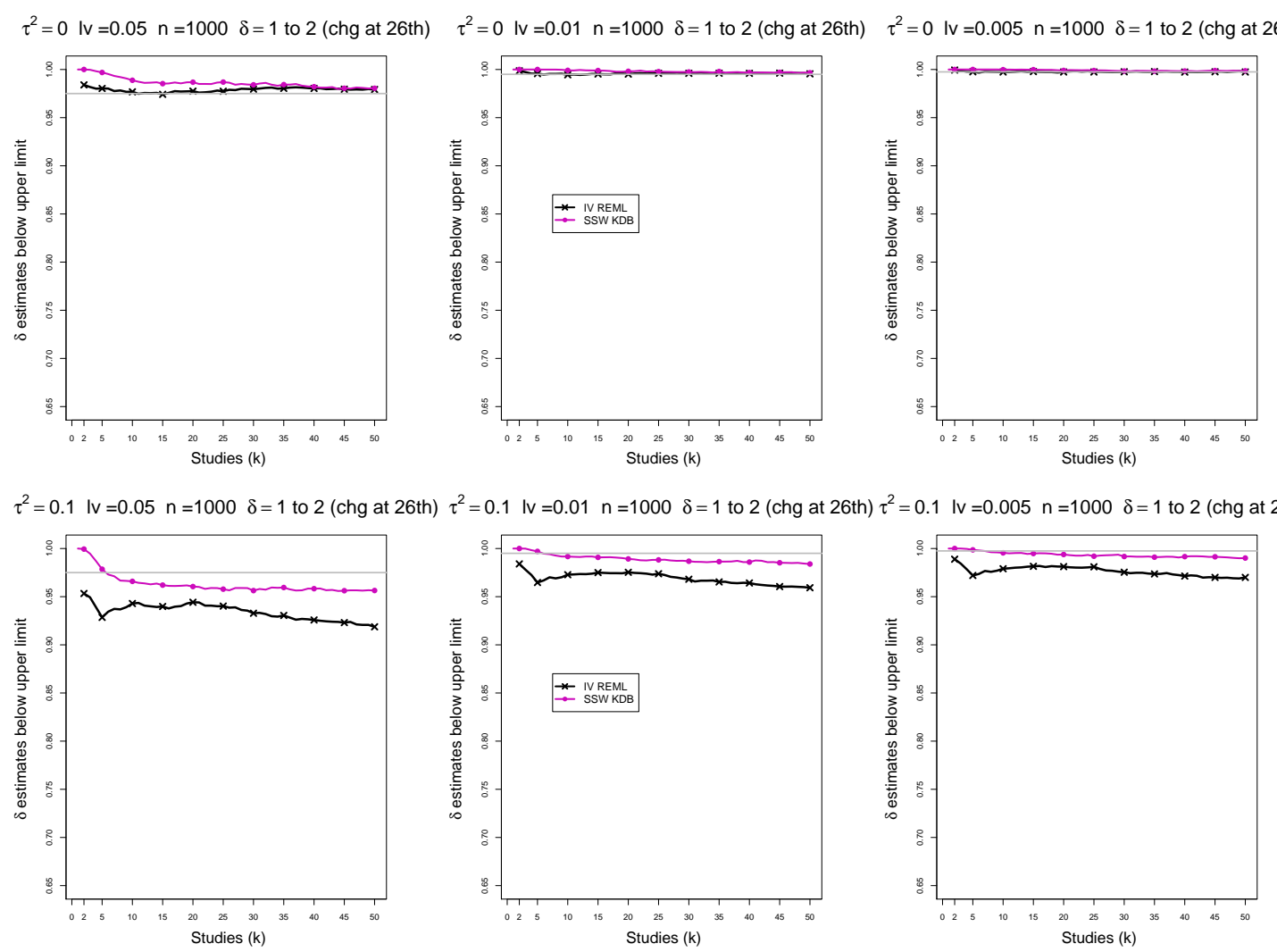

$\tau^{2}=0.25 \mathrm{lv}=0.05 \mathrm{n}=1000 \delta=1$ to 2 (chg at 26 th) $\tau^{2}=0.25 \mathrm{IV}=0.01 \mathrm{n}=1000 \quad \delta=1$ to 2 (chg at 26 th) $:^{2}=0.25 \mathrm{IV}=0.005 \mathrm{n}=1000 \quad \delta=1$ to 2 (chg at 26 th
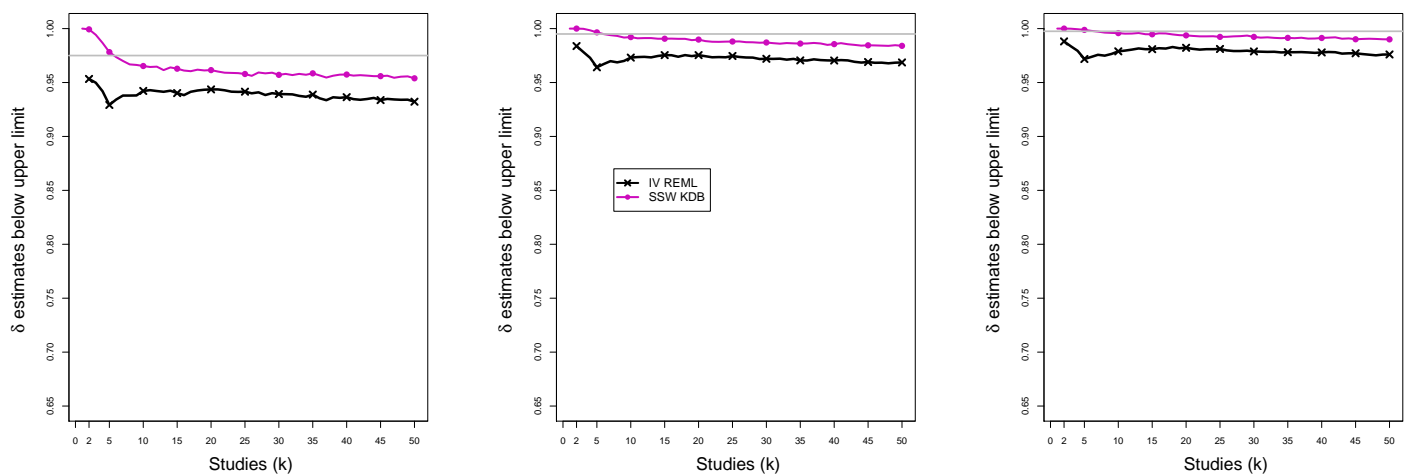

$\tau^{2}=1 \mathrm{IV}=0.05 \mathrm{n}=1000 \delta=1$ to 2 (chg at 26th)
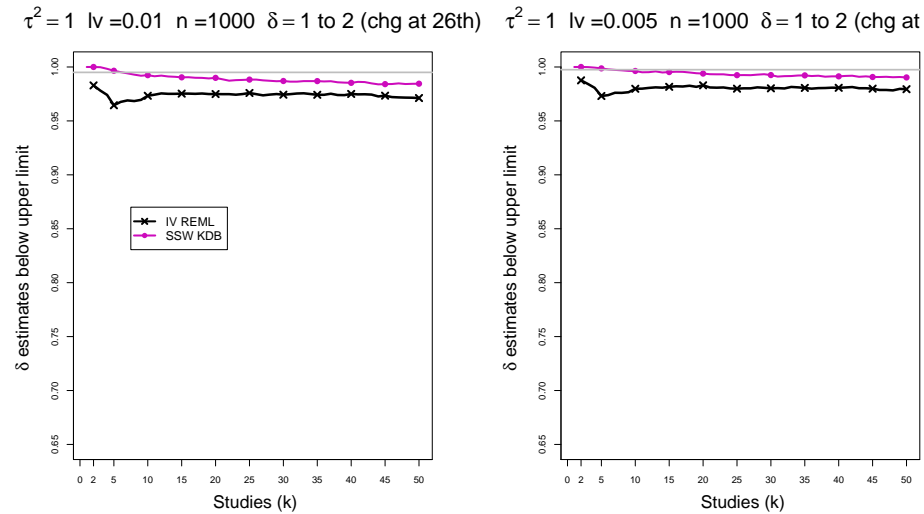

Figure D4. 25: 2-Stage CMA - Coverage of the upper confidence interval of $\delta$ (below upper limit) at the confidence level $1-\alpha / 2$ for $\alpha=0.05,0.01,0.005$ when $\delta=1$ for $k \leq 25$ followed by a shift to $\delta=2$ for $k \geq 26, \tau^{2}=0,0.1,0.25,1, n=1000, K=50$. Light grey line at $0.975,0.995,0.9975$. 

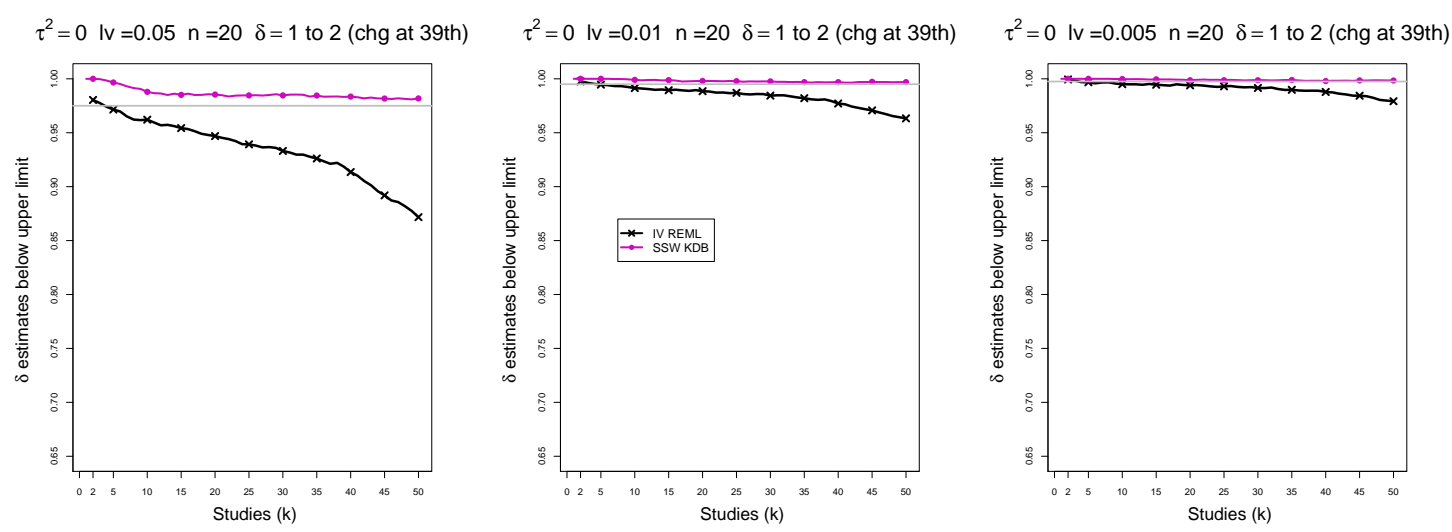

$\tau^{2}=0.1 \mathrm{lv}=0.05 \mathrm{n}=20 \delta=1$ to 2 (chg at 39 th)

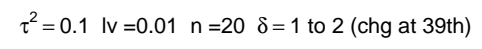

$\tau^{2}=0.1 \mathrm{lv}=0.005 \mathrm{n}=20 \quad \delta=1$ to 2 (chg at 39 th)
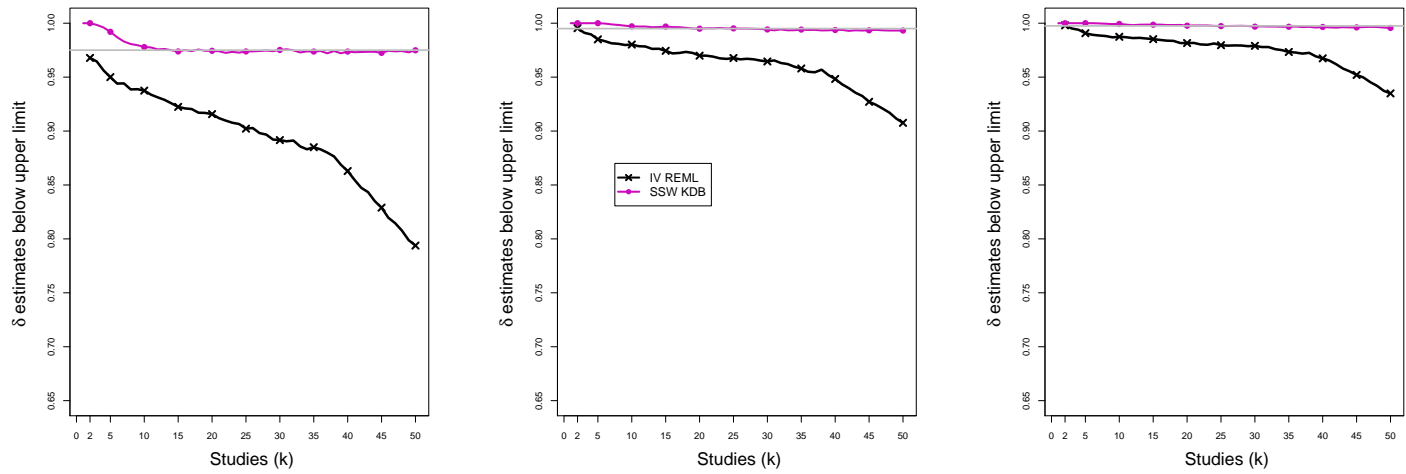

$\tau^{2}=0.25 \mathrm{lv}=0.05 \mathrm{n}=20 \quad \delta=1$ to 2 (chg at 39th)
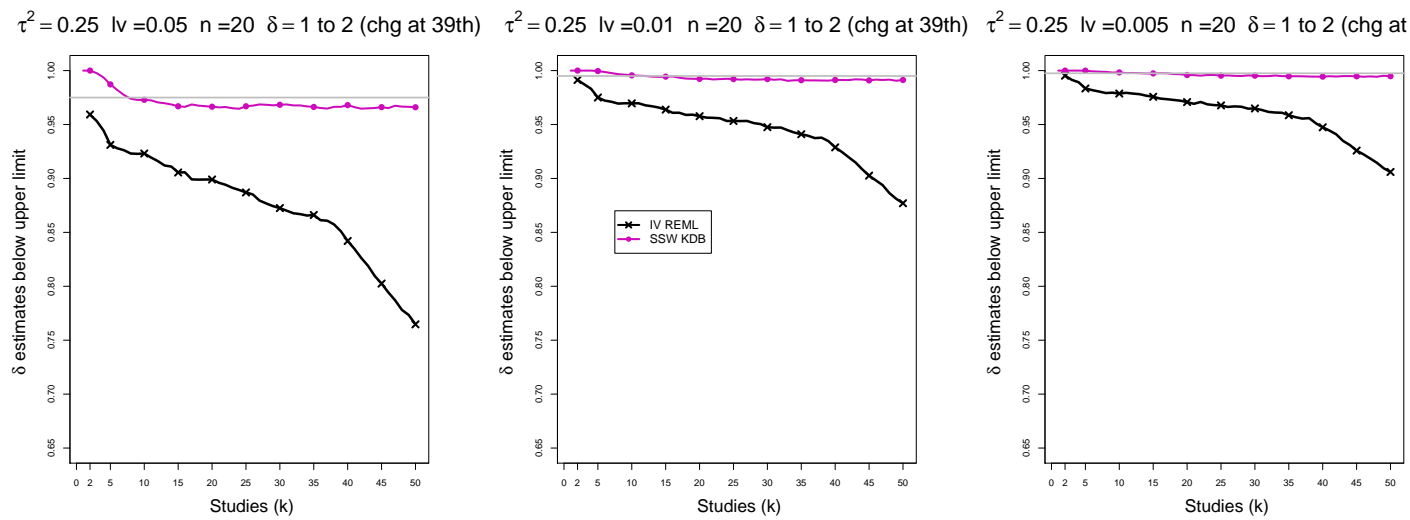

$\tau^{2}=1 \mathrm{IV}=0.01 \mathrm{n}=20 \quad \delta=1$ to 2 (chg at 39th)

$\tau^{2}=1 \mathrm{lv}=0.005 \mathrm{n}=20 \delta=1$ to 2 (chg at 39th)
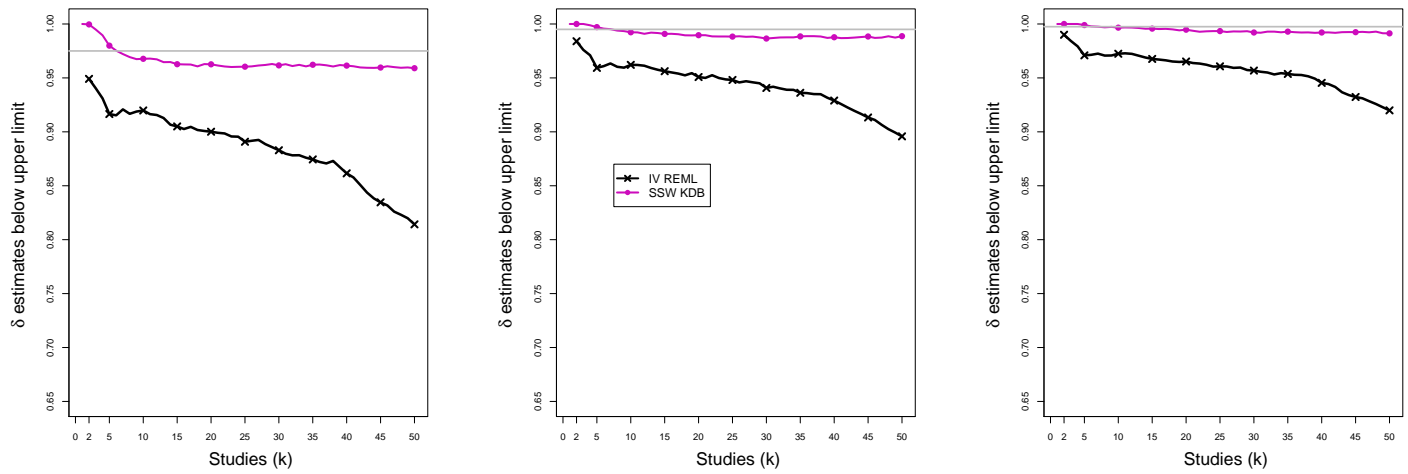

Figure D4. 26: 2-Stage CMA - Coverage of the upper confidence interval of $\delta$ (below upper limit) at the confidence level $1-\alpha / 2$ for $\alpha=0.05,0.01,0.005$ when $\delta=1$ for $k \leq 38$ followed by a shift to $\delta=2$ for $k \geq 39, \tau^{2}=0,0.1,0.25,1, n=20, K=50$. Light grey line at $0.975,0.995,0.9975$. 

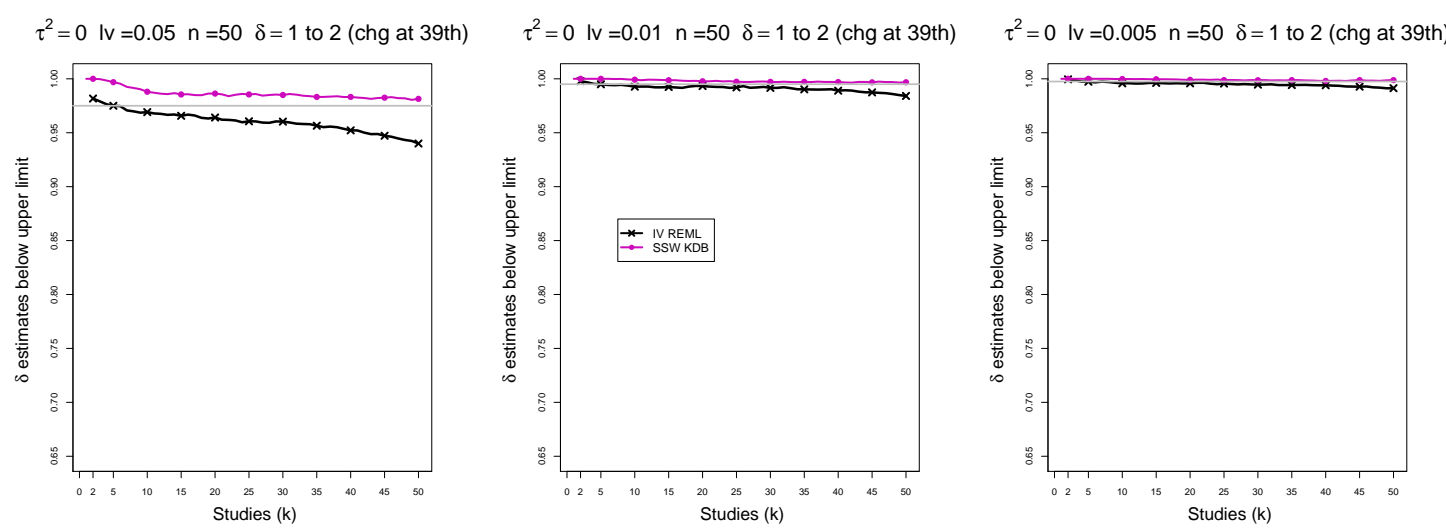

$\tau^{2}=0.1 \mathrm{IV}=0.05 \mathrm{n}=50 \delta=1$ to 2 (chg at 39 th)

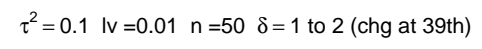

$\tau^{2}=0.1 \mathrm{lv}=0.005 \mathrm{n}=50 \quad \delta=1$ to 2 (chg at 39 th)
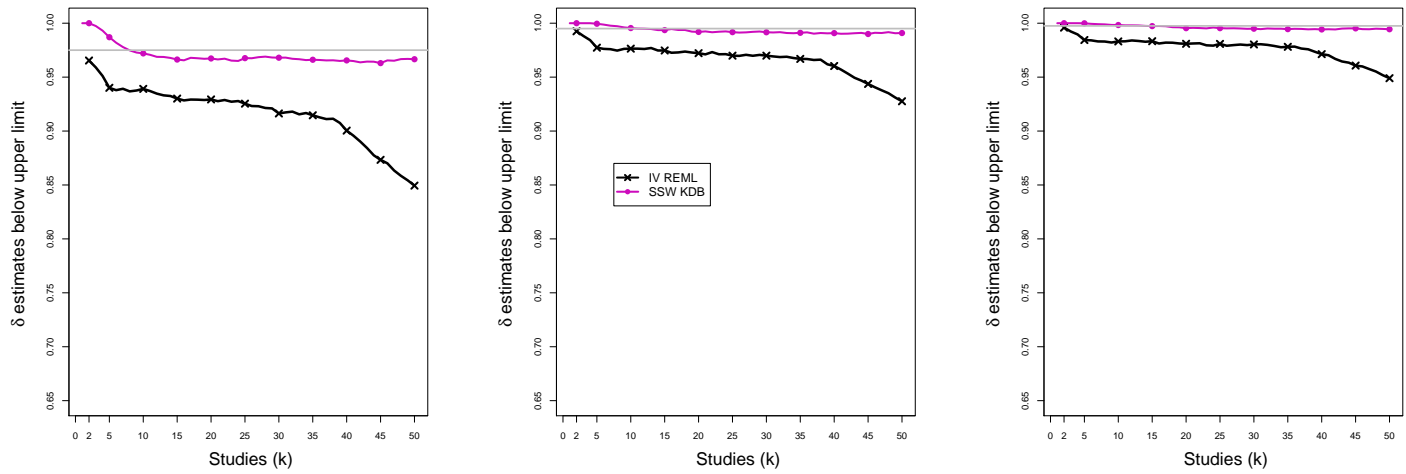

$\tau^{2}=0.25 \mathrm{lv}=0.05 \mathrm{n}=50 \quad \delta=1$ to 2 (chg at 39th)
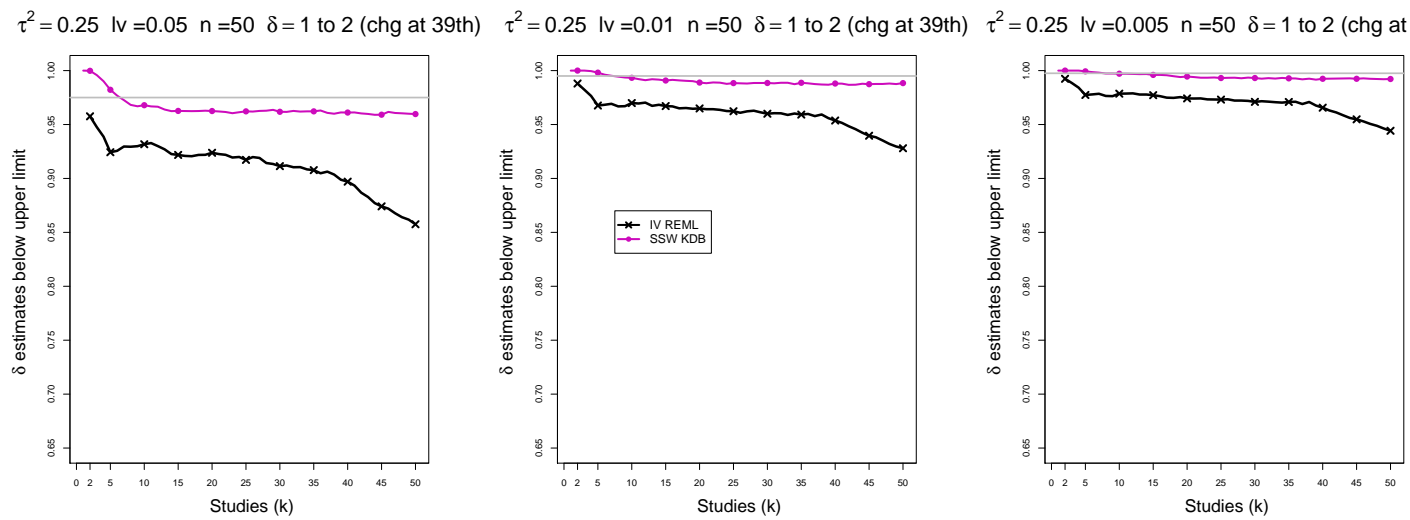

$\tau^{2}=1 \mathrm{IV}=0.01 \mathrm{n}=50 \quad \delta=1$ to 2 (chg at 39 th)

$\tau^{2}=1 \mathrm{lv}=0.005 \mathrm{n}=50 \delta=1$ to 2 (chg at 39th)
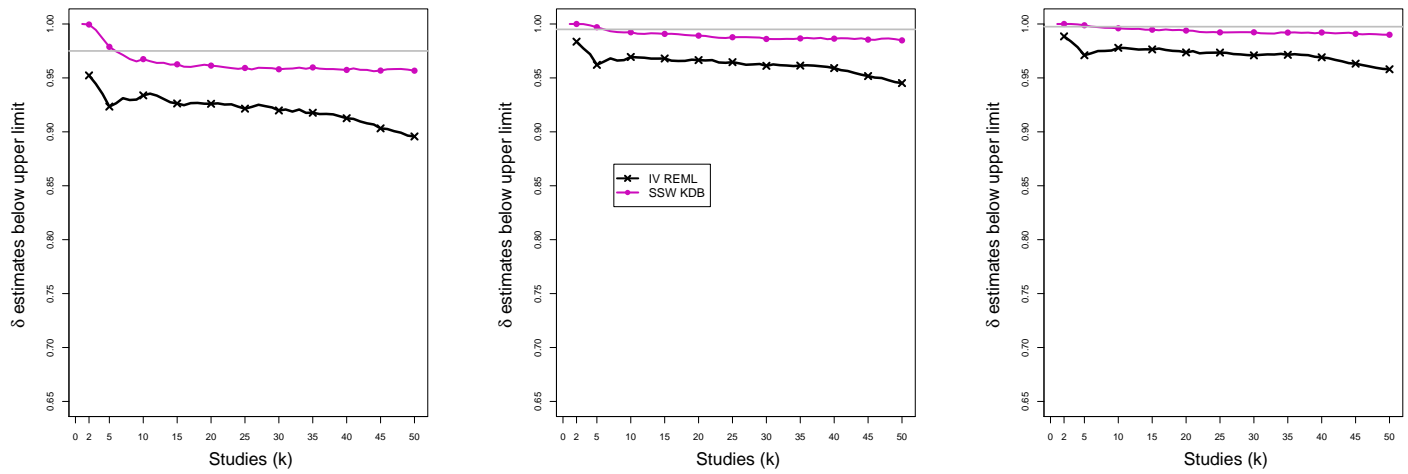

Figure D4. 27: 2-Stage CMA - Coverage of the upper confidence interval of $\delta$ (below upper limit) at the confidence level $1-\alpha / 2$ for $\alpha=0.05,0.01,0.005$ when $\delta=1$ for $k \leq 38$ followed by a shift to $\delta=2$ for $k \geq 39, \tau^{2}=0,0.1,0.25,1, n=50, K=50$. Light grey line at $0.975,0.995,0.9975$. 

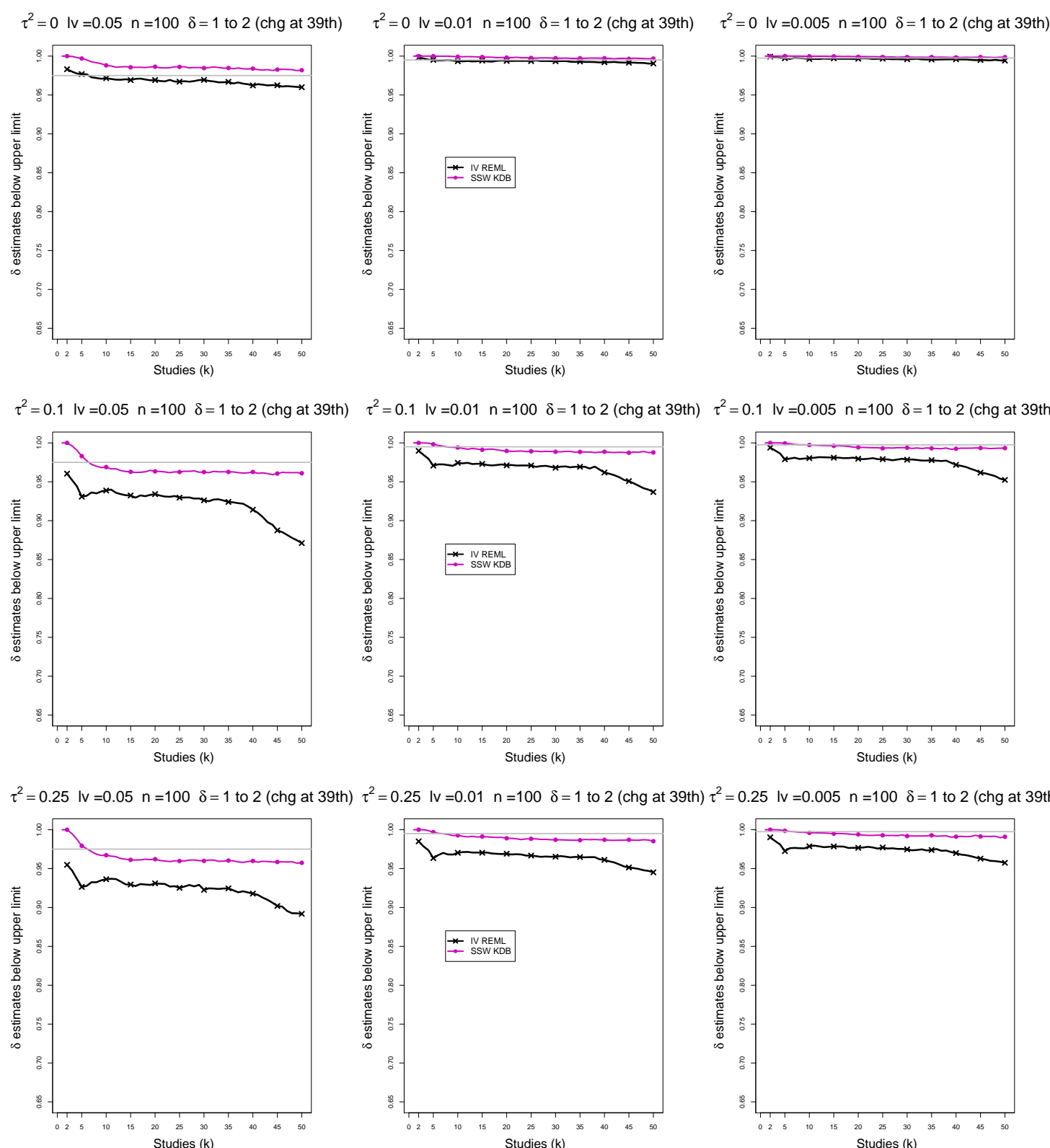

$\tau^{2}=1 \mathrm{lv}=0.05 \quad \mathrm{n}=100 \quad \delta=1$ to 2 (chg at 39th)

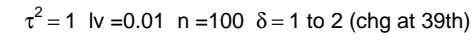

$\tau^{2}=1$ IV $=0.005 \quad n=100 \quad \delta=1$ to 2 (chg at 39th)
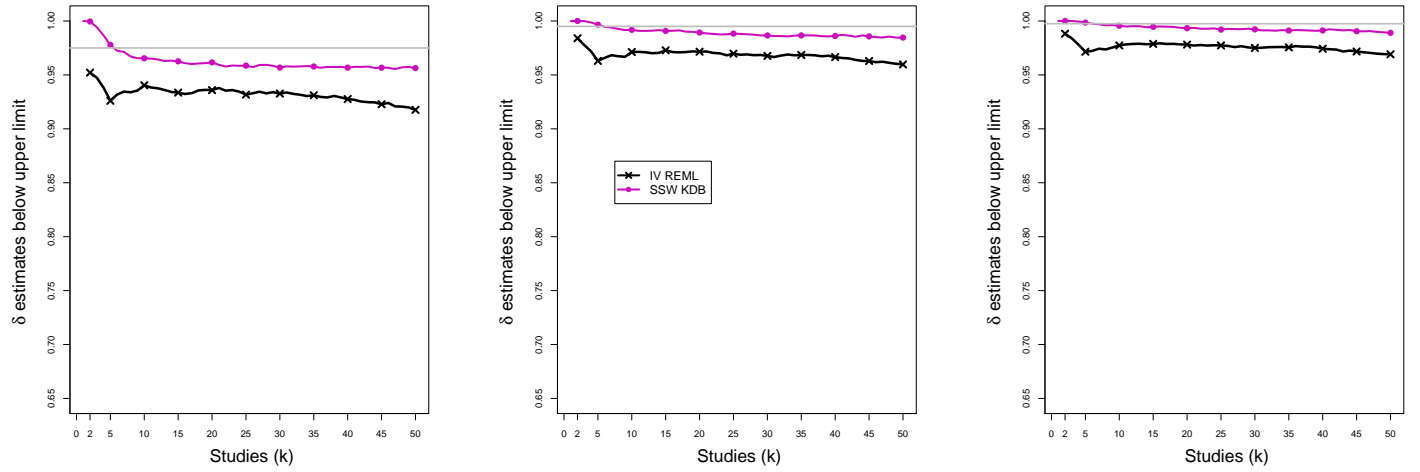

Figure D4. 28: 2-Stage CMA - Coverage of the upper confidence interval of $\delta$ (below upper limit) at the confidence level $1-\alpha / 2$ for $\alpha=0.05,0.01,0.005$ when $\delta=1$ for $k \leq 38$ followed by a shift to $\delta=2$ for $k \geq 39, \tau^{2}=0,0.1,0.25,1, n=100, K=50$. Light grey line at $0.975,0.995,0.9975$. 

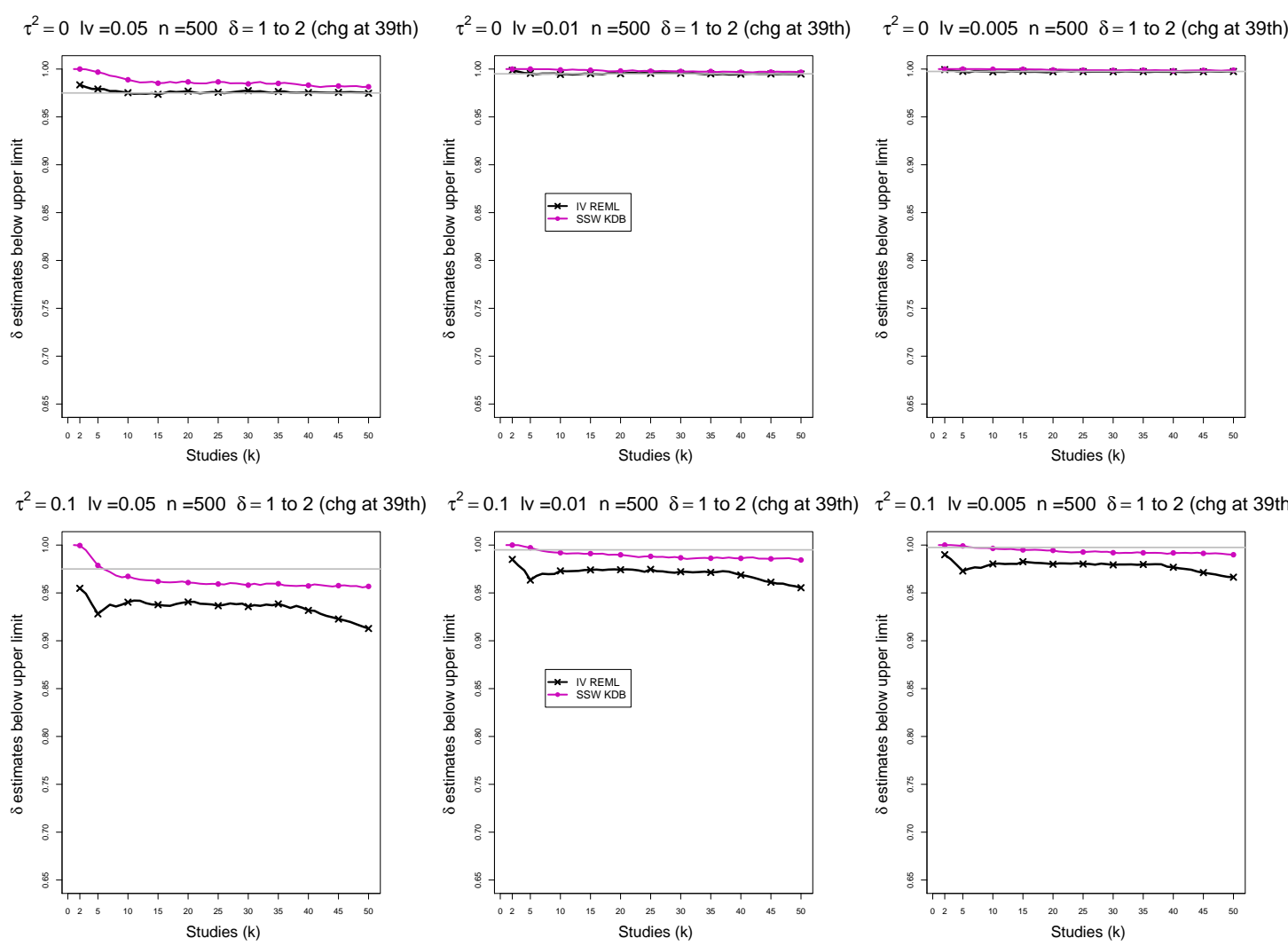

$\tau^{2}=0.25 \mathrm{lv}=0.05 \mathrm{n}=500 \quad \delta=1$ to 2 (chg at 39 th) $\tau^{2}=0.25 \mathrm{lv}=0.01 \mathrm{n}=500 \quad \delta=1$ to 2 (chg at 39 th) $\tau^{2}=0.25 \mathrm{lv}=0.005 \mathrm{n}=500 \quad \delta=1$ to 2 (chg at 39 th)
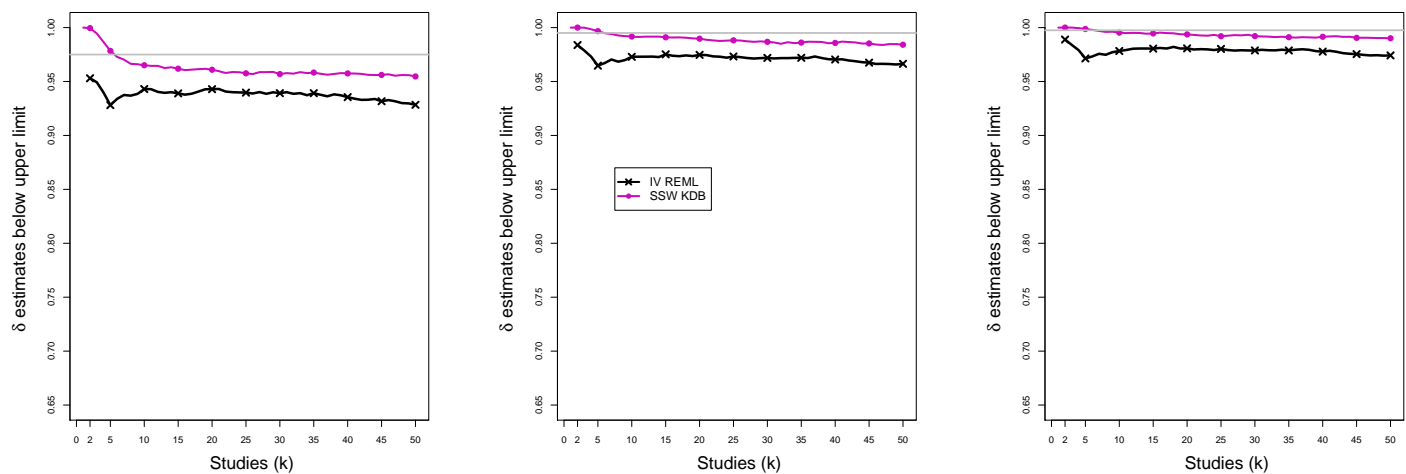

$\tau^{2}=1 \mathrm{lv}=0.05 \quad \mathrm{n}=500 \quad \delta=1$ to 2 (chg at 39th)

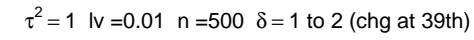

$\tau^{2}=1$ IV $=0.005 \quad n=500 \delta=1$ to 2 (chg at 39th)
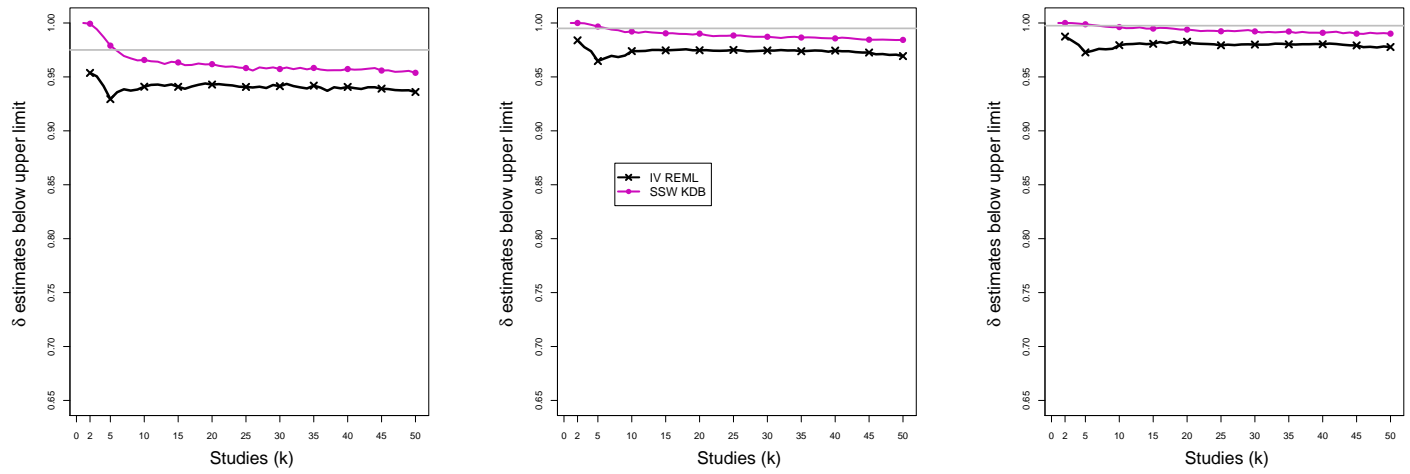

Figure D4. 29: 2-Stage CMA - Coverage of the upper confidence interval of $\delta$ (below upper limit) at the confidence level $1-\alpha / 2$ for $\alpha=0.05,0.01,0.005$ when $\delta=1$ for $k \leq 38$ followed by a shift to $\delta=2$ for $k \geq 39, \tau^{2}=0,0.1,0.25,1, n=500, K=50$. Light grey line at $0.975,0.995,0.9975$. 

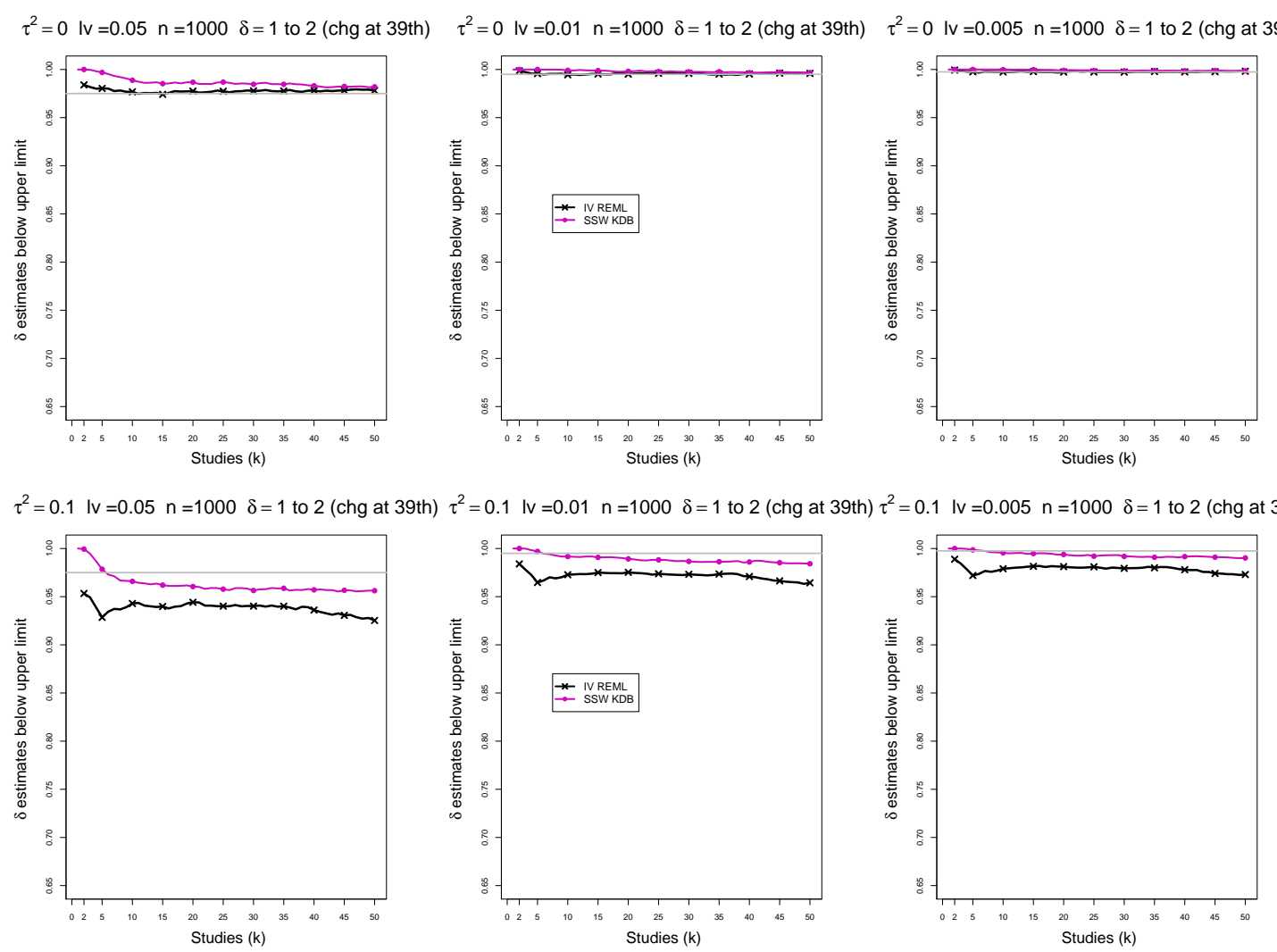

$\tau^{2}=0.25 \mathrm{lv}=0.05 \mathrm{n}=1000 \delta=1$ to 2 (chg at 39 th) $\tau^{2}=0.25 \mathrm{IV}=0.01 \mathrm{n}=1000 \quad \delta=1$ to 2 (chg at 39 th) $:^{2}=0.25 \mathrm{IV}=0.005 \mathrm{n}=1000 \quad \delta=1$ to 2 (chg at 39 th
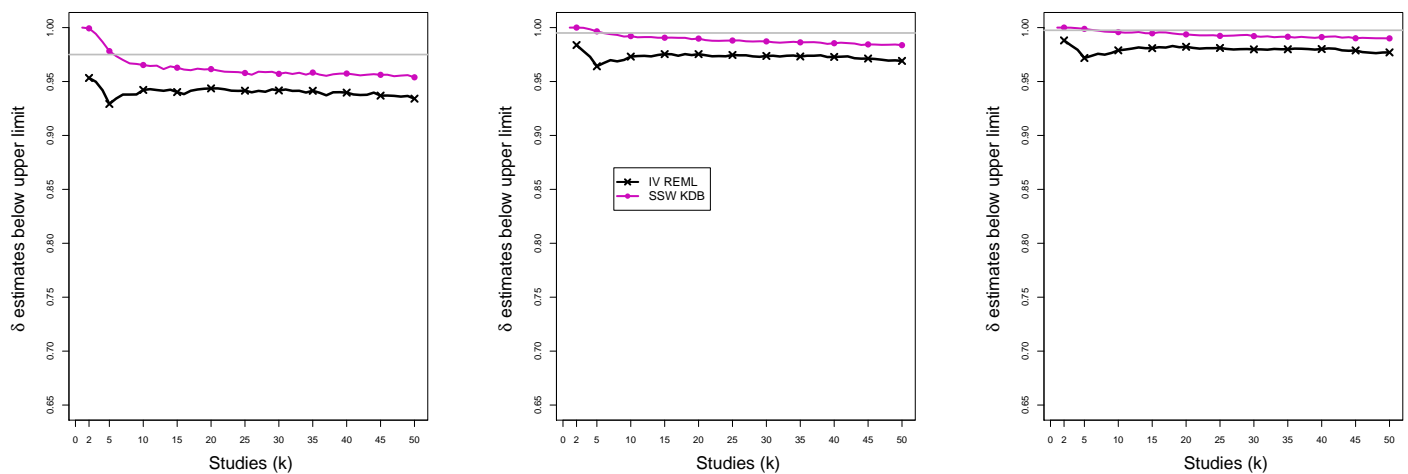

$\tau^{2}=1 \mathrm{IV}=0.05 \mathrm{n}=1000 \delta=1$ to 2 (chg at 39 th)

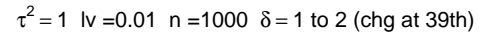

$\tau^{2}=1$ Iv $=0.005 \mathrm{n}=1000 \delta=1$ to 2 (chg at 39 th)
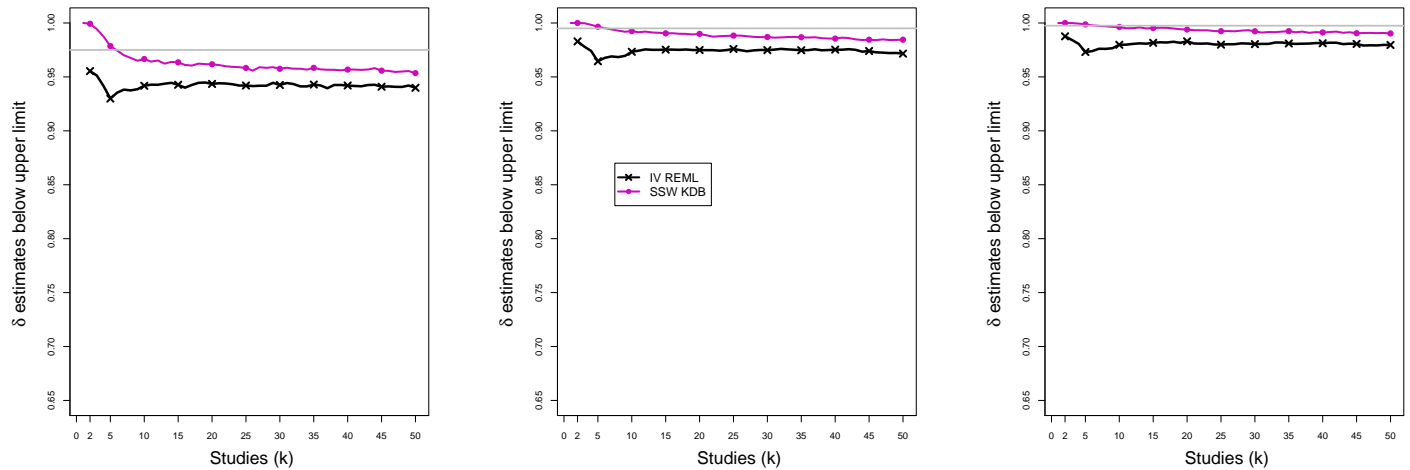

Figure D4. 30: 2-Stage CMA - Coverage of the upper confidence interval of $\delta$ (below upper limit) at the confidence level $1-\alpha / 2$ for $\alpha=0.05,0.01,0.005$ when $\delta=1$ for $k \leq 38$ followed by a shift to $\delta=2$ for $k \geq 39, \tau^{2}=0,0.1,0.25,1, n=1000, K=50$. Light grey line at $0.975,0.995,0.9975$. 


\section{D5. Empirical levels and power of 2-Stage CMA tests for shift in $\delta$}

For empirical levels and power of tests for shift in known $\delta$ in this section, each figure corresponds to one of the two tested meta-analysis methods, a set of values of $\tau^{2}(=0$, $0.1,0.25,1)$, a value of $n(=20,50,100,500,1000)$, a set of values of significance level $(=0.05,0.01,0.005)$ and a value of point of shift $f(=13,26,39)$ while $\delta$ shifts from 1 to 2 and maximum number of studies $K=50$.

Each figure contains a panel (with study $k$ on the horizontal axis) for each combination of parameters.

The tested methods are

- IV REML (Restricted maximum likelihood, inverse variance weighted)

- SSW KDB (Kulinskaya-Dollinger-Bjørkestøl estimator of $\tau^{2}$, sample size weighted) 

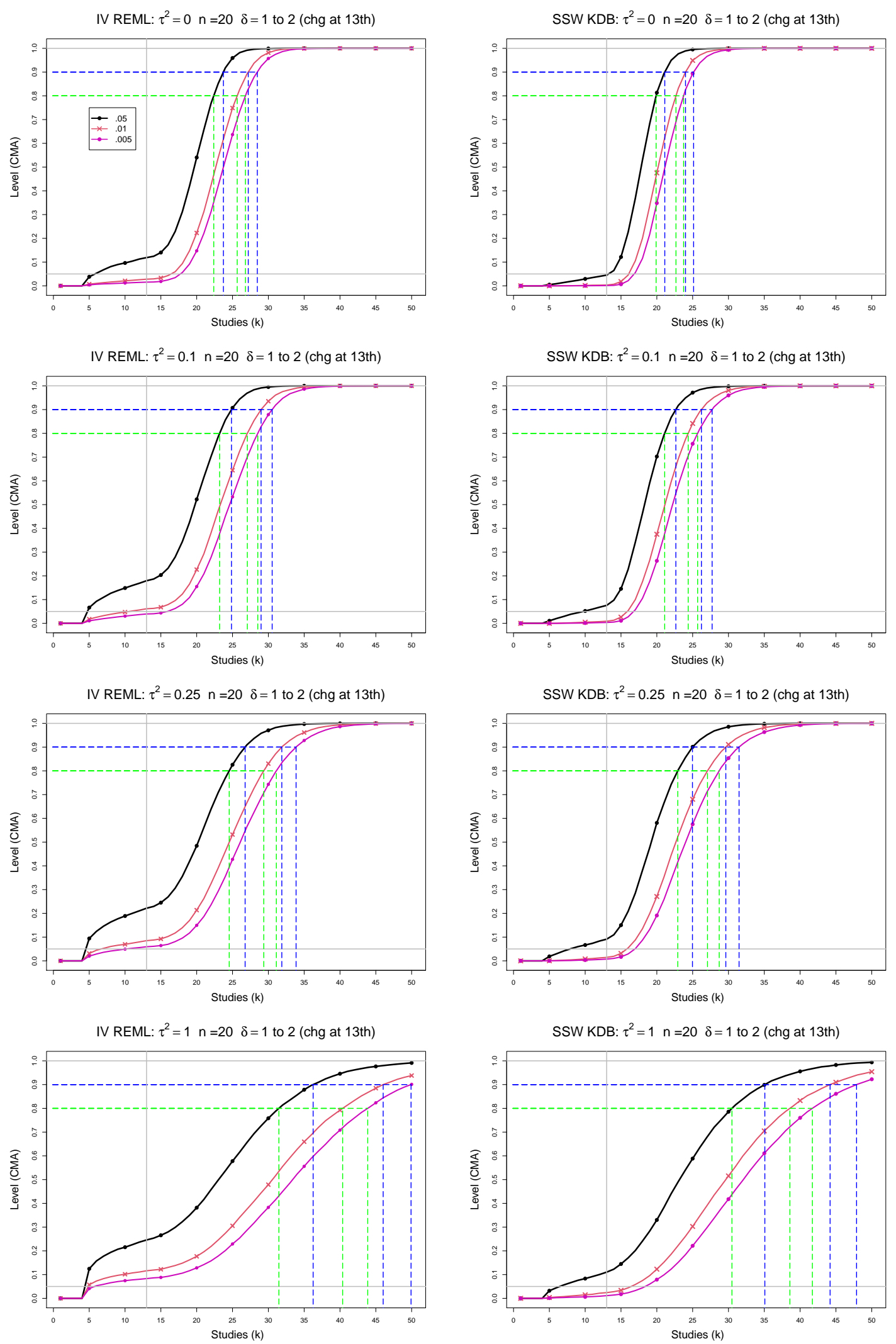

Figure D5. 1: Empirical levels of 2-Stage CMA tests for shift in $\delta$ based on SSW KDB and IV REML at .05, .01 and .005 nominal levels for equal sample sizes $n_{i C}+n_{i T}=n=20$, $\tau^{2}=0,0.1,0.25,1$ and a shift from $\delta=1$ to $\delta=2$ at study number 13 . Light grey line at .05 . Green and blue dashed lines correspond to power $80 \%$ and $90 \%$, respectively. 

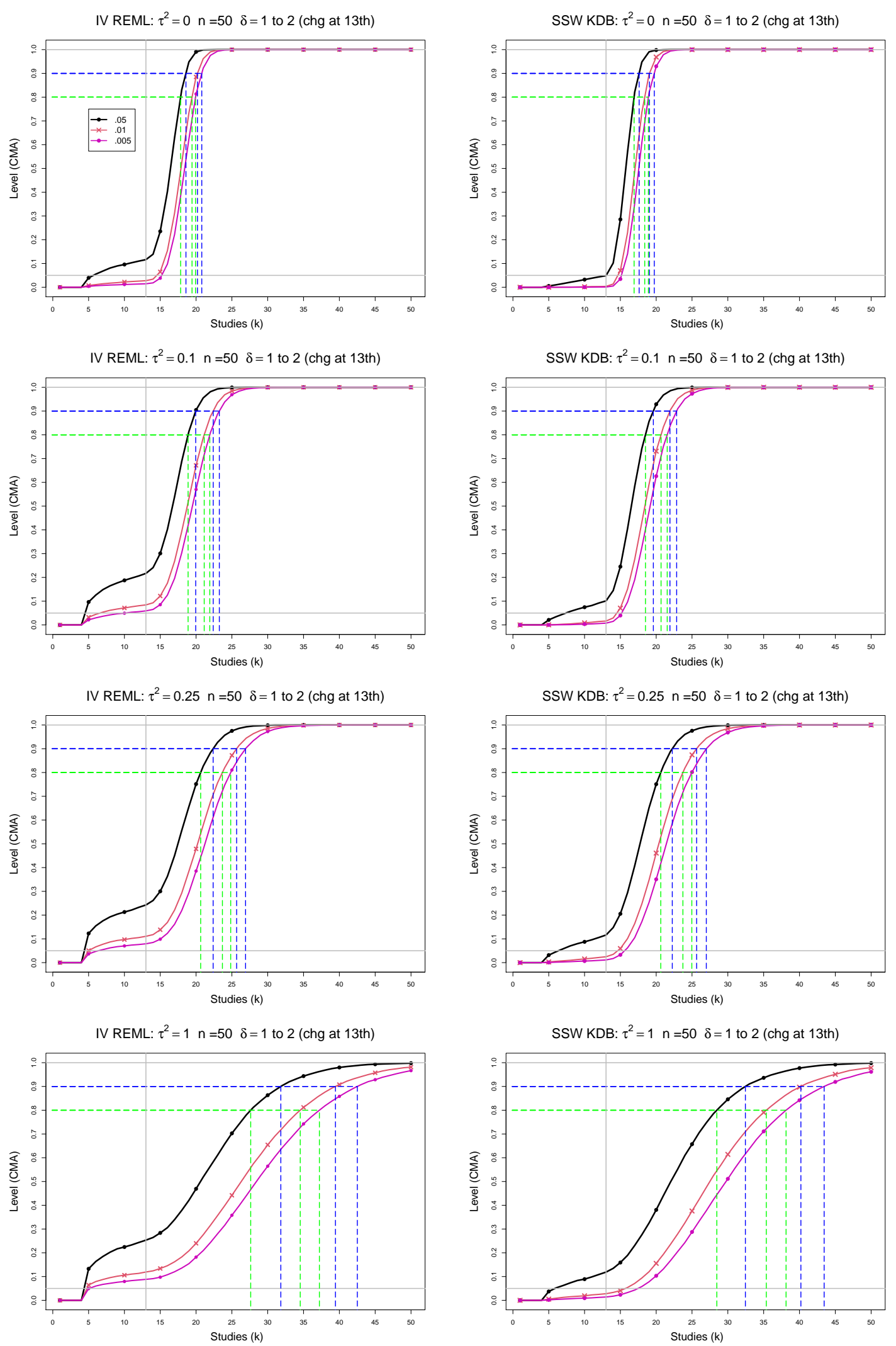

Figure D5. 2: Empirical levels of 2-Stage CMA tests for shift in $\delta$ based on SSW KDB and IV REML at .05, .01 and .005 nominal levels for equal sample sizes $n_{i C}+n_{i T}=n=50$, $\tau^{2}=0,0.1,0.25,1$ and a shift from $\delta=1$ to $\delta=2$ at study number 13 . Light grey line at .05. Green and blue dashed lines correspond to power $80 \%$ and $90 \%$, respectively. 

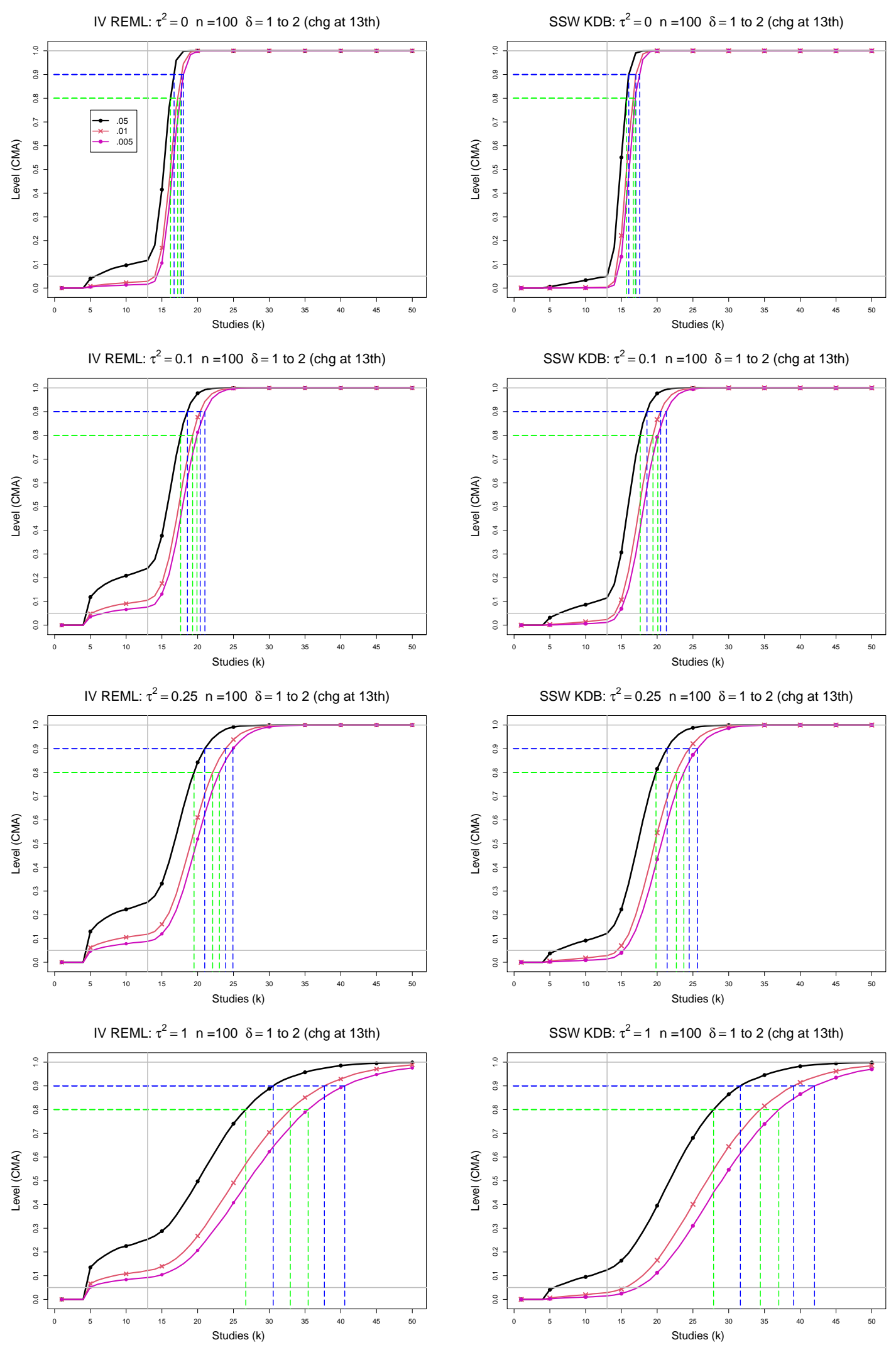

Figure D5. 3: Empirical levels of 2-Stage CMA tests for shift in $\delta$ based on SSW KDB and IV REML at .05, .01 and .005 nominal levels for equal sample sizes $n_{i C}+n_{i T}=n=100$, $\tau^{2}=0,0.1,0.25,1$ and a shift from $\delta=1$ to $\delta=2$ at study number 13 . Light grey line at .05. Green and blue dashed lines correspond to power $80 \%$ and $90 \%$, respectively. 

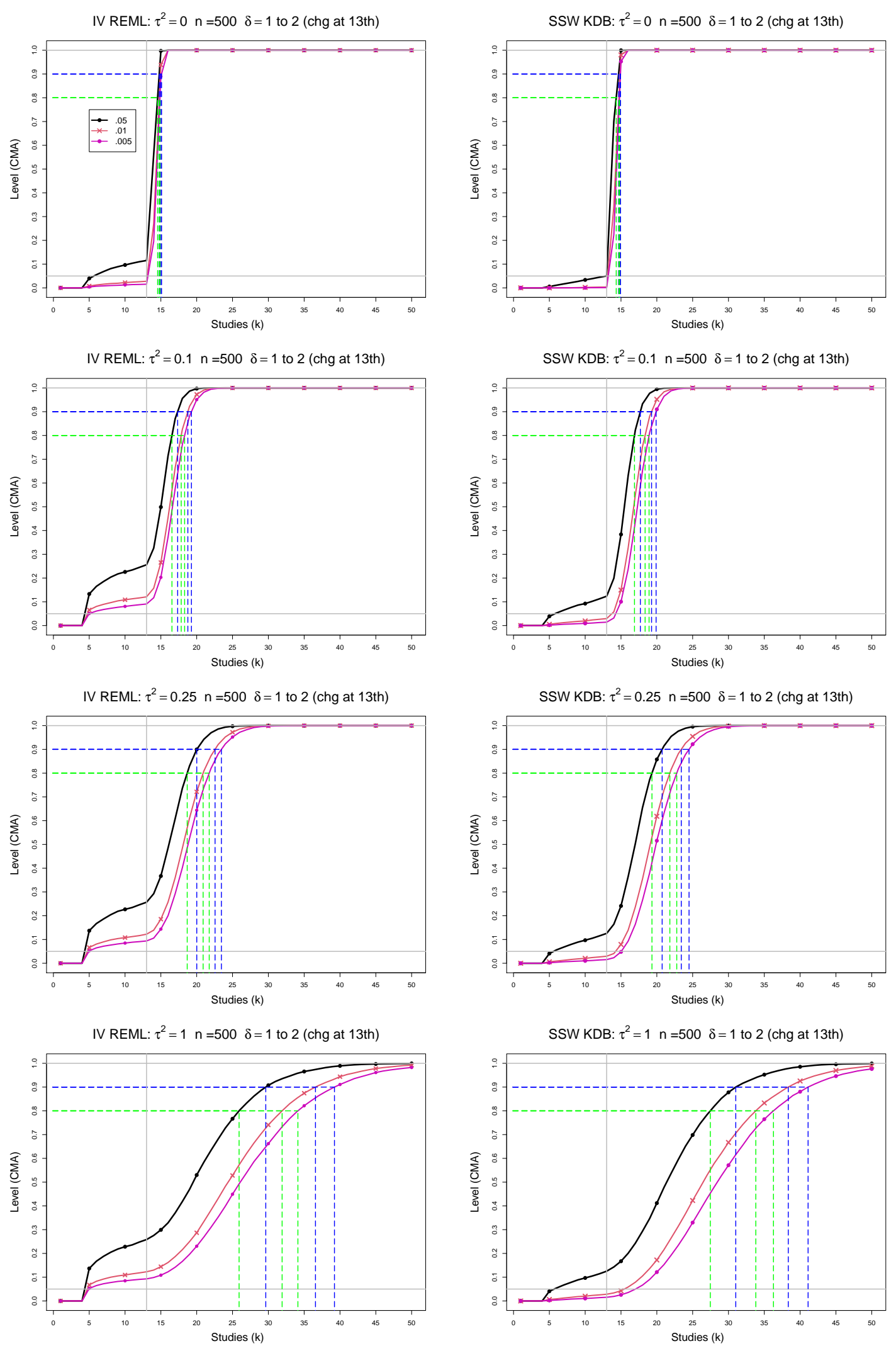

Figure D5. 4: Empirical levels of 2-Stage CMA tests for shift in $\delta$ based on SSW KDB and IV REML at .05, .01 and .005 nominal levels for equal sample sizes $n_{i C}+n_{i T}=n=500$, $\tau^{2}=0,0.1,0.25,1$ and a shift from $\delta=1$ to $\delta=2$ at study number 13 . Light grey line at .05. Green and blue dashed lines correspond to power $80 \%$ and $90 \%$, respectively. 

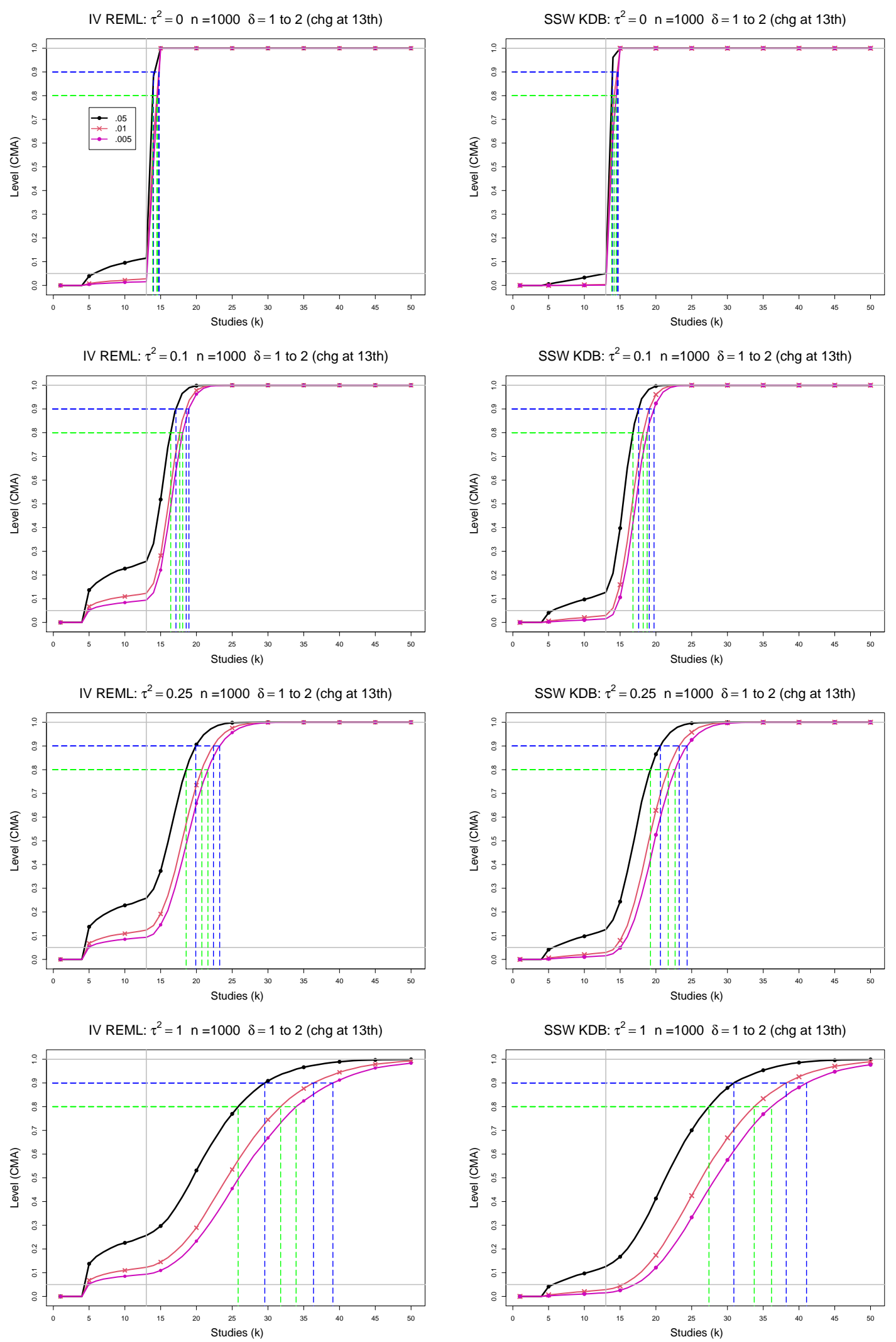

Figure D5. 5: Empirical levels of 2-Stage CMA tests for shift in $\delta$ based on SSW KDB and IV REML at .05, .01 and .005 nominal levels for equal sample sizes $n_{i C}+n_{i T}=n=1000$, $\tau^{2}=0,0.1,0.25,1$ and a shift from $\delta=1$ to $\delta=2$ at study number 13 . Light grey line at .05. Green and blue dashed lines correspond to power $80 \%$ and $90 \%$, respectively. 

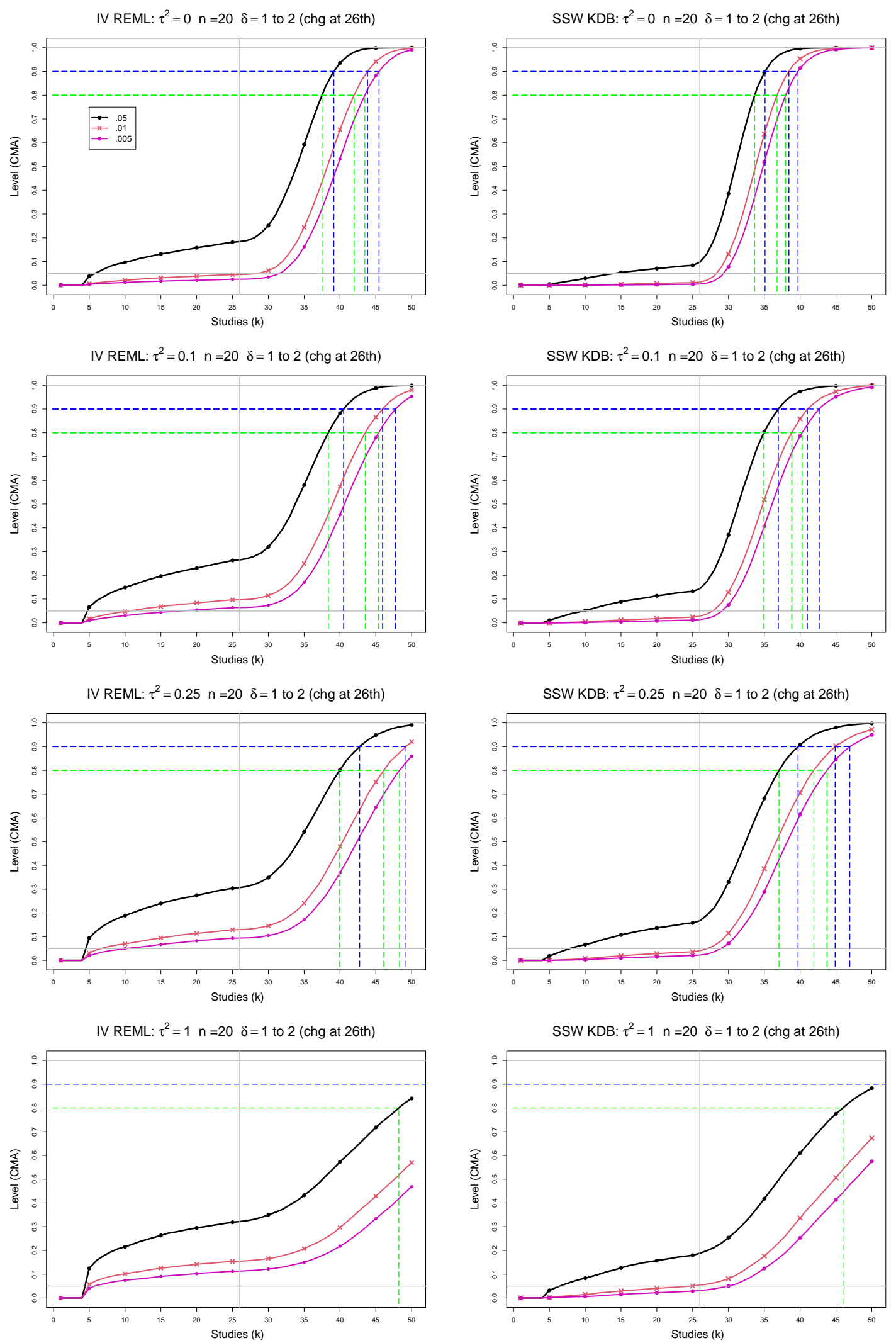

Figure D5. 6: Empirical levels of 2-Stage CMA tests for shift in $\delta$ based on SSW KDB and IV REML at .05, .01 and .005 nominal levels for equal sample sizes $n_{i C}+n_{i T}=n=20$, $\tau^{2}=0,0.1,0.25,1$ and a shift from $\delta=1$ to $\delta=2$ at study number 26. Light grey line at .05. Green and blue dashed lines correspond to power $80 \%$ and $90 \%$, respectively. 

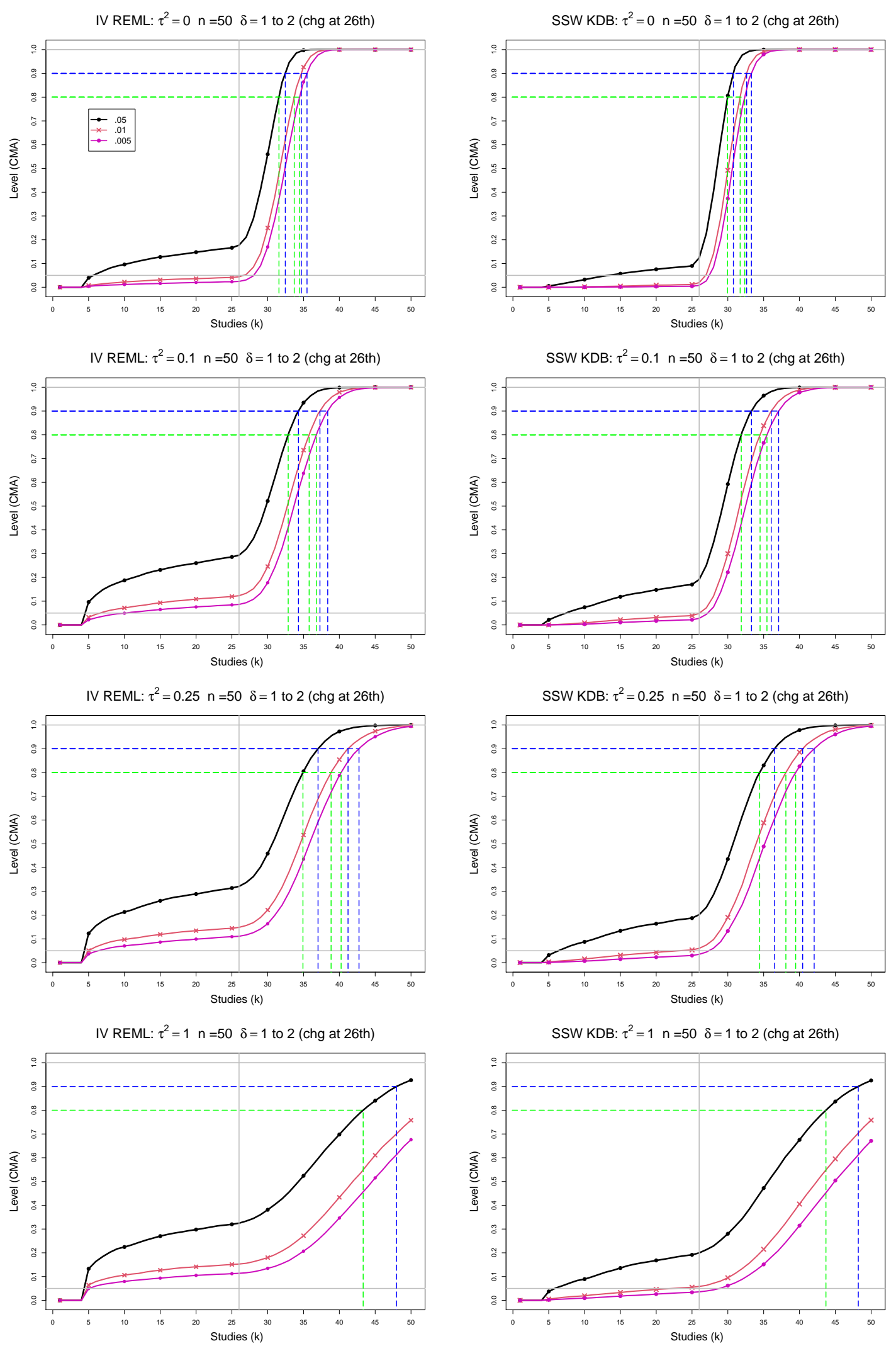

Figure D5. 7: Empirical levels of 2-Stage CMA tests for shift in $\delta$ based on SSW KDB and IV REML at .05, .01 and .005 nominal levels for equal sample sizes $n_{i C}+n_{i T}=n=50$, $\tau^{2}=0,0.1,0.25,1$ and a shift from $\delta=1$ to $\delta=2$ at study number 26. Light grey line at .05. Green and blue dashed lines correspond to power $80 \%$ and $90 \%$, respectively. 

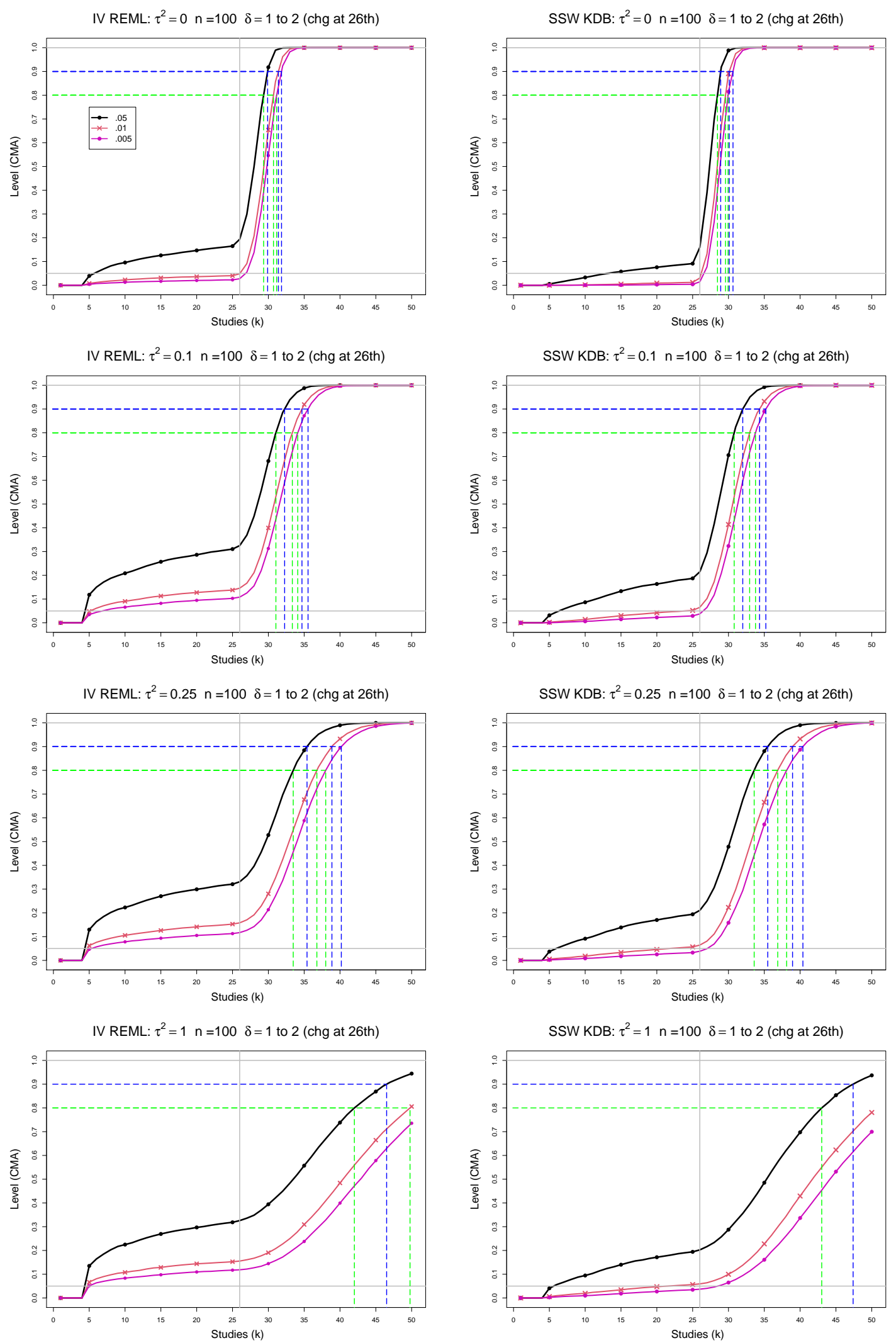

Figure D5. 8: Empirical levels of 2-Stage CMA tests for shift in $\delta$ based on SSW KDB and IV REML at .05, .01 and .005 nominal levels for equal sample sizes $n_{i C}+n_{i T}=n=100$, $\tau^{2}=0,0.1,0.25,1$ and a shift from $\delta=1$ to $\delta=2$ at study number 26. Light grey line at .05. Green and blue dashed lines correspond to power $80 \%$ and $90 \%$, respectively. 

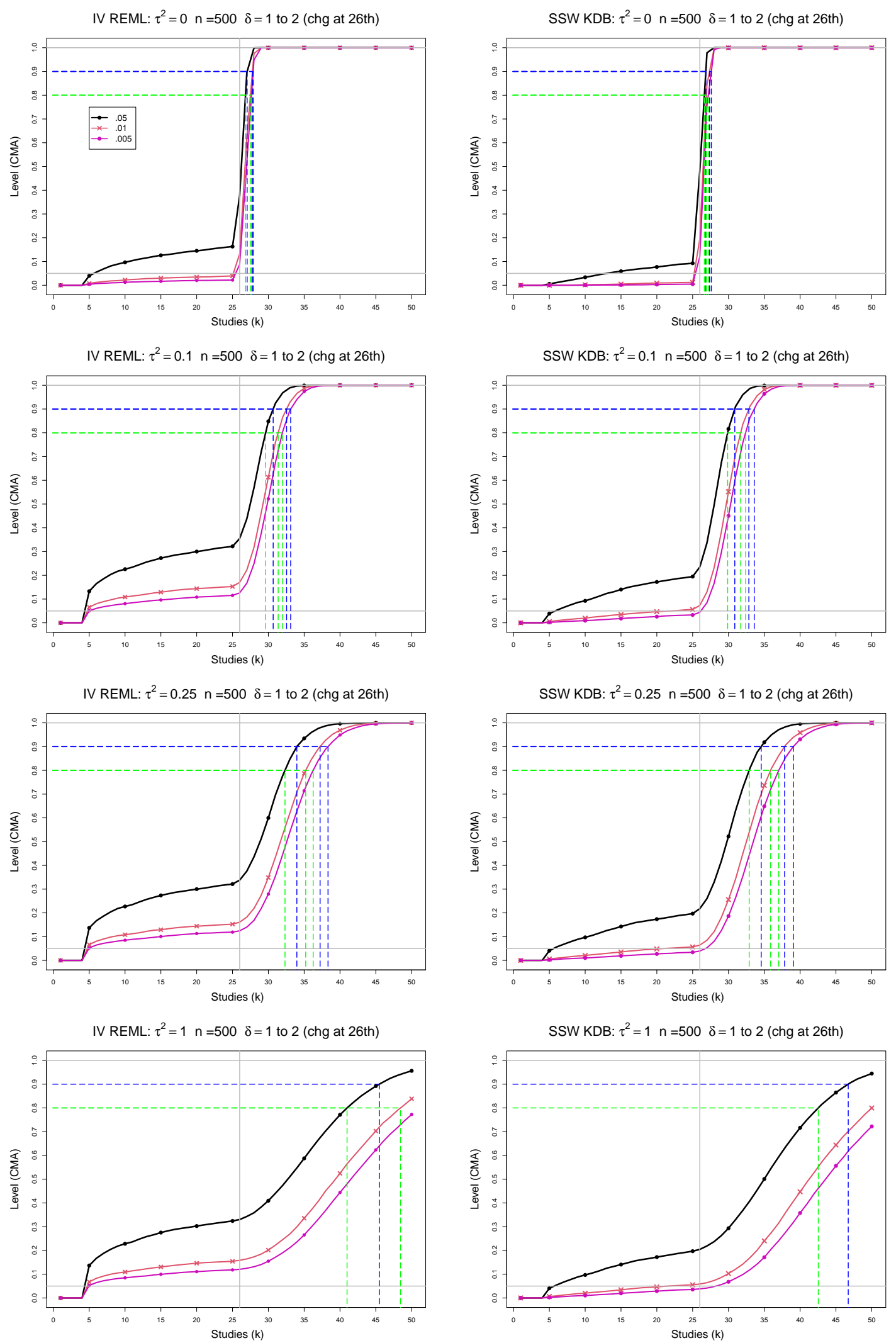

Figure D5. 9: Empirical levels of 2-Stage CMA tests for shift in $\delta$ based on SSW KDB and IV REML at .05, .01 and .005 nominal levels for equal sample sizes $n_{i C}+n_{i T}=n=500$, $\tau^{2}=0,0.1,0.25,1$ and a shift from $\delta=1$ to $\delta=2$ at study number 26. Light grey line at .05. Green and blue dashed lines correspond to power $80 \%$ and $90 \%$, respectively. 

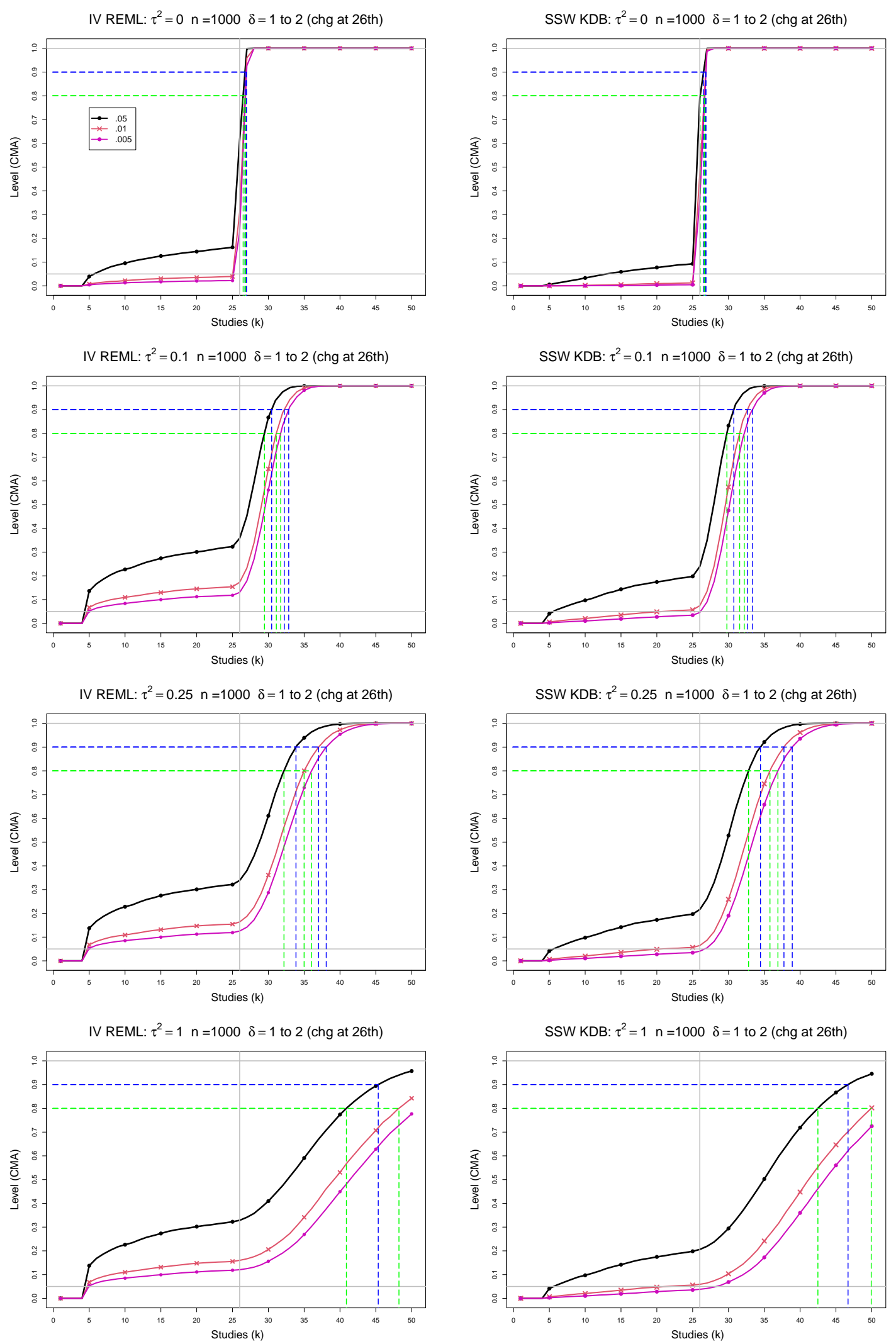

Figure D5. 10: Empirical levels of 2-Stage CMA tests for shift in $\delta$ based on SSW KDB and IV REML at .05, .01 and .005 nominal levels for equal sample sizes $n_{i C}+n_{i T}=n=1000$, $\tau^{2}=0,0.1,0.25,1$ and a shift from $\delta=1$ to $\delta=2$ at study number 26. Light grey line at .05. Green and blue dashed lines correspond to power $80 \%$ and $90 \%$, respectively. 

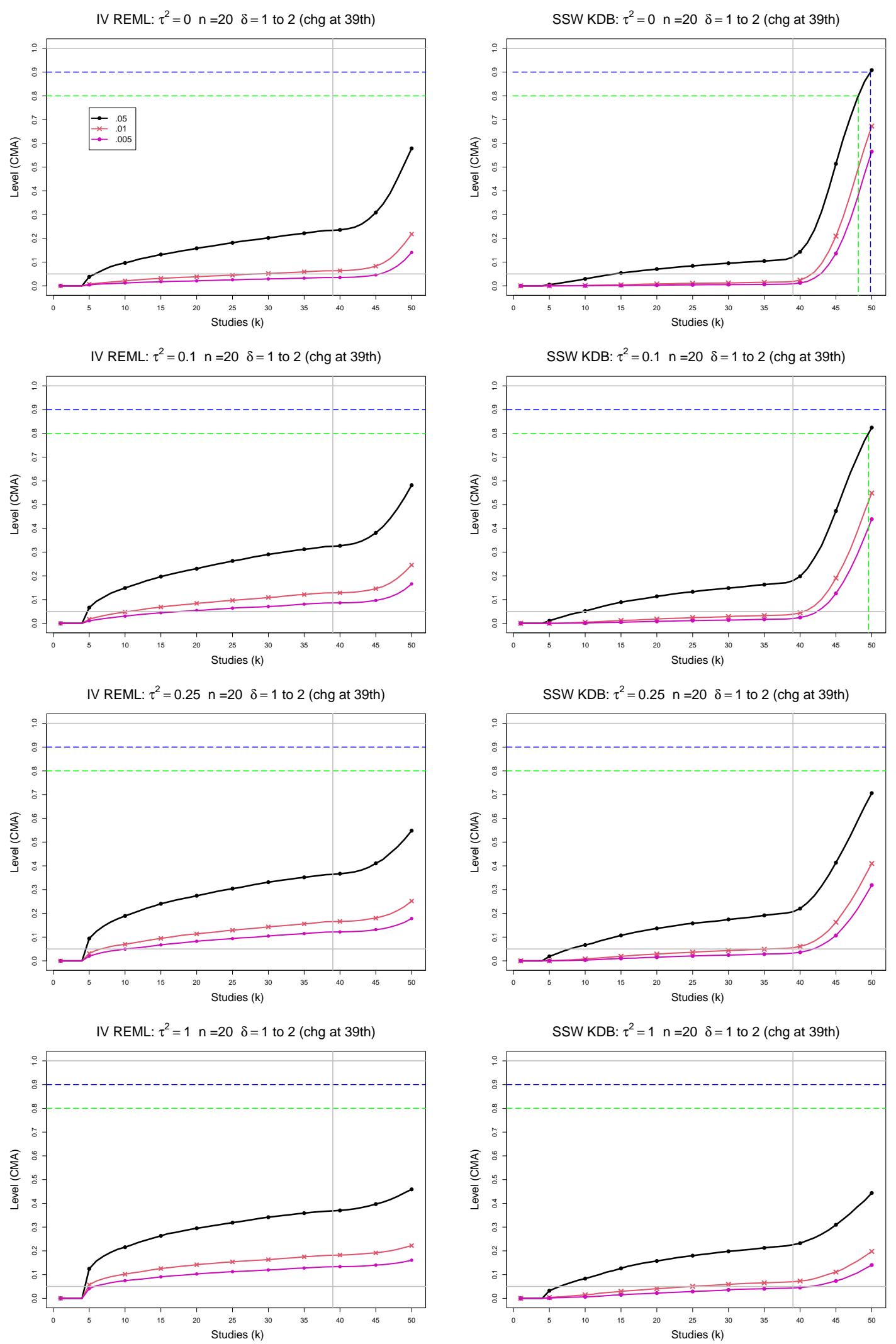

Figure D5. 11: Empirical levels of 2-Stage CMA tests for shift in $\delta$ based on SSW KDB and IV REML at .05, .01 and .005 nominal levels for equal sample sizes $n_{i C}+n_{i T}=n=20$, $\tau^{2}=0,0.1,0.25,1$ and a shift from $\delta=1$ to $\delta=2$ at study number 39. Light grey line at .05. Green and blue dashed lines correspond to power $80 \%$ and $90 \%$, respectively. 

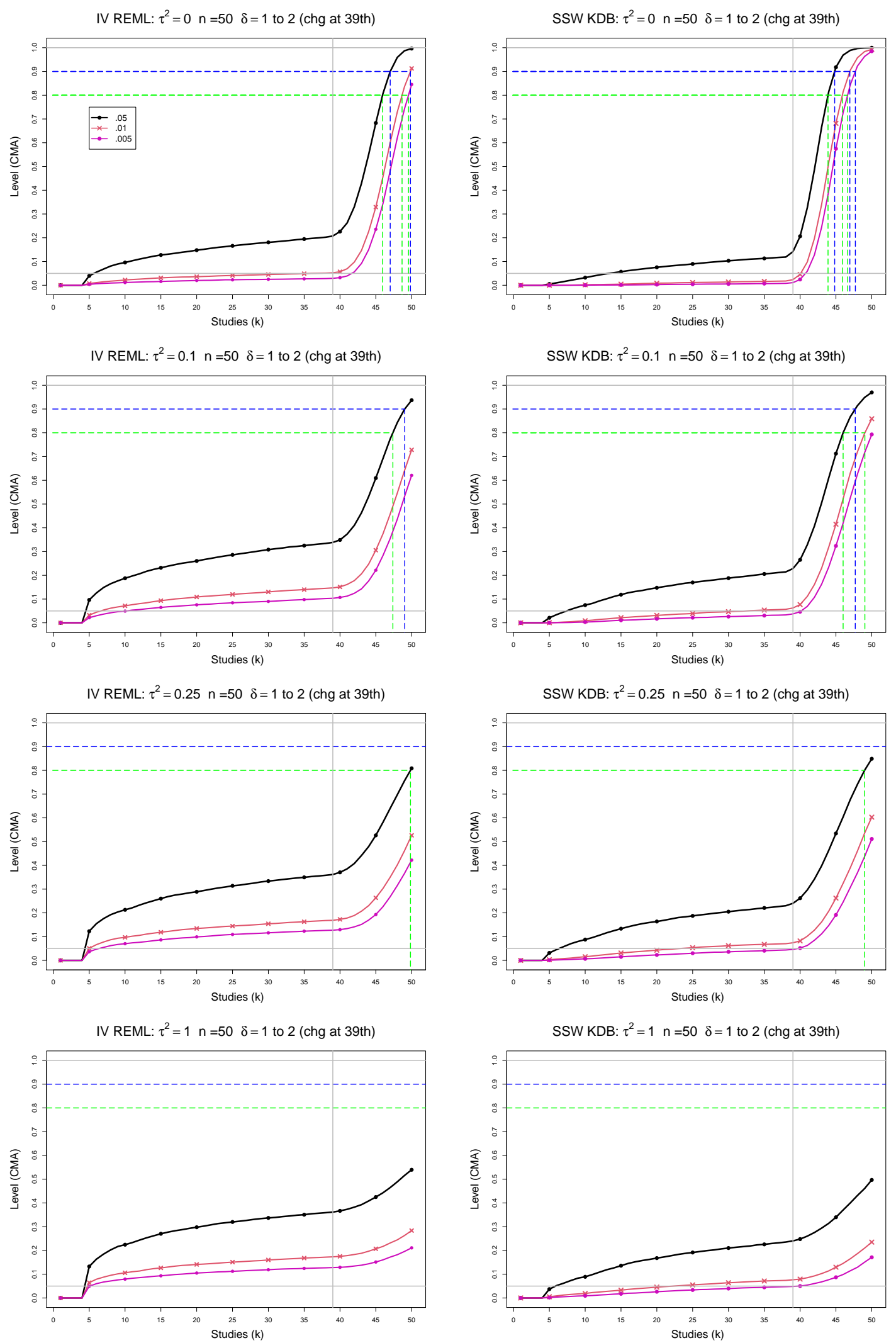

Figure D5. 12: Empirical levels of 2-Stage CMA tests for shift in $\delta$ based on SSW KDB and IV REML at .05, .01 and .005 nominal levels for equal sample sizes $n_{i C}+n_{i T}=n=50$, $\tau^{2}=0,0.1,0.25,1$ and a shift from $\delta=1$ to $\delta=2$ at study number 39. Light grey line at .05. Green and blue dashed lines correspond to power $80 \%$ and $90 \%$, respectively. 

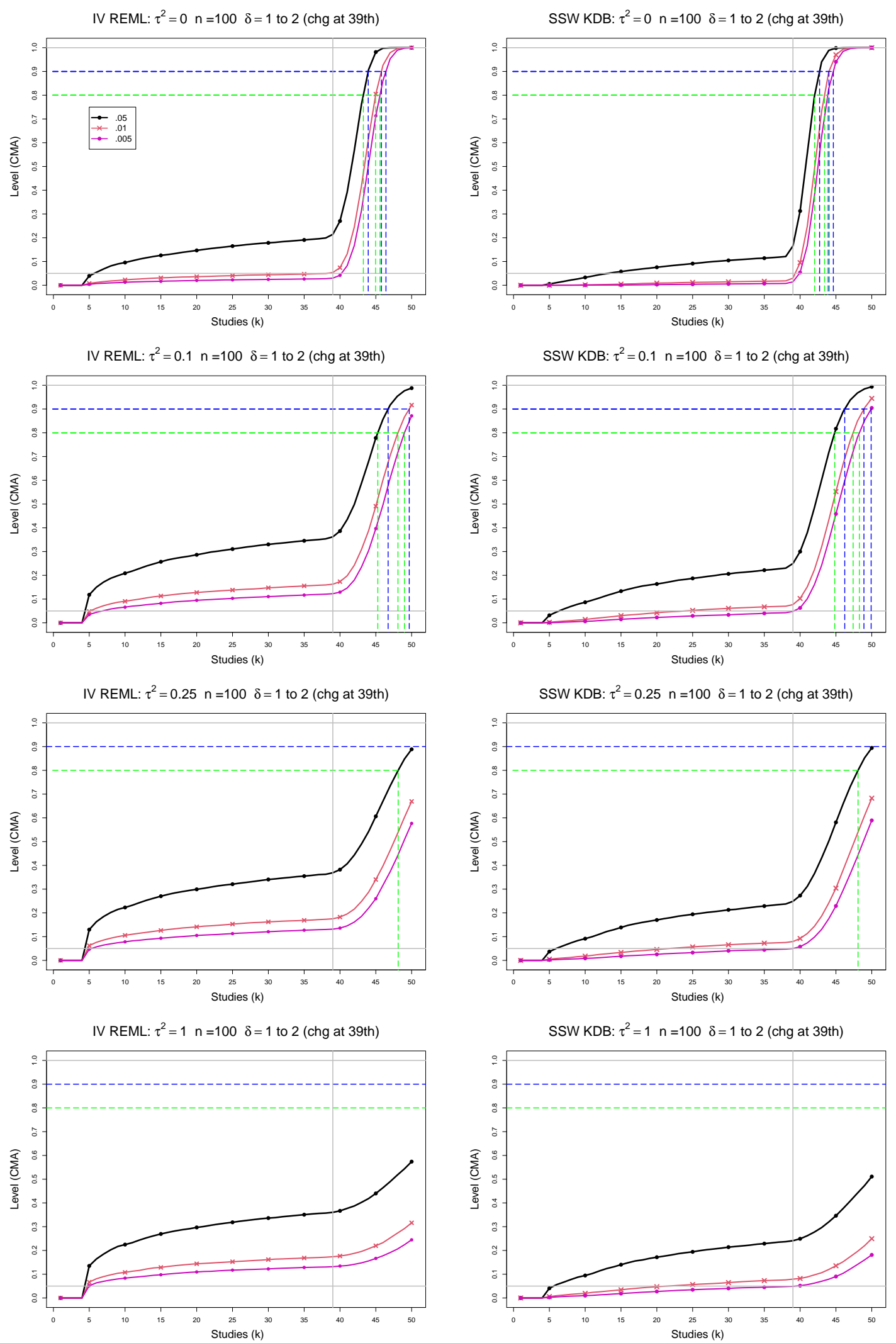

Figure D5. 13: Empirical levels of 2-Stage CMA tests for shift in $\delta$ based on SSW KDB and IV REML at .05, .01 and .005 nominal levels for equal sample sizes $n_{i C}+n_{i T}=n=100$, $\tau^{2}=0,0.1,0.25,1$ and a shift from $\delta=1$ to $\delta=2$ at study number 39. Light grey line at .05. Green and blue dashed lines correspond to power $80 \%$ and $90 \%$, respectively. 

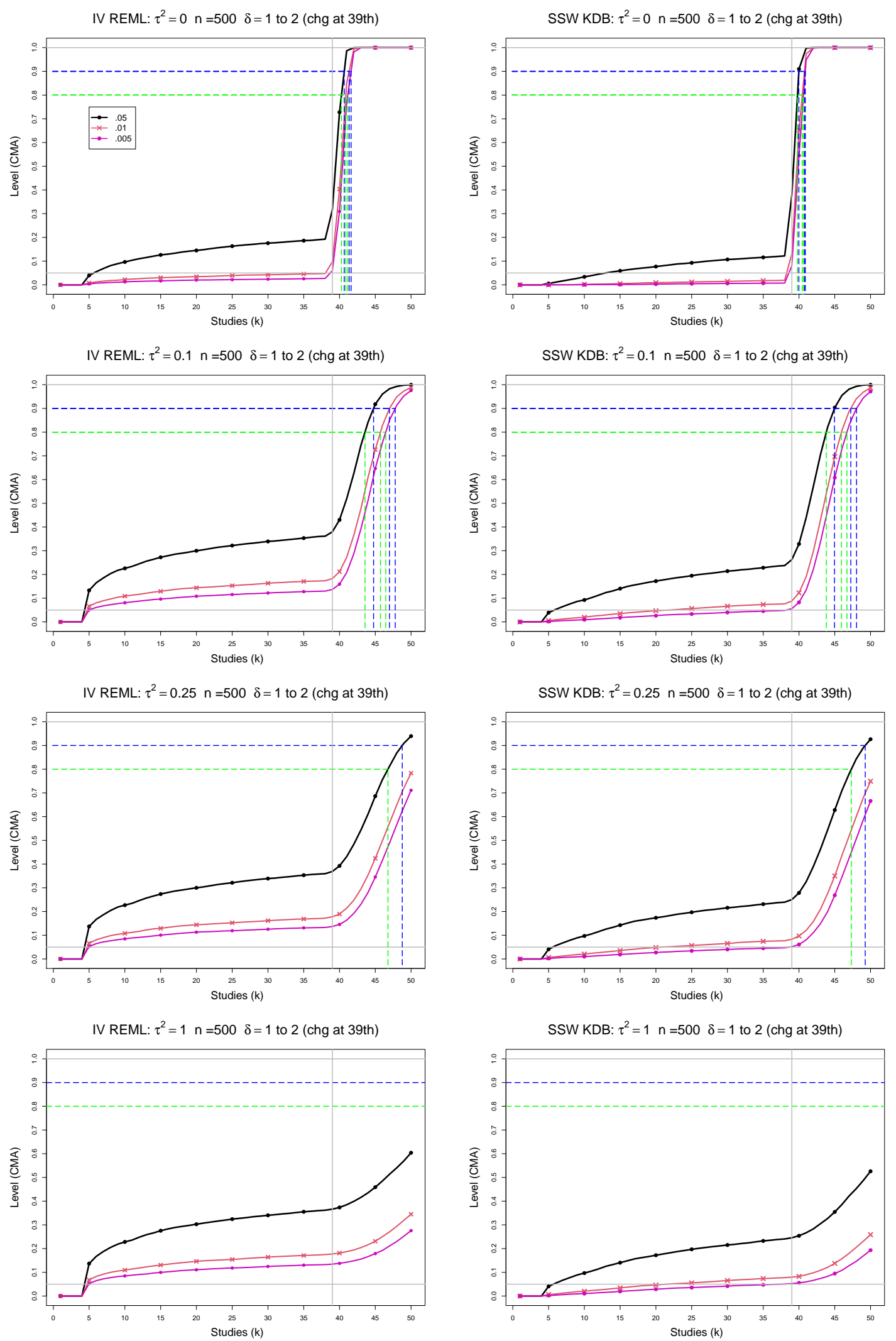

Figure D5. 14: Empirical levels of 2-Stage CMA tests for shift in $\delta$ based on SSW KDB and IV REML at .05, .01 and .005 nominal levels for equal sample sizes $n_{i C}+n_{i T}=n=500$, $\tau^{2}=0,0.1,0.25,1$ and a shift from $\delta=1$ to $\delta=2$ at study number 39. Light grey line at .05. Green and blue dashed lines correspond to power $80 \%$ and $90 \%$, respectively. 

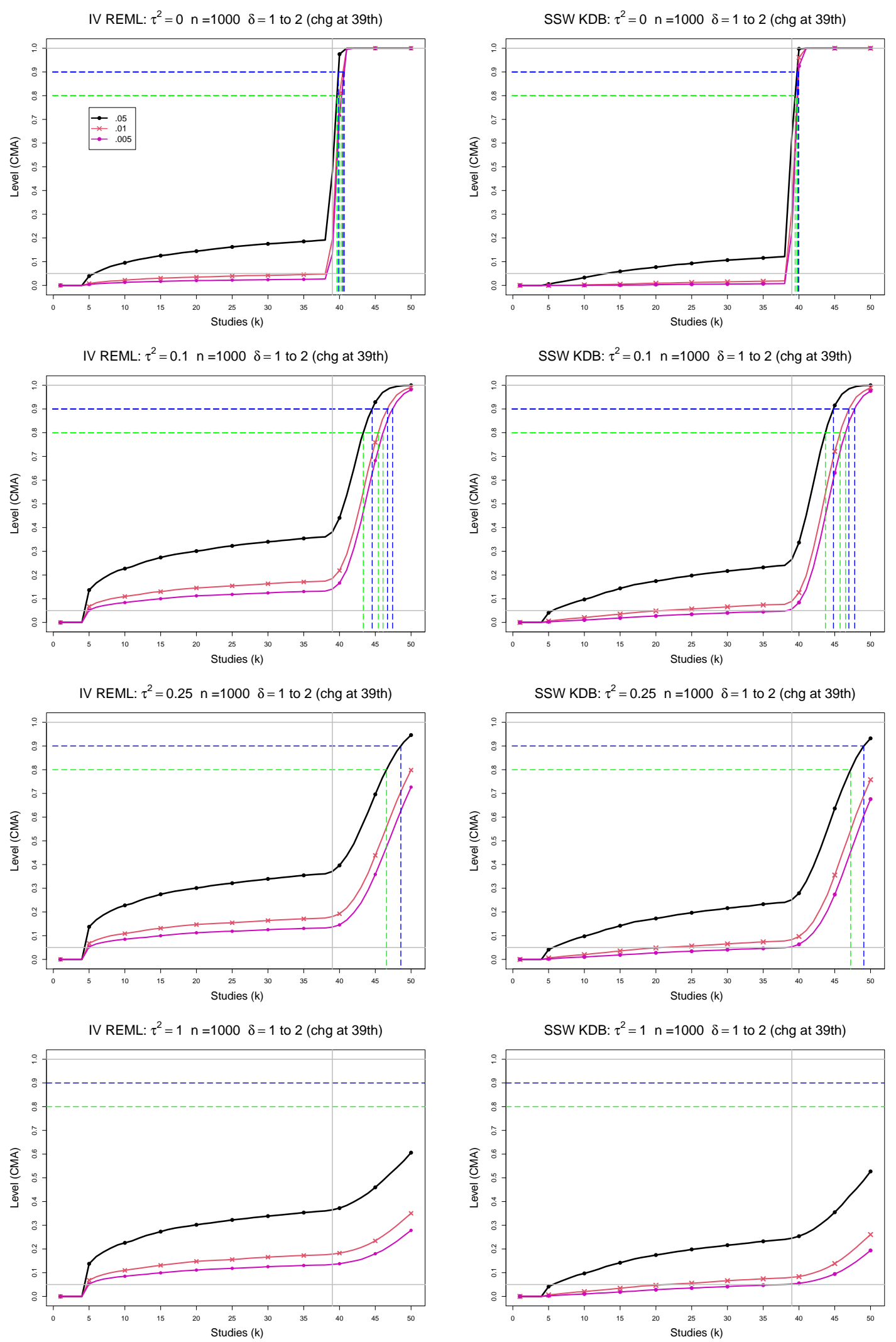

Figure D5. 15: Empirical levels of 2-Stage CMA tests for shift in $\delta$ based on SSW KDB and IV REML at .05, .01 and .005 nominal levels for equal sample sizes $n_{i C}+n_{i T}=n=1000$, $\tau^{2}=0,0.1,0.25,1$ and a shift from $\delta=1$ to $\delta=2$ at study number 39. Light grey line at .05. Green and blue dashed lines correspond to power $80 \%$ and $90 \%$, respectively. 


\section{D6. Empirical levels and power of 2-Stage CMA tests for shift in estimated from stage 1 mean effect $\hat{\delta}_{0}$}

For empirical levels of test for shift in $\hat{\delta}_{0}$ in this section, each figure corresponds to one of the two tested meta-analysis methods, a set of values of $\tau^{2}(=0,0.1,0.25,1)$, a value of $n(=20,50,100,500,1000)$, a set of values of significance level $(=0.05,0.01$, $0.005)$ and a value of point of shift $f(=13,26,39)$ while $\delta$ shifts from 1 to 2 and maximum number of studies $K=50$.

Each figure contains a panel (with study $k$ on the horizontal axis) for each combinations.

The tested methods are

- IV REML (Restricted maximum likelihood, inverse variance weighted)

- SSW KDB (Kulinskaya-Dollinger-Bjørkestøl estimator of $\tau^{2}$, sample size weighted) 

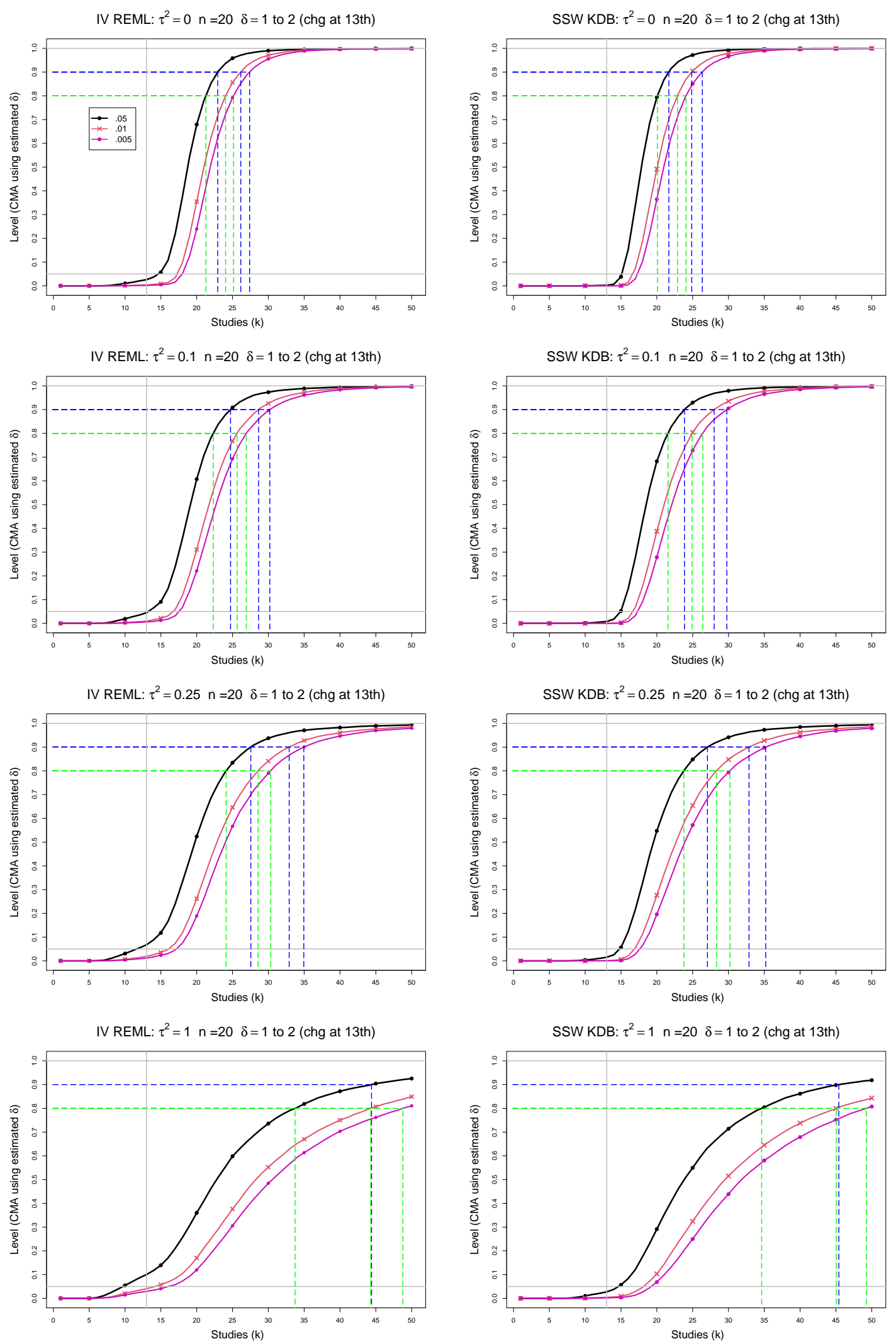

Figure D6. 1: Empirical levels of 2-Stage CMA tests for shift in $\hat{\delta}_{0}$ based on SSW KDB and IV REML at .05, .01 and .005 nominal levels for equal sample sizes $n_{i C}+n_{i T}=n=20$, $\tau^{2}=0,0.1,0.25,1$ and a shift from $\delta=1$ to $\delta=2$ at study number 13 . Light grey line at .05. Green and blue dashed lines correspond to power $80 \%$ and $90 \%$, respectively. 

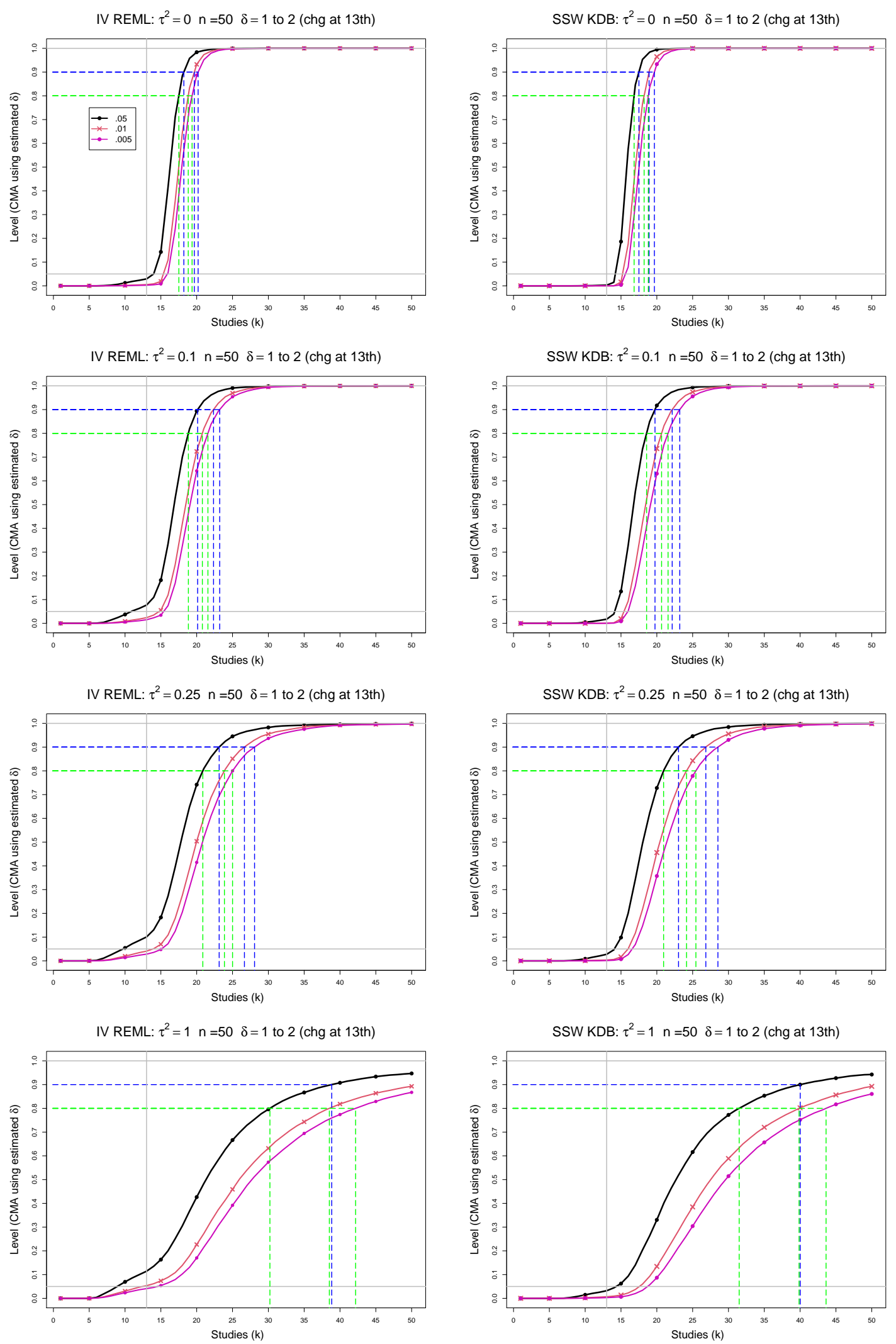

Figure D6. 2: Empirical levels of 2-Stage CMA tests for shift in $\hat{\delta}_{0}$ based on SSW KDB and IV REML at .05, .01 and .005 nominal levels for equal sample sizes $n_{i C}+n_{i T}=n=50$, $\tau^{2}=0,0.1,0.25,1$ and a shift from $\delta=1$ to $\delta=2$ at study number 13 . Light grey line at .05. Green and blue dashed lines correspond to power $80 \%$ and $90 \%$, respectively. 

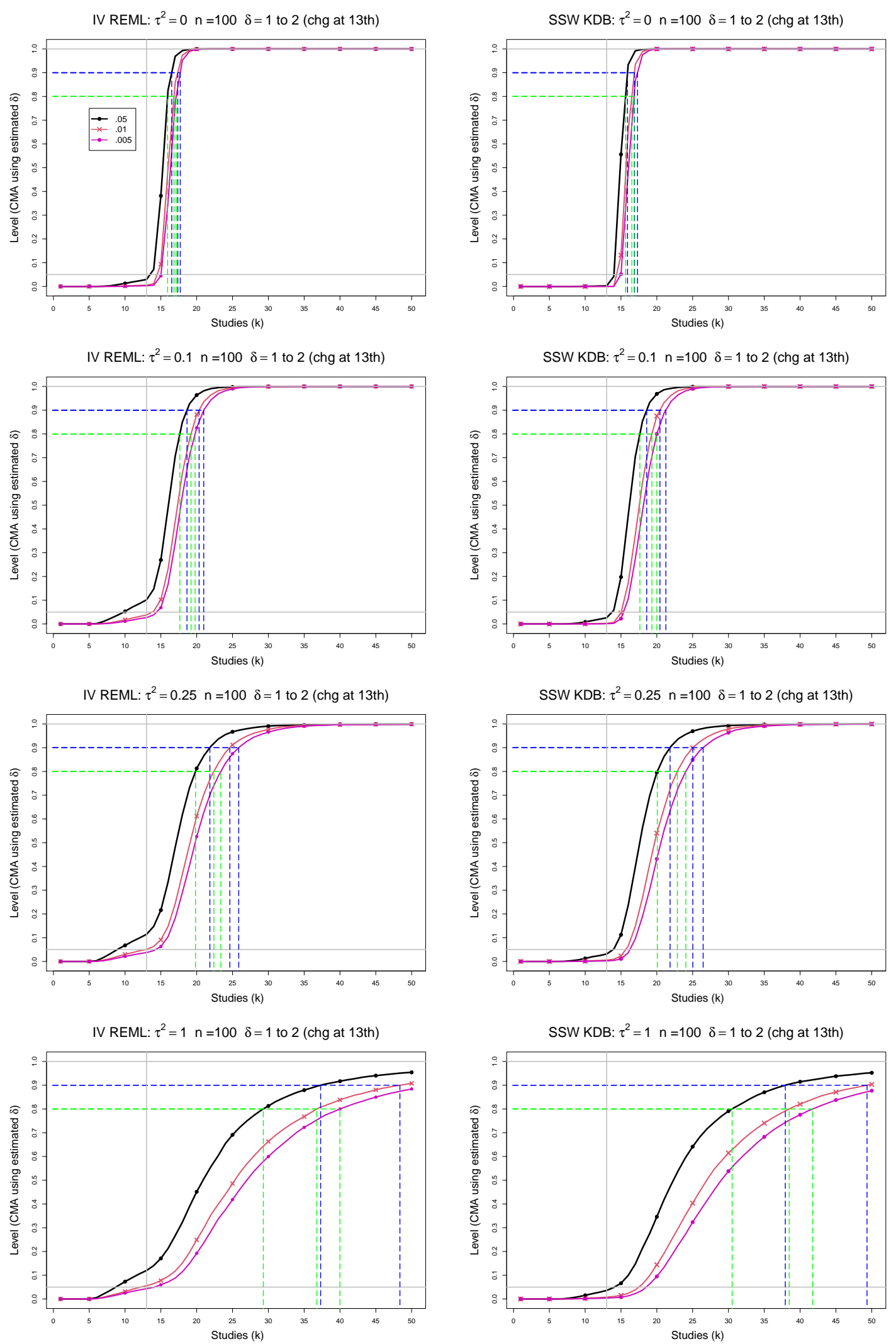

Figure D6. 3: Empirical levels of 2-Stage CMA tests for shift in $\hat{\delta}_{0}$ based on SSW KDB and IV REML at .05, .01 and .005 nominal levels for equal sample sizes $n_{i C}+n_{i T}=n=100$, $\tau^{2}=0,0.1,0.25,1$ and a shift from $\delta=1$ to $\delta=2$ at study number 13 . Light grey line at .05. Green and blue dashed lines correspond to power $80 \%$ and $90 \%$, respectively. 

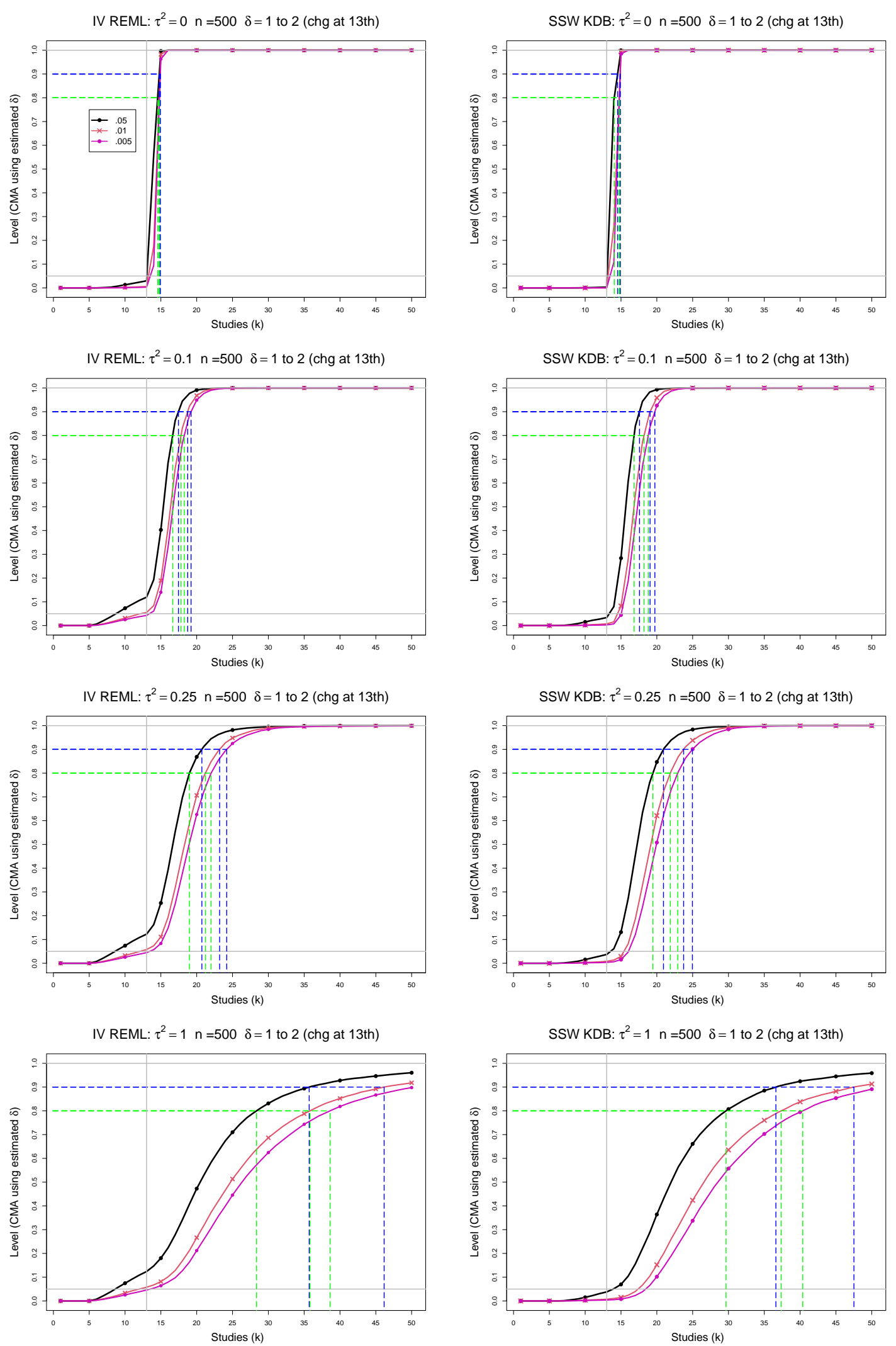

Figure D6. 4: Empirical levels of 2-Stage CMA tests for shift in $\hat{\delta}_{0}$ based on SSW KDB and IV REML at .05, .01 and .005 nominal levels for equal sample sizes $n_{i C}+n_{i T}=n=500$, $\tau^{2}=0,0.1,0.25,1$ and a shift from $\delta=1$ to $\delta=2$ at study number 13 . Light grey line at .05. Green and blue dashed lines correspond to power $80 \%$ and $90 \%$, respectively. 

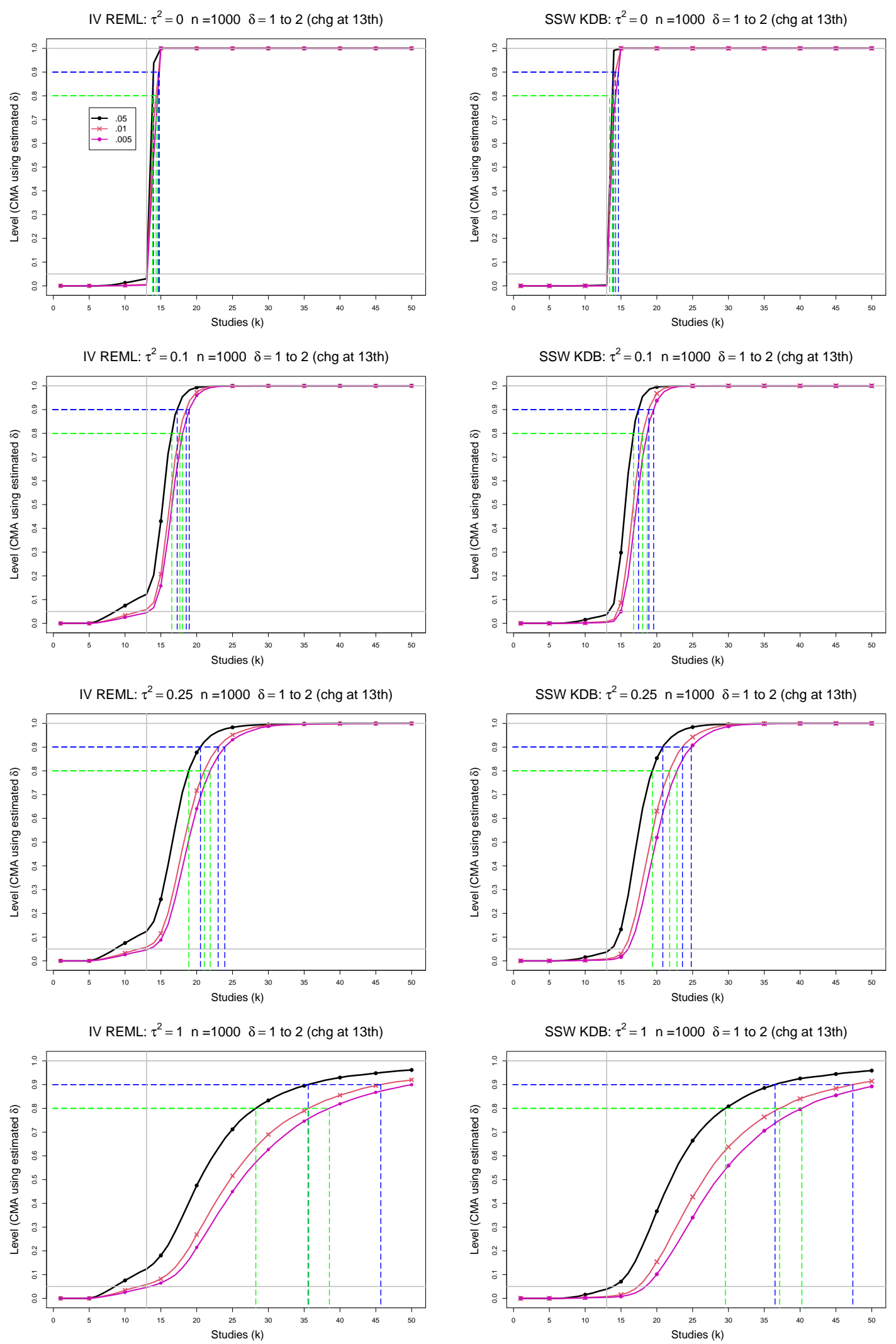

Figure D6. 5: Empirical levels of 2-Stage CMA tests for shift in $\hat{\delta}_{0}$ based on SSW KDB and IV REML at .05, .01 and .005 nominal levels for equal sample sizes $n_{i C}+n_{i T}=n=1000$, $\tau^{2}=0,0.1,0.25,1$ and a shift from $\delta=1$ to $\delta=2$ at study number 13 . Light grey line at .05. Green and blue dashed lines correspond to power $80 \%$ and $90 \%$, respectively. 

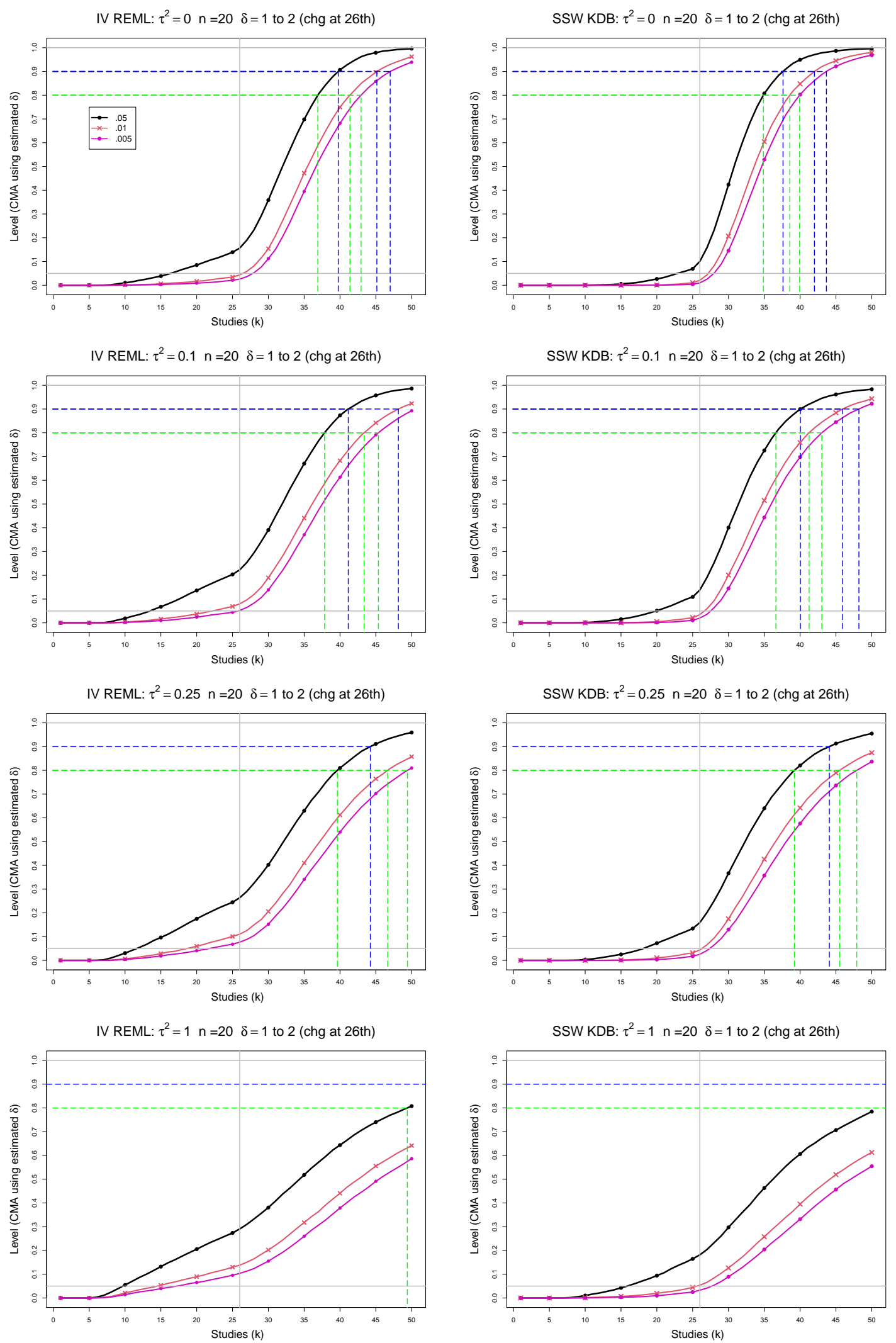

Figure D6. 6: Empirical levels of 2-Stage CMA tests for shift in $\hat{\delta}_{0}$ based on SSW KDB and IV REML at .05, .01 and .005 nominal levels for equal sample sizes $n_{i C}+n_{i T}=n=20$, $\tau^{2}=0,0.1,0.25,1$ and a shift from $\delta=1$ to $\delta=2$ at study number 26. Light grey line at .05. Green and blue dashed lines correspond to power $80 \%$ and $90 \%$, respectively. 

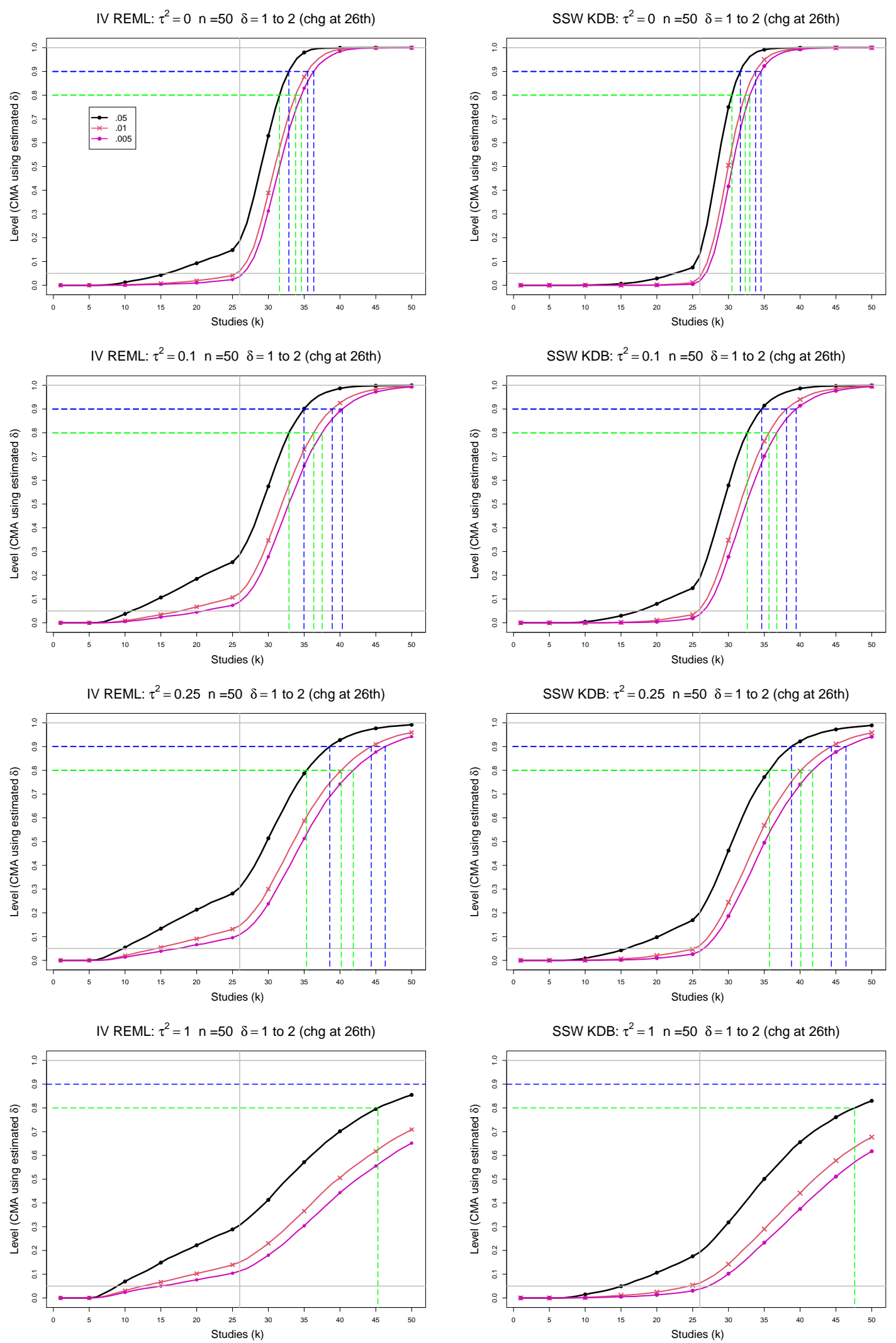

Figure D6. 7: Empirical levels of 2-Stage CMA tests for shift in $\hat{\delta}_{0}$ based on SSW KDB and IV REML at .05, .01 and .005 nominal levels for equal sample sizes $n_{i C}+n_{i T}=n=50$, $\tau^{2}=0,0.1,0.25,1$ and a shift from $\delta=1$ to $\delta=2$ at study number 26. Light grey line at .05. Green and blue dashed lines correspond to power $80 \%$ and $90 \%$, respectively. 

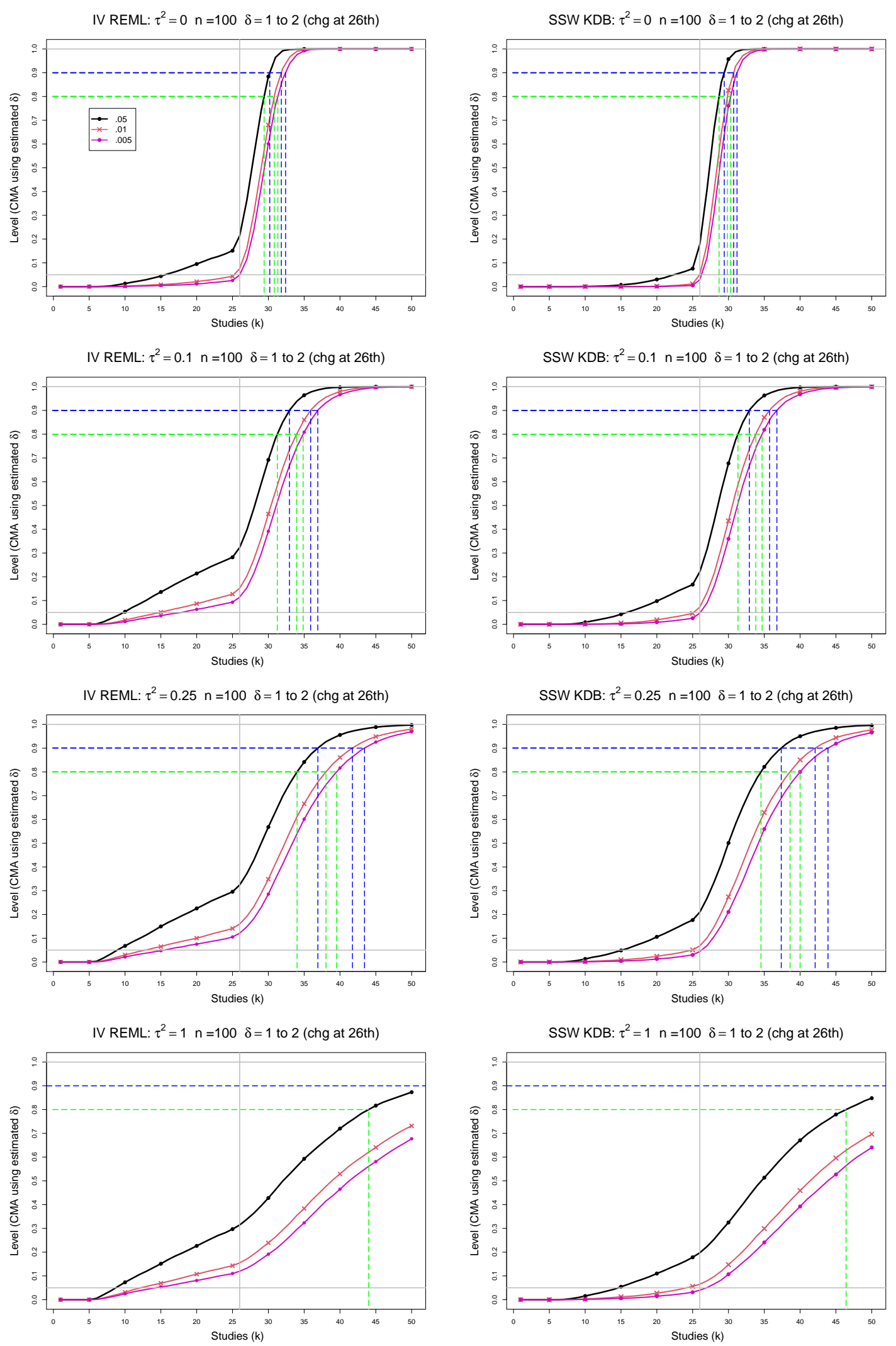

Figure D6. 8: Empirical levels of 2-Stage CMA tests for shift in $\hat{\delta}_{0}$ based on SSW KDB and IV REML at .05, .01 and .005 nominal levels for equal sample sizes $n_{i C}+n_{i T}=n=100$, $\tau^{2}=0,0.1,0.25,1$ and a shift from $\delta=1$ to $\delta=2$ at study number 26. Light grey line at .05. Green and blue dashed lines correspond to power $80 \%$ and $90 \%$, respectively. 

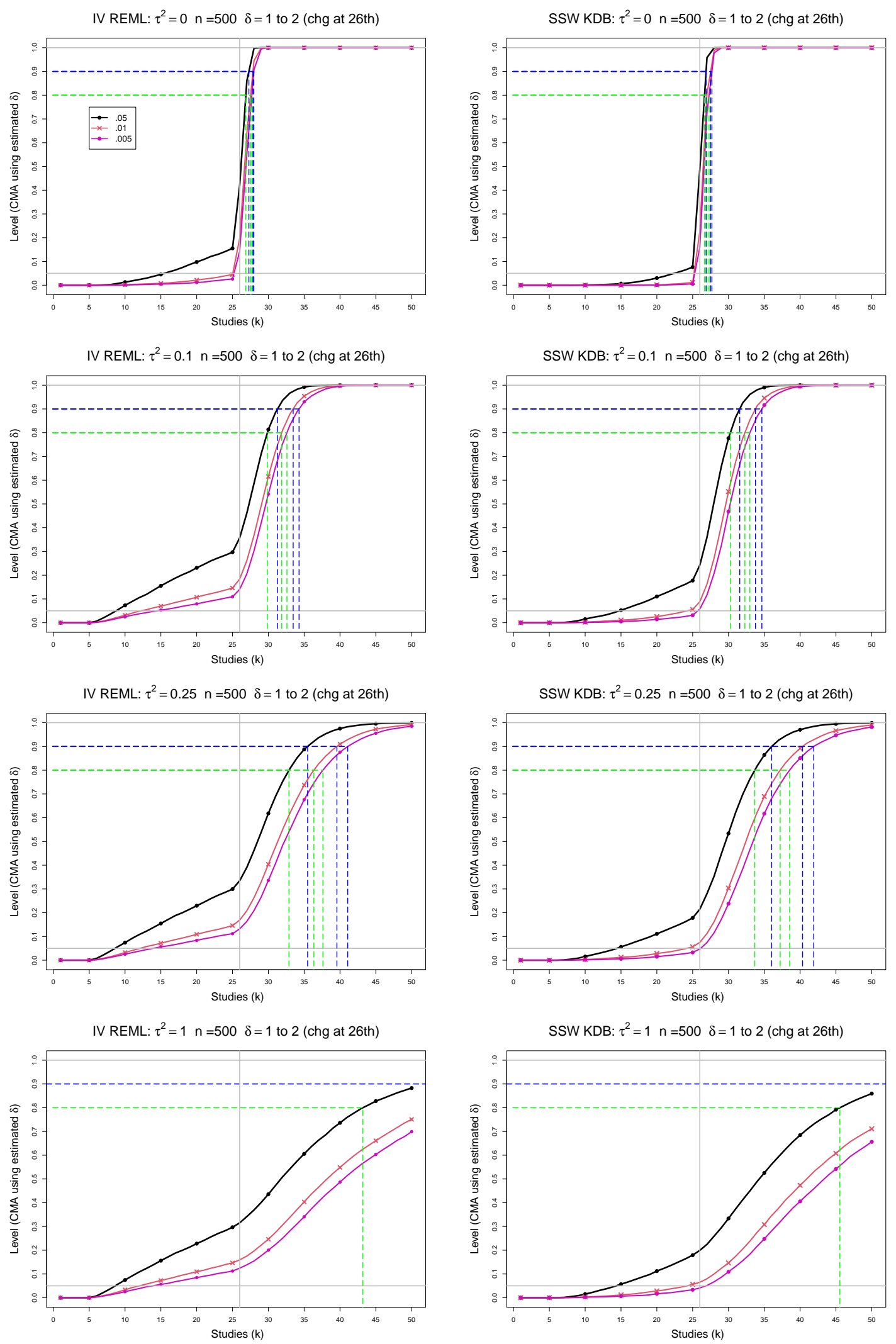

Figure D6. 9: Empirical levels of 2-Stage CMA tests for shift in $\hat{\delta}_{0}$ based on SSW KDB and IV REML at .05, .01 and .005 nominal levels for equal sample sizes $n_{i C}+n_{i T}=n=500$, $\tau^{2}=0,0.1,0.25,1$ and a shift from $\delta=1$ to $\delta=2$ at study number 26. Light grey line at .05. Green and blue dashed lines correspond to power $80 \%$ and $90 \%$, respectively. 

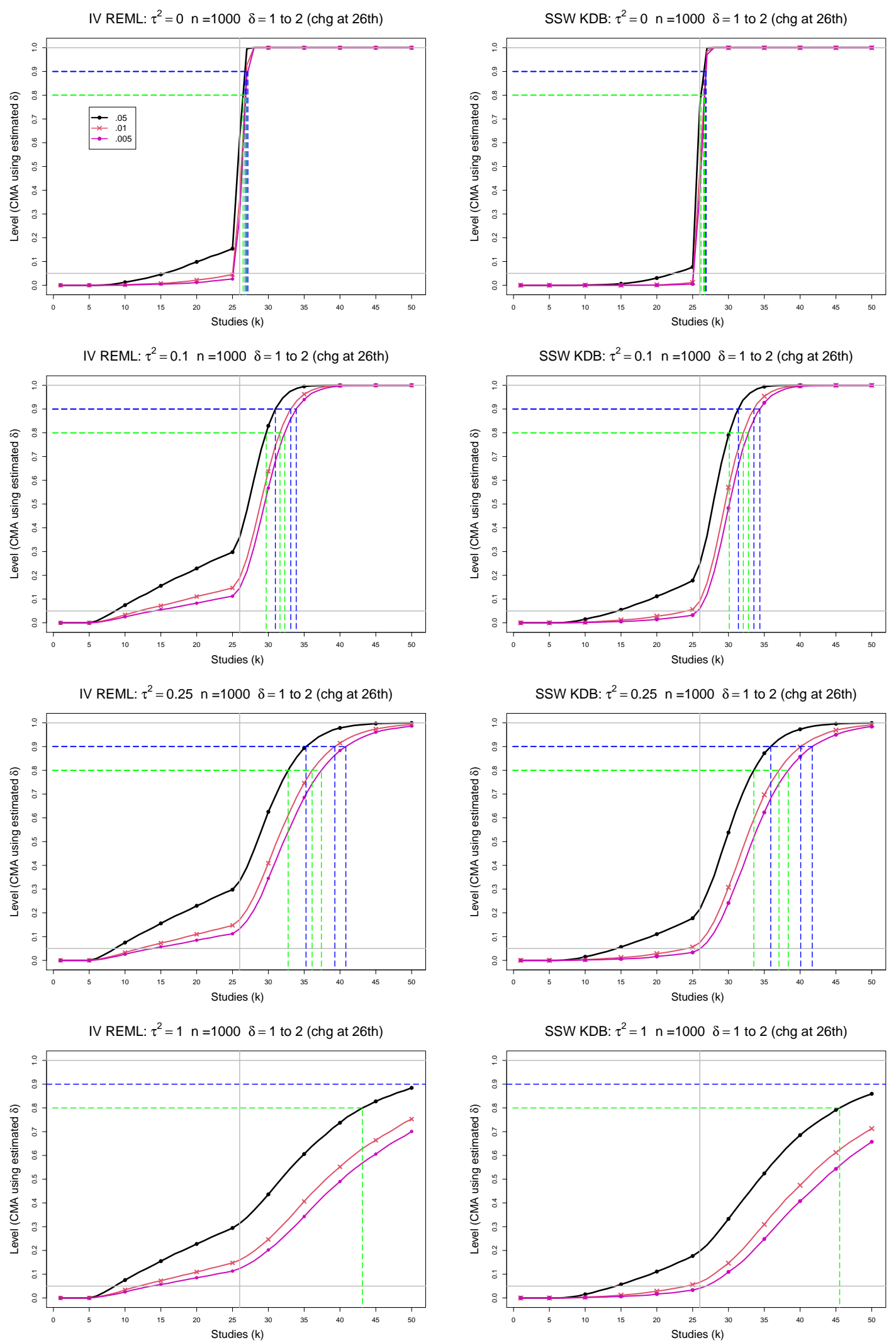

Figure D6. 10: Empirical levels of 2-Stage CMA tests for shift in $\hat{\delta}_{0}$ based on SSW KDB and IV REML at .05, .01 and .005 nominal levels for equal sample sizes $n_{i C}+n_{i T}=n=1000$, $\tau^{2}=0,0.1,0.25,1$ and a shift from $\delta=1$ to $\delta=2$ at study number 26. Light grey line at .05. Green and blue dashed lines correspond to power $80 \%$ and $90 \%$, respectively. 

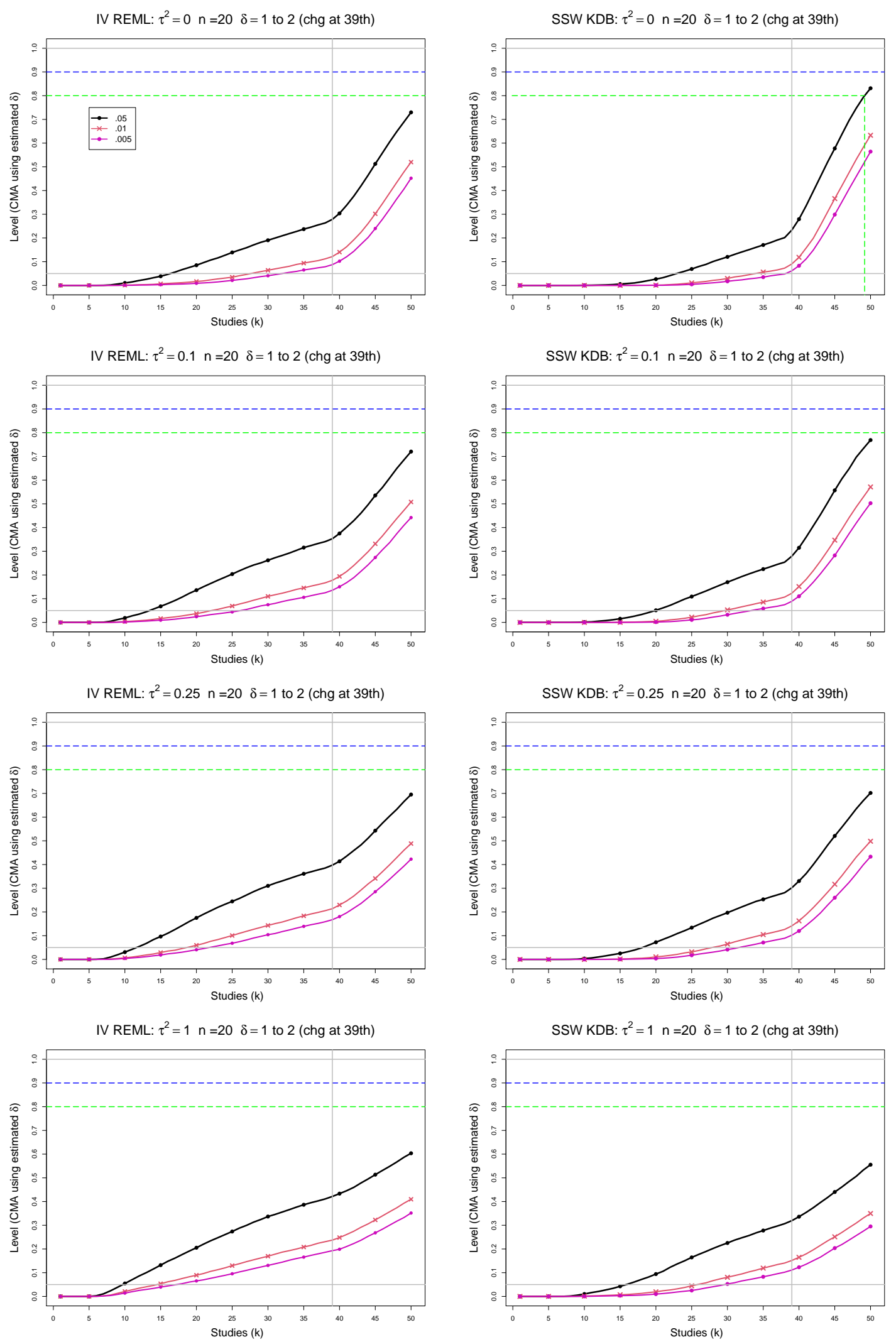

Figure D6. 11: Empirical levels of 2-Stage CMA tests for shift in $\hat{\delta}_{0}$ based on SSW KDB and IV REML at .05, .01 and .005 nominal levels for equal sample sizes $n_{i C}+n_{i T}=n=20$, $\tau^{2}=0,0.1,0.25,1$ and a shift from $\delta=1$ to $\delta=2$ at study number 39. Light grey line at .05. Green and blue dashed lines correspond to power $80 \%$ and $90 \%$, respectively. 

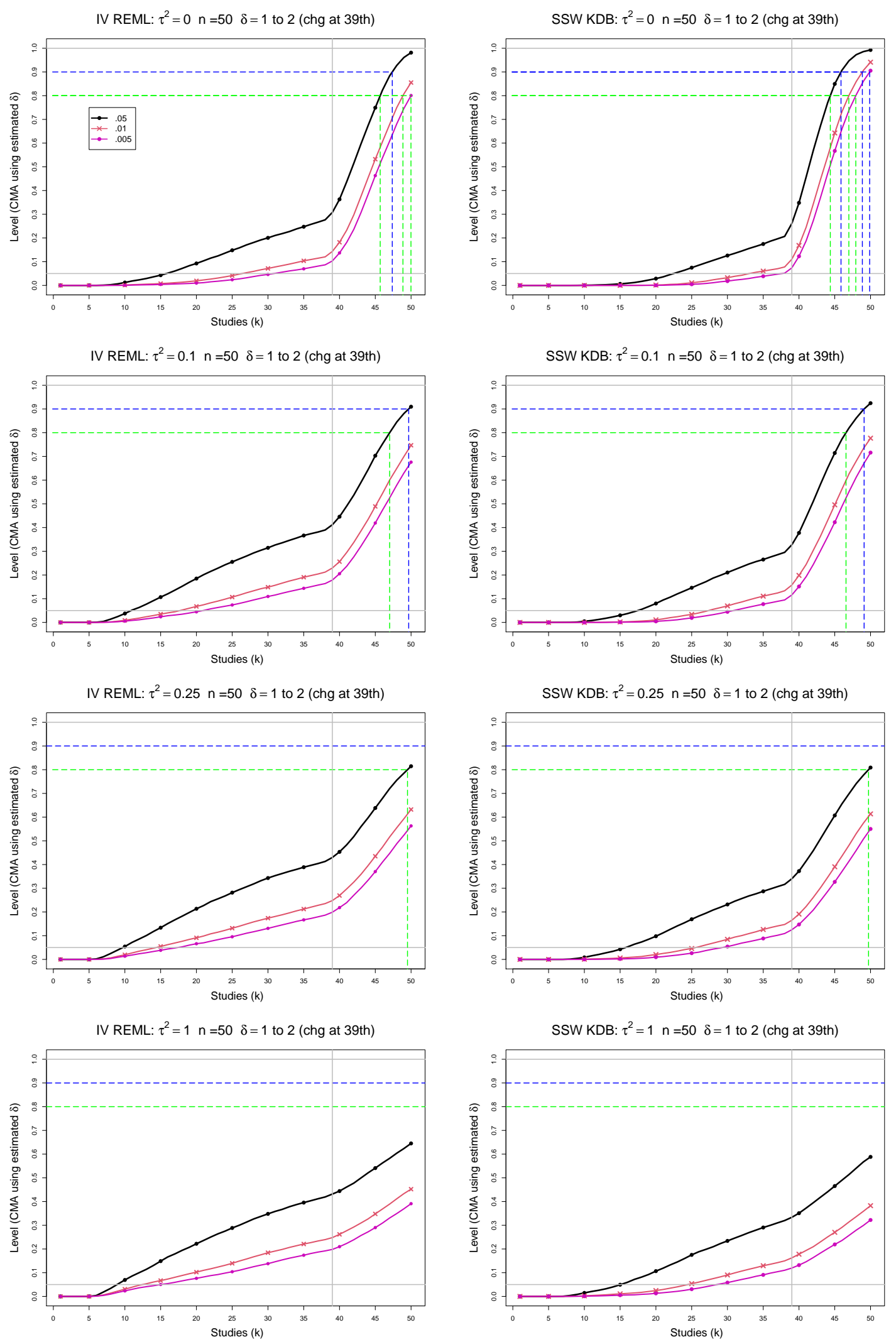

Figure D6. 12: Empirical levels of 2-Stage CMA tests for shift in $\hat{\delta}_{0}$ based on SSW KDB and IV REML at .05, .01 and .005 nominal levels for equal sample sizes $n_{i C}+n_{i T}=n=50$, $\tau^{2}=0,0.1,0.25,1$ and a shift from $\delta=1$ to $\delta=2$ at study number 39. Light grey line at .05. Green and blue dashed lines correspond to power $80 \%$ and $90 \%$, respectively. 

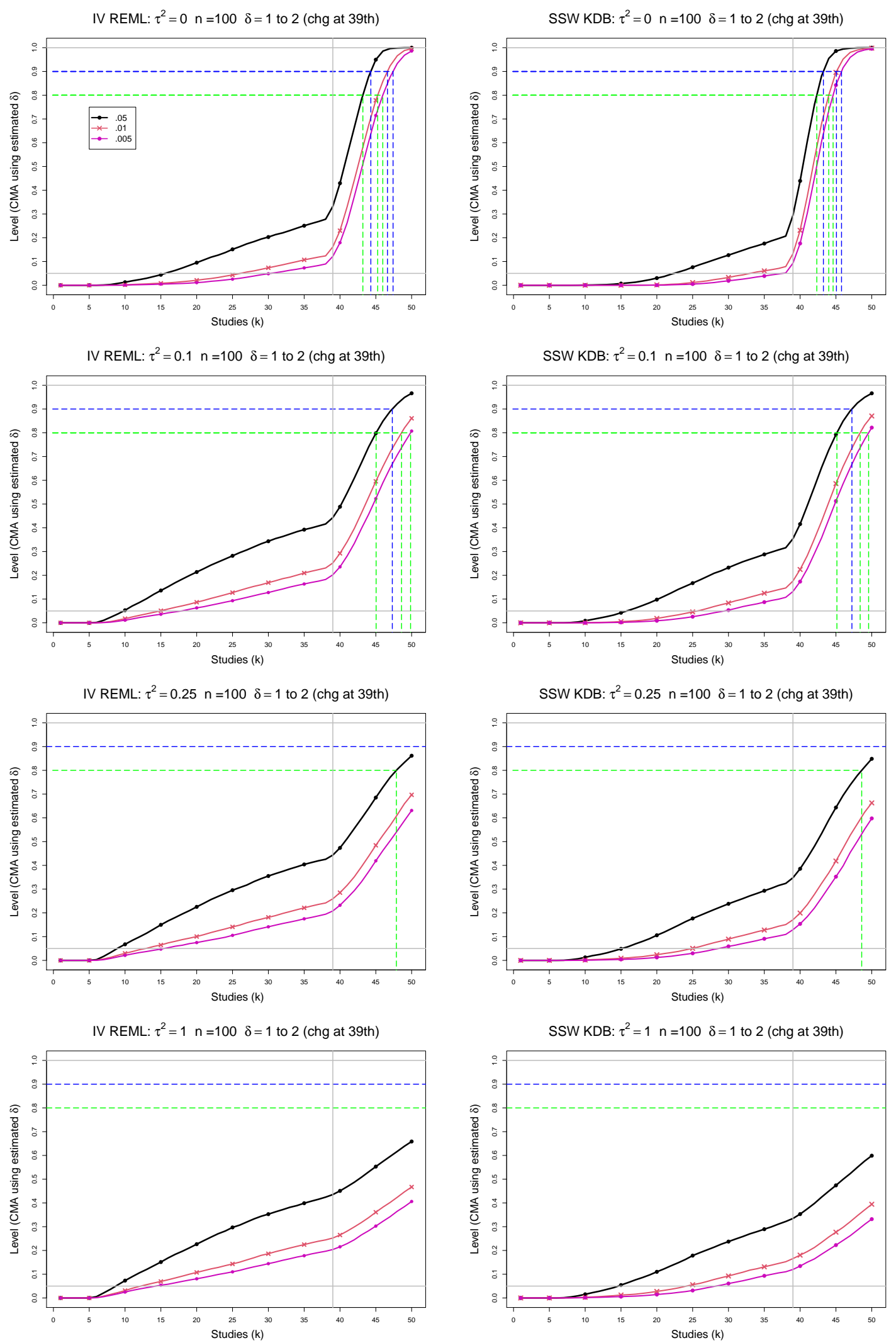

Figure D6. 13: Empirical levels of 2-Stage CMA tests for shift in $\hat{\delta}_{0}$ based on SSW KDB and IV REML at .05, .01 and .005 nominal levels for equal sample sizes $n_{i C}+n_{i T}=n=100$, $\tau^{2}=0,0.1,0.25,1$ and a shift from $\delta=1$ to $\delta=2$ at study number 39. Light grey line at .05. Green and blue dashed lines correspond to power $80 \%$ and $90 \%$, respectively. 

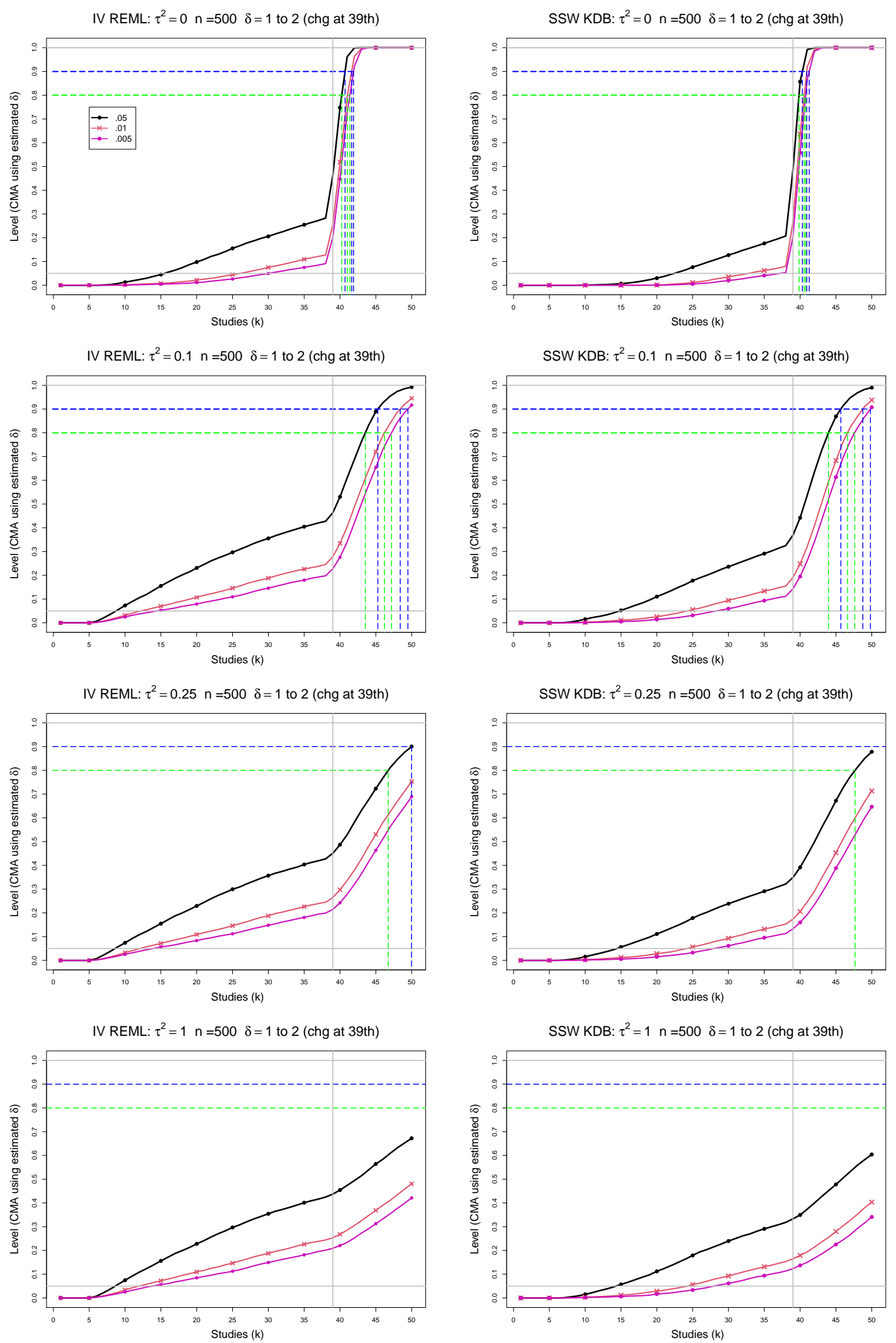

Figure D6. 14: Empirical levels of 2-Stage CMA tests for shift in $\hat{\delta}_{0}$ based on SSW KDB and IV REML at .05, .01 and .005 nominal levels for equal sample sizes $n_{i C}+n_{i T}=n=500$, $\tau^{2}=0,0.1,0.25,1$ and a shift from $\delta=1$ to $\delta=2$ at study number 39. Light grey line at .05. Green and blue dashed lines correspond to power $80 \%$ and $90 \%$, respectively. 

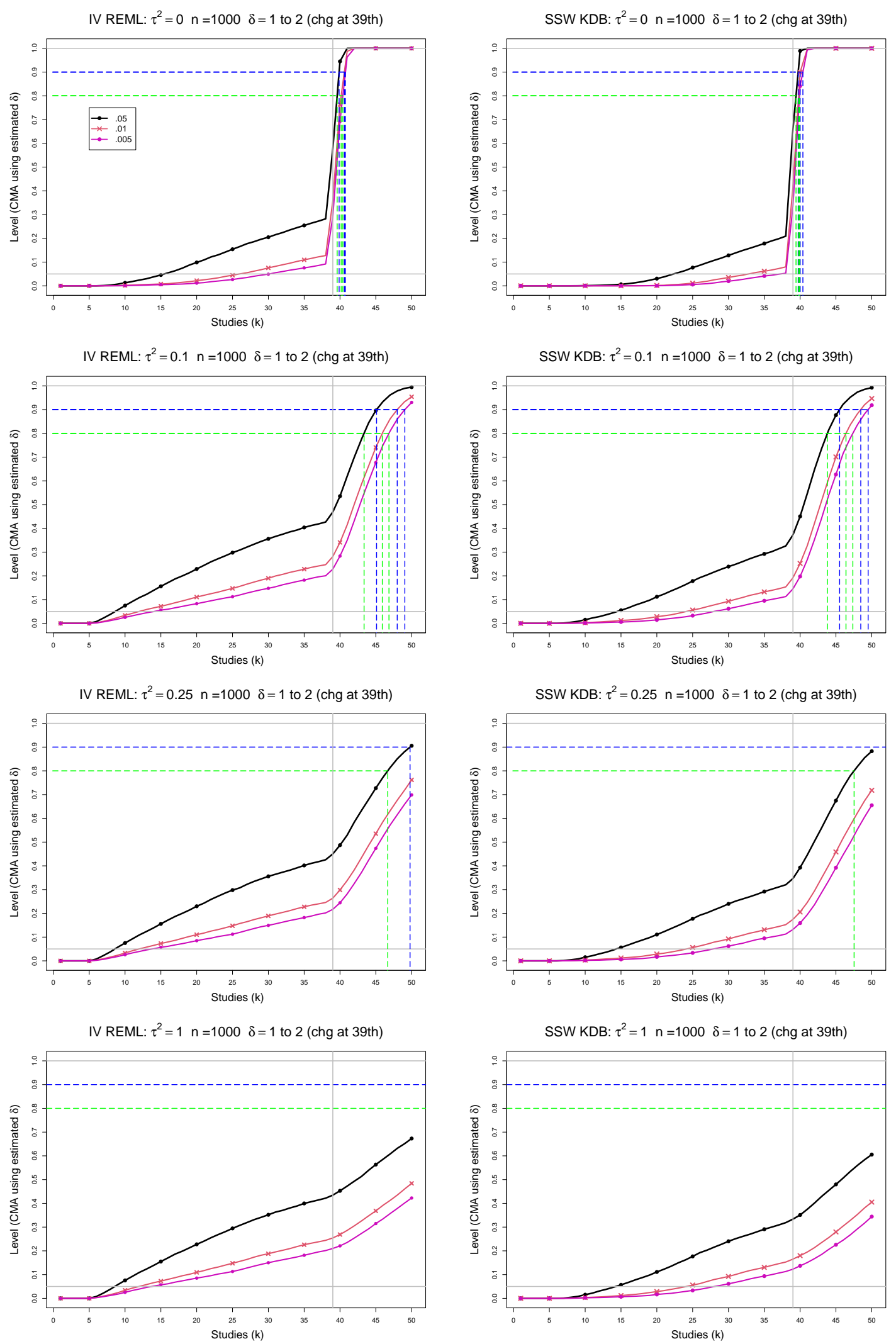

Figure D6. 15: Empirical levels of 2-Stage CMA tests for shift in $\hat{\delta}_{0}$ based on SSW KDB and IV REML at .05, .01 and .005 nominal levels for equal sample sizes $n_{i C}+n_{i T}=n=1000$, $\tau^{2}=0,0.1,0.25,1$ and a shift from $\delta=1$ to $\delta=2$ at study number 39. Light grey line at .05. Green and blue dashed lines correspond to power $80 \%$ and $90 \%$, respectively. 


\section{D7. Empirical levels of CUSUM tests for shift in $\hat{\delta}_{0}$}

For empirical levels of tests for shift in $\delta$ in this section, each figure corresponds to one of the two tested meta-analysis methods, a set of values of $\tau^{2}(=0,0.1,0.25,1)$, a value of $n(=20,50,100,500,1000)$, a set of values of decision interval $h(=4,5,6)$ and a value of point of shift $f(=13,26,39)$ while $\delta$ shifts from 1 to 2 and maximum number of studies $K=50$. CUSUM-based tests use estimated in stage 1 values of $\hat{\delta}_{0}$ and $\hat{\tau}^{2}$.

Each figure contains a panel (with study $k$ on the horizontal axis) for each combination of parameters.

The tested methods are

- IV REML (Restricted maximum likelihood, inverse variance weighted)

- SSW KDB (Kulinskaya-Dollinger-Bjørkestøl estimator of $\tau^{2}$, sample size weighted) 

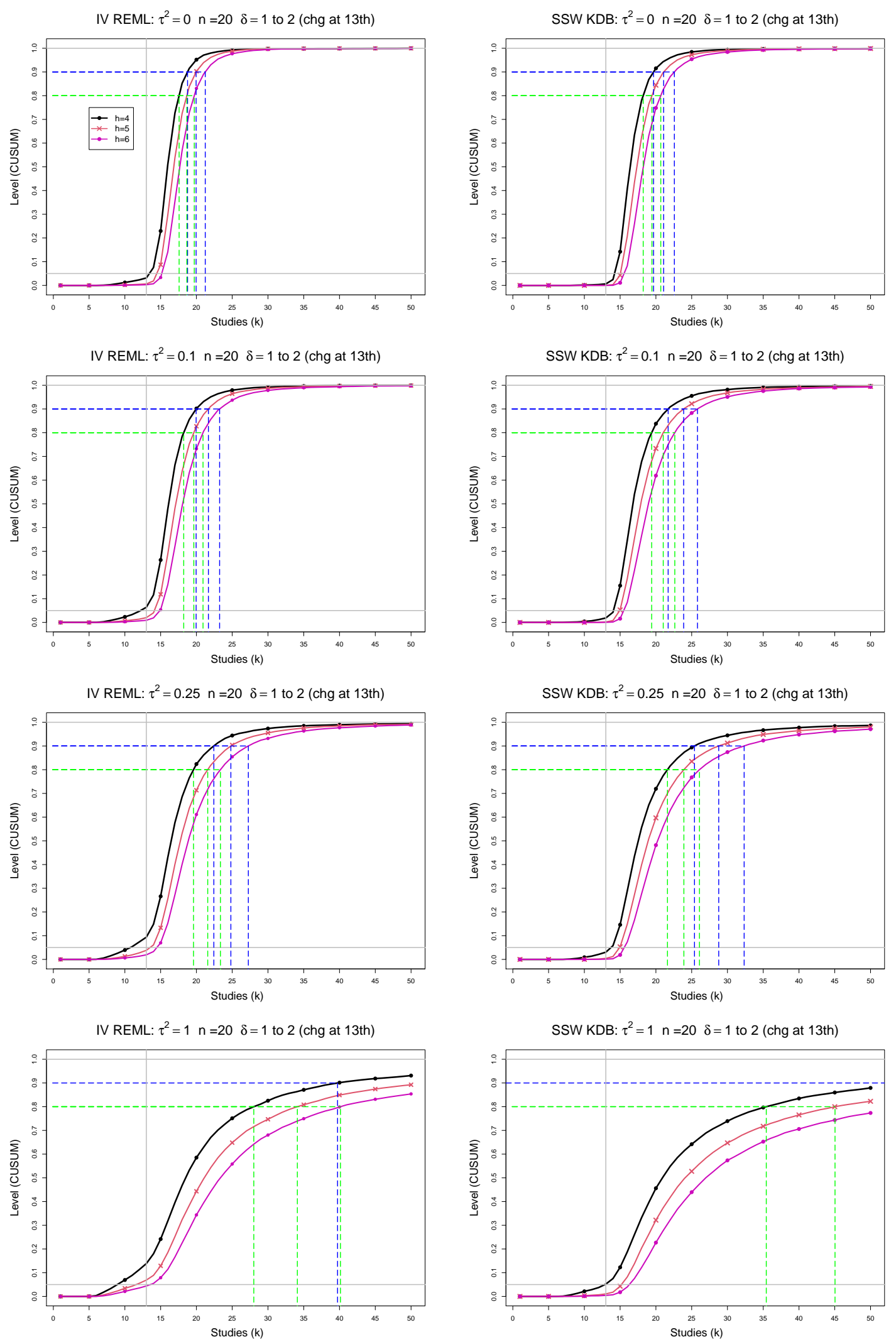

Figure D7. 1: Empirical levels of CUSUM tests for shift in $\delta$ based on SSW KDB and IV REML at $h=4,5,6$ for equal sample sizes $n_{i C}+n_{i T}=n=20, \tau^{2}=0,0.1,0.25,1$ and a shift from $\delta=1$ to $\delta=2$ at study number 13. Light grey line at .05 . Green and blue dashed lines correspond to power $80 \%$ and $90 \%$, respectively. 

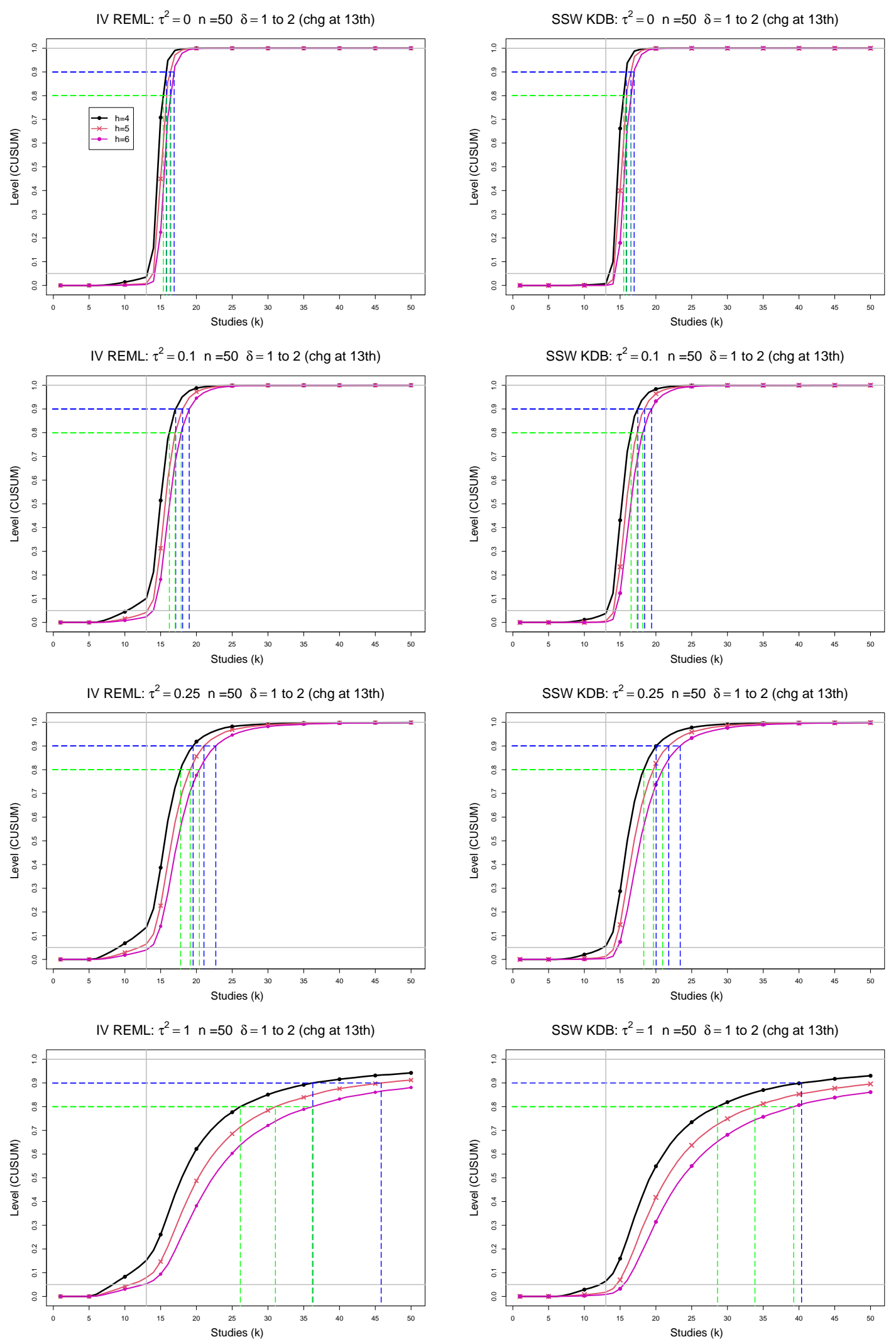

Figure D7. 2: Empirical levels of CUSUM tests for shift in $\delta$ based on SSW KDB and IV REML at $h=4,5,6$ for equal sample sizes $n_{i C}+n_{i T}=n=50, \tau^{2}=0,0.1,0.25,1$ and a shift from $\delta=1$ to $\delta=2$ at study number 13. Light grey line at .05. Green and blue dashed lines correspond to power $80 \%$ and $90 \%$, respectively. 

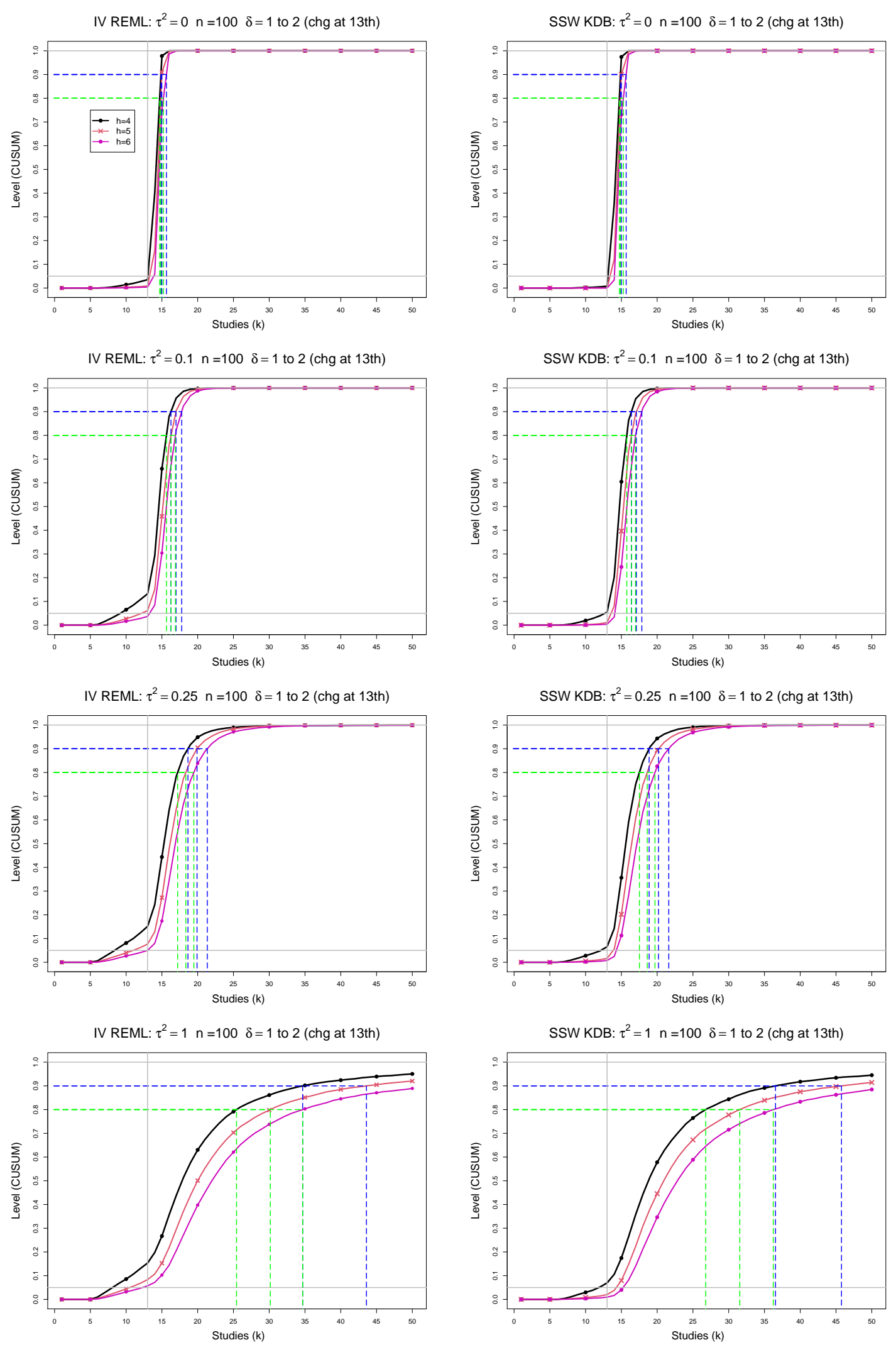

Figure D7. 3: Empirical levels of CUSUM tests for shift in $\delta$ based on SSW KDB and IV REML at $h=4,5,6$ for equal sample sizes $n_{i C}+n_{i T}=n=100, \tau^{2}=0,0.1,0.25,1$ and a shift from $\delta=1$ to $\delta=2$ at study number 13. Light grey line at .05 . Green and blue dashed lines correspond to power $80 \%$ and $90 \%$, respectively. 

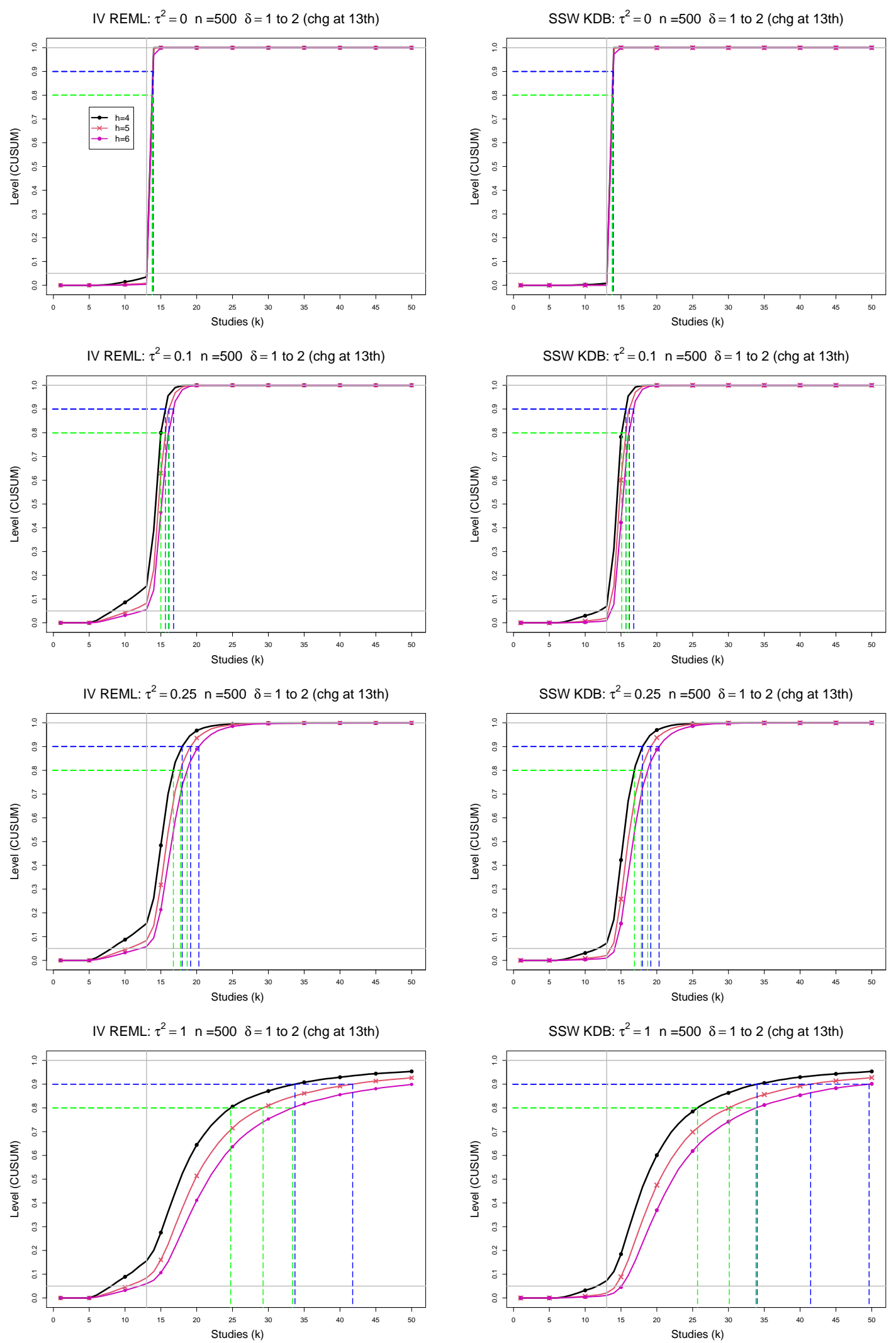

Figure D7. 4: Empirical levels of CUSUM tests for shift in $\delta$ based on SSW KDB and IV REML at $h=4,5,6$ for equal sample sizes $n_{i C}+n_{i T}=n=500, \tau^{2}=0,0.1,0.25,1$ and a shift from $\delta=1$ to $\delta=2$ at study number 13. Light grey line at .05 . Green and blue dashed lines correspond to power $80 \%$ and $90 \%$, respectively. 

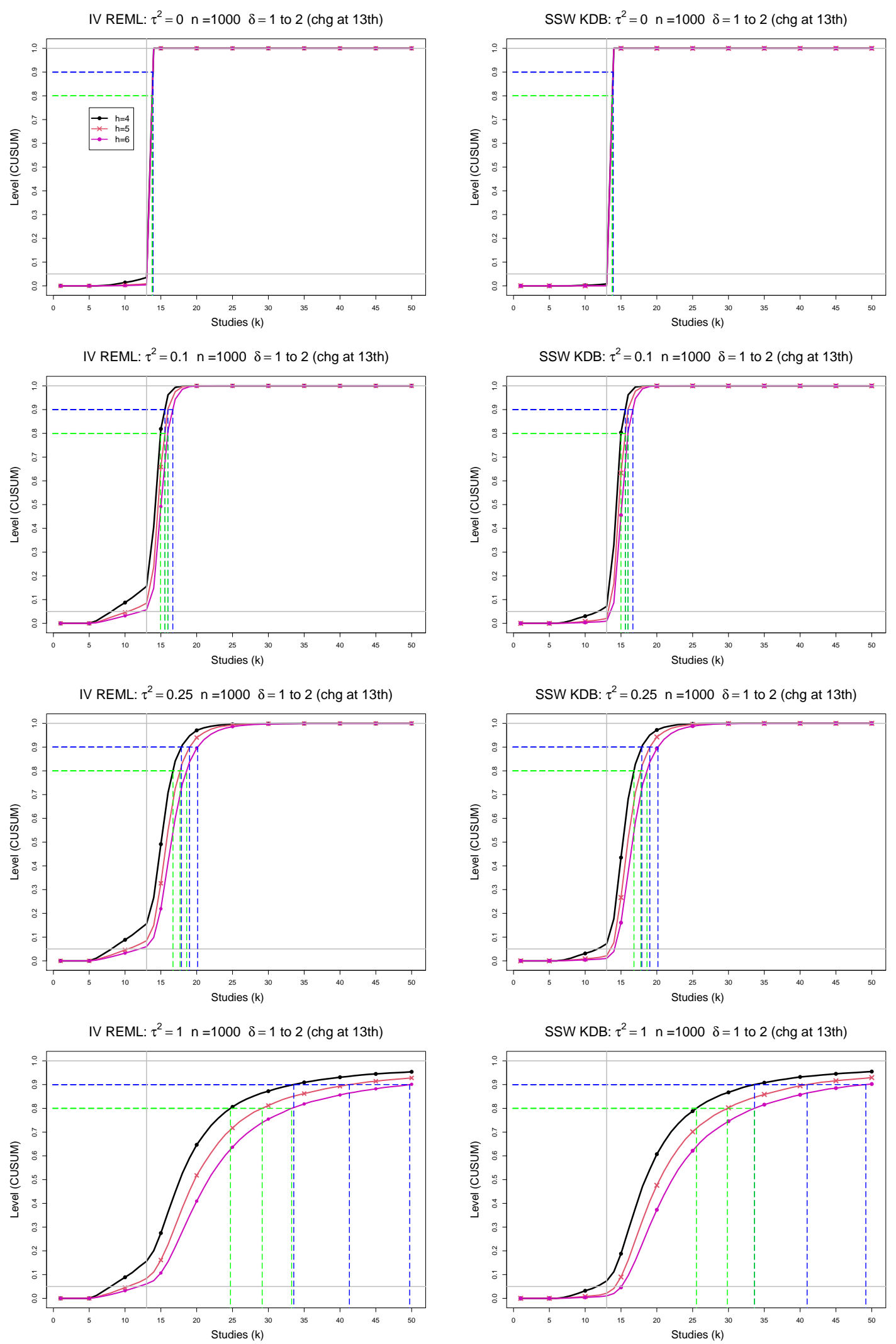

Figure D7. 5: Empirical levels of CUSUM tests for shift in $\delta$ based on SSW KDB and IV REML at $h=4,5,6$ for equal sample sizes $n_{i C}+n_{i T}=n=1000, \tau^{2}=0,0.1,0.25,1$ and a shift from $\delta=1$ to $\delta=2$ at study number 13. Light grey line at .05. Green and blue dashed lines correspond to power $80 \%$ and $90 \%$, respectively. 

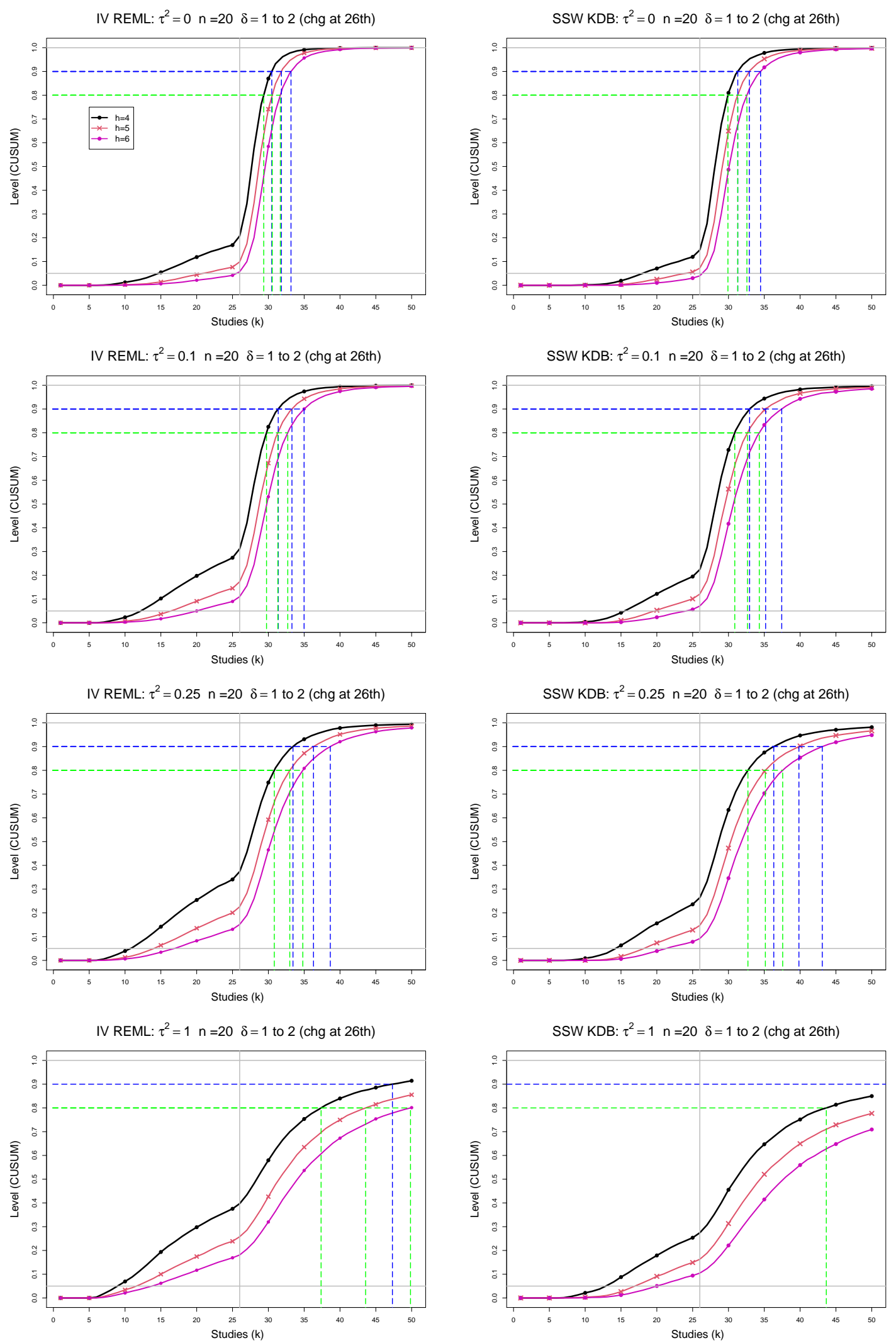

Figure D7. 6: Empirical levels of CUSUM tests for shift in $\delta$ based on SSW KDB and IV REML at $h=4,5,6$ for equal sample sizes $n_{i C}+n_{i T}=n=20, \tau^{2}=0,0.1,0.25,1$ and a shift from $\delta=1$ to $\delta=2$ at study number 26. Light grey line at .05 . Green and blue dashed lines correspond to power $80 \%$ and $90 \%$, respectively. 

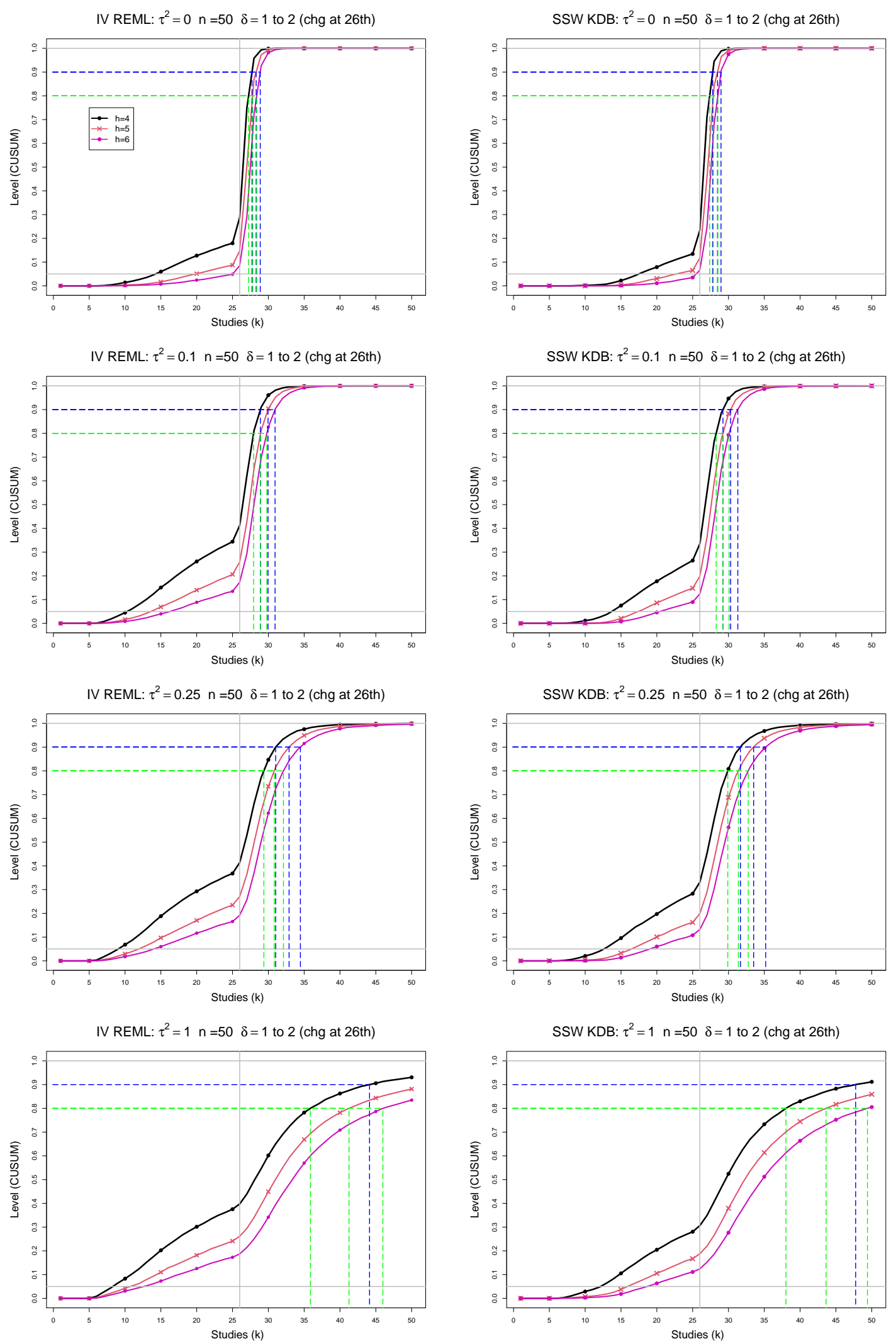

Figure D7. 7: Empirical levels of CUSUM tests for shift in $\delta$ based on SSW KDB and IV REML at $h=4,5,6$ for equal sample sizes $n_{i C}+n_{i T}=n=50, \tau^{2}=0,0.1,0.25,1$ and a shift from $\delta=1$ to $\delta=2$ at study number 26. Light grey line at .05 . Green and blue dashed lines correspond to power $80 \%$ and $90 \%$, respectively. 

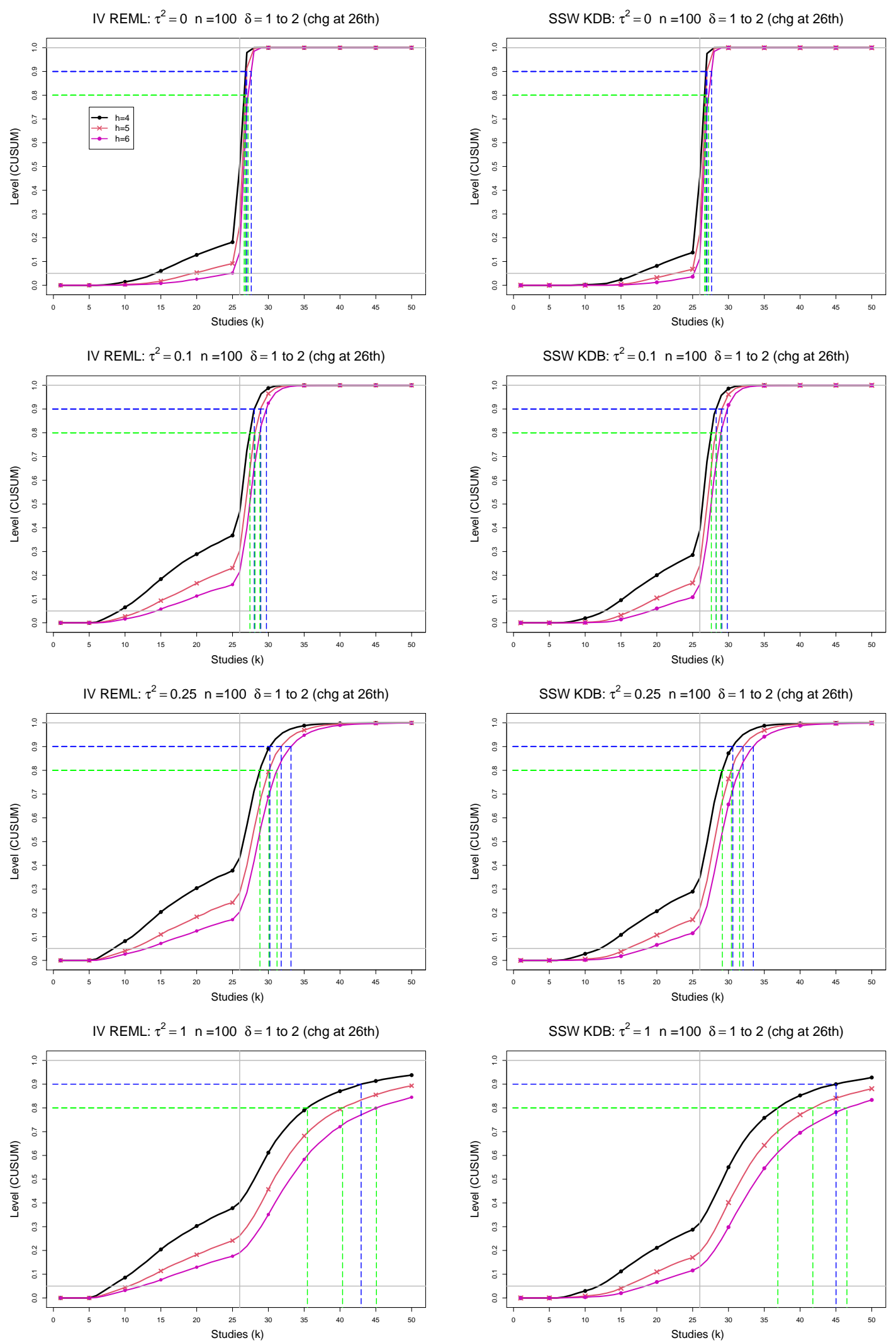

Figure D7. 8: Empirical levels of CUSUM tests for shift in $\delta$ based on SSW KDB and IV REML at $h=4,5,6$ for equal sample sizes $n_{i C}+n_{i T}=n=100, \tau^{2}=0,0.1,0.25,1$ and a shift from $\delta=1$ to $\delta=2$ at study number 26. Light grey line at .05 . Green and blue dashed lines correspond to power $80 \%$ and $90 \%$, respectively. 

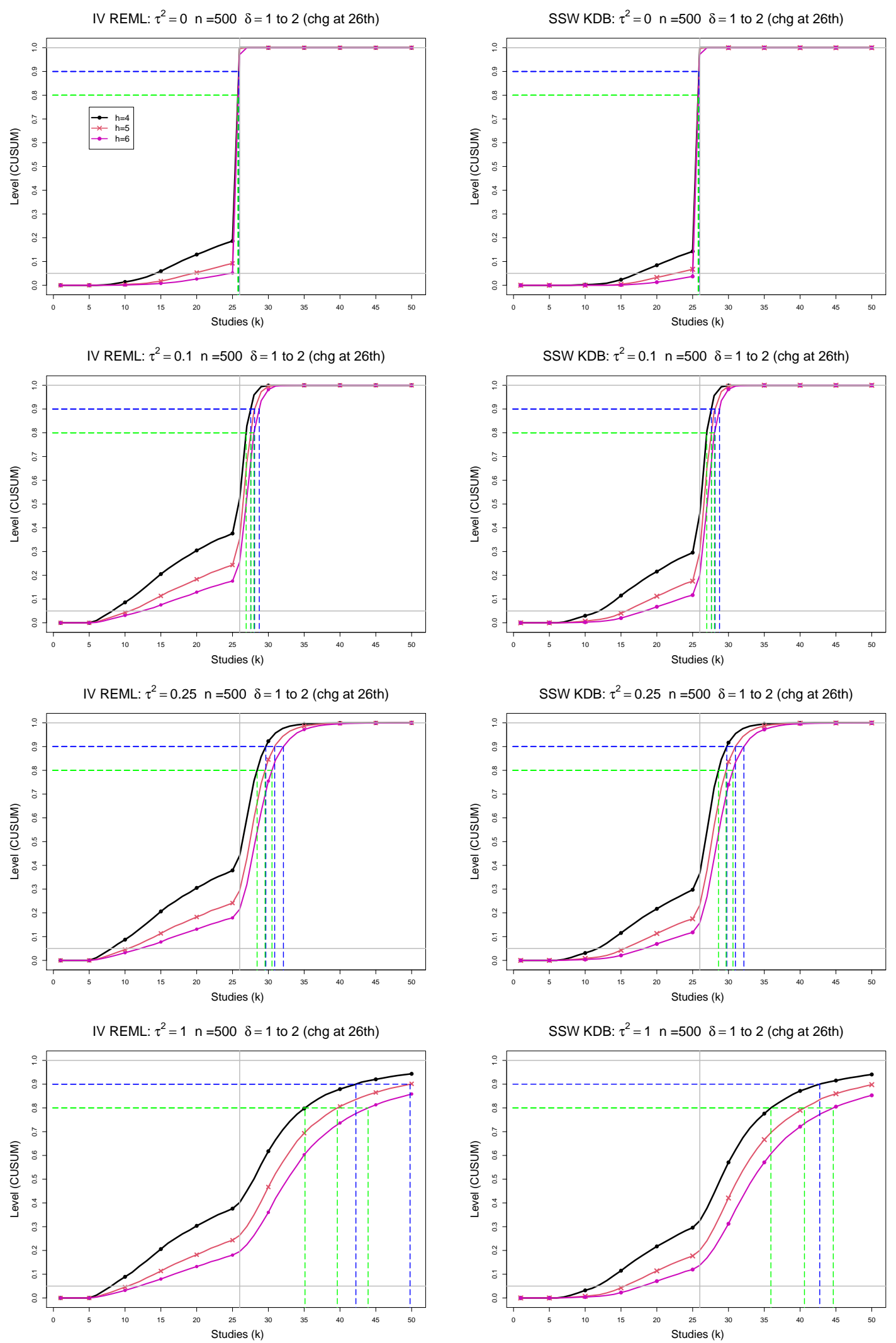

Figure D7. 9: Empirical levels of CUSUM tests for shift in $\delta$ based on SSW KDB and IV REML at $h=4,5,6$ for equal sample sizes $n_{i C}+n_{i T}=n=500, \tau^{2}=0,0.1,0.25,1$ and a shift from $\delta=1$ to $\delta=2$ at study number 26. Light grey line at .05. Green and blue dashed lines correspond to power $80 \%$ and $90 \%$, respectively. 

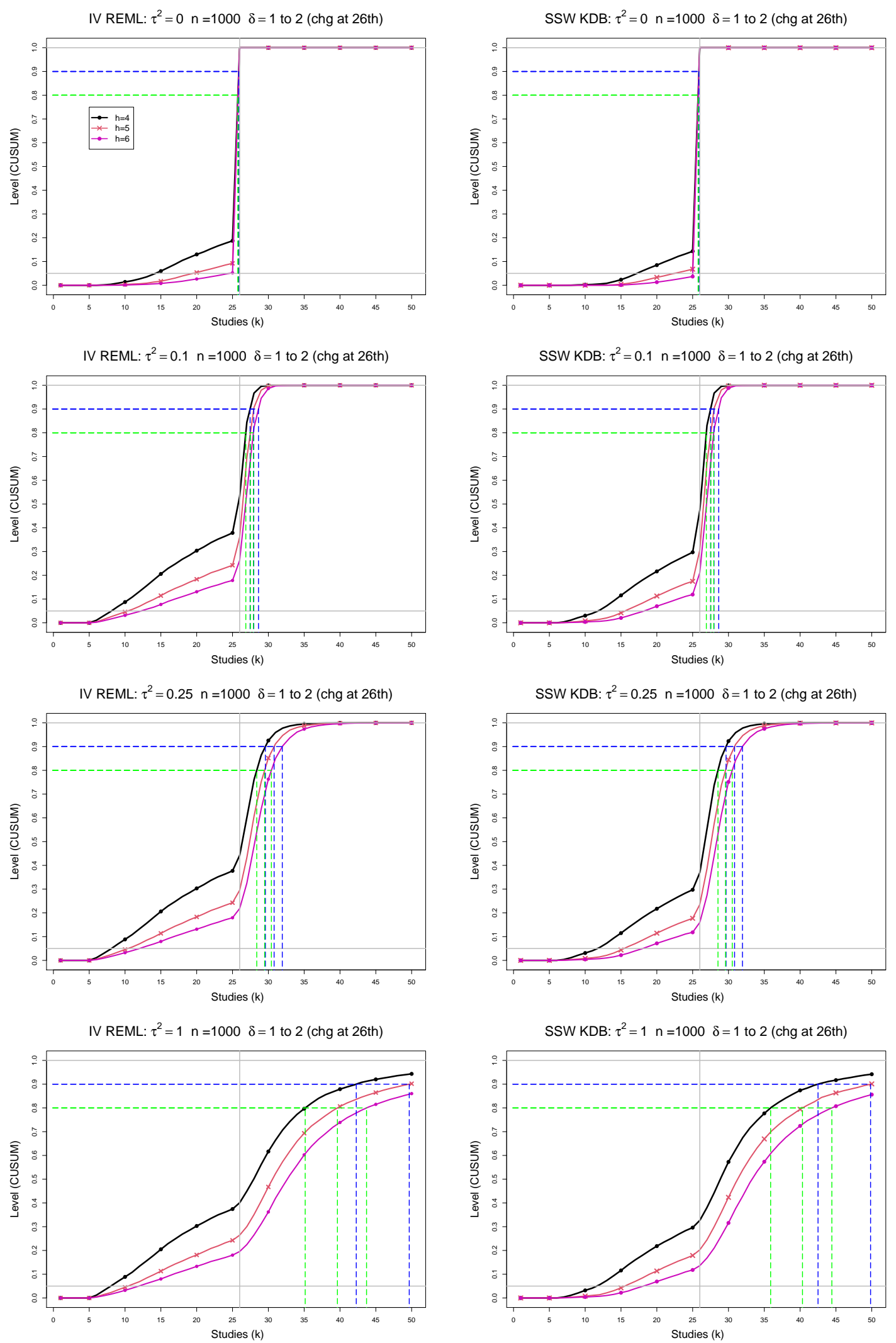

Figure D7. 10: Empirical levels of CUSUM tests for shift in $\delta$ based on SSW KDB and IV REML at $h=4,5,6$ for equal sample sizes $n_{i C}+n_{i T}=n=1000, \tau^{2}=0,0.1,0.25,1$ and a shift from $\delta=1$ to $\delta=2$ at study number 26. Light grey line at .05. Green and blue dashed lines correspond to power $80 \%$ and $90 \%$, respectively. 

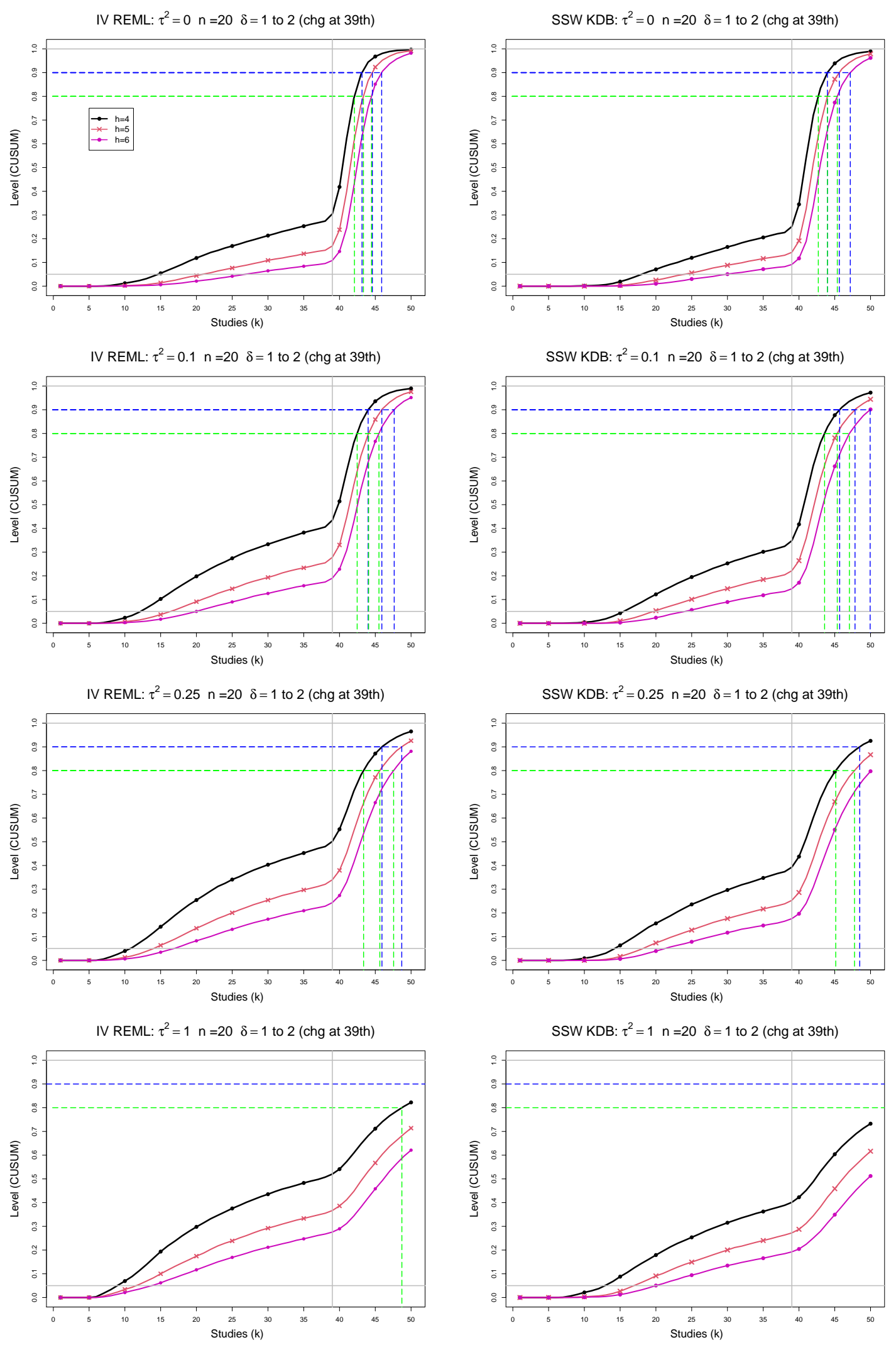

Figure D7. 11: Empirical levels of CUSUM tests for shift in $\delta$ based on SSW KDB and IV REML at $h=4,5,6$ for equal sample sizes $n_{i C}+n_{i T}=n=20, \tau^{2}=0,0.1,0.25,1$ and a shift from $\delta=1$ to $\delta=2$ at study number 39. Light grey line at .05. Green and blue dashed lines correspond to power $80 \%$ and $90 \%$, respectively. 

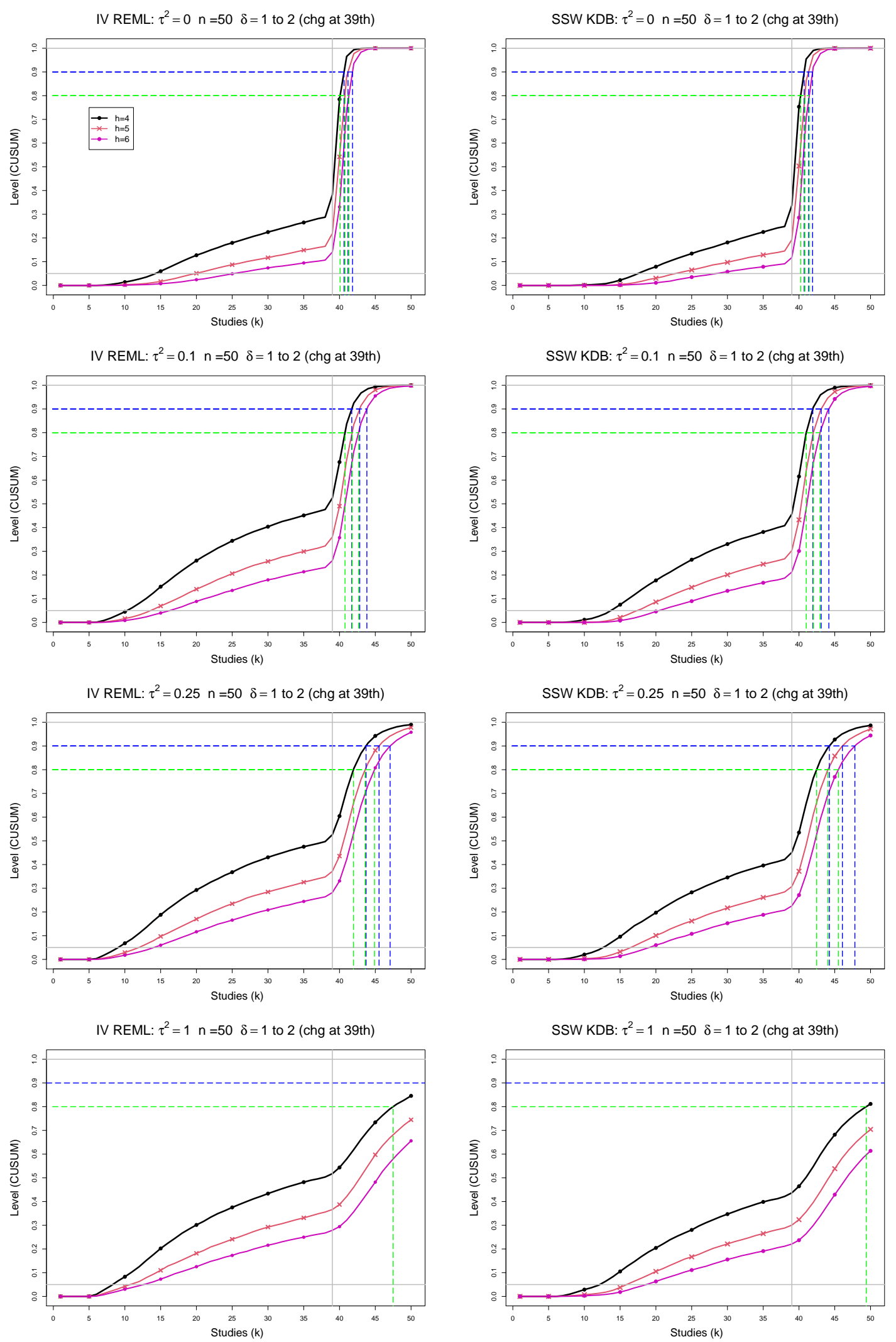

Figure D7. 12: Empirical levels of CUSUM tests for shift in $\delta$ based on SSW KDB and IV REML at $h=4,5,6$ for equal sample sizes $n_{i C}+n_{i T}=n=50, \tau^{2}=0,0.1,0.25,1$ and a shift from $\delta=1$ to $\delta=2$ at study number 39. Light grey line at .05. Green and blue dashed lines correspond to power $80 \%$ and $90 \%$, respectively. 

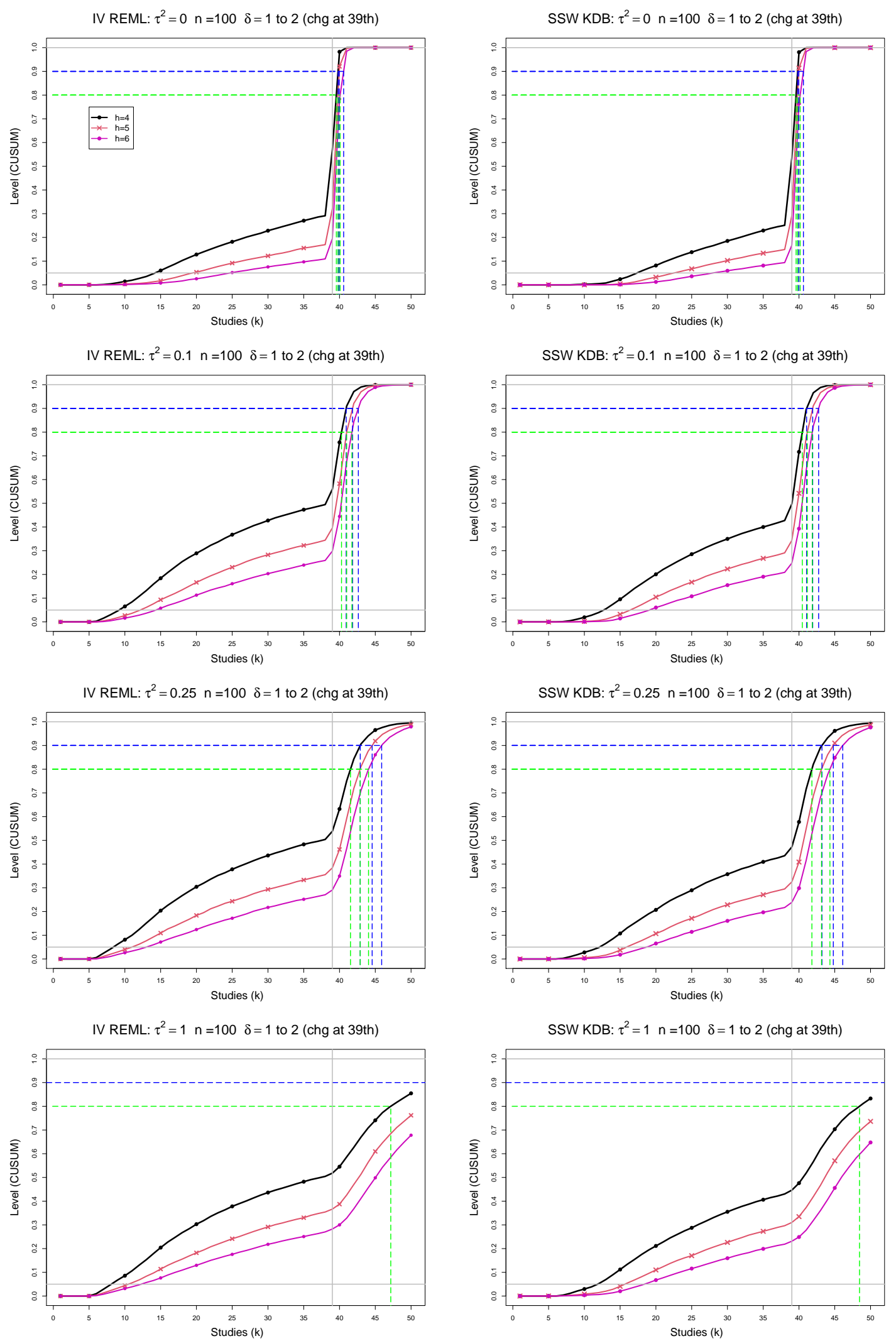

Figure D7. 13: Empirical levels of CUSUM tests for shift in $\delta$ based on SSW KDB and IV REML at $h=4,5,6$ for equal sample sizes $n_{i C}+n_{i T}=n=100, \tau^{2}=0,0.1,0.25,1$ and a shift from $\delta=1$ to $\delta=2$ at study number 39. Light grey line at .05. Green and blue dashed lines correspond to power $80 \%$ and $90 \%$, respectively. 

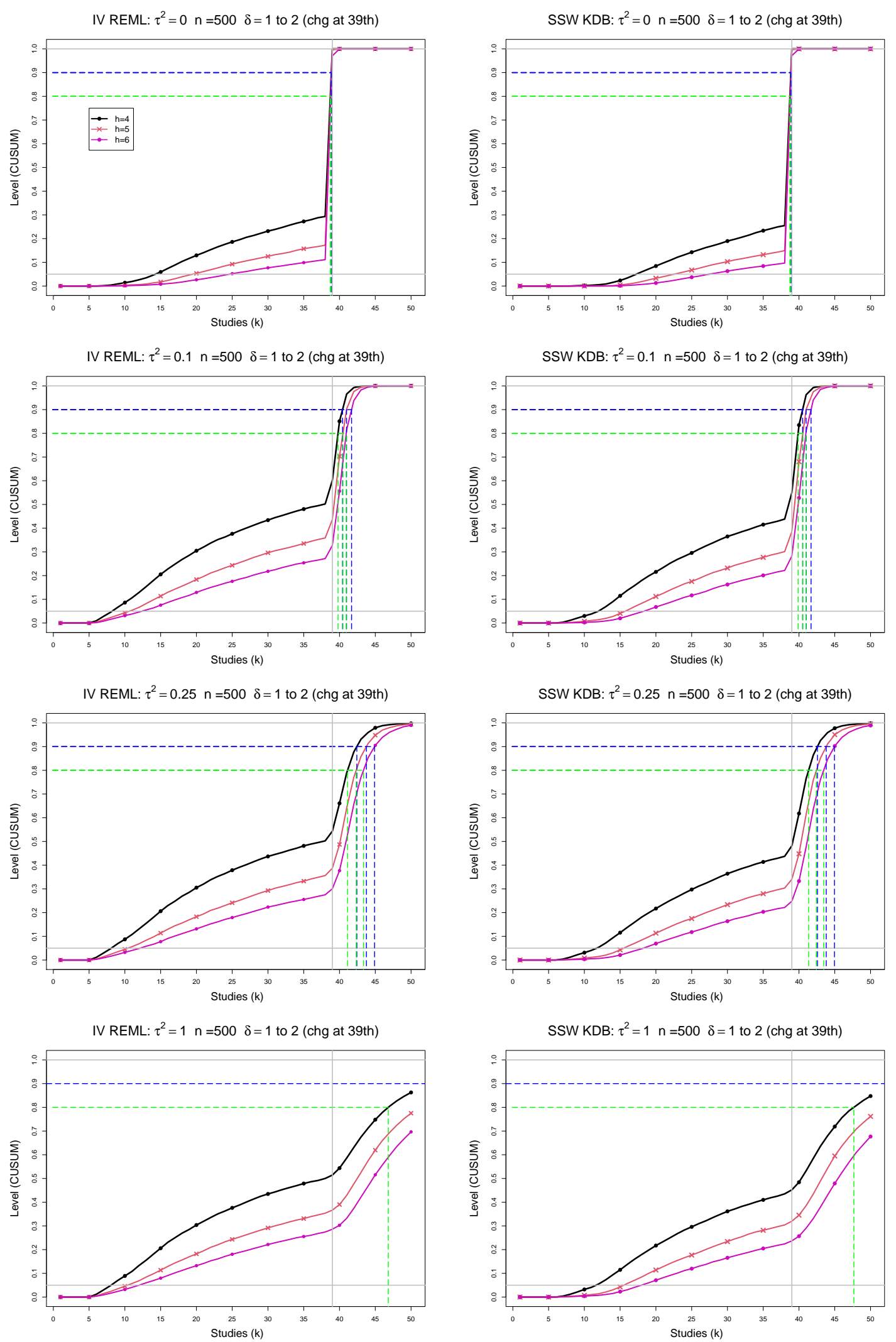

Figure D7. 14: Empirical levels of CUSUM tests for shift in $\delta$ based on SSW KDB and IV REML at $h=4,5,6$ for equal sample sizes $n_{i C}+n_{i T}=n=500, \tau^{2}=0,0.1,0.25,1$ and a shift from $\delta=1$ to $\delta=2$ at study number 39. Light grey line at .05 . Green and blue dashed lines correspond to power $80 \%$ and $90 \%$, respectively. 

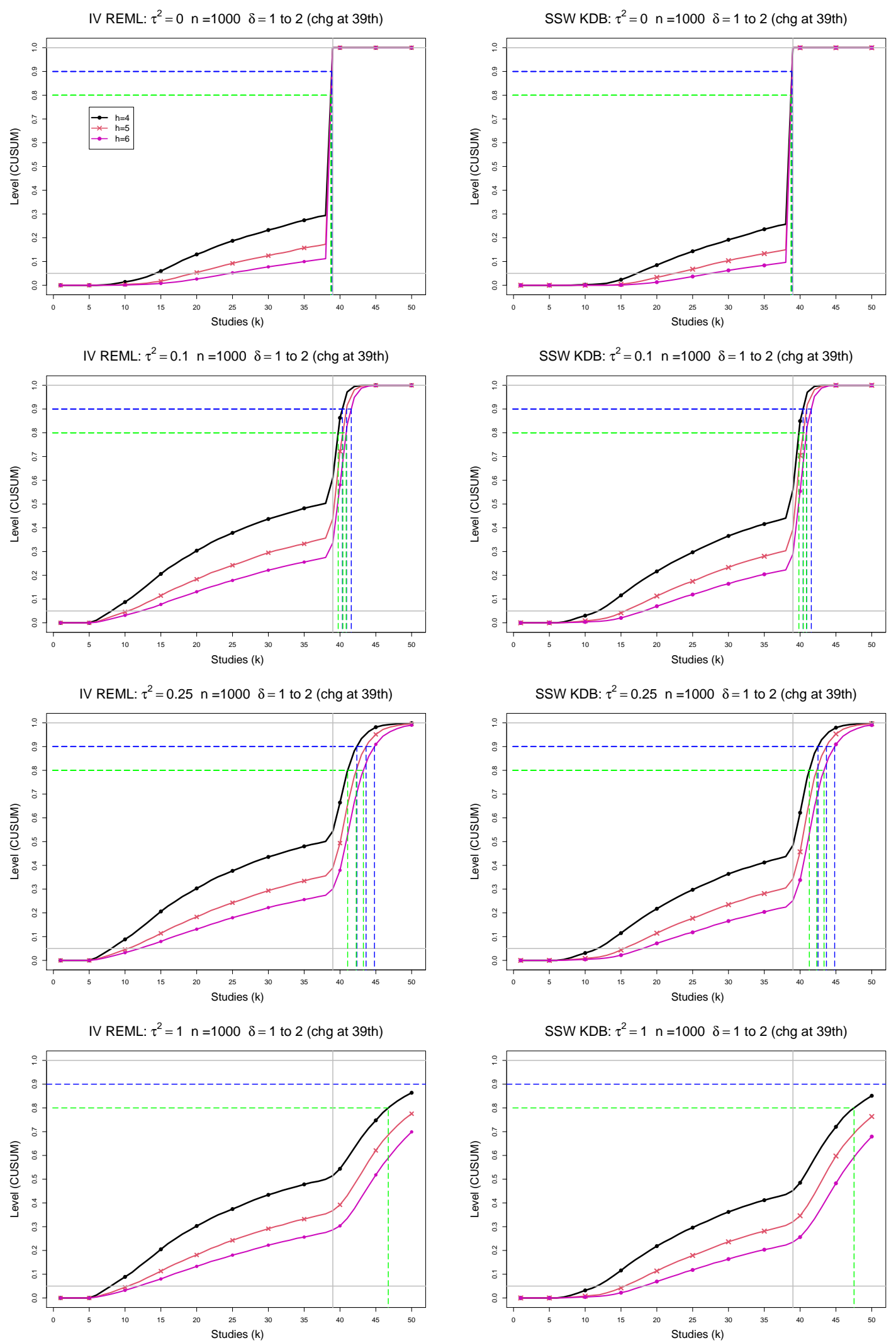

Figure D7. 15: Empirical levels of CUSUM tests for shift in $\delta$ based on SSW KDB and IV REML at $h=4,5,6$ for equal sample sizes $n_{i C}+n_{i T}=n=1000, \tau^{2}=0,0.1,0.25,1$ and a shift from $\delta=1$ to $\delta=2$ at study number 39. Light grey line at .05. Green and blue dashed lines correspond to power $80 \%$ and $90 \%$, respectively. 


\section{E: Additional materials}




\section{E1. Nominal cumulative effects}

For nominal cumulative effects in this section, each figure corresponds to a set of values of $\tau^{2}(=0,0.1,0.25,1)$, a set of values of $n(=20,50,100$ or 500,1000$)$ and a value of point of shift $f(=13,26,39)$ while $\delta$ shifts from 1 to 2 and maximum number of studies $K=50$.

Each figure contains a panel (with study $k$ on the horizontal axis) for each combination of parameters.

The nominal $\delta$ values are calculated with

- $\delta$ IV (inverse variance weights)

- $\delta$ SSW (effective sample size weights) 

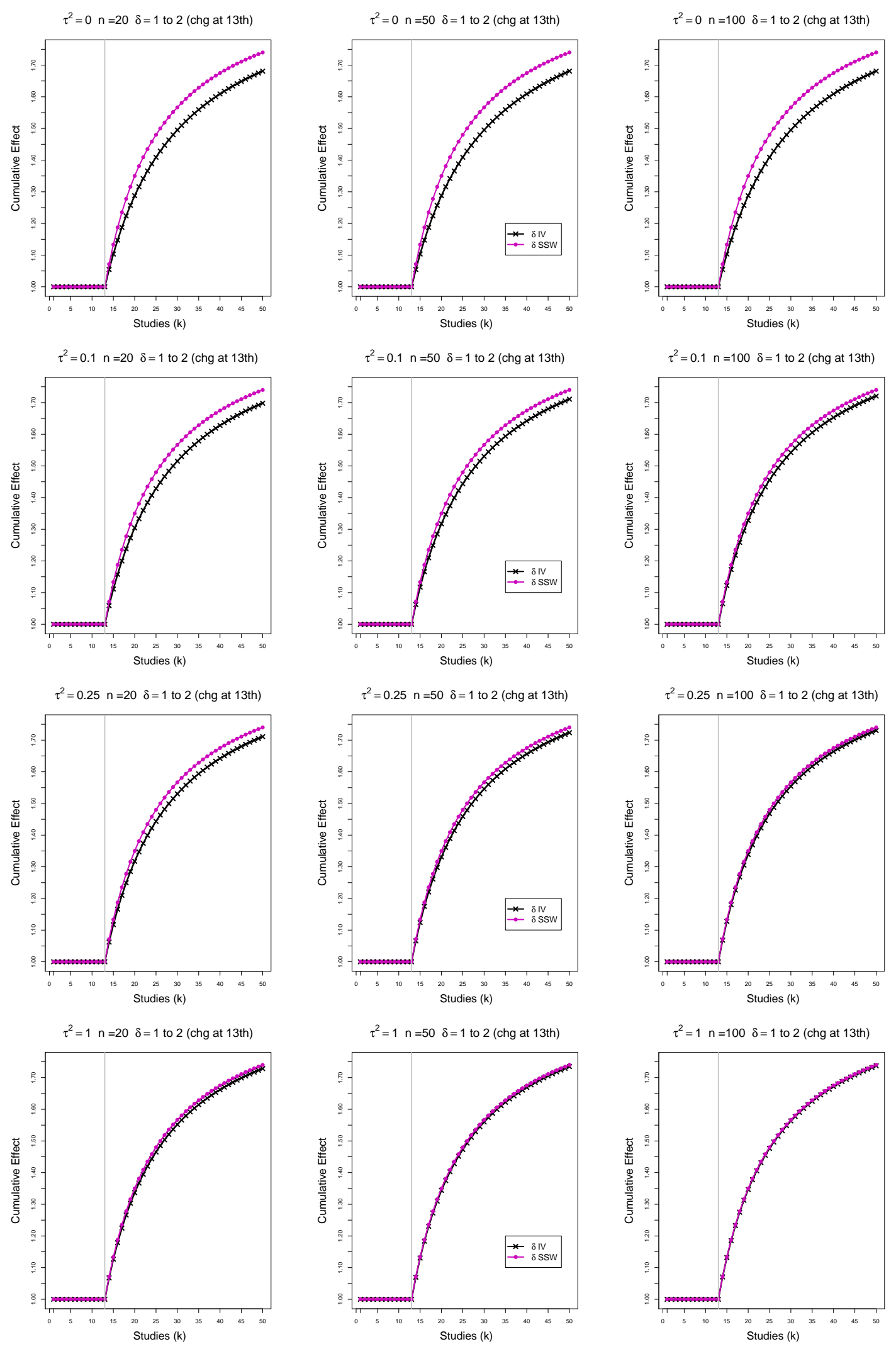

Figure E1. 1: Nominal cumulative mean effect $\delta_{(k)}$ for SSW and IV weights for equal sample sizes $n_{i C}+n_{i T}=n=20,50,100$ and a shift from $\delta_{1}=1$ to $\delta_{2}=2$ at study number 13 . 

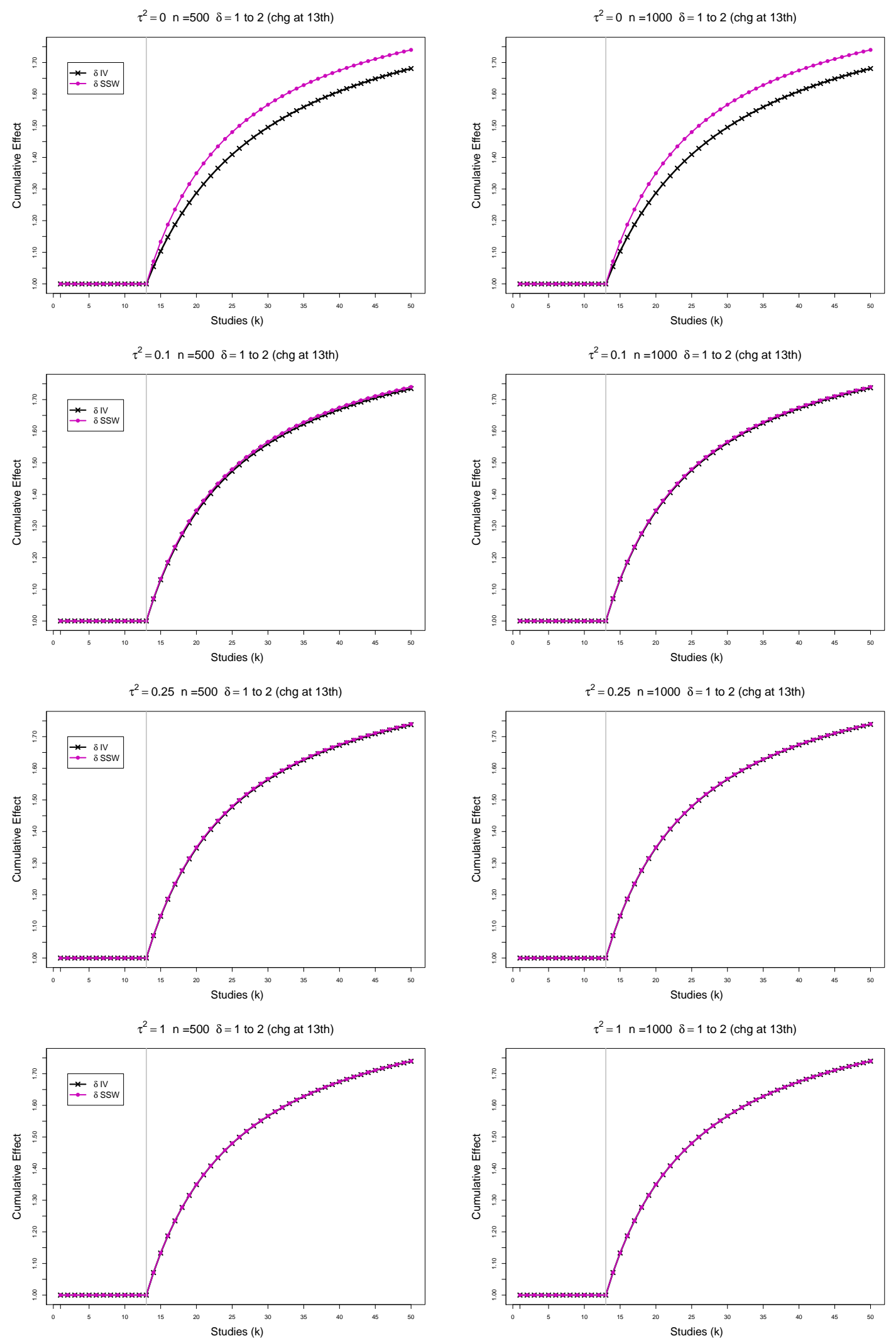

Figure E1. 2: Nominal cumulative mean effect $\delta_{(k)}$ for SSW and IV weights for equal sample sizes $n_{i C}+n_{i T}=n=500,1000$ and a shift from $\delta_{1}=1$ to $\delta_{2}=2$ at study number 13 . 

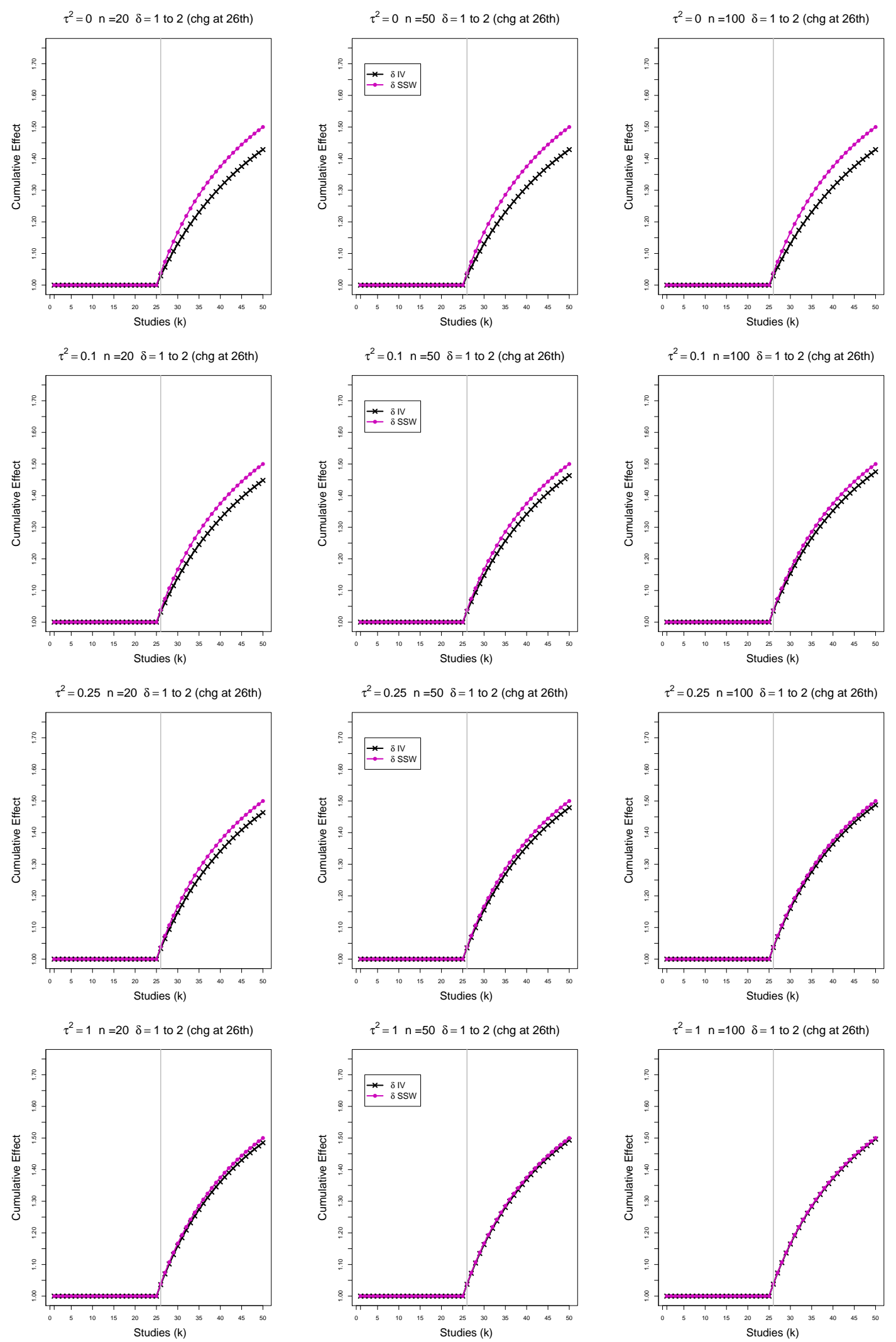

Figure E1. 3: Nominal cumulative mean effect $\delta_{(k)}$ for SSW and IV weights for equal sample sizes $n_{i C}+n_{i T}=n=20,50,100$ and a shift from $\delta_{1}=1$ to $\delta_{2}=2$ at study number 26 . 

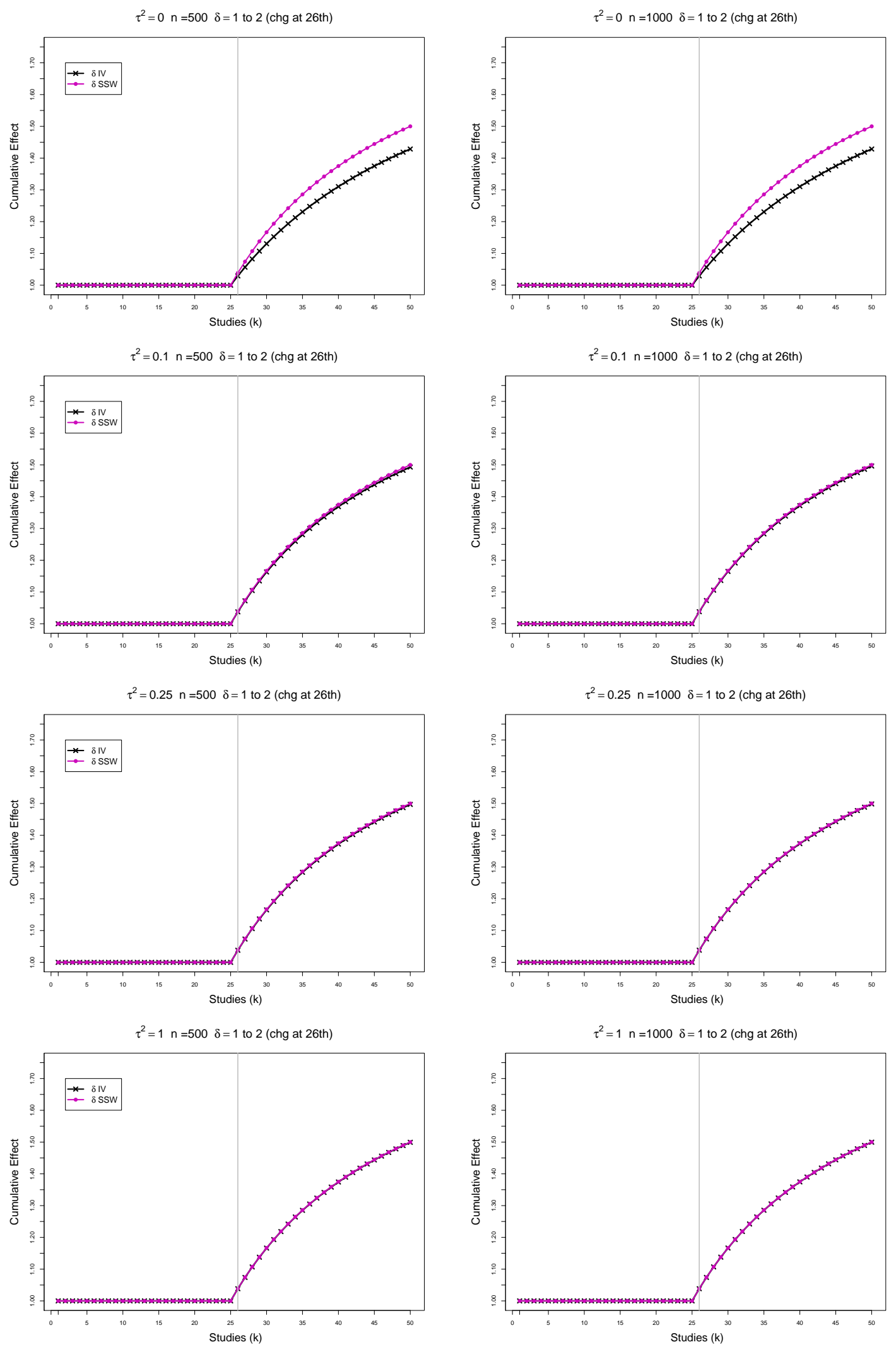

Figure E1. 4: Nominal cumulative mean effect $\delta_{(k)}$ for SSW and IV weights for equal sample sizes $n_{i C}+n_{i T}=n=500,1000$ and a shift from $\delta_{1}=1$ to $\delta_{2}=2$ at study number 26 . 

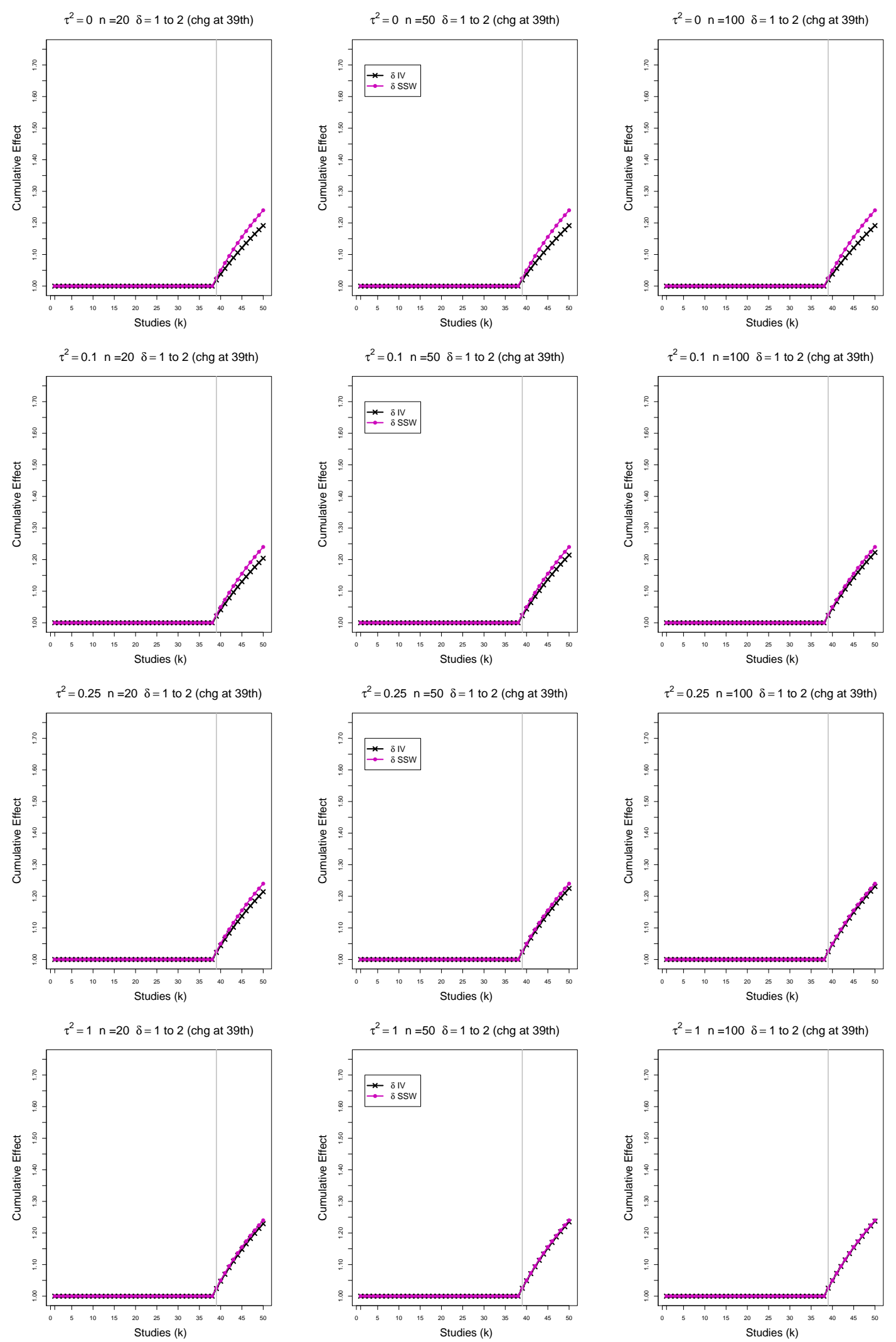

Figure E1. 5: Nominal cumulative mean effect $\delta_{(k)}$ for SSW and IV weights for equal sample sizes $n_{i C}+n_{i T}=n=20,50,100$ and a shift from $\delta_{1}=1$ to $\delta_{2}=2$ at study number 39 . 

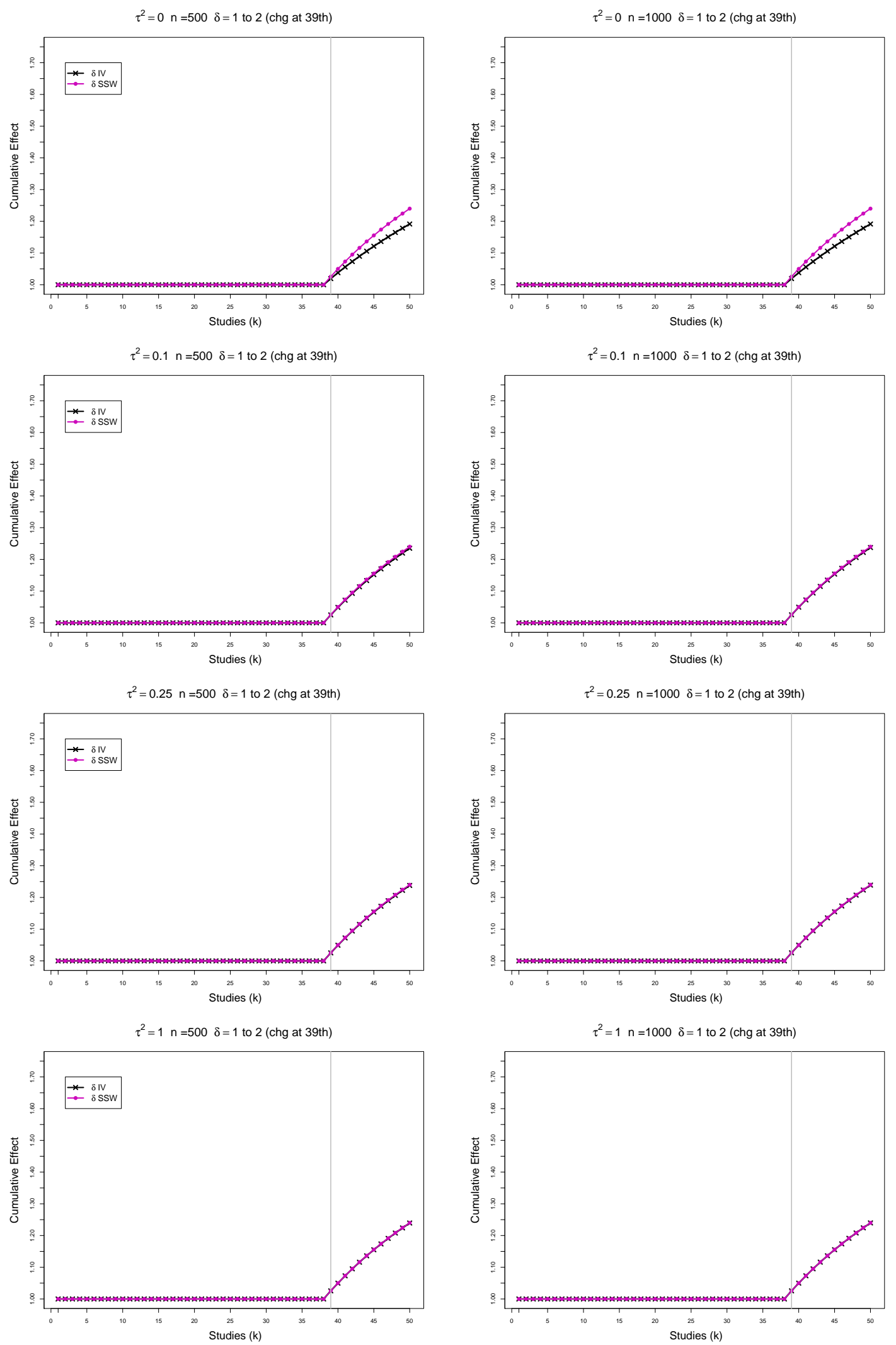

Figure E1. 6: Nominal cumulative mean effect $\delta_{(k)}$ for SSW and IV weights for equal sample sizes $n_{i C}+n_{i T}=n=500,1000$ and a shift from $\delta_{1}=1$ to $\delta_{2}=2$ at study number 39 . 


\section{E2. Comparison plots - Empirical levels of tests for shift-in-the-mean}

For empirical levels of tests for shift-in-the-mean in this section, each figure corresponds to one of the two tested meta-analysis methods, a set of values of $\tau^{2}(=0,0.1$, $0.25,1)$, a value of $n(=20,50,100,500,1000)$, a value of significance level $(=0.05$, $0.01,0.005)$, a maximum number of studies $K=50$, a value of $\delta$ which shifts from 1 to 2 with a point of shift $f=(13,26,39)$, a set of values of $\delta$ which shifts from ( 1 to 0 ), from (1 to 0.5$)$ and from (1 to 1.5$)$ with a point of shift $f=26$.

Each figure contains a panel (with study $k$ on the horizontal axis) for each combination of parameters.

The compared tests are

- CMA (standard CMA test)

- CMA $\left(\hat{\delta}_{0}\right)$ (standard CMA test with estimated from stage 1 value of $\hat{\delta}_{0}$ )

- 2-Stage CMA $\left(\tau_{0}^{2}\right)$ (2-Stage CMA test with estimated in stage 1 value of $\tau^{2}$ )

- 2-Stage CMA $\hat{\delta}_{0}, \tau_{0}^{2}$ (2-Stage CMA test with estimated from stage $1 \hat{\delta}_{0}$ and $\tau^{2}$ )

- CUSUM $\tau_{0}^{2}$ (estimated in stage 1value of $\tau^{2}$

- QP (test of positive shift in $\tau^{2}$ based on Q-profile confidence interval)

- KDB (test of positive shift in $\tau^{2}$ based on improved Q-profile method by Kulinskaya et.al.(2011) )

And the used meta-analysis methods are

- IV REML (Restricted maximum likelihood, inverse variance weighted)

- SSW KDB (Kulinskaya-Dollinger-Bjørkestøl estimator of $\tau^{2}$, sample size weighted) 


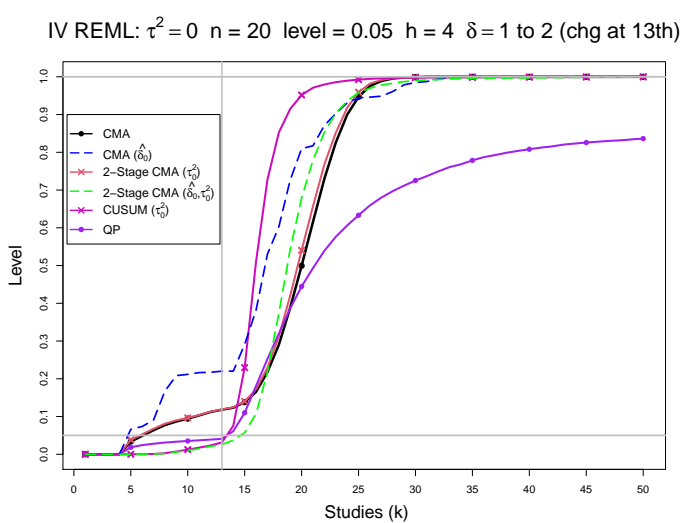

IV REML: $\tau^{2}=0.1 \mathrm{n}=20$ level $=0.05 \mathrm{~h}=4 \delta=1$ to 2 (chg at 13 th)

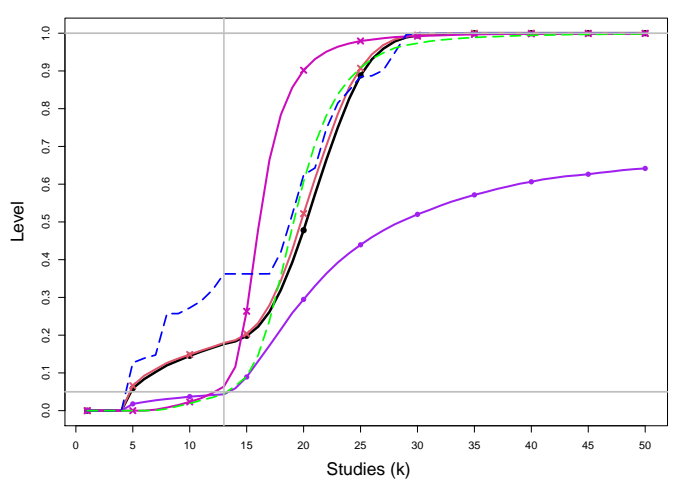

IV REML: $\tau^{2}=0.25 \mathrm{n}=20$ level $=0.05 \mathrm{~h}=4 \delta=1$ to 2 (chg at 13th)

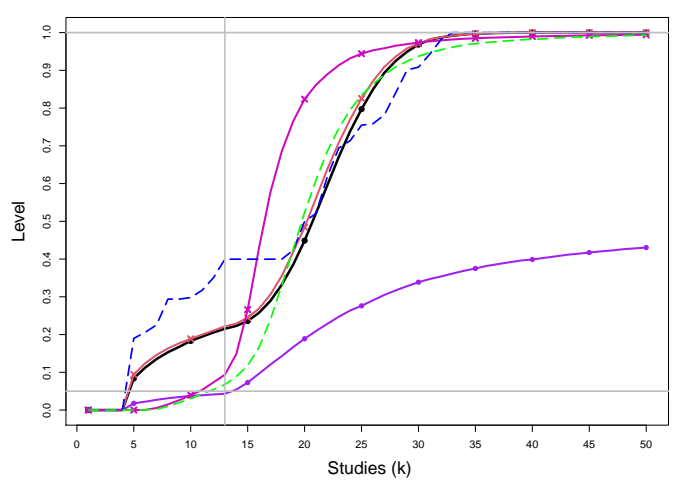

IV REML: $\tau^{2}=1 \mathrm{n}=20$ level $=0.05 \mathrm{~h}=4 \delta=1$ to 2 (chg at 13 th)

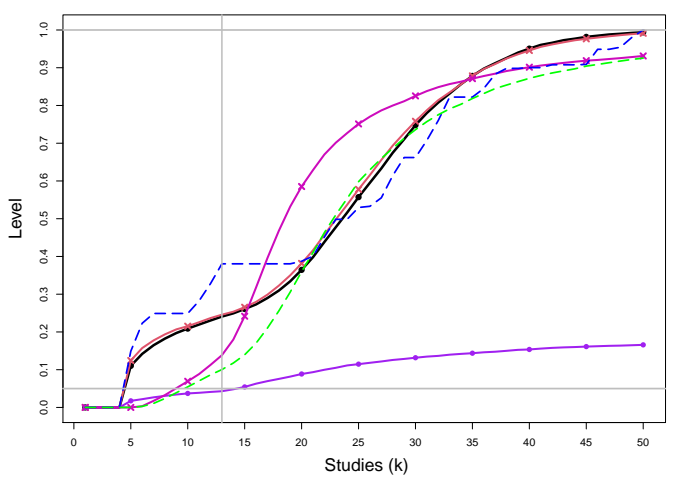

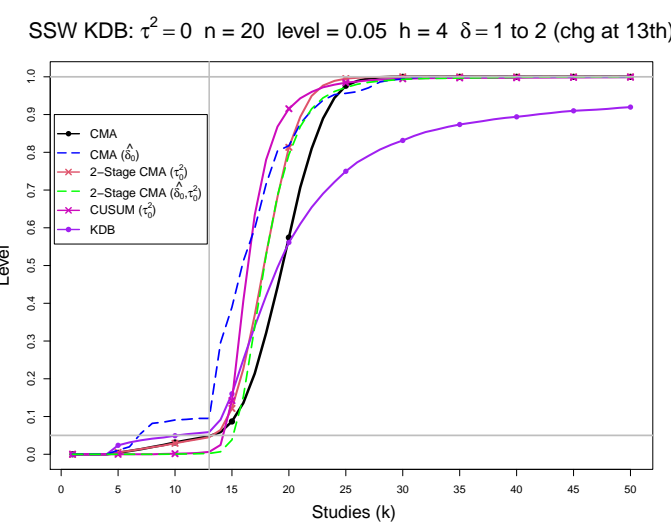

SSW KDB: $\tau^{2}=0.1 \mathrm{n}=20$ level $=0.05 \mathrm{~h}=4 \delta=1$ to 2 (chg at 13th)

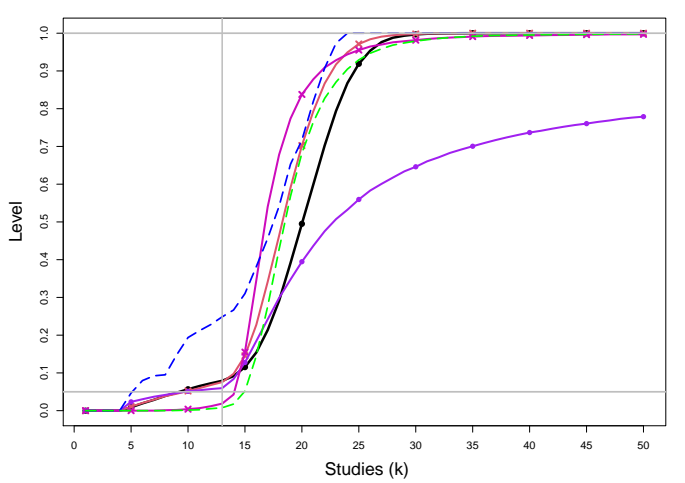

SSW KDB: $\tau^{2}=0.25 \mathrm{n}=20$ level $=0.05 \mathrm{~h}=4 \delta=1$ to 2 (chg at 13 th)

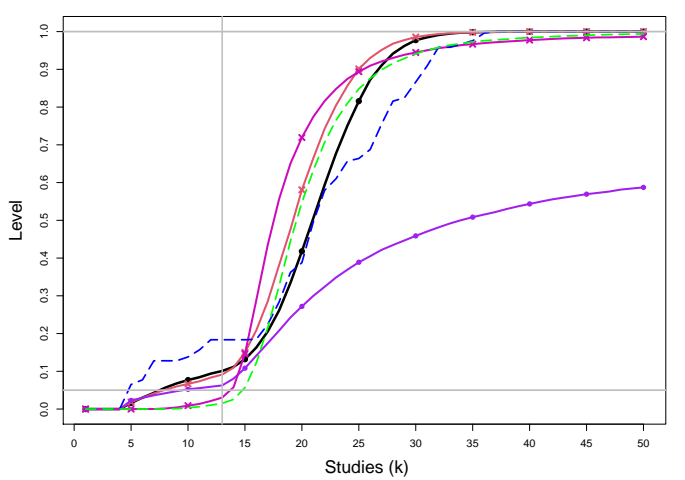

SSW KDB: $\tau^{2}=1 \mathrm{n}=20$ level $=0.05 \mathrm{~h}=4 \delta=1$ to 2 (chg at 13 th)

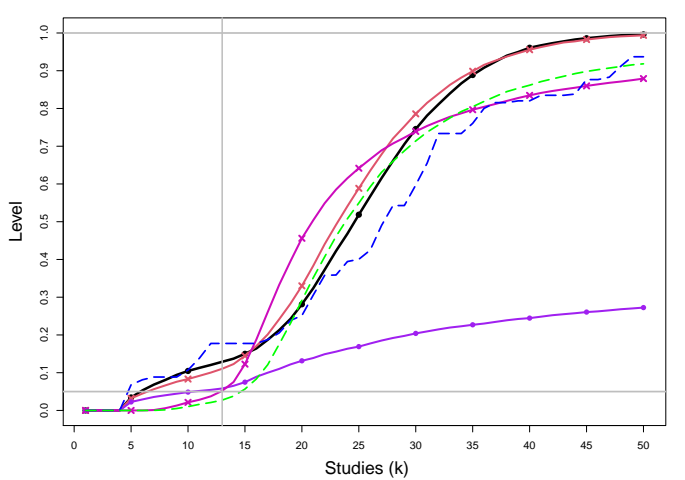

Figure E2. 1: Empirical levels of 1- and 2-stage CMA tests for shift in $\delta$ at .05 nominal level, shift in $\tau^{2}$ at .025 nominal levels and of CUSUM with $h=4$ for equal sample sizes $n_{i C}+n_{i T}=n=20, \tau^{2}=0,0.1,0.25,1$ and a shift from $\delta=1$ to $\delta=2$ at study number 13. Light grey line at .05. 


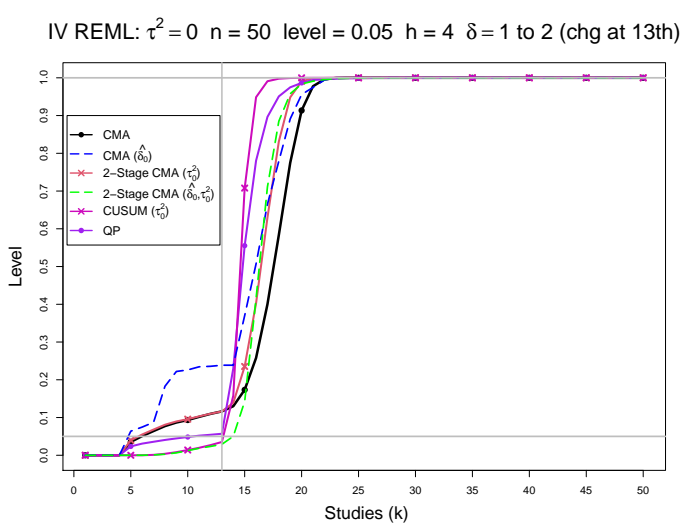

IV REML: $\tau^{2}=0.1 \mathrm{n}=50$ level $=0.05 \mathrm{~h}=4 \delta=1$ to 2 (chg at 13 th)

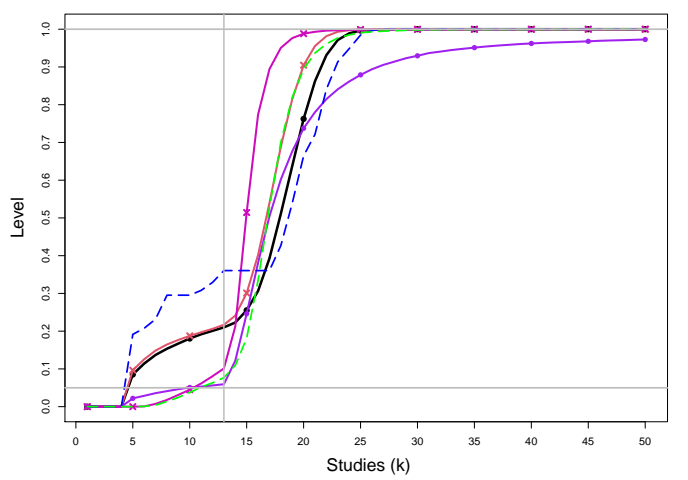

IV REML: $\tau^{2}=0.25 \mathrm{n}=50$ level $=0.05 \mathrm{~h}=4 \delta=1$ to 2 (chg at 13th)

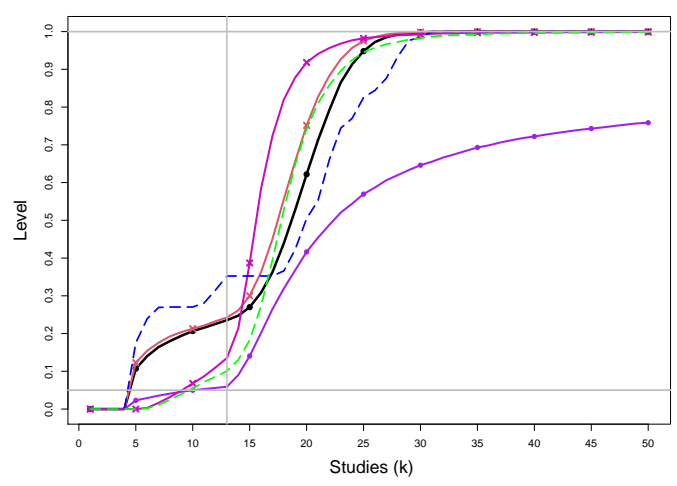

IV REML: $\tau^{2}=1 \mathrm{n}=50$ level $=0.05 \mathrm{~h}=4 \delta=1$ to 2 (chg at 13 th)

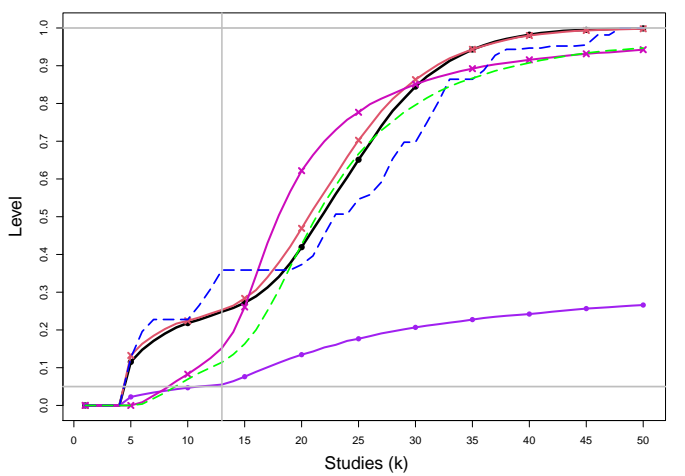

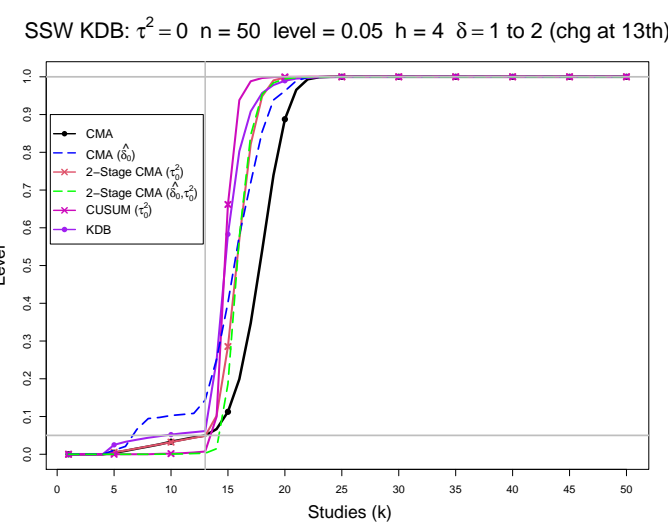

SSW KDB: $\tau^{2}=0.1 \mathrm{n}=50$ level $=0.05 \mathrm{~h}=4 \delta=1$ to 2 (chg at 13th)

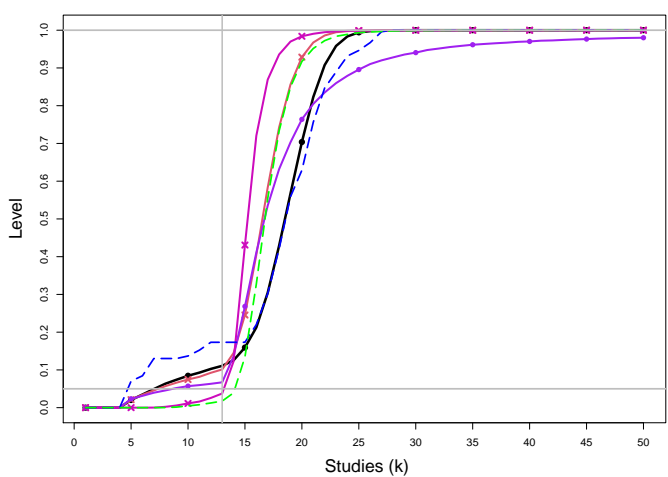

SSW KDB: $\tau^{2}=0.25 \mathrm{n}=50$ level $=0.05 \mathrm{~h}=4 \quad \delta=1$ to 2 (chg at 13 th)

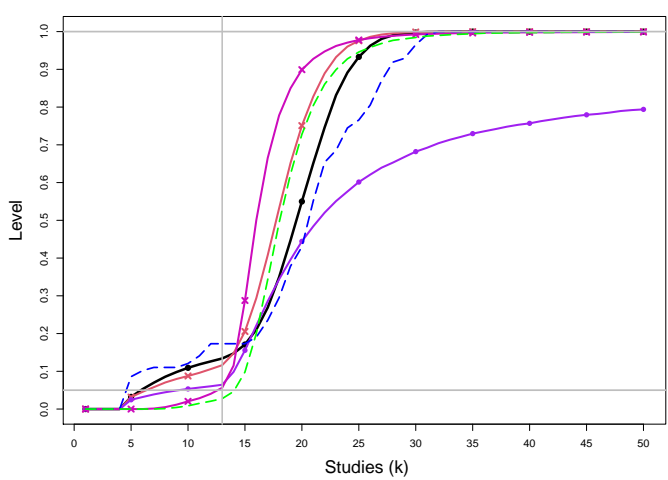

SSW KDB: $\tau^{2}=1 \mathrm{n}=50$ level $=0.05 \mathrm{~h}=4 \delta=1$ to 2 (chg at 13 th)

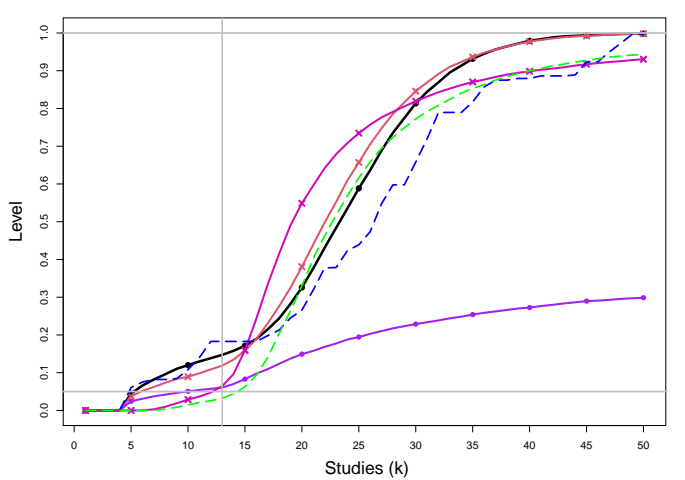

Figure E2. 2: Empirical levels of 1- and 2-stage CMA tests for shift in $\delta$ at .05 nominal level, shift in $\tau^{2}$ at .025 nominal levels and of CUSUM with $h=4$ for equal sample sizes $n_{i C}+n_{i T}=n=50, \tau^{2}=0,0.1,0.25,1$ and a shift from $\delta=1$ to $\delta=2$ at study number 13. Light grey line at .05. 


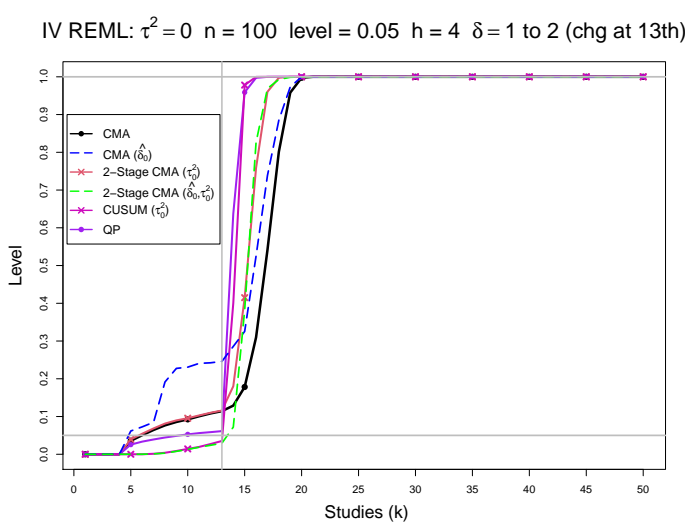

IV REML: $\tau^{2}=0.1 \mathrm{n}=100$ level $=0.05 \mathrm{~h}=4 \delta=1$ to 2 (chg at 13th)

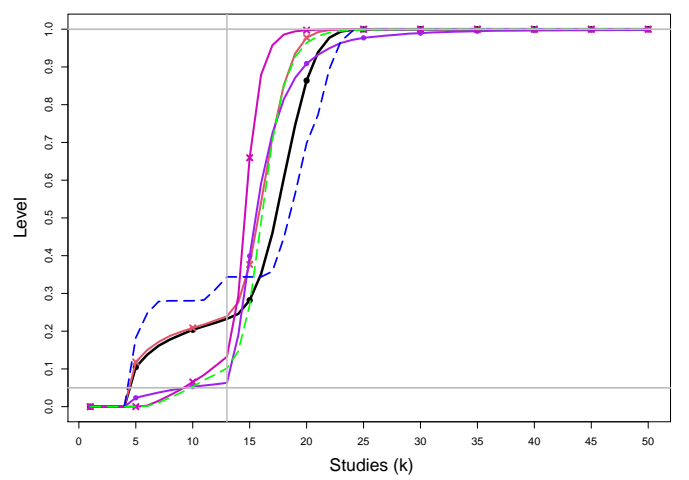

IV REML: $\tau^{2}=0.25 \mathrm{n}=100$ level $=0.05 \mathrm{~h}=4 \delta=1$ to 2 (chg at 13 th)

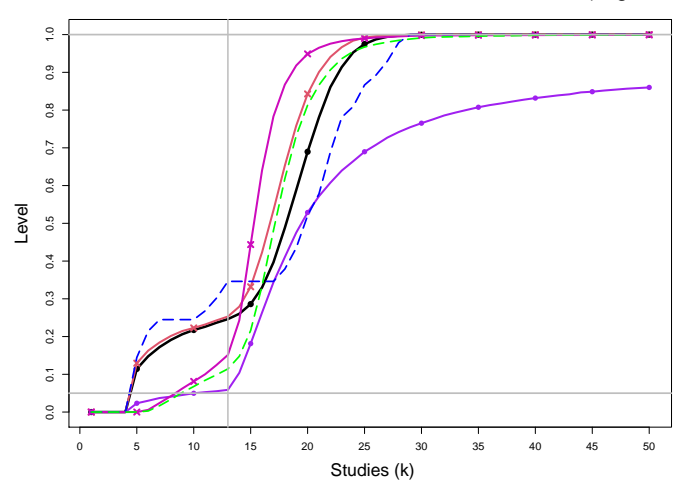

IV REML: $\tau^{2}=1 \mathrm{n}=100$ level $=0.05 \mathrm{~h}=4 \delta=1$ to 2 (chg at 13 th)

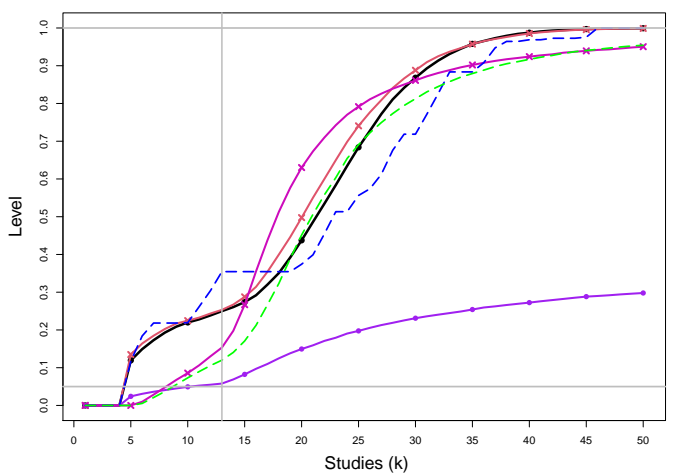

SSW KDB: $\tau^{2}=0 \mathrm{n}=100$ level $=0.05 \mathrm{~h}=4 \delta=1$ to 2 (chg at 13 th)

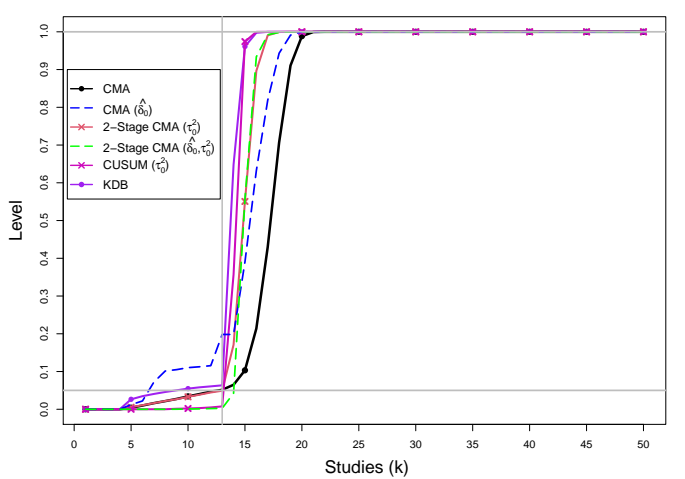

SSW KDB: $\tau^{2}=0.1 \mathrm{n}=100$ level $=0.05 \mathrm{~h}=4 \delta=1$ to 2 (chg at 13 th)

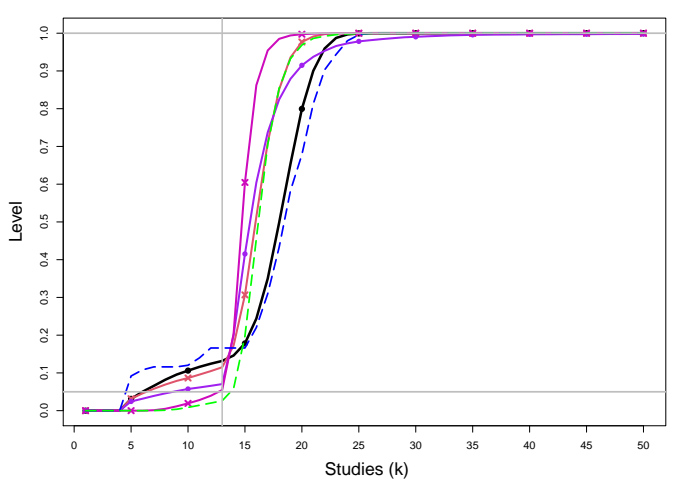

SSW KDB: $\tau^{2}=0.25 \mathrm{n}=100$ level $=0.05 \mathrm{~h}=4 \delta=1$ to 2 (chg at 13th)

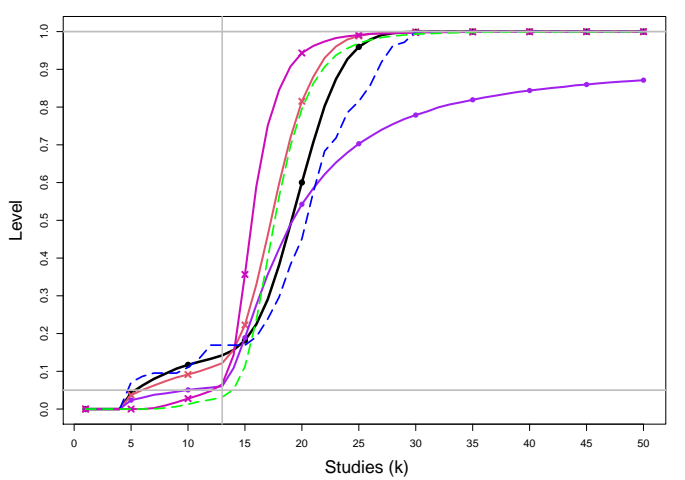

SSW KDB: $\tau^{2}=1 \mathrm{n}=100$ level $=0.05 \mathrm{~h}=4 \delta=1$ to 2 (chg at 13th)

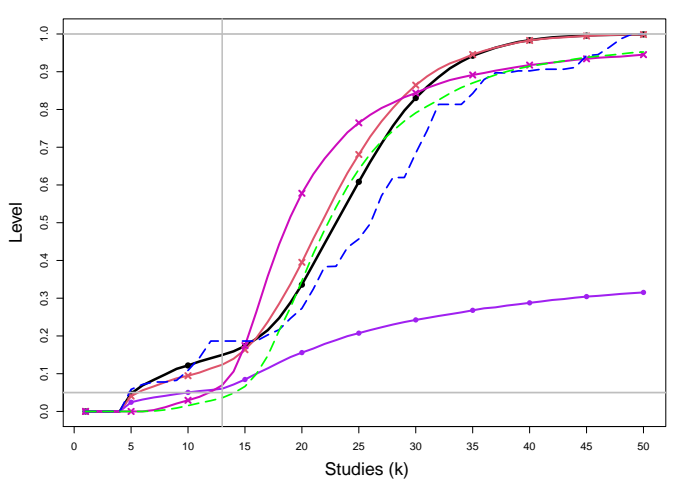

Figure E2. 3: Empirical levels of 1- and 2-stage CMA tests for shift in $\delta$ at .05 nominal level, shift in $\tau^{2}$ at .025 nominal levels and of CUSUM with $h=4$ for equal sample sizes $n_{i C}+n_{i T}=n=100, \tau^{2}=0,0.1,0.25,1$ and a shift from $\delta=1$ to $\delta=2$ at study number 13. Light grey line at .05. 


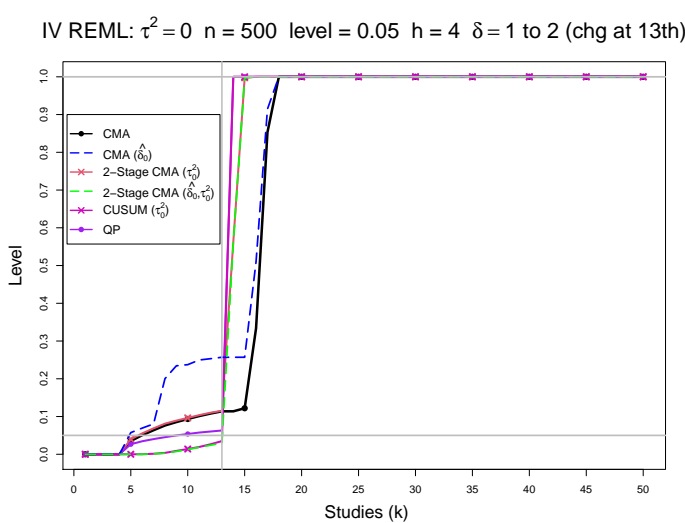

IV REML: $\tau^{2}=0.1 \mathrm{n}=500$ level $=0.05 \mathrm{~h}=4 \delta=1$ to 2 (chg at 13th)

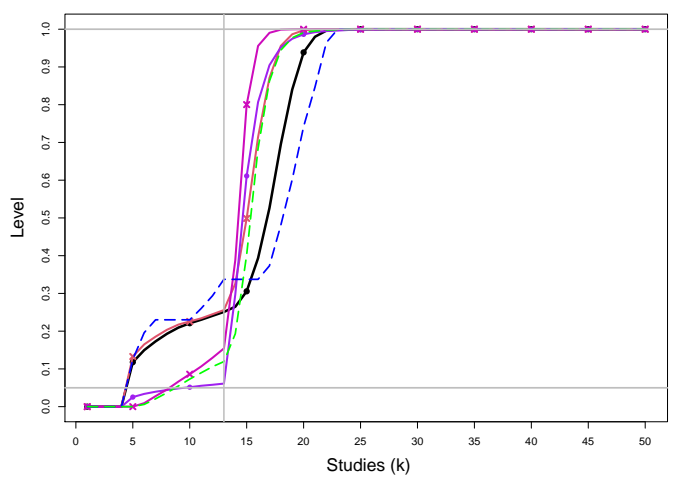

IV REML: $\tau^{2}=0.25 \mathrm{n}=500$ level $=0.05 \mathrm{~h}=4 \delta=1$ to 2 (chg at 13 th)

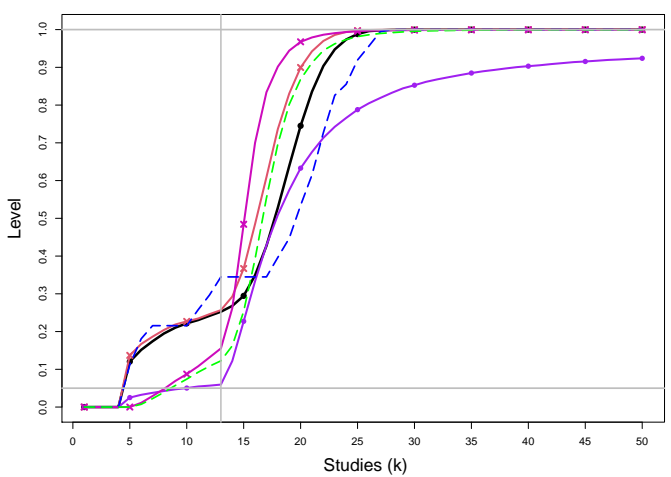

IV REML: $\tau^{2}=1 \mathrm{n}=500$ level $=0.05 \mathrm{~h}=4 \delta=1$ to 2 (chg at 13 th)

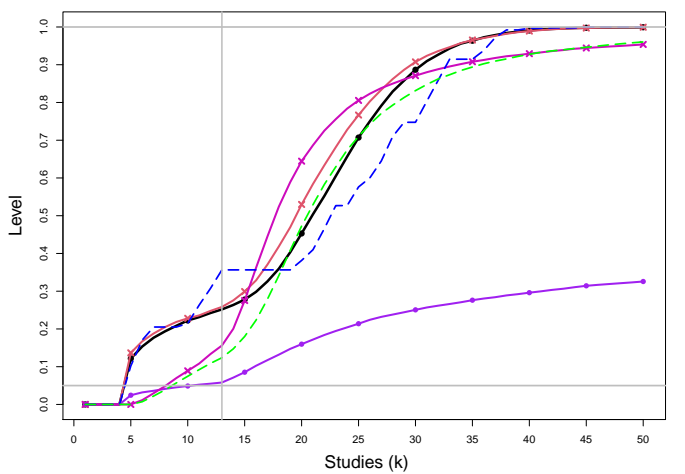

SSW KDB: $\tau^{2}=0 \mathrm{n}=500$ level $=0.05 \mathrm{~h}=4 \delta=1$ to 2 (chg at 13 th)

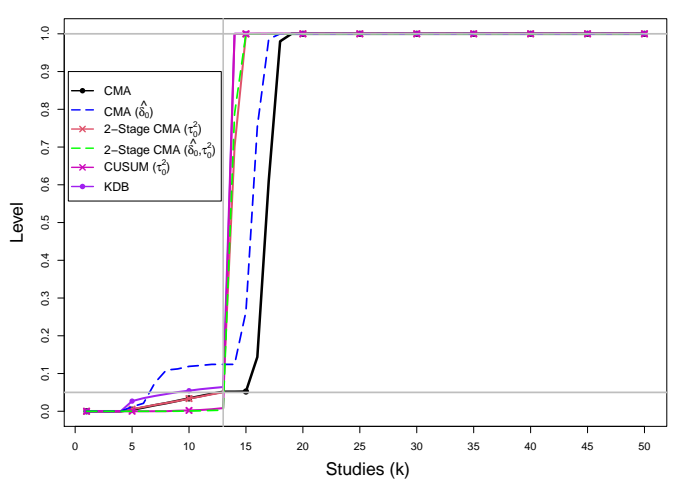

SSW KDB: $\tau^{2}=0.1 \mathrm{n}=500$ level $=0.05 \mathrm{~h}=4 \delta=1$ to 2 (chg at 13 th)

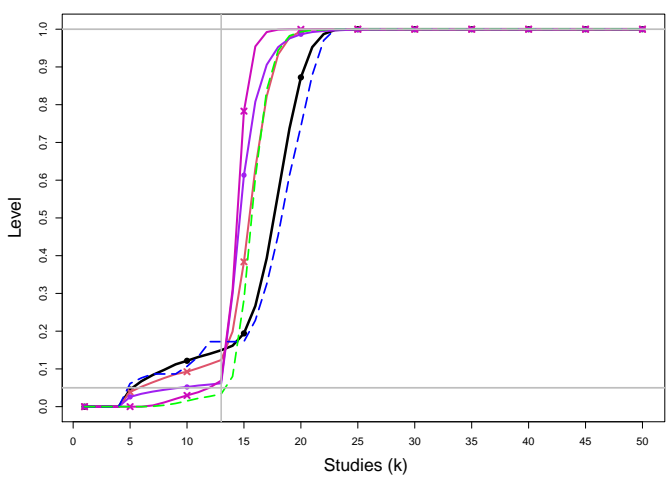

SSW KDB: $\tau^{2}=0.25 \mathrm{n}=500$ level $=0.05 \mathrm{~h}=4 \delta=1$ to 2 (chg at 13 th)

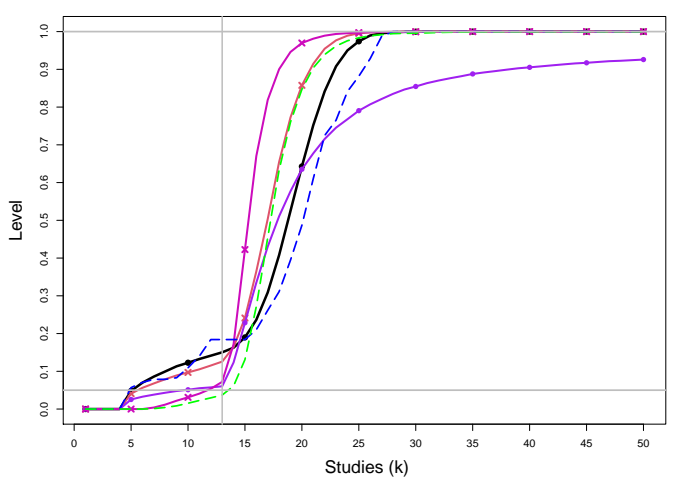

SSW KDB: $\tau^{2}=1 \mathrm{n}=500$ level $=0.05 \mathrm{~h}=4 \delta=1$ to 2 (chg at 13th)

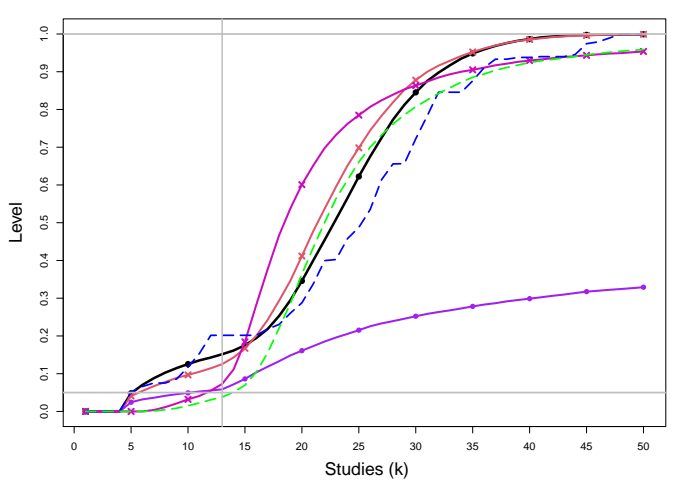

Figure E2. 4: Empirical levels of 1- and 2-stage CMA tests for shift in $\delta$ at .05 nominal level, shift in $\tau^{2}$ at .025 nominal levels and of CUSUM with $h=4$ for equal sample sizes $n_{i C}+n_{i T}=n=500, \tau^{2}=0,0.1,0.25,1$ and a shift from $\delta=1$ to $\delta=2$ at study number 13. Light grey line at .05. 
IV REML: $\tau^{2}=0 \mathrm{n}=1000$ level $=0.05 \mathrm{~h}=4 \delta=1$ to 2 (chg at 13 th)

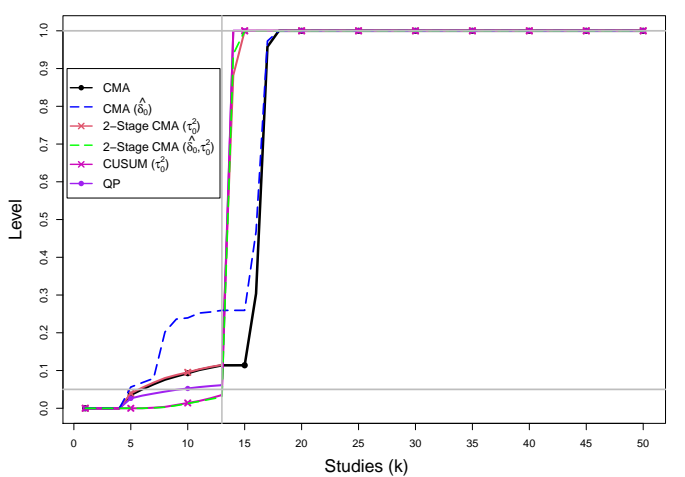

IV REML: $\tau^{2}=0.1 \mathrm{n}=1000$ level $=0.05 \mathrm{~h}=4 \delta=1$ to 2 (chg at 13 th)

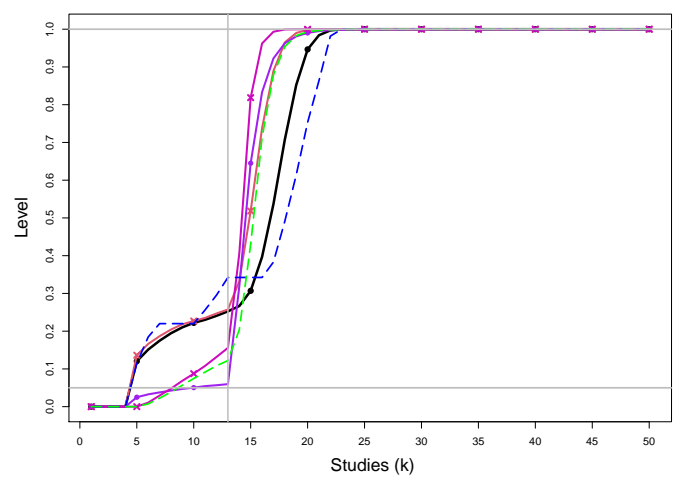

IV REML: $\tau^{2}=0.25 \mathrm{n}=1000$ level $=0.05 \mathrm{~h}=4 \delta=1$ to 2 (chg at 13 th)

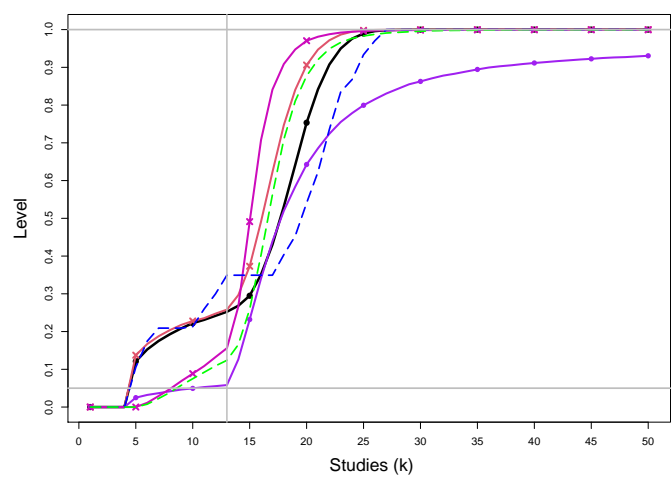

IV REML: $\tau^{2}=1 \mathrm{n}=1000$ level $=0.05 \mathrm{~h}=4 \delta=1$ to 2 (chg at 13 th)

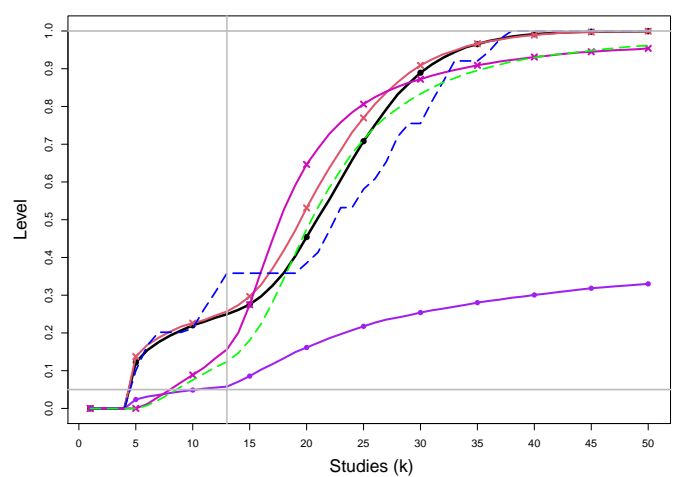

SSW KDB: $\tau^{2}=0 \mathrm{n}=1000$ level $=0.05 \mathrm{~h}=4 \quad \delta=1$ to 2 (chg at 13 th)

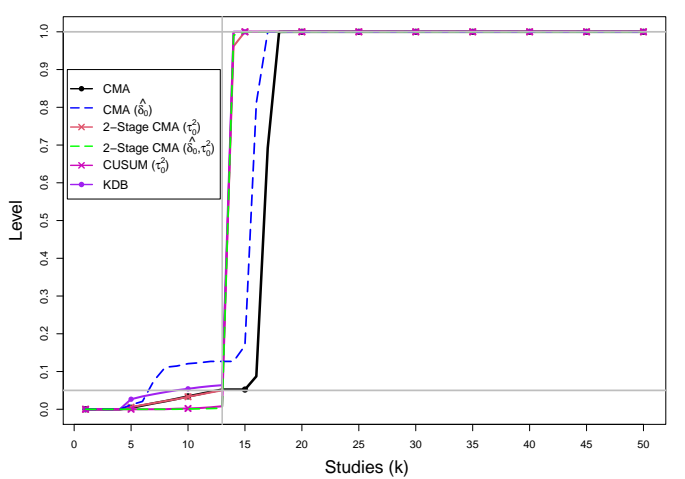

SSW KDB: $\tau^{2}=0.1 \mathrm{n}=1000$ level $=0.05 \mathrm{~h}=4 \delta=1$ to 2 (chg at 13th)

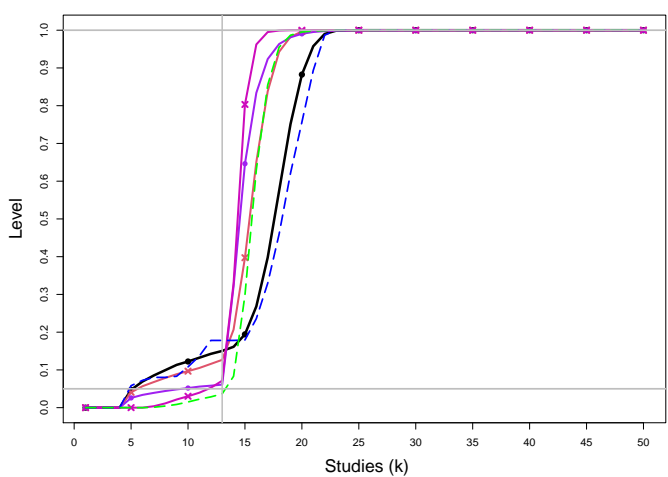

SSW KDB: $\tau^{2}=0.25 \mathrm{n}=1000$ level $=0.05 \mathrm{~h}=4 \delta=1$ to 2 (chg at 13th)

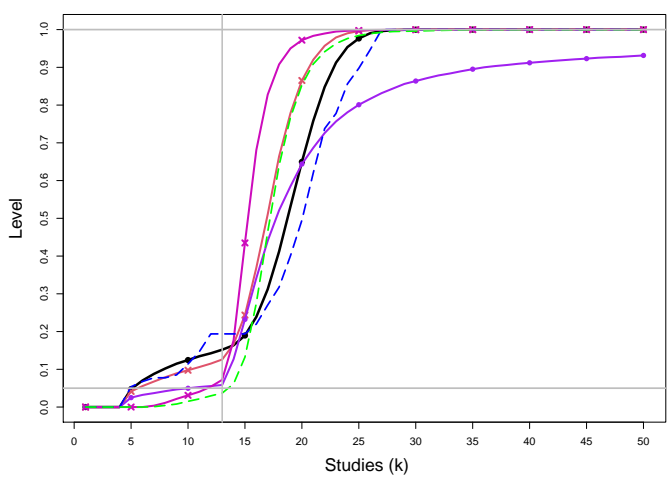

SSW KDB: $\tau^{2}=1 \mathrm{n}=1000$ level $=0.05 \mathrm{~h}=4 \delta=1$ to 2 (chg at 13th)

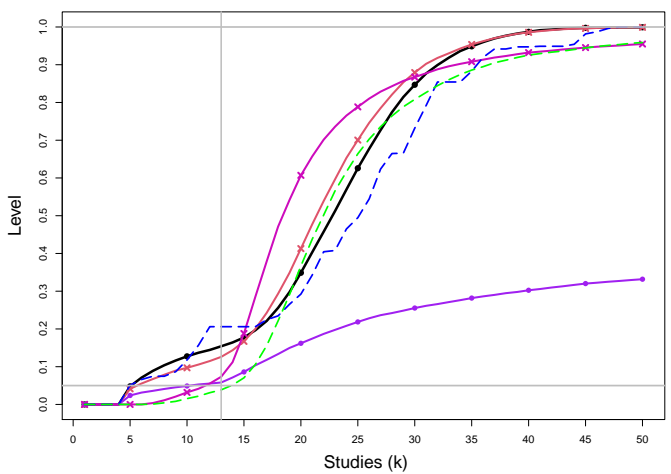

Figure E2. 5: Empirical levels of 1- and 2-stage CMA tests for shift in $\delta$ at .05 nominal level, shift in $\tau^{2}$ at .025 nominal levels and of CUSUM with $h=4$ for equal sample sizes $n_{i C}+n_{i T}=n=1000, \tau^{2}=0,0.1,0.25,1$ and a shift from $\delta=1$ to $\delta=2$ at study number 13. Light grey line at .05. 


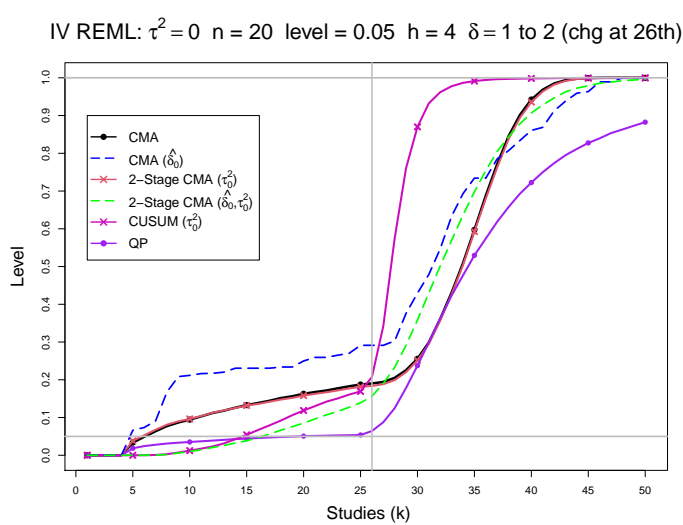

IV REML: $\tau^{2}=0.1 \mathrm{n}=20$ level $=0.05 \mathrm{~h}=4 \delta=1$ to 2 (chg at 26 th)

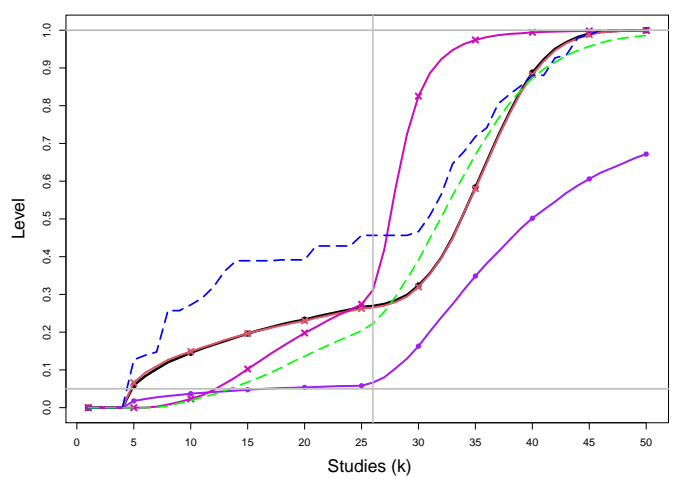

IV REML: $\tau^{2}=0.25 \mathrm{n}=20$ level $=0.05 \mathrm{~h}=4 \delta=1$ to 2 (chg at 26th)

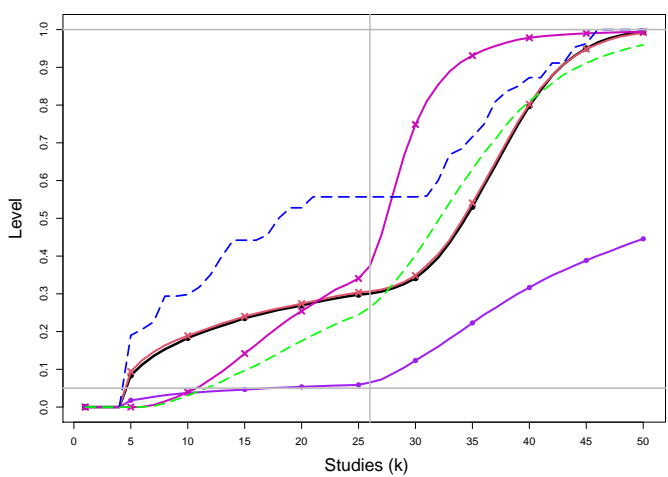

IV REML: $\tau^{2}=1 \mathrm{n}=20$ level $=0.05 \mathrm{~h}=4 \delta=1$ to 2 (chg at 26 th)

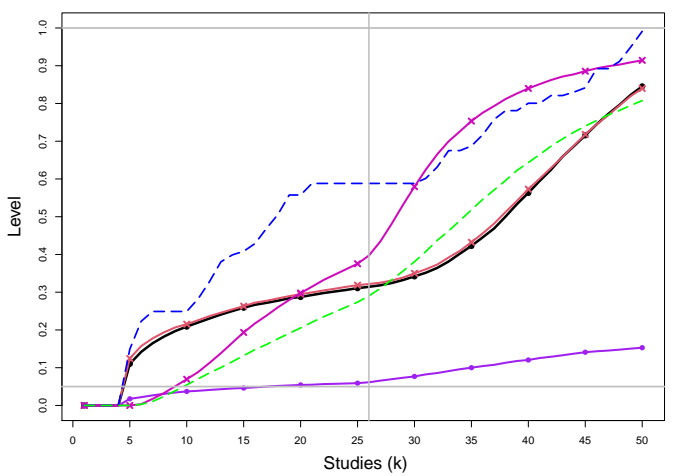

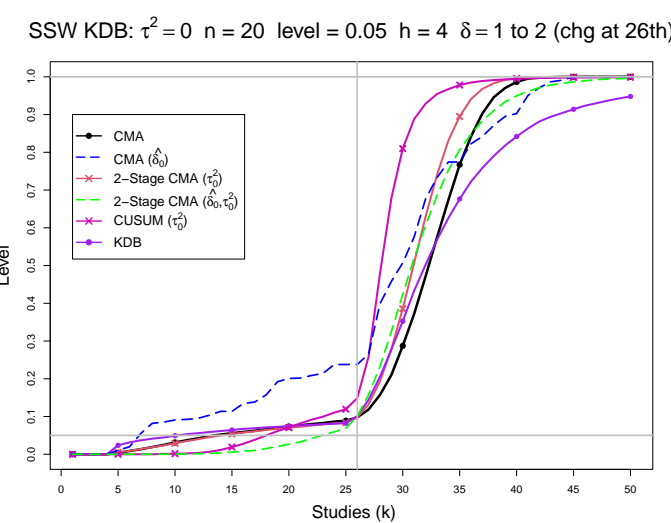

SSW KDB: $\tau^{2}=0.1 \mathrm{n}=20$ level $=0.05 \mathrm{~h}=4 \delta=1$ to 2 (chg at 26th)

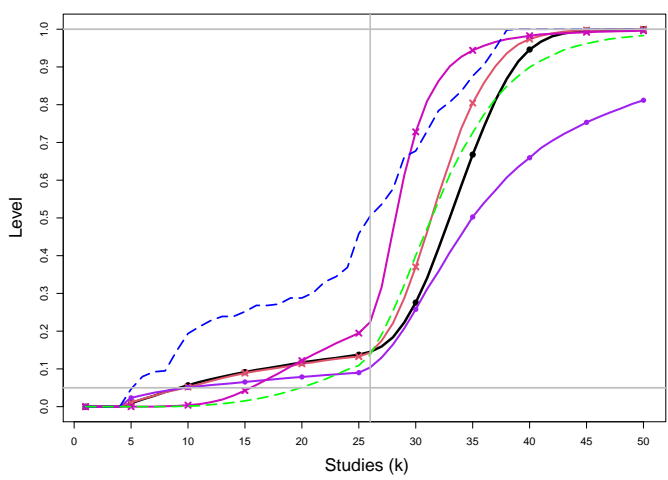

SSW KDB: $\tau^{2}=0.25 \mathrm{n}=20$ level $=0.05 \mathrm{~h}=4 \quad \delta=1$ to 2 (chg at 26th)

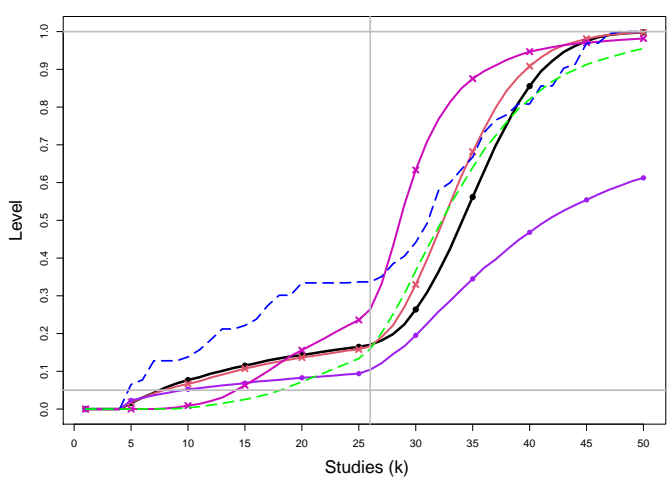

SSW KDB: $\tau^{2}=1 \mathrm{n}=20$ level $=0.05 \mathrm{~h}=4 \delta=1$ to 2 (chg at 26th)

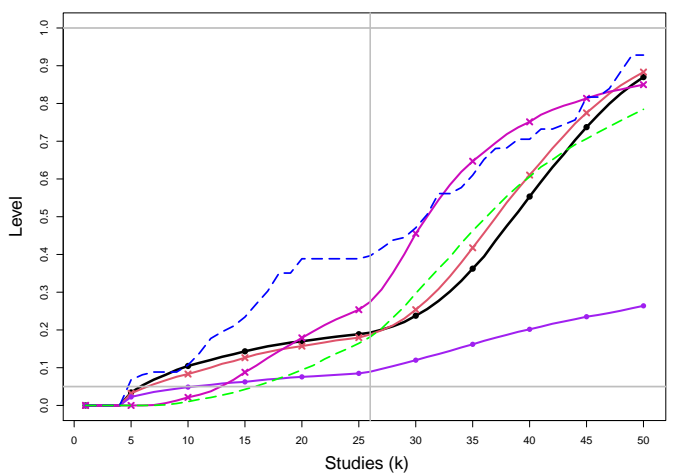

Figure E2. 6: Empirical levels of 1- and 2-stage CMA tests for shift in $\delta$ at .05 nominal level, shift in $\tau^{2}$ at .025 nominal levels and of CUSUM with $h=4$ for equal sample sizes $n_{i C}+n_{i T}=n=20, \tau^{2}=0,0.1,0.25,1$ and a shift from $\delta=1$ to $\delta=2$ at study number 26. Light grey line at .05. 


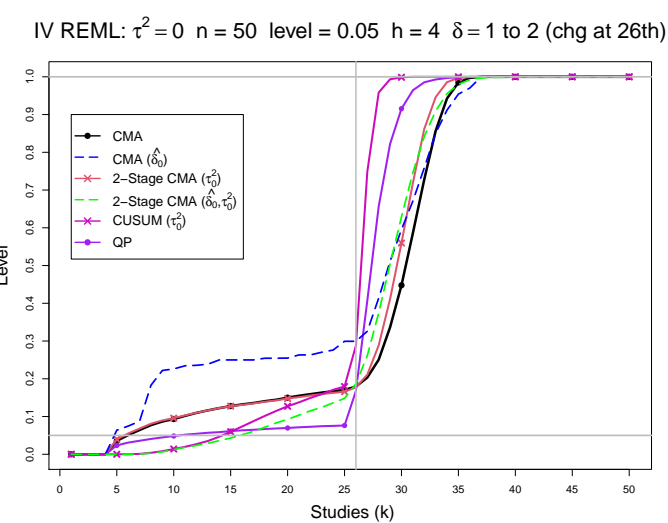

IV REML: $\tau^{2}=0.1 \mathrm{n}=50$ level $=0.05 \mathrm{~h}=4 \delta=1$ to 2 (chg at 26 th)

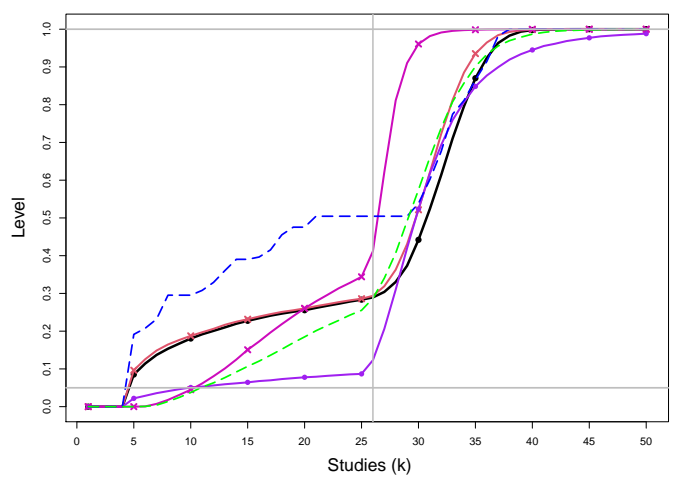

IV REML: $\tau^{2}=0.25 \mathrm{n}=50$ level $=0.05 \mathrm{~h}=4 \delta=1$ to 2 (chg at 26th)

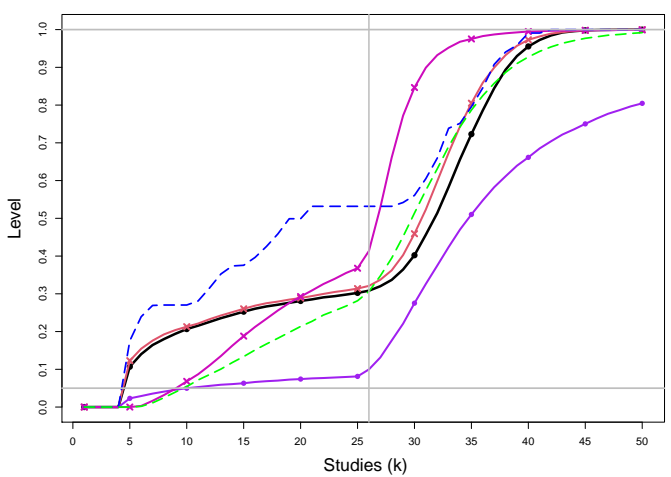

IV REML: $\tau^{2}=1 \mathrm{n}=50$ level $=0.05 \mathrm{~h}=4 \delta=1$ to 2 (chg at 26 th)

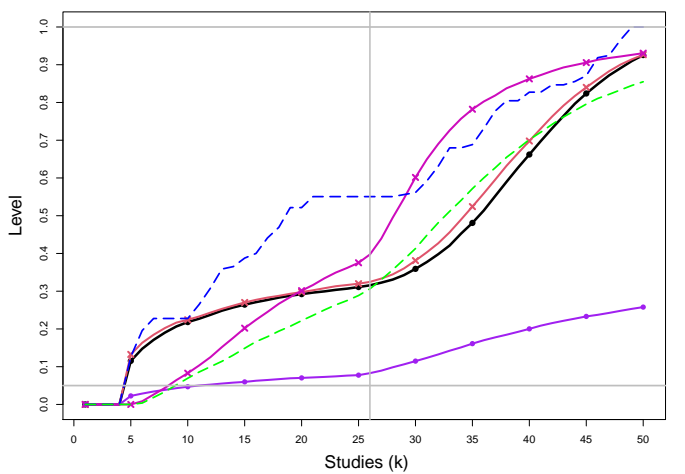

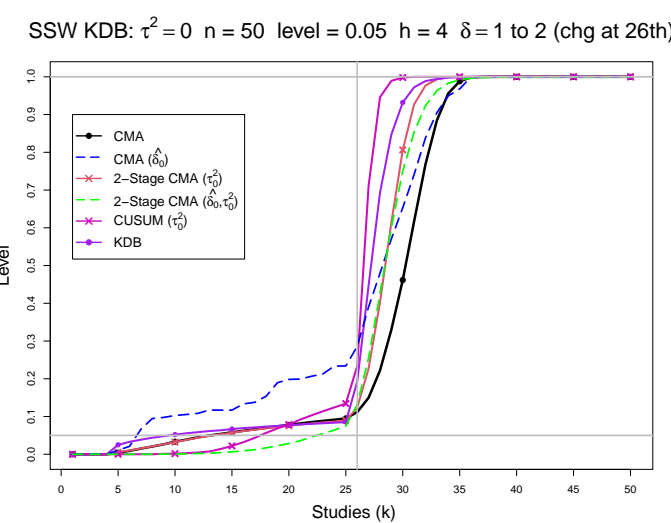

SSW KDB: $\tau^{2}=0.1 \mathrm{n}=50$ level $=0.05 \mathrm{~h}=4 \delta=1$ to 2 (chg at 26th)

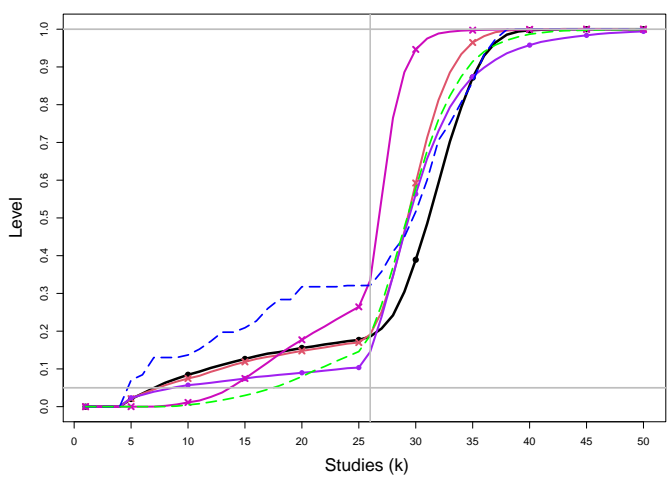

SSW KDB: $\tau^{2}=0.25 \mathrm{n}=50$ level $=0.05 \mathrm{~h}=4 \quad \delta=1$ to 2 (chg at 26th)

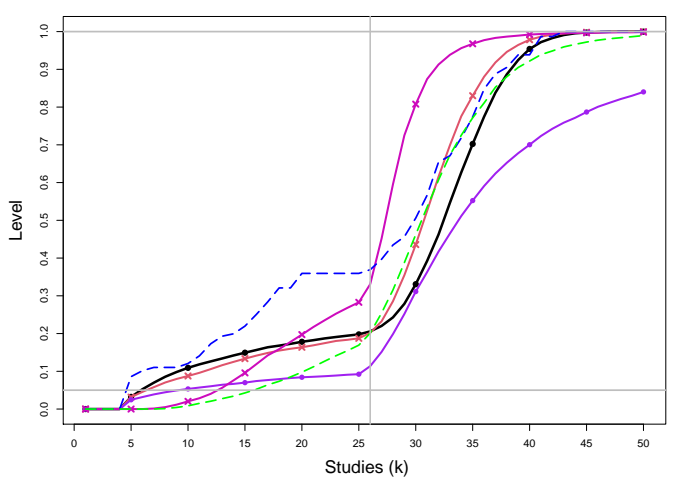

SSW KDB: $\tau^{2}=1 \mathrm{n}=50$ level $=0.05 \mathrm{~h}=4 \delta=1$ to 2 (chg at 26th)

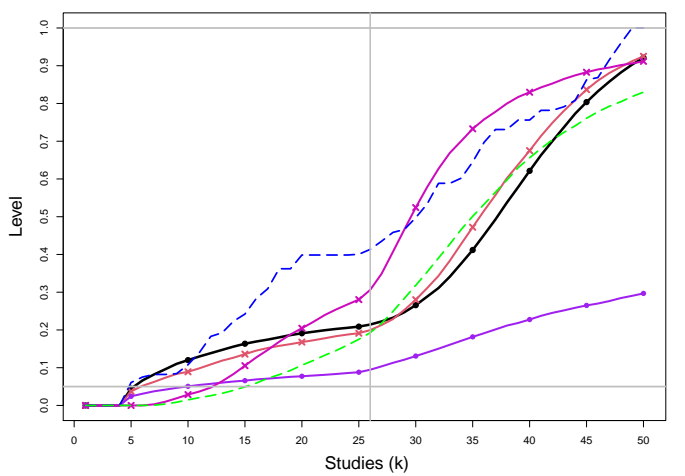

Figure E2. 7: Empirical levels of 1- and 2-stage CMA tests for shift in $\delta$ at .05 nominal level, shift in $\tau^{2}$ at .025 nominal levels and of CUSUM with $h=4$ for equal sample sizes $n_{i C}+n_{i T}=n=50, \tau^{2}=0,0.1,0.25,1$ and a shift from $\delta=1$ to $\delta=2$ at study number 26. Light grey line at .05. 


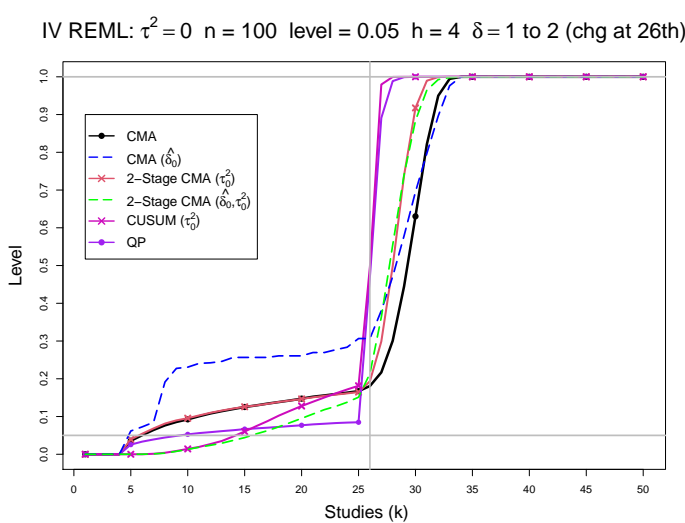

IV REML: $\tau^{2}=0.1 \mathrm{n}=100$ level $=0.05 \mathrm{~h}=4 \delta=1$ to 2 (chg at 26th)

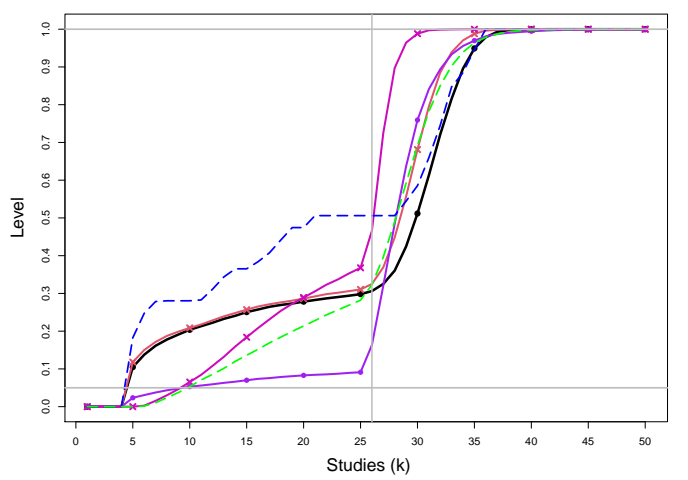

IV REML: $\tau^{2}=0.25 \mathrm{n}=100$ level $=0.05 \mathrm{~h}=4 \delta=1$ to 2 (chg at 26 th)

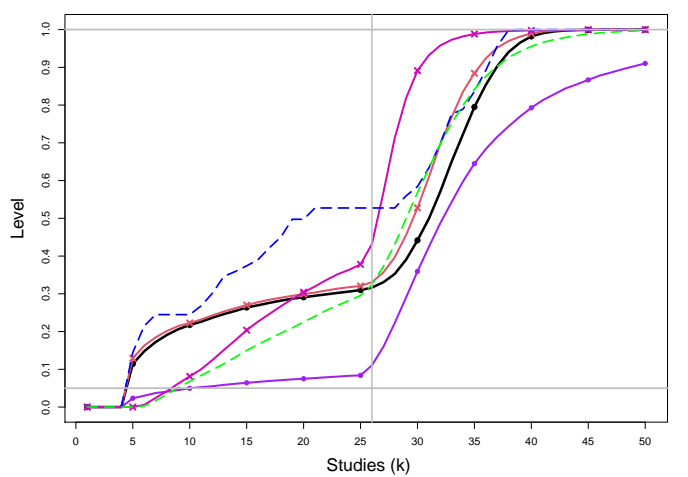

IV REML: $\tau^{2}=1 \mathrm{n}=100$ level $=0.05 \mathrm{~h}=4 \delta=1$ to 2 (chg at 26 th)

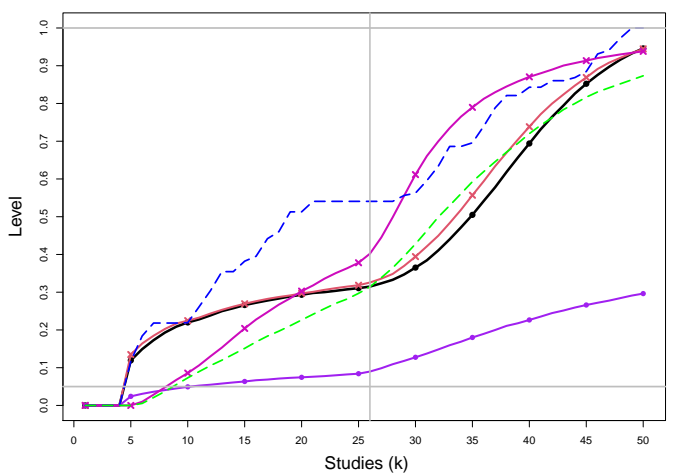

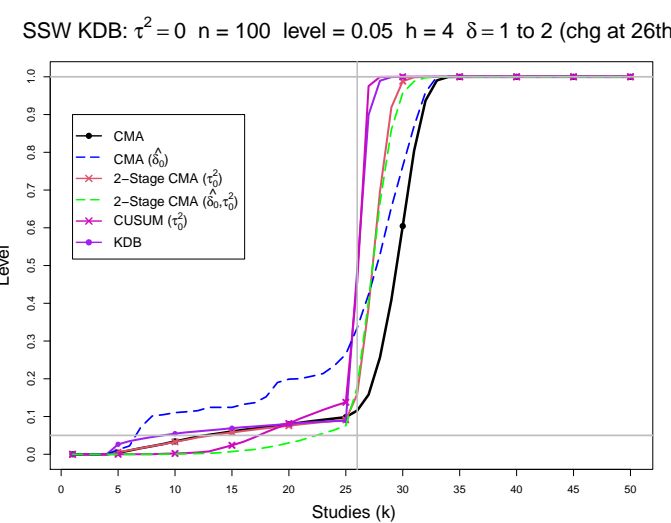

SSW KDB: $\tau^{2}=0.1 \mathrm{n}=100$ level $=0.05 \mathrm{~h}=4 \delta=1$ to 2 (chg at 26th)

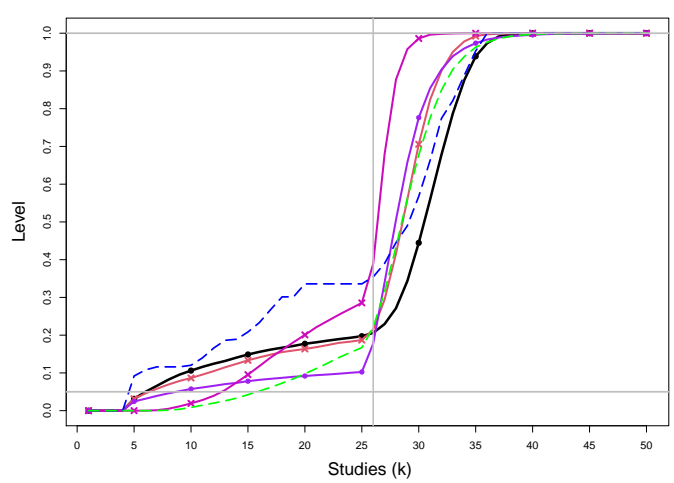

SSW KDB: $\tau^{2}=0.25 \mathrm{n}=100$ level $=0.05 \mathrm{~h}=4 \delta=1$ to 2 (chg at 26th)

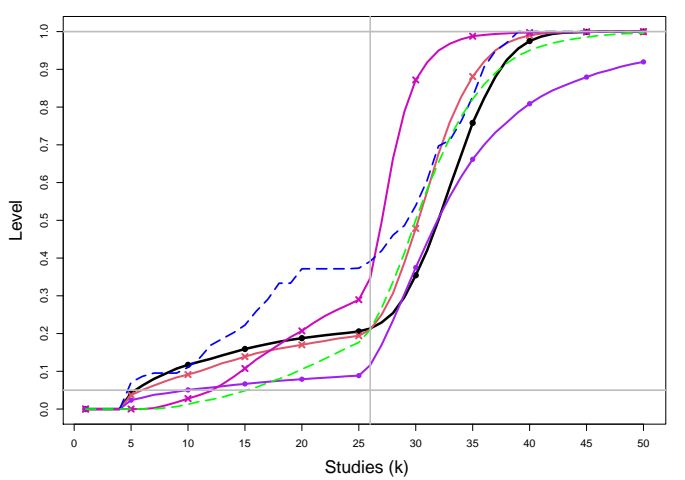

SSW KDB: $\tau^{2}=1 \mathrm{n}=100$ level $=0.05 \mathrm{~h}=4 \delta=1$ to 2 (chg at 26th)

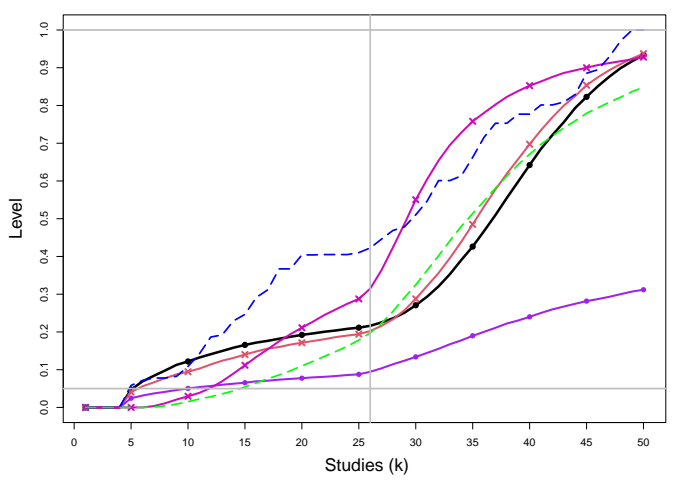

Figure E2. 8: Empirical levels of 1- and 2-stage CMA tests for shift in $\delta$ at .05 nominal level, shift in $\tau^{2}$ at .025 nominal levels and of CUSUM with $h=4$ for equal sample sizes $n_{i C}+n_{i T}=n=100, \tau^{2}=0,0.1,0.25,1$ and a shift from $\delta=1$ to $\delta=2$ at study number 26. Light grey line at .05. 


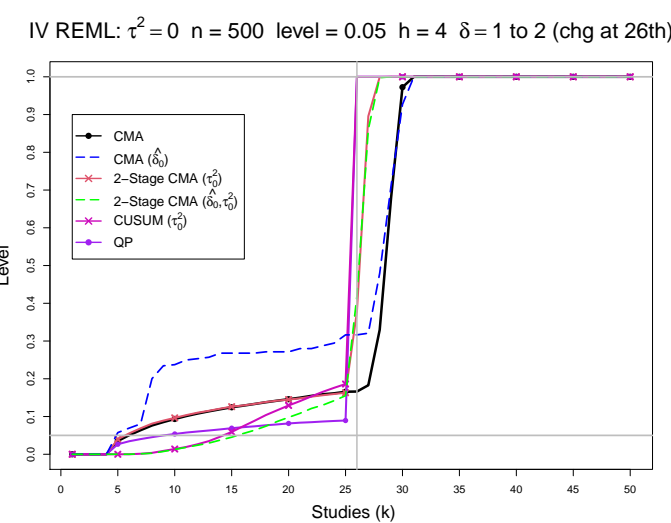

IV REML: $\tau^{2}=0.1 \mathrm{n}=500$ level $=0.05 \mathrm{~h}=4 \delta=1$ to 2 (chg at 26th)

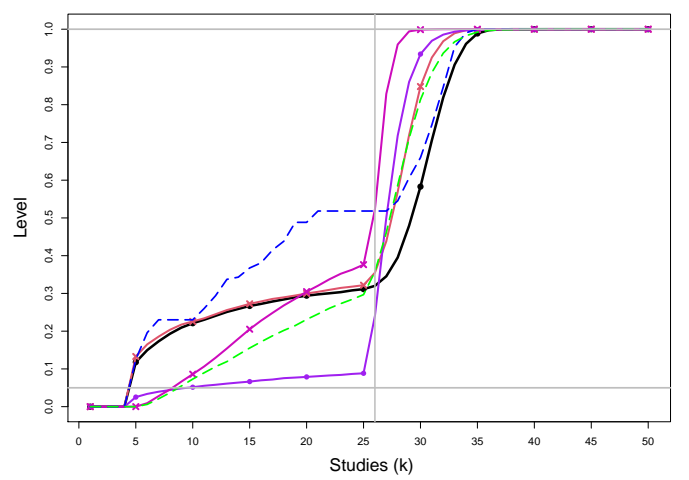

IV REML: $\tau^{2}=0.25 \mathrm{n}=500$ level $=0.05 \mathrm{~h}=4 \delta=1$ to 2 (chg at 26 th)

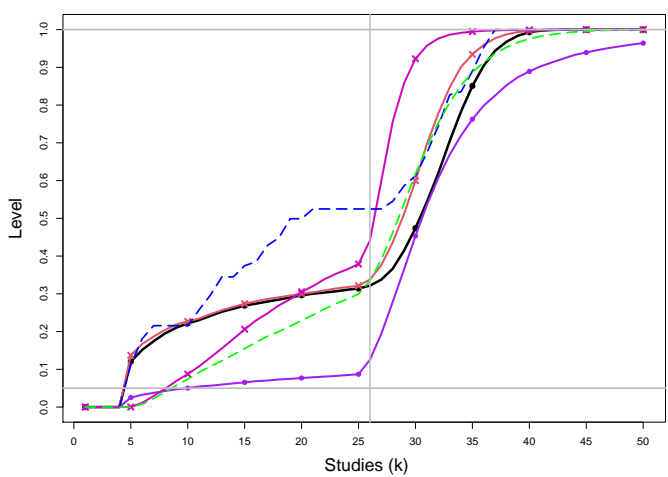

IV REML: $\tau^{2}=1 \mathrm{n}=500$ level $=0.05 \mathrm{~h}=4 \delta=1$ to 2 (chg at 26 th)

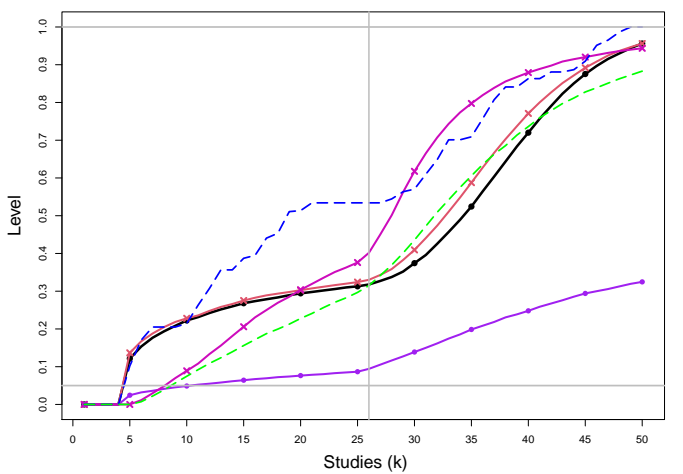

SSW KDB: $\tau^{2}=0 \mathrm{n}=500$ level $=0.05 \mathrm{~h}=4 \delta=1$ to 2 (chg at 26th)

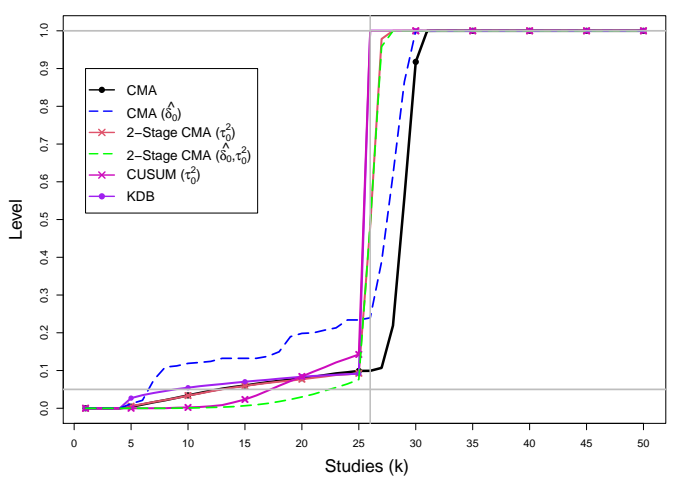

SSW KDB: $\tau^{2}=0.1 \mathrm{n}=500$ level $=0.05 \mathrm{~h}=4 \delta=1$ to 2 (chg at 26th)

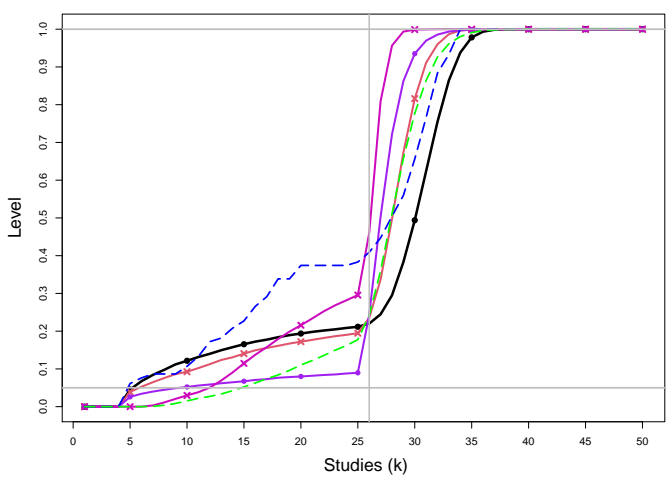

SSW KDB: $\tau^{2}=0.25 \mathrm{n}=500$ level $=0.05 \mathrm{~h}=4 \delta=1$ to 2 (chg at 26th)

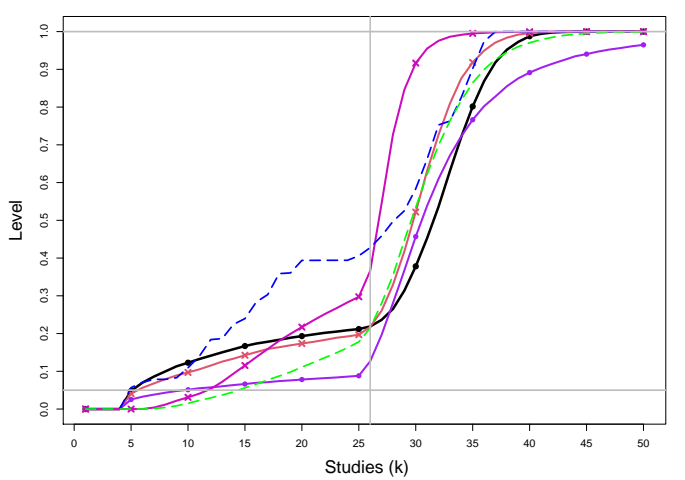

SSW KDB: $\tau^{2}=1 \mathrm{n}=500$ level $=0.05 \mathrm{~h}=4 \delta=1$ to 2 (chg at 26th)

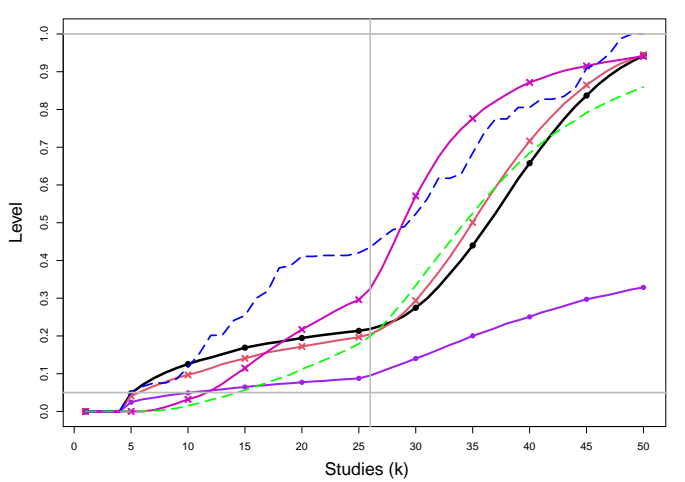

Figure E2. 9: Empirical levels of 1- and 2-stage CMA tests for shift in $\delta$ at .05 nominal level, shift in $\tau^{2}$ at .025 nominal levels and of CUSUM with $h=4$ for equal sample sizes $n_{i C}+n_{i T}=n=500, \tau^{2}=0,0.1,0.25,1$ and a shift from $\delta=1$ to $\delta=2$ at study number 26. Light grey line at .05. 
IV REML: $\tau^{2}=0 \mathrm{n}=1000$ level $=0.05 \mathrm{~h}=4 \delta=1$ to 2 (chg at 26 th)

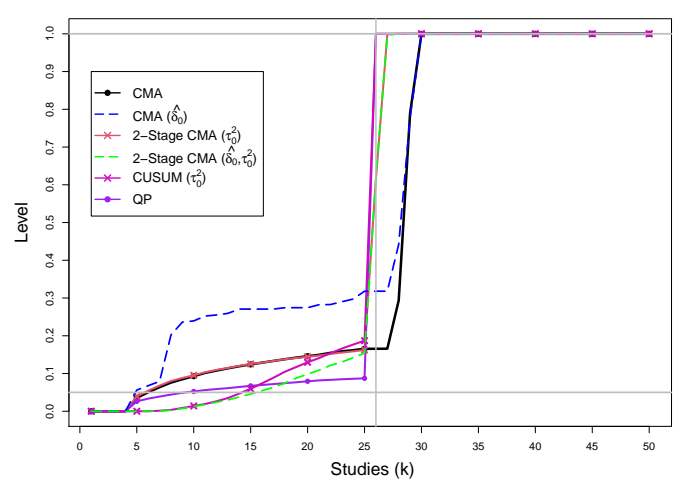

IV REML: $\tau^{2}=0.1 \mathrm{n}=1000$ level $=0.05 \mathrm{~h}=4 \quad \delta=1$ to 2 (chg at 26 th)

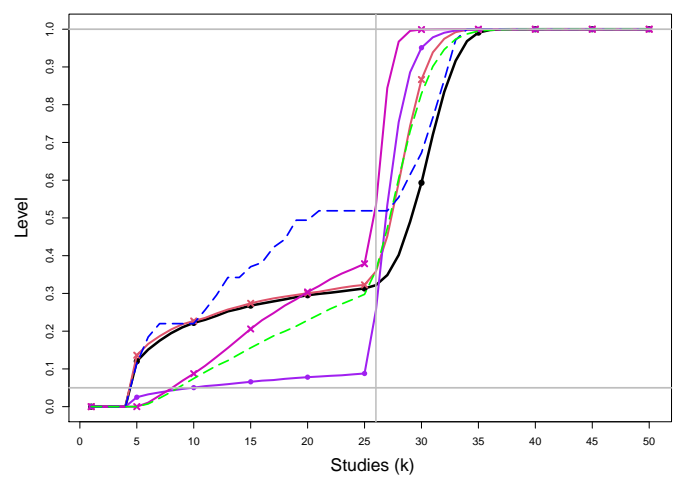

IV REML: $\tau^{2}=0.25 \mathrm{n}=1000$ level $=0.05 \mathrm{~h}=4 \delta=1$ to 2 (chg at 26th)

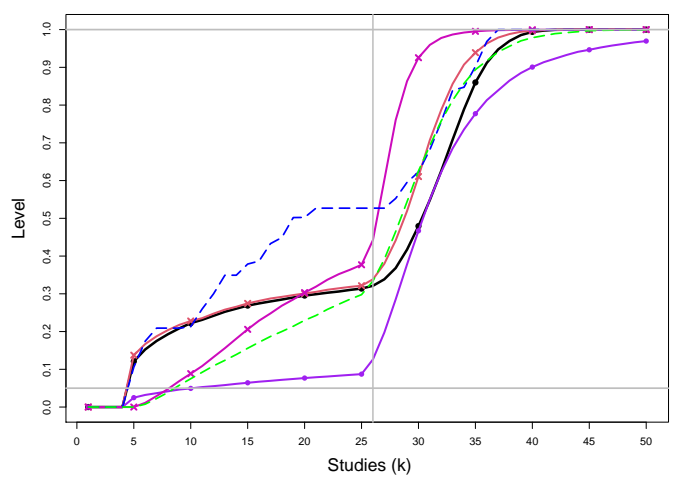

IV REML: $\tau^{2}=1 \mathrm{n}=1000$ level $=0.05 \mathrm{~h}=4 \quad \delta=1$ to 2 (chg at 26 th)

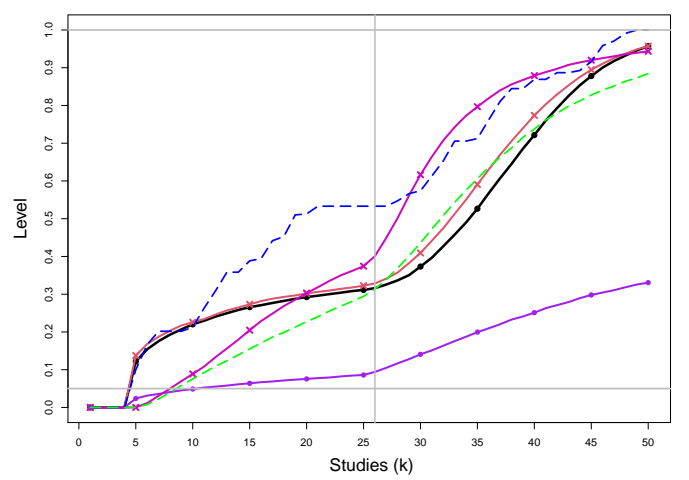

SSW KDB: $\tau^{2}=0 \mathrm{n}=1000$ level $=0.05 \mathrm{~h}=4 \quad \delta=1$ to 2 (chg at 26th)

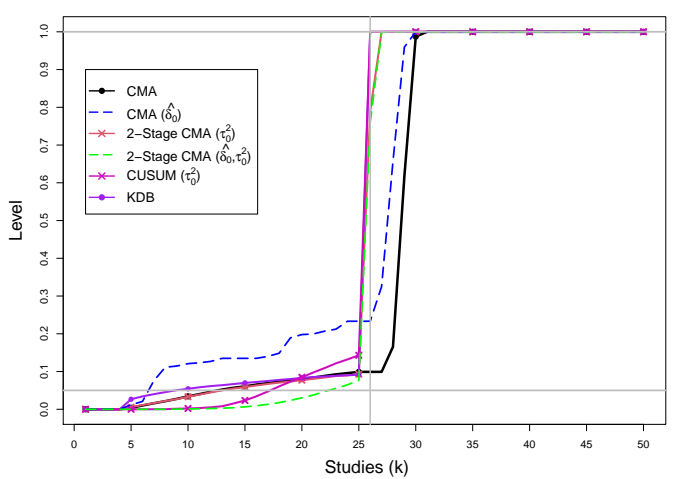

SSW KDB: $\tau^{2}=0.1 \mathrm{n}=1000$ level $=0.05 \mathrm{~h}=4 \delta=1$ to 2 (chg at 26 th)

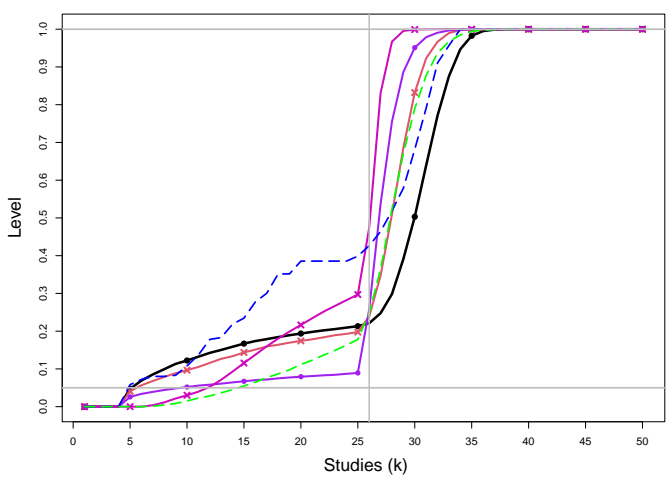

SSW KDB: $\tau^{2}=0.25 \mathrm{n}=1000$ level $=0.05 \mathrm{~h}=4 \delta=1$ to 2 (chg at 26th)

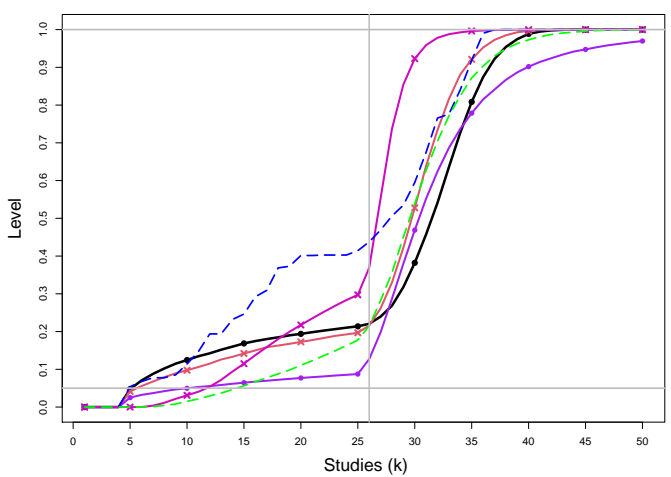

SSW KDB: $\tau^{2}=1 \mathrm{n}=1000$ level $=0.05 \mathrm{~h}=4 \delta=1$ to 2 (chg at 26th)

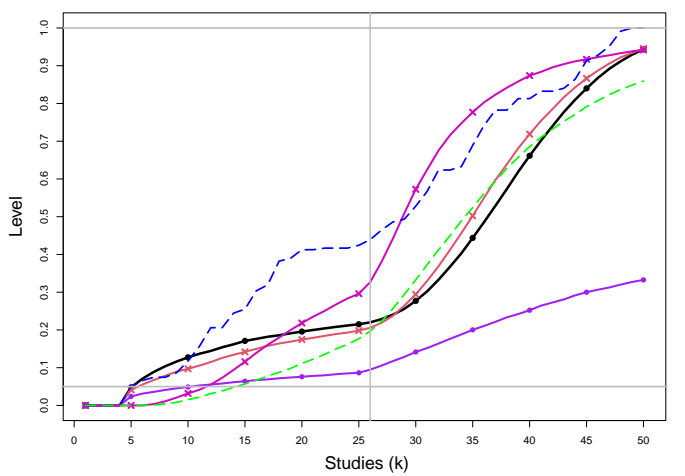

Figure E2. 10: Empirical levels of 1- and 2-stage CMA tests for shift in $\delta$ at .05 nominal level, shift in $\tau^{2}$ at .025 nominal levels and of CUSUM with $h=4$ for equal sample sizes $n_{i C}+n_{i T}=n=1000, \tau^{2}=0,0.1,0.25,1$ and a shift from $\delta=1$ to $\delta=2$ at study number 26. Light grey line at .05. 


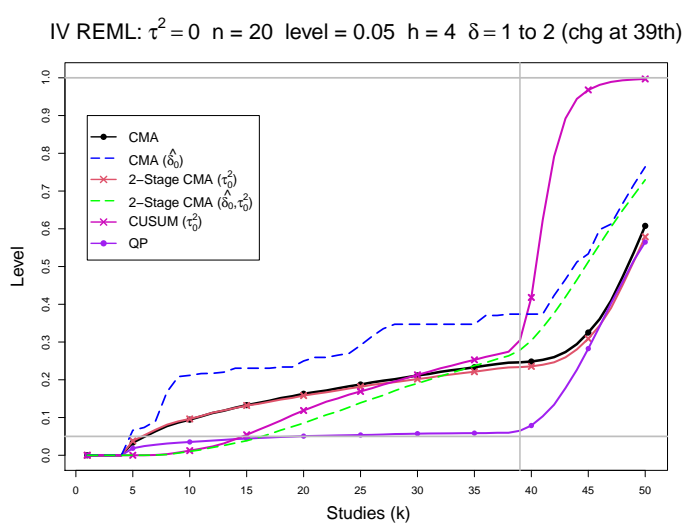

IV REML: $\tau^{2}=0.1 \mathrm{n}=20$ level $=0.05 \mathrm{~h}=4 \delta=1$ to 2 (chg at 39 th)

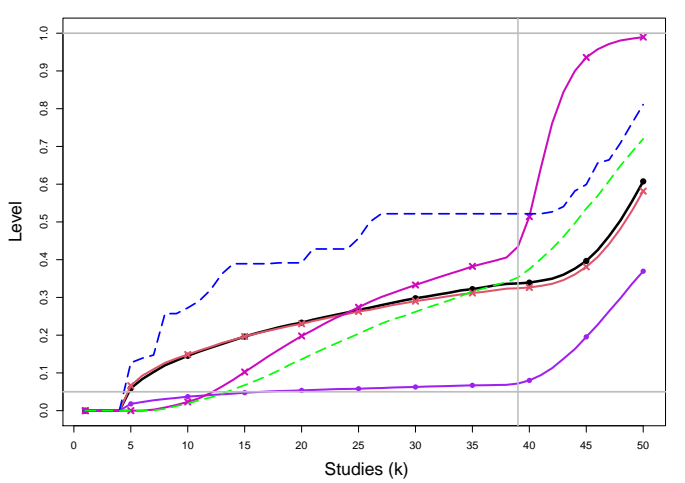

IV REML: $\tau^{2}=0.25 \mathrm{n}=20$ level $=0.05 \mathrm{~h}=4 \delta=1$ to 2 (chg at 39 th)

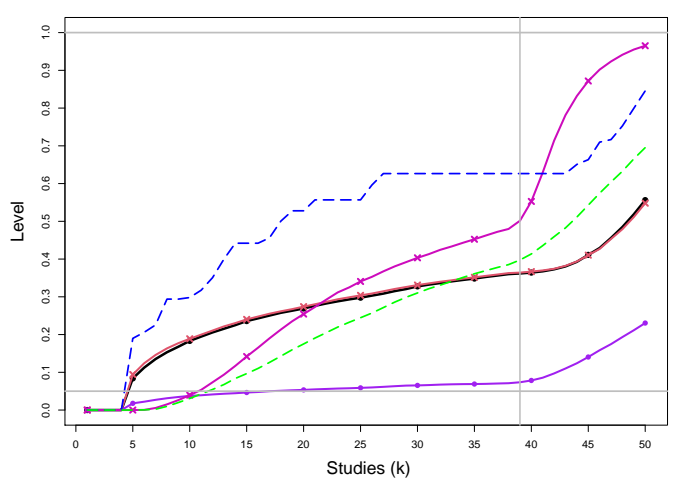

IV REML: $\tau^{2}=1 \mathrm{n}=20$ level $=0.05 \mathrm{~h}=4 \delta=1$ to 2 (chg at 39 th)

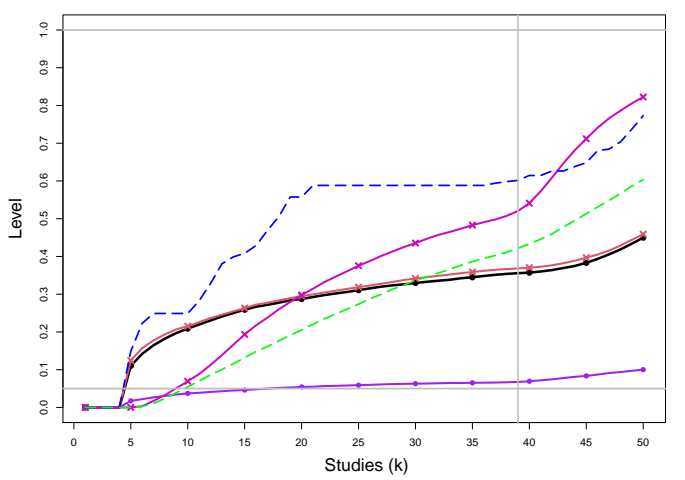

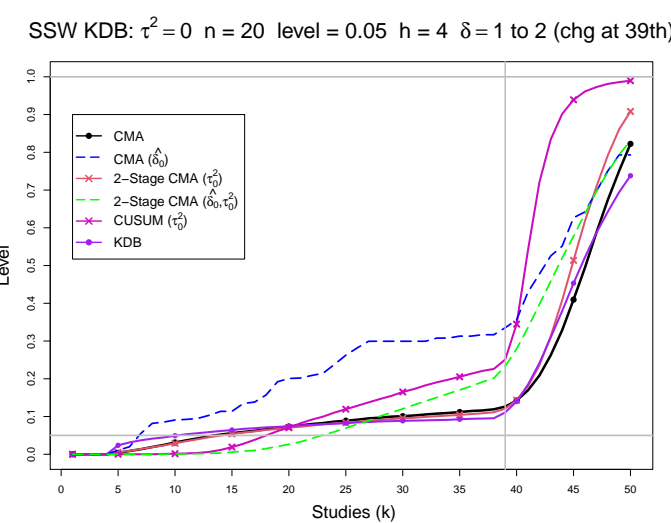

SSW KDB: $\tau^{2}=0.1 \mathrm{n}=20$ level $=0.05 \mathrm{~h}=4 \delta=1$ to 2 (chg at 39 th)

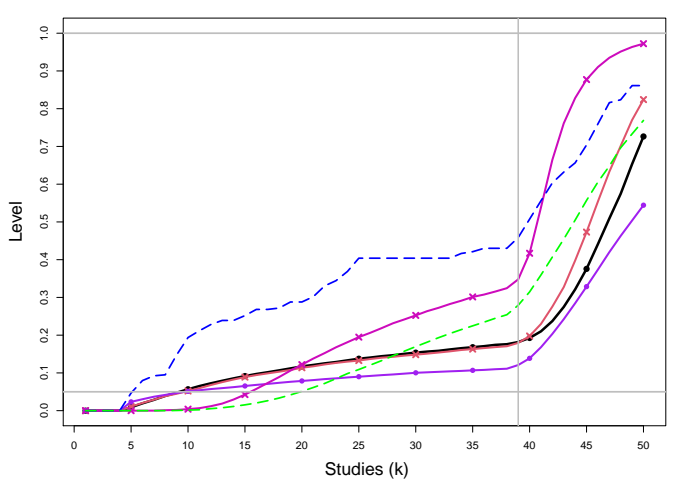

SSW KDB: $\tau^{2}=0.25 \mathrm{n}=20$ level $=0.05 \mathrm{~h}=4 \delta=1$ to 2 (chg at 39th)

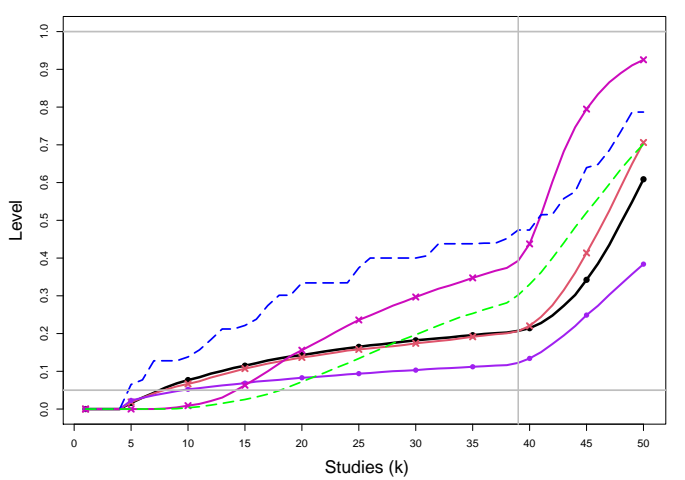

SSW KDB: $\tau^{2}=1 \mathrm{n}=20$ level $=0.05 \mathrm{~h}=4 \delta=1$ to 2 (chg at 39th)

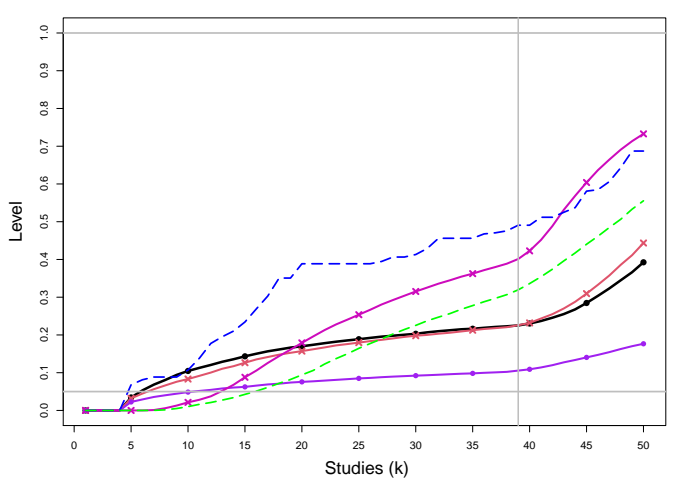

Figure E2. 11: Empirical levels of 1- and 2-stage CMA tests for shift in $\delta$ at .05 nominal level, shift in $\tau^{2}$ at .025 nominal levels and of CUSUM with $h=4$ for equal sample sizes $n_{i C}+n_{i T}=n=20, \tau^{2}=0,0.1,0.25,1$ and a shift from $\delta=1$ to $\delta=2$ at study number 39. Light grey line at .05. 


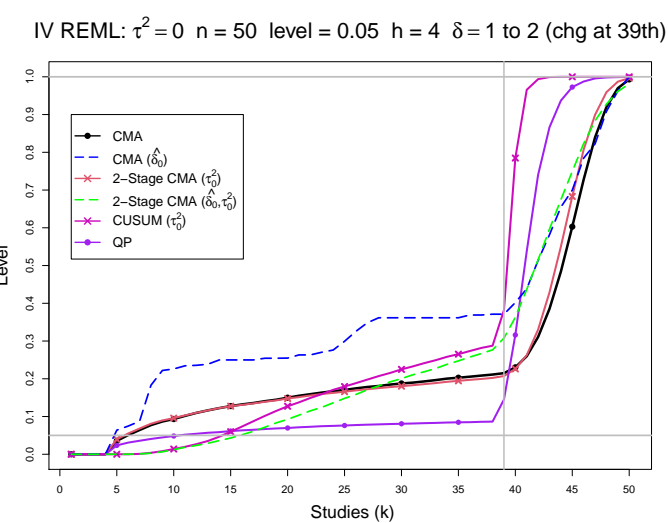

IV REML: $\tau^{2}=0.1 \mathrm{n}=50$ level $=0.05 \mathrm{~h}=4 \delta=1$ to 2 (chg at 39 th)

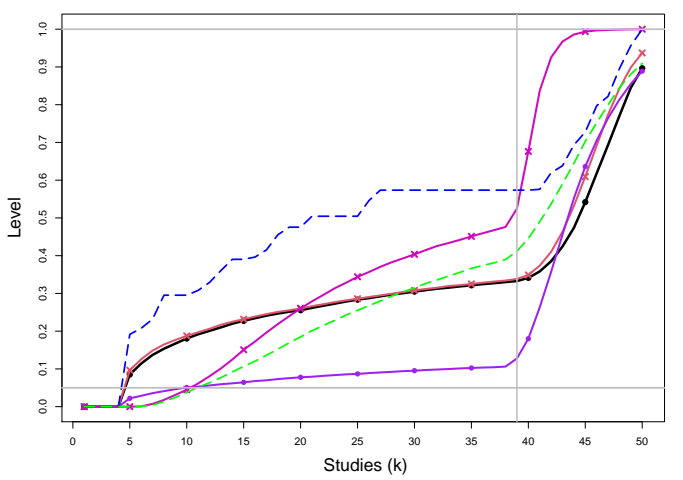

IV REML: $\tau^{2}=0.25 \mathrm{n}=50$ level $=0.05 \mathrm{~h}=4 \delta=1$ to 2 (chg at 39th)

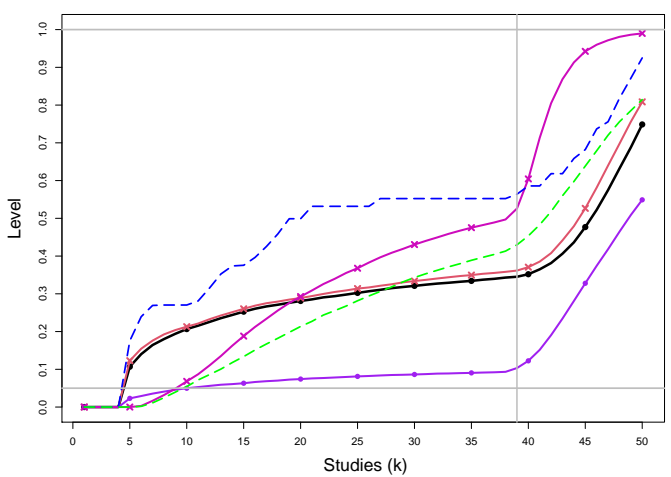

IV REML: $\tau^{2}=1 \mathrm{n}=50$ level $=0.05 \mathrm{~h}=4 \delta=1$ to 2 (chg at 39 th)

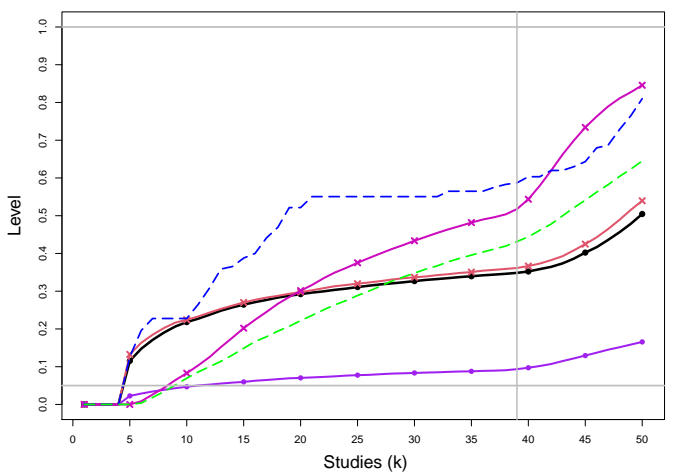

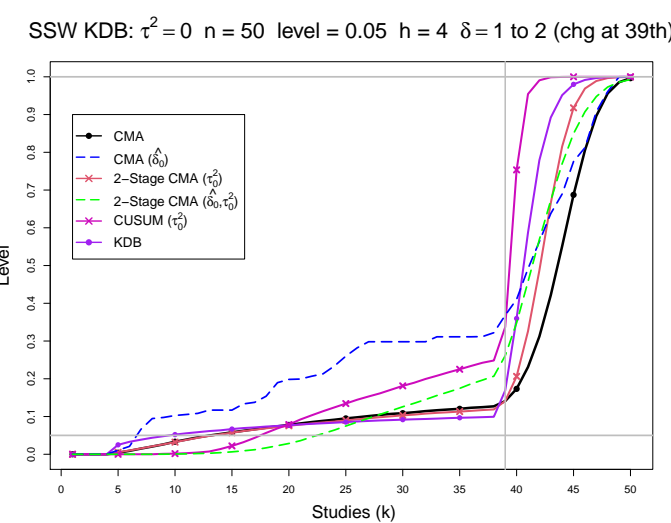

SSW KDB: $\tau^{2}=0.1 \mathrm{n}=50$ level $=0.05 \mathrm{~h}=4 \delta=1$ to 2 (chg at 39th)

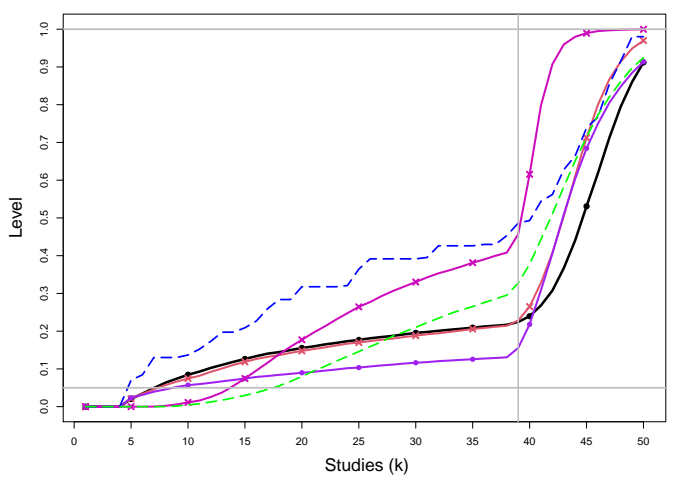

SSW KDB: $\tau^{2}=0.25 \mathrm{n}=50$ level $=0.05 \mathrm{~h}=4 \quad \delta=1$ to 2 (chg at 39 th)

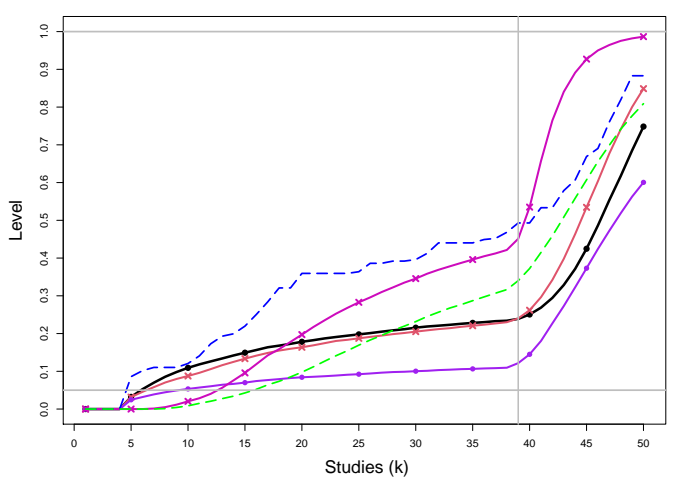

SSW KDB: $\tau^{2}=1 \mathrm{n}=50$ level $=0.05 \mathrm{~h}=4 \delta=1$ to 2 (chg at 39th)

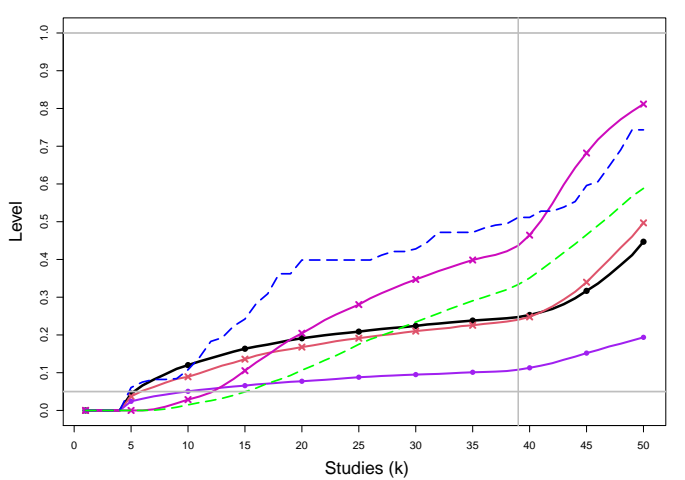

Figure E2. 12: Empirical levels of 1- and 2-stage CMA tests for shift in $\delta$ at .05 nominal level, shift in $\tau^{2}$ at .025 nominal levels and of CUSUM with $h=4$ for equal sample sizes $n_{i C}+n_{i T}=n=50, \tau^{2}=0,0.1,0.25,1$ and a shift from $\delta=1$ to $\delta=2$ at study number 39. Light grey line at .05. 


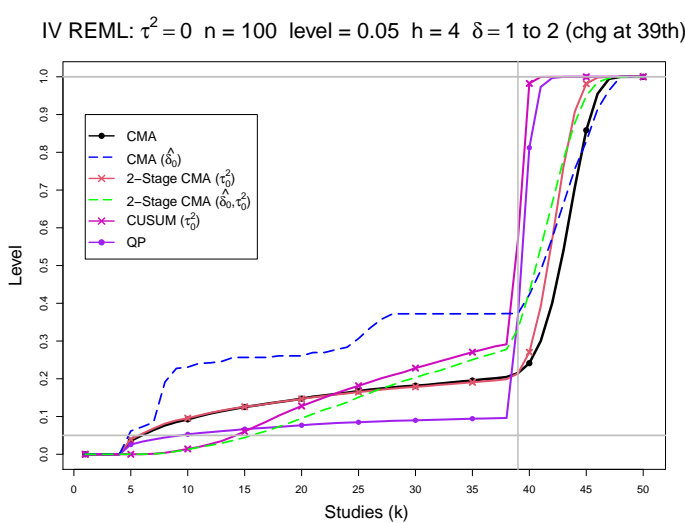

IV REML: $\tau^{2}=0.1 \mathrm{n}=100$ level $=0.05 \mathrm{~h}=4 \delta=1$ to 2 (chg at 39 th)

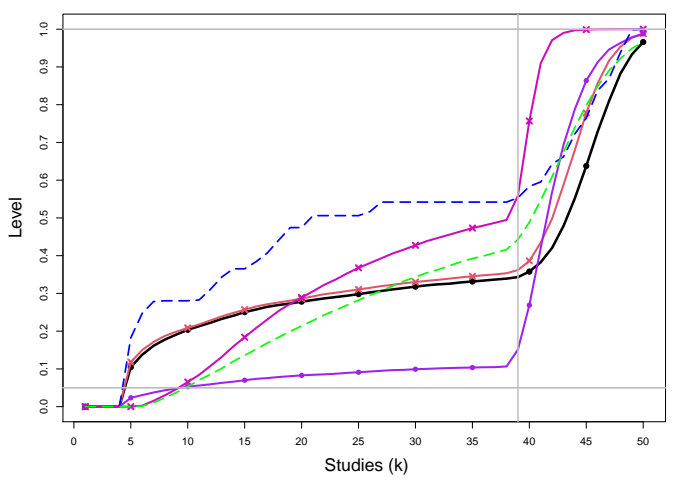

IV REML: $\tau^{2}=0.25 \mathrm{n}=100$ level $=0.05 \mathrm{~h}=4 \delta=1$ to 2 (chg at 39 th)

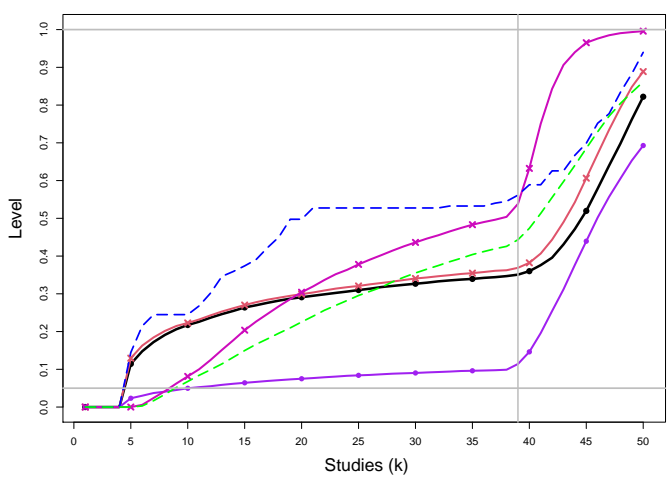

IV REML: $\tau^{2}=1 \mathrm{n}=100$ level $=0.05 \mathrm{~h}=4 \delta=1$ to 2 (chg at 39 th)

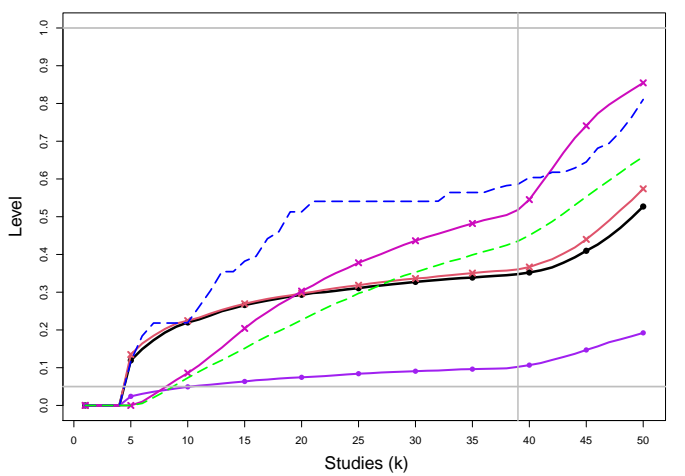

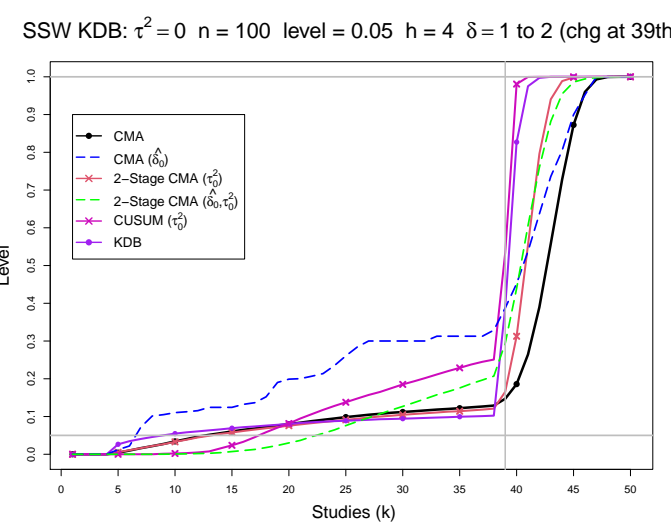

SSW KDB: $\tau^{2}=0.1 \mathrm{n}=100$ level $=0.05 \mathrm{~h}=4 \delta=1$ to 2 (chg at 39 th)

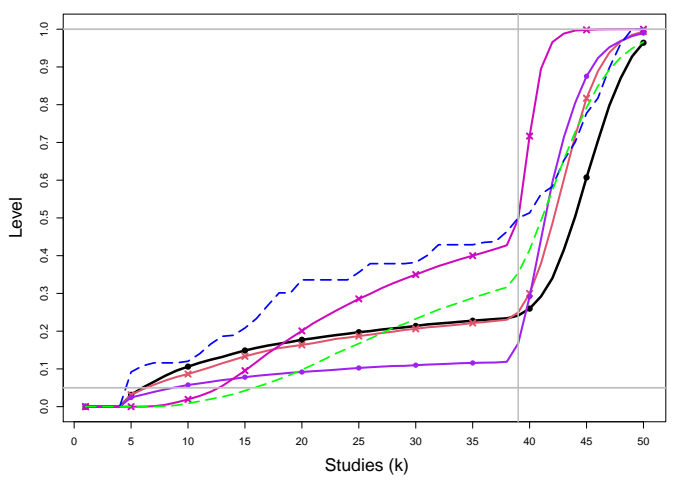

SSW KDB: $\tau^{2}=0.25 \mathrm{n}=100$ level $=0.05 \mathrm{~h}=4 \delta=1$ to 2 (chg at 39 th)

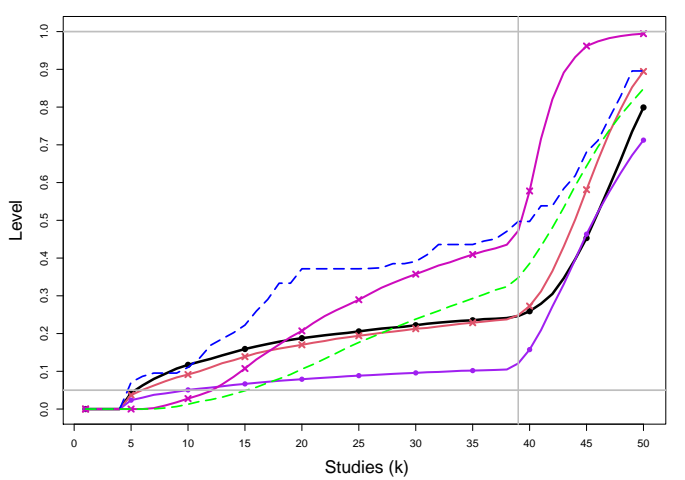

SSW KDB: $\tau^{2}=1 \mathrm{n}=100$ level $=0.05 \mathrm{~h}=4 \delta=1$ to 2 (chg at 39 th)

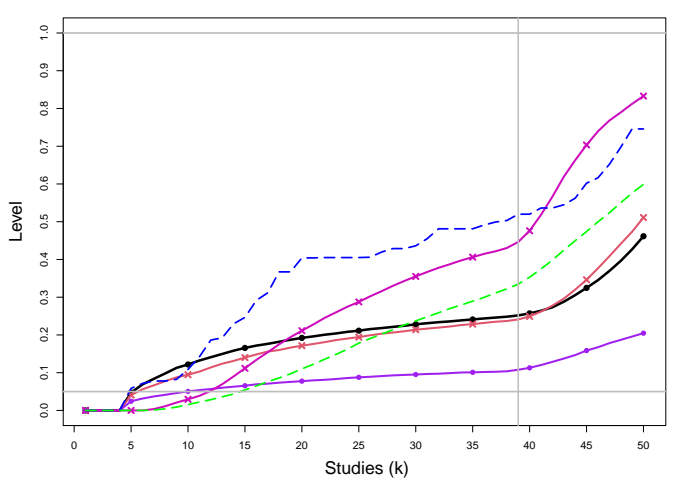

Figure E2. 13: Empirical levels of 1- and 2-stage CMA tests for shift in $\delta$ at .05 nominal level, shift in $\tau^{2}$ at .025 nominal levels and of CUSUM with $h=4$ for equal sample sizes $n_{i C}+n_{i T}=n=100, \tau^{2}=0,0.1,0.25,1$ and a shift from $\delta=1$ to $\delta=2$ at study number 39. Light grey line at .05. 


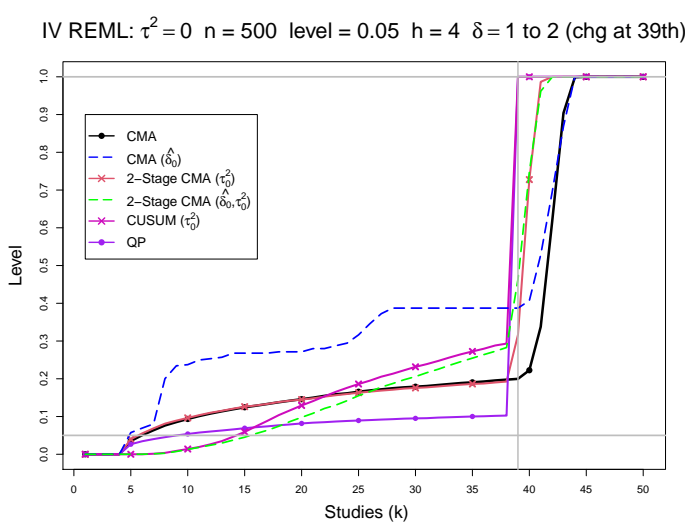

IV REML: $\tau^{2}=0.1 \mathrm{n}=500$ level $=0.05 \mathrm{~h}=4 \delta=1$ to 2 (chg at 39 th)

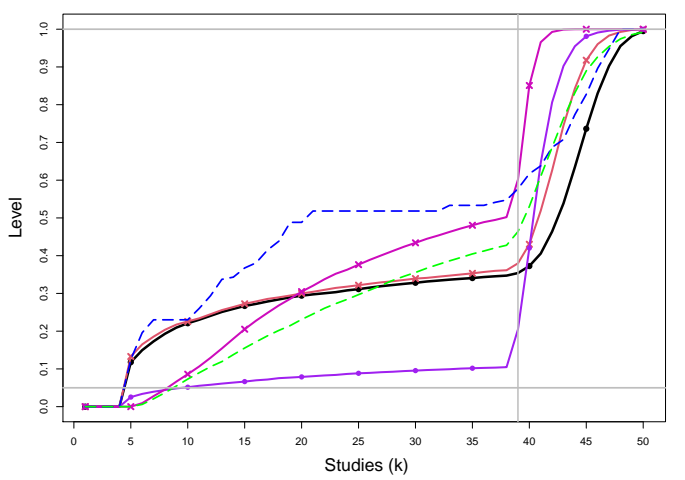

IV REML: $\tau^{2}=0.25 \mathrm{n}=500$ level $=0.05 \mathrm{~h}=4 \delta=1$ to 2 (chg at 39 th)

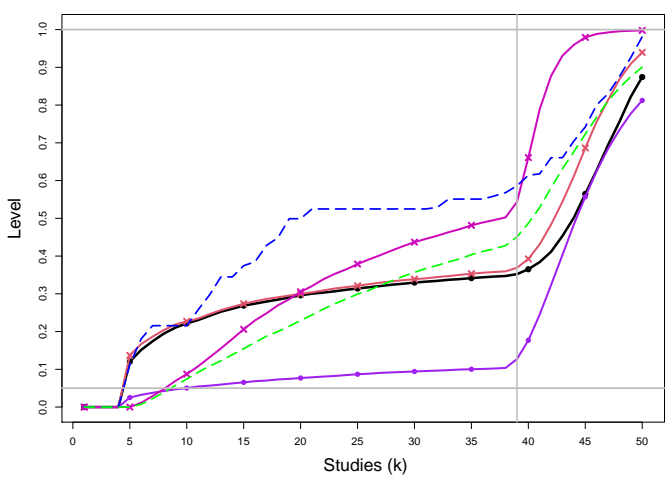

IV REML: $\tau^{2}=1 \mathrm{n}=500$ level $=0.05 \mathrm{~h}=4 \delta=1$ to 2 (chg at 39 th)

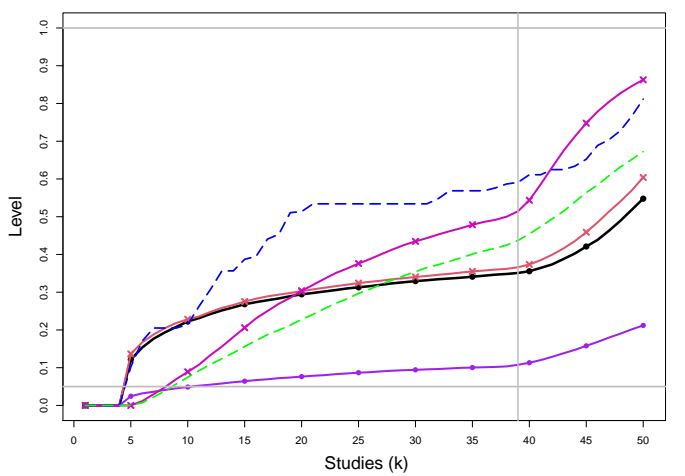

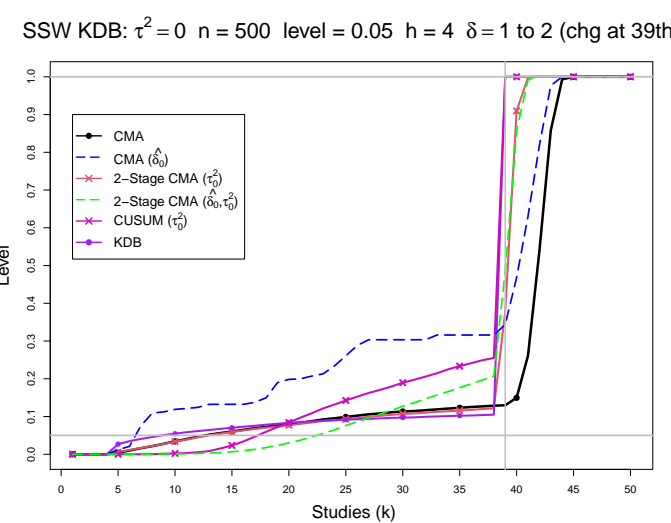

SSW KDB: $\tau^{2}=0.1 \mathrm{n}=500$ level $=0.05 \mathrm{~h}=4 \delta=1$ to 2 (chg at 39 th)

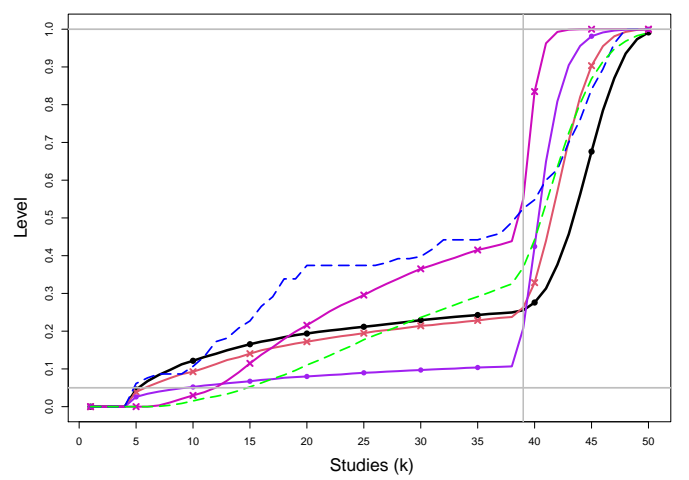

SSW KDB: $\tau^{2}=0.25 \mathrm{n}=500$ level $=0.05 \mathrm{~h}=4 \delta=1$ to 2 (chg at 39 th)

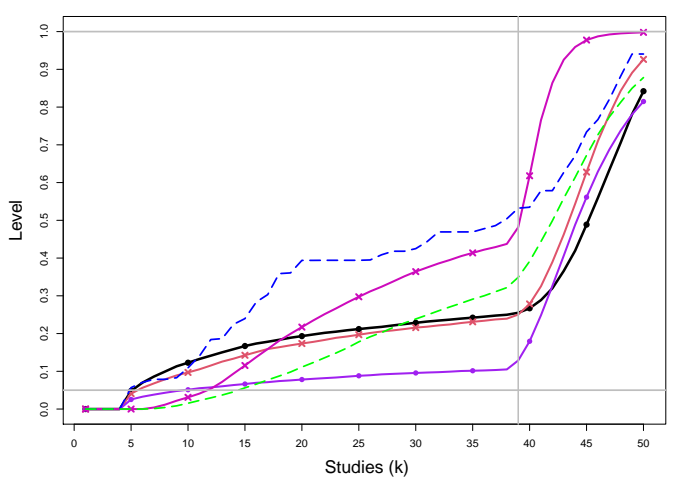

SSW KDB: $\tau^{2}=1 \mathrm{n}=500$ level $=0.05 \mathrm{~h}=4 \delta=1$ to 2 (chg at 39 th)

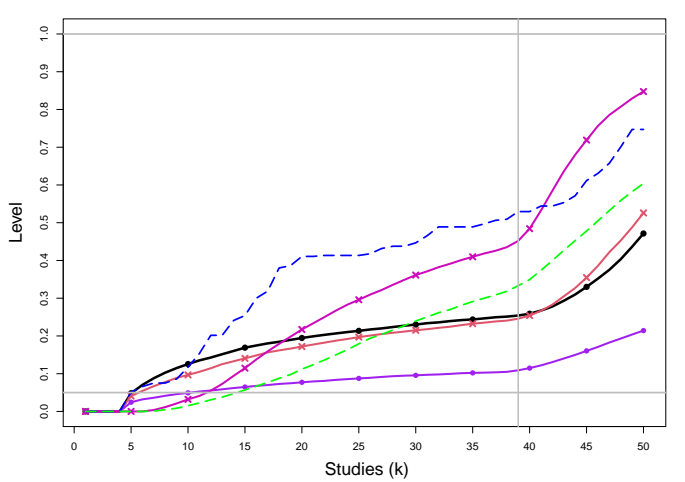

Figure E2. 14: Empirical levels of 1- and 2-stage CMA tests for shift in $\delta$ at .05 nominal level, shift in $\tau^{2}$ at .025 nominal levels and of CUSUM with $h=4$ for equal sample sizes $n_{i C}+n_{i T}=n=500, \tau^{2}=0,0.1,0.25,1$ and a shift from $\delta=1$ to $\delta=2$ at study number 39. Light grey line at .05. 
IV REML: $\tau^{2}=0 \mathrm{n}=1000$ level $=0.05 \mathrm{~h}=4 \delta=1$ to 2 (chg at 39 th)

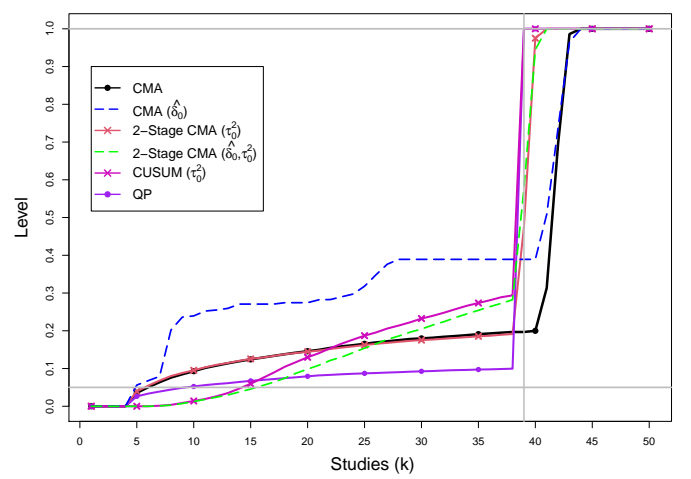

IV REML: $\tau^{2}=0.1 \mathrm{n}=1000$ level $=0.05 \mathrm{~h}=4 \delta=1$ to 2 (chg at 39th)

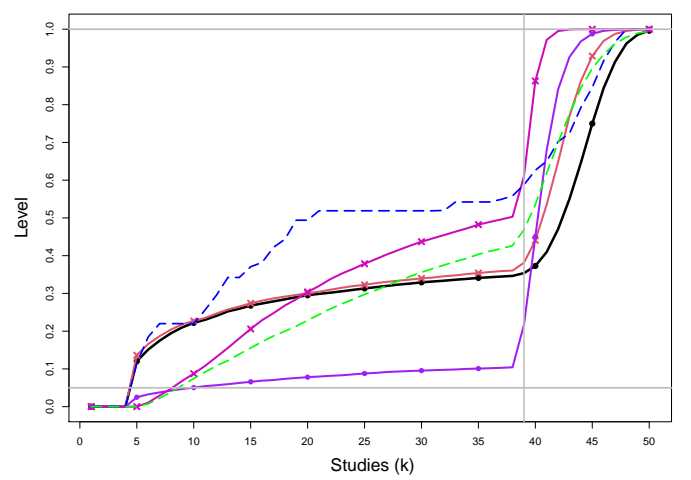

IV REML: $\tau^{2}=0.25 \mathrm{n}=1000$ level $=0.05 \mathrm{~h}=4 \delta=1$ to 2 (chg at 39 th)

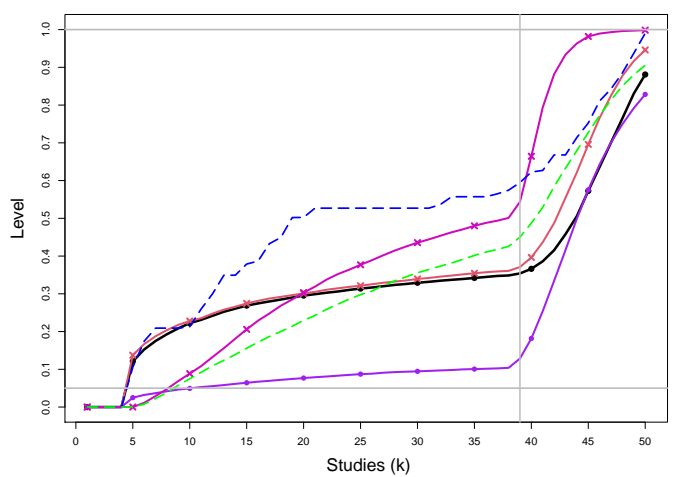

IV REML: $\tau^{2}=1 \mathrm{n}=1000$ level $=0.05 \mathrm{~h}=4 \delta=1$ to 2 (chg at 39 th)

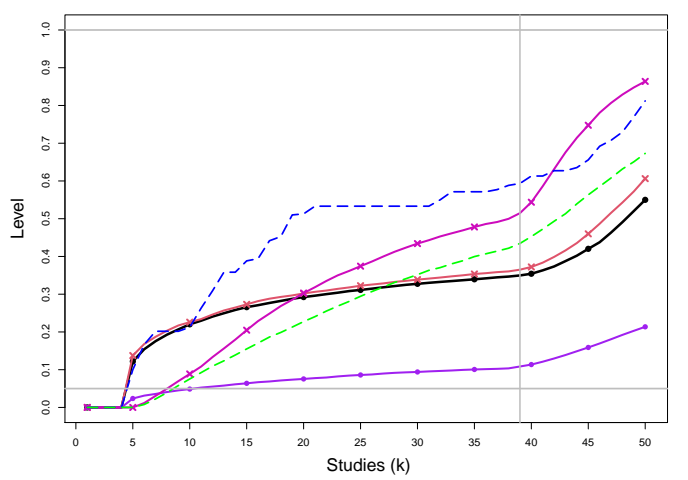

SSW KDB: $\tau^{2}=0 \quad n=1000$ level $=0.05 h=4 \delta=1$ to 2 (chg at 39th)

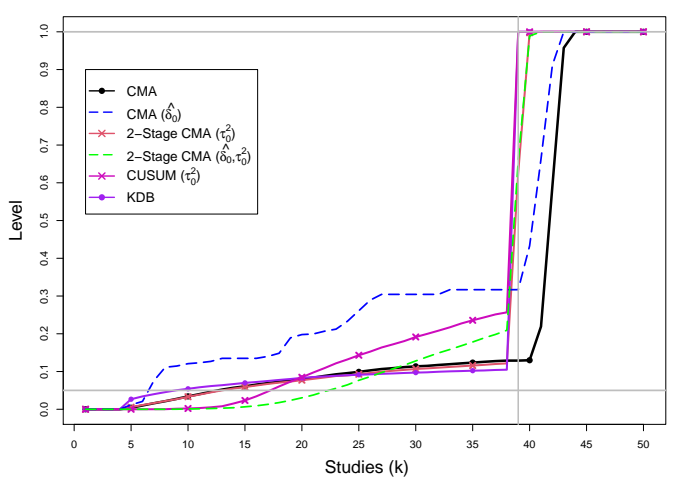

SSW KDB: $\tau^{2}=0.1 \mathrm{n}=1000$ level $=0.05 \mathrm{~h}=4 \delta=1$ to 2 (chg at 39th)

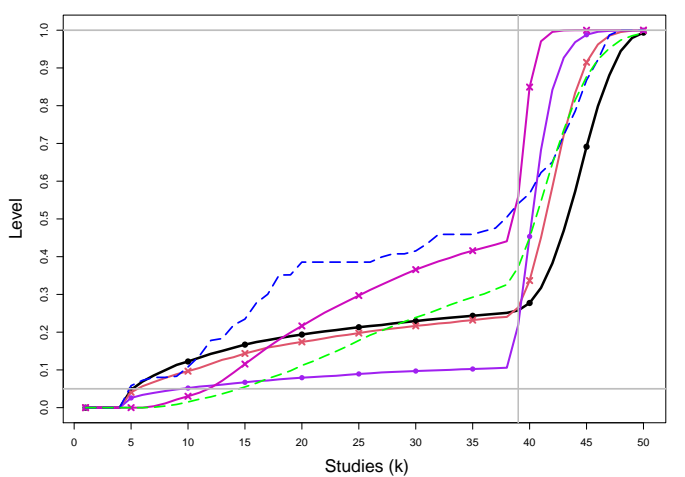

SSW KDB: $\tau^{2}=0.25 \mathrm{n}=1000$ level $=0.05 \mathrm{~h}=4 \delta=1$ to 2 (chg at 39th)

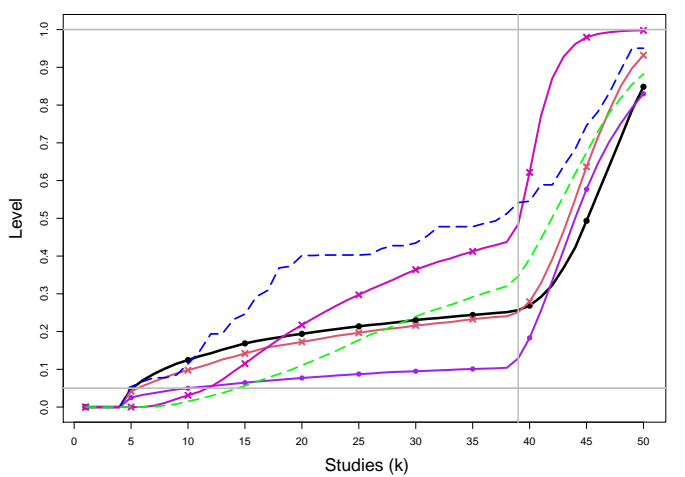

SSW KDB: $\tau^{2}=1 \mathrm{n}=1000$ level $=0.05 \mathrm{~h}=4 \delta=1$ to 2 (chg at 39th)

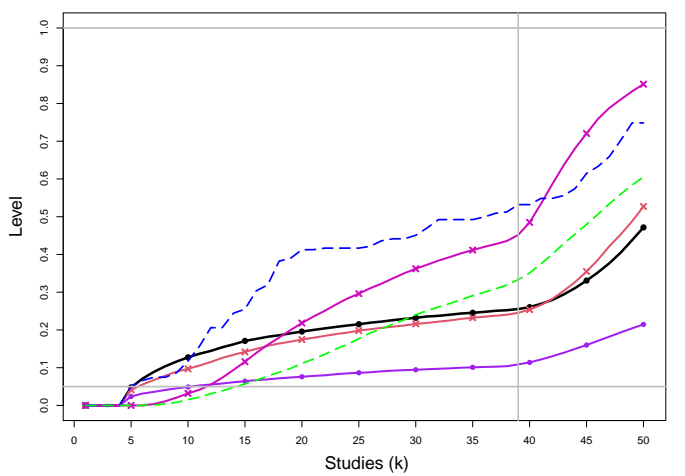

Figure E2. 15: Empirical levels of 1- and 2-stage CMA tests for shift in $\delta$ at .05 nominal level, shift in $\tau^{2}$ at .025 nominal levels and of CUSUM with $h=4$ for equal sample sizes $n_{i C}+n_{i T}=n=1000, \tau^{2}=0,0.1,0.25,1$ and a shift from $\delta=1$ to $\delta=2$ at study number 39. Light grey line at .05. 


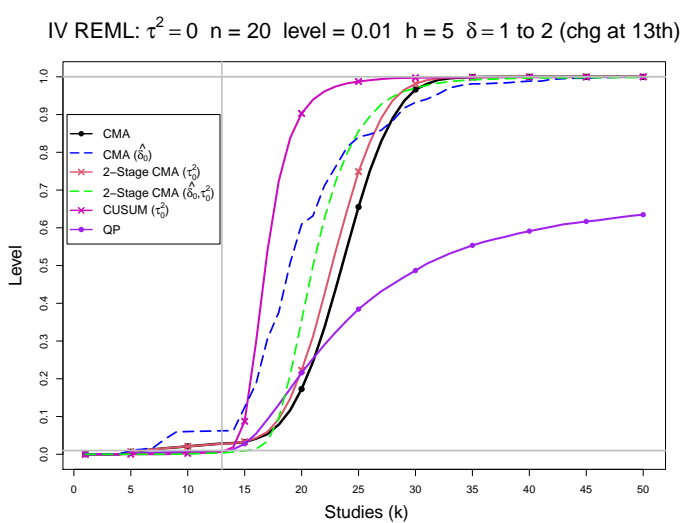

IV REML: $\tau^{2}=0.1 \mathrm{n}=20$ level $=0.01 \mathrm{~h}=5 \delta=1$ to 2 (chg at 13 th)

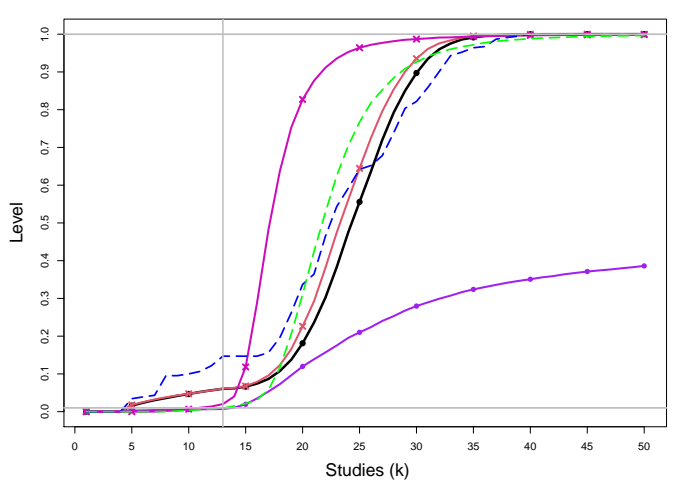

IV REML: $\tau^{2}=0.25 \mathrm{n}=20$ level $=0.01 \mathrm{~h}=5 \delta=1$ to 2 (chg at 13 th)

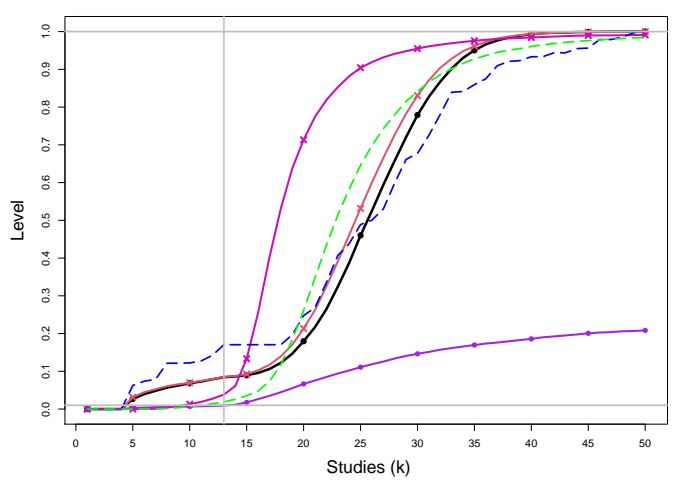

IV REML: $\tau^{2}=1 \mathrm{n}=20$ level $=0.01 \mathrm{~h}=5 \delta=1$ to 2 (chg at 13 th)

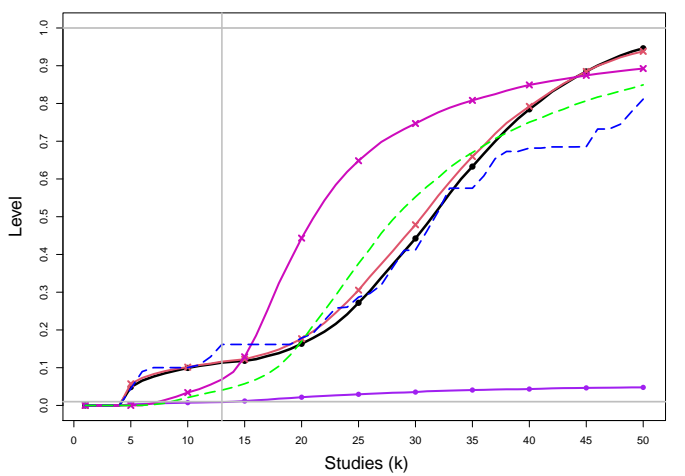

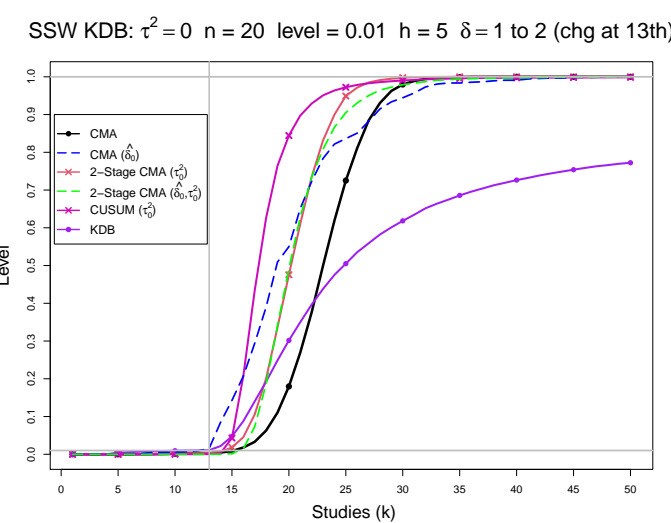

SSW KDB: $\tau^{2}=0.1 \mathrm{n}=20$ level $=0.01 \mathrm{~h}=5 \delta=1$ to 2 (chg at 13th)

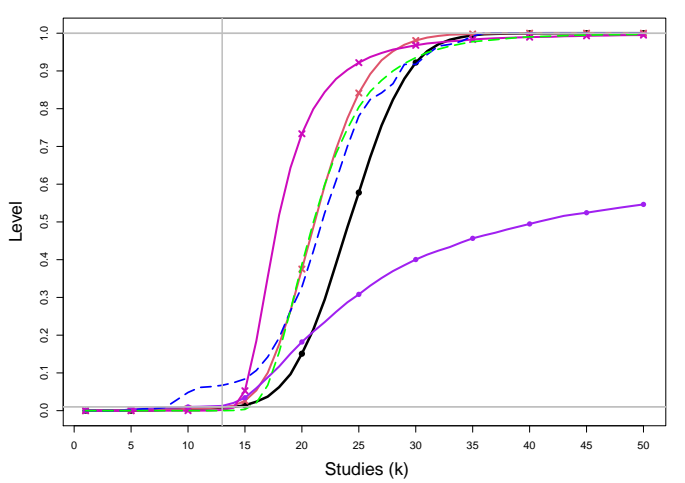

SSW KDB: $\tau^{2}=0.25 \mathrm{n}=20$ level $=0.01 \mathrm{~h}=5 \quad \delta=1$ to 2 (chg at 13 th)

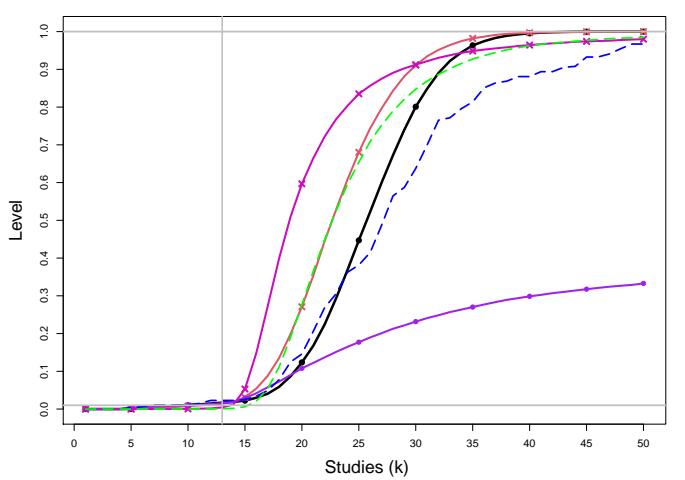

SSW KDB: $\tau^{2}=1 \mathrm{n}=20$ level $=0.01 \mathrm{~h}=5 \delta=1$ to 2 (chg at 13 th)

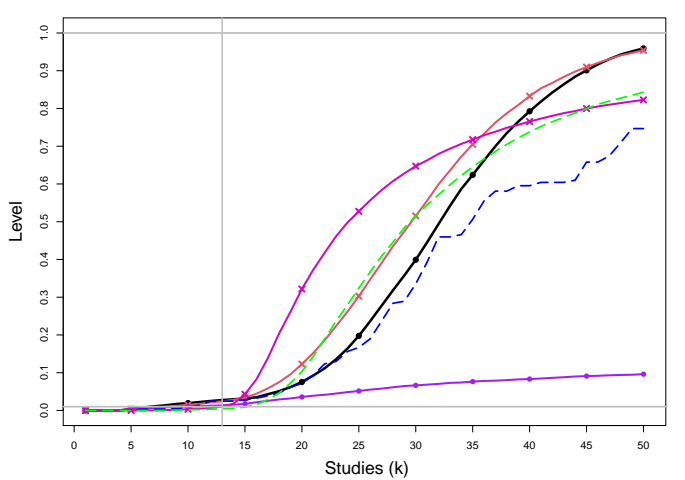

Figure E2. 16: Empirical levels of 1- and 2-stage CMA tests for shift in $\delta$ at .01 nominal level, shift in $\tau^{2}$ at .005 nominal levels and of CUSUM with $h=5$ for equal sample sizes $n_{i C}+n_{i T}=n=20, \tau^{2}=0,0.1,0.25,1$ and a shift from $\delta=1$ to $\delta=2$ at study number 13. Light grey line at .01. 


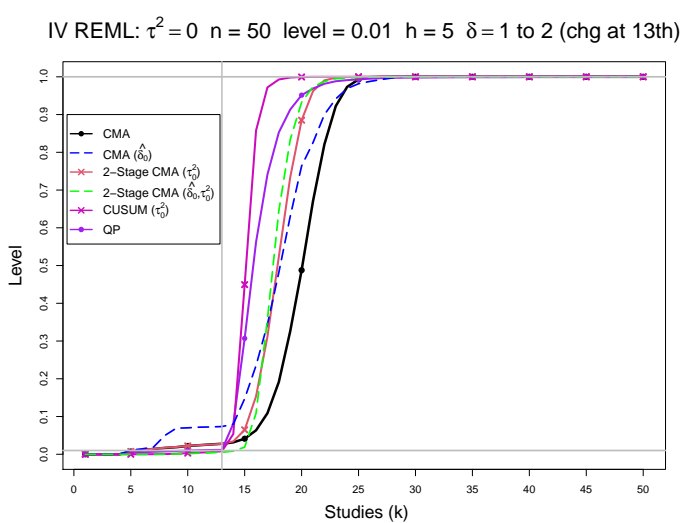

IV REML: $\tau^{2}=0.1 \mathrm{n}=50$ level $=0.01 \mathrm{~h}=5 \delta=1$ to 2 (chg at 13 th)

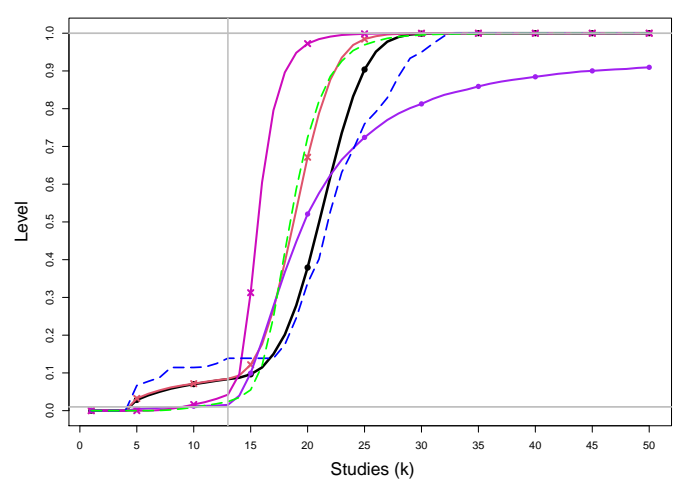

IV REML: $\tau^{2}=0.25 \mathrm{n}=50$ level $=0.01 \mathrm{~h}=5 \delta=1$ to 2 (chg at 13th)

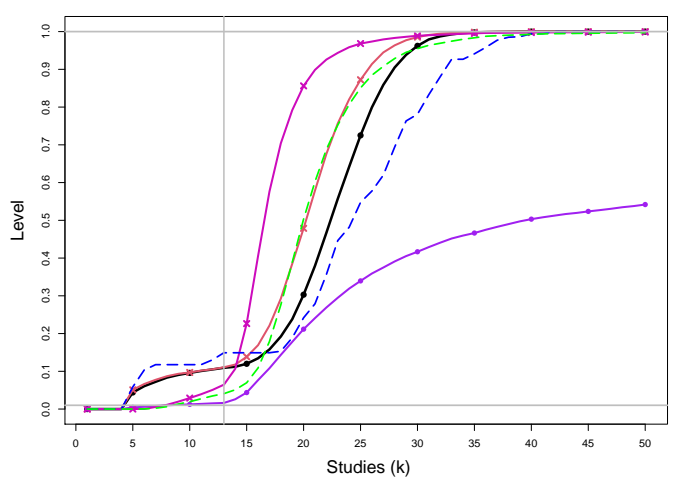

IV REML: $\tau^{2}=1 \mathrm{n}=50$ level $=0.01 \mathrm{~h}=5 \delta=1$ to 2 (chg at 13 th)

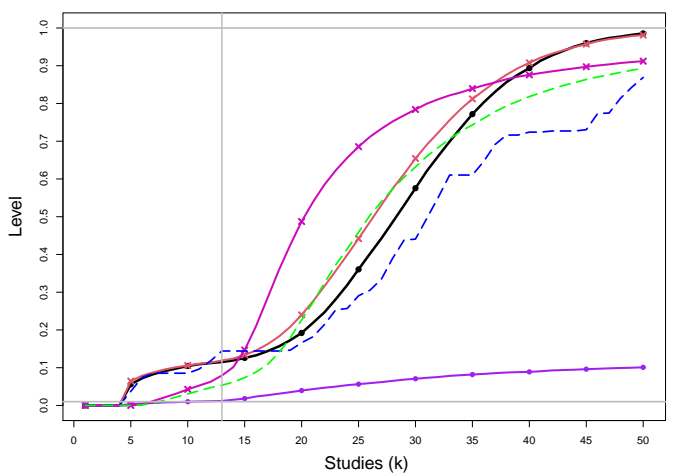

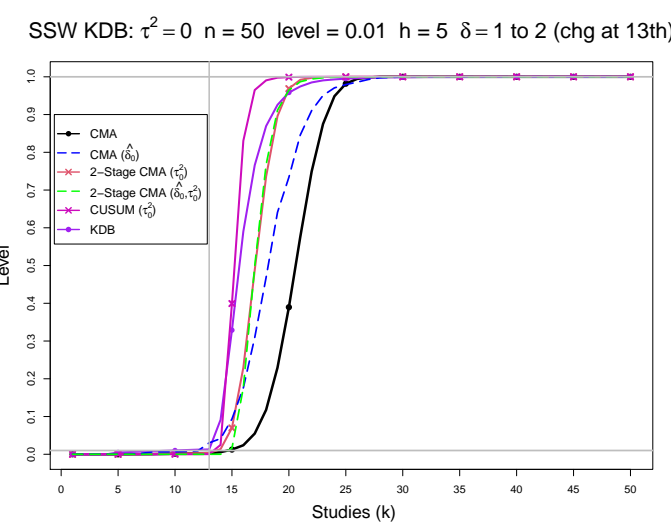

SSW KDB: $\tau^{2}=0.1 \mathrm{n}=50$ level $=0.01 \mathrm{~h}=5 \delta=1$ to 2 (chg at 13th)

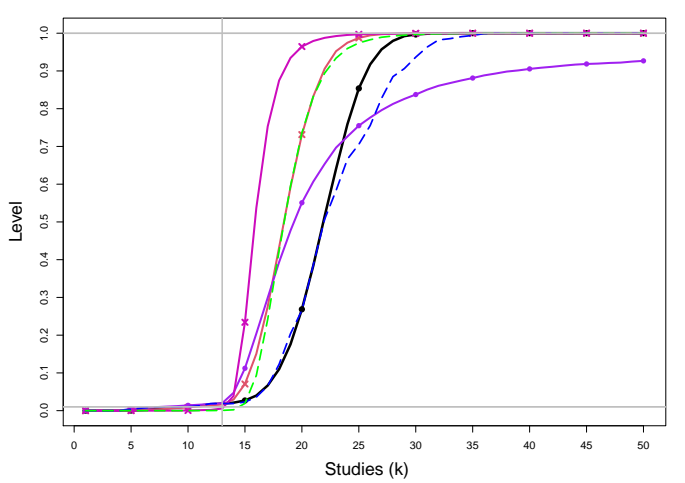

SSW KDB: $\tau^{2}=0.25 \mathrm{n}=50$ level $=0.01 \mathrm{~h}=5 \quad \delta=1$ to 2 (chg at 13 th)

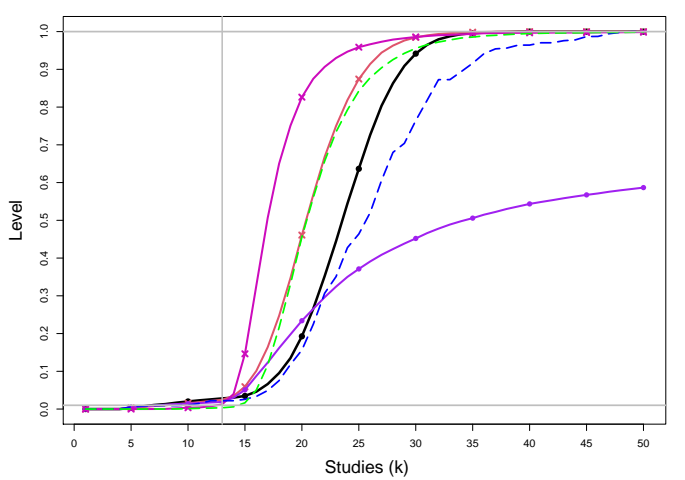

SSW KDB: $\tau^{2}=1 \mathrm{n}=50$ level $=0.01 \mathrm{~h}=5 \delta=1$ to 2 (chg at 13 th)

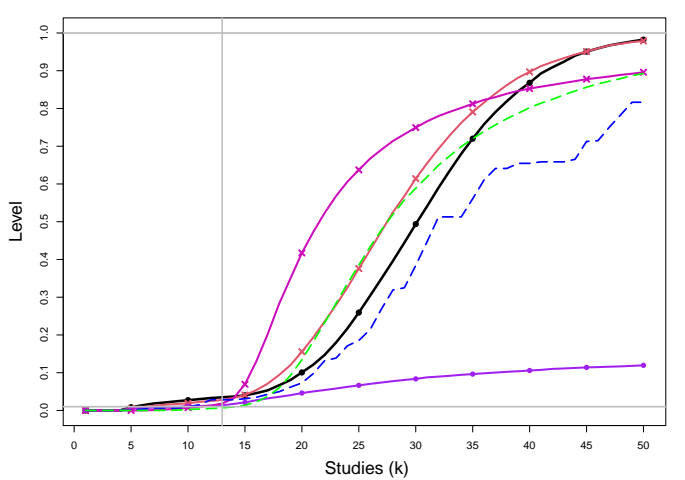

Figure E2. 17: Empirical levels of 1- and 2-stage CMA tests for shift in $\delta$ at .01 nominal level, shift in $\tau^{2}$ at .005 nominal levels and of CUSUM with $h=5$ for equal sample sizes $n_{i C}+n_{i T}=n=50, \tau^{2}=0,0.1,0.25,1$ and a shift from $\delta=1$ to $\delta=2$ at study number 13. Light grey line at .01. 


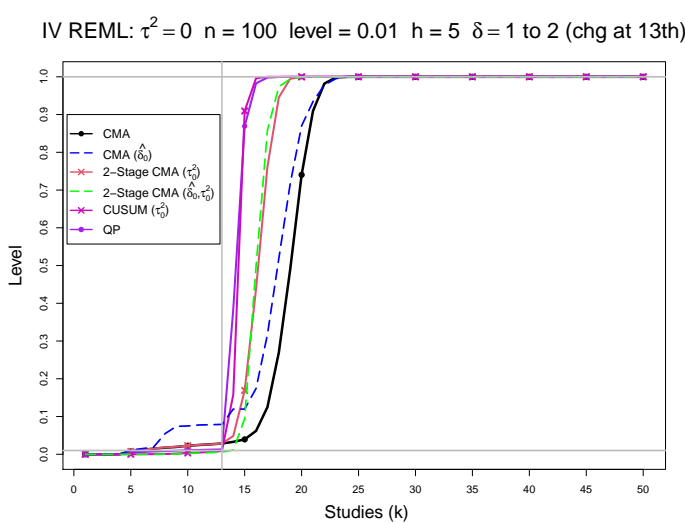

IV REML: $\tau^{2}=0.1 \mathrm{n}=100$ level $=0.01 \mathrm{~h}=5 \delta=1$ to 2 (chg at 13th)

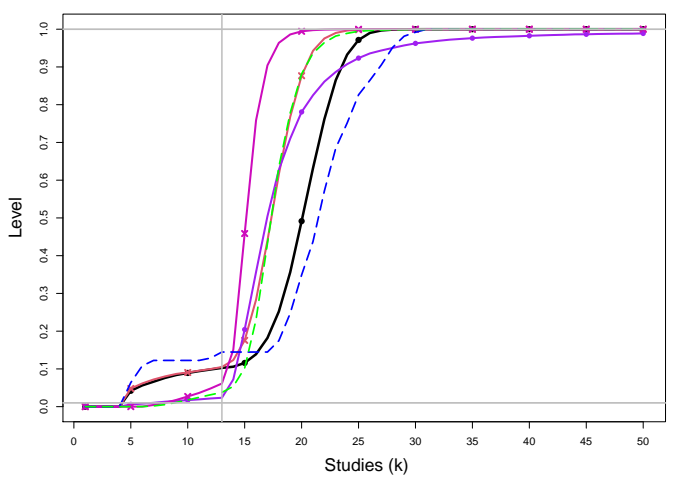

IV REML: $\tau^{2}=0.25 \mathrm{n}=100$ level $=0.01 \mathrm{~h}=5 \delta=1$ to 2 (chg at 13 th)

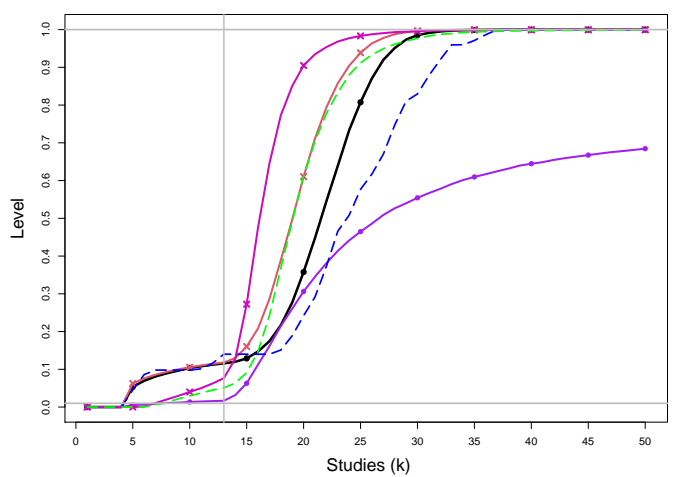

IV REML: $\tau^{2}=1 \mathrm{n}=100$ level $=0.01 \mathrm{~h}=5 \delta=1$ to 2 (chg at 13 th)

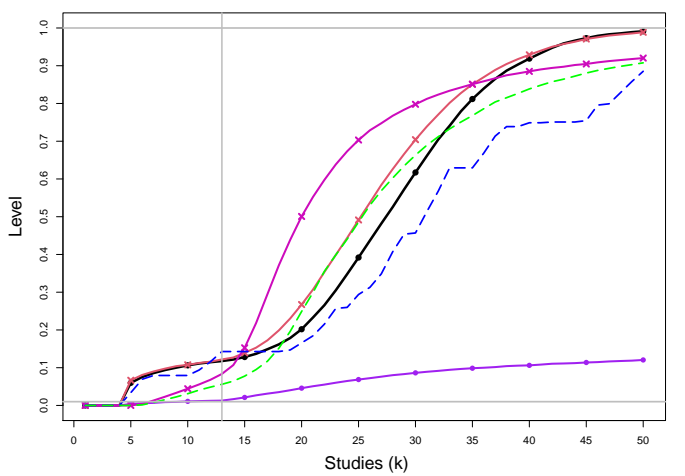

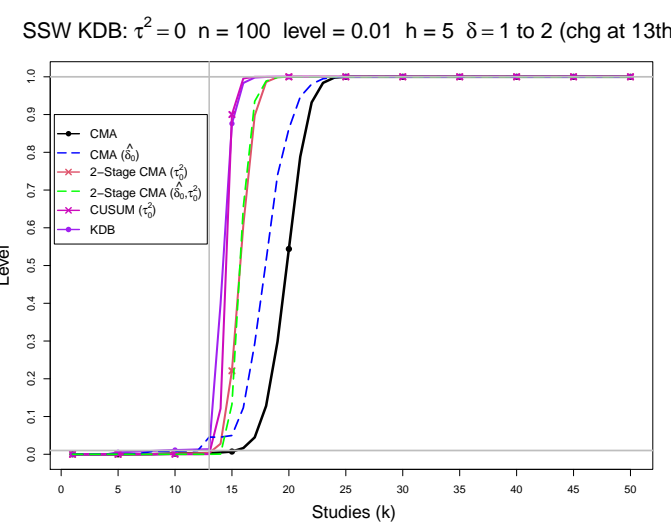

SSW KDB: $\tau^{2}=0.1 \mathrm{n}=100$ level $=0.01 \mathrm{~h}=5 \delta=1$ to 2 (chg at 13 th)

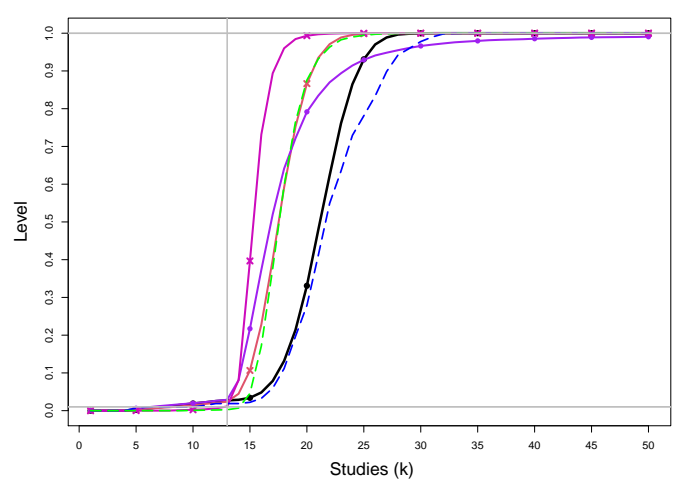

SSW KDB: $\tau^{2}=0.25 \mathrm{n}=100$ level $=0.01 \mathrm{~h}=5 \delta=1$ to 2 (chg at 13 th)

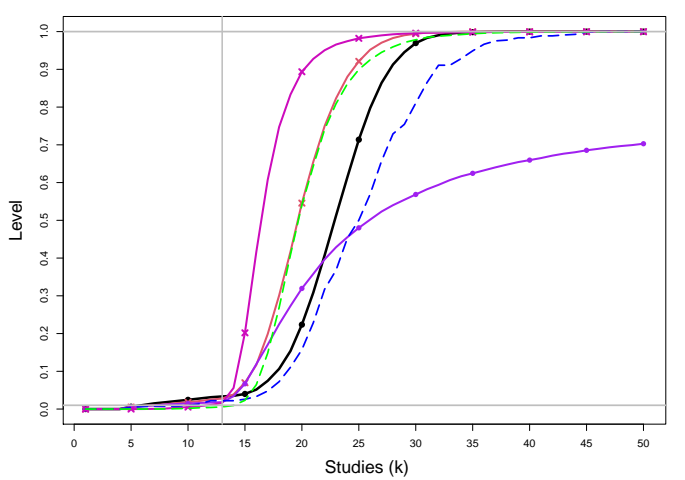

SSW KDB: $\tau^{2}=1 \mathrm{n}=100$ level $=0.01 \mathrm{~h}=5 \delta=1$ to 2 (chg at 13 th)

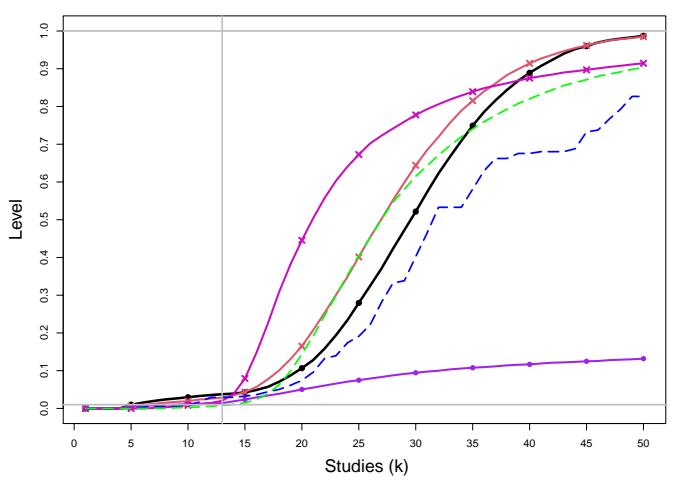

Figure E2. 18: Empirical levels of 1- and 2-stage CMA tests for shift in $\delta$ at .01 nominal level, shift in $\tau^{2}$ at .005 nominal levels and of CUSUM with $h=5$ for equal sample sizes $n_{i C}+n_{i T}=n=100, \tau^{2}=0,0.1,0.25,1$ and a shift from $\delta=1$ to $\delta=2$ at study number 13. Light grey line at .01. 


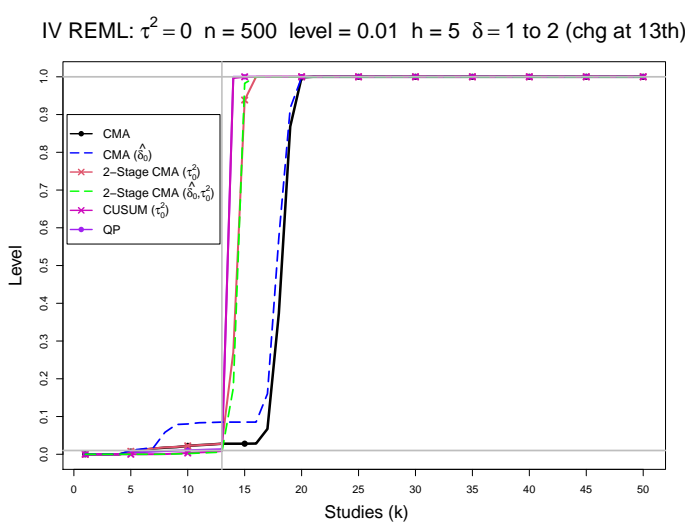

IV REML: $\tau^{2}=0.1 \mathrm{n}=500$ level $=0.01 \mathrm{~h}=5 \delta=1$ to 2 (chg at 13th)

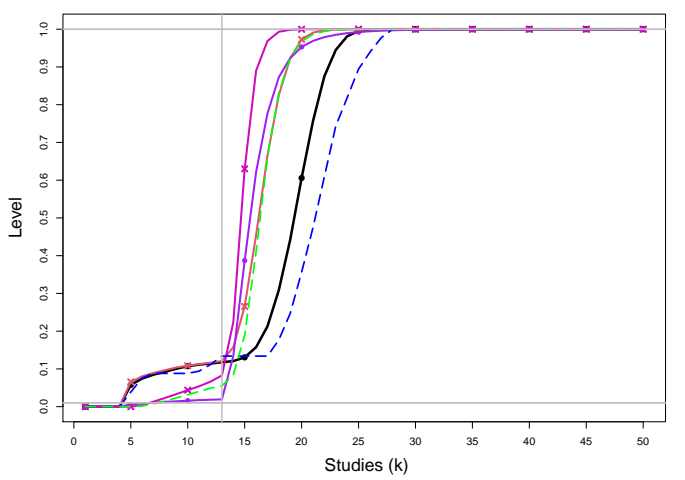

IV REML: $\tau^{2}=0.25 \mathrm{n}=500$ level $=0.01 \mathrm{~h}=5 \delta=1$ to 2 (chg at 13 th)

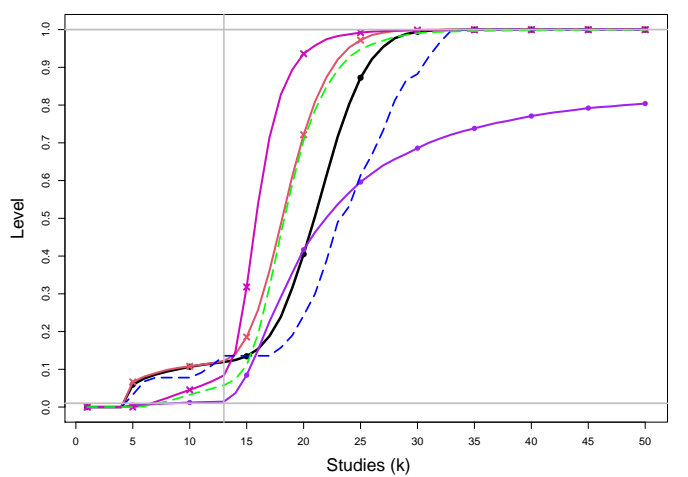

IV REML: $\tau^{2}=1 \mathrm{n}=500$ level $=0.01 \mathrm{~h}=5 \delta=1$ to 2 (chg at 13 th)

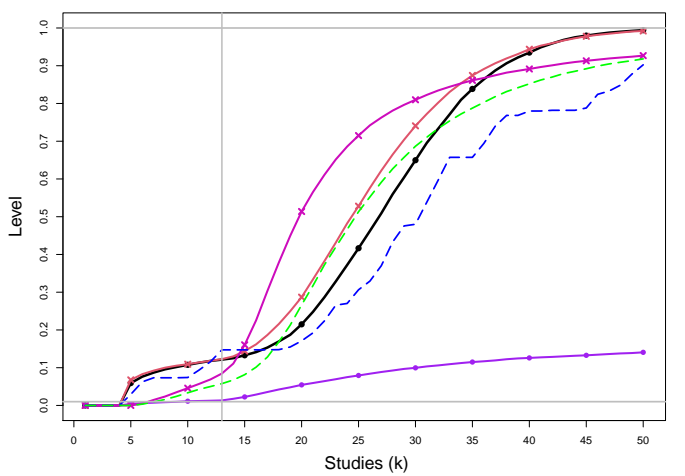

SSW KDB: $\tau^{2}=0 \mathrm{n}=500$ level $=0.01 \mathrm{~h}=5 \quad \delta=1$ to 2 (chg at 13 th)

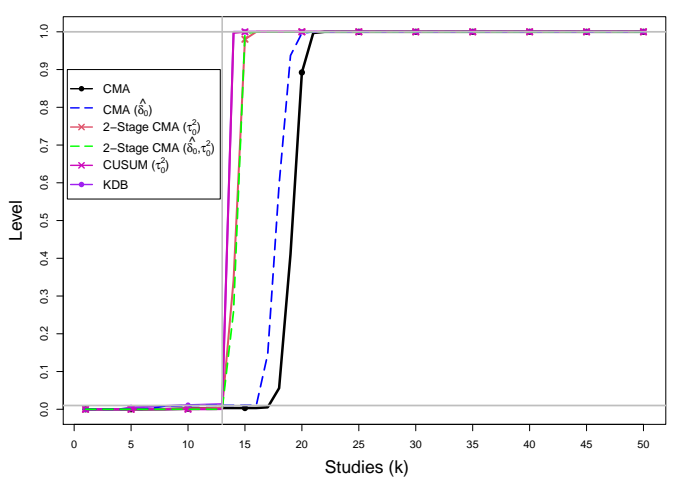

SSW KDB: $\tau^{2}=0.1 \mathrm{n}=500$ level $=0.01 \mathrm{~h}=5 \quad \delta=1$ to 2 (chg at 13 th)

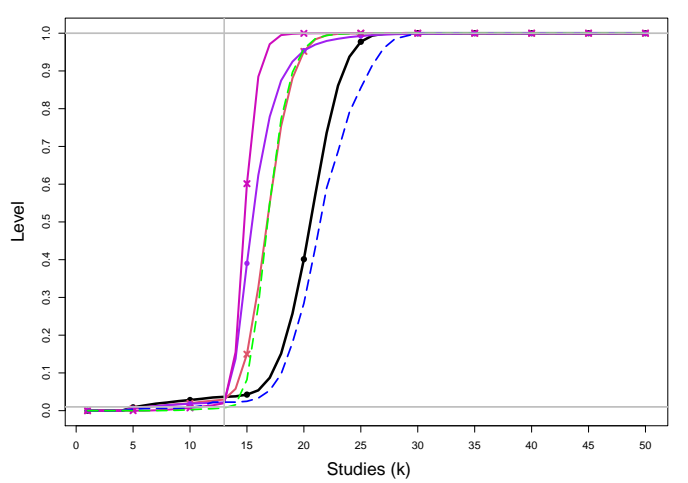

SSW KDB: $\tau^{2}=0.25 \mathrm{n}=500$ level $=0.01 \mathrm{~h}=5 \delta=1$ to 2 (chg at 13th)

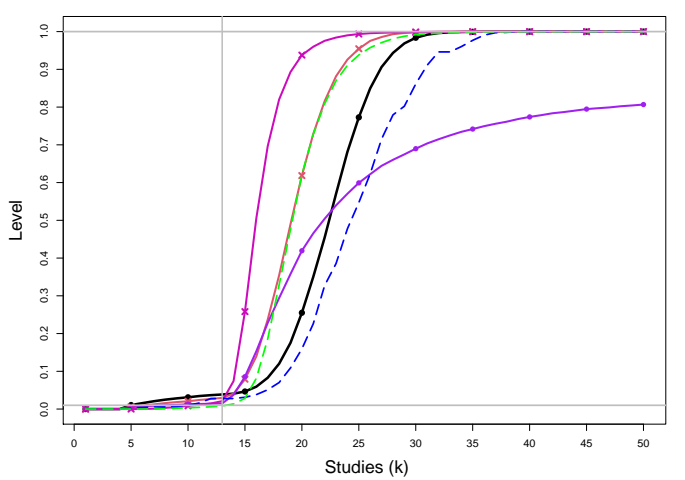

SSW KDB: $\tau^{2}=1 \mathrm{n}=500$ level $=0.01 \mathrm{~h}=5 \delta=1$ to 2 (chg at 13th)

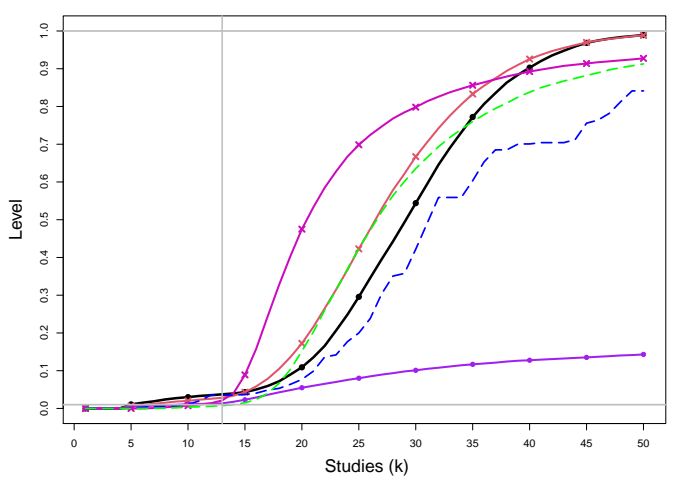

Figure E2. 19: Empirical levels of 1- and 2-stage CMA tests for shift in $\delta$ at .01 nominal level, shift in $\tau^{2}$ at .005 nominal levels and of CUSUM with $h=5$ for equal sample sizes $n_{i C}+n_{i T}=n=500, \tau^{2}=0,0.1,0.25,1$ and a shift from $\delta=1$ to $\delta=2$ at study number 13. Light grey line at .01. 
IV REML: $\tau^{2}=0 \mathrm{n}=1000$ level $=0.01 \mathrm{~h}=5 \quad \delta=1$ to 2 (chg at 13 th)

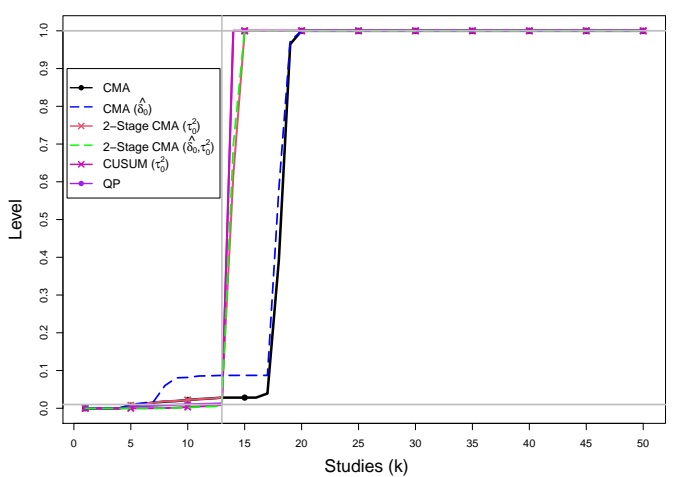

IV REML: $\tau^{2}=0.1 \mathrm{n}=1000$ level $=0.01 \mathrm{~h}=5 \quad \delta=1$ to 2 (chg at 13 th)

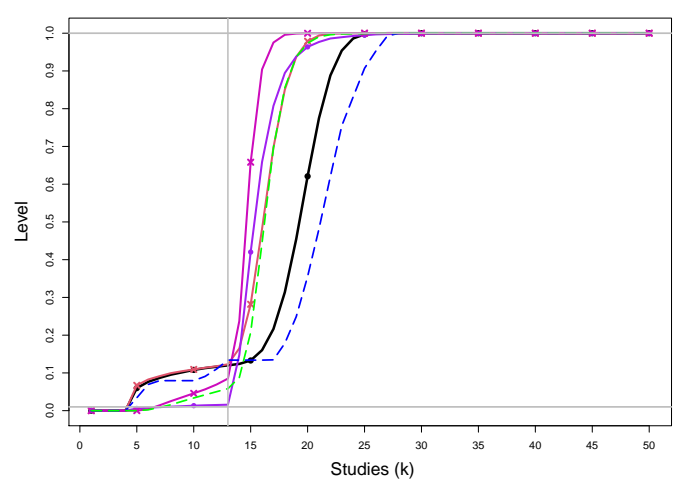

IV REML: $\tau^{2}=0.25 \mathrm{n}=1000$ level $=0.01 \mathrm{~h}=5 \quad \delta=1$ to 2 (chg at 13 th)

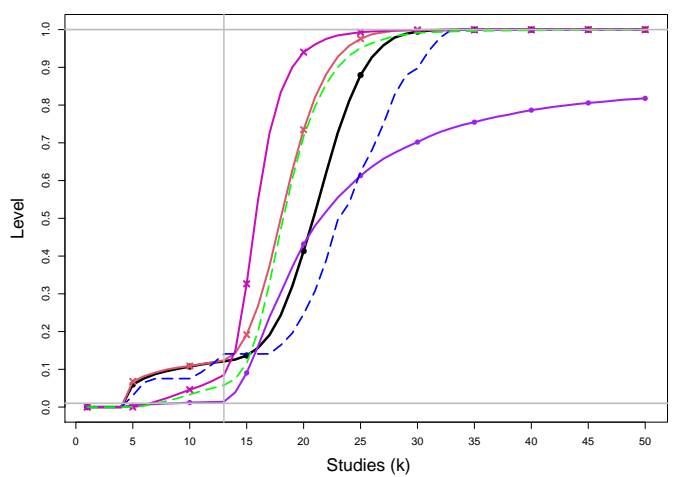

IV REML: $\tau^{2}=1 \mathrm{n}=1000$ level $=0.01 \mathrm{~h}=5 \quad \delta=1$ to 2 (chg at 13 th)

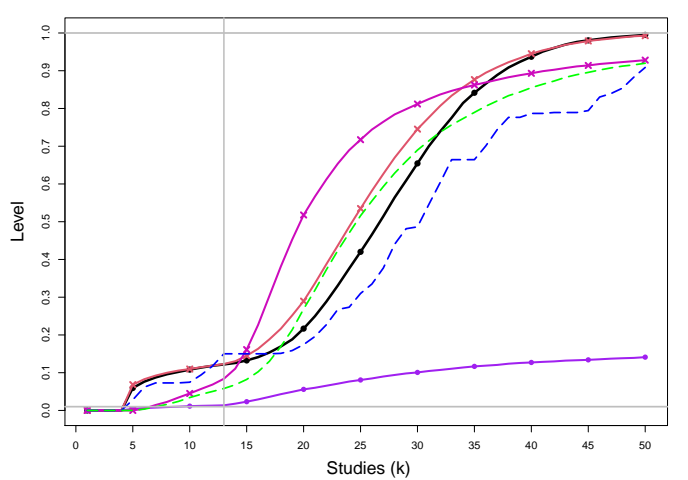

SSW KDB: $\tau^{2}=0 \quad \mathrm{n}=1000$ level $=0.01 \mathrm{~h}=5 \quad \delta=1$ to 2 (chg at 13th)

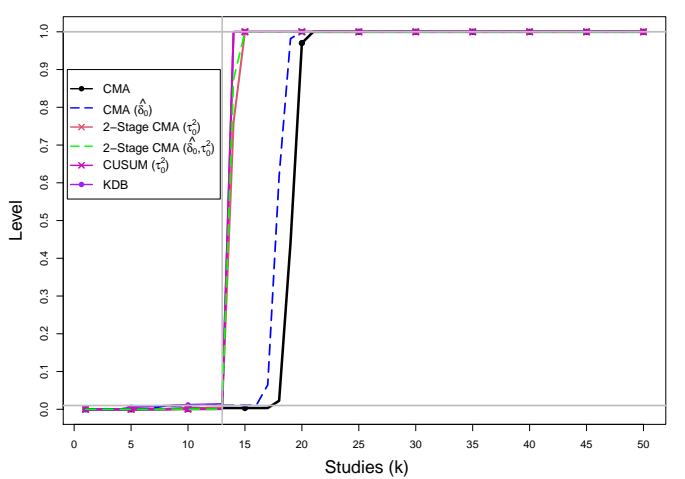

SSW KDB: $\tau^{2}=0.1 \mathrm{n}=1000$ level $=0.01 \mathrm{~h}=5 \delta=1$ to 2 (chg at 13th)

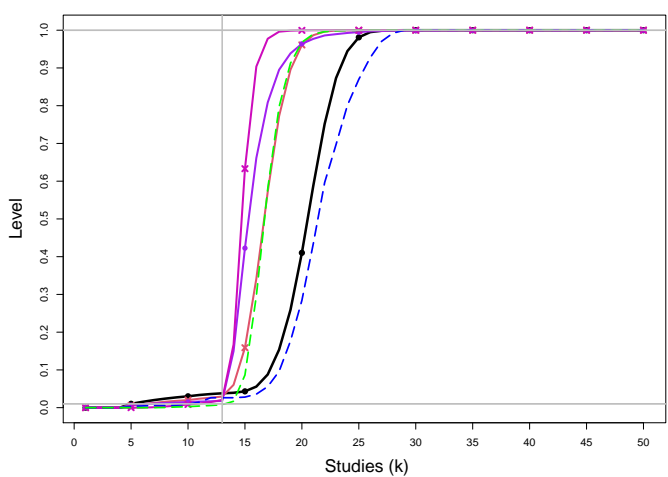

SSW KDB: $\tau^{2}=0.25 \mathrm{n}=1000$ level $=0.01 \mathrm{~h}=5 \delta=1$ to 2 (chg at 13th)

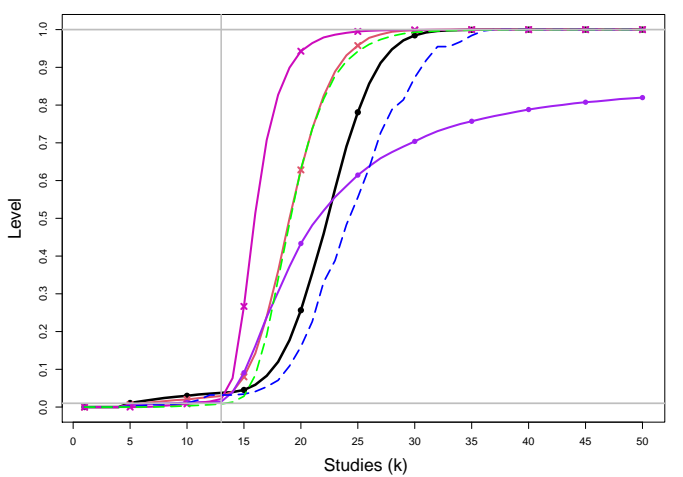

SSW KDB: $\tau^{2}=1 \mathrm{n}=1000$ level $=0.01 \mathrm{~h}=5 \delta=1$ to 2 (chg at 13th)

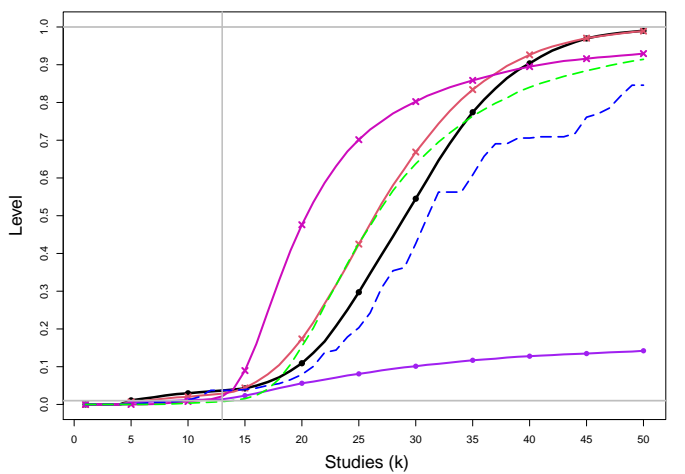

Figure E2. 20: Empirical levels of 1- and 2-stage CMA tests for shift in $\delta$ at .01 nominal level, shift in $\tau^{2}$ at .005 nominal levels and of CUSUM with $h=5$ for equal sample sizes $n_{i C}+n_{i T}=n=1000, \tau^{2}=0,0.1,0.25,1$ and a shift from $\delta=1$ to $\delta=2$ at study number 13. Light grey line at .01. 


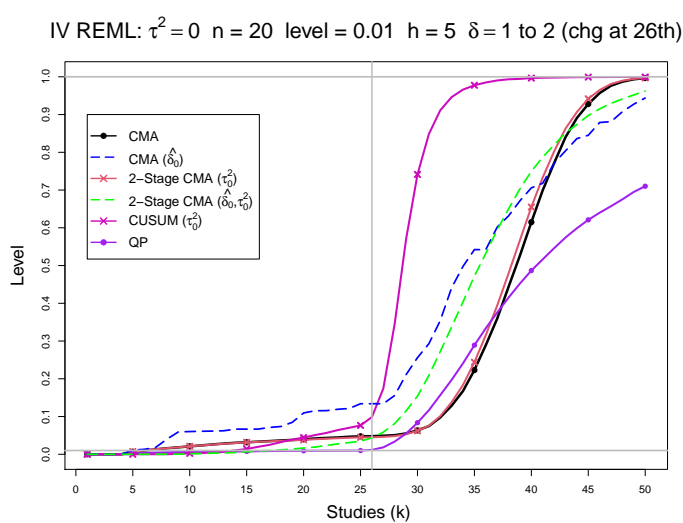

IV REML: $\tau^{2}=0.1 \mathrm{n}=20$ level $=0.01 \mathrm{~h}=5 \delta=1$ to 2 (chg at 26 th)

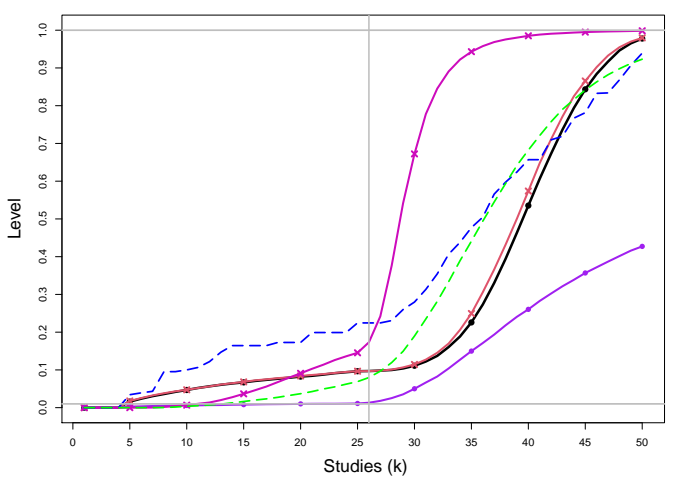

IV REML: $\tau^{2}=0.25 \mathrm{n}=20$ level $=0.01 \mathrm{~h}=5 \delta=1$ to 2 (chg at 26th)

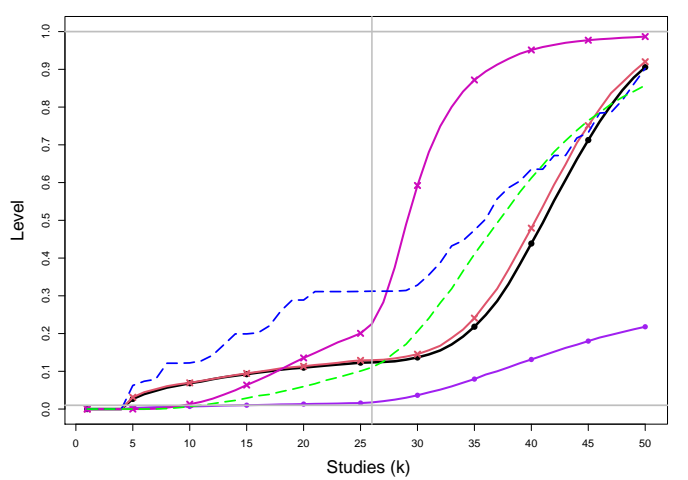

IV REML: $\tau^{2}=1 \mathrm{n}=20$ level $=0.01 \mathrm{~h}=5 \delta=1$ to 2 (chg at 26 th)

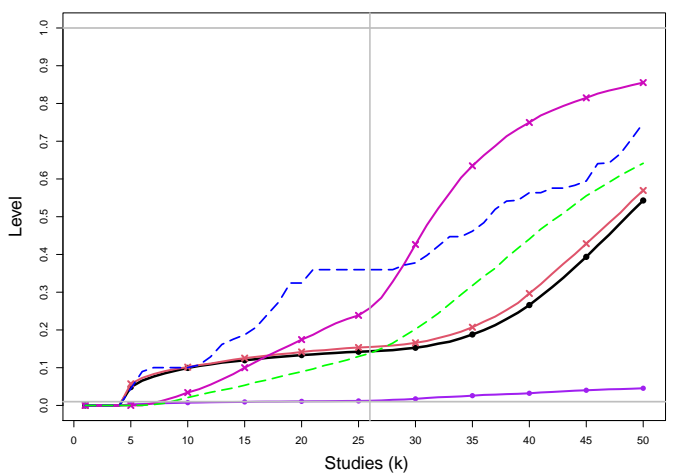

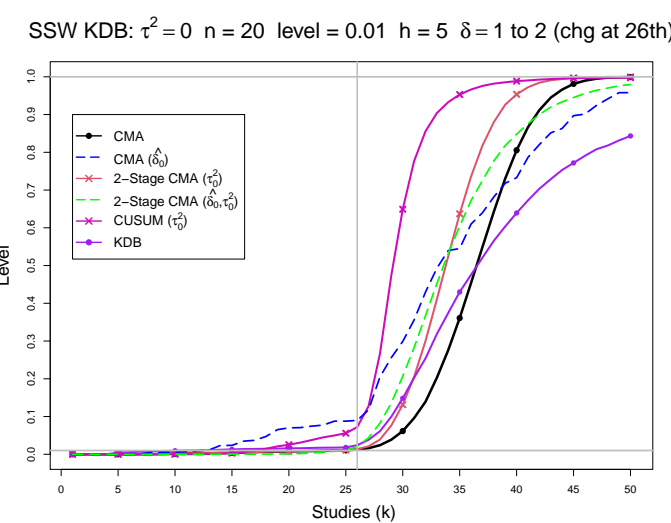

SSW KDB: $\tau^{2}=0.1 \mathrm{n}=20$ level $=0.01 \mathrm{~h}=5 \delta=1$ to 2 (chg at 26th)

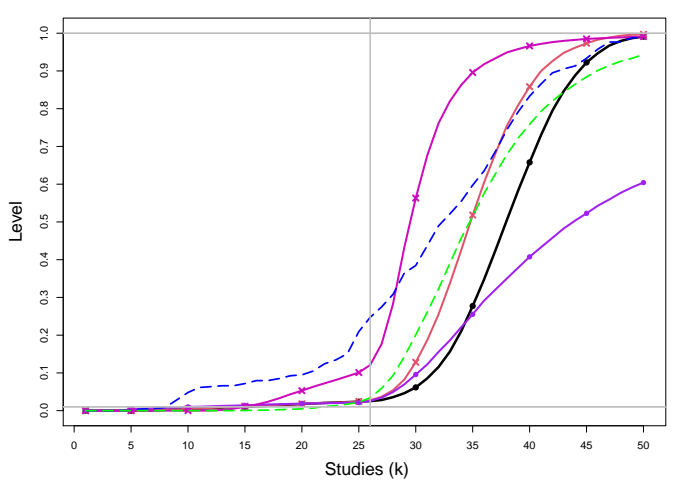

SSW KDB: $\tau^{2}=0.25 \mathrm{n}=20$ level $=0.01 \mathrm{~h}=5 \quad \delta=1$ to 2 (chg at 26th)

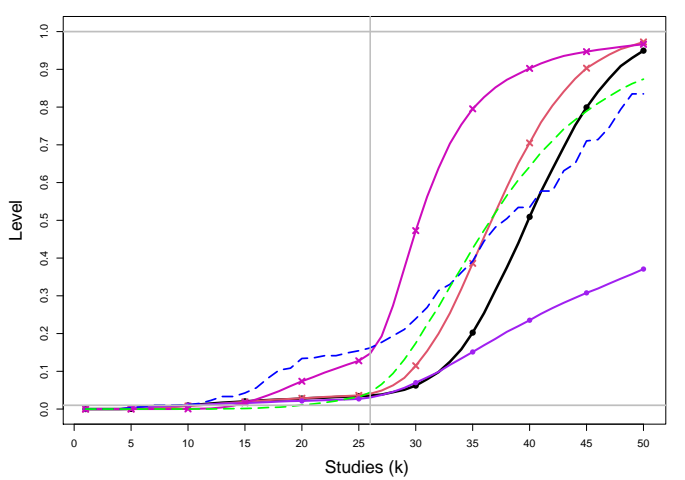

SSW KDB: $\tau^{2}=1 \mathrm{n}=20$ level $=0.01 \mathrm{~h}=5 \delta=1$ to 2 (chg at 26 th)

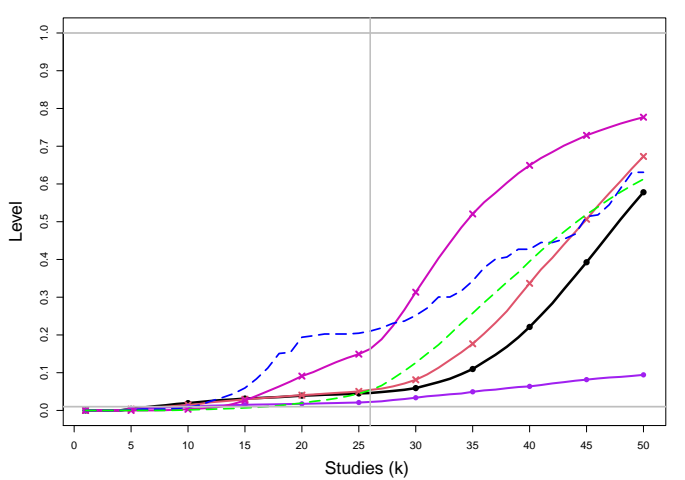

Figure E2. 21: Empirical levels of 1- and 2-stage CMA tests for shift in $\delta$ at .01 nominal level, shift in $\tau^{2}$ at .005 nominal levels and of CUSUM with $h=5$ for equal sample sizes $n_{i C}+n_{i T}=n=20, \tau^{2}=0,0.1,0.25,1$ and a shift from $\delta=1$ to $\delta=2$ at study number 26. Light grey line at .01. 


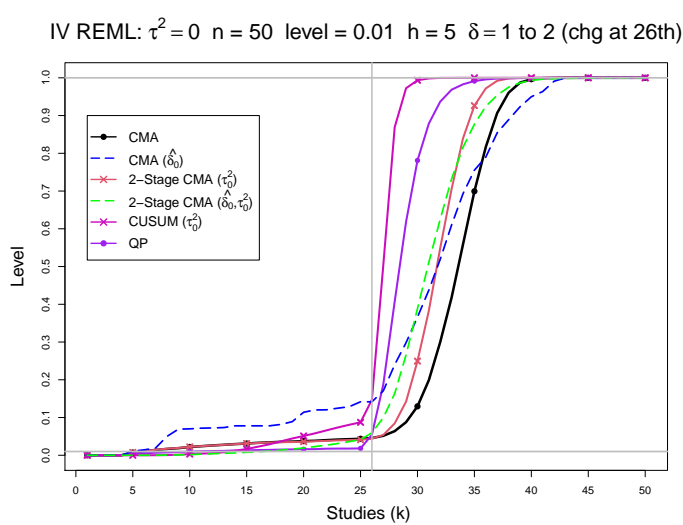

IV REML: $\tau^{2}=0.1 \mathrm{n}=50$ level $=0.01 \mathrm{~h}=5 \delta=1$ to 2 (chg at 26 th)

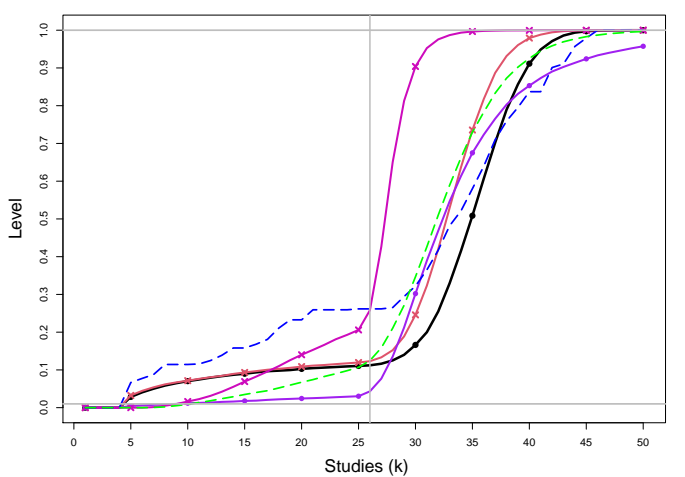

IV REML: $\tau^{2}=0.25 \mathrm{n}=50$ level $=0.01 \mathrm{~h}=5 \delta=1$ to 2 (chg at 26th)

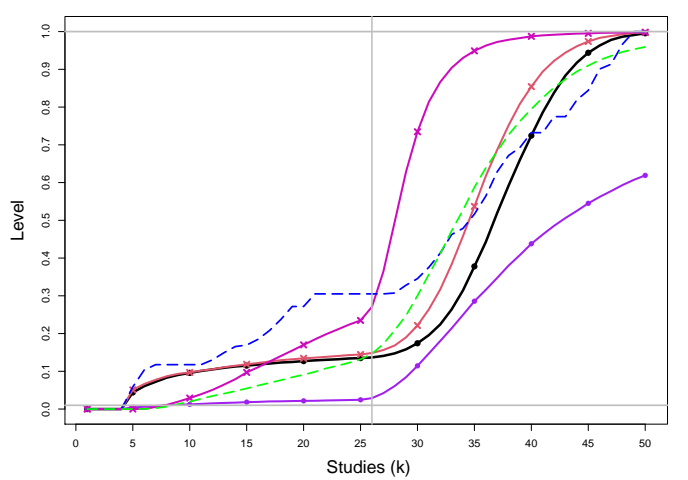

IV REML: $\tau^{2}=1 \mathrm{n}=50$ level $=0.01 \mathrm{~h}=5 \delta=1$ to 2 (chg at 26 th)

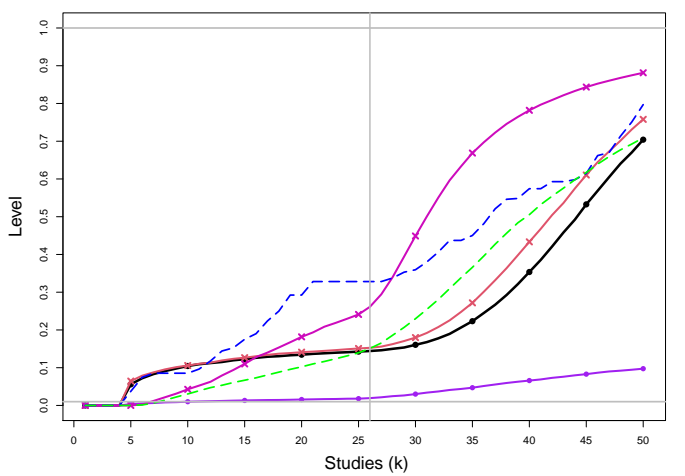

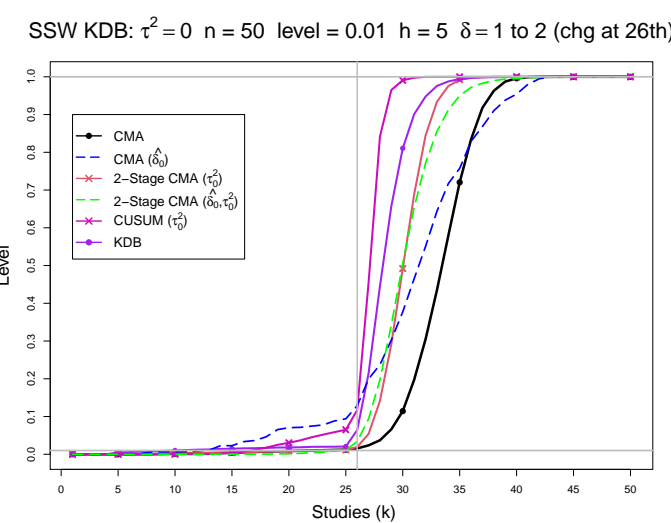

SSW KDB: $\tau^{2}=0.1 \mathrm{n}=50$ level $=0.01 \mathrm{~h}=5 \delta=1$ to 2 (chg at 26th)

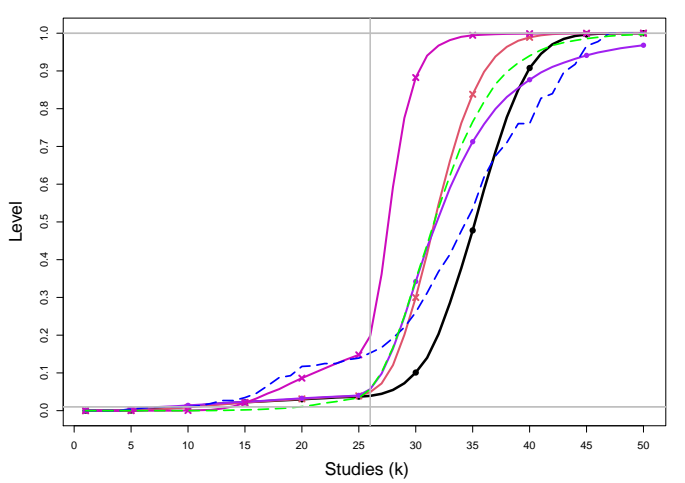

SSW KDB: $\tau^{2}=0.25 \mathrm{n}=50$ level $=0.01 \mathrm{~h}=5 \quad \delta=1$ to 2 (chg at 26th)

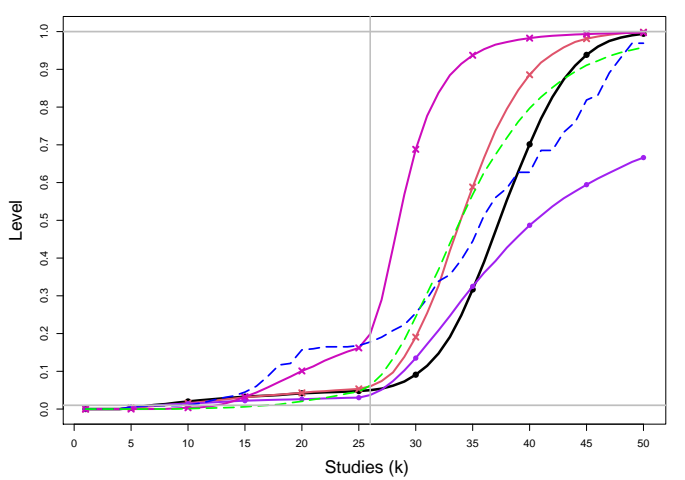

SSW KDB: $\tau^{2}=1 \mathrm{n}=50$ level $=0.01 \mathrm{~h}=5 \delta=1$ to 2 (chg at 26 th)

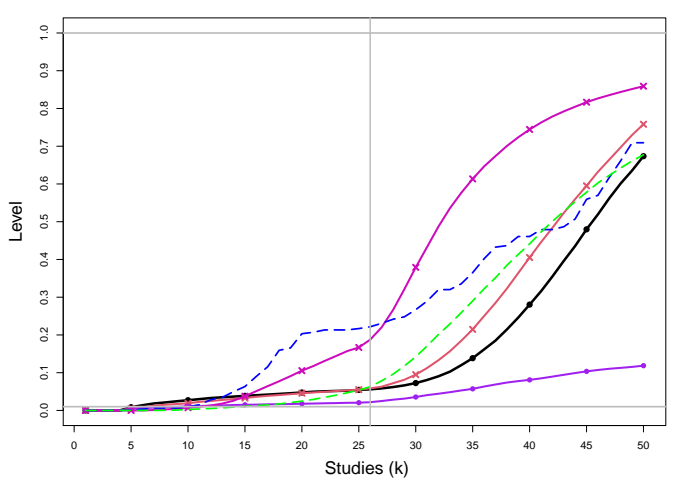

Figure E2. 22: Empirical levels of 1- and 2-stage CMA tests for shift in $\delta$ at .01 nominal level, shift in $\tau^{2}$ at .005 nominal levels and of CUSUM with $h=5$ for equal sample sizes $n_{i C}+n_{i T}=n=50, \tau^{2}=0,0.1,0.25,1$ and a shift from $\delta=1$ to $\delta=2$ at study number 26. Light grey line at .01. 


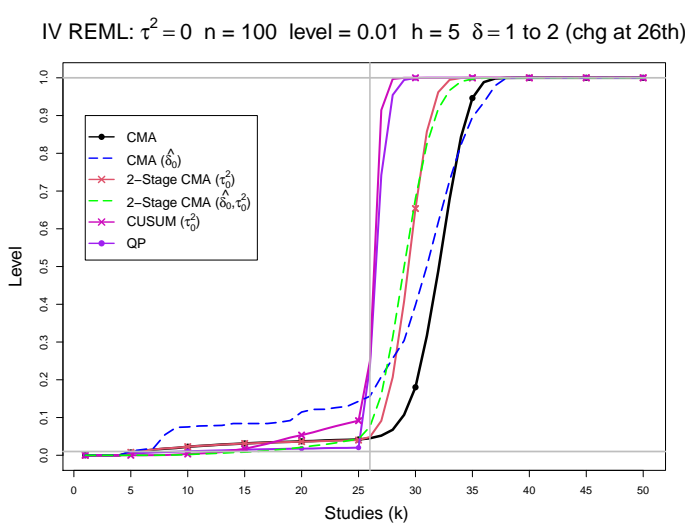

IV REML: $\tau^{2}=0.1 \mathrm{n}=100$ level $=0.01 \mathrm{~h}=5 \delta=1$ to 2 (chg at 26th)

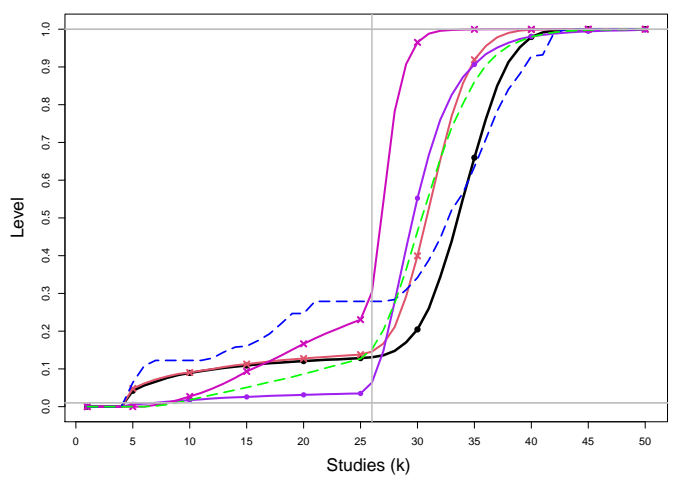

IV REML: $\tau^{2}=0.25 \mathrm{n}=100$ level $=0.01 \mathrm{~h}=5 \quad \delta=1$ to 2 (chg at 26 th)

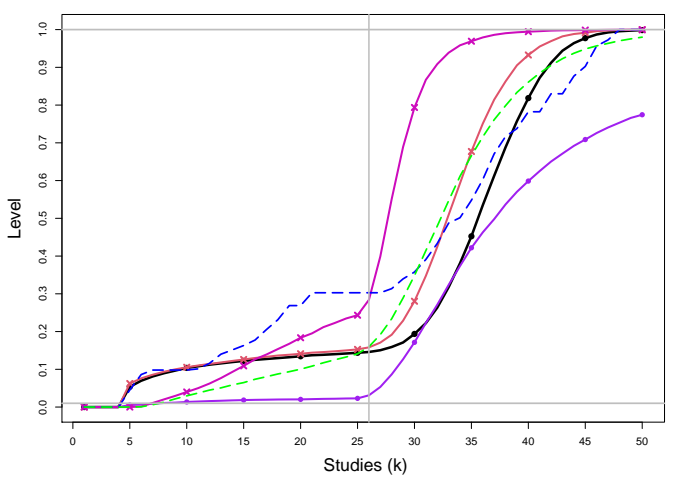

IV REML: $\tau^{2}=1 \mathrm{n}=100$ level $=0.01 \mathrm{~h}=5 \delta=1$ to 2 (chg at 26 th)

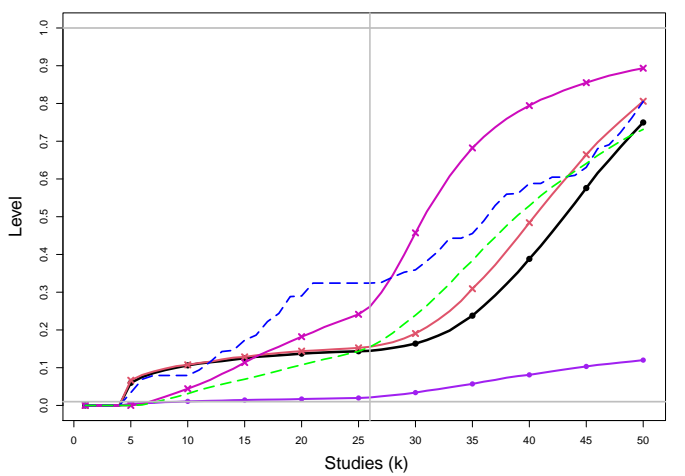

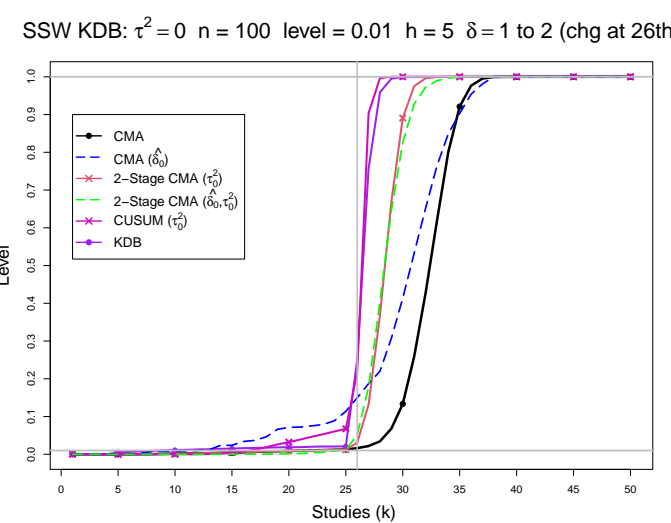

SSW KDB: $\tau^{2}=0.1 \mathrm{n}=100$ level $=0.01 \mathrm{~h}=5 \quad \delta=1$ to 2 (chg at 26th)

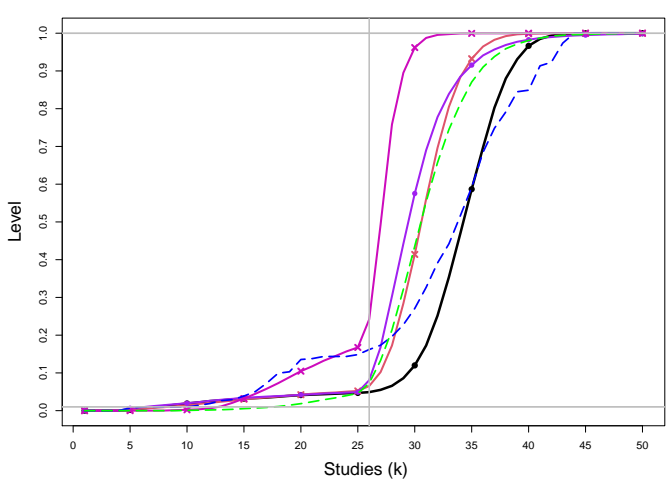

SSW KDB: $\tau^{2}=0.25 \mathrm{n}=100$ level $=0.01 \mathrm{~h}=5 \delta=1$ to 2 (chg at 26th)

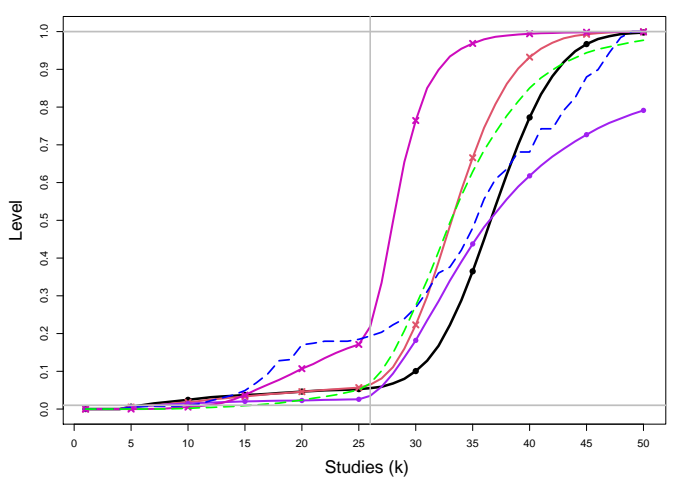

SSW KDB: $\tau^{2}=1 \mathrm{n}=100$ level $=0.01 \mathrm{~h}=5 \delta=1$ to 2 (chg at 26th)

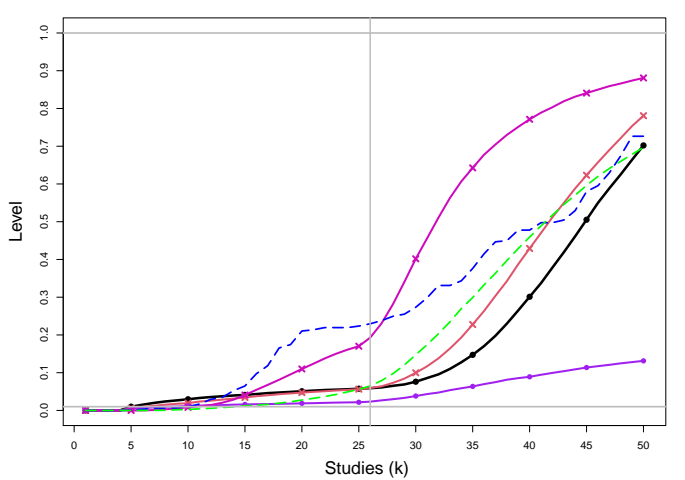

Figure E2. 23: Empirical levels of 1- and 2-stage CMA tests for shift in $\delta$ at .01 nominal level, shift in $\tau^{2}$ at .005 nominal levels and of CUSUM with $h=5$ for equal sample sizes $n_{i C}+n_{i T}=n=100, \tau^{2}=0,0.1,0.25,1$ and a shift from $\delta=1$ to $\delta=2$ at study number 26. Light grey line at .01. 


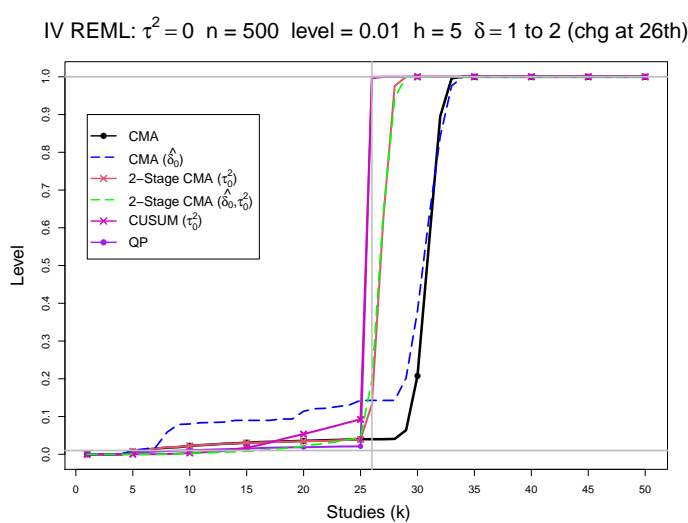

IV REML: $\tau^{2}=0.1 \mathrm{n}=500$ level $=0.01 \mathrm{~h}=5 \delta=1$ to 2 (chg at 26th)

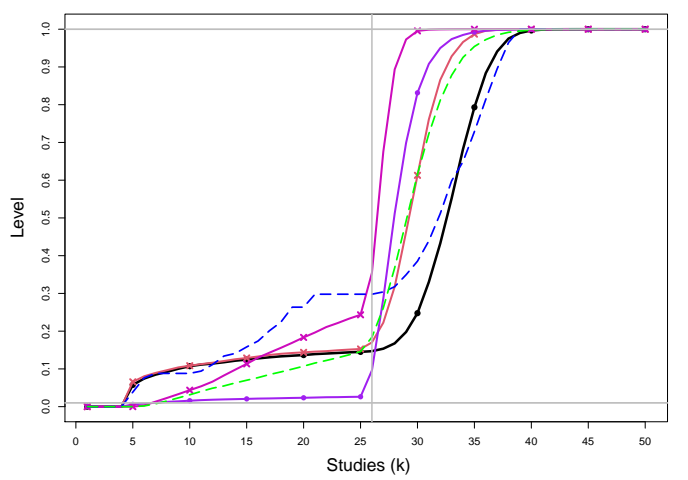

IV REML: $\tau^{2}=0.25 \mathrm{n}=500$ level $=0.01 \mathrm{~h}=5 \quad \delta=1$ to 2 (chg at 26 th)

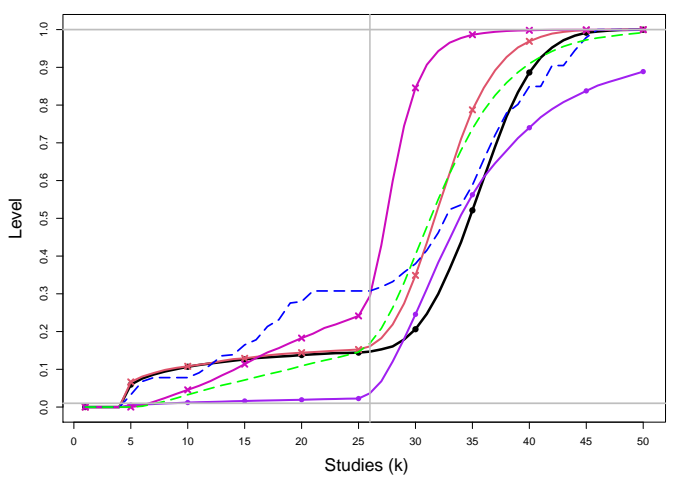

IV REML: $\tau^{2}=1 \mathrm{n}=500$ level $=0.01 \mathrm{~h}=5 \delta=1$ to 2 (chg at 26 th)

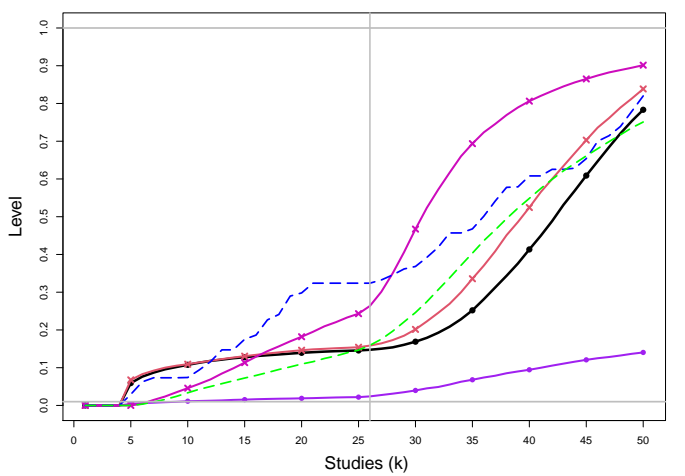

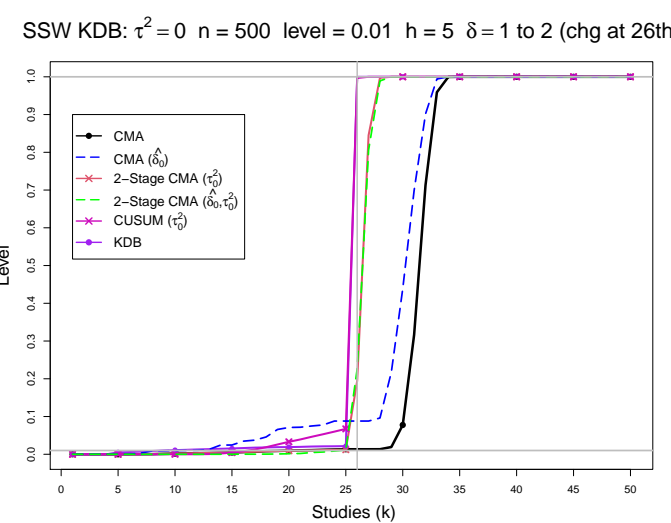

SSW KDB: $\tau^{2}=0.1 \mathrm{n}=500$ level $=0.01 \mathrm{~h}=5 \quad \delta=1$ to 2 (chg at 26th)

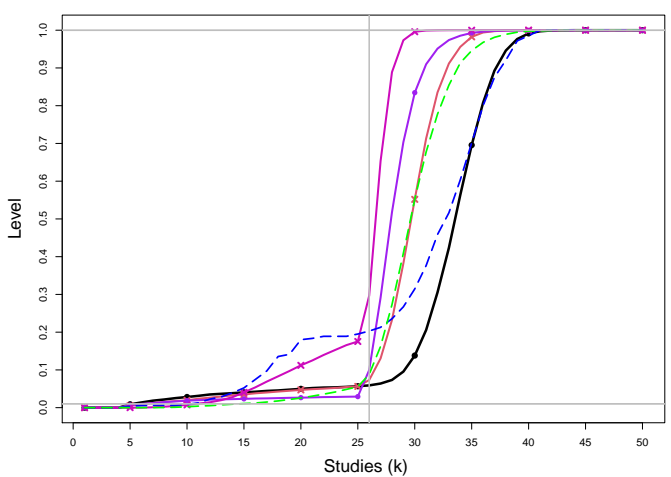

SSW KDB: $\tau^{2}=0.25 \mathrm{n}=500$ level $=0.01 \mathrm{~h}=5 \delta=1$ to 2 (chg at 26th)

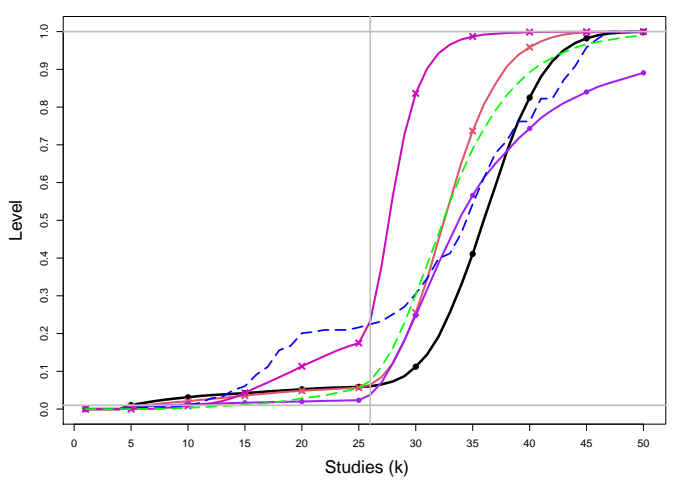

SSW KDB: $\tau^{2}=1 \mathrm{n}=500$ level $=0.01 \mathrm{~h}=5 \delta=1$ to 2 (chg at 26th)

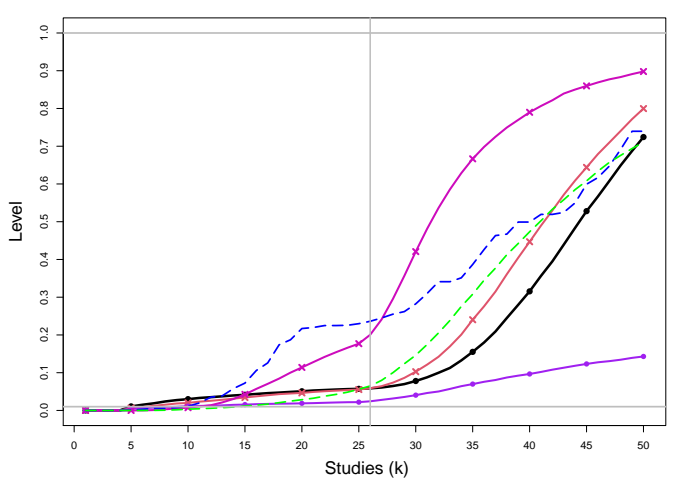

Figure E2. 24: Empirical levels of 1- and 2-stage CMA tests for shift in $\delta$ at .01 nominal level, shift in $\tau^{2}$ at .005 nominal levels and of CUSUM with $h=5$ for equal sample sizes $n_{i C}+n_{i T}=n=500, \tau^{2}=0,0.1,0.25,1$ and a shift from $\delta=1$ to $\delta=2$ at study number 26. Light grey line at .01. 
IV REML: $\tau^{2}=0 \mathrm{n}=1000$ level $=0.01 \mathrm{~h}=5 \quad \delta=1$ to 2 (chg at 26 th)

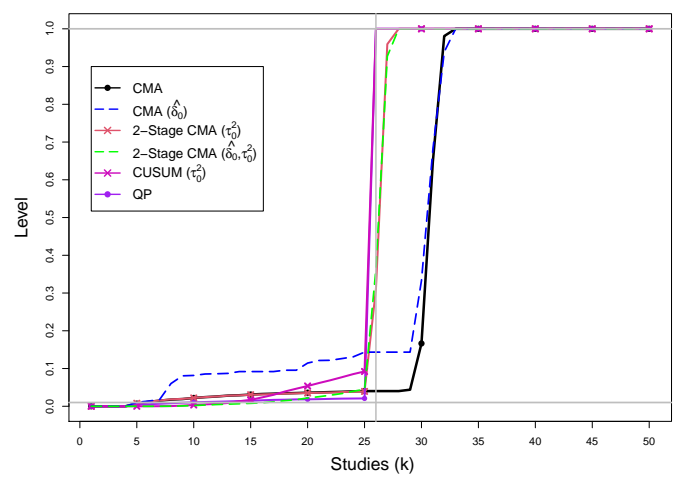

IV REML: $\tau^{2}=0.1 \mathrm{n}=1000$ level $=0.01 \mathrm{~h}=5 \quad \delta=1$ to 2 (chg at 26 th)

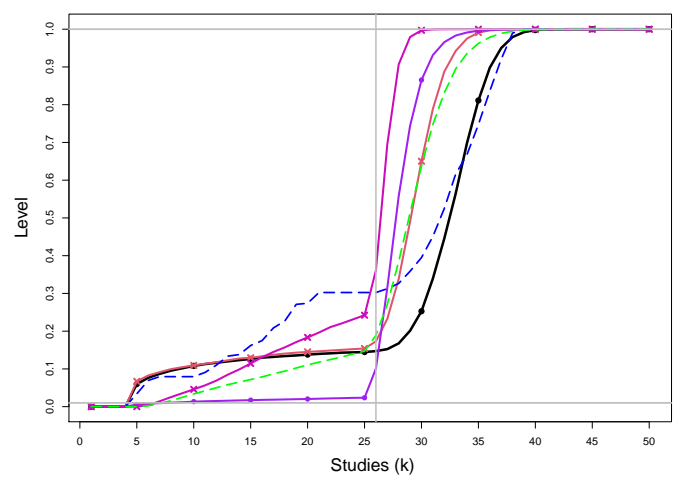

IV REML: $\tau^{2}=0.25 \mathrm{n}=1000$ level $=0.01 \mathrm{~h}=5 \quad \delta=1$ to 2 (chg at 26th)

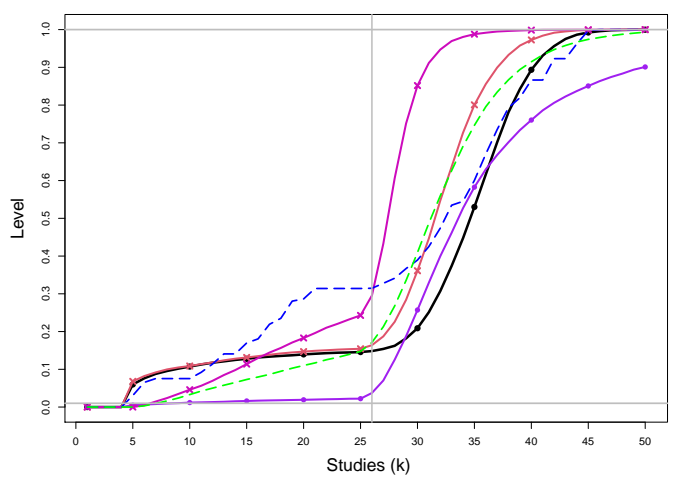

IV REML: $\tau^{2}=1 \mathrm{n}=1000$ level $=0.01 \mathrm{~h}=5 \quad \delta=1$ to 2 (chg at 26 th)

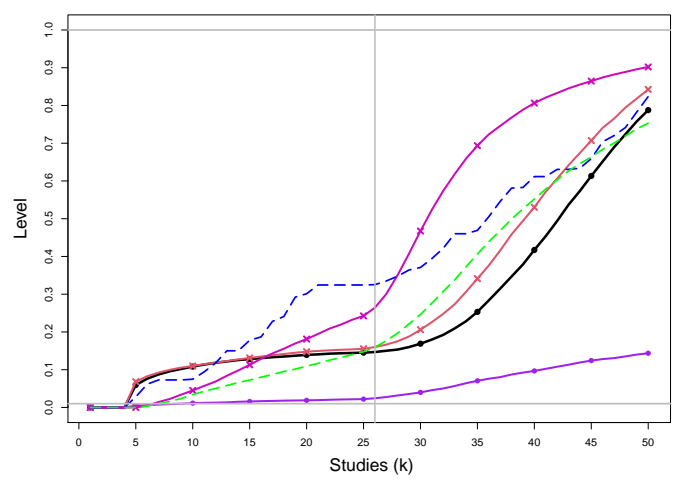

SSW KDB: $\tau^{2}=0 \mathrm{n}=1000$ level $=0.01 \mathrm{~h}=5 \quad \delta=1$ to 2 (chg at 26th)

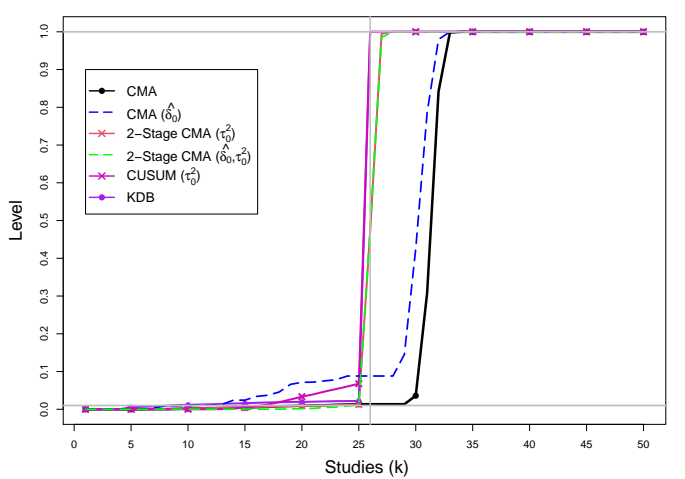

SSW KDB: $\tau^{2}=0.1 \mathrm{n}=1000$ level $=0.01 \mathrm{~h}=5 \quad \delta=1$ to 2 (chg at 26 th)

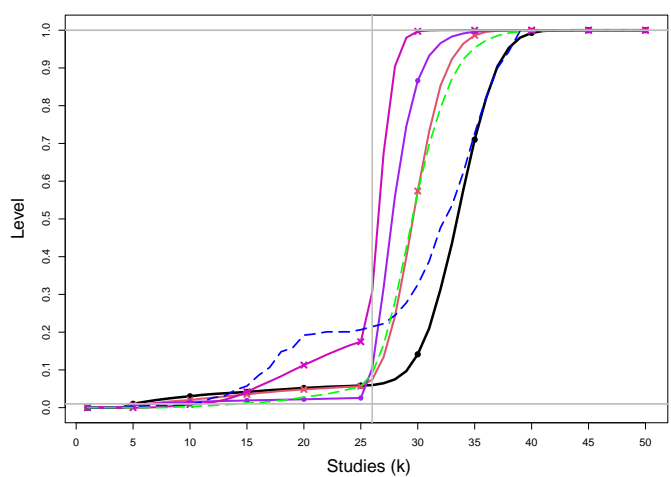

SSW KDB: $\tau^{2}=0.25 \mathrm{n}=1000$ level $=0.01 \mathrm{~h}=5 \delta=1$ to 2 (chg at 26th)

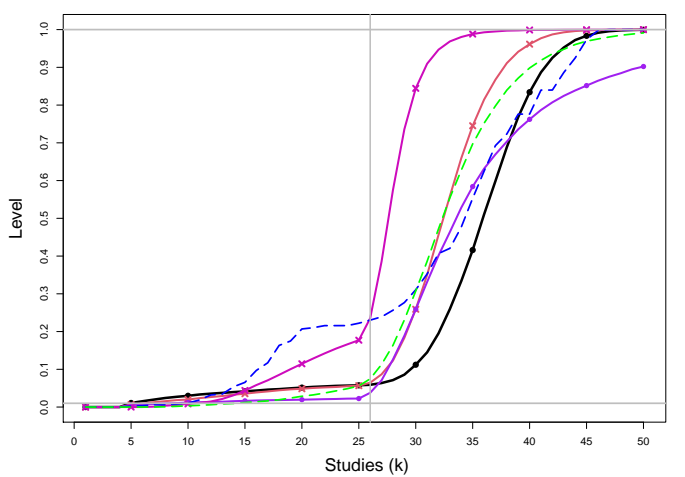

SSW KDB: $\tau^{2}=1 \mathrm{n}=1000$ level $=0.01 \mathrm{~h}=5 \delta=1$ to 2 (chg at 26th)

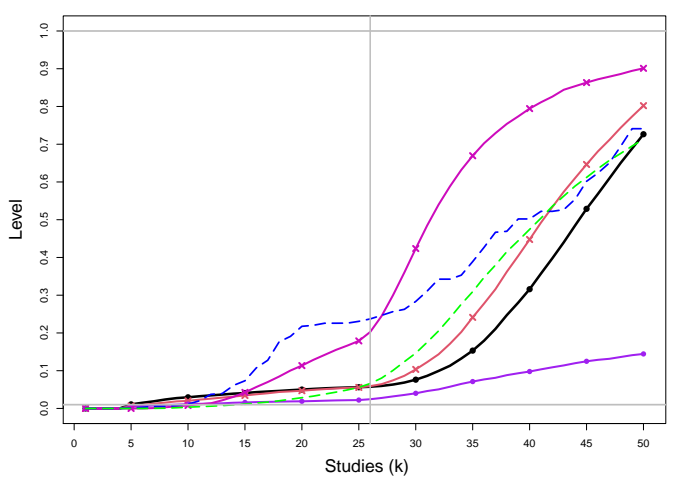

Figure E2. 25: Empirical levels of 1- and 2-stage CMA tests for shift in $\delta$ at .01 nominal level, shift in $\tau^{2}$ at .005 nominal levels and of CUSUM with $h=5$ for equal sample sizes $n_{i C}+n_{i T}=n=1000, \tau^{2}=0,0.1,0.25,1$ and a shift from $\delta=1$ to $\delta=2$ at study number 26. Light grey line at .01. 


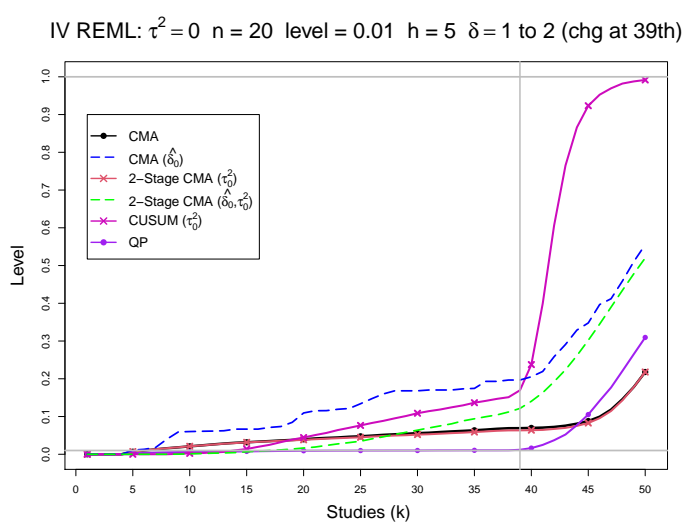

IV REML: $\tau^{2}=0.1 \mathrm{n}=20$ level $=0.01 \mathrm{~h}=5 \delta=1$ to 2 (chg at 39 th)

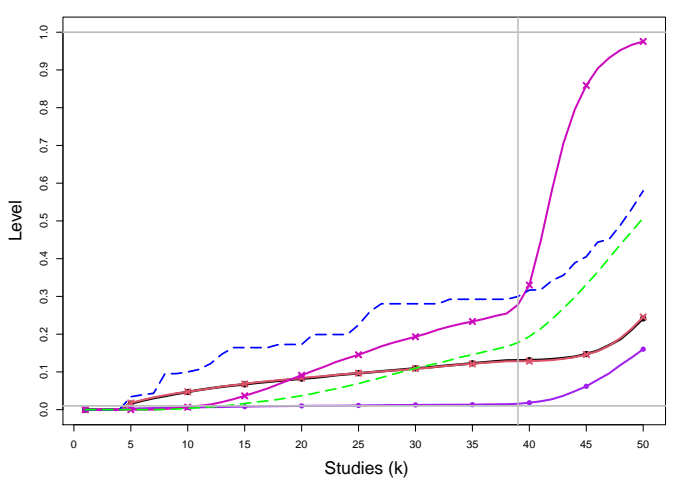

IV REML: $\tau^{2}=0.25 \mathrm{n}=20$ level $=0.01 \mathrm{~h}=5 \delta=1$ to 2 (chg at 39th)

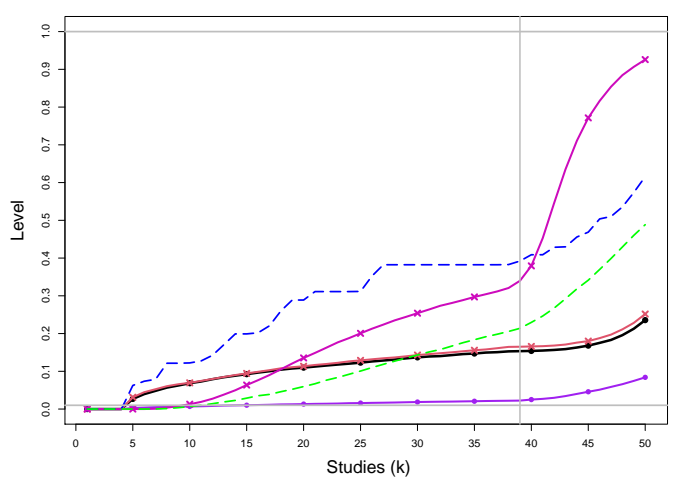

IV REML: $\tau^{2}=1 \mathrm{n}=20$ level $=0.01 \mathrm{~h}=5 \delta=1$ to 2 (chg at 39 th)

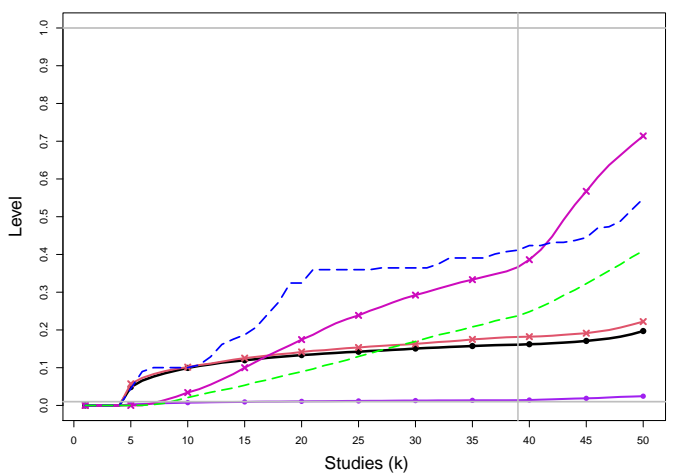

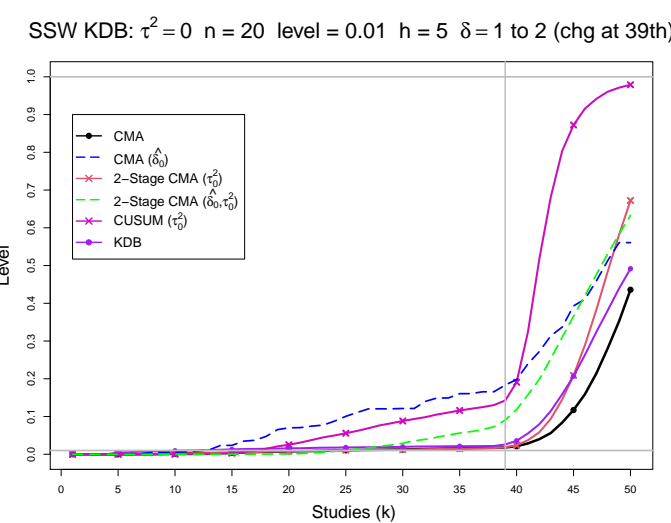

SSW KDB: $\tau^{2}=0.1 \mathrm{n}=20$ level $=0.01 \mathrm{~h}=5 \delta=1$ to 2 (chg at 39 th)

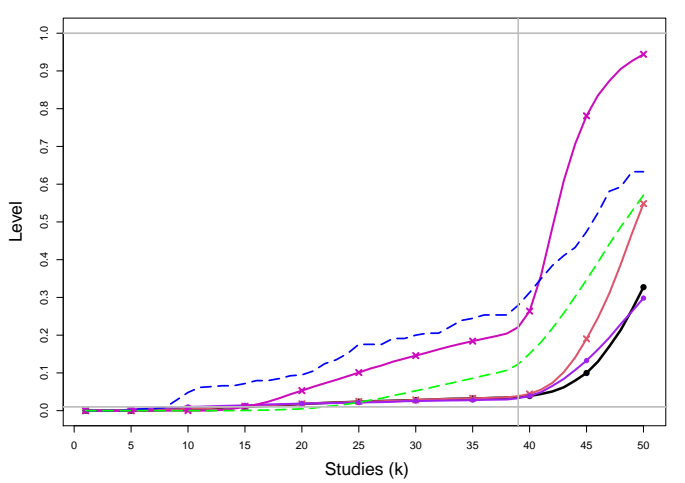

SSW KDB: $\tau^{2}=0.25 \mathrm{n}=20$ level $=0.01 \mathrm{~h}=5 \quad \delta=1$ to 2 (chg at 39th)

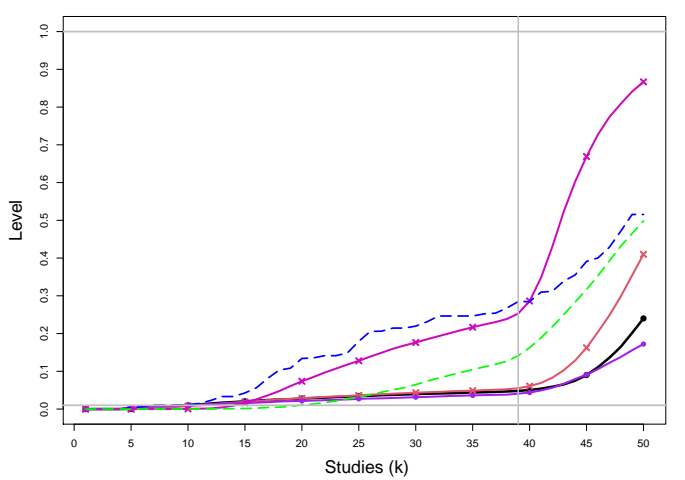

SSW KDB: $\tau^{2}=1 \mathrm{n}=20$ level $=0.01 \mathrm{~h}=5 \delta=1$ to 2 (chg at 39th)

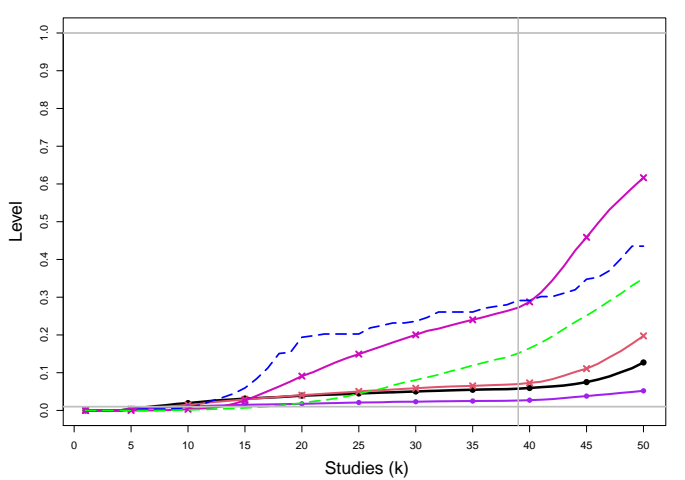

Figure E2. 26: Empirical levels of 1- and 2-stage CMA tests for shift in $\delta$ at .01 nominal level, shift in $\tau^{2}$ at .005 nominal levels and of CUSUM with $h=5$ for equal sample sizes $n_{i C}+n_{i T}=n=20, \tau^{2}=0,0.1,0.25,1$ and a shift from $\delta=1$ to $\delta=2$ at study number 39. Light grey line at .01. 


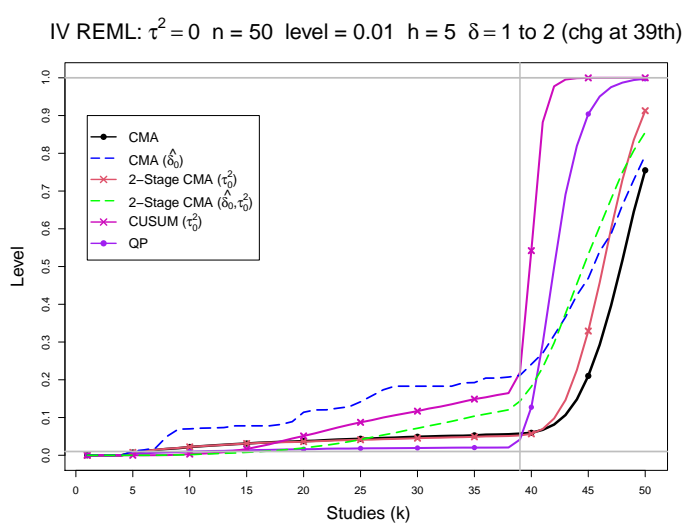

IV REML: $\tau^{2}=0.1 \mathrm{n}=50$ level $=0.01 \mathrm{~h}=5 \delta=1$ to 2 (chg at 39 th)

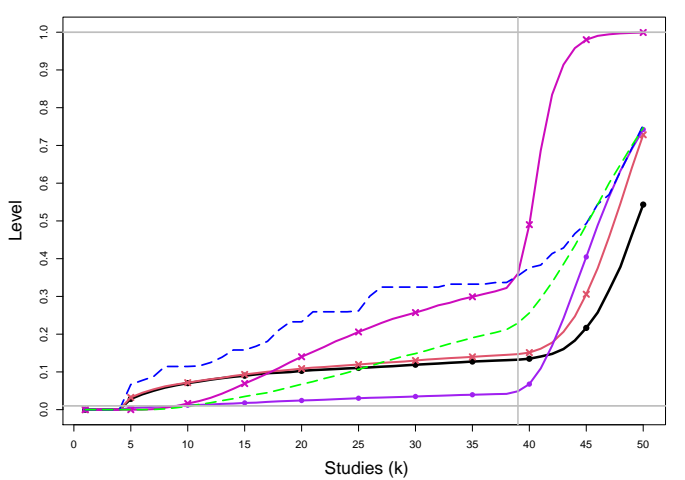

IV REML: $\tau^{2}=0.25 \mathrm{n}=50$ level $=0.01 \mathrm{~h}=5 \delta=1$ to 2 (chg at 39th)

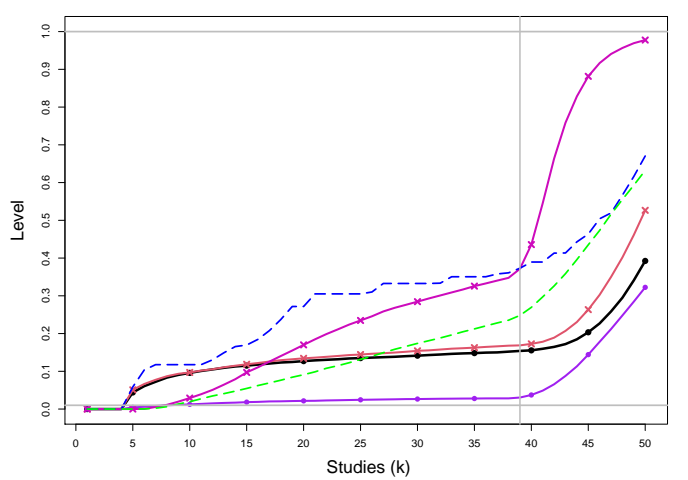

IV REML: $\tau^{2}=1 \mathrm{n}=50$ level $=0.01 \mathrm{~h}=5 \delta=1$ to 2 (chg at 39 th)

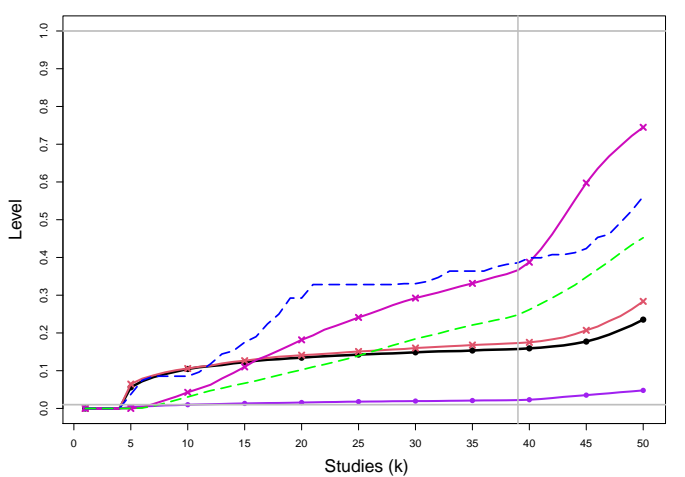

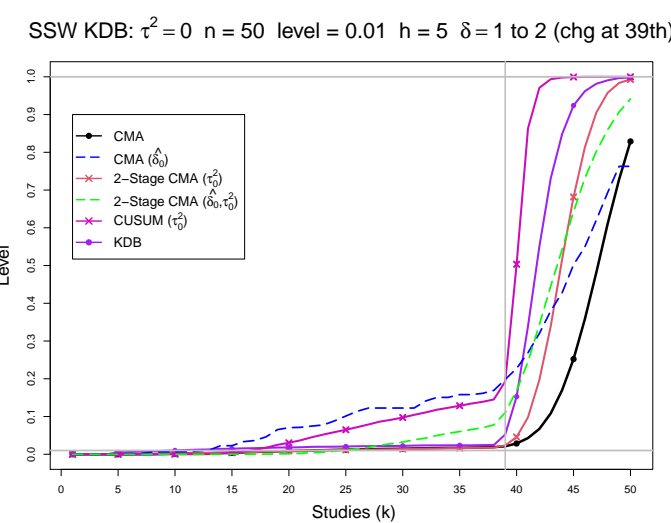

SSW KDB: $\tau^{2}=0.1 \mathrm{n}=50$ level $=0.01 \mathrm{~h}=5 \delta=1$ to 2 (chg at 39 th)

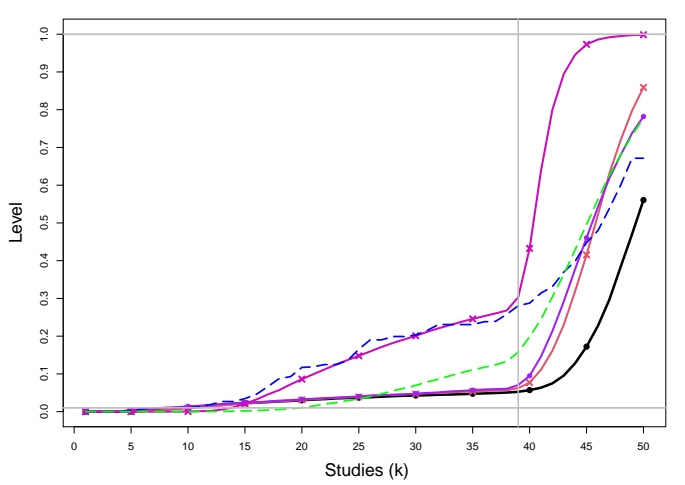

SSW KDB: $\tau^{2}=0.25 \mathrm{n}=50$ level $=0.01 \mathrm{~h}=5 \quad \delta=1$ to 2 (chg at 39th)

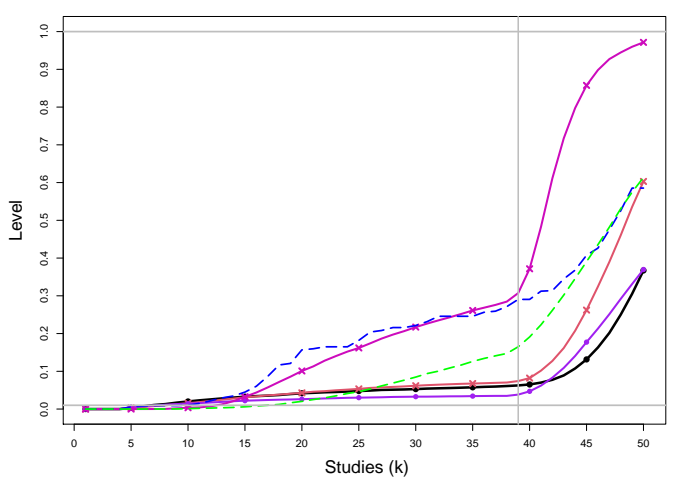

SSW KDB: $\tau^{2}=1 \mathrm{n}=50$ level $=0.01 \mathrm{~h}=5 \delta=1$ to 2 (chg at 39th)

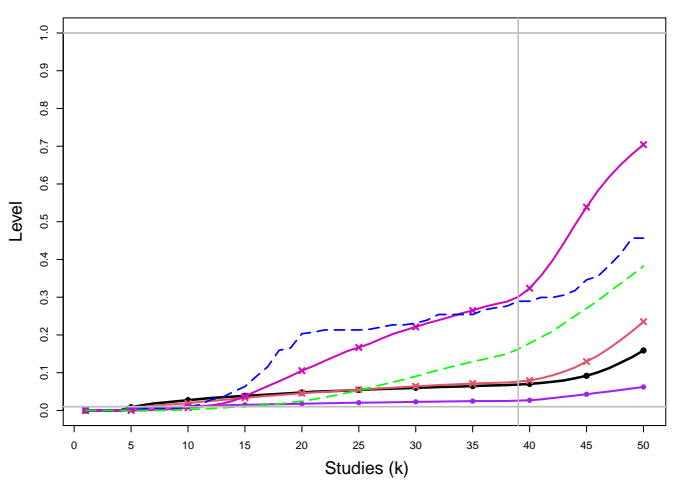

Figure E2. 27: Empirical levels of 1- and 2-stage CMA tests for shift in $\delta$ at .01 nominal level, shift in $\tau^{2}$ at .005 nominal levels and of CUSUM with $h=5$ for equal sample sizes $n_{i C}+n_{i T}=n=50, \tau^{2}=0,0.1,0.25,1$ and a shift from $\delta=1$ to $\delta=2$ at study number 39. Light grey line at .01. 


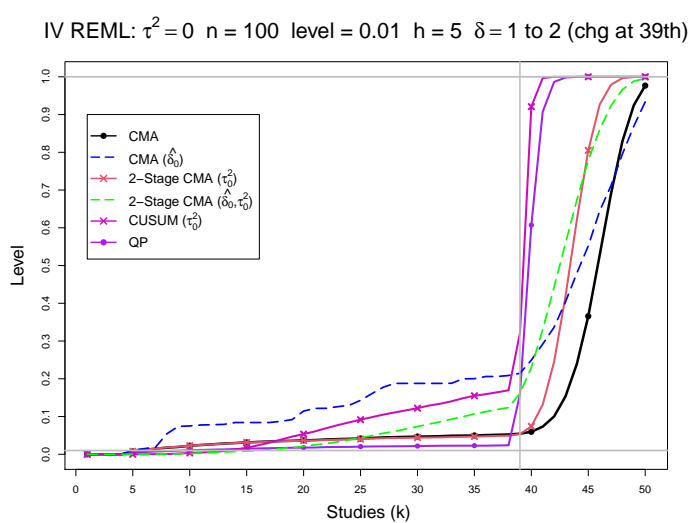

IV REML: $\tau^{2}=0.1 \mathrm{n}=100$ level $=0.01 \mathrm{~h}=5 \delta=1$ to 2 (chg at 39th)

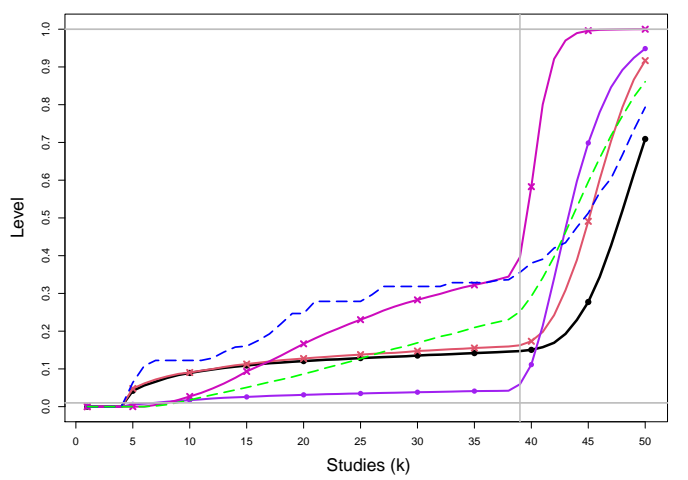

IV REML: $\tau^{2}=0.25 \mathrm{n}=100$ level $=0.01 \mathrm{~h}=5 \delta=1$ to 2 (chg at 39 th)

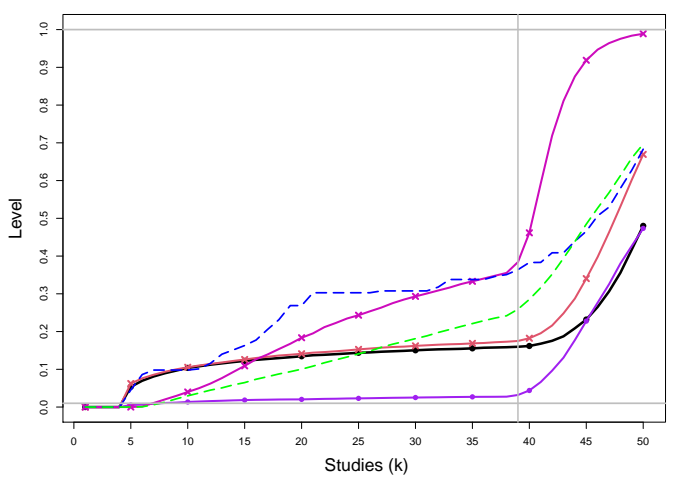

IV REML: $\tau^{2}=1 \mathrm{n}=100$ level $=0.01 \mathrm{~h}=5 \delta=1$ to 2 (chg at 39th)

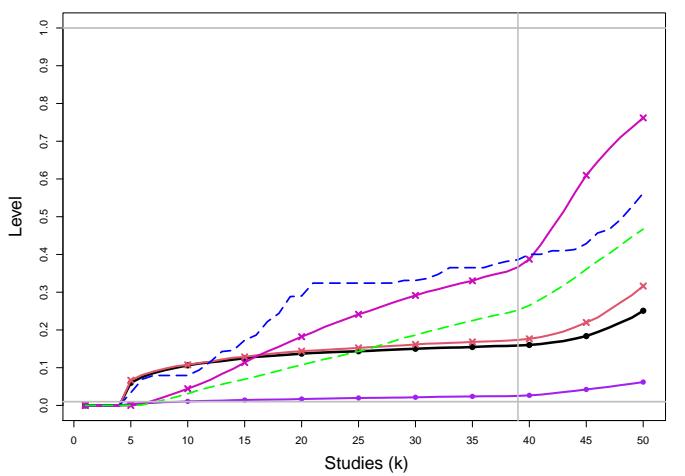

SSW KDB: $\tau^{2}=0 \mathrm{n}=100$ level $=0.01 \mathrm{~h}=5 \quad \delta=1$ to 2 (chg at 39th)

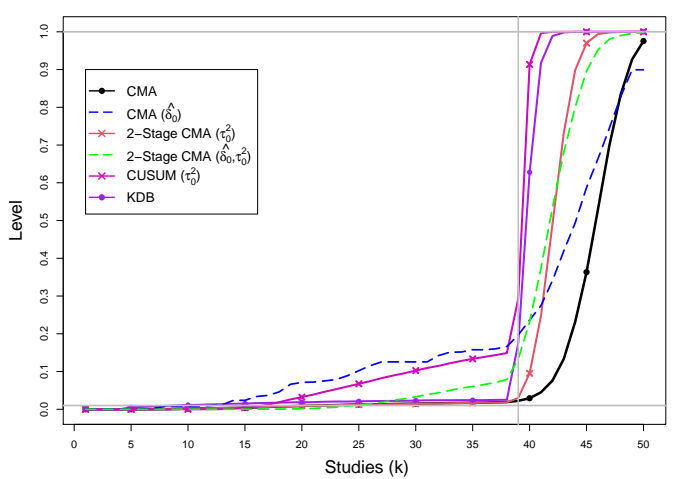

SSW KDB: $\tau^{2}=0.1 \mathrm{n}=100$ level $=0.01 \mathrm{~h}=5 \quad \delta=1$ to 2 (chg at 39th)

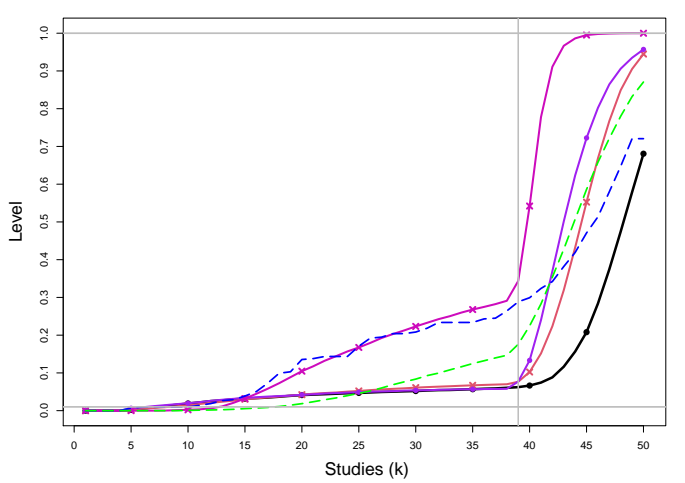

SSW KDB: $\tau^{2}=0.25 \mathrm{n}=100$ level $=0.01 \mathrm{~h}=5 \delta=1$ to 2 (chg at 39th)

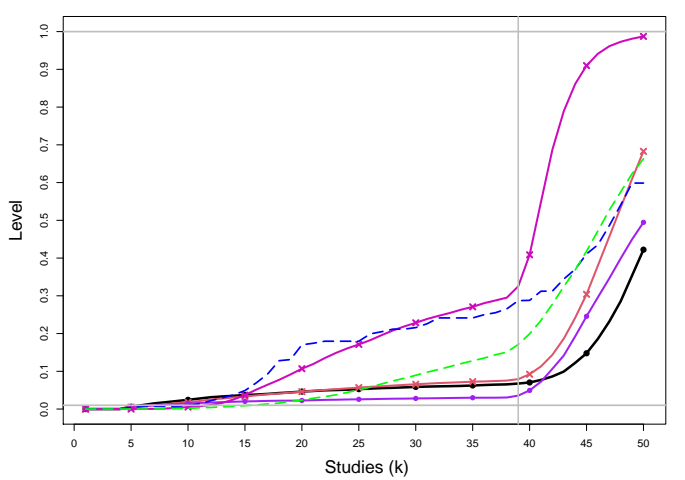

SSW KDB: $\tau^{2}=1 \mathrm{n}=100$ level $=0.01 \mathrm{~h}=5 \delta=1$ to 2 (chg at 39th)

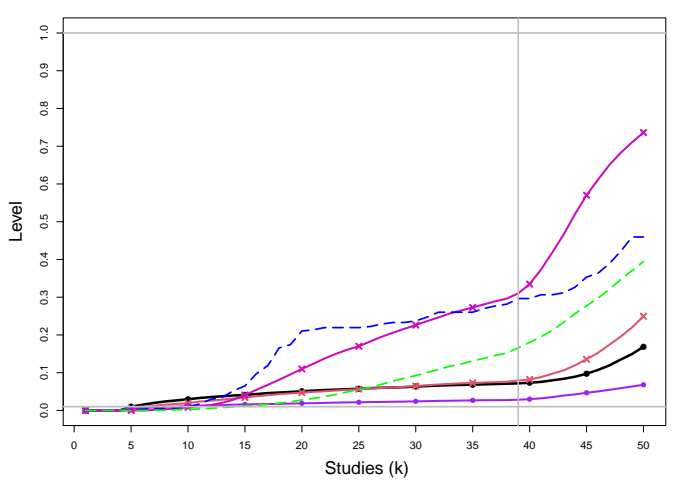

Figure E2. 28: Empirical levels of 1- and 2-stage CMA tests for shift in $\delta$ at .01 nominal level, shift in $\tau^{2}$ at .005 nominal levels and of CUSUM with $h=5$ for equal sample sizes $n_{i C}+n_{i T}=n=100, \tau^{2}=0,0.1,0.25,1$ and a shift from $\delta=1$ to $\delta=2$ at study number 39. Light grey line at .01. 


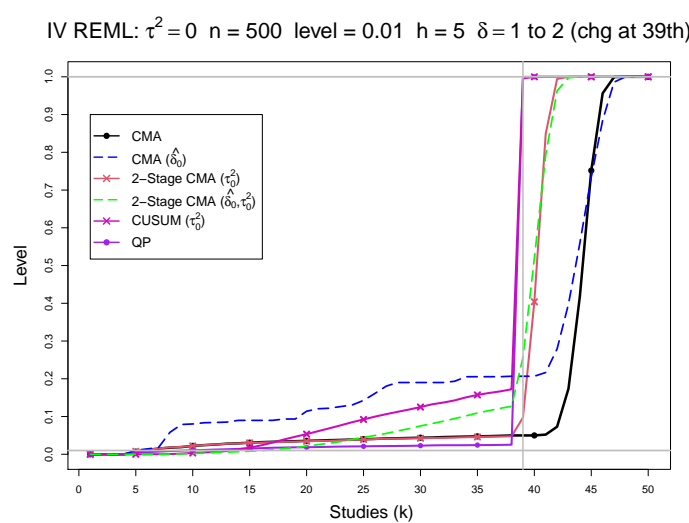

IV REML: $\tau^{2}=0.1 \mathrm{n}=500$ level $=0.01 \mathrm{~h}=5 \delta=1$ to 2 (chg at 39 th)

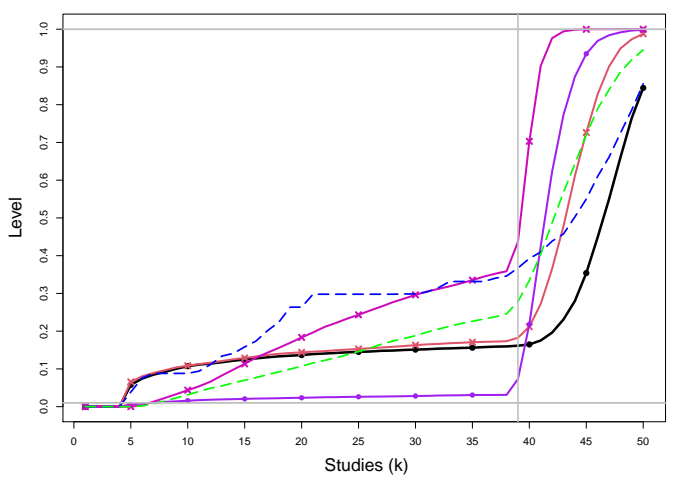

IV REML: $\tau^{2}=0.25 \mathrm{n}=500$ level $=0.01 \mathrm{~h}=5 \delta=1$ to 2 (chg at 39 th)

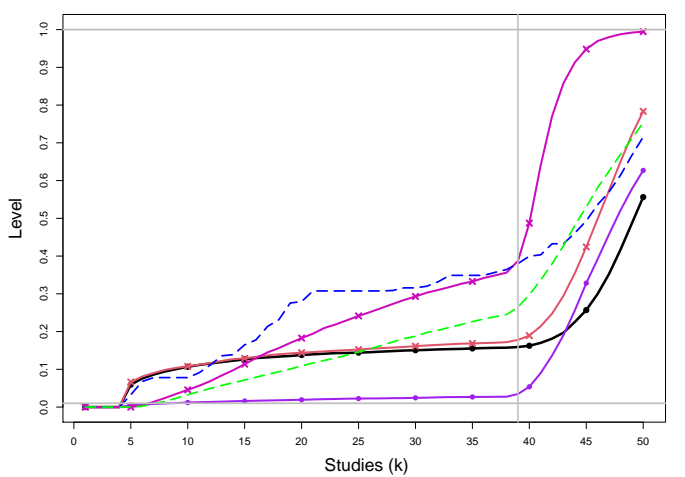

IV REML: $\tau^{2}=1 \mathrm{n}=500$ level $=0.01 \mathrm{~h}=5 \delta=1$ to 2 (chg at 39 th)

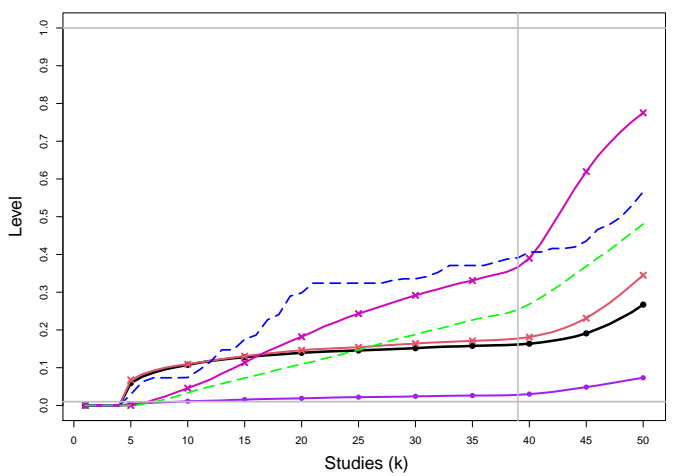

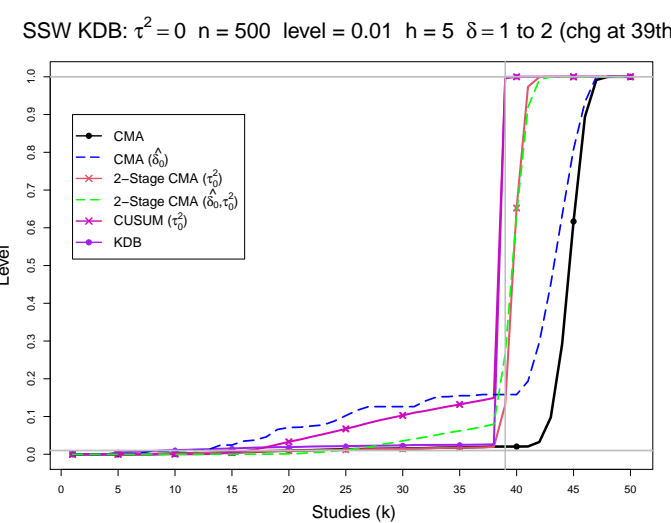

SSW KDB: $\tau^{2}=0.1 \mathrm{n}=500$ level $=0.01 \mathrm{~h}=5 \delta=1$ to 2 (chg at 39 th)

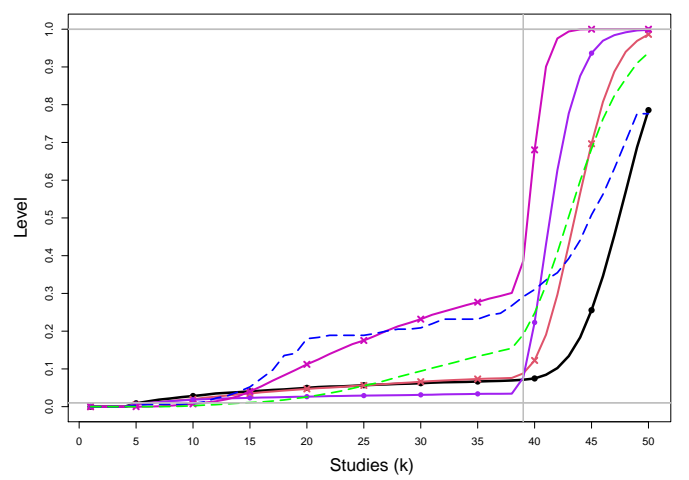

SSW KDB: $\tau^{2}=0.25 \mathrm{n}=500$ level $=0.01 \mathrm{~h}=5 \delta=1$ to 2 (chg at 39 th)

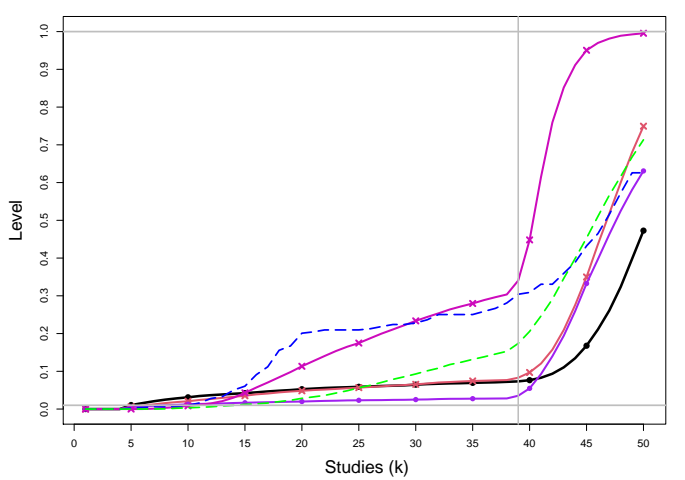

SSW KDB: $\tau^{2}=1 \mathrm{n}=500$ level $=0.01 \mathrm{~h}=5 \delta=1$ to 2 (chg at 39 th)

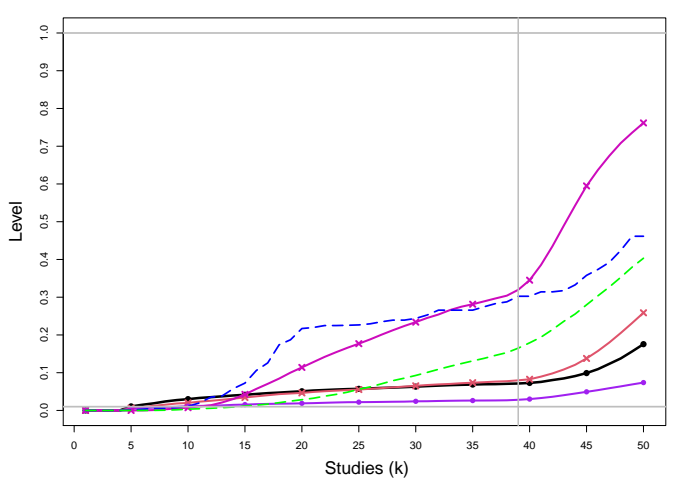

Figure E2. 29: Empirical levels of 1- and 2-stage CMA tests for shift in $\delta$ at .01 nominal level, shift in $\tau^{2}$ at .005 nominal levels and of CUSUM with $h=5$ for equal sample sizes $n_{i C}+n_{i T}=n=500, \tau^{2}=0,0.1,0.25,1$ and a shift from $\delta=1$ to $\delta=2$ at study number 39. Light grey line at .01. 
IV REML: $\tau^{2}=0 \mathrm{n}=1000$ level $=0.01 \mathrm{~h}=5 \quad \delta=1$ to 2 (chg at 39 th)

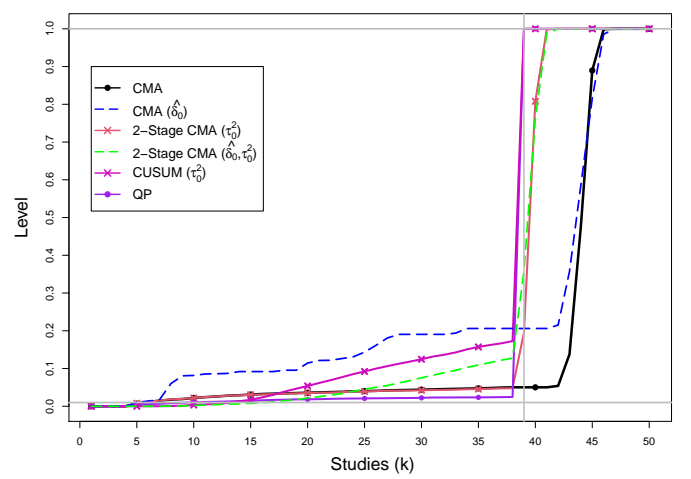

IV REML: $\tau^{2}=0.1 \mathrm{n}=1000$ level $=0.01 \mathrm{~h}=5 \quad \delta=1$ to 2 (chg at 39th)

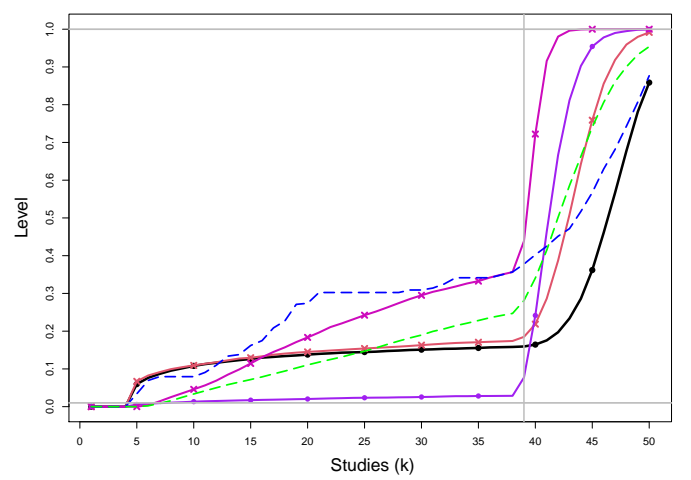

IV REML: $\tau^{2}=0.25 \mathrm{n}=1000$ level $=0.01 \mathrm{~h}=5 \quad \delta=1$ to 2 (chg at 39 th)

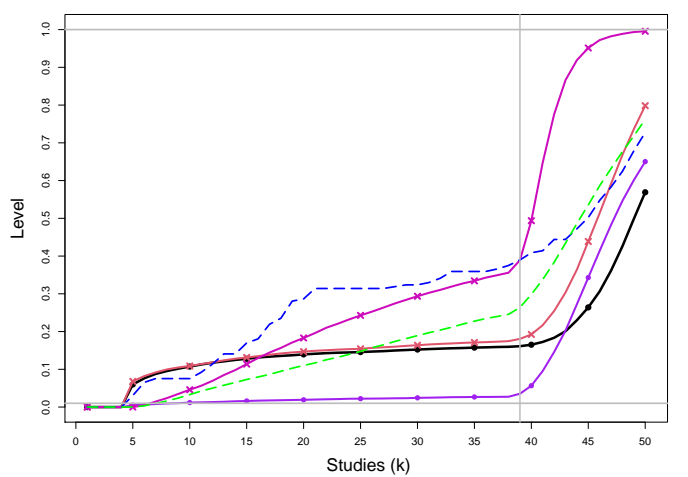

IV REML: $\tau^{2}=1 \mathrm{n}=1000$ level $=0.01 \mathrm{~h}=5 \quad \delta=1$ to 2 (chg at 39 th)

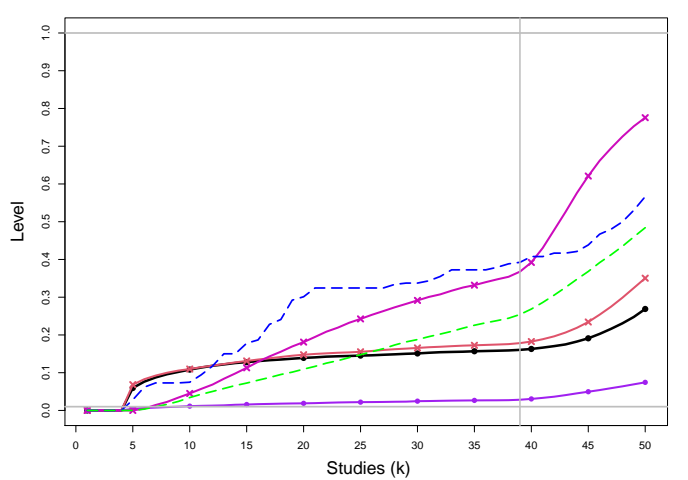

SSW KDB: $\tau^{2}=0 \quad n=1000$ level $=0.01 \quad h=5 \delta=1$ to 2 (chg at 39th)

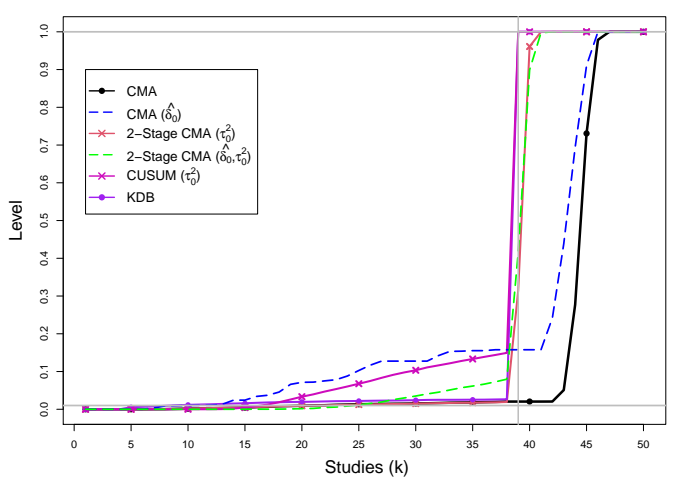

SSW KDB: $\tau^{2}=0.1 \mathrm{n}=1000$ level $=0.01 \mathrm{~h}=5 \quad \delta=1$ to 2 (chg at 39th)

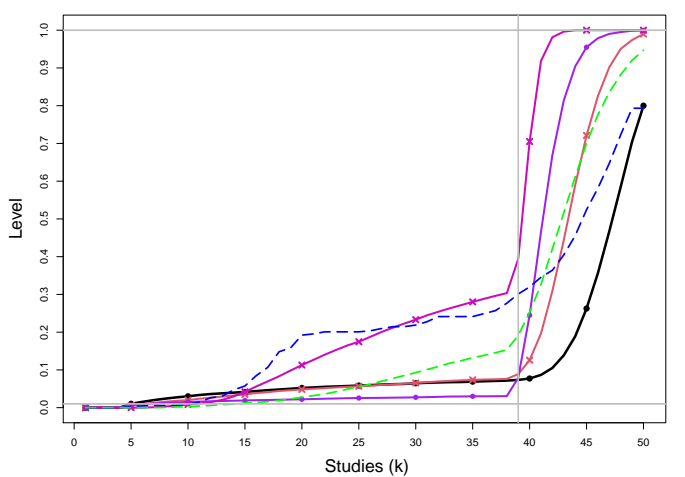

SSW KDB: $\tau^{2}=0.25 \mathrm{n}=1000$ level $=0.01 \mathrm{~h}=5 \delta=1$ to 2 (chg at 39th)

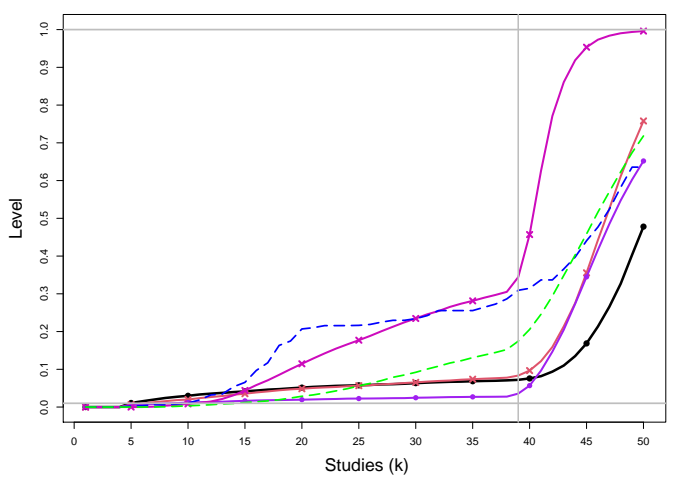

SSW KDB: $\tau^{2}=1 \mathrm{n}=1000$ level $=0.01 \mathrm{~h}=5 \delta=1$ to 2 (chg at 39th)

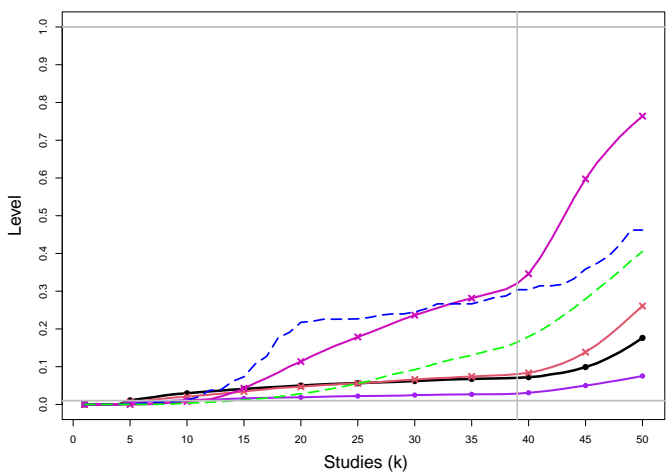

Figure E2. 30: Empirical levels of 1- and 2-stage CMA tests for shift in $\delta$ at .01 nominal level, shift in $\tau^{2}$ at .005 nominal levels and of CUSUM with $h=5$ for equal sample sizes $n_{i C}+n_{i T}=n=1000, \tau^{2}=0,0.1,0.25,1$ and a shift from $\delta=1$ to $\delta=2$ at study number 39. Light grey line at .01. 


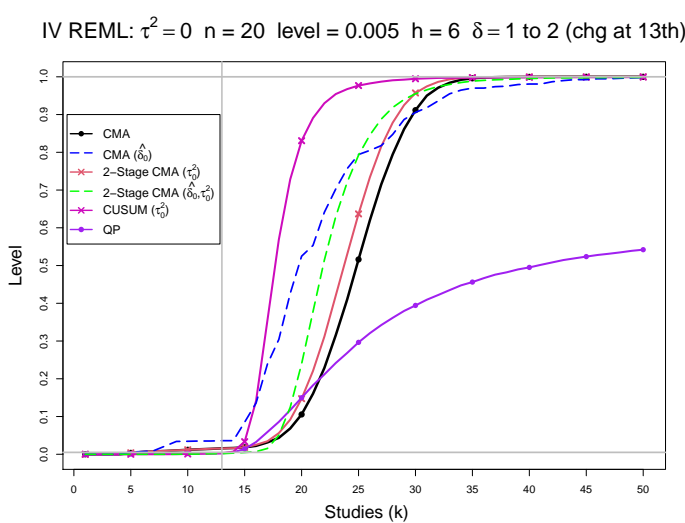

IV REML: $\tau^{2}=0.1 \mathrm{n}=20$ level $=0.005 \mathrm{~h}=6 \delta=1$ to 2 (chg at 13 th)

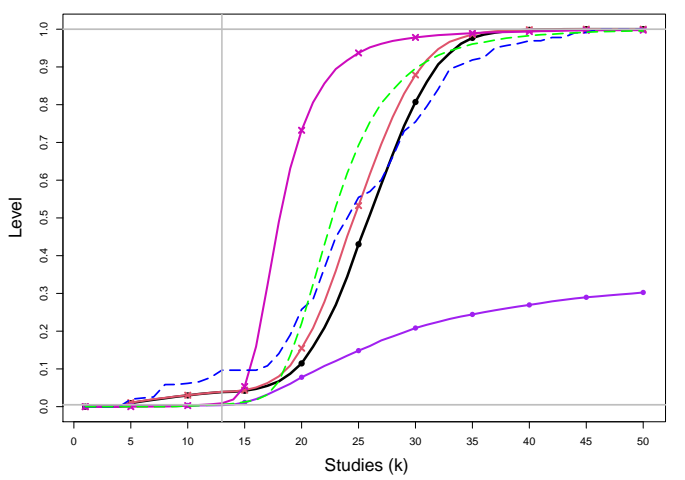

IV REML: $\tau^{2}=0.25 \mathrm{n}=20$ level $=0.005 \mathrm{~h}=6 \delta=1$ to 2 (chg at 13 th)

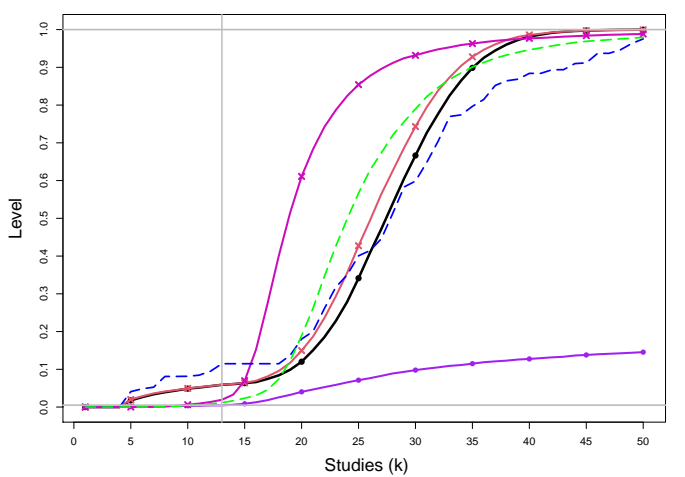

IV REML: $\tau^{2}=1 \mathrm{n}=20$ level $=0.005 \mathrm{~h}=6 \delta=1$ to 2 (chg at 13 th)

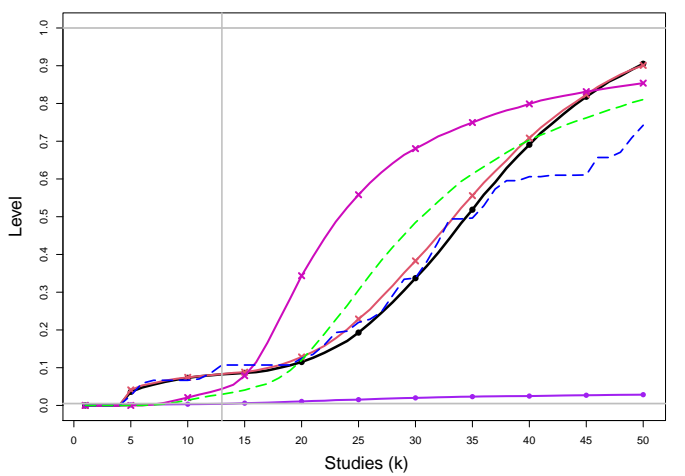

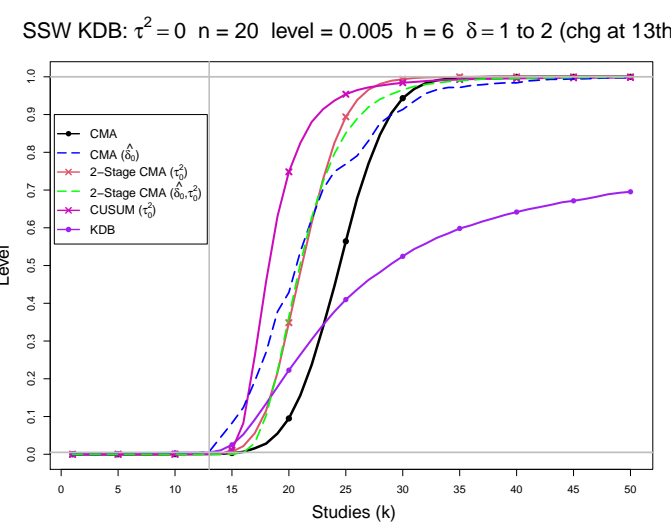

SSW KDB: $\tau^{2}=0.1 \mathrm{n}=20$ level $=0.005 \mathrm{~h}=6 \delta=1$ to 2 (chg at 13 th)

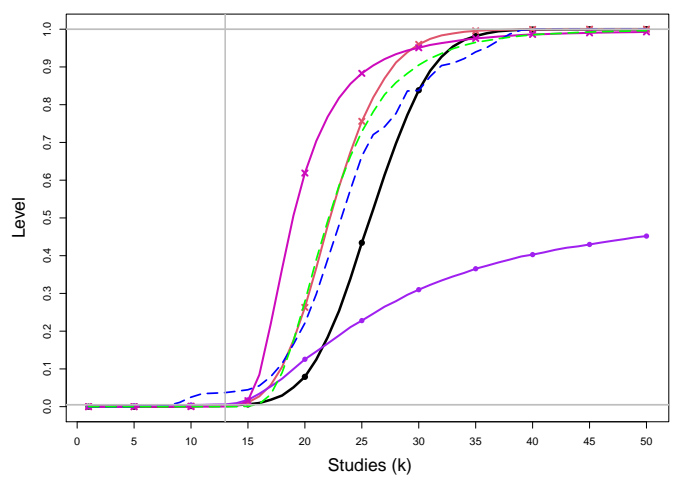

SSW KDB: $\tau^{2}=0.25 \mathrm{n}=20$ level $=0.005 \mathrm{~h}=6 \delta=1$ to 2 (chg at 13 th)

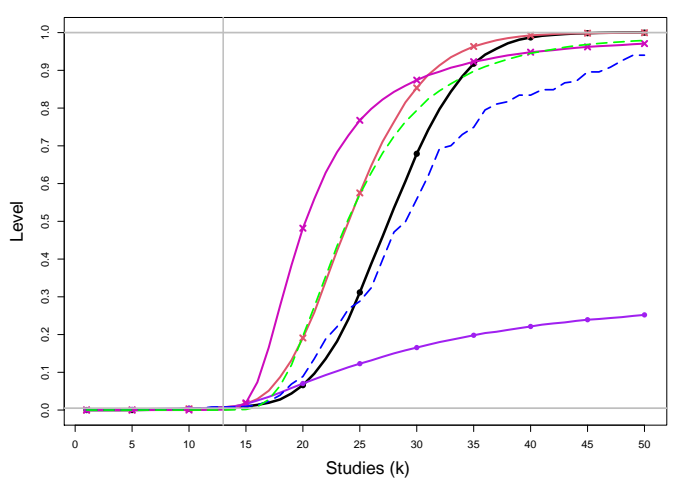

SSW KDB: $\tau^{2}=1 \mathrm{n}=20$ level $=0.005 \mathrm{~h}=6 \delta=1$ to 2 (chg at 13 th)

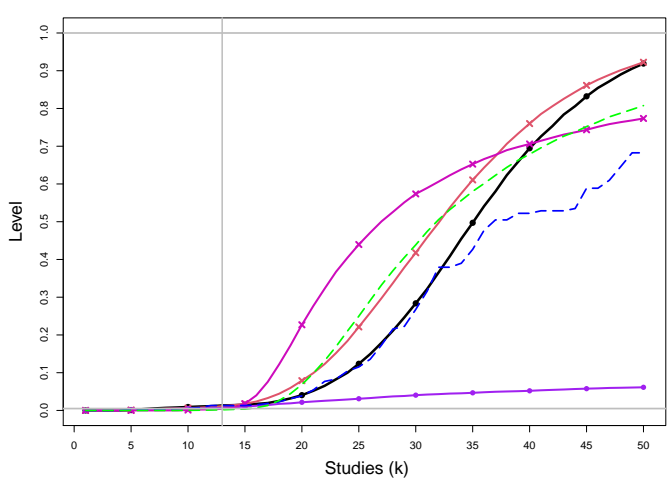

Figure E2. 31: Empirical levels of 1- and 2-stage CMA tests for shift in $\delta$ at .005 nominal level, shift in $\tau^{2}$ at .0025 nominal levels and of CUSUM with $h=6$ for equal sample sizes $n_{i C}+n_{i T}=n=20, \tau^{2}=0,0.1,0.25,1$ and a shift from $\delta=1$ to $\delta=2$ at study number 13. Light grey line at .005. 


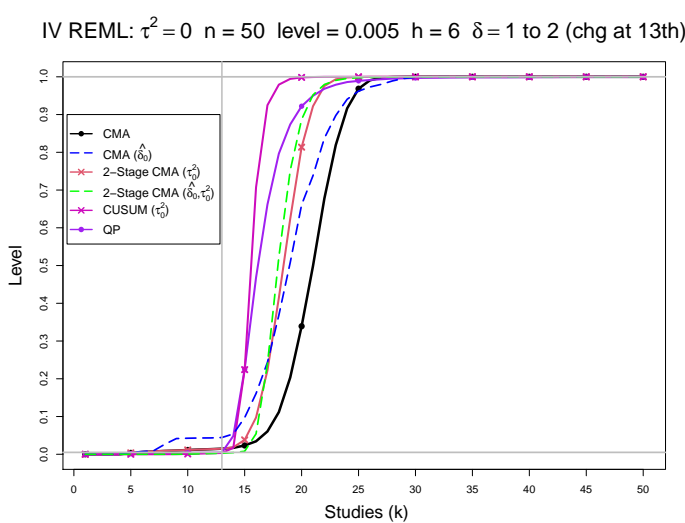

IV REML: $\tau^{2}=0.1 \mathrm{n}=50$ level $=0.005 \mathrm{~h}=6 \delta=1$ to 2 (chg at 13th)

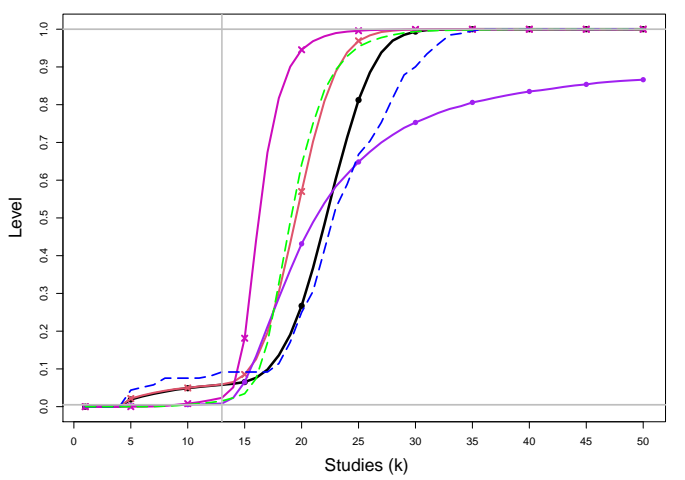

IV REML: $\tau^{2}=0.25 \mathrm{n}=50$ level $=0.005 \mathrm{~h}=6 \delta=1$ to 2 (chg at 13 th)

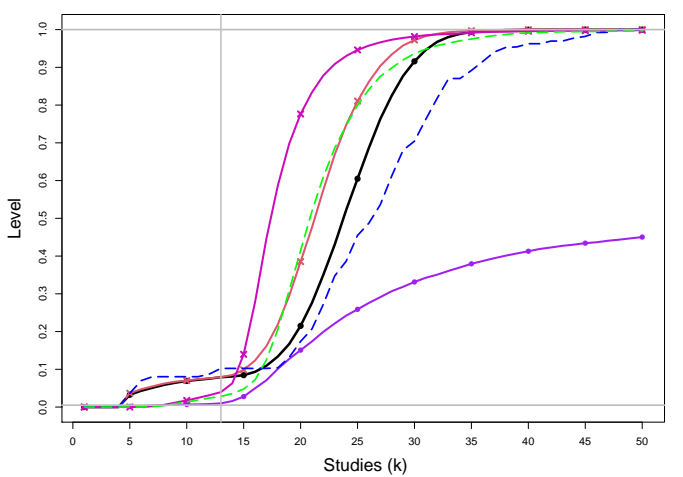

IV REML: $\tau^{2}=1 \mathrm{n}=50$ level $=0.005 \mathrm{~h}=6 \delta=1$ to 2 (chg at 13 th)

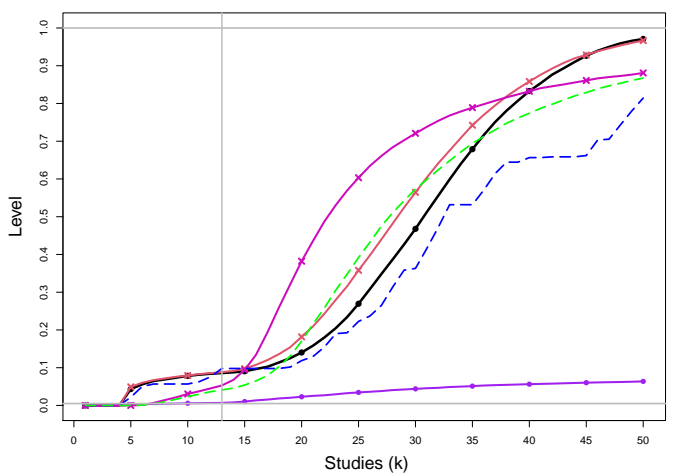

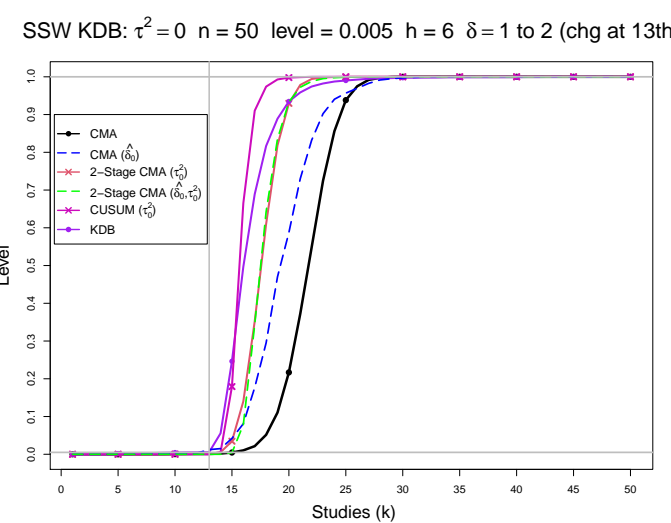

SSW KDB: $\tau^{2}=0.1 \mathrm{n}=50$ level $=0.005 \mathrm{~h}=6 \delta=1$ to 2 (chg at 13 th)

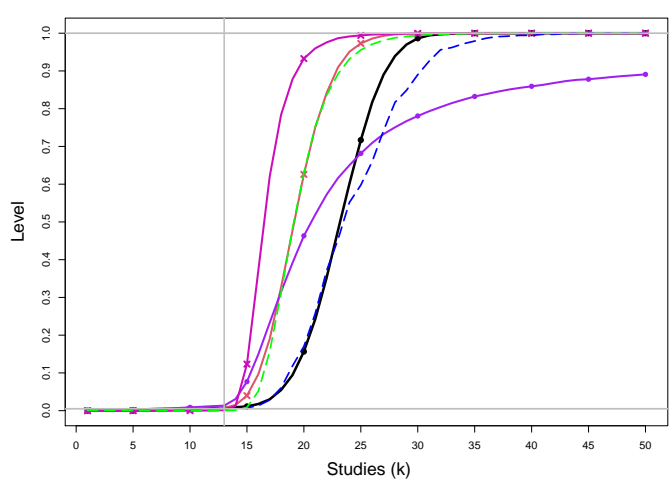

SSW KDB: $\tau^{2}=0.25 \mathrm{n}=50$ level $=0.005 \mathrm{~h}=6 \delta=1$ to 2 (chg at 13 th)

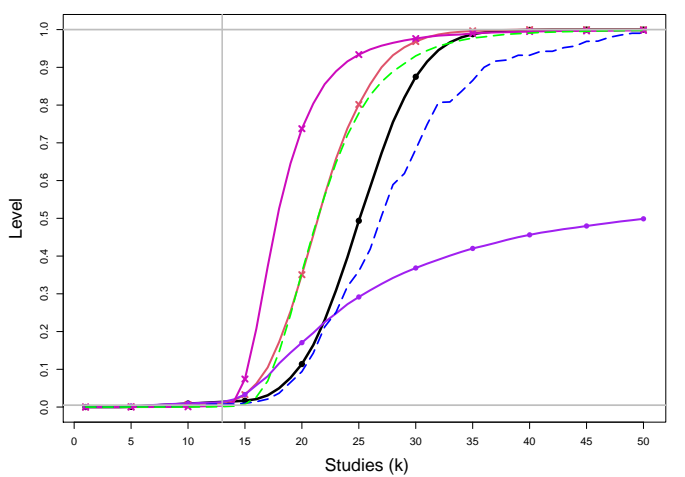

SSW KDB: $\tau^{2}=1 \mathrm{n}=50$ level $=0.005 \mathrm{~h}=6 \delta=1$ to 2 (chg at 13 th)

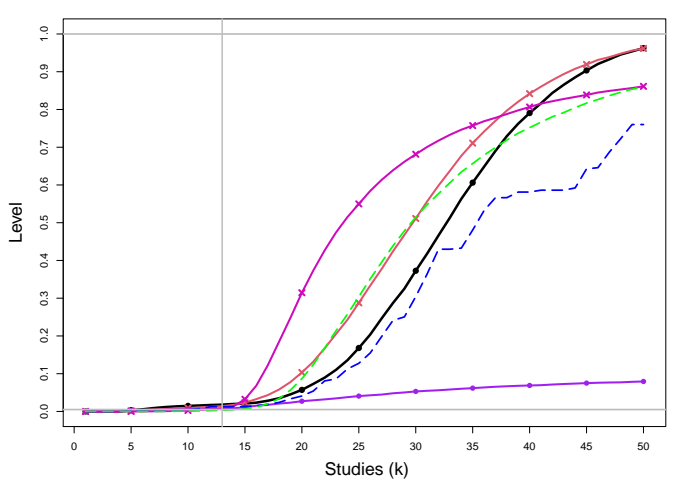

Figure E2. 32: Empirical levels of 1- and 2-stage CMA tests for shift in $\delta$ at .005 nominal level, shift in $\tau^{2}$ at .0025 nominal levels and of CUSUM with $h=6$ for equal sample sizes $n_{i C}+n_{i T}=n=50, \tau^{2}=0,0.1,0.25,1$ and a shift from $\delta=1$ to $\delta=2$ at study number 13. Light grey line at .005. 


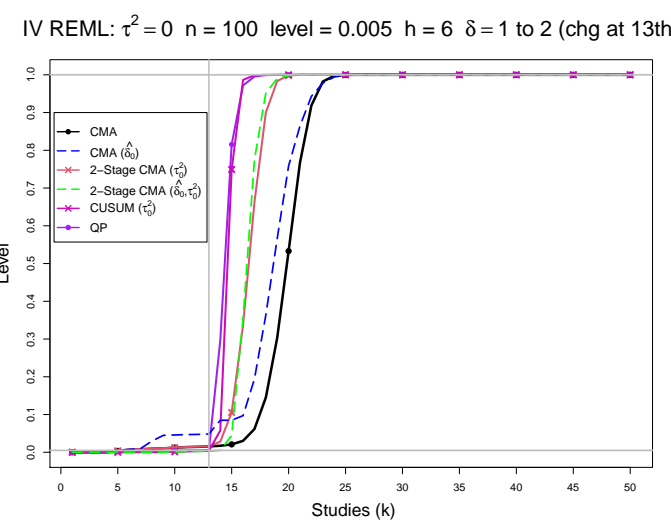

IV REML: $\tau^{2}=0.1 \mathrm{n}=100$ level $=0.005 \mathrm{~h}=6 \delta=1$ to 2 (chg at 13 th)

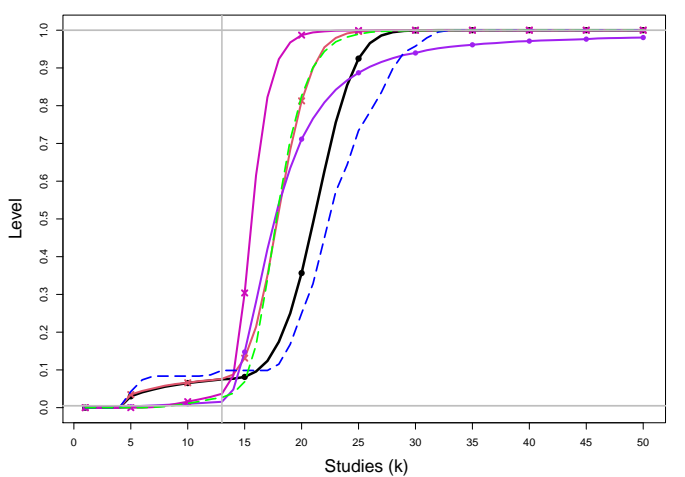

IV REML: $\tau^{2}=0.25 \mathrm{n}=100$ level $=0.005 \mathrm{~h}=6 \delta=1$ to 2 (chg at 13 th)

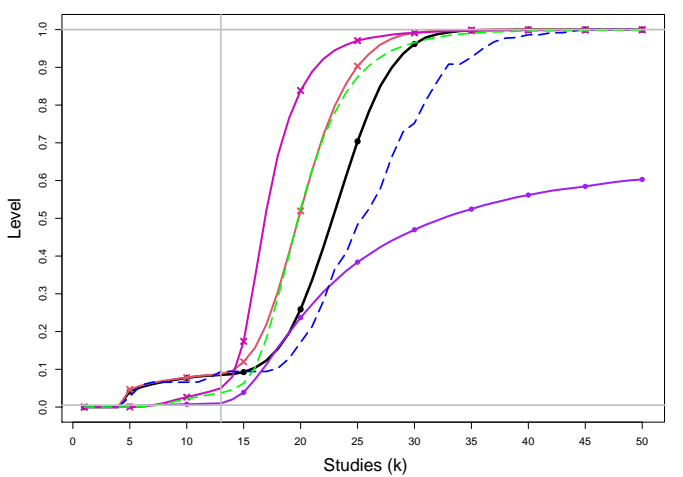

IV REML: $\tau^{2}=1 \mathrm{n}=100$ level $=0.005 \mathrm{~h}=6 \delta=1$ to 2 (chg at 13 th)

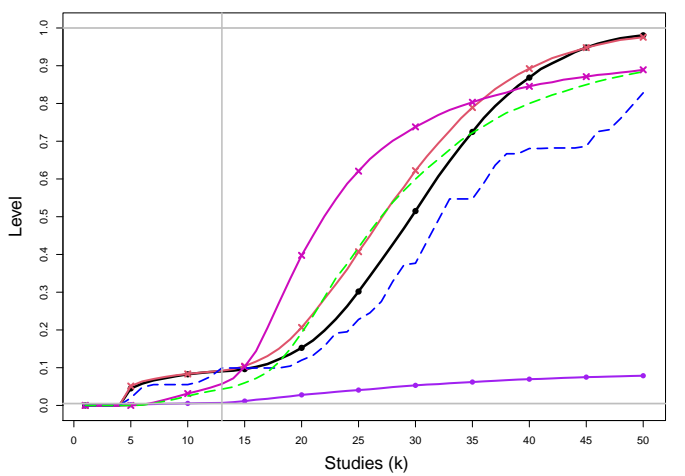

SSW KDB: $\tau^{2}=0 \mathrm{n}=100$ level $=0.005 \mathrm{~h}=6 \delta=1$ to 2 (chg at 13 th)

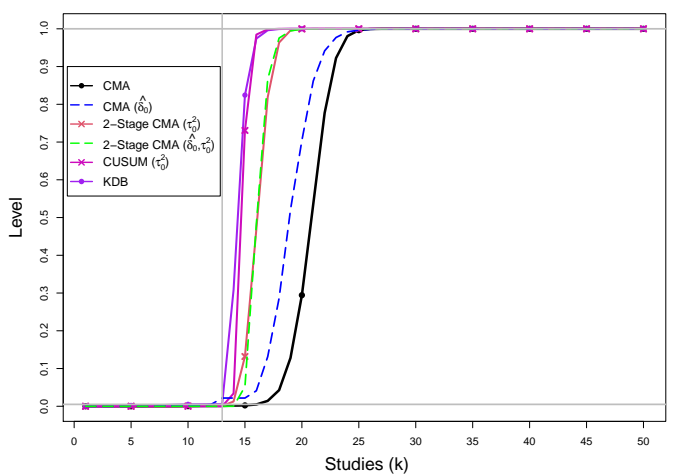

SSW KDB: $\tau^{2}=0.1 \mathrm{n}=100$ level $=0.005 \mathrm{~h}=6 \delta=1$ to 2 (chg at 13 th)

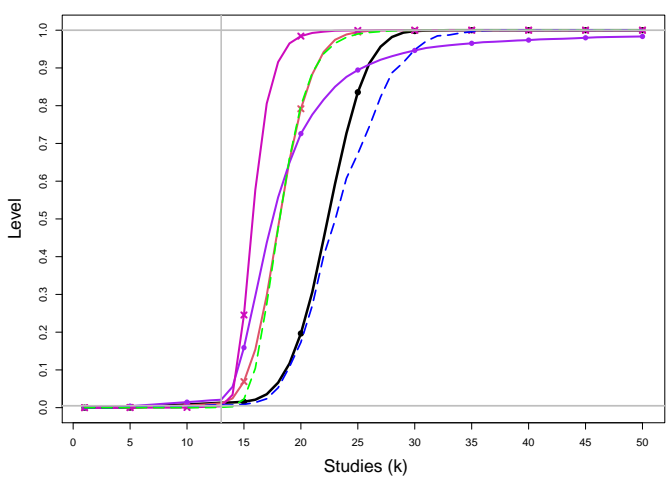

SSW KDB: $\tau^{2}=0.25 \mathrm{n}=100$ level $=0.005 \mathrm{~h}=6 \delta=1$ to 2 (chg at 13 th)

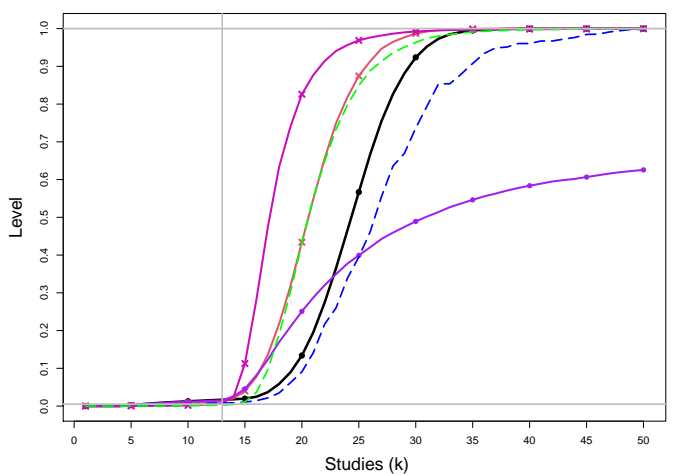

SSW KDB: $\tau^{2}=1 \mathrm{n}=100$ level $=0.005 \mathrm{~h}=6 \delta=1$ to 2 (chg at 13 th)

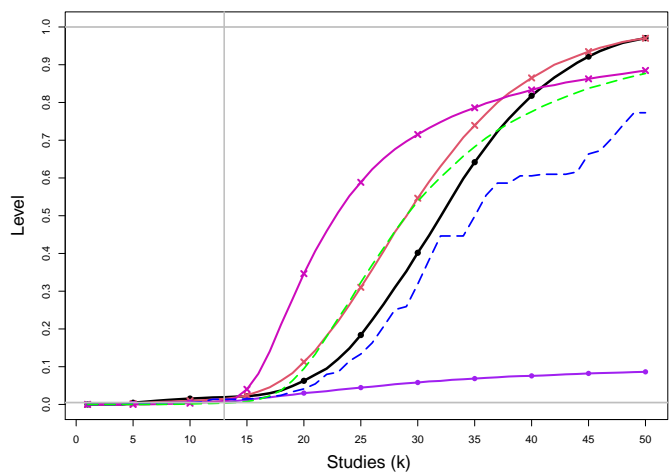

Figure E2. 33: Empirical levels of 1- and 2-stage CMA tests for shift in $\delta$ at .005 nominal level, shift in $\tau^{2}$ at .0025 nominal levels and of CUSUM with $h=6$ for equal sample sizes $n_{i C}+n_{i T}=n=100, \tau^{2}=0,0.1,0.25,1$ and a shift from $\delta=1$ to $\delta=2$ at study number 13. Light grey line at .005. 


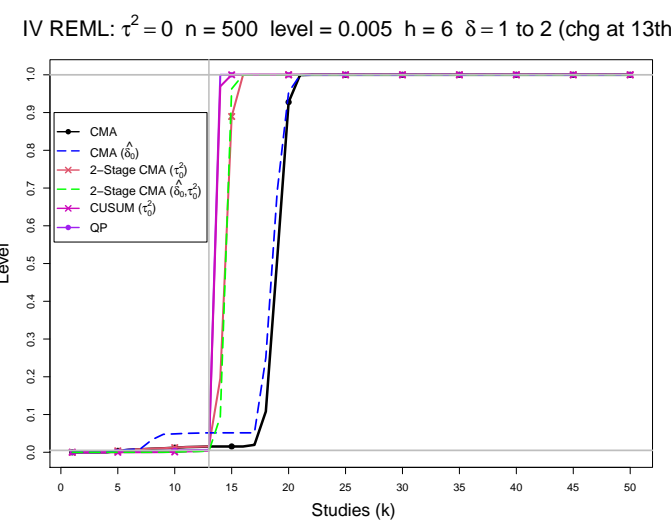

IV REML: $\tau^{2}=0.1 \mathrm{n}=500$ level $=0.005 \mathrm{~h}=6 \delta=1$ to 2 (chg at 13 th)

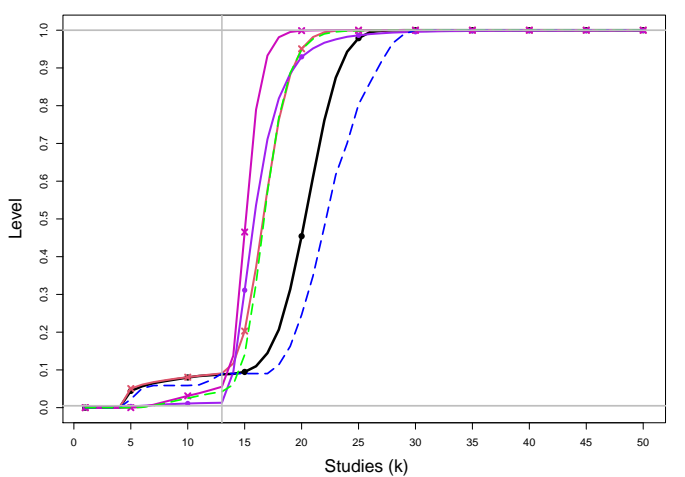

IV REML: $\tau^{2}=0.25 \mathrm{n}=500$ level $=0.005 \mathrm{~h}=6 \delta=1$ to 2 (chg at 13 th)

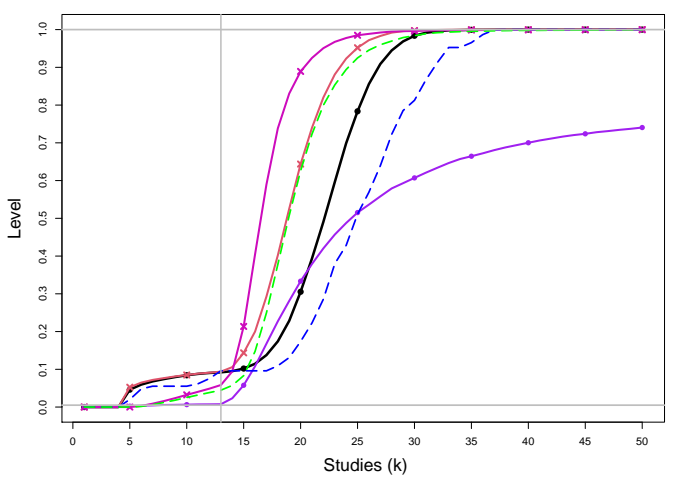

IV REML: $\tau^{2}=1 \mathrm{n}=500$ level $=0.005 \mathrm{~h}=6 \delta=1$ to 2 (chg at 13 th)

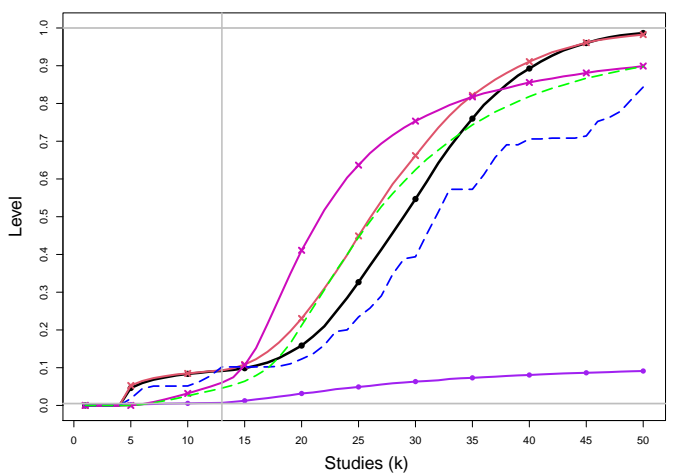

SSW KDB: $\tau^{2}=0 \mathrm{n}=500$ level $=0.005 \mathrm{~h}=6 \delta=1$ to 2 (chg at 13 th)

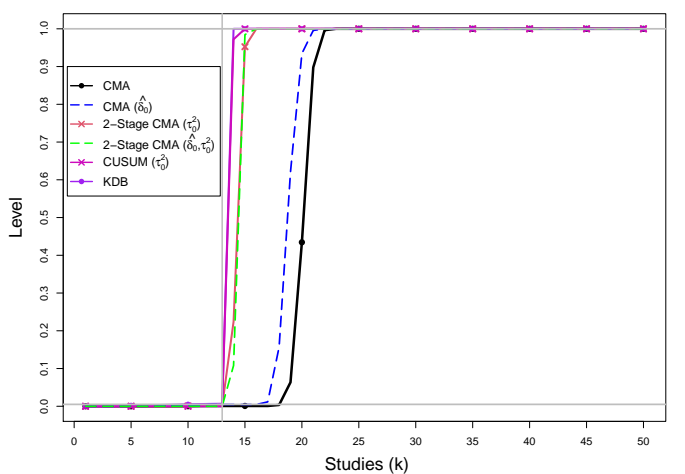

SSW KDB: $\tau^{2}=0.1 \mathrm{n}=500$ level $=0.005 \mathrm{~h}=6 \delta=1$ to 2 (chg at 13 th)

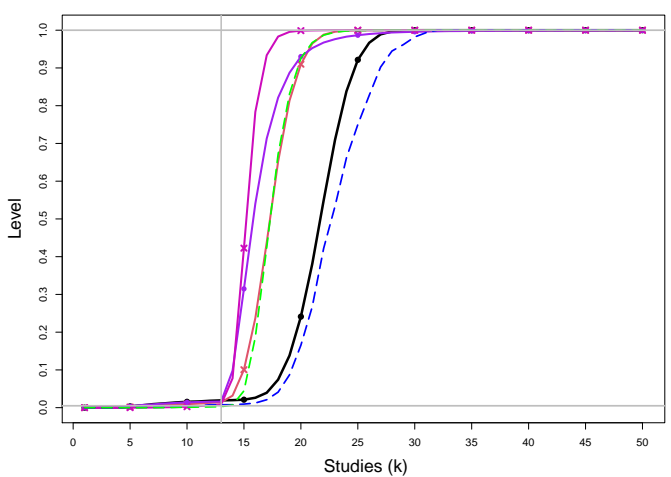

SSW KDB: $\tau^{2}=0.25 \mathrm{n}=500$ level $=0.005 \mathrm{~h}=6 \delta=1$ to 2 (chg at 13 th)

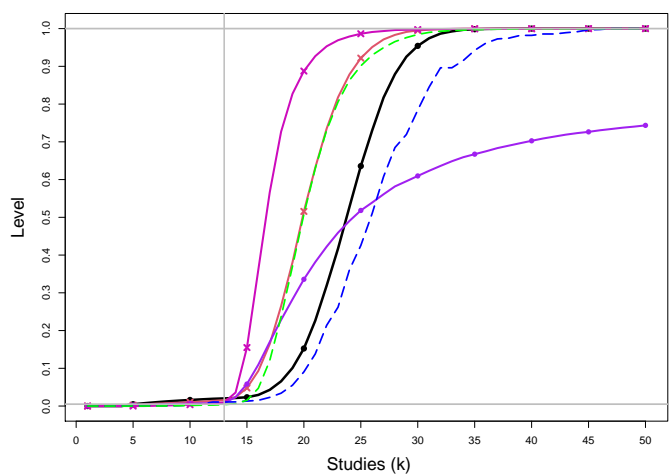

SSW KDB: $\tau^{2}=1 \mathrm{n}=500$ level $=0.005 \mathrm{~h}=6 \delta=1$ to 2 (chg at 13 th)

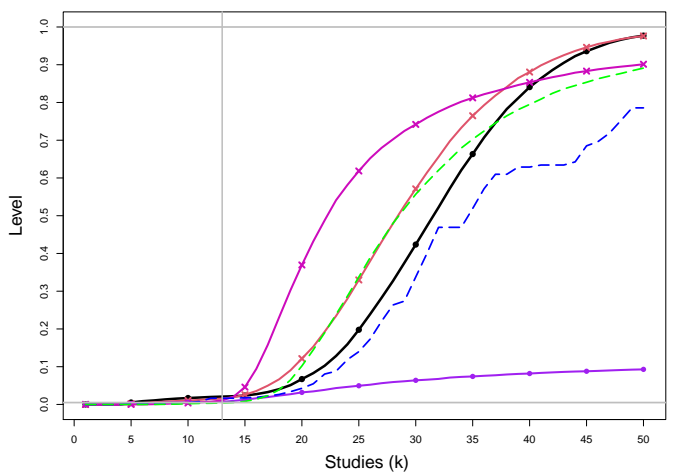

Figure E2. 34: Empirical levels of 1- and 2-stage CMA tests for shift in $\delta$ at .005 nominal level, shift in $\tau^{2}$ at .0025 nominal levels and of CUSUM with $h=6$ for equal sample sizes $n_{i C}+n_{i T}=n=500, \tau^{2}=0,0.1,0.25,1$ and a shift from $\delta=1$ to $\delta=2$ at study number 13. Light grey line at .005. 
IV REML: $\tau^{2}=0 \mathrm{n}=1000$ level $=0.005 \mathrm{~h}=6 \delta=1$ to 2 (chg at 13 th)

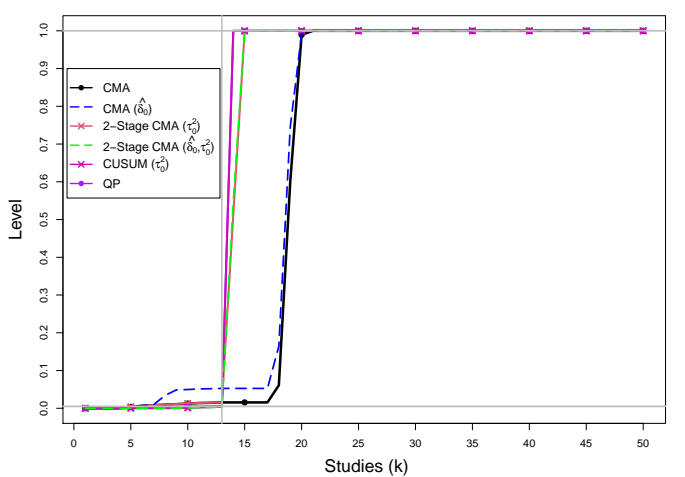

IV REML: $\tau^{2}=0.1 \mathrm{n}=1000$ level $=0.005 \mathrm{~h}=6 \delta=1$ to 2 (chg at 13 th)

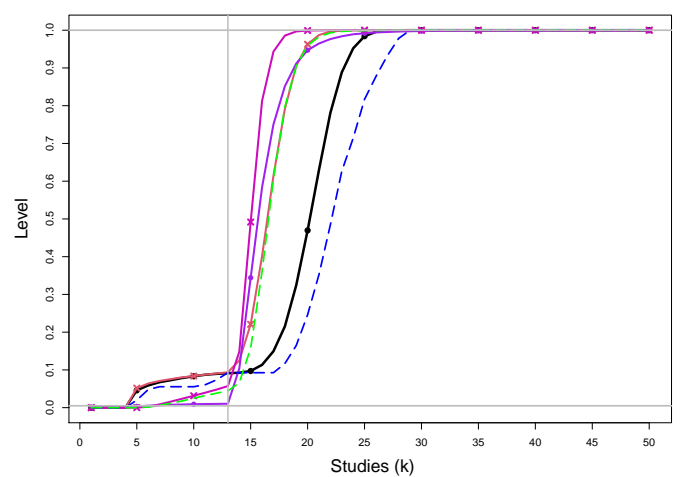

IV REML: $\tau^{2}=0.25 \mathrm{n}=1000$ level $=0.005 \mathrm{~h}=6 \delta=1$ to 2 (chg at 13 th)

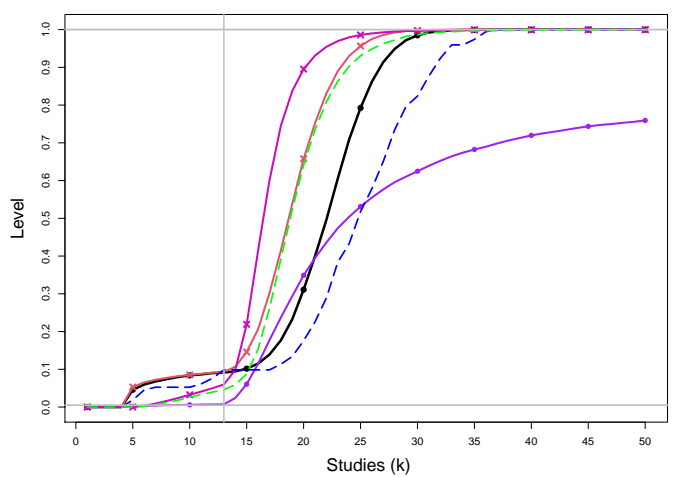

IV REML: $\tau^{2}=1 \mathrm{n}=1000$ level $=0.005 \mathrm{~h}=6 \delta=1$ to 2 (chg at 13 th)

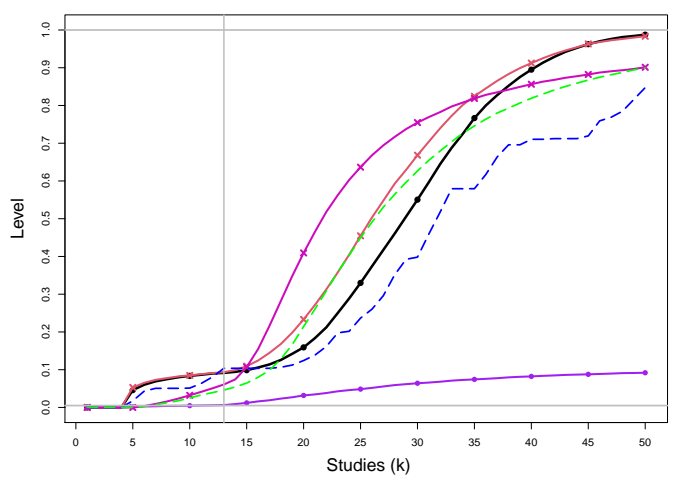

SSW KDB: $\tau^{2}=0 \mathrm{n}=1000$ level $=0.005 \mathrm{~h}=6 \delta=1$ to 2 (chg at 13th)

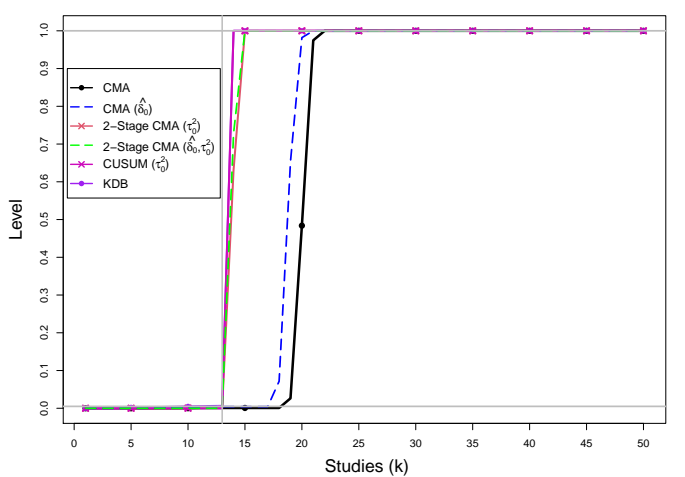

SSW KDB: $\tau^{2}=0.1 \mathrm{n}=1000$ level $=0.005 \mathrm{~h}=6 \quad \delta=1$ to 2 (chg at 13 th)

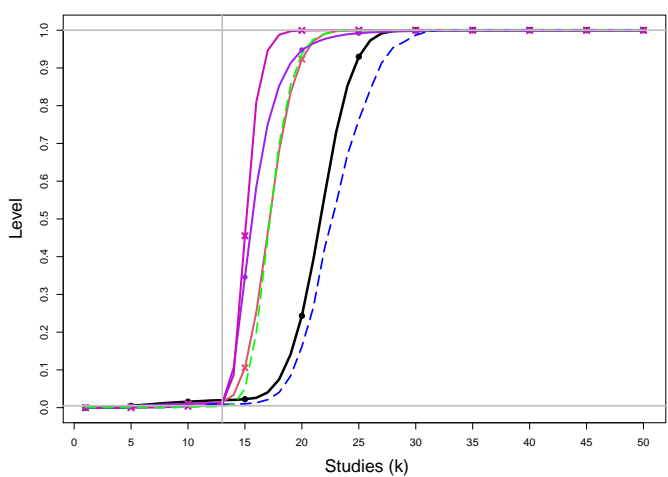

SSW KDB: $\tau^{2}=0.25 \mathrm{n}=1000$ level $=0.005 \mathrm{~h}=6 \delta=1$ to 2 (chg at 13th)

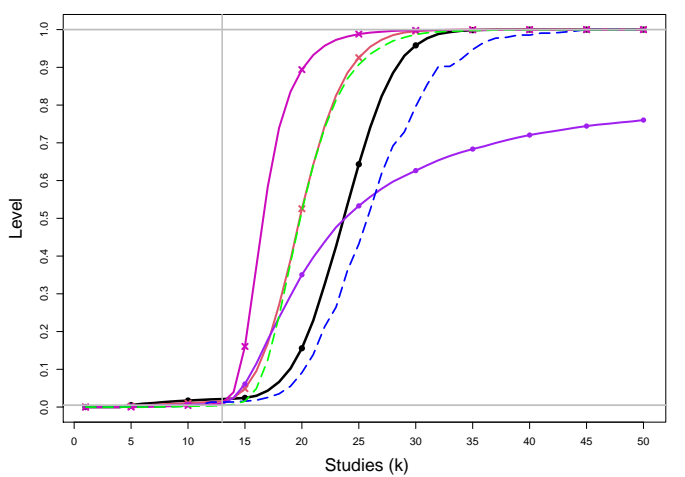

SSW KDB: $\tau^{2}=1 \mathrm{n}=1000$ level $=0.005 \mathrm{~h}=6 \delta=1$ to 2 (chg at 13th)

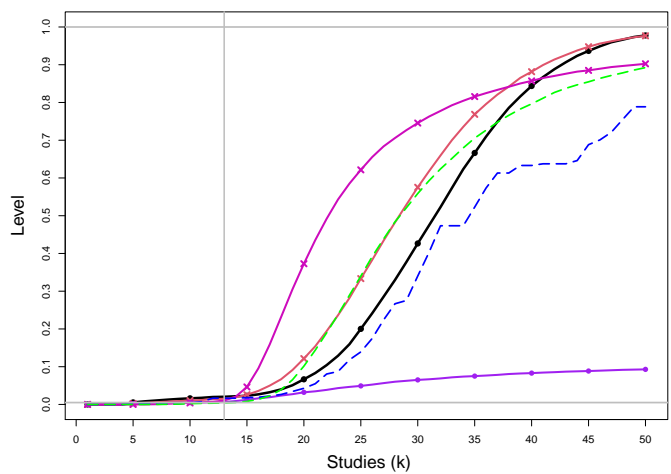

Figure E2. 35: Empirical levels of 1- and 2-stage CMA tests for shift in $\delta$ at .005 nominal level, shift in $\tau^{2}$ at .0025 nominal levels and of CUSUM with $h=6$ for equal sample sizes $n_{i C}+n_{i T}=n=1000, \tau^{2}=0,0.1,0.25,1$ and a shift from $\delta=1$ to $\delta=2$ at study number 13. Light grey line at .005. 


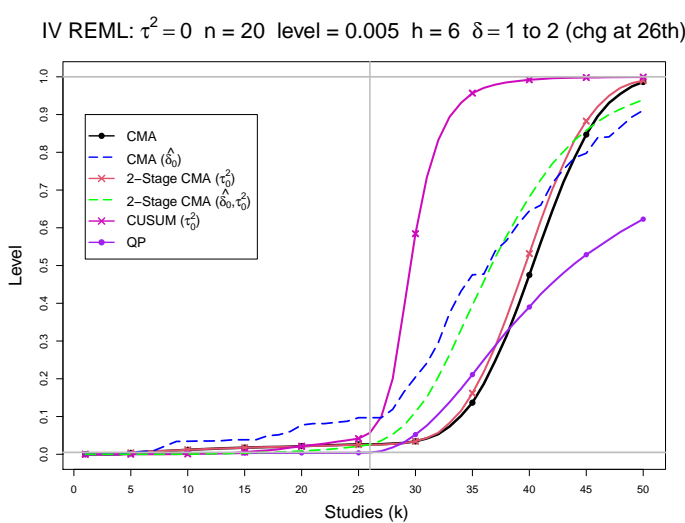

IV REML: $\tau^{2}=0.1 \mathrm{n}=20$ level $=0.005 \mathrm{~h}=6 \delta=1$ to 2 (chg at 26th)

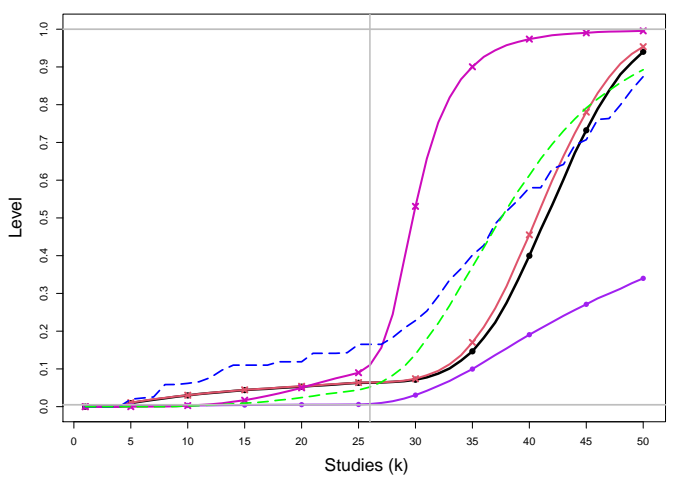

IV REML: $\tau^{2}=0.25 \mathrm{n}=20$ level $=0.005 \mathrm{~h}=6 \delta=1$ to 2 (chg at 26 th)

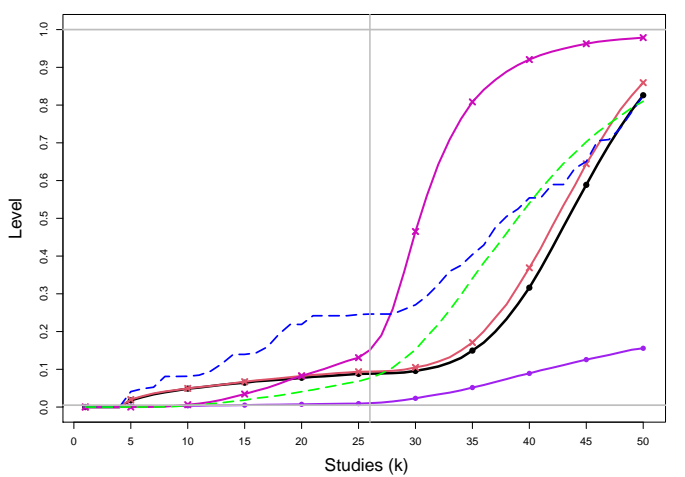

IV REML: $\tau^{2}=1 \mathrm{n}=20$ level $=0.005 \mathrm{~h}=6 \delta=1$ to 2 (chg at 26 th)

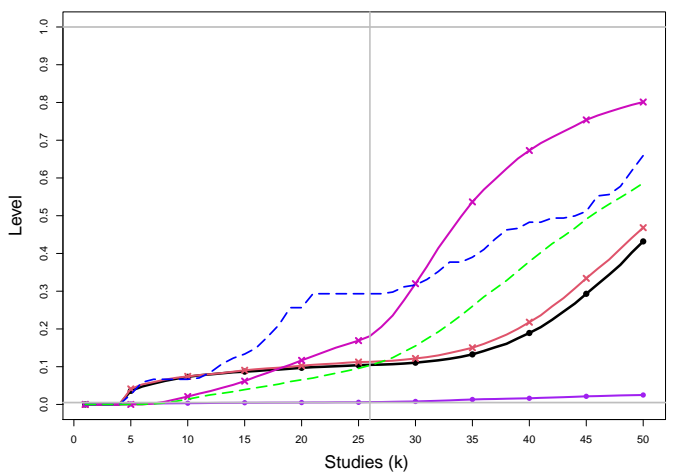

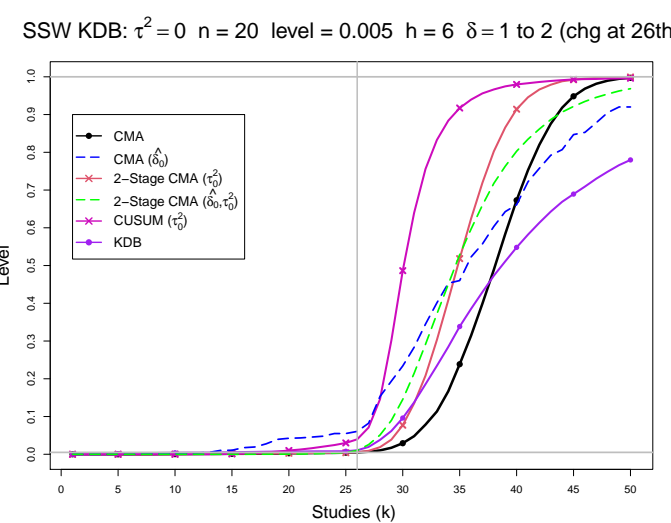

SSW KDB: $\tau^{2}=0.1 \mathrm{n}=20$ level $=0.005 \mathrm{~h}=6 \delta=1$ to 2 (chg at 26th)

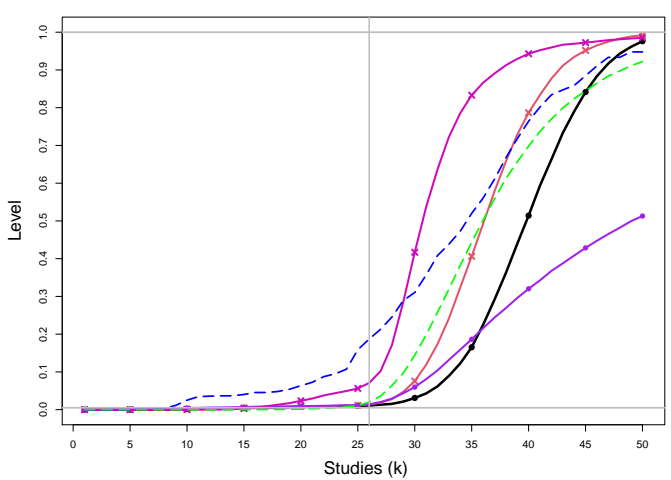

SSW KDB: $\tau^{2}=0.25 \mathrm{n}=20$ level $=0.005 \mathrm{~h}=6 \delta=1$ to 2 (chg at 26th)

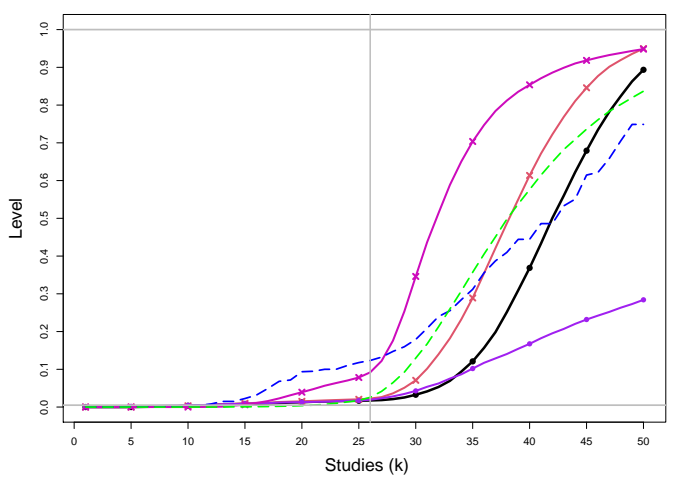

SSW KDB: $\tau^{2}=1 \mathrm{n}=20$ level $=0.005 \mathrm{~h}=6 \delta=1$ to 2 (chg at 26th)

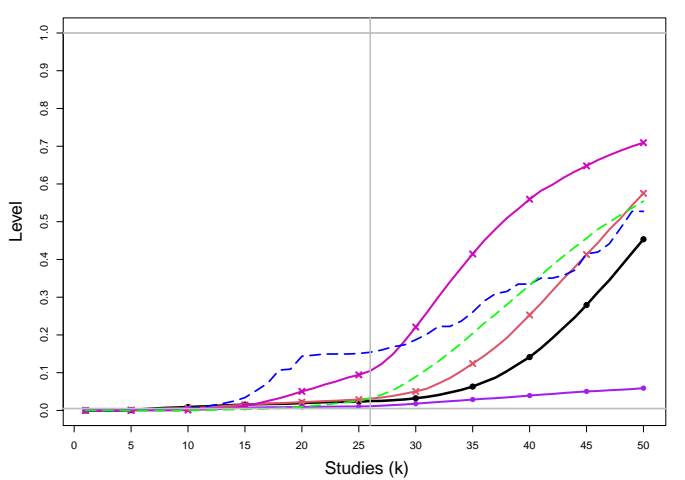

Figure E2. 36: Empirical levels of 1- and 2-stage CMA tests for shift in $\delta$ at .005 nominal level, shift in $\tau^{2}$ at .0025 nominal levels and of CUSUM with $h=6$ for equal sample sizes $n_{i C}+n_{i T}=n=20, \tau^{2}=0,0.1,0.25,1$ and a shift from $\delta=1$ to $\delta=2$ at study number 26. Light grey line at .005. 


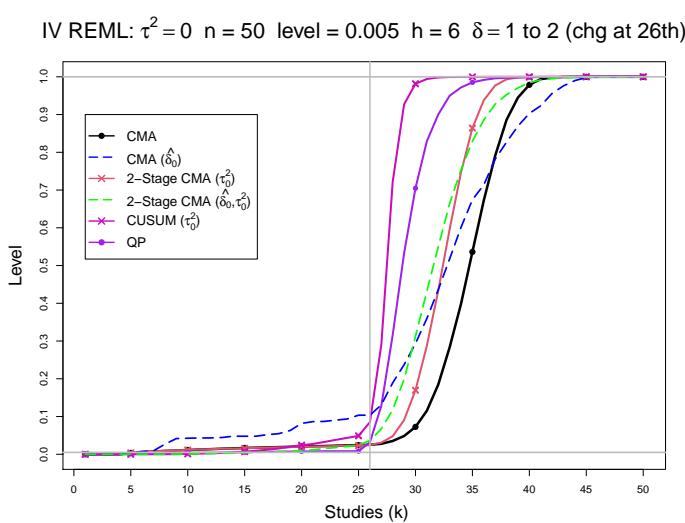

IV REML: $\tau^{2}=0.1 \mathrm{n}=50$ level $=0.005 \mathrm{~h}=6 \delta=1$ to 2 (chg at 26th)

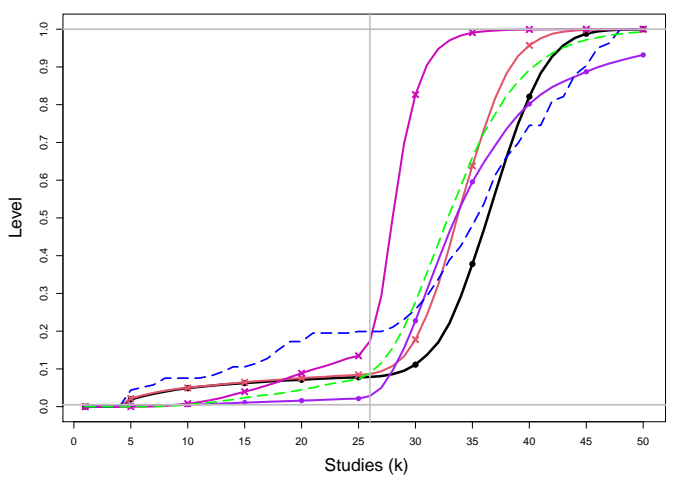

IV REML: $\tau^{2}=0.25 \mathrm{n}=50$ level $=0.005 \mathrm{~h}=6 \delta=1$ to 2 (chg at 26 th)

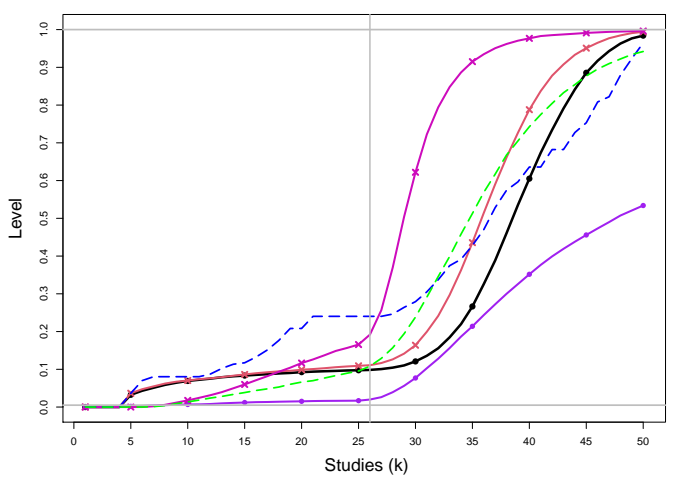

IV REML: $\tau^{2}=1 \mathrm{n}=50$ level $=0.005 \mathrm{~h}=6 \delta=1$ to 2 (chg at 26 th)

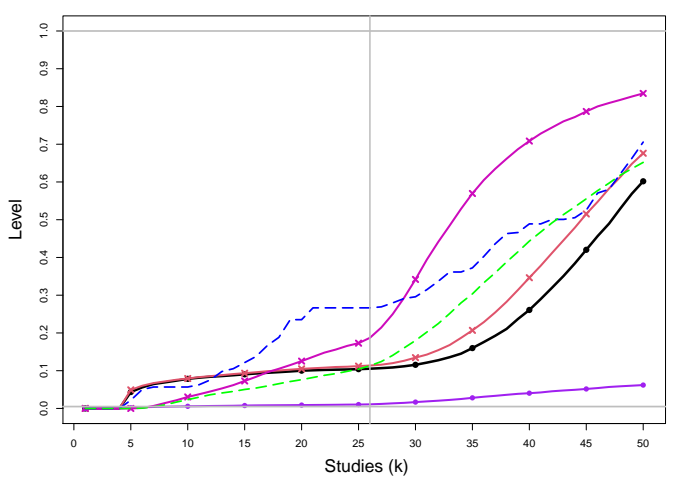

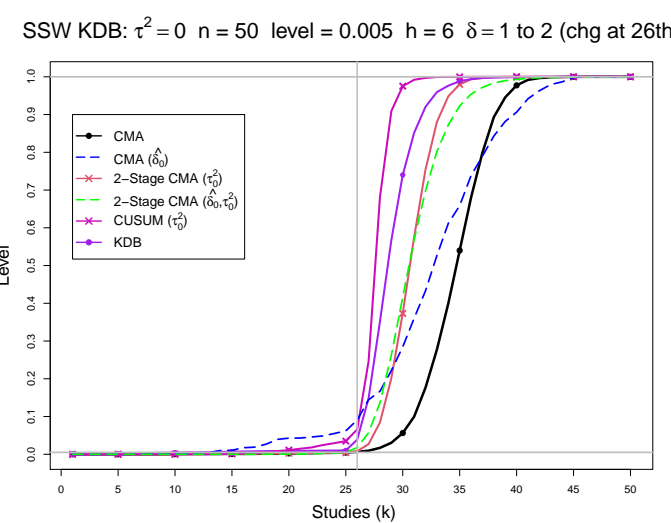

SSW KDB: $\tau^{2}=0.1 \mathrm{n}=50$ level $=0.005 \mathrm{~h}=6 \delta=1$ to 2 (chg at 26th)

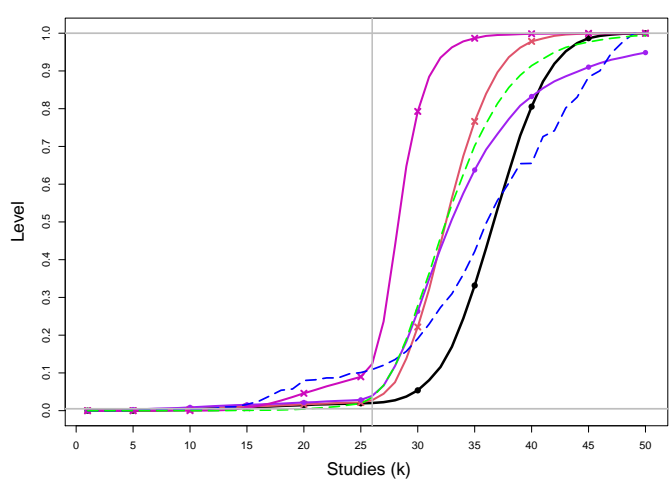

SSW KDB: $\tau^{2}=0.25 \mathrm{n}=50$ level $=0.005 \mathrm{~h}=6 \delta=1$ to 2 (chg at 26th)

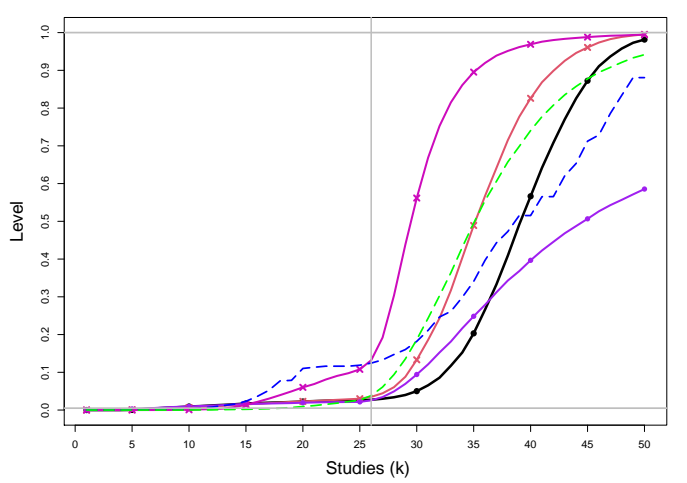

SSW KDB: $\tau^{2}=1 \mathrm{n}=50$ level $=0.005 \mathrm{~h}=6 \delta=1$ to 2 (chg at 26 th)

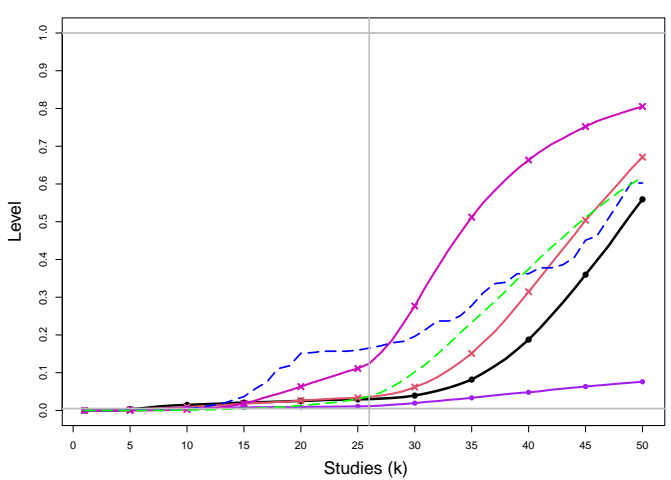

Figure E2. 37: Empirical levels of 1- and 2-stage CMA tests for shift in $\delta$ at .005 nominal level, shift in $\tau^{2}$ at .0025 nominal levels and of CUSUM with $h=6$ for equal sample sizes $n_{i C}+n_{i T}=n=50, \tau^{2}=0,0.1,0.25,1$ and a shift from $\delta=1$ to $\delta=2$ at study number 26. Light grey line at .005. 


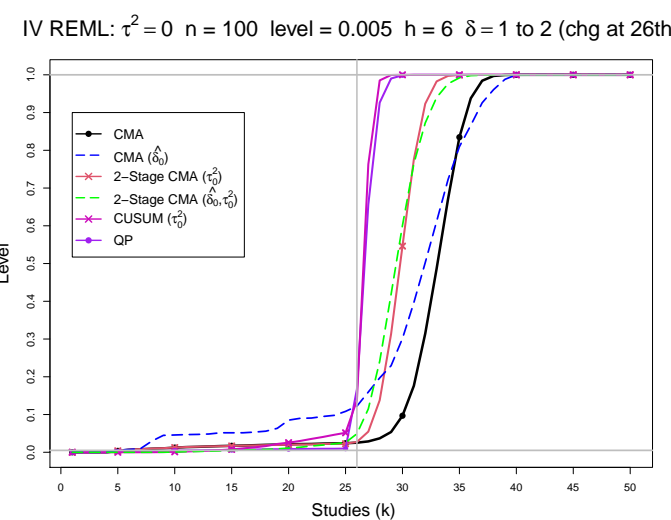

IV REML: $\tau^{2}=0.1 \mathrm{n}=100$ level $=0.005 \mathrm{~h}=6 \delta=1$ to 2 (chg at 26th)

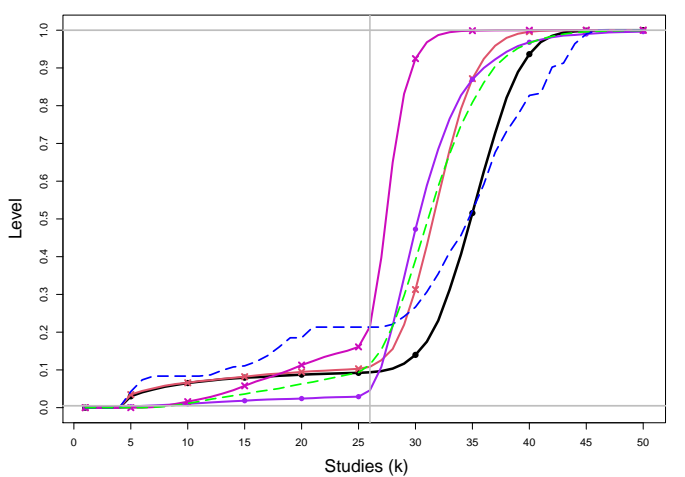

IV REML: $\tau^{2}=0.25 \mathrm{n}=100$ level $=0.005 \mathrm{~h}=6 \delta=1$ to 2 (chg at 26th)

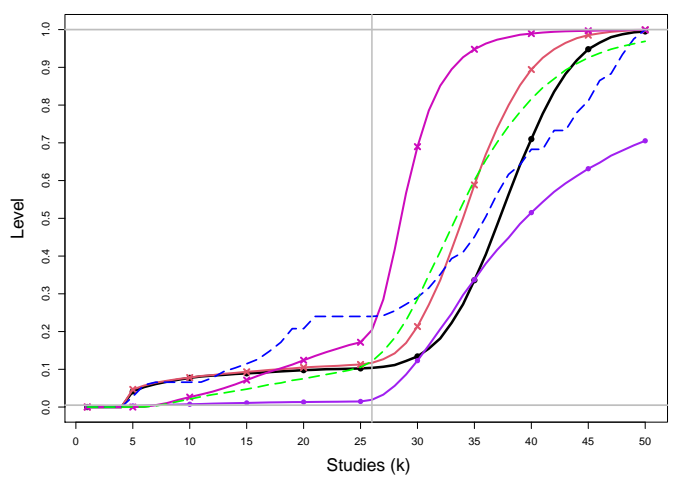

IV REML: $\tau^{2}=1 \mathrm{n}=100$ level $=0.005 \mathrm{~h}=6 \delta=1$ to 2 (chg at 26th)

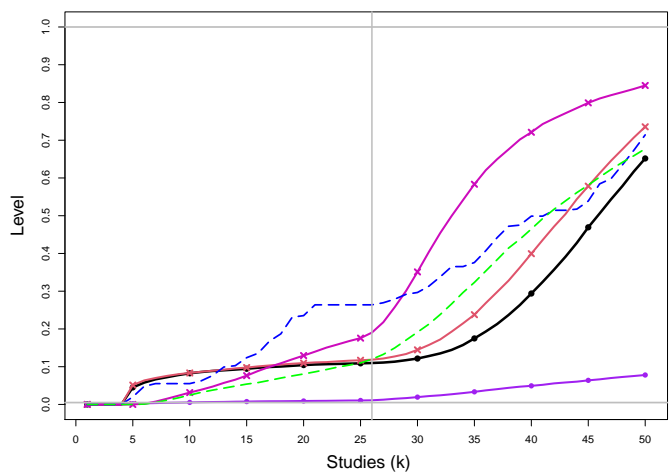

SSW KDB: $\tau^{2}=0 \mathrm{n}=100$ level $=0.005 \mathrm{~h}=6 \delta=1$ to 2 (chg at 26 th)

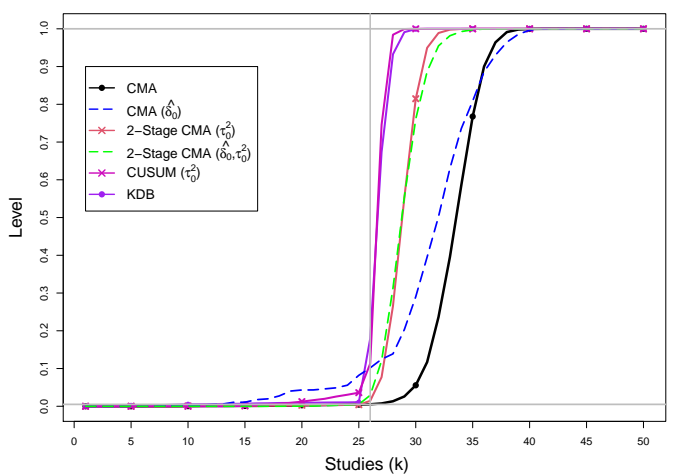

SSW KDB: $\tau^{2}=0.1 \mathrm{n}=100$ level $=0.005 \mathrm{~h}=6 \delta=1$ to 2 (chg at 26th)

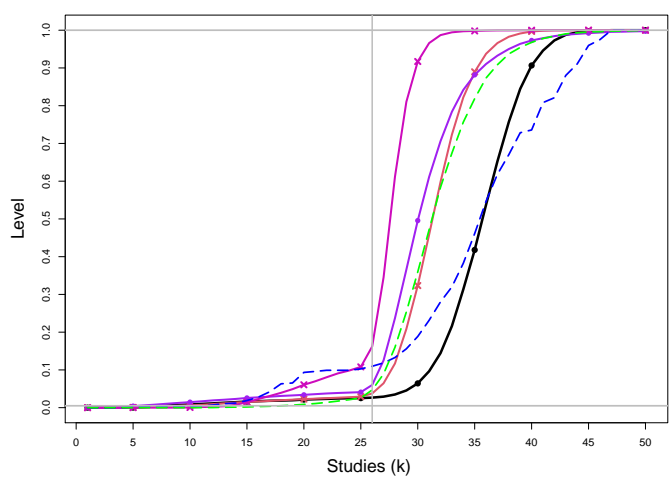

SSW KDB: $\tau^{2}=0.25 \mathrm{n}=100$ level $=0.005 \mathrm{~h}=6 \delta=1$ to 2 (chg at 26th)

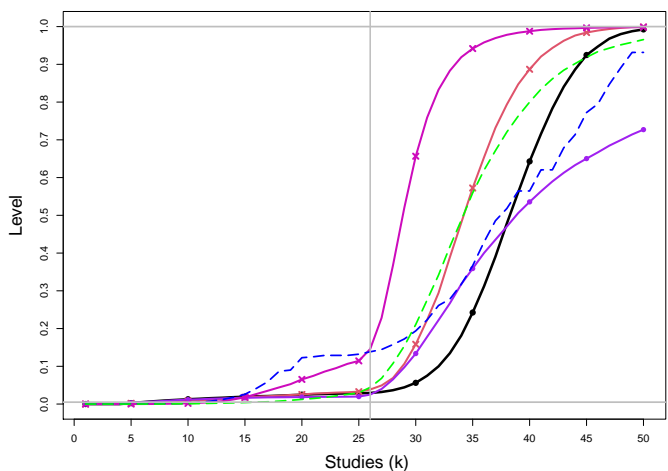

SSW KDB: $\tau^{2}=1 \mathrm{n}=100$ level $=0.005 \mathrm{~h}=6 \delta=1$ to 2 (chg at 26th)

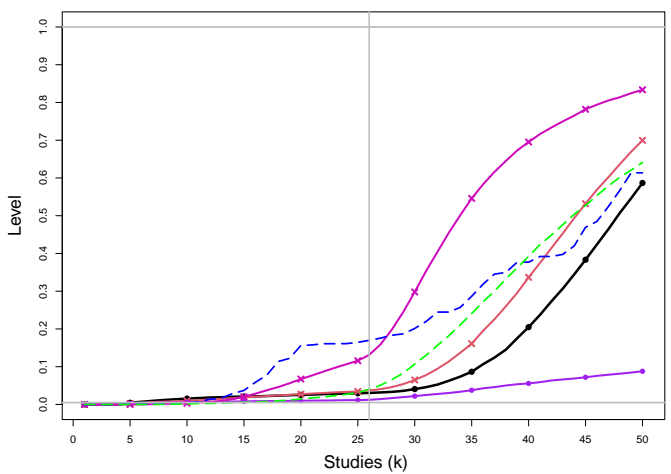

Figure E2. 38: Empirical levels of 1- and 2-stage CMA tests for shift in $\delta$ at .005 nominal level, shift in $\tau^{2}$ at .0025 nominal levels and of CUSUM with $h=6$ for equal sample sizes $n_{i C}+n_{i T}=n=100, \tau^{2}=0,0.1,0.25,1$ and a shift from $\delta=1$ to $\delta=2$ at study number 26. Light grey line at .005. 
IV REML: $\tau^{2}=0 \mathrm{n}=500$ level $=0.005 \mathrm{~h}=6 \quad \delta=1$ to 2 (chg at 26 th)

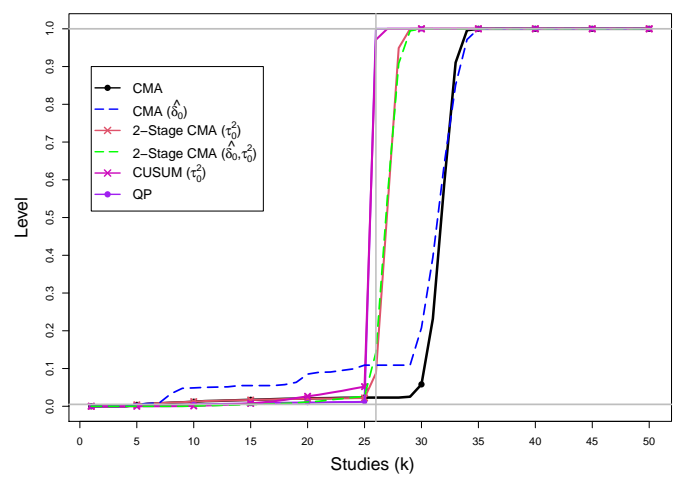

IV REML: $\tau^{2}=0.1 \mathrm{n}=500$ level $=0.005 \mathrm{~h}=6 \quad \delta=1$ to 2 (chg at 26 th)

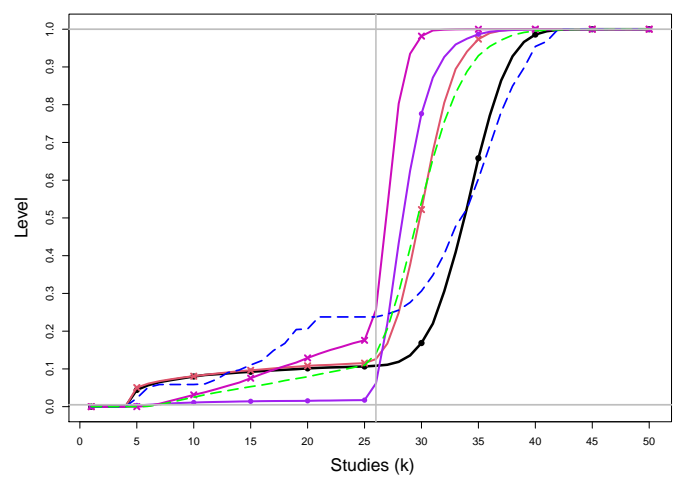

IV REML: $\tau^{2}=0.25 \mathrm{n}=500$ level $=0.005 \mathrm{~h}=6 \quad \delta=1$ to 2 (chg at 26 th)

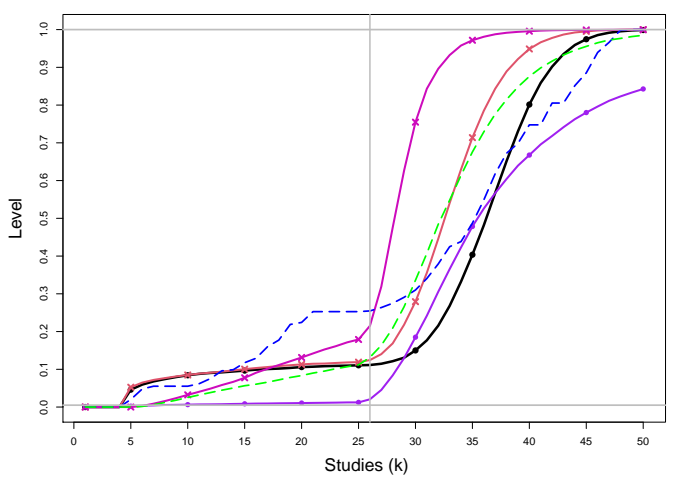

IV REML: $\tau^{2}=1 \mathrm{n}=500$ level $=0.005 \mathrm{~h}=6 \quad \delta=1$ to 2 (chg at 26 th)

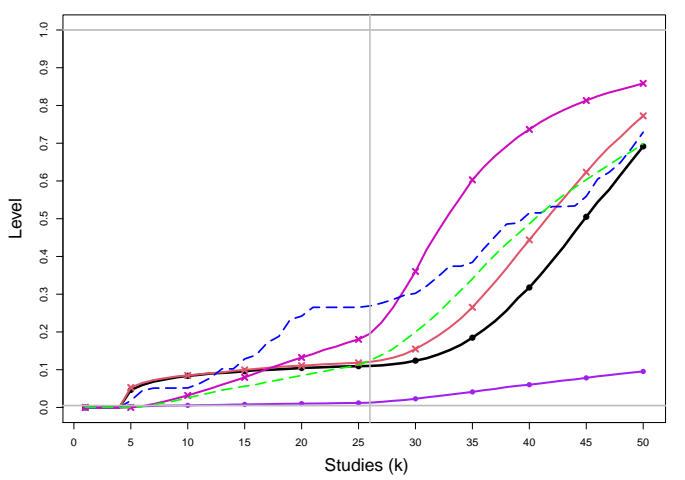

SSW KDB: $\tau^{2}=0 \mathrm{n}=500$ level $=0.005 \mathrm{~h}=6 \quad \delta=1$ to 2 (chg at 26 th)

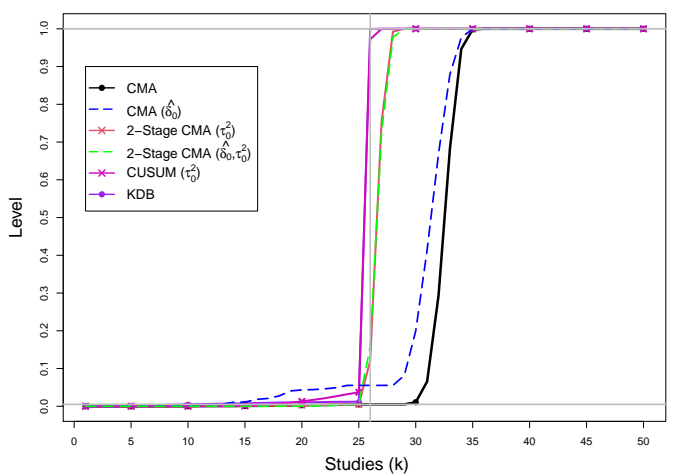

SSW KDB: $\tau^{2}=0.1 \mathrm{n}=500$ level $=0.005 \mathrm{~h}=6 \quad \delta=1$ to 2 (chg at 26 th)

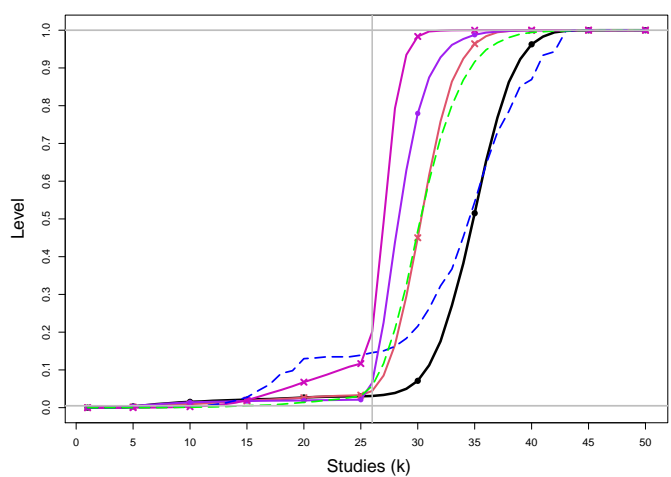

SSW KDB: $\tau^{2}=0.25 \mathrm{n}=500$ level $=0.005 \mathrm{~h}=6 \delta=1$ to 2 (chg at 26 th)

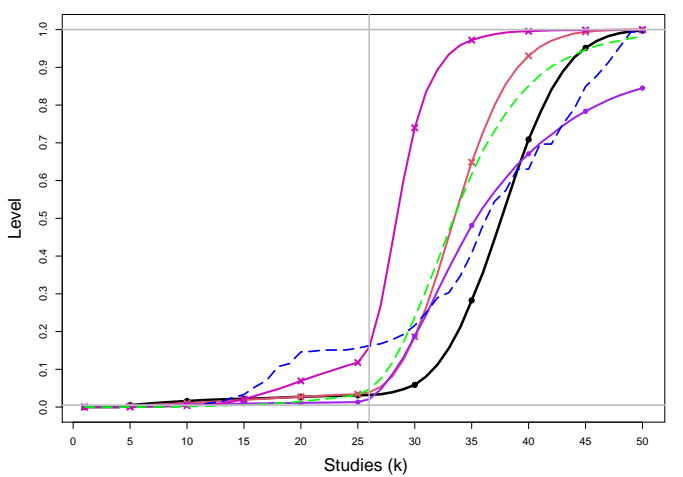

SSW KDB: $\tau^{2}=1 \mathrm{n}=500$ level $=0.005 \mathrm{~h}=6 \delta=1$ to 2 (chg at 26th)

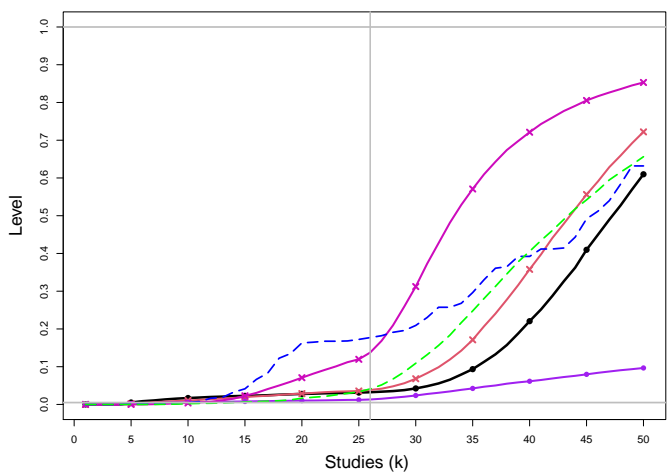

Figure E2. 39: Empirical levels of 1- and 2-stage CMA tests for shift in $\delta$ at .005 nominal level, shift in $\tau^{2}$ at .0025 nominal levels and of CUSUM with $h=6$ for equal sample sizes $n_{i C}+n_{i T}=n=500, \tau^{2}=0,0.1,0.25,1$ and a shift from $\delta=1$ to $\delta=2$ at study number 26. Light grey line at .005. 
IV REML: $\tau^{2}=0 \mathrm{n}=1000$ level $=0.005 \mathrm{~h}=6 \delta=1$ to 2 (chg at 26th)

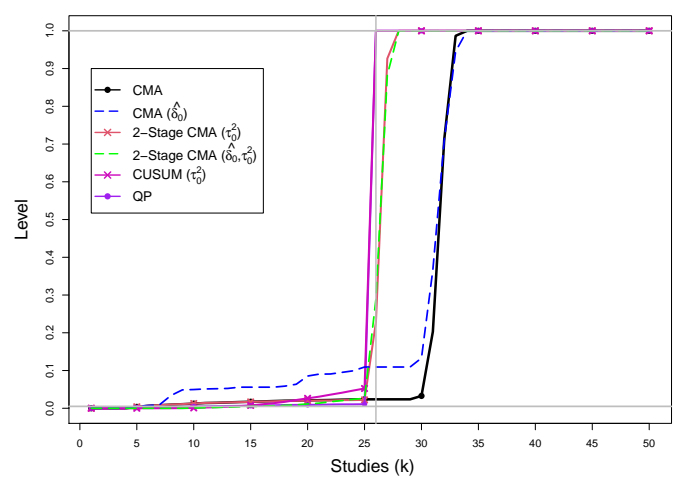

IV REML: $\tau^{2}=0.1 \mathrm{n}=1000$ level $=0.005 \mathrm{~h}=6 \delta=1$ to 2 (chg at 26 th)

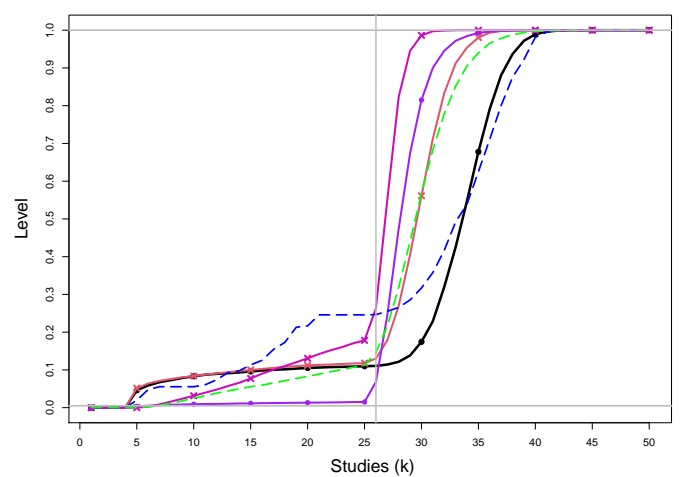

IV REML: $\tau^{2}=0.25 \mathrm{n}=1000$ level $=0.005 \mathrm{~h}=6 \delta=1$ to 2 (chg at 26th)

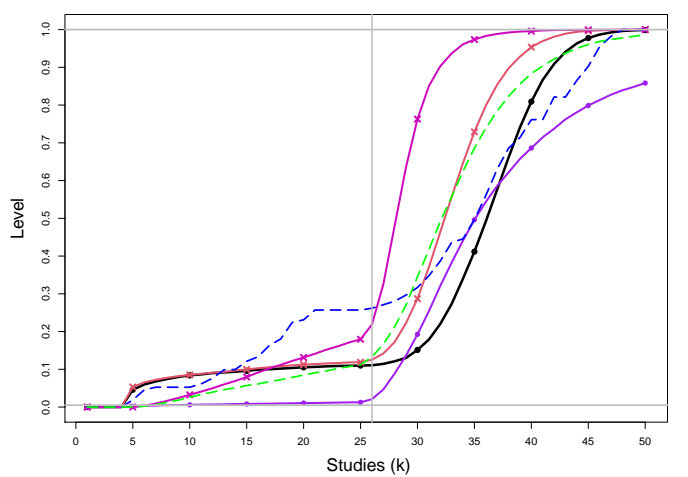

IV REML: $\tau^{2}=1 \mathrm{n}=1000$ level $=0.005 \mathrm{~h}=6 \delta=1$ to 2 (chg at 26 th)

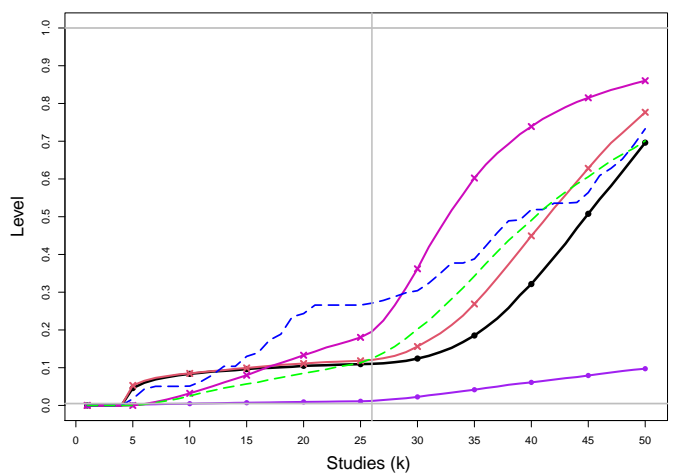

SSW KDB: $\tau^{2}=0 \mathrm{n}=1000$ level $=0.005 \mathrm{~h}=6 \quad \delta=1$ to 2 (chg at 26th)

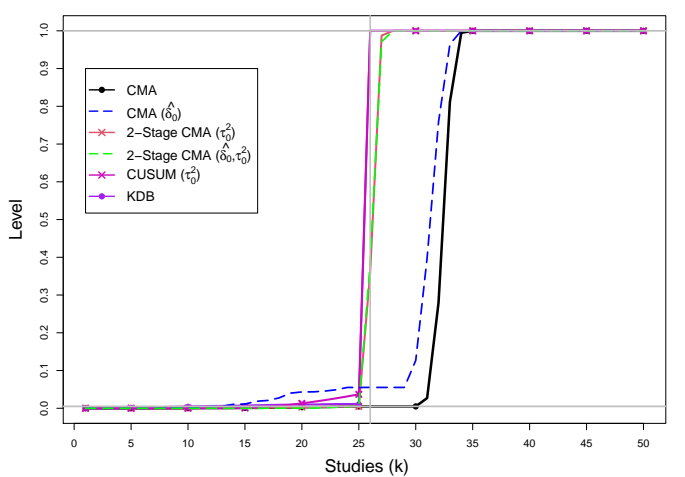

SSW KDB: $\tau^{2}=0.1 \mathrm{n}=1000$ level $=0.005 \mathrm{~h}=6 \quad \delta=1$ to 2 (chg at 26th)

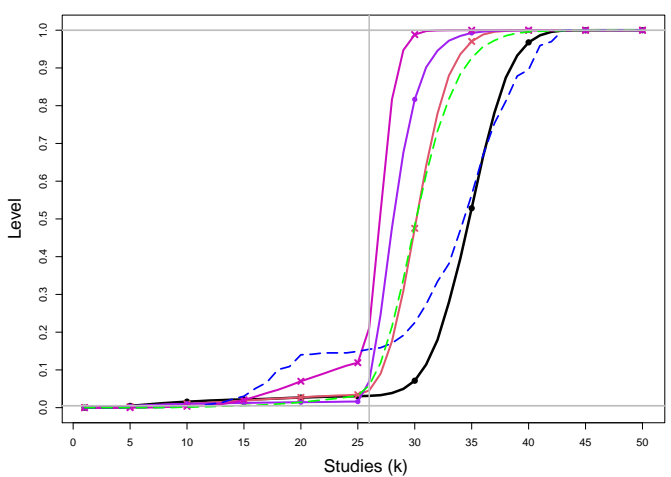

SSW KDB: $\tau^{2}=0.25 \mathrm{n}=1000$ level $=0.005 \mathrm{~h}=6 \delta=1$ to 2 (chg at 26th)

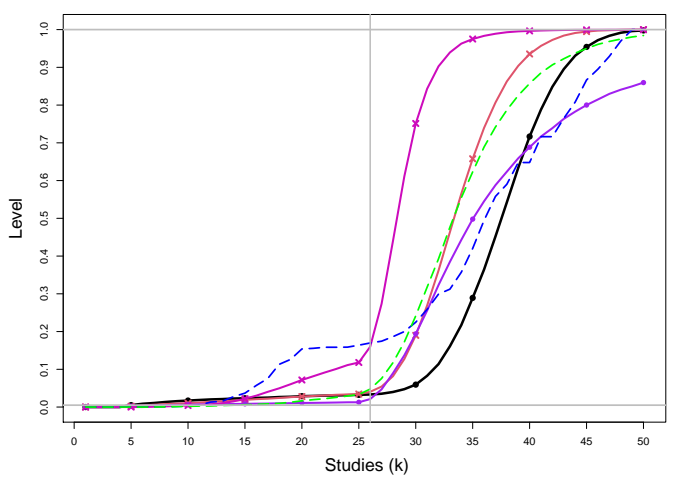

SSW KDB: $\tau^{2}=1 \mathrm{n}=1000$ level $=0.005 \mathrm{~h}=6 \delta=1$ to 2 (chg at 26th)

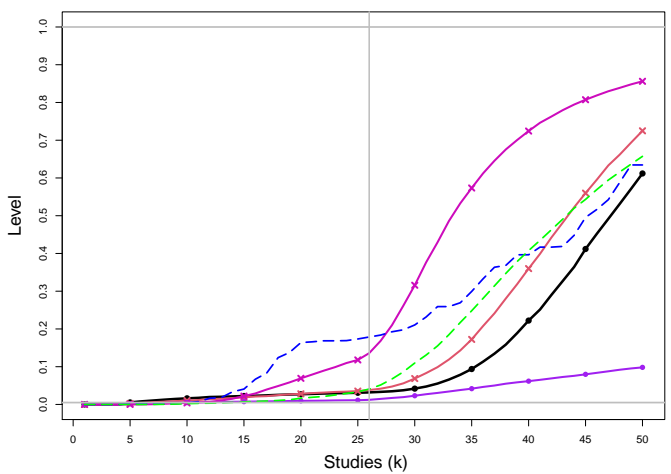

Figure E2. 40: Empirical levels of 1- and 2-stage CMA tests for shift in $\delta$ at .005 nominal level, shift in $\tau^{2}$ at .0025 nominal levels and of CUSUM with $h=6$ for equal sample sizes $n_{i C}+n_{i T}=n=1000, \tau^{2}=0,0.1,0.25,1$ and a shift from $\delta=1$ to $\delta=2$ at study number 26. Light grey line at .005. 


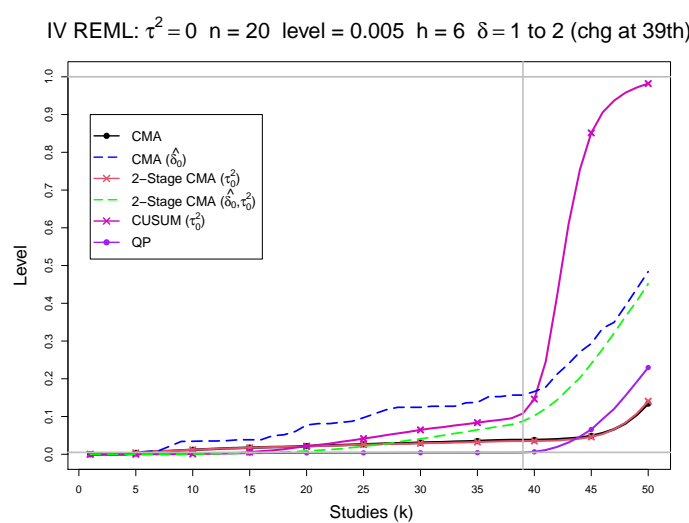

IV REML: $\tau^{2}=0.1 \mathrm{n}=20$ level $=0.005 \mathrm{~h}=6 \delta=1$ to 2 (chg at 39 th)

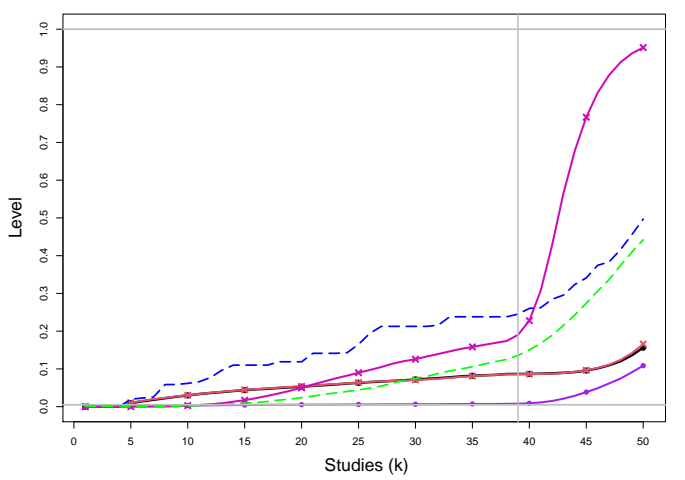

IV REML: $\tau^{2}=0.25 \mathrm{n}=20$ level $=0.005 \mathrm{~h}=6 \delta=1$ to 2 (chg at 39 th)

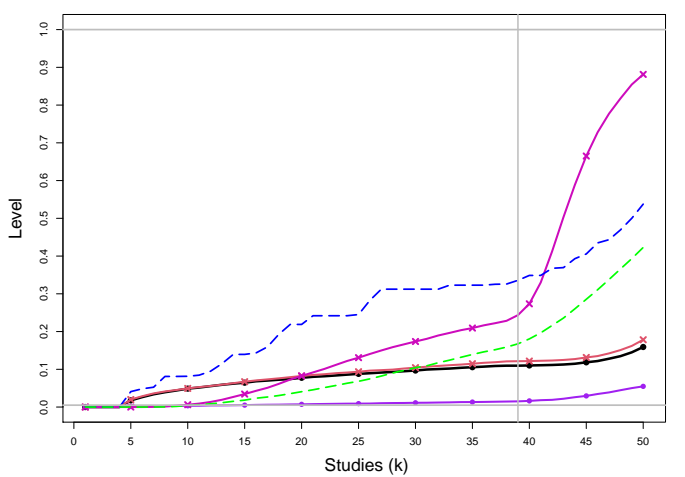

IV REML: $\tau^{2}=1 \mathrm{n}=20$ level $=0.005 \mathrm{~h}=6 \delta=1$ to 2 (chg at 39 th)

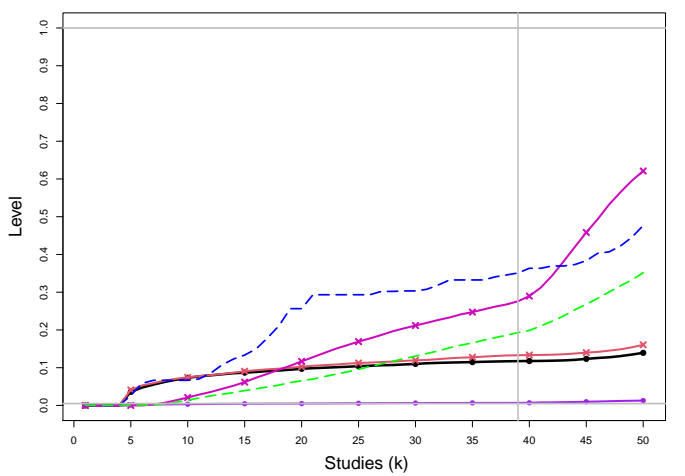

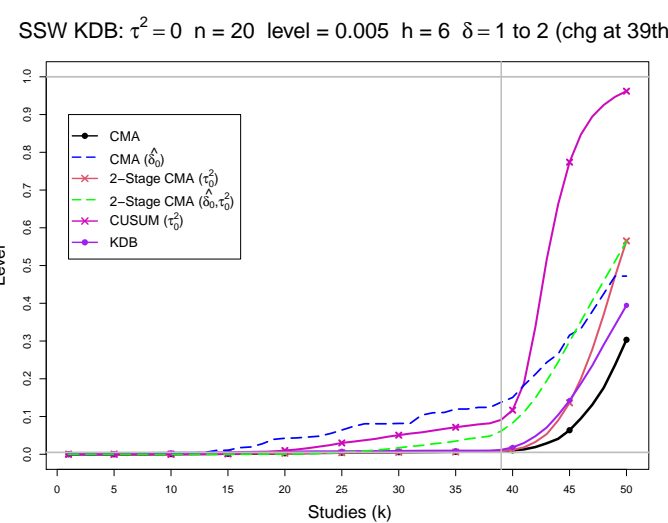

SSW KDB: $\tau^{2}=0.1 \mathrm{n}=20$ level $=0.005 \mathrm{~h}=6 \delta=1$ to 2 (chg at 39 th)

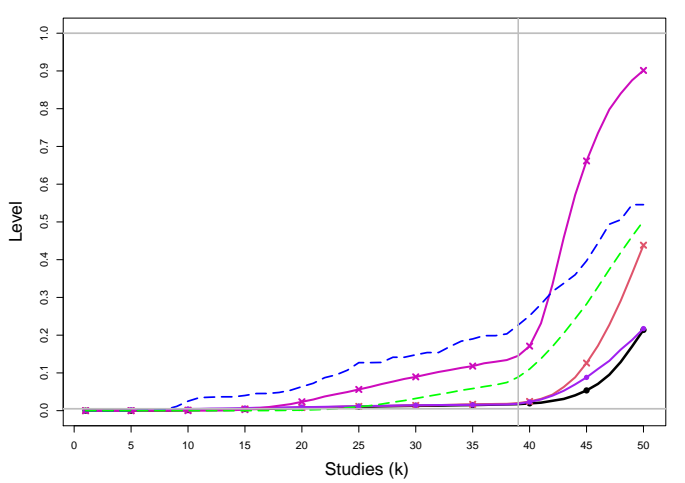

SSW KDB: $\tau^{2}=0.25 \mathrm{n}=20$ level $=0.005 \mathrm{~h}=6 \delta=1$ to 2 (chg at 39 th)

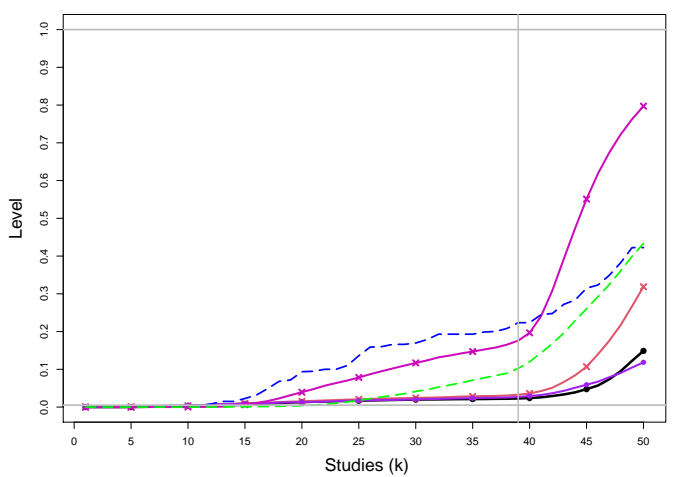

SSW KDB: $\tau^{2}=1 \mathrm{n}=20$ level $=0.005 \mathrm{~h}=6 \delta=1$ to 2 (chg at 39 th)

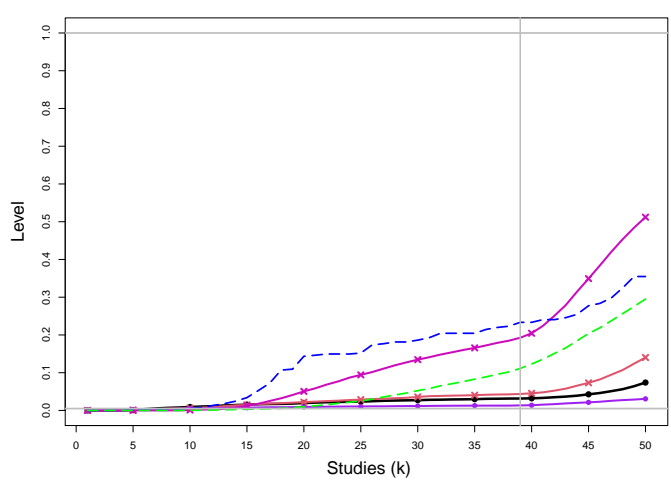

Figure E2. 41: Empirical levels of 1- and 2-stage CMA tests for shift in $\delta$ at .005 nominal level, shift in $\tau^{2}$ at .0025 nominal levels and of CUSUM with $h=6$ for equal sample sizes $n_{i C}+n_{i T}=n=20, \tau^{2}=0,0.1,0.25,1$ and a shift from $\delta=1$ to $\delta=2$ at study number 39. Light grey line at .005. 


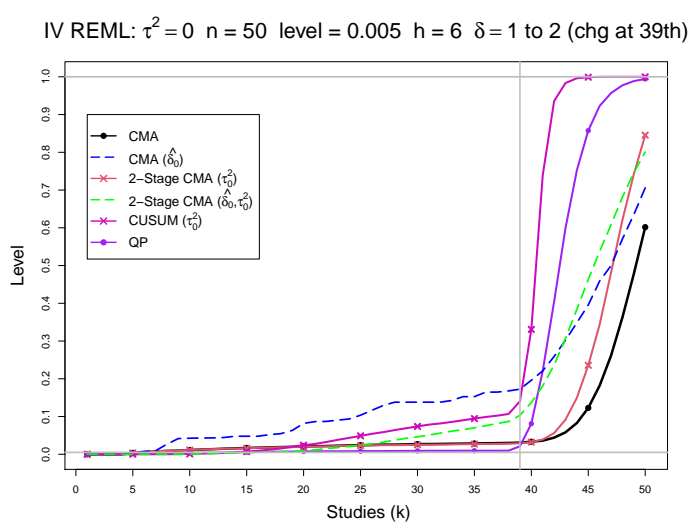

IV REML: $\tau^{2}=0.1 \mathrm{n}=50$ level $=0.005 \mathrm{~h}=6 \delta=1$ to 2 (chg at 39 th)

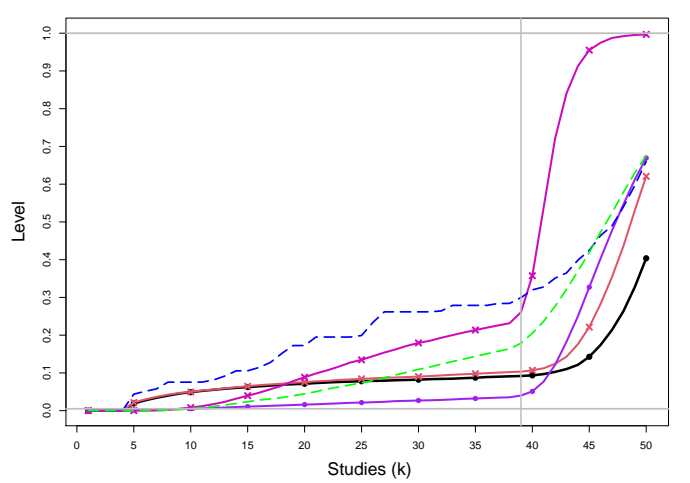

IV REML: $\tau^{2}=0.25 \mathrm{n}=50$ level $=0.005 \mathrm{~h}=6 \delta=1$ to 2 (chg at 39 th)

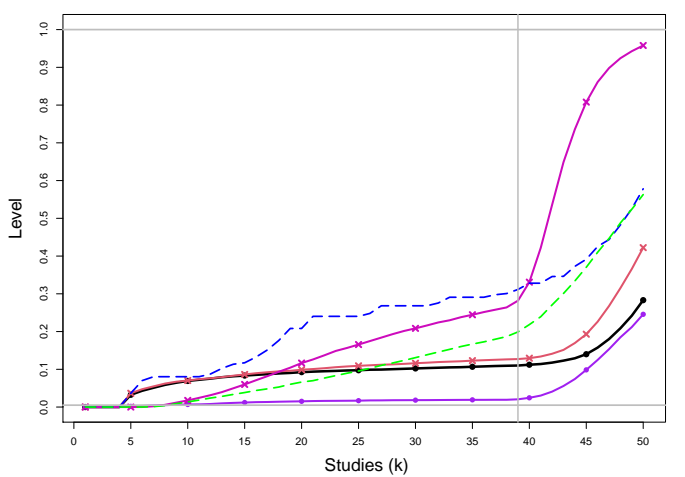

IV REML: $\tau^{2}=1 \mathrm{n}=50$ level $=0.005 \mathrm{~h}=6 \delta=1$ to 2 (chg at 39 th)

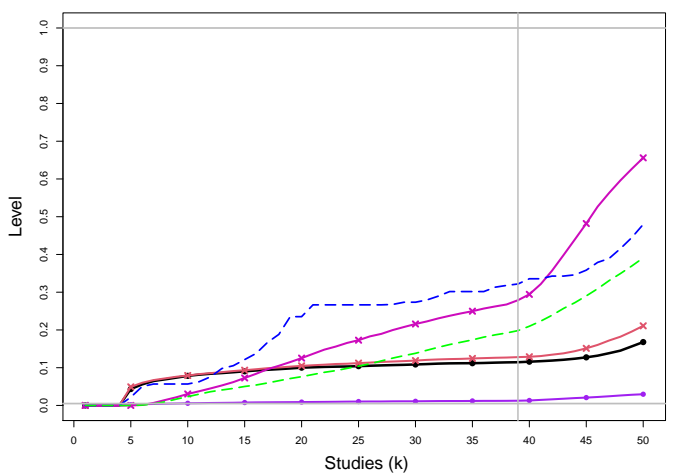

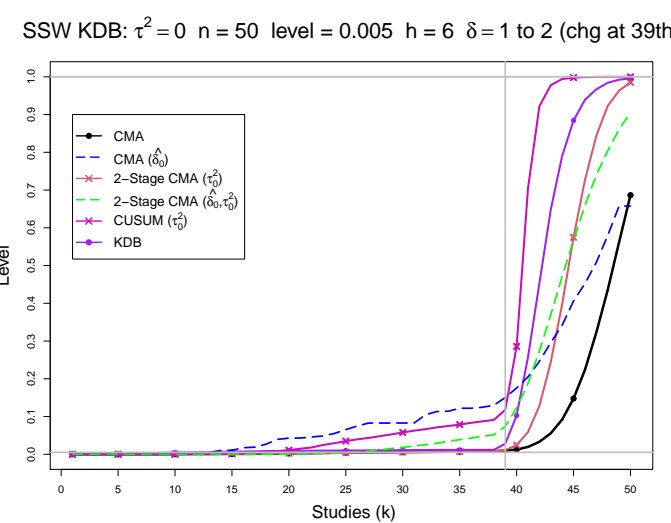

SSW KDB: $\tau^{2}=0.1 \mathrm{n}=50$ level $=0.005 \mathrm{~h}=6 \delta=1$ to 2 (chg at 39 th)

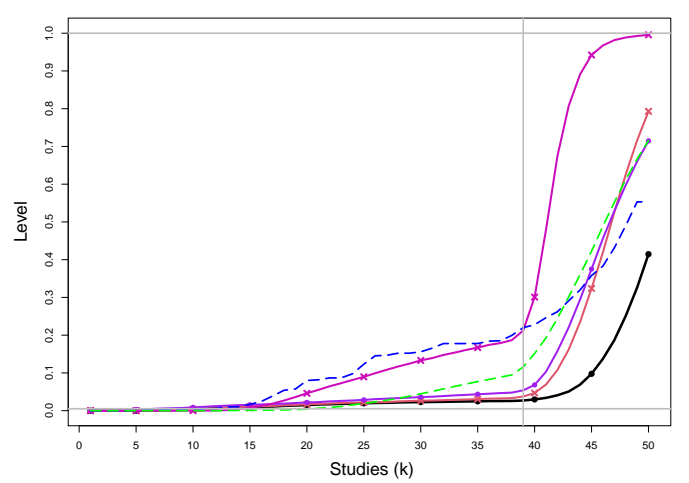

SSW KDB: $\tau^{2}=0.25 \mathrm{n}=50$ level $=0.005 \mathrm{~h}=6 \delta=1$ to 2 (chg at 39 th)

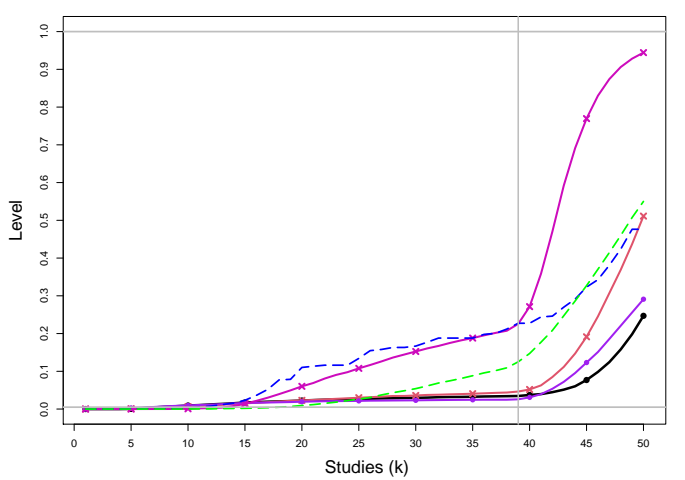

SSW KDB: $\tau^{2}=1 \mathrm{n}=50$ level $=0.005 \mathrm{~h}=6 \delta=1$ to 2 (chg at 39 th)

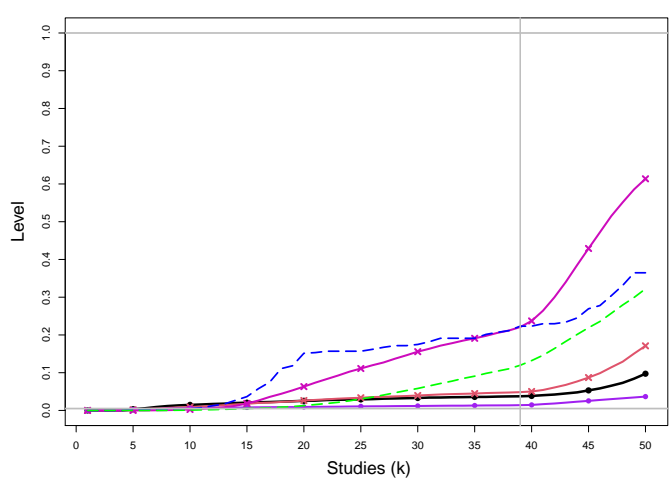

Figure E2. 42: Empirical levels of 1- and 2-stage CMA tests for shift in $\delta$ at .005 nominal level, shift in $\tau^{2}$ at .0025 nominal levels and of CUSUM with $h=6$ for equal sample sizes $n_{i C}+n_{i T}=n=50, \tau^{2}=0,0.1,0.25,1$ and a shift from $\delta=1$ to $\delta=2$ at study number 39. Light grey line at .005. 
IV REML: $\tau^{2}=0 \mathrm{n}=100$ level $=0.005 \mathrm{~h}=6 \delta=1$ to 2 (chg at 39th)

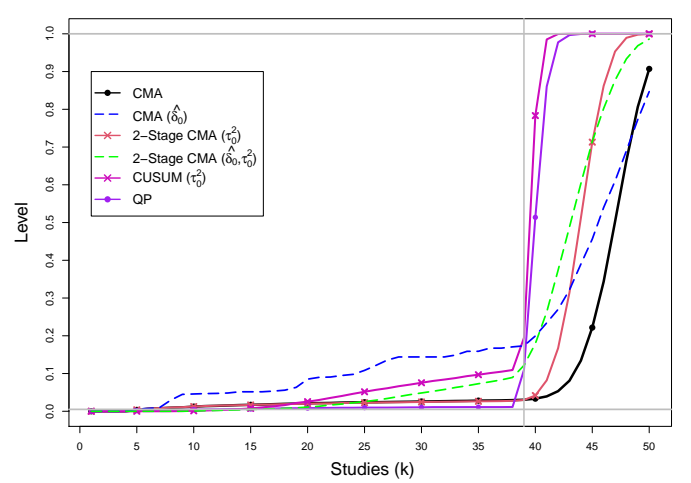

IV REML: $\tau^{2}=0.1 \mathrm{n}=100$ level $=0.005 \mathrm{~h}=6 \delta=1$ to 2 (chg at 39 th)

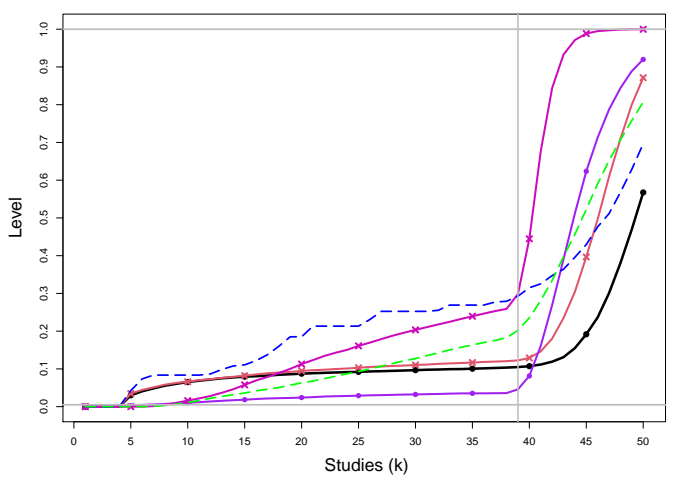

IV REML: $\tau^{2}=0.25 \mathrm{n}=100$ level $=0.005 \mathrm{~h}=6 \delta=1$ to 2 (chg at 39 th)

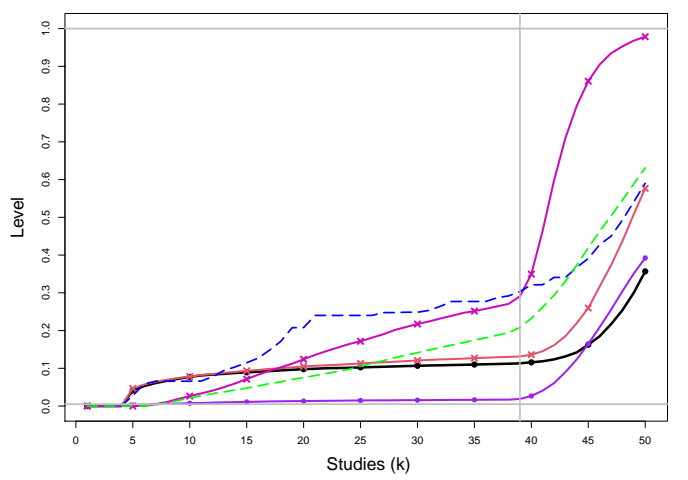

IV REML: $\tau^{2}=1 \mathrm{n}=100$ level $=0.005 \mathrm{~h}=6 \delta=1$ to 2 (chg at 39 th)

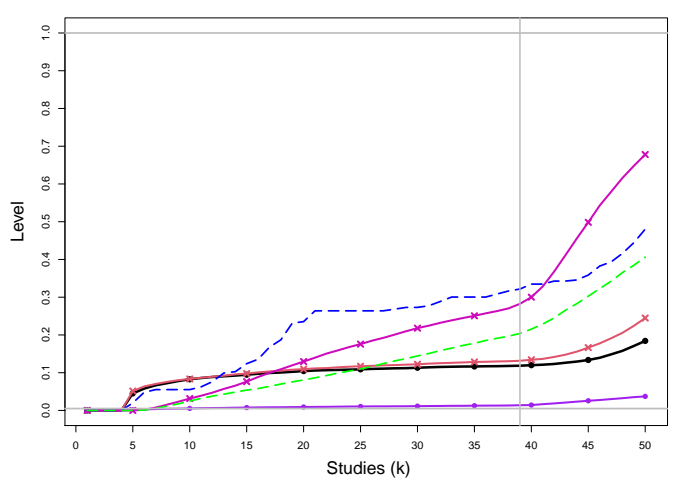

SSW KDB: $\tau^{2}=0 \mathrm{n}=100$ level $=0.005 \mathrm{~h}=6 \delta=1$ to 2 (chg at 39th)

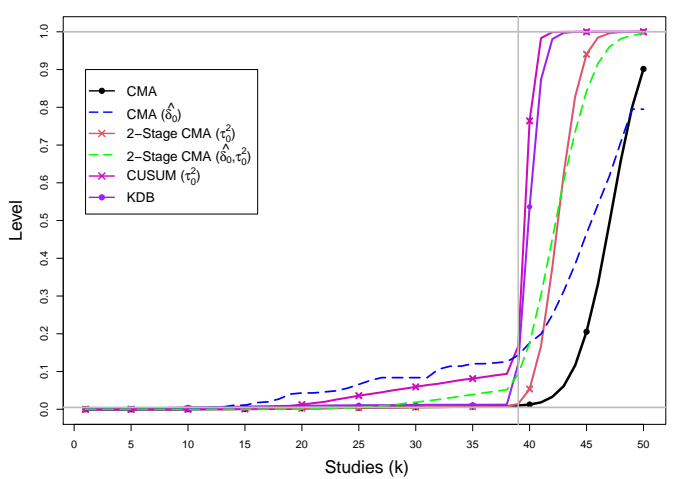

SSW KDB: $\tau^{2}=0.1 \mathrm{n}=100$ level $=0.005 \mathrm{~h}=6 \delta=1$ to 2 (chg at 39th)

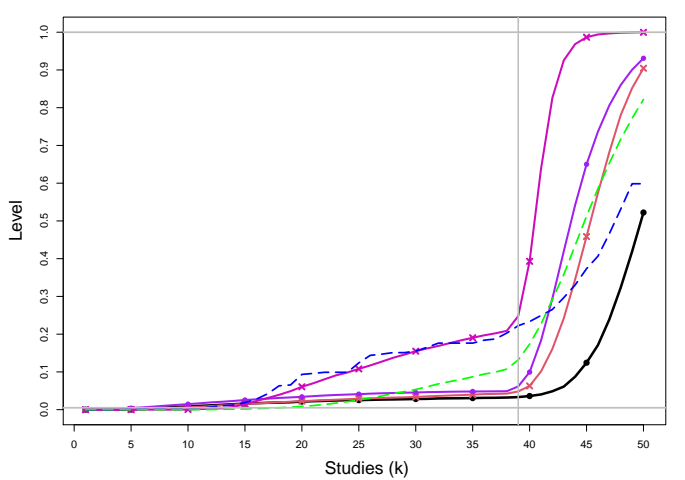

SSW KDB: $\tau^{2}=0.25 \mathrm{n}=100$ level $=0.005 \mathrm{~h}=6 \delta=1$ to 2 (chg at 39th)

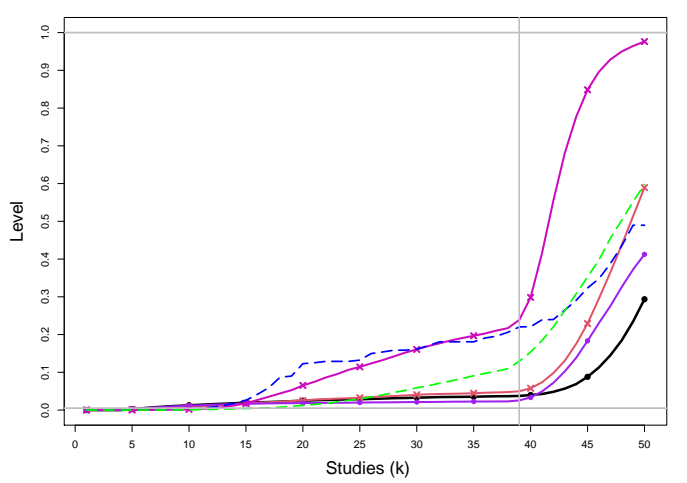

SSW KDB: $\tau^{2}=1 \mathrm{n}=100$ level $=0.005 \mathrm{~h}=6 \delta=1$ to 2 (chg at 39 th)

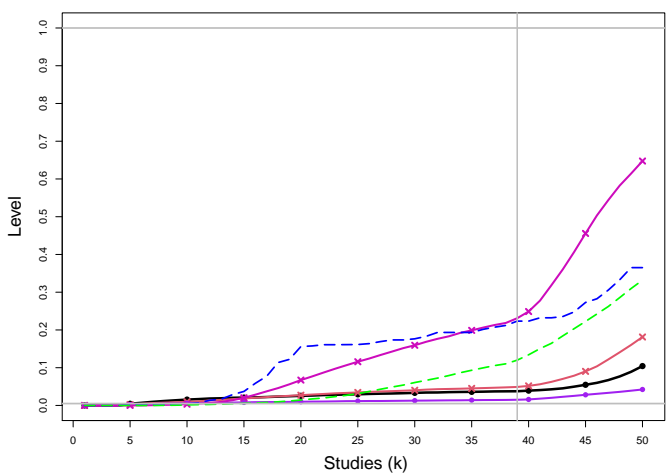

Figure E2. 43: Empirical levels of 1- and 2-stage CMA tests for shift in $\delta$ at .005 nominal level, shift in $\tau^{2}$ at .0025 nominal levels and of CUSUM with $h=6$ for equal sample sizes $n_{i C}+n_{i T}=n=100, \tau^{2}=0,0.1,0.25,1$ and a shift from $\delta=1$ to $\delta=2$ at study number 39. Light grey line at .005. 


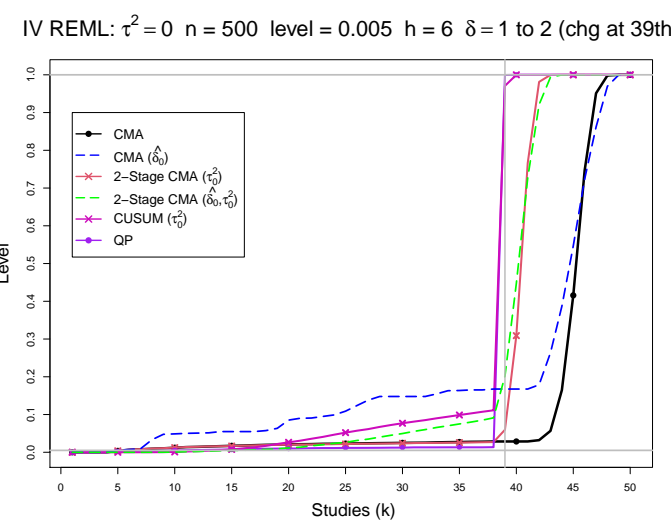

IV REML: $\tau^{2}=0.1 \mathrm{n}=500$ level $=0.005 \mathrm{~h}=6 \delta=1$ to 2 (chg at 39 th)

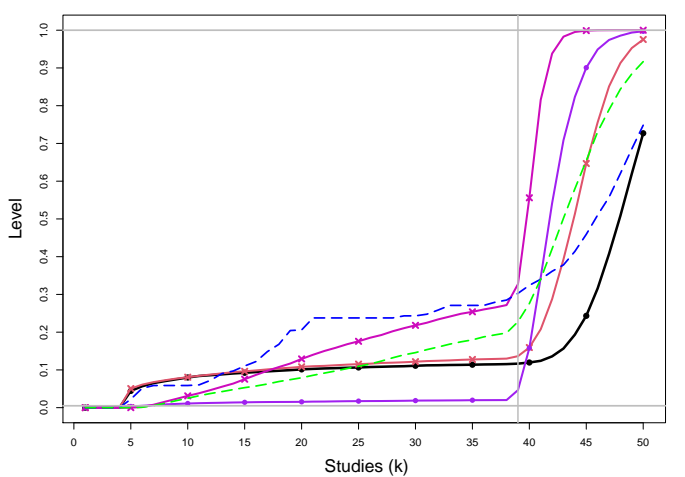

IV REML: $\tau^{2}=0.25 \mathrm{n}=500$ level $=0.005 \mathrm{~h}=6 \delta=1$ to 2 (chg at 39th)

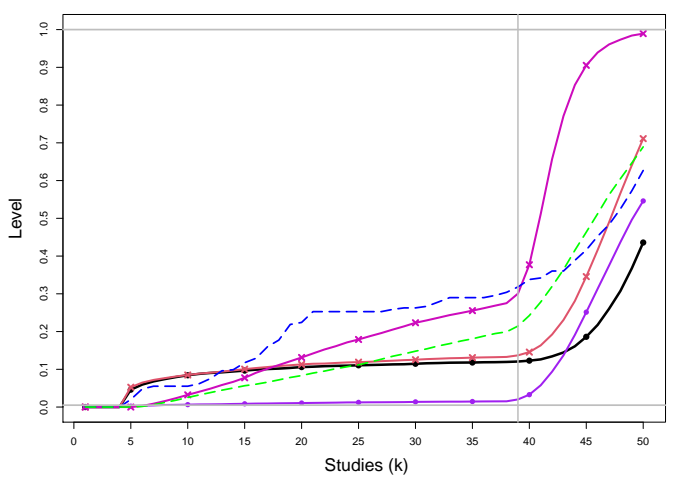

IV REML: $\tau^{2}=1 \mathrm{n}=500$ level $=0.005 \mathrm{~h}=6 \delta=1$ to 2 (chg at 39th)

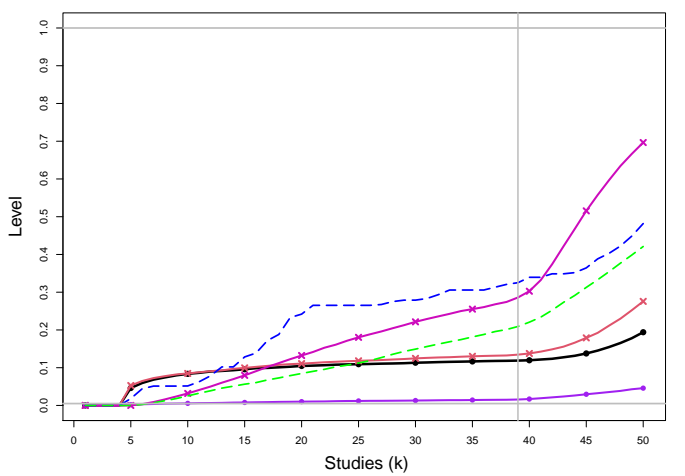

SSW KDB: $\tau^{2}=0 \mathrm{n}=500$ level $=0.005 \mathrm{~h}=6 \delta=1$ to 2 (chg at 39th)

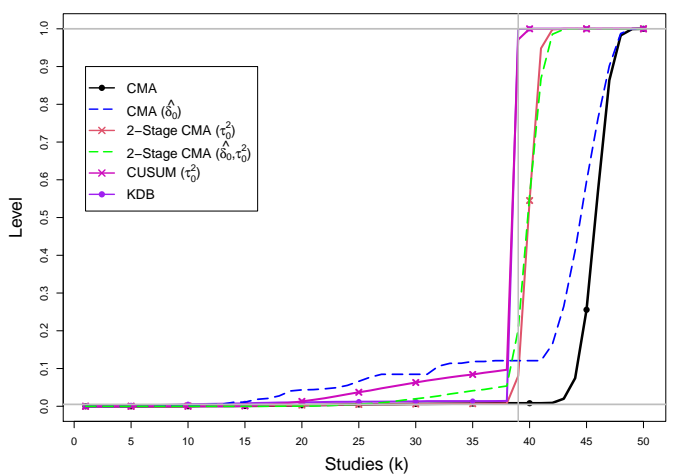

SSW KDB: $\tau^{2}=0.1 \mathrm{n}=500$ level $=0.005 \mathrm{~h}=6 \delta=1$ to 2 (chg at 39th)

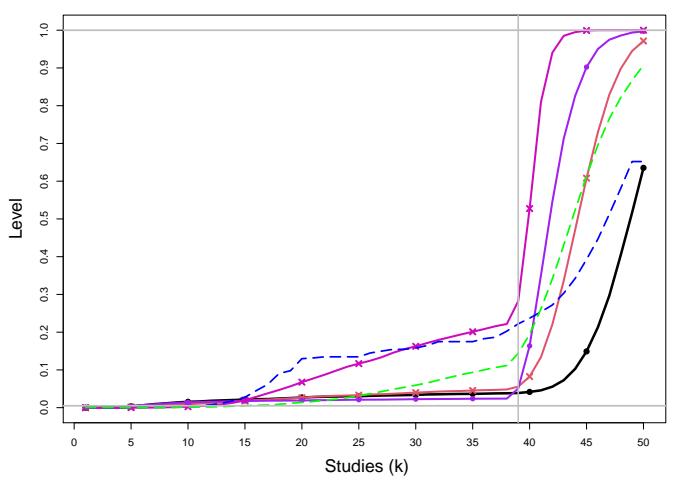

SSW KDB: $\tau^{2}=0.25 \mathrm{n}=500$ level $=0.005 \mathrm{~h}=6 \delta=1$ to 2 (chg at 39th)

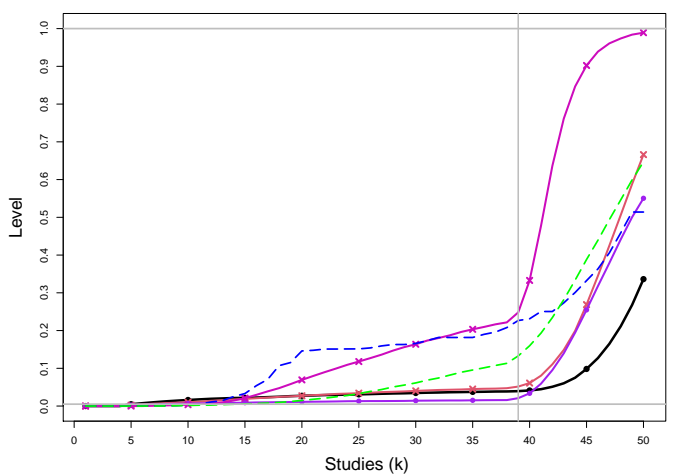

SSW KDB: $\tau^{2}=1 \mathrm{n}=500$ level $=0.005 \mathrm{~h}=6 \delta=1$ to 2 (chg at 39 th)

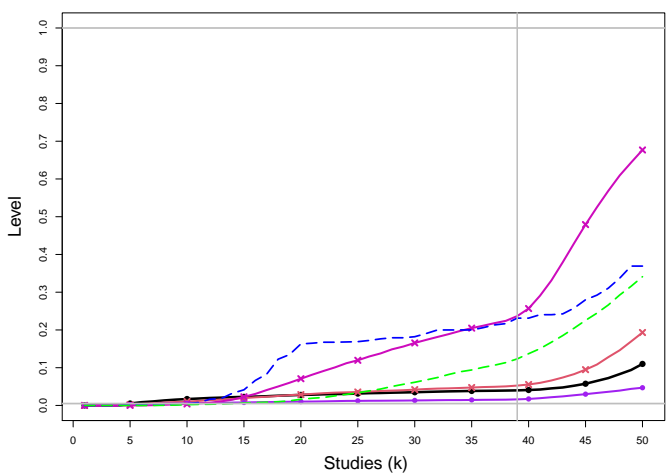

Figure E2. 44: Empirical levels of 1- and 2-stage CMA tests for shift in $\delta$ at .005 nominal level, shift in $\tau^{2}$ at .0025 nominal levels and of CUSUM with $h=6$ for equal sample sizes $n_{i C}+n_{i T}=n=500, \tau^{2}=0,0.1,0.25,1$ and a shift from $\delta=1$ to $\delta=2$ at study number 39. Light grey line at .005. 
IV REML: $\tau^{2}=0 \mathrm{n}=1000$ level $=0.005 \mathrm{~h}=6 \quad \delta=1$ to 2 (chg at 39th)

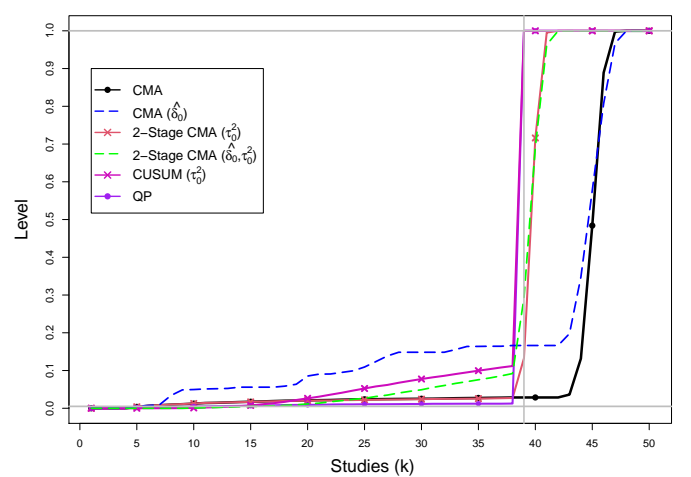

IV REML: $\tau^{2}=0.1 \mathrm{n}=1000$ level $=0.005 \mathrm{~h}=6 \quad \delta=1$ to 2 (chg at 39th)

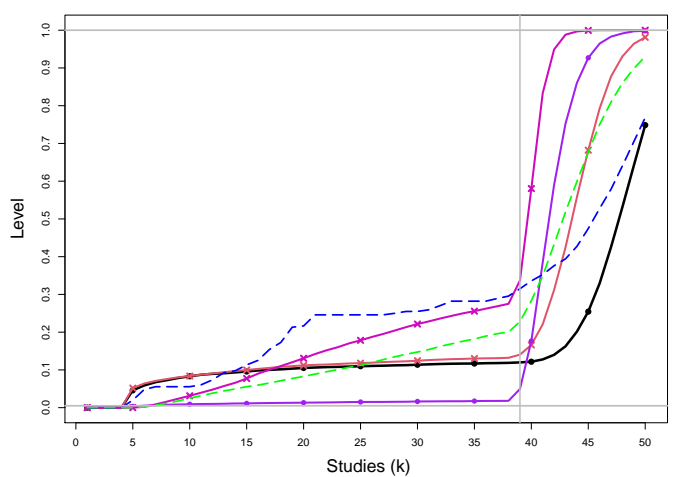

IV REML: $\tau^{2}=0.25 \mathrm{n}=1000$ level $=0.005 \mathrm{~h}=6 \delta=1$ to 2 (chg at 39 th)

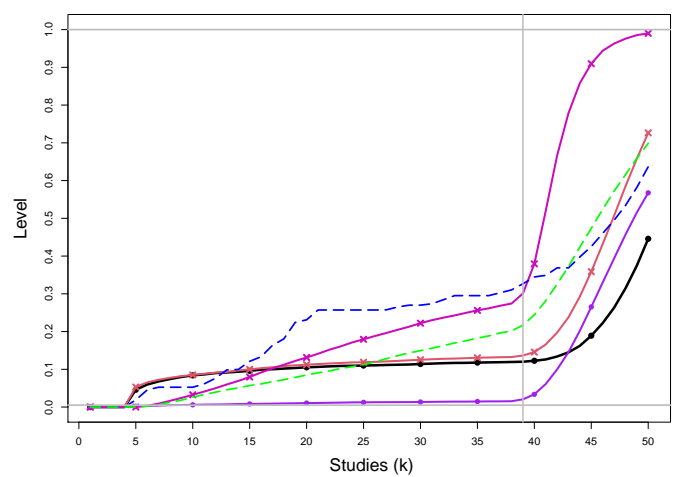

IV REML: $\tau^{2}=1 \mathrm{n}=1000$ level $=0.005 \mathrm{~h}=6 \delta=1$ to 2 (chg at 39 th)

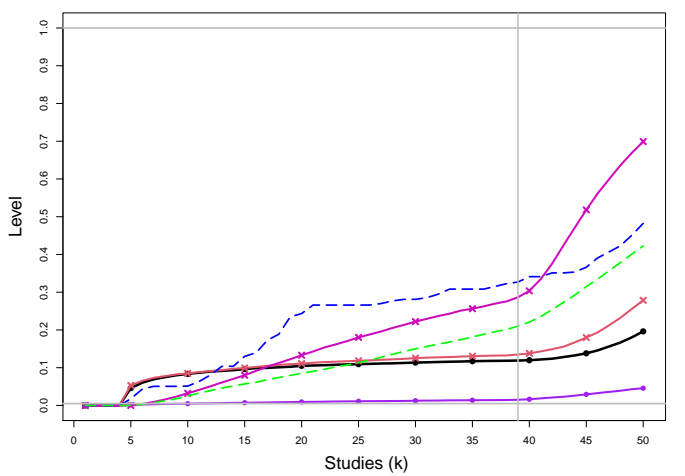

SSW KDB: $\tau^{2}=0 \mathrm{n}=1000$ level $=0.005 \mathrm{~h}=6 \delta=1$ to 2 (chg at 39th)

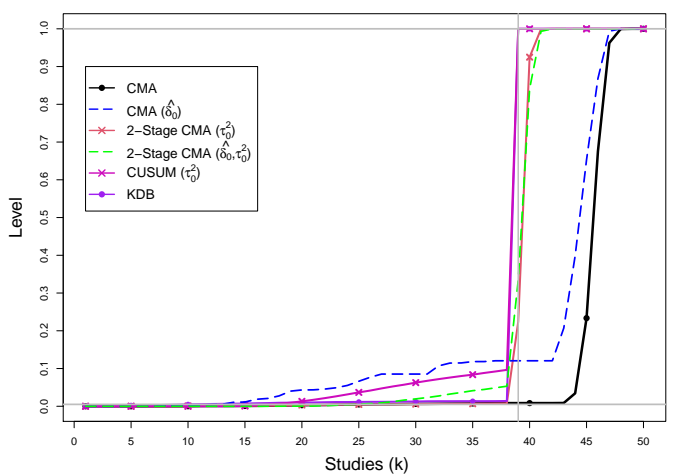

SSW KDB: $\tau^{2}=0.1 \mathrm{n}=1000$ level $=0.005 \mathrm{~h}=6 \quad \delta=1$ to 2 (chg at 39 th)

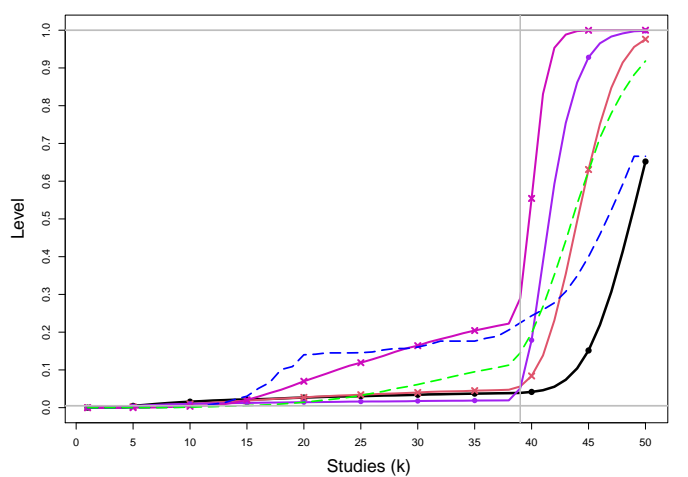

SSW KDB: $\tau^{2}=0.25 \mathrm{n}=1000$ level $=0.005 \mathrm{~h}=6 \delta=1$ to 2 (chg at 39th)

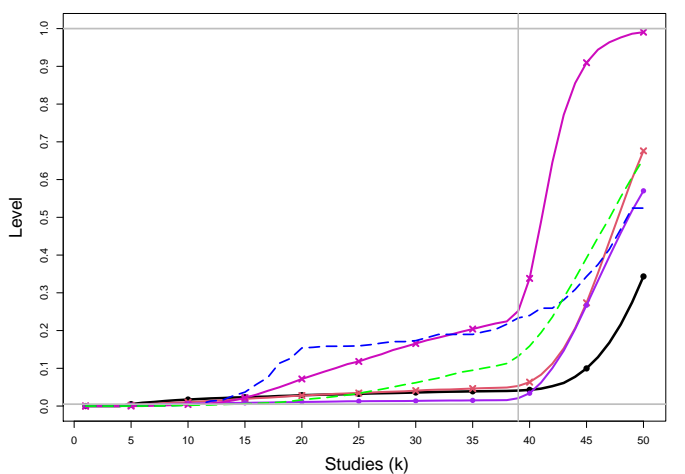

SSW KDB: $\tau^{2}=1 \mathrm{n}=1000$ level $=0.005 \mathrm{~h}=6 \delta=1$ to 2 (chg at 39th)

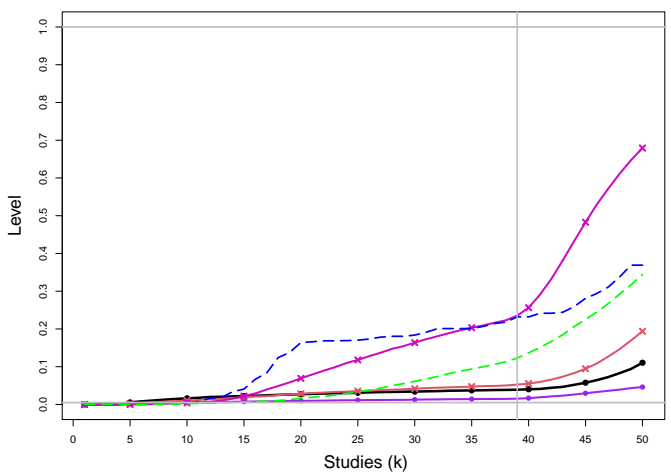

Figure E2. 45: Empirical levels of 1- and 2-stage CMA tests for shift in $\delta$ at .005 nominal level, shift in $\tau^{2}$ at .0025 nominal levels and of CUSUM with $h=6$ for equal sample sizes $n_{i C}+n_{i T}=n=1000, \tau^{2}=0,0.1,0.25,1$ and a shift from $\delta=1$ to $\delta=2$ at study number 39. Light grey line at .005. 


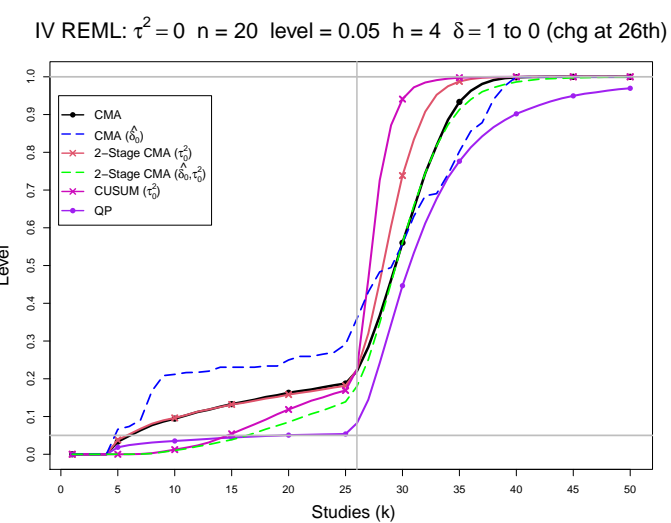

IV REML: $\tau^{2}=0.1 \mathrm{n}=20$ level $=0.05 \mathrm{~h}=4 \delta=1$ to 0 (chg at 26 th)

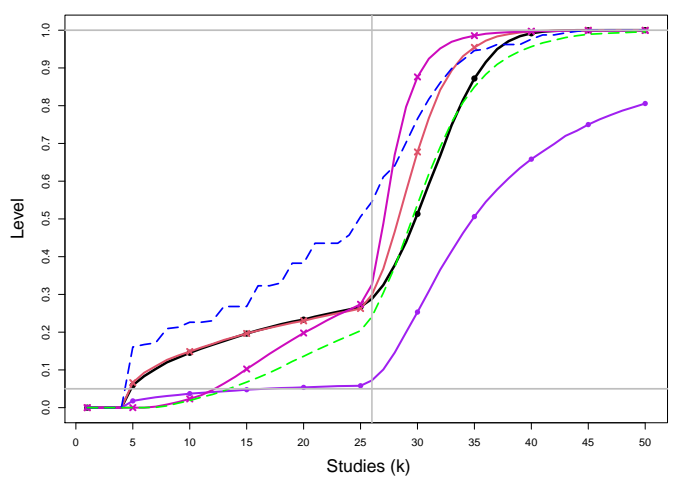

IV REML: $\tau^{2}=0.25 \mathrm{n}=20$ level $=0.05 \mathrm{~h}=4 \delta=1$ to 0 (chg at 26th)

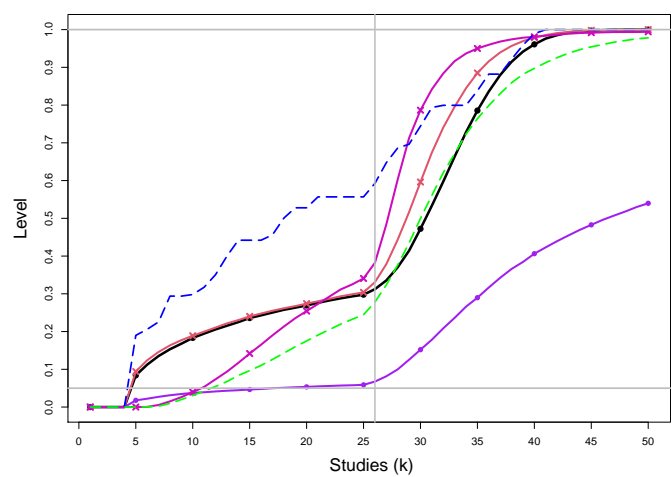

IV REML: $\tau^{2}=1 \mathrm{n}=20$ level $=0.05 \mathrm{~h}=4 \delta=1$ to 0 (chg at 26th)

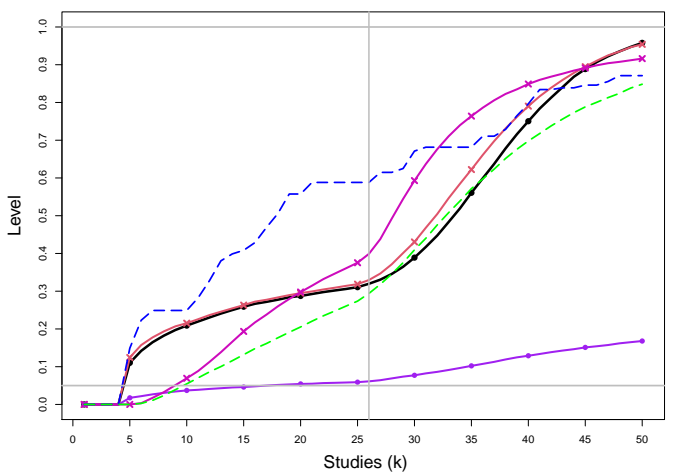

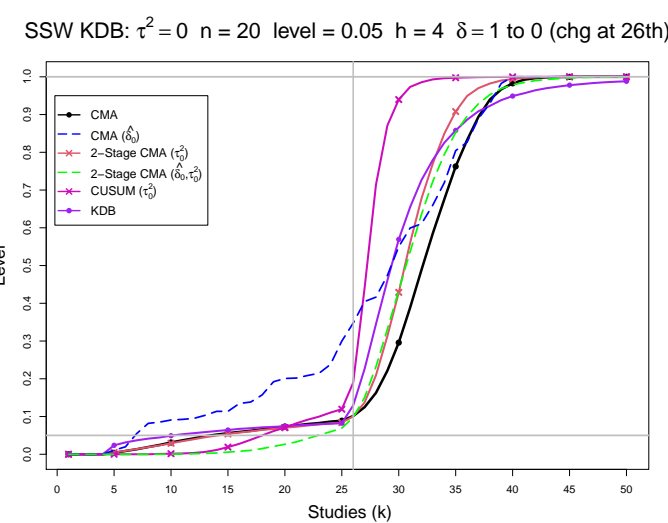

SSW KDB: $\tau^{2}=0.1 \mathrm{n}=20$ level $=0.05 \mathrm{~h}=4 \delta=1$ to 0 (chg at 26th)

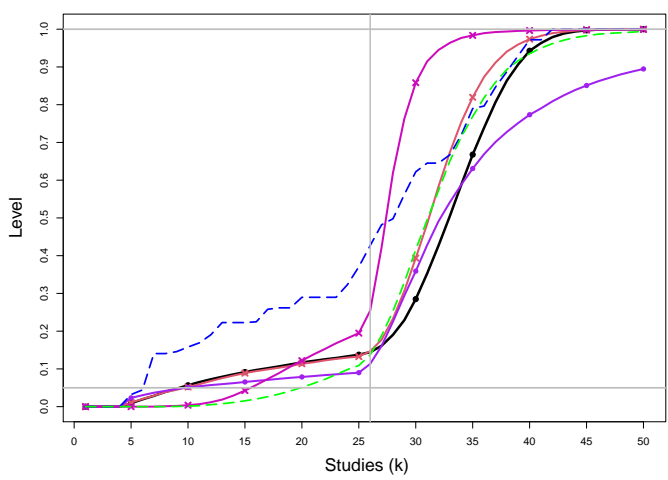

SSW KDB: $\tau^{2}=0.25 \mathrm{n}=20$ level $=0.05 \mathrm{~h}=4 \delta=1$ to 0 (chg at 26th)

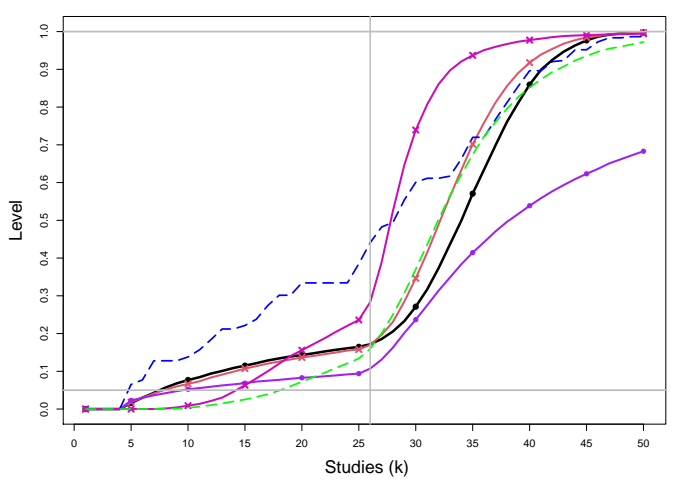

SSW KDB: $\tau^{2}=1 \mathrm{n}=20$ level $=0.05 \mathrm{~h}=4 \delta=1$ to 0 (chg at 26th)

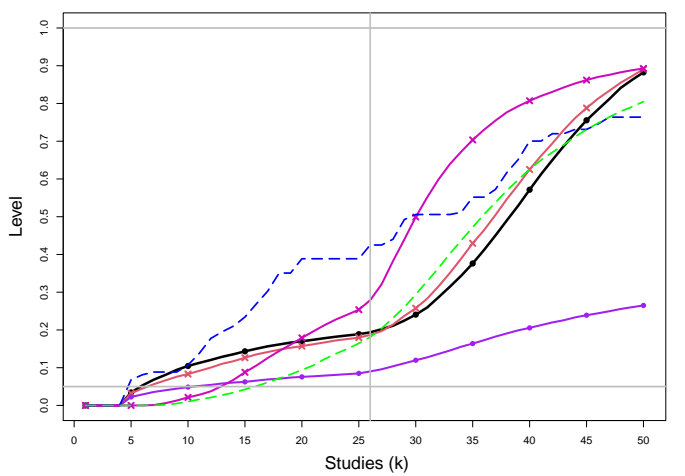

Figure E2. 46: Empirical levels of 1- and 2-stage CMA tests for shift in $\delta$ at .05 nominal level, shift in $\tau^{2}$ at .025 nominal levels and of CUSUM with $h=4$ for equal sample sizes $n_{i C}+n_{i T}=n=20, \tau^{2}=0,0.1,0.25,1$ and a shift from $\delta=1$ to $\delta=0$ at study number 26. Light grey line at .05. 


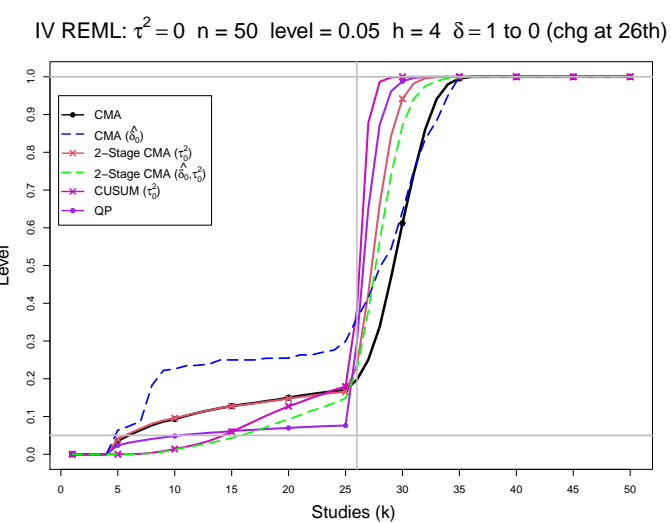

IV REML: $\tau^{2}=0.1 \mathrm{n}=50$ level $=0.05 \mathrm{~h}=4 \delta=1$ to 0 (chg at 26 th)

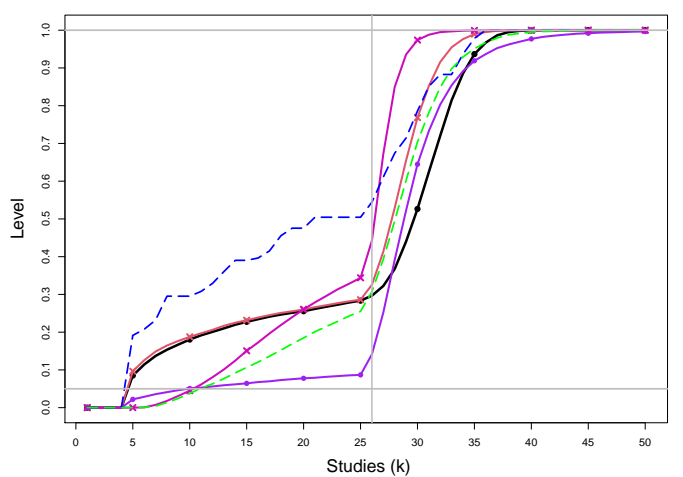

IV REML: $\tau^{2}=0.25 \mathrm{n}=50$ level $=0.05 \mathrm{~h}=4 \delta=1$ to 0 (chg at 26th)

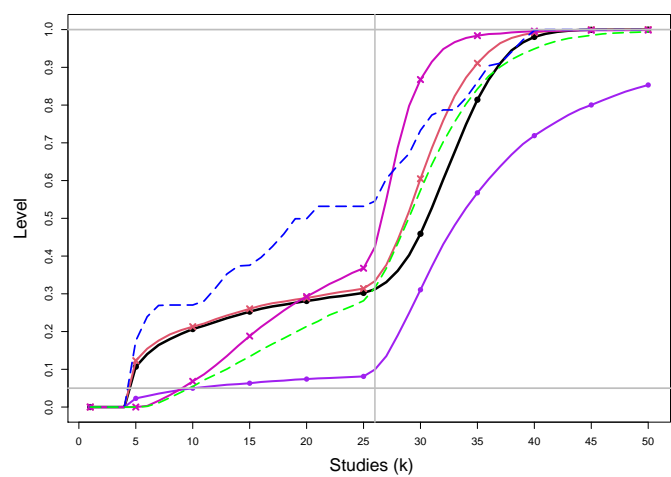

IV REML: $\tau^{2}=1 \mathrm{n}=50$ level $=0.05 \mathrm{~h}=4 \delta=1$ to 0 (chg at 26th)

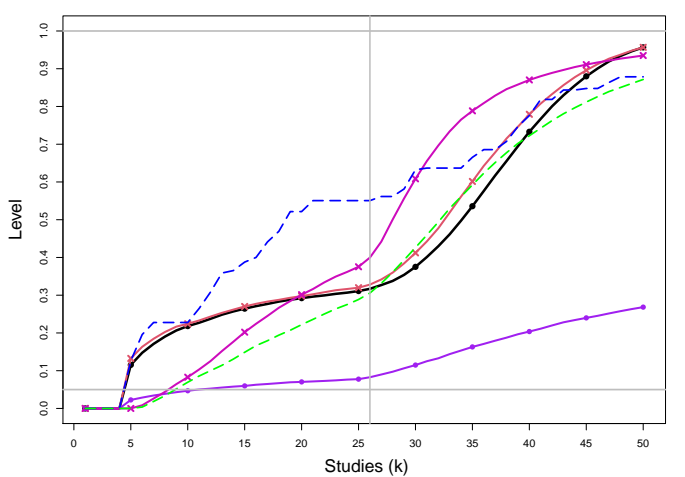

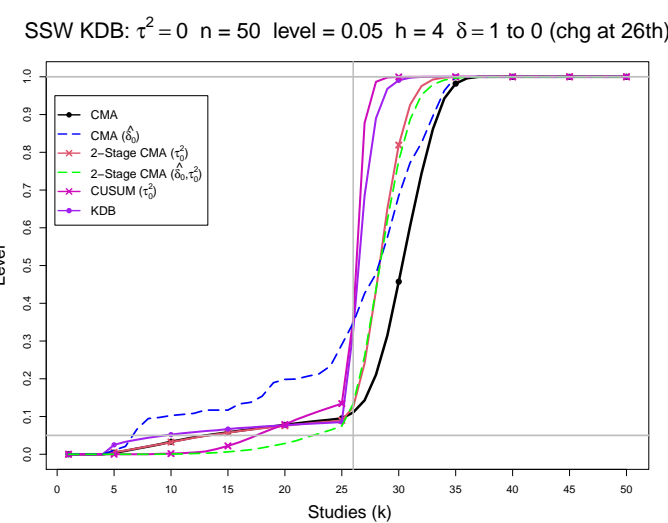

SSW KDB: $\tau^{2}=0.1 \mathrm{n}=50$ level $=0.05 \mathrm{~h}=4 \delta=1$ to 0 (chg at 26th)

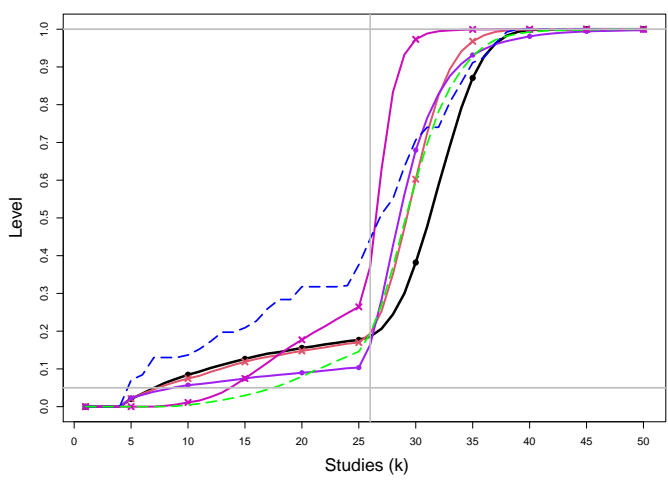

SSW KDB: $\tau^{2}=0.25 \mathrm{n}=50$ level $=0.05 \mathrm{~h}=4 \delta=1$ to 0 (chg at 26th)

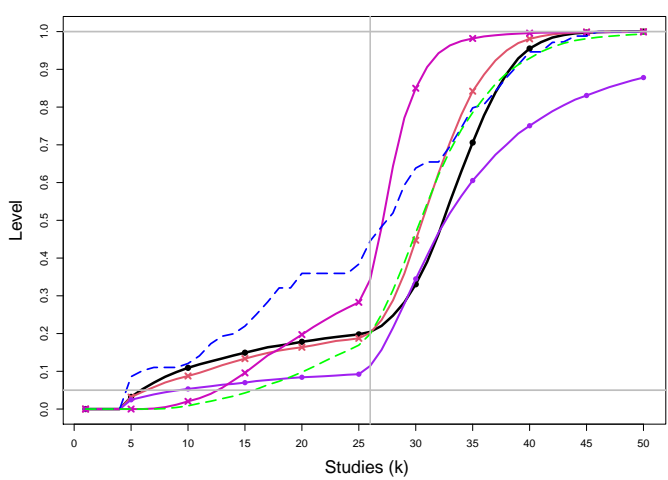

SSW KDB: $\tau^{2}=1 \mathrm{n}=50$ level $=0.05 \mathrm{~h}=4 \delta=1$ to 0 (chg at 26th)

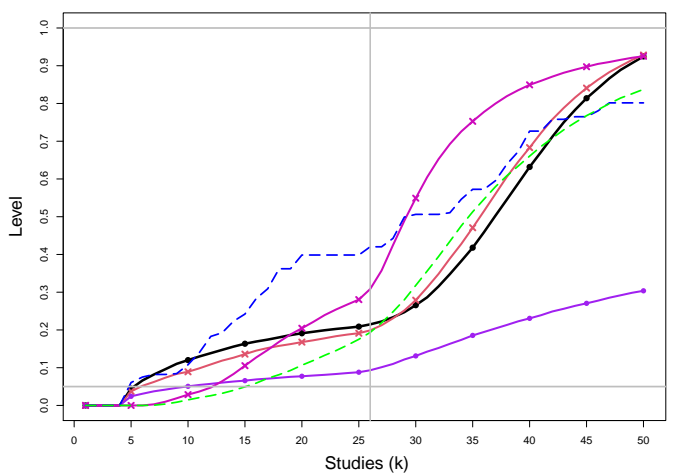

Figure E2. 47: Empirical levels of 1- and 2-stage CMA tests for shift in $\delta$ at .05 nominal level, shift in $\tau^{2}$ at .025 nominal levels and of CUSUM with $h=4$ for equal sample sizes $n_{i C}+n_{i T}=n=50, \tau^{2}=0,0.1,0.25,1$ and a shift from $\delta=1$ to $\delta=0$ at study number 26. Light grey line at .05. 


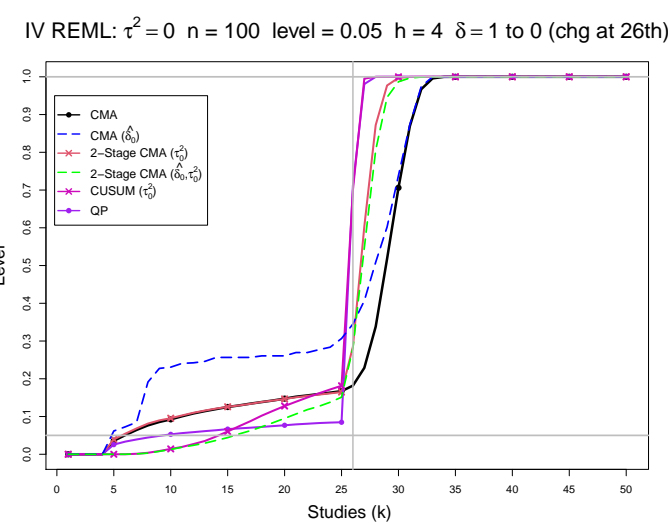

IV REML: $\tau^{2}=0.1 \mathrm{n}=100$ level $=0.05 \mathrm{~h}=4 \delta=1$ to 0 (chg at 26th)

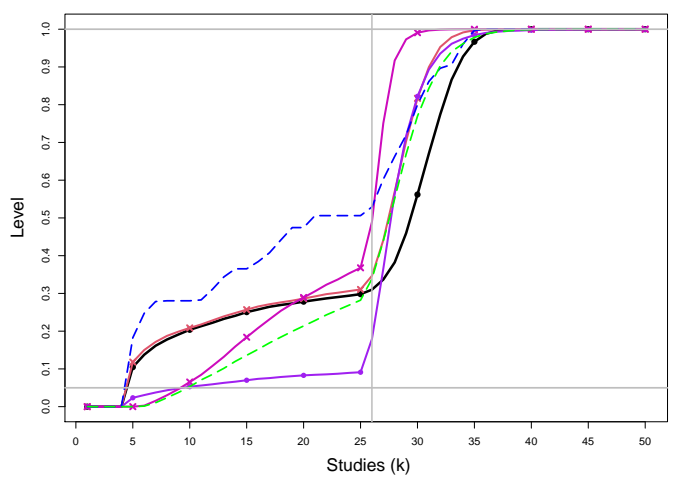

IV REML: $\tau^{2}=0.25 \mathrm{n}=100$ level $=0.05 \mathrm{~h}=4 \delta=1$ to 0 (chg at 26 th)

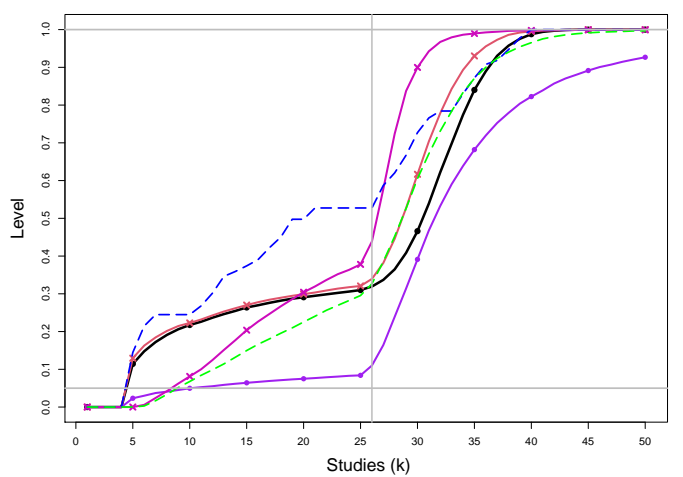

IV REML: $\tau^{2}=1 \mathrm{n}=100$ level $=0.05 \mathrm{~h}=4 \delta=1$ to 0 (chg at 26 th)

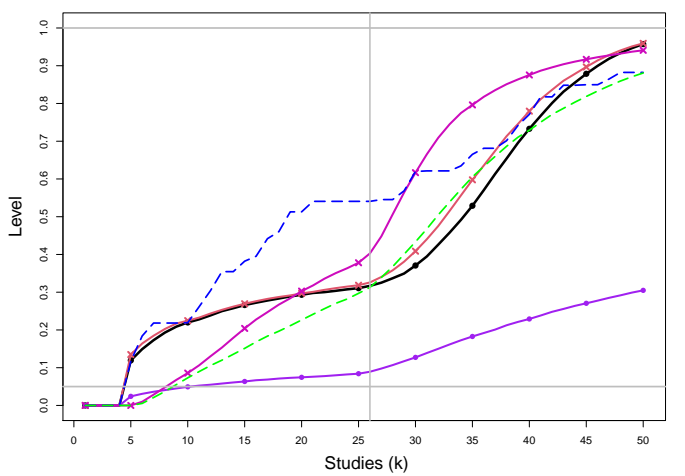

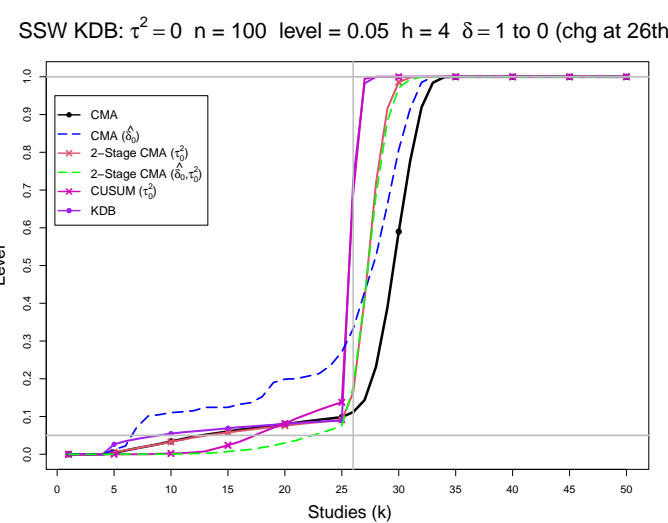

SSW KDB: $\tau^{2}=0.1 \mathrm{n}=100$ level $=0.05 \mathrm{~h}=4 \delta=1$ to 0 (chg at 26th)

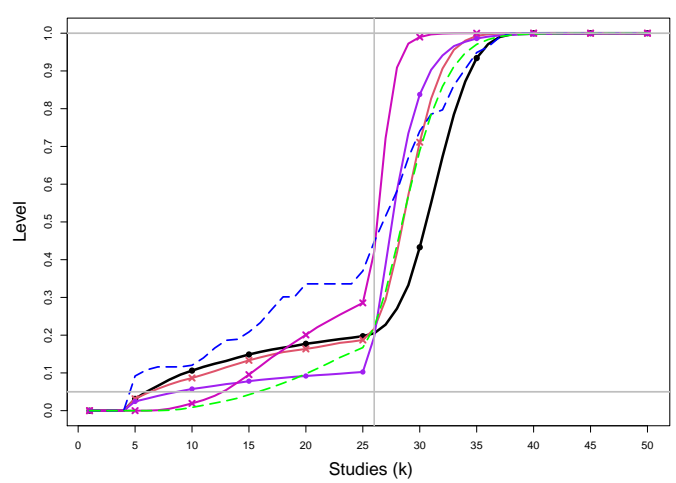

SSW KDB: $\tau^{2}=0.25 \mathrm{n}=100$ level $=0.05 \mathrm{~h}=4 \delta=1$ to 0 (chg at 26th)

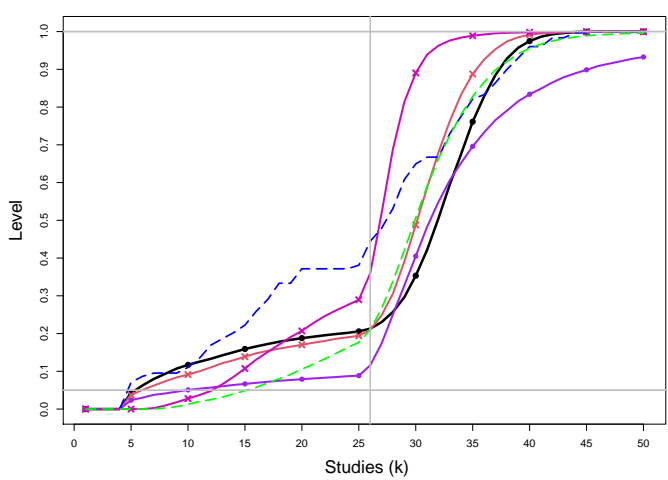

SSW KDB: $\tau^{2}=1 \mathrm{n}=100$ level $=0.05 \mathrm{~h}=4 \delta=1$ to 0 (chg at 26th)

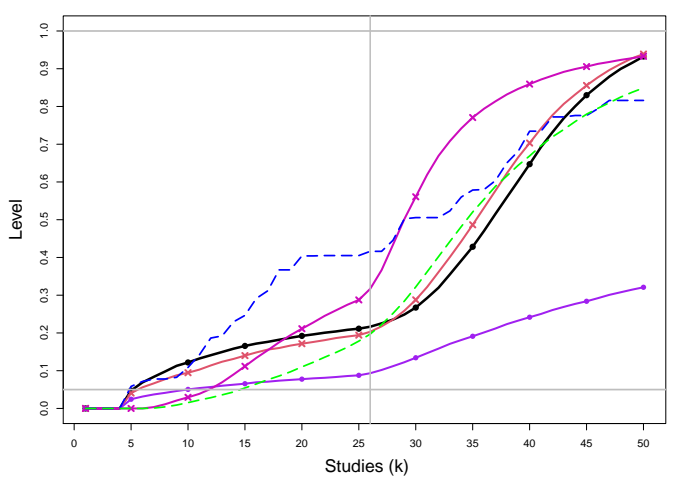

Figure E2. 48: Empirical levels of 1- and 2-stage CMA tests for shift in $\delta$ at .05 nominal level, shift in $\tau^{2}$ at .025 nominal levels and of CUSUM with $h=4$ for equal sample sizes $n_{i C}+n_{i T}=n=100, \tau^{2}=0,0.1,0.25,1$ and a shift from $\delta=1$ to $\delta=0$ at study number 26. Light grey line at .05. 


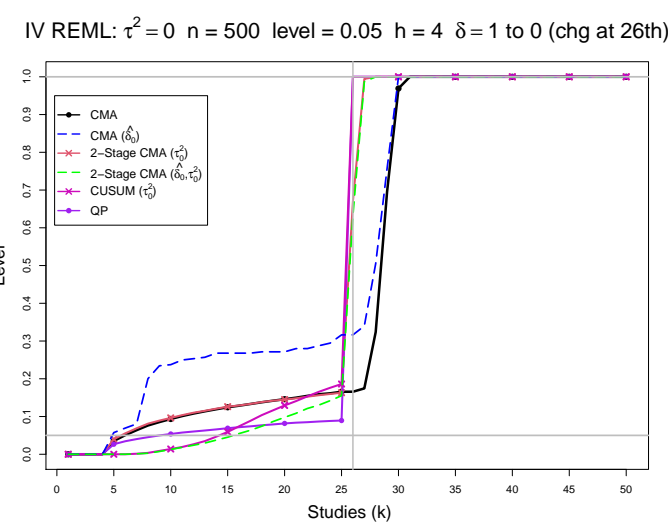

IV REML: $\tau^{2}=0.1 \mathrm{n}=500$ level $=0.05 \mathrm{~h}=4 \delta=1$ to 0 (chg at 26th)

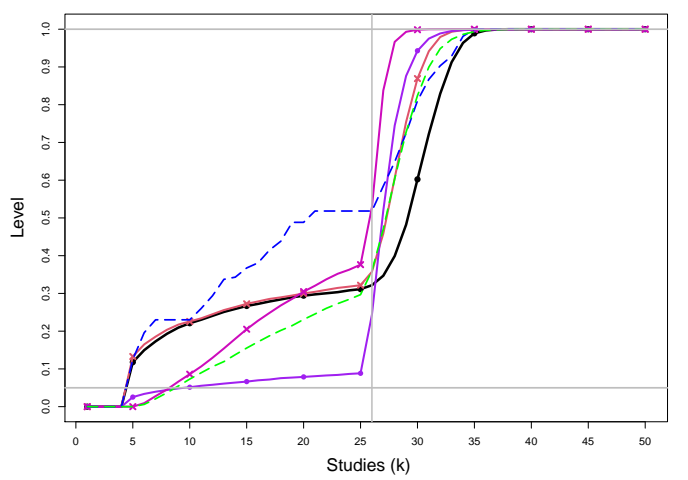

IV REML: $\tau^{2}=0.25 \mathrm{n}=500$ level $=0.05 \mathrm{~h}=4 \delta=1$ to 0 (chg at 26 th)

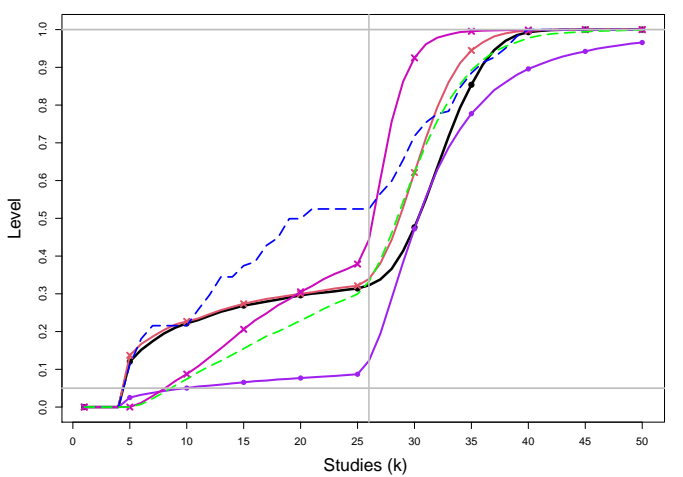

IV REML: $\tau^{2}=1 \mathrm{n}=500$ level $=0.05 \mathrm{~h}=4 \delta=1$ to 0 (chg at 26th)

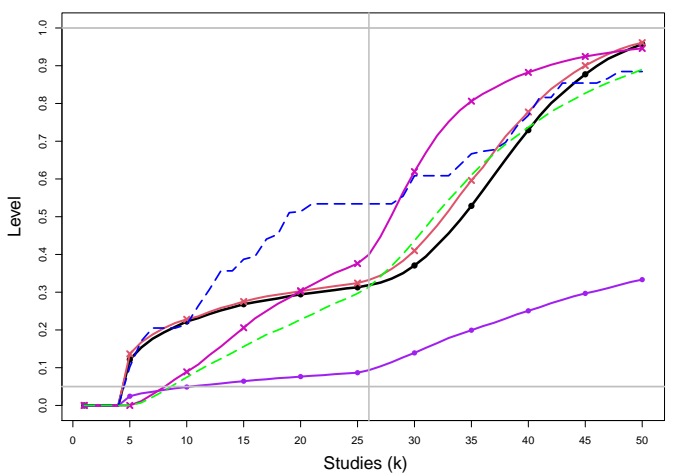

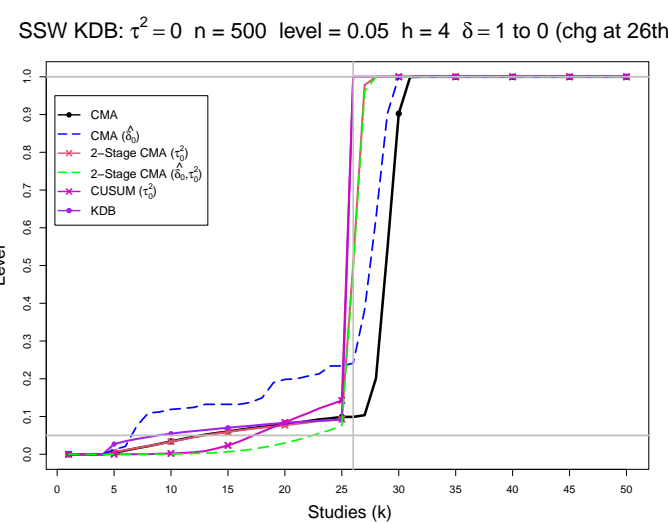

SSW KDB: $\tau^{2}=0.1 \mathrm{n}=500$ level $=0.05 \mathrm{~h}=4 \delta=1$ to 0 (chg at 26th)

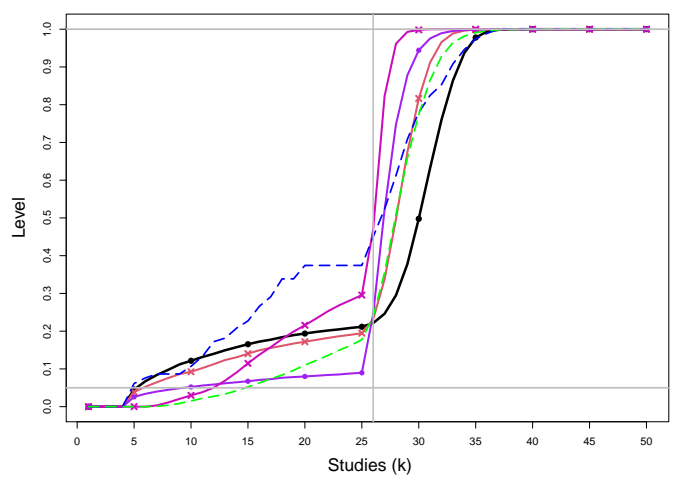

SSW KDB: $\tau^{2}=0.25 \mathrm{n}=500$ level $=0.05 \mathrm{~h}=4 \delta=1$ to 0 (chg at 26th)

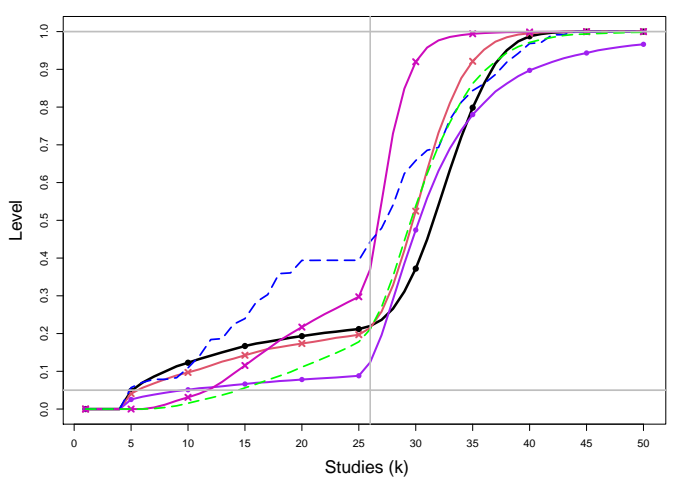

SSW KDB: $\tau^{2}=1 \mathrm{n}=500$ level $=0.05 \mathrm{~h}=4 \delta=1$ to 0 (chg at 26th)

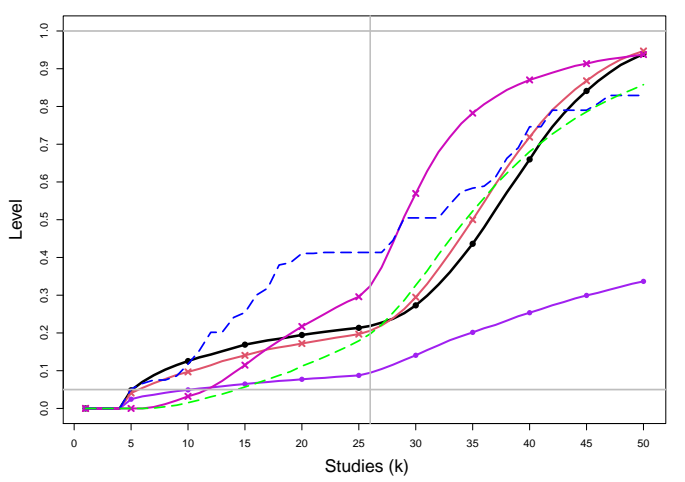

Figure E2. 49: Empirical levels of 1- and 2-stage CMA tests for shift in $\delta$ at .05 nominal level, shift in $\tau^{2}$ at .025 nominal levels and of CUSUM with $h=4$ for equal sample sizes $n_{i C}+n_{i T}=n=500, \tau^{2}=0,0.1,0.25,1$ and a shift from $\delta=1$ to $\delta=0$ at study number 26. Light grey line at .05. 
IV REML: $\tau^{2}=0 \mathrm{n}=1000$ level $=0.05 \mathrm{~h}=4 \delta=1$ to 0 (chg at 26 th)

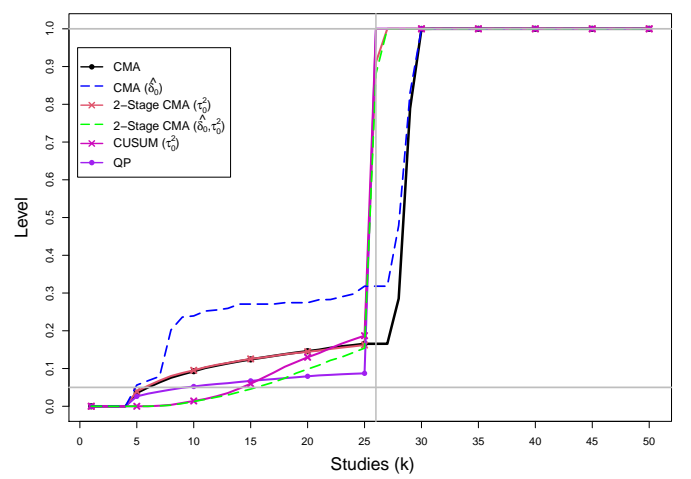

IV REML: $\tau^{2}=0.1 \mathrm{n}=1000$ level $=0.05 \mathrm{~h}=4 \delta=1$ to 0 (chg at 26 th)

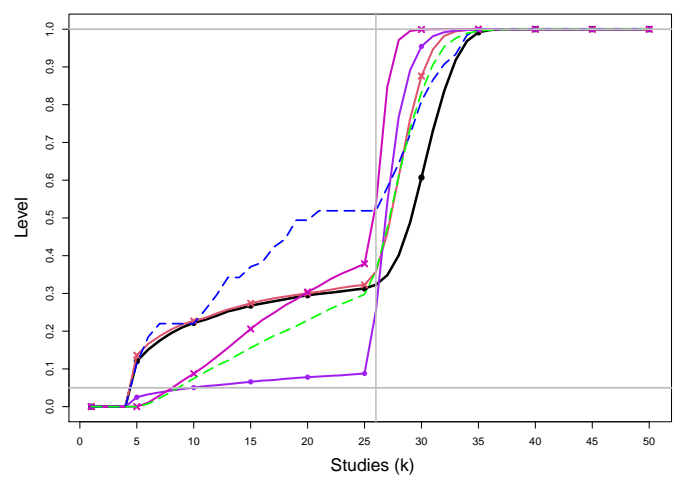

IV REML: $\tau^{2}=0.25 \mathrm{n}=1000$ level $=0.05 \mathrm{~h}=4 \quad \delta=1$ to 0 (chg at 26th)

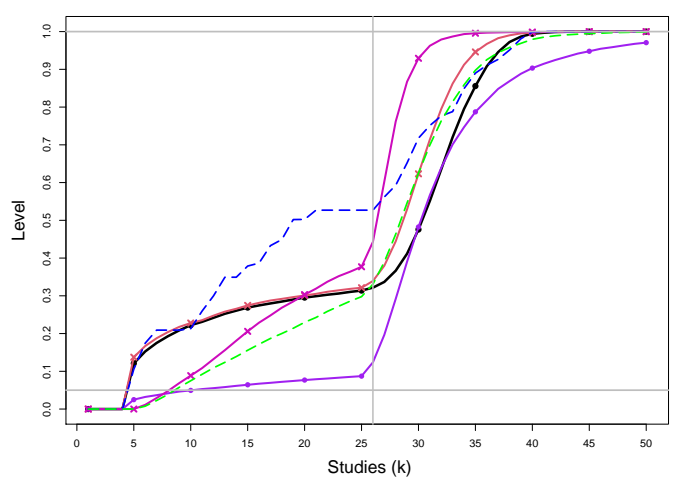

IV REML: $\tau^{2}=1 \mathrm{n}=1000$ level $=0.05 \mathrm{~h}=4 \delta=1$ to 0 (chg at 26th)

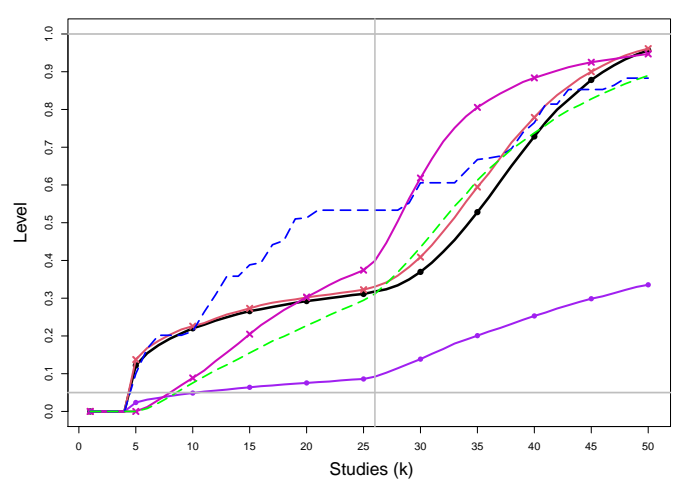

SSW KDB: $\tau^{2}=0 \mathrm{n}=1000$ level $=0.05 \mathrm{~h}=4 \delta=1$ to 0 (chg at 26 th)

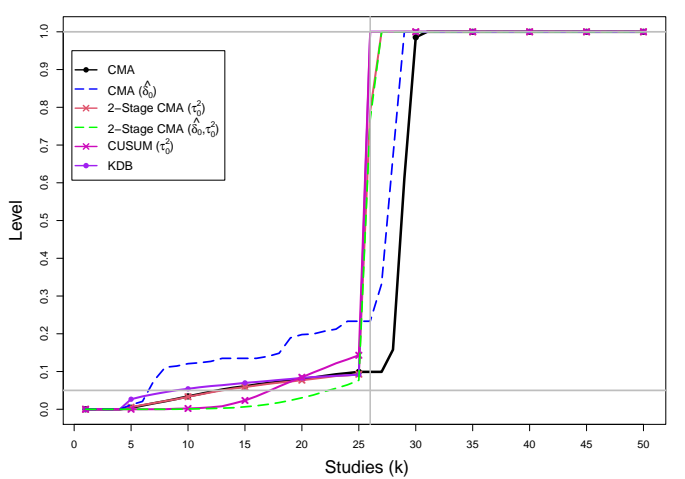

SSW KDB: $\tau^{2}=0.1 \mathrm{n}=1000$ level $=0.05 \mathrm{~h}=4 \delta=1$ to 0 (chg at 26 th)

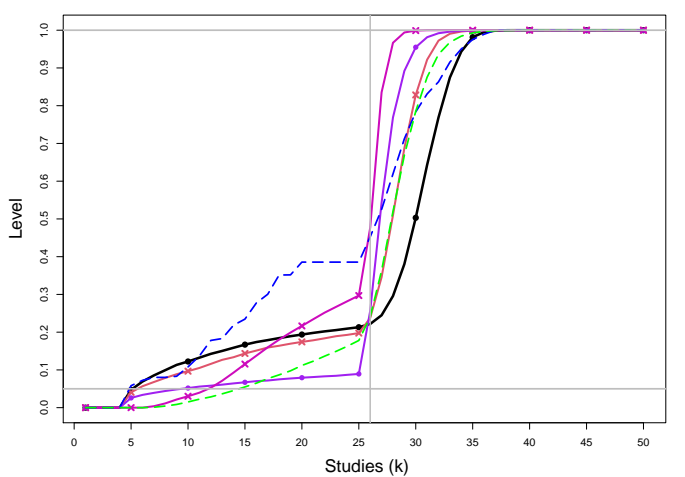

SSW KDB: $\tau^{2}=0.25 \mathrm{n}=1000$ level $=0.05 \mathrm{~h}=4 \delta=1$ to 0 (chg at 26th)

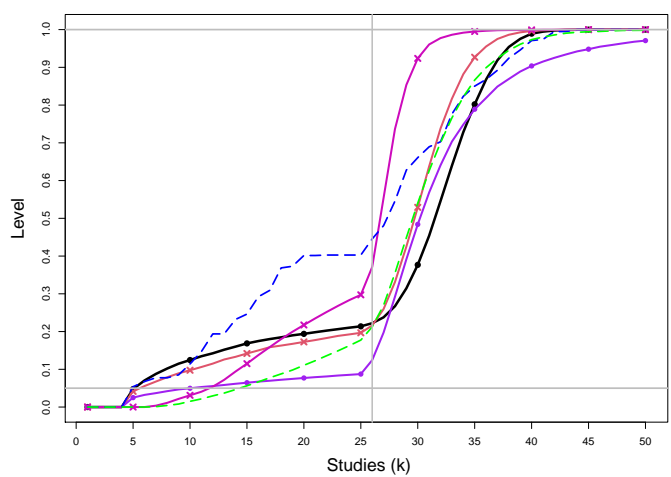

SSW KDB: $\tau^{2}=1 \mathrm{n}=1000$ level $=0.05 \mathrm{~h}=4 \delta=1$ to 0 (chg at 26th)

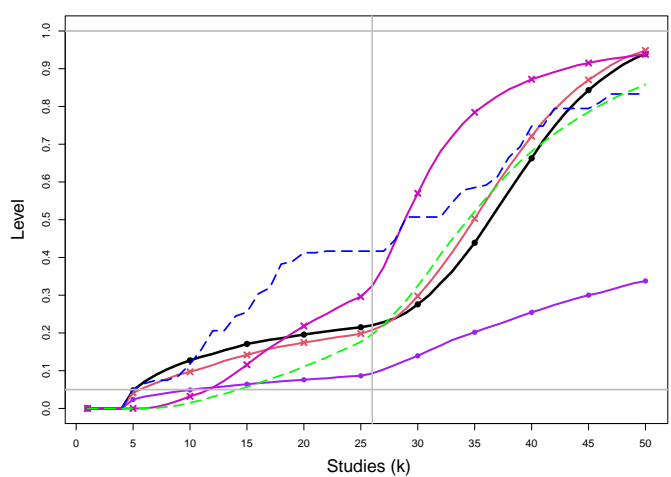

Figure E2. 50: Empirical levels of 1- and 2-stage CMA tests for shift in $\delta$ at .05 nominal level, shift in $\tau^{2}$ at .025 nominal levels and of CUSUM with $h=4$ for equal sample sizes $n_{i C}+n_{i T}=n=1000, \tau^{2}=0,0.1,0.25,1$ and a shift from $\delta=1$ to $\delta=0$ at study number 26. Light grey line at .05. 


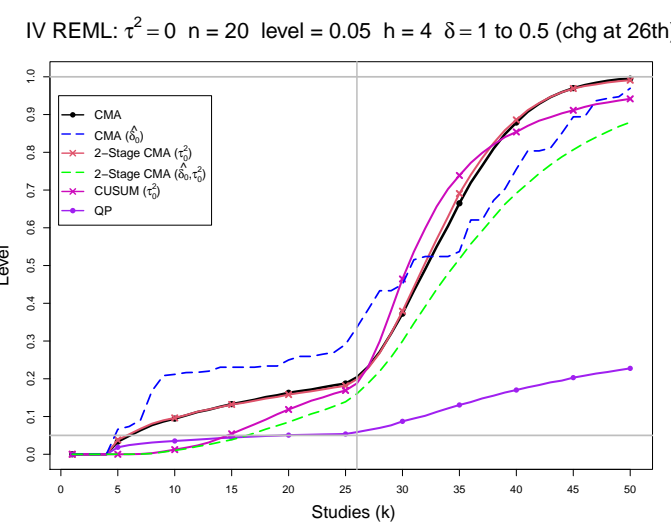

IV REML: $\tau^{2}=0.1 \mathrm{n}=20$ level $=0.05 \mathrm{~h}=4 \delta=1$ to 0.5 (chg at 26th)

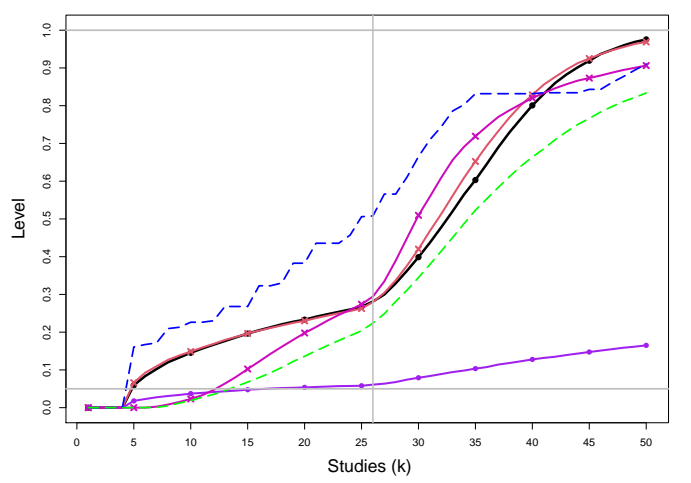

IV REML: $\tau^{2}=0.25 \mathrm{n}=20$ level $=0.05 \mathrm{~h}=4 \delta=1$ to 0.5 (chg at 26th)

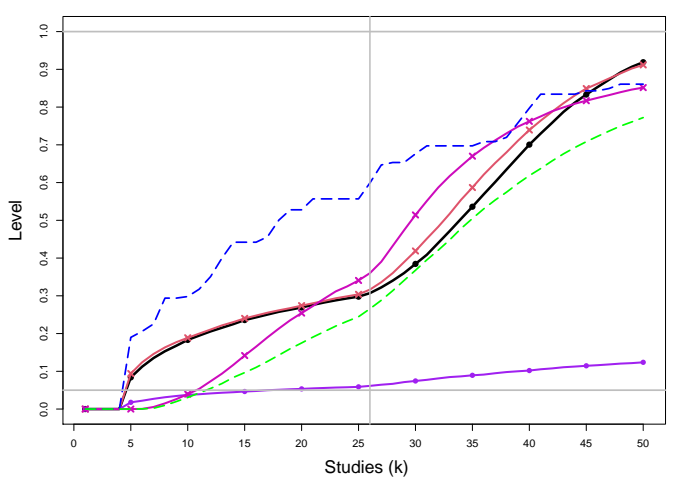

IV REML: $\tau^{2}=1 \mathrm{n}=20$ level $=0.05 \mathrm{~h}=4 \delta=1$ to 0.5 (chg at 26th)

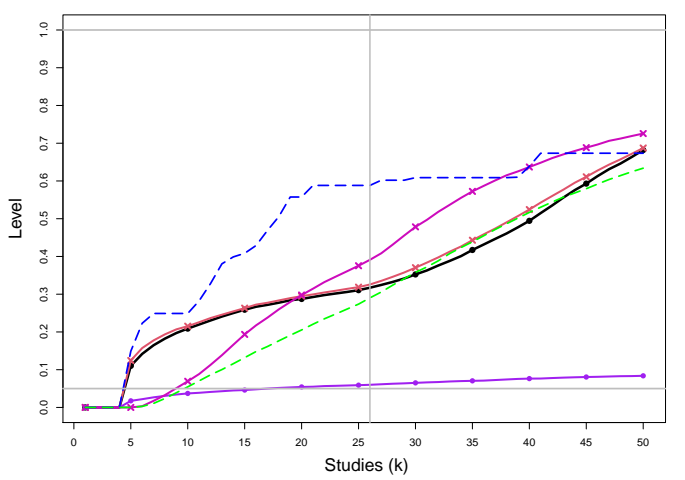

SSW KDB: $\tau^{2}=0 \mathrm{n}=20$ level $=0.05 \mathrm{~h}=4 \delta=1$ to 0.5 (chg at 26th)

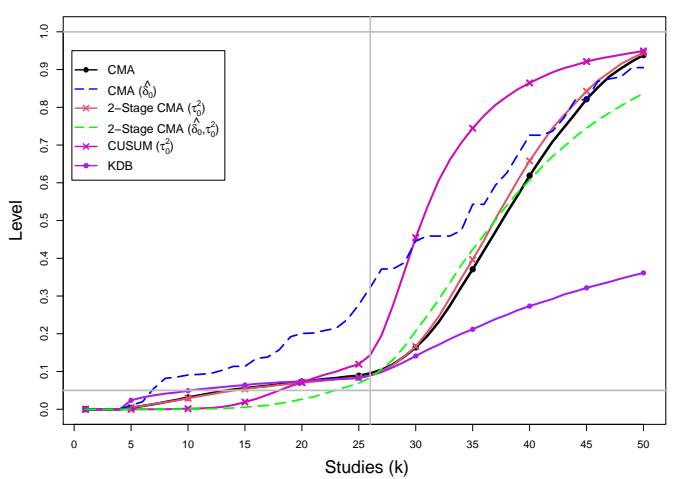

SSW KDB: $\tau^{2}=0.1 \mathrm{n}=20$ level $=0.05 \mathrm{~h}=4 \delta=1$ to 0.5 (chg at 26th)

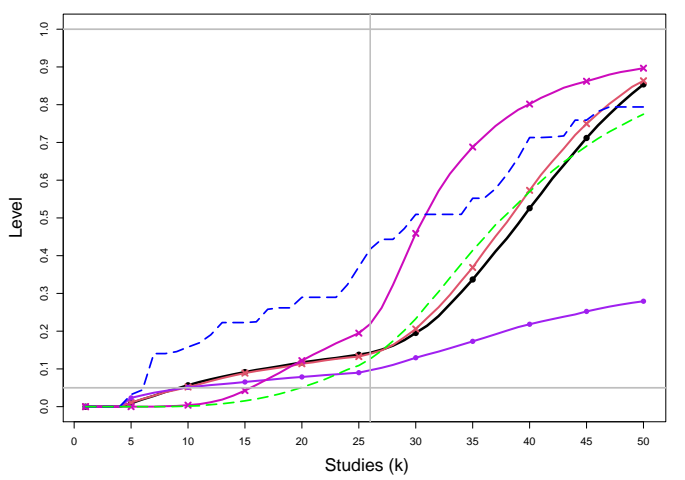

SSW KDB: $\tau^{2}=0.25 \mathrm{n}=20$ level $=0.05 \mathrm{~h}=4 \delta=1$ to 0.5 (chg at 26th)

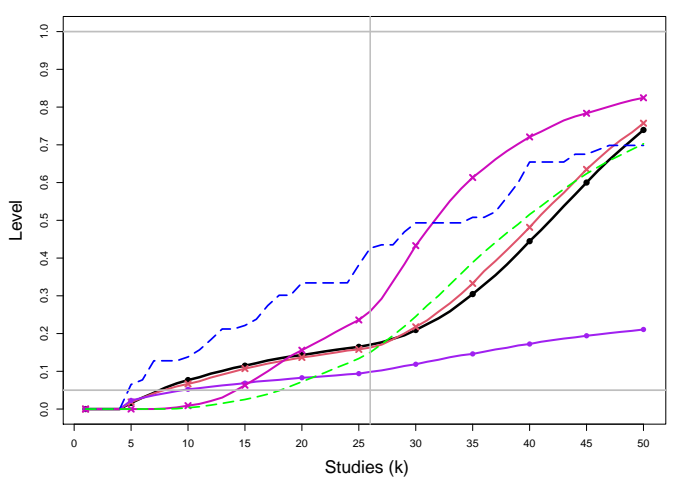

SSW KDB: $\tau^{2}=1 \mathrm{n}=20$ level $=0.05 \mathrm{~h}=4 \delta=1$ to 0.5 (chg at 26th)

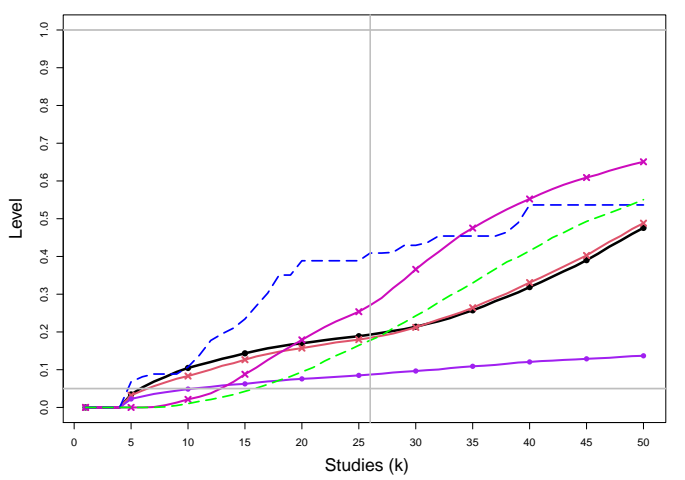

Figure E2. 51: Empirical levels of 1- and 2-stage CMA tests for shift in $\delta$ at .05 nominal level, shift in $\tau^{2}$ at .025 nominal levels and of CUSUM with $h=4$ for equal sample sizes $n_{i C}+n_{i T}=n=20, \tau^{2}=0,0.1,0.25,1$ and a shift from $\delta=1$ to $\delta=0.5$ at study number 26. Light grey line at .05. 


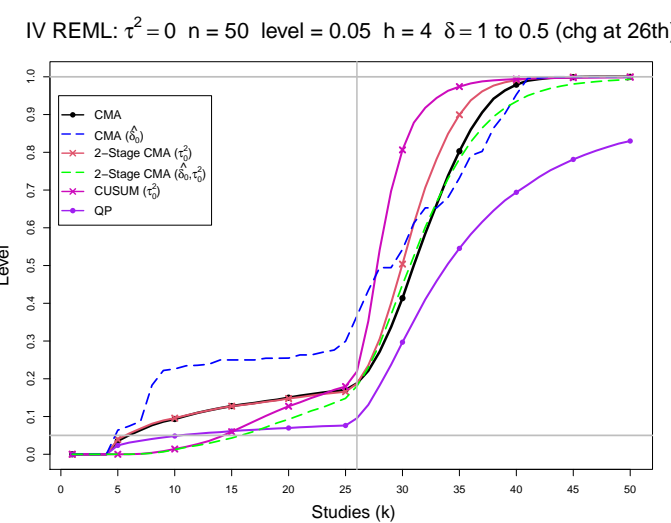

IV REML: $\tau^{2}=0.1 \mathrm{n}=50$ level $=0.05 \mathrm{~h}=4 \delta=1$ to 0.5 (chg at 26th)

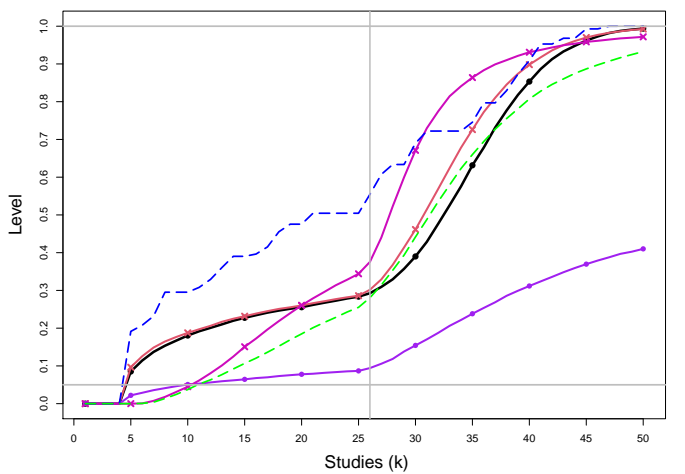

IV REML: $\tau^{2}=0.25 \mathrm{n}=50$ level $=0.05 \mathrm{~h}=4 \delta=1$ to 0.5 (chg at 26th)

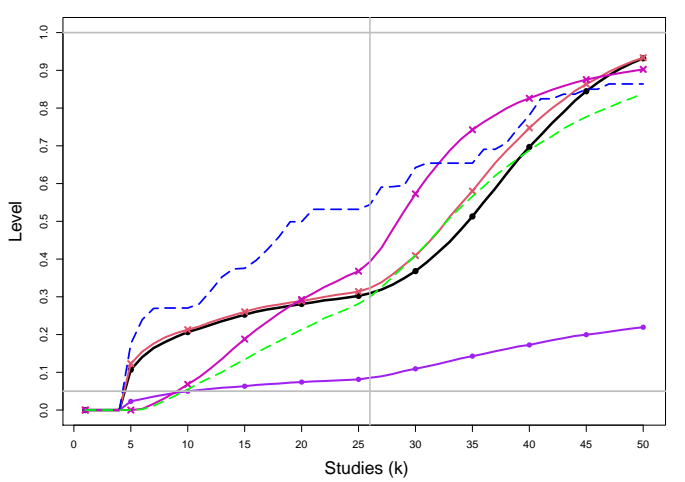

IV REML: $\tau^{2}=1 \mathrm{n}=50$ level $=0.05 \mathrm{~h}=4 \delta=1$ to 0.5 (chg at 26th)

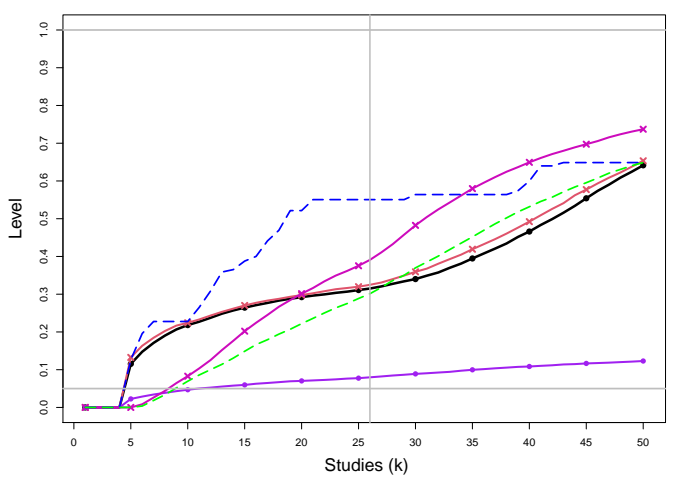

SSW KDB: $\tau^{2}=0 \mathrm{n}=50$ level $=0.05 \mathrm{~h}=4 \delta=1$ to 0.5 (chg at 26th)

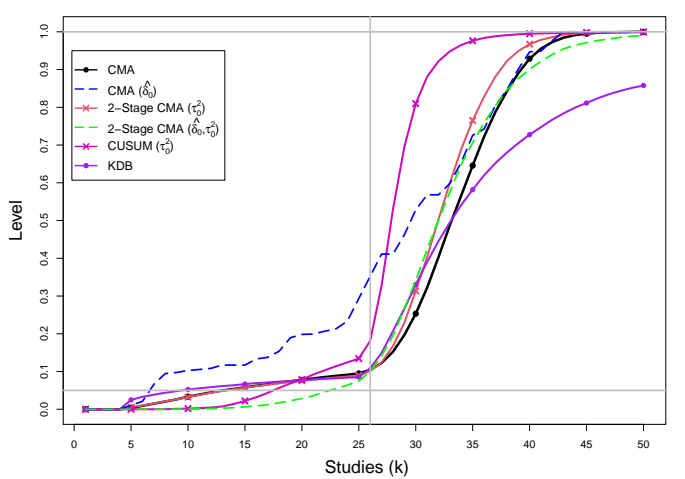

SSW KDB: $\tau^{2}=0.1 \mathrm{n}=50$ level $=0.05 \mathrm{~h}=4 \delta=1$ to 0.5 (chg at 26th)

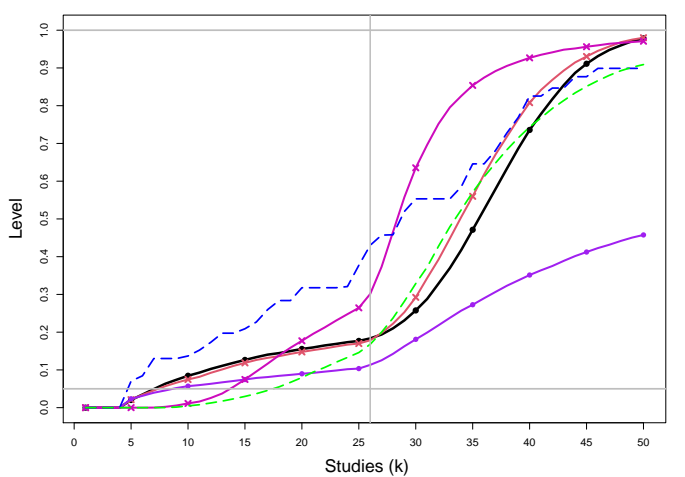

SSW KDB: $\tau^{2}=0.25 \mathrm{n}=50$ level $=0.05 \mathrm{~h}=4 \delta=1$ to 0.5 (chg at 26th)

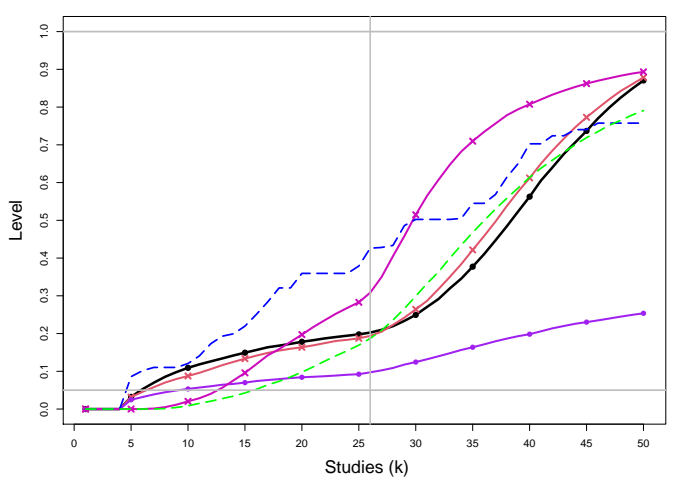

SSW KDB: $\tau^{2}=1 \mathrm{n}=50$ level $=0.05 \mathrm{~h}=4 \delta=1$ to 0.5 (chg at 26th)

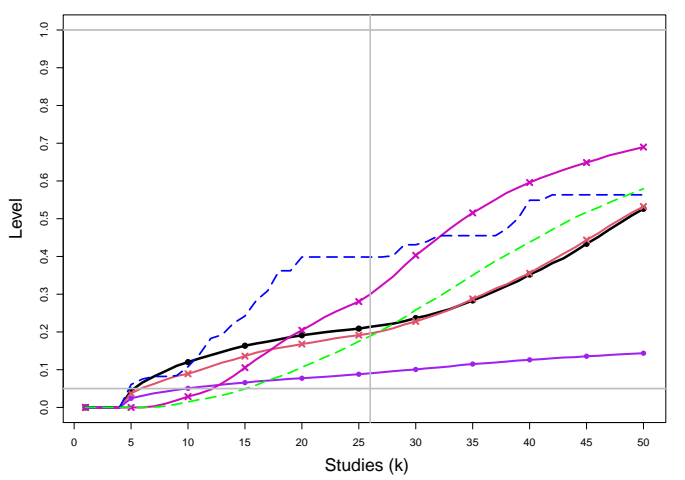

Figure E2. 52: Empirical levels of 1- and 2-stage CMA tests for shift in $\delta$ at .05 nominal level, shift in $\tau^{2}$ at .025 nominal levels and of CUSUM with $h=4$ for equal sample sizes $n_{i C}+n_{i T}=n=50, \tau^{2}=0,0.1,0.25,1$ and a shift from $\delta=1$ to $\delta=0.5$ at study number 26. Light grey line at .05. 
IV REML: $\tau^{2}=0 \mathrm{n}=100$ level $=0.05 \mathrm{~h}=4 \delta=1$ to 0.5 (chg at 26th)

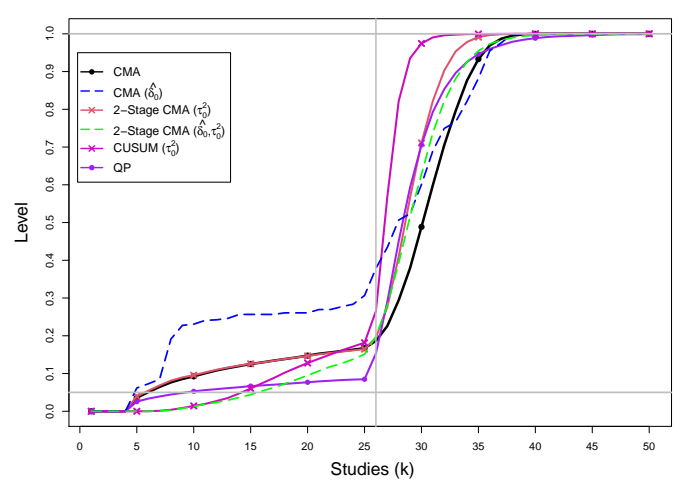

IV REML: $\tau^{2}=0.1 \mathrm{n}=100$ level $=0.05 \mathrm{~h}=4 \delta=1$ to 0.5 (chg at 26th)

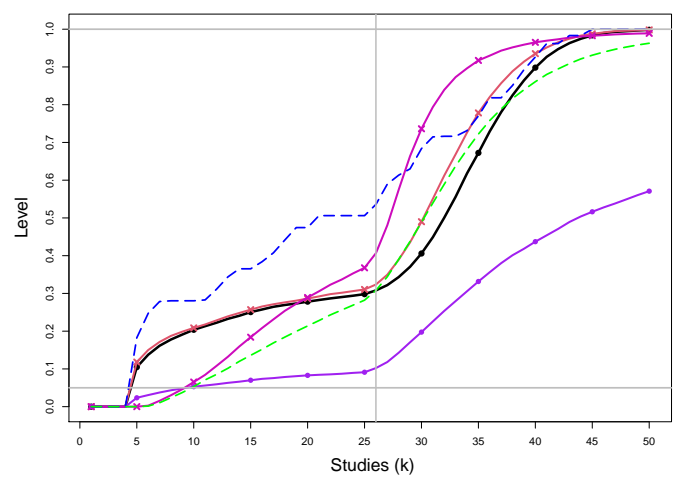

IV REML: $\tau^{2}=0.25 \mathrm{n}=100$ level $=0.05 \mathrm{~h}=4 \delta=1$ to 0.5 (chg at 26th)

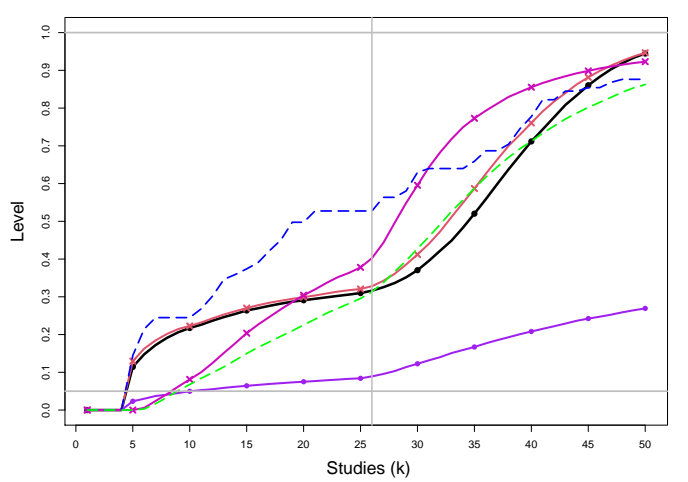

IV REML: $\tau^{2}=1 \mathrm{n}=100$ level $=0.05 \mathrm{~h}=4 \delta=1$ to 0.5 (chg at 26th)

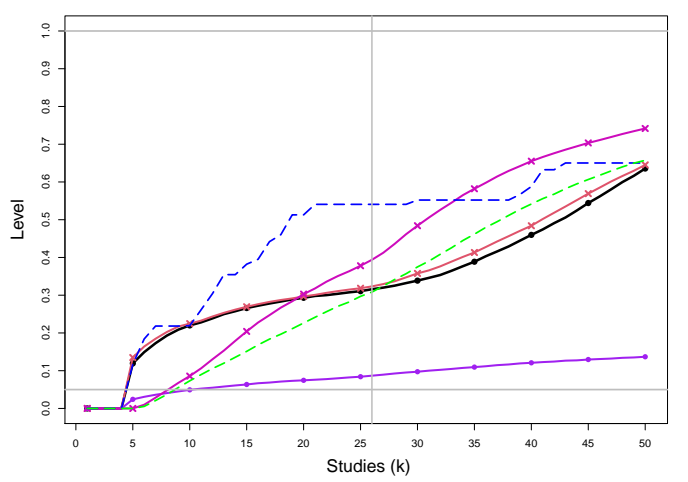

SSW KDB: $\tau^{2}=0 \mathrm{n}=100$ level $=0.05 \mathrm{~h}=4 \delta=1$ to 0.5 (chg at 26th)

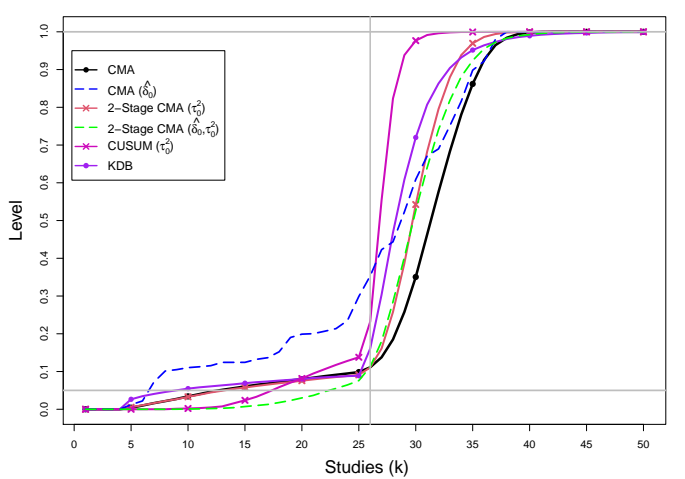

SSW KDB: $\tau^{2}=0.1 \mathrm{n}=100$ level $=0.05 \mathrm{~h}=4 \delta=1$ to 0.5 (chg at 26th)

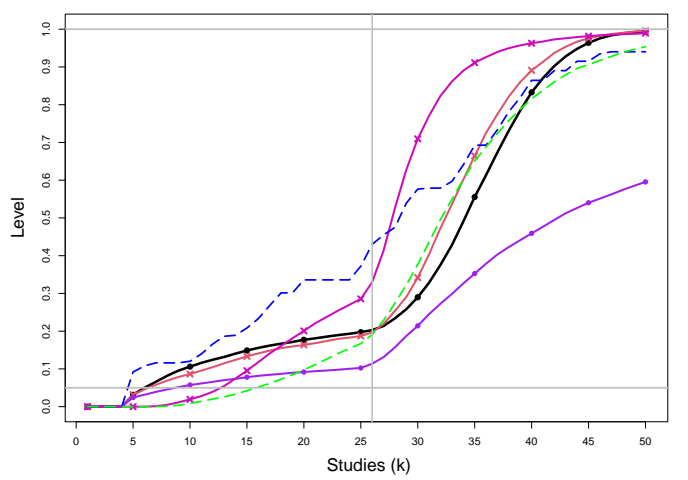

SSW KDB: $\tau^{2}=0.25 \mathrm{n}=100$ level $=0.05 \mathrm{~h}=4 \delta=1$ to 0.5 (chg at 26th)

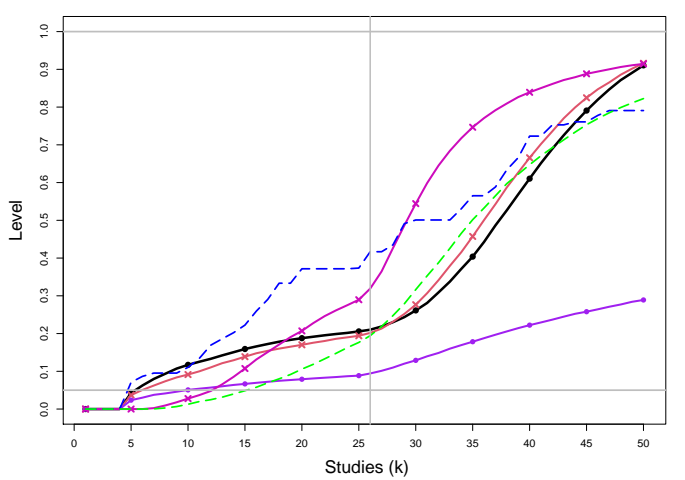

SSW KDB: $\tau^{2}=1 \mathrm{n}=100$ level $=0.05 \mathrm{~h}=4 \delta=1$ to 0.5 (chg at 26th)

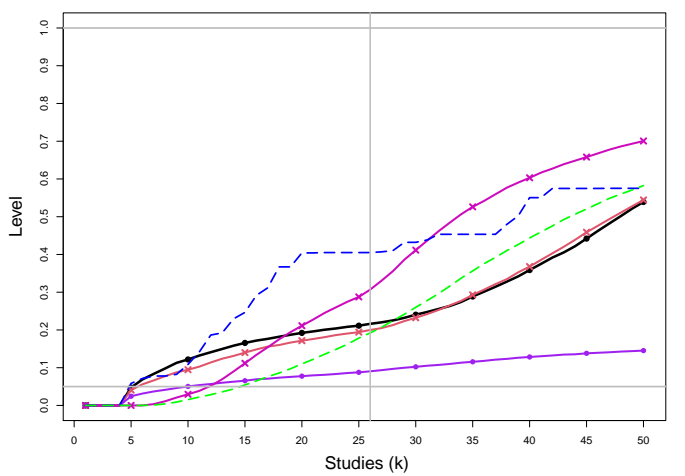

Figure E2. 53: Empirical levels of 1- and 2-stage CMA tests for shift in $\delta$ at .05 nominal level, shift in $\tau^{2}$ at .025 nominal levels and of CUSUM with $h=4$ for equal sample sizes $n_{i C}+n_{i T}=n=100, \tau^{2}=0,0.1,0.25,1$ and a shift from $\delta=1$ to $\delta=0.5$ at study number 26. Light grey line at .05. 
IV REML: $\tau^{2}=0 \quad \mathrm{n}=500$ level $=0.05 \mathrm{~h}=4 \quad \delta=1$ to 0.5 (chg at 26 th)

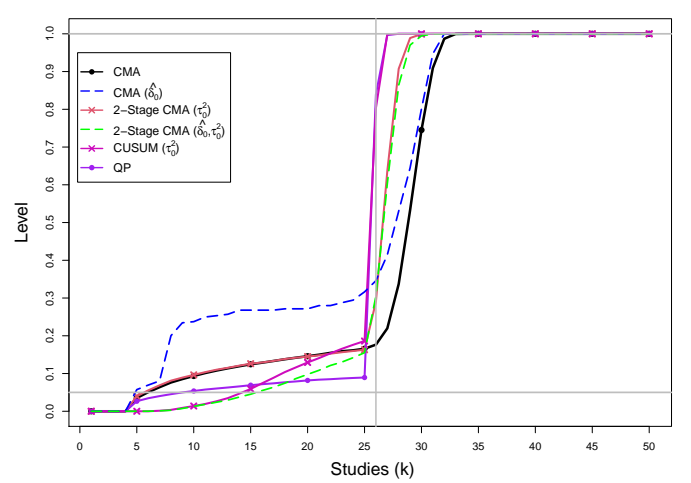

IV REML: $\tau^{2}=0.1 \mathrm{n}=500$ level $=0.05 \mathrm{~h}=4 \delta=1$ to 0.5 (chg at 26 th)

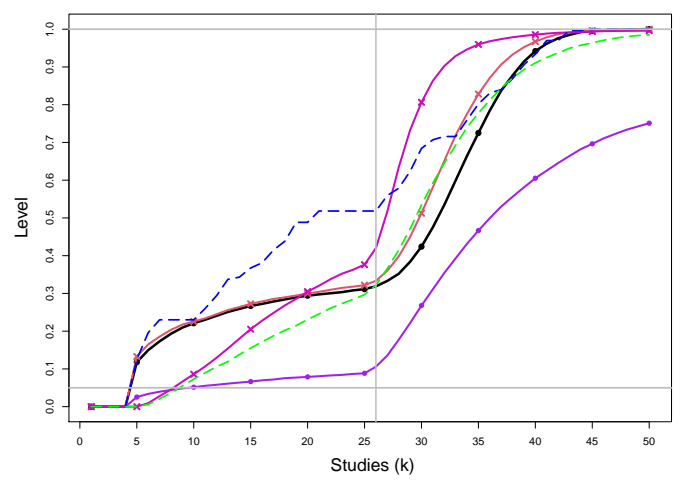

IV REML: $\tau^{2}=0.25 \mathrm{n}=500$ level $=0.05 \mathrm{~h}=4 \quad \delta=1$ to 0.5 (chg at 26 th)

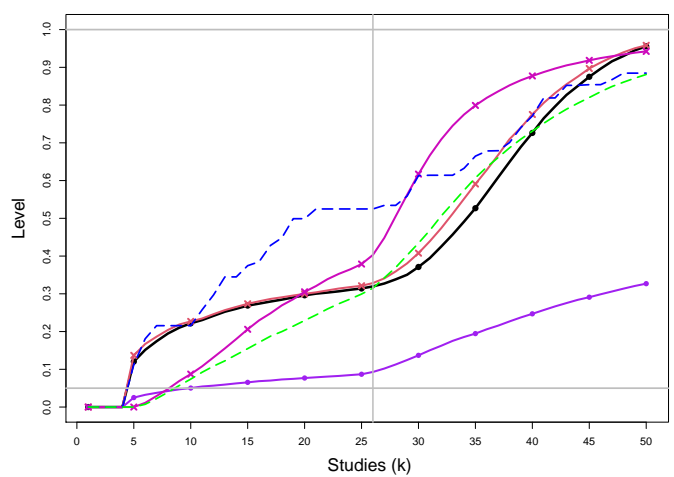

IV REML: $\tau^{2}=1 \mathrm{n}=500$ level $=0.05 \mathrm{~h}=4 \quad \delta=1$ to 0.5 (chg at 26 th)

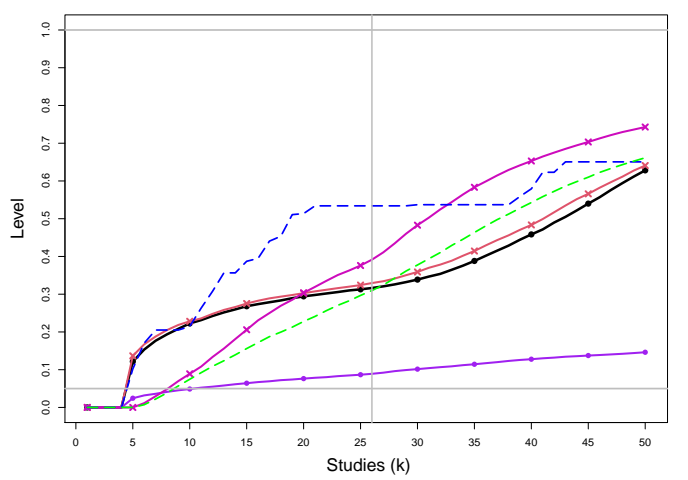

SSW KDB: $\tau^{2}=0 \mathrm{n}=500$ level $=0.05 \mathrm{~h}=4 \delta=1$ to 0.5 (chg at 26th)

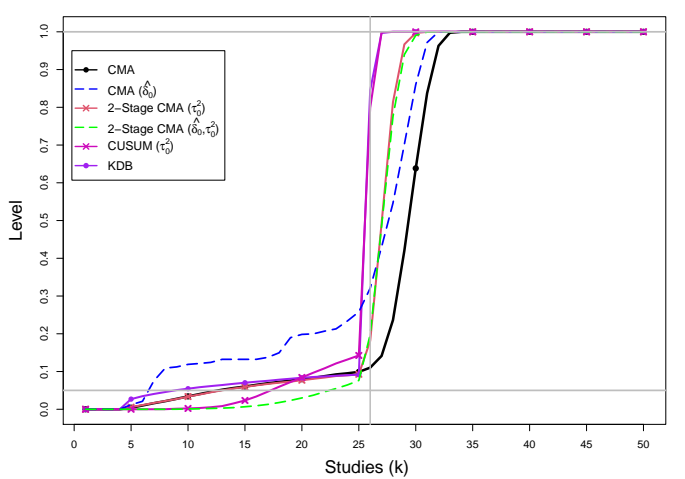

SSW KDB: $\tau^{2}=0.1 \mathrm{n}=500$ level $=0.05 \mathrm{~h}=4 \delta=1$ to 0.5 (chg at 26 th)

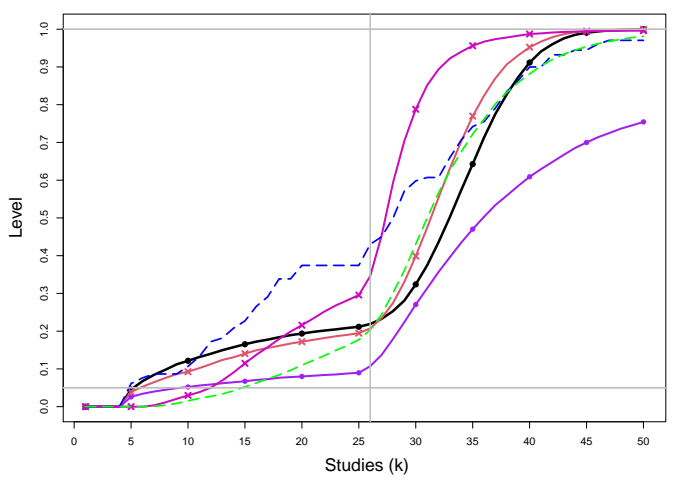

SSW KDB: $\tau^{2}=0.25 \mathrm{n}=500$ level $=0.05 \mathrm{~h}=4 \delta=1$ to 0.5 (chg at 26th)

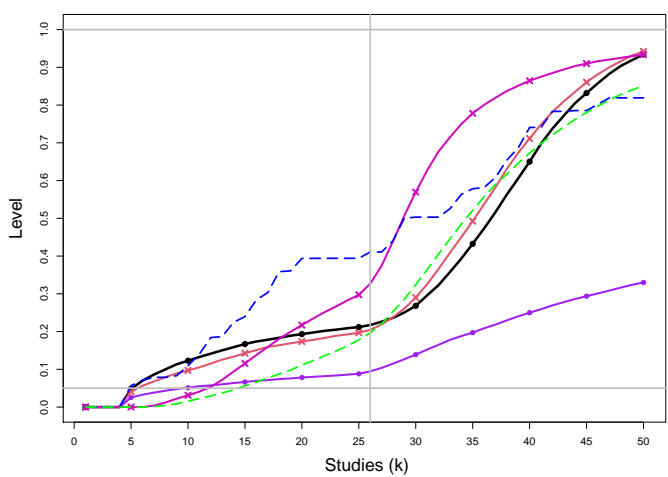

SSW KDB: $\tau^{2}=1 \mathrm{n}=500$ level $=0.05 \mathrm{~h}=4 \delta=1$ to 0.5 (chg at 26 th)

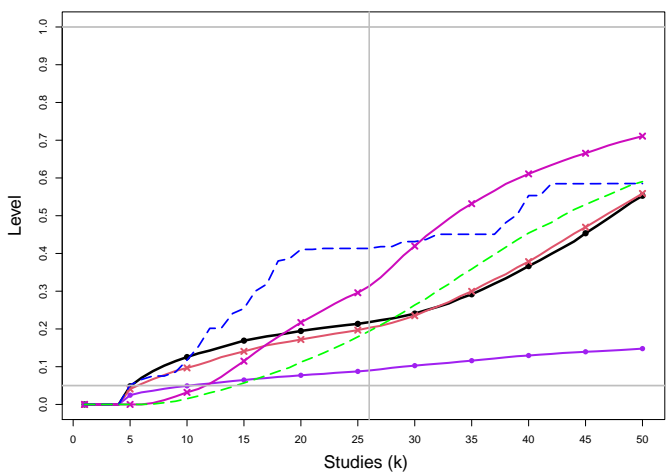

Figure E2. 54: Empirical levels of 1- and 2-stage CMA tests for shift in $\delta$ at .05 nominal level, shift in $\tau^{2}$ at .025 nominal levels and of CUSUM with $h=4$ for equal sample sizes $n_{i C}+n_{i T}=n=500, \tau^{2}=0,0.1,0.25,1$ and a shift from $\delta=1$ to $\delta=0.5$ at study number 26. Light grey line at .05. 
IV REML: $\tau^{2}=0 \mathrm{n}=1000$ level $=0.05 \mathrm{~h}=4 \quad \delta=1$ to 0.5 (chg at 26 th)

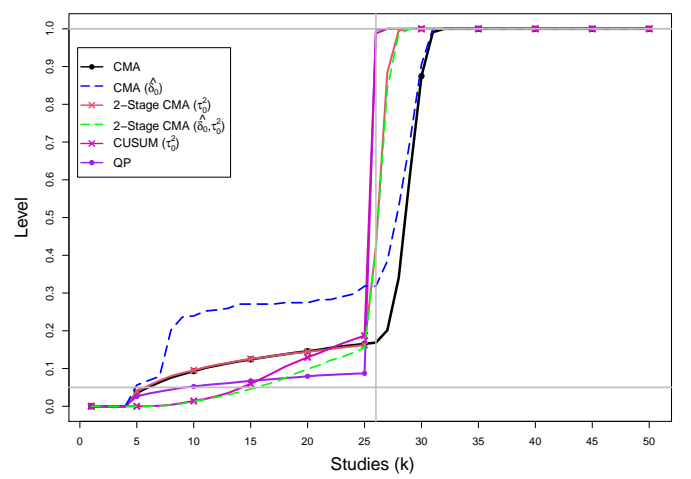

IV REML: $\tau^{2}=0.1 \mathrm{n}=1000$ level $=0.05 \mathrm{~h}=4 \delta=1$ to 0.5 (chg at 26 th)

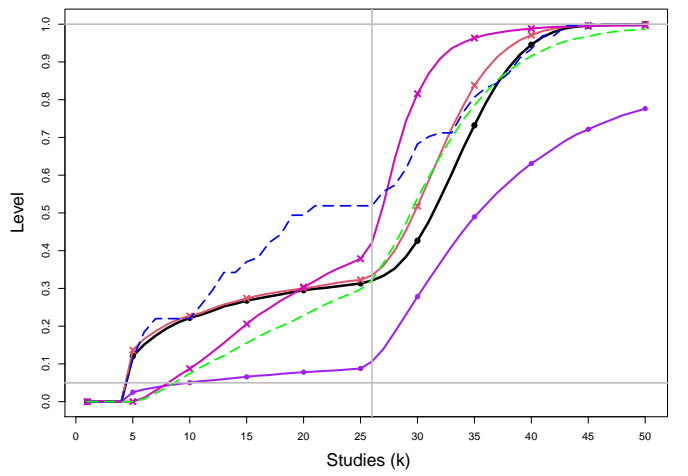

IV REML: $\tau^{2}=0.25 \mathrm{n}=1000$ level $=0.05 \mathrm{~h}=4 \delta=1$ to 0.5 (chg at 26 th) SSW KDB: $\tau^{2}=0.25 \mathrm{n}=1000$ level $=0.05 \mathrm{~h}=4 \delta=1$ to 0.5 (chg at 26 th)

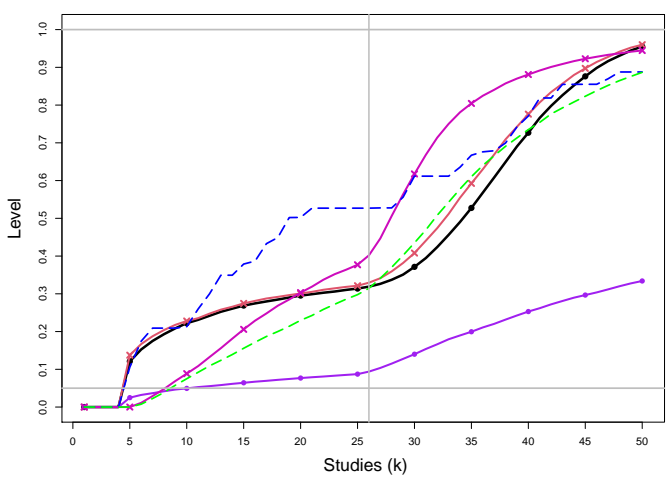

IV REML: $\tau^{2}=1 \mathrm{n}=1000$ level $=0.05 \mathrm{~h}=4 \delta=1$ to 0.5 (chg at 26 th)

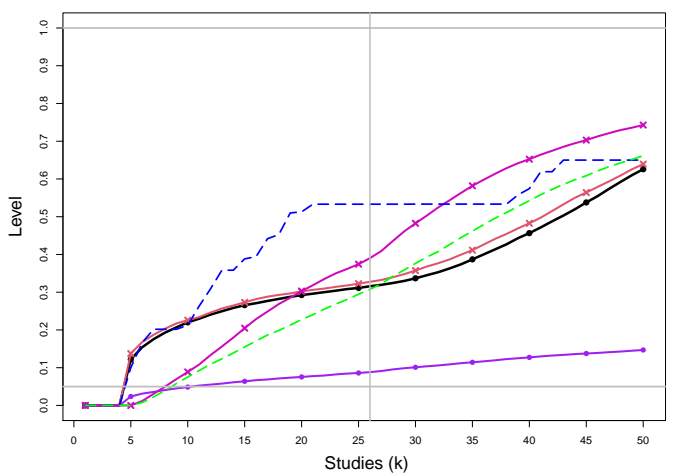

SSW KDB: $\tau^{2}=0 \mathrm{n}=1000$ level $=0.05 \mathrm{~h}=4 \delta=1$ to 0.5 (chg at 26 th)

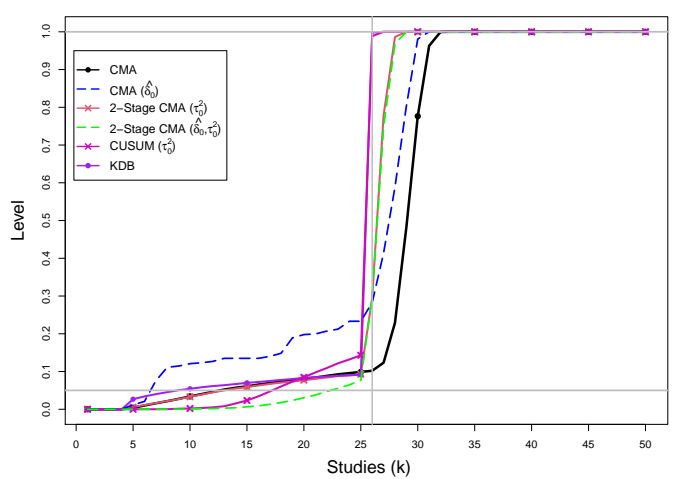

SSW KDB: $\tau^{2}=0.1 \mathrm{n}=1000$ level $=0.05 \mathrm{~h}=4 \delta=1$ to 0.5 (chg at 26th)

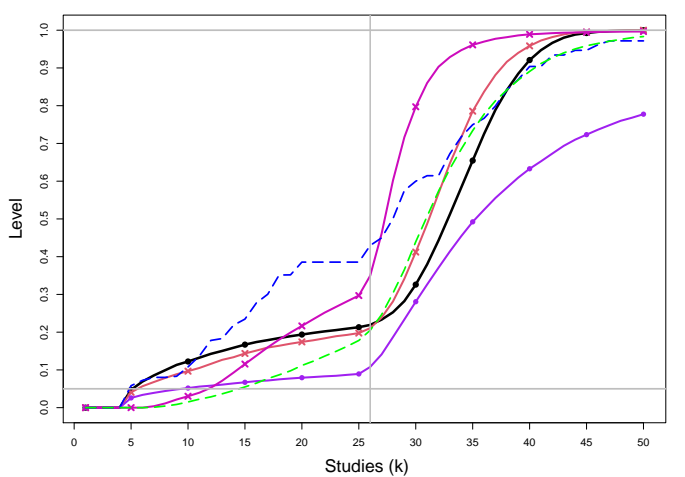

SSW KDB: $\tau^{2}=1 \mathrm{n}=1000$ level $=0.05 \mathrm{~h}=4 \delta=1$ to 0.5 (chg at 26th)

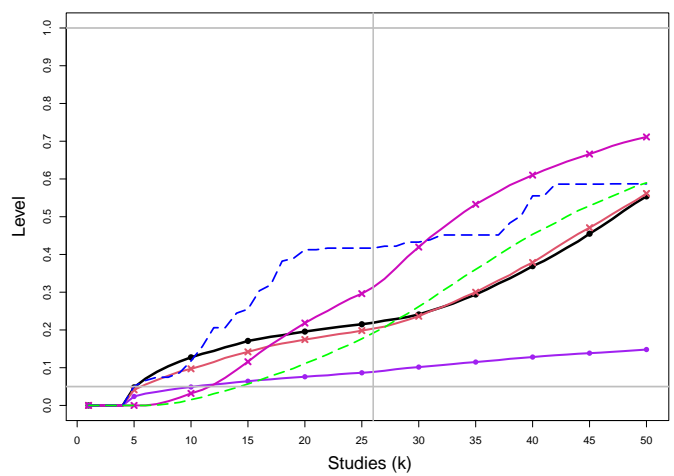

Figure E2. 55: Empirical levels of 1- and 2-stage CMA tests for shift in $\delta$ at .05 nominal level, shift in $\tau^{2}$ at .025 nominal levels and of CUSUM with $h=4$ for equal sample sizes $n_{i C}+n_{i T}=n=1000, \tau^{2}=0,0.1,0.25,1$ and a shift from $\delta=1$ to $\delta=0.5$ at study number 26. Light grey line at .05. 


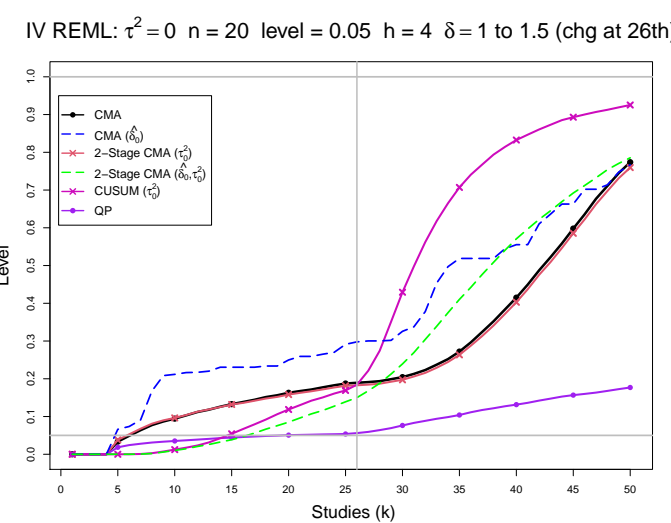

IV REML: $\tau^{2}=0.1 \mathrm{n}=20$ level $=0.05 \mathrm{~h}=4 \delta=1$ to 1.5 (chg at 26 th)

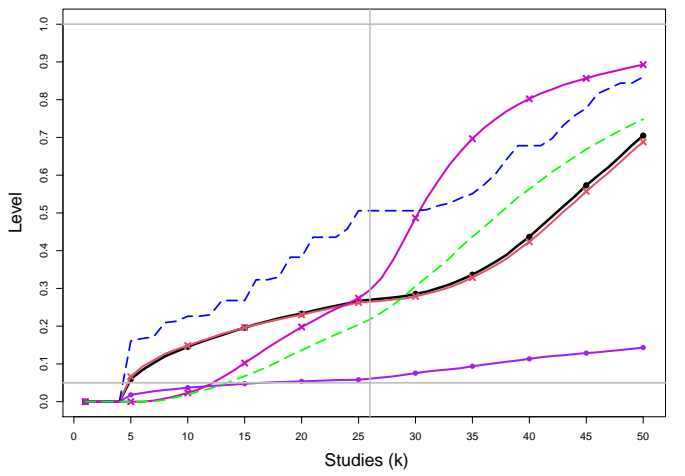

IV REML: $\tau^{2}=0.25 \mathrm{n}=20$ level $=0.05 \mathrm{~h}=4 \delta=1$ to 1.5 (chg at 26th)

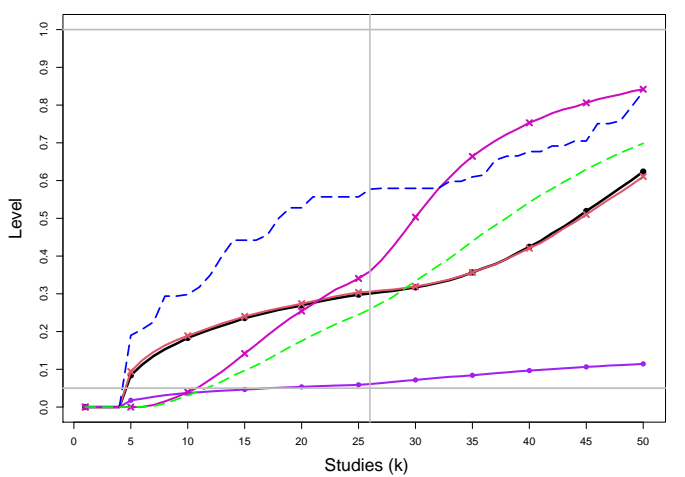

IV REML: $\tau^{2}=1 \mathrm{n}=20$ level $=0.05 \mathrm{~h}=4 \delta=1$ to 1.5 (chg at 26th)

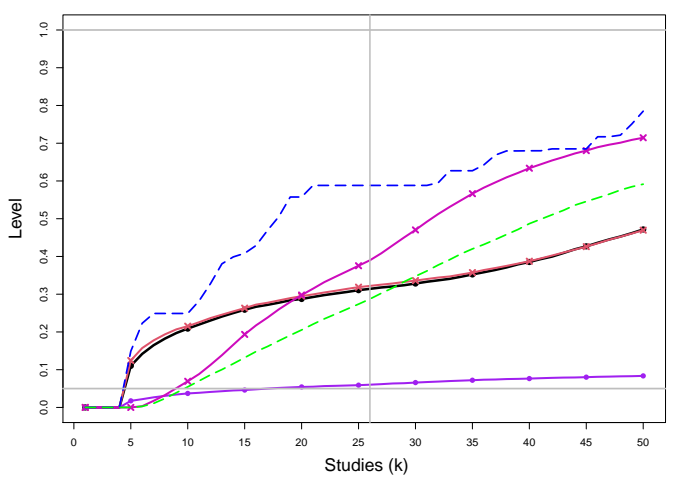

SSW KDB: $\tau^{2}=0 \mathrm{n}=20$ level $=0.05 \mathrm{~h}=4 \delta=1$ to 1.5 (chg at 26th)

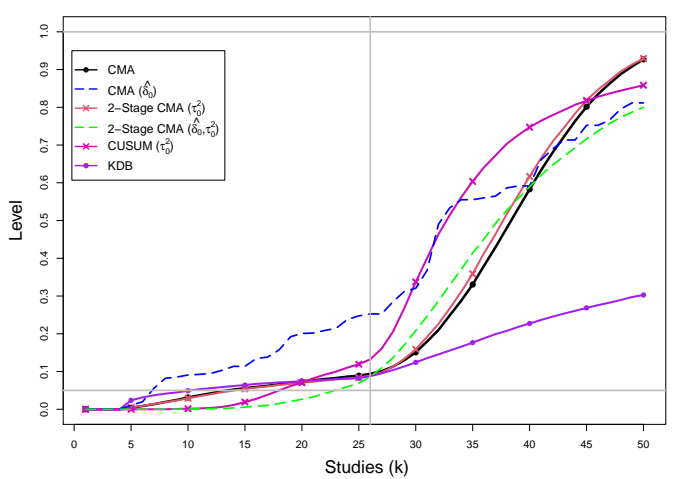

SSW KDB: $\tau^{2}=0.1 \mathrm{n}=20$ level $=0.05 \mathrm{~h}=4 \delta=1$ to 1.5 (chg at 26th)

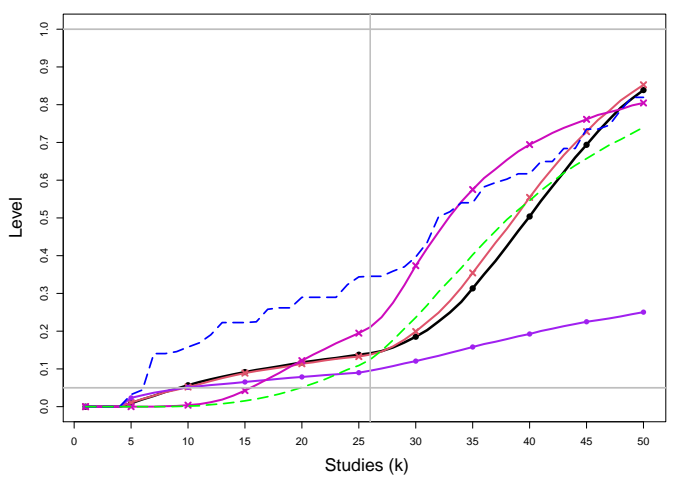

SSW KDB: $\tau^{2}=0.25 \mathrm{n}=20$ level $=0.05 \mathrm{~h}=4 \delta=1$ to 1.5 (chg at 26th)

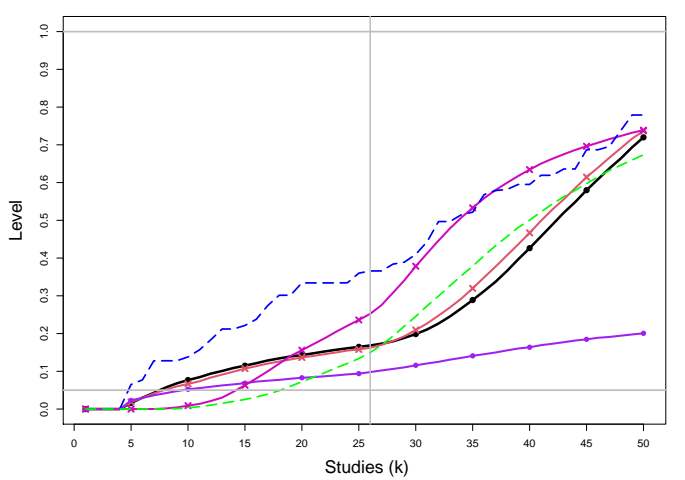

SSW KDB: $\tau^{2}=1 \mathrm{n}=20$ level $=0.05 \mathrm{~h}=4 \delta=1$ to 1.5 (chg at 26th)

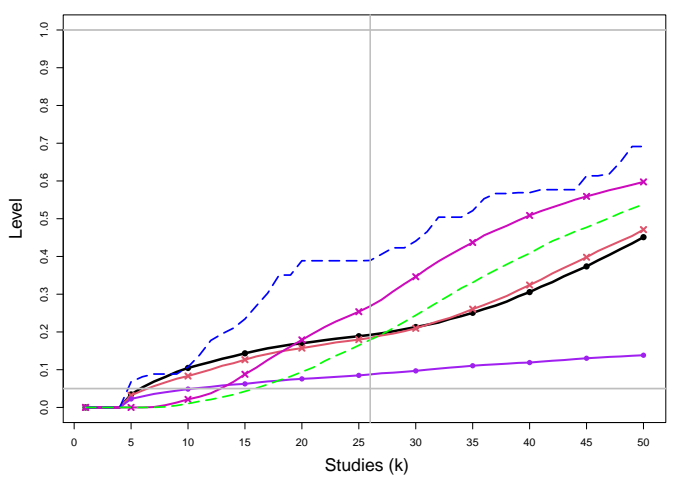

Figure E2. 56: Empirical levels of 1- and 2-stage CMA tests for shift in $\delta$ at .05 nominal level, shift in $\tau^{2}$ at .025 nominal levels and of CUSUM with $h=4$ for equal sample sizes $n_{i C}+n_{i T}=n=20, \tau^{2}=0,0.1,0.25,1$ and a shift from $\delta=1$ to $\delta=1.5$ at study number 26. Light grey line at .05. 


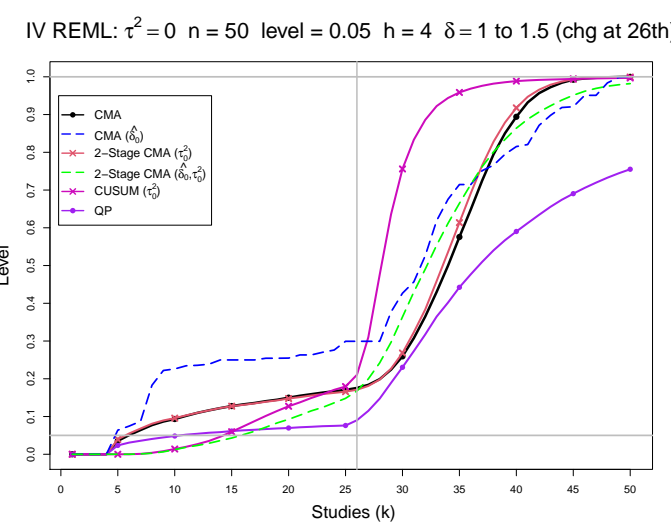

IV REML: $\tau^{2}=0.1 \mathrm{n}=50$ level $=0.05 \mathrm{~h}=4 \delta=1$ to 1.5 (chg at 26 th)

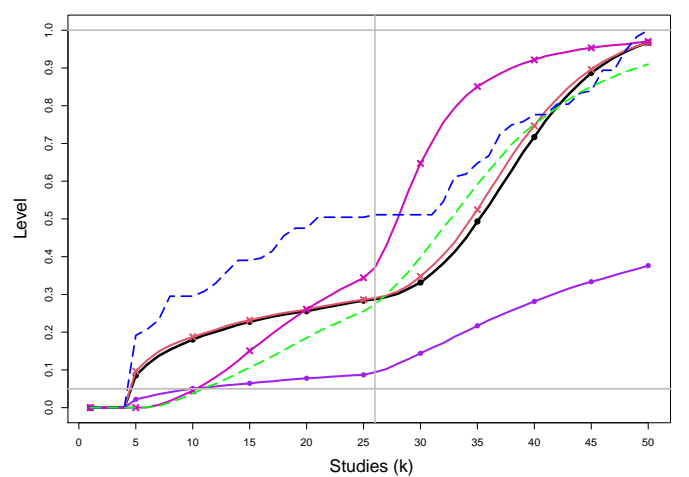

IV REML: $\tau^{2}=0.25 \mathrm{n}=50$ level $=0.05 \mathrm{~h}=4 \delta=1$ to 1.5 (chg at 26th)

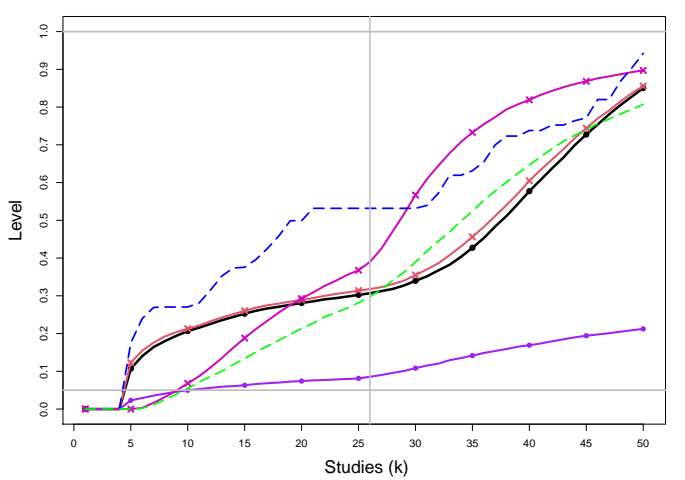

IV REML: $\tau^{2}=1 \mathrm{n}=50$ level $=0.05 \mathrm{~h}=4 \delta=1$ to 1.5 (chg at 26th)

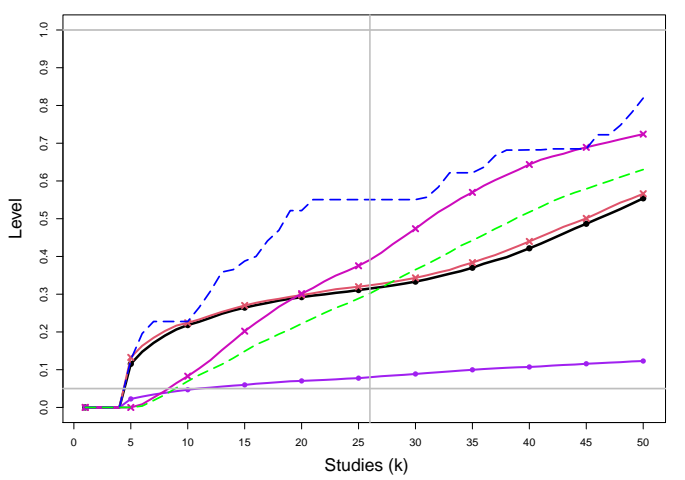

SSW KDB: $\tau^{2}=0 \mathrm{n}=50$ level $=0.05 \mathrm{~h}=4 \delta=1$ to 1.5 (chg at 26th)

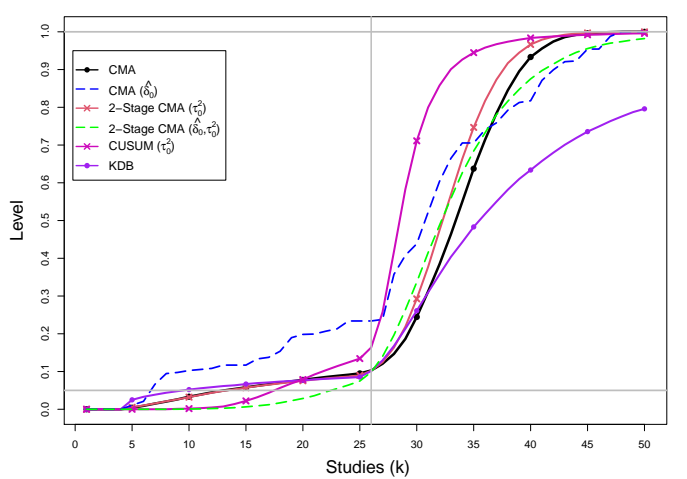

SSW KDB: $\tau^{2}=0.1 \mathrm{n}=50$ level $=0.05 \mathrm{~h}=4 \delta=1$ to 1.5 (chg at 26th)

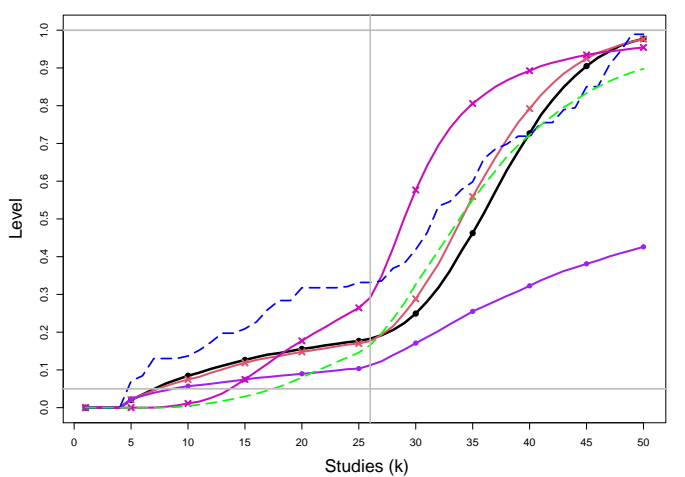

SSW KDB: $\tau^{2}=0.25 \mathrm{n}=50$ level $=0.05 \mathrm{~h}=4 \delta=1$ to 1.5 (chg at 26th)

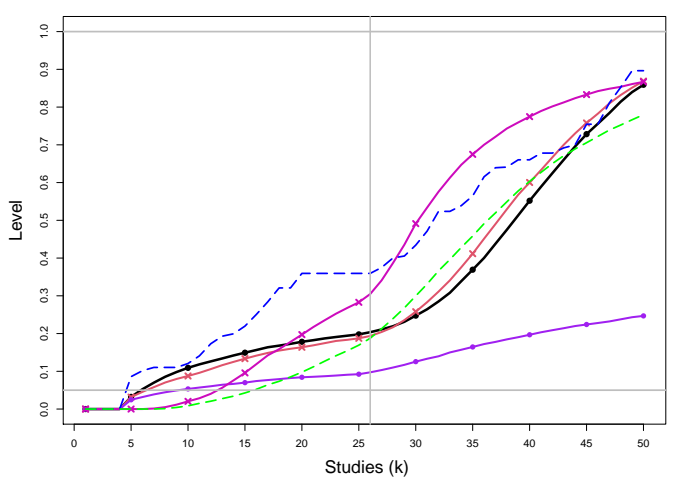

SSW KDB: $\tau^{2}=1 \mathrm{n}=50$ level $=0.05 \mathrm{~h}=4 \delta=1$ to 1.5 (chg at 26th)

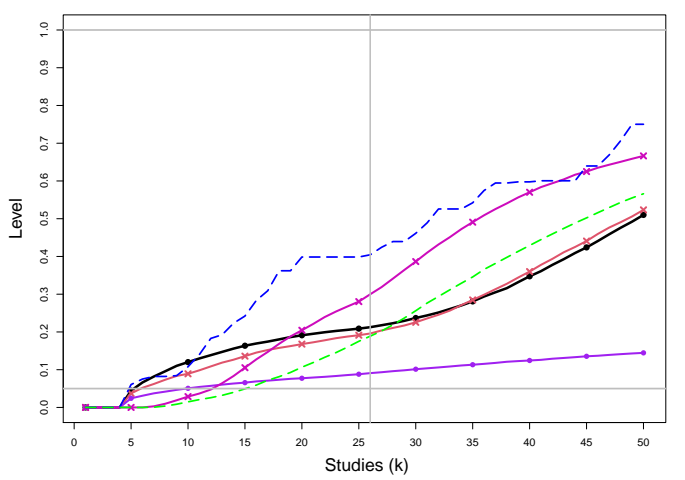

Figure E2. 57: Empirical levels of 1- and 2-stage CMA tests for shift in $\delta$ at .05 nominal level, shift in $\tau^{2}$ at .025 nominal levels and of CUSUM with $h=4$ for equal sample sizes $n_{i C}+n_{i T}=n=50, \tau^{2}=0,0.1,0.25,1$ and a shift from $\delta=1$ to $\delta=1.5$ at study number 26. Light grey line at .05. 
IV REML: $\tau^{2}=0 \mathrm{n}=100$ level $=0.05 \mathrm{~h}=4 \delta=1$ to 1.5 (chg at 26th)

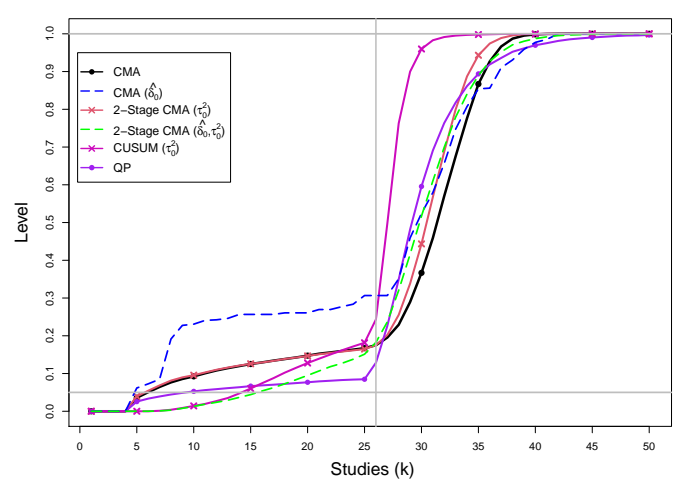

IV REML: $\tau^{2}=0.1 \mathrm{n}=100$ level $=0.05 \mathrm{~h}=4 \delta=1$ to 1.5 (chg at 26 th)

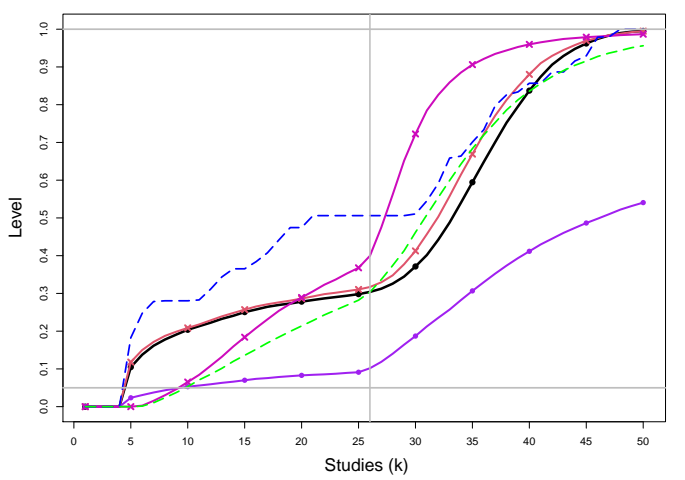

IV REML: $\tau^{2}=0.25 \mathrm{n}=100$ level $=0.05 \mathrm{~h}=4 \delta=1$ to 1.5 (chg at 26th)

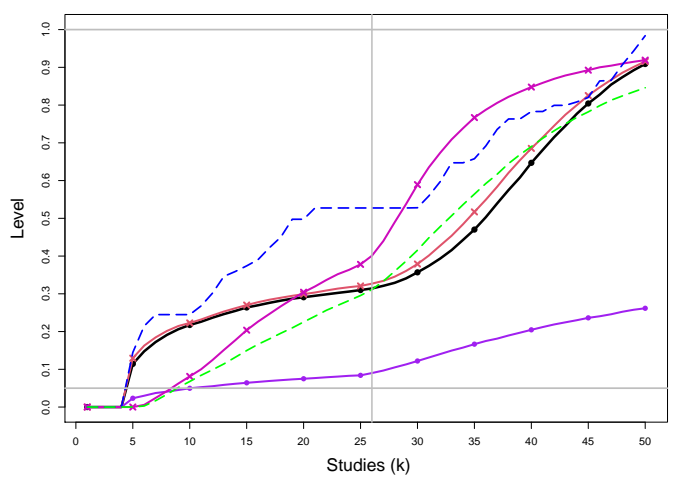

IV REML: $\tau^{2}=1 \mathrm{n}=100$ level $=0.05 \mathrm{~h}=4 \delta=1$ to 1.5 (chg at 26th)

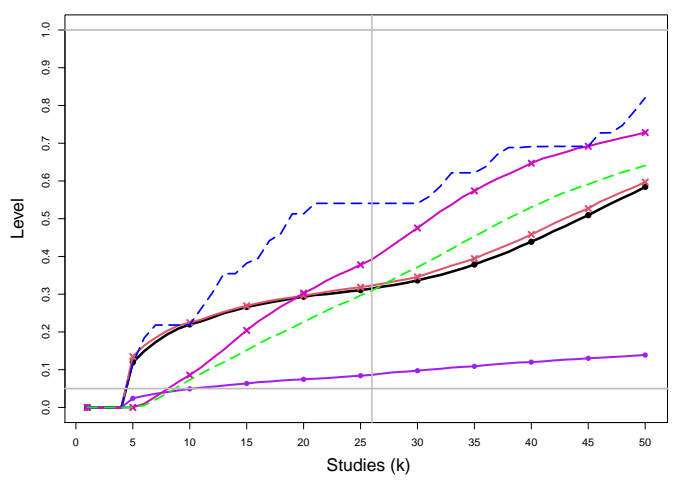

SSW KDB: $\tau^{2}=0 \mathrm{n}=100$ level $=0.05 \mathrm{~h}=4 \delta=1$ to 1.5 (chg at 26th)

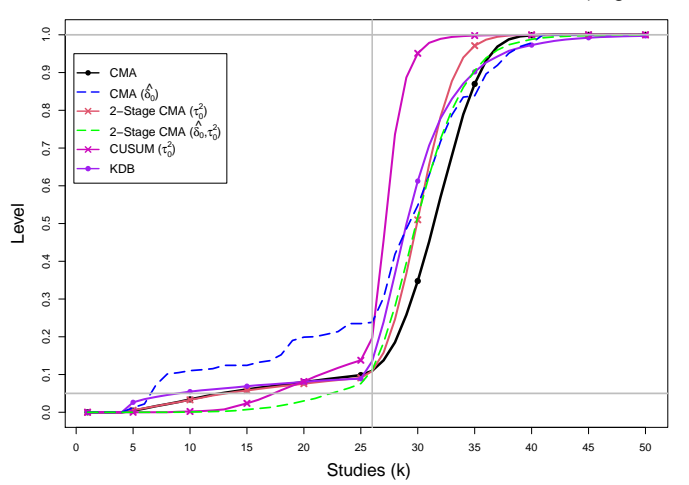

SSW KDB: $\tau^{2}=0.1 \mathrm{n}=100$ level $=0.05 \mathrm{~h}=4 \delta=1$ to 1.5 (chg at 26th)

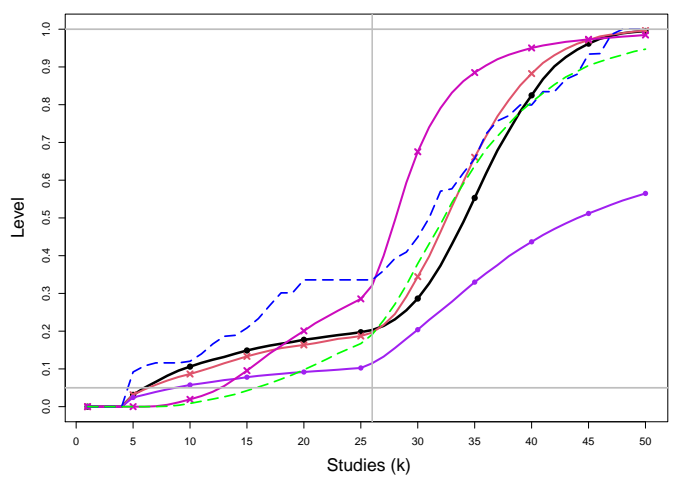

SSW KDB: $\tau^{2}=0.25 \mathrm{n}=100$ level $=0.05 \mathrm{~h}=4 \delta=1$ to 1.5 (chg at 26th)

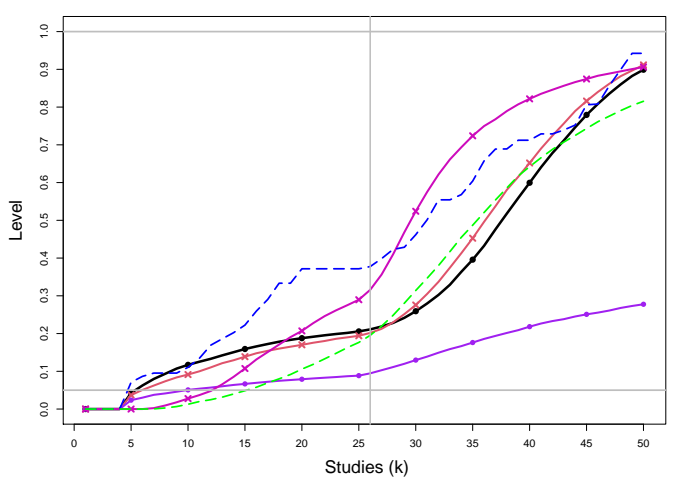

SSW KDB: $\tau^{2}=1 \mathrm{n}=100$ level $=0.05 \mathrm{~h}=4 \delta=1$ to 1.5 (chg at 26th)

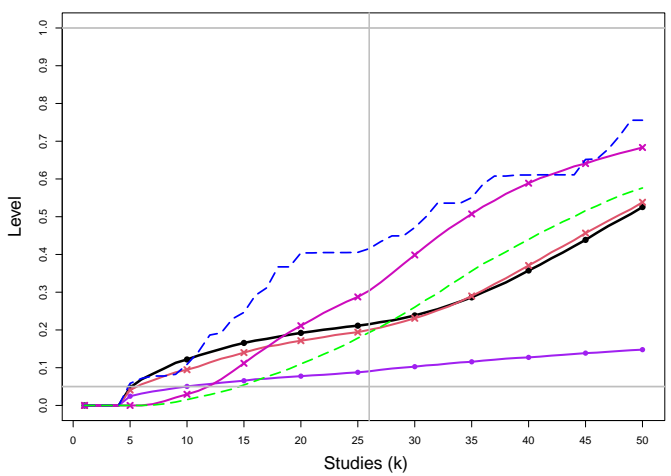

Figure E2. 58: Empirical levels of 1- and 2-stage CMA tests for shift in $\delta$ at .05 nominal level, shift in $\tau^{2}$ at .025 nominal levels and of CUSUM with $h=4$ for equal sample sizes $n_{i C}+n_{i T}=n=100, \tau^{2}=0,0.1,0.25,1$ and a shift from $\delta=1$ to $\delta=1.5$ at study number 26. Light grey line at .05. 
IV REML: $\tau^{2}=0 \quad \mathrm{n}=500$ level $=0.05 \mathrm{~h}=4 \quad \delta=1$ to 1.5 (chg at 26 th)

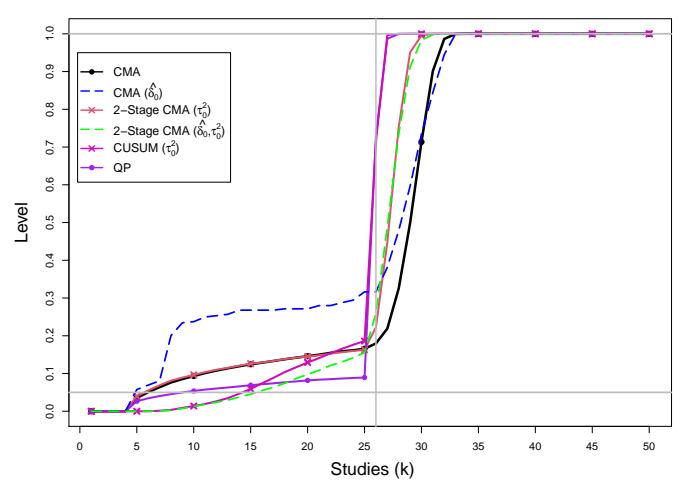

IV REML: $\tau^{2}=0.1 \mathrm{n}=500$ level $=0.05 \mathrm{~h}=4 \delta=1$ to 1.5 (chg at 26 th)

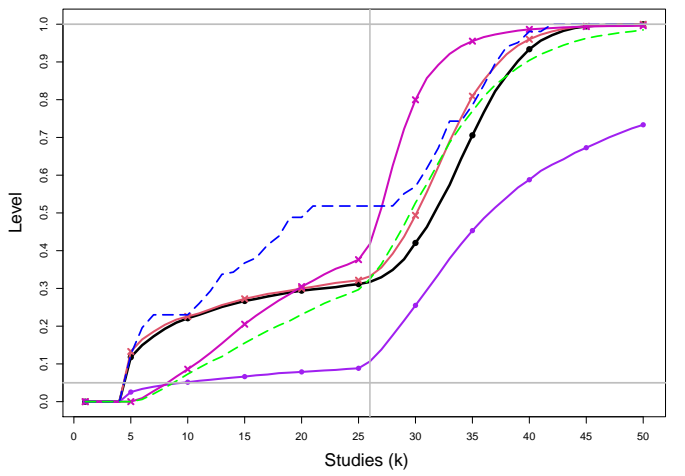

IV REML: $\tau^{2}=0.25 \mathrm{n}=500$ level $=0.05 \mathrm{~h}=4 \quad \delta=1$ to 1.5 (chg at 26 th)

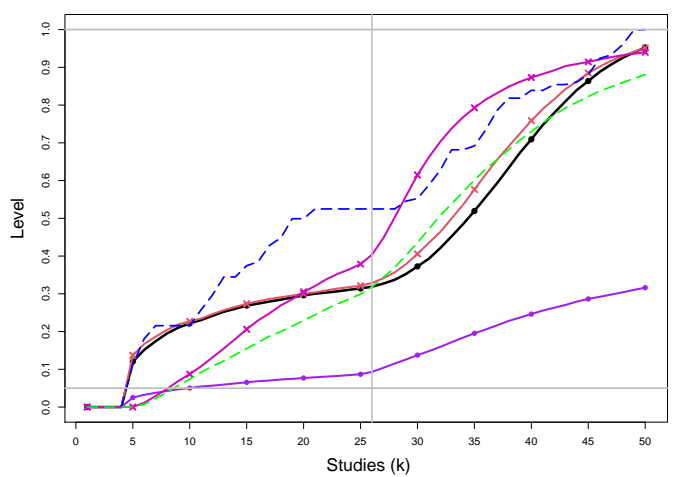

IV REML: $\tau^{2}=1 \mathrm{n}=500$ level $=0.05 \mathrm{~h}=4 \delta=1$ to 1.5 (chg at 26 th)

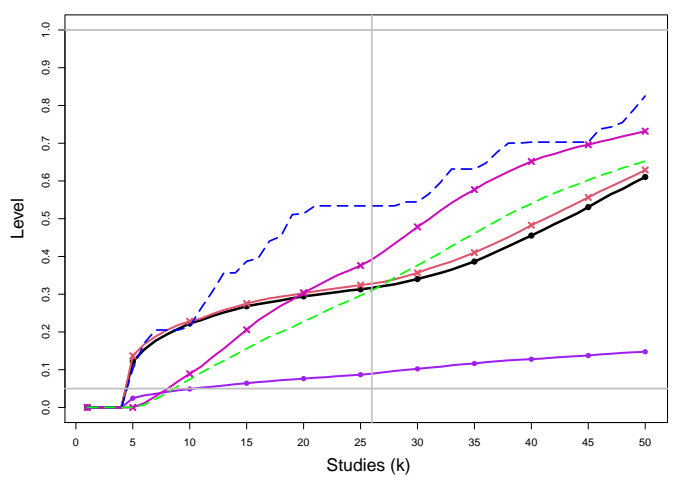

SSW KDB: $\tau^{2}=0 \mathrm{n}=500$ level $=0.05 \mathrm{~h}=4 \delta=1$ to 1.5 (chg at 26th)

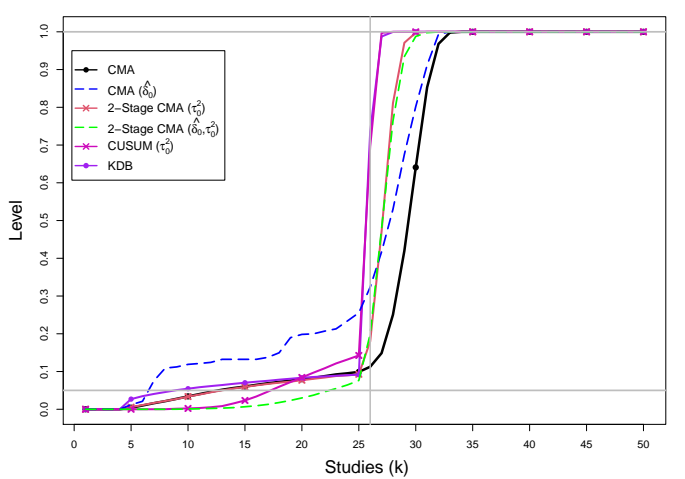

SSW KDB: $\tau^{2}=0.1 \mathrm{n}=500$ level $=0.05 \mathrm{~h}=4 \delta=1$ to 1.5 (chg at 26 th)

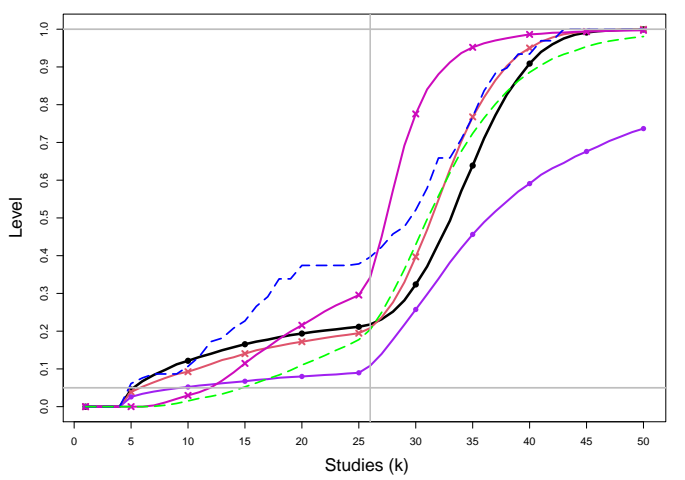

SSW KDB: $\tau^{2}=0.25 \mathrm{n}=500$ level $=0.05 \mathrm{~h}=4 \delta=1$ to 1.5 (chg at 26th)

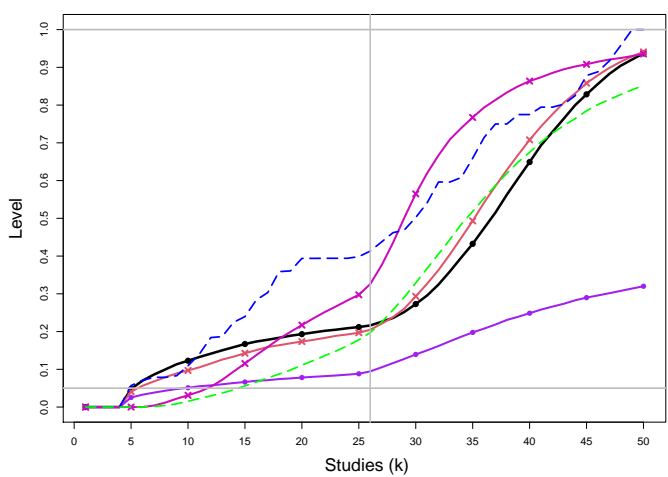

SSW KDB: $\tau^{2}=1 \mathrm{n}=500$ level $=0.05 \mathrm{~h}=4 \delta=1$ to 1.5 (chg at 26 th)

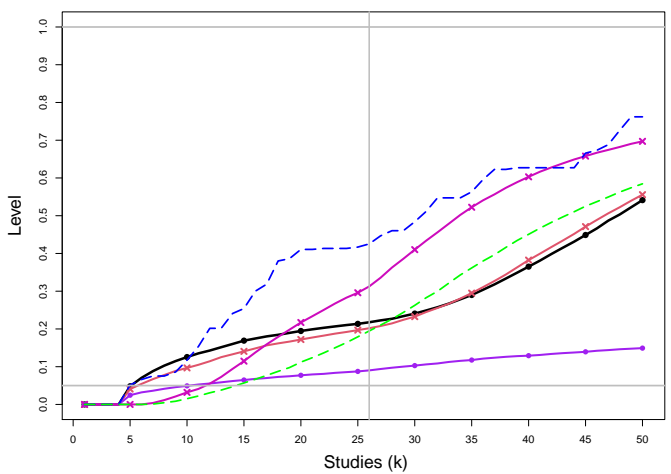

Figure E2. 59: Empirical levels of 1- and 2-stage CMA tests for shift in $\delta$ at .05 nominal level, shift in $\tau^{2}$ at .025 nominal levels and of CUSUM with $h=4$ for equal sample sizes $n_{i C}+n_{i T}=n=500, \tau^{2}=0,0.1,0.25,1$ and a shift from $\delta=1$ to $\delta=1.5$ at study number 26. Light grey line at .05. 
IV REML: $\tau^{2}=0 \mathrm{n}=1000$ level $=0.05 \mathrm{~h}=4 \quad \delta=1$ to 1.5 (chg at 26 th)

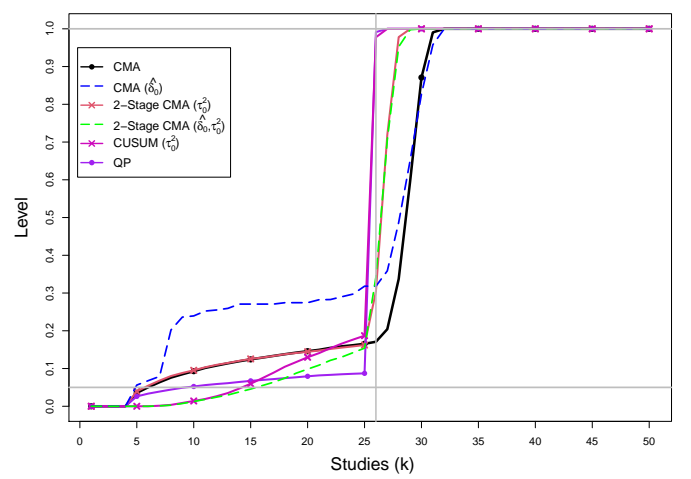

IV REML: $\tau^{2}=0.1 \mathrm{n}=1000$ level $=0.05 \mathrm{~h}=4 \delta=1$ to 1.5 (chg at 26th)

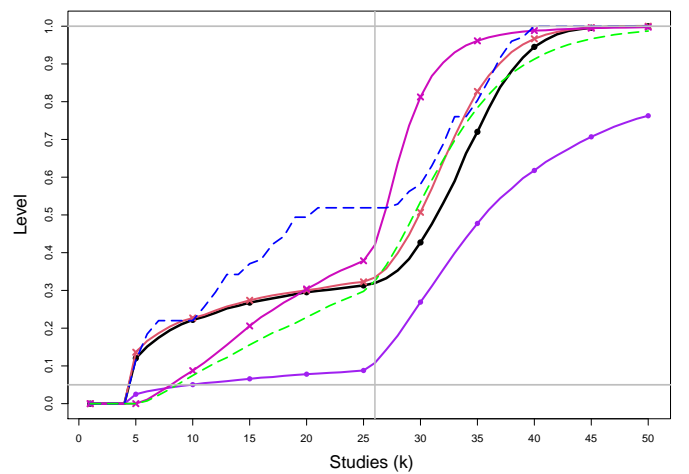

IV REML: $\tau^{2}=0.25 \mathrm{n}=1000$ level $=0.05 \mathrm{~h}=4 \delta=1$ to 1.5 (chg at 26 th) SSW KDB: $\tau^{2}=0.25 \mathrm{n}=1000$ level $=0.05 \mathrm{~h}=4 \delta=1$ to 1.5 (chg at 26 th)

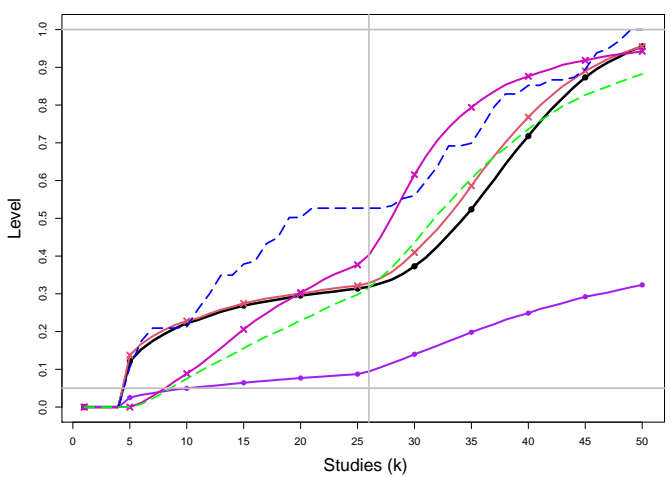

IV REML: $\tau^{2}=1 \mathrm{n}=1000$ level $=0.05 \mathrm{~h}=4 \delta=1$ to 1.5 (chg at 26 th)

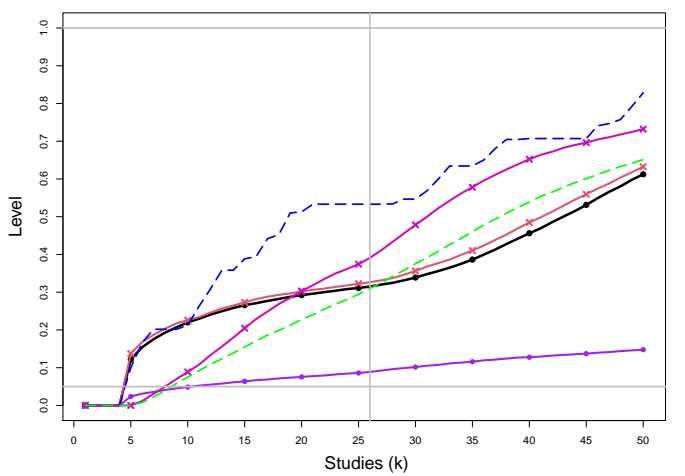

SSW KDB: $\tau^{2}=0 \mathrm{n}=1000$ level $=0.05 \mathrm{~h}=4 \delta=1$ to 1.5 (chg at 26 th)

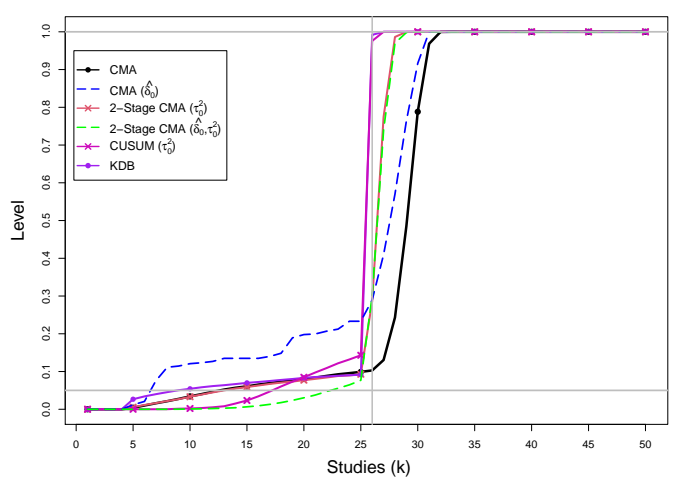

SSW KDB: $\tau^{2}=0.1 \mathrm{n}=1000$ level $=0.05 \mathrm{~h}=4 \delta=1$ to 1.5 (chg at 26th)

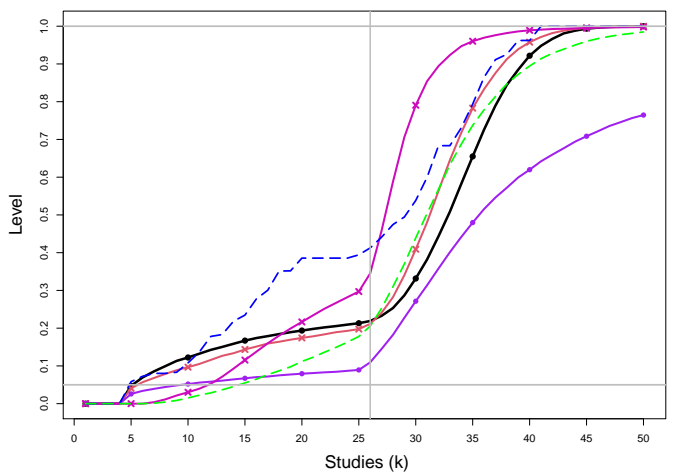

SSW KDB: $\tau^{2}=1 \mathrm{n}=1000$ level $=0.05 \mathrm{~h}=4 \delta=1$ to 1.5 (chg at 26th)

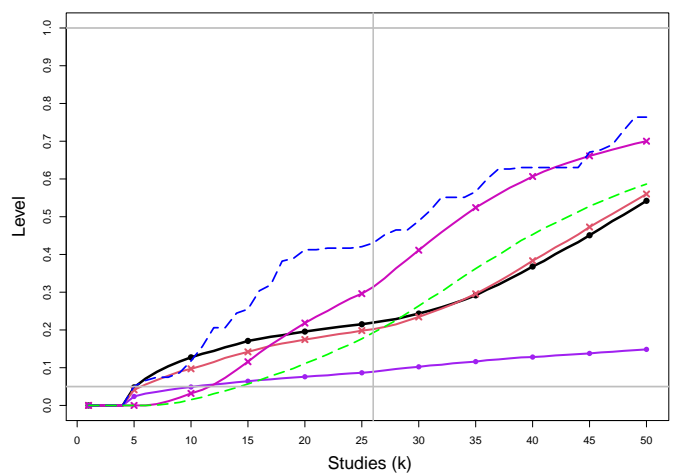

Figure E2. 60: Empirical levels of 1- and 2-stage CMA tests for shift in $\delta$ at .05 nominal level, shift in $\tau^{2}$ at .025 nominal levels and of CUSUM with $h=4$ for equal sample sizes $n_{i C}+n_{i T}=n=1000, \tau^{2}=0,0.1,0.25,1$ and a shift from $\delta=1$ to $\delta=1.5$ at study number 26. Light grey line at .05. 


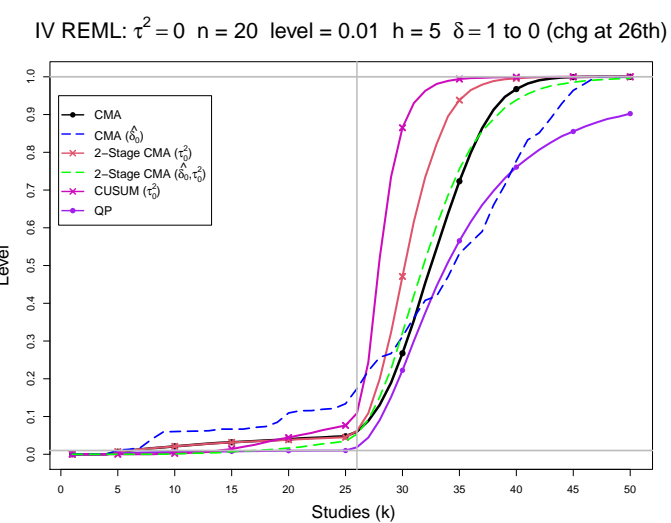

IV REML: $\tau^{2}=0.1 \mathrm{n}=20$ level $=0.01 \mathrm{~h}=5 \delta=1$ to 0 (chg at 26 th)

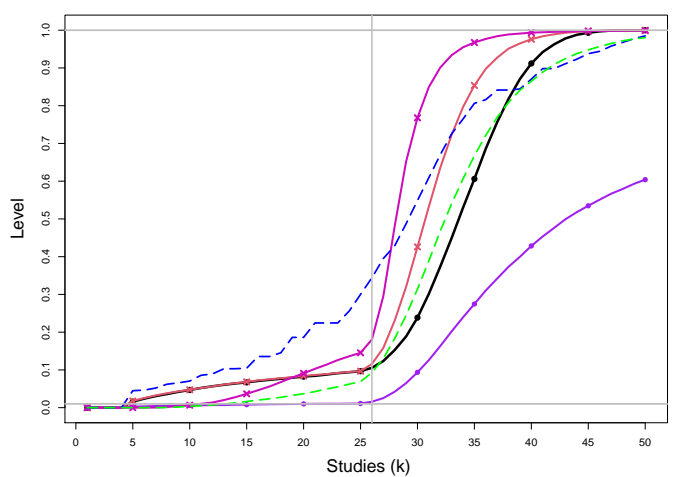

IV REML: $\tau^{2}=0.25 \mathrm{n}=20$ level $=0.01 \mathrm{~h}=5 \delta=1$ to 0 (chg at 26th)

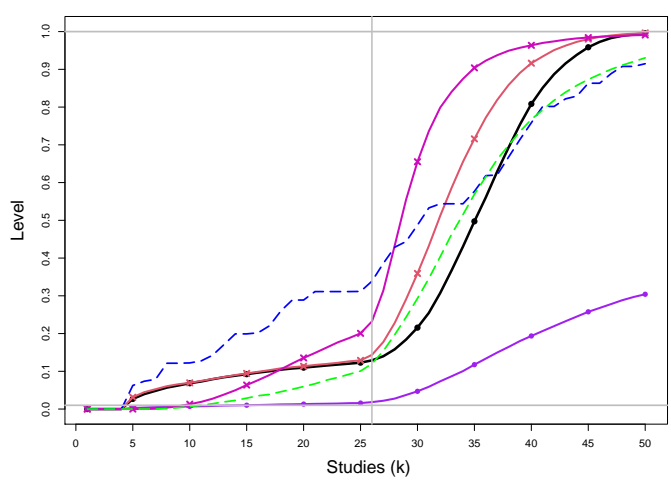

IV REML: $\tau^{2}=1 \mathrm{n}=20$ level $=0.01 \mathrm{~h}=5 \delta=1$ to 0 (chg at 26th)

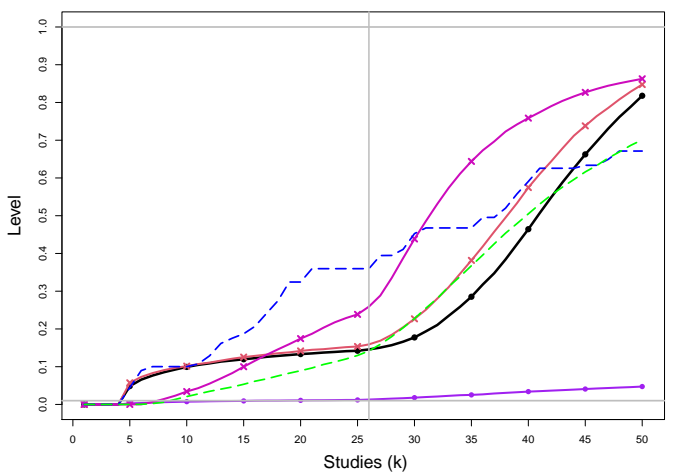

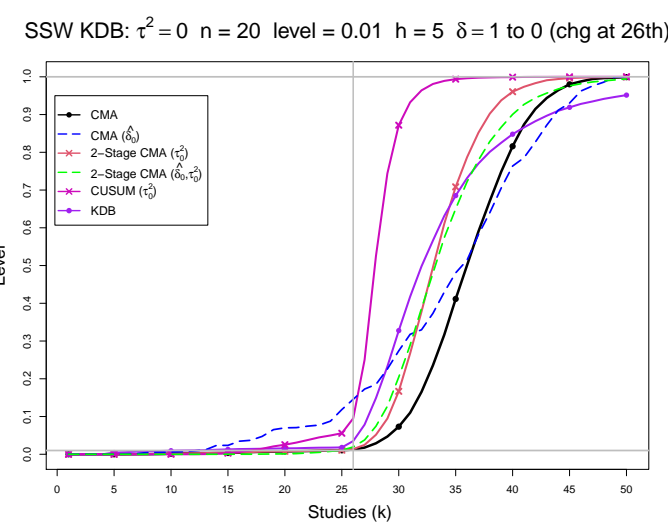

SSW KDB: $\tau^{2}=0.1 \mathrm{n}=20$ level $=0.01 \mathrm{~h}=5 \delta=1$ to 0 (chg at 26th)

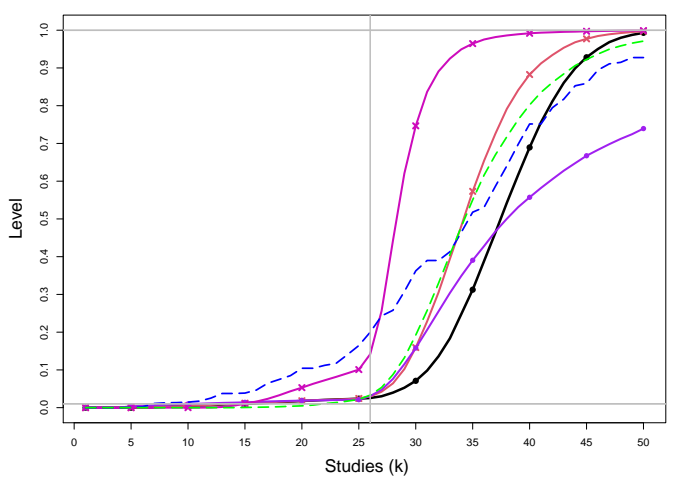

SSW KDB: $\tau^{2}=0.25 \mathrm{n}=20$ level $=0.01 \mathrm{~h}=5 \quad \delta=1$ to 0 (chg at 26th)

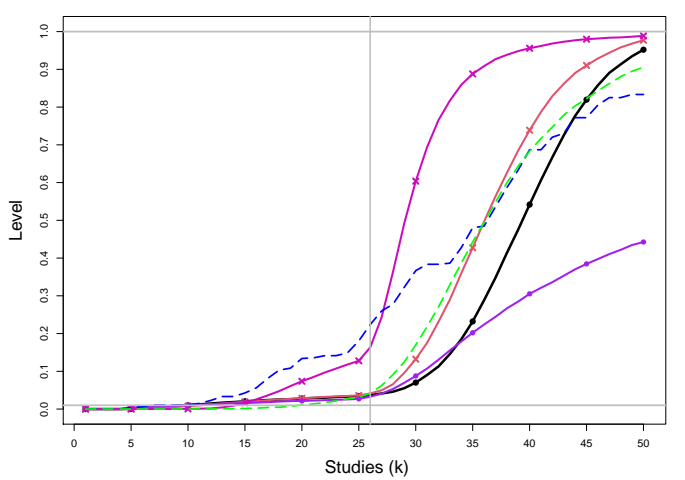

SSW KDB: $\tau^{2}=1 \mathrm{n}=20$ level $=0.01 \mathrm{~h}=5 \delta=1$ to 0 (chg at 26th)

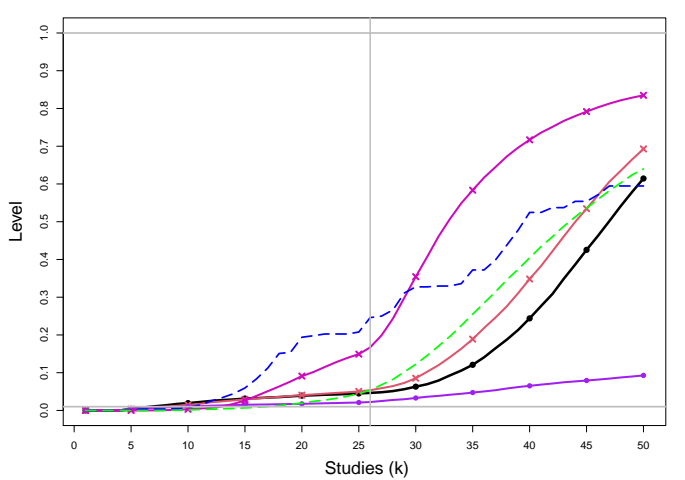

Figure E2. 61: Empirical levels of 1- and 2-stage CMA tests for shift in $\delta$ at .01 nominal level, shift in $\tau^{2}$ at .005 nominal levels and of CUSUM with $h=4$ for equal sample sizes $n_{i C}+n_{i T}=n=20, \tau^{2}=0,0.1,0.25,1$ and a shift from $\delta=1$ to $\delta=0$ at study number 26. Light grey line at .01. 


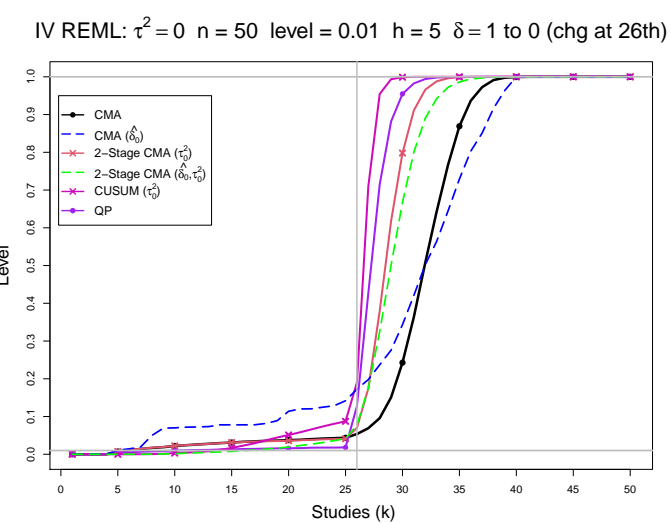

IV REML: $\tau^{2}=0.1 \mathrm{n}=50$ level $=0.01 \mathrm{~h}=5 \delta=1$ to 0 (chg at 26 th)

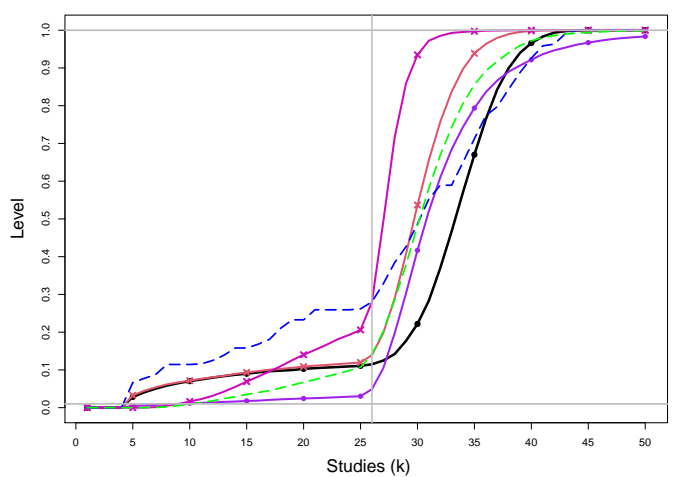

IV REML: $\tau^{2}=0.25 \mathrm{n}=50$ level $=0.01 \mathrm{~h}=5 \delta=1$ to 0 (chg at 26th)

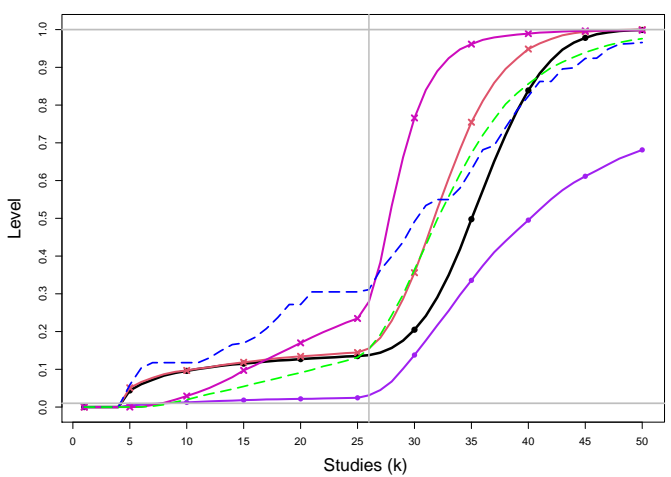

IV REML: $\tau^{2}=1 \mathrm{n}=50$ level $=0.01 \mathrm{~h}=5 \delta=1$ to 0 (chg at 26th)

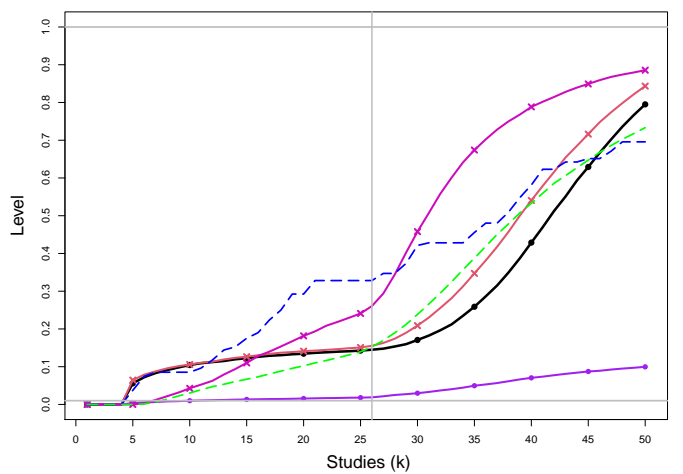

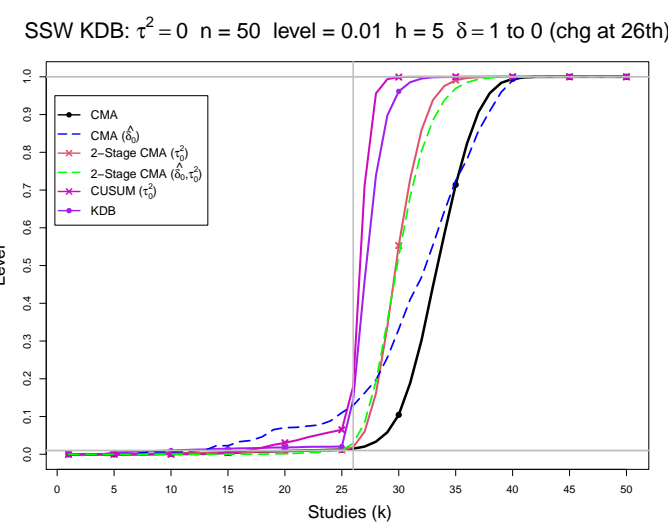

SSW KDB: $\tau^{2}=0.1 \mathrm{n}=50$ level $=0.01 \mathrm{~h}=5 \delta=1$ to 0 (chg at 26th)

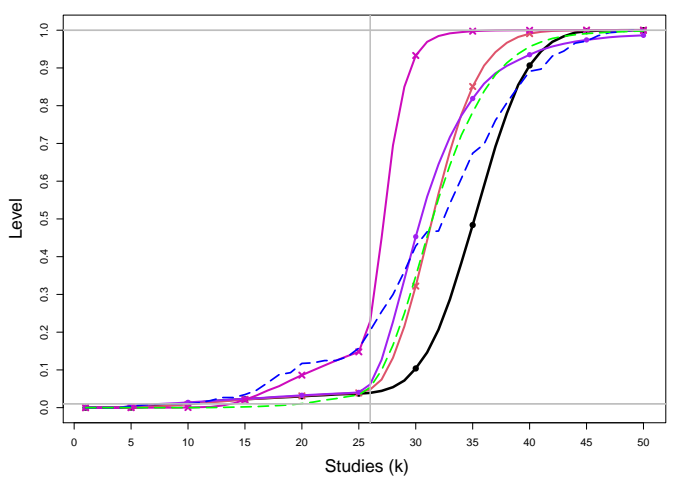

SSW KDB: $\tau^{2}=0.25 \mathrm{n}=50$ level $=0.01 \mathrm{~h}=5 \quad \delta=1$ to 0 (chg at 26th)

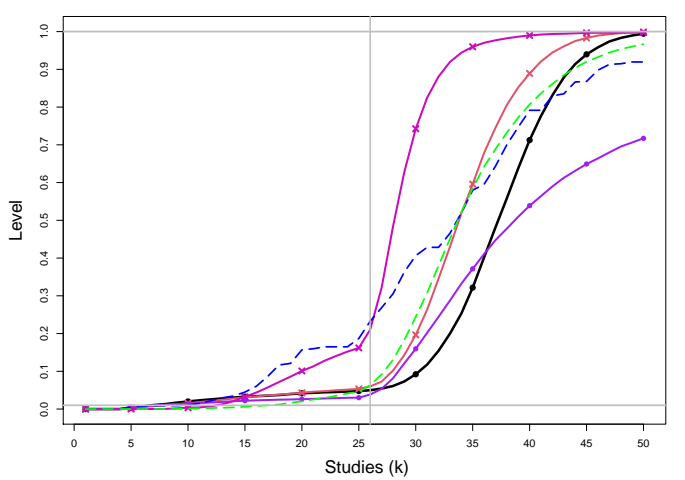

SSW KDB: $\tau^{2}=1 \mathrm{n}=50$ level $=0.01 \mathrm{~h}=5 \delta=1$ to 0 (chg at 26th)

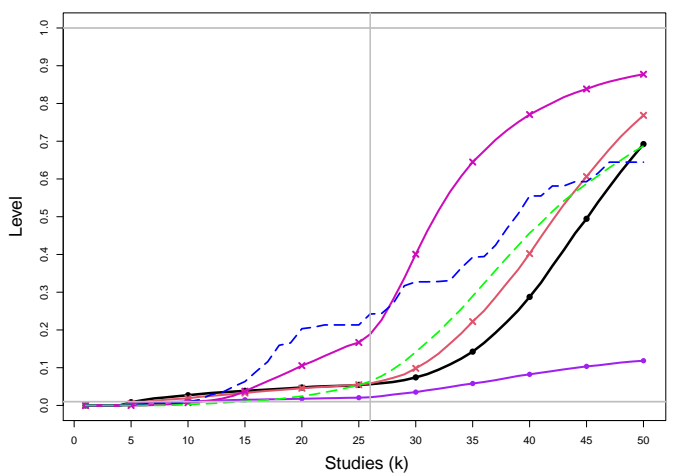

Figure E2. 62: Empirical levels of 1- and 2-stage CMA tests for shift in $\delta$ at .01 nominal level, shift in $\tau^{2}$ at .005 nominal levels and of CUSUM with $h=4$ for equal sample sizes $n_{i C}+n_{i T}=n=50, \tau^{2}=0,0.1,0.25,1$ and a shift from $\delta=1$ to $\delta=0$ at study number 26. Light grey line at .01. 


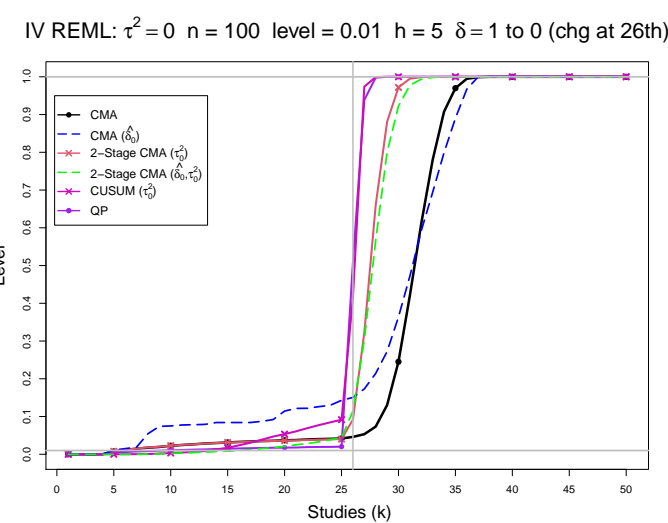

IV REML: $\tau^{2}=0.1 \mathrm{n}=100$ level $=0.01 \mathrm{~h}=5 \delta=1$ to 0 (chg at 26th)

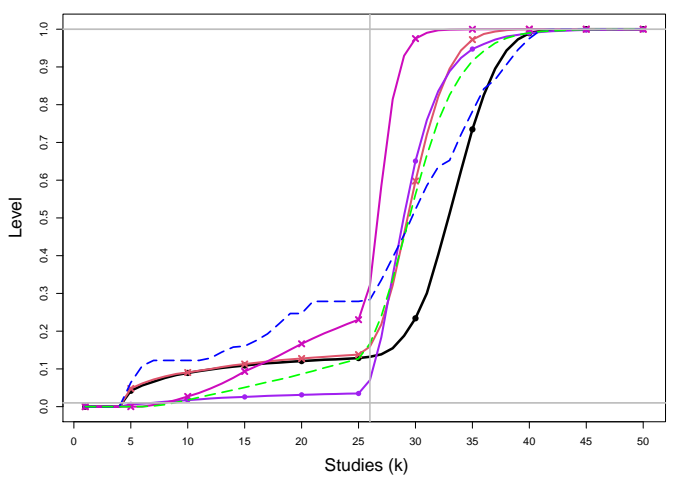

IV REML: $\tau^{2}=0.25 \mathrm{n}=100$ level $=0.01 \mathrm{~h}=5 \delta=1$ to 0 (chg at 26 th)

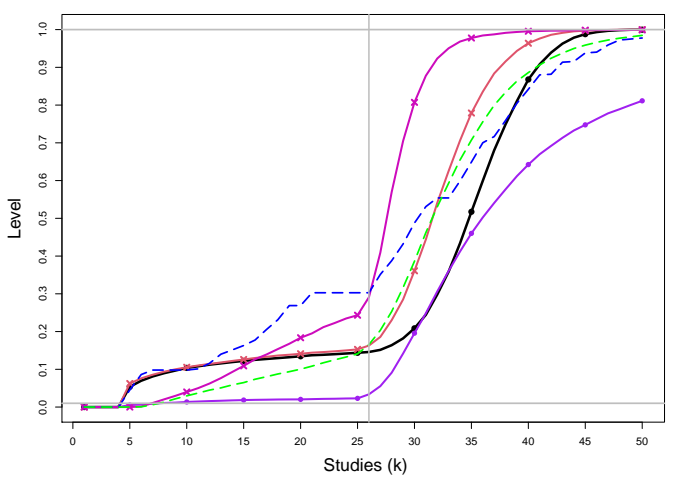

IV REML: $\tau^{2}=1 \mathrm{n}=100$ level $=0.01 \mathrm{~h}=5 \delta=1$ to 0 (chg at 26 th)

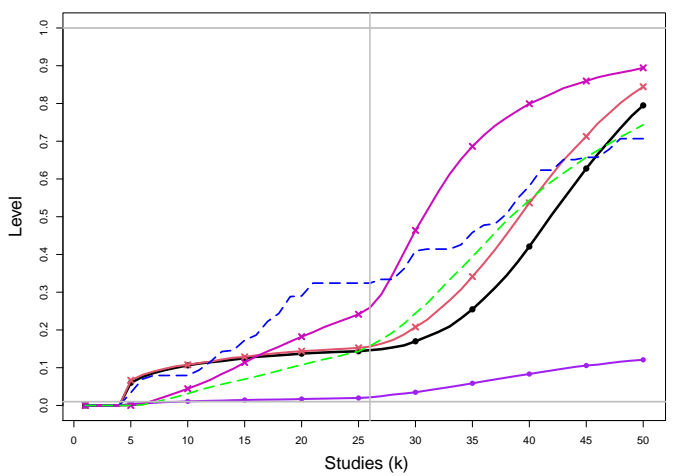

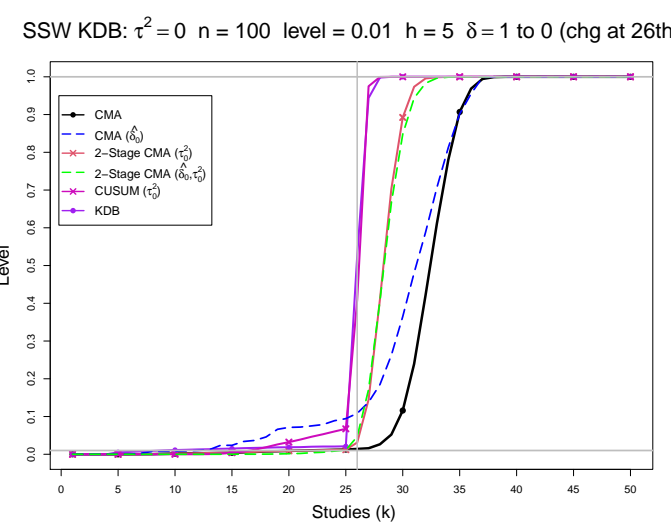

SSW KDB: $\tau^{2}=0.1 \mathrm{n}=100$ level $=0.01 \mathrm{~h}=5 \delta=1$ to 0 (chg at 26th)

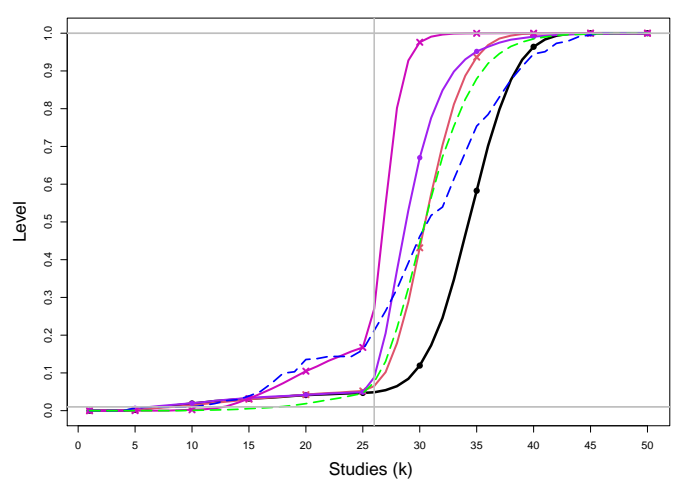

SSW KDB: $\tau^{2}=0.25 \mathrm{n}=100$ level $=0.01 \mathrm{~h}=5 \delta=1$ to 0 (chg at 26th)

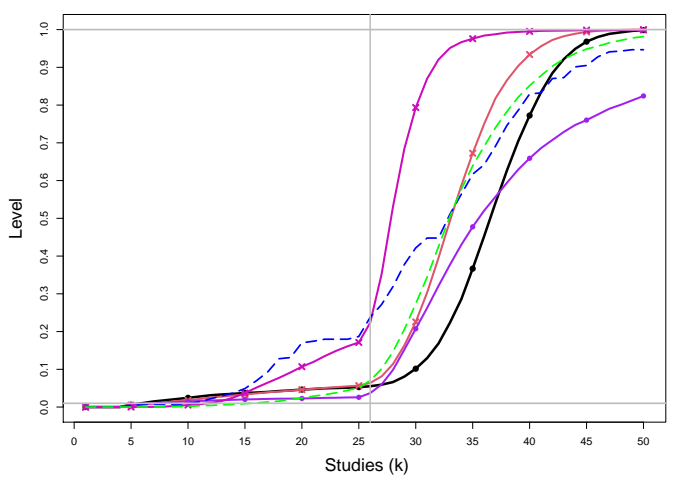

SSW KDB: $\tau^{2}=1 \mathrm{n}=100$ level $=0.01 \mathrm{~h}=5 \delta=1$ to 0 (chg at 26th)

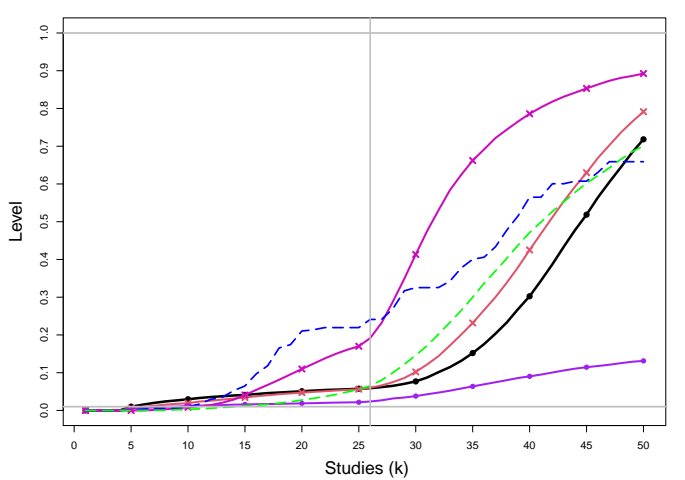

Figure E2. 63: Empirical levels of 1- and 2-stage CMA tests for shift in $\delta$ at .01 nominal level, shift in $\tau^{2}$ at .005 nominal levels and of CUSUM with $h=4$ for equal sample sizes $n_{i C}+n_{i T}=n=100, \tau^{2}=0,0.1,0.25,1$ and a shift from $\delta=1$ to $\delta=0$ at study number 26. Light grey line at .01. 


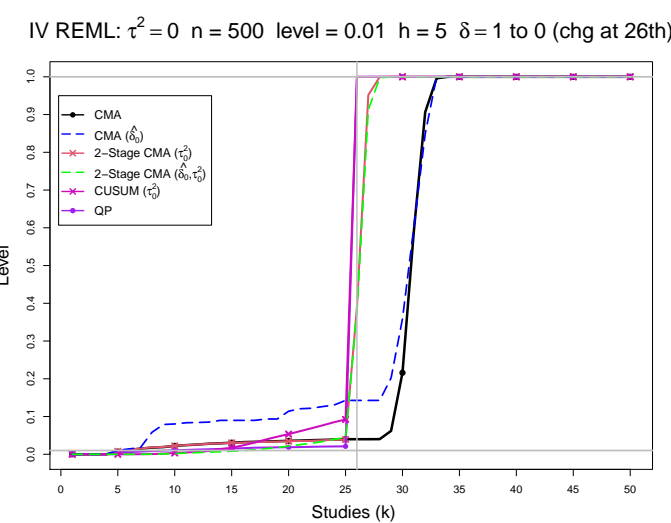

IV REML: $\tau^{2}=0.1 \mathrm{n}=500$ level $=0.01 \mathrm{~h}=5 \delta=1$ to 0 (chg at 26th)

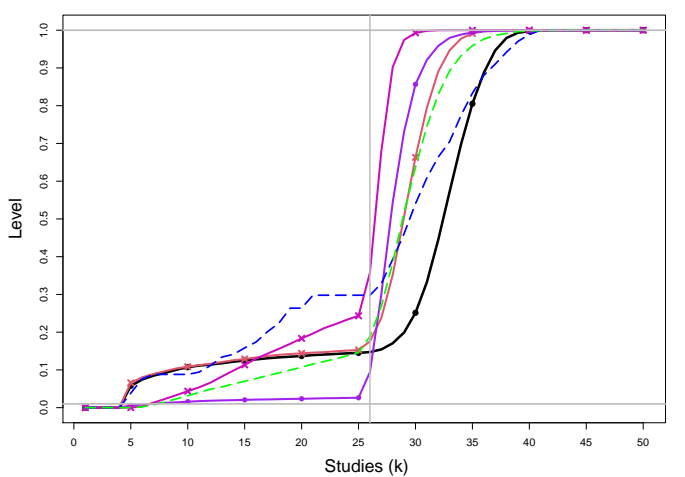

IV REML: $\tau^{2}=0.25 \mathrm{n}=500$ level $=0.01 \mathrm{~h}=5 \delta=1$ to 0 (chg at 26 th)

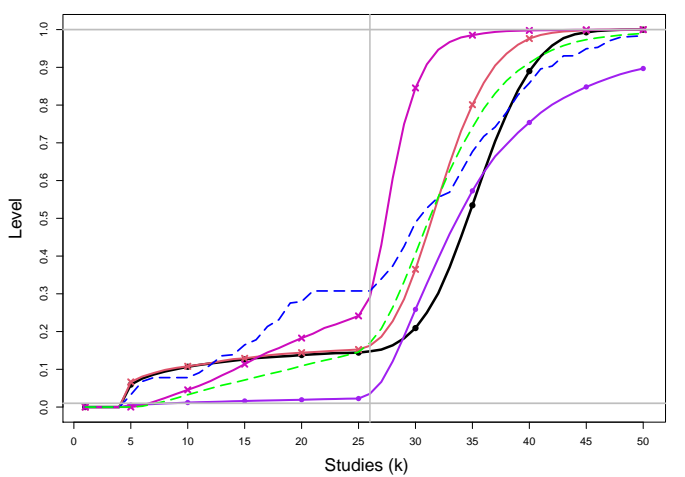

IV REML: $\tau^{2}=1 \mathrm{n}=500$ level $=0.01 \mathrm{~h}=5 \delta=1$ to 0 (chg at 26th)

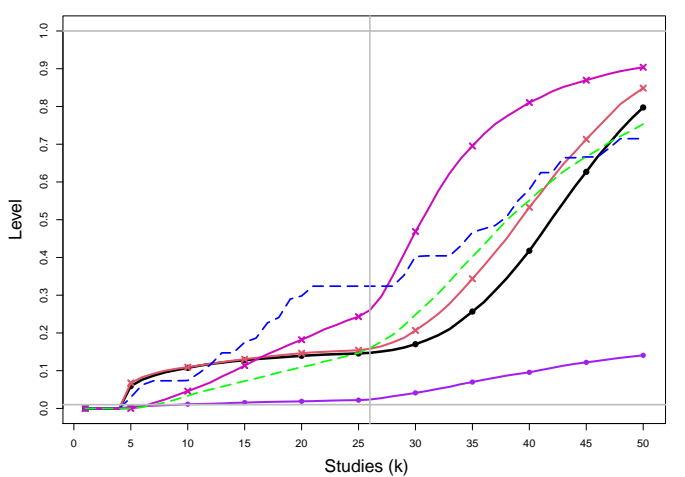

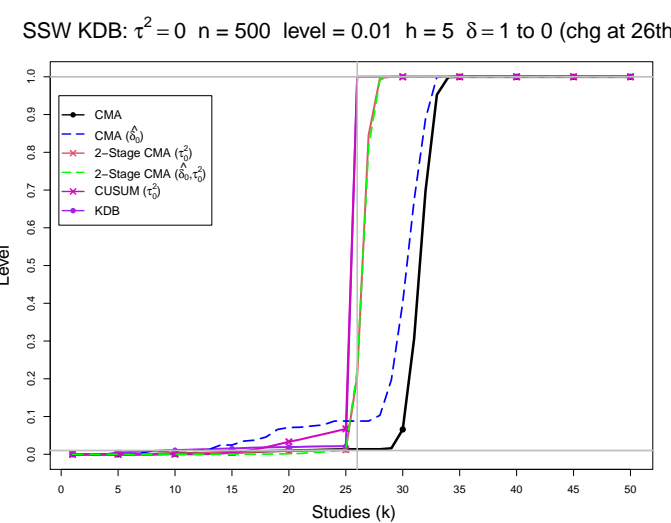

SSW KDB: $\tau^{2}=0.1 \mathrm{n}=500$ level $=0.01 \mathrm{~h}=5 \delta=1$ to 0 (chg at 26th)

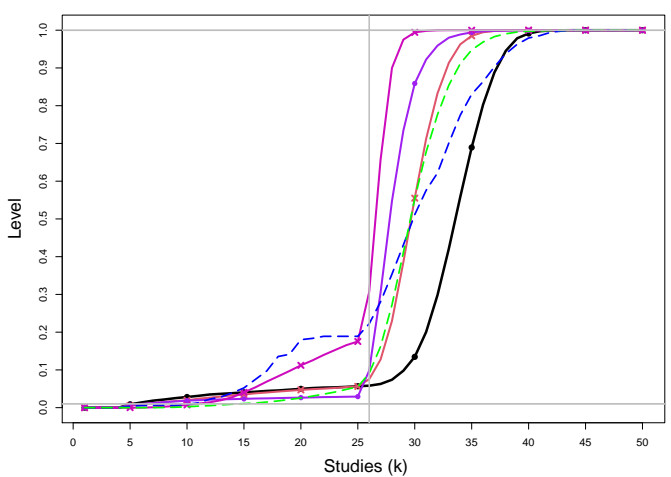

SSW KDB: $\tau^{2}=0.25 \mathrm{n}=500$ level $=0.01 \mathrm{~h}=5 \delta=1$ to 0 (chg at 26th)

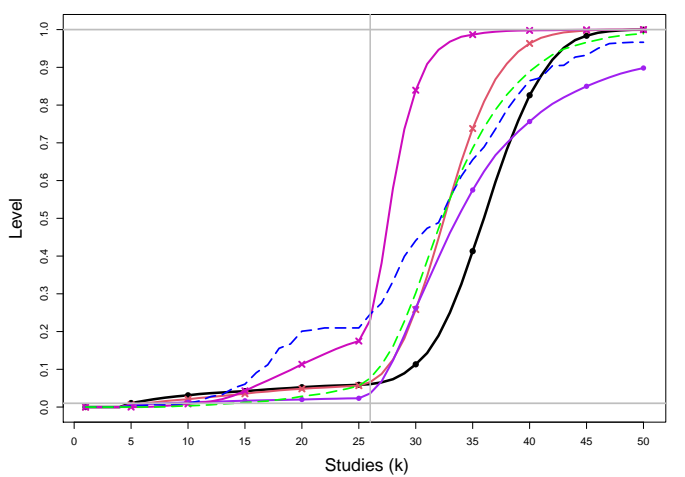

SSW KDB: $\tau^{2}=1 \mathrm{n}=500$ level $=0.01 \mathrm{~h}=5 \delta=1$ to 0 (chg at 26th)

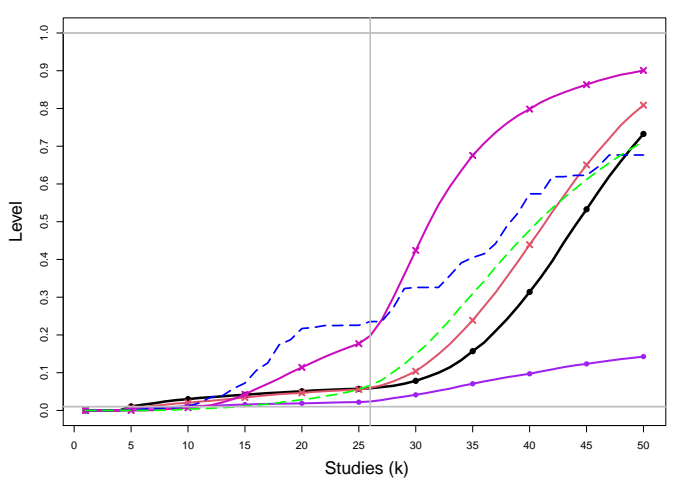

Figure E2. 64: Empirical levels of 1- and 2-stage CMA tests for shift in $\delta$ at .01 nominal level, shift in $\tau^{2}$ at .005 nominal levels and of CUSUM with $h=4$ for equal sample sizes $n_{i C}+n_{i T}=n=500, \tau^{2}=0,0.1,0.25,1$ and a shift from $\delta=1$ to $\delta=0$ at study number 26. Light grey line at .01. 
IV REML: $\tau^{2}=0 \mathrm{n}=1000$ level $=0.01 \mathrm{~h}=5 \quad \delta=1$ to 0 (chg at 26 th)

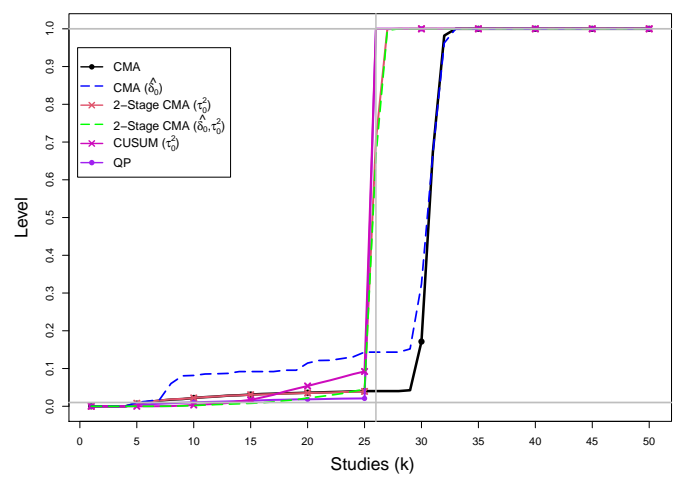

IV REML: $\tau^{2}=0.1 \mathrm{n}=1000$ level $=0.01 \mathrm{~h}=5 \quad \delta=1$ to 0 (chg at 26th)

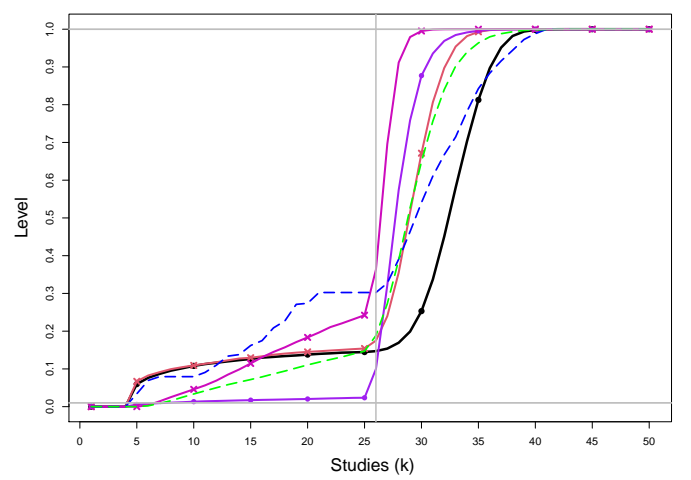

IV REML: $\tau^{2}=0.25 \mathrm{n}=1000$ level $=0.01 \mathrm{~h}=5 \quad \delta=1$ to 0 (chg at 26th)

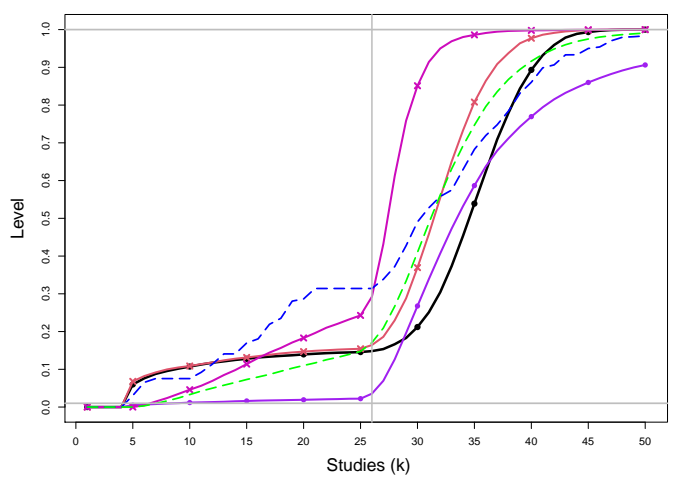

IV REML: $\tau^{2}=1 \mathrm{n}=1000$ level $=0.01 \mathrm{~h}=5 \quad \delta=1$ to 0 (chg at 26th)

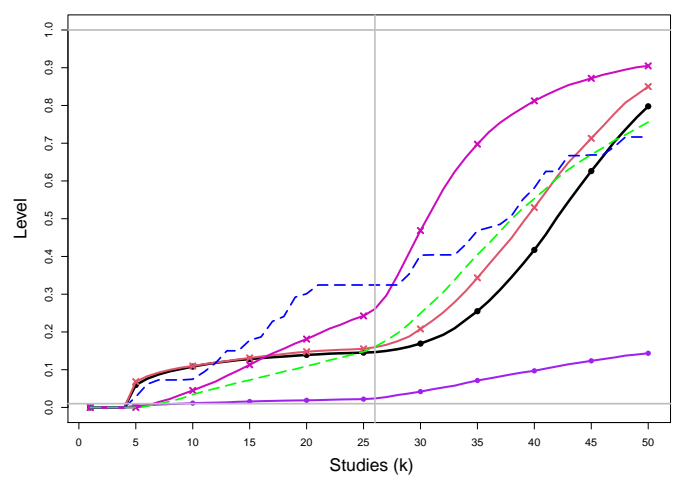

SSW KDB: $\tau^{2}=0 \quad n=1000$ level $=0.01 h=5 \delta=1$ to 0 (chg at 26th)

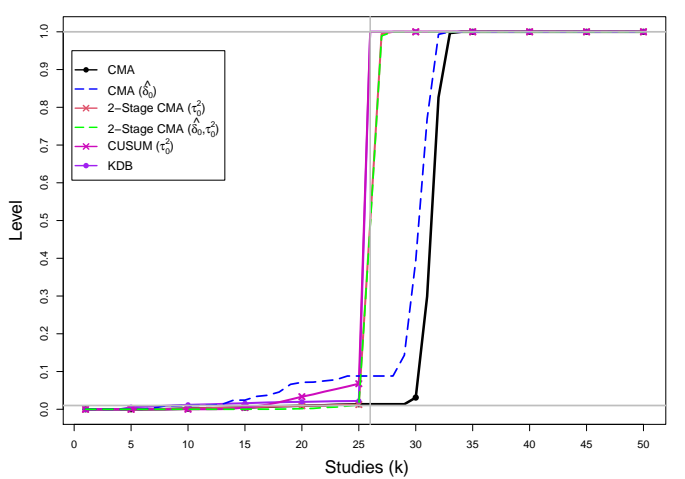

SSW KDB: $\tau^{2}=0.1 \mathrm{n}=1000$ level $=0.01 \mathrm{~h}=5 \delta=1$ to 0 (chg at 26 th)

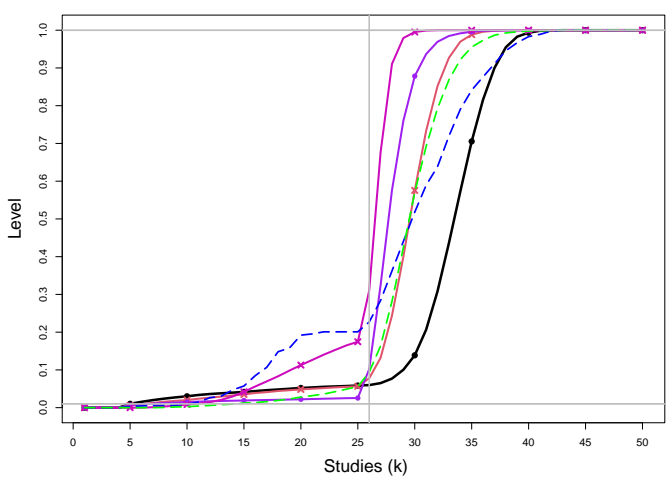

SSW KDB: $\tau^{2}=0.25 \mathrm{n}=1000$ level $=0.01 \mathrm{~h}=5 \delta=1$ to 0 (chg at 26th)

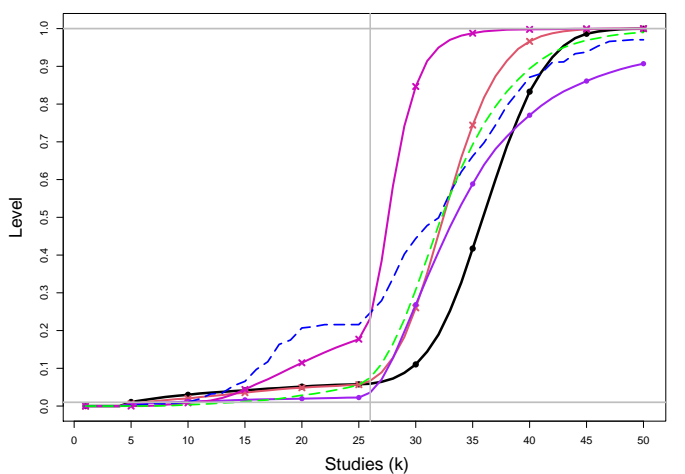

SSW KDB: $\tau^{2}=1 \mathrm{n}=1000$ level $=0.01 \mathrm{~h}=5 \delta=1$ to 0 (chg at 26th)

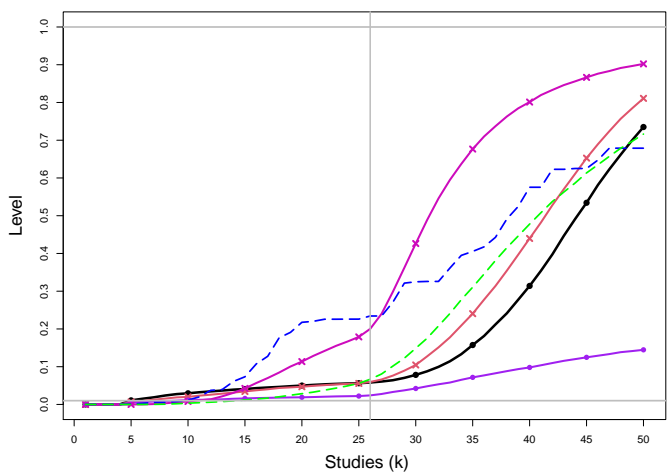

Figure E2. 65: Empirical levels of 1- and 2-stage CMA tests for shift in $\delta$ at .01 nominal level, shift in $\tau^{2}$ at .005 nominal levels and of CUSUM with $h=4$ for equal sample sizes $n_{i C}+n_{i T}=n=1000, \tau^{2}=0,0.1,0.25,1$ and a shift from $\delta=1$ to $\delta=0$ at study number 26. Light grey line at .01. 


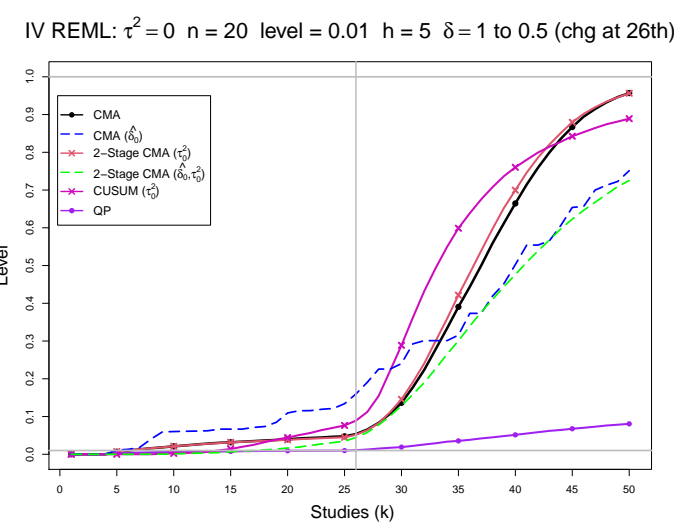

IV REML: $\tau^{2}=0.1 \mathrm{n}=20$ level $=0.01 \mathrm{~h}=5 \delta=1$ to 0.5 (chg at 26 th)

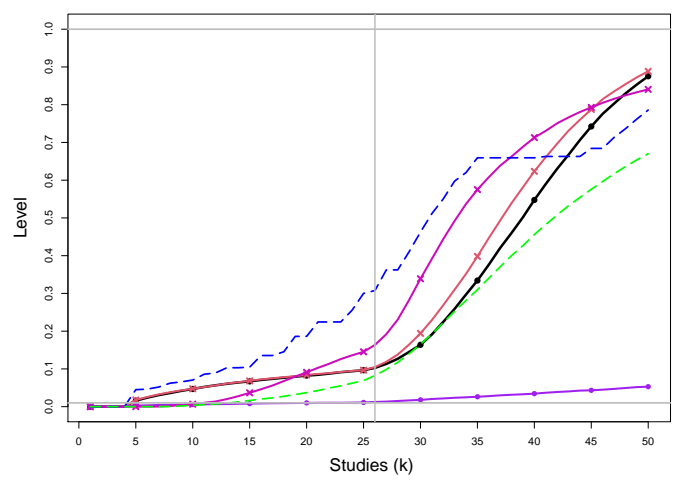

IV REML: $\tau^{2}=0.25 \mathrm{n}=20$ level $=0.01 \mathrm{~h}=5 \delta=1$ to 0.5 (chg at 26th)

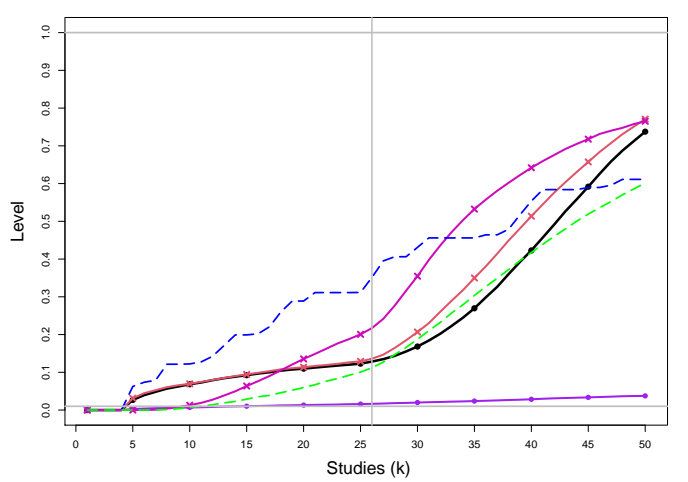

IV REML: $\tau^{2}=1 \mathrm{n}=20$ level $=0.01 \mathrm{~h}=5 \delta=1$ to 0.5 (chg at 26 th)

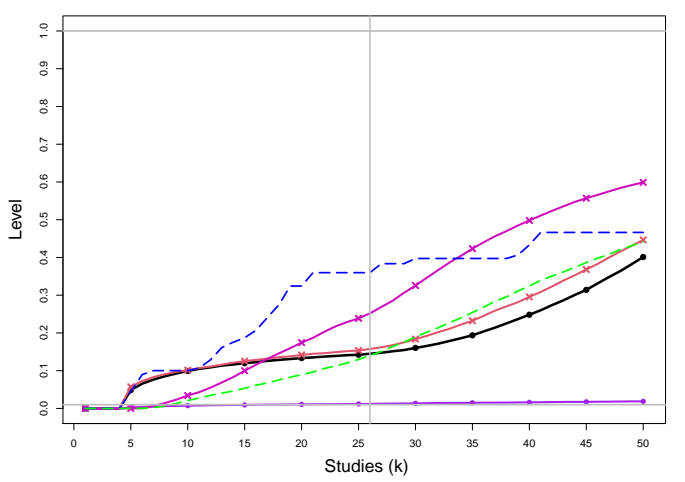

SSW KDB: $\tau^{2}=0 \mathrm{n}=20$ level $=0.01 \mathrm{~h}=5 \quad \delta=1$ to 0.5 (chg at 26th)

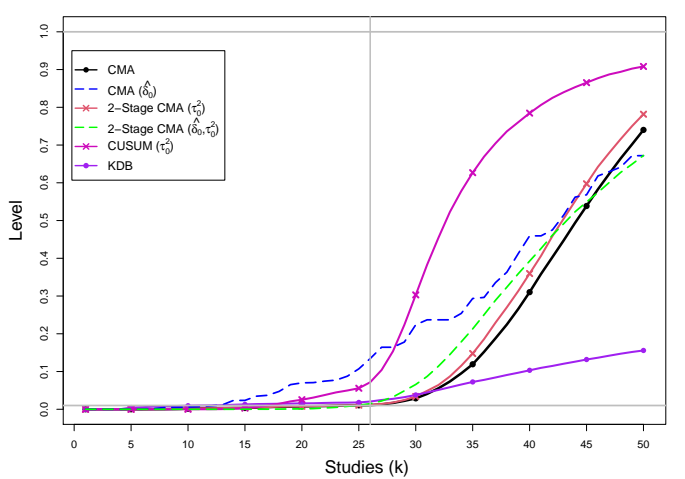

SSW KDB: $\tau^{2}=0.1 \mathrm{n}=20$ level $=0.01 \mathrm{~h}=5 \delta=1$ to 0.5 (chg at 26th)

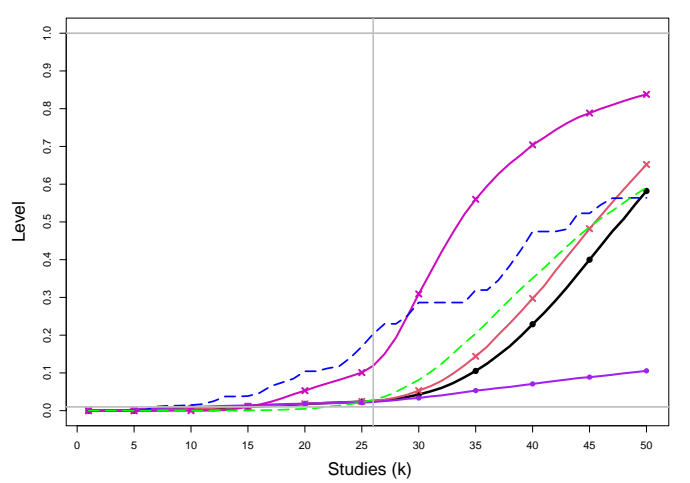

SSW KDB: $\tau^{2}=0.25 \mathrm{n}=20$ level $=0.01 \mathrm{~h}=5 \delta=1$ to 0.5 (chg at 26th)

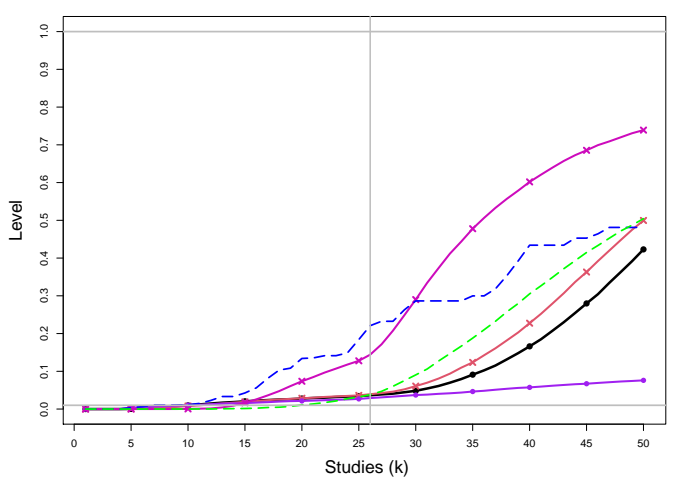

SSW KDB: $\tau^{2}=1 \mathrm{n}=20$ level $=0.01 \mathrm{~h}=5 \quad \delta=1$ to 0.5 (chg at 26th)

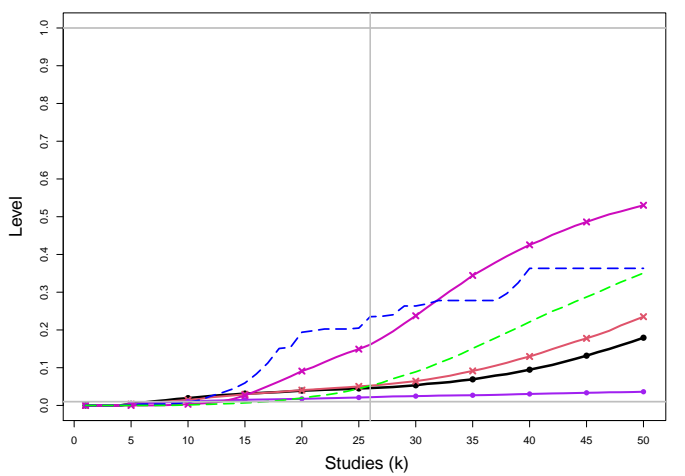

Figure E2. 66: Empirical levels of 1- and 2-stage CMA tests for shift in $\delta$ at .01 nominal level, shift in $\tau^{2}$ at .005 nominal levels and of CUSUM with $h=4$ for equal sample sizes $n_{i C}+n_{i T}=n=20, \tau^{2}=0,0.1,0.25,1$ and a shift from $\delta=1$ to $\delta=0.5$ at study number 26. Light grey line at .01. 


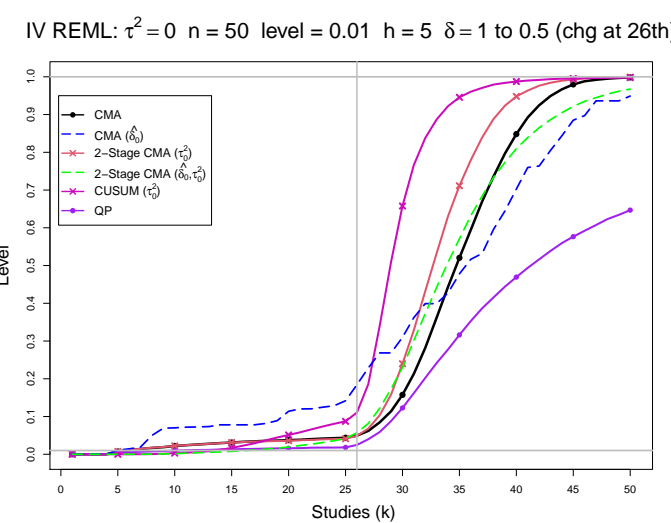

IV REML: $\tau^{2}=0.1 \mathrm{n}=50$ level $=0.01 \mathrm{~h}=5 \delta=1$ to 0.5 (chg at 26th)

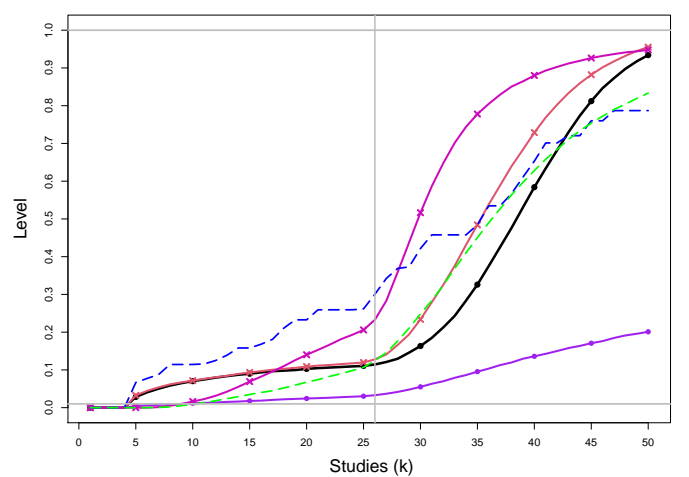

IV REML: $\tau^{2}=0.25 \mathrm{n}=50$ level $=0.01 \mathrm{~h}=5 \delta=1$ to 0.5 (chg at 26th)

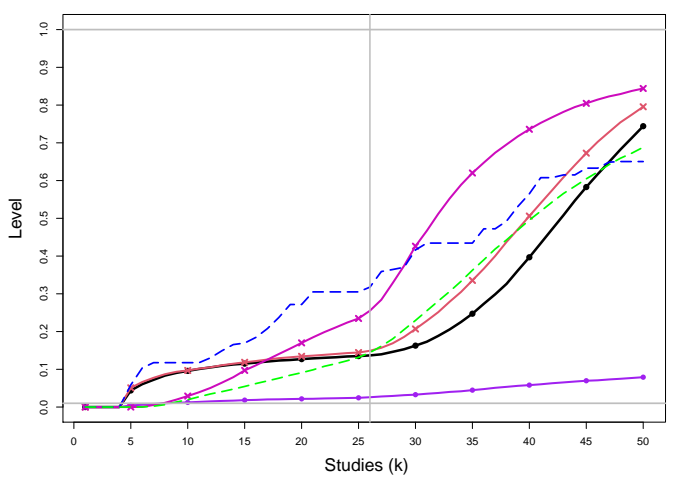

IV REML: $\tau^{2}=1 \mathrm{n}=50$ level $=0.01 \mathrm{~h}=5 \delta=1$ to 0.5 (chg at 26th)

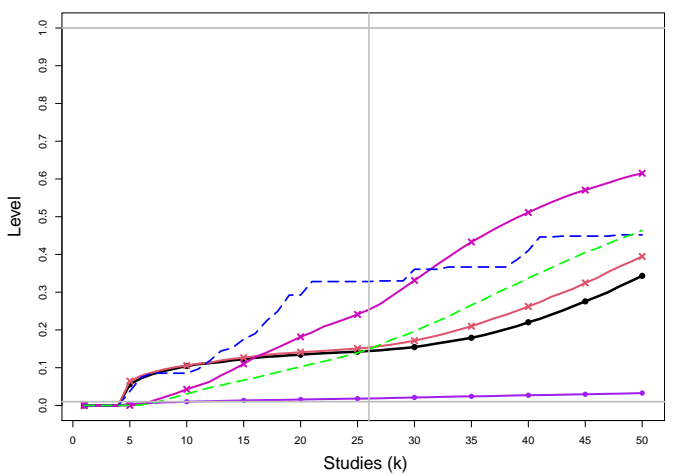

SSW KDB: $\tau^{2}=0 \mathrm{n}=50$ level $=0.01 \mathrm{~h}=5 \quad \delta=1$ to 0.5 (chg at 26th)

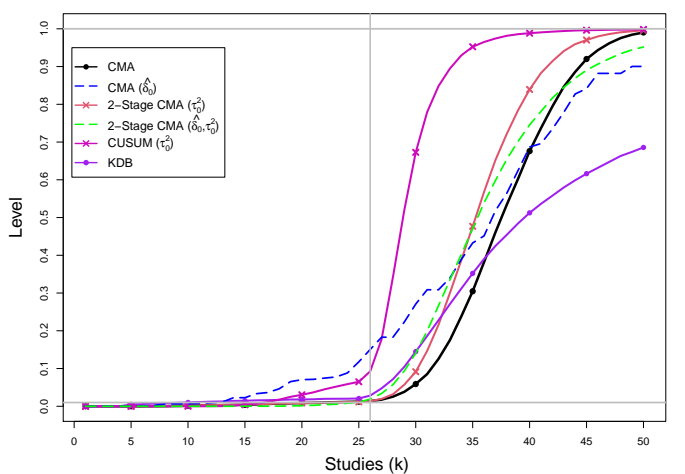

SSW KDB: $\tau^{2}=0.1 \mathrm{n}=50$ level $=0.01 \mathrm{~h}=5 \delta=1$ to 0.5 (chg at 26th)

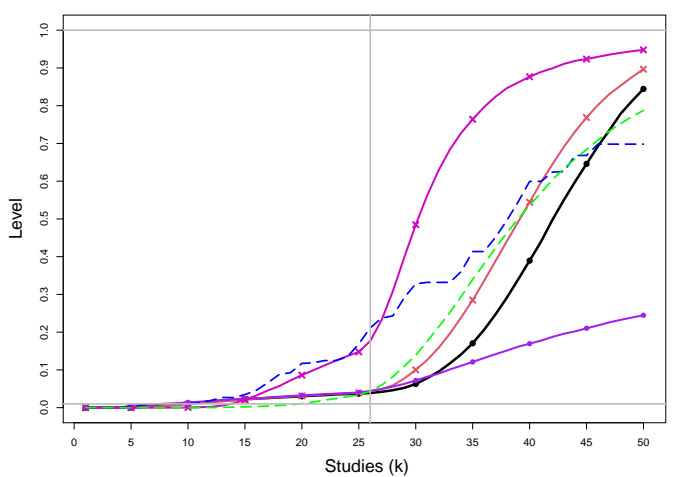

SSW KDB: $\tau^{2}=0.25 \mathrm{n}=50$ level $=0.01 \mathrm{~h}=5 \delta=1$ to 0.5 (chg at 26th)

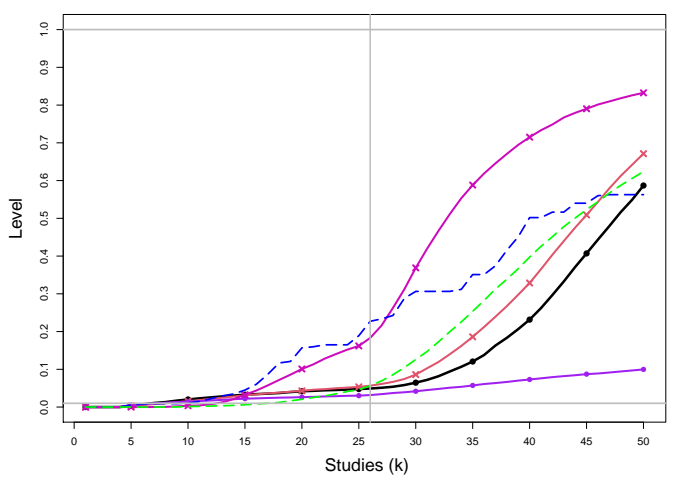

SSW KDB: $\tau^{2}=1 \mathrm{n}=50$ level $=0.01 \mathrm{~h}=5 \quad \delta=1$ to 0.5 (chg at 26th)

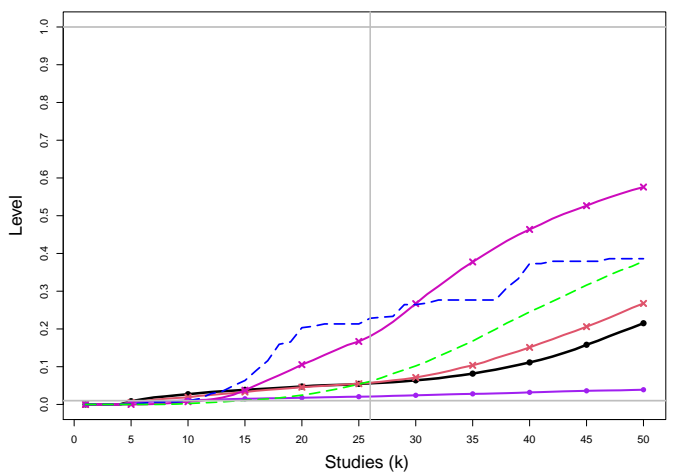

Figure E2. 67: Empirical levels of 1- and 2-stage CMA tests for shift in $\delta$ at .01 nominal level, shift in $\tau^{2}$ at .005 nominal levels and of CUSUM with $h=4$ for equal sample sizes $n_{i C}+n_{i T}=n=50, \tau^{2}=0,0.1,0.25,1$ and a shift from $\delta=1$ to $\delta=0.5$ at study number 26. Light grey line at .01. 
IV REML: $\tau^{2}=0 \quad \mathrm{n}=100$ level $=0.01 \mathrm{~h}=5 \quad \delta=1$ to 0.5 (chg at 26 th)

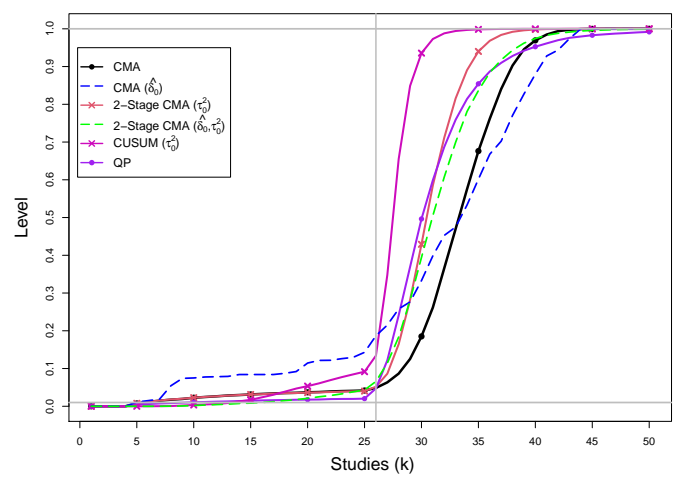

IV REML: $\tau^{2}=0.1 \mathrm{n}=100$ level $=0.01 \mathrm{~h}=5 \quad \delta=1$ to 0.5 (chg at 26 th)

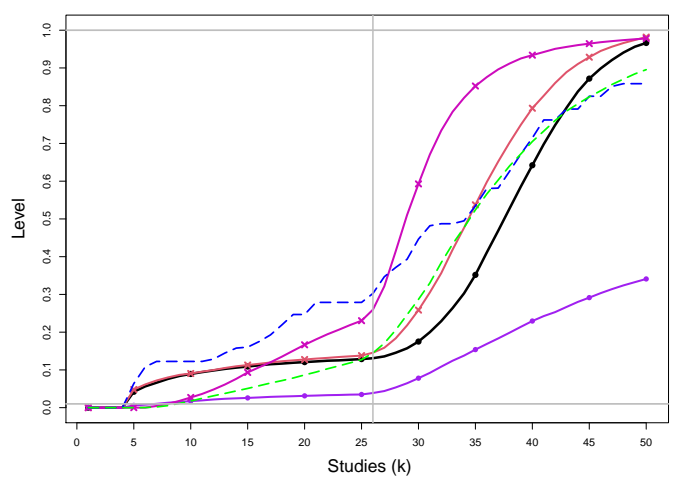

IV REML: $\tau^{2}=0.25 \mathrm{n}=100$ level $=0.01 \mathrm{~h}=5 \quad \delta=1$ to 0.5 (chg at 26 th)

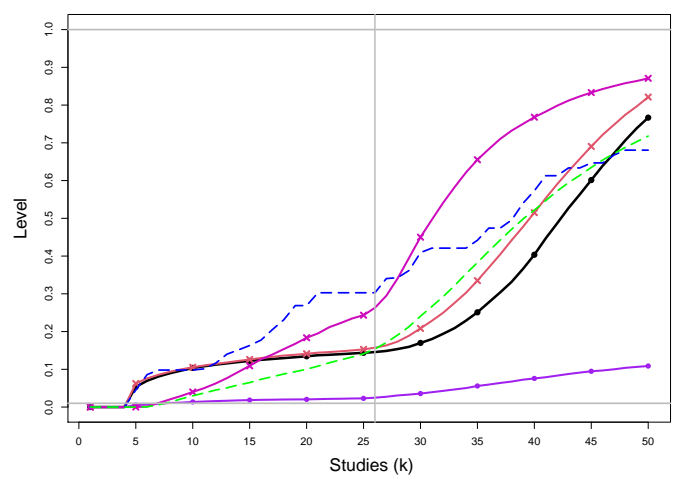

IV REML: $\tau^{2}=1 \mathrm{n}=100$ level $=0.01 \mathrm{~h}=5 \delta=1$ to 0.5 (chg at 26th)

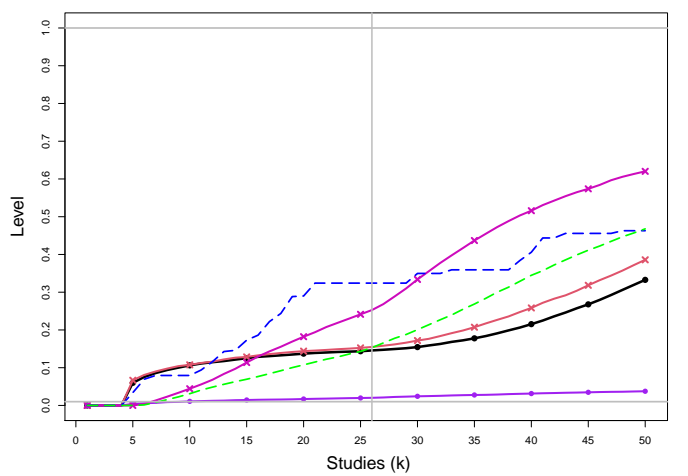

SSW KDB: $\tau^{2}=0 \mathrm{n}=100$ level $=0.01 \mathrm{~h}=5 \quad \delta=1$ to 0.5 (chg at 26th)

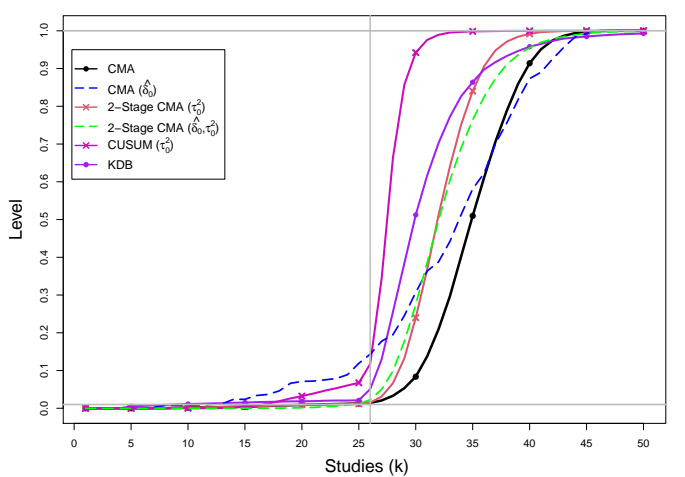

SSW KDB: $\tau^{2}=0.1 \mathrm{n}=100$ level $=0.01 \mathrm{~h}=5 \delta=1$ to 0.5 (chg at 26th)

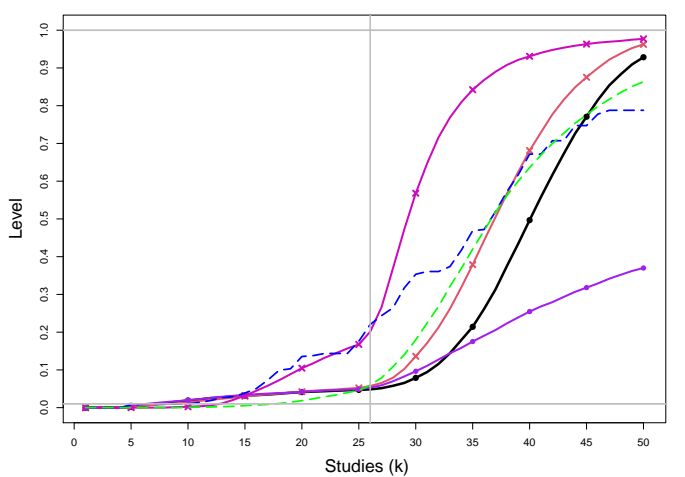

SSW KDB: $\tau^{2}=0.25 \mathrm{n}=100$ level $=0.01 \mathrm{~h}=5 \quad \delta=1$ to 0.5 (chg at 26th)

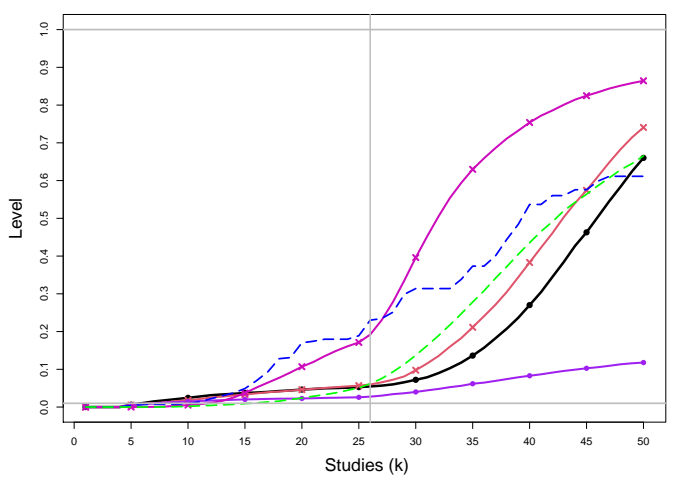

SSW KDB: $\tau^{2}=1 \mathrm{n}=100$ level $=0.01 \mathrm{~h}=5 \delta=1$ to 0.5 (chg at 26 th)

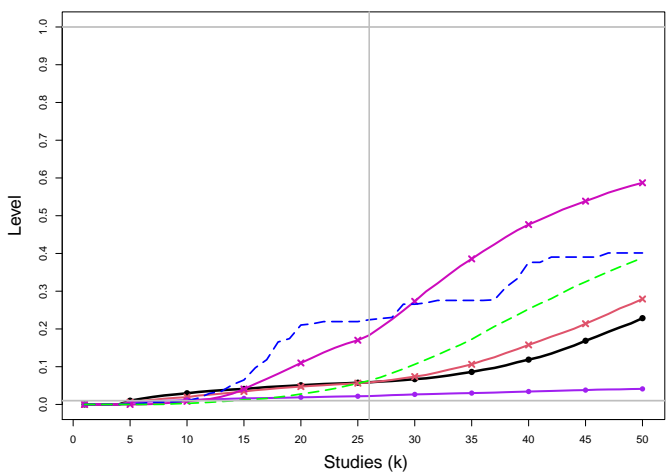

Figure E2. 68: Empirical levels of 1- and 2-stage CMA tests for shift in $\delta$ at .01 nominal level, shift in $\tau^{2}$ at .005 nominal levels and of CUSUM with $h=4$ for equal sample sizes $n_{i C}+n_{i T}=n=100, \tau^{2}=0,0.1,0.25,1$ and a shift from $\delta=1$ to $\delta=0.5$ at study number 26. Light grey line at .01. 
IV REML: $\tau^{2}=0 \mathrm{n}=500$ level $=0.01 \mathrm{~h}=5 \quad \delta=1$ to 0.5 (chg at 26 th)

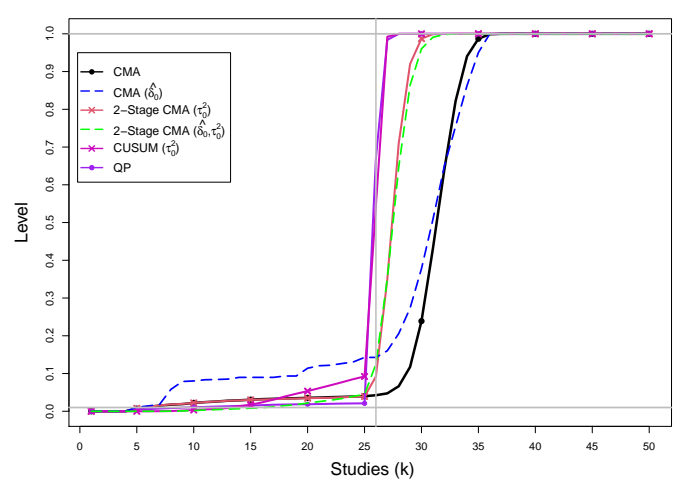

IV REML: $\tau^{2}=0.1 \mathrm{n}=500$ level $=0.01 \mathrm{~h}=5 \quad \delta=1$ to 0.5 (chg at 26 th)

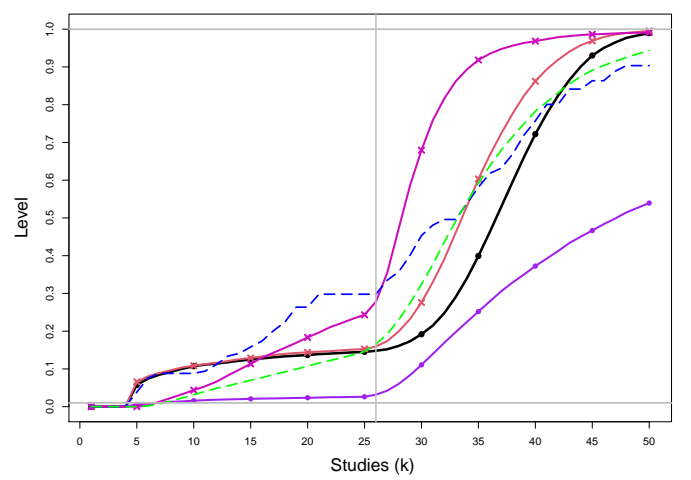

IV REML: $\tau^{2}=0.25 \mathrm{n}=500$ level $=0.01 \mathrm{~h}=5 \quad \delta=1$ to 0.5 (chg at 26 th)

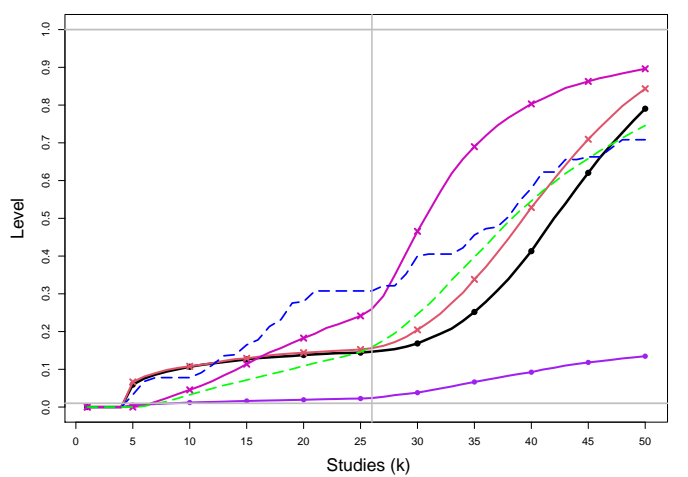

IV REML: $\tau^{2}=1 \mathrm{n}=500$ level $=0.01 \mathrm{~h}=5 \quad \delta=1$ to 0.5 (chg at 26 th)

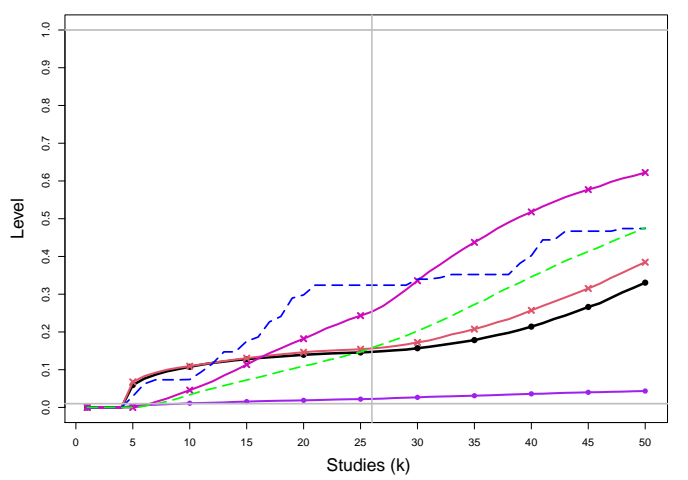

SSW KDB: $\tau^{2}=0 \mathrm{n}=500$ level $=0.01 \mathrm{~h}=5 \quad \delta=1$ to 0.5 (chg at 26th)

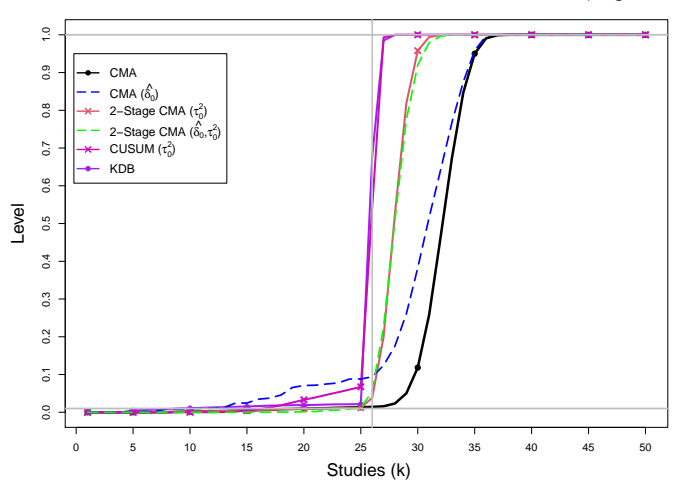

SSW KDB: $\tau^{2}=0.1 \mathrm{n}=500$ level $=0.01 \mathrm{~h}=5 \delta=1$ to 0.5 (chg at 26th)

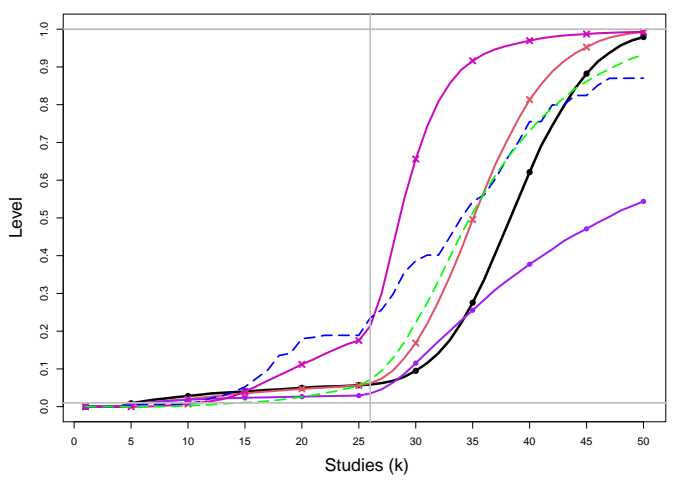

SSW KDB: $\tau^{2}=0.25 \mathrm{n}=500$ level $=0.01 \mathrm{~h}=5 \delta=1$ to 0.5 (chg at 26th)

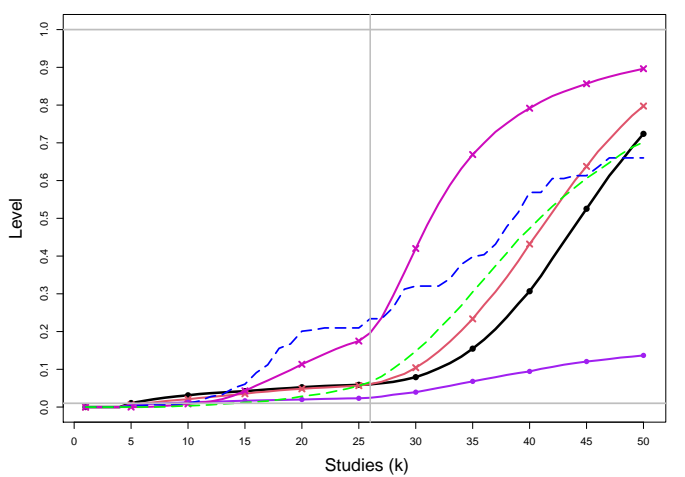

SSW KDB: $\tau^{2}=1 \mathrm{n}=500$ level $=0.01 \mathrm{~h}=5 \delta=1$ to 0.5 (chg at 26 th)

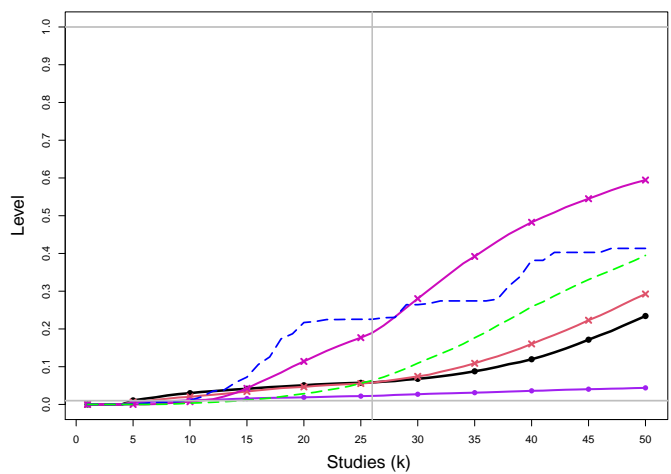

Figure E2. 69: Empirical levels of 1- and 2-stage CMA tests for shift in $\delta$ at .01 nominal level, shift in $\tau^{2}$ at .005 nominal levels and of CUSUM with $h=4$ for equal sample sizes $n_{i C}+n_{i T}=n=500, \tau^{2}=0,0.1,0.25,1$ and a shift from $\delta=1$ to $\delta=0.5$ at study number 26. Light grey line at .01. 
IV REML: $\tau^{2}=0 \mathrm{n}=1000$ level $=0.01 \mathrm{~h}=5 \quad \delta=1$ to 0.5 (chg at 26th)

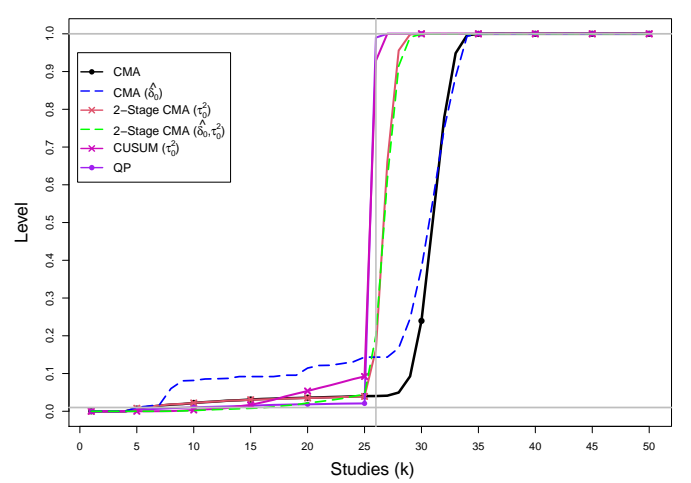

IV REML: $\tau^{2}=0.1 \mathrm{n}=1000$ level $=0.01 \mathrm{~h}=5 \delta=1$ to 0.5 (chg at 26th)
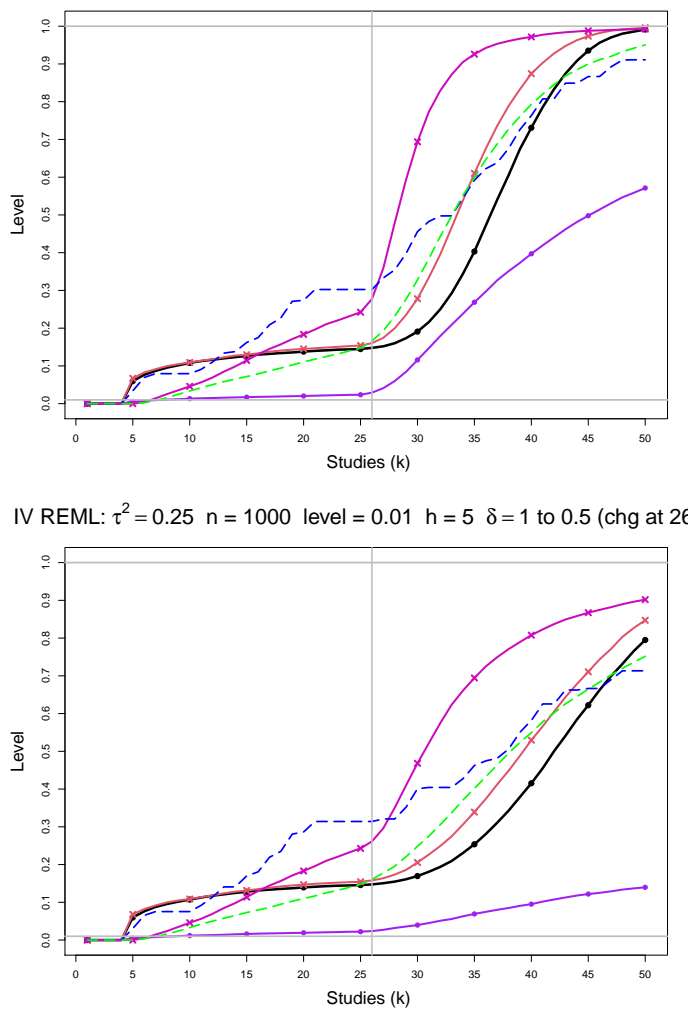

IV REML: $\tau^{2}=1 \mathrm{n}=1000$ level $=0.01 \mathrm{~h}=5 \delta=1$ to 0.5 (chg at 26th)

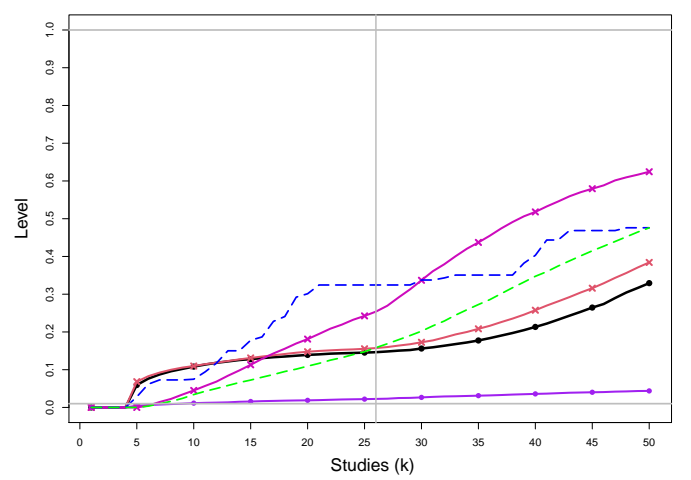

SSW KDB: $\tau^{2}=0 \mathrm{n}=1000$ level $=0.01 \mathrm{~h}=5 \quad \delta=1$ to 0.5 (chg at 26 th)

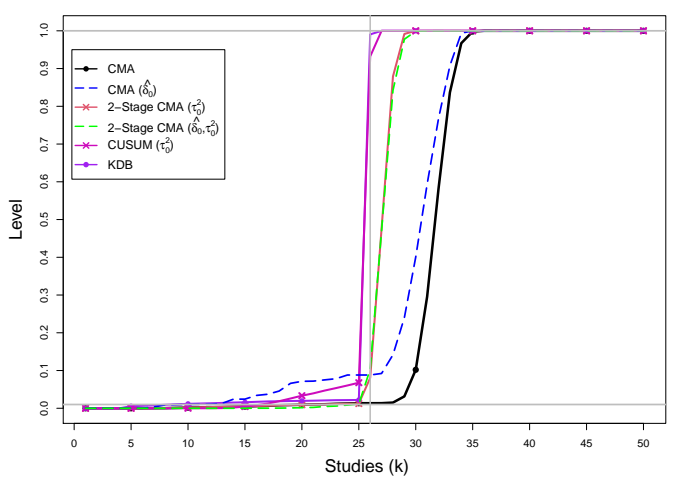

SSW KDB: $\tau^{2}=0.1 \mathrm{n}=1000$ level $=0.01 \mathrm{~h}=5 \delta=1$ to 0.5 (chg at 26th)

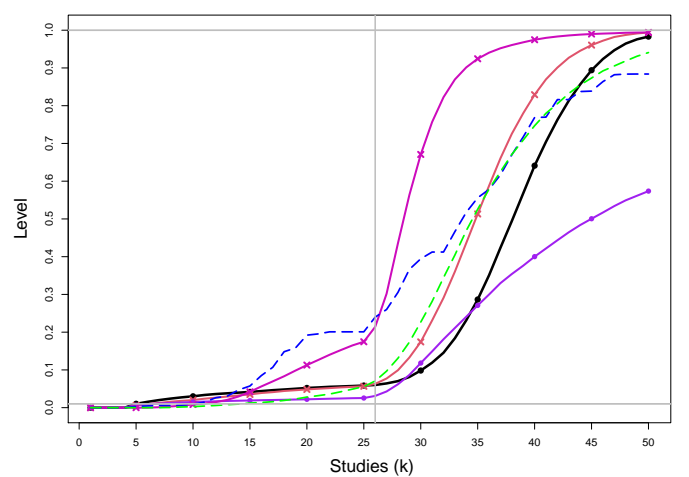

SSW KDB: $\tau^{2}=1 \mathrm{n}=1000$ level $=0.01 \mathrm{~h}=5 \quad \delta=1$ to 0.5 (chg at 26th)

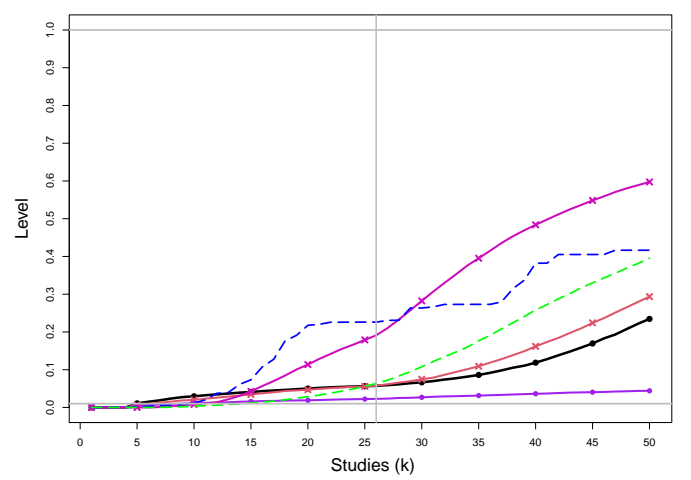

Figure E2. 70: Empirical levels of 1- and 2-stage CMA tests for shift in $\delta$ at .01 nominal level, shift in $\tau^{2}$ at .005 nominal levels and of CUSUM with $h=4$ for equal sample sizes $n_{i C}+n_{i T}=n=1000, \tau^{2}=0,0.1,0.25,1$ and a shift from $\delta=1$ to $\delta=0.5$ at study number 26. Light grey line at .01. 


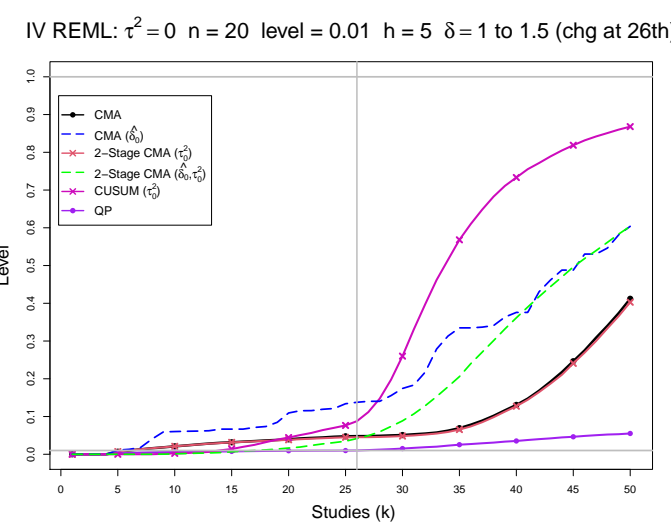

IV REML: $\tau^{2}=0.1 \mathrm{n}=20$ level $=0.01 \mathrm{~h}=5 \delta=1$ to 1.5 (chg at 26th)

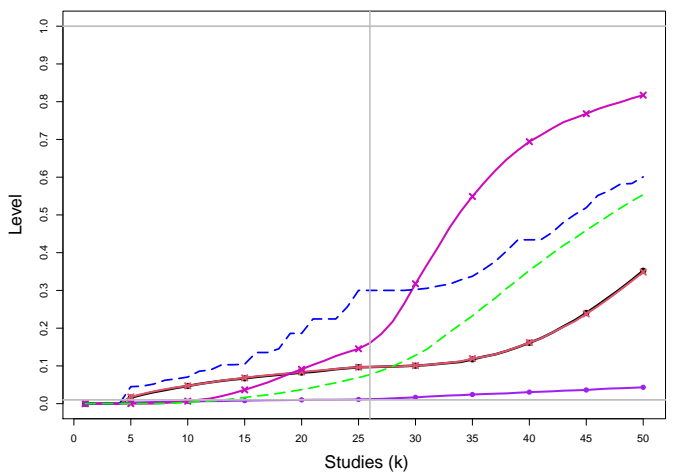

IV REML: $\tau^{2}=0.25 \mathrm{n}=20$ level $=0.01 \mathrm{~h}=5 \delta=1$ to 1.5 (chg at 26th)

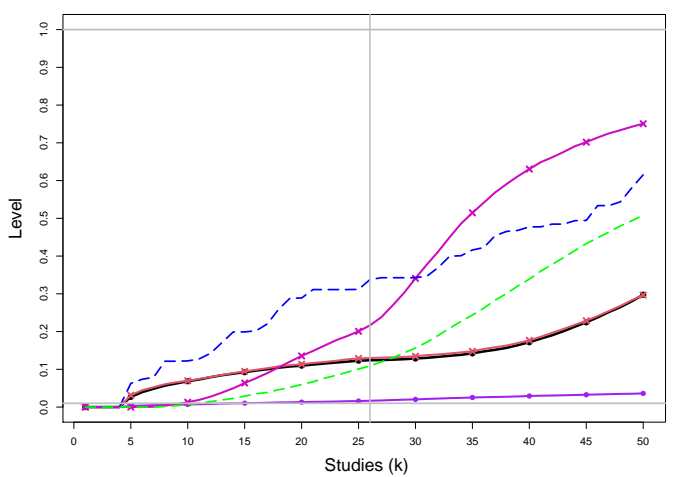

IV REML: $\tau^{2}=1 \mathrm{n}=20$ level $=0.01 \mathrm{~h}=5 \delta=1$ to 1.5 (chg at 26th)

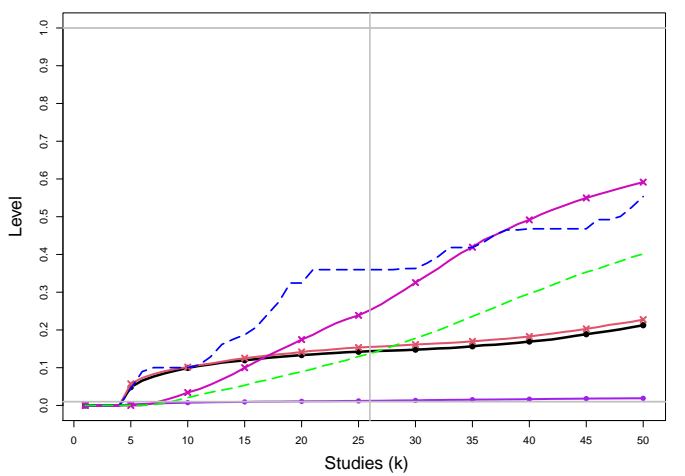

SSW KDB: $\tau^{2}=0 \mathrm{n}=20$ level $=0.01 \mathrm{~h}=5 \quad \delta=1$ to 1.5 (chg at 26th)

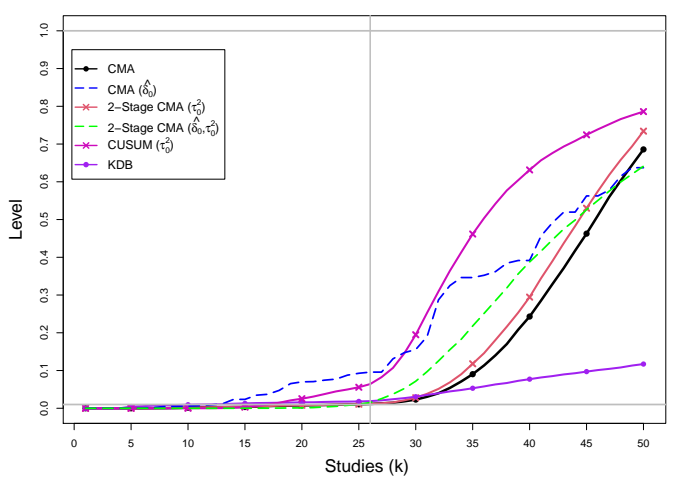

SSW KDB: $\tau^{2}=0.1 \mathrm{n}=20$ level $=0.01 \mathrm{~h}=5 \delta=1$ to 1.5 (chg at 26th)

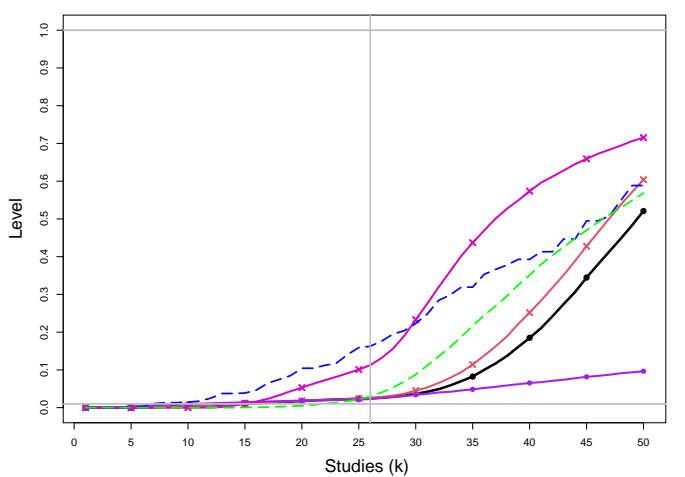

SSW KDB: $\tau^{2}=0.25 \mathrm{n}=20$ level $=0.01 \mathrm{~h}=5 \delta=1$ to 1.5 (chg at 26th)

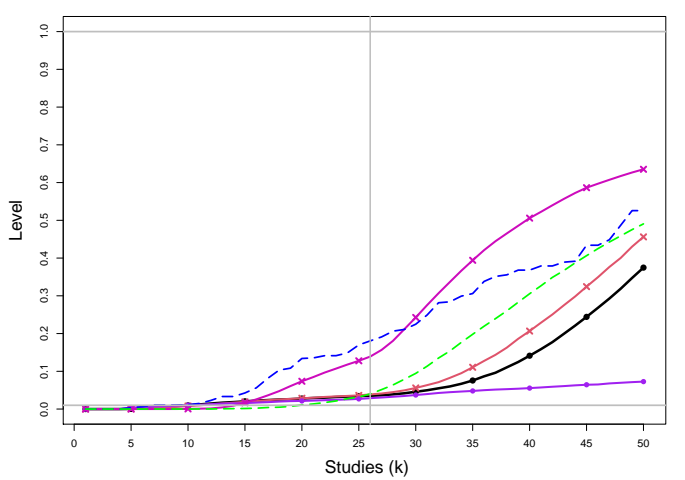

SSW KDB: $\tau^{2}=1 \mathrm{n}=20$ level $=0.01 \mathrm{~h}=5 \quad \delta=1$ to 1.5 (chg at 26th)

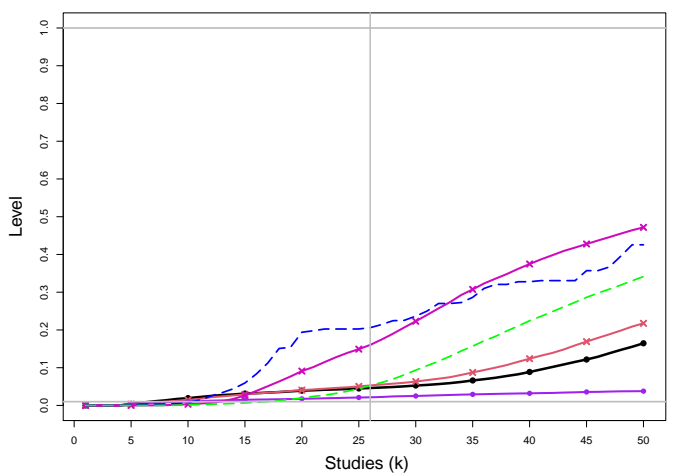

Figure E2. 71: Empirical levels of 1- and 2-stage CMA tests for shift in $\delta$ at .01 nominal level, shift in $\tau^{2}$ at .005 nominal levels and of CUSUM with $h=4$ for equal sample sizes $n_{i C}+n_{i T}=n=20, \tau^{2}=0,0.1,0.25,1$ and a shift from $\delta=1$ to $\delta=1.5$ at study number 26. Light grey line at .01. 


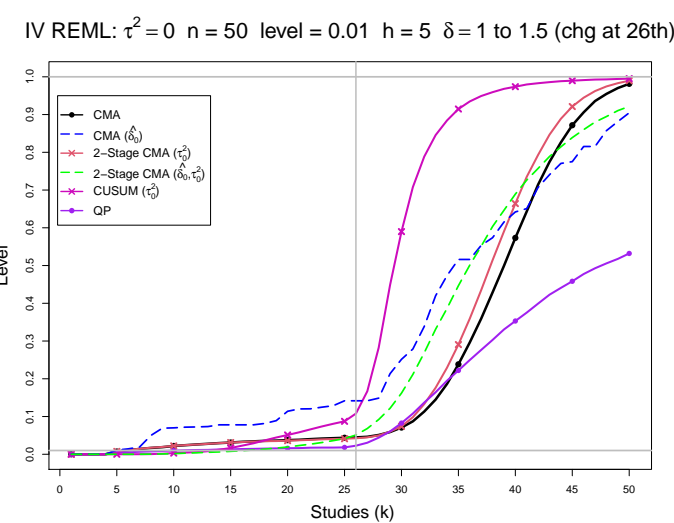

IV REML: $\tau^{2}=0.1 \mathrm{n}=50$ level $=0.01 \mathrm{~h}=5 \delta=1$ to 1.5 (chg at 26 th)

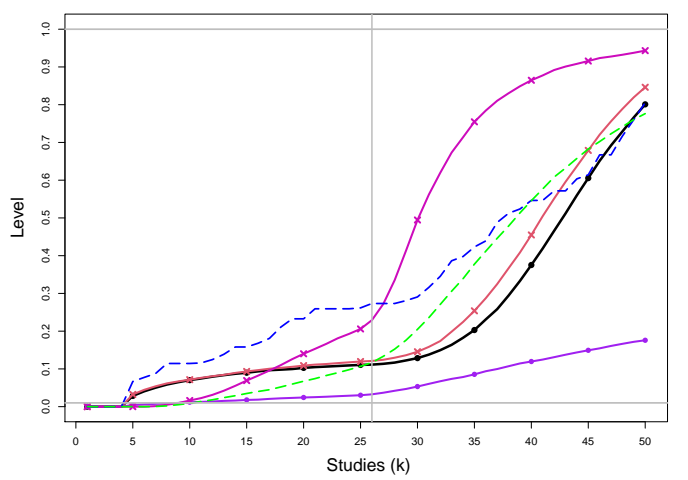

IV REML: $\tau^{2}=0.25 \mathrm{n}=50$ level $=0.01 \mathrm{~h}=5 \delta=1$ to 1.5 (chg at 26th)

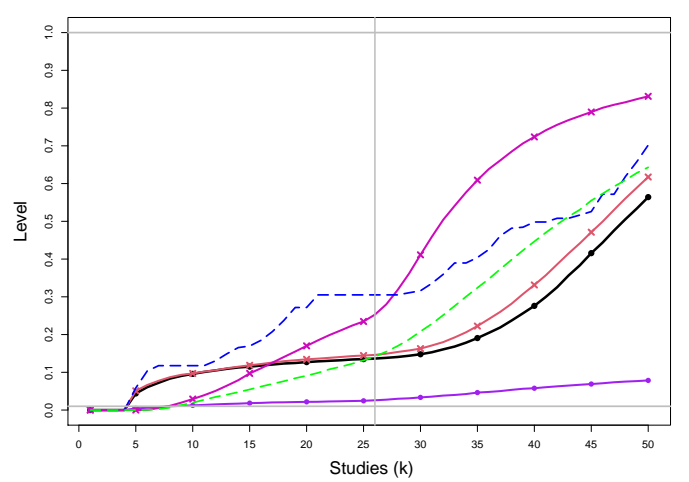

IV REML: $\tau^{2}=1 \mathrm{n}=50$ level $=0.01 \mathrm{~h}=5 \delta=1$ to 1.5 (chg at 26th)

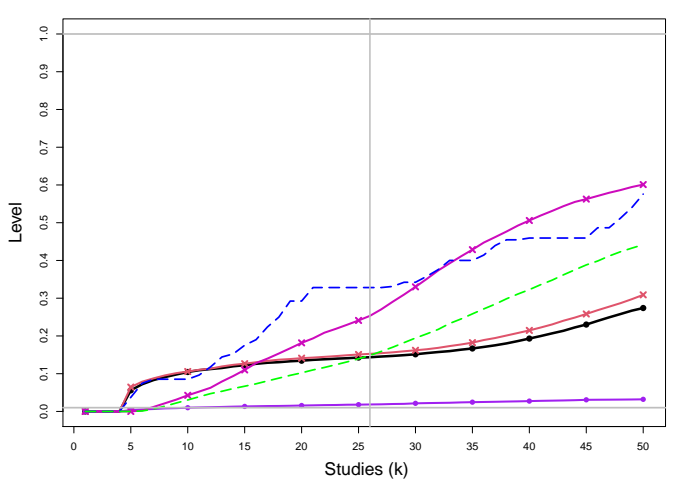

SSW KDB: $\tau^{2}=0 \mathrm{n}=50$ level $=0.01 \mathrm{~h}=5 \quad \delta=1$ to 1.5 (chg at 26th)

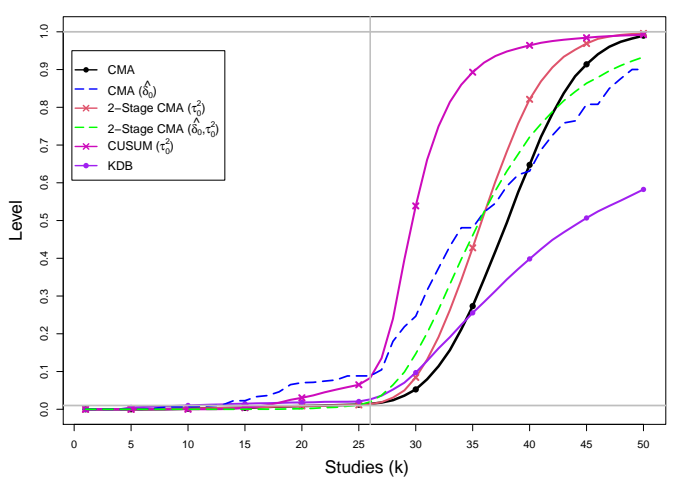

SSW KDB: $\tau^{2}=0.1 \mathrm{n}=50$ level $=0.01 \mathrm{~h}=5 \delta=1$ to 1.5 (chg at 26th)

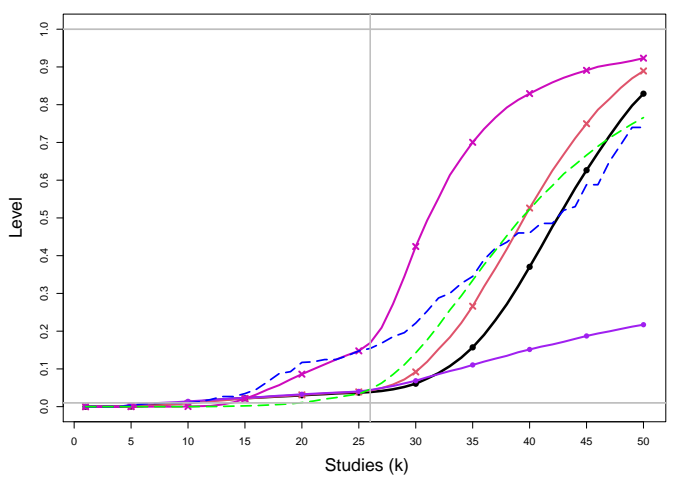

SSW KDB: $\tau^{2}=0.25 \mathrm{n}=50$ level $=0.01 \mathrm{~h}=5 \delta=1$ to 1.5 (chg at 26th)

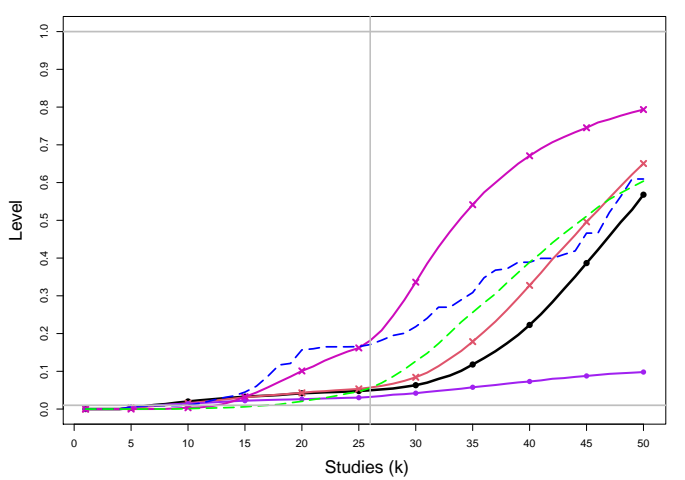

SSW KDB: $\tau^{2}=1 \mathrm{n}=50$ level $=0.01 \mathrm{~h}=5 \quad \delta=1$ to 1.5 (chg at 26th)

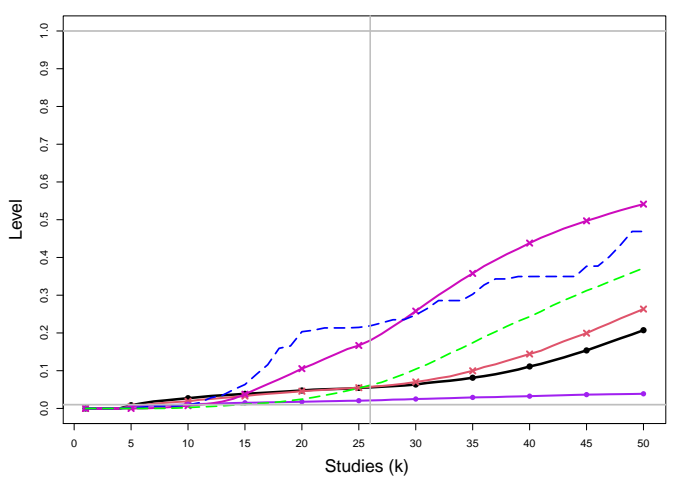

Figure E2. 72: Empirical levels of 1- and 2-stage CMA tests for shift in $\delta$ at .01 nominal level, shift in $\tau^{2}$ at .005 nominal levels and of CUSUM with $h=4$ for equal sample sizes $n_{i C}+n_{i T}=n=50, \tau^{2}=0,0.1,0.25,1$ and a shift from $\delta=1$ to $\delta=1.5$ at study number 26. Light grey line at .01. 
IV REML: $\tau^{2}=0 \mathrm{n}=100$ level $=0.01 \mathrm{~h}=5 \quad \delta=1$ to 1.5 (chg at 26 th)

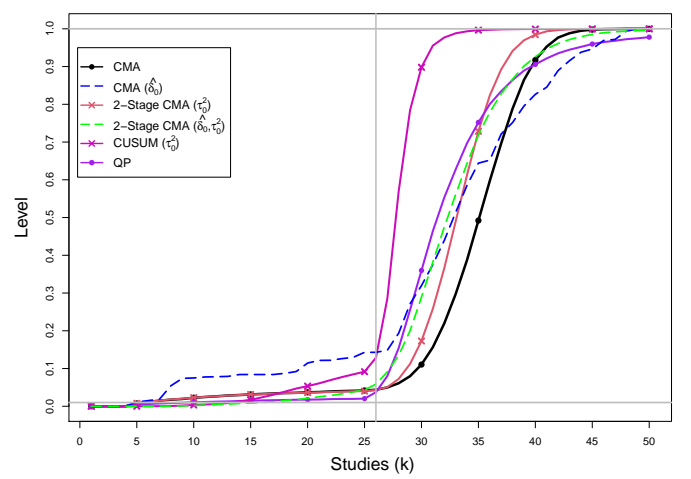

IV REML: $\tau^{2}=0.1 \mathrm{n}=100$ level $=0.01 \mathrm{~h}=5 \quad \delta=1$ to 1.5 (chg at 26 th)

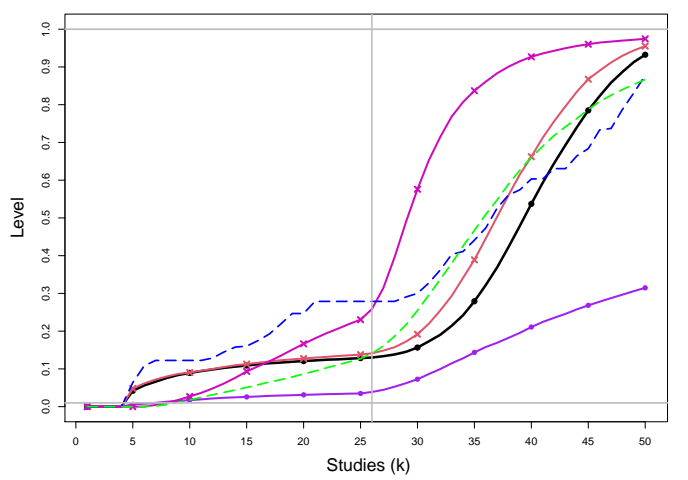

IV REML: $\tau^{2}=0.25 \mathrm{n}=100$ level $=0.01 \mathrm{~h}=5 \quad \delta=1$ to 1.5 (chg at 26 th)

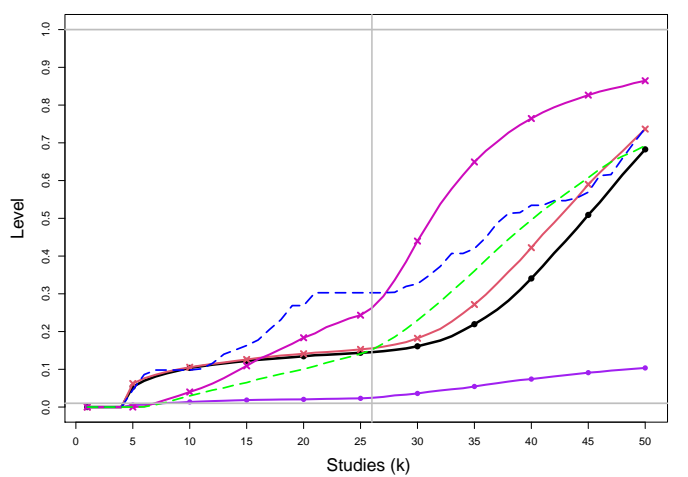

IV REML: $\tau^{2}=1 \mathrm{n}=100$ level $=0.01 \mathrm{~h}=5 \delta=1$ to 1.5 (chg at 26th)

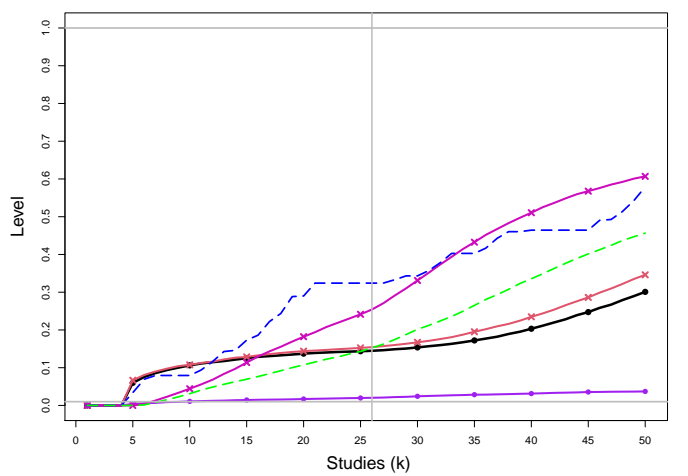

SSW KDB: $\tau^{2}=0 \mathrm{n}=100$ level $=0.01 \mathrm{~h}=5 \quad \delta=1$ to 1.5 (chg at 26th)

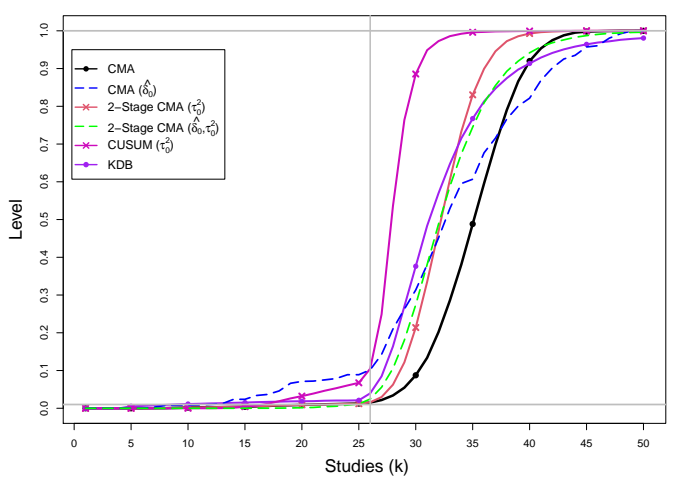

SSW KDB: $\tau^{2}=0.1 \mathrm{n}=100$ level $=0.01 \mathrm{~h}=5 \quad \delta=1$ to 1.5 (chg at 26th)

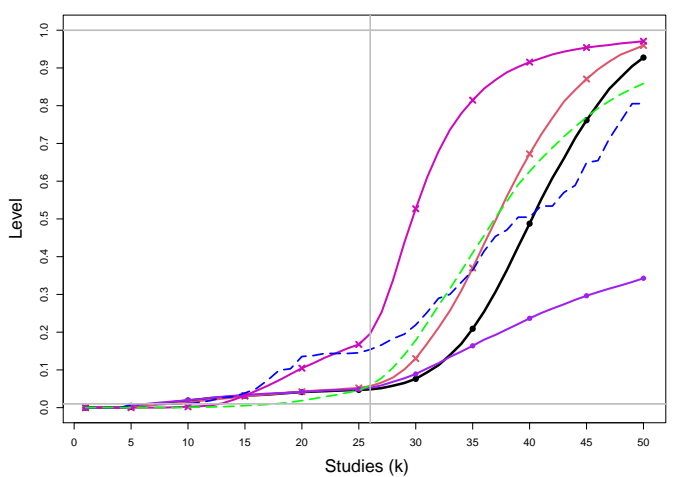

SSW KDB: $\tau^{2}=0.25 \mathrm{n}=100$ level $=0.01 \mathrm{~h}=5 \quad \delta=1$ to 1.5 (chg at 26th)

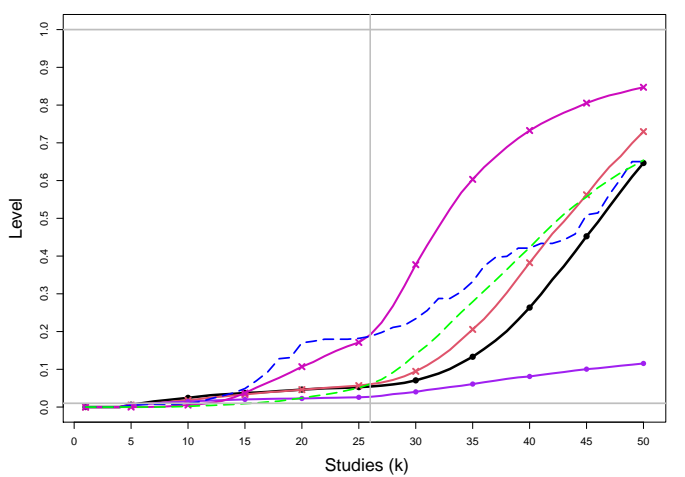

SSW KDB: $\tau^{2}=1 \mathrm{n}=100$ level $=0.01 \mathrm{~h}=5 \delta=1$ to 1.5 (chg at 26 th)

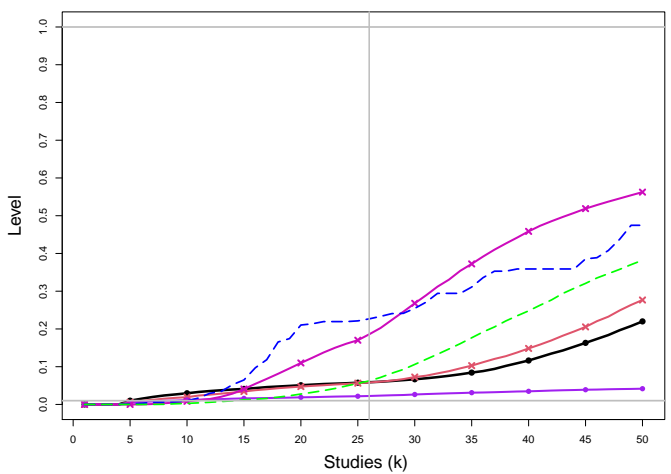

Figure E2. 73: Empirical levels of 1- and 2-stage CMA tests for shift in $\delta$ at .01 nominal level, shift in $\tau^{2}$ at .005 nominal levels and of CUSUM with $h=4$ for equal sample sizes $n_{i C}+n_{i T}=n=100, \tau^{2}=0,0.1,0.25,1$ and a shift from $\delta=1$ to $\delta=1.5$ at study number 26. Light grey line at .01. 
IV REML: $\tau^{2}=0 \quad \mathrm{n}=500$ level $=0.01 \mathrm{~h}=5 \quad \delta=1$ to 1.5 (chg at 26 th)

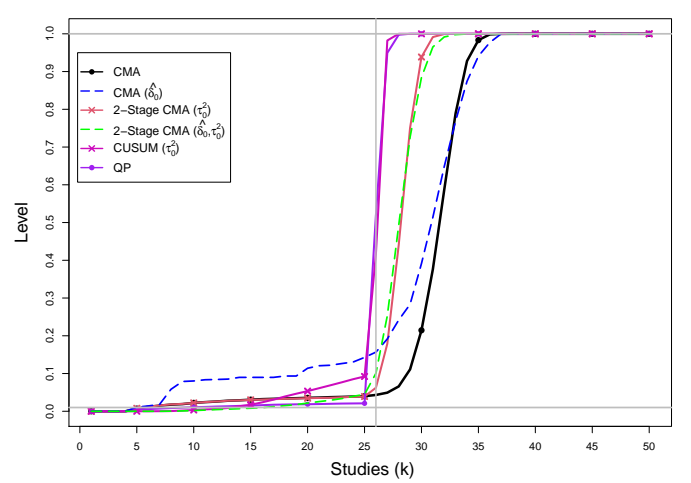

IV REML: $\tau^{2}=0.1 \mathrm{n}=500$ level $=0.01 \mathrm{~h}=5 \quad \delta=1$ to 1.5 (chg at 26 th)

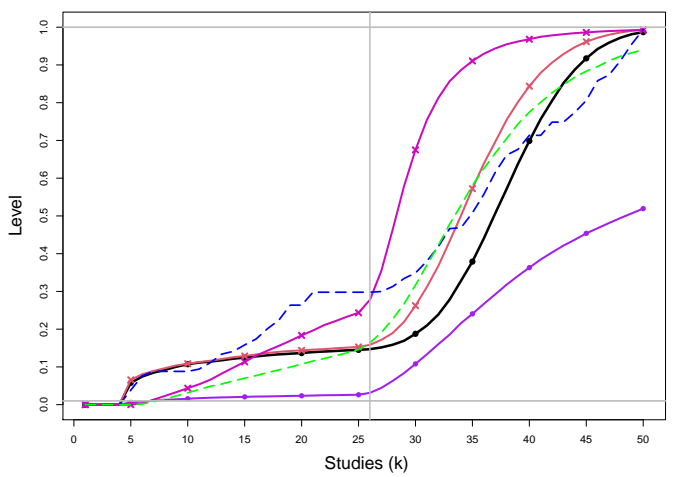

IV REML: $\tau^{2}=0.25 \mathrm{n}=500$ level $=0.01 \mathrm{~h}=5 \quad \delta=1$ to 1.5 (chg at 26 th)

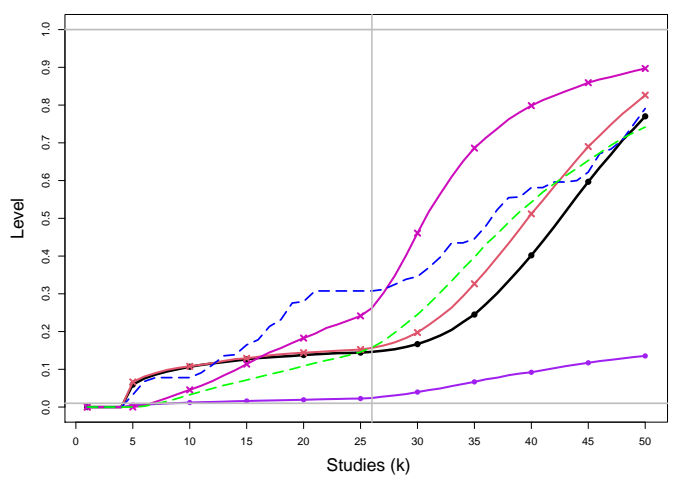

IV REML: $\tau^{2}=1 \mathrm{n}=500$ level $=0.01 \mathrm{~h}=5 \delta=1$ to 1.5 (chg at 26th)

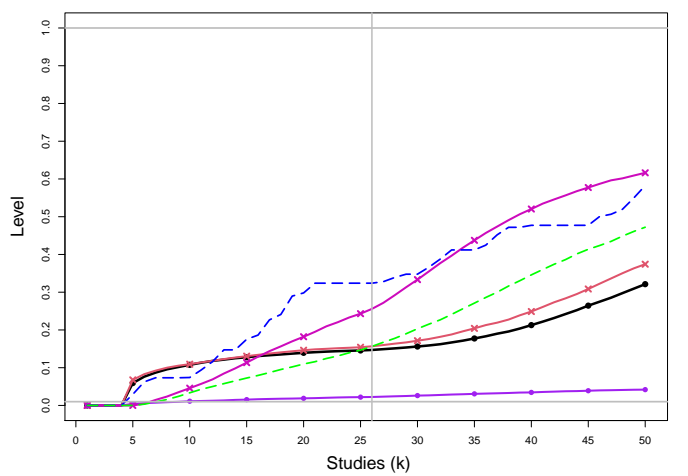

SSW KDB: $\tau^{2}=0 \mathrm{n}=500$ level $=0.01 \mathrm{~h}=5 \quad \delta=1$ to 1.5 (chg at 26th)

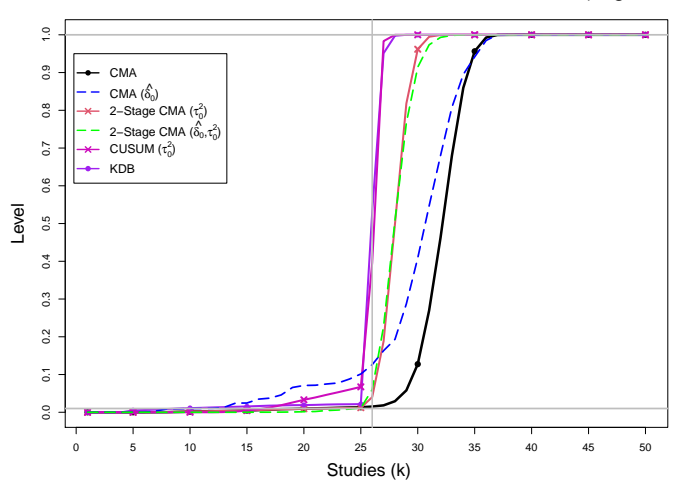

SSW KDB: $\tau^{2}=0.1 \mathrm{n}=500$ level $=0.01 \mathrm{~h}=5 \delta=1$ to 1.5 (chg at 26 th)

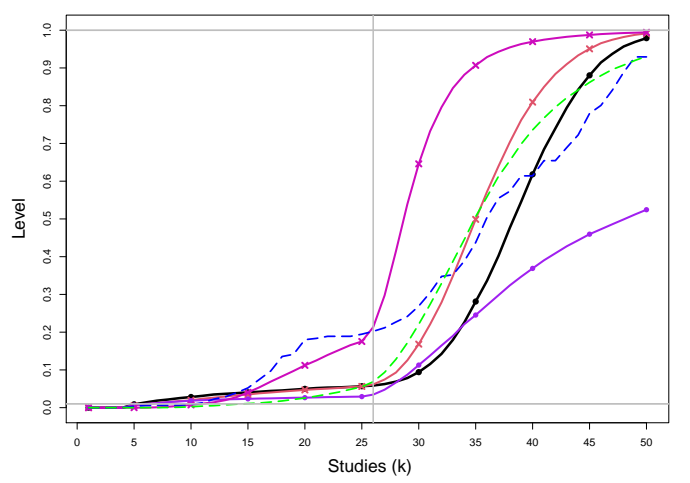

SSW KDB: $\tau^{2}=0.25 \mathrm{n}=500$ level $=0.01 \mathrm{~h}=5 \quad \delta=1$ to 1.5 (chg at 26th)

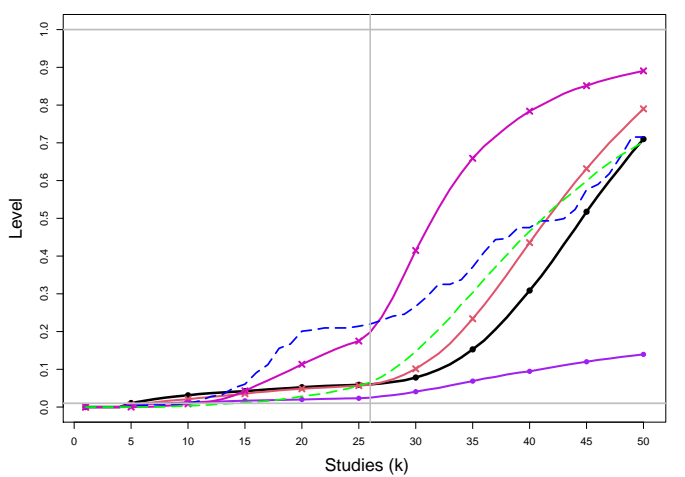

SSW KDB: $\tau^{2}=1 \mathrm{n}=500$ level $=0.01 \mathrm{~h}=5 \delta=1$ to 1.5 (chg at 26 th)

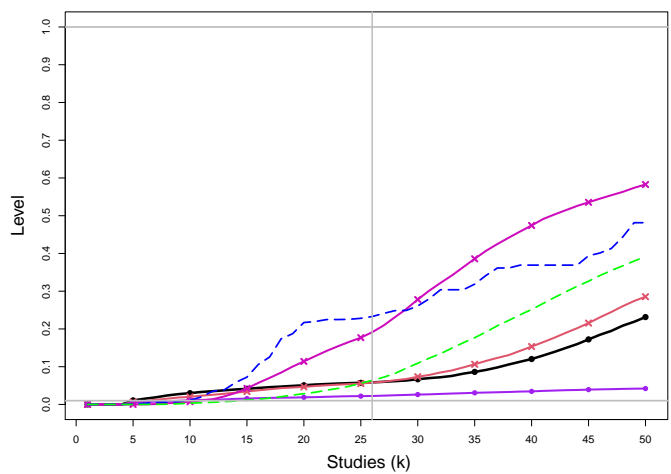

Figure E2. 74: Empirical levels of 1- and 2-stage CMA tests for shift in $\delta$ at .01 nominal level, shift in $\tau^{2}$ at .005 nominal levels and of CUSUM with $h=4$ for equal sample sizes $n_{i C}+n_{i T}=n=500, \tau^{2}=0,0.1,0.25,1$ and a shift from $\delta=1$ to $\delta=1.5$ at study number 26. Light grey line at .01. 
IV REML: $\tau^{2}=0 \mathrm{n}=1000$ level $=0.01 \mathrm{~h}=5 \quad \delta=1$ to 1.5 (chg at 26 th)

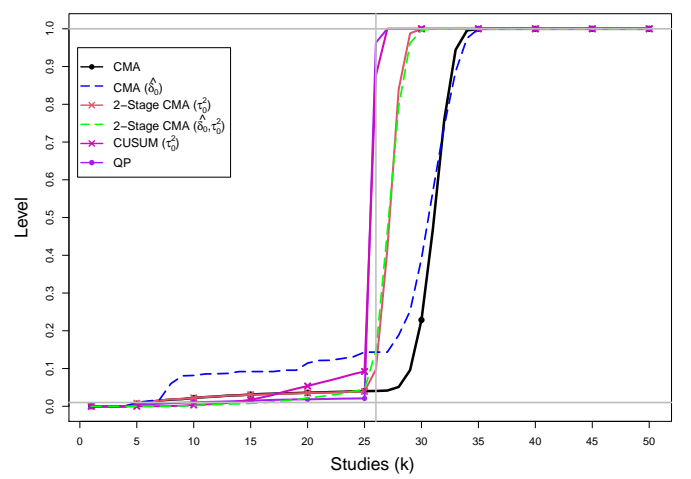

IV REML: $\tau^{2}=0.1 \mathrm{n}=1000$ level $=0.01 \mathrm{~h}=5 \delta=1$ to 1.5 (chg at 26th)

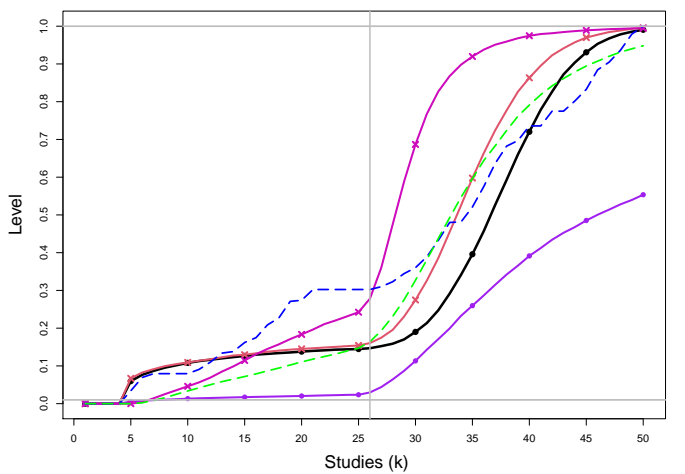

IV REML: $\tau^{2}=0.25 \mathrm{n}=1000$ level $=0.01 \mathrm{~h}=5 \delta=1$ to 1.5 (chg at 26th) SSW KDB: $\tau^{2}=0.25 \mathrm{n}=1000$ level $=0.01 \mathrm{~h}=5 \delta=1$ to 1.5 (chg at 26 th)

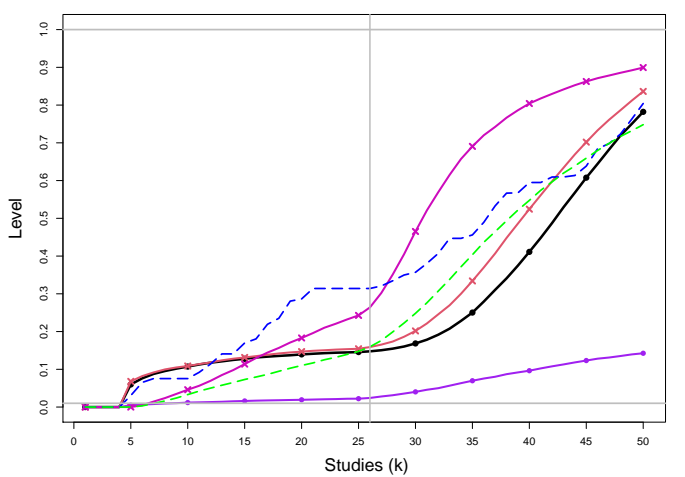

IV REML: $\tau^{2}=1 \mathrm{n}=1000$ level $=0.01 \mathrm{~h}=5 \delta=1$ to 1.5 (chg at 26 th)

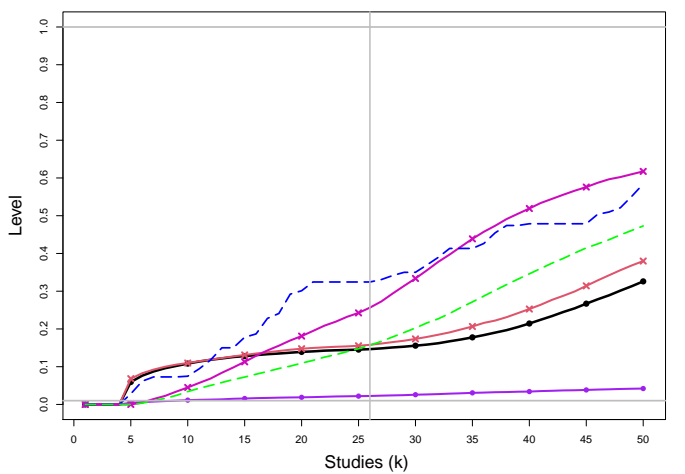

SSW KDB: $\tau^{2}=0 \mathrm{n}=1000$ level $=0.01 \mathrm{~h}=5 \quad \delta=1$ to 1.5 (chg at 26 th)

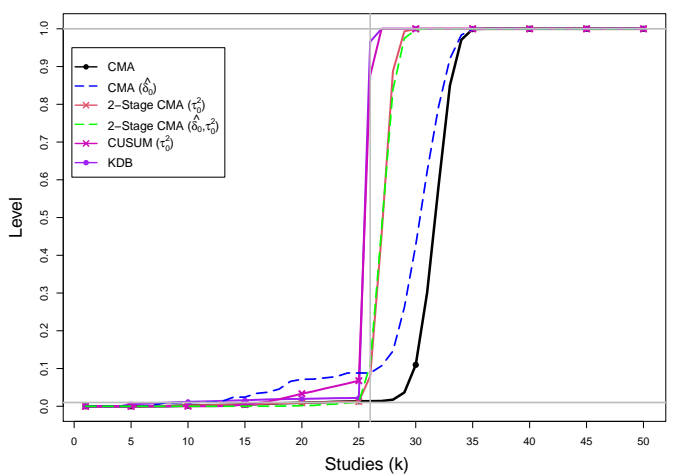

SSW KDB: $\tau^{2}=0.1 \mathrm{n}=1000$ level $=0.01 \mathrm{~h}=5 \delta=1$ to 1.5 (chg at 26th)

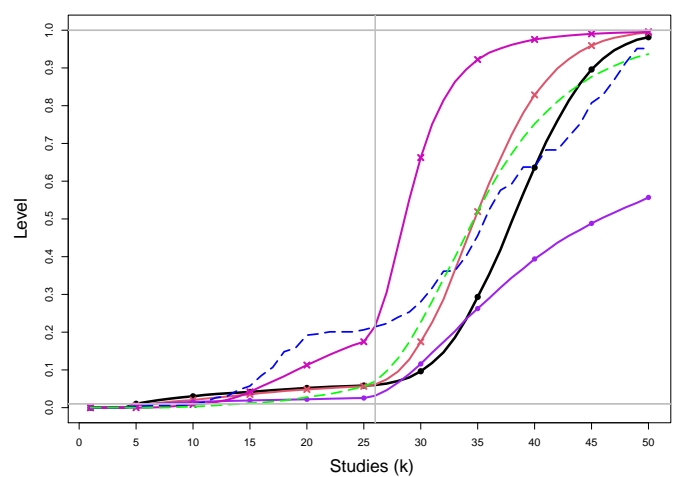

SSW KDB: $\tau^{2}=1 \mathrm{n}=1000$ level $=0.01 \mathrm{~h}=5 \delta=1$ to 1.5 (chg at 26th)

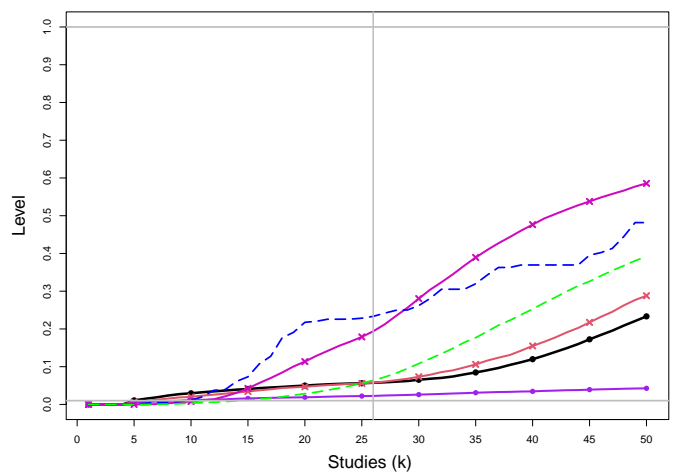

Figure E2. 75: Empirical levels of 1- and 2-stage CMA tests for shift in $\delta$ at .01 nominal level, shift in $\tau^{2}$ at .005 nominal levels and of CUSUM with $h=4$ for equal sample sizes $n_{i C}+n_{i T}=n=1000, \tau^{2}=0,0.1,0.25,1$ and a shift from $\delta=1$ to $\delta=1.5$ at study number 26. Light grey line at .01. 


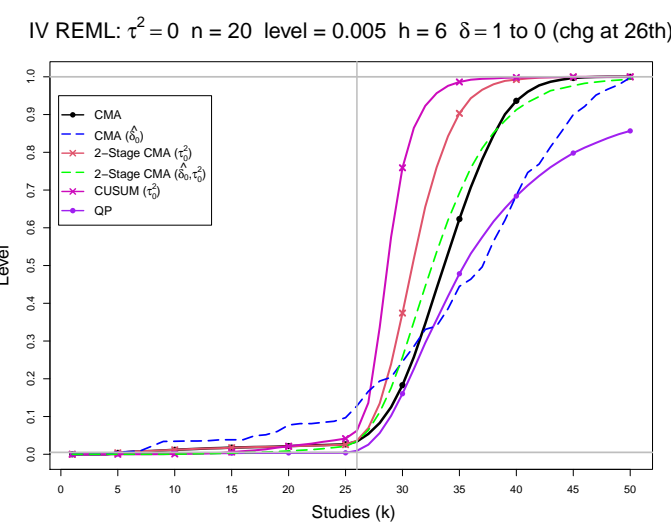

IV REML: $\tau^{2}=0.1 \mathrm{n}=20$ level $=0.005 \mathrm{~h}=6 \delta=1$ to 0 (chg at 26th)

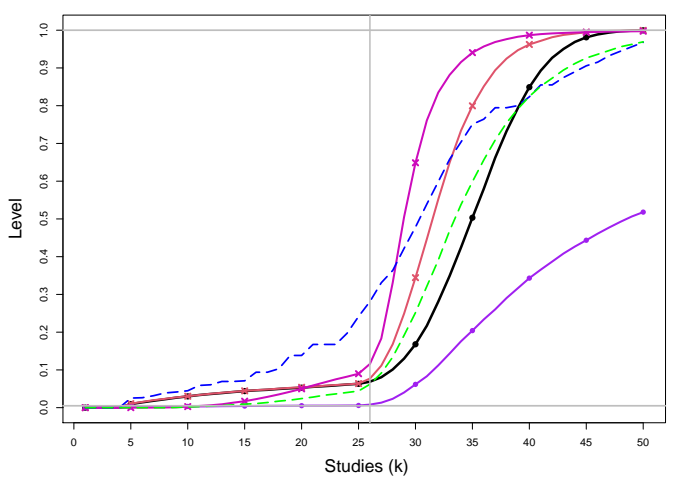

IV REML: $\tau^{2}=0.25 \mathrm{n}=20$ level $=0.005 \mathrm{~h}=6 \delta=1$ to 0 (chg at 26 th)

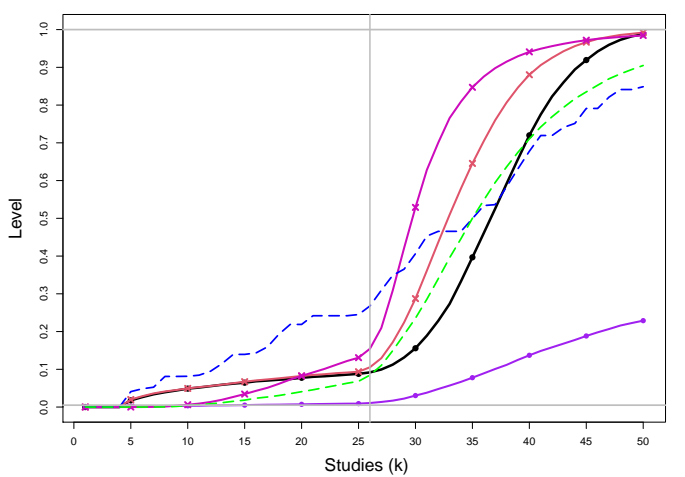

IV REML: $\tau^{2}=1 \mathrm{n}=20$ level $=0.005 \mathrm{~h}=6 \delta=1$ to 0 (chg at 26 th)

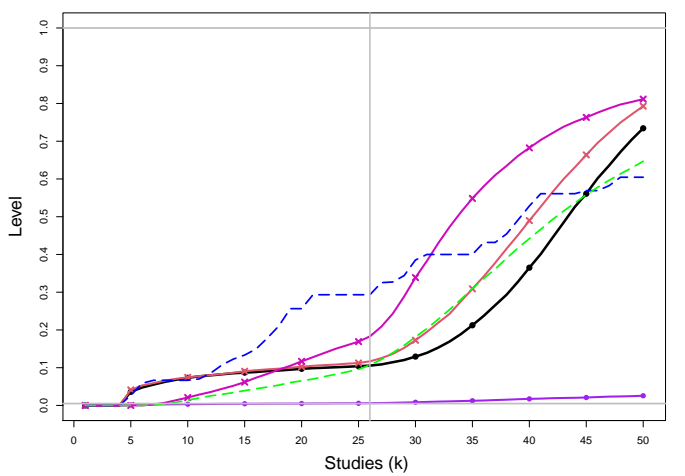

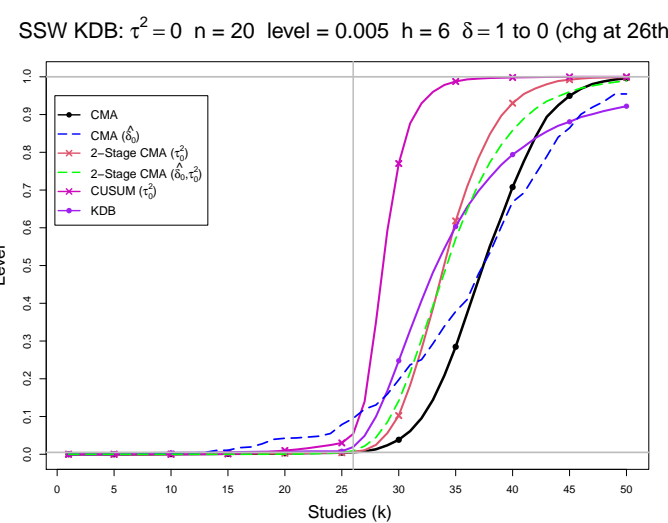

SSW KDB: $\tau^{2}=0.1 \mathrm{n}=20$ level $=0.005 \mathrm{~h}=6 \delta=1$ to 0 (chg at 26th)

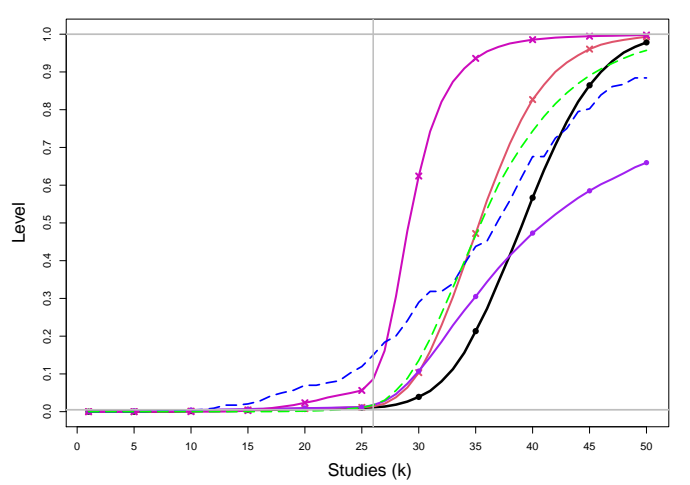

SSW KDB: $\tau^{2}=0.25 \mathrm{n}=20$ level $=0.005 \mathrm{~h}=6 \delta=1$ to 0 (chg at 26th)

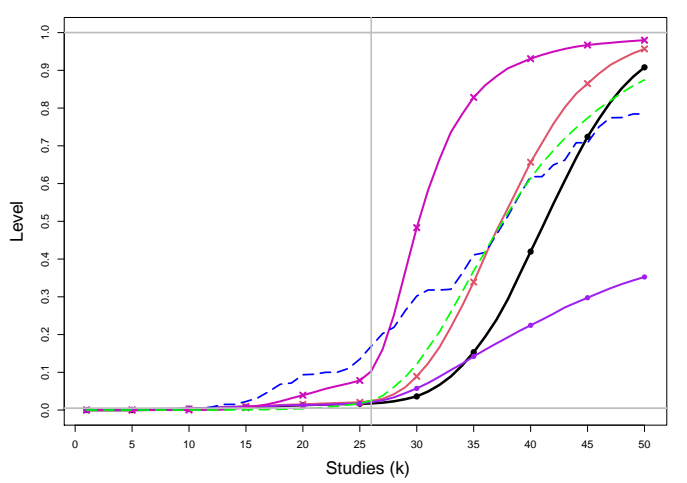

SSW KDB: $\tau^{2}=1 \mathrm{n}=20$ level $=0.005 \mathrm{~h}=6 \delta=1$ to 0 (chg at 26th)

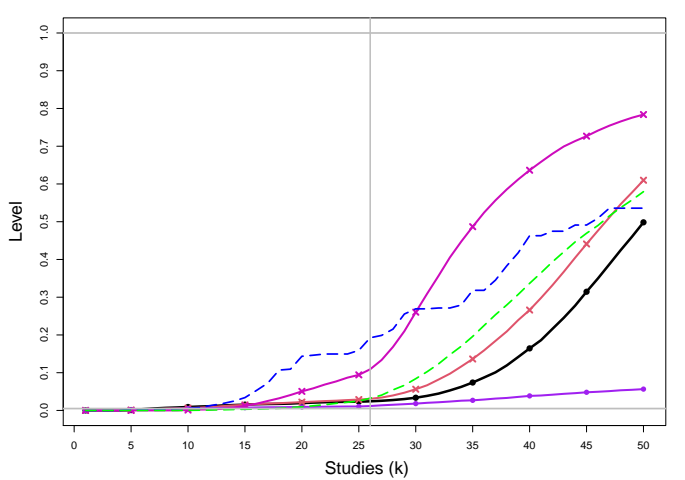

Figure E2. 76: Empirical levels of 1- and 2-stage CMA tests for shift in $\delta$ at .005 nominal level, shift in $\tau^{2}$ at .0025 nominal levels and of CUSUM with $h=4$ for equal sample sizes $n_{i C}+n_{i T}=n=20, \tau^{2}=0,0.1,0.25,1$ and a shift from $\delta=1$ to $\delta=0$ at study number 26. Light grey line at .005. 


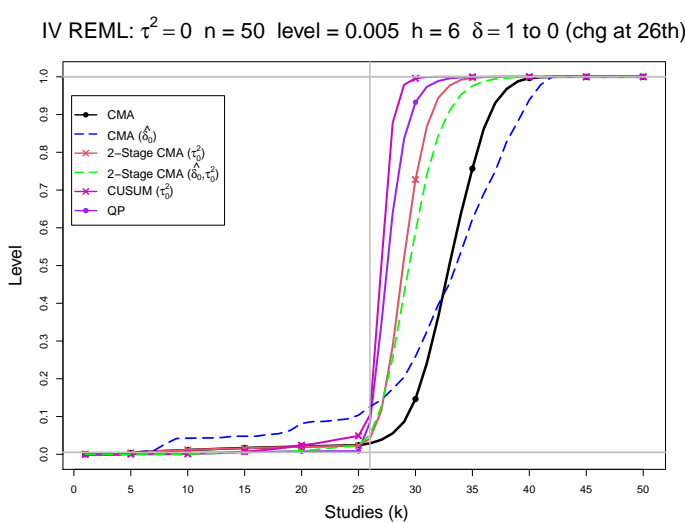

IV REML: $\tau^{2}=0.1 \mathrm{n}=50$ level $=0.005 \mathrm{~h}=6 \delta=1$ to 0 (chg at 26th)

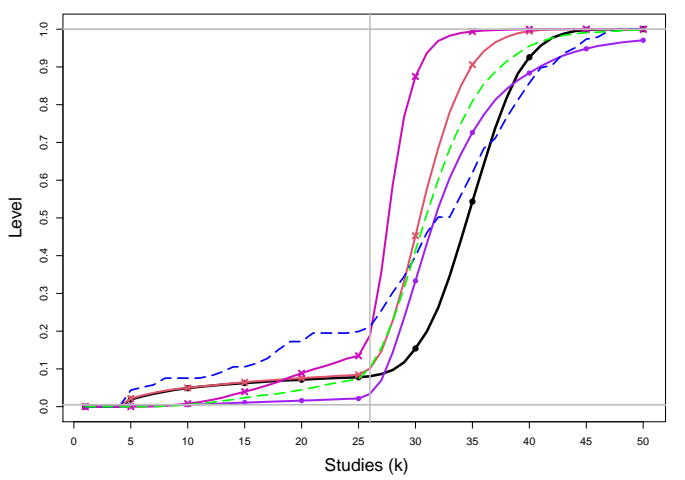

IV REML: $\tau^{2}=0.25 \mathrm{n}=50$ level $=0.005 \mathrm{~h}=6 \delta=1$ to 0 (chg at 26 th)

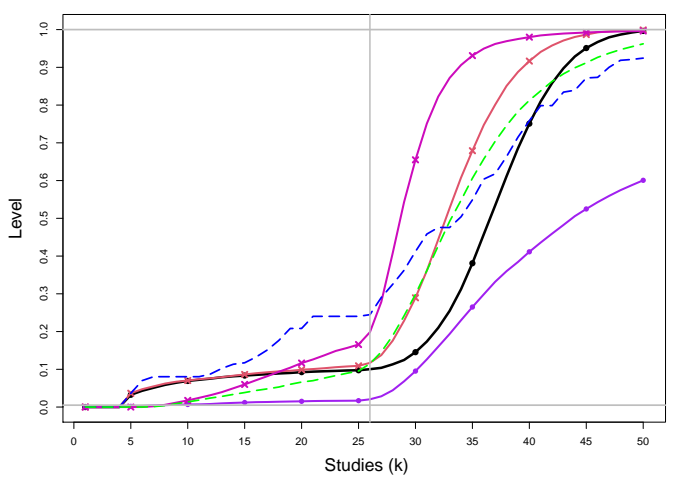

IV REML: $\tau^{2}=1 \mathrm{n}=50$ level $=0.005 \mathrm{~h}=6 \delta=1$ to 0 (chg at 26 th)

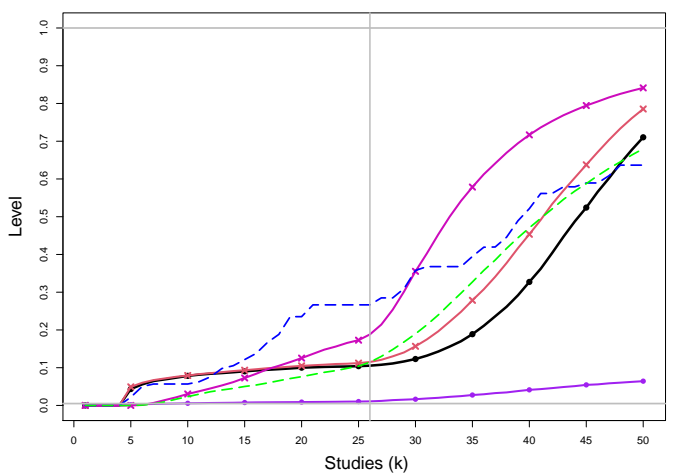

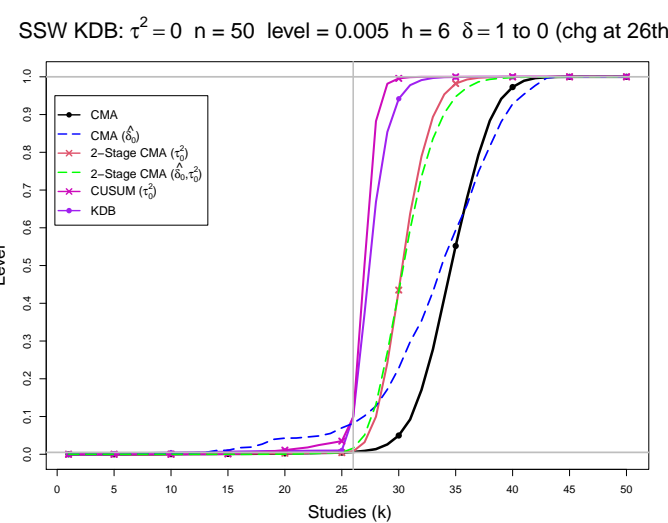

SSW KDB: $\tau^{2}=0.1 \mathrm{n}=50$ level $=0.005 \mathrm{~h}=6 \delta=1$ to 0 (chg at 26th)

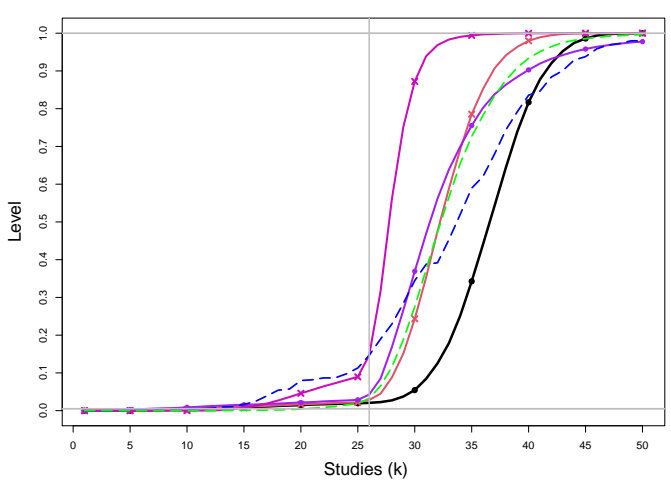

SSW KDB: $\tau^{2}=0.25 \mathrm{n}=50$ level $=0.005 \mathrm{~h}=6 \delta=1$ to 0 (chg at 26th)

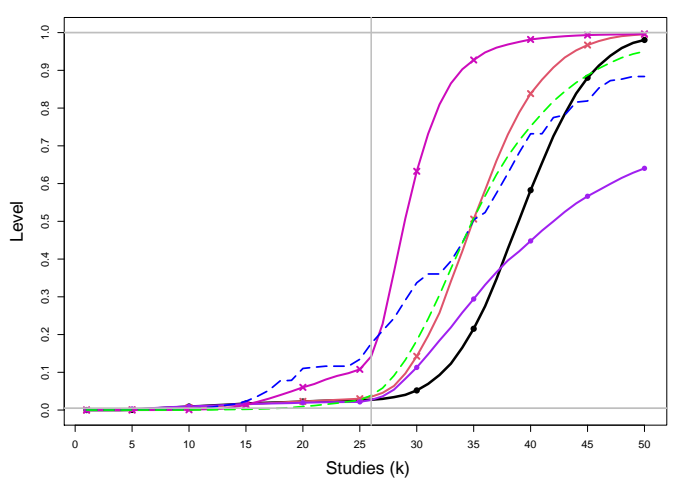

SSW KDB: $\tau^{2}=1 \mathrm{n}=50$ level $=0.005 \mathrm{~h}=6 \delta=1$ to 0 (chg at 26th)

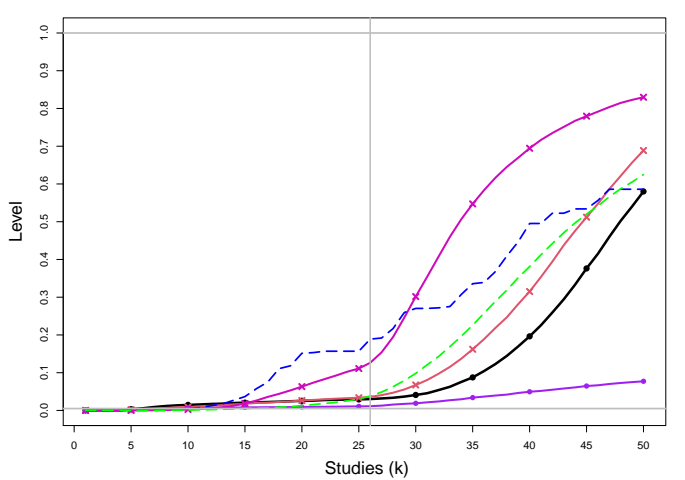

Figure E2. 77: Empirical levels of 1- and 2-stage CMA tests for shift in $\delta$ at .005 nominal level, shift in $\tau^{2}$ at .0025 nominal levels and of CUSUM with $h=4$ for equal sample sizes $n_{i C}+n_{i T}=n=50, \tau^{2}=0,0.1,0.25,1$ and a shift from $\delta=1$ to $\delta=0$ at study number 26. Light grey line at .005. 


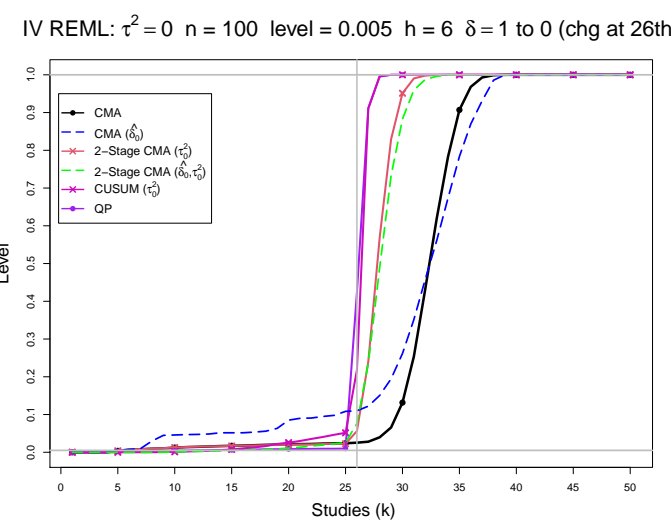

IV REML: $\tau^{2}=0.1 \mathrm{n}=100$ level $=0.005 \mathrm{~h}=6 \delta=1$ to 0 (chg at 26th)

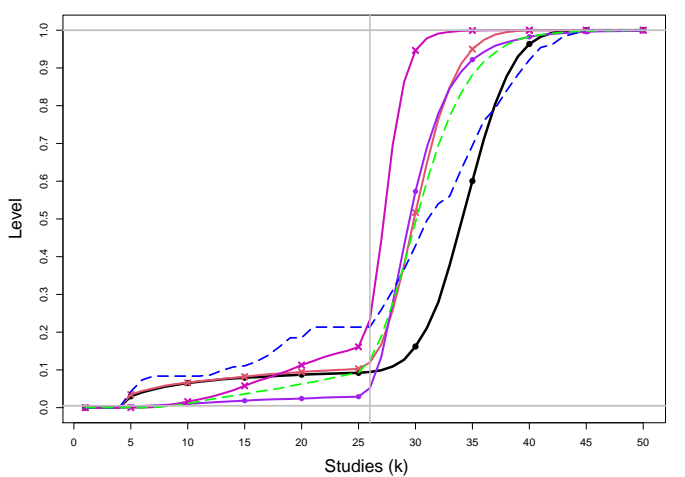

IV REML: $\tau^{2}=0.25 \mathrm{n}=100$ level $=0.005 \mathrm{~h}=6 \delta=1$ to 0 (chg at 26th)

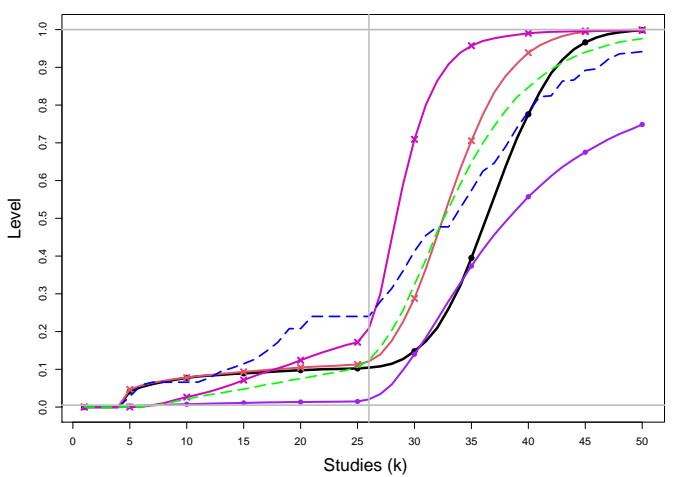

IV REML: $\tau^{2}=1 \mathrm{n}=100$ level $=0.005 \mathrm{~h}=6 \delta=1$ to 0 (chg at 26th)

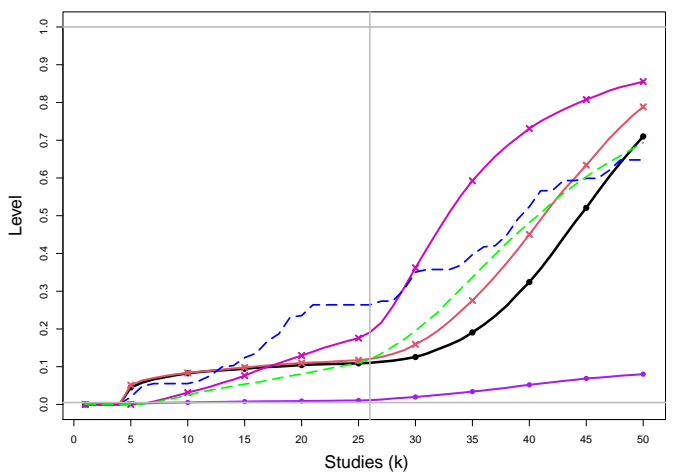

SSW KDB: $\tau^{2}=0 \mathrm{n}=100$ level $=0.005 \mathrm{~h}=6 \quad \delta=1$ to 0 (chg at 26th)

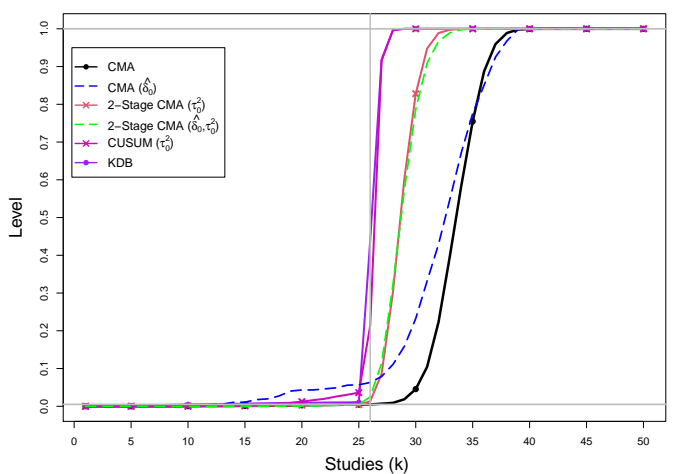

SSW KDB: $\tau^{2}=0.1 \mathrm{n}=100$ level $=0.005 \mathrm{~h}=6 \delta=1$ to 0 (chg at 26th)

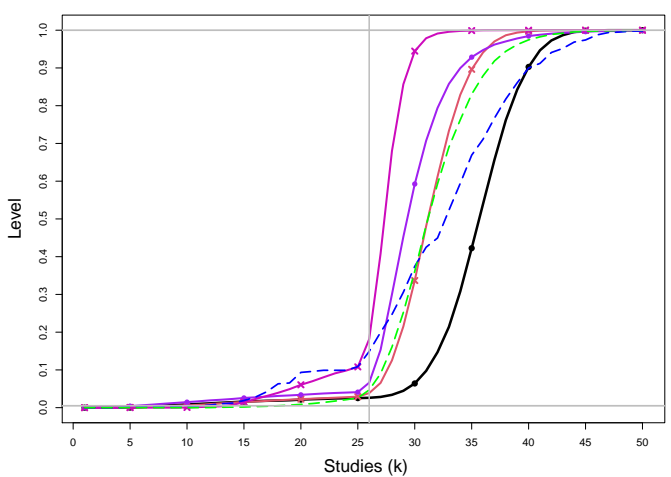

SSW KDB: $\tau^{2}=0.25 \mathrm{n}=100$ level $=0.005 \mathrm{~h}=6 \delta=1$ to 0 (chg at 26th)

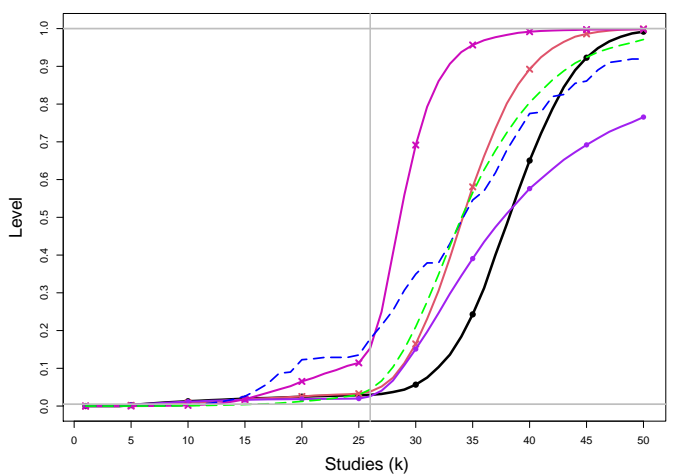

SSW KDB: $\tau^{2}=1 \mathrm{n}=100$ level $=0.005 \mathrm{~h}=6 \delta=1$ to 0 (chg at 26th)

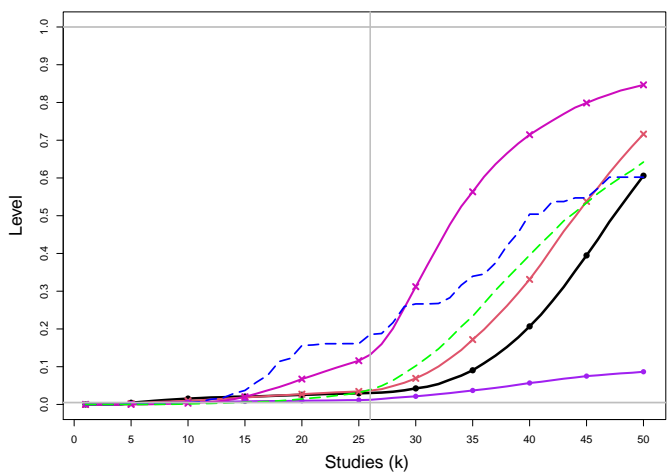

Figure E2. 78: Empirical levels of 1- and 2-stage CMA tests for shift in $\delta$ at .005 nominal level, shift in $\tau^{2}$ at .0025 nominal levels and of CUSUM with $h=4$ for equal sample sizes $n_{i C}+n_{i T}=n=100, \tau^{2}=0,0.1,0.25,1$ and a shift from $\delta=1$ to $\delta=0$ at study number 26. Light grey line at .005. 


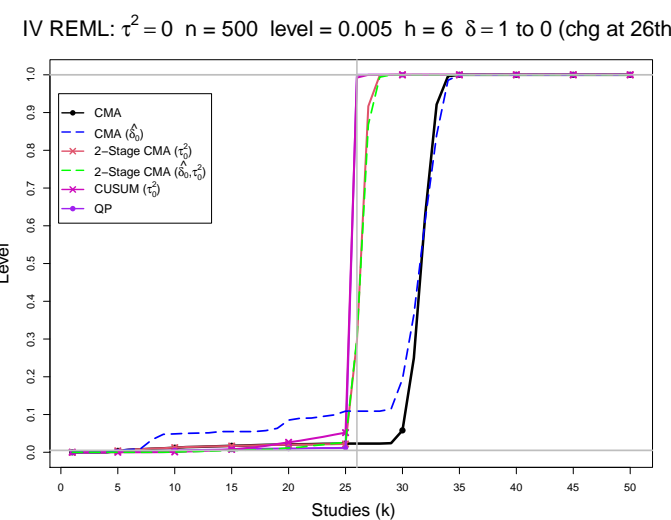

IV REML: $\tau^{2}=0.1 \mathrm{n}=500$ level $=0.005 \mathrm{~h}=6 \delta=1$ to 0 (chg at 26th)

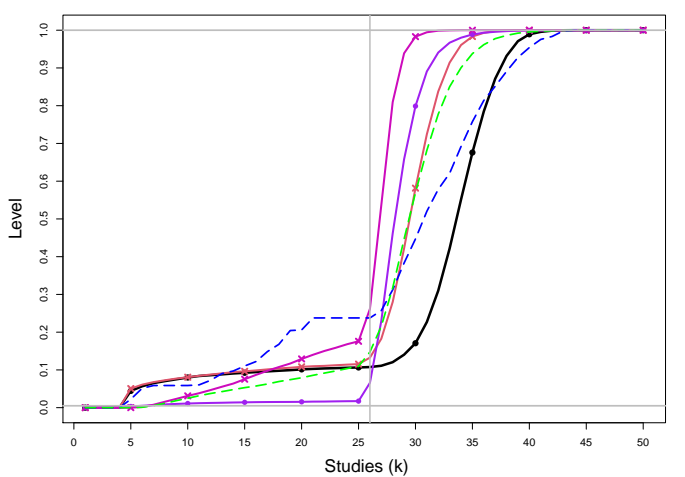

IV REML: $\tau^{2}=0.25 \mathrm{n}=500$ level $=0.005 \mathrm{~h}=6 \delta=1$ to 0 (chg at 26th)

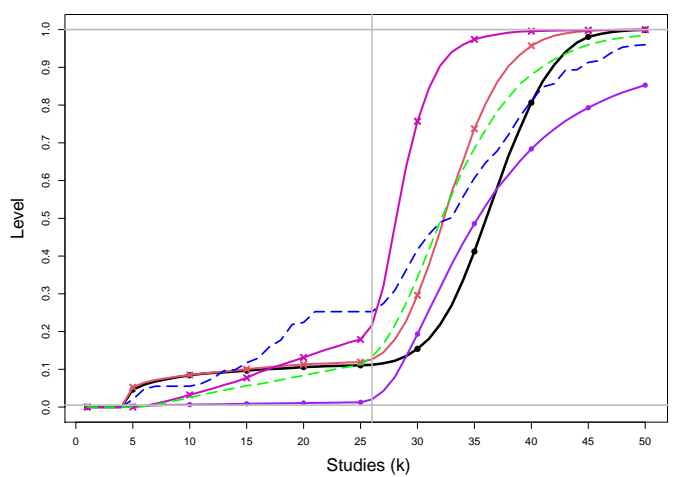

IV REML: $\tau^{2}=1 \mathrm{n}=500$ level $=0.005 \mathrm{~h}=6 \delta=1$ to 0 (chg at 26th)

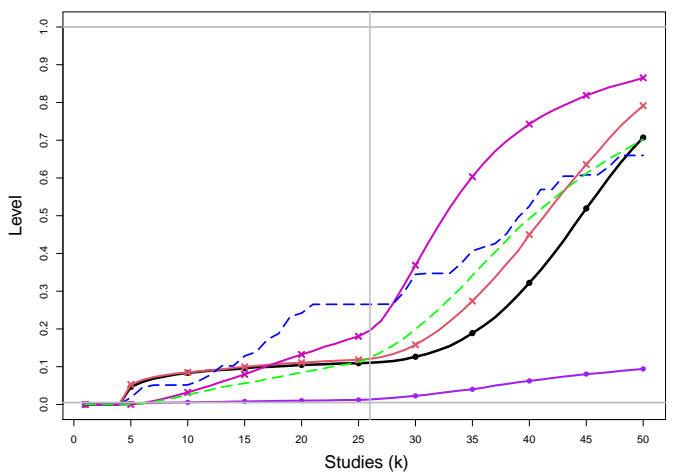

SSW KDB: $\tau^{2}=0 \mathrm{n}=500$ level $=0.005 \mathrm{~h}=6 \quad \delta=1$ to 0 (chg at 26th)

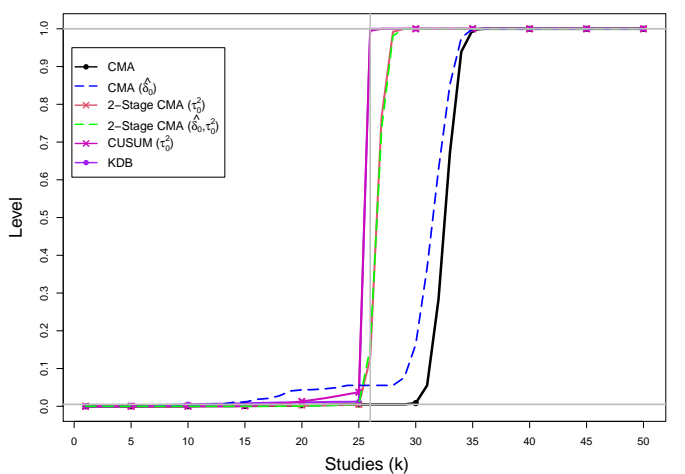

SSW KDB: $\tau^{2}=0.1 \mathrm{n}=500$ level $=0.005 \mathrm{~h}=6 \delta=1$ to 0 (chg at 26th)

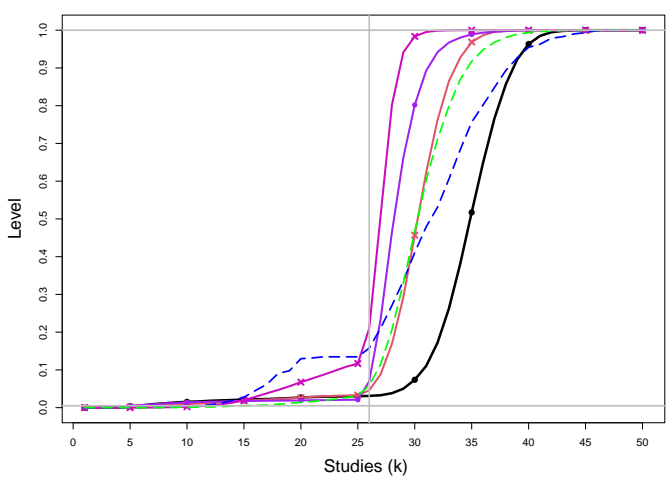

SSW KDB: $\tau^{2}=0.25 \mathrm{n}=500$ level $=0.005 \mathrm{~h}=6 \delta=1$ to 0 (chg at 26th)

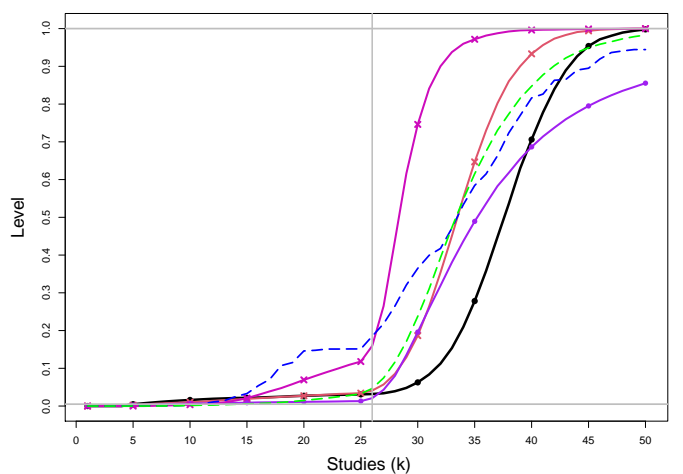

SSW KDB: $\tau^{2}=1 \mathrm{n}=500$ level $=0.005 \mathrm{~h}=6 \delta=1$ to 0 (chg at 26th)

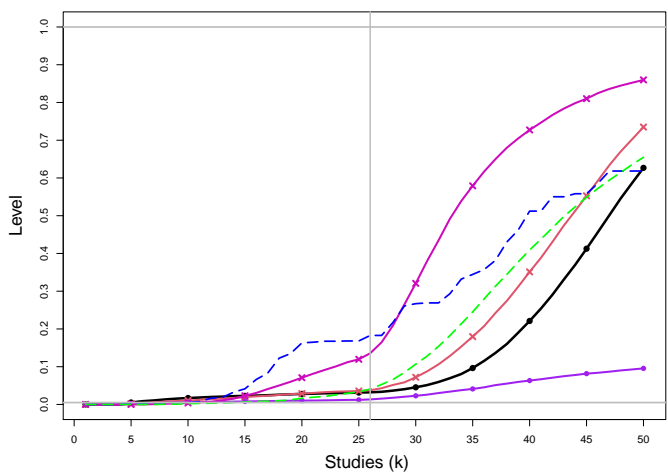

Figure E2. 79: Empirical levels of 1- and 2-stage CMA tests for shift in $\delta$ at .005 nominal level, shift in $\tau^{2}$ at .0025 nominal levels and of CUSUM with $h=4$ for equal sample sizes $n_{i C}+n_{i T}=n=500, \tau^{2}=0,0.1,0.25,1$ and a shift from $\delta=1$ to $\delta=0$ at study number 26. Light grey line at .005. 
IV REML: $\tau^{2}=0 \mathrm{n}=1000$ level $=0.005 \mathrm{~h}=6 \delta=1$ to 0 (chg at 26th)

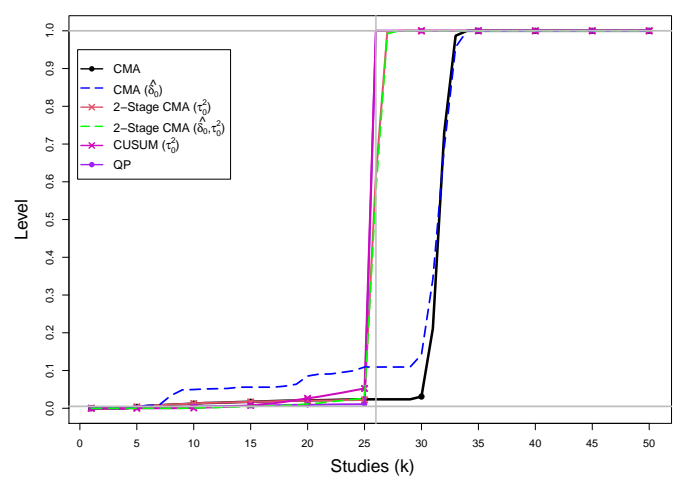

IV REML: $\tau^{2}=0.1 \mathrm{n}=1000$ level $=0.005 \mathrm{~h}=6 \delta=1$ to 0 (chg at 26th)

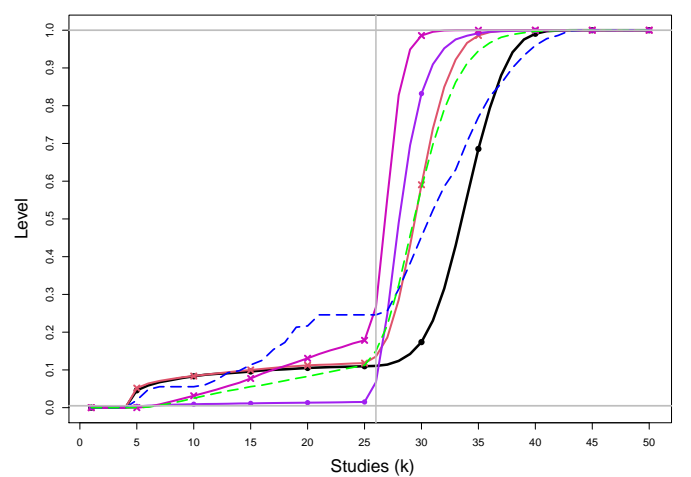

IV REML: $\tau^{2}=0.25 \mathrm{n}=1000$ level $=0.005 \mathrm{~h}=6 \delta=1$ to 0 (chg at 26th)

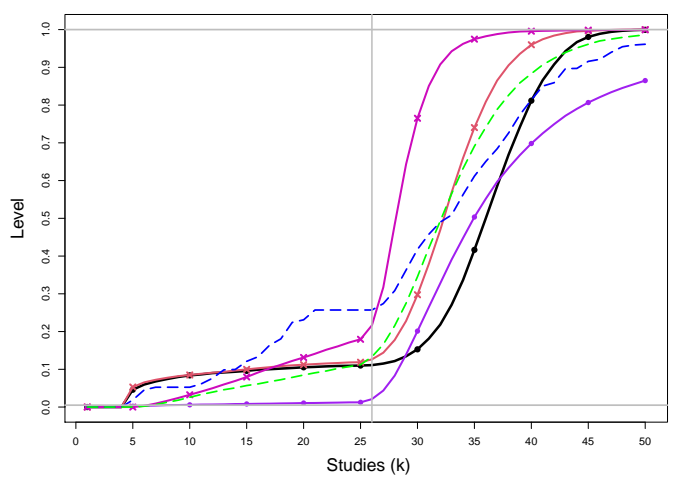

IV REML: $\tau^{2}=1 \mathrm{n}=1000$ level $=0.005 \mathrm{~h}=6 \delta=1$ to 0 (chg at 26 th)

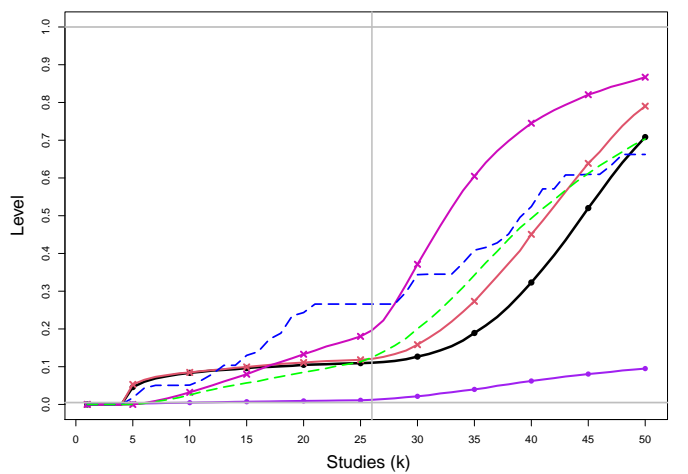

SSW KDB: $\tau^{2}=0 \mathrm{n}=1000$ level $=0.005 \mathrm{~h}=6 \delta=1$ to 0 (chg at 26th)

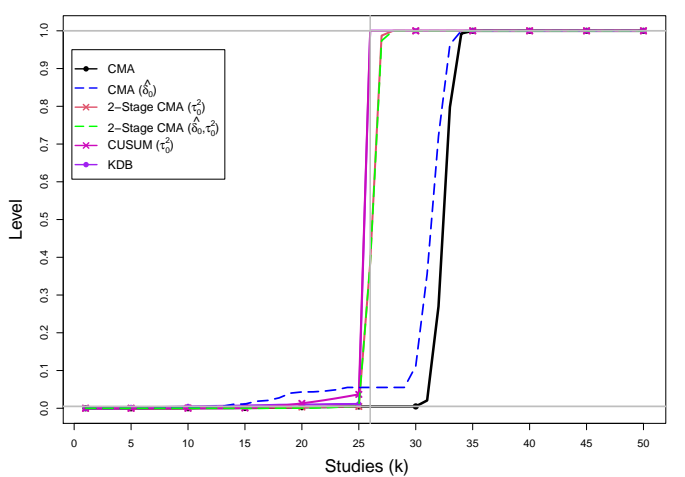

SSW KDB: $\tau^{2}=0.1 \mathrm{n}=1000$ level $=0.005 \mathrm{~h}=6 \quad \delta=1$ to 0 (chg at 26th)

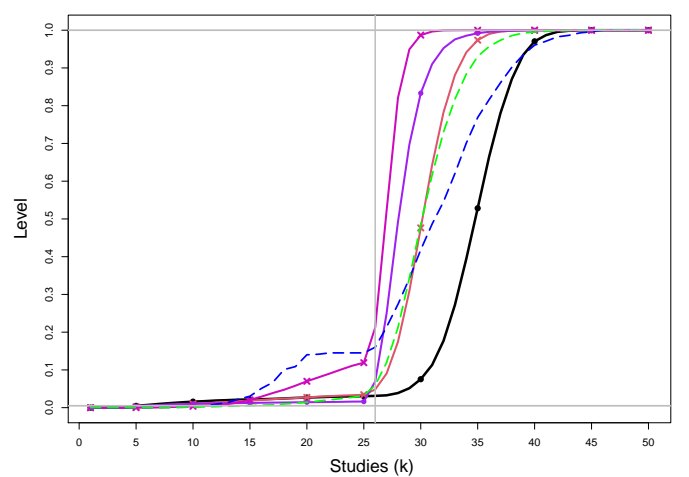

SSW KDB: $\tau^{2}=0.25 \mathrm{n}=1000$ level $=0.005 \mathrm{~h}=6 \delta=1$ to 0 (chg at 26th)

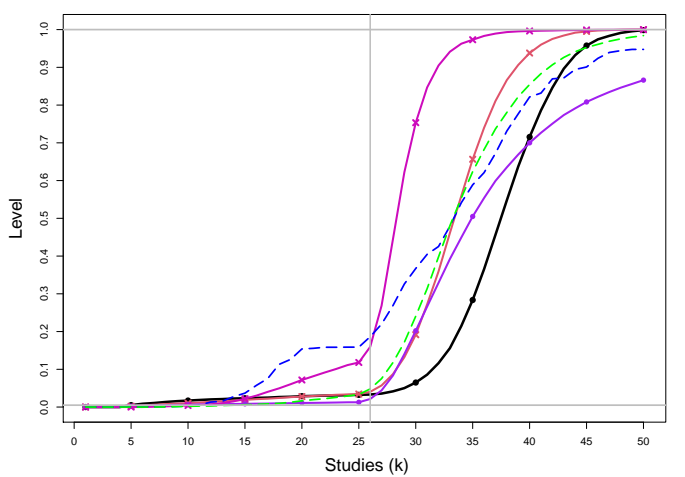

SSW KDB: $\tau^{2}=1 \mathrm{n}=1000$ level $=0.005 \mathrm{~h}=6 \delta=1$ to 0 (chg at 26th)

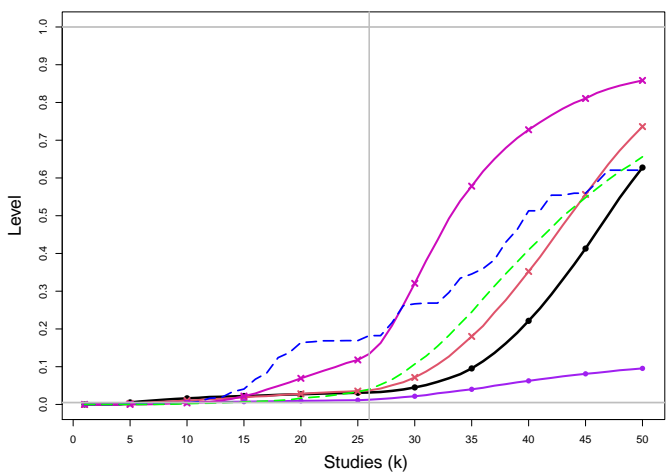

Figure E2. 80: Empirical levels of 1- and 2-stage CMA tests for shift in $\delta$ at .005 nominal level, shift in $\tau^{2}$ at .0025 nominal levels and of CUSUM with $h=4$ for equal sample sizes $n_{i C}+n_{i T}=n=1000, \tau^{2}=0,0.1,0.25,1$ and a shift from $\delta=1$ to $\delta=0$ at study number 26. Light grey line at .005. 
IV REML: $\tau^{2}=0 \mathrm{n}=20$ level $=0.005 \mathrm{~h}=6 \quad \delta=1$ to 0.5 (chg at 26th)

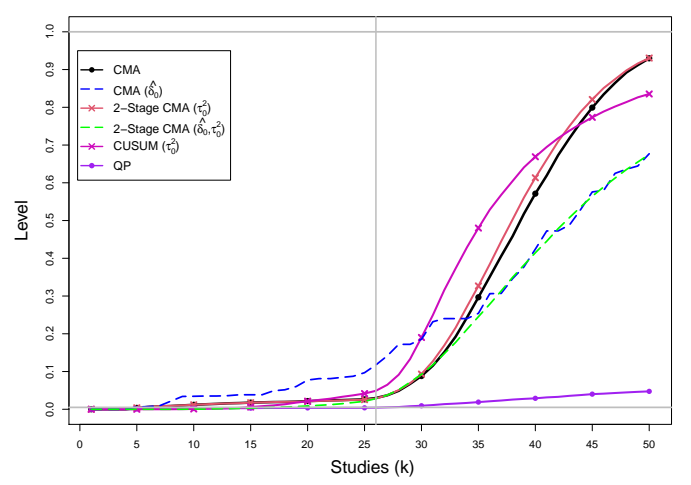

IV REML: $\tau^{2}=0.1 \mathrm{n}=20$ level $=0.005 \mathrm{~h}=6 \delta=1$ to 0.5 (chg at 26th)

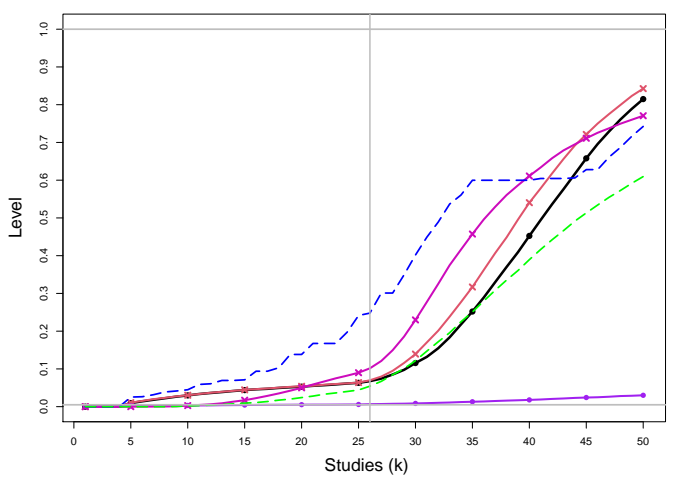

IV REML: $\tau^{2}=0.25 \mathrm{n}=20$ level $=0.005 \mathrm{~h}=6 \delta=1$ to 0.5 (chg at 26th)

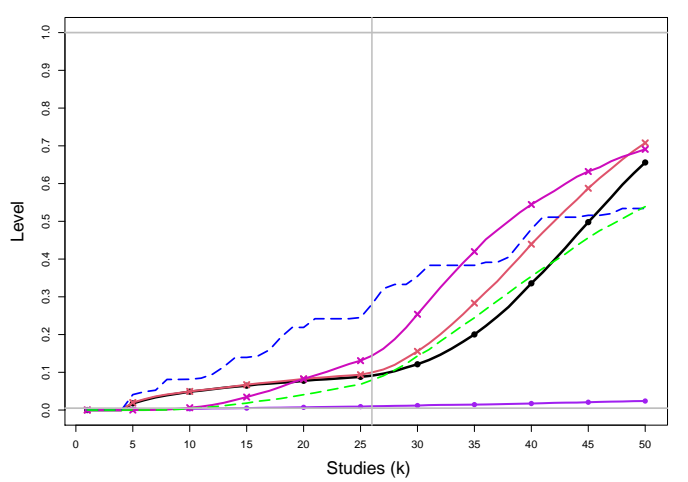

IV REML: $\tau^{2}=1 \mathrm{n}=20$ level $=0.005 \mathrm{~h}=6 \delta=1$ to 0.5 (chg at 26th)

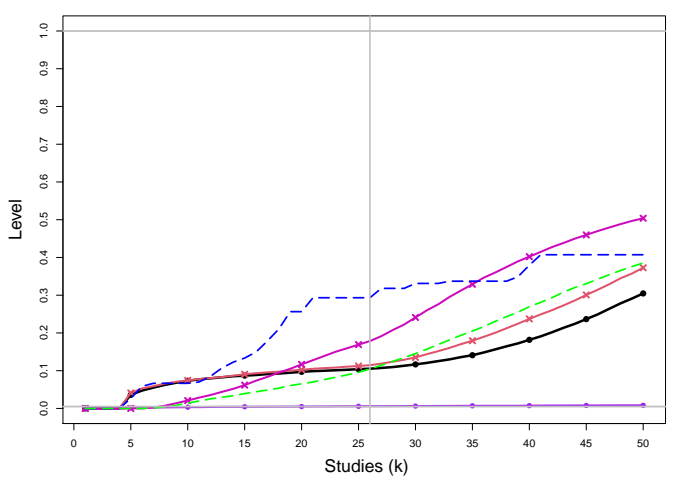

SSW KDB: $\tau^{2}=0 \mathrm{n}=20$ level $=0.005 \mathrm{~h}=6 \delta=1$ to 0.5 (chg at 26th)

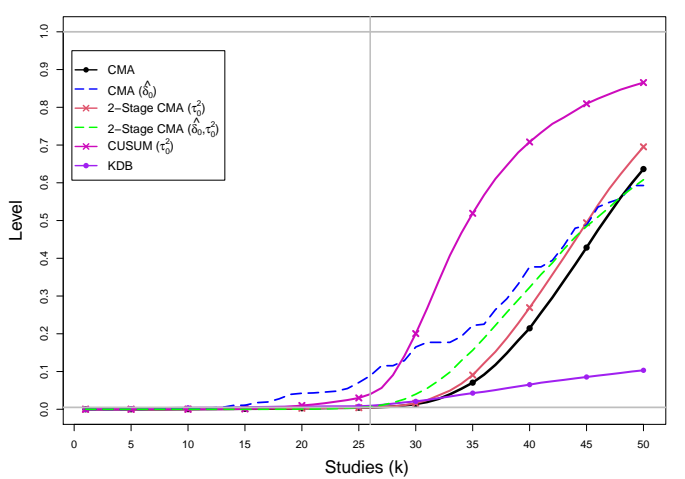

SSW KDB: $\tau^{2}=0.1 \mathrm{n}=20$ level $=0.005 \mathrm{~h}=6 \delta=1$ to 0.5 (chg at 26th)

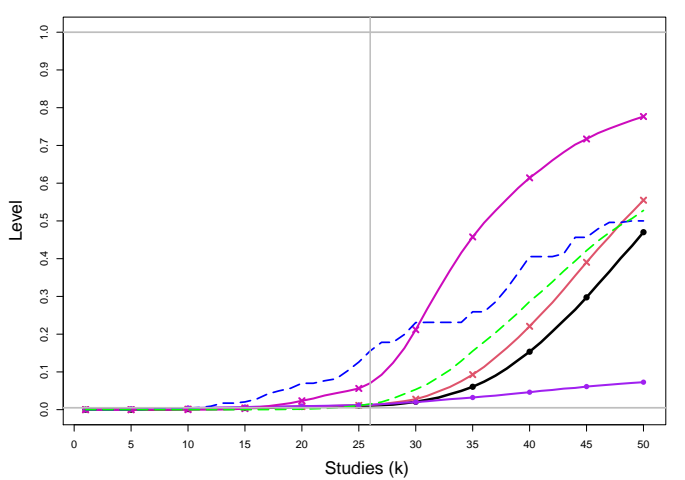

SSW KDB: $\tau^{2}=0.25 \mathrm{n}=20$ level $=0.005 \mathrm{~h}=6 \delta=1$ to 0.5 (chg at 26th)

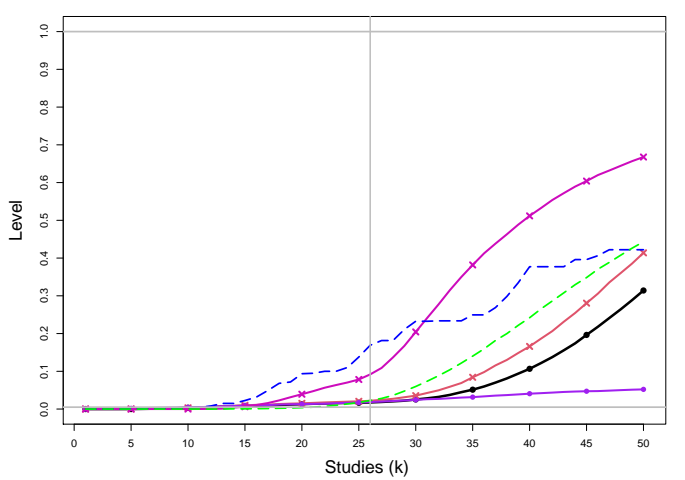

SSW KDB: $\tau^{2}=1 \mathrm{n}=20$ level $=0.005 \mathrm{~h}=6 \delta=1$ to 0.5 (chg at 26th)

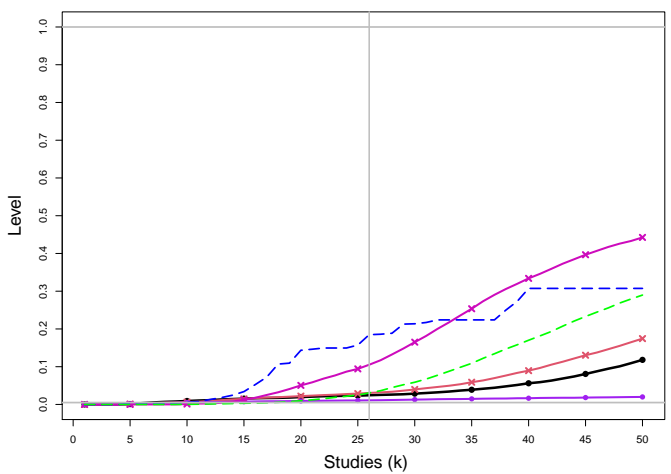

Figure E2. 81: Empirical levels of 1- and 2-stage CMA tests for shift in $\delta$ at .005 nominal level, shift in $\tau^{2}$ at .0025 nominal levels and of CUSUM with $h=4$ for equal sample sizes $n_{i C}+n_{i T}=n=20, \tau^{2}=0,0.1,0.25,1$ and a shift from $\delta=1$ to $\delta=0.5$ at study number 26. Light grey line at .005. 
IV REML: $\tau^{2}=0 \mathrm{n}=50$ level $=0.005 \mathrm{~h}=6 \quad \delta=1$ to 0.5 (chg at 26th)

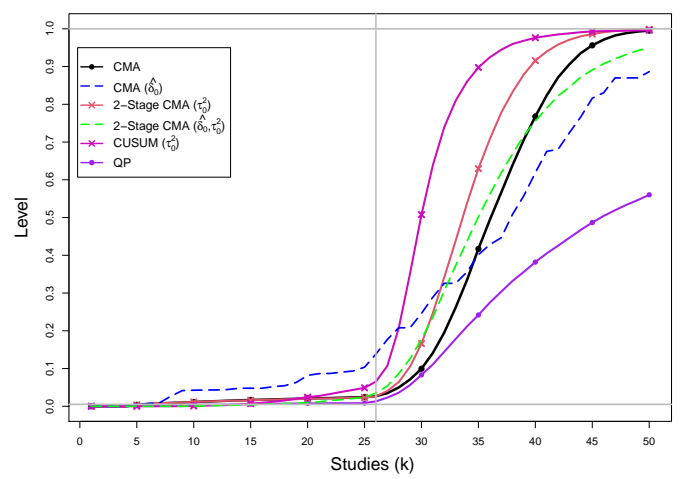

IV REML: $\tau^{2}=0.1 \mathrm{n}=50$ level $=0.005 \mathrm{~h}=6 \delta=1$ to 0.5 (chg at 26 th)

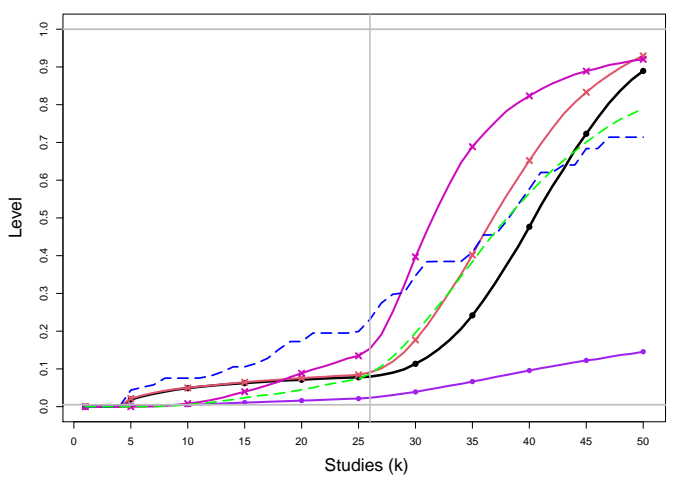

IV REML: $\tau^{2}=0.25 \mathrm{n}=50$ level $=0.005 \mathrm{~h}=6 \delta=1$ to 0.5 (chg at 26th)

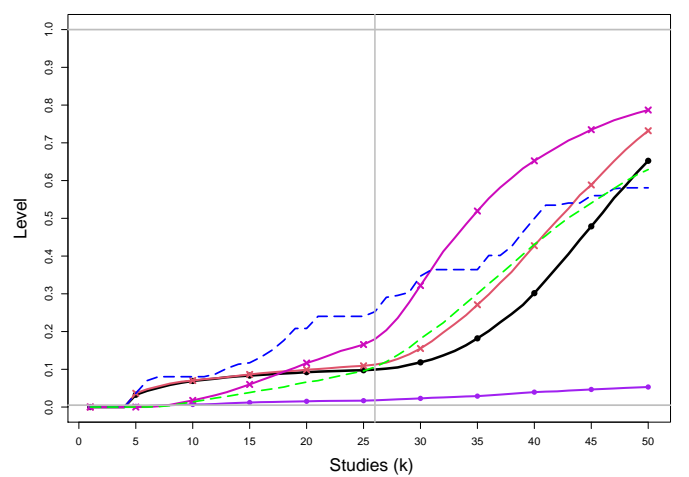

IV REML: $\tau^{2}=1 \mathrm{n}=50$ level $=0.005 \mathrm{~h}=6 \delta=1$ to 0.5 (chg at 26th)

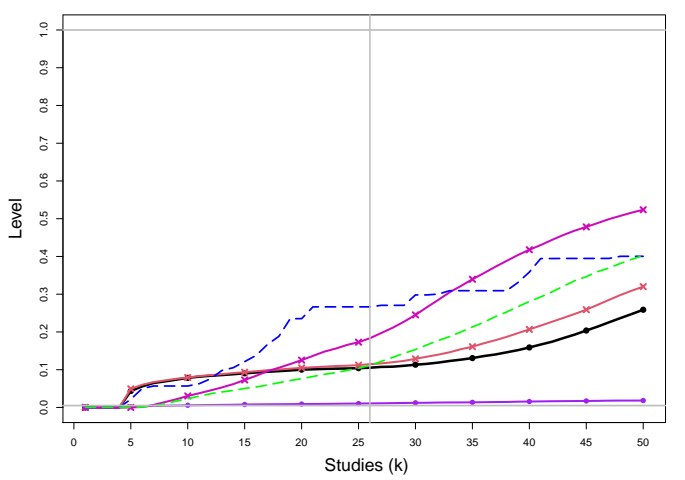

SSW KDB: $\tau^{2}=0 \mathrm{n}=50$ level $=0.005 \mathrm{~h}=6 \delta=1$ to 0.5 (chg at 26th)

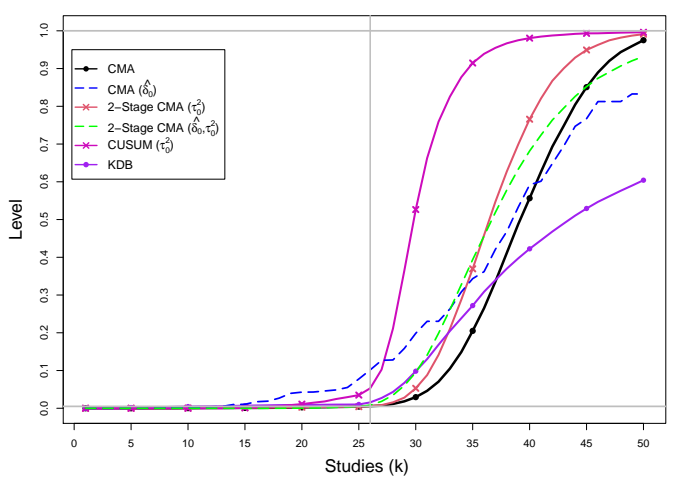

SSW KDB: $\tau^{2}=0.1 \mathrm{n}=50$ level $=0.005 \mathrm{~h}=6 \delta=1$ to 0.5 (chg at 26th)

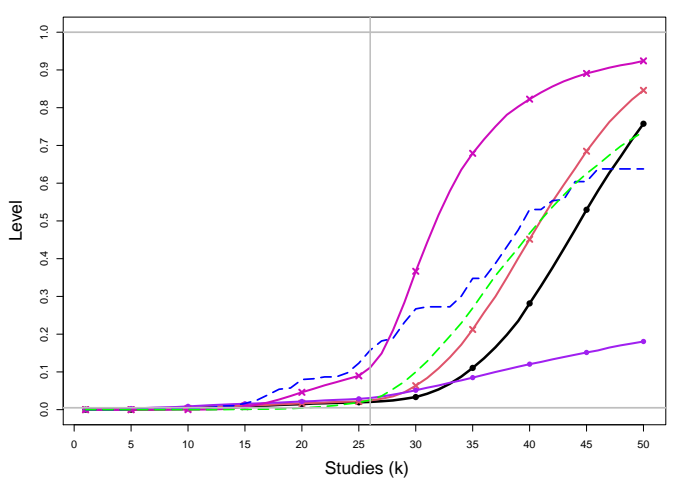

SSW KDB: $\tau^{2}=0.25 \mathrm{n}=50$ level $=0.005 \mathrm{~h}=6 \delta=1$ to 0.5 (chg at 26th)

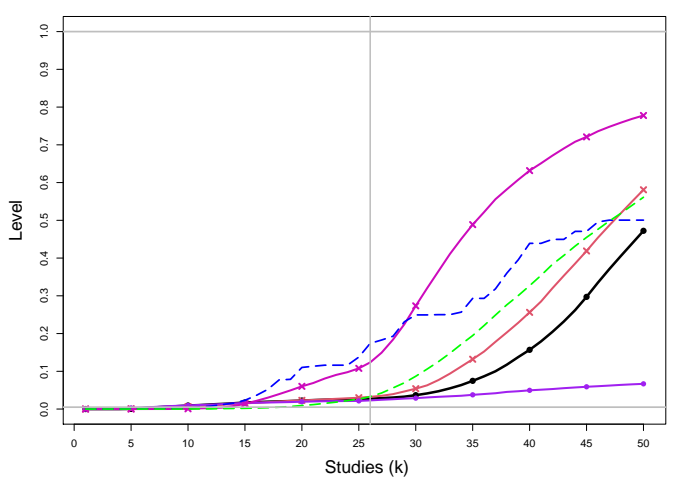

SSW KDB: $\tau^{2}=1 \mathrm{n}=50$ level $=0.005 \mathrm{~h}=6 \delta=1$ to 0.5 (chg at 26th)

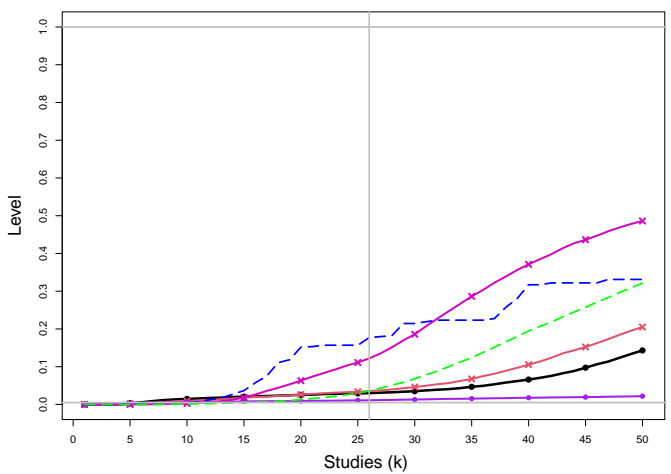

Figure E2. 82: Empirical levels of 1- and 2-stage CMA tests for shift in $\delta$ at .005 nominal level, shift in $\tau^{2}$ at .0025 nominal levels and of CUSUM with $h=4$ for equal sample sizes $n_{i C}+n_{i T}=n=50, \tau^{2}=0,0.1,0.25,1$ and a shift from $\delta=1$ to $\delta=0.5$ at study number 26. Light grey line at .005. 
IV REML: $\tau^{2}=0 \mathrm{n}=100$ level $=0.005 \mathrm{~h}=6 \delta=1$ to 0.5 (chg at 26 th)

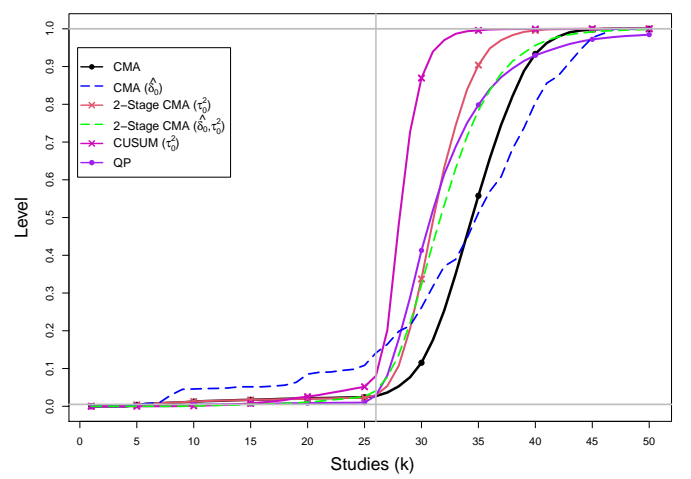

IV REML: $\tau^{2}=0.1 \mathrm{n}=100$ level $=0.005 \mathrm{~h}=6 \delta=1$ to 0.5 (chg at 26 th)

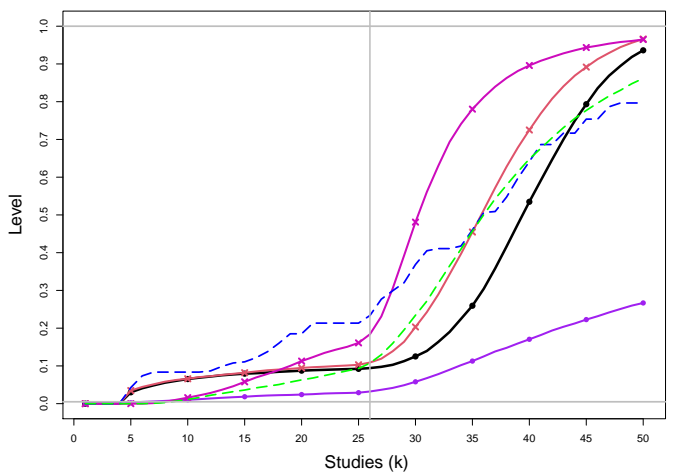

IV REML: $\tau^{2}=0.25 \mathrm{n}=100$ level $=0.005 \mathrm{~h}=6 \delta=1$ to 0.5 (chg at 26 th) SSW KDB: $\tau^{2}=0.25 \mathrm{n}=100$ level $=0.005 \mathrm{~h}=6 \quad \delta=1$ to 0.5 (chg at 26 th)

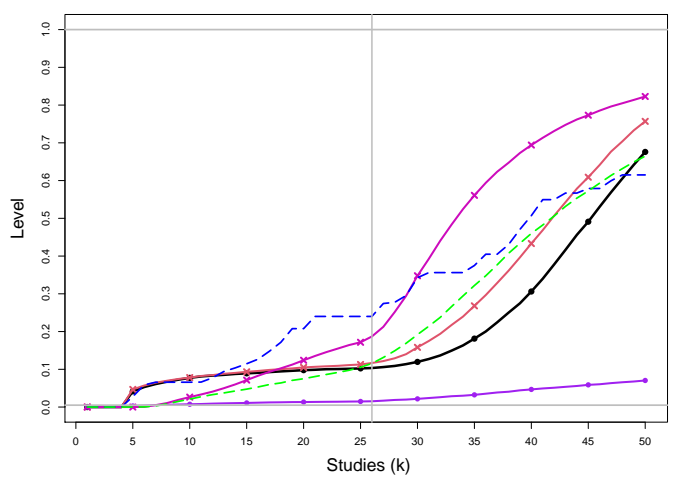

IV REML: $\tau^{2}=1 \mathrm{n}=100$ level $=0.005 \mathrm{~h}=6 \delta=1$ to 0.5 (chg at 26th)

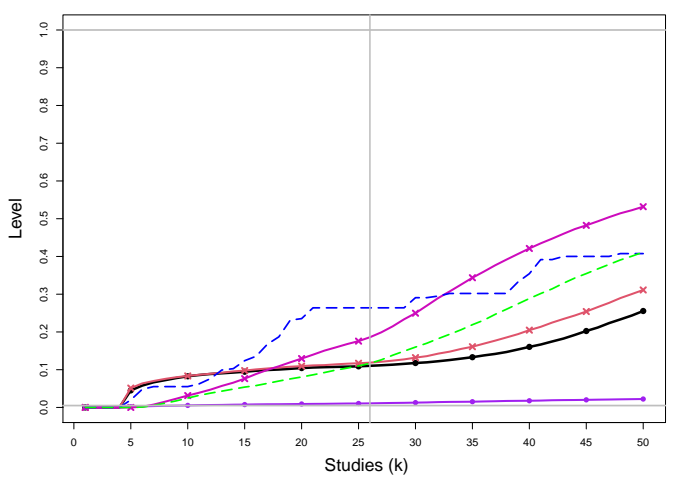

SSW KDB: $\tau^{2}=0 \mathrm{n}=100$ level $=0.005 \mathrm{~h}=6 \quad \delta=1$ to 0.5 (chg at 26 th)

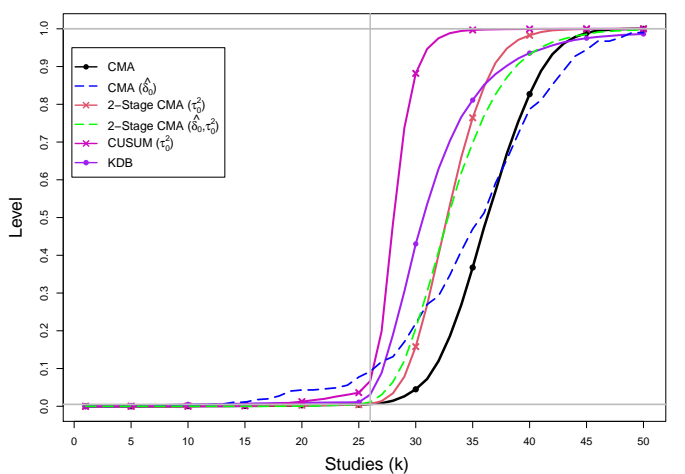

SSW KDB: $\tau^{2}=0.1 \mathrm{n}=100$ level $=0.005 \mathrm{~h}=6 \delta=1$ to 0.5 (chg at 26th)

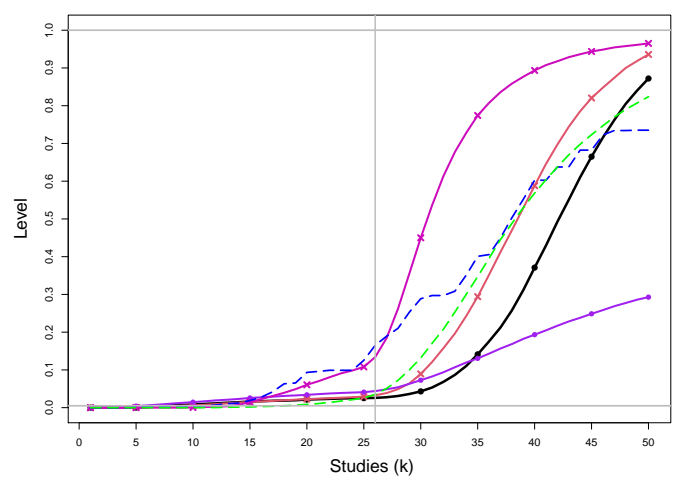

SSW KDB: $\tau^{2}=1 \mathrm{n}=100$ level $=0.005 \mathrm{~h}=6 \delta=1$ to 0.5 (chg at 26th)

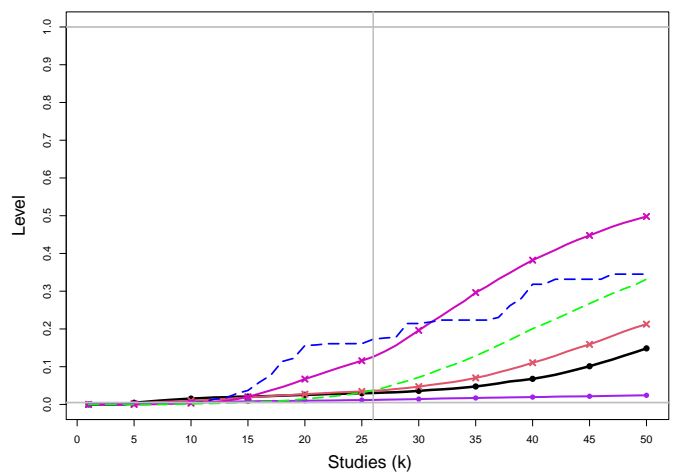

Figure E2. 83: Empirical levels of 1- and 2-stage CMA tests for shift in $\delta$ at .005 nominal level, shift in $\tau^{2}$ at .0025 nominal levels and of CUSUM with $h=4$ for equal sample sizes $n_{i C}+n_{i T}=n=100, \tau^{2}=0,0.1,0.25,1$ and a shift from $\delta=1$ to $\delta=0.5$ at study number 26. Light grey line at .005. 
IV REML: $\tau^{2}=0 \quad \mathrm{n}=500$ level $=0.005 \mathrm{~h}=6 \quad \delta=1$ to 0.5 (chg at 26 th)

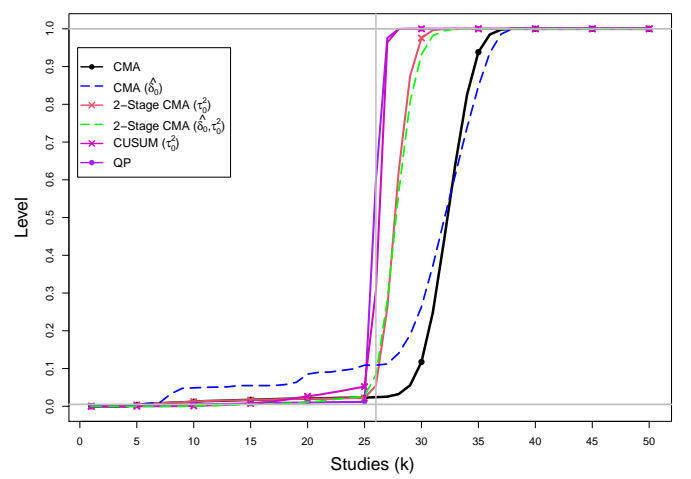

IV REML: $\tau^{2}=0.1 \mathrm{n}=500$ level $=0.005 \mathrm{~h}=6 \delta=1$ to 0.5 (chg at 26th)

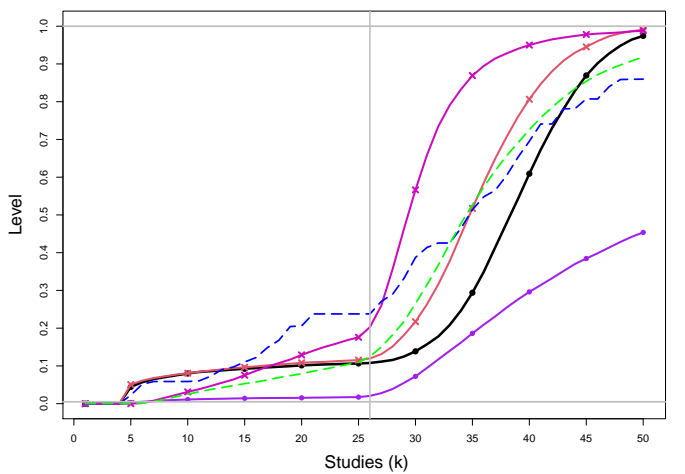

IV REML: $\tau^{2}=0.25 \mathrm{n}=500$ level $=0.005 \mathrm{~h}=6 \delta=1$ to 0.5 (chg at 26 th) SSW KDB: $\tau^{2}=0.25 \mathrm{n}=500$ level $=0.005 \mathrm{~h}=6 \delta=1$ to 0.5 (chg at 26 th)

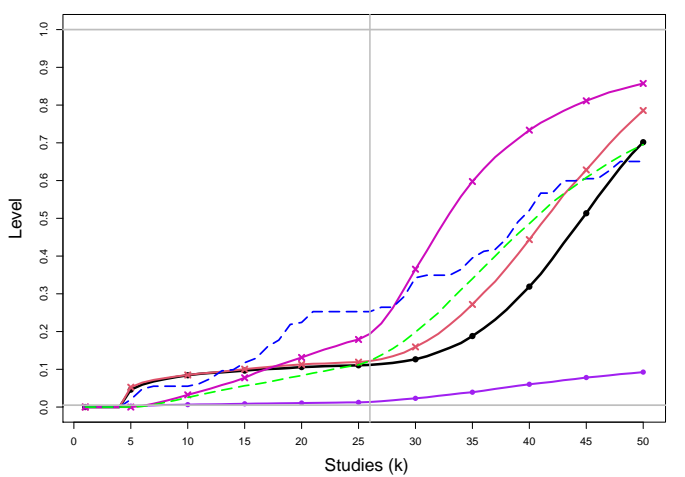

IV REML: $\tau^{2}=1 \mathrm{n}=500$ level $=0.005 \mathrm{~h}=6 \delta=1$ to 0.5 (chg at 26th)

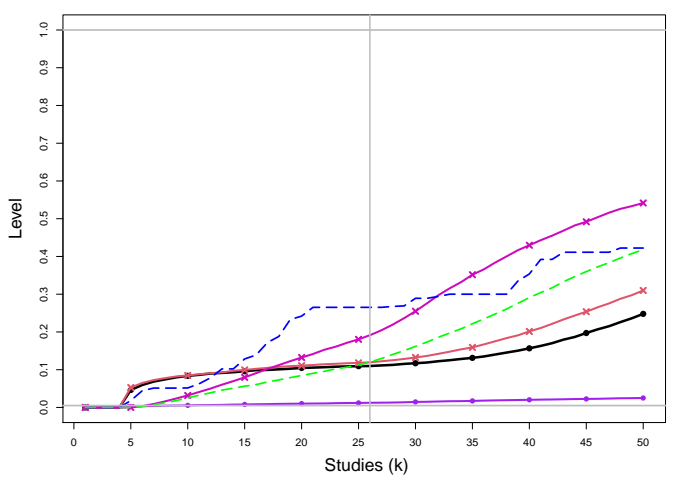

SSW KDB: $\tau^{2}=0 \mathrm{n}=500$ level $=0.005 \mathrm{~h}=6 \quad \delta=1$ to 0.5 (chg at 26 th)

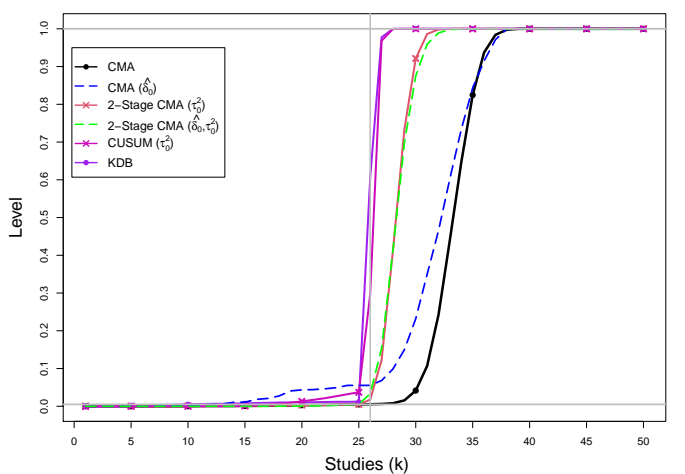

SSW KDB: $\tau^{2}=0.1 \mathrm{n}=500$ level $=0.005 \mathrm{~h}=6 \delta=1$ to 0.5 (chg at 26th)

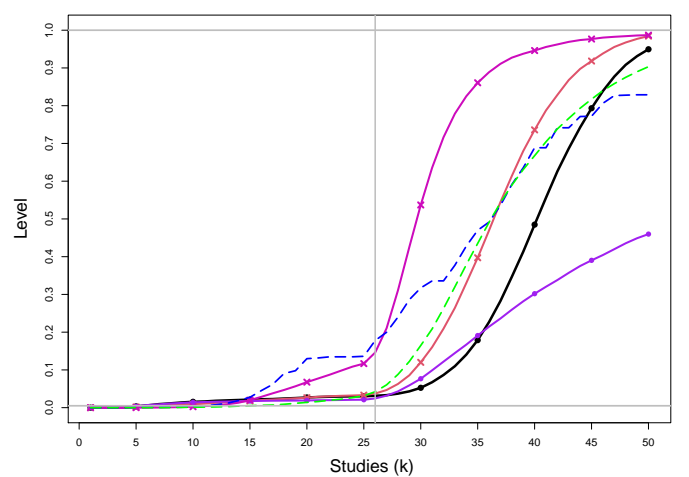

SSW KDB: $\tau^{2}=1 \mathrm{n}=500$ level $=0.005 \mathrm{~h}=6 \delta=1$ to 0.5 (chg at 26th)

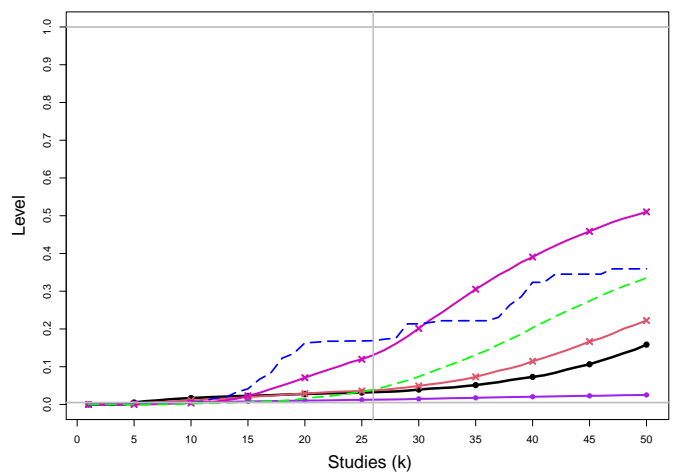

Figure E2. 84: Empirical levels of 1- and 2-stage CMA tests for shift in $\delta$ at .005 nominal level, shift in $\tau^{2}$ at .0025 nominal levels and of CUSUM with $h=4$ for equal sample sizes $n_{i C}+n_{i T}=n=500, \tau^{2}=0,0.1,0.25,1$ and a shift from $\delta=1$ to $\delta=0.5$ at study number 26. Light grey line at .005. 
IV REML: $\tau^{2}=0 \mathrm{n}=1000$ level $=0.005 \mathrm{~h}=6 \delta=1$ to 0.5 (chg at 26 th)

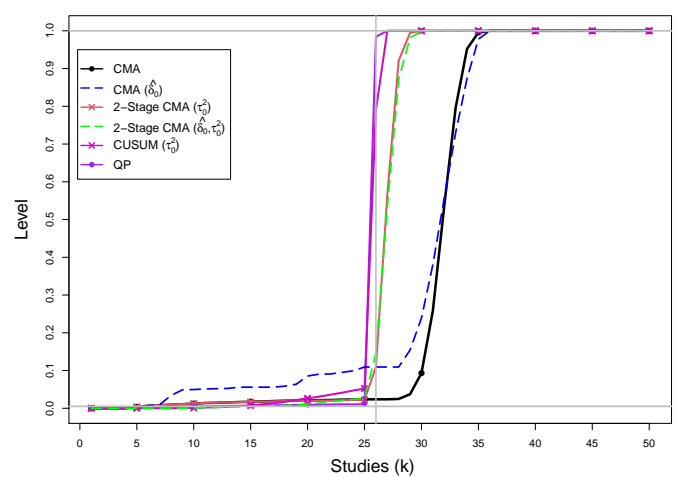

IV REML: $\tau^{2}=0.1 \mathrm{n}=1000$ level $=0.005 \mathrm{~h}=6 \delta=1$ to 0.5 (chg at 26th) SSW KDB: $\tau^{2}=0.1 \mathrm{n}=1000$ level $=0.005 \mathrm{~h}=6 \delta=1$ to 0.5 (chg at 26th)

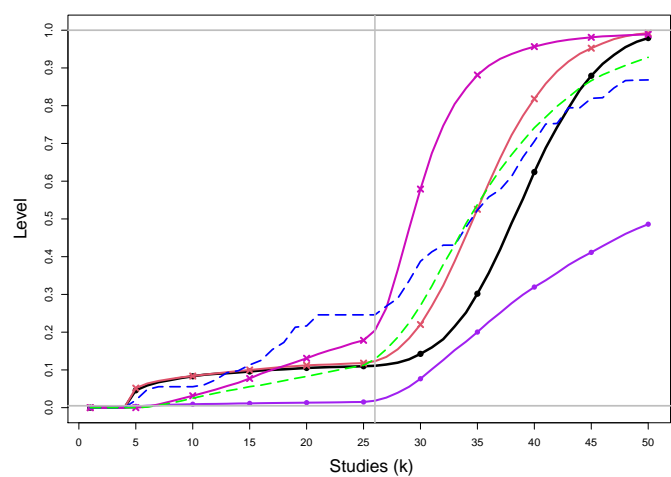

IV REML: $\tau^{2}=0.25 \mathrm{n}=1000$ level $=0.005 \mathrm{~h}=6 \quad \delta=1$ to 0.5 (chg at 26th) SSW KDB: $\tau^{2}=0.25 \mathrm{n}=1000$ level $=0.005 \mathrm{~h}=6 \quad \delta=1$ to 0.5 (chg at 26 th)

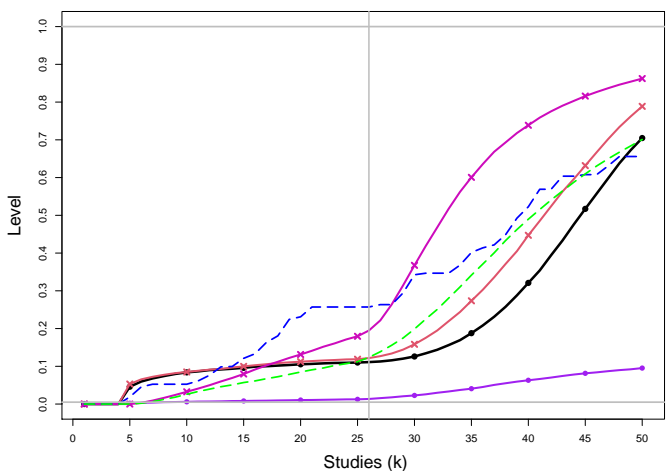

IV REML: $\tau^{2}=1 \mathrm{n}=1000$ level $=0.005 \mathrm{~h}=6 \delta=1$ to 0.5 (chg at 26 th)

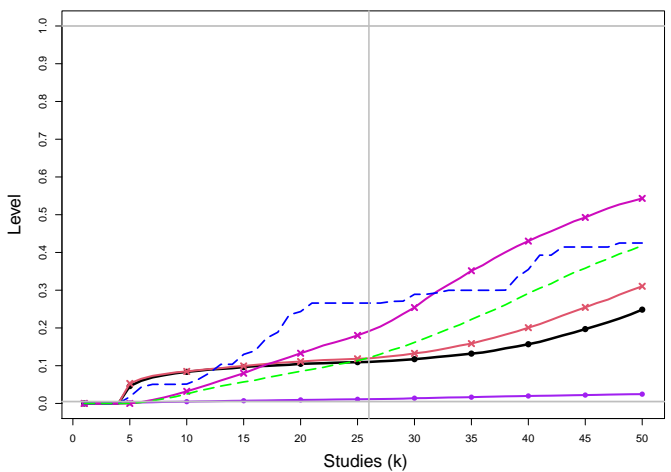

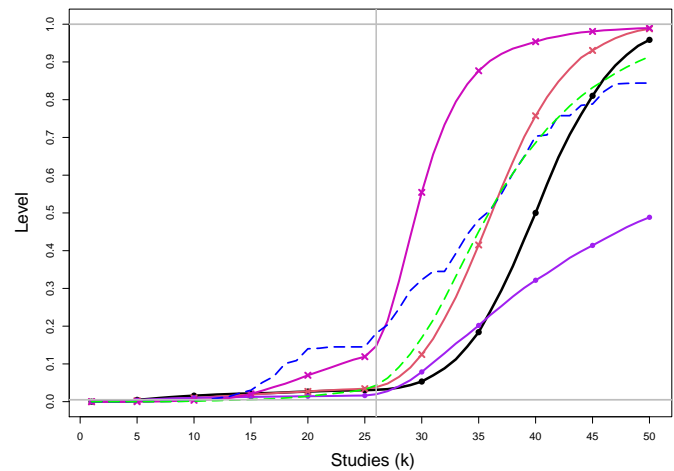

SSW KDB: $\tau^{2}=0 \mathrm{n}=1000$ level $=0.005 \mathrm{~h}=6 \delta=1$ to 0.5 (chg at 26 th)
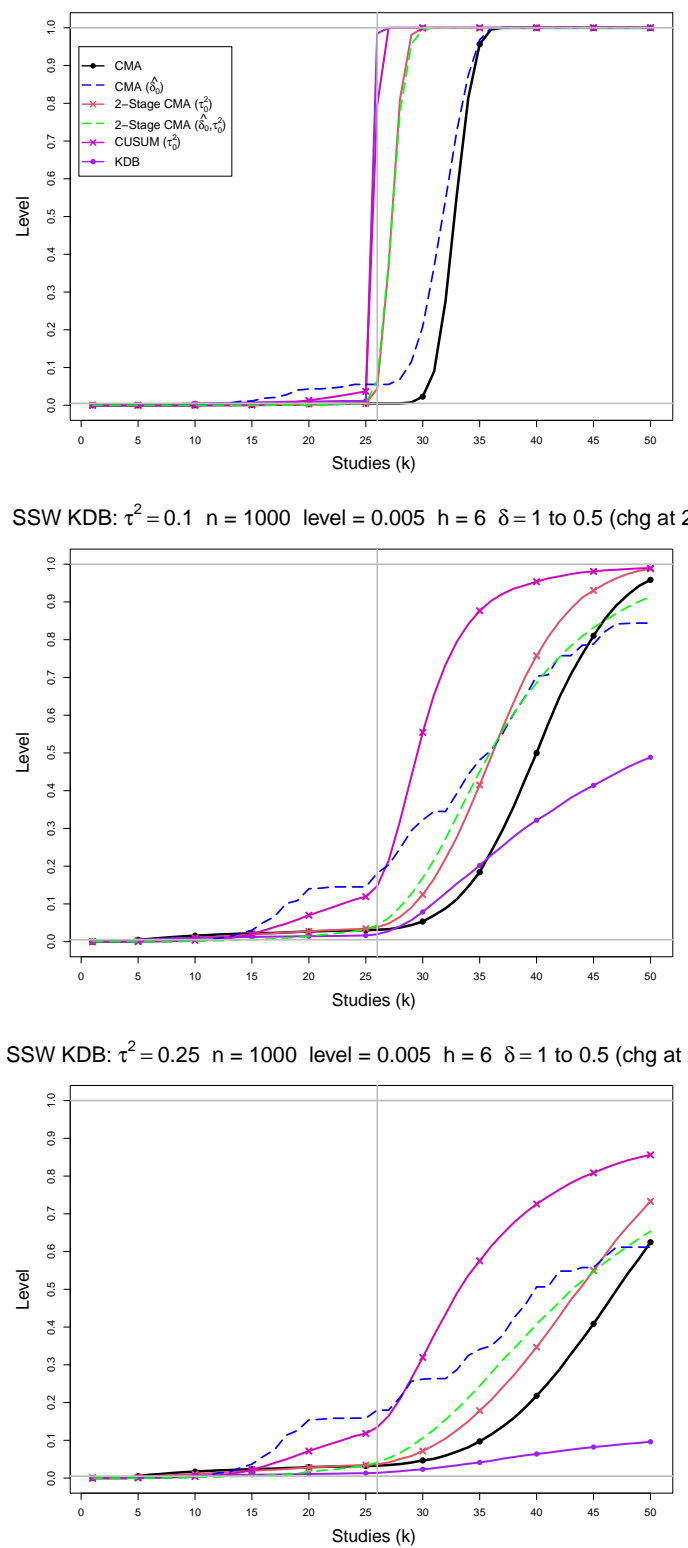

SSW KDB: $\tau^{2}=1 \mathrm{n}=1000$ level $=0.005 \mathrm{~h}=6 \quad \delta=1$ to 0.5 (chg at 26 th)

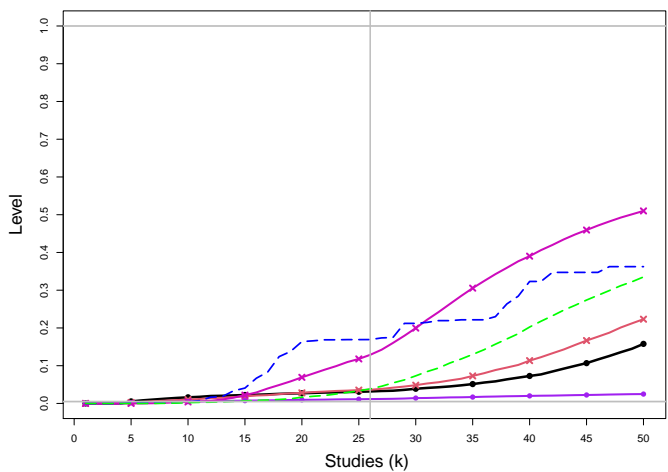

Figure E2. 85: Empirical levels of 1- and 2-stage CMA tests for shift in $\delta$ at .005 nominal level, shift in $\tau^{2}$ at .0025 nominal levels and of CUSUM with $h=4$ for equal sample sizes $n_{i C}+n_{i T}=n=1000, \tau^{2}=0,0.1,0.25,1$ and a shift from $\delta=1$ to $\delta=0.5$ at study number 26. Light grey line at .005. 
IV REML: $\tau^{2}=0 \mathrm{n}=20$ level $=0.005 \mathrm{~h}=6 \quad \delta=1$ to 1.5 (chg at 26th)

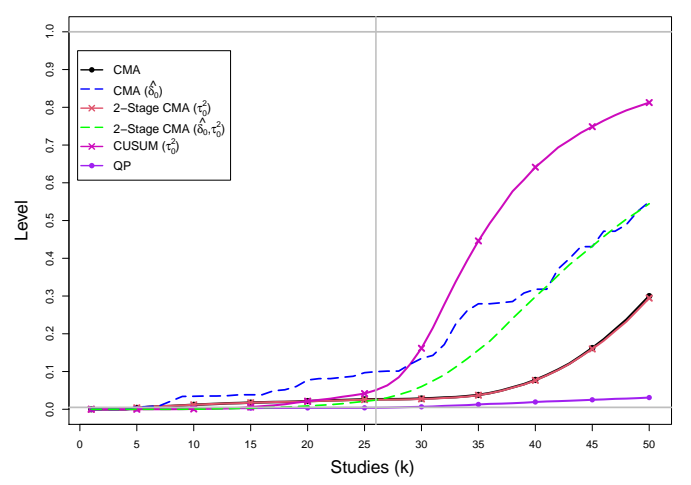

IV REML: $\tau^{2}=0.1 \mathrm{n}=20$ level $=0.005 \mathrm{~h}=6 \delta=1$ to 1.5 (chg at 26th)

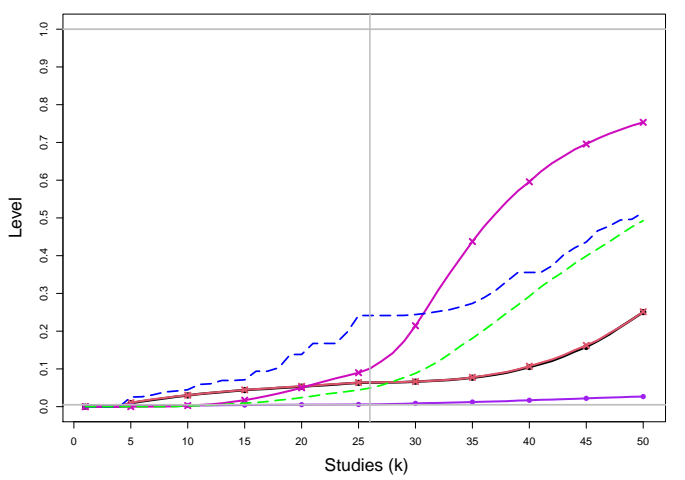

IV REML: $\tau^{2}=0.25 \mathrm{n}=20$ level $=0.005 \mathrm{~h}=6 \delta=1$ to 1.5 (chg at 26th)

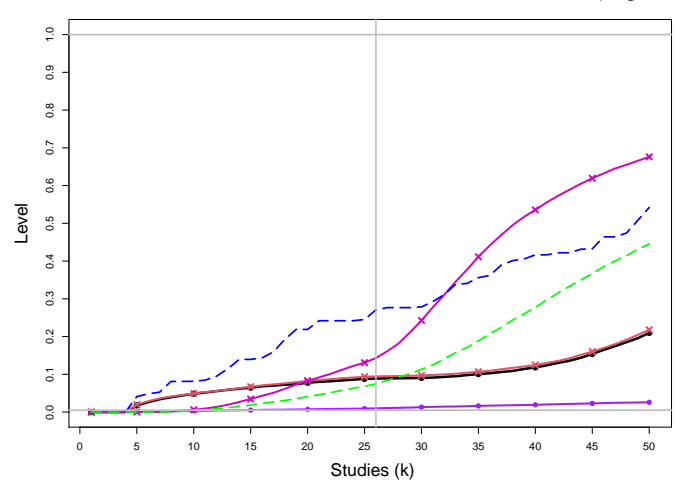

IV REML: $\tau^{2}=1 \mathrm{n}=20$ level $=0.005 \mathrm{~h}=6 \delta=1$ to 1.5 (chg at 26th)

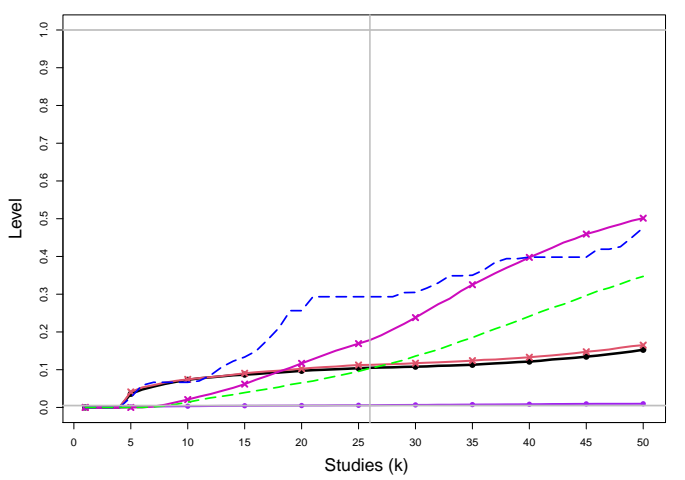

SSW KDB: $\tau^{2}=0 \mathrm{n}=20$ level $=0.005 \mathrm{~h}=6 \delta=1$ to 1.5 (chg at 26 th)

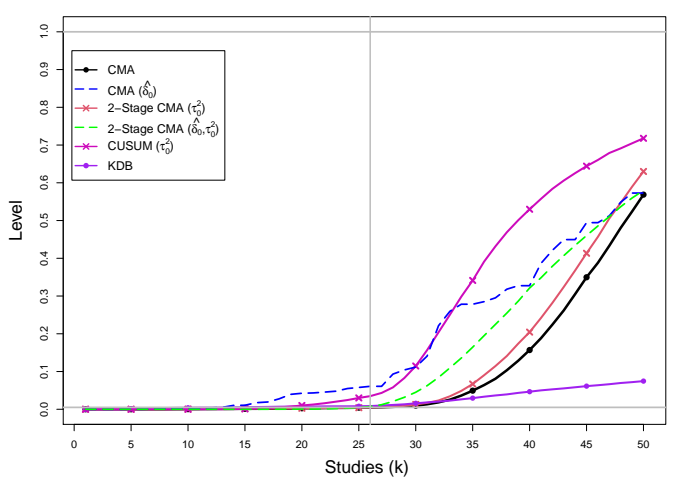

SSW KDB: $\tau^{2}=0.1 \mathrm{n}=20$ level $=0.005 \mathrm{~h}=6 \delta=1$ to 1.5 (chg at 26th)

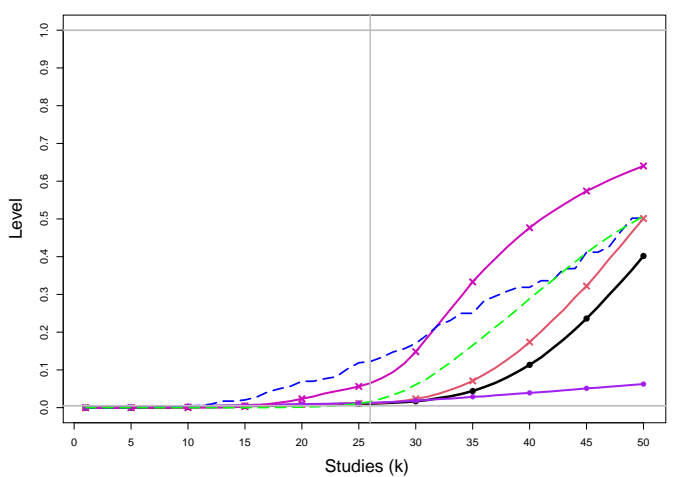

SSW KDB: $\tau^{2}=0.25 \mathrm{n}=20$ level $=0.005 \mathrm{~h}=6 \delta=1$ to 1.5 (chg at 26th)

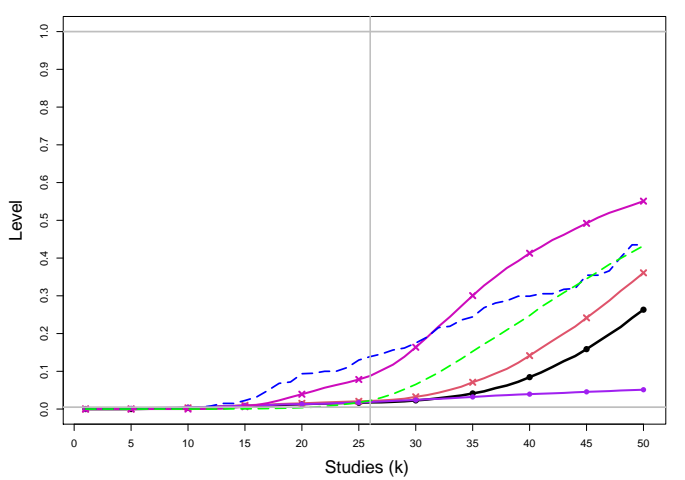

SSW KDB: $\tau^{2}=1 \mathrm{n}=20$ level $=0.005 \mathrm{~h}=6 \delta=1$ to 1.5 (chg at 26th)

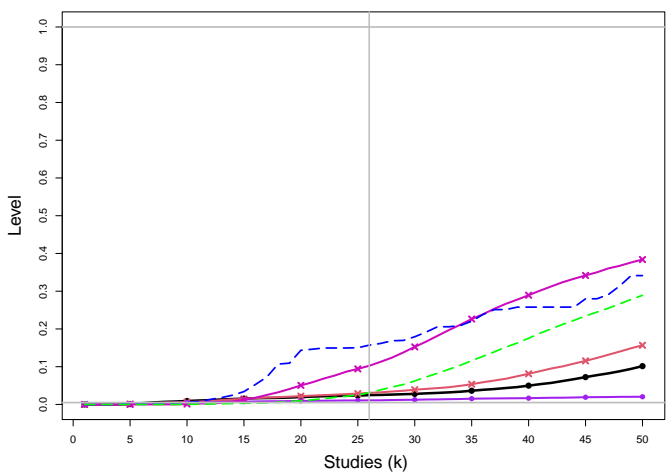

Figure E2. 86: Empirical levels of 1- and 2-stage CMA tests for shift in $\delta$ at .005 nominal level, shift in $\tau^{2}$ at .0025 nominal levels and of CUSUM with $h=4$ for equal sample sizes $n_{i C}+n_{i T}=n=20, \tau^{2}=0,0.1,0.25,1$ and a shift from $\delta=1$ to $\delta=1.5$ at study number 26. Light grey line at .005. 
IV REML: $\tau^{2}=0 \mathrm{n}=50$ level $=0.005 \mathrm{~h}=6 \delta=1$ to 1.5 (chg at 26th)

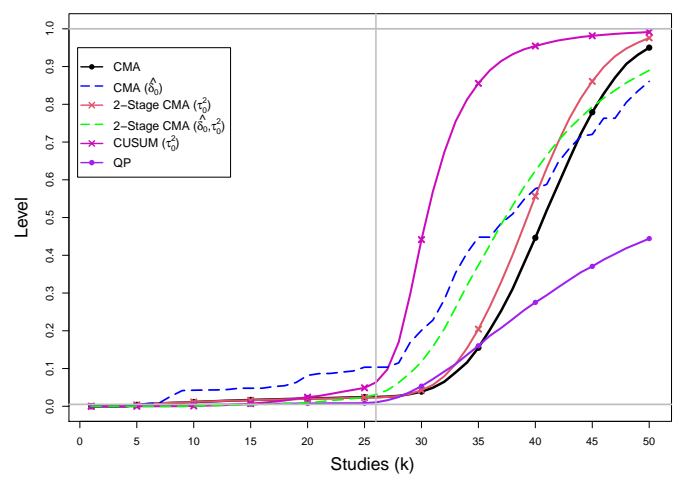

IV REML: $\tau^{2}=0.1 \mathrm{n}=50$ level $=0.005 \mathrm{~h}=6 \delta=1$ to 1.5 (chg at 26 th)

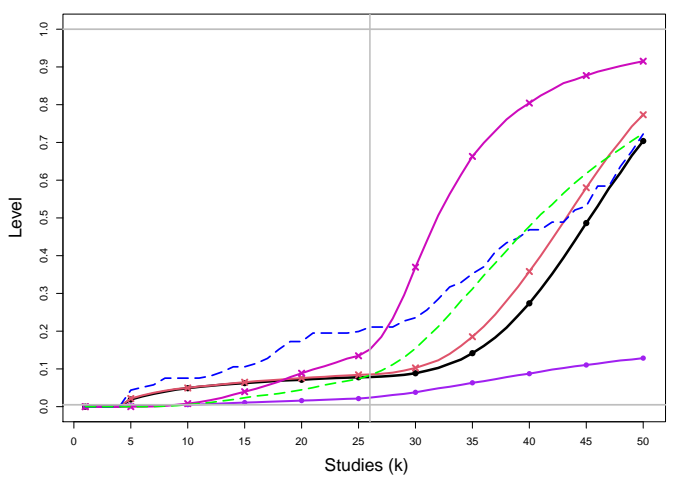

IV REML: $\tau^{2}=0.25 \mathrm{n}=50$ level $=0.005 \mathrm{~h}=6 \delta=1$ to 1.5 (chg at 26th)

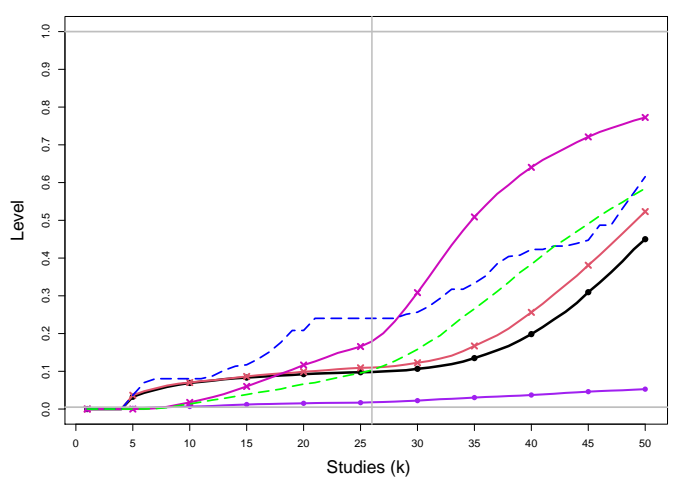

IV REML: $\tau^{2}=1 \mathrm{n}=50$ level $=0.005 \mathrm{~h}=6 \delta=1$ to 1.5 (chg at 26th)

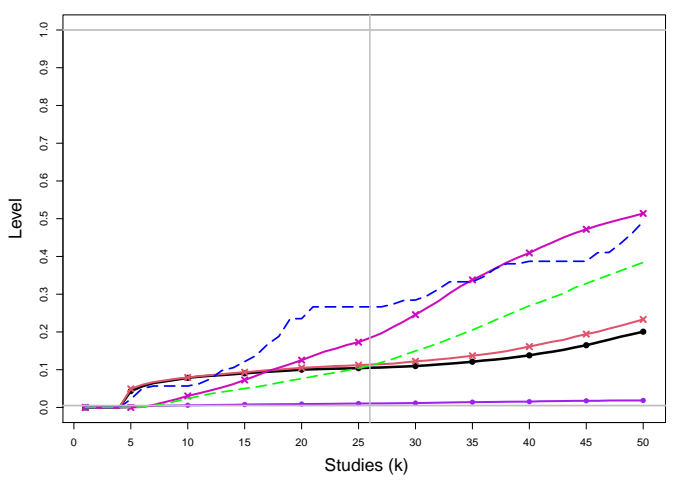

SSW KDB: $\tau^{2}=0 \mathrm{n}=50$ level $=0.005 \mathrm{~h}=6 \delta=1$ to 1.5 (chg at 26th)

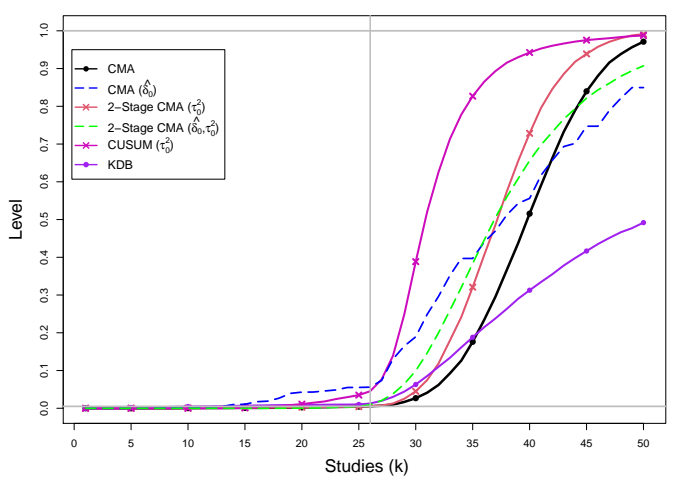

SSW KDB: $\tau^{2}=0.1 \mathrm{n}=50$ level $=0.005 \mathrm{~h}=6 \delta=1$ to 1.5 (chg at 26th)

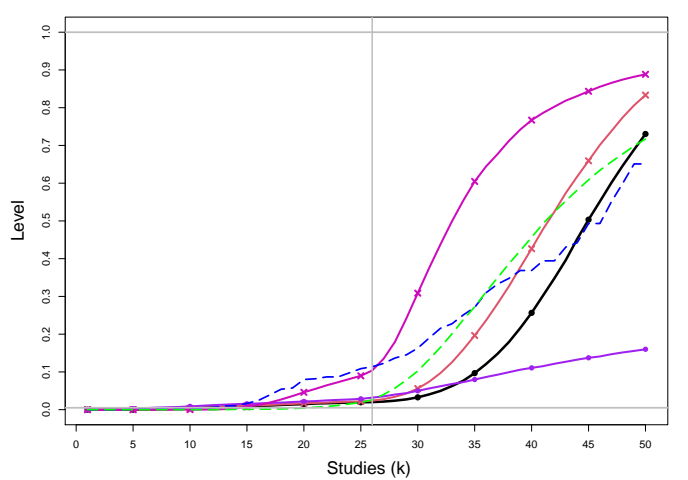

SSW KDB: $\tau^{2}=0.25 \mathrm{n}=50$ level $=0.005 \mathrm{~h}=6 \delta=1$ to 1.5 (chg at 26th)

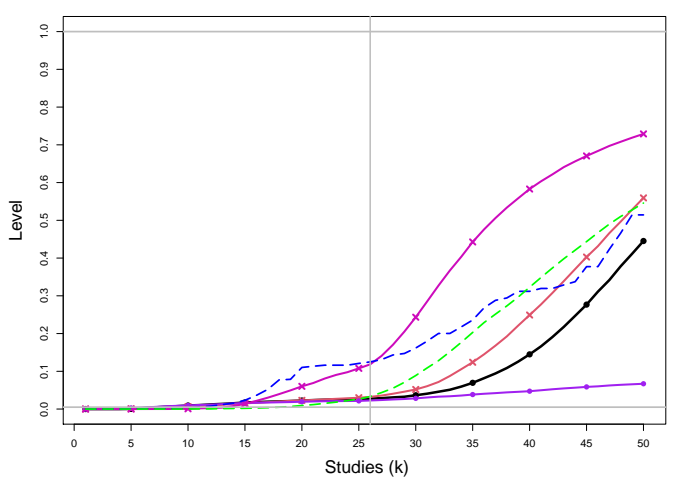

SSW KDB: $\tau^{2}=1 \mathrm{n}=50$ level $=0.005 \mathrm{~h}=6 \delta=1$ to 1.5 (chg at 26th)

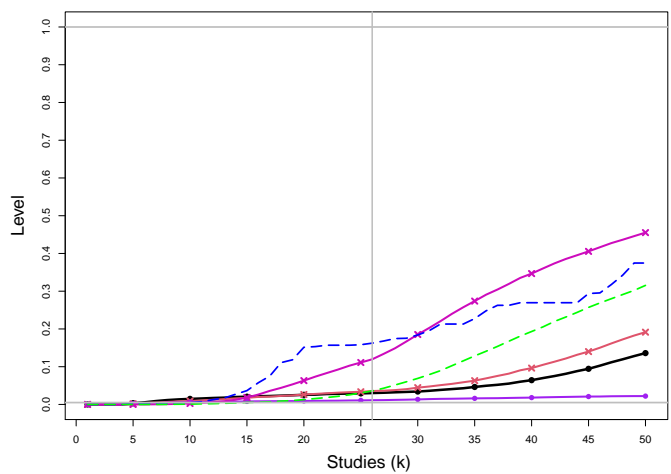

Figure E2. 87: Empirical levels of 1- and 2-stage CMA tests for shift in $\delta$ at .005 nominal level, shift in $\tau^{2}$ at .0025 nominal levels and of CUSUM with $h=4$ for equal sample sizes $n_{i C}+n_{i T}=n=50, \tau^{2}=0,0.1,0.25,1$ and a shift from $\delta=1$ to $\delta=1.5$ at study number 26. Light grey line at .005. 
IV REML: $\tau^{2}=0 \mathrm{n}=100$ level $=0.005 \mathrm{~h}=6 \delta=1$ to 1.5 (chg at 26 th)

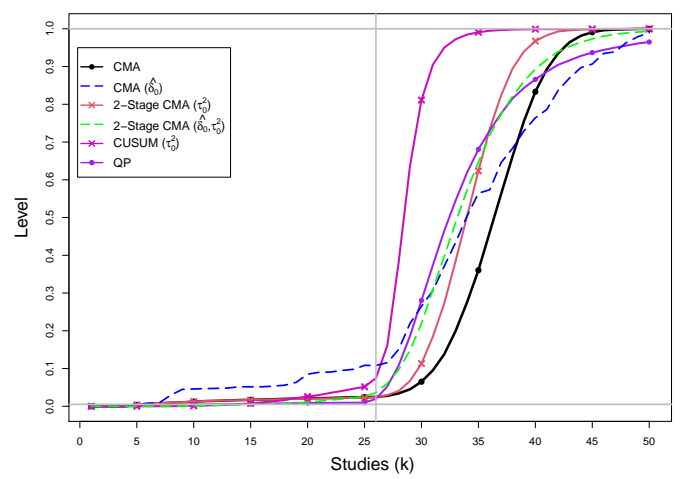

IV REML: $\tau^{2}=0.1 \mathrm{n}=100$ level $=0.005 \mathrm{~h}=6 \delta=1$ to 1.5 (chg at 26 th)

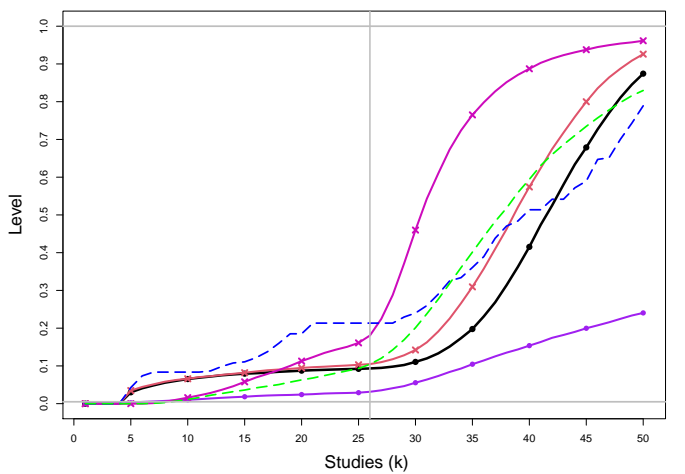

IV REML: $\tau^{2}=0.25 \mathrm{n}=100$ level $=0.005 \mathrm{~h}=6 \delta=1$ to 1.5 (chg at 26 th) SSW KDB: $\tau^{2}=0.25 \mathrm{n}=100$ level $=0.005 \mathrm{~h}=6 \quad \delta=1$ to 1.5 (chg at 26 th)

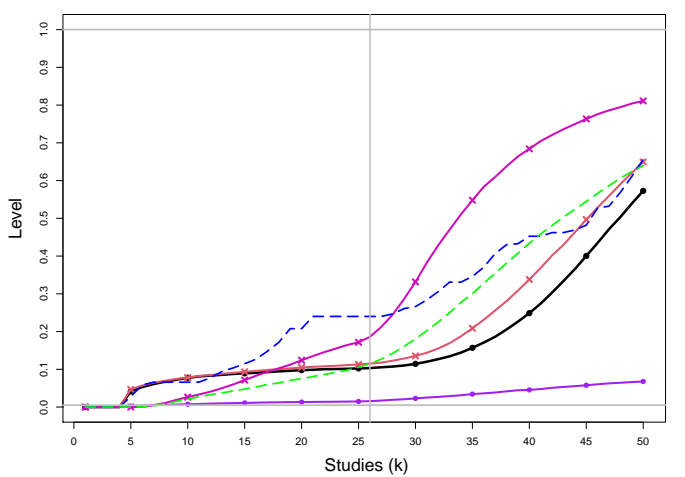

IV REML: $\tau^{2}=1 \mathrm{n}=100$ level $=0.005 \mathrm{~h}=6 \delta=1$ to 1.5 (chg at 26 th)

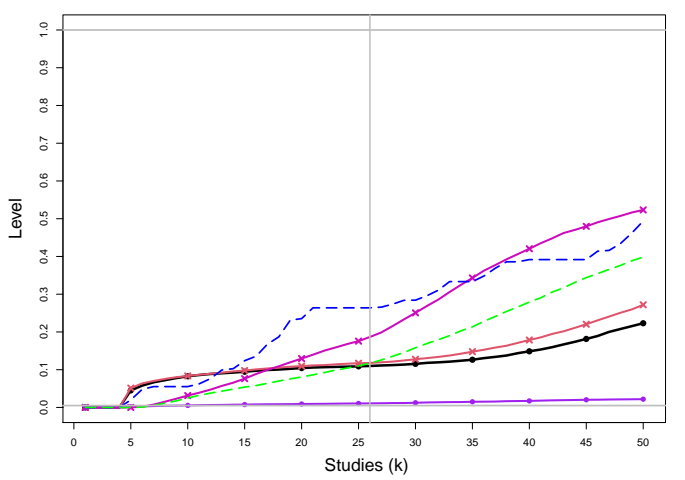

SSW KDB: $\tau^{2}=0 \mathrm{n}=100$ level $=0.005 \mathrm{~h}=6 \quad \delta=1$ to 1.5 (chg at 26 th)

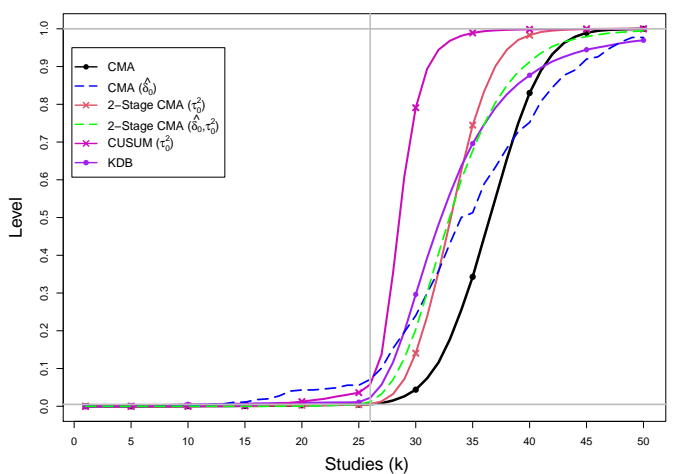

SSW KDB: $\tau^{2}=0.1 \mathrm{n}=100$ level $=0.005 \mathrm{~h}=6 \delta=1$ to 1.5 (chg at 26th)

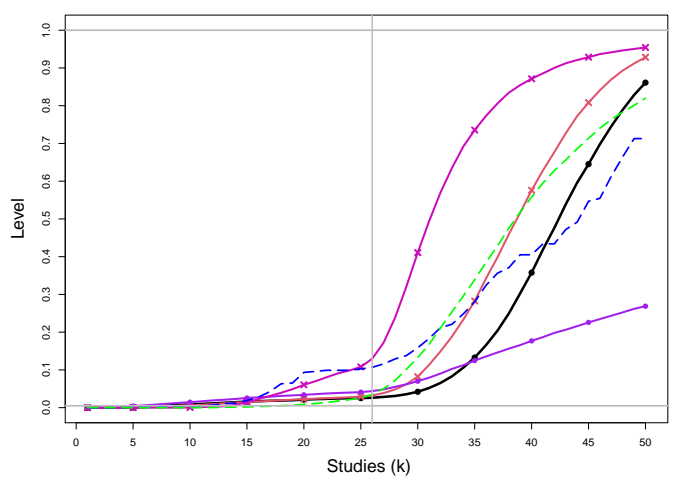

SSW KDB: $\tau^{2}=1 \mathrm{n}=100$ level $=0.005 \mathrm{~h}=6 \delta=1$ to 1.5 (chg at 26th)

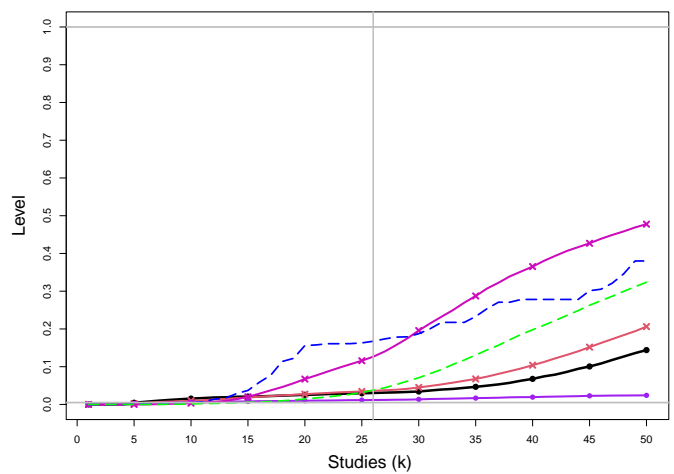

Figure E2. 88: Empirical levels of 1- and 2-stage CMA tests for shift in $\delta$ at .005 nominal level, shift in $\tau^{2}$ at .0025 nominal levels and of CUSUM with $h=4$ for equal sample sizes $n_{i C}+n_{i T}=n=100, \tau^{2}=0,0.1,0.25,1$ and a shift from $\delta=1$ to $\delta=1.5$ at study number 26. Light grey line at .005. 
IV REML: $\tau^{2}=0 \mathrm{n}=500$ level $=0.005 \mathrm{~h}=6 \delta=1$ to 1.5 (chg at 26 th)

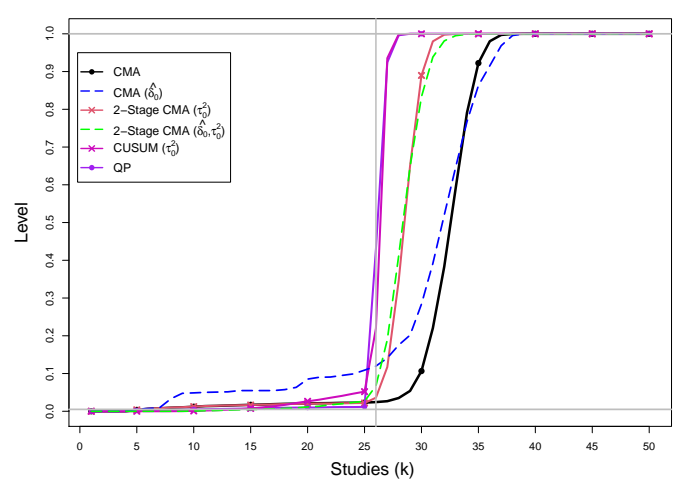

IV REML: $\tau^{2}=0.1 \mathrm{n}=500$ level $=0.005 \mathrm{~h}=6 \delta=1$ to 1.5 (chg at 26th)

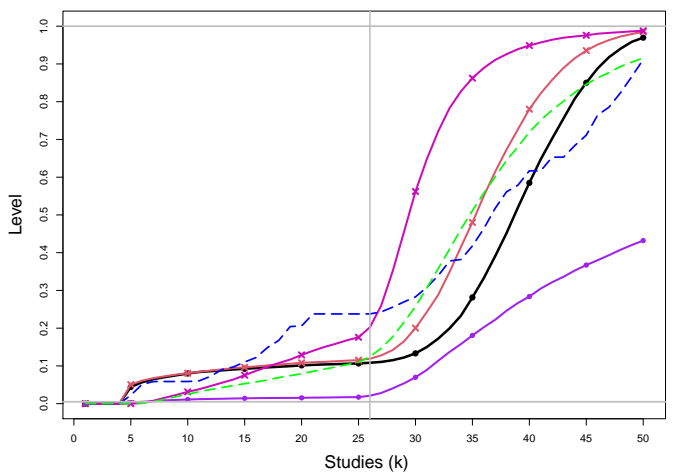

IV REML: $\tau^{2}=0.25 \mathrm{n}=500$ level $=0.005 \mathrm{~h}=6 \delta=1$ to 1.5 (chg at 26 th) SSW KDB: $\tau^{2}=0.25 \mathrm{n}=500$ level $=0.005 \mathrm{~h}=6 \quad \delta=1$ to 1.5 (chg at 26 th)

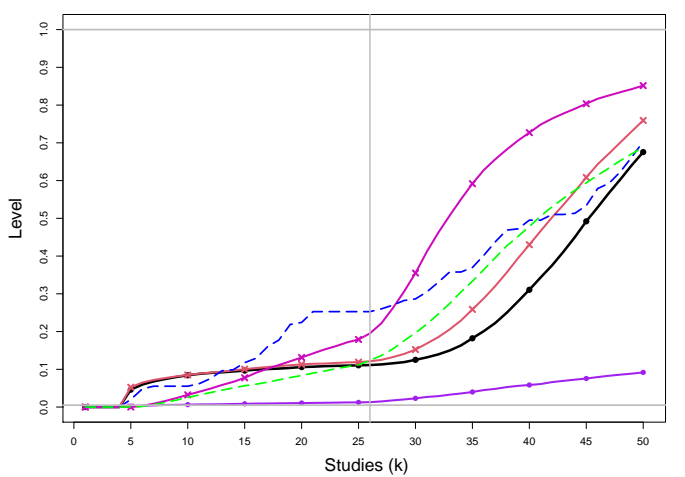

IV REML: $\tau^{2}=1 \mathrm{n}=500$ level $=0.005 \mathrm{~h}=6 \delta=1$ to 1.5 (chg at 26th)

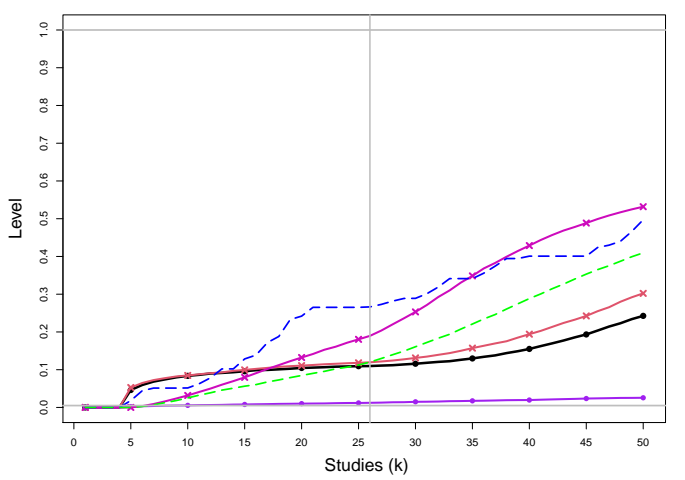

SSW KDB: $\tau^{2}=0 \mathrm{n}=500$ level $=0.005 \mathrm{~h}=6 \quad \delta=1$ to 1.5 (chg at 26 th)

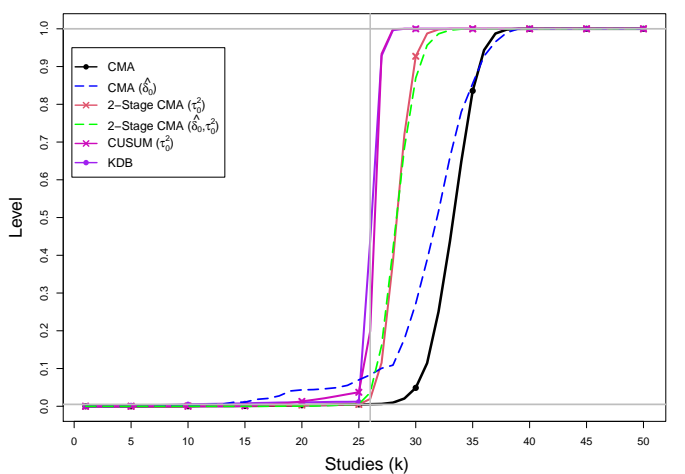

SSW KDB: $\tau^{2}=0.1 \mathrm{n}=500$ level $=0.005 \mathrm{~h}=6 \delta=1$ to 1.5 (chg at 26th)

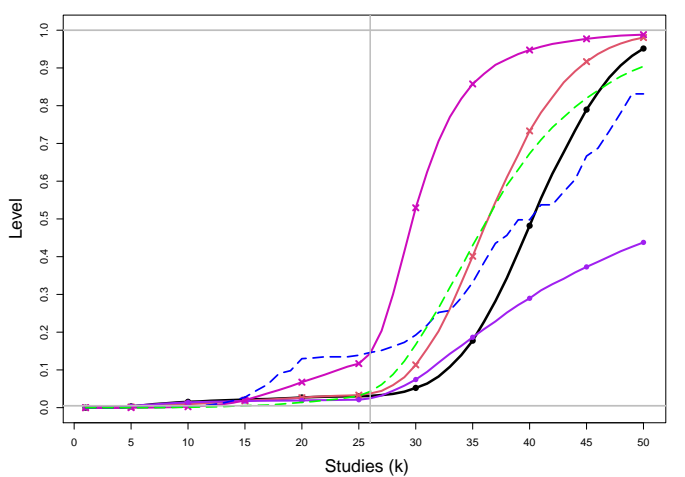

SSW KDB: $\tau^{2}=1 \mathrm{n}=500$ level $=0.005 \mathrm{~h}=6 \delta=1$ to 1.5 (chg at 26th)

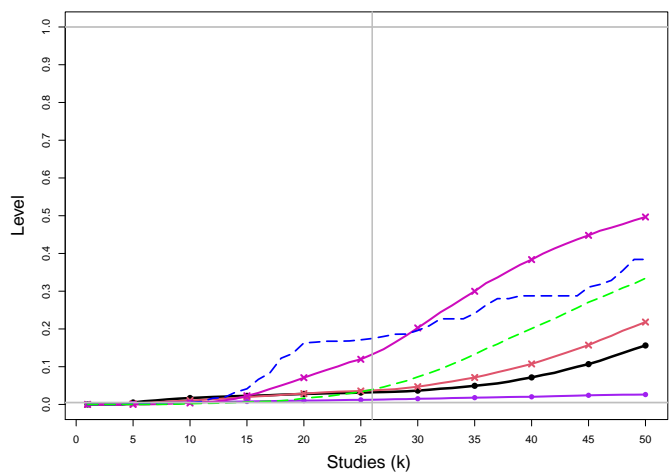

Figure E2. 89: Empirical levels of 1- and 2-stage CMA tests for shift in $\delta$ at .005 nominal level, shift in $\tau^{2}$ at .0025 nominal levels and of CUSUM with $h=4$ for equal sample sizes $n_{i C}+n_{i T}=n=500, \tau^{2}=0,0.1,0.25,1$ and a shift from $\delta=1$ to $\delta=1.5$ at study number 26. Light grey line at .005. 
IV REML: $\tau^{2}=0 \mathrm{n}=1000$ level $=0.005 \mathrm{~h}=6 \delta=1$ to 1.5 (chg at 26 th)

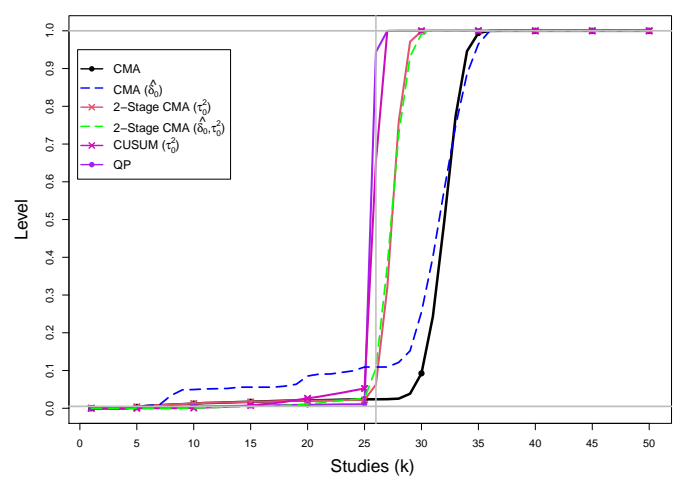

IV REML: $\tau^{2}=0.1 \mathrm{n}=1000$ level $=0.005 \mathrm{~h}=6 \delta=1$ to 1.5 (chg at 26th) SSW KDB: $\tau^{2}=0.1 \mathrm{n}=1000$ level $=0.005 \mathrm{~h}=6 \delta=1$ to 1.5 (chg at 26th)

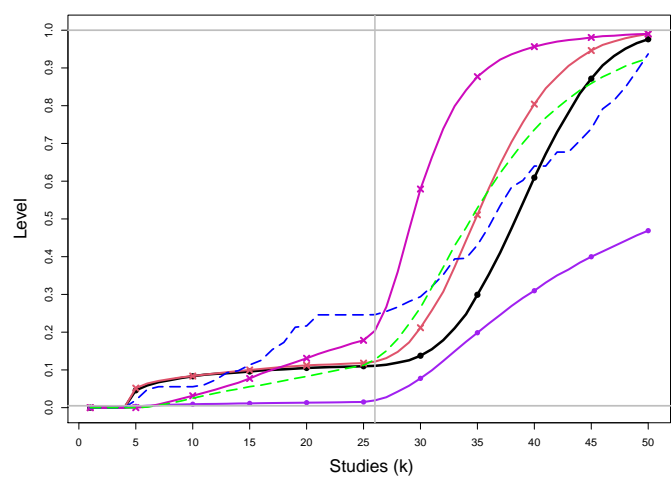

IV REML: $\tau^{2}=0.25 \mathrm{n}=1000$ level $=0.005 \mathrm{~h}=6 \quad \delta=1$ to 1.5 (chg at 26th) SSW KDB: $\tau^{2}=0.25 \mathrm{n}=1000$ level $=0.005 \mathrm{~h}=6 \quad \delta=1$ to 1.5 (chg at 26th)

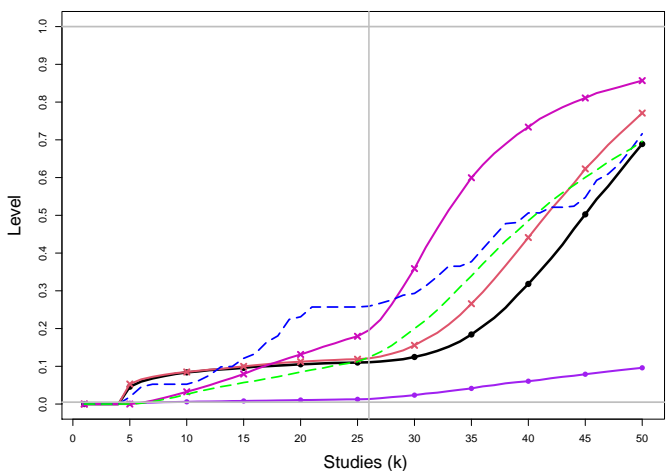

IV REML: $\tau^{2}=1 \mathrm{n}=1000$ level $=0.005 \mathrm{~h}=6 \delta=1$ to 1.5 (chg at 26 th)

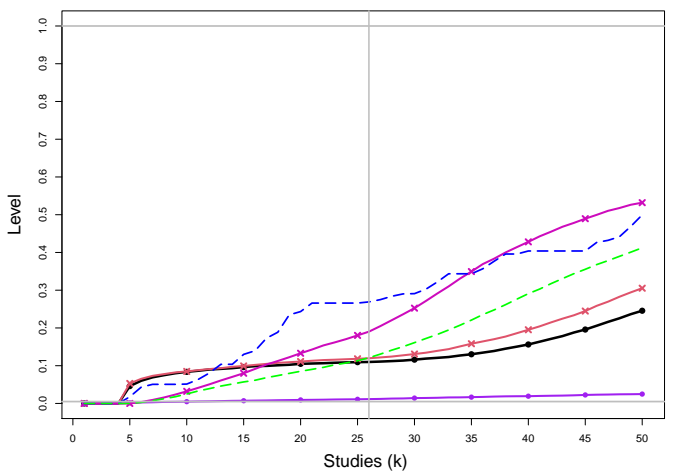

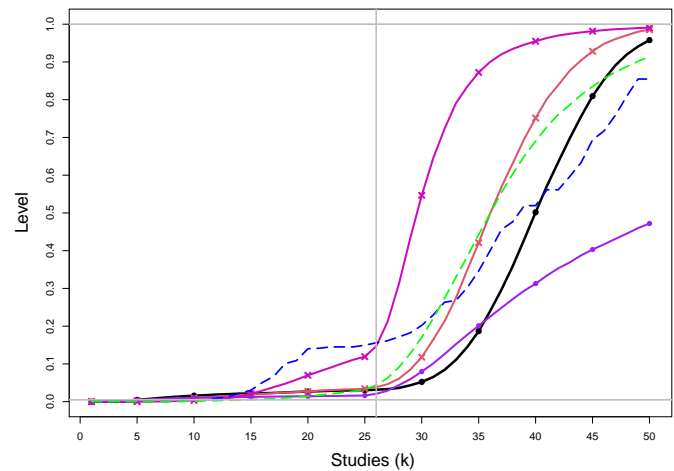

SSW KDB: $\tau^{2}=0 \mathrm{n}=1000$ level $=0.005 \mathrm{~h}=6 \delta=1$ to 1.5 (chg at 26 th)
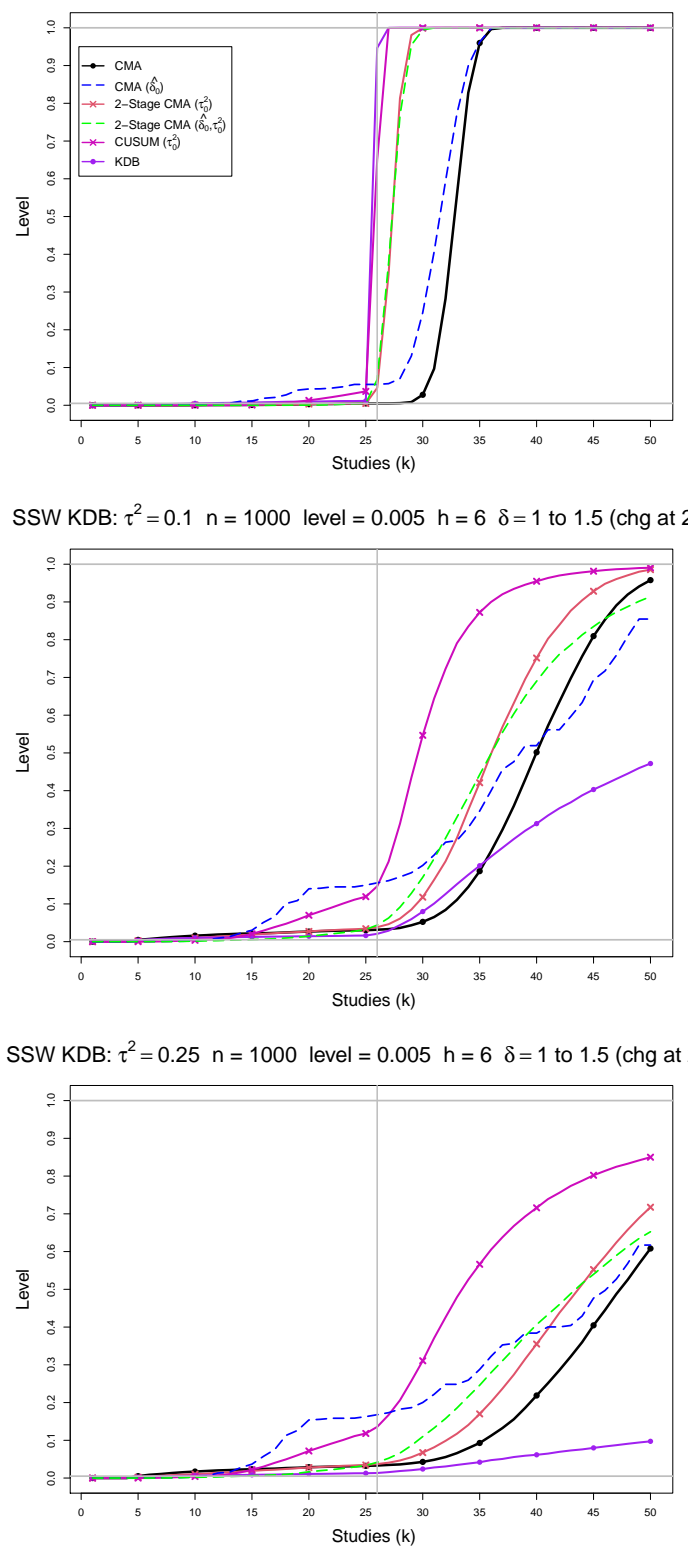

SSW KDB: $\tau^{2}=1 \mathrm{n}=1000$ level $=0.005 \mathrm{~h}=6 \delta=1$ to 1.5 (chg at 26 th)

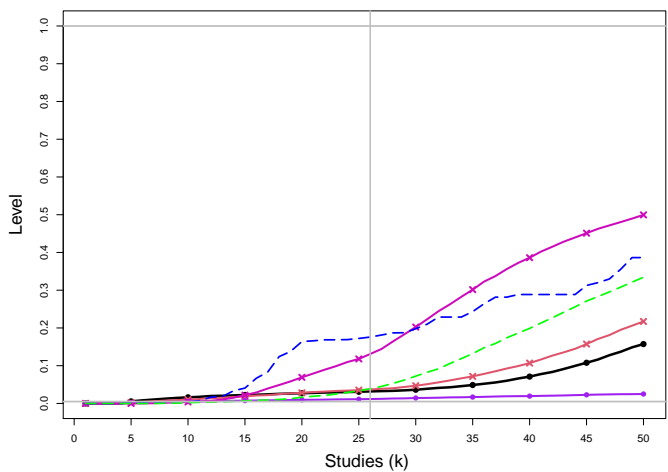

Figure E2. 90: Empirical levels of 1- and 2-stage CMA tests for shift in $\delta$ at .005 nominal level, shift in $\tau^{2}$ at .0025 nominal levels and of CUSUM with $h=4$ for equal sample sizes $n_{i C}+n_{i T}=n=1000, \tau^{2}=0,0.1,0.25,1$ and a shift from $\delta=1$ to $\delta=1.5$ at study number 26. Light grey line at .005. 